Portland State University

PDXScholar

7-16-2021

\title{
Field Trials and Long-Term Monitoring of Microbially- Induced Desaturation for the Treatment of Liquefiable Silty Soils
}

Kayla Rae Sorenson

Portland State University

Follow this and additional works at: https://pdxscholar.library.pdx.edu/open_access_etds

Part of the Civil Engineering Commons, and the Soil Science Commons Let us know how access to this document benefits you.

Recommended Citation

Sorenson, Kayla Rae, "Field Trials and Long-Term Monitoring of Microbially-Induced Desaturation for the Treatment of Liquefiable Silty Soils" (2021). Dissertations and Theses. Paper 5772.

https://doi.org/10.15760/etd.7643

This Thesis is brought to you for free and open access. It has been accepted for inclusion in Dissertations and Theses by an authorized administrator of PDXScholar. Please contact us if we can make this document more accessible: pdxscholar@pdx.edu. 
Field Trials and Long-Term Monitoring of Microbially-Induced Desaturation for the Treatment of Liquefiable Silty Soils

by

Kayla Rae Sorenson

A thesis submitted in partial fulfillment of the

requirements for the degree of

\author{
Master of Science \\ in \\ Civil and Environmental Engineering
}

Thesis Committee:

Arash Khosravifar, Chair

Diane Moug

Peter Dusicka

Portland State University

2021 
(C) 2021 Kayla Rae Sorenson 


\begin{abstract}
Earthquake liquefaction hazards in silty soils are a critical problem in Portland, Oregon, and other areas around the world. This is a particular problem for existing facilities founded on liquefiable soils, for which there exists no cost-effective liquefaction mitigation solution at the present time. It is essential from both a seismic safety and a seismic resilience perspective to be able to mitigate potentially liquefiable soils underneath existing structures, and to do so in a cost-effective manner. Recent studies suggest that liquefaction mitigation using microbially-induced desaturation (MID) may provide this capability.
\end{abstract}

The objective of MID is to reduce earthquake-induced excess pore water pressure generation compared to saturated soil, and thereby reduce the potential for triggering liquefaction. A field study of liquefaction mitigation using MID was performed at two sites in Portland in the summer of 2019. Low plasticity liquefiable silts were treated with MID by injecting a treatment solution to stimulate native bacteria for a duration of four weeks.

Monitoring at both sites indicated that the liquefiable soils were successfully desaturated. The persistence of desaturation was monitored for eight months post-treatment at one site and is ongoing at the other site (20 months so far). The monitoring data indicate that the induced desaturation has persisted through seasonal fluctuations of the ground water table since the end of treatment. These data, which document MID longevity, are particularly important to establish MID as a viable option for liquefaction mitigation for use not only in silty soils, but also underneath existing structures for which there is no other mitigation method available. 


\section{Acknowledgements}

I would like to express my gratitude to my advisor Dr. Arash Khosravifar. This journey started when you stopped, listened, and thought about the question I asked you, instead of giving a vague reply or dismissing it. You have continued to guide me and provided invaluable assistance throughout my undergraduate and graduate studies. Thank you.

I would also like to thank Dr. Diane Moug for her guidance and continued support, both inside and outside the classroom. Your encouragement, patience, and wisdom are behind many of the endeavors that I have achieved. Thank you.

My graduate studies would have been immensely more difficult were it not for the CEE staff. Your support, laughter, and friendship cannot be seen on the following pages, but without you there would be no following pages.

Finally, I would like to thank my family and my friends. Although you probably did not understand what I was talking about most of the time, you were there and you listened. I am only successful because of you. 


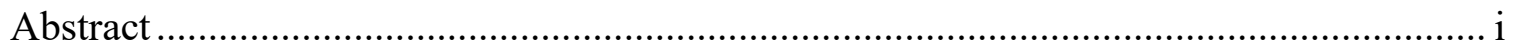

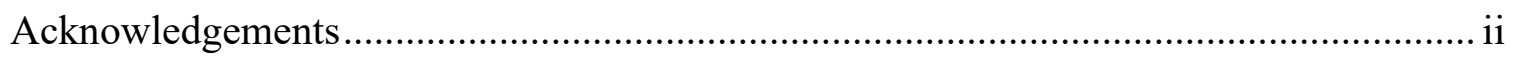

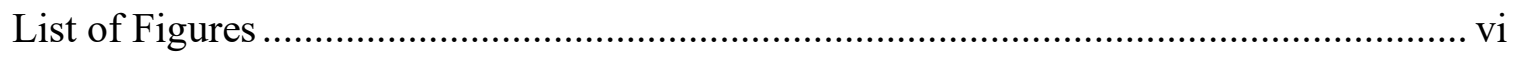

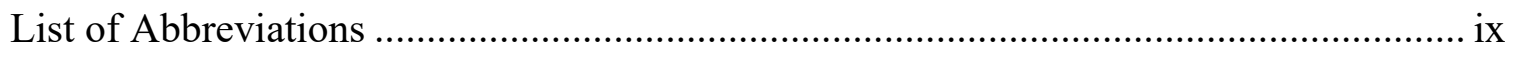

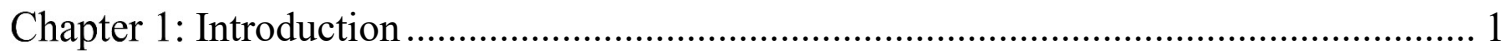

1.1: Seismic Hazards in Pacific Northwest.............................................................. 1

1.2: Liquefaction Hazard in Oregon ................................................................. 2

1.3: Existing Ground Improvement Methods for Liquefaction Mitigation ................... 4

1.4: Microbially Induced Desaturation for Liquefaction Mitigation ........................... 5

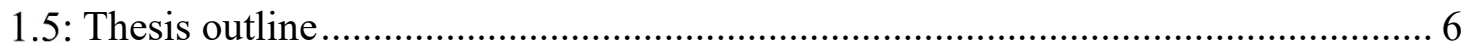

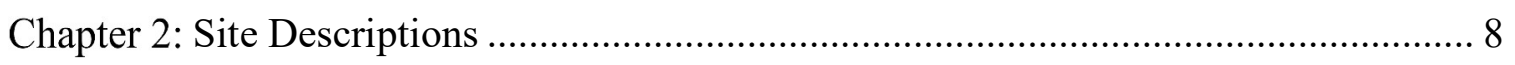

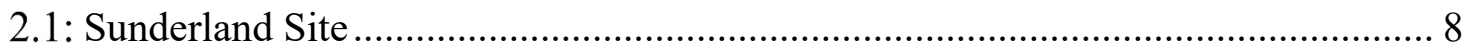

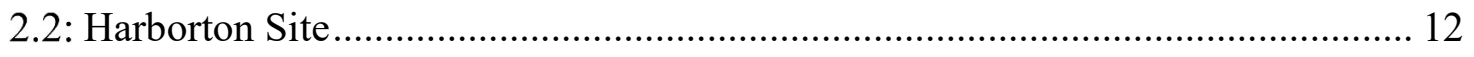

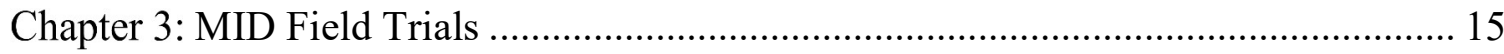

3.1: Treatment Solution and Chemical Reactions................................................... 15

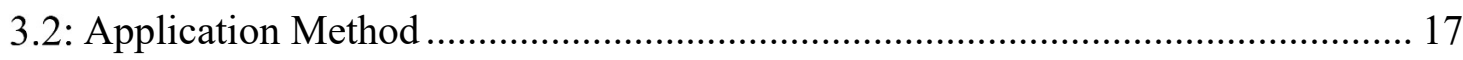

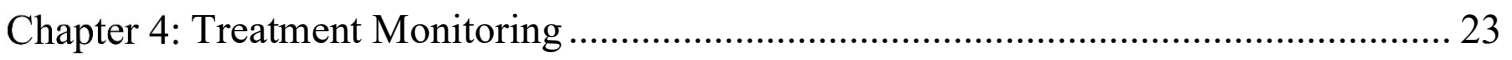

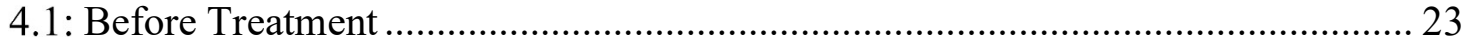

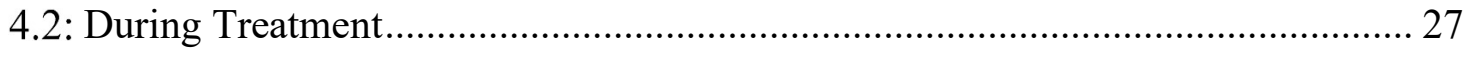




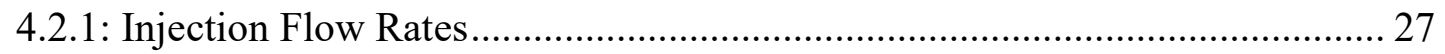

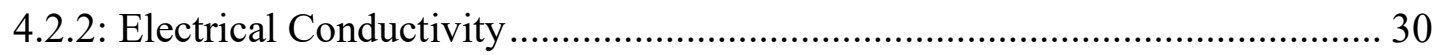

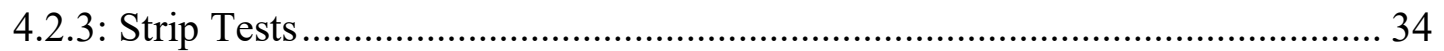

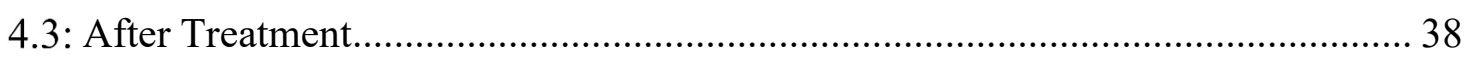

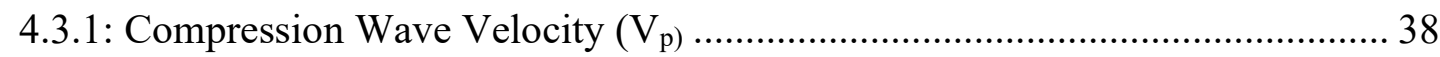

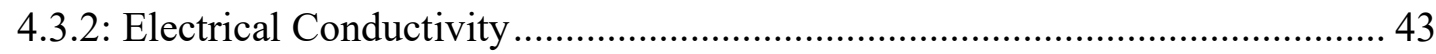

4.3.3: Direct measurements of saturation ratio ...................................................... 51

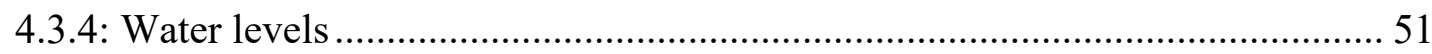

Chapter 5: Summary, Conclusions, and Recommendations for Future Work.................. 54

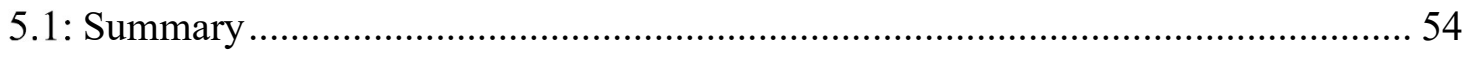

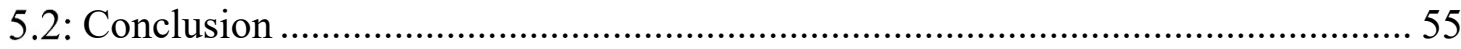

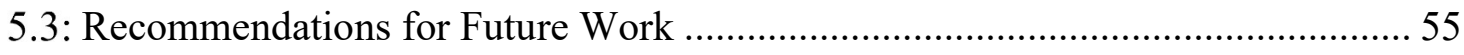

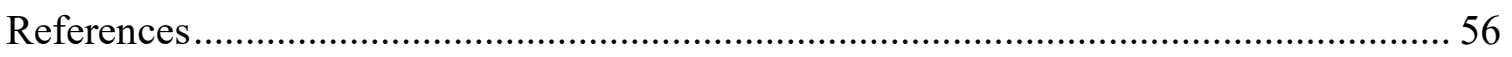

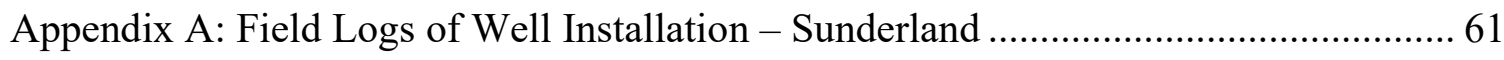

Appendix B: Finished Boring Logs - Sunderland ....................................................... 74

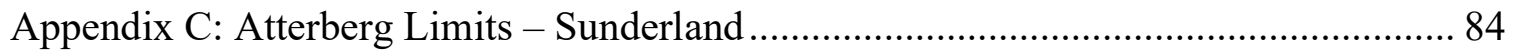

Appendix D: Hydrometer and P200 Washes - Sunderland ......................................... 132

Appendix E: Field Logs of Well Installation - Harborton ............................................ 167

Appendix F: Finished Boring Logs - Harborton ....................................................... 178

Appendix G: Atterberg Limits - Harborton.................................................................. 184 
Appendix H: Hydrometer and P200 Washes - Harborton.

Appendix I: $V_{s} / V_{p}$ Acquisition Software Installation Instructions .................................. 248

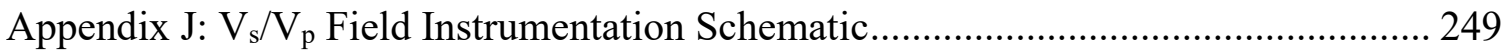

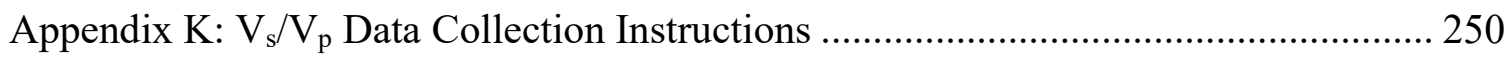

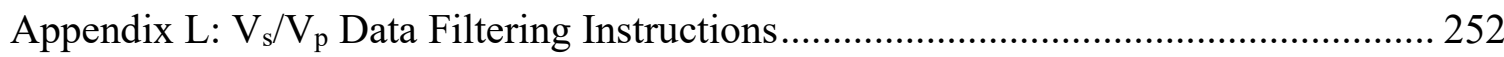

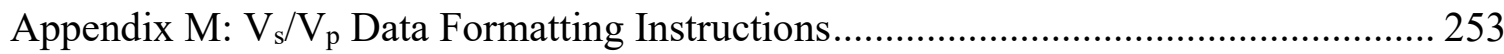

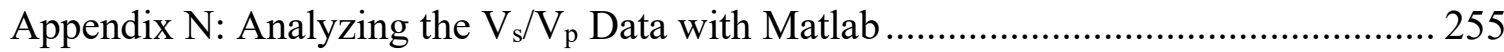

Appendix O: Before treatment: Crosshole geophone voltage time series for $V_{s}$ and $V_{p}$ arrival selection.

Appendix P: During treatment: Crosshole geophone voltage time series for $\mathrm{V}_{\mathrm{s}}$ and $\mathrm{V}_{\mathrm{p}}$ arrival selection

Appendix Q: After treatment: Crosshole geophone voltage time series for $V_{s}$ and $V_{p}$ arrival selection. 


\section{List of Figures}

Figure 1.1: The CSZ in relation to the Pacific Northwest of the United States, and the tectonic plates that make up the CSZ. Image adapted from Atwater et al. 2005............... 1

Figure 1.2: Shallow inter-crustal faults located within the Portland, Oregon Metro area. Image source: Washington County Emergency Management...................................... 2

Figure 1.3: Liquefaction susceptibility of Portland area soils as defined by DOGAMI 2012, in relation to the two field trial sites, Sunderland and Harborton............................ 3

Figure 2.1: Areal view of the Sunderland site, including geotechnical explorations, MID treatment system, and the targeted treatment zone.

Figure 2.2: Sunderland split-spoon sampler collected from $1.98 \mathrm{~m}$ to $2.44 \mathrm{~m}$ bgs showing the delineation between brown and gray silt. 10

Figure 2.3: Sunderland (a) idealized soil profile, (b) Atterberg limits, (c) clay, silt, and coarse-grained content by percentage with depth, (d) USCS classification using the plasticity chart, and (e) liquefaction susceptibility according to Bray and Sancio 2006. . 11

Figure 2.4: Areal view of the Harborton site, including geotechnical explorations, MID treatment system, and the targeted treatment zone.

Figure 2.5: Harborton (a) idealized soil profile, (b) Atterberg limits, (c) clay, silt, and coarse-grained content by percentage with depth, (d) USCS classification using the plasticity chart, and (e) liquefaction susceptibility according to Bray and Sancio 2006. . 14 Figure 3.1: Schematic showing the MID system, which included an extraction tank for collecting the extracted groundwater, a mixing tote where the calcium acetate and calcium nitrate were added, and an injection tank from which the treatment solution was injected into the subsurface. 
Figure 3.2: Left - a perspective shot of the submersible pump and right - the submersible pump as set up in the field. 19

Figure 3.3: Left - a perspective shot of the float switch and right - the float switch as set up in the field. 20

Figure 3.4: The automated control system which controlled the extraction pumps, comprised of motor controllers and delay timers. 20

Figure 4.1: The Vp, Vs, and Ic values of Sunderland $(a-c)$ and Harborton $(d-f) \ldots . . .25$

Figure 4.2: Top - top view of the embedded sensor array. Bottom - cross-section view of the 3D geophones and PPTs with depth. 26

Figure 4.3: The Sunderland screening test locations relative to the TREX baseplate and the targeted treatment zone. 27

Figure 4.4: Injection treatment solution flow rate estimated from change of water levels in injection tank and flow gauge (a) Sunderland site, (b) Harborton site. 28 Figure 4.5: Cumulative volume of injected treatment solution at (a) Sunderland site, and

(b) Harborton site. 29

Figure 4.6: Injection pressures for (a) Sunderland site, and (b) Harborton site. 30

Figure 4.7: Electrical conductivity measured by suspended divers in the treatment solution and the extracted water at (a) Sunderland and (b) Harborton. 32 Figure 4.8: Electrical conductivity in the injection and extraction tanks 24 hours (Sunderland)

Figure 4.9: Strip test results to indicate progress of MID reaction and water quality for the injected solution and extracted groundwater for (a-e) Sunderland and (f-j) Harborton sites. 37 
Figure 4.10: Vp measurements using SPCTs, DPCH, and crosshole methods at (a) Sunderland and (b) Harborton. 39

Figure 4.11: Travel-time records of pressure-wave measured during the crosshole testing at $2.55 \mathrm{~m}$ depth performed pre-treatment ( 5 days before start of treatment) and posttreatment (43 days after the start of treatment).................................................... 41

Figure 4.12: Vp crosshole time history measurements at Sunderland......................... 42 Figure 4.13: Side view of the TEROS sensors at (a) Sunderland and (b) Harborton, in relation to their respective target treatment zone.

Figure 4.14: Bulk EC measured by in-situ sensors at (a) Sunderland and (b) Harborton.

Figure 4.15: $w_{n}$ interpreted from in-situ sensors at (a) Sunderland and (b) Harborton. .. 49

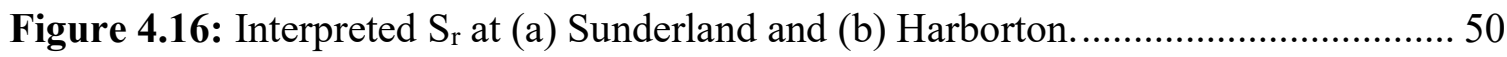

Figure 4.17: Water levels over time at the Sunderland site.................................... 53 


\section{List of Abbreviations}

\begin{tabular}{|c|c|}
\hline ASCE & American Society of Civil Engineers \\
\hline bgs & below ground surface \\
\hline CEI & Critical Energy Infrastructure \\
\hline $\mathrm{CL}$ & low plasticity clay \\
\hline $\mathrm{cm}$ & centimeter \\
\hline CSZ & Cascadia Subduction Zone \\
\hline DOGAMI & Oregon Department of Geology and Mineral Industries \\
\hline $\mathrm{DPCH}$ & direct push crosshole \\
\hline$\Delta u$ & pore water pressure \\
\hline $\mathrm{EC}$ & electrical conductivity \\
\hline$g$ & gram \\
\hline $\mathrm{GH}$ & general hardness \\
\hline GWT & ground water table \\
\hline HSA & hollow stem auger \\
\hline $\mathrm{I}_{\mathrm{c}}$ & soil behavior index \\
\hline $\mathrm{kg}$ & kilogram \\
\hline $\mathrm{KH}$ & carbonate hardness \\
\hline$k P a$ & kilopascal \\
\hline $\mathrm{L}$ & liter \\
\hline M & magnitude \\
\hline $\mathrm{m}$ & meter \\
\hline$m g$ & milligram \\
\hline
\end{tabular}




\begin{tabular}{ll} 
MID & microbially induced desaturation \\
MIDP & microbially induced desaturation and precipitation \\
ML & low plasticity silt \\
mol & mole \\
$m S$ & millisiemen \\
PDX & Portland International Airport \\
PI & plasticity index \\
PPT & pore pressure transducer \\
PVC & polyvinyl chloride \\
$\rho_{\text {dry }}$ & dry density \\
SC & screening test \\
SCPT & seismic cone penetration test \\
SPT & standard penetration test \\
S $_{\mathrm{r}}$ & saturation ratio \\
USCS & United Soil Classification System \\
$\mathrm{V}_{\mathrm{s}}$ & shear wave velocity \\
$\mathrm{V}_{\mathrm{p}}$ & pressure wave velocity \\
$\mathrm{w}_{\mathrm{L}}$ & liquid limit \\
$\mathrm{W}_{\mathrm{n}}$ & in-situ water content \\
WP & plastic limit \\
\hline
\end{tabular}




\section{Chapter 1: Introduction}

\section{1: Seismic Hazards in Pacific Northwest}

The primary seismic hazard for the pacific northwest is an earthquake produced by the Cascadia Subduction Zone (CSZ). The CSZ is a $965 \mathrm{~km}$ (600 mile) fault that runs from northern Vancouver Island in Canada to northern California as seen in Figure 1.1 and is located between $100 \mathrm{~km}$ and $160 \mathrm{~km}(110-160$ miles) off the Oregon coast (OOEM). Historical evidence indicates at least 40 major (Magnitude $[\mathrm{M}]>7.5$ ) $\mathrm{CSZ}$ earthquakes since 9845 BCE (Goldfinger et al. 2012), with the last major earthquake being a M9.0 occurring on Jan 26, 1700 (Satake et el. 1996).

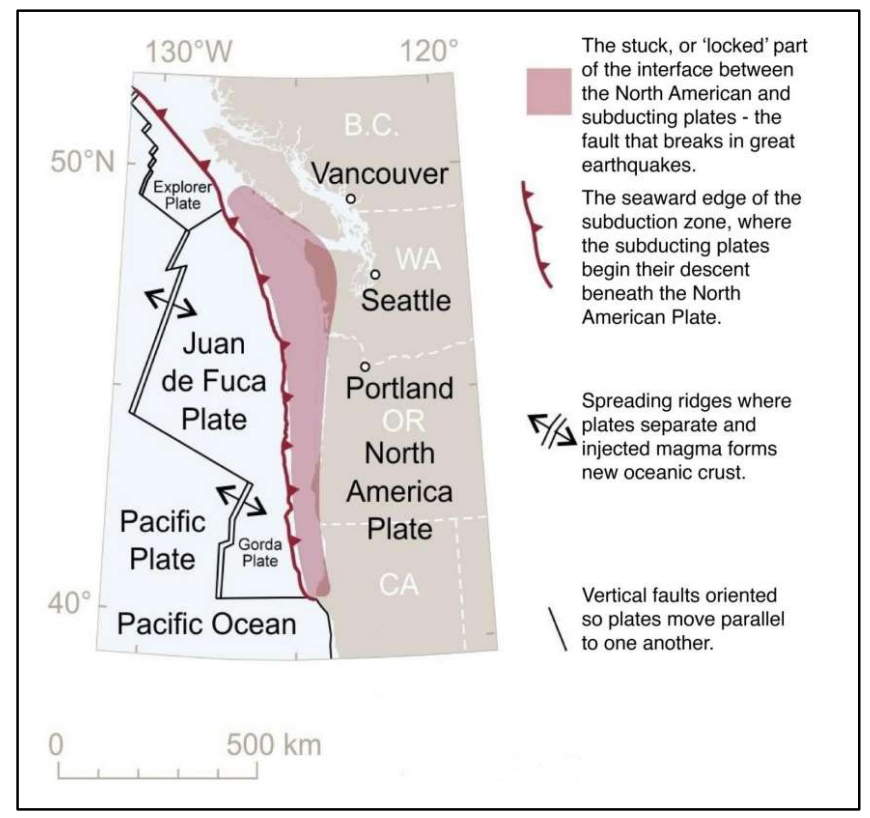

Figure 1.1: The CSZ in relation to the Pacific Northwest of the United States, and the tectonic plates that make up the CSZ. Image adapted from Atwater et al. 2005.

In addition to the CSZ, the seismicity in Oregon is also affected by shallow crustal faults. Portland, Oregon has three known inter-crustal faults that run throughout the metro area 
that are referred to as the Portland Hills Fault zone, as seen in Figure 1.2. There is indirect evidence that this fault zone can produce large magnitude earthquakes (M6.8 - M7.2) every 1,000 years (Wong et al. 2001).

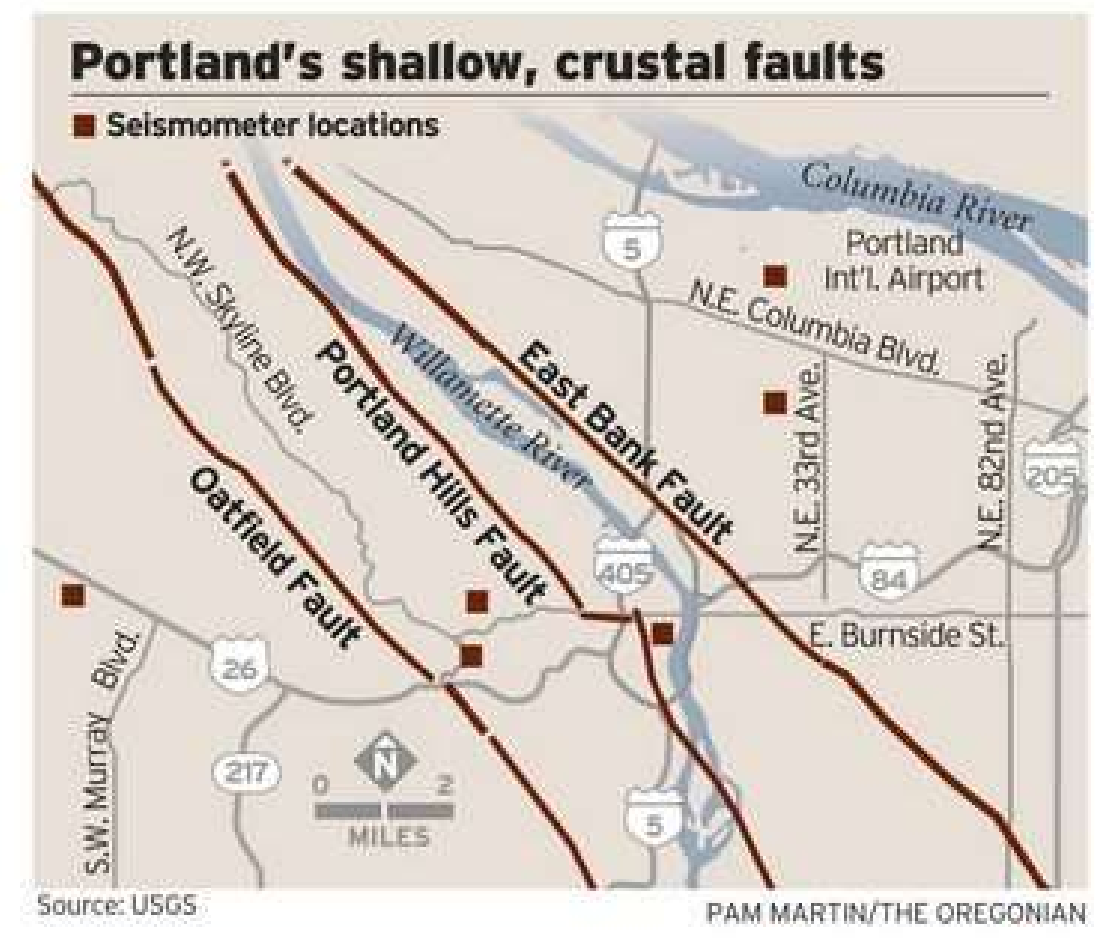

Figure 1.2: Shallow inter-crustal faults located within the Portland, Oregon Metro area. Image source: Washington County Emergency Management.

\section{2: Liquefaction Hazard in Oregon}

Liquefaction (or cyclic softening) is a term that is used when saturated, loosely compacted soils temporarily lose their strength and stiffness, and exhibit the characteristic of a liquid during earthquake shaking. The applied stress increases the soil's pore water pressure $(\Delta u)$, which in turn decreases the shear strength of the soil. 
Portland, Oregon, and the surrounding Willamette Valley were subjected to the Missoula Floods, a series of flood events that occurred between 15,000 and 13,000 years ago. These flooding events left behind substantial layers of alluvial sediments, primarily composed of silt, sand, and organic-rich clays. The maximum thickness of the combined sediment layers is approximately 45 meters (Hull 1991). These Quaternary sediments, combined with dredged soils from the Columbia and Willamette rivers, have high liquefaction susceptibility as seen in Figure 1.3 and as categorized by the Oregon Department of Geology and Mineral Industries (DOGAMI) (Bauer et al. 2012).
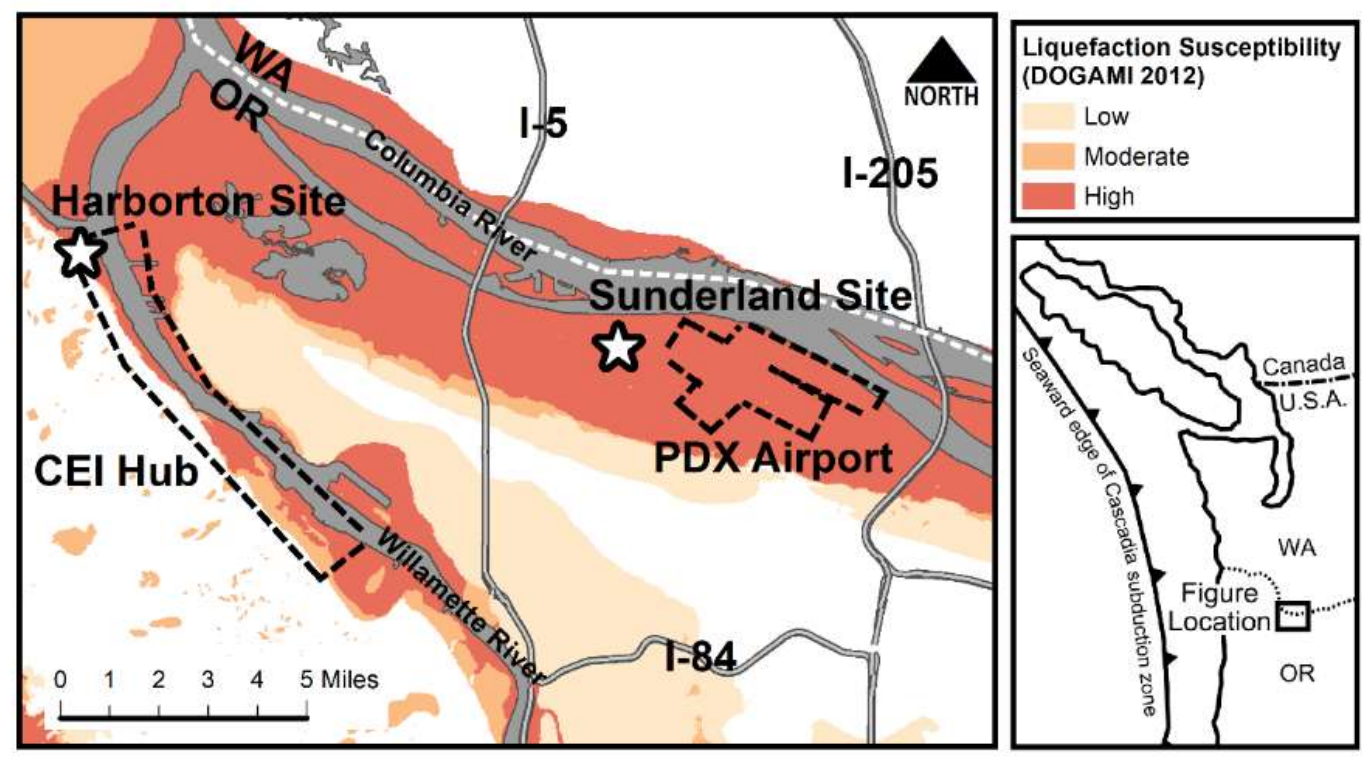

Figure 1.3: Liquefaction susceptibility of Portland area soils as defined by DOGAMI 2012, in relation to the two field trial sites, Sunderland and Harborton.

Several factors are used to determine whether or not a fine-grained soil is liquefiable, the most notable being the soil's liquid limit $\left(\mathrm{w}_{\mathrm{L}}\right)$, the in-situ water content $\left(\mathrm{w}_{\mathrm{n}}\right)$, and the plasticity index $(\mathrm{PI}) \mathrm{w}_{\mathrm{L}}$ is defined as the water content at which soil changes from a plastic 
state to a liquid state; $\mathrm{w}_{\mathrm{n}}$ is the ratio of the weight of water to the weight of soil solids; and $\mathrm{PI}$ is the difference between $\mathrm{W}_{\mathrm{L}}$ and the plastic limit ( $\mathrm{WP}_{\mathrm{P}}$ ) (the plastic limit being defined as the water content at which soil changes from a solid state to a plastic state). Low plasticity soils $(\mathrm{PI}<18)$ are considered moderately susceptible to liquefaction, and soils where PI < 12 are considered to be susceptible to highly susceptible to liquefaction (Bray and Sancio 2006).

\section{3: Existing Ground Improvement Methods for Liquefaction Mitigation}

Current liquefaction mitigation methods aim to either (i) increase the soil density or (ii) increase the soil strength. Increasing the density through methods such as vibrocompaction or surcharge loading aims to reduce the amount of $\Delta u$ that can be generated

during a shaking event - the soil will dilate creating larger voids that the pore fluid can inhabit, allowing the soil to have a higher threshold for applied stress before $\Delta u$ generation occurs. Strengthening the soil works in several ways, from allowing $\Delta u$ to quickly drain from the soil (stone columns), to increasing the soil's stiffness (soil cement mixing).

There are several methods for liquefaction mitigation besides those previously mentioned. However, only a fraction of these methods are suitable to mitigate fine-grained soils, and even fewer are suitable to treat fine-grained soils beneath existing structures. The lack of a sufficient mitigation method is a cause for concern for areas around the globe. Sites that have not been properly mitigated against liquefaction (or mitigated at all) face liquefactioninduced ground deformations, lateral spreading, and site-specific liquefaction concerns. 
The critical energy infrastructure (CEI) hub in Portland has the concern of significant environmental damage due to spilled fuel contamination in the Willamette River (Wang et al. 2012). The methods that can treat fine-grained soils under existing structures are usually cost-prohibitive and often cannot be used due to unique challenges at induvial sites; the CEI hub is composed of aging fuel tanks which strong vibrations may damage. Therefore, there is a strong need for a non-disruptive ground improvement method that is costeffective for fine-grained liquefiable soils and can be applied beneath existing structures.

\section{4: Microbially Induced Desaturation for Liquefaction Mitigation}

An emerging method to non-disruptively treat low plasticity fine-grained soils underneath existing structures is microbially-induced desaturation (MID). MID is performed by injecting a solution that contains calcium nitrate, calcium acetate, and trace elements into the subsurface. The treatment solution stimulates the growth of native bacteria that oxidize the acetate by reducing the nitrate, producing dissolved inorganic carbon and nitrogen gas as a byproduct (O’Donnell et al. 2017a). The relatively insoluble nitrogen gas displaces a small percentage of the fluid's pore water, which in turn reduces the soil's saturation ratio $\left(\mathrm{S}_{\mathrm{r}}\right)$. Liquefaction only occurs when the $\mathrm{S}_{\mathrm{r}}>99.5 \%$ (Valle-Molina and Stokoe 2012); previous laboratory tests on sand show that cyclically induced $\Delta u$ may be reduced to values between $95 \%$ and $50 \%$ when $\mathrm{S}_{\mathrm{r}}$ reduces from $100 \%$ to $95 \%$ (He et al. 2013 , Vega-Posada et al. 2014). 
Field trials utilizing MID were performed from August to September 2019 at two sites in Portland, Oregon. This was the first time MID was performed at the field scale in the United States.

\section{5: Thesis outline}

The contents in this thesis have been submitted as a conference paper to the 2021 ASCE Lifelines conference and a journal paper to be submitted to the ASCE Geotechnical and Geoenvironmental Engineering Journal with the following citations:

Sorenson, K., Preciado, A.M., Moug, D., Khosravifar, K., van Paassen, L., Kavazanjian, E., Stokoe, K., and Menq, F. "Field Monitoring of the Persistence of Microbially Induced Desaturation for Mitigation of Earthquake Induced Soil Liquefaction in Silty Soil”. Submitted to ASCE Lifelines Conference, 2021.

Moug, D., Sorenson, K., Khosravifar, A., Preciado, A.M., Stallings Young, E., van Paassen, L.,

Kavazanjian, E., Zhang, B., Stokoe, K., Menq, F., Wang, Y. "Field Trials of Microbially Induced Desaturation in Low Plasticity Silt”. Submitted to the Journal of Geotechnical and Geoenvironmental Engineering, 2021

Chapter 2 of this thesis describes the field trial site locations including the geotechnical explorations, the MID field setup, and the soil classification and liquefaction susceptibility of subsurface soil units at each site.

Chapter 3 of this thesis describes the chemical composition of the treatment solution, how the treatment solution was applied, and the automated control system that was used to 
induce a seepage gradient to facilitate the application of the treatment solution throughout the subsurface.

Chapter 4 of this thesis describes the various monitoring data that were collected before, during, and after the completion of the MID field trials. These data can be broadly defined as (a) pertaining to the application of the treatment solution during the four-week field trial period and (b) evaluating the effectiveness of the treatment after the four-week field trial period. Data categorized as (a) include injection rates and pressures, electrical conductivity of the treatment solution as a means of monitoring the chemical reaction and as a means of and observing the subsurface seepage path, and strip-tests to qualitatively observe larger trends in the progress of the MID reaction between the injection well and the extraction wells. Data categorized as (b) include crosshole pressure-wave velocity measurements, a vertical array of embedded sensors that measure water content and bulk electrical conductivity, pre- and post-treatment seismic cone penetration tests, and direct soil sample of the treated soils.

Chapter 5 of this thesis presents the conclusions of these field trials up until 20 months post-treatment, and recommendations for future tests to better understand and assess the viability of MID as a liquefaction mitigation method. 


\section{Chapter 2: Site Descriptions}

The two sites chosen for MID treatment are located by critical lifelines: the Sunderland site is located by the Portland International Airport (PDX) and the Harborton site is located by the CEI hub. The CEI hub handles $90 \%$ of all the liquid fuel (gasoline, diesel, natural gas, etc.) for the entire state of Oregon and 100\% of the jet fuel for PDX (Wang et al. 2012). Both sites are underlain by low plasticity soils that are considered susceptible to liquefaction and damaging ground deformations during strong earthquake shaking. Both sites and their proximity to nearby critical lifelines can be seen in Figure 1.3; the Sunderland site is underlain by soils similar to PDX and the Columbia river levees, and the Harborton stie is underlain by soils similar to the CEI hub.

\section{1: Sunderland Site}

The Sunderland site is underlain by Holocene-aged floodplain alluvium from the Columbia River (Hull 1991). These floodplain deposits typically overlay coarse river sand deposits. However, the coarse-grained deposits were not encountered in this study.

Geotechnical characterization activities for the Sunderland site included sampling, lab testing, and in-situ testing. The layout of the geotechnical explorations are shown in Figure 2.1. Hollow-stem auger (HSA) drilling was performed for installation of the injection and extraction wells as part of the MID treatment system. Appendix A contains the field logs that were fill out during the well installation and Appendix B contains the finished boring logs. During HSA drilling, soil samples were obtained with split-spoon samplers from 
standard penetration tests (SPTs) and by Shelby tube sampling. Hand-augured samples were obtained near the targeted treatment zone.

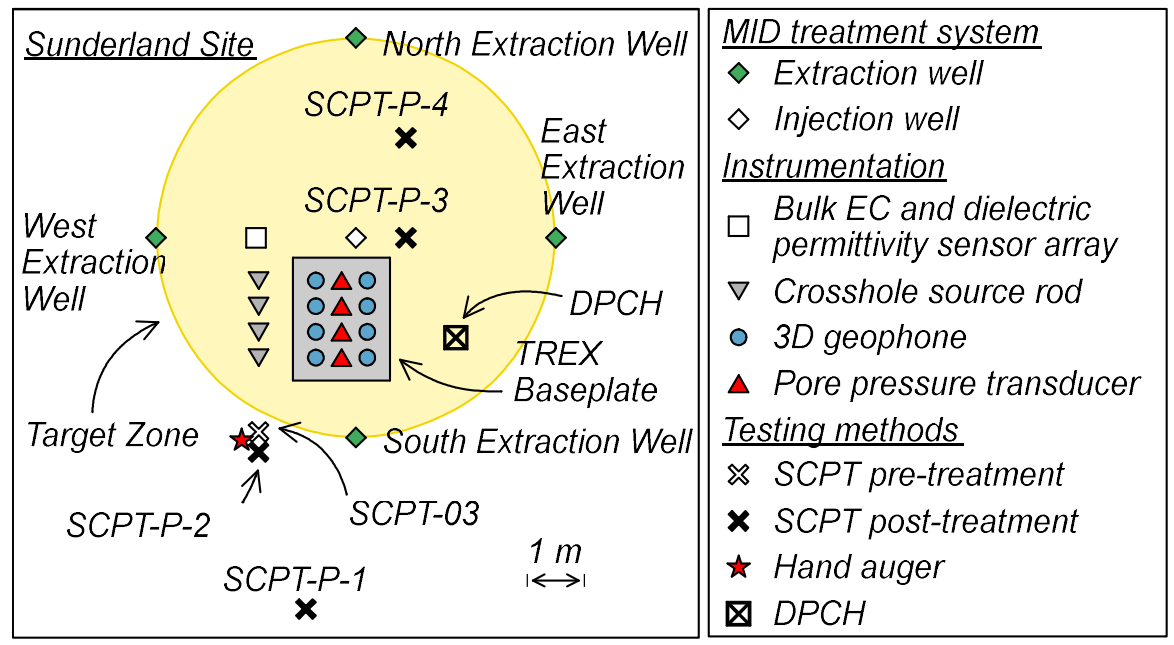

Figure 2.1: Areal view of the Sunderland site, including geotechnical explorations, MID treatment system, and the targeted treatment zone.

The interpreted site conditions are summarized in Figure 2.3a. The site is primarily composed of low plasticity silt to about $6.1 \mathrm{~m}$ below the ground surface (bgs). The boundary between brown and gray silt at about $2.25 \mathrm{~m}$ bgs is likely due to oxidationinduced soil color change in the vadose zone above the water table. This boundary can be seen in Figure 2.2. A summary of the measured $w_{P}, w_{L}$, and $w_{n}$ values obtained from soil samples is shown in Figure 2.3b. The $\mathrm{w}_{\mathrm{L}}, \mathrm{w}_{\mathrm{P}}, \mathrm{w}_{\mathrm{n}}$, and corresponding PI values for individual tests can be found in Appendix C. Generally, the $\mathrm{w}_{\mathrm{n}}$ at the site is close to or larger than $\mathrm{w}_{\mathrm{L}}$ (the ratio of $\mathrm{w}_{\mathrm{n}} / \mathrm{w}_{\mathrm{L}}$ ranges from 0.89 to 1.27 ). The average PI of the soils is 14. Grain size distribution was characterized using hydrometer tests and No. 200 sieve washes to further classify the soil via the Unified Soil Classification System (USCS) as presented in Figure 2.3c. The individual hydrometer and No. 200 wash values for each test 
can be found in Appendix D. Fining upward sequences with larger coarse contents at about $2.5 \mathrm{~m}$ and $6.5 \mathrm{~m}$ bgs are interpreted to be soils deposited during flooding events. The soils classify as low plasticity silt (ML) or lean clay (CL) as indicated by the plasticity chart in Figure 2.3d. Based on index testing, 9 out of 48 samples of the soils sampled are classified as susceptible to liquefaction, 26 out of 48 samples are classified as moderately susceptible to liquefaction, and 13 out of 48 samples are not susceptible based on Bray and Sancio (2006) as seen in Figure 2.3e. 46 out of 48 samples are susceptible to cyclic softening based on Boulanger and Idriss (2006).

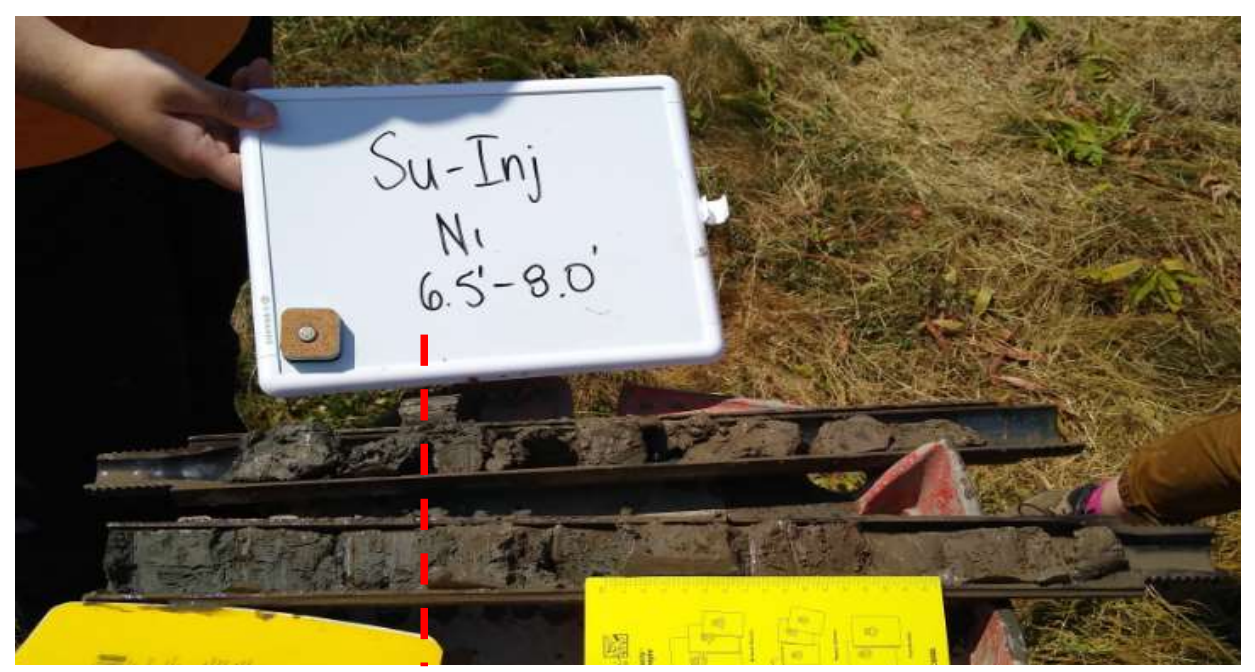

Figure 2.2: Sunderland split-spoon sampler collected from $1.98 \mathrm{~m}$ to $2.44 \mathrm{~m}$ bgs showing the delineation between brown and gray silt.

The water table at the site fluctuates seasonally but is also influenced by the water level in an adjacent drainage canal. Over the course of 23 months the measured groundwater table (GWT) depth ranged from $1.5 \mathrm{~m}$ to $0.0 \mathrm{~m}$ bgs with observed ponding of up to $0.43 \mathrm{~m}$ on site; the water table depth was $1.1 \mathrm{~m}$ bgs at the time of MID application. 


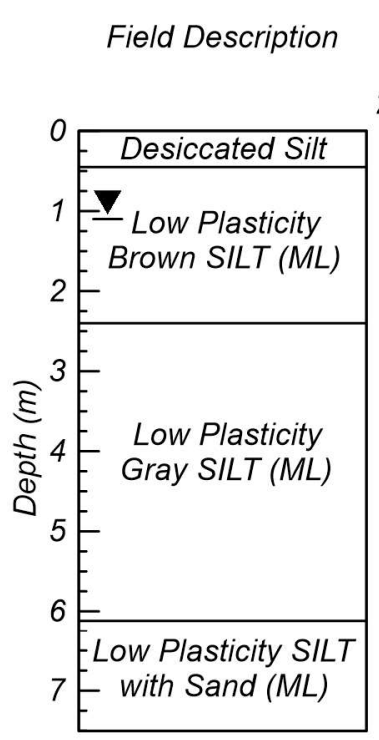

(a)

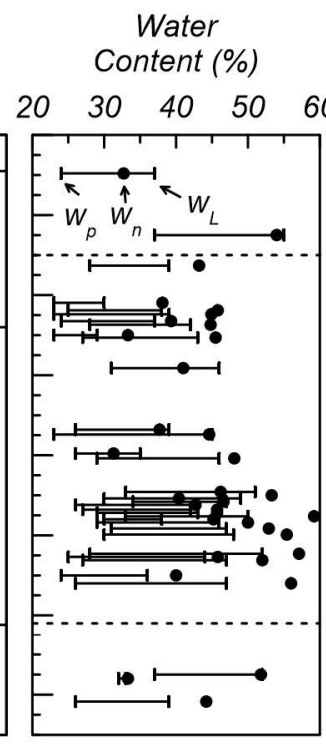

(b)

Coarse Content (\%)

$\begin{array}{llllll}0 \quad 20 \quad 40 & 60 & 80 & 100\end{array}$

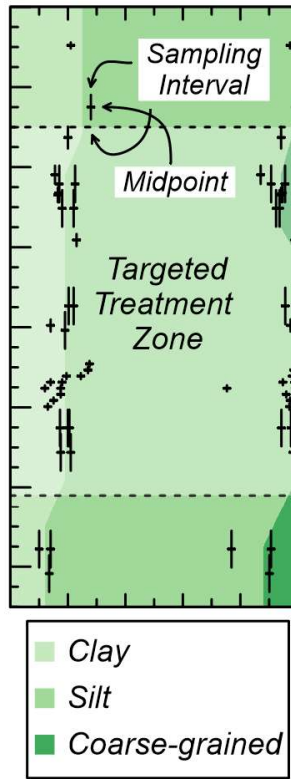

(c)

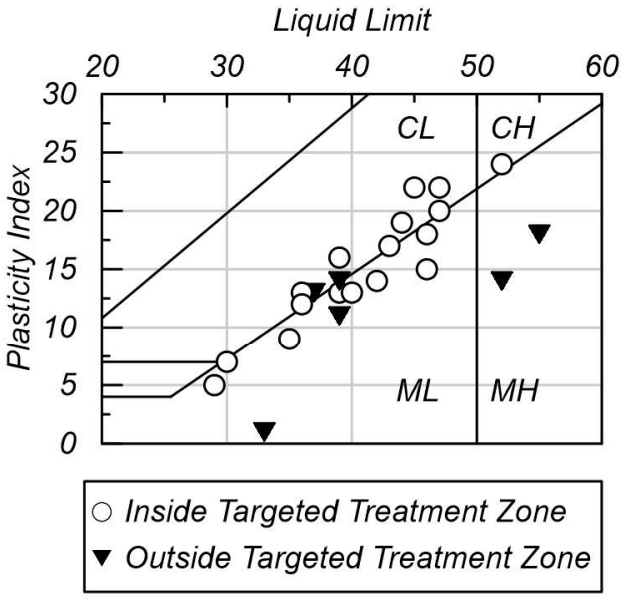

(d)

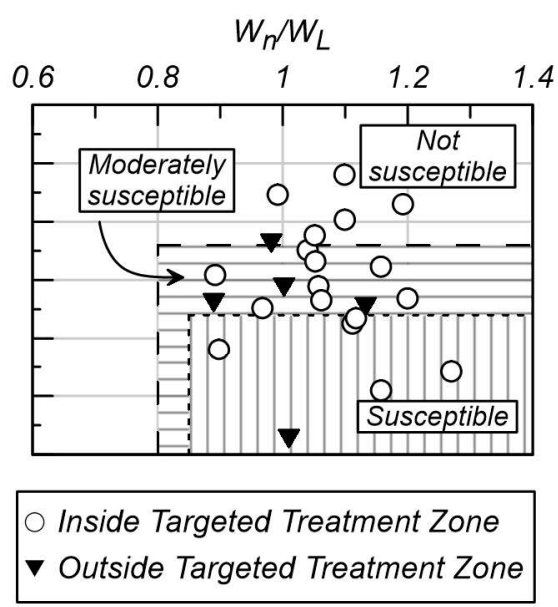

(e)

Figure 2.3: Sunderland (a) idealized soil profile, (b) Atterberg limits, (c) clay, silt, and coarse-grained content by percentage with depth, (d) USCS classification using the plasticity chart, and (e) liquefaction susceptibility according to Bray and Sancio 2006. 


\section{2: Harborton Site}

The soils in the area of the Harborton site (considered to be representative of the CEI hub) are composed of Willamette river-deposited soils and fill soils dredged from the river (Wang et al. 2012). Similar to the Sunderland site, geotechnical characterization activities for the Harborton site included sampling, lab testing, and in-situ testing. HSA drilling was performed for installation of injection and extraction wells as part of the MID treatment system, and during HSA drilling soil samples were obtained with split-spoon samplers from SPTs; Shelby tubes and hand-augured samples were obtained within the targeted treatment zone. Figure 2.4 shows the layout of the geotechnical explorations at the Harborton site. Appendix E contains the field logs that were fill out during the well installation and Appendix F contains the finished boring logs.

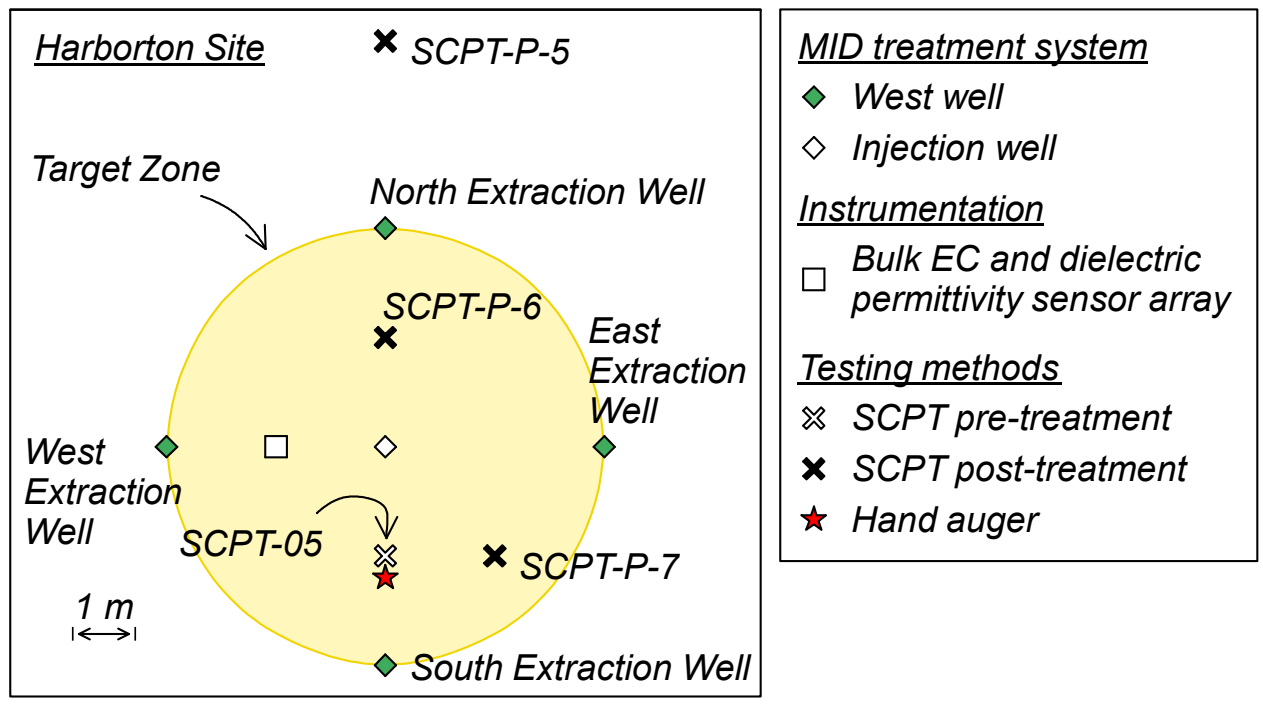

Figure 2.4: Areal view of the Harborton site, including geotechnical explorations, MID treatment system, and the targeted treatment zone. 
The interpreted site conditions at Harborton are summarized in Figure 2.5a. The site is primarily composed of interbedded non-plastic and low plasticity silt to about $5.25 \mathrm{~m}$ bgs. Over half of the specimens tested were classified as non-plastic. Atterberg index testing was performed; the resulting $\mathrm{w}_{\mathrm{p}}, \mathrm{w}_{\mathrm{L}}$ and $\mathrm{w}_{\mathrm{n}}$ values are shown in Figure 2.5b. Hollow symbols represent $\mathrm{w}_{\mathrm{n}}$ of non-plastic specimens. The $\mathrm{w}_{\mathrm{L}}, \mathrm{w}_{\mathrm{p}}$, and $\mathrm{w}_{\mathrm{n}}$ values for individual tests can be found in Appendix G. $\mathrm{w}_{\mathrm{n}}$ at the Harborton site is close to or less than $\mathrm{w}_{\mathrm{L}}$ (the ratio of $\mathrm{w}_{\mathrm{n}} / \mathrm{w}_{\mathrm{L}}$ ranges from 0.78 to 1.28 ). The low plasticity silt had an average PI of 13 , which was interbedded with non-plastic silt. The grain size distribution was characterized using hydrometer tests and No. 200 sieve wash tests to further classify the soil via the USCS, the results of which are presented in Figure 2.5c. The individual hydrometer and No. 200 wash values for each test can be found in Appendix H. The soils classify as nonplastic or as indicated on the plasticity chart in Figure 2.5d; non-plastic soils are not plotted. Based on index testing, 6 out of 13 soil samples are classified as susceptible to liquefaction, 4 out of 13 samples are classified as moderately susceptible, and 3 out of 13 samples are not susceptible based on Bray and Sancio (2006) as seen in Figure 2.5e. An additional 19 soil samples could not be classified according to this criterion, as no $\mathrm{w}_{\mathrm{L}}$ value could be obtained; these samples are not plotted. 11 out of 13 samples are susceptible to cyclic softening based on Boulanger and Idriss (2006).

The water table at the time of well installation in July was measured at approximately $2.3 \mathrm{~m}$ bgs. The GWT at the site likely undergoes fluctuations with the Willamette River water level and seasonal seepage from hills located to the south-west of the site. 


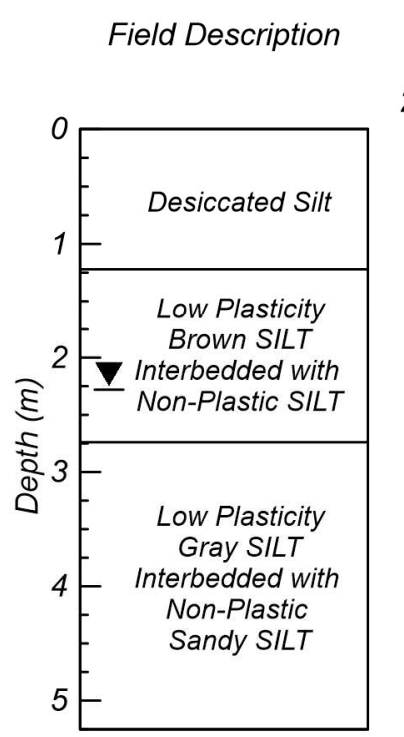

(a)
Water
Content (\%) $203040 \quad 506070$

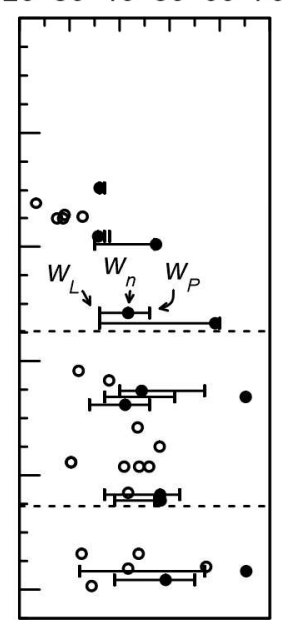

(b)

Fine and

coarse content (\%)

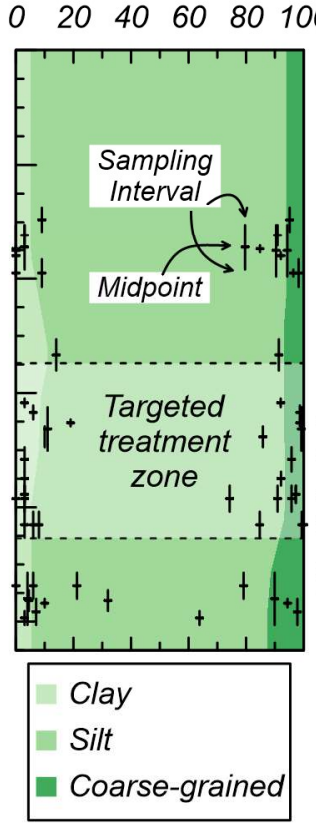

(c)

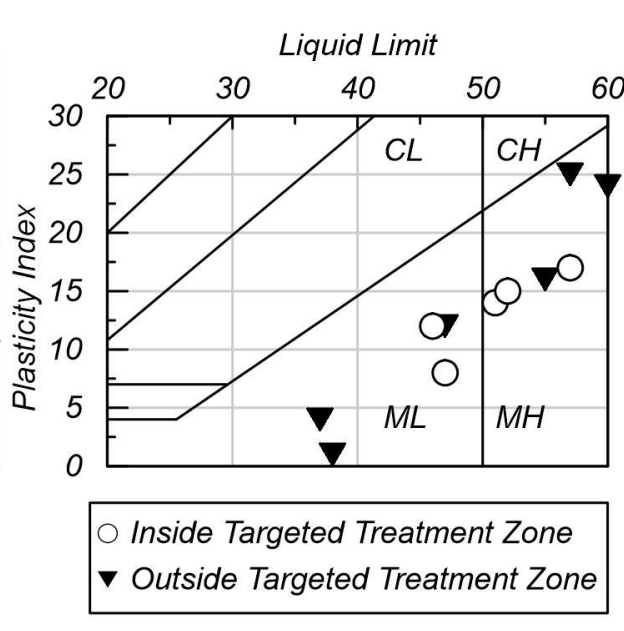

(d)

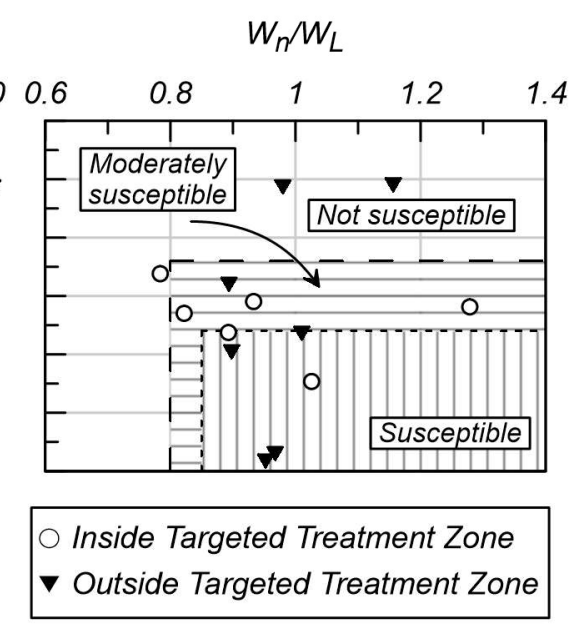

(e)

Figure 2.5: Harborton (a) idealized soil profile, (b) Atterberg limits, (c) clay, silt, and coarse-grained content by percentage with depth,

(d) USCS classification using the plasticity chart, and (e) liquefaction susceptibility according to Bray and Sancio 2006. 


\section{Chapter 3: MID Field Trials}

MID field trials for liquefaction mitigation of fine-grained soils were performed at both the Sunderland and Harborton sites. The MID treatment was performed by injecting a substrate solution into the subsurface. The treatment method and field trial design are described herein.

\section{1: Treatment Solution and Chemical Reactions}

MID relies on native denitrifying bacteria, which oxidize organic carbon (electron donor) in conditions where the groundwater is depleted of dissolved oxygen by reducing nitrate (electron acceptor), and produces $\mathrm{N}_{2}(\mathrm{~g})$, biomass, and dissolved inorganic carbon. Dissolved inorganic carbon may form carbon dioxide gas $\left(\mathrm{CO}_{2}(\mathrm{~g})\right)$ or, in the presence of dissolved calcium ions $\left(\mathrm{Ca}^{2+}\right)$, precipitate as calcium carbonate $\left(\mathrm{CaCO}_{3}\right)$. The MID process is the first stage of the microbially induced desaturation and precipitation (MIDP) process as detailed in O'Donnell et al. (2017a,b) and Stallings Young et al. (2020), in which ground improvement is achieved through biogenic gas formation and mineral precipitation. Although both desaturation and precipitation improve the cyclic resistance ratio for loose granular soils, the amount of substrates needed to sufficiently reduce $S_{r}$ may be 10 to 40 times less than the required amount to reach a sufficient level of cementation (O'Donnell et al. 2017a,b, Pham et al. 2017, 2018b). MID can mitigate the triggering of liquefaction by increasing the compressibility of the pore fluid and preventing $\Delta u$ build up during cyclic loading. Studies on desaturated sand showed a reduction in cyclic loading-induced $\Delta u$ when compared to saturated conditions (O’Donnell et al. 2017b, Yegian et al. 2007, Wang et al. 2020). 
The stoichiometry of the denitrification reaction varies depending on the carbon source and bacteria growth rate. Most reported studies on MIDP used calcium acetate as an organic carbon and calcium source. However, other organic carbon sources, such as ethanol in solution (Mousavi \& Ghayoomi 2021), may be used. The reduction of nitrate $\left(\mathrm{NO}_{3}{ }^{-}\right)$to $\mathrm{N}_{2(\mathrm{~g})}$ involves several steps, which can result in the accumulation of the intermediate products nitrite $\left(\mathrm{NO}_{2}^{-}\right)$, nitric oxide $(\mathrm{NO})$, and nitrous oxide $\left(\mathrm{N}_{2} \mathrm{O}\right)$. By using acetate $\left(\mathrm{C}_{2} \mathrm{H}_{3} \mathrm{O}_{2}{ }^{-}\right)$as the carbon source, and assuming full conversion of $\mathrm{NO}_{3}^{-}$to $\mathrm{N}_{2}(\mathrm{~g})$ and a maximum growth rate of biomass $\left(\mathrm{CH}_{1.8} \mathrm{O}_{0.5} \mathrm{~N}_{0.2}\right)$ the stoichiometry of the reaction is estimated by Pham et al. (2017) as:

$$
1.21 \mathrm{C}_{2} \mathrm{H}_{3} \mathrm{O}_{2}^{-}+0.97 \mathrm{NO}_{3}^{-} \rightarrow \mathrm{CH}_{1.8} \mathrm{O}_{0.5} \mathrm{~N}_{0.2}+0.39 \mathrm{~N}_{2}+1.41 \mathrm{HCO}_{3}^{-}+0.31 \mathrm{H}_{2} \mathrm{O}+0.28 \mathrm{OH}^{-}
$$

When biomass growth is limited, for example due to lack of nutrients or inhibited by accumulation of intermediate nitrogen compounds, the reaction may follow the stoichiometry of the catabolic reaction (zero biomass growth):

$$
\mathrm{C}_{2} \mathrm{H}_{3} \mathrm{O}_{2}^{-}+1.6 \mathrm{NO}_{3}^{-} \rightarrow 0.8 \mathrm{~N}_{2}+2 \mathrm{HCO}_{3}^{-}+0.2 \mathrm{H}_{2} \mathrm{O}+0.6 \mathrm{OH}^{-}
$$

Depending on the biomass growth rate, the molar substrate ratio $\left(\mathrm{C}_{2} \mathrm{H}_{3} \mathrm{O}_{2}{ }^{-}: \mathrm{NO}_{3}^{-}\right)$may vary roughly between 1.2:1 to 0.6:1 (van Paassen et al. 2010, Pham et al. 2018a). 
Accumulation of intermediate products should be avoided, since $\mathrm{NO}_{2}{ }^{-}$and $\mathrm{NO}$ are environmental toxins and inhibit the denitrification reaction, and $\mathrm{NO}$ is a strong greenhouse

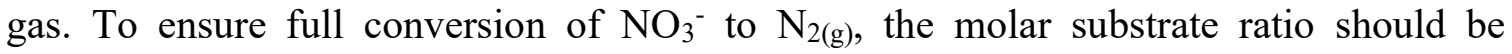
sufficiently high to ensure the reaction is not $\mathrm{C}_{2} \mathrm{H}_{3} \mathrm{O}_{2}{ }^{-}$(electron donor) limited. At the same time the total substrate concentrations should not be too high, as high $\mathrm{NO}_{3}{ }^{-}$concentrations can also inhibit the denitrification reaction (Van Paassen et al. 2010; Pham et al., 2018a,b; Glass \& Silverstein, 1998).

Both maximum and zero biomass growth conditions result in an increase in $\mathrm{pH}$ and alkalinity. At neutral $\mathrm{pH}$, bicarbonate $\left(\mathrm{HCO}_{3}^{-}\right)$is the dominate inorganic carbon product from the above reactions. If available, $\mathrm{Ca}^{2+}$ may react with $\mathrm{HCO}_{3}{ }^{-}$to precipitate $\mathrm{CaCO}_{3}$ :

$$
\mathrm{Ca}^{2+}+\mathrm{HCO}_{3}^{-} \rightarrow \mathrm{CaCO}_{3(\mathrm{~s})}+\mathrm{H}^{+}
$$

Release of protons $\left(\mathrm{H}^{+}\right)$due to the precipitation of $\mathrm{CaCO}_{3}$ buffers the alkalinity production of the denitrification reaction, allowing the $\mathrm{pH}$ to remain close to neutral. Under acidic conditions some of the $\mathrm{HCO}_{3}{ }^{-}$that remains in the solution may speciate to form $\mathrm{CO}_{2(\mathrm{~g})}$ :

$$
\mathrm{HCO}_{3}^{-}+\mathrm{H}^{+} \rightarrow \mathrm{CO}_{2(\mathrm{~g})}+\mathrm{H}_{2} \mathrm{O}
$$

\section{2: Application Method}

The MID treatment solution was applied to the subsurface through a central injection well. The injection well was surrounded by four equally spaced extraction wells located $3.5 \mathrm{~m}$ 
from the injection well. The layout of the wells and the associated instrumentation for both sites is shown in Figure 2.1 and Figure 2.4. The wells were installed in holes drilled with a 10-inch HSA. 2-inch diameter schedule 40 PVC casings were installed and were screened with 0.01 -inch slot size. The wells at Sunderland were screened from $1.5 \mathrm{~m}$ to $6.1 \mathrm{~m}$ depth and at Harborton were screened from $3.0 \mathrm{~m}$ to $4.6 \mathrm{~m}$ depth. The extraction wells pumped out groundwater that was used for (i) the treatment solutions and (ii) to induce seepage gradients to encourage migration of the treatment solution. The target zone for treatment was the circular area centered around the injection well with a radius of $3.5 \mathrm{~m}$, and with a height equal to the screened interval at each site.

The extracted groundwater was stored in a $3780 \mathrm{~L}$ (1000 gallon) tank that was referred to as the "extraction tank" as seen in Figure 3.1. Groundwater was extracted using plastic submersible pumps with capacities up to $7.6 \mathrm{~L} / \mathrm{min}$, which were activated via float switches as seen in Figure 3.2 and Figure 3.3. The pumps turned on and off through an automated control system. This control system was comprised of motor speed controllers, delay timers, and float switches as shown in Figure 3.4. The float switches were used to cutoff the power source to the pump when the water level dropped below the top of the well screen. This ensured that desaturation was not induced by drawing the GWT into the target zone (i.e., below the well screen), and that the pumps did not run dry and burn out. The motor controls reduced the voltage supplied to the pumps for lower, more sustained pumping rates in these fine-grained soils. The delay timers were used to turn pumps on after a time interval following water level recovery above the float switch. These timers 
ensured that the on/off signals to the pump were not dominated by rapid drawdown and recovery from pumping well loss.

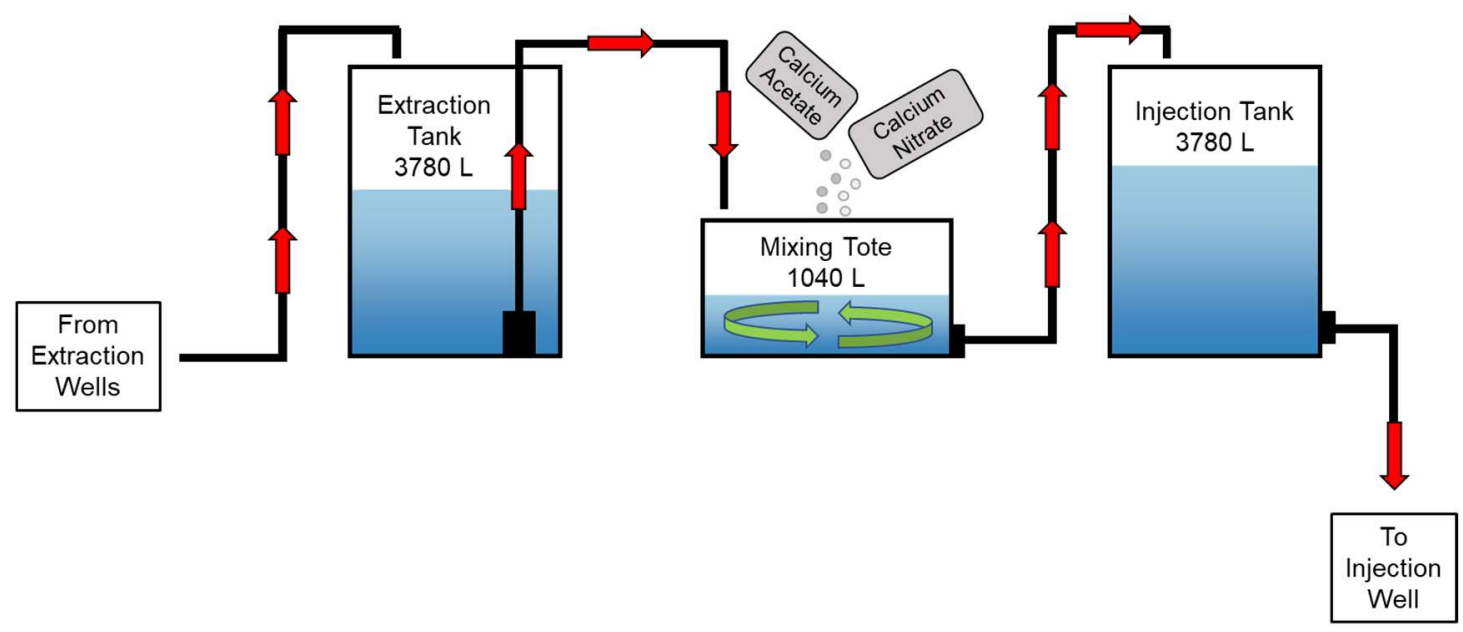

Figure 3.1: Schematic showing the MID system, which included an extraction tank for collecting the extracted groundwater, a mixing tote where the calcium acetate and calcium nitrate were added, and an injection tank from which the treatment solution was injected into the subsurface.
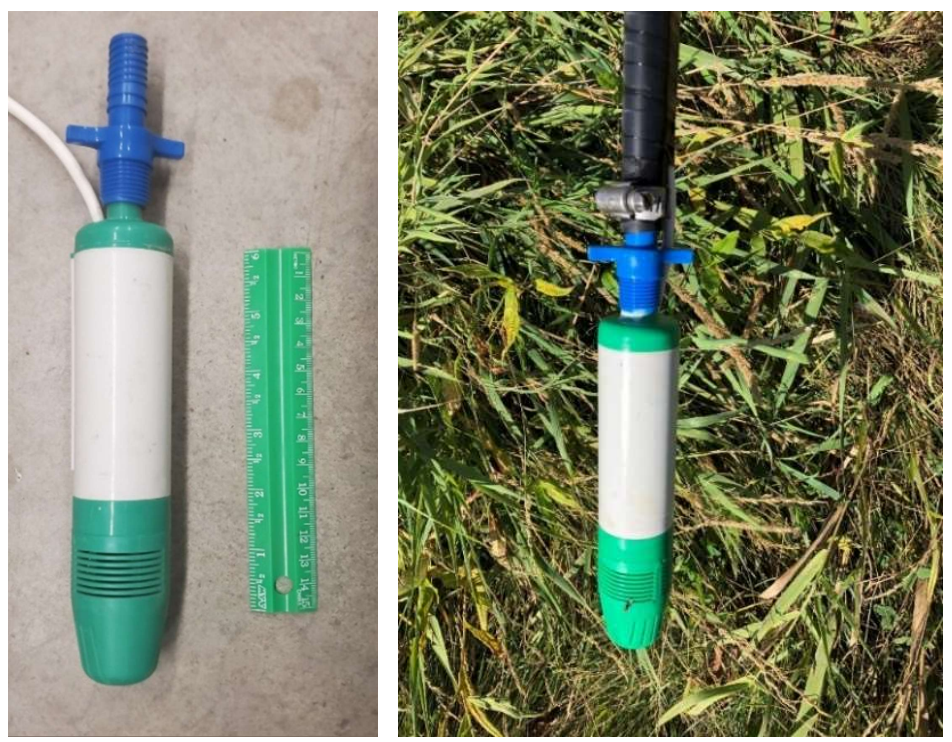

Figure 3.2: Left - a perspective shot of the submersible pump and right-the submersible pump as set up in the field. 

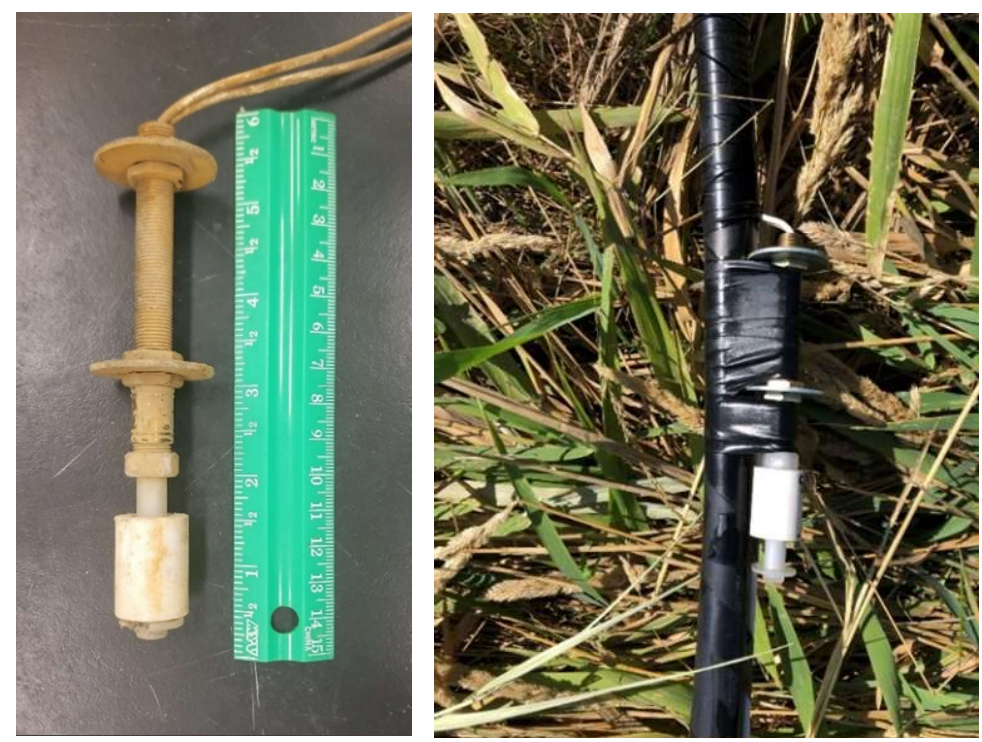

Figure 3.3: Left-a perspective shot of the float switch and right - the float switch as set up in the field.

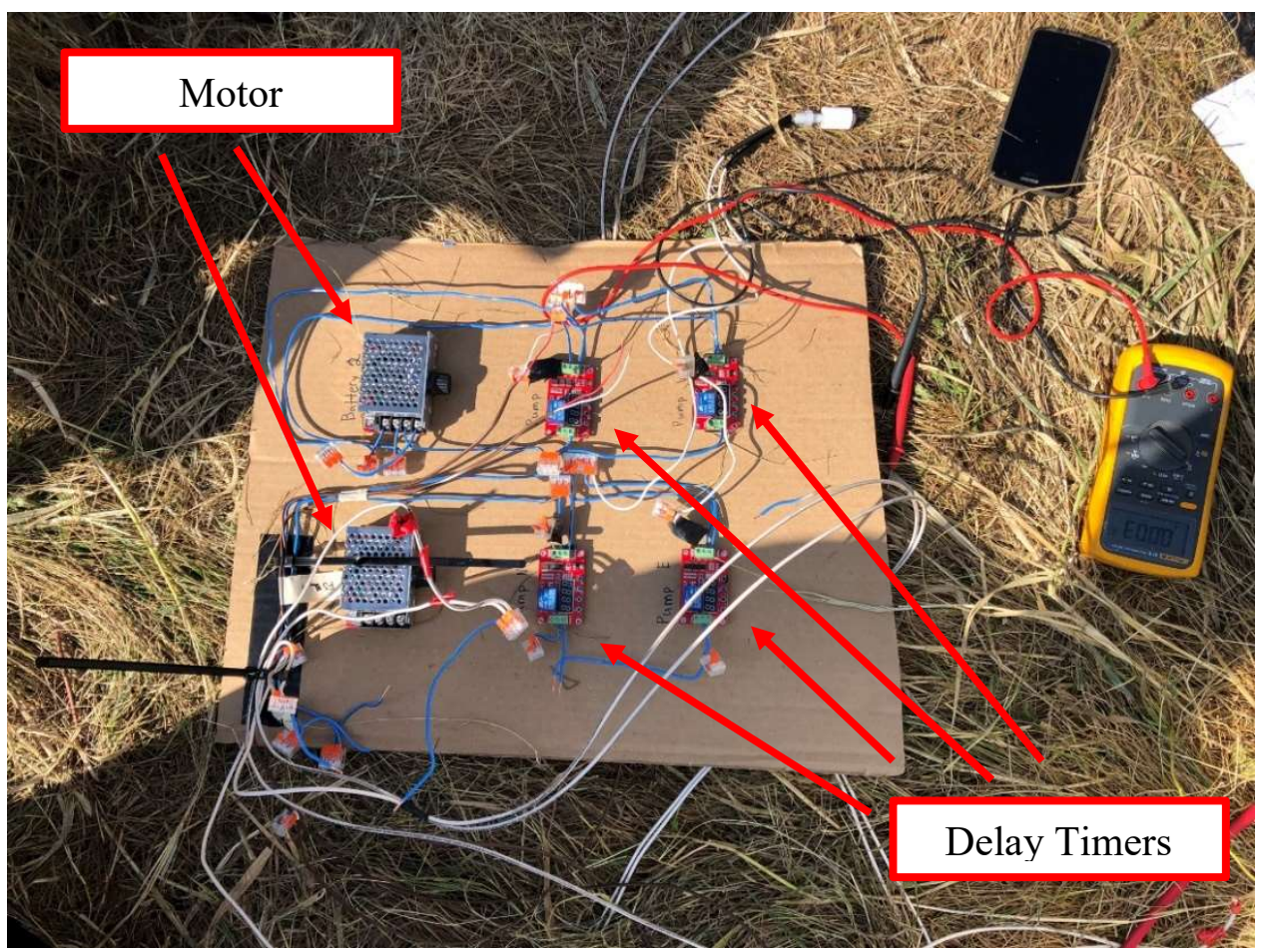

Figure 3.4: The automated control system which controlled the extraction pumps, comprised of motor controllers and delay timers. 
To prepare the substrate solutions, a 1040 L (275 gallon) tote was filled with water. A high flow trash pump circulated water as one $22.7 \mathrm{~kg}$ bag of calcium nitrate (YaraLiva ${ }^{\circledR}$ Calcinit ${ }^{\circledR}$, CAS No: 15245-12-2), then one $20 \mathrm{~kg}$ bag of calcium acetate (Niacet, CAS No: 62-54-4) were added. Circulation continued until the substrates were in solution. The mixed solutions were transferred to a $3780 \mathrm{~L}$ tank called the "injection tank" that was filled with water by the trash pump to uniformly dilute the solution to the target concentration. The initial target nitrate concentration at both sites was $0.05 \mathrm{~mol} / \mathrm{L}$ with a molar substrate ratio of approximately 1.08:1. City water was used in addition to the extracted groundwater at the Sunderland site for dilution, and only extracted groundwater was used at the Harborton site. At the Sunderland site $0.25 \mathrm{mg} / \mathrm{L}$ Miracle-Gro ${ }^{\circledR}$ All Purpose Plant Food and $0.06 \mathrm{mg} / \mathrm{L}$ Epsom Salt were initially added to provide nutrients and trace minerals to stimulate growth of indigenous denitrifying bacteria. However, after several days of treatment the reaction rate in the substrate solution became very high and a significant portion of the reaction occurred in the injection tank. Therefore, after treatment day 7 the additional nutrients and trace minerals were left out, and after day 12 the concentration was changed from about $0.05 \mathrm{~mol} / \mathrm{L}$ to $0.10 \mathrm{~mol} / \mathrm{L}$ ( 2 bags of each substrate per injection tank) to lower the reaction rate and allow the substrates to migrate a longer distance from the injection well before being consumed. The treatment solution at the Harborton site was consistently prepared at $0.05 \mathrm{~mol} / \mathrm{L}$ and without addition of nutrients or trace minerals.

The treatment solution was gravity fed into the injection well. Additional injection pressures were applied when the researchers were on-site through an in-line pump installed near the well head at the Sunderland site, or a sump pump in the injection tank at the 
Harborton site. It was advantageous to inject the treatment solution within 12 hours of mixing so that the MID reactions would take place predominately in the subsurface. The project team also aimed to inject the treatment solution without applying too much injection pressure to prevent soil fracturing, however this was challenging as biomass built up within the injection well and the surrounding filter pack. To reduce biomass clogging, the injection wells were purged and plunged using a surge block tool when the injection flow rates decreased and injection pressure increased. Additionally, the injection wells were treated with about $0.5 \mathrm{~L}$ of $3 \%$ hydrogen peroxide daily to dissolve the accumulating biomass.

At Sunderland, the treatment solution was applied for 33 days (from 14 August 2019 to 16 September 2019), and a total of $55 \mathrm{~m}^{3}$ of solution was injected replacing $58 \%$ of the native pore fluid in the targeted treatment zone. The treatment solution was applied for 34 days at Harborton (21 August 2019 to 22 September 2019), and a total of $17.6 \mathrm{~m}^{3}$ of solution was injected replacing $58 \%$ of the native pore fluid in the targeted treatment zone; the volume of pore fluid was calculated assuming a porosity of $50 \%$ and fully saturated initial conditions. 


\section{Chapter 4: Treatment Monitoring}

Several different methods of monitoring were used before, during, and after MID application to (i) obtain baseline values, (ii) monitor the seepage path and effectiveness of MID treatment, and (iii) check the longevity and viability of MID as a liquefaction mitigation method. These methods include measurements of pressure wave velocity, bulk electrical conductivity, flow rates and injection pressures, chemical analyses, in-situ moisture contents through instrumentation, sampling and lab testing, and measurements of the water table over time.

\section{1: Before Treatment}

Seismic cone penetration tests (SCPTs) were performed at both the Sunderland site and the Harborton site before the start of treatment to obtain baseline values of shear wave $\left(\mathrm{V}_{\mathrm{s}}\right)$ and pressure wave $\left(V_{p}\right)$ velocities, the GWT, and the soil's behavior type index $\left(I_{c}\right)$. These values were critical in determining which of these two sites would be better suited for additional in-situ testing using NHERI@UTexas large mobile shakers and the corresponding embedded sensor array. The results of the large mobile shaker tests are described in Preciado et al. 2021. Both Sunderland and Harborton profiles are shown in Figure 4.1.

Using the $\mathrm{I}_{\mathrm{c}}$ profiles, it was decided that the embedded sensor array would be installed at the Sunderland site. Sunderland had consistent $I_{c}$ values ranging between 2.6 and 2.95 with few outliers; Robertson 2009 correlates this $I_{c}$ range as silt mixtures - clayey silt to silty 
clay. Harborton had a range of values, indicating that the soil profile consisted of a mixture of sands, silts, clays, and organic soils.

Upon determining that the embedded sensor array was going to be installed at the Sunderland site, a direct push crosshole test (DPCH) and liquefaction screening tests (SC) were performed according to Stokoe et al. 2020; the screening test locations are seen in Figure 4.3. The embedded sensor array is composed of four pairs of 3D geophones located at different depths, with each pair having a metal source rod driven to the same depth. Four pore pressure transducers (PPTs) were embedded between each pair of 3D geophones, but at shallower depths as seen in Figure 4.2. Each source rod and 3D geophone pair act as a crosshole test, allowing for the collection of $\mathrm{V}_{\mathrm{s}}$ and $\mathrm{V}_{\mathrm{p}}$ data, and each PPT allows for GWT and $\Delta u$ measurements. $\mathrm{V}_{\mathrm{s}}, \mathrm{V}_{\mathrm{p}}$, and GWT data will be presented later; $\Delta u$ is not included in this paper. 


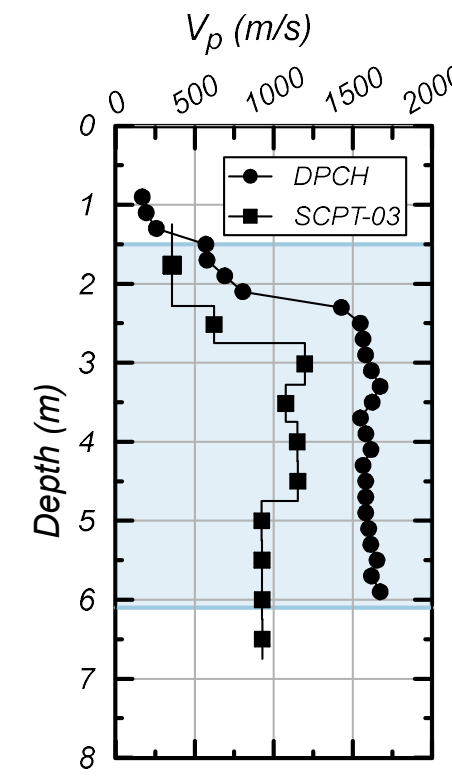

(a)
$V_{s}(\mathrm{~m} / \mathrm{s})$

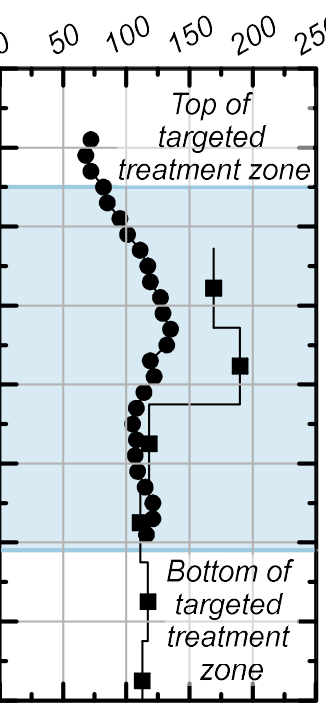

(b)
$I_{C}$

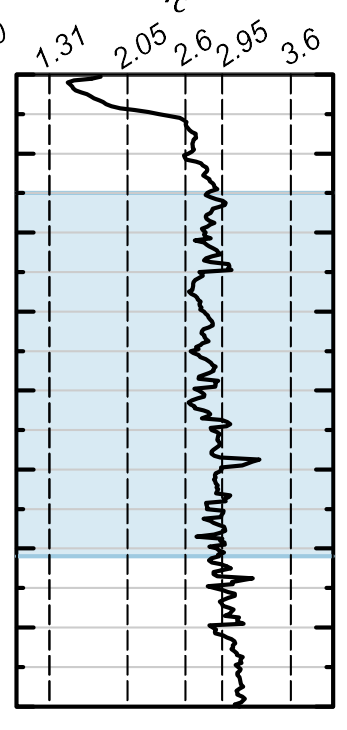

(c)

Sunderland

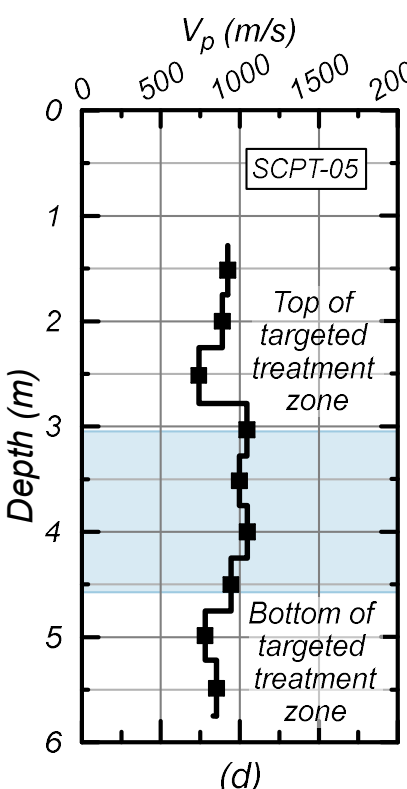

(d)
$V_{s}(m / s)$

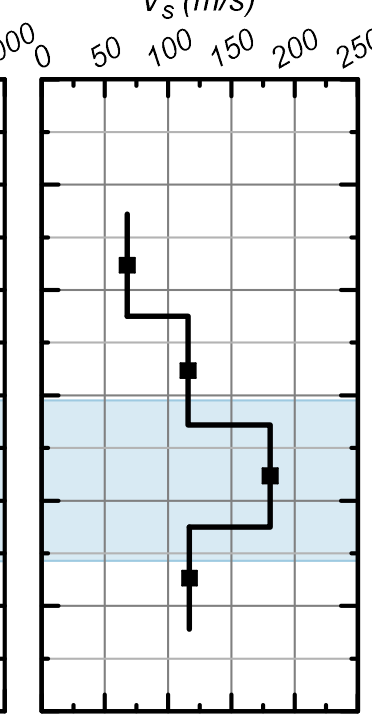

(e)

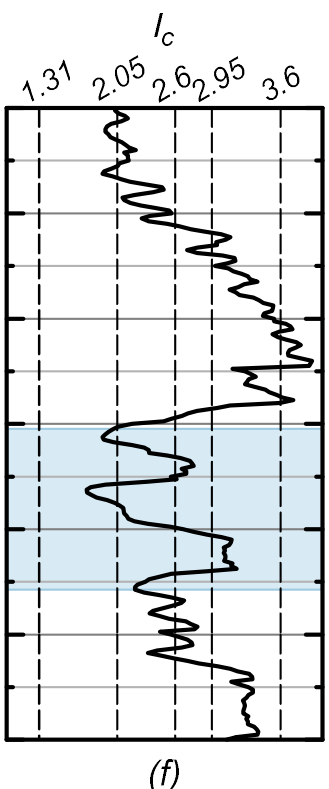

Harborton

Figure 4.1: The Vp, Vs, and Ic values of Sunderland $(a-c)$ and Harborton $(d-f)$. 

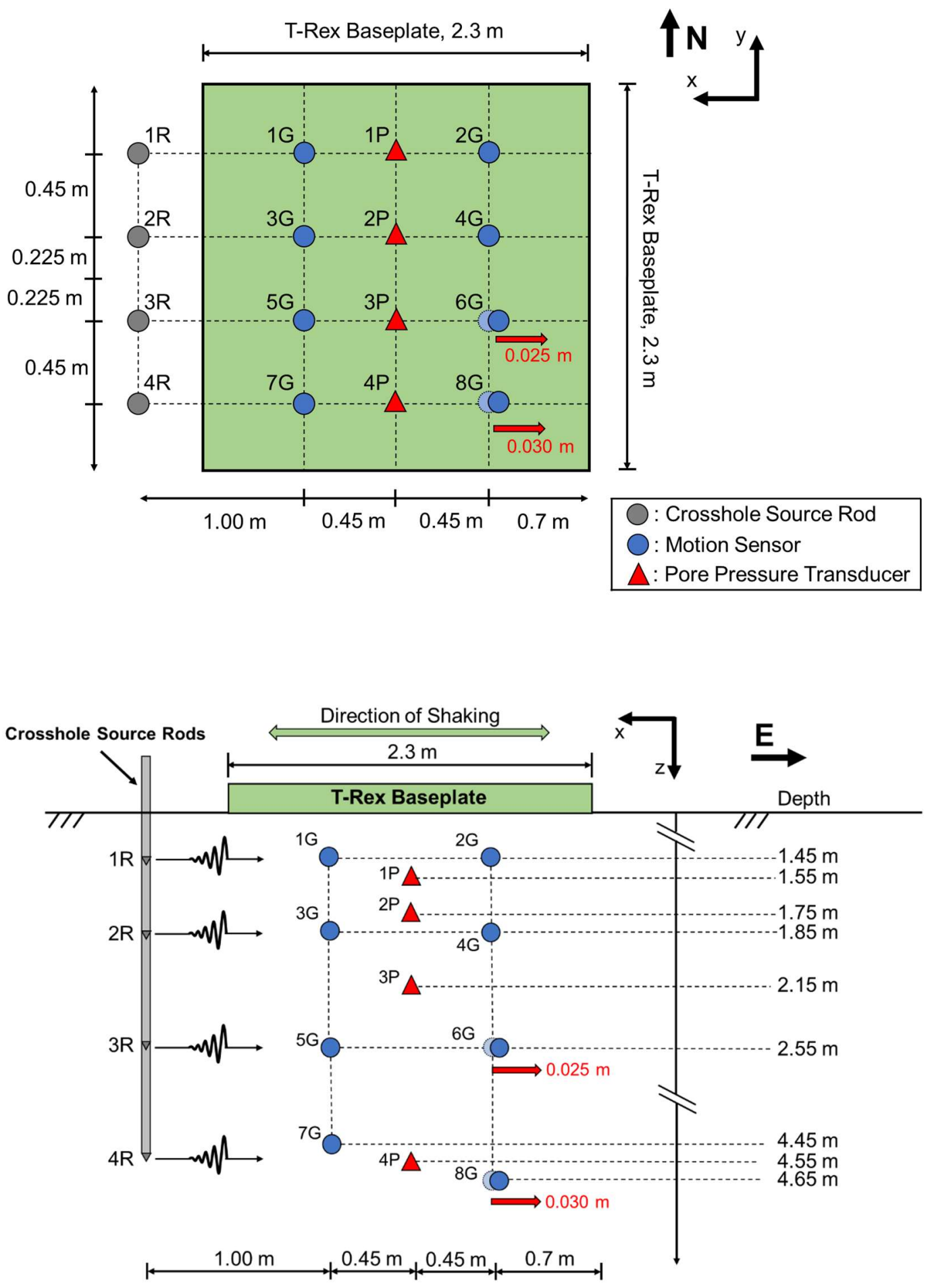

Figure 4.2: Top - top view of the embedded sensor array. Bottom - cross-section view of the $3 D$ geophones and PPTs with depth. 


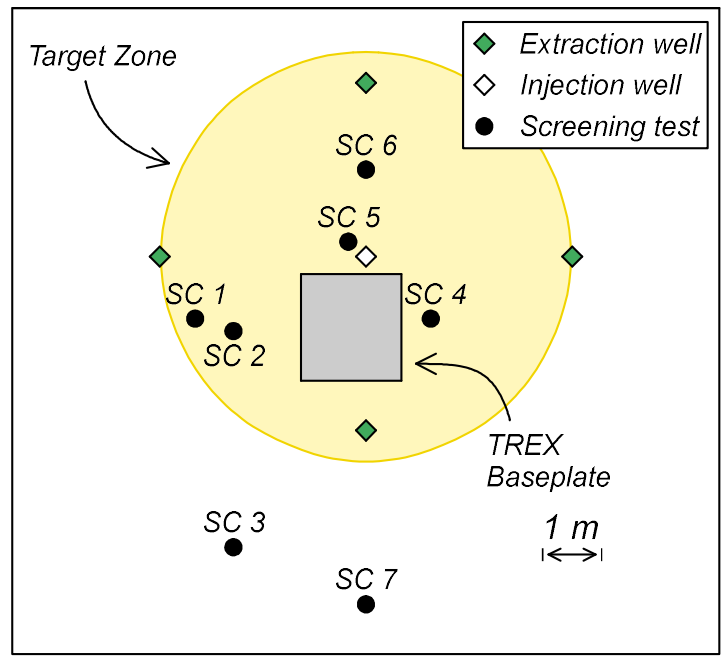

Figure 4.3: The Sunderland screening test locations relative to the TREX baseplate and the targeted treatment zone.

Once the array was in place, wells were drilled at both Sunderland and Harborton, although only Sunderland has the sensor array. The specifications were described previously in the Application Method section.

\section{2: During Treatment}

Injection flow rates, treatment solution volumes, injection pressures, electrical conductivity, and the chemical breakdown of the treatment solution were measured and recorded to monitor the effectiveness of the MID treatment and to track the seepage path and subsurface progress of the treatment solution.

\subsection{1: Injection Flow Rates}

Injection flow rates and volumes were recorded to study the relationship between volume of treatment solution injected and when MID reactions were observed. The flow rates at 
both sites are shown in Figure 4.4 and are estimated from (i) changes in water pressure over a one-hour moving average measured by a submersible datalogger (CTD-divers, Van Essen instruments) in the injection tank (solid line), and (ii) observed values at an in-line flowmeter (green circles). In several cases the flowmeter values are larger than those estimated from change in water pressure. These discrepancies are most common soon after starting pumping injection.

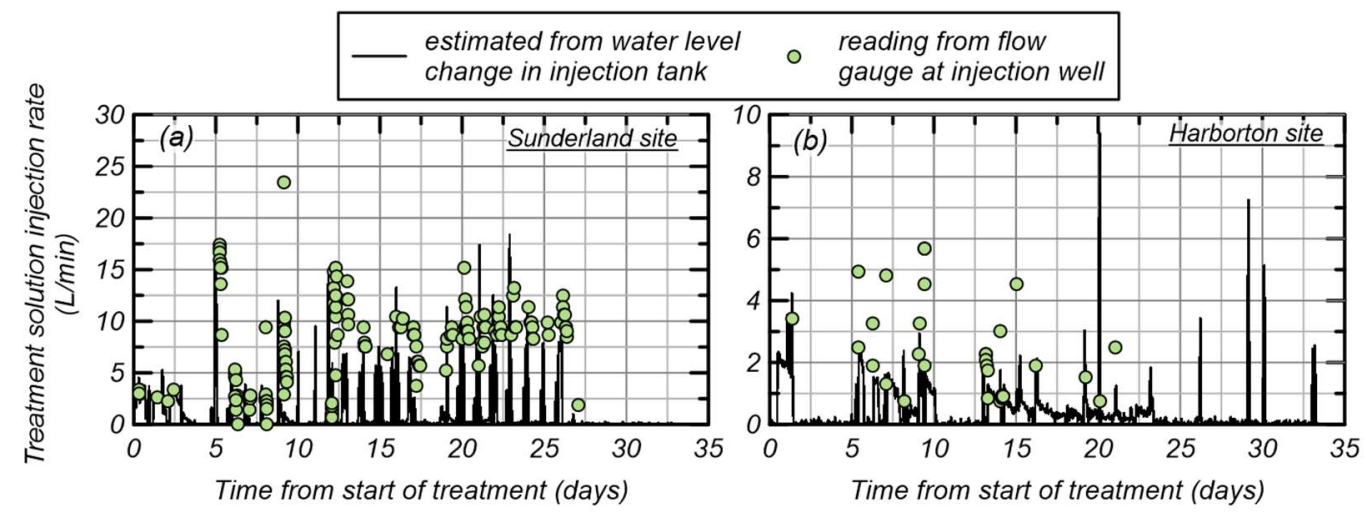

Figure 4.4: Injection treatment solution flow rate estimated from change of water levels in injection tank and flow gauge (a) Sunderland site, (b) Harborton site.

At Sunderland, the injection flow rates from day 0 to 5 were less than $4 \mathrm{~L} / \mathrm{min}$. During this period, the treatment solution was gravity fed from the injection tank that was placed on a hill approximately $2 \mathrm{~m}$ higher than the injection well. Initial flow rates decreased after day 3, likely due to biomass buildup at the well screens and filter pack. Injection pumping was implemented on day 5 at Sunderland, and after day 12 the additional measures to mitigate biomass clogging were fully implemented. Pumped injection was used from the start of treatment at Harborton and methods to prevent biomass clogging were used after day 5. With injection by pumping and biomass clogging mitigation measures, the flow rates were 
about 6 to $15 \mathrm{~L} / \mathrm{min}$ at Sunderland and 1 to $2 \mathrm{~L} / \mathrm{min}$ at Harborton. The difference in flow rates between the two sites is attributed to the different length of the well screens and distinct hydrogeologic properties.

The cumulative volumes of injected treatment solution are presented in Figure 4.5. The total volume was approximately $55 \mathrm{~m}^{3}$ at Sunderland and $18 \mathrm{~m}^{3}$ at Harborton. These volumes are equivalent to approximately $60 \%$ of the total pore volume of the target zone, assuming $50 \%$ porosity at both sites. The end of treatment was primarily driven by schedule constraints; however, these injection volumes were sufficient to achieve desaturation at both sites.
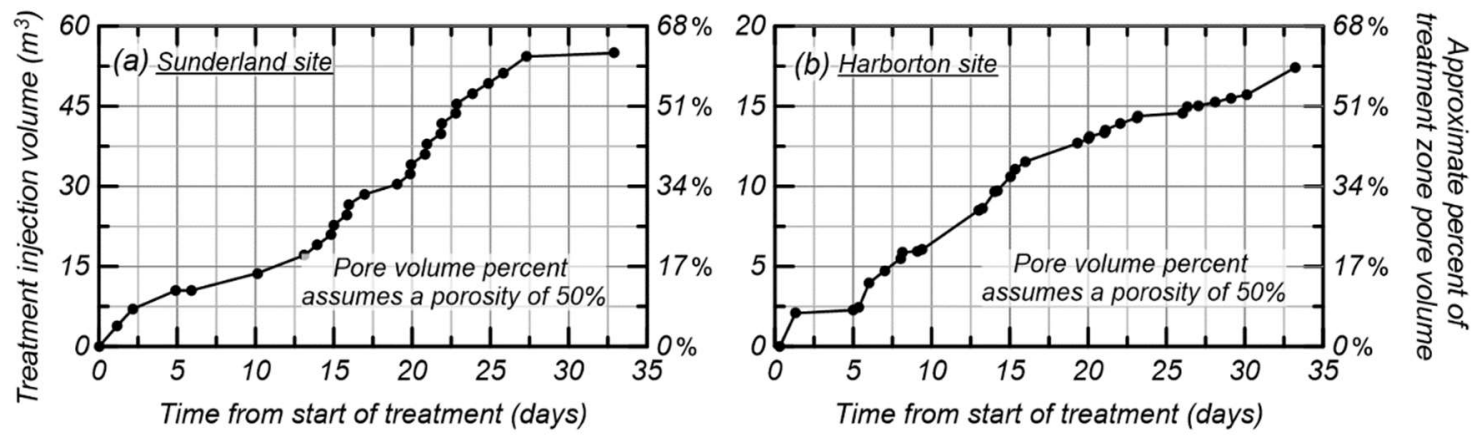

Figure 4.5: Cumulative volume of injected treatment solution at (a) Sunderland site, and

\section{(b) Harborton site.}

Pressures at the injection well head are shown in Figure 4.6. The solid line shows the pressure contribution from the treatment solution height in the injection tank. The green symbols show the pressure reading at the well head from a pressure gauge, which was composed of pressure from the treatment solution height and injection pumping. There are 
elevated pressures at Sunderland for days 0 to 12 that then reduced after day 12 when biomass mitigation measures were implemented. There are instances when the measured pressure is less than the pressure from the solution height; this may be due to biomass buildup and hydraulic head loss in the plumbing from the injection tank. Injection pressures measured at the well head at Sunderland after day 12 remained below $30 \mathrm{kPa}$, at Harborton throughout treatment values remained below $25 \mathrm{kPa}$.

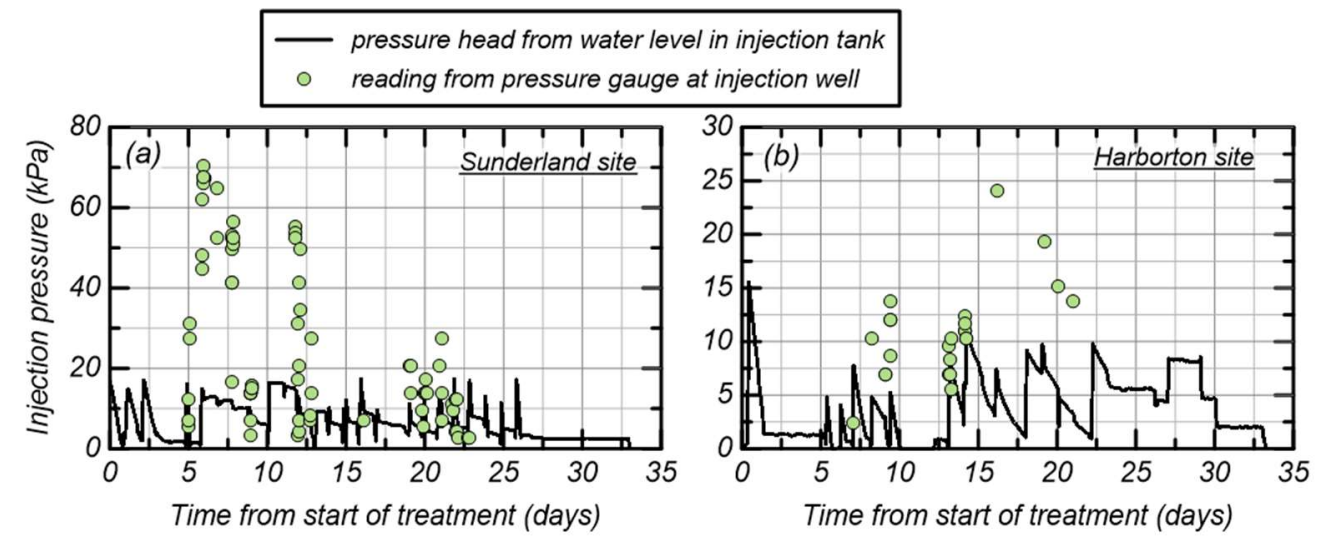

Figure 4.6: Injection pressures for (a) Sunderland site, and (b) Harborton site.

\subsection{2: Electrical Conductivity}

Electrical conductivity (EC) measurements provided information on subsurface migration of the treatment solution and progress of the MID reaction. The EC relates to fluid salinity. Since the treatment solution had a larger salinity than the native groundwater, the EC measured in the treatment solution was larger than the EC measured for native groundwater. The treatment solution substrates are all dissolved salts and the MID reaction products are either gas, solid minerals, or biomass; therefore, the EC is also a direct 
measure of substrate consumption. Specifically, decreasing EC in the treatment solution was a sign that the MID reaction was taking place.

EC was measured in the injection and extraction tank water with the submersible dataloggers, these results are plotted in Figure 4.7. At Sunderland, the initial EC in the treatment solution (Figure 4.7a) was approximately $10 \mathrm{mS} / \mathrm{cm}$ from day 0 to day 12 and then increased to approximately $20 \mathrm{mS} / \mathrm{cm}$ when the solution concentration increased from 0.05 to $0.10 \mathrm{~mol} / \mathrm{L}$. Decreasing EC in the Sunderland injection solution from day 2 to day 12 indicated that the MID reaction was occurring in the injection tank prior to injection. After day 12 the treatment solution concentration was increased, and city water was used to mix the treatment solution which slowed the biochemical conversion in the injection tank. EC of the treatment solution at Harborton (Figure 4.7b) was about $10 \mathrm{mS} / \mathrm{cm}$ for the $0.05 \mathrm{~mol} / \mathrm{L}$ treatment solution. Decreasing EC of the treatment solution in the injection tank was observed after day 12 at Harborton, which indicated an increased metabolic conversion rate. Considering no additional nutrients or trace minerals were added to Harborton treatment solutions, it is likely that the extracted groundwater used for the treatment solution was the source of these nutrients, allowing accumulation of indigenous denitrifying microbes and increased microbial activity in the injection tank. 


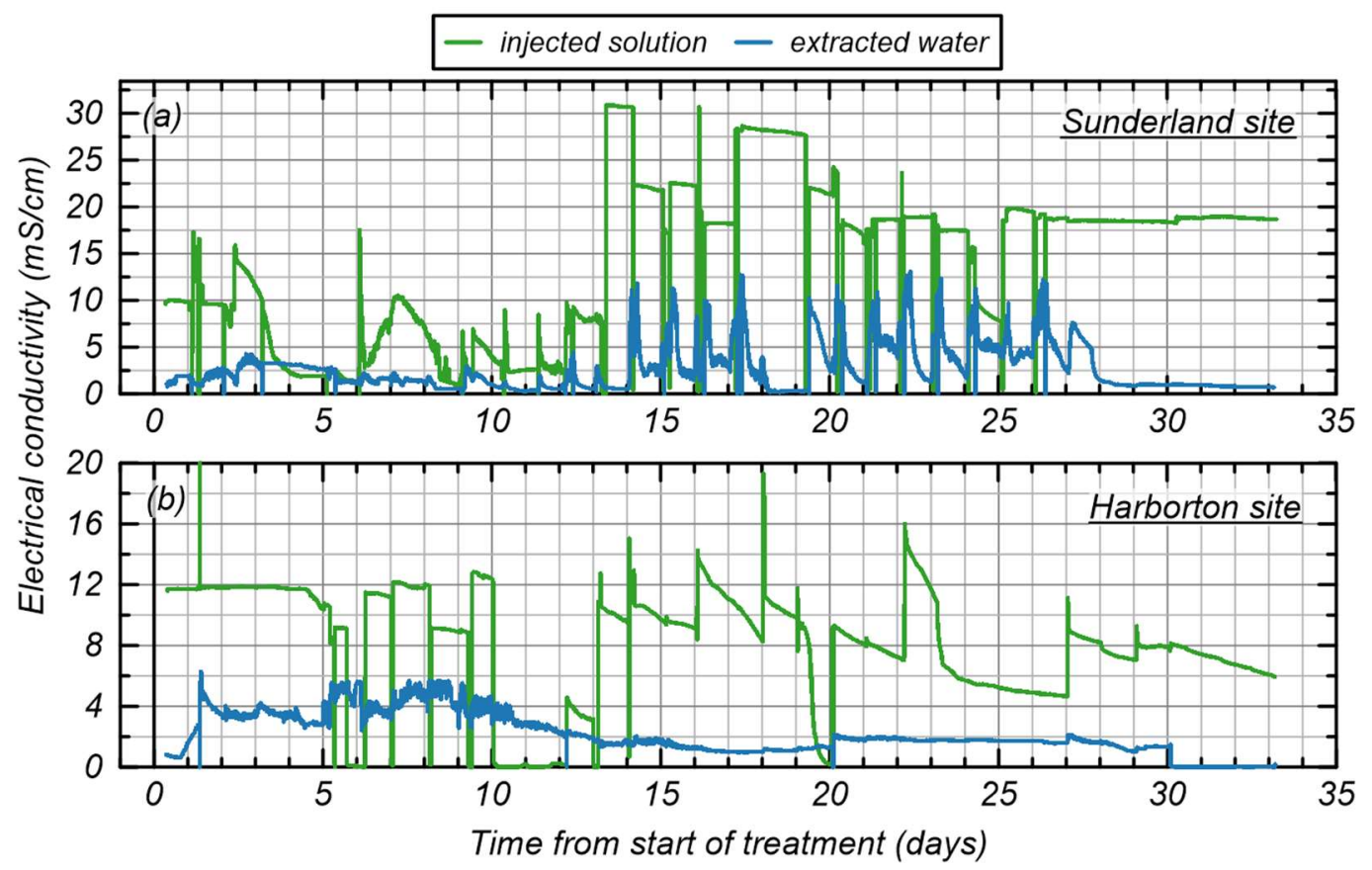

Figure 4.7: Electrical conductivity measured by suspended divers in the treatment solution and the extracted water at (a) Sunderland and (b) Harborton.

EC at the extraction tank reflects the average value of water from the four extraction wells. The datalogger was installed in a $1 \mathrm{~L}$ container that collected extracted water before the water overflowed to fill the extraction tank. This allowed changes in EC to be constrained over a narrower time range than if the datalogger was suspended in the extraction tank water. The background level of EC was characterized with groundwater extracted to mix the first treatment solution and was approximately $1.0 \mathrm{mS} / \mathrm{cm}$ at Sunderland and $0.7 \mathrm{mS} / \mathrm{cm}$ at Harborton. Increased EC of the extracted groundwater signaled that the treatment solution had traveled from the injection well to one or more extraction wells. At both sites, the extracted groundwater throughout treatment had lower EC than the injected treatment 
solution, likely due to the combined effects of substrate consumption during the MID reaction and mixing with native groundwater.

Throughout treatment at Sunderland, EC of the extracted water increased during injection pumping and decreased under gravity fed injection. The relationship is closely examined in Figure 4.8 which shows injection volume, and EC of extracted and injected water from 12:00 on day 14 to $12: 00$ on day 15 . The average injection rate was $6 \mathrm{~L} /$ min during injection pumping (black line), and $0.22 \mathrm{~L} / \mathrm{min}$ during gravity feed (dashed black line). Injection pumping started at 13:30 on day 14, and an increase in extracted water EC was measured at 14:00 (i.e., 30 min after injection pumping started). At 15:30 the injection was switched to gravity feed and extracted water EC decreased until 19:00. A similar pattern is observed the following day when the remaining treatment solution was injected by pumping starting at 07:30 and extracted water EC started increasing at 08:05. The relation between injection flow rate and EC in the extracted groundwater illustrates how the reactive transport is affected by the hydrological boundary conditions. The increase in EC in the extraction wells indicates the presence of highly preferential pathways during injection pumping that channel the treatment solution between the injection well and at least one extraction well. This is also supported by Figure 4.7a that shows a notable increase in extracted water EC following the increase of the treatment solution concentration after day 12 . 


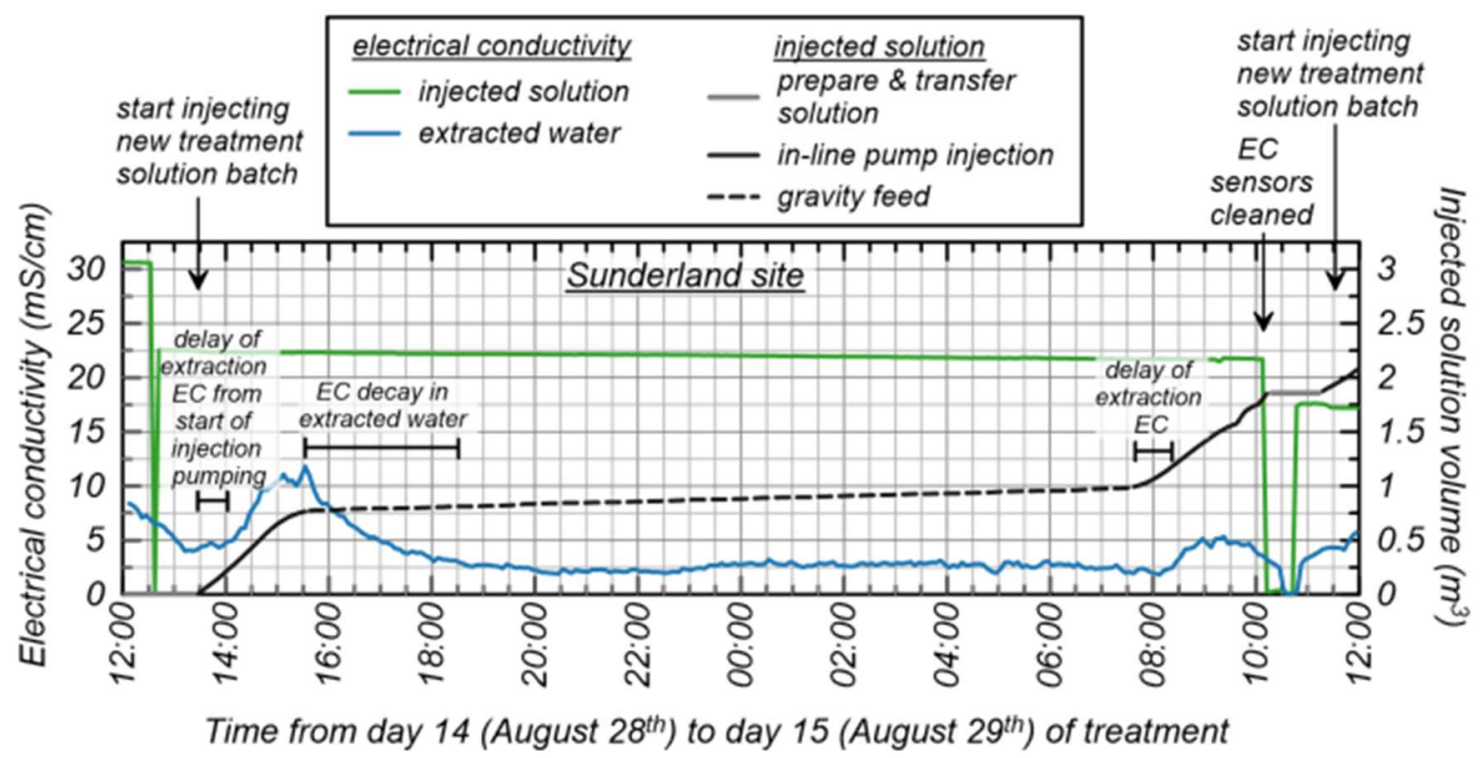

Figure 4.8: Electrical conductivity in the injection and extraction tanks 24 hours (Sunderland).

At the Harborton site, extracted water EC does not appear to be related to injection pumping. As shown in Figure 4.7b, extracted water EC increases from background EC about 0.5 days into treatment, and remains between 3 and $6 \mathrm{mS} / \mathrm{cm}$ until day 10 when the EC decreases to 1 to $2 \mathrm{mS} / \mathrm{cm}$. The decrease in EC is likely due to the increased MID reaction rate in the subsurface, where after 10 days of treatment the MID reaction was occurring at a sufficiently fast rate that most substrates were consumed by the point the injected solution reached the extraction wells.

\subsection{3: Strip Tests}

Additional monitoring of the treatment solution and extracted groundwater was performed to estimate concentrations of $\mathrm{NO}_{3}{ }^{-}$and $\mathrm{NO}_{2}{ }^{-}$, carbonate hardness $(\mathrm{KH})$, general hardness $(\mathrm{GH})$, and $\mathrm{pH}$. Measurements were performed with color changing strip tests (API ${ }^{\circledR}$ 5-in- 
1 Test Strips) by submersing the strips into fluid and matching the resultant color to a value range specified by the manufacturer. Often the fluid was diluted to bring concentrations within the test range, and the results were corrected for the dilution factor. The strip tests do not have high accuracy; however, they were performed regularly to qualitatively observe larger trends in the progress of the MID reaction between the injection well and extraction wells.

The strip test results for the injected solution and extracted water at the Sunderland site are summarized in Figure 4.9(a-e). Generally, the $\mathrm{NO}_{3}{ }^{-}$concentration decreased from 800$8000 \mathrm{mg} / \mathrm{L}$ in the injected solution to $0-3200 \mathrm{mg} / \mathrm{L}$ in the extracted water (Figure $4.9 \mathrm{a}$ ). Figure $4.9 \mathrm{~b}$ shows some presence of $\mathrm{NO}_{2}^{-}$, an intermediate product of reactions (1) and (2), in the extracted water. Slightly elevated $\mathrm{NO}_{3}{ }^{-}$and $\mathrm{NO}_{2}{ }^{-}$concentrations in extracted water may be due to seepage through a highly preferential pathways that did not complete the MID reaction before reaching the extraction well. Many of these elevated concentrations were measured during injection pumping with similar trends as observed for EC and discussed for Figure 4.8. Since values decrease during gravity feed, the treatment solution outside of this highly preferential pathway likely completed the conversion from $\mathrm{NO}_{3}{ }^{-}$to $\mathrm{N}_{2(\mathrm{~g})}$. $\mathrm{pH}$ values in Figure 4.9 do not show overall differences between the injected solution and extracted groundwater, which likely reflects that the MID reaction is self-buffering. Data for $\mathrm{KH}$ and $\mathrm{GH}$ were obtained during the later period of treatment (Figure 4.9d,e). $\mathrm{KH}$ is a measure of $\mathrm{HCO}_{3}{ }^{-}$and $\mathrm{CO}_{3}{ }^{2-}$ in solution and may increase due to reactions (1) and (2), then decrease due to reactions (3) and (4). GH closely relates to the concentration of $\mathrm{Ca}^{2+}$ and other divalent cations. The reduction of GH and $\mathrm{KH}$ between the injection and 
extraction wells may be attributed to consumption of inorganic carbon and $\mathrm{Ca}^{2+}$ by bioprecipitate (reactions 3 and 4), alternatively cation exchange (discussed above) may be consistent with decreasing GH. Final indicator strip tests were performed at all the Sunderland wells on day 33. These final measurements report very little $\mathrm{NO}_{3}^{-}, \mathrm{NO}_{2}^{-}, \mathrm{KH}$, and $\mathrm{GH}$, and a $\mathrm{pH}$ about 7.5 , signaling that the MID reaction had completed in the subsurface by this time. However, it should be noted that the wells were not completely purged according to standard sampling procedures (EPA 2013). Instead, approximately 15 L of water was extracted before collecting water for a strip test.

Strip test data from the Harborton site generally indicate that the MID reaction completed in the subsurface. The results in Figure 4.9f show that $\mathrm{NO}^{-}$concentrations were reduced to 0 between the injection and extraction wells. The presence of $\mathrm{NO}_{2}^{-}($Figure $4.9 \mathrm{~g})$ in the injected solution may be an intermediate product of the MID reaction taking place in the injection tank; however, the concentration decreased to 0 in the extracted water, likely due to subsurface completion of the denitrification reaction. Figure $4.9 \mathrm{~h}$ shows a general $\mathrm{pH}$ increase between injection and extraction wells; while a $\mathrm{pH}$ increase is expected from the $\mathrm{OH}^{-}$product in reaction (1), the slight increase in $\mathrm{pH}$ indicates possible $\mathrm{CaCO}_{3}$ precipitation and $\mathrm{CO}_{2}$ gas formation (reactions 3 and 4) that buffered a larger $\mathrm{pH}$ increase. $\mathrm{KH}$ and $\mathrm{GH}$ decreased between injection and extraction water (Figure 4.9i,j) which is attributed to possible occurrence of reactions (3) and (4). 


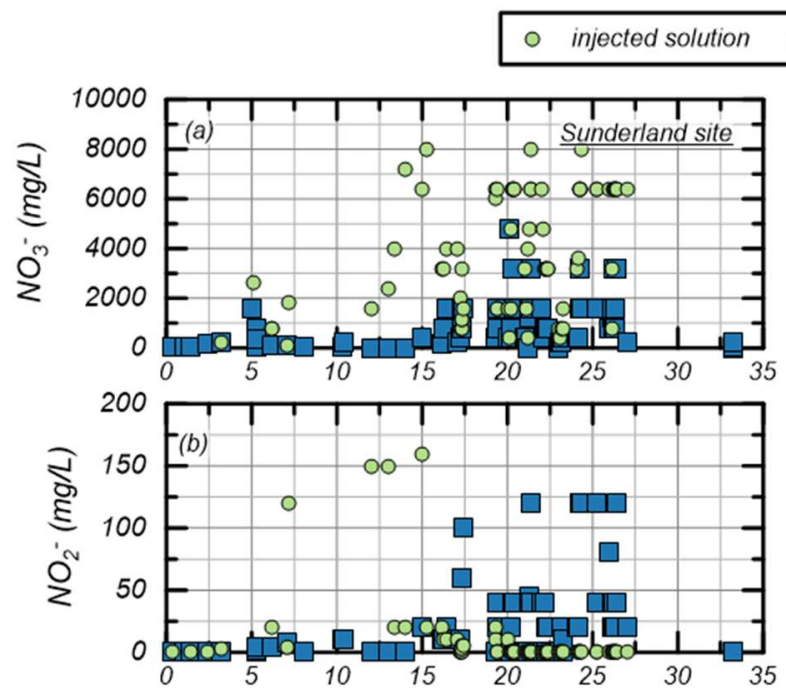

extracted water
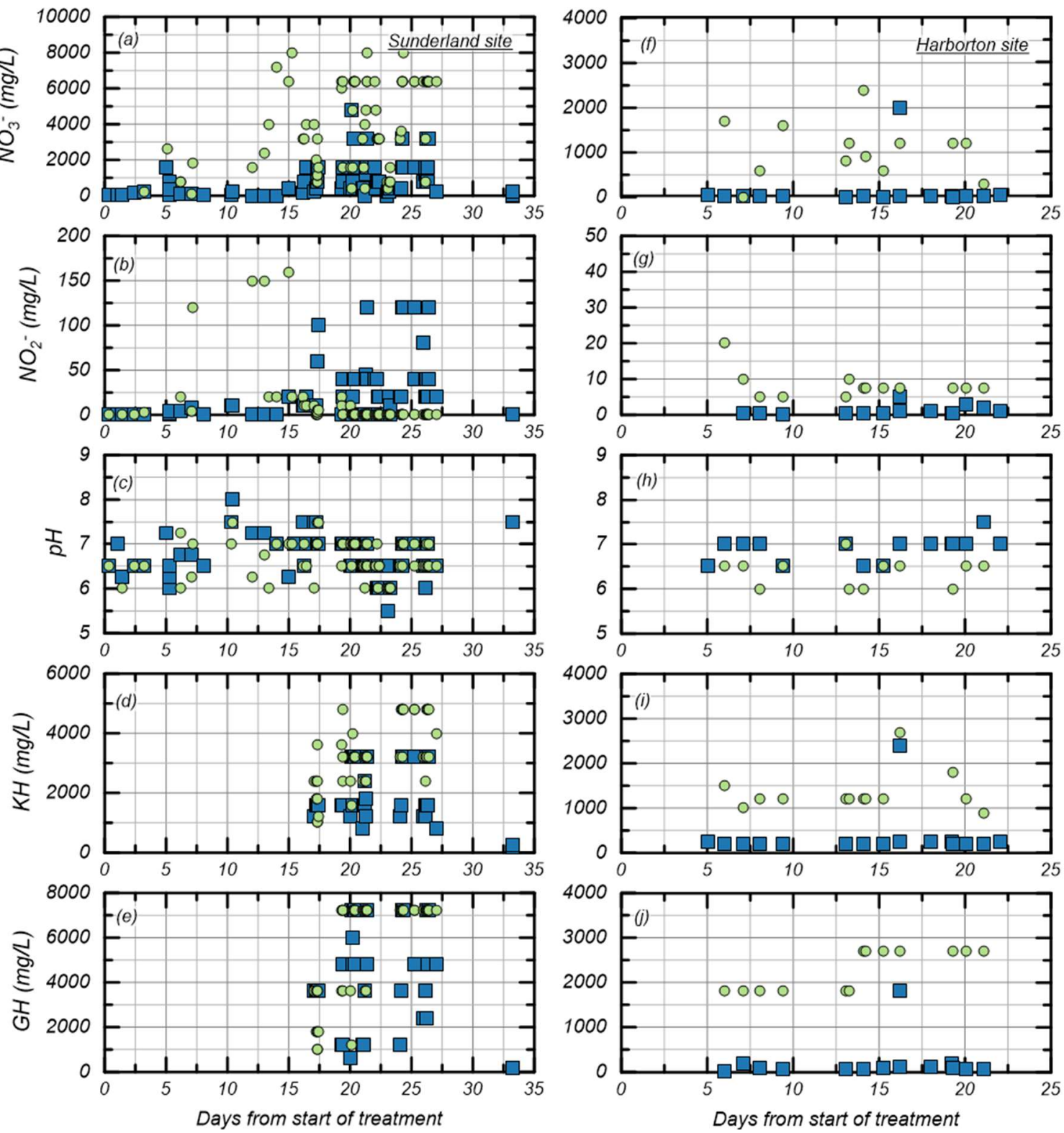

Figure 4.9: Strip test results to indicate progress of MID reaction and water quality for the injected solution and extracted groundwater for (a-e) Sunderland and $(f-j)$ Harborton sites. 


\section{3: After Treatment}

To check the longevity and viability of MID as a liquefaction mitigation method, $\mathrm{V}_{\mathrm{p}}$, subsurface bulk EC, and in-situ moisture contents were monitored both during and after the MID field trials, while direct saturation ratio measurements and GWT levels were monitored solely after treatment.

\subsection{1:Compression Wave Velocity $\left(V_{p}\right)$}

$\mathrm{V}_{\mathrm{p}}$ was monitored at both sites, as an indication of change in $\mathrm{S}_{\mathrm{r}}$. For soils with $\mathrm{S}_{\mathrm{r}}>99.5 \%$, the measured $\mathrm{V}_{\mathrm{p}}$ will be approximately $1500 \mathrm{~m} / \mathrm{s}$. Theoretically, when $\mathrm{S}_{\mathrm{r}}$ drops below approximately $98.5 \%, \mathrm{~V}_{\mathrm{p}}$ drops to about $400 \mathrm{~m} / \mathrm{s}$ (Valle-Molina and Stokoe, 2012). Posttreatment $\mathrm{V}_{\mathrm{p}}$ measurements were performed with SCPT both inside and outside of the target treatment zone to (i) evaluate MID in various locations in the target zone, and (ii) see if the treatment solution had travelled beyond the target treatment zone. The location of post-treatment SCPT-measured seismic velocity profiles are shown in Figure 2.1 and Figure 2.4 for both sites; Figure 4.10 shows the SCPT $V_{p}$ profiles for both sites. At Sunderland, post-treatment $V_{p}$ measurements consistently show $V_{p}$ around $400 \mathrm{~m} / \mathrm{s}$ in the target zone, indicating the MID successfully desaturated the soil at these profile locations. The SCPT profile outside the treatment zone at Sunderland shows $V_{p}<600 \mathrm{~m} / \mathrm{s}$ until $5 \mathrm{~m}$ bgs; this may indicate that there was migration of the treatment solution or the produced gas outside the target zone. Similarly, the post-treatment $V_{p}$ profiles at the Harborton site indicate reduced $V_{p}$ from pre-treatment conditions both inside and outside the target treatment zone. 


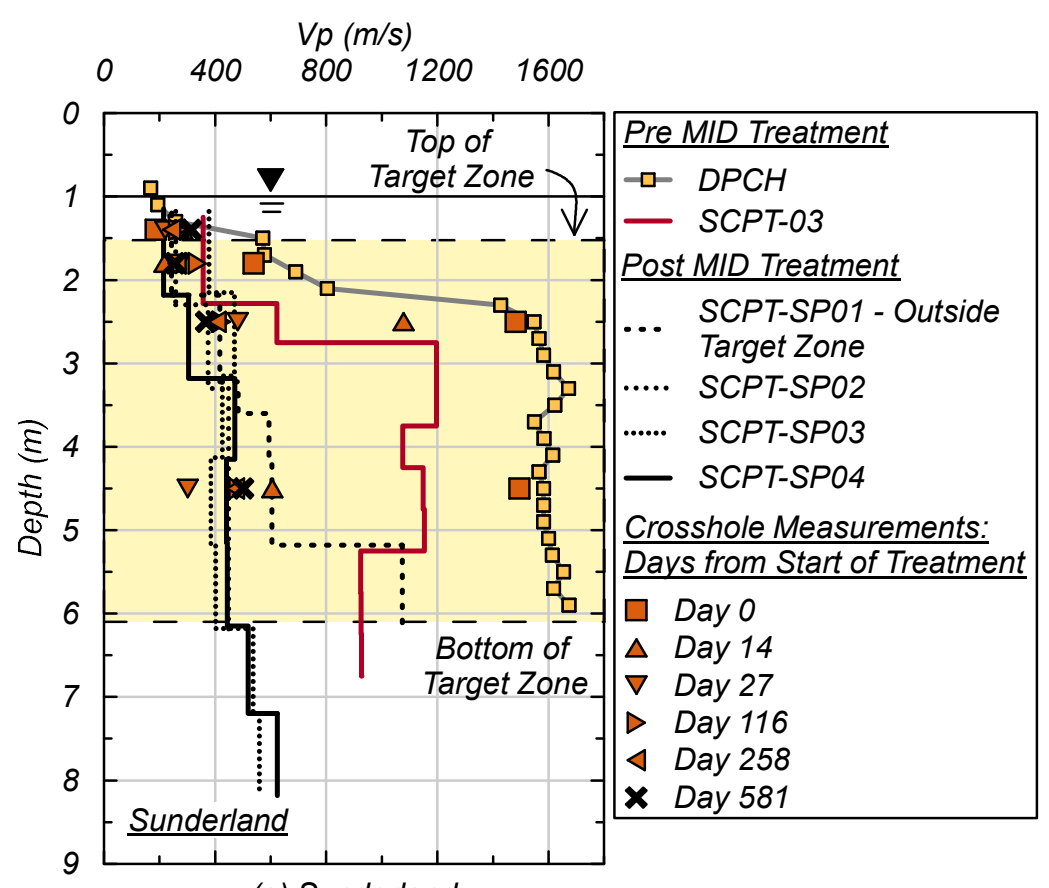

(a) Sunderland

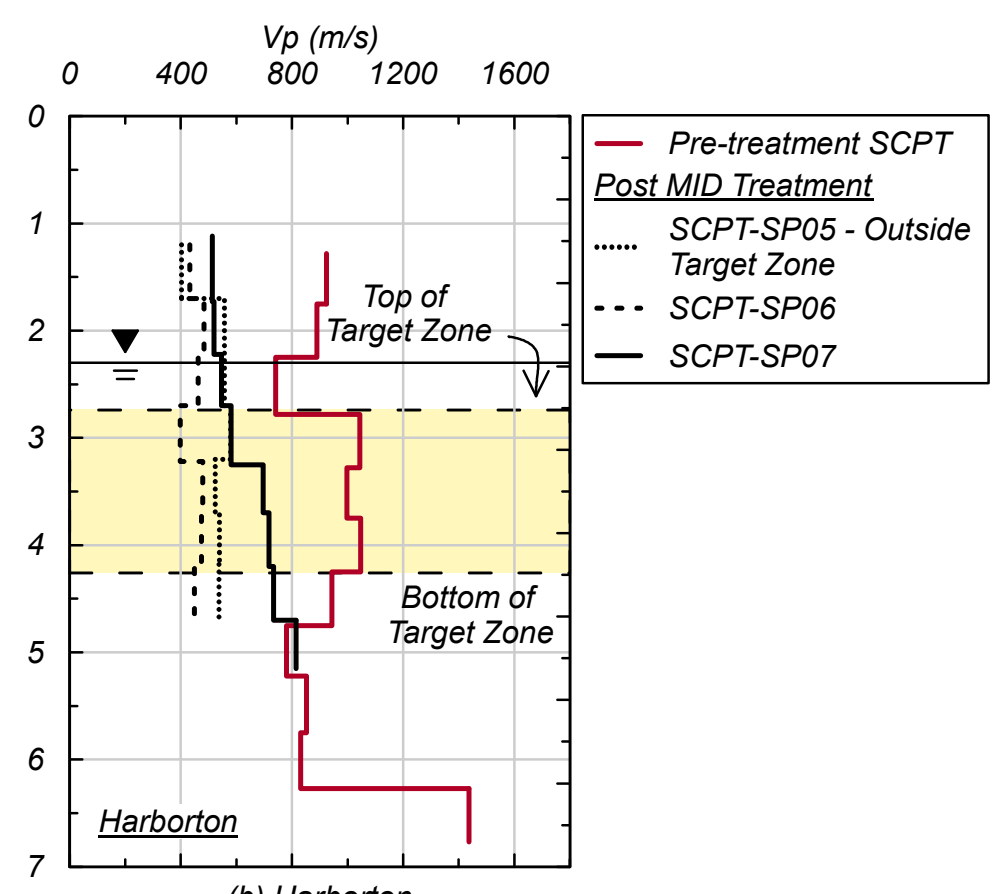

(b) Harborton

Figure 4.10: Vp measurements using SPCTs, DPCH, and crosshole methods at (a) Sunderland and (b) Harborton. 
The crosshole array installed at the Sunderland site allowed for regular $V_{p}$ measurements. Instructions for how to install the required software, set up the equipment, collect, filter, and format the data, and how to analyze the data can be found in Appendices I - N. The array consists of seismic source rods and geophones within the target treatment zone (Figure 2.1 and Figure 4.2). The array measured $V_{p}$ at $1.45 \mathrm{~m}, 1.85 \mathrm{~m}, 2.55 \mathrm{~m}$, and $4.55 \mathrm{~m}$ bgs with the distance between geophones being $0.90 \mathrm{~m}$ (i.e., $\mathrm{V}_{\mathrm{p}}$ is averaged over $0.90 \mathrm{~m}$ ).

The $V_{p}$ and $V_{s}$ arrival times at each receiver were manually selected based on the geophone voltage time series. In this study, both upward and downward impacts were imposed on the source rod to create reversed polarities in the time series, which helps determine wave arrival times. These arrival times and the manual selections can be seen in Appendices O, $\mathrm{P}$, and Q, for data collected before, during, and after treatment, respectively. Examples of these time series and selection of pressure wave arrival times are shown in Figure 4.11 for measurements taken at $2.55 \mathrm{~m}$ bgs performed 5 days before treatment and 43 days after the start of treatment. The data in Figure 4.11 show how the pressure wave travel time between the two receivers increased over the course of treatment. It can also be noted that the pressure wave amplitude reduced following MID treatment, which is also an indication of decreasing $\mathrm{S}_{\mathrm{r}}$ (Valle Molina \& Stokoe, 2012).

In addition to $V_{p}$ measurements, the sensor array was used for in-situ cyclic testing with the NHERI@UTexas shaker trucks T-Rex and Rattler. Pre-treatment analysis of the soil is described in Preciado et al. 2021; post-treatment analyses are still ongoing at this time. 
Crosshole $\mathrm{V}_{\mathrm{p}}$ measurements were taken before the treatment solution was applied, daily during the treatment application, and at least monthly since the treatment ended. The time series of $V_{p}$ is shown in Figure 4.12. Measured $V_{p}$ dropped to about $400 \mathrm{~m} / \mathrm{s}$ in all sensors about 15 days into MID treatment. $V_{p}$ remained between $200 \mathrm{~m} / \mathrm{s}$ and $600 \mathrm{~m} / \mathrm{s}$ for 20 months after treatment indicating that $S_{\text {r }}$ persists at less than $98.5 \%$.

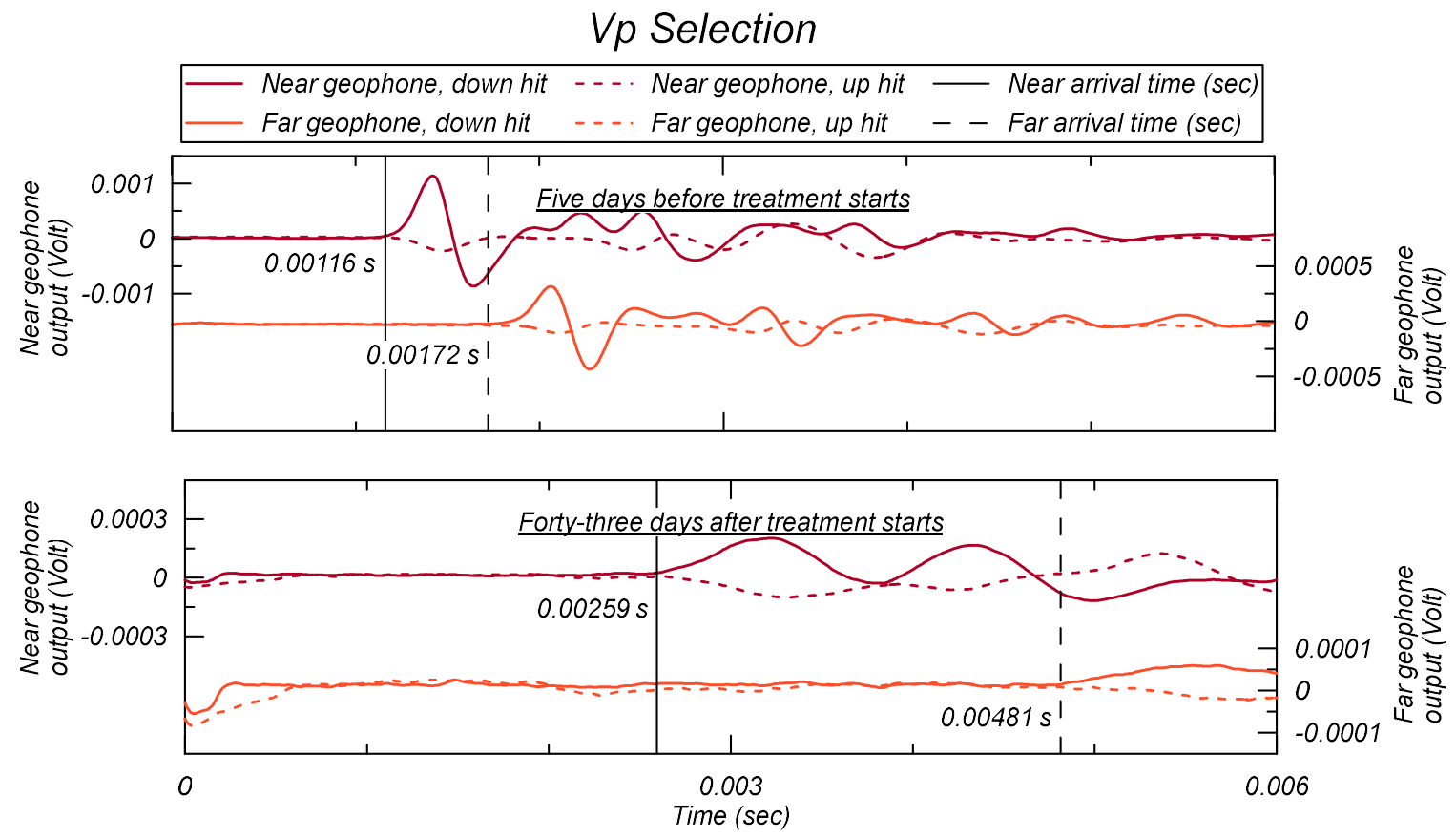

Figure 4.11: Travel-time records of pressure-wave measured during the crosshole testing at $2.55 \mathrm{~m}$ depth performed pre-treatment (5 days before start of treatment) and post-treatment (43 days after the start of treatment). 


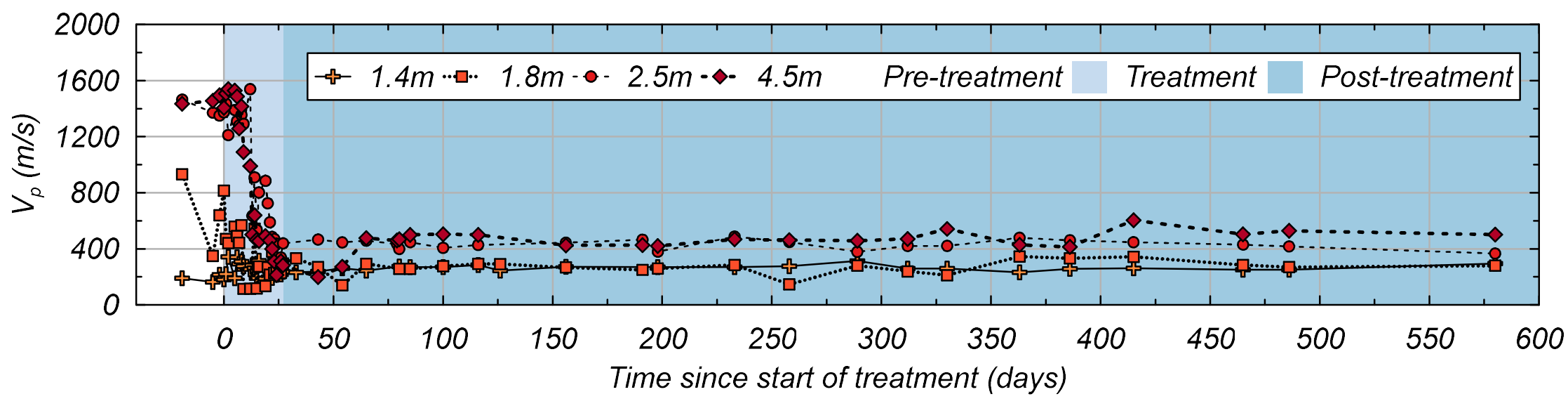

Figure 4.12: Vp crosshole time history measurements at Sunderland. 


\subsection{2:Electrical Conductivity}

TEROS 12 (Metergroup) sensors were installed at both sites in a vertical array to measure the bulk EC and the dielectric capacitance of the soil and pore fluid. The sensors were installed at discrete depths between $0.9 \mathrm{~m}$ and $5.8 \mathrm{~m}$ mid-way between the injection well and an extraction well (Figure 2.1 and Figure 2.4). The individual TEROS sensors with regards to their respective targeted treatment zone are shown in Figure 4.13. Six sensors were installed at Sunderland with all located within the target zone except for the $0.9 \mathrm{~m}$ depth sensor; five sensors were installed at Harborton with the sensors at $3.4 \mathrm{~m}$ and $4.0 \mathrm{~m}$ depths located inside the target zone. Sensors at Sunderland were installed by lowering the sensors into the borehole and backfilling with native soil, whereas at Harborton the sensor pins were inserted into the borehole sidewall which is the proposed methodology by the manufacturer. It is expected that the installation method affected the measurements as discussed below. The TEROS 12 sensors rely on a battery source to relay the captured data to a cloud storage system. These batteries were stolen for a period of time; a linear interpolation was done to fill in the lost data. 

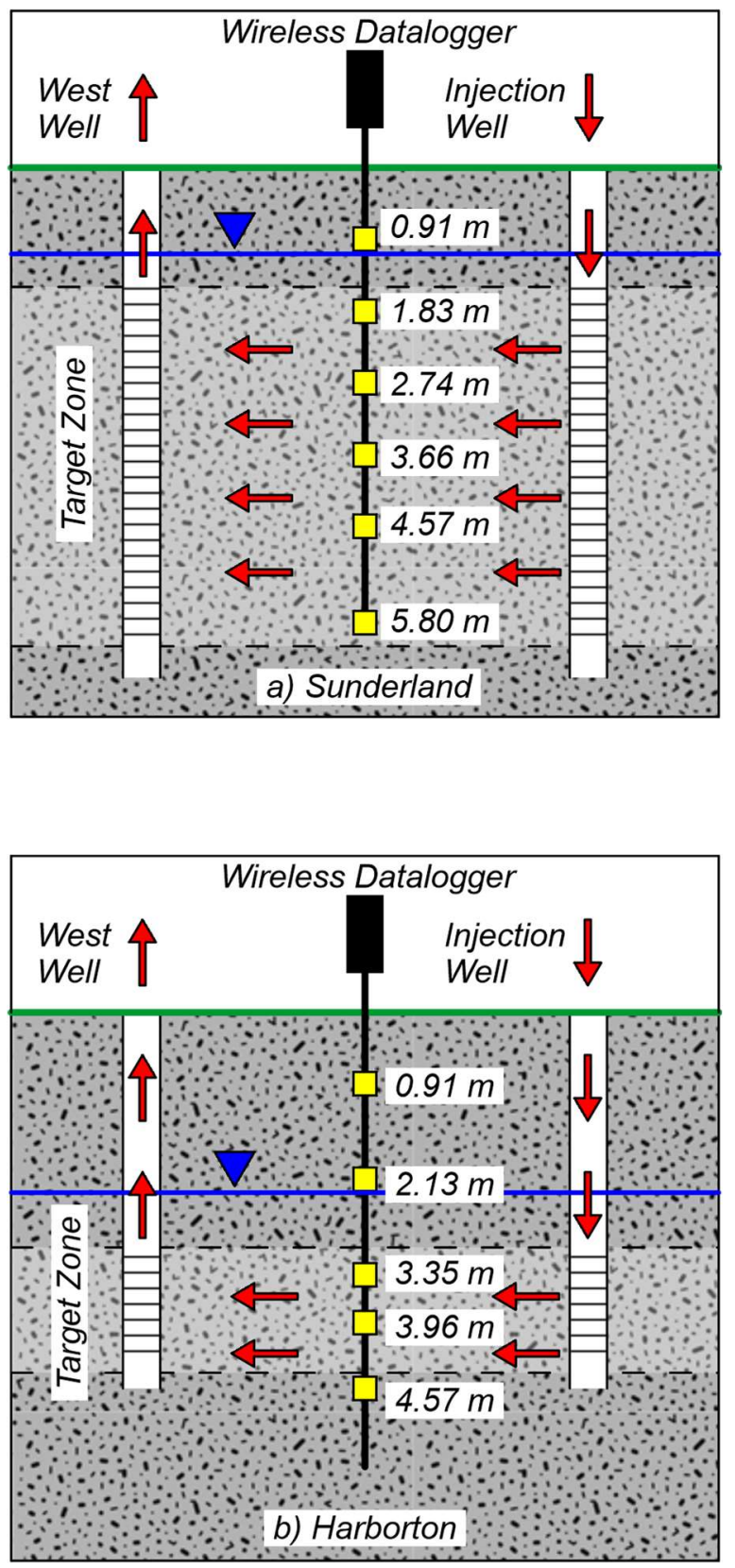

Figure 4.13: Side view of the TEROS sensors at (a) Sunderland and (b) Harborton, in relation to their respective target treatment zone. 
The bulk EC measured by the sensors relate to changes of groundwater salinity, as previously described. The bulk EC over time measured by the TEROS sensor array are plotted in Figure 4.14. At the Sunderland site (Figure 4.14a), the seepage front arrived at the sensor array at approximately day 22 as indicated by the sharp increase in bulk EC. A rise in bulk EC was also observed at the shallowest sensor that was above the treatment interval, indicating the injected substrate solution seeped above the screened interval. It appears that the treatment solution did not reach all the sensors in the targeted treatment interval; for example, there is no noticeable increase in bulk EC at $2.7 \mathrm{~m}$ that can be attributed to the treatment solution. The variability in the observed EC indicates that the treatment solution was not homogeneously distributed throughout the target zone, likely due to preferential flow paths. The bulk EC continued to rise even after the injection of treatment solution was stopped, which was unexpected. The gradual increase in EC after injection could be the result of migration of unreacted substrates from the high permeable flow paths to zones of lower permeability through diffusion or buoyancy driven convective transport. The observation that EC did not drop to background values could be the result of an incomplete conversion, an excess of calcium acetate remaining, or due to cation exchange, which is common in fine-grained soils. For example, calcium ions in the substrate solution may replace sodium ions in clay minerals, leaving highly soluble sodium bicarbonate in the groundwater.

At Harborton, the treatment solution arrived at the sensor at $4.6 \mathrm{~m}$ depth around day 6 of treatment, as shown in Figure 4.14b. Measured bulk EC increased at the $4.0 \mathrm{~m}$ and $3.4 \mathrm{~m}$ sensors on day 10 and at the $2.1 \mathrm{~m}$ sensor on day 15 . Following the increase in bulk EC 
there is a decrease that can be attributed to the MID reaction reducing salinity. The different arrival times of the treatment solution indicate that although there existed preferential seepage pathways, the treatment solution also migrated through non-preferential areas. It is notable that the treatment solution reached the $2.1 \mathrm{~m}$ sensor although it is outside the target treatment depth interval.

The TEROS sensors were also able to estimate the volumetric moisture contents, which were then converted to gravimetric moisture contents $\left(\mathrm{W}_{\mathrm{n}}\right)$ using the average dry density measured on post-treatment Shelby tube samples. The estimated Sunderland $\rho_{\text {dry }}$ is 1.09 $\mathrm{g} / \mathrm{cm}^{3}$ and $1.19 \mathrm{~g} / \mathrm{cm}^{3}$ for Harborton. Figure 4.15 shows $\mathrm{w}_{\mathrm{n}}$ interpreted on this basis.

The change in $\mathrm{w}_{\mathrm{n}}$ interpreted by the sensors was used to interpret corresponding changes in $S_{r}$. The estimated $S_{r}$ values over time are shown in Figure 4.16. For the sensors installed at depths where measured $V_{p}$ was greater than $1500 \mathrm{~m} / \mathrm{s}$, the initial $S_{r}$ was assumed to be $100 \%$. All other sensors were plotted at an initial $\mathrm{S}_{\mathrm{r}}$ of $98.5 \%$, assuming that $\mathrm{S}_{\mathrm{r}}$ is no greater than $98.5 \%$ for $\mathrm{V}_{\mathrm{p}}$ smaller than $1500 \mathrm{~m} / \mathrm{s}$ (Valle-Molina \& Stokoe 2012). Note that there is considerable uncertainty in estimating the initial $S_{r}$ based on $V_{p}$ for the very shallow sensors close to the ground water table, as the $\mathrm{S}_{\mathrm{r}}-\mathrm{V}_{\mathrm{p}}$ relationships are highly nonlinear and not well-established for silts. All sensors, including those outside the target zone, showed a decrease in $\mathrm{S}_{\mathrm{r}}$ during the treatment period (based upon a decrease in $\mathrm{V}_{\mathrm{p}}$ ). At Sunderland, $\mathrm{S}_{\mathrm{r}}$ decreased by $2 \%$ to $8 \%$ during the treatment period, and between $6 \%$ and $9 \%$ at the Harborton site. At the Sunderland site $\mathrm{w}_{\mathrm{n}}$ continued to drop even after treatment. This could be due to a redistribution of the generated gas, but considering the sensors at Sunderland 
were lowered into the borehole, the decrease in $\mathrm{w}_{\mathrm{n}}$ could also be attributed to the consolidation of the backfill material. However, for many of the sensors a sharp decrease in the $\mathrm{w}_{\mathrm{n}}$ corresponded to an increase then decrease in bulk EC, indicating that the arrival and conversion of substrates had a direct effect on the $\mathrm{w}_{\mathrm{n}}$ and $\mathrm{S}_{\mathrm{r}} \mathrm{S}_{\mathrm{r}}$ decreases outside the target intervals were likely due to migration of the treatment solution or produced gas outside the target zone. All sensors show that $\mathrm{w}_{\mathrm{n}}$ remained below pre-treatment values from the end of treatment. The $4.57 \mathrm{~m}$ bgs sensor at Harborton shows an anomaly with $\mathrm{S}_{\mathrm{r}}$ $>100 \%$; similar anomalies in volumetric moisture content measurements have been observed at the lab scale and are still under investigation (Stallings-Young et al. 2020). As of July 9, 2020, the Harborton site is under construction and data collection has ceased; measurements continue to be taken at the Sunderland site. 


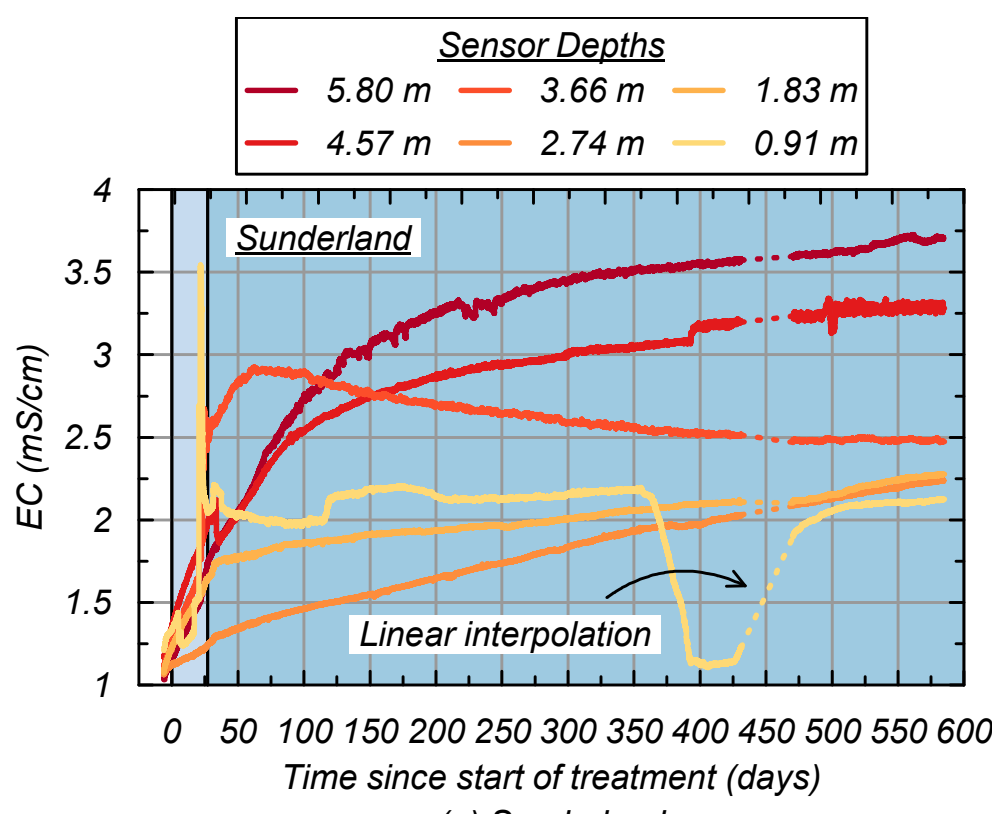

(a) Sunderland

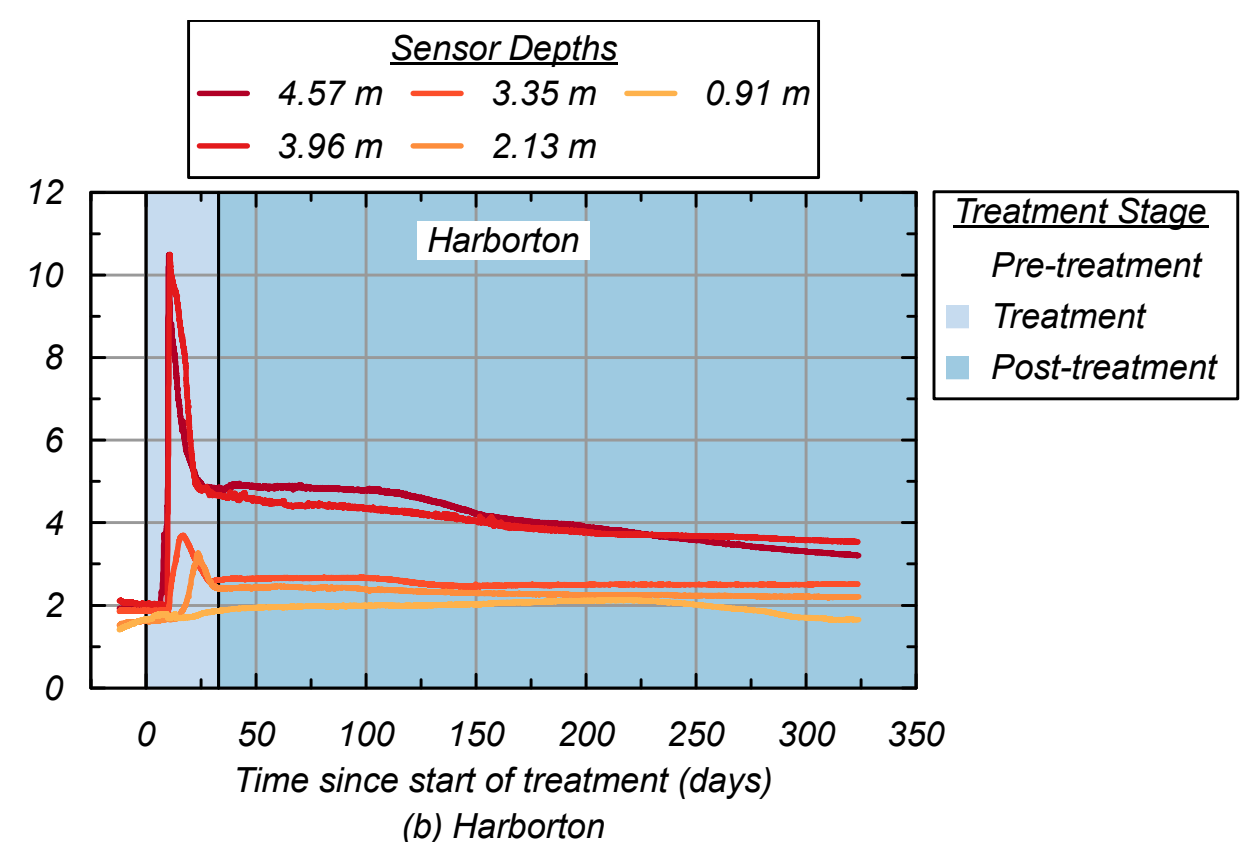

(b) Harborton

Figure 4.14: Bulk EC measured by in-situ sensors at (a) Sunderland and (b) Harborton. 


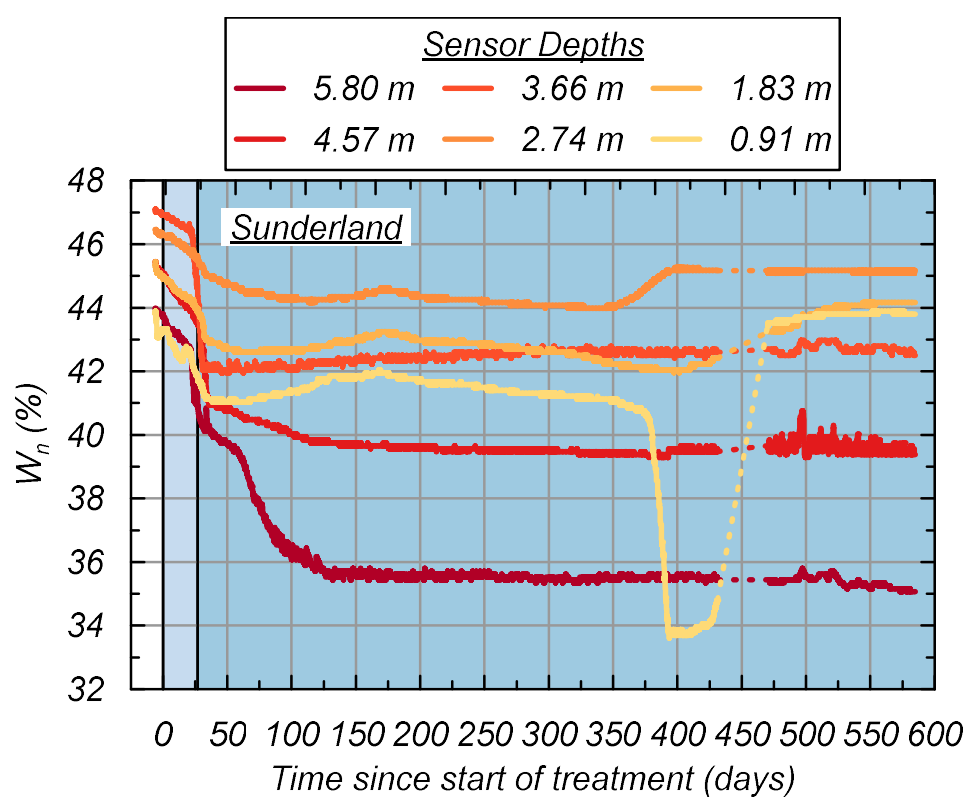

(a) Sunderland

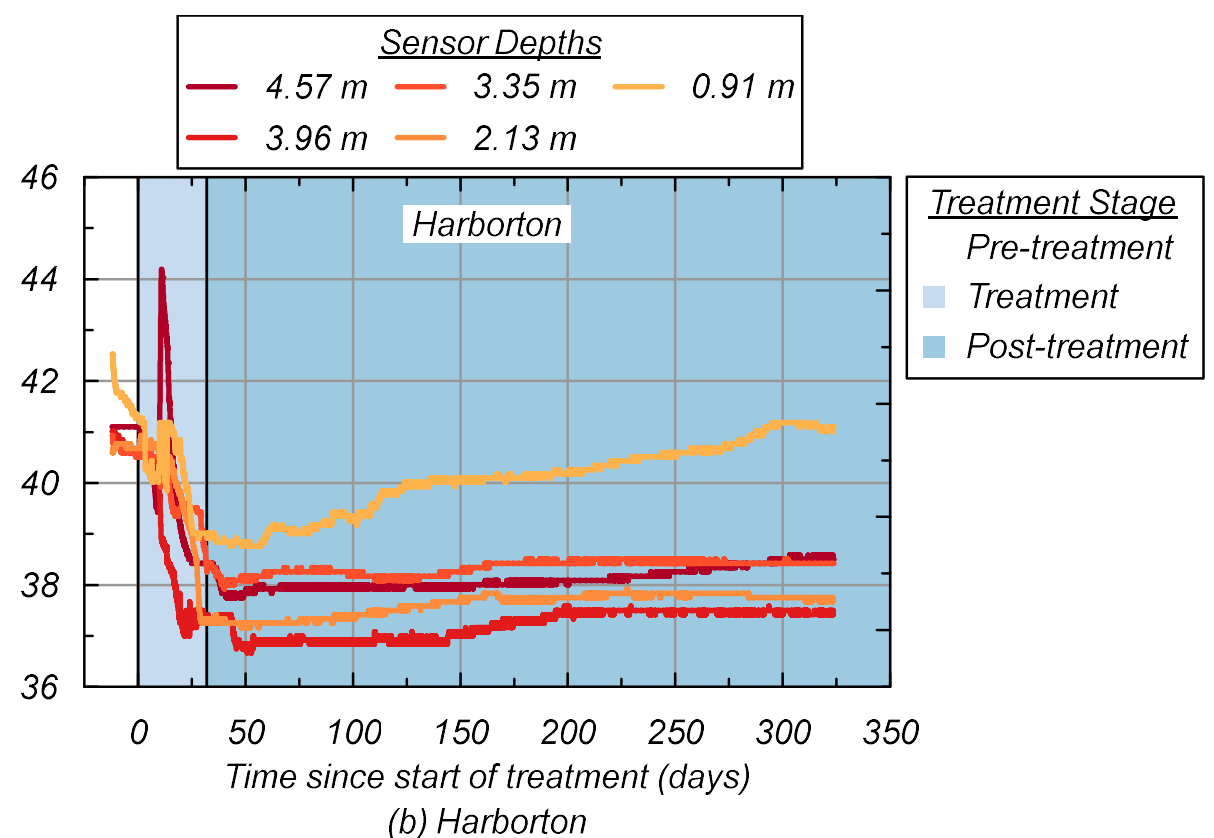

(b) Harborton

Figure 4.15: $w_{n}$ interpreted from in-situ sensors at (a) Sunderland and (b) Harborton. 


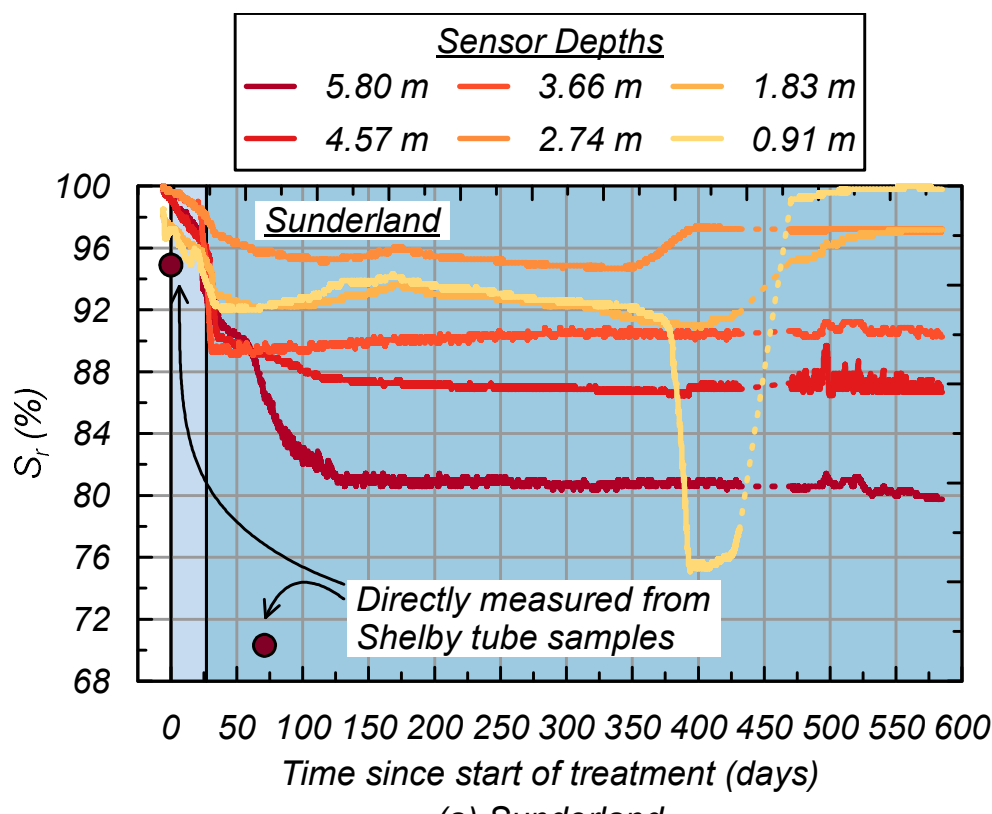

(a) Sunderland

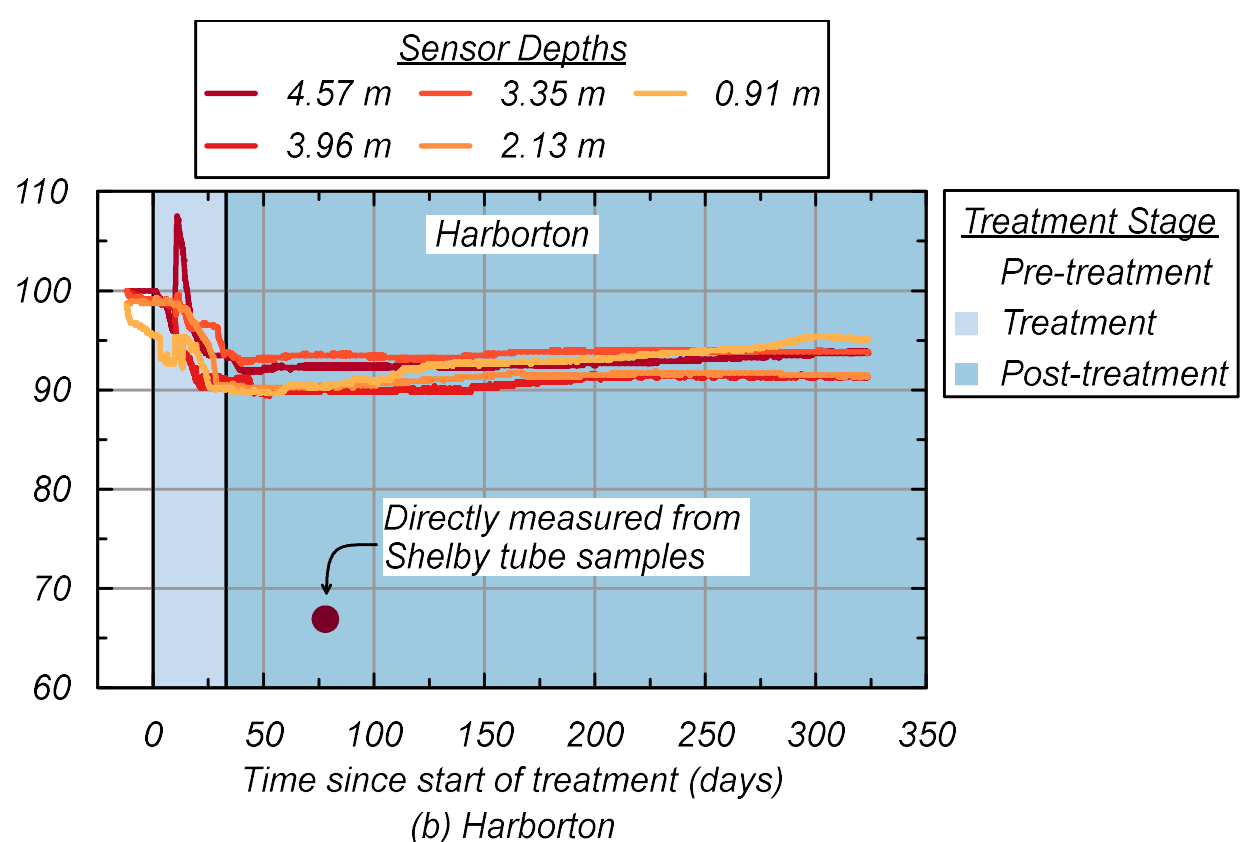

(b) Harborton

Figure 4.16: Interpreted $S_{r}$ at (a) Sunderland and (b) Harborton. 


\subsection{3: Direct measurements of saturation ratio}

Shelby tube samples were collected pre- and post-treatment at both sites. $\mathrm{S}_{\mathrm{r}}$ was directly measured by measuring the bulk density, $\mathrm{w}_{\mathrm{n}}$ and an assumed specific gravity of 2.65 . Measured $\mathrm{S}_{\mathrm{r}}$ was corrected for gas volume changes between in-situ total vertical stress and atmospheric pressure with the Ideal Gas Law (Gallant and Finno 2017).

Figure 4.16 shows the average measured $S_{r}$ plotted on the sampling date. The $S_{r}$ from 25 pre-treatment samples from depths $4.26 \mathrm{~m}$ to $5.24 \mathrm{~m}$ at Sunderland is $95.4 \%$; the $\mathrm{S}_{\mathrm{r}}$ from 18 post-treatment samples from depths $3.29 \mathrm{~m}-5.27 \mathrm{~m}$ at Sunderland is $70.1 \%$. The $\mathrm{S}_{\mathrm{r}}$ from three post-treatment samples from depths $2.70 \mathrm{~m}-2.92 \mathrm{~m}$ at Harborton is $67 \%$; additional testing is planned using pre-treatment Harborton samples. While the samplemeasured $\mathrm{S}_{\mathrm{r}}$ values differ from the interpreted $\mathrm{S}_{\mathrm{r}}$ values from in-situ sensors, they both indicate a notable decrease due to MID treatment, which is also supported by the $V_{p}$ measurements shown in Figure 4.12. The difference between the sample-measured $\mathrm{S}_{\mathrm{r}}$ values and the in-situ sensors could be due to moisture loss during transportation and storage of the Shelby tubes.

\subsection{4: Water levels}

Ground water levels were measured to observe if the persistence of MID was affected by seasonal water table fluctuations. Water levels at the Sunderland site were directly measured in the injection and extraction wells and calculated using the PPTs installed within the sensor array and from the dissipation tests performed during SCPT testing. The water level measurements over time are shown in Figure 4.17. Seasonal water table 
fluctuations were between $1.5 \mathrm{~m}$ bgs and the ground surface (with observed ponding). These data and the monitoring data shown in previous figures show that the induced desaturation was not affected by the seasonal water table fluctuations, as $V_{p}$ and $w_{n}$ remain below pre-treatment values 23 months after treatment. Water levels were not monitored at the Harborton site. 


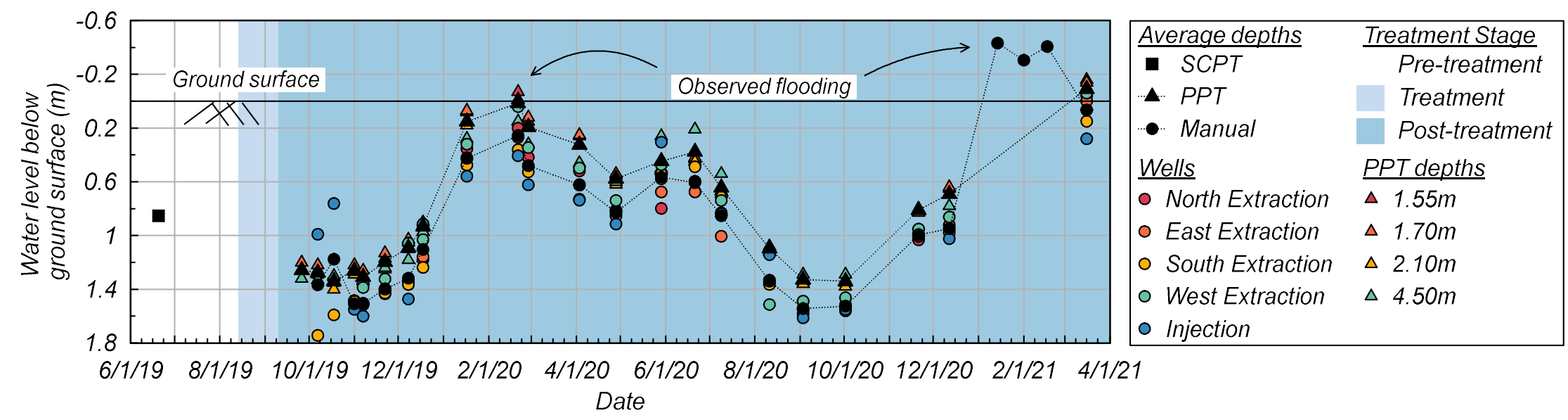

Figure 4.17: Water levels over time at the Sunderland site. 


\section{Chapter 5: Summary, Conclusions, and Recommendations for Future Work}

\section{1: Summary}

MID field trials were performed at two sites of liquefiable low plasticity fine-grained soils in Portland, Oregon in an effort to find a non-disruptive liquefaction mitigation method for essential lifelines. The field trial sites were located close to PDX International Airport and the CEI hub; both sites having soils that are reflective of the general conditions of nearby critical infrastructure.

The MID treatment solution of calcium nitrate and calcium acetate was applied through injection and extraction wells that were installed $3.5 \mathrm{~m}$ apart and screened over the target treatment depth interval (between $1.5 \mathrm{~m}$ to $6.1 \mathrm{~m} \mathrm{bgs}$ ). The treatment was applied for one month at each site.

$\mathrm{S}_{\mathrm{r}}$ was monitored by several methods before, during, and after MID treatment with the goal of evaluating the effectiveness and longevity of MID treatment at the field-scale. Monitoring included pre- and post-treatment SCPTs from both field trial sites, measurements of $\mathrm{S}_{\mathrm{r}}$ on soil samples from both sites, interpreted $\mathrm{S}_{\mathrm{r}}$ from in-situ sensors, and crosshole $V_{p}$ measurements at the Sunderland site. Although exact values of $S_{r}$ are difficult to determine, all methods show a decrease in $\mathrm{S}_{\mathrm{r}}$ between pre- and post-treatment conditions in the targeted treatment zone. Additionally, SCPTs performed outside the targeted zone indicates that MID extended beyond the extraction wells. 


\section{2: Conclusion}

$\mathrm{V}_{\mathrm{p}}$ and $\mathrm{w}_{\mathrm{n}}$ remained at values that indicate $\mathrm{S}_{\mathrm{r}}<98.5 \%$ persisted for 20 months following MID treatment at Sunderland and 8 months following MID treatment at Harborton (the site is now under construction, so ongoing monitoring is not possible). Furthermore, at the Sunderland site, $\mathrm{S}_{\mathrm{r}}$ does not appear to be affected by water table fluctuations, including flooding at the ground surface.

\section{3: Recommendations for Future Work}

Continued research from these field trial sites may inform future liquefaction mitigation efforts. Monitoring at Sunderland will continue for the next several years, including measurements of $\mathrm{V}_{\mathrm{p}}$, bulk EC and conductivity, and additional lab testing. This lab testing will include developing a $\mathrm{V}_{\mathrm{p}}-\mathrm{S}_{\mathrm{r}}$ plot for non-plastic and low-plasticity soils to better estimate the percentage of desaturation in these types of fine-grained soil. It will also include further analyses of the sub-surface distribution of the MID treatment via direct measurements of $\mathrm{S}_{\mathrm{r}}$.

These efforts will provide important data to assess the long-term persistence of MID as a viable, non-invasive option for mitigating liquefaction in low plasticity fine-grained soils. If proved effective, this method can provide a solution to reduce liquefaction risk at existing infrastructure and lifelines. 


\section{References}

Atwater, Brian F., et al. The orphan tsunami of 1700: Japanese clues to a parent earthquake in North America. No. 1707. US Geological Survey, 2005.

Bauer, J. M., Burns, W. J., and Madin, I. P. (2012). "Open-File Report O-18-02, Earthquake regional impact analysis for Clackamas, Multnomah, and Washington counties, Oregon."

Bray, J. D., and Sancio, R. B. (2006). "Assessment of the liquefaction susceptibility of finegrained soils." Journal of geotechnical and geoenvironmental engineering, ASCE, 132(9), 1165-1177.

Boulanger, Ross W., and Izzat M. Idriss. Evaluating the potential for liquefaction or cyclic failure of silts and clays. Davis, California: Center for Geotechnical Modeling, 2004.

Boulanger, R. W., and Idriss, I. M. (2006). "Liquefaction susceptibility criteria for silts and clays." Journal of Geotechnical and Geoenvironmental Engineering, ASCE, 132(11), 1413-1426.

"Cascadia Subduction Zone." Oregon Office of Emergency Management (OOEM)t: Cascadia Subduction Zone: Hazards and Preparedness: State of Oregon, www.oregon.gov/oem/hazardsprep/Pages/Cascadia-Subduction-Zone.aspx.

“Community Lifelines.” Community Lifelines | FEMA.gov, 27 July 2020, www.fema.gov/emergency-managers/practitioners/lifelines.

Gallant, A. P., and Finno, R. J. (2017). "Measurement of gas released during blast densification.” Geotechnical Testing Journal, 40(6), 1011-1025. 
Glass, C. and Silverstein, J. "Denitrification kinetics of high nitrate concentration water: pH effect on inhibition and nitrite accumulation." Water Research 32.3 (1998): 831839.

Goldfinger, Chris, et al. Turbidite event history—Methods and implications for Holocene paleoseismicity of the Cascadia subduction zone. No. 1661-F. US Geological Survey, 2012.

He, J., Chu, J., and Ivanov, V. (2013). "Mitigation of liquefaction of saturated sand using biogas." Géotechnique, 63(4), 267-275.

Hull, D. A. (1991). "Geologic Map of the Portland Quadrangle, Multnomah and Washington Counties, Oregon, and Clark County, Washington.” Oregon Geology, map, Department of Geology and Mineral Industries.

"Local Earthquakes." Washington County Oregon Emergency Management: Local Earthquakes: Fault Zones in and Near Washing County. https://www.co.washington.or.us/EmergencyManagement/Hazards/Earthquake/lo cal.cfm

Mousavi, S., and Ghayoomi, M. (2021). "Liquefaction mitigation of sands with nonplastic fines via microbial-induced partial saturation." Journal of Geotechnical and. Geoenvironmental Engineering, 147(2): 04020156.

O'Donnell, S. T., Kavazanjian, E., and Rittmann, B E. (2017). "MIDP: Liquefaction Mitigation via Microbial Denitrification as a Two-Stage Process. II: MICP.” Journal of Geotechnical and Geoenvironmental Engineering, 143(12), 04017095. 
O'Donnell, S. T., Rittmann, B. E., and Kavazanjian, E. (2017). "MIDP: Liquefaction Mitigation via Microbial Denitrification as a Two-Stage Process. I: Desaturation.” Journal of Geotechnical and Geoenvironmental Engineering, 143(12), 04017094.

Pham, V. P., Nakano, A., Van Der Star, W. R., Heimovaara, T. J., \& Van Paassen, L. A. (2017). Applying MICP by denitrification in soils: a process analysis. Environmental Geotechnics, 5(2), 79-93.

Pham, V. P., van Paassen, L. A., van der Star, W. R., \& Heimovaara, T. J. (2018b). Evaluating strategies to improve process efficiency of denitrification-based MICP. $J$. Geotech. Geoenviron. Eng., 144(8), 04018049.

Pham, V.P., van Paassen, L.A., \& van der Star, W.R L. (2018a). "Quantifying the desaturation effect of biogenic gas formation in sandy soil". Proceedings of the Seventh International Conference on Unsaturated Soils, Hong Kong, 3-5 August, 2018.

Preciado, A.M., Sorenson, K.R., Khosravifar, A., Moug, D., Stokoe, K., Menq, F., and Zhang, B. "Evaluating Cyclic Loading Response of a Low Plasticity Silt with Laboratory and In-Situ Cyclic Loading Tests." Submitted to ASCE Lifelines Conference 2021 (under review)

Robertson, Peter K. "Interpretation of cone penetration tests-a unified approach." Canadian geotechnical journal 46.11 (2009): 1337-1355.

Satake, K., Shimazaki, K., Tsuji, Y. et al. Time and size of a giant earthquake in Cascadia inferred from Japanese tsunami records of January 1700. Nature 379, 246-249 (1996). https://doi.org/10.1038/379246a0 
Stallings Young, E.G., Zapata, C.E. \& van Paassen, L.A. (2020). "Unsaturated Fluid Flow through Granular Soils Treated with Microbial Induced Desaturation and Precipitation.” Proceedings of the 4th European Conference on Unsaturated Soil Mechanics, Lisbon, 24-26 June, 2020.

Stokoe II, K., Zhang, B. and Menq, F., 2020. Field Assessment of the microbially Induced Desaturation (MID) Method to Mitigate Liquefaction in Silty Soils with Plasticity. Design Safe project PRJ-2482.

Valle-Molina, C., and Stokoe, K. H. (2012). "Seismic measurements in sand specimens with varying degrees of saturation using piezoelectric transducers." Canadian Geotechnical Journal, 49(6), 671-685.

Van Paassen, Leon A., et al. "Potential soil reinforcement by biological denitrification." Ecological Engineering 36.2 (2010): 168-175.

Vega-Posada, C. A., Finno, R. J., and Zapata-Medina, D. G. (2014). "Effect of Gas on the Mechanical Behavior of Medium-Dense Sands." Journal of Geotechnical and Geoenvironmental Engineering, 140(11), 04014063.

Wang, L., van Paassen, L., Gao, Y., He, J., Gao, Y., \& Kim, D. (2020). "Laboratory Tests on Mitigation of Soil Liquefaction Using Microbial Induced Desaturation and Precipitation”. Geotech. Test. J., 44(2).

Wang, Y., Bartlett, S. F., and Miles, S. B. (2012). Earthquake Risk Study for Oregon's Critical Energy Infrastructure Hub, State of Oregon, Salem, OR.

Wong, Ivan G., et al. "The Portland Hills fault: an earthquake generator or just another old fault." Oregon Geology 63.2 (2001): 39-50. 
Yegian, M. K., Eseller-Bayat, E., Alshawabkeh, A., and Ali, S. (2007). "Induced-Partial Saturation for Liquefaction Mitigation: Experimental Investigation."Journal of Geotechnical and Geoenvironmental Engineering, 133(4), 372-380. 


\section{Appendix A: Field Logs of Well Installation - Sunderland}

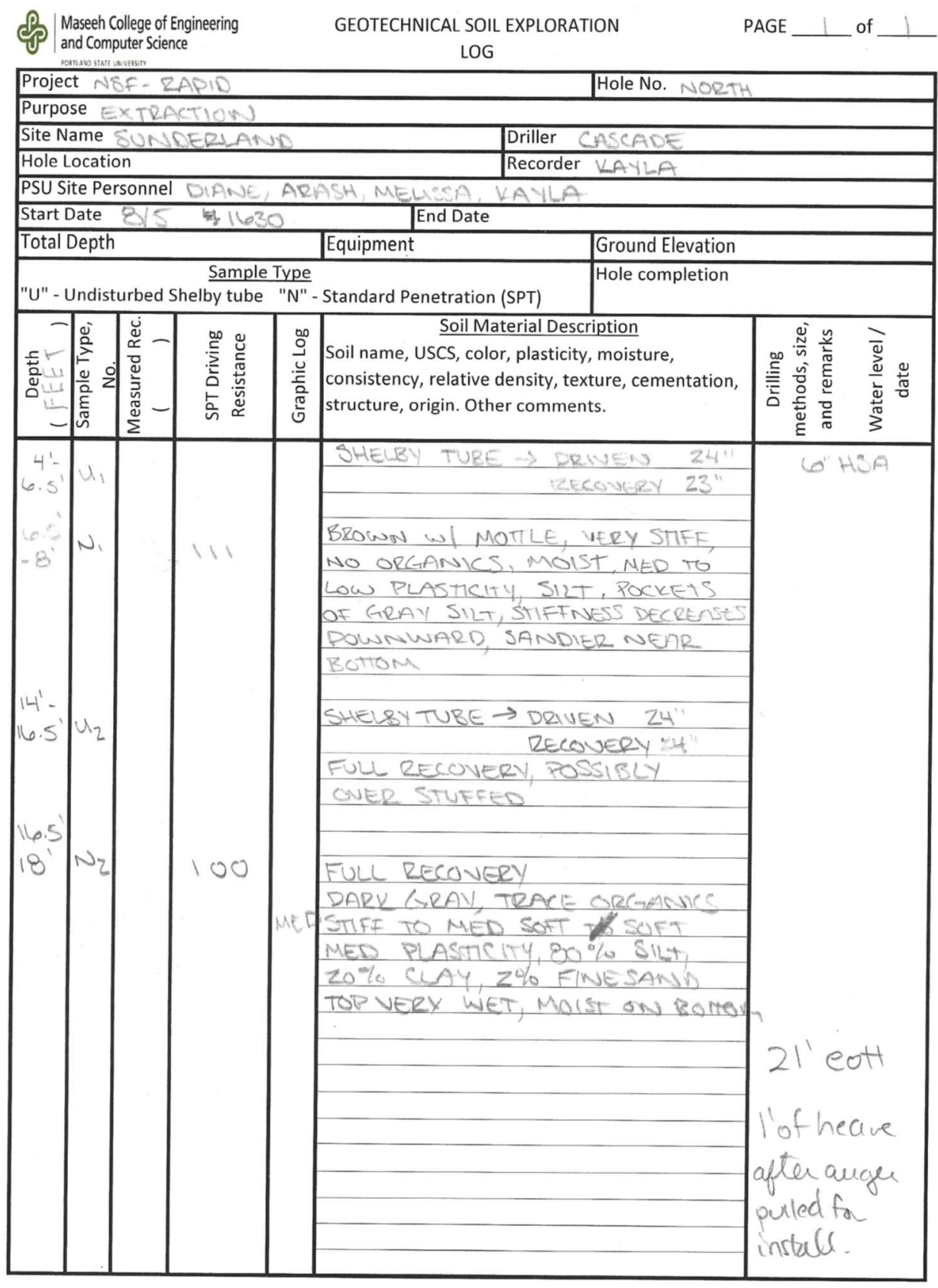




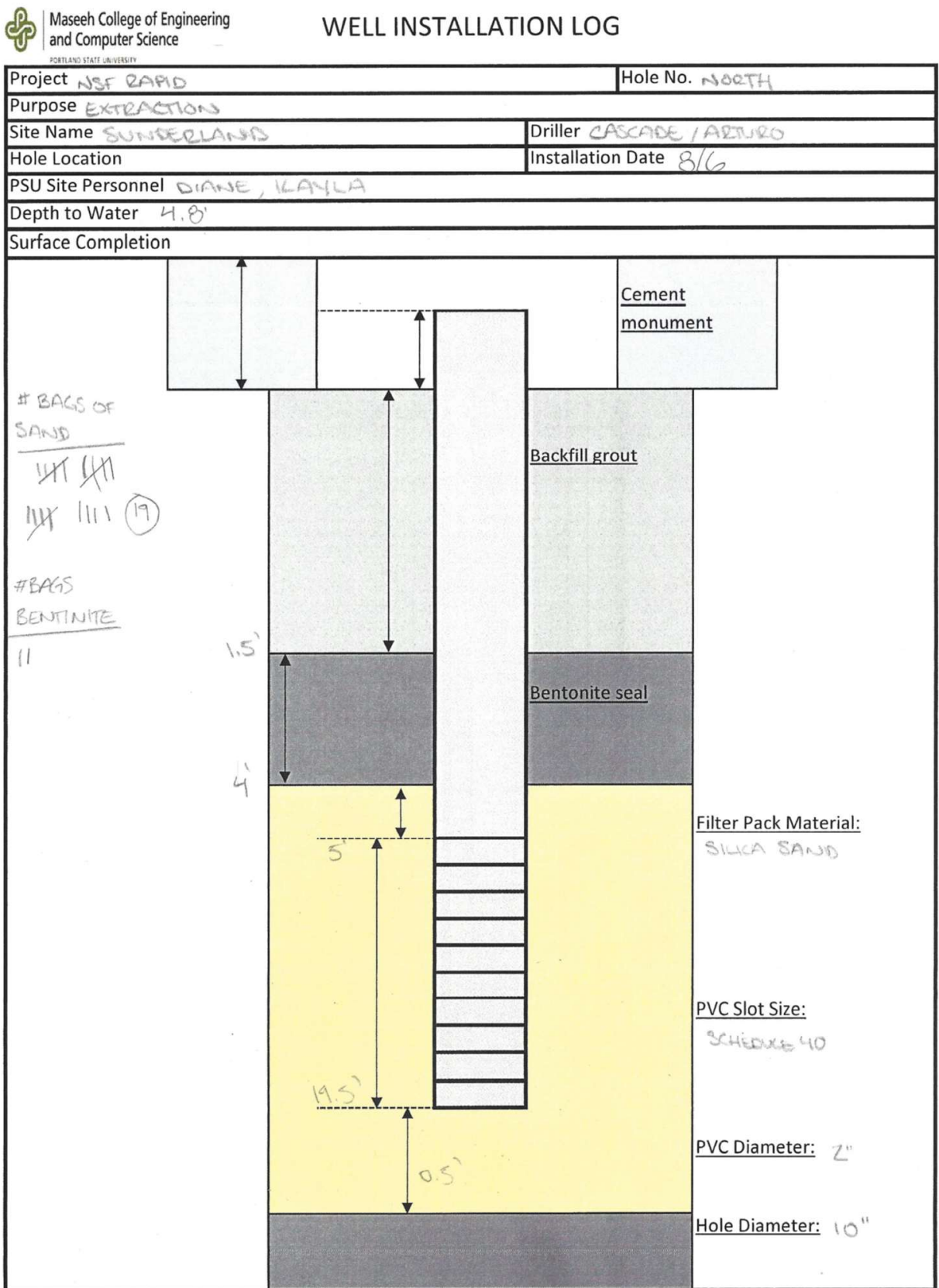




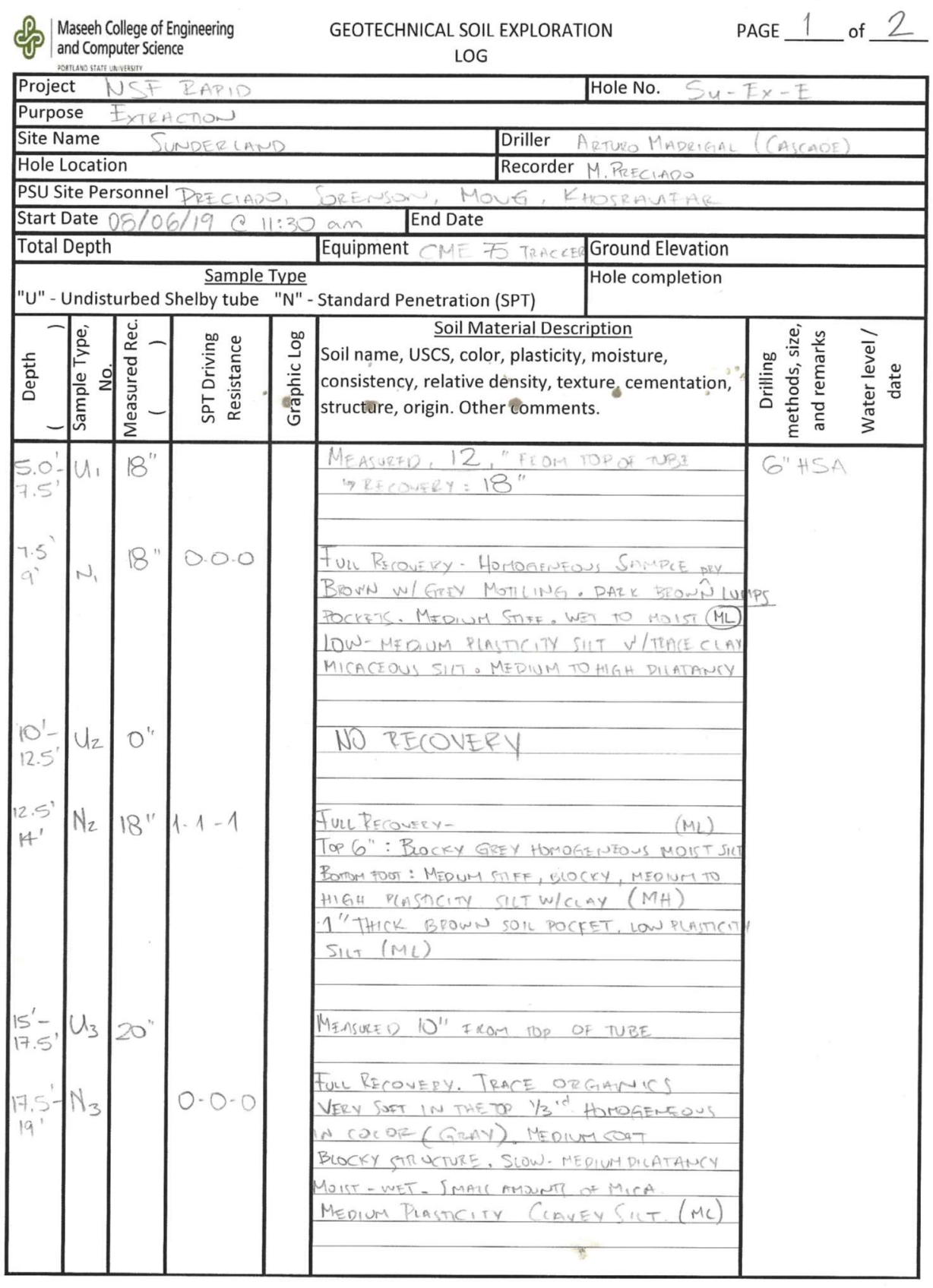




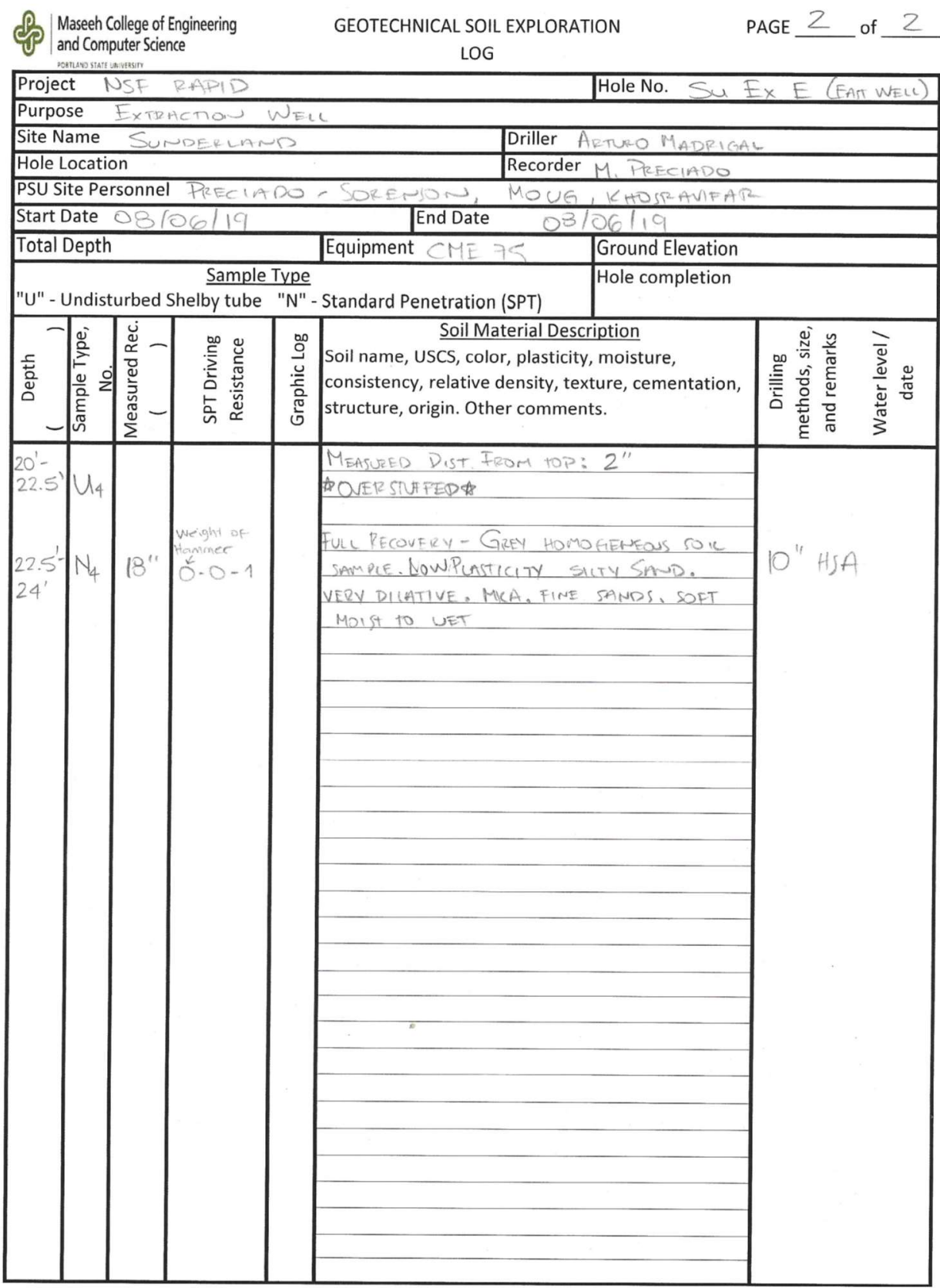




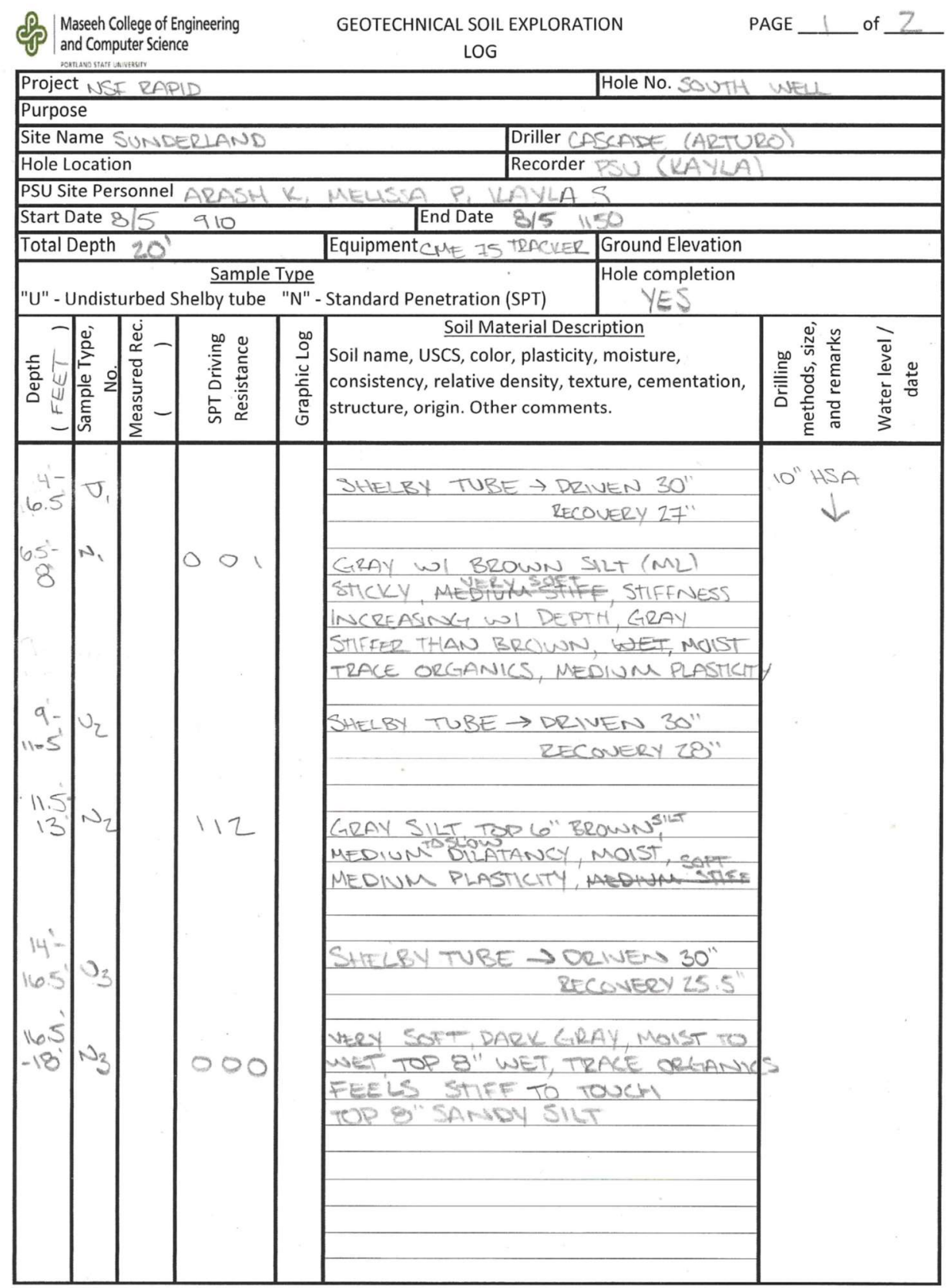




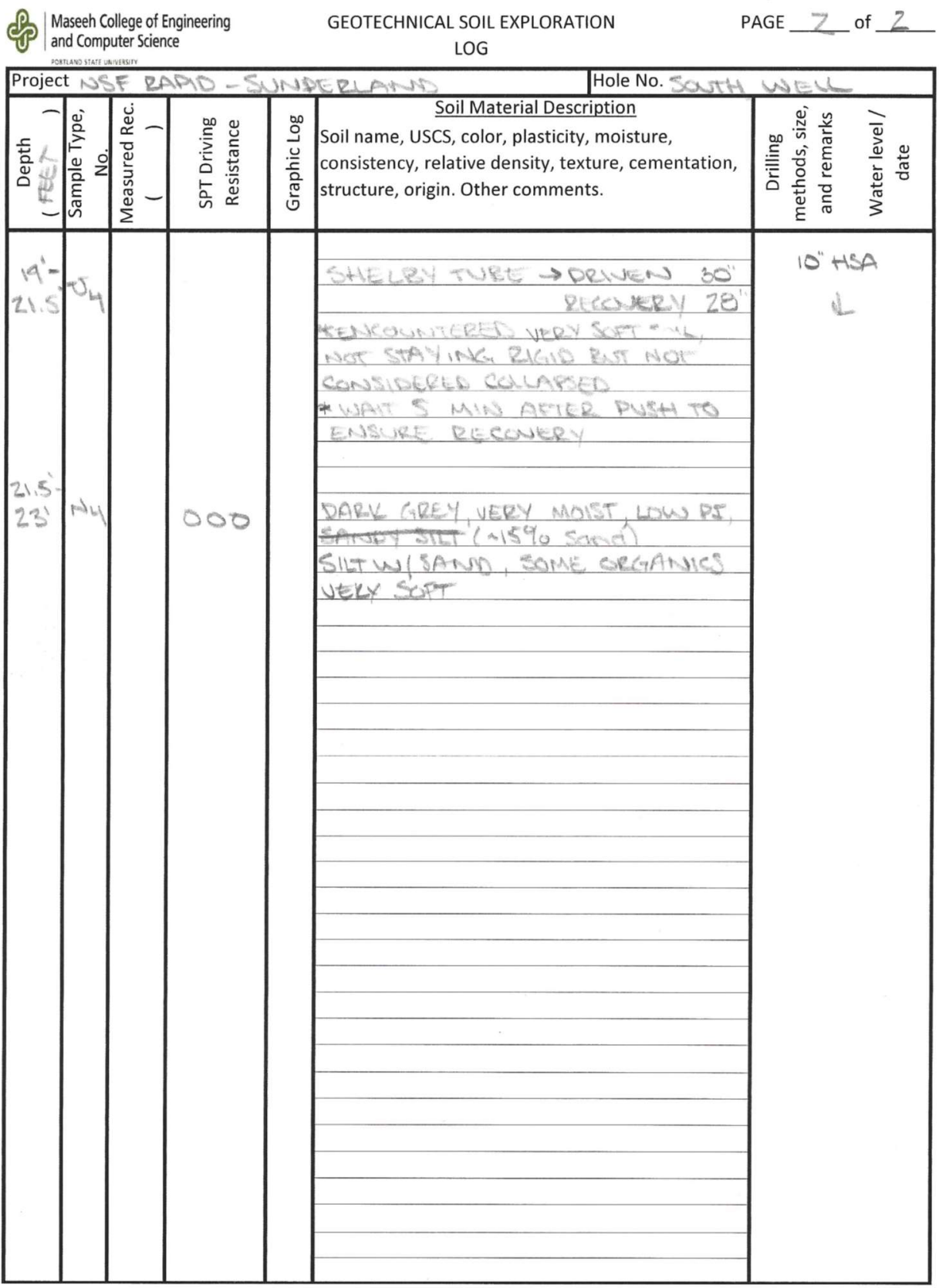




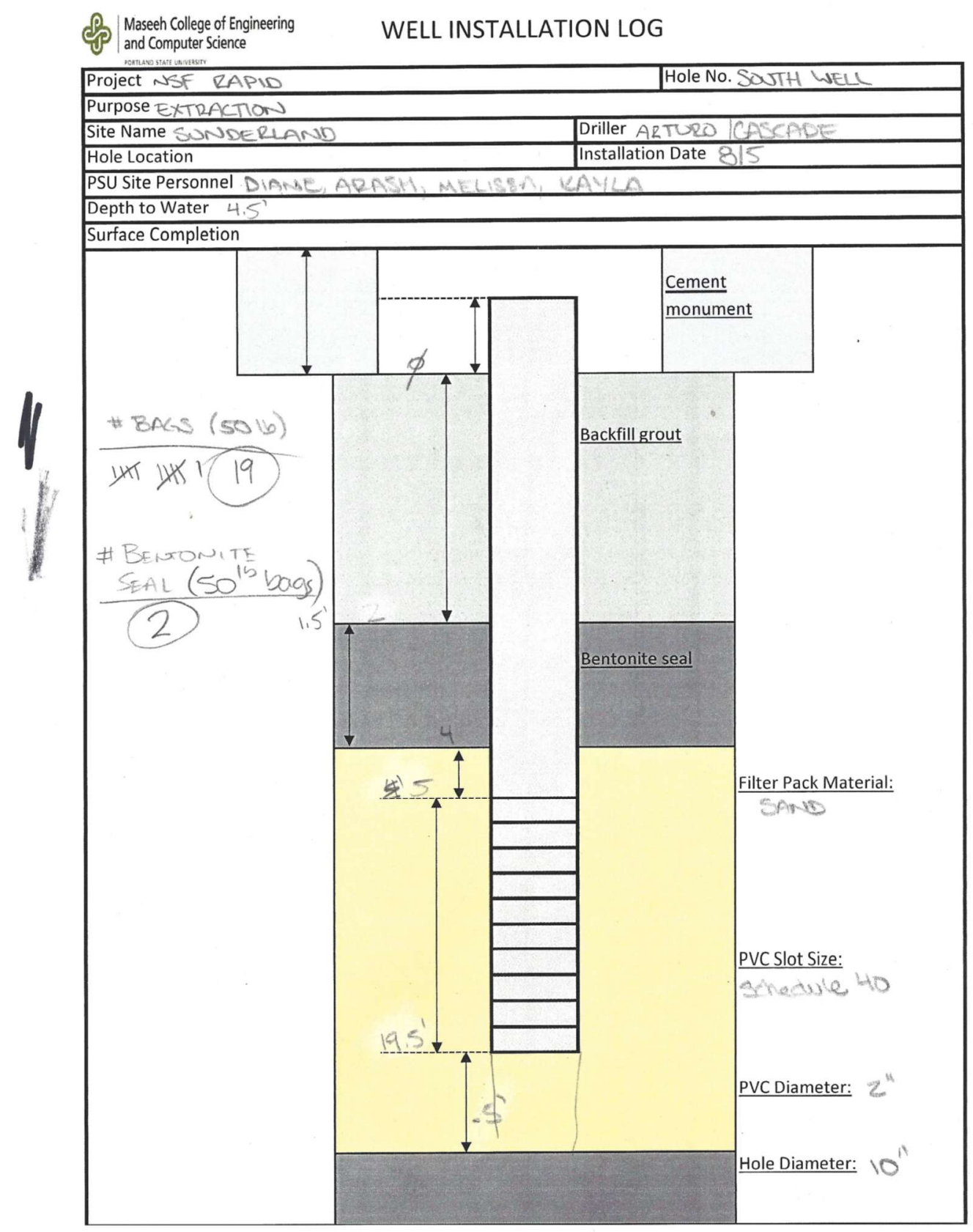




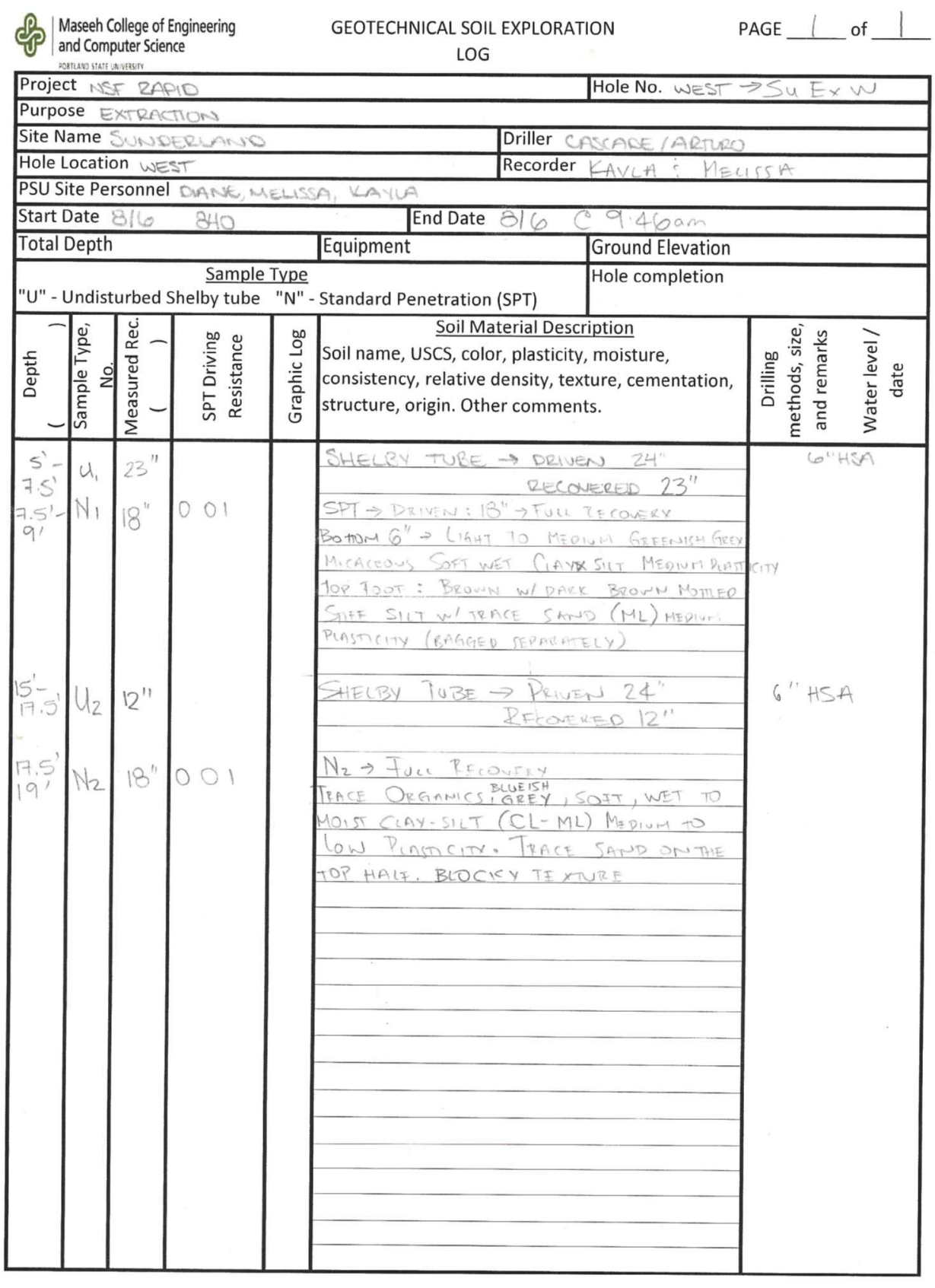




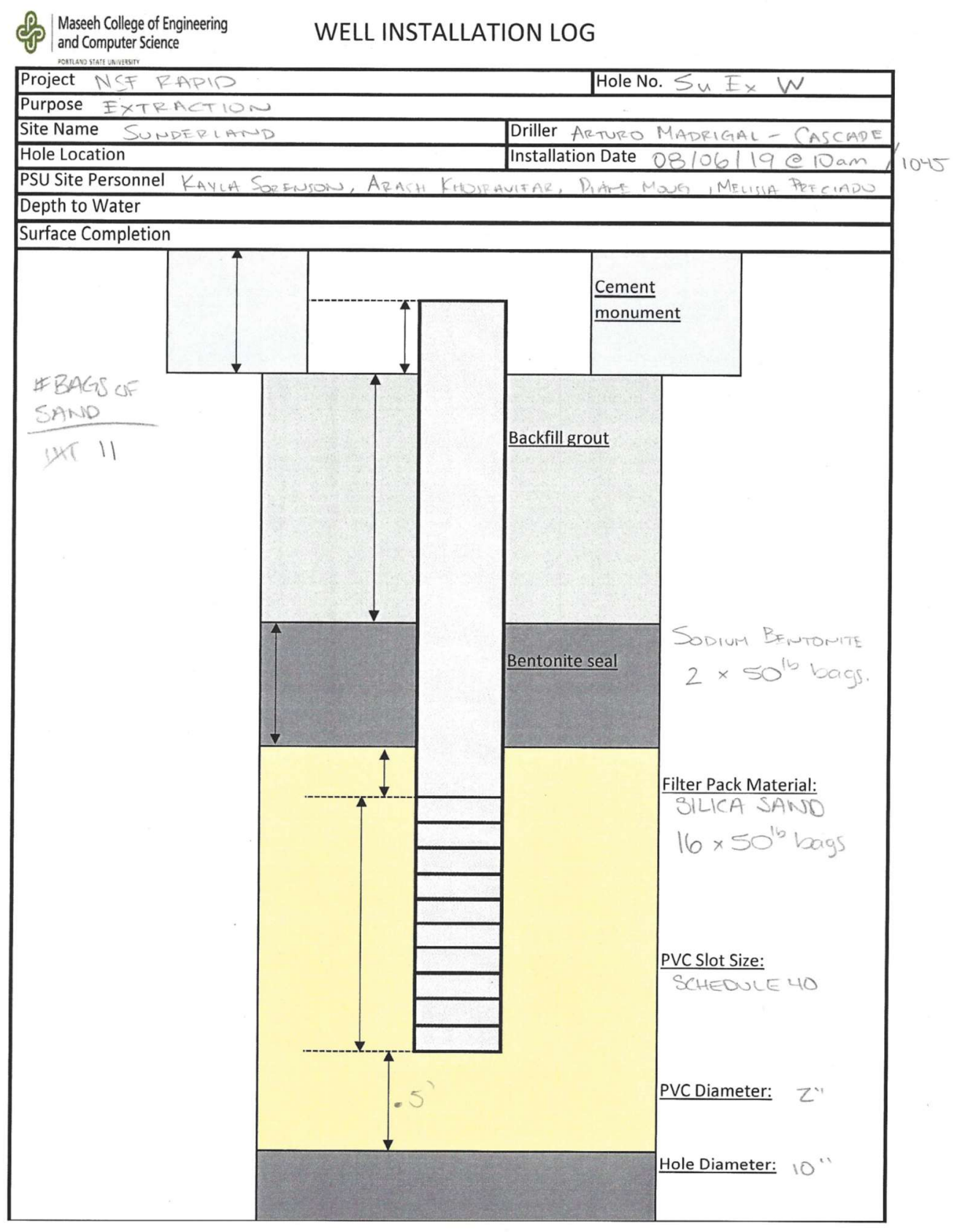




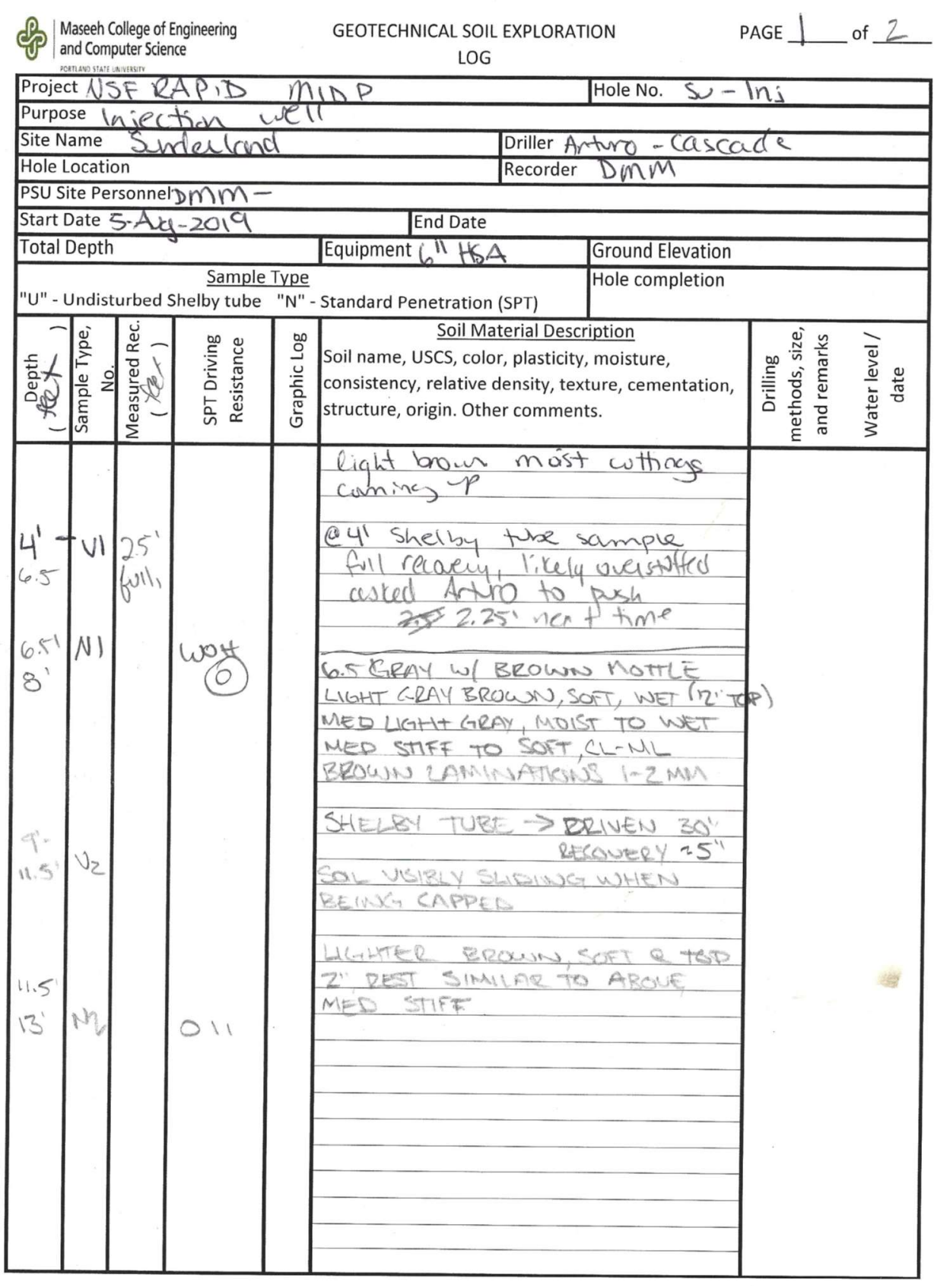




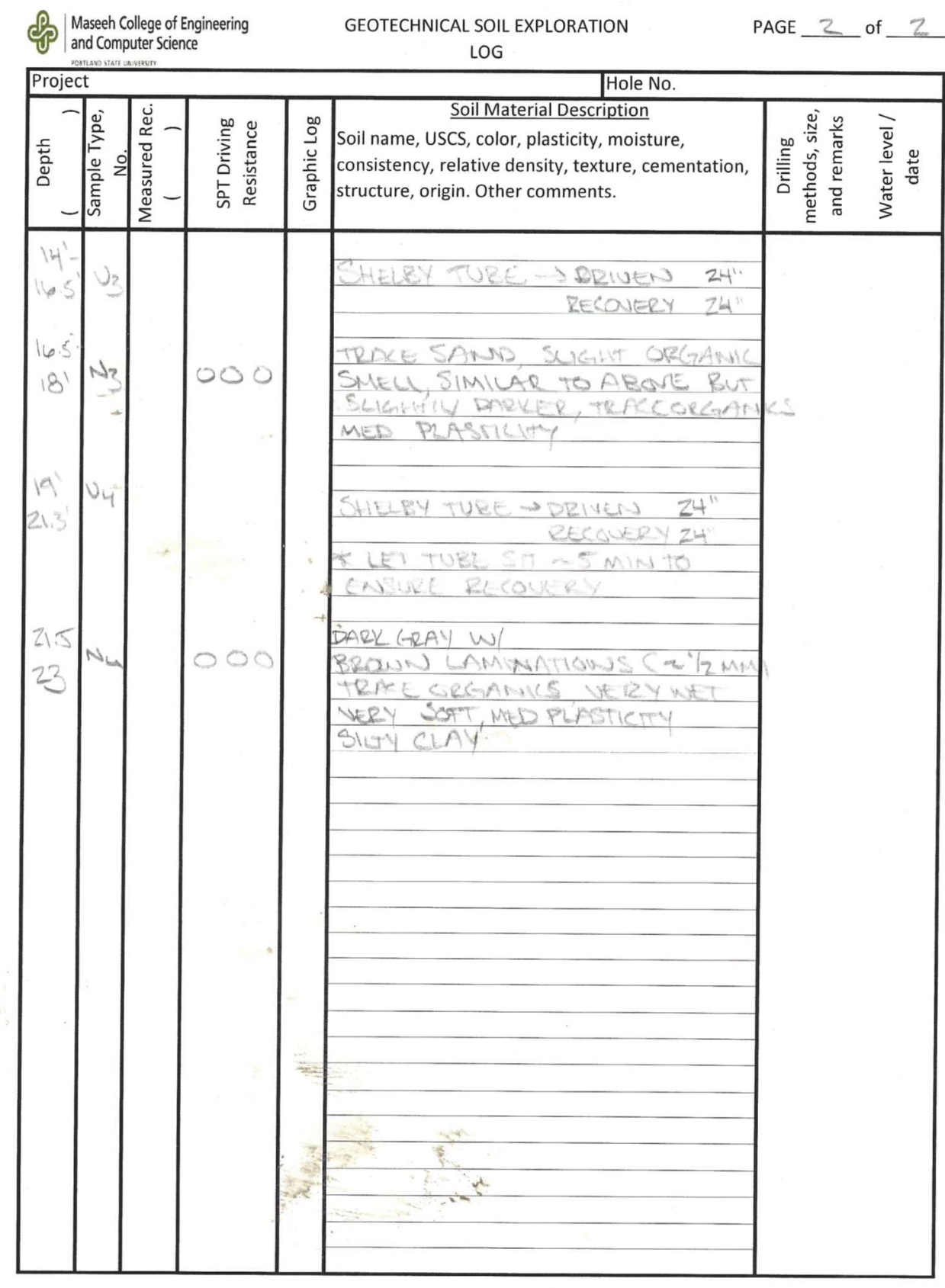




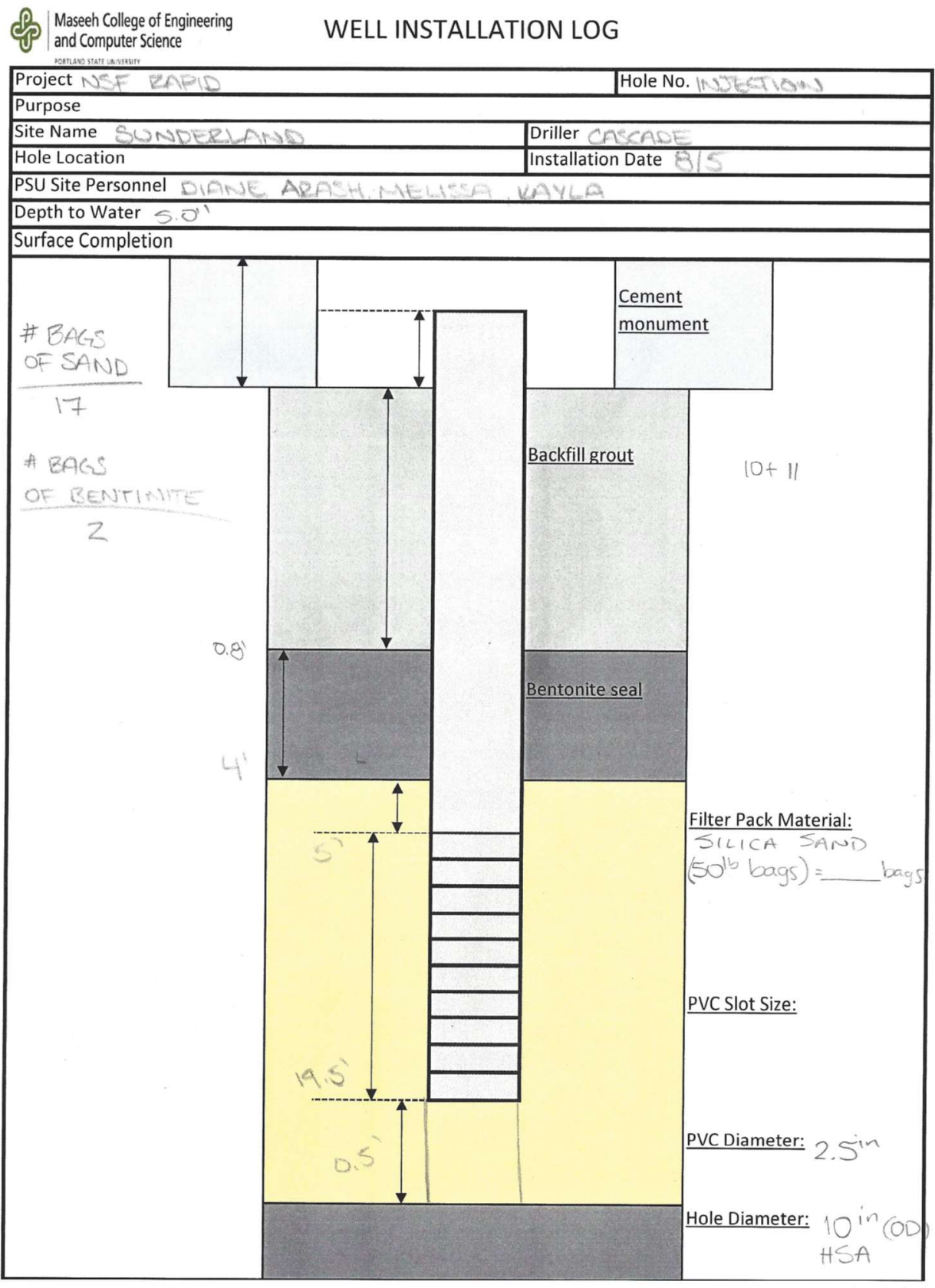




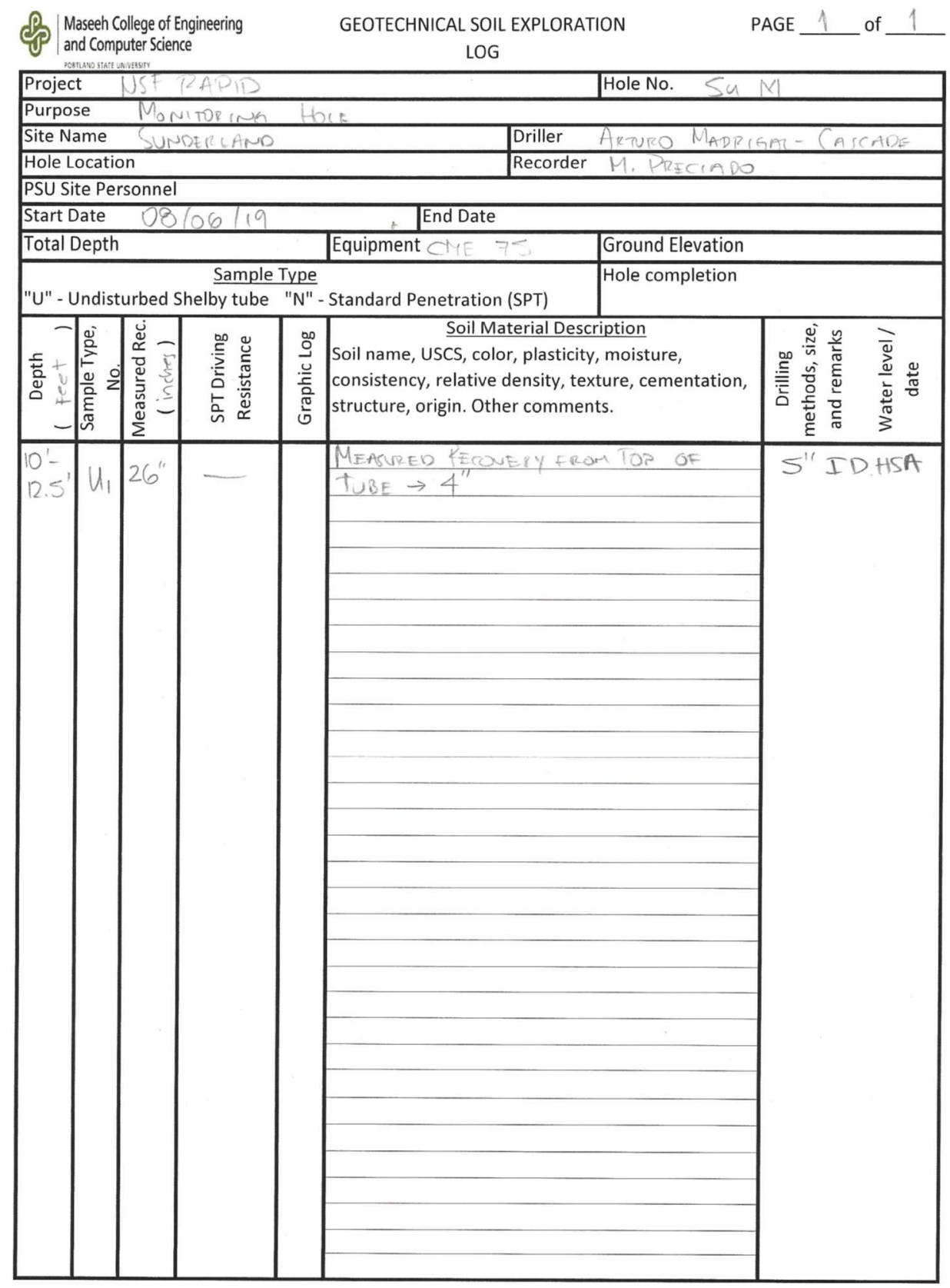




\section{Appendix B: Finished Boring Logs - Sunderland}

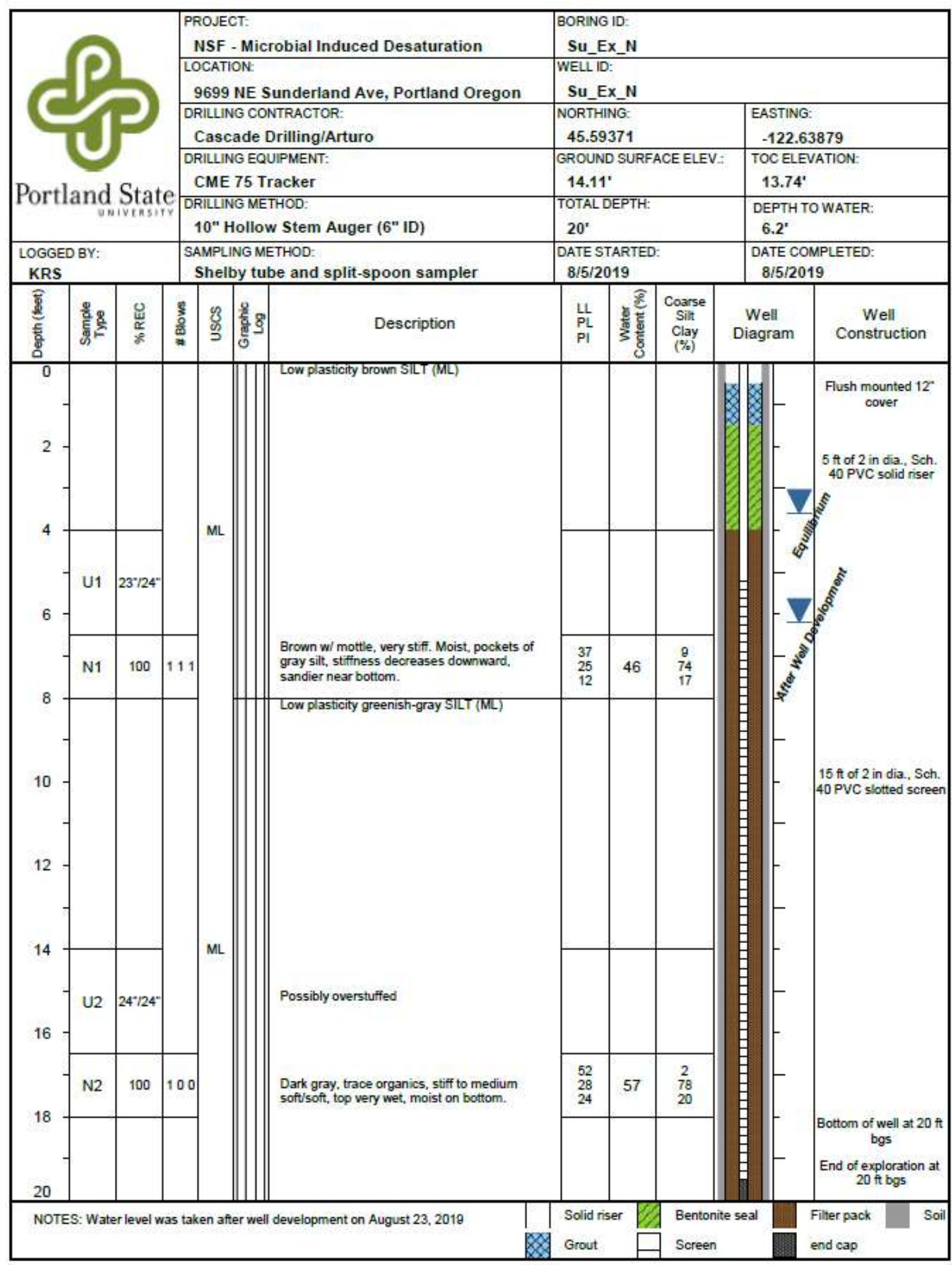




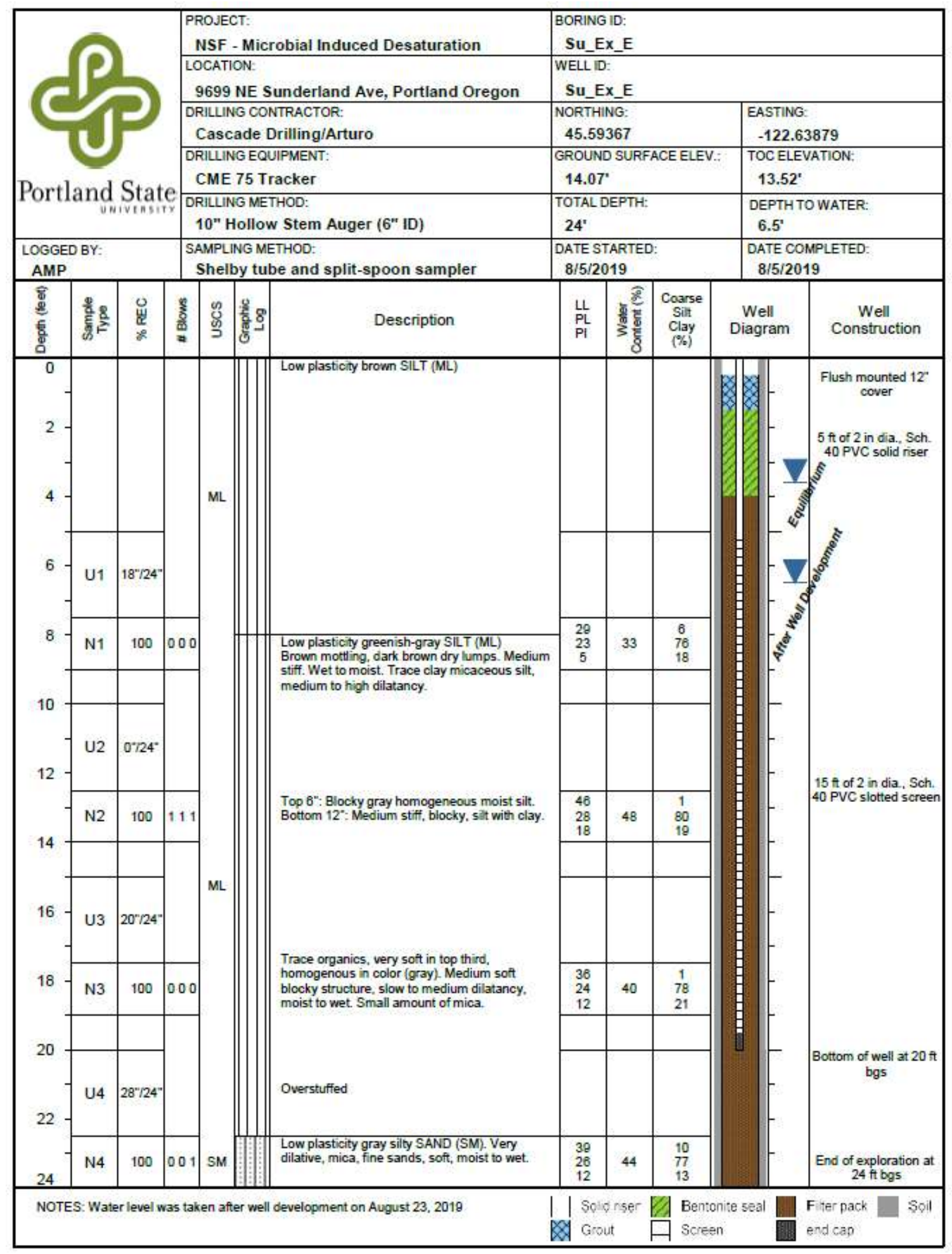




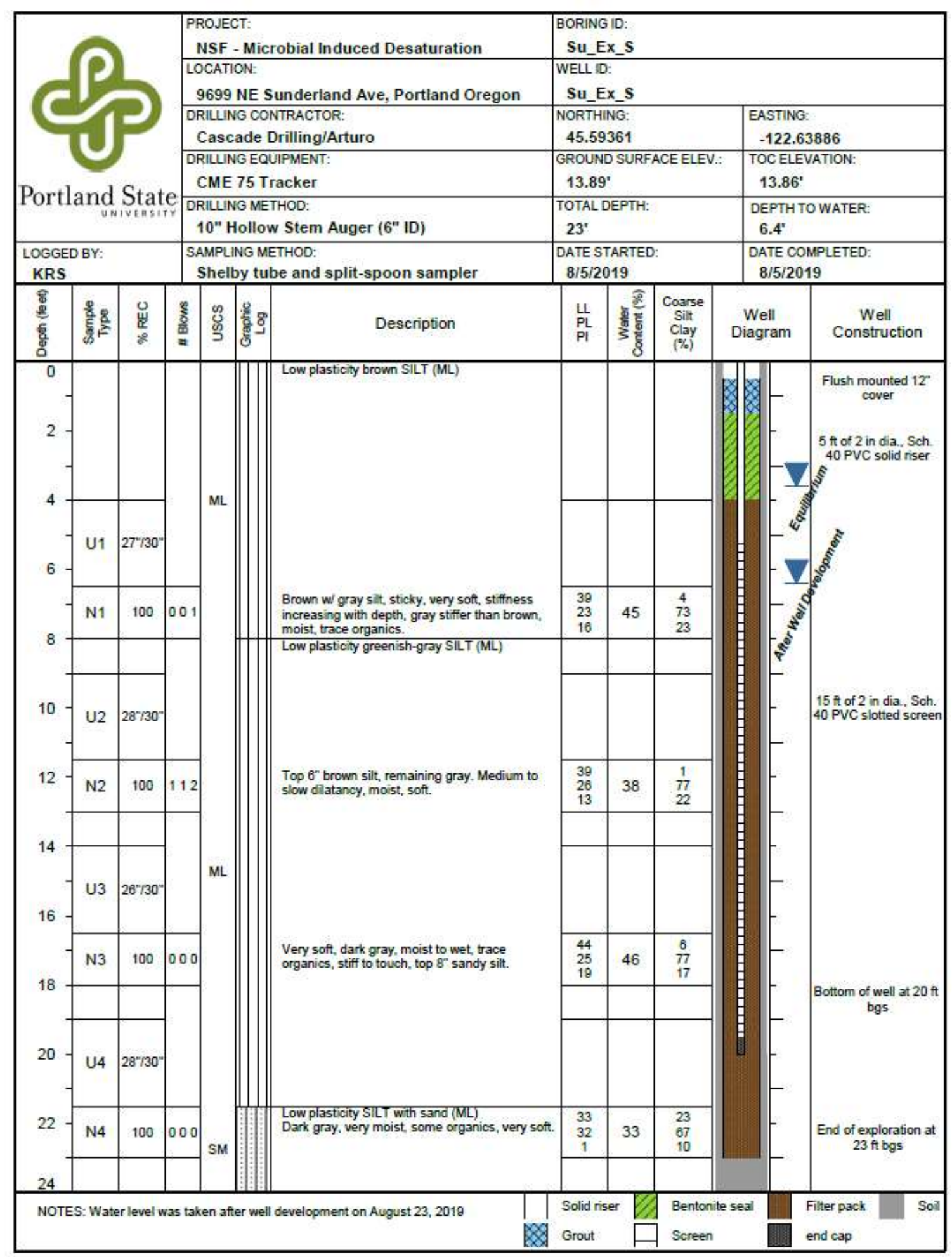




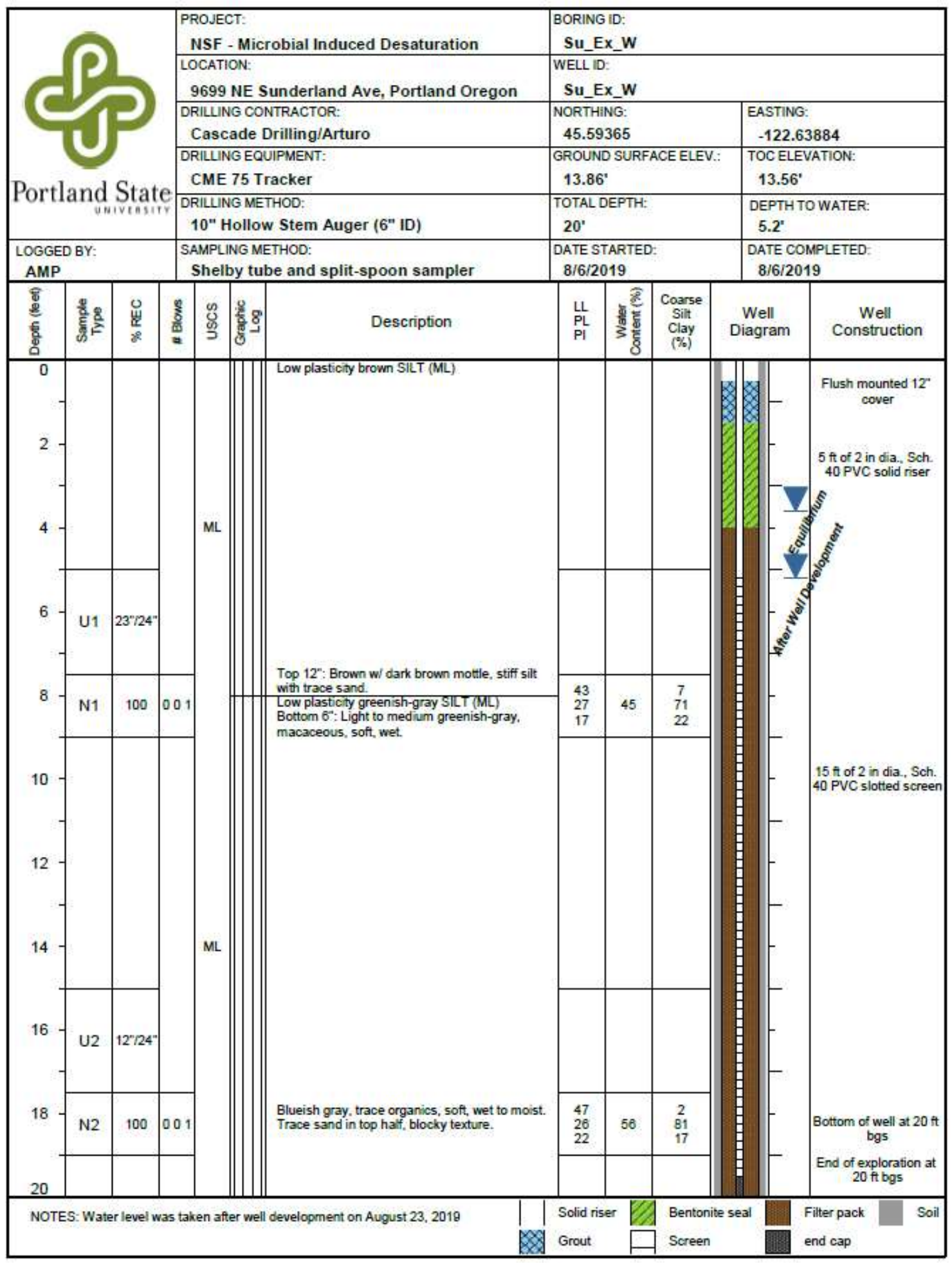




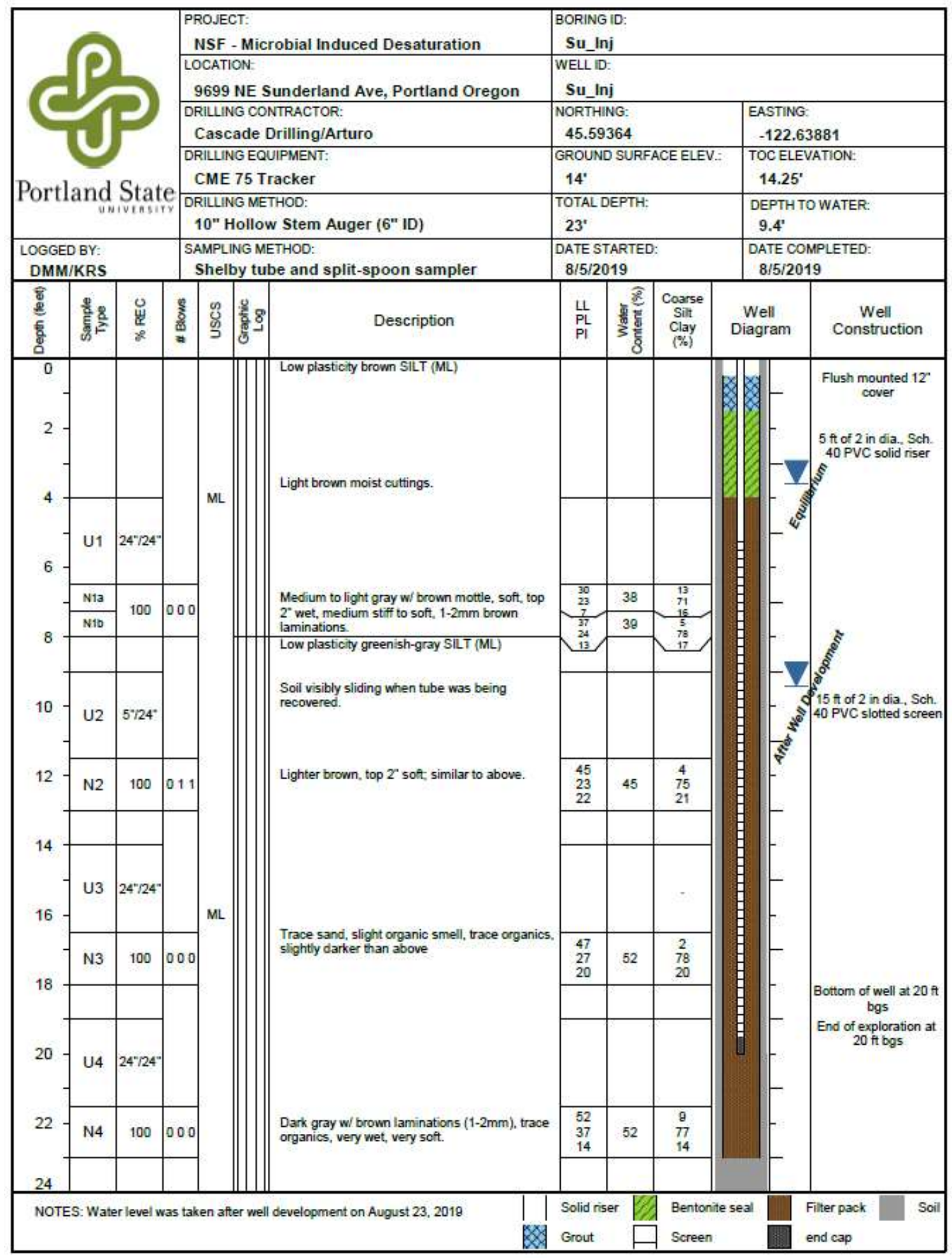




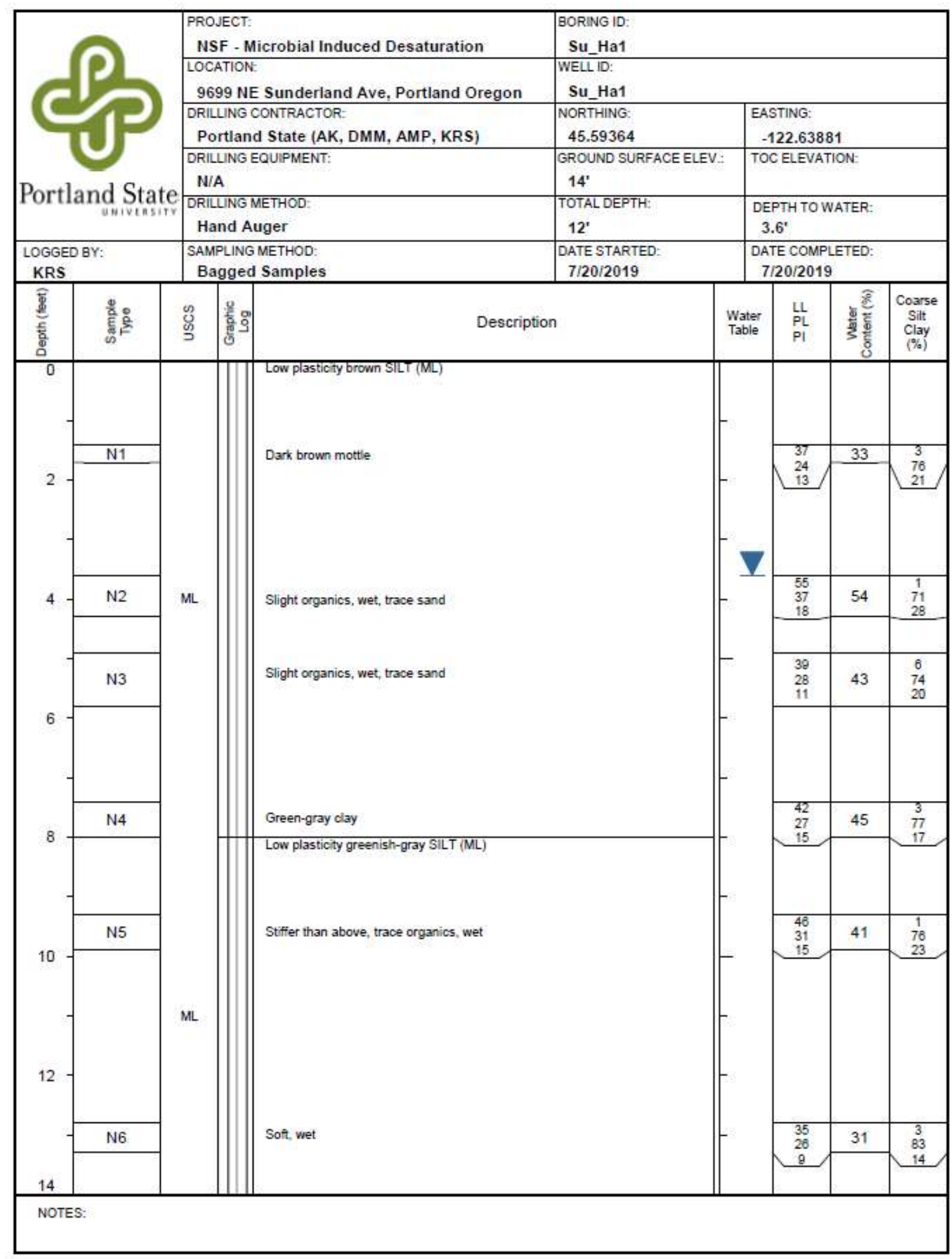




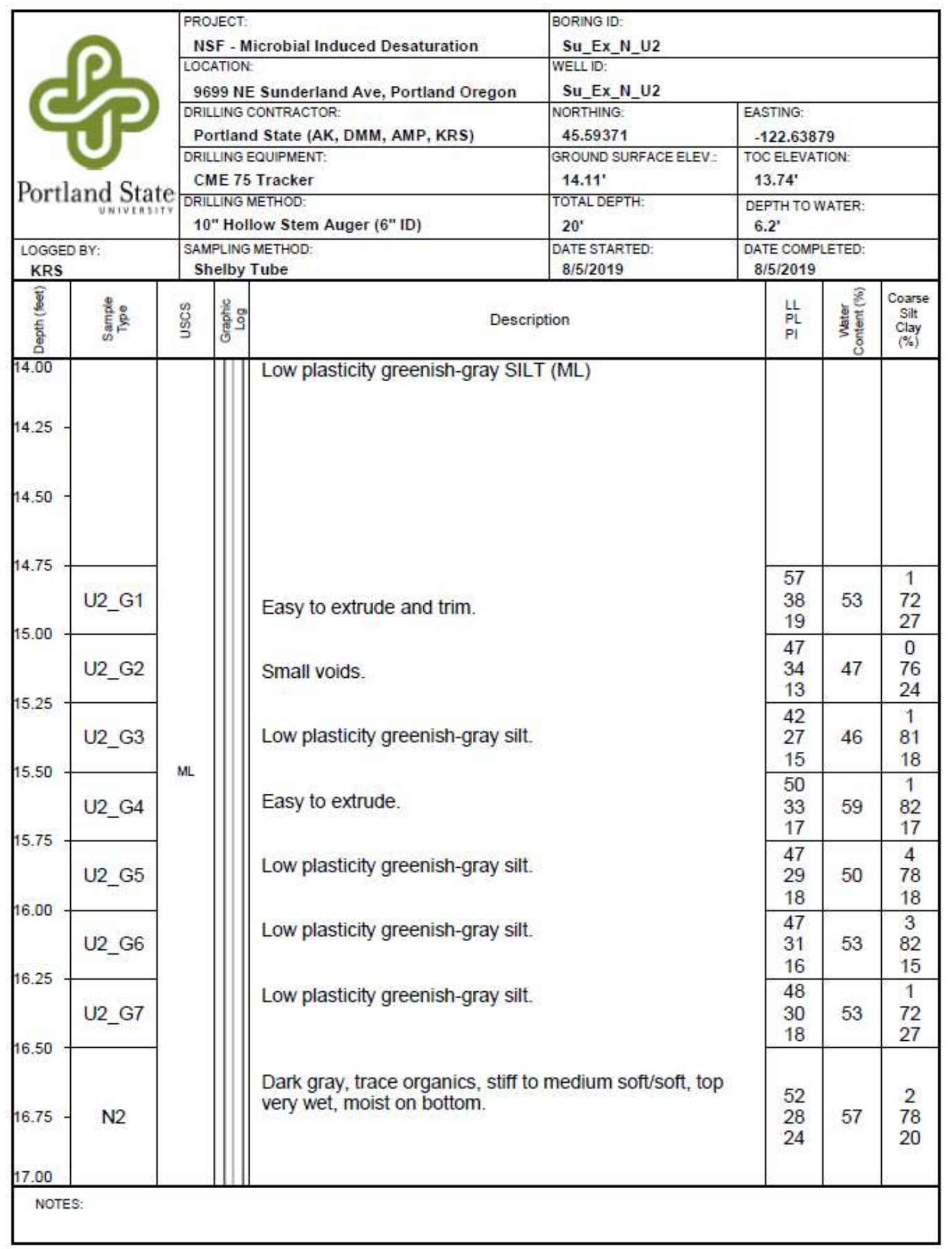




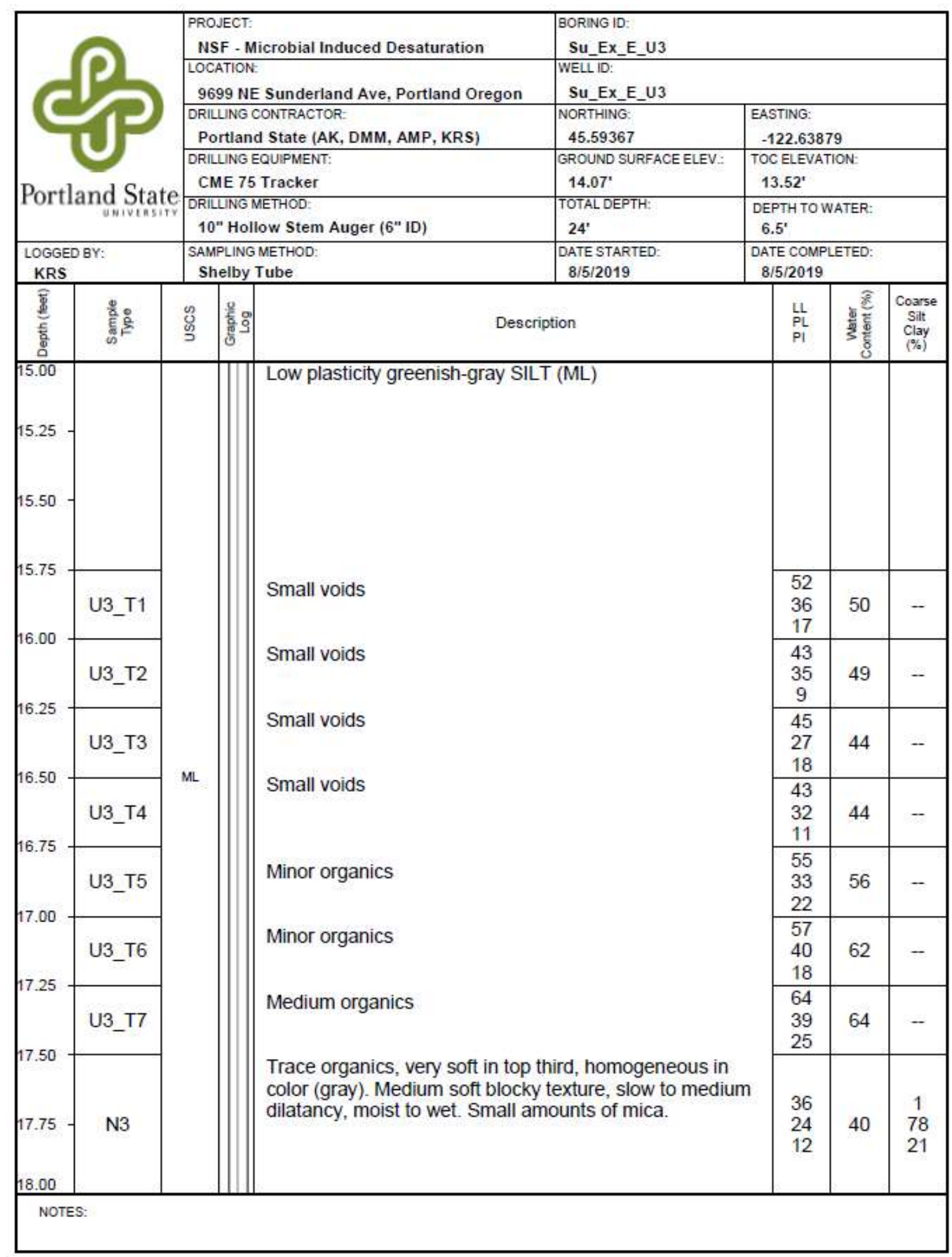




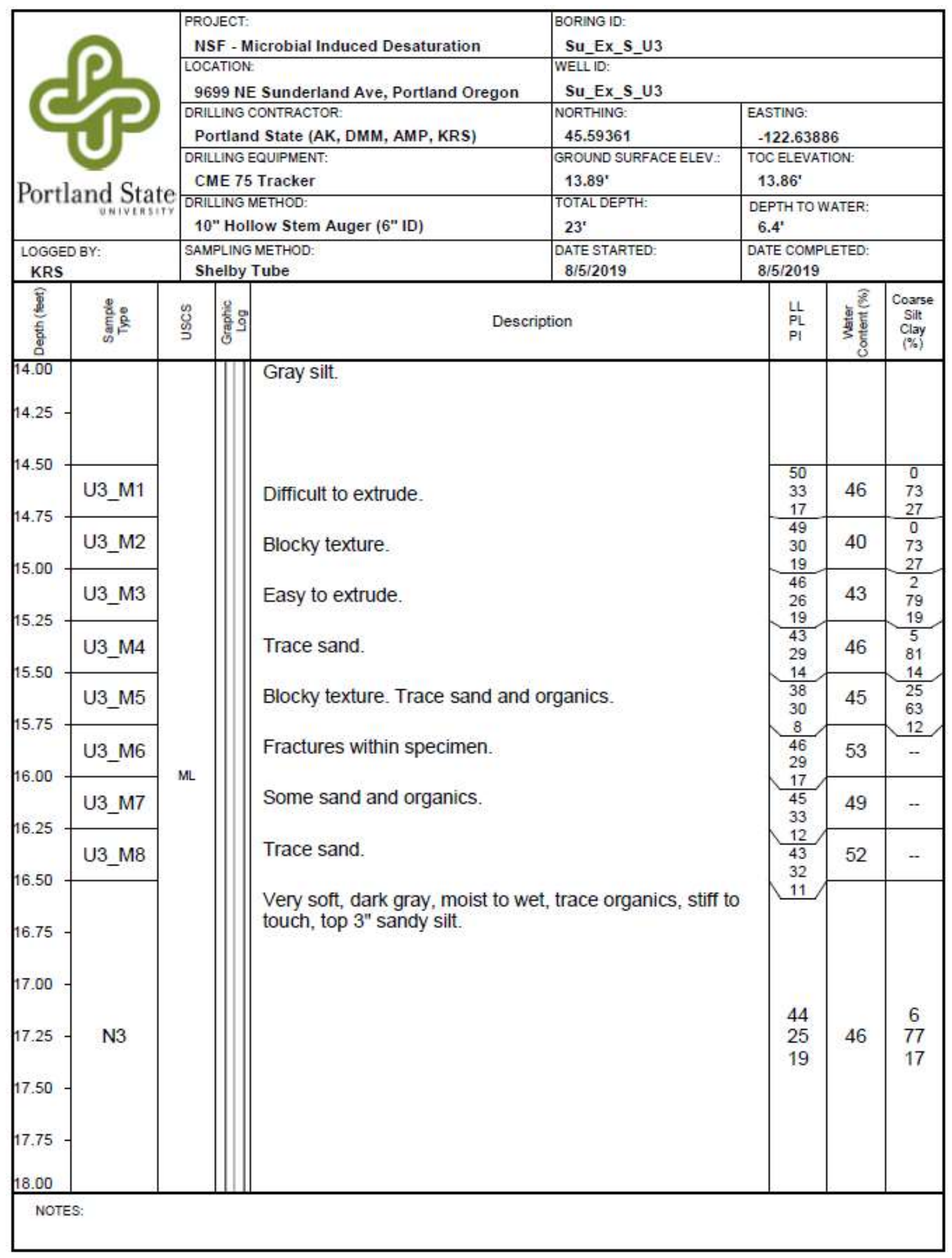




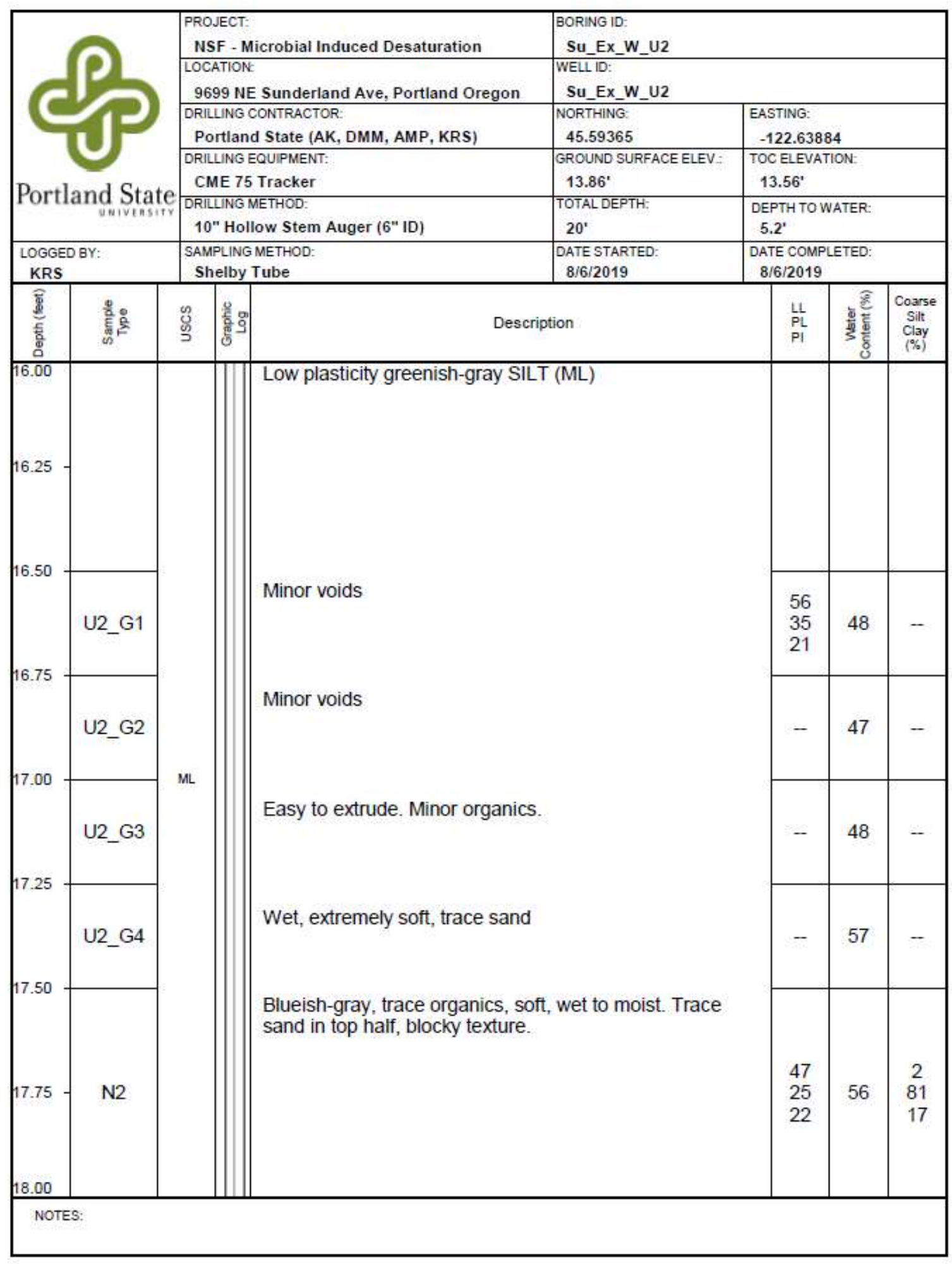




\section{Appendix C: Atterberg Limits - Sunderland}

\section{胥 Portland State}

\begin{tabular}{|c|c|c|c|c|c|c|c|c|c|c|c|}
\hline \multirow{3}{*}{\multicolumn{2}{|c|}{$\begin{array}{l}\text { Project: } \\
\text { Location: } \\
\text { Borehole: }\end{array}$}} & \multicolumn{10}{|c|}{ NSF - Microbial Induced Desaturation } \\
\hline & & \multicolumn{10}{|c|}{ Sunderland } \\
\hline & & \multicolumn{2}{|c|}{ Su_Ha1 } & \multicolumn{4}{|c|}{ Sample Number: } & \multicolumn{2}{|l|}{ N1 } & \multicolumn{2}{|c|}{ Depth (ft-ft): } \\
\hline \multicolumn{7}{|c|}{ Liquid Limit } & \multicolumn{5}{|c|}{ Plastic Limit } \\
\hline 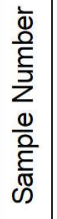 & 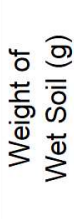 & 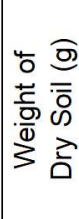 & 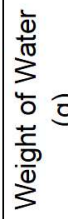 & & 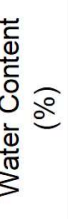 & 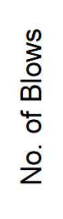 & 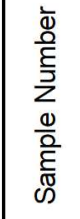 & 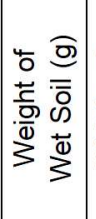 & 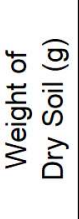 & 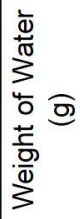 & 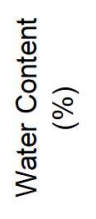 \\
\hline 1 & 6.5 & 4.9 & 1. & & 32.7 & 85 & 1 & 6.8 & 5.5 & 1.3 & 23.6 \\
\hline 2 & 3.0 & 2.2 & 0. & & 36.4 & 27 & 2 & 7.0 & 5.5 & 1.5 & 27 \\
\hline 3 & 5.1 & 3.7 & 1. & & 37.8 & 19 & 3 & 6.7 & 5.4 & & \\
\hline 4 & 2.8 & 2.0 & 0. & & 40.0 & $\S$ & & & & & \\
\hline & & & & & & & & & & & \\
\hline & & & & & & & & & & & \\
\hline
\end{tabular}
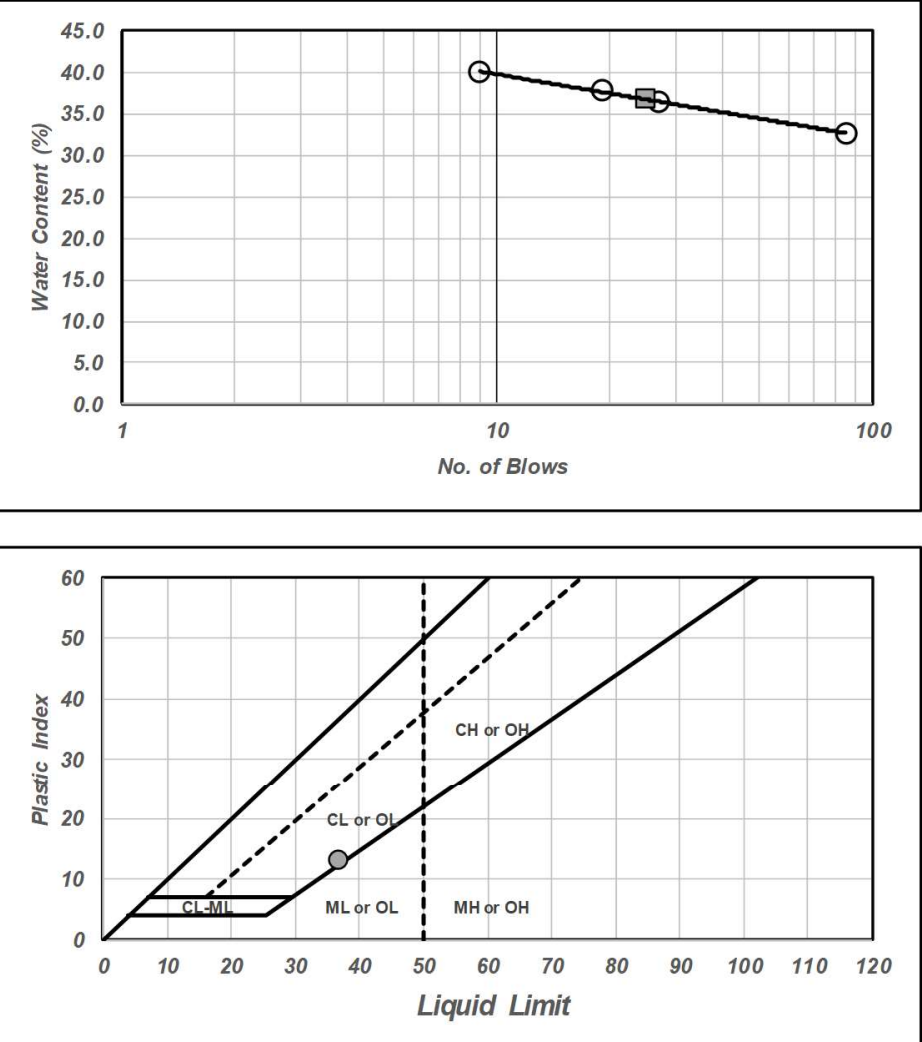

Liquid Limit:

Plastic Limit:

Plasticity Index:

Total amount of materials passing sieve No. 40 : $100 \%$

Fines

Classification: $\mathrm{CL}$

Procedure Done By: Kayla 


\section{\& Portland State}

\begin{tabular}{|c|c|c|c|c|c|c|c|c|c|c|}
\hline \multicolumn{2}{|c|}{ Project: } & \multicolumn{9}{|c|}{ NSF - Microbial Induced Desaturation } \\
\hline \multicolumn{2}{|c|}{ Location: } & \multicolumn{9}{|c|}{ Sunderland } \\
\hline \multicolumn{2}{|c|}{ Borehole: } & \multicolumn{2}{|c|}{ Su Ha1 } & \multicolumn{3}{|c|}{ Sample Number: } & \multicolumn{2}{|l|}{ N2 } & \multicolumn{2}{|c|}{ Depth (ft-ft): } \\
\hline \multicolumn{6}{|c|}{ Liquid Limit } & \multicolumn{5}{|c|}{ Plastic Limit } \\
\hline 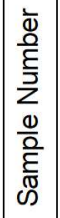 & 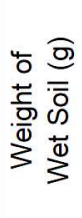 & 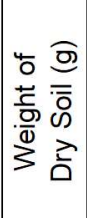 & 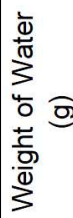 & 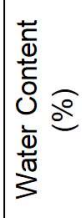 & 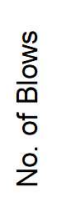 & 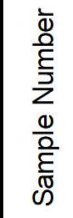 & 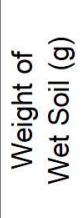 & 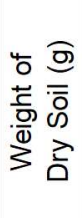 & 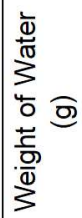 & 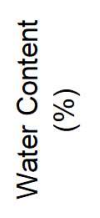 \\
\hline 1 & 15.4 & 10.0 & 5.4 & 54.0 & 14 & 1 & 7.8 & 5.7 & 2.1 & 36.8 \\
\hline 2 & 12.5 & 7.9 & 4.6 & 58.2 & 8 & 2 & 7.4 & 5.7 & 1.7 & 29.8 \\
\hline 3 & 14.6 & 9.5 & 5.13 & 54.0 & 75 & 3 & 7.5 & 5.5 & & \\
\hline 4 & 15.7 & 10.2 & 5.5 & 53.9 & 38 & 4 & 7.7 & 5.4 & & \\
\hline & & & & & & & & & & \\
\hline & & & & & & & & & & \\
\hline
\end{tabular}

Date Collected: July 18, 2019

3646 Average Depth (ft):

4.10

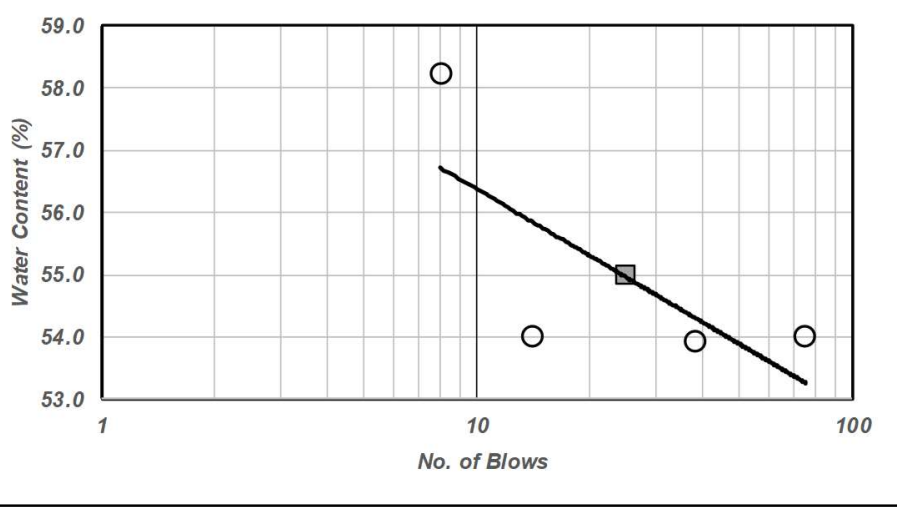

Liquid Limit:

Plastic Limit:

Plasticity Index:

\begin{tabular}{l}
55 \\
\hline 37 \\
\hline 18 \\
\hline
\end{tabular}

Total amount of materials passing sieve No. 40 : $100 \%$

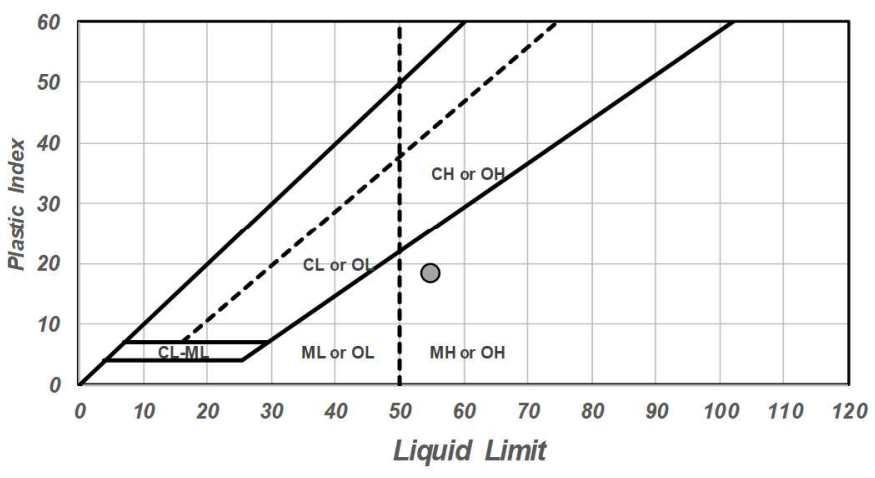

Fines

Classification: $\mathrm{MH}$ 


\section{\& Portland State}

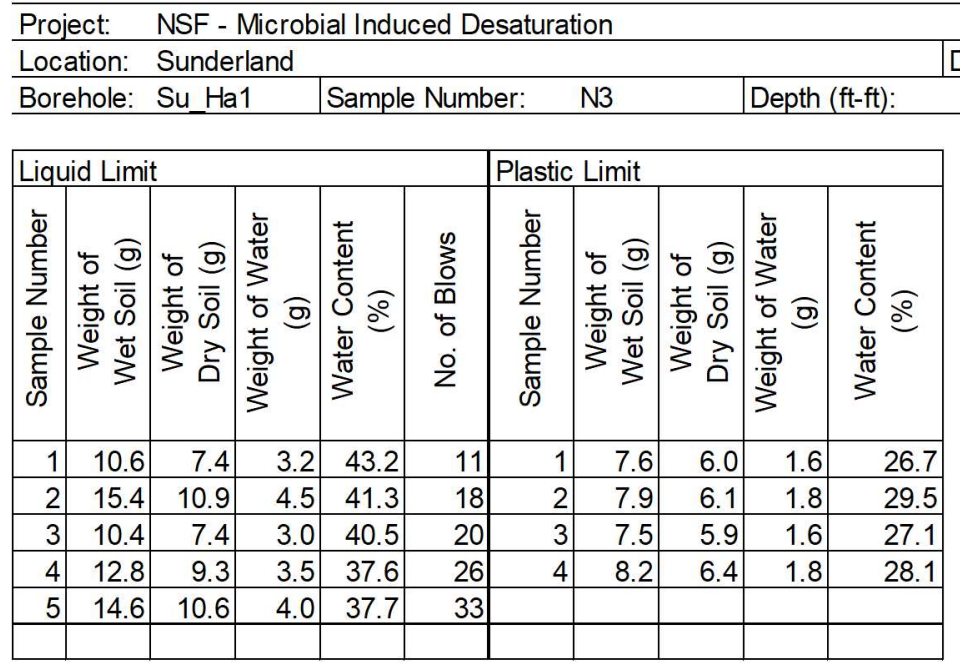
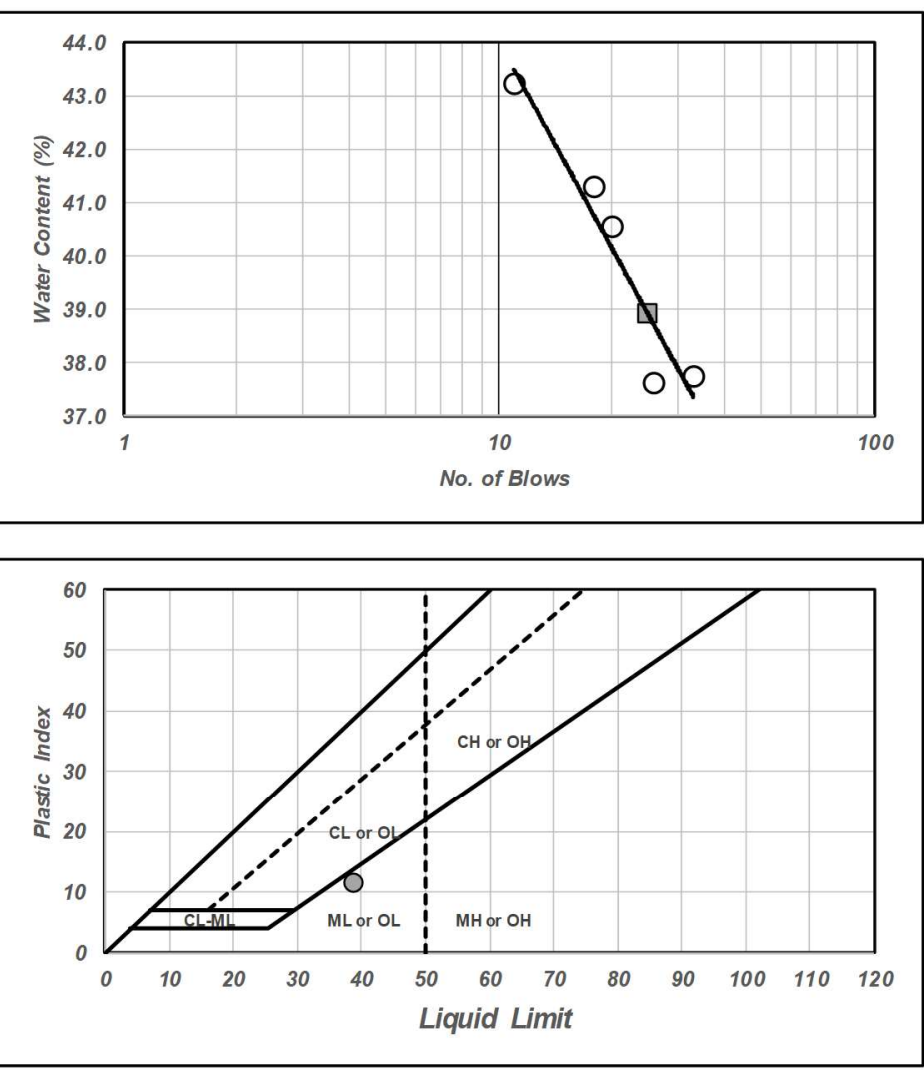

Liquid Limit:

Plastic Limit:

Plasticity Index:
Total amount of materials passing sieve No. 40: $100 \%$

Procedure Done By: Kayla

Fines

Classification: ML 


\section{\& Portland State}

\begin{tabular}{|c|c|c|c|c|c|c|c|c|c|c|}
\hline \multicolumn{2}{|c|}{ Project: } & \multicolumn{9}{|c|}{ NSF - Microbial Induced Desaturation } \\
\hline \multicolumn{2}{|c|}{ Location: } & \multicolumn{9}{|c|}{ Sunderland } \\
\hline \multicolumn{2}{|c|}{ Borehole: } & \multicolumn{2}{|c|}{ Su Ha1 } & \multicolumn{3}{|c|}{ Sample Number: } & \multicolumn{2}{|l|}{$\mathrm{N} 4$} & \multicolumn{2}{|c|}{ Depth (ft-ft): } \\
\hline \multicolumn{6}{|c|}{ Liquid Limit } & \multicolumn{5}{|c|}{ Plastic Limit } \\
\hline 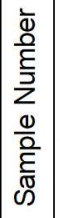 & 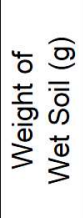 & 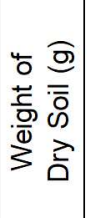 & 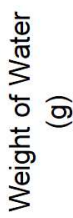 & 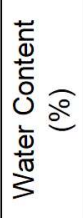 & 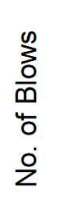 & 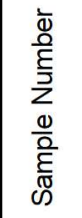 & 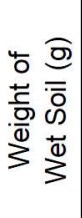 & 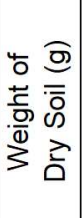 & 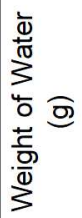 & 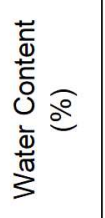 \\
\hline 1 & 4.2 & 2.9 & 1.3 & 44.8 & 43 & 1 & 6.3 & 4.9 & 1.4 & 28.6 \\
\hline 2 & 5.1 & 3.4 & 1.7 & 50.0 & 14 & 2 & 12.2 & 9.2 & 3 & 32.6 \\
\hline 3 & 6.2 & 3.9 & 2.3 & 59.0 & 7 & 3 & 9.8 & 7.7 & 2.1 & 27.3 \\
\hline 4 & 5.3 & 3.8 & 1.5 & 39.5 & 13 & & & & & \\
\hline 5 & 5.8 & 4.3 & 1.5 & 34.9 & 30 & & & & & \\
\hline & & & & & & & & & & \\
\hline
\end{tabular}

Date Collected: July 18, 2019

\begin{tabular}{ll|l}
7.4 & 8.0 & Average Depth $(\mathrm{ft}):$
\end{tabular}

7.70

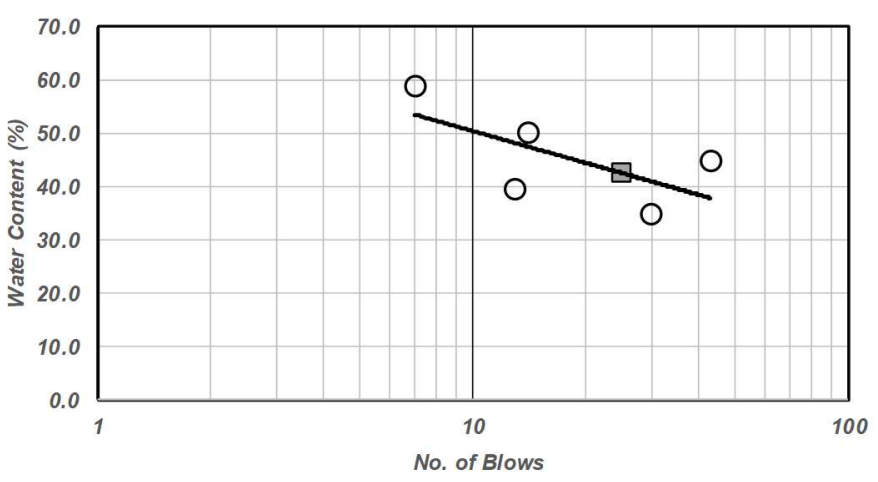

Liquid Limit:

Plastic Limit:

Plasticity Index:

\begin{tabular}{l}
42 \\
\hline 28 \\
\hline 14
\end{tabular}

Total amount of materials passing sieve No. 40: $100 \%$

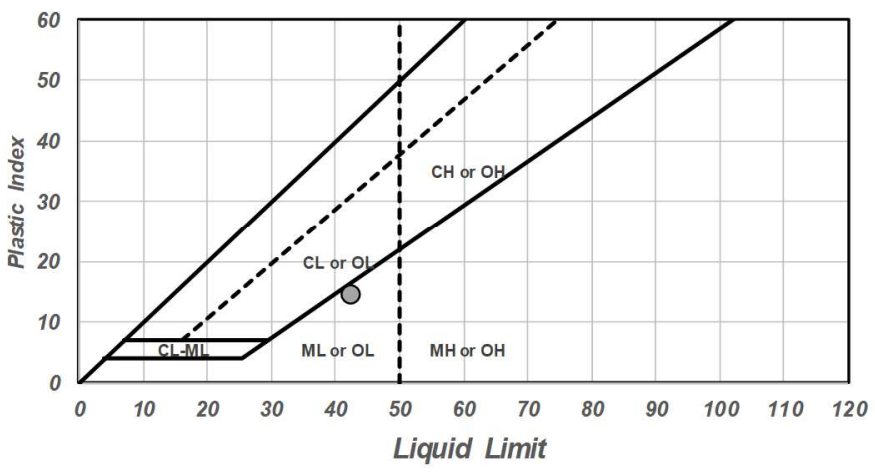

Fines

Classification: ML

Procedure Done By: Kayla 


\section{\& Portland State}

\begin{tabular}{|c|c|c|c|c|c|c|c|c|c|c|}
\hline \multicolumn{2}{|c|}{ Project: } & \multicolumn{9}{|c|}{ NSF - Microbial Induced Desaturation } \\
\hline \multicolumn{2}{|c|}{ Location: } & \multicolumn{9}{|c|}{ Sunderland } \\
\hline \multicolumn{2}{|c|}{ Borehole: } & \multicolumn{2}{|c|}{ Su Ha1 } & \multicolumn{3}{|c|}{ Sample Number: } & \multicolumn{2}{|l|}{ N5 } & \multicolumn{2}{|c|}{ Depth (ft-ft): } \\
\hline \multicolumn{6}{|c|}{ Liquid Limit } & \multicolumn{5}{|c|}{ Plastic Limit } \\
\hline 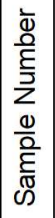 & 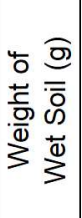 & 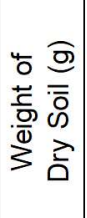 & 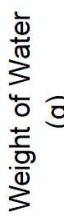 & 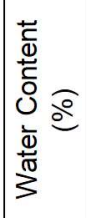 & 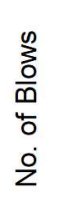 & 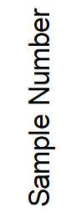 & 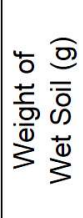 & 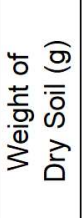 & 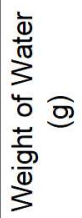 & 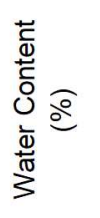 \\
\hline 1 & 5.5 & 3.9 & 1. & 41.0 & 120 & 1 & 11.3 & 8.6 & 2.7 & 31.4 \\
\hline 2 & 4.2 & 2.9 & 1. & 44.8 & 48 & 2 & 6.3 & 5.0 & 1.3 & 26.0 \\
\hline 3 & 5.0 & 3.5 & 1. & 42.9 & 16 & 3 & 9.6 & 7.4 & 2.2 & 29.7 \\
\hline 4 & 6.5 & 4.2 & 2. & 54.8 & 6 & & & & & \\
\hline & & & & & & & & & & \\
\hline & & & & & & & & & & \\
\hline
\end{tabular}

Date Collected: July 18, 2019

939.8 Average Depth (ft):

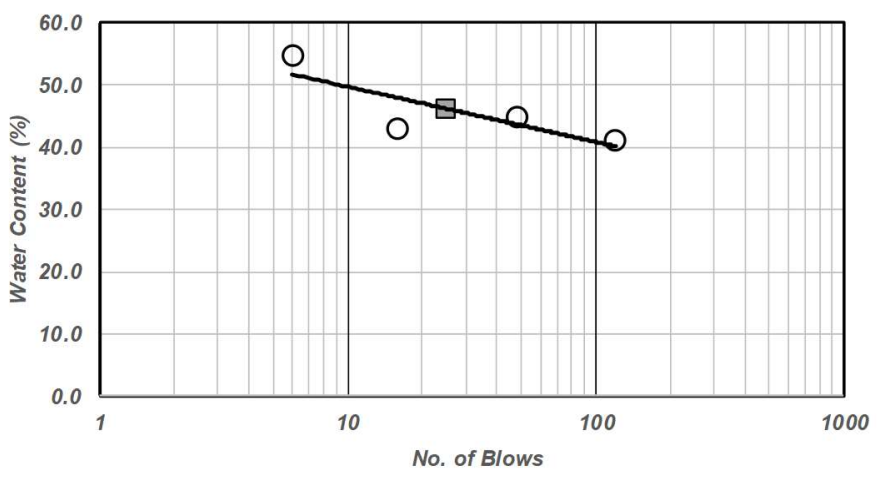

Liquid Limit:

Plastic Limit:

Plasticity Index:

\begin{tabular}{l}
46 \\
\hline 31 \\
\hline 15
\end{tabular}

Total amount of materials passing sieve No. 40: $100 \%$

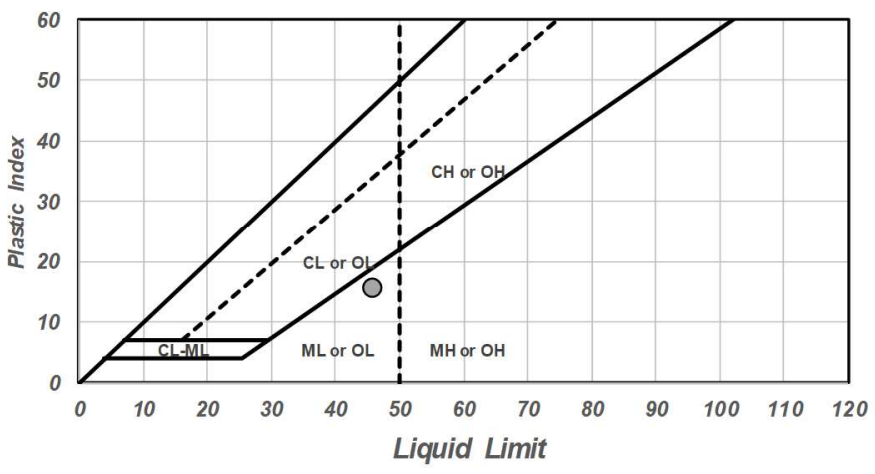

Fines

Classification: ML

Procedure Done By: Kayla 


\section{\& Portland State}

\begin{tabular}{|c|c|c|c|c|c|c|c|c|c|c|}
\hline \multicolumn{2}{|c|}{ Project: } & \multicolumn{9}{|c|}{ NSF - Microbial Induced Desaturation } \\
\hline \multicolumn{2}{|c|}{ Location: } & \multicolumn{9}{|c|}{ Sunderland } \\
\hline \multicolumn{2}{|c|}{ Borehole: } & \multicolumn{2}{|c|}{ Su Ha1 } & \multicolumn{3}{|c|}{ Sample Number: } & \multicolumn{2}{|l|}{ N6 } & \multicolumn{2}{|c|}{ Depth (ft-ft): } \\
\hline \multicolumn{6}{|c|}{ Liquid Limit } & \multicolumn{5}{|c|}{ Plastic Limit } \\
\hline 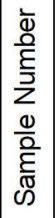 & 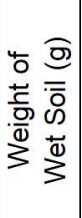 & 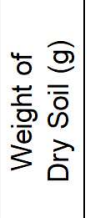 & 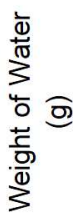 & 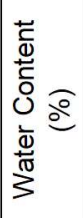 & 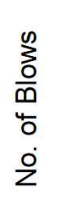 & 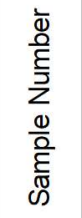 & 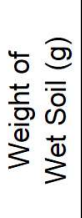 & 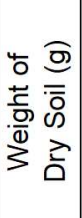 & 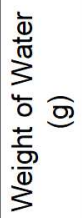 & 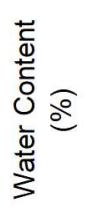 \\
\hline 1 & 2.1 & 1.6 & 0.5 & 31.3 & 21 & 1 & 8.2 & 6.5 & 1.7 & 26.2 \\
\hline 2 & 4.9 & 3.5 & 1.4 & 40.0 & 8 & 2 & 7.9 & 6.3 & 1.6 & 25.4 \\
\hline 3 & 3.0 & 2.3 & 0.7 & 30.4 & 216 & & & & & \\
\hline 4 & 3.5 & 2.6 & 0.9 & 34.6 & 39 & & & & & \\
\hline & & & & & & & & & & \\
\hline & & & & & & & & & & \\
\hline
\end{tabular}

Date Collected: July 18, 2019

12813.3 Average Depth $(\mathrm{ft})$.

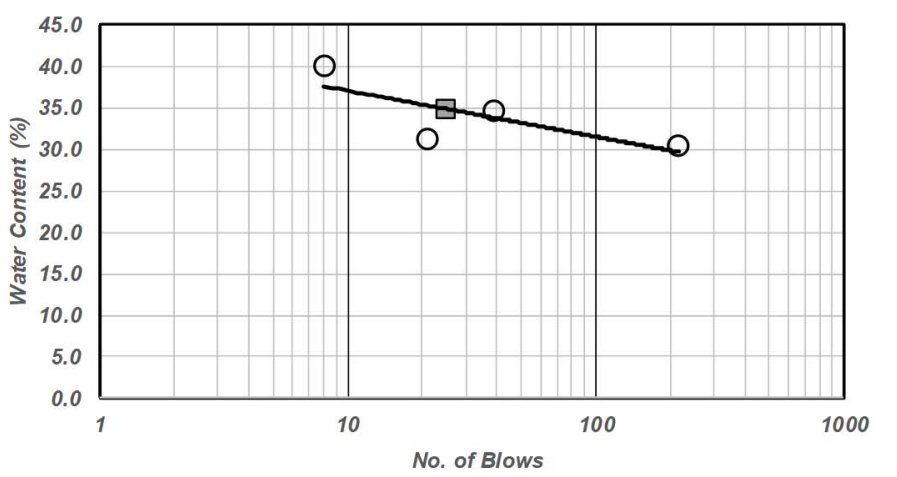

Liquid Limit:

Plastic Limit:

Plasticity Index:
Total amount of materials passing sieve No. 40: $100 \%$

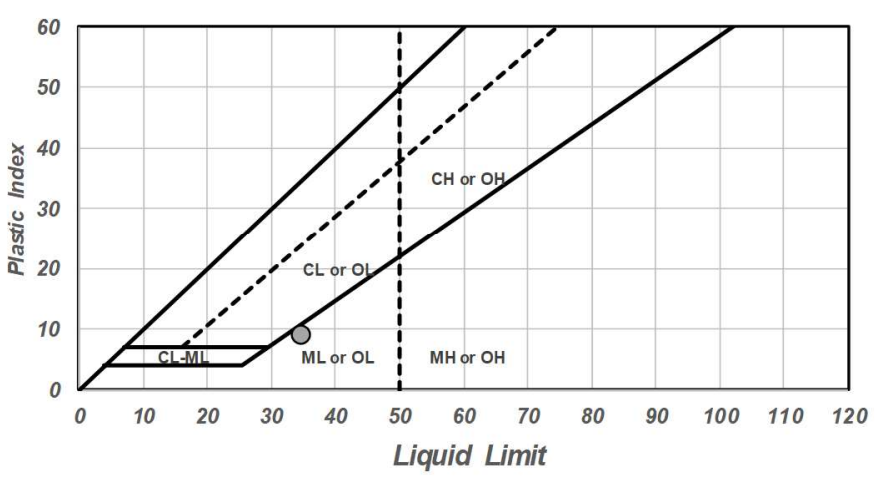

Procedure Done By:
Fines

Classification: $\mathrm{ML}$ 


\section{S Portland State}

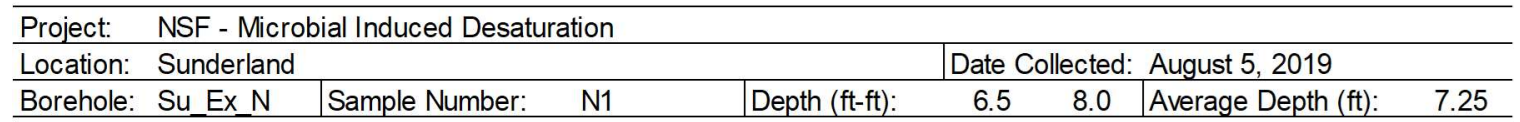

\begin{tabular}{|c|c|c|c|c|c|c|c|c|c|c|}
\hline \multicolumn{6}{|c|}{ Liquid Limit } & \multicolumn{5}{|c|}{ Plastic Limit } \\
\hline 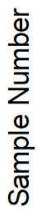 & 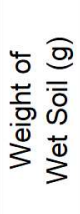 & 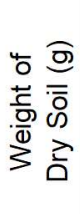 & 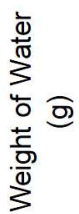 & 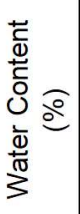 & 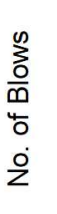 & 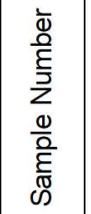 & 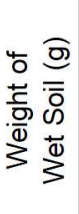 & 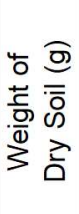 & 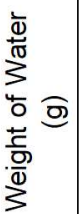 & 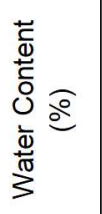 \\
\hline 1 & 14.0 & 9.6 & 4.4 & 45.8 & 2 & 1 & 6.2 & 5.0 & 1.2 & 24.0 \\
\hline 2 & 11.0 & 7.7 & 3.3 & 42.9 & 11 & 2 & 5.9 & 4.7 & 1.2 & 25.5 \\
\hline 3 & 13.2 & 9.8 & 3.4 & 34.7 & 35 & & & & & \\
\hline 4 & 15.5 & 11.4 & 4.1 & 36.0 & 75 & & & & & \\
\hline & & & & & & & & & & \\
\hline & & & & & & & & & & \\
\hline
\end{tabular}

Liquid Limit:

Plastic Limit:

Plasticity Index:

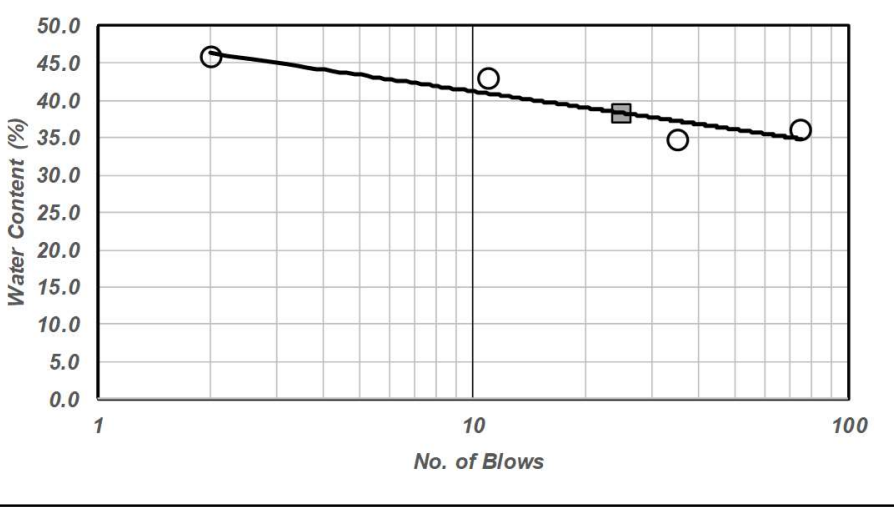

Total amount of materials passing sieve No. 40: $100 \%$

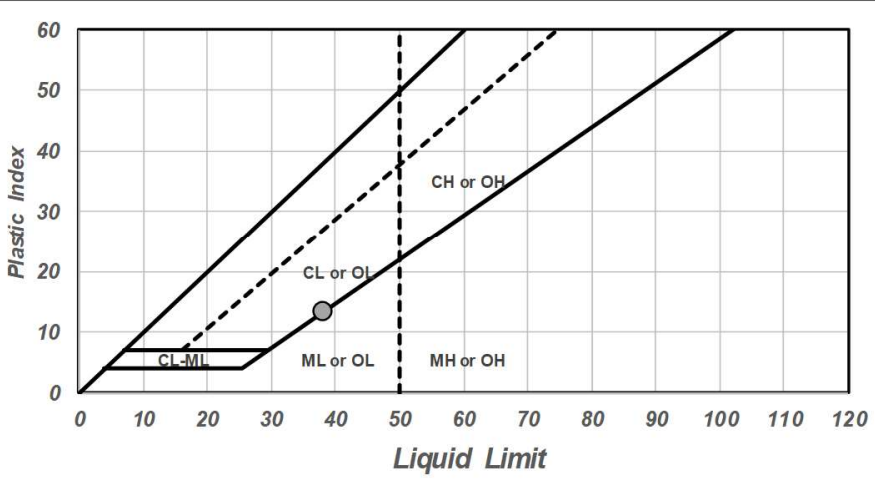

Procedure Done By
Fines

Classification: $\mathrm{CL}$ 


\section{\& Portland State}

\begin{tabular}{|c|c|c|c|c|c|c|c|c|c|c|c|}
\hline Proj & ect: & NSF & Micro & ial & I Indu & ced D & satura & ation & & & \\
\hline LOC & ation: & Sund & rland & & & & & & & & \\
\hline Bor & ehole: & Su_E & $\mathrm{N}$ & & Sample & Num & & $\mathrm{N} 2$ & & Depth & $\mathrm{ft}-\mathrm{ft}):$ \\
\hline Liqu & iid Lim & & & & & & & c Limit & & & \\
\hline 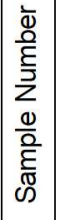 & 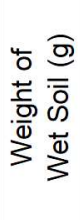 & 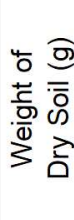 & 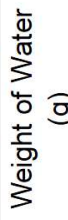 & & 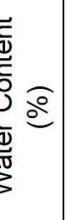 & 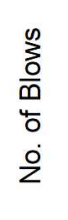 & 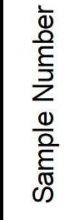 & 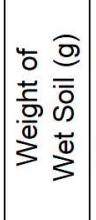 & 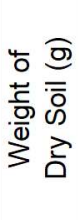 & 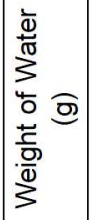 & 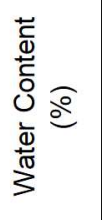 \\
\hline 1 & 14.3 & 9.1 & 5. & & 57.1 & 17 & 1 & 6.2 & 4.9 & 1.3 & 26.5 \\
\hline 2 & 6.5 & 4.3 & 2. & & 51.2 & 23 & 2 & 6.6 & 5.1 & 1.5 & 29.4 \\
\hline 3 & 8.3 & 5.6 & 2. & & 48.2 & 31 & & & & & \\
\hline 4 & 9.5 & 6.4 & 3. & & 48.4 & 46 & & & & & \\
\hline 5 & 2.6 & 1.8 & 0.8 & & 44.4 & 85 & & & & & \\
\hline & & & & & & & & & & & \\
\hline
\end{tabular}
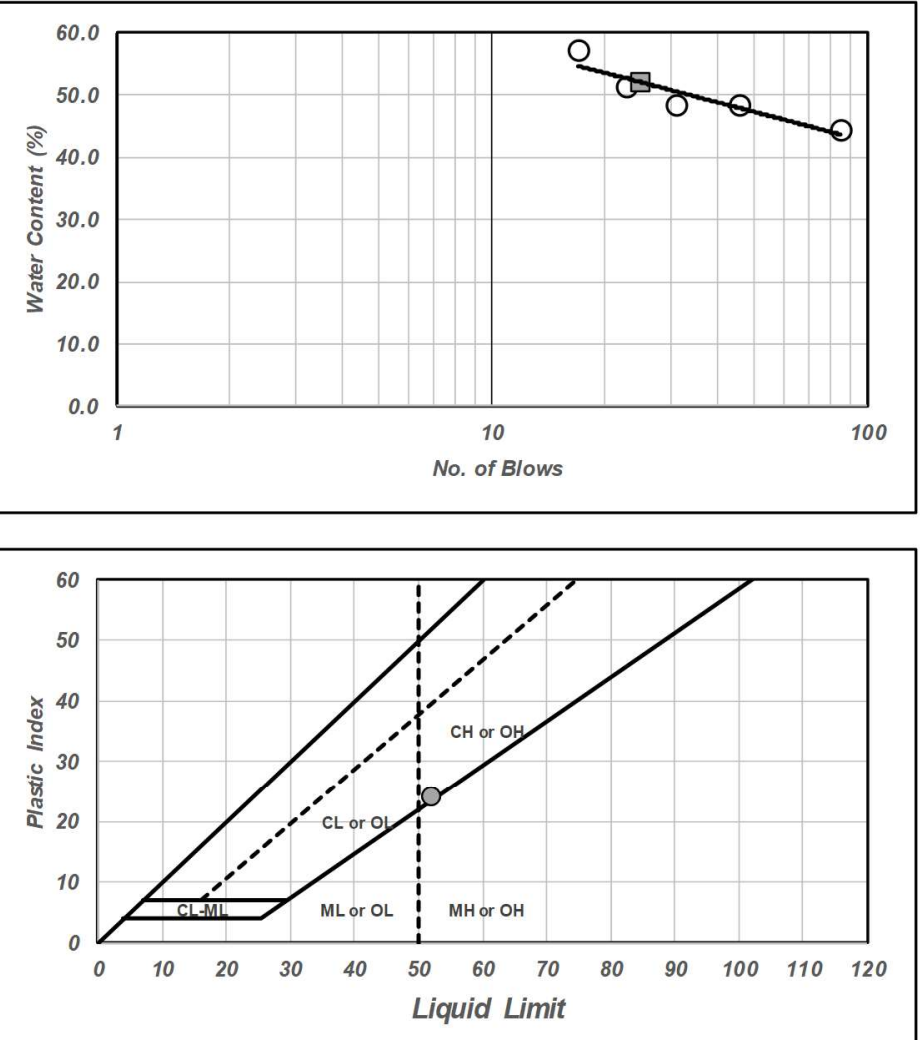

Date Collected: August 5, 2019

\begin{tabular}{ll|l}
16.5 & 18.0 & Average Depth $(\mathrm{ft}):$ \\
& 17.25
\end{tabular}
Total amount of materials passing sieve No. 40: $100 \%$
Fines

Classification: $\mathrm{CH}$ 


\section{S Portland State}

\begin{tabular}{|c|c|c|c|c|c|c|c|c|c|c|}
\hline \multicolumn{2}{|c|}{ Project: } & \multicolumn{9}{|c|}{ NSF - Microbial Induced Desaturation } \\
\hline \multicolumn{2}{|c|}{ Location: } & \multicolumn{9}{|c|}{ Sunderland } \\
\hline \multicolumn{2}{|c|}{ Borehole: } & \multicolumn{2}{|c|}{ Su Ex E } & \multicolumn{3}{|c|}{ Sample Number: } & \multicolumn{2}{|l|}{ N1 } & \multicolumn{2}{|c|}{ Depth (ft-ft): } \\
\hline \multicolumn{6}{|c|}{ Liquid Limit } & \multicolumn{5}{|c|}{ Plastic Limit } \\
\hline 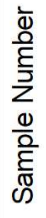 & 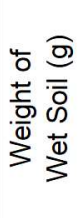 & 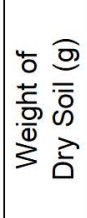 & 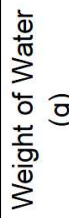 & 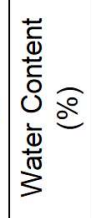 & 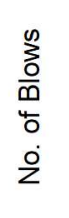 & 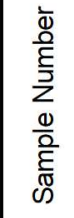 & 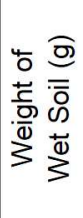 & 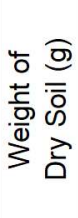 & 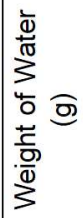 & 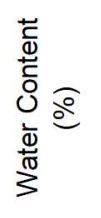 \\
\hline 1 & 2.4 & 1.8 & 0. & 33.3 & 2 & 1 & 6.1 & 5.4 & 0.7 & 13.0 \\
\hline 2 & 2.1 & 1.7 & 0. & 23.5 & 65 & 2 & 6.7 & 5.4 & 1.3 & 24.1 \\
\hline 3 & 3.0 & 2.3 & 0. & 30.4 & 59 & 3 & 7.6 & 6.2 & 1.4 & 22.6 \\
\hline 4 & 2.1 & 1.6 & 0. & 31.3 & 47 & & & & & \\
\hline 5 & 1.9 & 1.5 & 0. & 26.7 & 16 & & & & & \\
\hline & & & & & & & & & & \\
\hline
\end{tabular}

Date Collected: August 6, 2019

\begin{tabular}{ll|l}
7.5 & 9.0 & Average Depth $(\mathrm{ft}):$
\end{tabular}

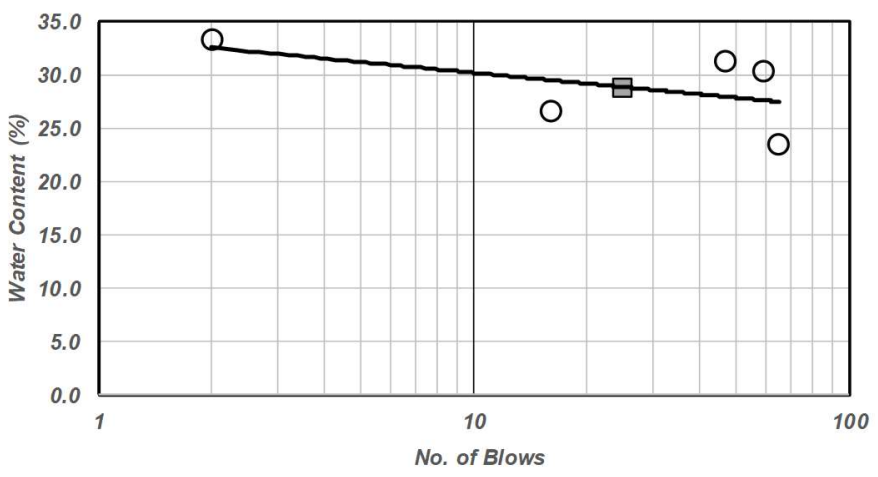

Liquid Limit:

Plastic Limit:

Plasticity Index:
Total amount of materials passing sieve No. 40: $100 \%$

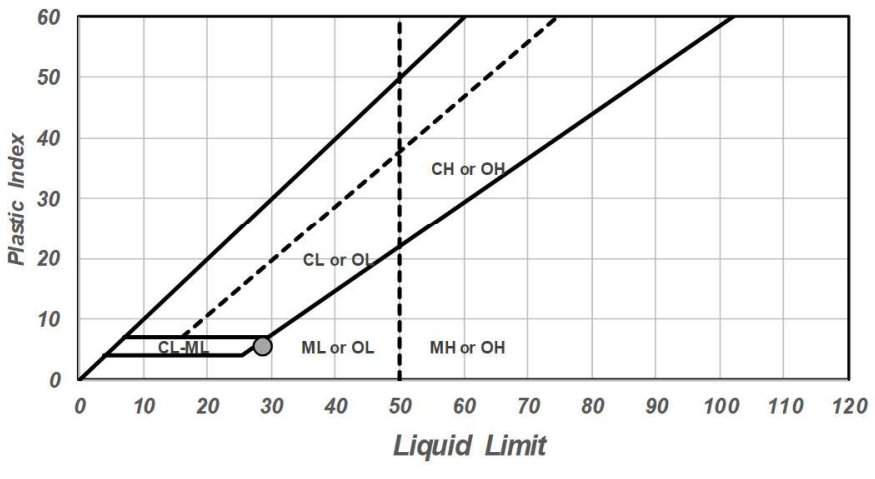

Procedure Done By:
Fines

Classification: $\mathrm{ML}$ 


\section{S Portland State}

\begin{tabular}{|c|c|c|c|c|c|c|c|c|c|c|c|}
\hline Proj & ect: & NSF & Micro & bia & al Ind & ed D & esatura & ation & & & \\
\hline LOC & ation: & Sund & rland & & & & & & & & \\
\hline Bor & ehole: & Su_E & $\underline{E} E$ & & Samp & Num & ber: & $\mathrm{N} 2$ & & Depth & $\mathrm{ft}-\mathrm{ft}):$ \\
\hline Liqu & iid Lim & & & & & & Plastic & c Limit & & & \\
\hline 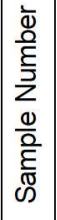 & 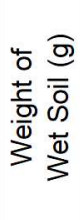 & 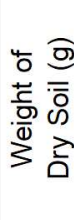 & 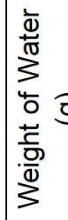 & & 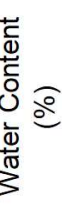 & 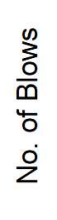 & 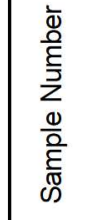 & 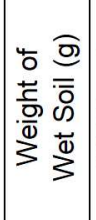 & 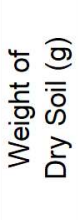 & 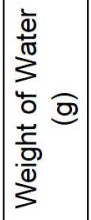 & 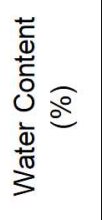 \\
\hline 1 & 4.0 & 2.7 & 1. & 3 & 48.1 & 22 & 1 & 6.2 & 4.8 & 1.4 & 29.2 \\
\hline 2 & 3.9 & 2.8 & 1. & 1 & 39.3 & 68 & 2 & 6.8 & 5.3 & 1.5 & 28.3 \\
\hline 3 & 5.0 & 3.5 & 1. & 5 & 42.9 & 46 & & & & & \\
\hline 4 & 3.1 & 2.1 & 1. & 0 & 47.6 & 2 & & & & & \\
\hline 5 & 6 & 4 & 2. & 0 & 50.0 & 1 & & & & & \\
\hline & & & & & & & & & & & \\
\hline
\end{tabular}
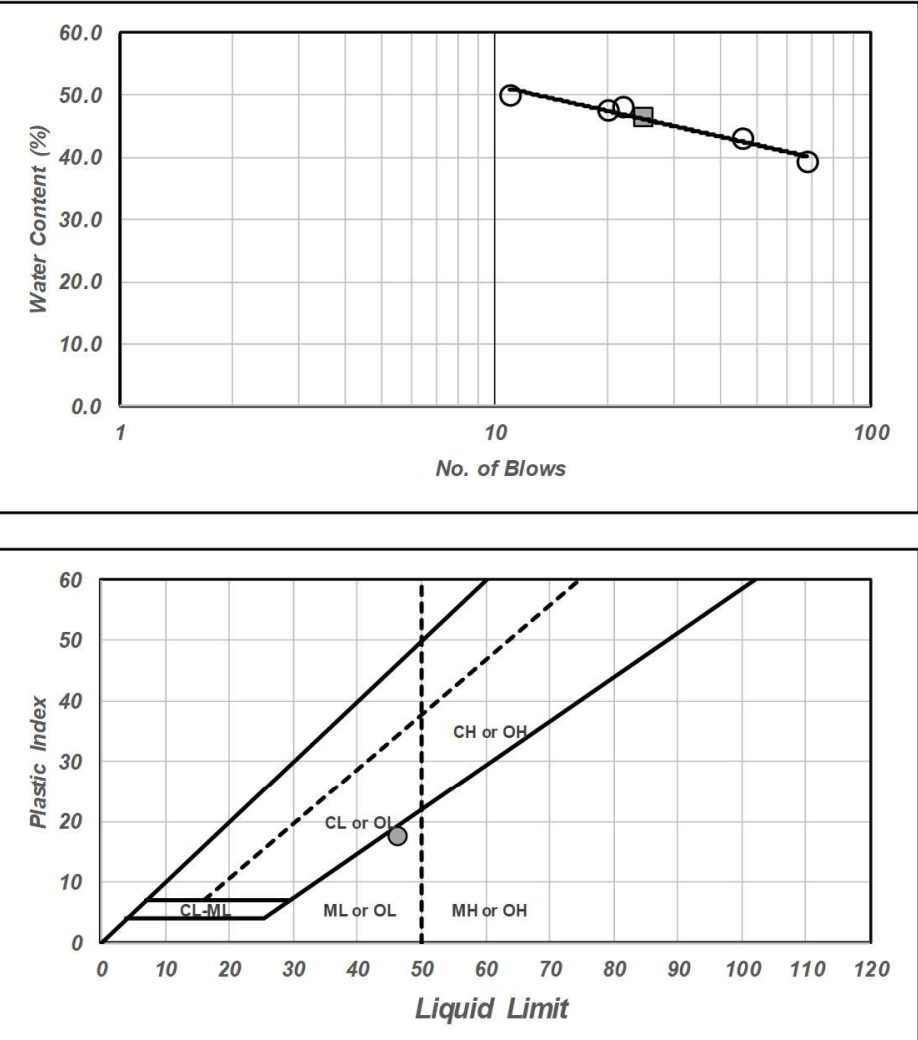

Liquid Limit:

Plastic Limit:

Plasticity Index:
Total amount of materials passing sieve No. 40: $100 \%$
Fines

Classification: ML 


\section{\& Portland State}

\begin{tabular}{|c|c|c|c|c|c|c|}
\hline oject: & NSF - Micr & Induced Desaturation & & & & \\
\hline Location: & Sunderlano & & & Date Collected: & August 6,2019 & \\
\hline Borehole: & Su Ex E & Sample Number: & Depth (ft-ft): & 19.0 & Average Depth (ft): & 18.25 \\
\hline
\end{tabular}

\begin{tabular}{|c|c|c|c|c|c|c|c|c|c|c|}
\hline \multicolumn{6}{|c|}{ Liquid Limit } & \multicolumn{5}{|c|}{ Plastic Limit } \\
\hline 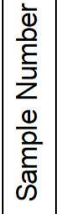 & 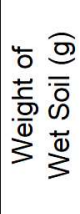 & 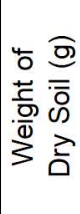 & 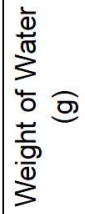 & 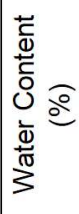 & $\begin{array}{l}0 \\
\frac{0}{0} \\
00 \\
40 \\
\dot{0} \\
\frac{0}{2}\end{array}$ & 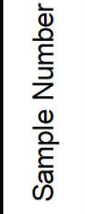 & 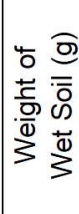 & 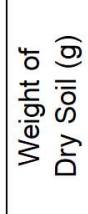 & 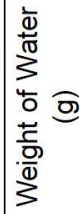 & 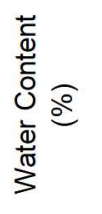 \\
\hline 1 & 2.1 & 1.5 & 0.6 & 40.0 & 18 & 1 & 6.1 & 4.9 & 1.2 & 24.5 \\
\hline 2 & 3.0 & 2.1 & 0.9 & 42.9 & 5 & 2 & 6.8 & 5.5 & 1.3 & 23.6 \\
\hline 3 & 1.7 & 1.3 & 0.4 & 30.8 & 48 & & & & & \\
\hline 4 & 4.3 & 3.2 & 1.1 & 34.4 & 34 & & & & & \\
\hline & & & & & & & & & & \\
\hline & & & & & & & & & & \\
\hline
\end{tabular}
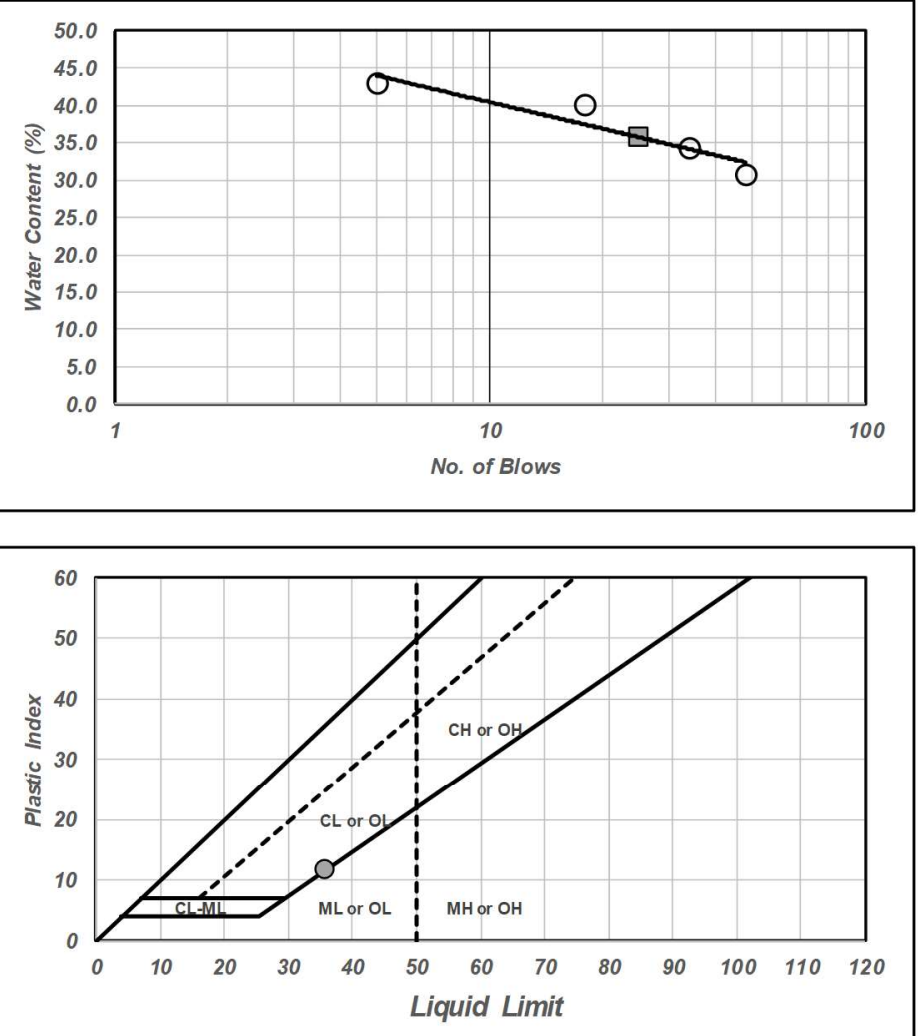

Procedure Done By:
Liquid Limit:

Plastic Limit:

Plasticity Index:
Total amount of materials passing sieve No. 40: $100 \%$
Fines

Classification: $\mathrm{CL}$ 


\section{S Portland State}

\begin{tabular}{|c|c|c|c|c|c|c|c|c|c|c|c|}
\hline Proj & ect: & NSF - & Micro & bial & Induc & ed De & esature & ation & & & \\
\hline LOC & ation: & Sund & rland & & & & & & & & \\
\hline Bor & ehole: & Su_E & & & ample & Numk & ber: & $\mathrm{N} 4$ & & Depth & $\mathrm{ft}-\mathrm{ft}$ : \\
\hline Liqu & iid Lim & & & & & & Plasti & c Limit & & & \\
\hline 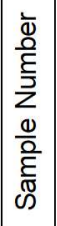 & 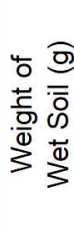 & 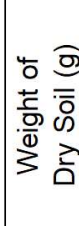 & 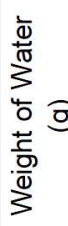 & & ஓ & 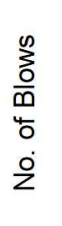 & 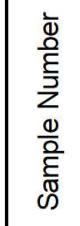 & 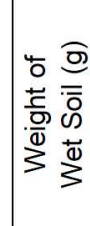 & 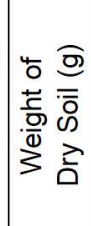 & 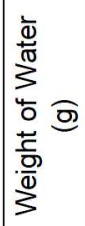 & 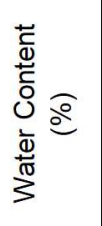 \\
\hline 1 & 6.2 & 4.3 & 1. & & 44.2 & 7 & 1 & 6.3 & 5.0 & 1.3 & 26.0 \\
\hline 2 & 6.7 & 4.7 & 2. & & 42.6 & 16 & 2 & 8.7 & 6.7 & 2.0 & 29.9 \\
\hline 3 & 7.8 & 5.7 & 2. & & 36.8 & 34 & 3 & 8.1 & 6.4 & 1.7 & 26.6 \\
\hline 4 & 9.0 & 6.6 & 2. & & 36.4 & 44 & & & & & \\
\hline & & & & & & & & & & & \\
\hline & & & & & & & & & & & \\
\hline
\end{tabular}
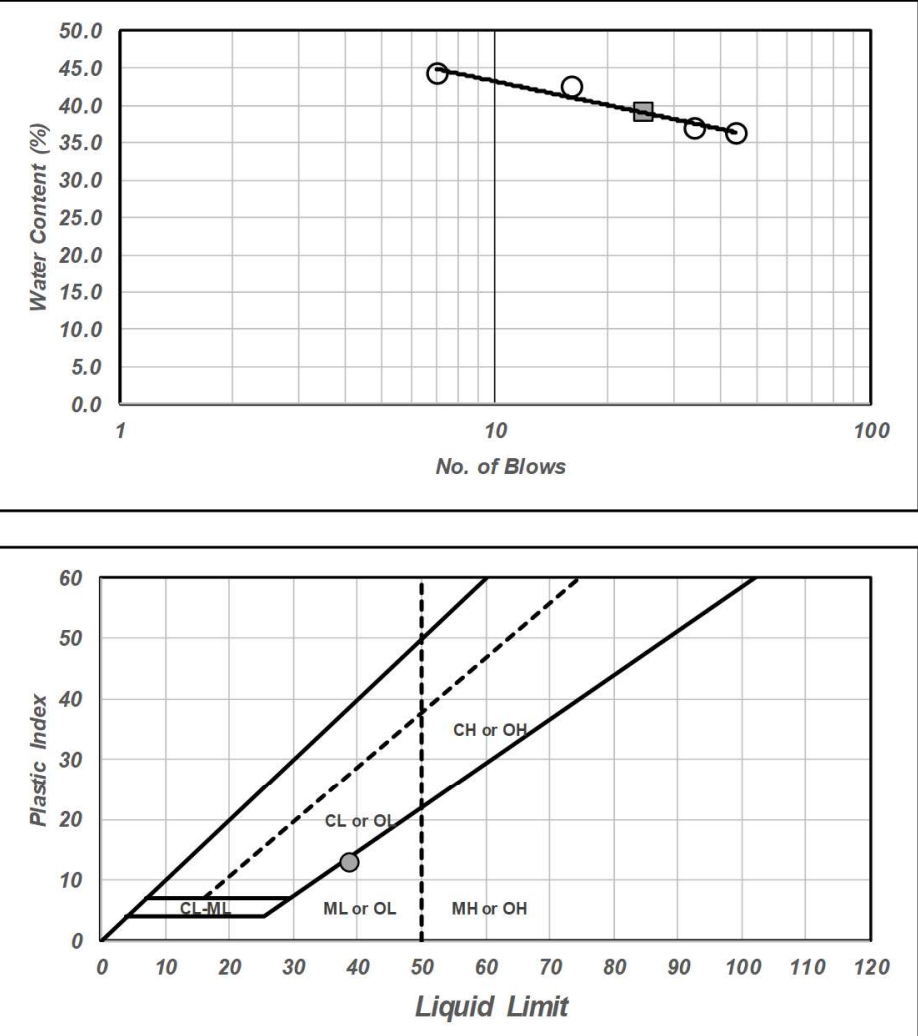

Date Collected: August 6, 2019

\begin{tabular}{ll|l}
225 & 24.0 & Average Depth $(\mathrm{ft}):$ \\
2325
\end{tabular}
Total amount of materials passing sieve No. 40: $100 \%$
Fines

Classification: ML

Procedure Done By: Kayla 


\section{\& Portland State}

\begin{tabular}{|c|c|c|c|c|c|c|c|c|c|c|}
\hline \multicolumn{2}{|c|}{ Project: } & \multicolumn{9}{|c|}{ NSF - Microbial Induced Desaturation } \\
\hline \multicolumn{2}{|c|}{ Location: } & \multicolumn{9}{|c|}{ Sunderland } \\
\hline \multicolumn{2}{|c|}{ Borehole: } & \multicolumn{2}{|c|}{ Su Ex S } & \multicolumn{3}{|c|}{ Sample Number: } & \multicolumn{2}{|l|}{ N1 } & \multicolumn{2}{|c|}{ Depth (ft-ft): } \\
\hline \multicolumn{6}{|c|}{ Liquid Limit } & \multicolumn{5}{|c|}{ Plastic Limit } \\
\hline 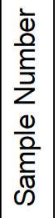 & 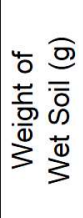 & 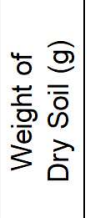 & 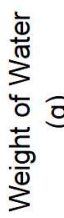 & 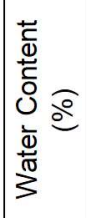 & 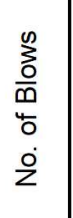 & 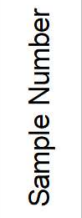 & 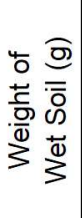 & 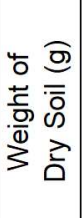 & 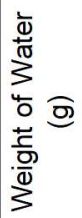 & 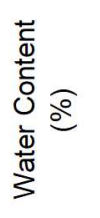 \\
\hline 1 & 7.1 & 4.9 & 2. & 44.9 & 9 & 1 & 6.7 & 5.8 & 0.9 & 15.5 \\
\hline 2 & 4.1 & 3.0 & 1. & 36.7 & 36 & 2 & 8.8 & 7.2 & 1.6 & 22.2 \\
\hline 3 & 4.1 & 3.1 & 1. & 32.3 & 65 & 3 & 9.6 & 7.8 & 1.8 & 23.1 \\
\hline 4 & 4.8 & 3.5 & 1. & 37.1 & 36 & & & & & \\
\hline 5 & 2.3 & 1.7 & 0. & 35.3 & 40 & & & & & \\
\hline & & & & & & & & & & \\
\hline
\end{tabular}

Date Collected: August 5, 2019

6.580 Average Depth $(\mathrm{ft})$

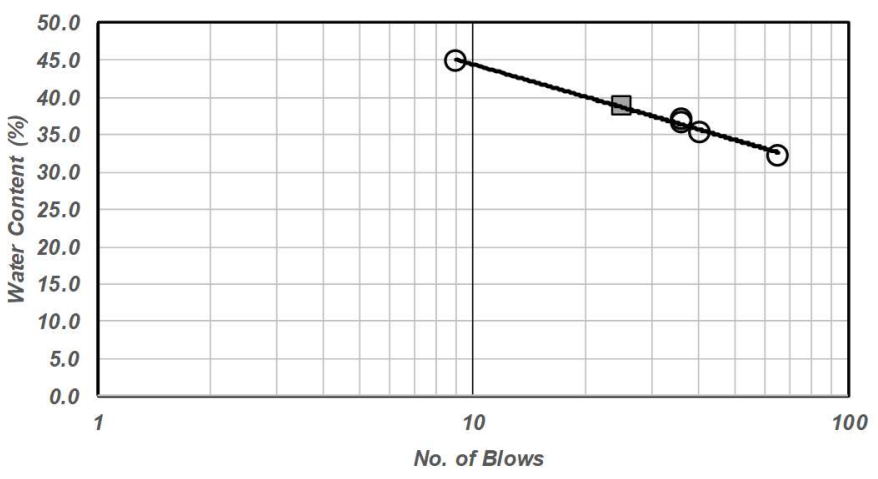

Liquid Limit:

Plastic Limit:

Plasticity Index:

\begin{tabular}{l}
39 \\
\hline 23 \\
\hline 16 \\
\hline
\end{tabular}

Total amount of materials passing sieve No. 40: $100 \%$

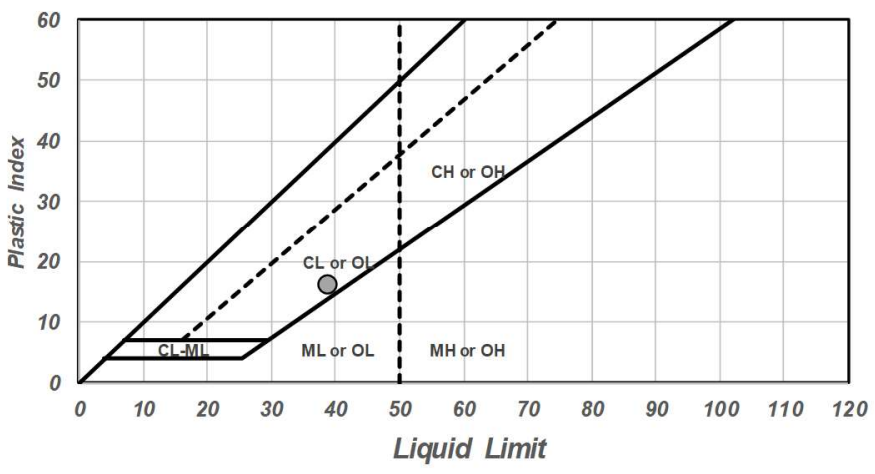

Fines

Classification: $\mathrm{CL}$ 


\section{\& Portland State}

\begin{tabular}{|c|c|c|c|c|c|c|c|c|c|c|}
\hline \multicolumn{2}{|c|}{ Project: } & \multicolumn{9}{|c|}{ NSF - Microbial Induced Desaturation } \\
\hline \multicolumn{2}{|c|}{ Location: } & \multicolumn{9}{|c|}{ Sunderland } \\
\hline \multicolumn{2}{|c|}{ Borehole: } & \multicolumn{2}{|c|}{ Su Ex S } & \multicolumn{3}{|c|}{ Sample Number: } & \multicolumn{2}{|l|}{ N2 } & \multicolumn{2}{|c|}{ Depth (ft-ft): } \\
\hline \multicolumn{6}{|c|}{ Liquid Limit } & \multicolumn{5}{|c|}{ Plastic Limit } \\
\hline 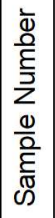 & 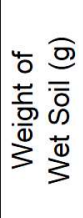 & 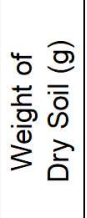 & 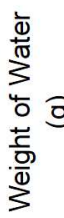 & 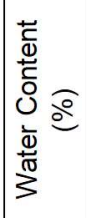 & 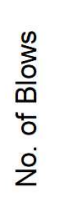 & 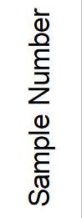 & 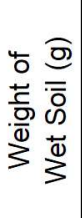 & 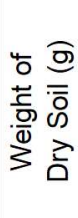 & 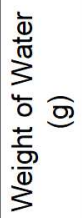 & 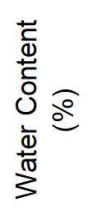 \\
\hline 1 & 7.3 & 5.3 & & 37.7 & 29 & 1 & 8.5 & 6.7 & 1.8 & 26.9 \\
\hline 2 & 14.1 & 9.8 & 4. & 43.9 & 14 & 2 & 6.8 & 5.4 & 1.4 & 25.9 \\
\hline 3 & 13.4 & 9.9 & 3. & 35.4 & 37 & & & & & \\
\hline 4 & 16.9 & 11.9 & 5. & 42.0 & 12 & & & & & \\
\hline 5 & 2.5 & 1.9 & 0. & 31.6 & 166 & & & & & \\
\hline 6 & 3.3 & 2.5 & 0. & 32.0 & 80 & & & & & \\
\hline
\end{tabular}

Date Collected: August 5, 2019

\begin{tabular}{ll|l}
11.5 & 13.0 & Average Depth $(\mathrm{ft}):$ \\
1225
\end{tabular}

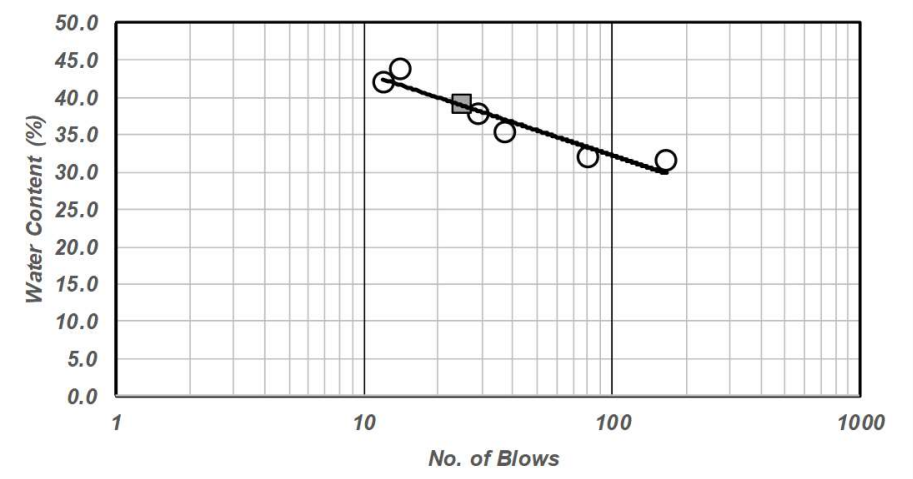

Liquid Limit:

Plastic Limit:

Plasticity Index:

$\begin{array}{r}39 \\ \hline 26 \\ \hline 13 \\ \hline\end{array}$

Total amount of materials passing sieve No. 40: $100 \%$

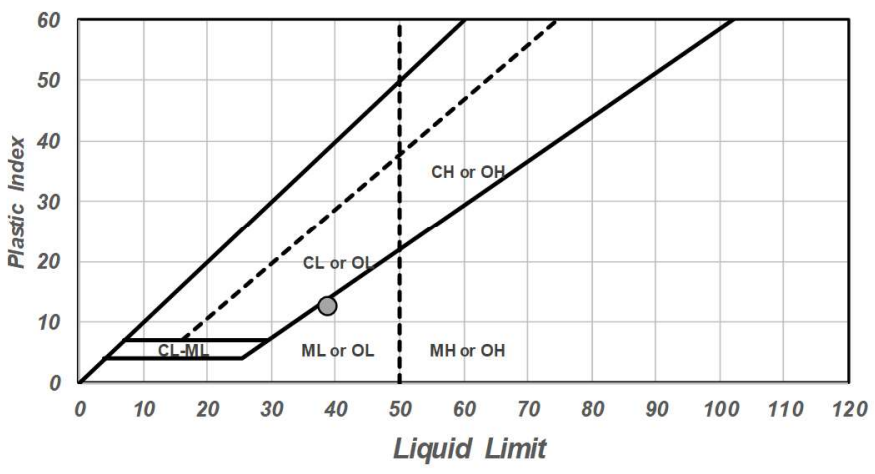

Fines

Classification: $\mathrm{ML}$ 


\section{S Portland State}

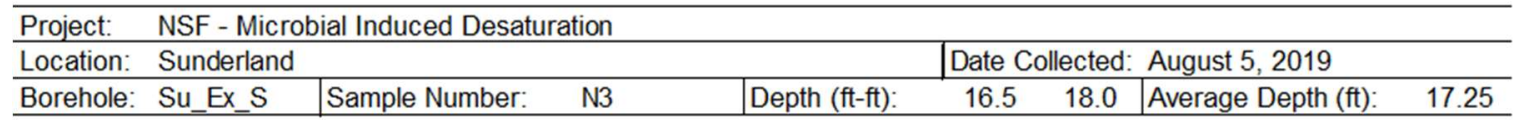

\begin{tabular}{|c|c|c|c|c|c|c|c|c|c|c|}
\hline \multicolumn{6}{|c|}{ Liquid Limit } & \multicolumn{5}{|c|}{ Plastic Limit } \\
\hline 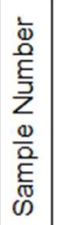 & 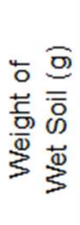 & 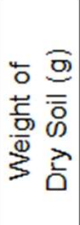 & 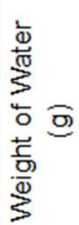 & 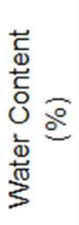 & 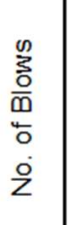 & 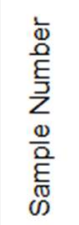 & 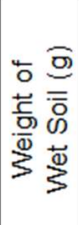 & 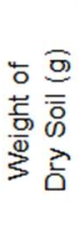 & 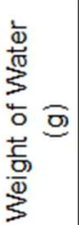 & 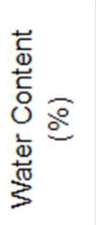 \\
\hline 1 & 3.5 & 2.4 & 1.1 & 45.8 & 18 & 1 & 6.2 & 5 & 1.2 & 24.0 \\
\hline 2 & 4.5 & 3.1 & 1.4 & 45.2 & 8 & 2 & 6.7 & 5.2 & 1.5 & 28.8 \\
\hline 3 & 3.6 & 2.6 & 1.0 & 38.5 & 140 & 3 & 5.9 & 4.7 & 1.2 & 25.5 \\
\hline 4 & 3.7 & 2.6 & 1.1 & 42.3 & 75 & & & & & \\
\hline & & & & & & & & & & \\
\hline & & & & & & & & & & \\
\hline
\end{tabular}

Liquid Limit:

Plastic Limit:

Plasticity Index
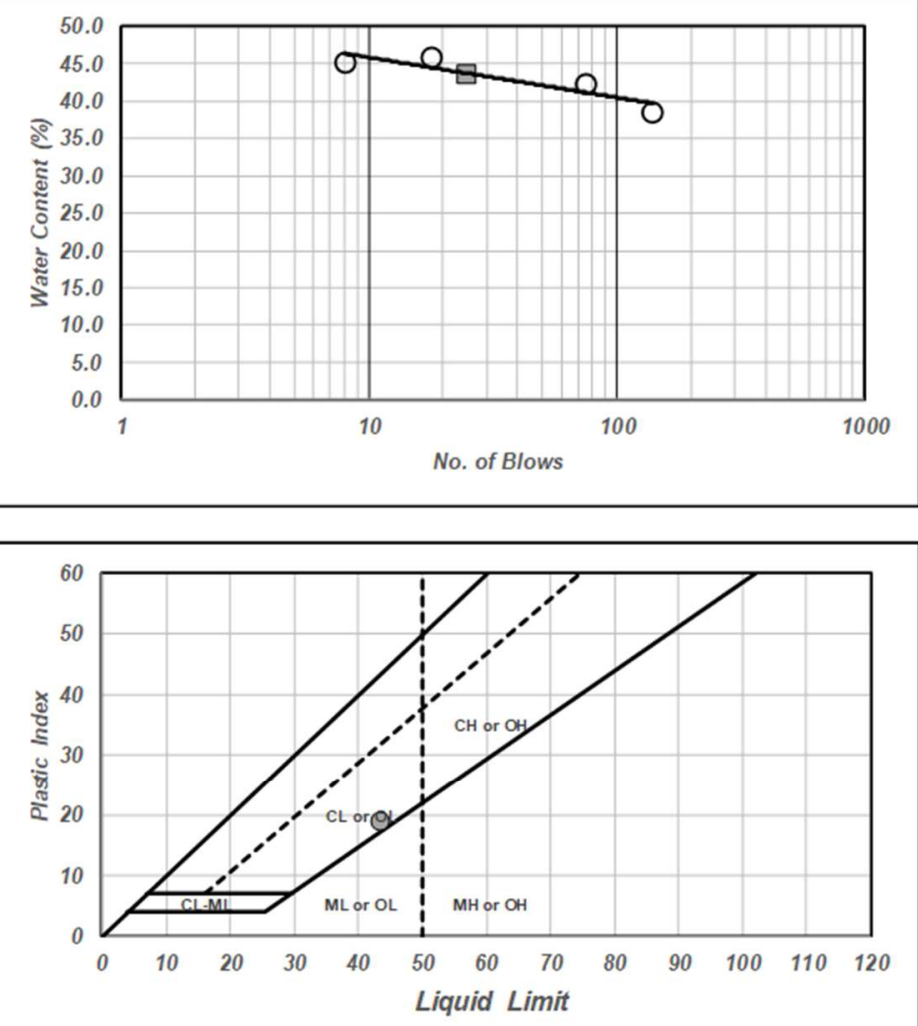

Total amount of materials passing sieve No. 40 : $100 \%$
Fines

Classification: $\mathrm{CL}$

Procedure Done By: Kayla 


\section{\& Portland State}

\begin{tabular}{|c|c|c|c|c|c|c|c|c|c|c|}
\hline \multicolumn{2}{|c|}{ Project: } & \multicolumn{9}{|c|}{ NSF - Microbial Induced Desaturation } \\
\hline \multicolumn{2}{|c|}{ Location: } & \multicolumn{9}{|c|}{ Sunderland } \\
\hline \multicolumn{2}{|c|}{ Borehole: } & \multicolumn{2}{|c|}{ Su_Ex S } & \multicolumn{3}{|c|}{ Sample Number: } & \multicolumn{2}{|l|}{$\mathrm{N} 4$} & \multicolumn{2}{|c|}{ Depth (ft-ft): } \\
\hline \multicolumn{6}{|c|}{ Liquid Limit } & \multicolumn{5}{|c|}{ Plastic Limit } \\
\hline 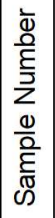 & 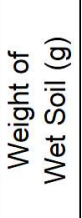 & 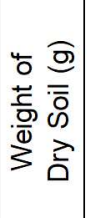 & 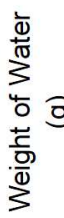 & 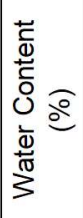 & 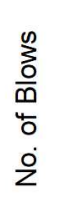 & 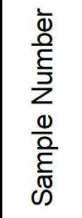 & 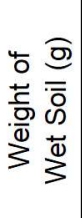 & 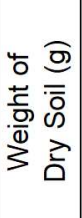 & 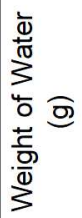 & 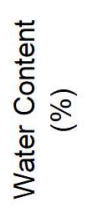 \\
\hline 1 & 1.6 & 1.2 & 0. & 33.3 & 20 & 1 & 8.2 & 6.2 & 2.0 & 32.3 \\
\hline 2 & 2.7 & 1.9 & 0. & 42.1 & 10 & 2 & 6.6 & 5.1 & 1.5 & 29.4 \\
\hline 3 & 3.5 & 2.7 & 0. & 29.6 & 38 & 3 & 7.6 & 5.8 & 1.8 & 31.0 \\
\hline 4 & 2.0 & 1.5 & 0. & 33.3 & 28 & & & & & \\
\hline & & & & & & & & & & \\
\hline & & & & & & & & & & \\
\hline
\end{tabular}

Date Collected: August 5, 2019

\begin{tabular}{ll|l}
215 & 230 & Average Depth (ft): 2225
\end{tabular}

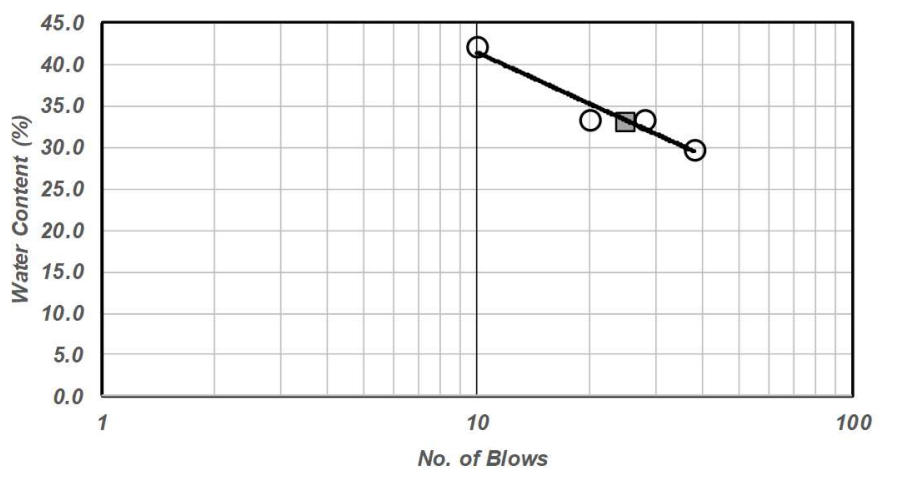

Liquid Limit:

Plastic Limit:

Plasticity Index:

\begin{tabular}{c}
$\frac{33}{32}$ \\
\hline 1
\end{tabular}

Total amount of materials passing sieve No. 40: $100 \%$

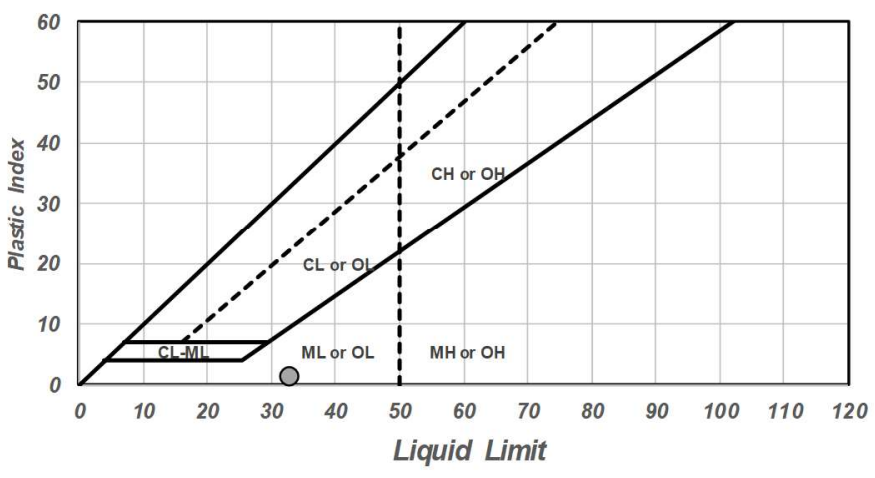

Fines

Classification: ML

Procedure Done By: Kayla 


\section{\& Portland State}

\begin{tabular}{|c|c|c|c|c|c|c|c|c|c|c|}
\hline \multicolumn{2}{|c|}{ Project: } & \multicolumn{9}{|c|}{ NSF - Microbial Induced Desaturation } \\
\hline \multicolumn{2}{|c|}{ Location: } & \multicolumn{9}{|c|}{ Sunderland } \\
\hline \multicolumn{2}{|c|}{ Borehole: } & \multicolumn{2}{|c|}{ Su Ex W } & \multicolumn{3}{|c|}{ Sample Number: } & \multicolumn{2}{|l|}{ N1 } & \multicolumn{2}{|c|}{ Depth (ft-ft): } \\
\hline \multicolumn{6}{|c|}{ Liquid Limit } & \multicolumn{5}{|c|}{ Plastic Limit } \\
\hline 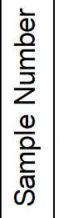 & 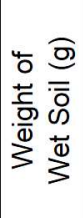 & 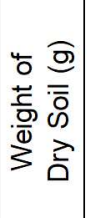 & 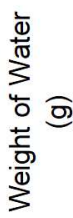 & 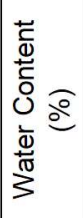 & 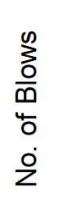 & 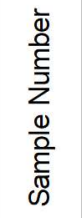 & 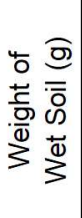 & 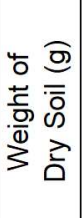 & 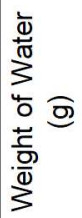 & 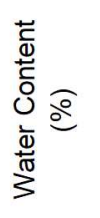 \\
\hline 1 & 4.8 & 3.3 & 1.5 & 45.5 & 7 & 1 & 6.8 & 5.4 & 1.4 & 25.9 \\
\hline 2 & 5.9 & 4.2 & 1.7 & 40.5 & 96 & 2 & 7.0 & 5.5 & 1.5 & 27.3 \\
\hline 3 & 3.7 & 2.6 & 1.1 & 42.3 & 54 & & & & & \\
\hline 4 & 6.9 & 4.8 & 2.1 & 43.8 & 16 & & & & & \\
\hline & & & & & & & & & & \\
\hline & & & & & & & & & & \\
\hline
\end{tabular}

Date Collected: August 6, 2019

\begin{tabular}{ll|l}
7.5 & 9.0 & Average Depth $(\mathrm{ft}):$
\end{tabular}

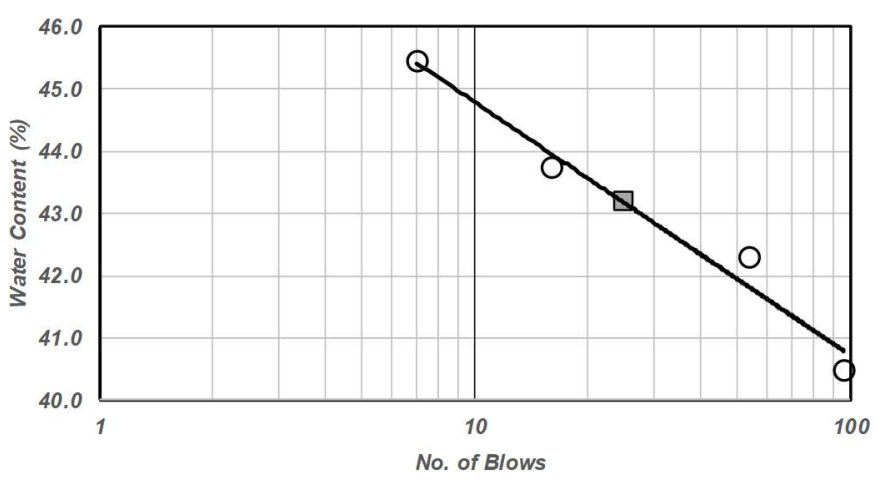

Liquid Limit:

Plastic Limit:

Plasticity Index:

\begin{tabular}{l}
43 \\
\hline 27 \\
\hline 17
\end{tabular}

Total amount of materials passing sieve No. 40: $100 \%$

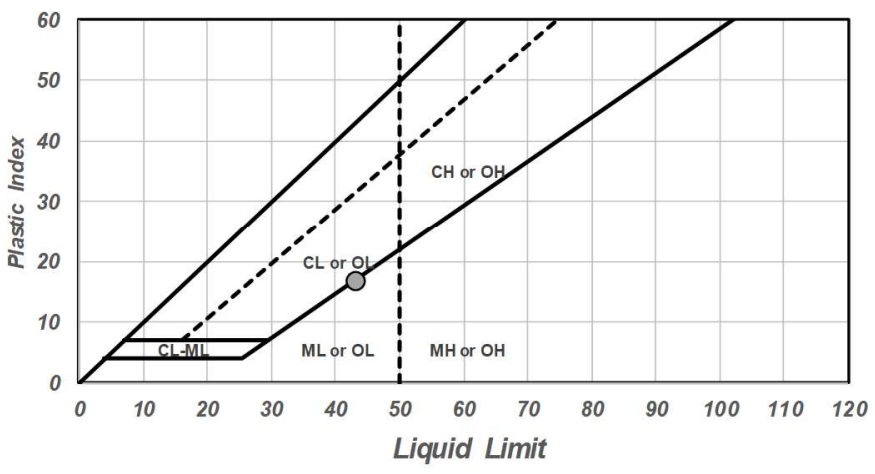

Fines

Classification: ML

Procedure Done By: Kayla 


\section{\& Portland State}

\begin{tabular}{|c|c|c|c|c|c|c|}
\hline oject: & NSF - Micr & nduced Desaturation & & & & \\
\hline Location: & Sunderland & & & Date Collected: & August 6,2019 & \\
\hline Borehole: & Su Ex W & Sample Number: & Depth (ft-ft): & 19.0 & Average Depth (ft): & 18.25 \\
\hline
\end{tabular}

\begin{tabular}{|c|c|c|c|c|c|c|c|c|c|c|}
\hline \multicolumn{6}{|c|}{ Liquid Limit } & \multicolumn{5}{|c|}{ Plastic Limit } \\
\hline 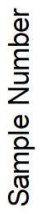 & 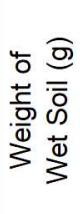 & 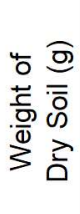 & 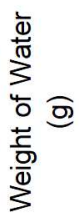 & 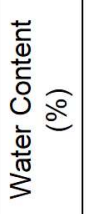 & 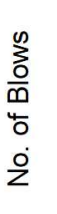 & 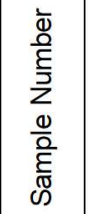 & 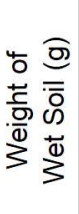 & 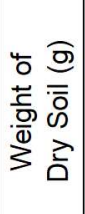 & 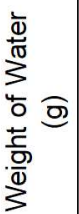 & 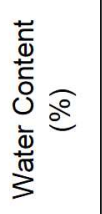 \\
\hline 1 & 14.2 & 9.1 & 5.1 & 56.0 & 6 & 1 & 6.5 & 5.2 & 1.3 & 25.0 \\
\hline 2 & 10.8 & 7.3 & 3.5 & 47.9 & 22 & 2 & 6.3 & 5.0 & 1.3 & 26.0 \\
\hline 3 & 10.2 & 7.0 & 3.2 & 45.7 & 16 & & & & & \\
\hline 4 & 8.0 & 5.6 & 2.4 & 42.9 & 90 & & & & & \\
\hline & & & & & & & & & & \\
\hline & & & & & & & & & & \\
\hline
\end{tabular}

Liquid Limit:

Plastic Limit:

Plasticity Index:
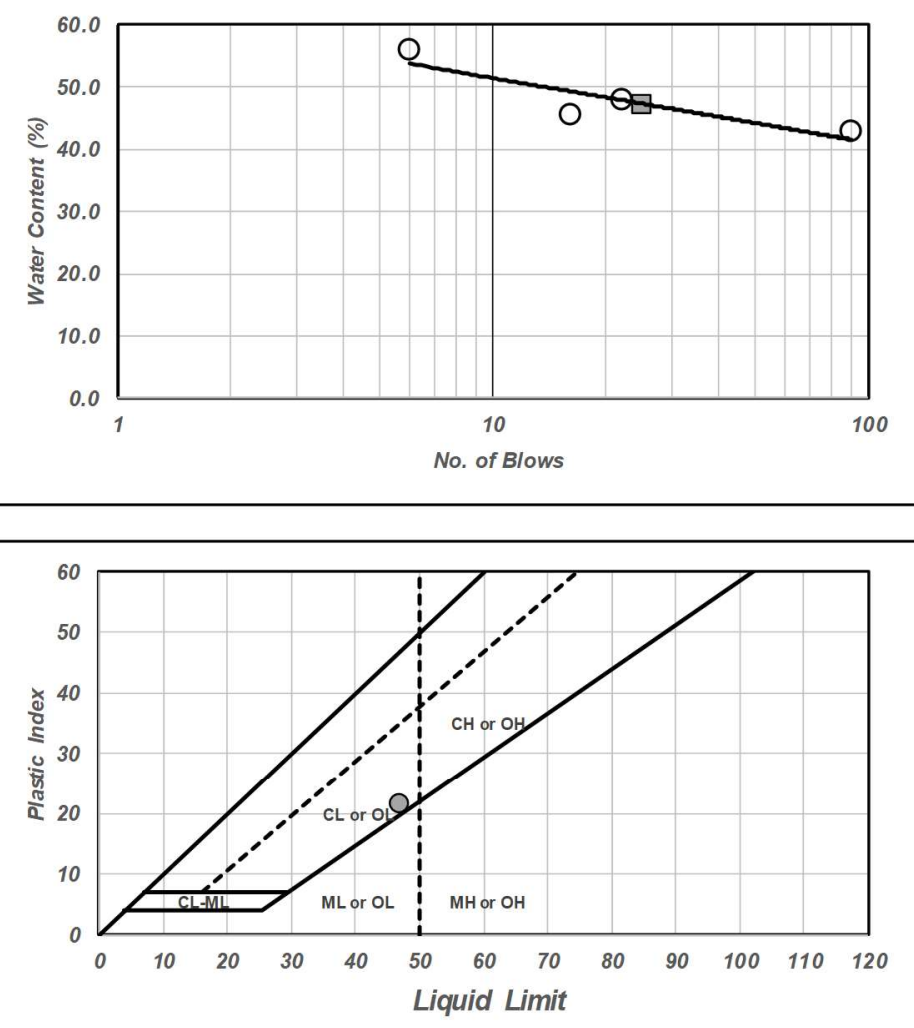

Procedure Done By:

Rawan
Total amount of materials passing sieve No. 40: $100 \%$
Fines

Classification: $\mathrm{CL}$ 


\section{S Portland State}

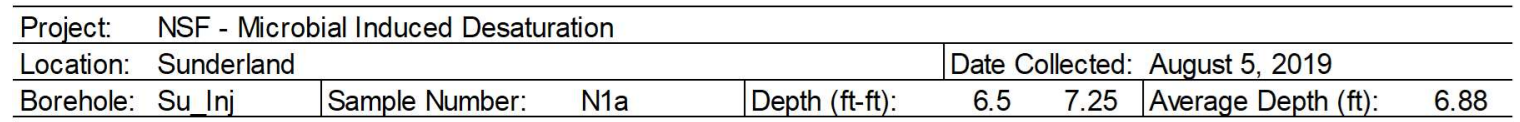

\begin{tabular}{|c|c|c|c|c|c|c|c|c|c|c|}
\hline \multicolumn{6}{|c|}{ Liquid Limit } & \multicolumn{5}{|c|}{ Plastic Limit } \\
\hline 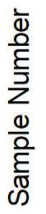 & 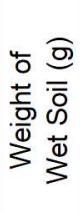 & 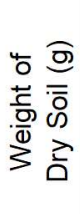 & 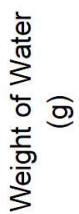 & 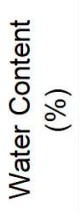 & 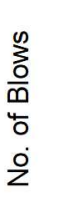 & 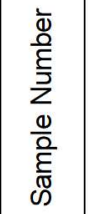 & 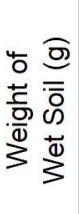 & 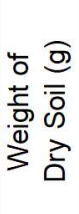 & 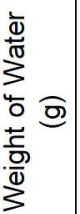 & 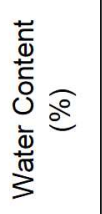 \\
\hline 1 & 2.9 & 2.1 & 0.8 & 38.1 & 2 & 1 & 10.7 & 8.7 & 2.0 & 23.0 \\
\hline 2 & 3.0 & 2.4 & 0.6 & 25.0 & 90 & 2 & 7.0 & 5.7 & 1.3 & 22.8 \\
\hline 3 & 5.0 & 3.8 & 1.2 & 31.6 & 22 & & & & & \\
\hline 4 & 5.5 & 4.3 & 1.2 & 27.9 & 39 & & & & & \\
\hline & & & & & & & & & & \\
\hline & & & & & & & & & & \\
\hline
\end{tabular}

$\begin{array}{ll}\text { Liquid Limit: } & \frac{30}{23} \\ \text { Plastic Limit: } & \frac{23}{7} \\ \text { Plasticity Index: } & \end{array}$

Total amount of materials passing sieve No. 40: $100 \%$
Fines

Classification: ML

Procedure Done By: Kayla 


\section{\& Portland State}

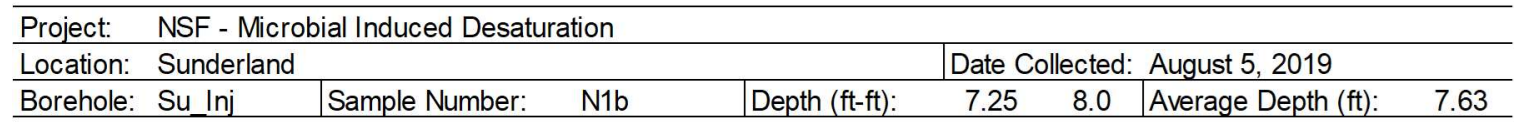

\begin{tabular}{|c|c|c|c|c|c|c|c|c|c|c|}
\hline \multicolumn{6}{|c|}{ Liquid Limit } & \multicolumn{5}{|c|}{ Plastic Limit } \\
\hline 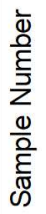 & 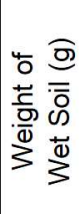 & 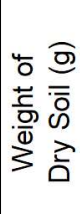 & 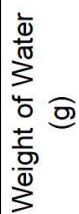 & 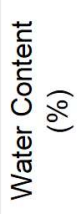 & 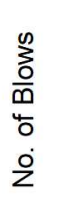 & 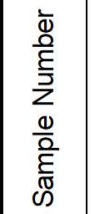 & 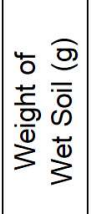 & 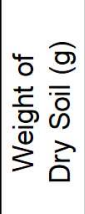 & 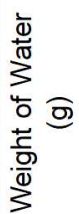 & 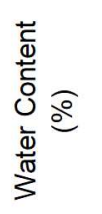 \\
\hline 1 & 3.9 & 2.8 & 1.1 & 39.3 & 4 & 1 & 9 & 7.3 & 1.7 & 23.3 \\
\hline 2 & 3.6 & 2.7 & 0.9 & 33.3 & 82 & 2 & 11.3 & 9.1 & 2.2 & 24.2 \\
\hline 3 & 4.8 & 3.5 & 1.3 & 37.1 & 36 & & & & & \\
\hline 4 & 7.1 & 5.1 & 2.0 & 39.2 & 16 & & & & & \\
\hline & & & & & & & & & & \\
\hline & & & & & & & & & & \\
\hline
\end{tabular}
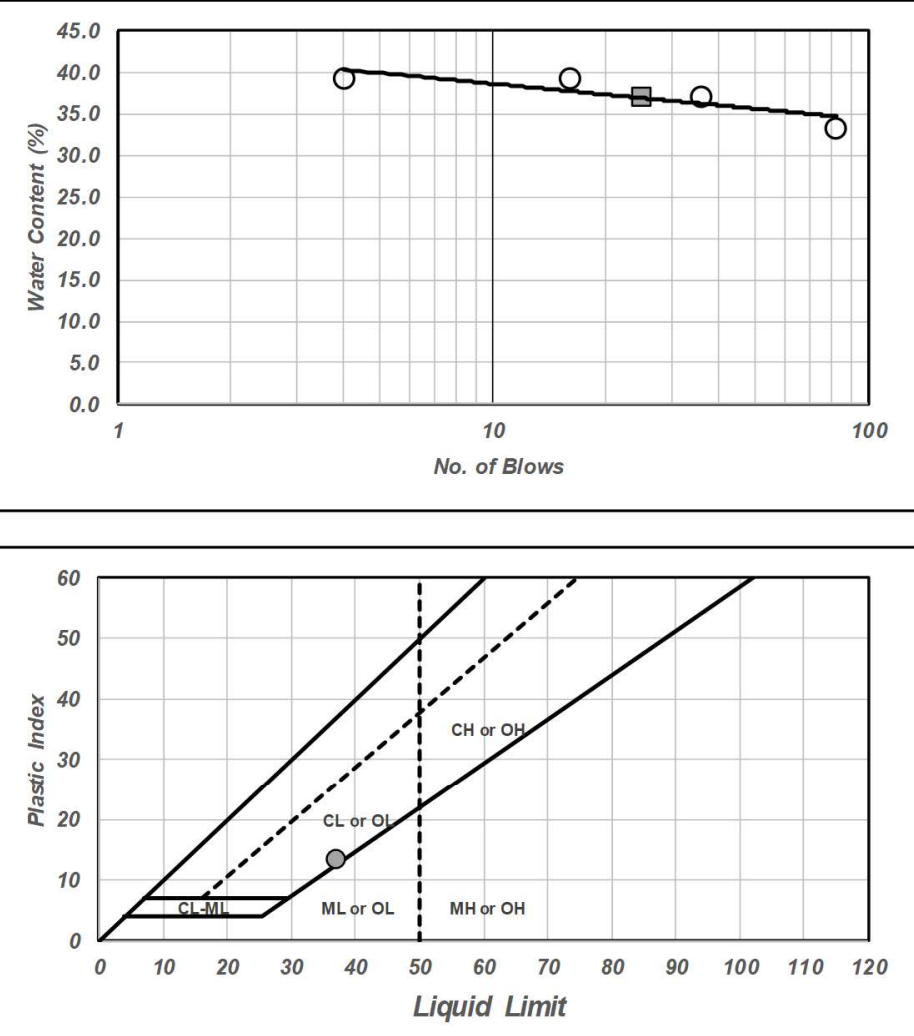

Procedure Done By:
Liquid Limit:

Plastic Limit:

Plasticity Index:
Total amount of materials passing sieve No. 40: $100 \%$
Fines

Classification: $\mathrm{CL}$ 


\section{S Portland State}

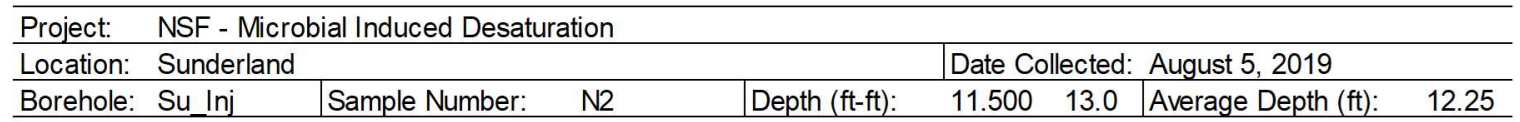

\begin{tabular}{|c|c|c|c|c|c|c|c|c|c|c|}
\hline \multicolumn{6}{|c|}{ Liquid Limit } & \multicolumn{5}{|c|}{ Plastic Limit } \\
\hline 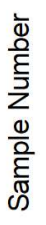 & 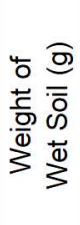 & 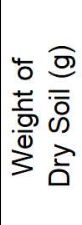 & 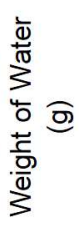 & 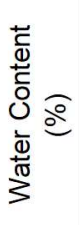 & 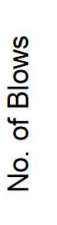 & $\begin{array}{l}\bar{\Phi} \\
\frac{0}{E} \\
\bar{\Sigma} \\
\bar{z} \\
\frac{0}{0} \\
\frac{0}{E} \\
\bar{N} \\
\infty\end{array}$ & 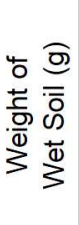 & 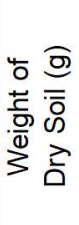 & 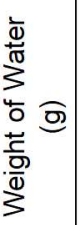 & 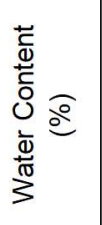 \\
\hline 1 & 8.1 & 5.6 & 2.5 & 44.6 & 23 & 1 & 6.5 & 5.3 & 1.2 & 22.6 \\
\hline 2 & 6.1 & 4.2 & 1.9 & 45.2 & 35 & 2 & 8.1 & 6.6 & 1.5 & 22.7 \\
\hline 3 & 6.2 & 4.3 & 1.9 & 44.2 & 22 & & & & & \\
\hline 4 & 3.5 & 2.6 & 0.9 & 34.6 & 235 & & & & & \\
\hline 5 & 7.7 & 5.5 & 2.2 & 40.0 & 152 & & & & & \\
\hline & & & & & & & & & & \\
\hline
\end{tabular}

Liquid Limit:

Plastic Limit:

Plasticity Index:
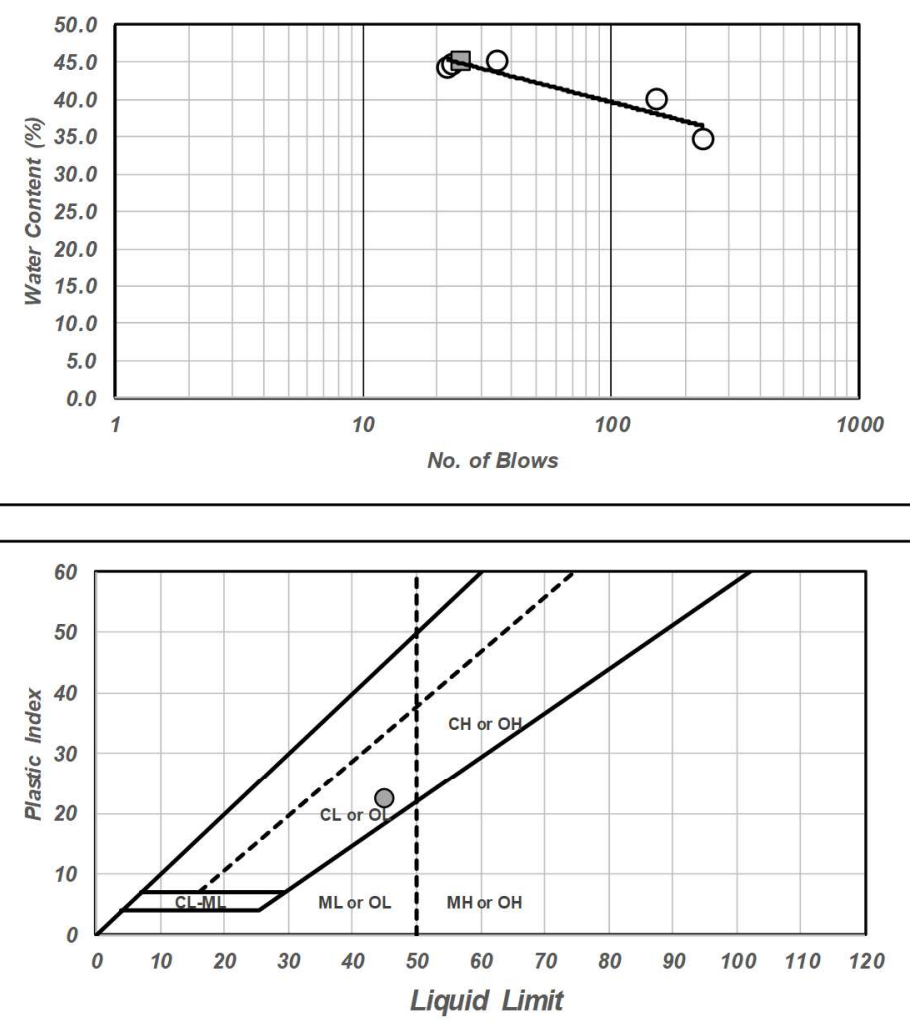

Procedure Done By:
Total amount of materials passing sieve No. 40: $100 \%$
Fines

Classification: $\mathrm{CL}$ 


\section{\& Portland State}

\begin{tabular}{|c|c|c|c|c|c|c|c|c|c|c|}
\hline \multicolumn{2}{|c|}{ Project: } & \multicolumn{9}{|c|}{ NSF - Microbial Induced Desaturation } \\
\hline \multicolumn{2}{|c|}{ Location: } & \multicolumn{9}{|c|}{ Sunderland } \\
\hline \multicolumn{2}{|c|}{ Borehole: } & \multicolumn{2}{|l|}{ Su Inj } & \multicolumn{3}{|c|}{ Sample Number: } & \multicolumn{2}{|l|}{ N3 } & \multicolumn{2}{|c|}{ Depth (ft-ft): } \\
\hline \multicolumn{6}{|c|}{ Liquid Limit } & \multicolumn{5}{|c|}{ Plastic Limit } \\
\hline 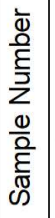 & 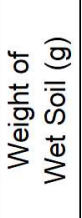 & 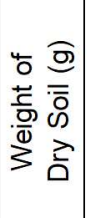 & 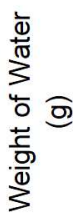 & 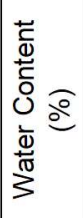 & 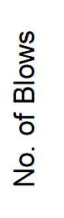 & 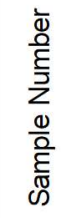 & 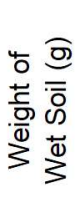 & 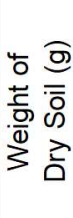 & 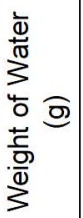 & 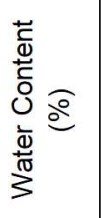 \\
\hline 1 & 7.6 & 5.0 & 2.6 & 52.0 & 13 & 1 & 8 & 6.3 & 1.7 & 27.0 \\
\hline 2 & 6.8 & 4.4 & 2.4 & 54.5 & 5 & 2 & 7 & 5.5 & 1.5 & 27.3 \\
\hline 3 & 6.2 & 4.4 & 1.8 & 40.9 & 135 & & & & & \\
\hline 4 & 5.8 & 4.1 & 1.7 & 41.5 & 60 & & & & & \\
\hline & & & & & & & & & & \\
\hline & & & & & & & & & & \\
\hline
\end{tabular}

Date Collected: August 5, 2019

\begin{tabular}{ll|l}
16.5 & 18.0 & Average Depth $(\mathrm{ft}):$ \\
1725
\end{tabular}

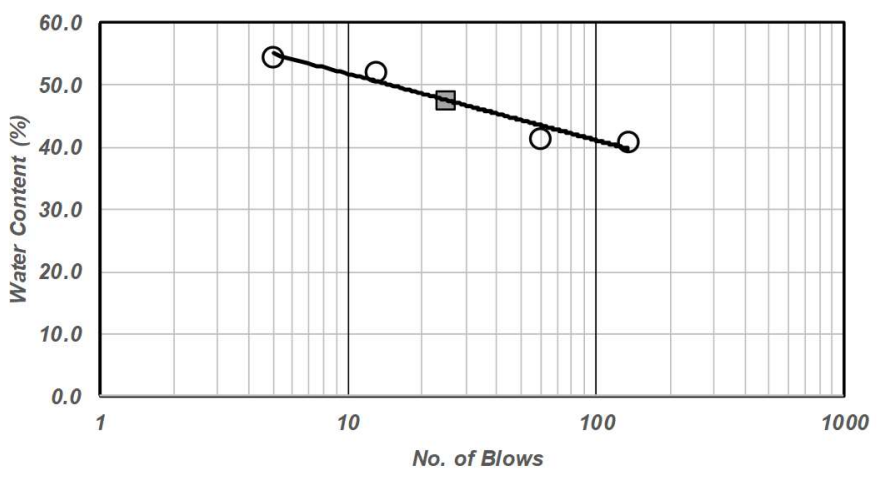

Liquid Limit:

Plastic Limit:

Plasticity Index:

\begin{tabular}{l}
47 \\
\hline 27 \\
\hline 20
\end{tabular}

Total amount of materials passing sieve No. 40: $100 \%$

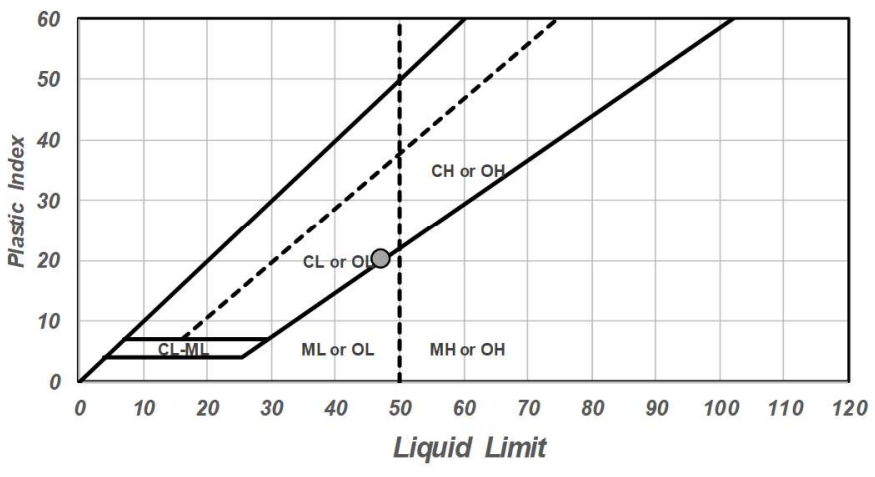

Fines

Classification: $\mathrm{CL}$ 


\section{\& Portland State}

\begin{tabular}{|c|c|c|c|c|c|c|c|c|c|c|}
\hline \multicolumn{2}{|c|}{ Project: } & \multicolumn{9}{|c|}{ NSF - Microbial Induced Desaturation } \\
\hline \multicolumn{2}{|c|}{ Location: } & \multicolumn{9}{|c|}{ Sunderland } \\
\hline \multicolumn{2}{|c|}{ Borehole: } & \multicolumn{2}{|l|}{ Su Inj } & \multicolumn{3}{|c|}{ Sample Number: } & \multicolumn{2}{|l|}{ N4 } & \multicolumn{2}{|c|}{ Depth (ft-ft): } \\
\hline \multicolumn{6}{|c|}{ Liquid Limit } & \multicolumn{5}{|c|}{ Plastic Limit } \\
\hline 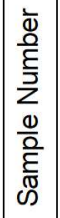 & 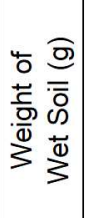 & 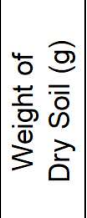 & 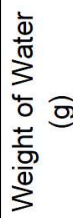 & 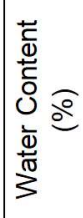 & 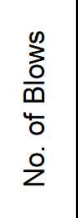 & 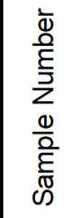 & 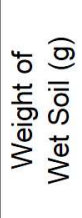 & 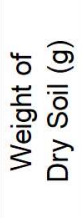 & 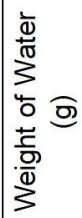 & 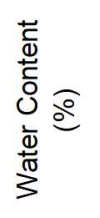 \\
\hline 1 & 16.7 & 11.0 & 5.7 & 51.8 & 25 & 1 & 8.0 & 6.1 & 1.9 & 31.1 \\
\hline 2 & 14.2 & 9.3 & 4.9 & 52.7 & 20 & 2 & 8.2 & 6.0 & 2.2 & 36.7 \\
\hline 3 & 17.2 & 11.2 & 6.0 & 53.6 & 15 & 3 & 8.0 & 5.8 & 2.2 & 37.9 \\
\hline 4 & 6.5 & 4.3 & 2.2 & 51.2 & 27 & & & & & \\
\hline & & & & & & & & & & \\
\hline & & & & & & & & & & \\
\hline
\end{tabular}

Date Collected: August 5, 2019

\begin{tabular}{ll|l}
215 & 230 & Average Depth (ft): 2225
\end{tabular}

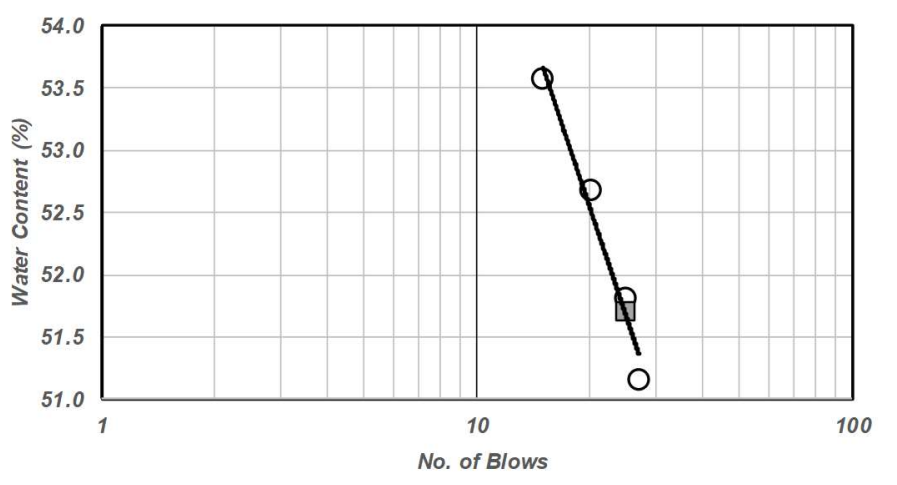

Liquid Limit:

Plastic Limit:

Plasticity Index:

\begin{tabular}{l}
52 \\
\hline 37 \\
\hline 14
\end{tabular}

Total amount of materials passing sieve No. 40: $100 \%$

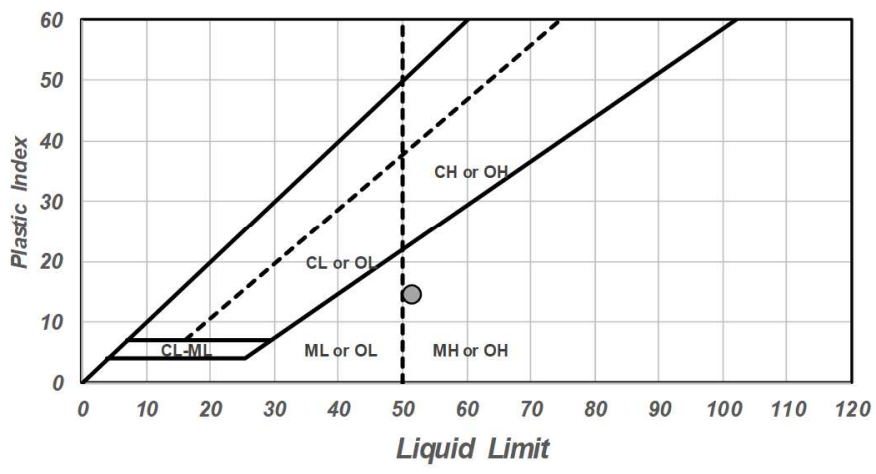

Fines

Classification: $\mathrm{MH}$ 


\section{S Portland State}

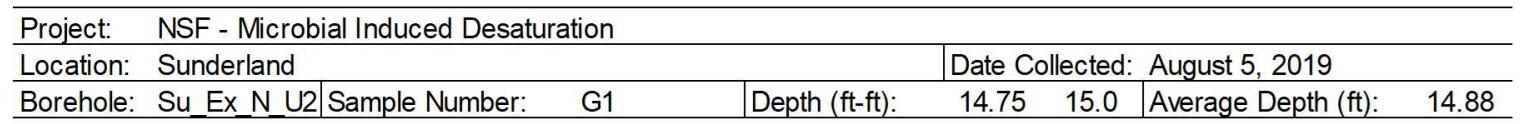

\begin{tabular}{|c|c|c|c|c|c|c|c|c|c|c|}
\hline \multicolumn{6}{|c|}{ Liquid Limit } & \multicolumn{5}{|c|}{ Plastic Limit } \\
\hline 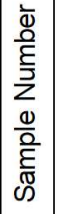 & 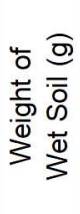 & 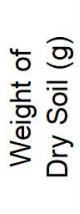 & 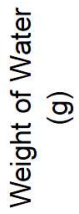 & 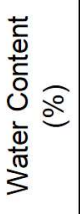 & $\begin{array}{l}n \\
\sum_{0}^{0} \\
0 \\
\text { to } \\
\dot{0} \\
\dot{2}\end{array}$ & 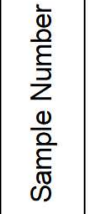 & 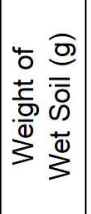 & 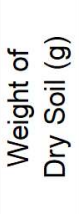 & 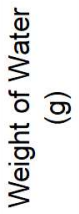 & 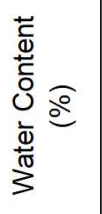 \\
\hline 1 & 4.6 & 3.0 & 1.6 & 53.3 & & 1 & 9.8 & 7.2 & 2.6 & 36.1 \\
\hline 2 & 7.9 & 5.2 & 2.7 & 51.9 & 100 & 2 & 10.0 & 7.3 & 2.7 & 37.0 \\
\hline 3 & 7.8 & 5.1 & 2.7 & 52.9 & 68 & & & & & \\
\hline 4 & 9.3 & 6.0 & 3.3 & 55.0 & 28 & & & & & \\
\hline 5 & 9.7 & 6.2 & 3.5 & 56.5 & 21 & & & & & \\
\hline 6 & 15.6 & 9.7 & 5.9 & 60.8 & 11 & & & & & \\
\hline
\end{tabular}

$\begin{array}{ll}\text { Liquid Limit: } & \frac{57}{36} \\ \text { Plastic Limit: } & \underline{20} \\ \text { Plasticity Index: } & \end{array}$

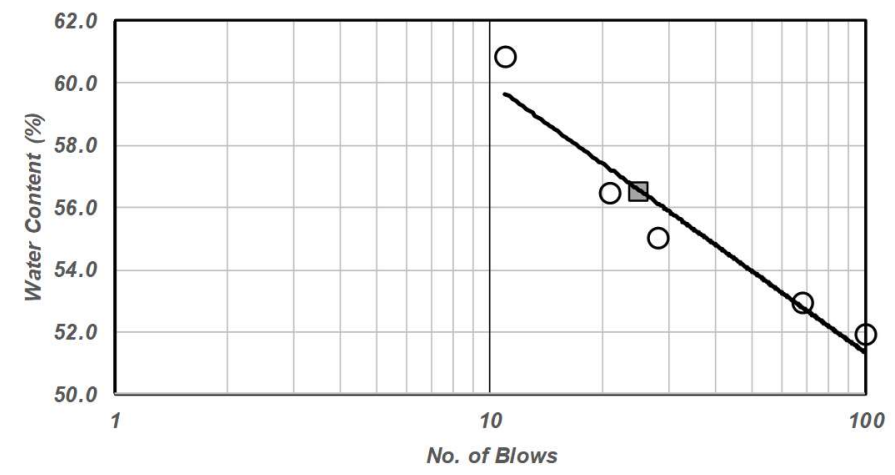

Total amount of materials passing sieve No. 40: $100 \%$

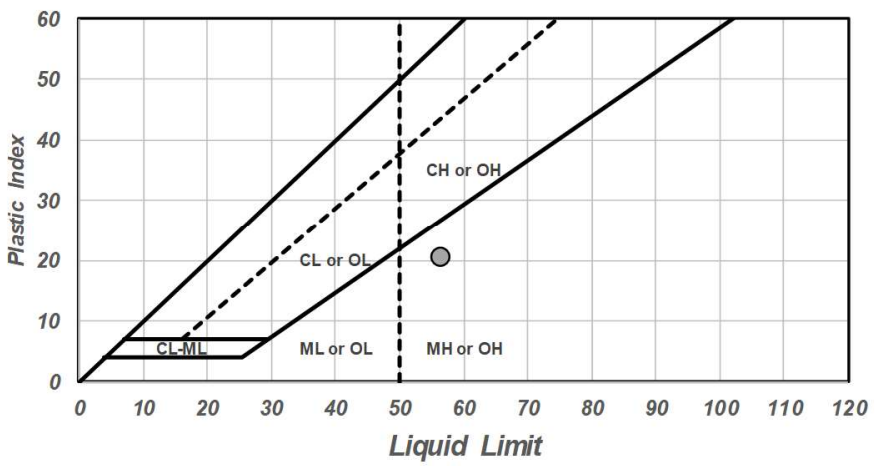

Fines

Classification: $\mathrm{MH}$

Procedure Done By: Kayla

Date Performed:

July 29, 2020




\section{S Portland State}

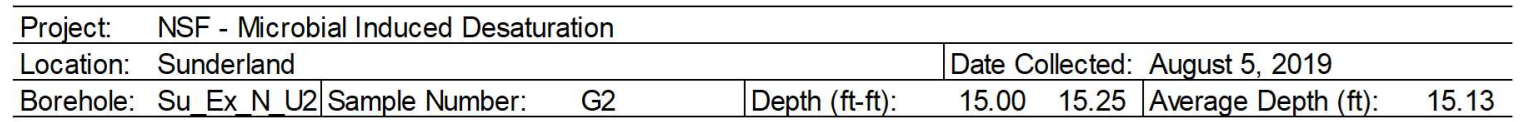

\begin{tabular}{|c|c|c|c|c|c|c|c|c|c|c|}
\hline \multicolumn{6}{|c|}{ Liquid Limit } & \multicolumn{5}{|c|}{ Plastic Limit } \\
\hline 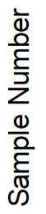 & 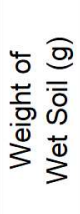 & 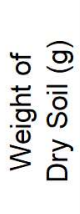 & 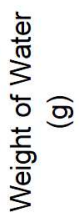 & 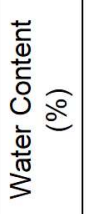 & 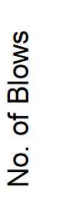 & 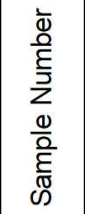 & 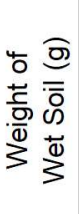 & 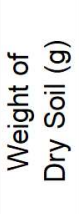 & 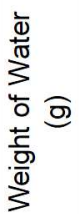 & 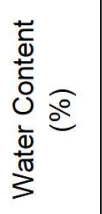 \\
\hline 1 & 12.9 & 8.8 & 4.1 & 46.6 & 58 & 1 & 7.8 & 6 & 1.8 & 30.0 \\
\hline 2 & 15.7 & 10.6 & 5.1 & 48.1 & 17 & 2 & 8.0 & 6.2 & 1.8 & 29.0 \\
\hline 3 & 12.6 & 8.3 & 4.3 & 51.8 & 12 & & & & & \\
\hline 4 & 16.4 & 11.4 & 5.0 & 43.9 & 30 & & & & & \\
\hline 5 & 17.8 & 12.5 & 5.3 & 42.4 & 43 & & & & & \\
\hline & & & & & & & & & & \\
\hline
\end{tabular}

Liquid Limit:

Plastic Limit:

Plasticity Index:
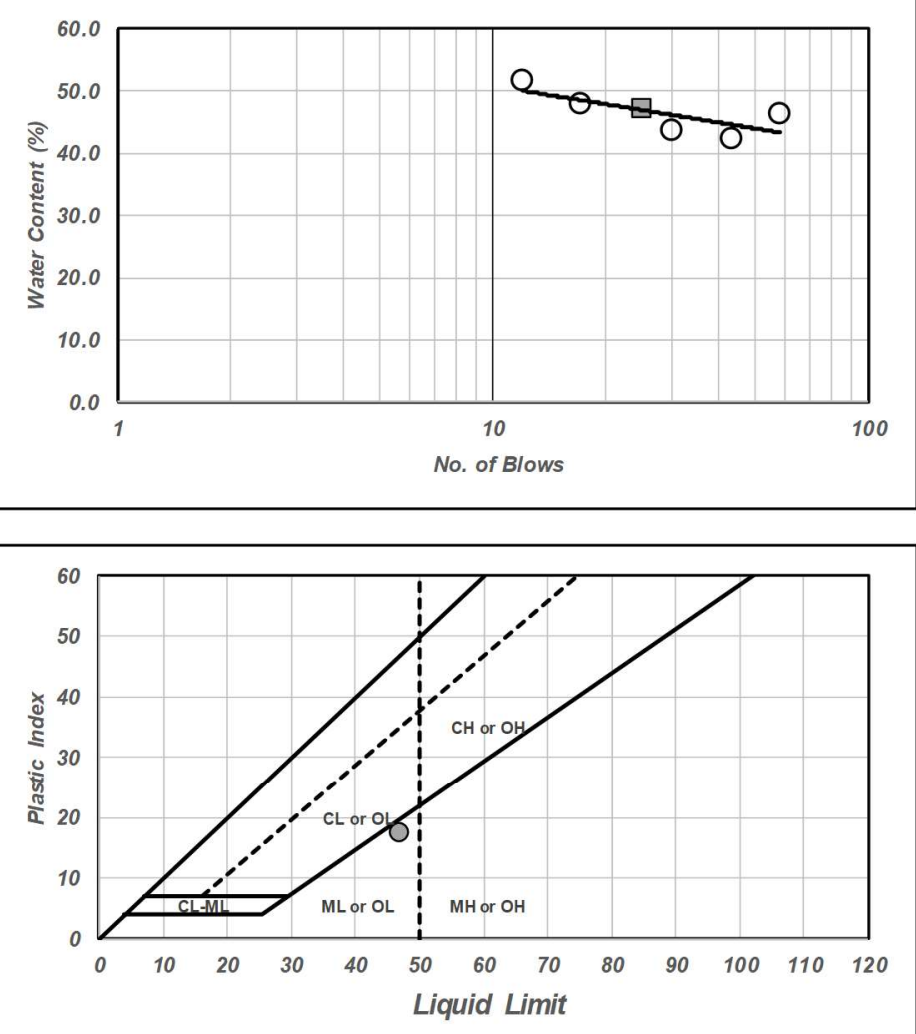

Procedure Done By: Max/Kayla
Total amount of materials passing sieve No. 40: $100 \%$
Fines

Classification: ML 


\section{S Portland State}

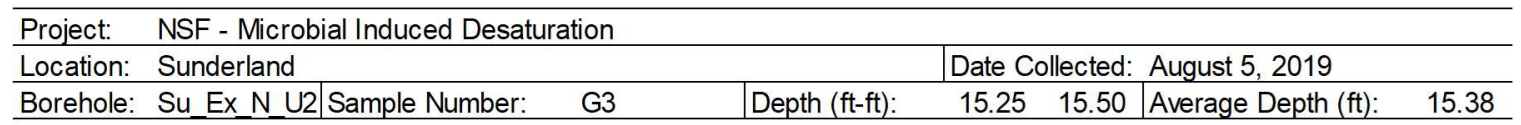

\begin{tabular}{|c|c|c|c|c|c|c|c|c|c|c|}
\hline \multicolumn{6}{|c|}{ Liquid Limit } & \multicolumn{5}{|c|}{ Plastic Limit } \\
\hline 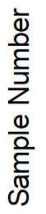 & 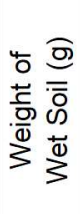 & 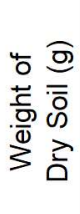 & 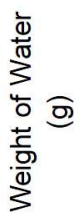 & 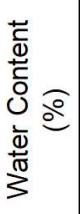 & 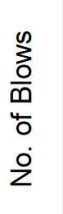 & 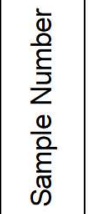 & 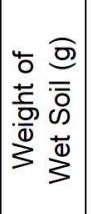 & 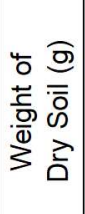 & 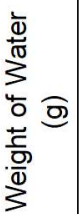 & 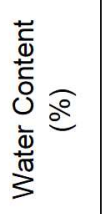 \\
\hline 1 & 6.7 & 4.6 & 2.1 & 45.7 & 10 & 1 & 7.8 & 6.2 & 1.6 & 25.8 \\
\hline 2 & 11.2 & 7.8 & 3.4 & 43.6 & 28 & 2 & 9.3 & 7.3 & 2 & 27.4 \\
\hline 3 & 10.8 & 7.4 & 3.4 & 45.9 & 10 & & & & & \\
\hline 4 & 10.5 & 7.8 & 2.7 & 34.6 & 98 & & & & & \\
\hline 5 & 19.9 & 14.2 & 5.7 & 40.1 & 24 & & & & & \\
\hline & & & & & & & & & & \\
\hline
\end{tabular}

Liquid Limit:

Plastic Limit:

Plasticity Index:

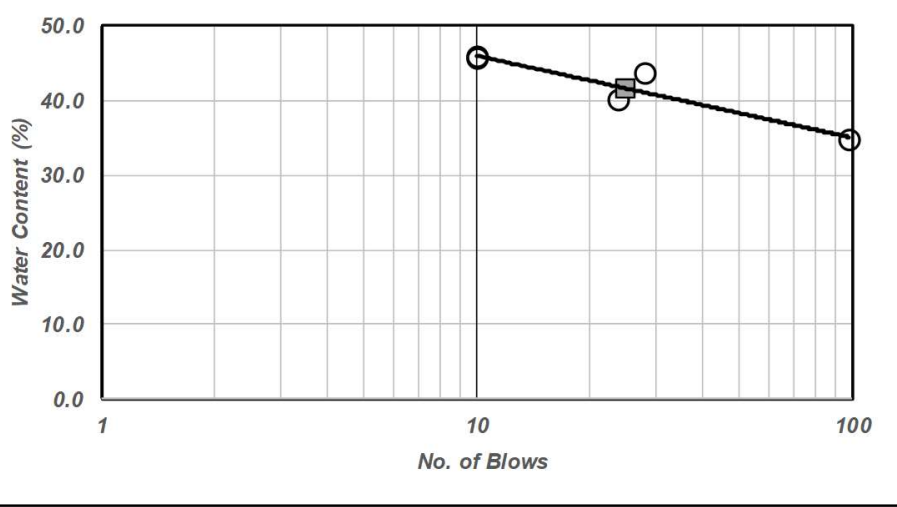

Total amount of materials passing sieve No. 40: $100 \%$

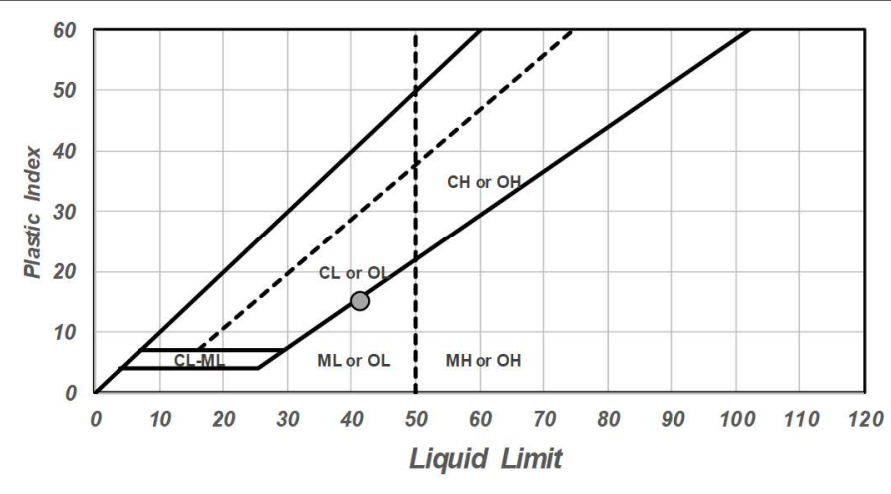

Procedure Done By:
Fines

Classification: ML 


\section{S Portland State}

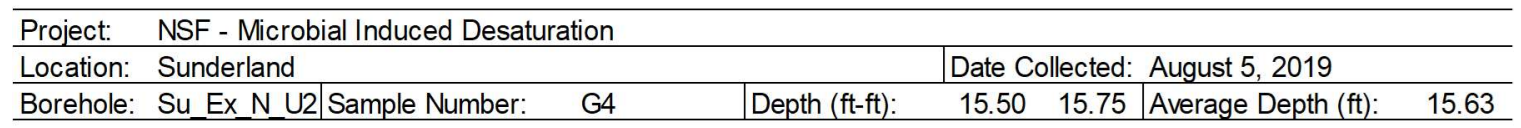

\begin{tabular}{|c|c|c|c|c|c|c|c|c|c|c|}
\hline \multicolumn{6}{|c|}{ Liquid Limit } & \multicolumn{5}{|c|}{ Plastic Limit } \\
\hline 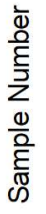 & 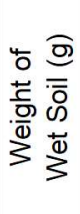 & 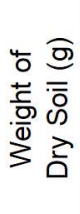 & 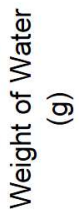 & 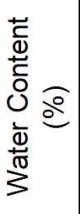 & 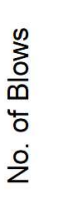 & 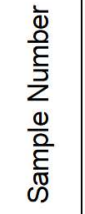 & 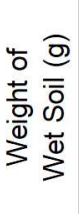 & 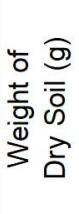 & 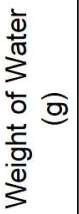 & 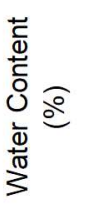 \\
\hline 1 & 16.4 & 10.3 & 6.1 & 59.2 & 6 & 1 & 6.2 & 4.7 & 1.5 & 31.9 \\
\hline 2 & 15.0 & 9.5 & 5.5 & 57.9 & 9 & 2 & 13.2 & 9.8 & 3.4 & 34.7 \\
\hline 3 & 13.6 & 9.2 & 4.4 & 47.8 & 36 & & & & & \\
\hline 4 & 11.3 & 8.1 & 3.2 & 39.5 & 110 & & & & & \\
\hline & & & & & & & & & & \\
\hline & & & & & & & & & & \\
\hline
\end{tabular}

\begin{tabular}{ll} 
Liquid Limit: & 50 \\
Plastic Limit: & $\quad 33$ \\
Plasticity Index: & \\
\hline
\end{tabular}

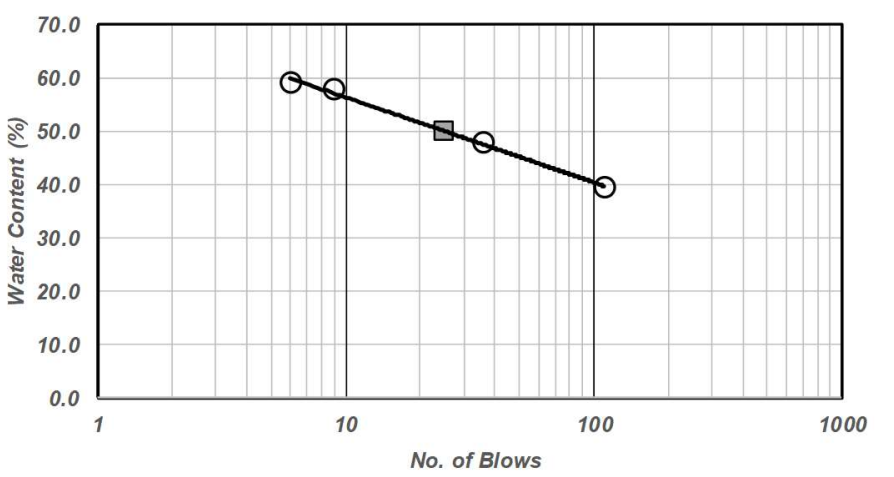

Total amount of materials passing sieve No. 40: $100 \%$

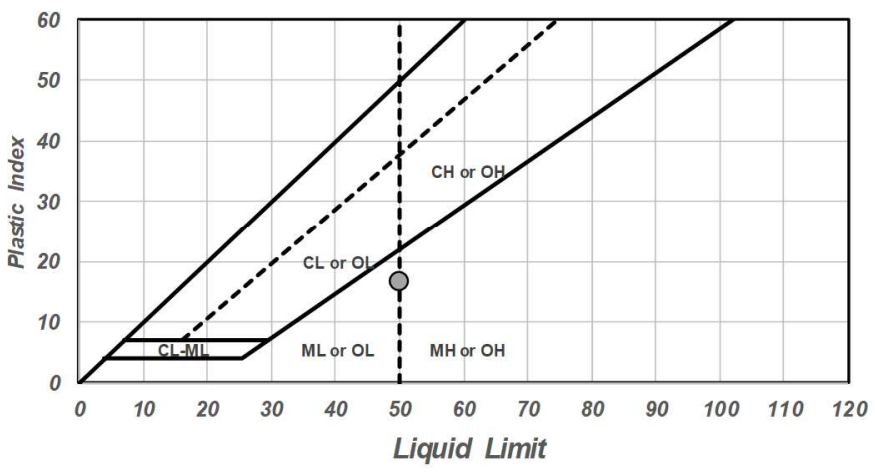

Fines

Classification: ML 


\section{S Portland State}

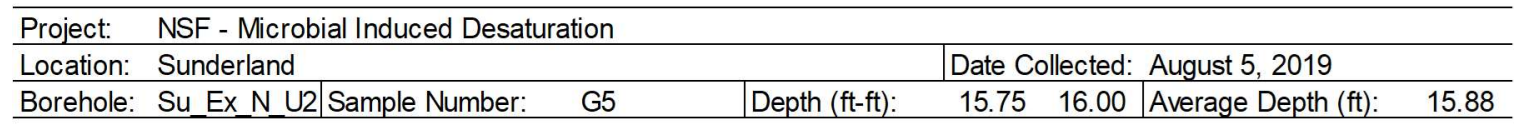

\begin{tabular}{|c|c|c|c|c|c|c|c|c|c|c|}
\hline \multicolumn{6}{|c|}{ Liquid Limit } & \multicolumn{5}{|c|}{ Plastic Limit } \\
\hline 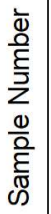 & 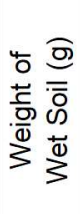 & 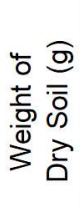 & 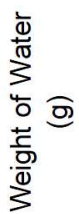 & 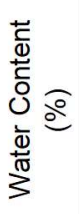 & 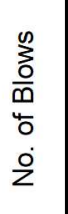 & 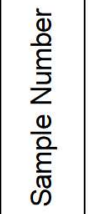 & 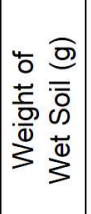 & 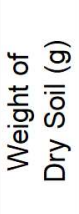 & 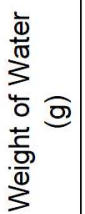 & 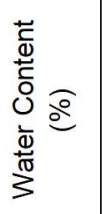 \\
\hline 1 & 9.3 & 6.2 & 3.1 & 50.0 & 18 & 1 & 9.5 & 7.3 & 2.2 & 30.1 \\
\hline 2 & 12.2 & 8.3 & 3.9 & 47.0 & 29 & 2 & 6.8 & 5.3 & 1.5 & 28.3 \\
\hline 3 & 9.5 & 6.9 & 2.6 & 37.7 & 93 & & & & & \\
\hline 4 & 11.6 & 8.0 & 3.6 & 45.0 & 11 & & & & & \\
\hline 5 & 10.9 & 7.2 & 3.7 & 51.4 & 32 & & & & & \\
\hline 6 & 10.4 & 7.3 & 3.1 & 42.5 & 39 & & & & & \\
\hline
\end{tabular}

Liquid Limit:

Plastic Limit:

Plasticity Index:
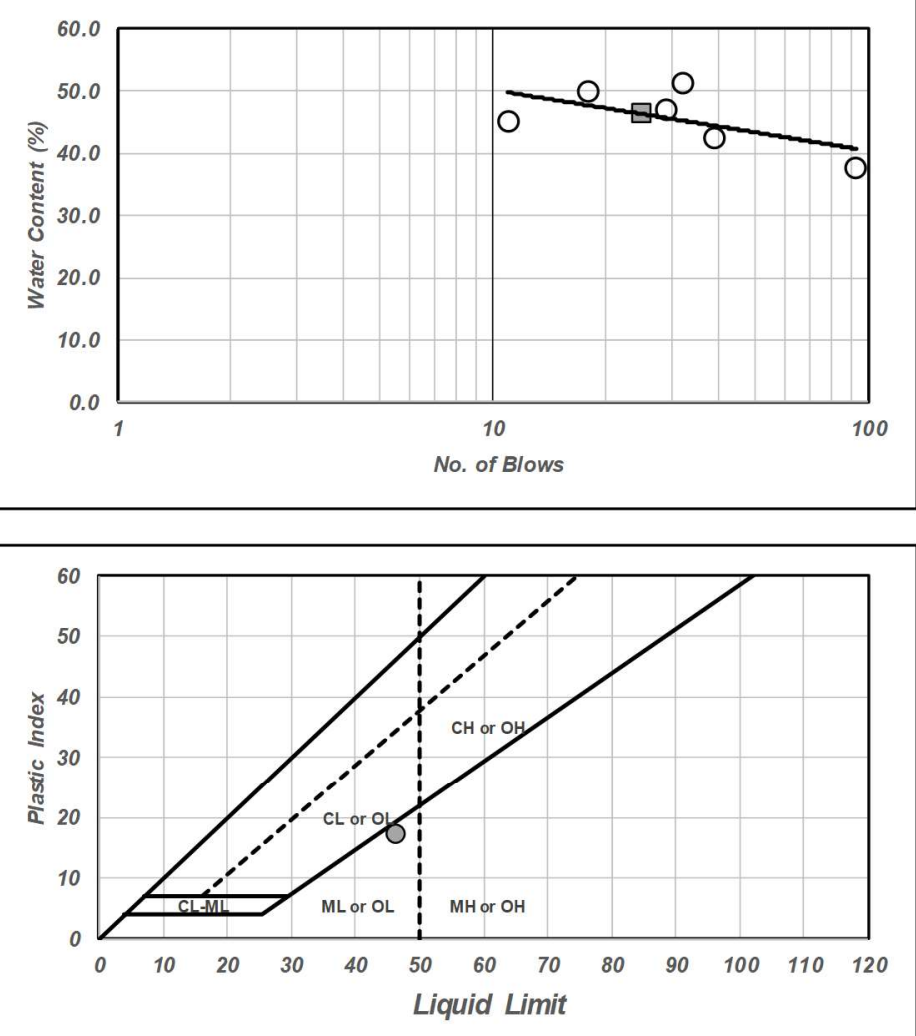

Procedure Done By:
Total amount of materials passing sieve No. 40: $100 \%$
Fines

Classification: ML 


\section{S Portland State}

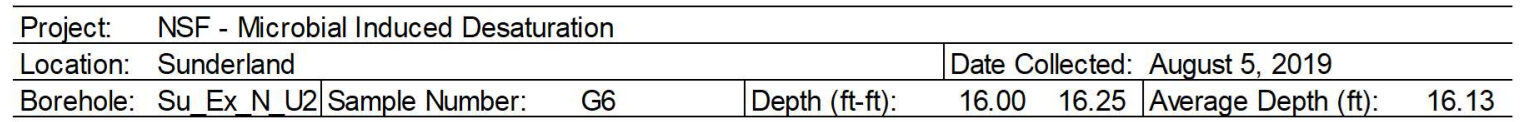

\begin{tabular}{|c|c|c|c|c|c|c|c|c|c|c|}
\hline \multicolumn{6}{|c|}{ Liquid Limit } & \multicolumn{5}{|c|}{ Plastic Limit } \\
\hline 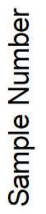 & 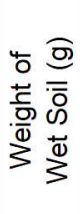 & 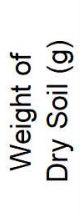 & 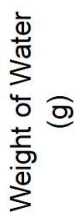 & 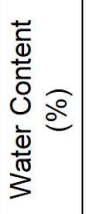 & 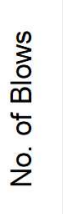 & 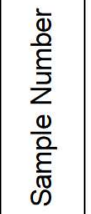 & 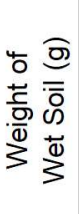 & 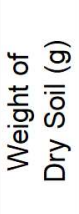 & 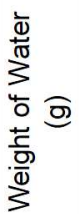 & 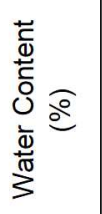 \\
\hline 1 & 15.6 & 10.2 & 5.4 & 52.9 & & 1 & 6.7 & 5.1 & 1.6 & 31.4 \\
\hline 2 & 11.3 & 7.4 & 3.9 & 52.7 & 8 & 2 & 6.1 & 4.7 & 1.4 & 29.8 \\
\hline 3 & 13.7 & 9.1 & 4.6 & 50.5 & 11 & & & & & \\
\hline 4 & 13.4 & 9.2 & 4.2 & 45.7 & 24 & & & & & \\
\hline 5 & 12.7 & 9.0 & 3.7 & 41.1 & 92 & & & & & \\
\hline & & & & & & & & & & \\
\hline
\end{tabular}

Liquid Limit:

Plastic Limit:

Plasticity Index:
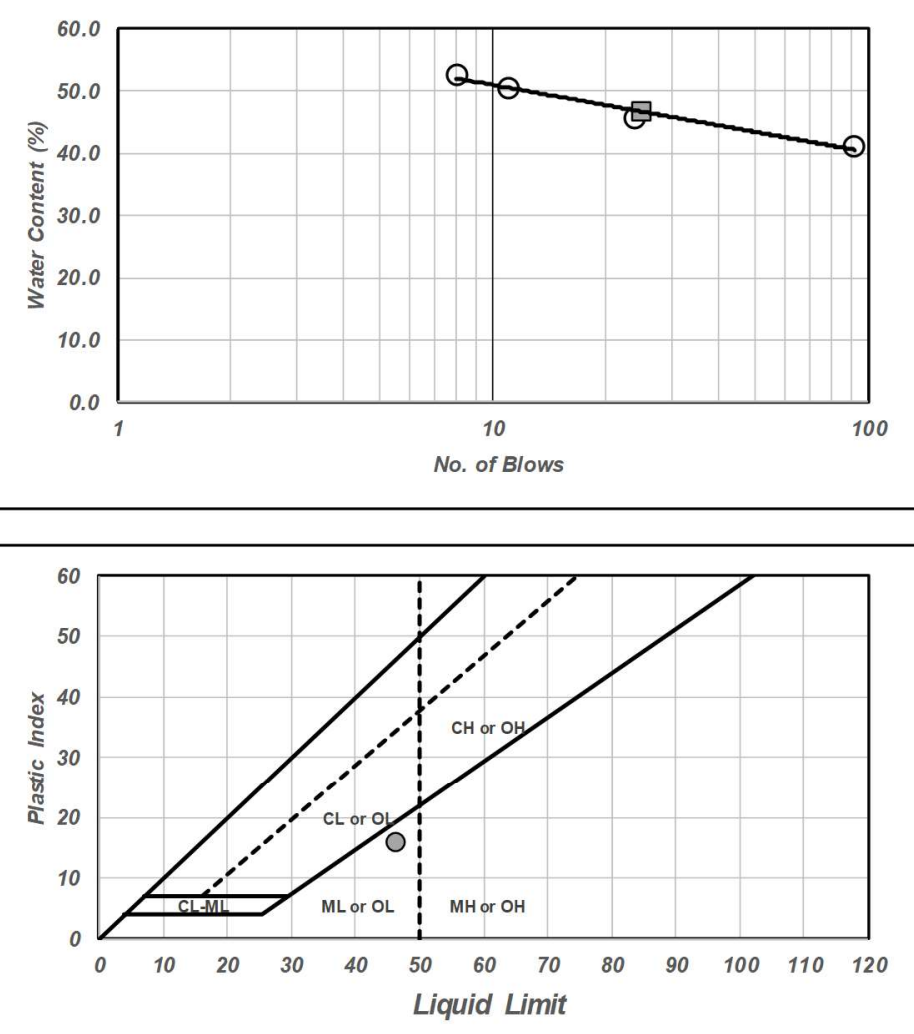

Procedure Done By:

$\operatorname{Max}$
Total amount of materials passing sieve No. 40: $100 \%$
Fines

Classification: ML 


\section{S Portland State}

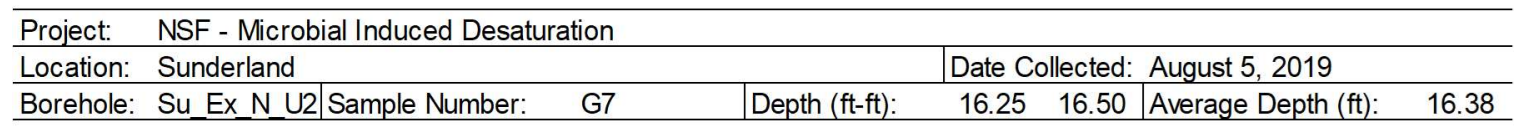

\begin{tabular}{|c|c|c|c|c|c|c|c|c|c|c|}
\hline \multicolumn{6}{|c|}{ Liquid Limit } & \multicolumn{5}{|c|}{ Plastic Limit } \\
\hline 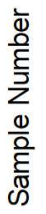 & 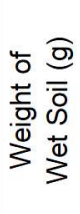 & 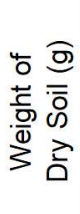 & 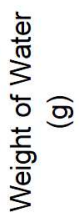 & 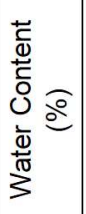 & 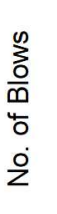 & 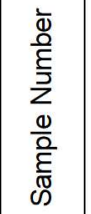 & 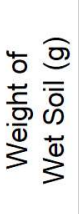 & 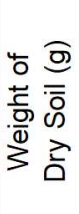 & 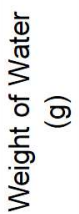 & 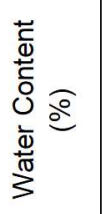 \\
\hline 1 & 12.9 & 8.3 & 4.6 & 55.4 & 7 & 1 & 7 & 5.4 & 1.6 & 29.6 \\
\hline 2 & 14.6 & 9.7 & 4.9 & 50.5 & 16 & 2 & 7.4 & 5.7 & 1.7 & 29.8 \\
\hline 3 & 13.2 & 9.0 & 4.2 & 46.7 & 28 & & & & & \\
\hline 4 & 12.3 & 8.5 & 3.8 & 44.7 & 57 & & & & & \\
\hline & & & & & & & & & & \\
\hline & & & & & & & & & & \\
\hline
\end{tabular}

Liquid Limit:

Plastic Limit:

Plasticity Index:

$\frac{48}{30}$

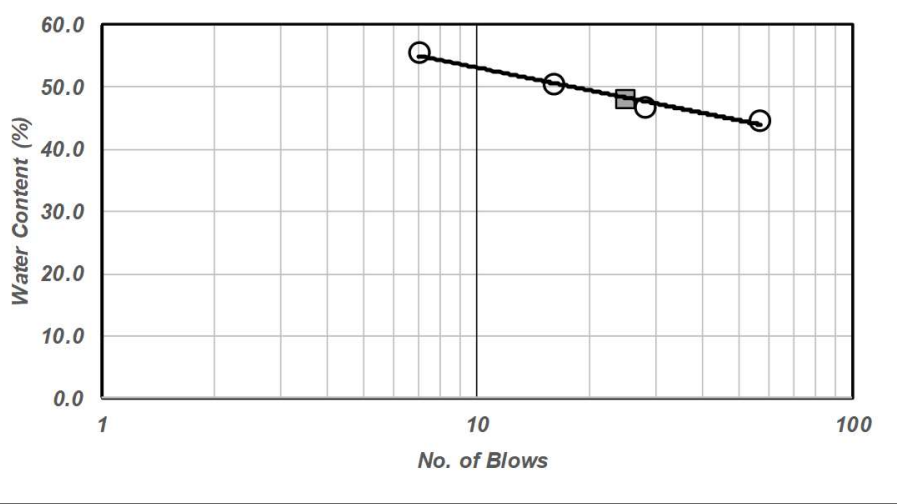

Total amount of materials passing sieve No. 40: $100 \%$

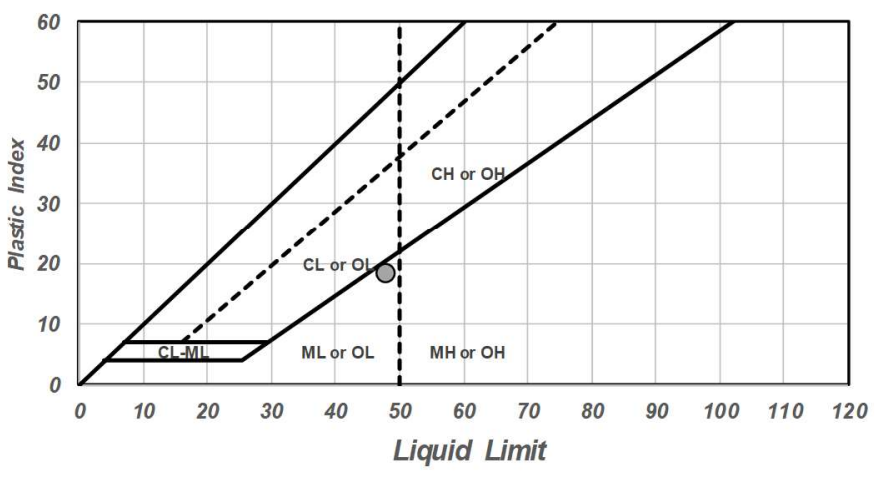

Fines

Classification: ML

Procedure Done By:

$\operatorname{Max}$ 


\section{S Portland State}

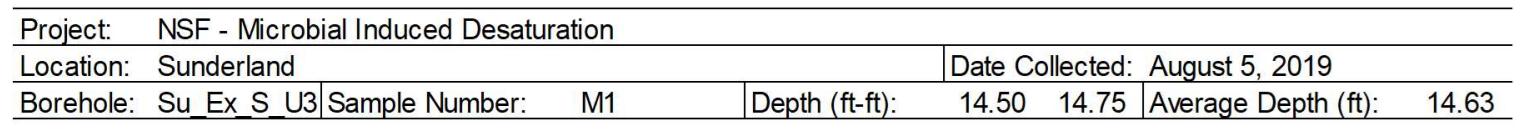

\begin{tabular}{|c|c|c|c|c|c|c|c|c|c|c|}
\hline \multicolumn{6}{|c|}{ Liquid Limit } & \multicolumn{5}{|c|}{ Plastic Limit } \\
\hline 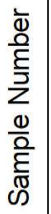 & 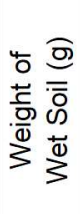 & 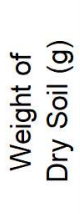 & 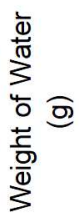 & 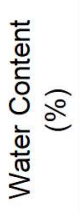 & 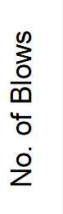 & 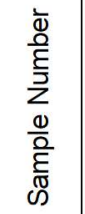 & 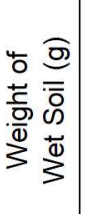 & 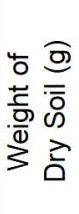 & 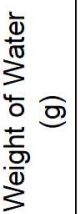 & 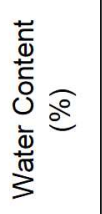 \\
\hline 1 & 22.8 & 15.6 & 7.2 & 46.2 & & 1 & 10.9 & 8.2 & 2.7 & 32.9 \\
\hline 2 & 13.4 & 8.8 & 4.6 & 52.3 & 17 & 2 & 10.1 & 7.6 & 2.5 & 32.9 \\
\hline 3 & 11.0 & 7.3 & 3.7 & 50.7 & 18 & & & & & \\
\hline 4 & 10.3 & 6.9 & 3.4 & 49.3 & 61 & & & & & \\
\hline 5 & 10.8 & 7.4 & 3.4 & 45.9 & 90 & & & & & \\
\hline & & & & & & & & & & \\
\hline
\end{tabular}
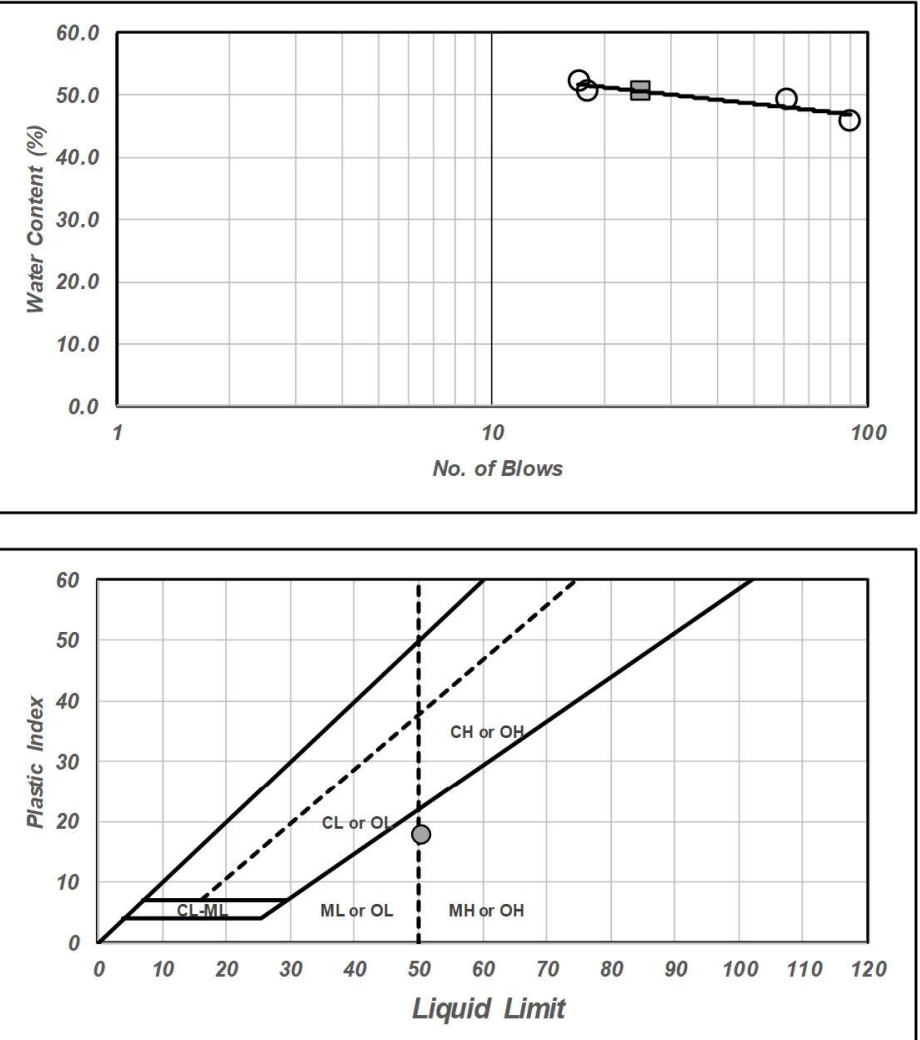

Procedure Done By:
Liquid Limit:

Plastic Limit:

Plasticity Index:
Total amount of materials passing sieve No. 40 : $100 \%$
Fines

Classification: $\mathrm{MH}$ 


\section{S Portland State}

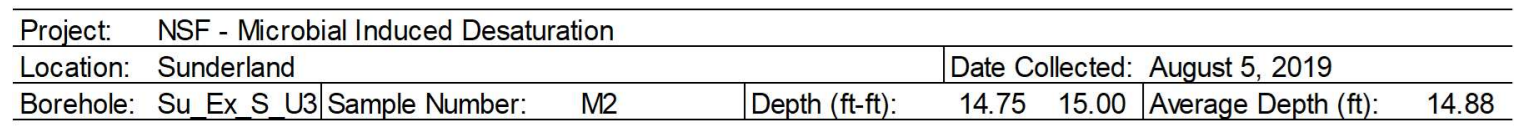

\begin{tabular}{|c|c|c|c|c|c|c|c|c|c|c|}
\hline \multicolumn{6}{|c|}{ Liquid Limit } & \multicolumn{5}{|c|}{ Plastic Limit } \\
\hline 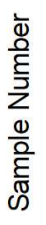 & 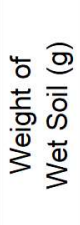 & 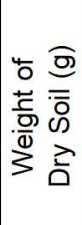 & 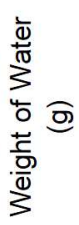 & 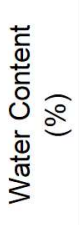 & 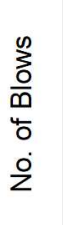 & $\begin{array}{l}\bar{\Phi} \\
\frac{0}{E} \\
\bar{\Sigma} \\
\bar{z} \\
\frac{0}{0} \\
\frac{0}{E} \\
\bar{N} \\
\infty\end{array}$ & 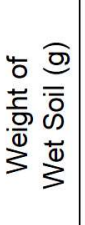 & 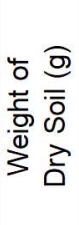 & 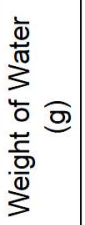 & 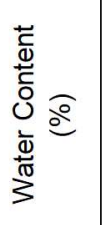 \\
\hline 1 & 8.0 & 5.7 & 2.3 & 40.4 & & 1 & 7.8 & 6 & 1.8 & 30.0 \\
\hline 2 & 10.1 & 7.0 & 3.1 & 44.3 & 96 & 2 & 7.8 & 6 & 1.8 & 30.0 \\
\hline 3 & 14.5 & 9.8 & 4.7 & 48.0 & 27 & & & & & \\
\hline 4 & 9.9 & 6.8 & 3.1 & 45.6 & 70 & & & & & \\
\hline 5 & 12.7 & 8.5 & 4.2 & 49.4 & 22 & & & & & \\
\hline & & & & & & & & & & \\
\hline
\end{tabular}
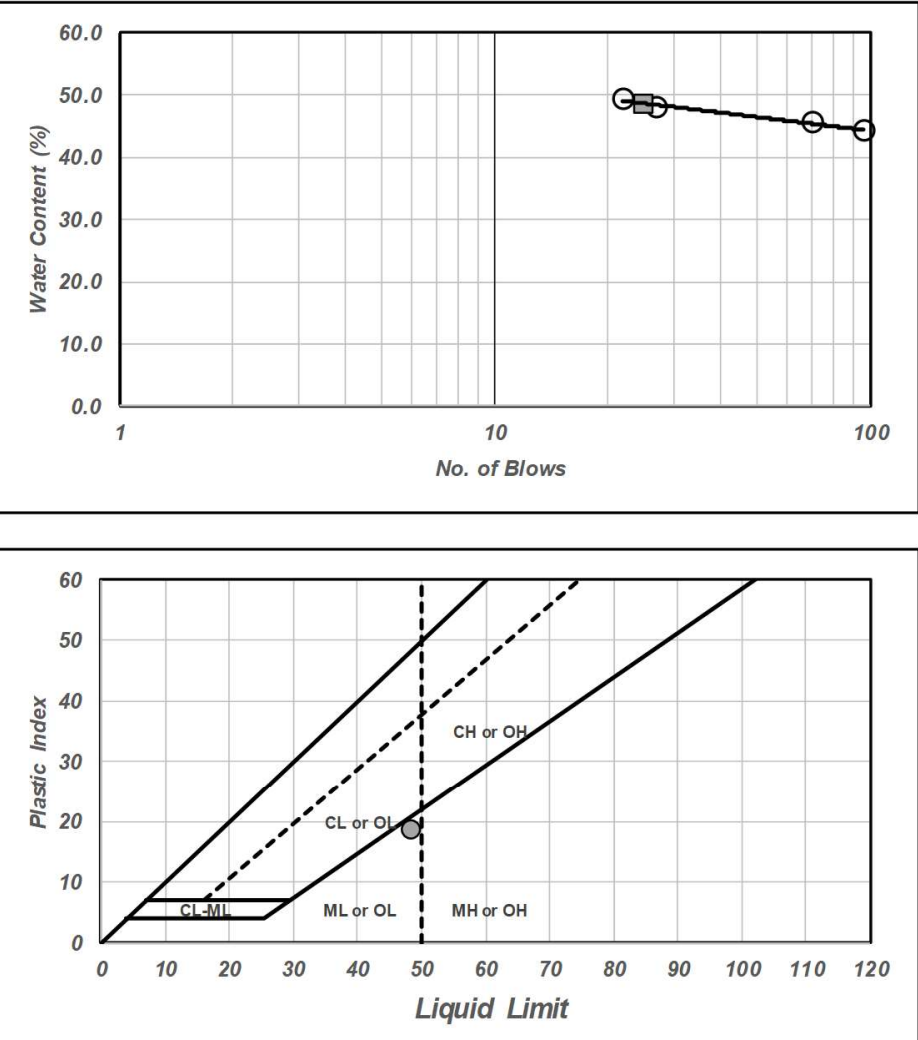

Procedure Done By:
Liquid Limit:

Plastic Limit:

Plasticity Index:
Total amount of materials passing sieve No. 40: $100 \%$
Fines

Classification: ML 


\section{S Portland State}

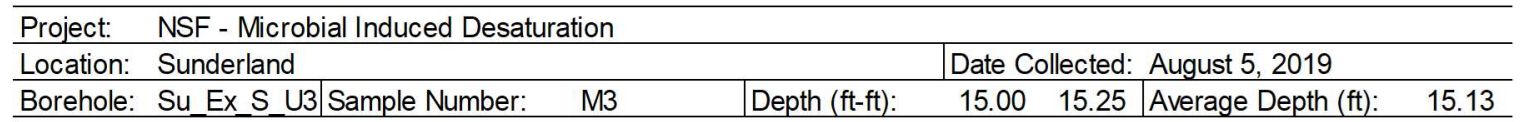

\begin{tabular}{|c|c|c|c|c|c|c|c|c|c|c|}
\hline \multicolumn{6}{|c|}{ Liquid Limit } & \multicolumn{5}{|c|}{ Plastic Limit } \\
\hline 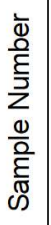 & 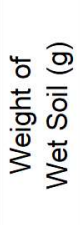 & 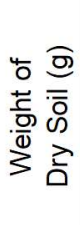 & 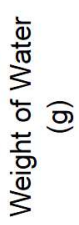 & 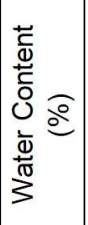 & 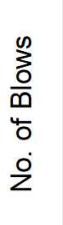 & $\begin{array}{l}\bar{\Phi} \\
\frac{0}{E} \\
\bar{\Sigma} \\
\bar{z} \\
\frac{0}{0} \\
\frac{0}{E} \\
\bar{N} \\
\infty\end{array}$ & 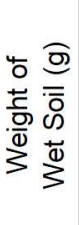 & 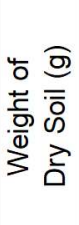 & 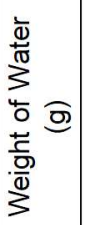 & 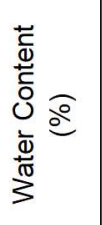 \\
\hline 1 & 13.7 & 9.6 & 4.1 & 42.7 & & 1 & 6 & 4.8 & 1.2 & 25.0 \\
\hline 2 & 14 & 9.7 & 4.3 & 44.3 & 20 & 2 & 8.7 & 6.9 & 1.8 & 26.1 \\
\hline 3 & 10.6 & 7.3 & 3.3 & 45.2 & 12 & & & & & \\
\hline 4 & 12.7 & 9.0 & 3.7 & 41.1 & 34 & & & & & \\
\hline 5 & 13.6 & 9.8 & 3.8 & 38.8 & 70 & & & & & \\
\hline & & & & & & & & & & \\
\hline
\end{tabular}

Liquid Limit:

Plastic Limit:

Plasticity Index:
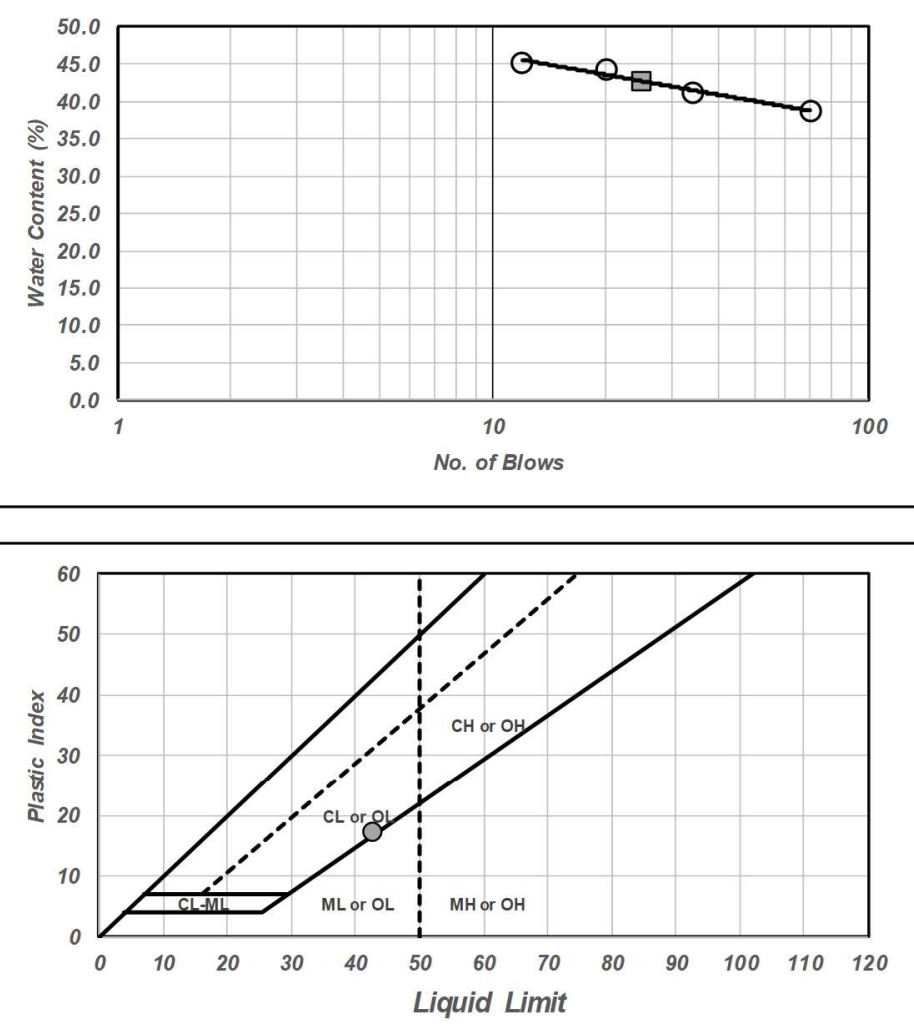

Procedure Done By:

$\operatorname{Max}$
Total amount of materials passing sieve No. 40: $100 \%$
Fines

Classification: $\mathrm{CL}$ 


\section{S Portland State}

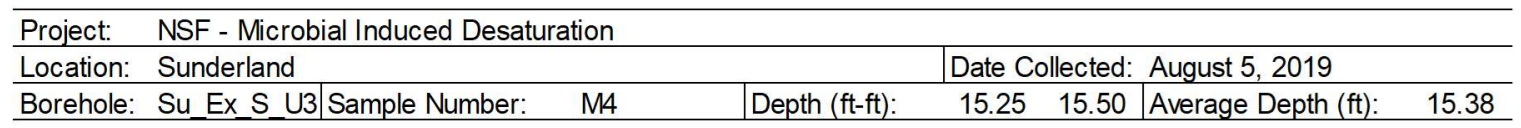

\begin{tabular}{|c|c|c|c|c|c|c|c|c|c|c|}
\hline \multicolumn{6}{|c|}{ Liquid Limit } & \multicolumn{5}{|c|}{ Plastic Limit } \\
\hline 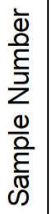 & 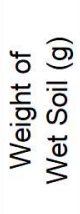 & 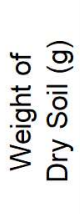 & 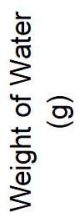 & 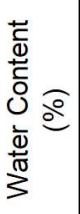 & 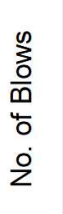 & 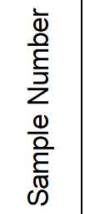 & 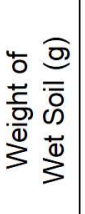 & 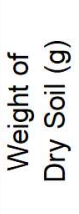 & 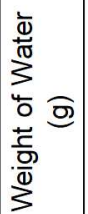 & 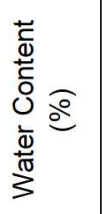 \\
\hline 1 & 10.2 & 7.0 & 3.2 & 45.7 & & 1 & 10.9 & 8.5 & 2.4 & 28.2 \\
\hline 2 & 12.9 & 8.8 & 4.1 & 46.6 & 14 & 2 & 12.2 & 9.4 & 2.8 & 29.8 \\
\hline 3 & 13.5 & 9.4 & 4.1 & 43.6 & 18 & & & & & \\
\hline 4 & 13.0 & 9.3 & 3.7 & 39.8 & 48 & & & & & \\
\hline 5 & 14.3 & 10.5 & 3.8 & 36.2 & 121 & & & & & \\
\hline & & & & & & & & & & \\
\hline
\end{tabular}

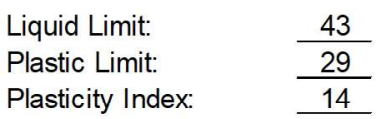

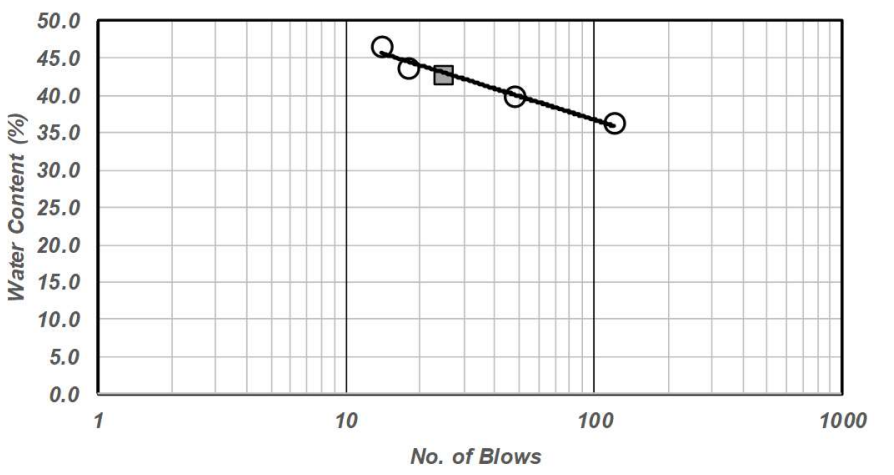

Total amount of materials passing sieve No. 40: $100 \%$

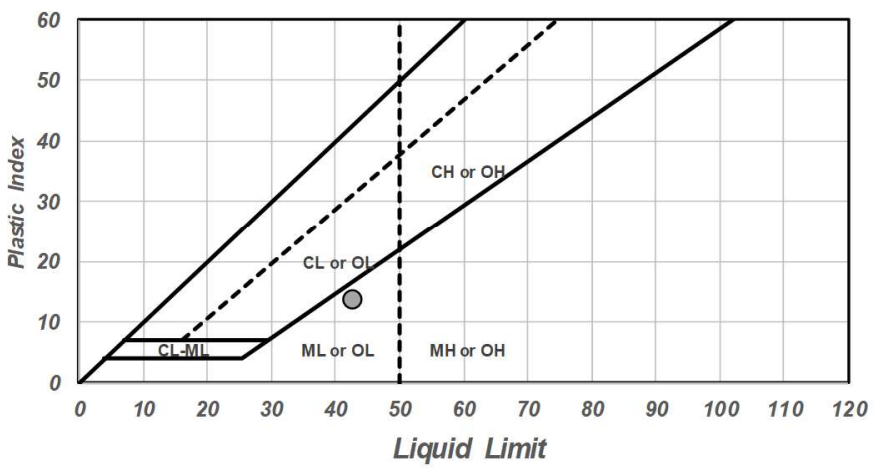

Fines

Classification: ML

Procedure Done By:

$\operatorname{Max}$ 


\section{S Portland State}

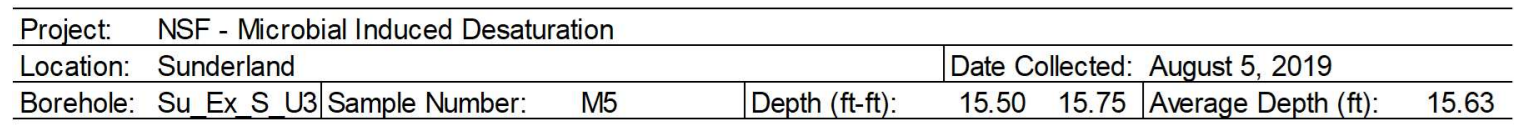

\begin{tabular}{|c|c|c|c|c|c|c|c|c|c|c|}
\hline \multicolumn{6}{|c|}{ Liquid Limit } & \multicolumn{5}{|c|}{ Plastic Limit } \\
\hline 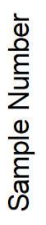 & 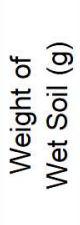 & 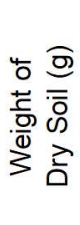 & 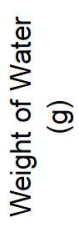 & 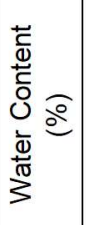 & 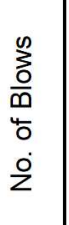 & 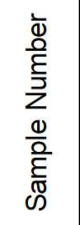 & 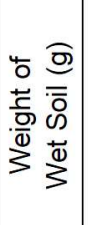 & 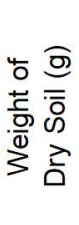 & 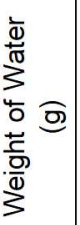 & 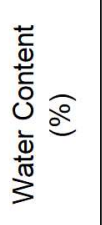 \\
\hline 1 & 13.5 & 9.3 & 4.2 & 45.2 & & 1 & 8.7 & 6.7 & 2 & 29.9 \\
\hline 2 & 12.9 & 8.6 & 4.3 & 50.0 & 3 & 2 & 6.1 & 4.7 & 1.4 & 29.8 \\
\hline 3 & 9.9 & 7.0 & 2.9 & 41.4 & 11 & & & & & \\
\hline 4 & 12.3 & 9.1 & 3.2 & 35.2 & 36 & & & & & \\
\hline 5 & 11 & 8.2 & 2.8 & 34.1 & 58 & & & & & \\
\hline & & & & & & & & & & \\
\hline
\end{tabular}
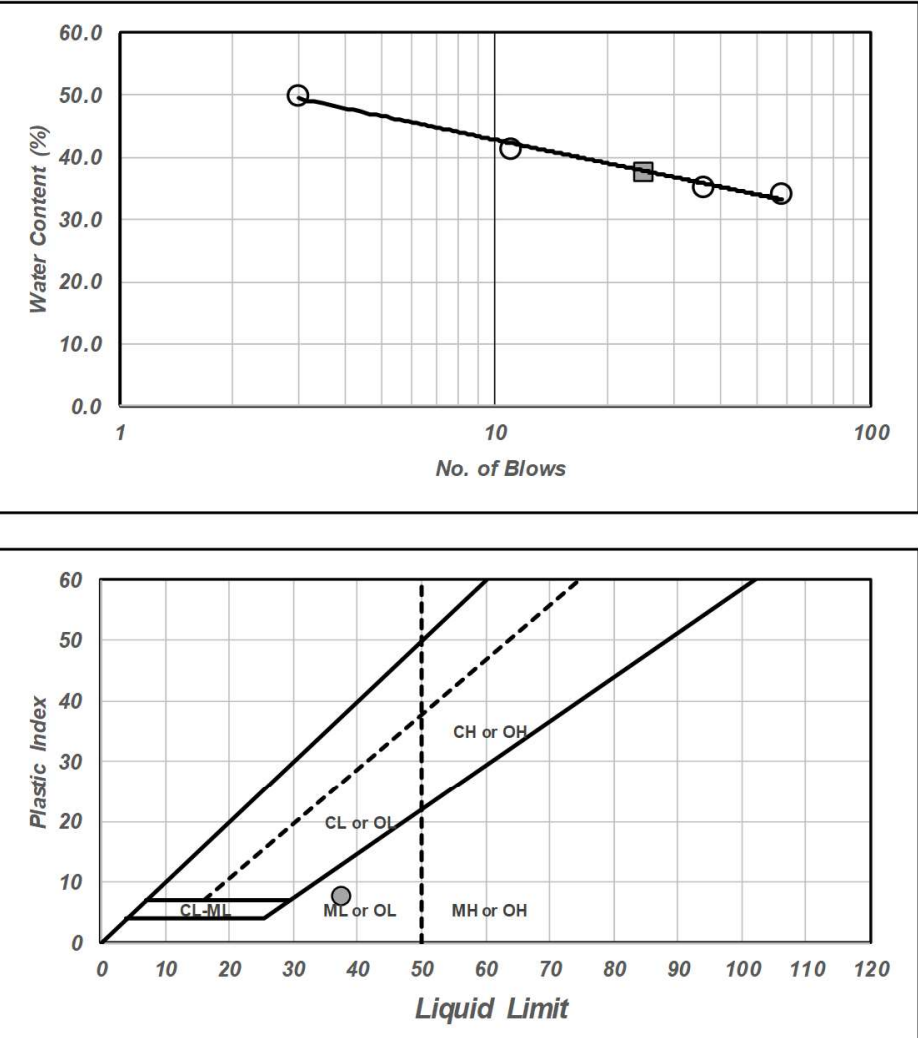

Procedure Done By:
Liquid Limit:

Plastic Limit:

Plasticity Index:
Total amount of materials passing sieve No. 40: $100 \%$
Fines

Classification: ML 


\section{S Portland State}

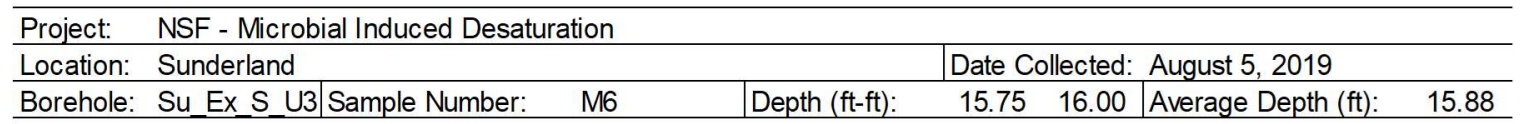

\begin{tabular}{|c|c|c|c|c|c|c|c|c|c|c|}
\hline \multicolumn{6}{|c|}{ Liquid Limit } & \multicolumn{5}{|c|}{ Plastic Limit } \\
\hline 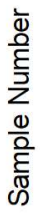 & 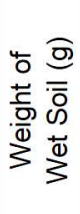 & 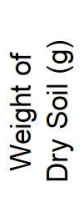 & 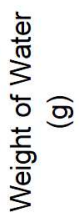 & 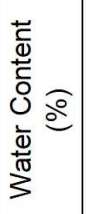 & 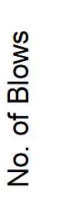 & 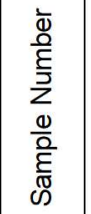 & 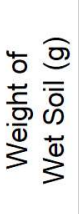 & 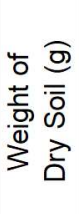 & 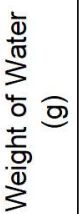 & 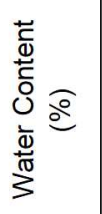 \\
\hline 1 & 10.1 & 6.6 & 3.5 & 53.0 & & 1 & 9.3 & 7.2 & 2.1 & 29.2 \\
\hline 2 & 13.1 & 8.9 & 4.2 & 47.2 & 34 & 2 & 9.2 & 7.1 & 2.1 & 29.6 \\
\hline 3 & 12.8 & 8.6 & 4.2 & 48.8 & 17 & & & & & \\
\hline 4 & 16.5 & 11.5 & 5.0 & 43.5 & 41 & & & & & \\
\hline 5 & 18.3 & 12.5 & 5.8 & 46.4 & 15 & & & & & \\
\hline & & & & & & & & & & \\
\hline
\end{tabular}

Liquid Limit:

Plastic Limit:

Plasticity Index: $\frac{46}{29}$
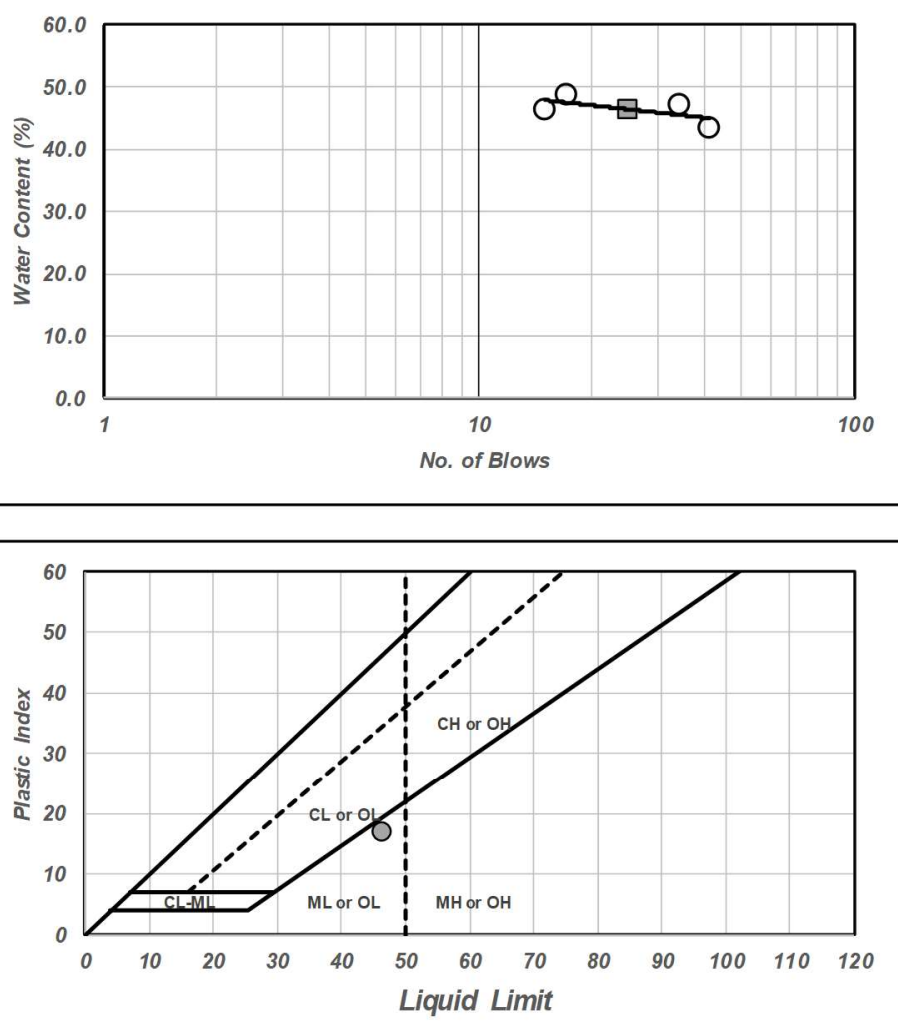

Procedure Done By: Date Tested:

Kayla

July 21,2020

Total amount of materials passing sieve No. 40: $100 \%$
Fines

Classification: ML 


\section{S Portland State}

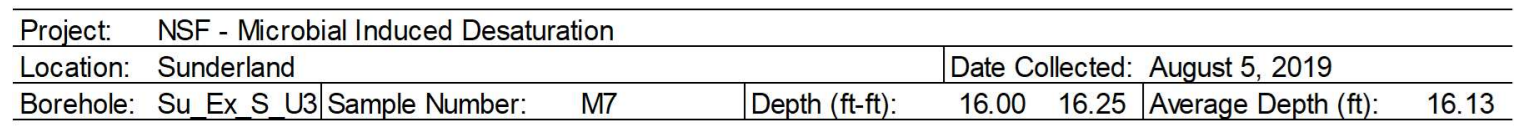

\begin{tabular}{|c|c|c|c|c|c|c|c|c|c|c|}
\hline \multicolumn{6}{|c|}{ Liquid Limit } & \multicolumn{5}{|c|}{ Plastic Limit } \\
\hline 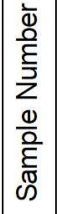 & 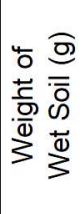 & 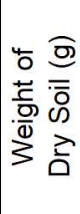 & 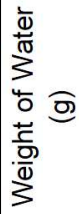 & 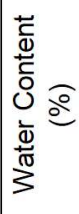 & $\begin{array}{l}0 \\
\frac{0}{0} \\
00 \\
40 \\
\dot{0} \\
\frac{0}{2}\end{array}$ & 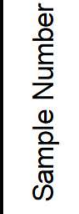 & 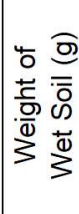 & 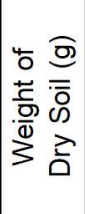 & 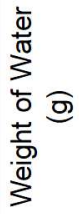 & 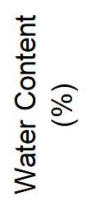 \\
\hline 1 & 27.0 & 18.1 & 8.9 & 49.2 & & 1 & 10.9 & 8.2 & 2.7 & 32.9 \\
\hline 2 & 16.6 & 11.3 & 5.3 & 46.9 & 23 & 2 & 10.7 & 8.1 & 2.6 & 32.1 \\
\hline 3 & 14.9 & 10.4 & 4.5 & 43.3 & 29 & & & & & \\
\hline 4 & 18.6 & 12.9 & 5.7 & 44.2 & 16 & & & & & \\
\hline & & & & & & & & & & \\
\hline & & & & & & & & & & \\
\hline
\end{tabular}
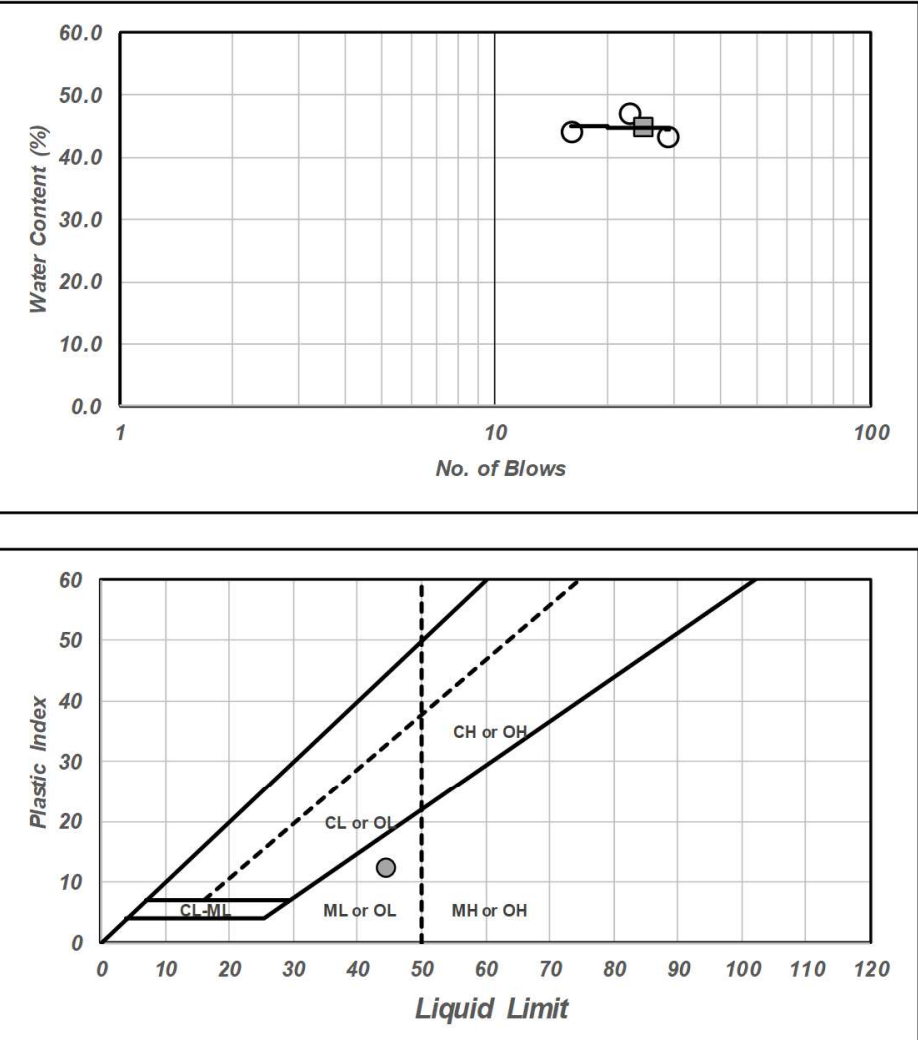

Procedure Done By: Date Tested:

Kayla

July 22, 2020

\begin{tabular}{ll} 
Liquid Limit: & 45 \\
Plastic Limit: & $\quad 33$ \\
Plasticity Index: & \multicolumn{12}{|c}{} \\
\hline
\end{tabular}

Total amount of materials passing sieve No. 40 : $100 \%$
Fines

Classification: ML 


\section{S Portland State}

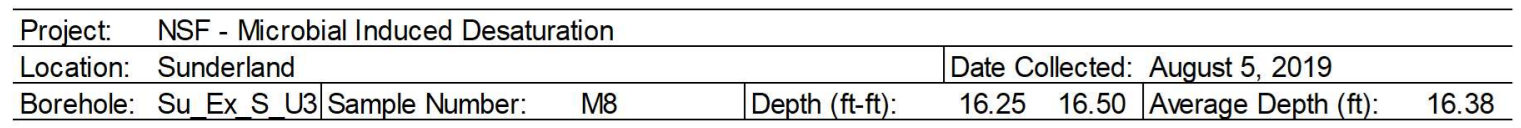

\begin{tabular}{|c|c|c|c|c|c|c|c|c|c|c|}
\hline \multicolumn{6}{|c|}{ Liquid Limit } & \multicolumn{5}{|c|}{ Plastic Limit } \\
\hline 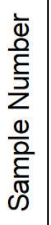 & 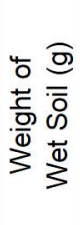 & 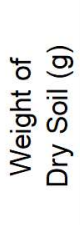 & 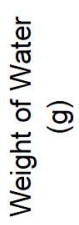 & 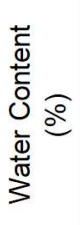 & 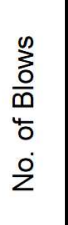 & 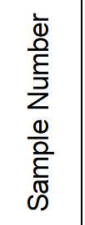 & 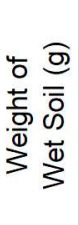 & 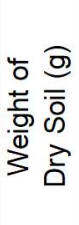 & 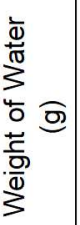 & 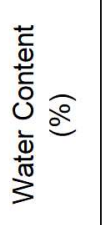 \\
\hline 1 & 9.9 & 6.5 & 3.4 & 52.3 & & 1 & 10.2 & 7.7 & 2.5 & 32.5 \\
\hline 2 & 18.7 & 12.3 & 6.4 & 52.0 & 8 & 2 & 9.8 & 7.4 & 2.4 & 32.4 \\
\hline 3 & 13.0 & 9.4 & 3.6 & 38.3 & 75 & & & & & \\
\hline 4 & 11.7 & 8.3 & 3.4 & 41.0 & 29 & & & & & \\
\hline 5 & 13.5 & 9.4 & 4.1 & 43.6 & 19 & & & & & \\
\hline & & & & & & & & & & \\
\hline
\end{tabular}
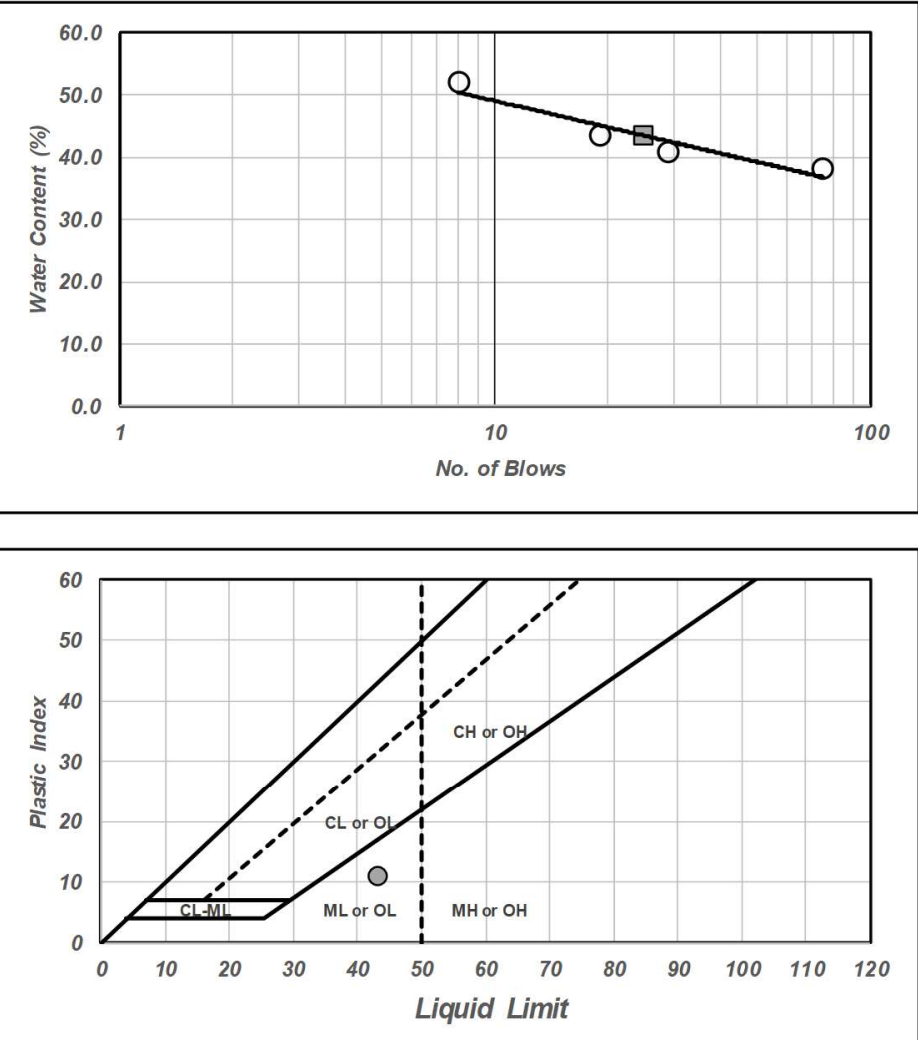

Procedure Done By: Date Tested:

Kayla

July 22, 2020

\begin{tabular}{ll} 
Liquid Limit: & 43 \\
Plastic Limit: & $\quad 32$ \\
Plasticity Index: & $\quad 11$ \\
\hline
\end{tabular}

Total amount of materials passing sieve No. 40 : $100 \%$
Fines

Classification: ML 


\section{S Portland State}

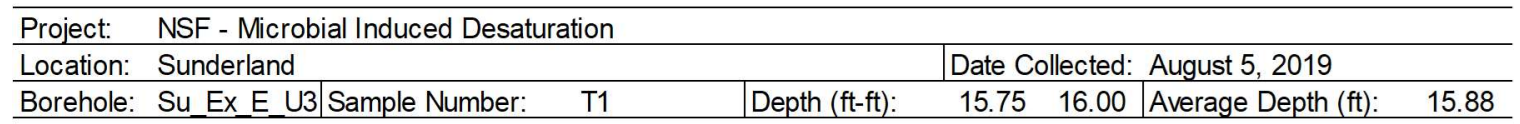

\begin{tabular}{|c|c|c|c|c|c|c|c|c|c|c|}
\hline \multicolumn{6}{|c|}{ Liquid Limit } & \multicolumn{5}{|c|}{ Plastic Limit } \\
\hline 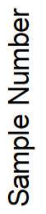 & 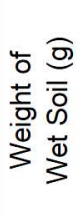 & 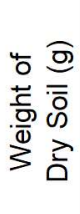 & 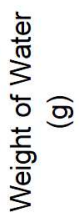 & 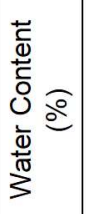 & 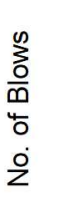 & 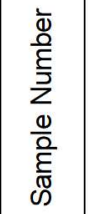 & 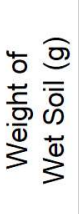 & 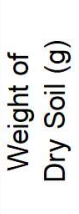 & 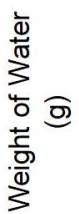 & 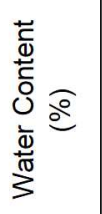 \\
\hline 1 & 15.7 & 10.5 & 5.2 & 49.5 & & 1 & 10.2 & 7.5 & 2.7 & 36.0 \\
\hline 2 & 13.3 & 8.8 & 4.5 & 51.1 & 42 & 2 & 10.4 & 7.7 & 2.7 & 35.1 \\
\hline 3 & 14.7 & 9.6 & 5.1 & 53.1 & 25 & & & & & \\
\hline 4 & 20.5 & 13.5 & 7.0 & 51.9 & 18 & & & & & \\
\hline 5 & 13.2 & 8.5 & 4.7 & 55.3 & 11 & & & & & \\
\hline & & & & & & & & & & \\
\hline
\end{tabular}

\begin{tabular}{ll} 
Liquid Limit: & $\frac{52}{36}$ \\
Plastic Limit: & $\quad$ \\
Plasticity Index: & \\
\hline
\end{tabular}

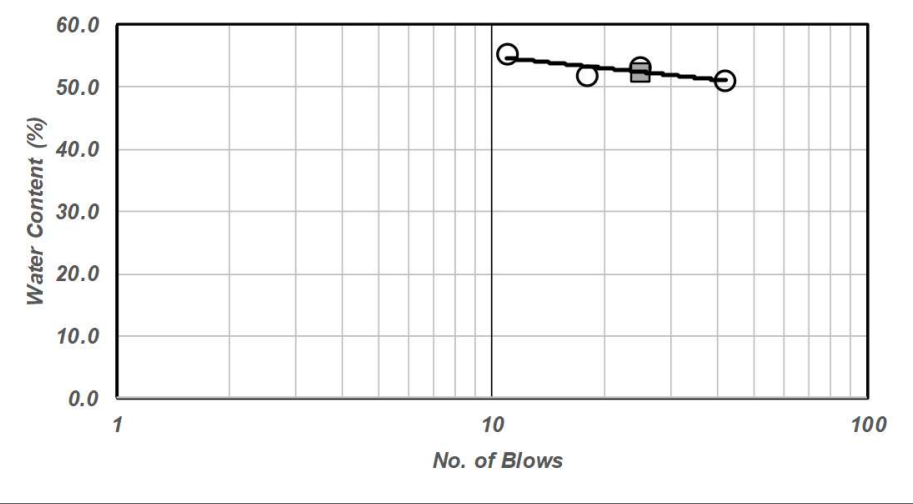

Total amount of materials passing sieve No. 40: $100 \%$

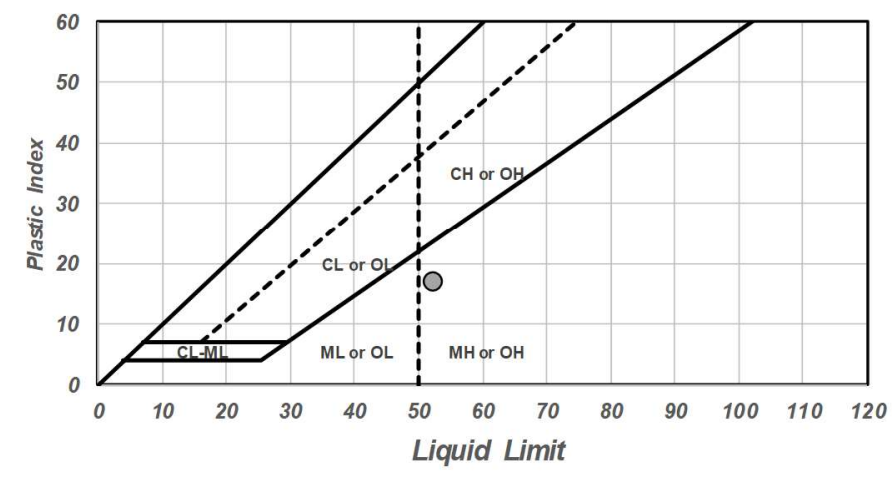

Fines

Classification: $\mathrm{MH}$

Procedure Done By:

Kayla

Date Tested:

July 22, 2020 


\section{\& Portland State}

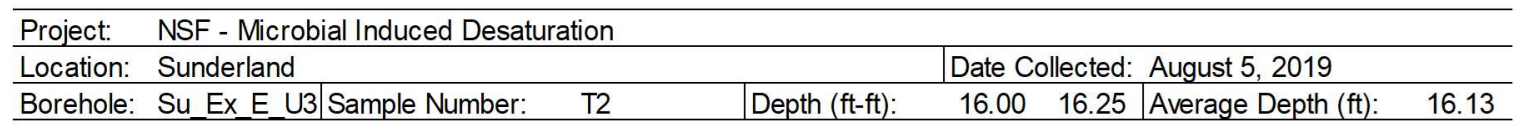

\begin{tabular}{|c|c|c|c|c|c|c|c|c|c|c|}
\hline \multicolumn{6}{|c|}{ Liquid Limit } & \multicolumn{5}{|c|}{ Plastic Limit } \\
\hline 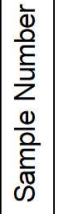 & 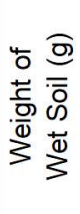 & 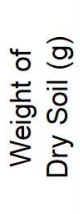 & 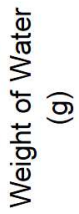 & 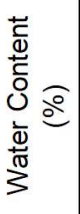 & $\begin{array}{l}n \\
\sum_{0}^{0} \\
0 \\
\text { to } \\
\dot{0} \\
\dot{2}\end{array}$ & 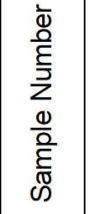 & 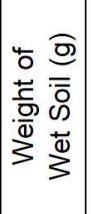 & 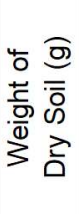 & 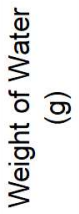 & 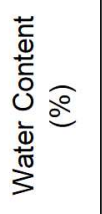 \\
\hline 1 & 11.6 & 7.8 & 3.8 & 48.7 & & 1 & 7.3 & 5.4 & 1.9 & 35.2 \\
\hline 2 & 6 & 4.1 & 1.9 & 46.3 & 14 & 2 & 7.8 & 5.8 & 2 & 34.5 \\
\hline 6 & 8.3 & 5.8 & 2.5 & 43.1 & 50 & & & & & \\
\hline 4 & 9.0 & 6.3 & 2.7 & 42.9 & 20 & & & & & \\
\hline 5 & 11.5 & 8.2 & 3.3 & 40.2 & 62 & & & & & \\
\hline 6 & 6.8 & 4.8 & 2.0 & 41.7 & 35 & & & & & \\
\hline
\end{tabular}

Liquid Limit:

Plastic Limit:

Plasticity Index: $\frac{43}{\frac{35}{9}}$
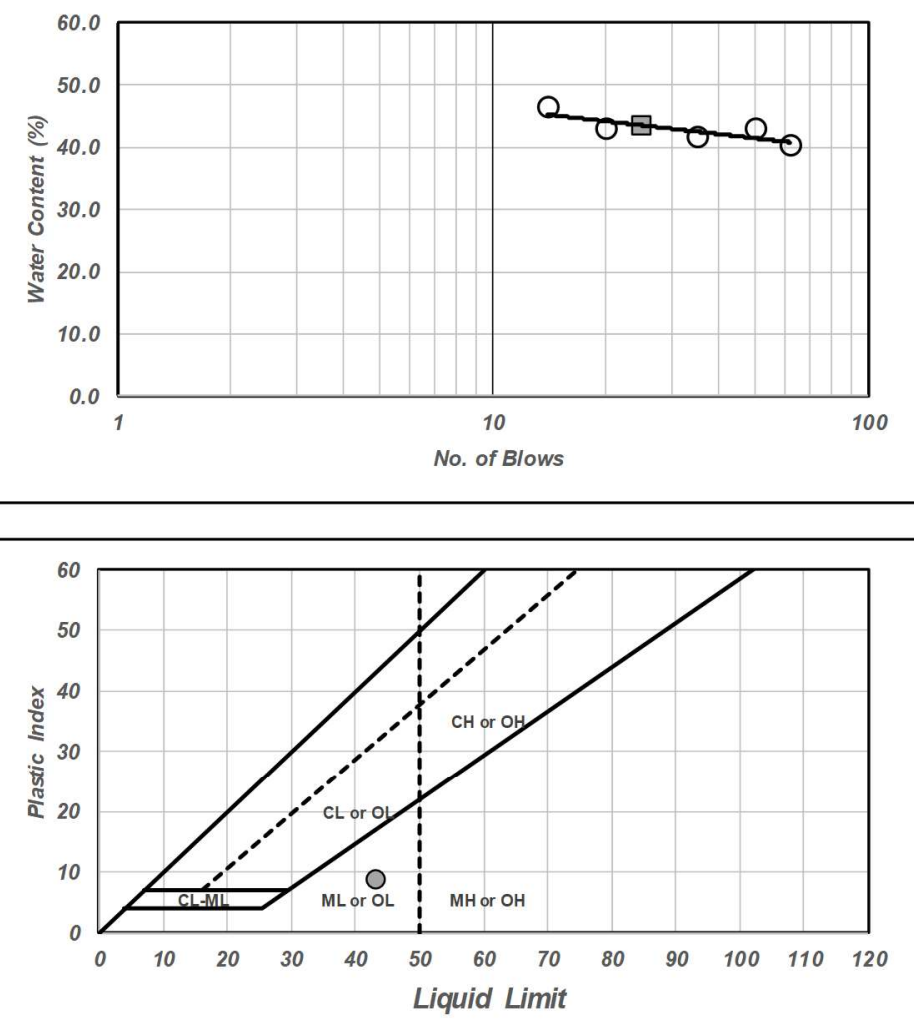

Procedure Done By: Date Tested:

Kayla

August 18/20, 2020
Total amount of materials passing sieve No. 40: $100 \%$
Fines

Classification: ML 


\section{\& Portland State}

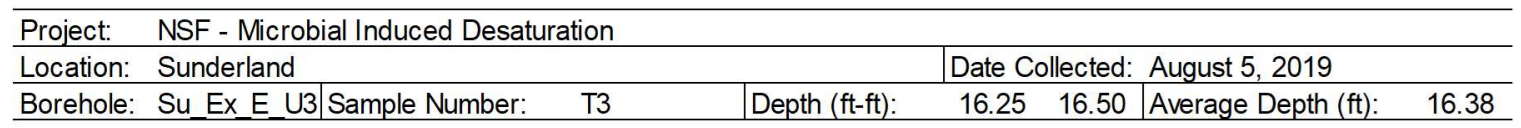

\begin{tabular}{|c|c|c|c|c|c|c|c|c|c|c|}
\hline \multicolumn{6}{|c|}{ Liquid Limit } & \multicolumn{5}{|c|}{ Plastic Limit } \\
\hline 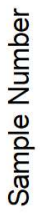 & 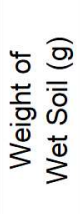 & 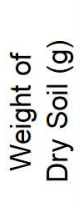 & 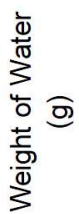 & 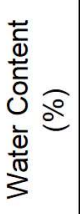 & 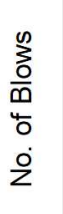 & 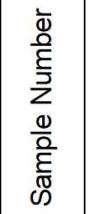 & 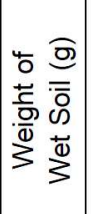 & 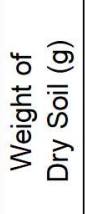 & 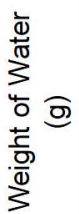 & 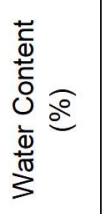 \\
\hline 1 & 7.1 & 5.0 & 2.1 & 42.0 & & 1 & 7.6 & 6 & 1.6 & 26.7 \\
\hline 2 & 9.9 & 6.8 & 3.1 & 45.6 & 68 & 2 & 7.9 & 6.2 & 1.7 & 27.4 \\
\hline 3 & 7.5 & 5.3 & 2.2 & 41.5 & 54 & & & & & \\
\hline 4 & 7.4 & 5.1 & 2.3 & 45.1 & 18 & & & & & \\
\hline 5 & 9.9 & 6.7 & 3.2 & 47.8 & 10 & & & & & \\
\hline & & & & & & & & & & \\
\hline
\end{tabular}

Liquid Limit:

Plastic Limit:

Plasticity Index: $\frac{45}{27}$
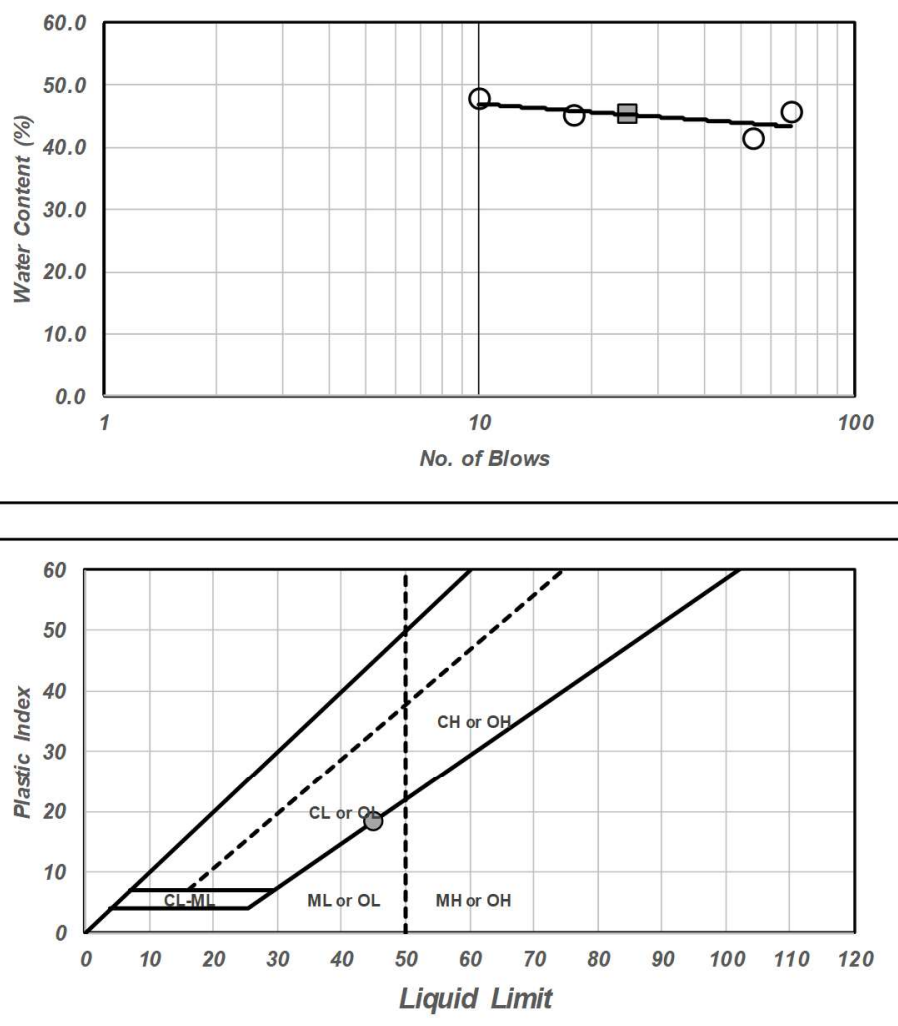

Procedure Done By: Date Tested:

Kayla

August 20, 2020
Total amount of materials passing sieve No. 40 : $100 \%$
Fines

Classification: $\mathrm{ML} / \mathrm{CL}$ 


\section{\& Portland State}

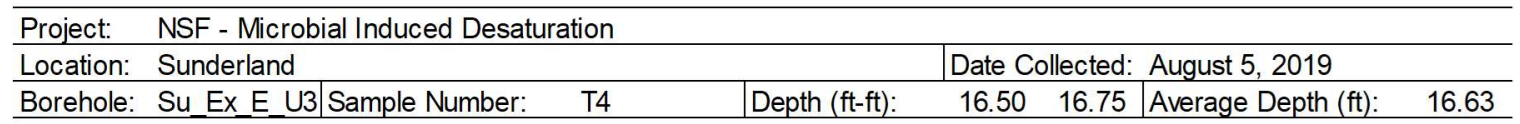

\begin{tabular}{|c|c|c|c|c|c|c|c|c|c|c|}
\hline \multicolumn{6}{|c|}{ Liquid Limit } & \multicolumn{5}{|c|}{ Plastic Limit } \\
\hline 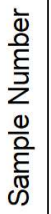 & 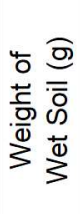 & 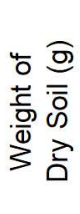 & 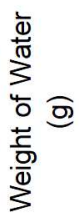 & 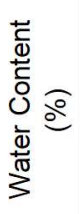 & 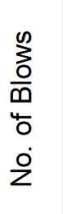 & 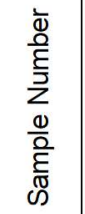 & 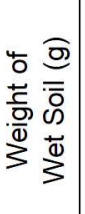 & 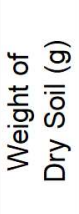 & 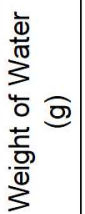 & 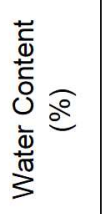 \\
\hline 1 & 15.7 & 10.9 & 4.8 & 44.0 & & 1 & 6.6 & 5 & 1.6 & 32.0 \\
\hline 2 & 6.4 & 4.4 & 2 & 45.5 & 17 & 2 & 6.6 & 5 & 1.6 & 32.0 \\
\hline 3 & 7.1 & 5.1 & 2 & 39.2 & 70 & & & & & \\
\hline 4 & 9.4 & 6.7 & 2.7 & 40.3 & 43 & & & & & \\
\hline 5 & 10.9 & 7.6 & 3.3 & 43.4 & 20 & & & & & \\
\hline & & & & & & & & & & \\
\hline
\end{tabular}
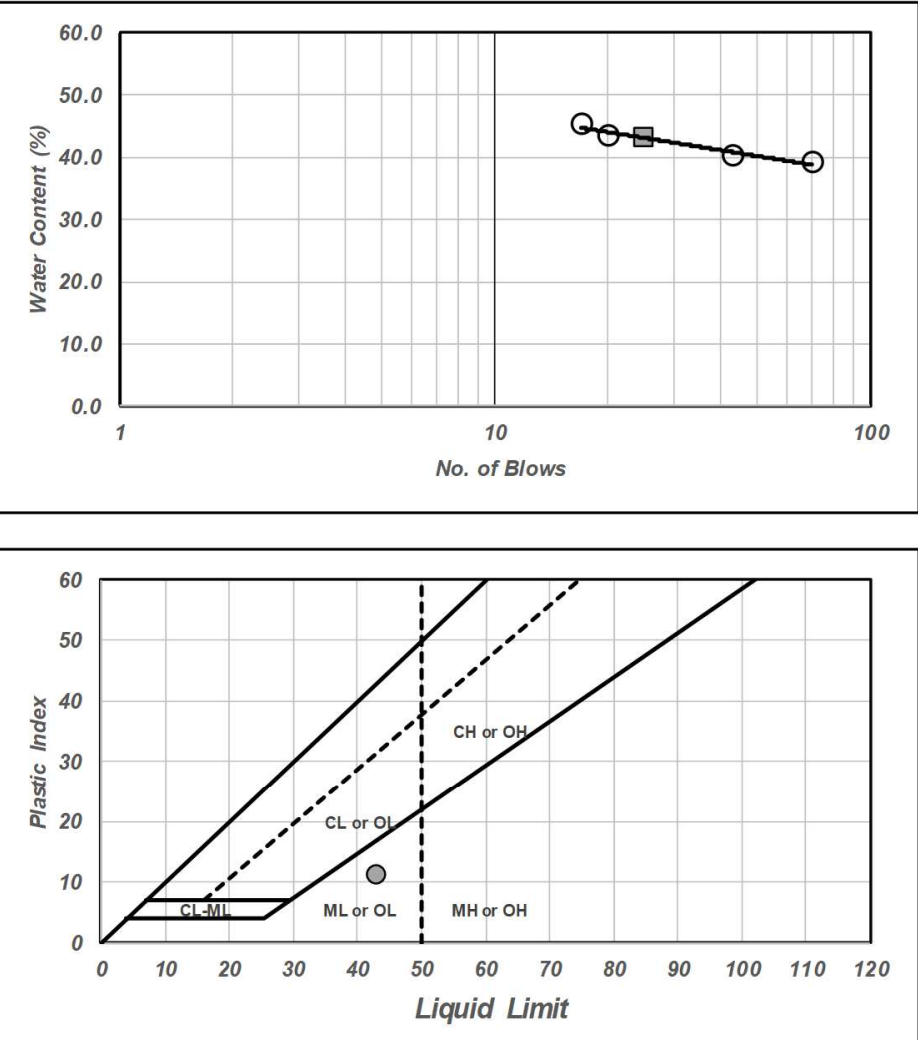

Procedure Done By: Date Tested:

Kayla

October 1, 2020

\begin{tabular}{ll} 
Liquid Limit: & 43 \\
Plastic Limit: & $\quad 32$ \\
Plasticity Index: & $\quad 11$ \\
\hline
\end{tabular}

Total amount of materials passing sieve No. 40 : $100 \%$
Fines

Classification: ML 


\section{\& Portland State}

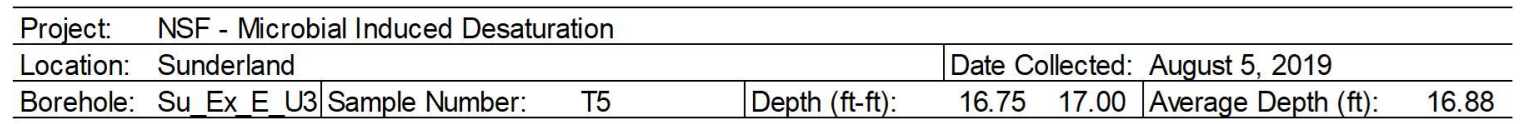

\begin{tabular}{|c|c|c|c|c|c|c|c|c|c|c|}
\hline \multicolumn{6}{|c|}{ Liquid Limit } & \multicolumn{5}{|c|}{ Plastic Limit } \\
\hline 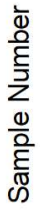 & 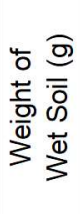 & 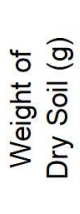 & 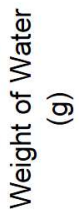 & 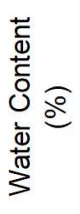 & 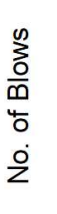 & 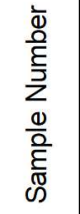 & 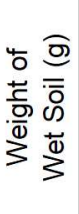 & 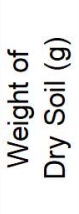 & 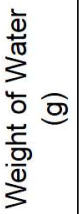 & 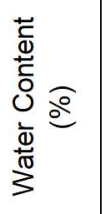 \\
\hline 1 & 8.6 & 5.5 & 3.1 & 56.4 & & 1 & 8.1 & 6.1 & 2 & 32.8 \\
\hline 2 & 14.5 & 9.5 & 5 & 52.6 & 54 & 2 & 8.0 & 6 & 2 & 33.3 \\
\hline 3 & 12.3 & 7.9 & 4.4 & 55.7 & 35 & & & & & \\
\hline 4 & 10.4 & 6.7 & 3.7 & 55.2 & 20 & & & & & \\
\hline 5 & 15.4 & 9.8 & 5.6 & 57.1 & 15 & & & & & \\
\hline & & & & & & & & & & \\
\hline
\end{tabular}

\begin{tabular}{ll} 
Liquid Limit: & 55 \\
Plastic Limit: & $\quad 33$ \\
Plasticity Index: & $\underline{22}$ \\
\hline
\end{tabular}

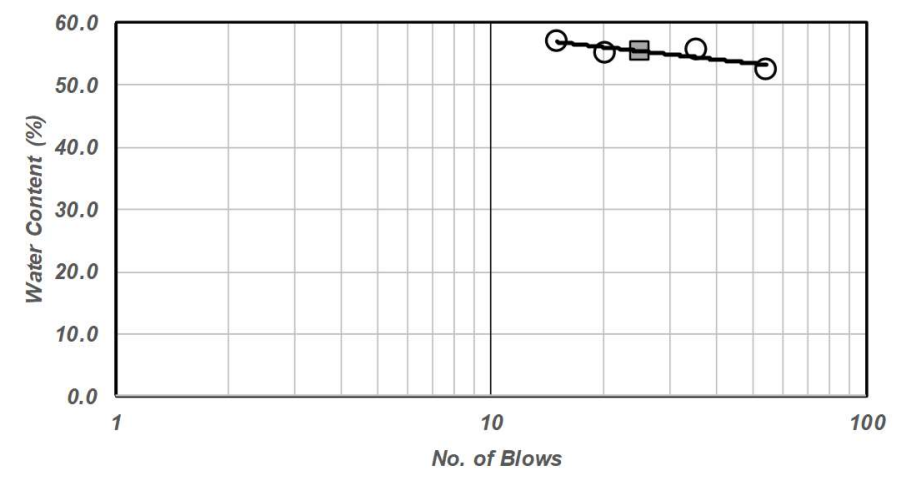

Total amount of materials passing sieve No. 40: $100 \%$

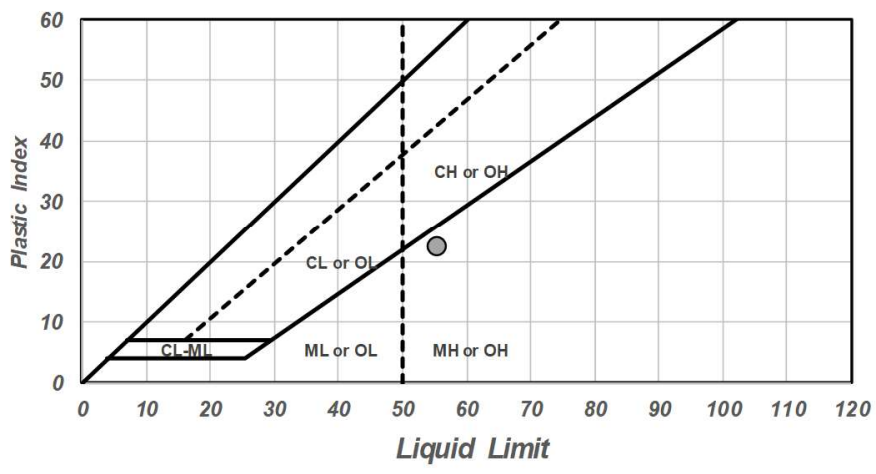

Fines

Classification: $\mathrm{MH}$

Procedure Done By: Date Tested:

Kayla

October 18, 2020




\section{S Portland Satee}

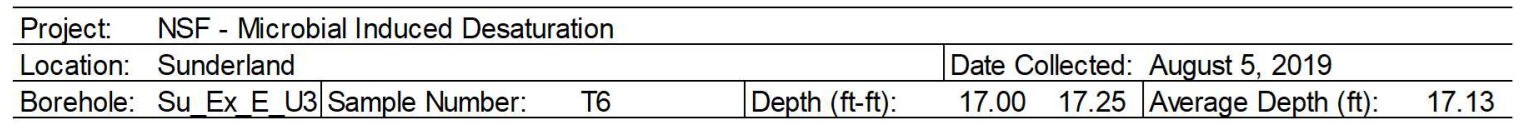

\begin{tabular}{|c|c|c|c|c|c|c|c|c|c|c|}
\hline \multicolumn{6}{|c|}{ Liquid Limit } & \multicolumn{5}{|c|}{ Plastic Limit } \\
\hline 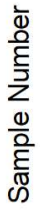 & 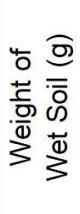 & 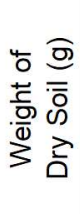 & 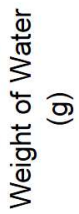 & 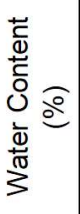 & 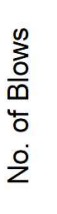 & 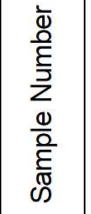 & 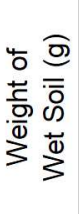 & 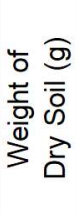 & 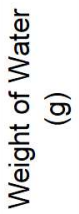 & 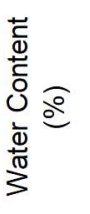 \\
\hline 1 & 12.6 & 7.8 & 4.8 & 61.5 & & 1 & 9.1 & 6.5 & 2.6 & 40.0 \\
\hline 2 & 11 & 7.4 & 3.6 & 48.6 & 106 & 2 & 9.2 & 6.6 & 2.6 & 39.4 \\
\hline 3 & 12.5 & 8.0 & 4.5 & 56.3 & 45 & & & & & \\
\hline 4 & 13.5 & 8.5 & 5.0 & 58.8 & 28 & & & & & \\
\hline 5 & 12.2 & 7.6 & 4.6 & 60.5 & 17 & & & & & \\
\hline 6 & 12.6 & 7.8 & 4.8 & 61.5 & 9 & & & & & \\
\hline
\end{tabular}

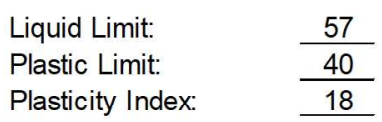

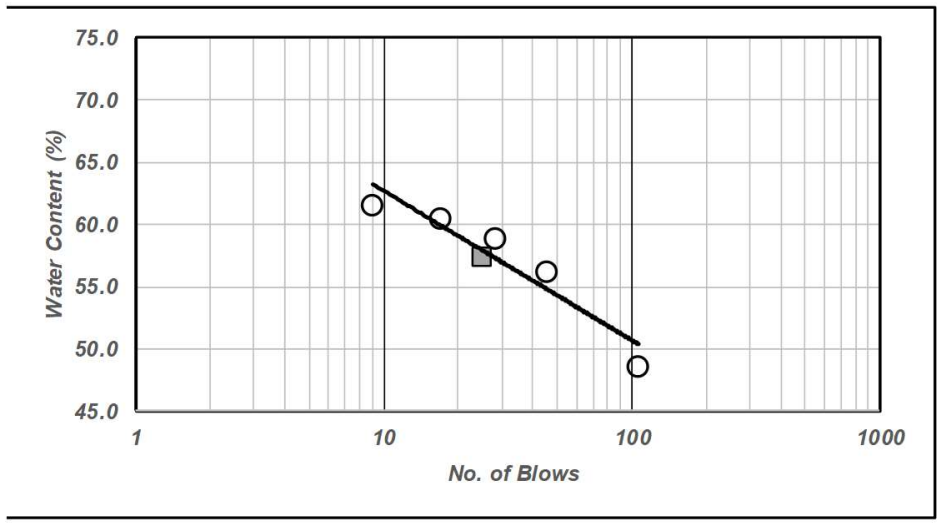

Total amount of materials passing sieve No. 40 : $100 \%$

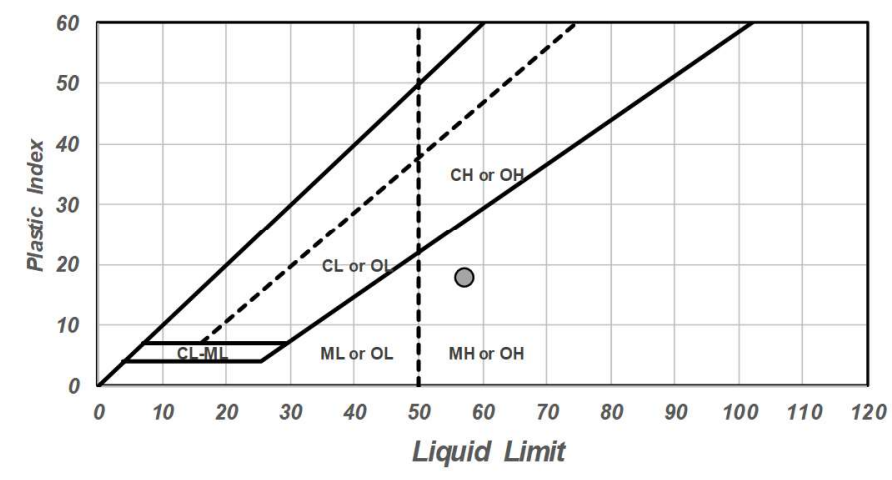

Fines

Classification: $\mathrm{MH}$

Procedure Done By: 


\section{\& Portland State}

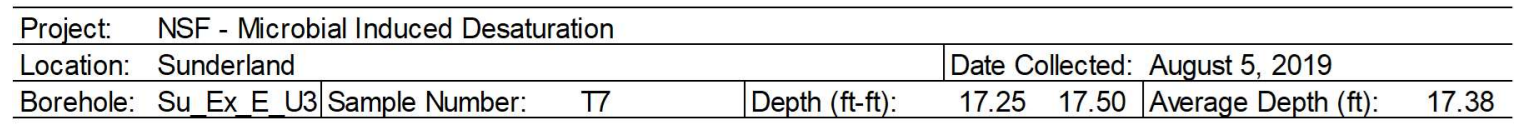

\begin{tabular}{|c|c|c|c|c|c|c|c|c|c|c|}
\hline \multicolumn{6}{|c|}{ Liquid Limit } & \multicolumn{5}{|c|}{ Plastic Limit } \\
\hline 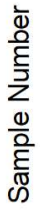 & 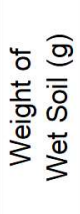 & 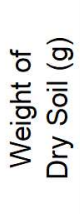 & 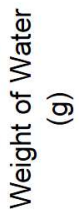 & 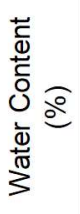 & 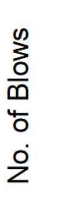 & 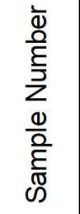 & 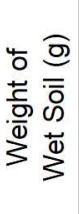 & 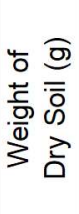 & 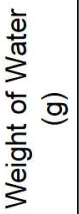 & 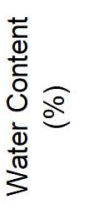 \\
\hline 1 & 10.8 & 6.6 & 4.2 & 63.6 & & 1 & 8.9 & 6.4 & 2.5 & 39.1 \\
\hline 2 & 7.8 & 4.9 & 2.9 & 59.2 & 45 & 2 & 8.9 & 6.4 & 2.5 & 39.1 \\
\hline 3 & 12.8 & 7.8 & 5 & 64.1 & 27 & & & & & \\
\hline 4 & 8.2 & 4.9 & 3.3 & 67.3 & 17 & & & & & \\
\hline 5 & 10.4 & 6.1 & 4.3 & 70.5 & 13 & & & & & \\
\hline & & & & & & & & & & \\
\hline
\end{tabular}

Liquid Limit:

Plastic Limit:

Plasticity Index: $\frac{64}{39}$

Total amount of materials passing sieve No. 40: $100 \%$

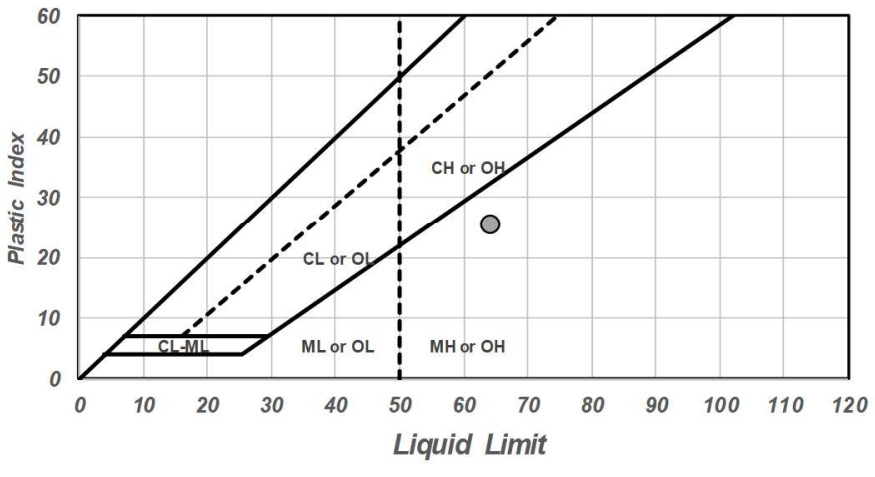

Procedure Done By: Date Tested:

Kayla

October 21, 2020
Fines

Classification: $\mathrm{MH}$ 


\section{\& Portland Sate}

\begin{tabular}{|c|c|c|c|c|c|c|c|c|c|c|c|}
\hline Pro & ect: & NSF - & icrol & bi & | Indu & $\mathrm{ed} \mathrm{D \epsilon}$ & saturat & ion & & & \\
\hline Loc & ation: & Sunde & land & & & & & & & & \\
\hline Bor & ehole: & Su E & W U & $2 \mid s$ & Samp & Num & oer: & $\mathrm{G} 1$ & & Depth & $(\mathrm{ft}-\mathrm{ft}):$ \\
\hline \begin{tabular}{|l|} 
Liqu \\
\end{tabular} & id Limi & & & & & & Plastic & Limit & & & \\
\hline 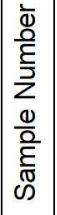 & 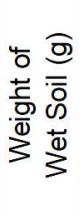 & 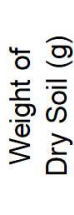 & 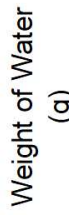 & & 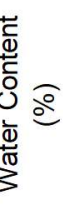 & $\begin{array}{l}\frac{0}{3} \\
\frac{0}{0} \\
\frac{0}{0} \\
\dot{0} \\
\frac{0}{2}\end{array}$ & 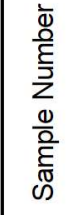 & 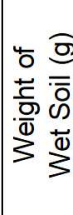 & 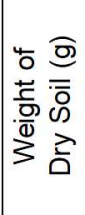 & 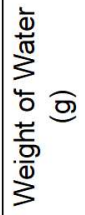 & 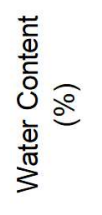 \\
\hline 1 & 18.2 & 12.3 & 5. & & 48.0 & & 1 & 8.9 & 6.6 & 2.3 & 34.8 \\
\hline 2 & 10.8 & 7.1 & 3. & & 52.1 & 53 & 2 & 9.0 & 6.7 & 2.3 & 34.3 \\
\hline 3 & 10.8 & 7.0 & 3. & & 54.3 & 25 & & & & & \\
\hline 4 & 12.9 & 8.2 & 4. & & 57.3 & 21 & & & & & \\
\hline 5 & & & 0. & & \#\#\# & & & & & & \\
\hline 6 & & & 0. & .07 & \#\# & & & & & & \\
\hline
\end{tabular}
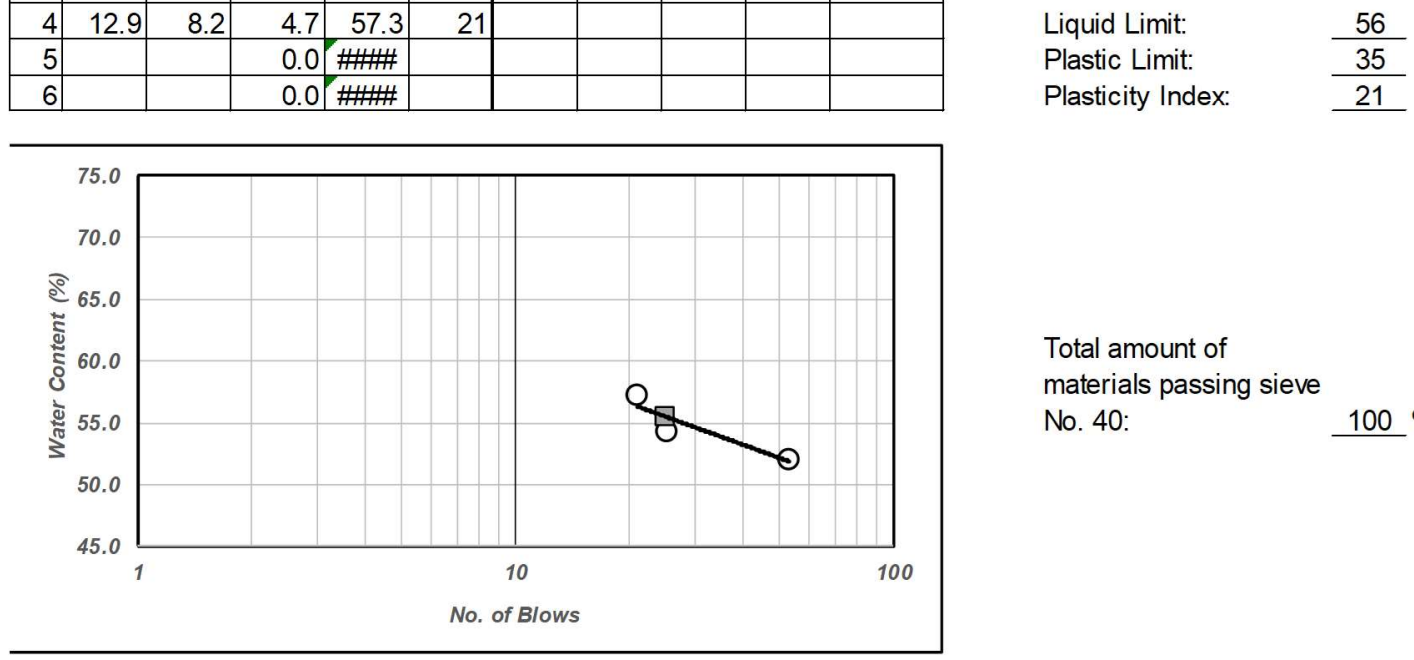

Total amount of materials passing sieve No. 40: $100 \%$

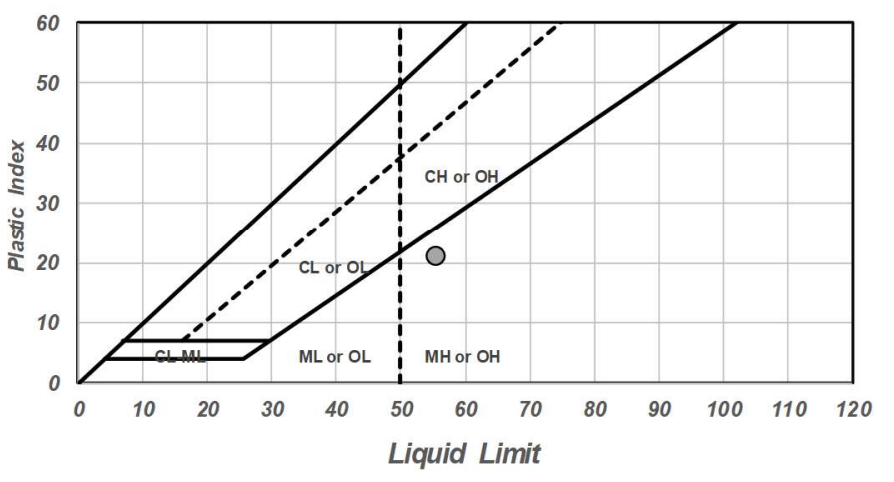

Fines

Classification: $\mathrm{MH}$

Procedure Done By: Kayla

Date Tested:

October 21, 2020 


\section{\& 8 Portland State}

\begin{tabular}{|c|c|c|c|c|c|c|c|c|c|c|}
\hline \multirow{3}{*}{\multicolumn{2}{|c|}{$\begin{array}{l}\text { Project: } \\
\text { Location: } \\
\text { Borehole: }\end{array}$}} & \multicolumn{9}{|c|}{ NSF - Microbial Induced Desaturation } \\
\hline & & \multicolumn{9}{|c|}{ Sunderland } \\
\hline & & \multicolumn{2}{|c|}{ Su_Ex_W_U2 } & \multicolumn{3}{|c|}{ Sample Number: } & \multicolumn{2}{|l|}{$\mathrm{G} 2$} & \multicolumn{2}{|c|}{ Depth (ft-ft): } \\
\hline \multicolumn{6}{|c|}{ Liquid Limit } & \multicolumn{5}{|c|}{ Plastic Limit } \\
\hline 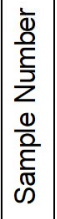 & 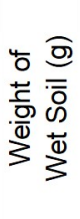 & 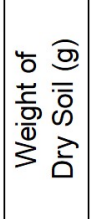 & 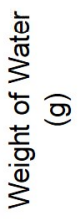 & 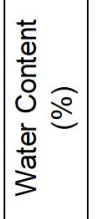 & $\begin{array}{l}\frac{0}{3} \\
\frac{0}{0} \\
40 \\
0 \\
2\end{array}$ & 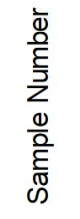 & 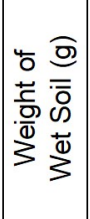 & 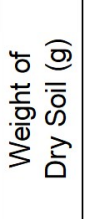 & 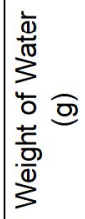 & 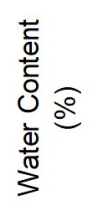 \\
\hline 1 & 15.7 & 10.7 & . & 46.7 & & 1 & 7.5 & 5.6 & 1.9 & 33.9 \\
\hline 2 & 11.4 & 7.6 & 3.8 & 50.0 & 145 & 2 & 7.6 & 5.7 & 1.9 & 33.3 \\
\hline 3 & 11.5 & 7.5 & 4 & 53.3 & 39 & & & & & \\
\hline 4 & 12.3 & 7.9 & 4.4 & 55.7 & 28 & & & & & \\
\hline 5 & 12 & 7.6 & 4.4 & 57.9 & 22 & & & & & \\
\hline 6 & & & 0.0 & \#\# & & & & & & \\
\hline
\end{tabular}
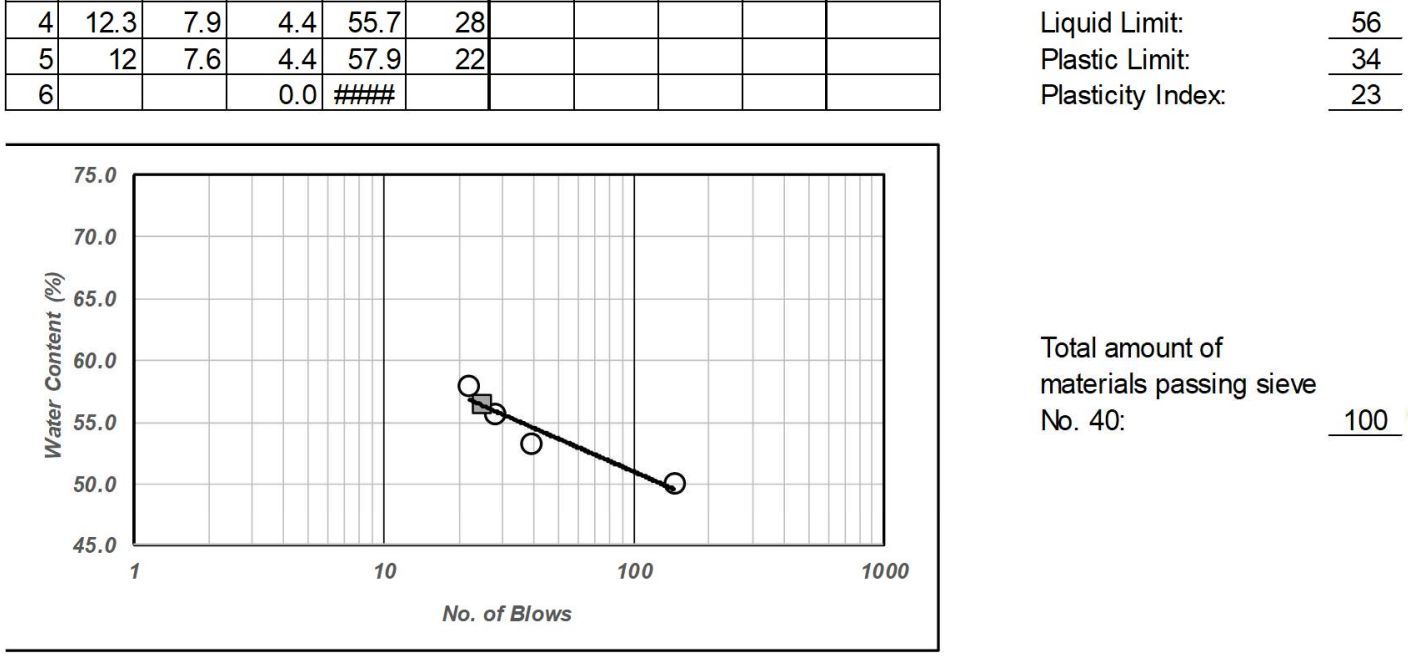

Total amount of materials passing sieve No. 40: $100 \%$

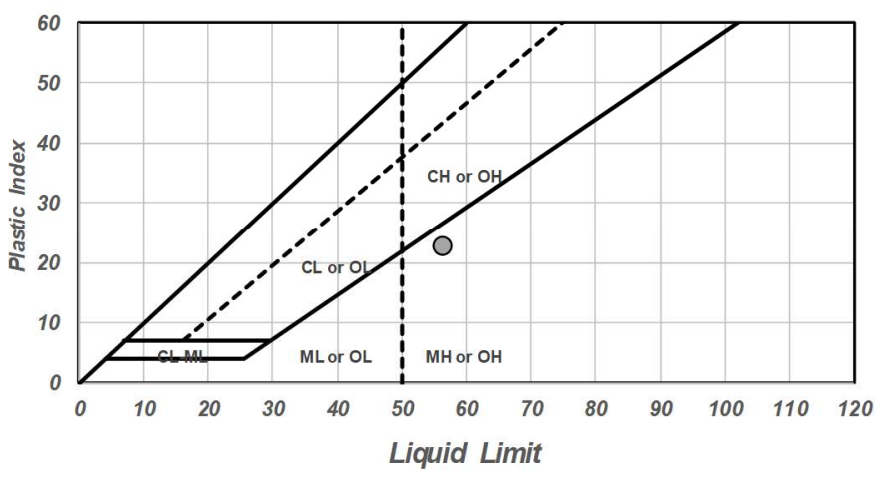

Fines

Classification: $\mathrm{MH}$

Procedure Done By: Kayla

Date Tested:

April 2, 2021 


\section{S Portland State}

\begin{tabular}{|c|c|c|c|c|c|c|c|c|c|c|c|}
\hline \multicolumn{2}{|c|}{ Project: } & \multicolumn{10}{|c|}{ NSF - Microbial Induced Desaturation } \\
\hline \multicolumn{2}{|c|}{ Location: } & \multicolumn{10}{|c|}{ Sunderland } \\
\hline \multicolumn{2}{|c|}{ Borehole: } & \multicolumn{3}{|c|}{ Su_Ex_W_U2 } & \multicolumn{3}{|c|}{ Sample Number: } & \multicolumn{2}{|l|}{ G3 } & \multicolumn{2}{|c|}{ Depth (ft-ft): } \\
\hline \multicolumn{7}{|c|}{ Liquid Limit } & \multicolumn{5}{|c|}{ Plastic Limit } \\
\hline 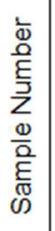 & 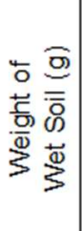 & 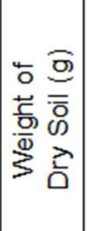 & 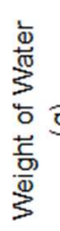 & $\widetilde{(0)}$ & 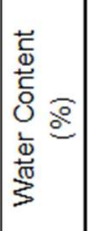 & 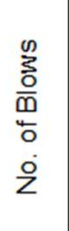 & 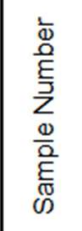 & 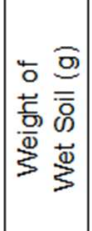 & 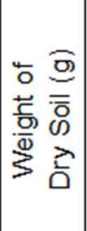 & 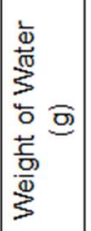 & 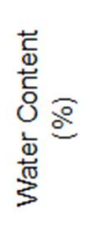 \\
\hline 1 & 12.3 & 8.3 & & 4 & 48.2 & & 1 & 6.4 & 4.8 & 1.6 & 33.3 \\
\hline 2 & 14.8 & 9.6 & & 5.2 & 54.2 & 5 & 2 & 6.6 & 4.9 & 1.7 & 34.7 \\
\hline 3 & 19.7 & 13.0 & & 6.7 & 51.5 & 11 & & & & & \\
\hline 4 & 16.8 & 11.6 & & 5.2 & 44.8 & 41 & & & & & \\
\hline 5 & & & & 0.0 & \#\#\# & & & & & & \\
\hline 6 & & & & 0.0 & \#\#\# & & & & & & \\
\hline
\end{tabular}

Date Collected: August 6, 2019

\begin{tabular}{ll|ll}
16.40 & 16.75 & Average Depth (ft): & 16.58
\end{tabular}

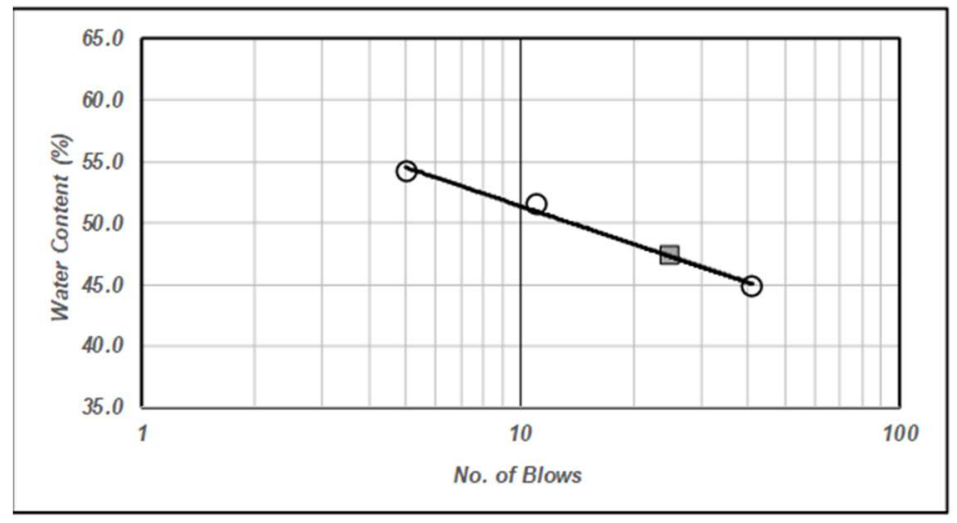

Liquid Limit:

Plastic Limit:

Plasticity Index:

$\begin{array}{r}47 \\ \hline 34 \\ \hline 13 \\ \hline\end{array}$

Total amount of materials passing sieve

No. 40 :

$100 \%$

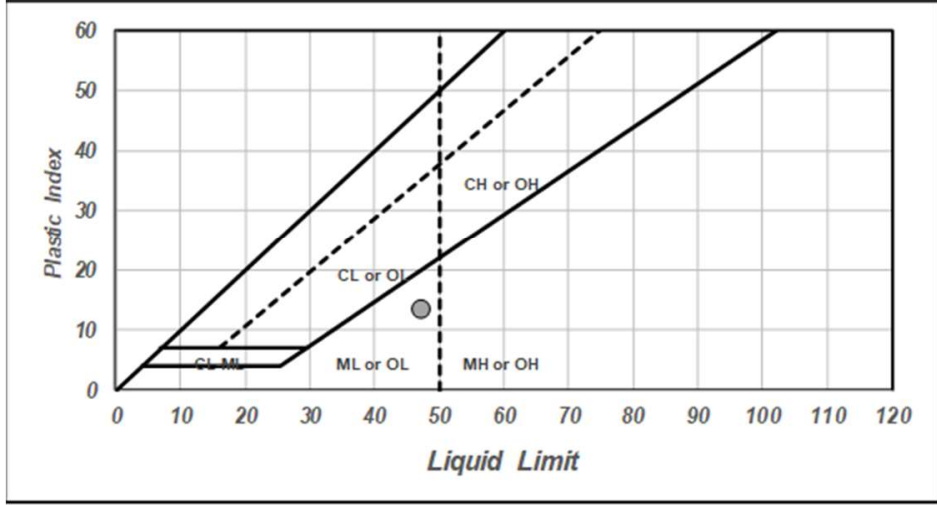

Fines

Classification: $\mathrm{ML}$ 


\section{Appendix D: Hydrometer and P200 Washes - Sunderland}

\section{蛋 Portland State}

Hydrometer Test

\begin{tabular}{|c|c|c|c|c|c|c|c|c|c|}
\hline \multirow[t]{2}{*}{ Boring: } & \multicolumn{2}{|c|}{ Su_Ha1 } & & \multirow[t]{2}{*}{ Sample: } & \multirow[t]{2}{*}{ N1 } & \multicolumn{2}{|l|}{ Depth (ft-ft): } & \multirow[t]{2}{*}{ Start Time: } & \multirow[t]{2}{*}{$10: 26: 00 \mathrm{AM}$} \\
\hline & & & & & & Ave. Depth ( $\mathrm{ft}$ & 1.58 & & \\
\hline & \multicolumn{2}{|c|}{ Constants } & & & & & & & \\
\hline & $\overline{M_{s}(g)}$ & 53.74 & & & & & & & \\
\hline & $G_{s}$ & 2.65 & & & & & & & \\
\hline & $G_{w}$ & 1 & & & & & & & \\
\hline & $\begin{array}{l}\text { Time } \\
(\min )\end{array}$ & $\begin{array}{c}\text { Soil } \\
\text { (slurry) }\end{array}$ & Control & $\begin{array}{l}\text { Temp } \\
\left({ }^{\circ} \mathrm{C}\right)\end{array}$ & $\begin{array}{c}\text { Temp } \\
\text { Correction (K) }\end{array}$ & \begin{tabular}{|c|} 
Hydrometer \\
Correction \\
$(R)$
\end{tabular} & $\begin{array}{l}\text { Effective } \\
\text { Depth, L } \\
\text { (cm) }\end{array}$ & Diameter (mm) & $\begin{array}{c}\text { Percent } \\
\text { Passing (\%) }\end{array}$ \\
\hline & 0.5 & 1.032 & 1.004 & 19.6 & 0.013718 & 1.028 & 7.8 & 0.054181759 & 84 \\
\hline & 1 & 1.031 & 1.004 & 19.6 & 0.013718 & 1.027 & 8.1 & 0.039042112 & 81 \\
\hline & 2 & 1.029 & 1.004 & 19.6 & 0.013718 & 1.025 & 8.6 & 0.02844625 & 75 \\
\hline & 4 & 1.026 & 1.004 & 19.6 & 0.013718 & 1.022 & 9.4 & 0.021029296 & 66 \\
\hline & 8 & 1.023 & 1.004 & 19.6 & 0.013718 & 1.019 & 10.2 & 0.015489803 & 57 \\
\hline & 18 & 1.020 & 1.004 & 19.5 & 0.013735 & 1.016 & 11.0 & 0.010737143 & 48 \\
\hline & 34 & 1.017 & 1.004 & 19.4 & 0.013752 & 1.013 & 11.8 & 0.008101537 & 39 \\
\hline & 68 & 1.015 & 1.004 & 19.1 & 0.013803 & 1.011 & 12.3 & 0.005870453 & 33 \\
\hline & 163 & 1.013 & 1.004 & 17.0 & 0.014170 & 1.009 & 12.9 & 0.003986308 & 27 \\
\hline & 300 & 1.012 & 1.004 & 17.0 & 0.014170 & 1.008 & 13.1 & 0.002961044 & 24 \\
\hline & 1447 & 1.010 & 1.004 & 16.0 & 0.014350 & 1.006 & 13.7 & 0.001396297 & 18 \\
\hline & 1616 & 1.010 & 1.004 & 16.0 & 0.014350 & 1.006 & 13.9 & 0.001330879 & 18 \\
\hline
\end{tabular}

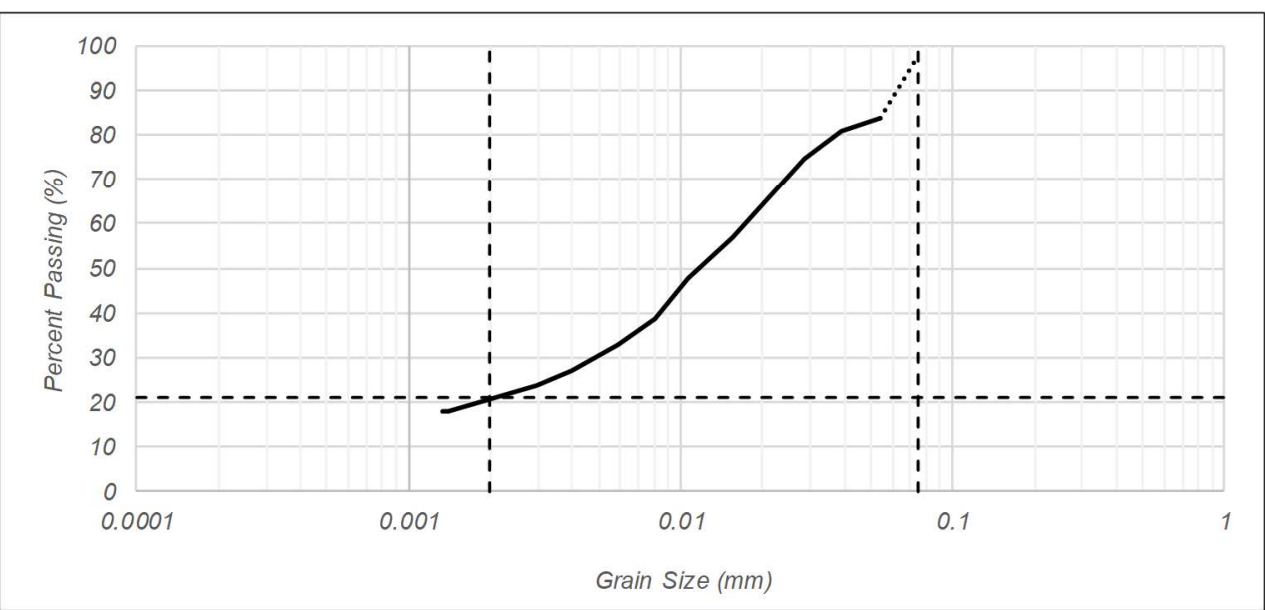

P200 Wash

Container ID: $\quad$ I1

Before - $M_{d}: \quad 29.3 \mathrm{~g}$

After - $\mathrm{M}_{\mathrm{d}}: \quad 0.8 \mathrm{~g}$

Fines Fraction: $\quad 97.27 \%$

Coarse Fraction: $\quad 2.73 \%$

Procedures Done By: Kayla/Melissa P.

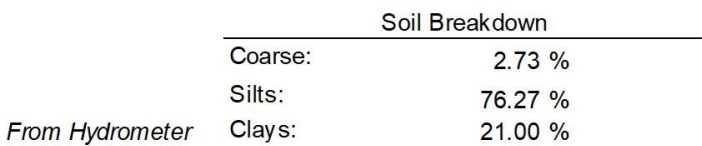

132 


\section{胥 Portland State}

Hydrometer Test

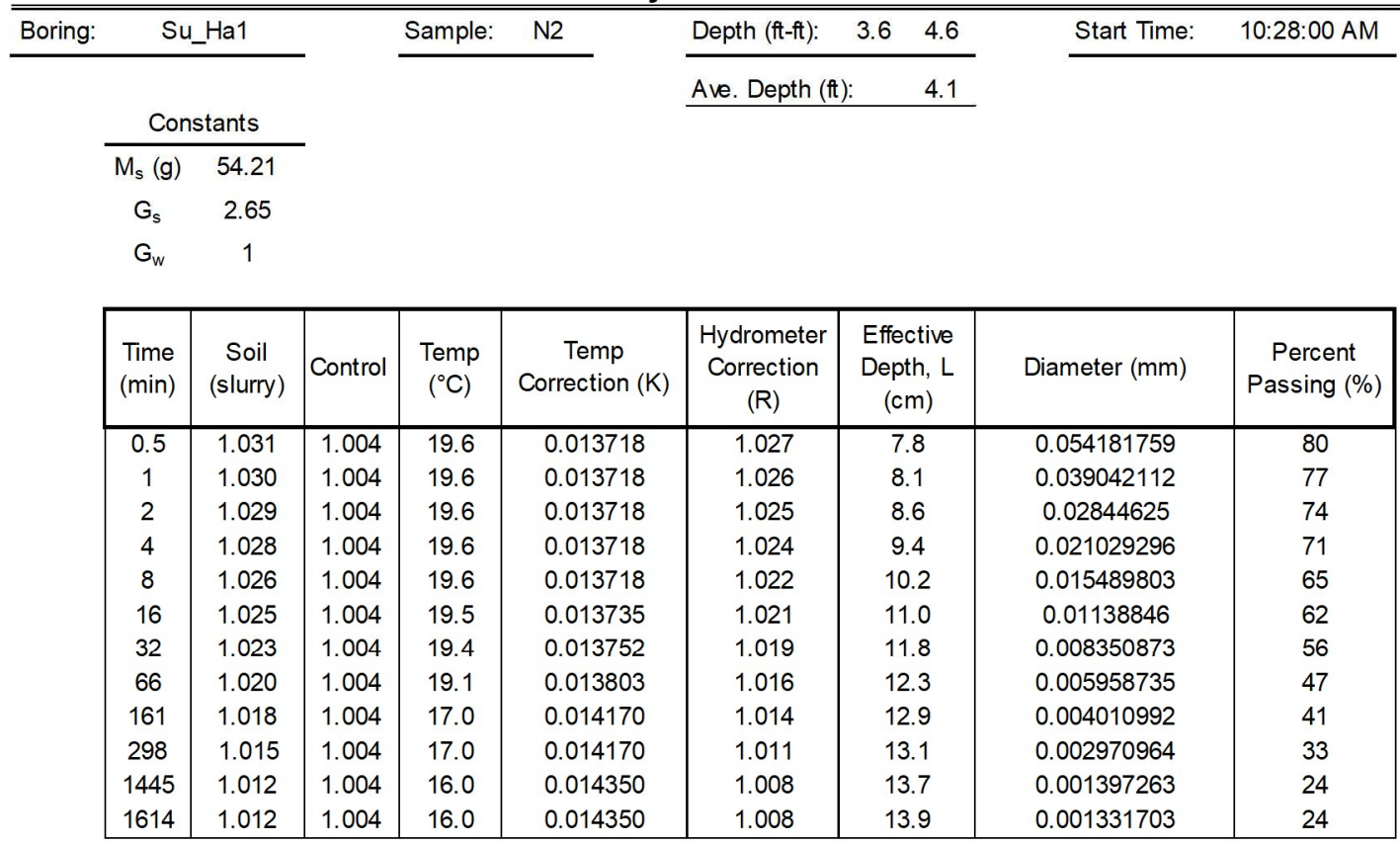

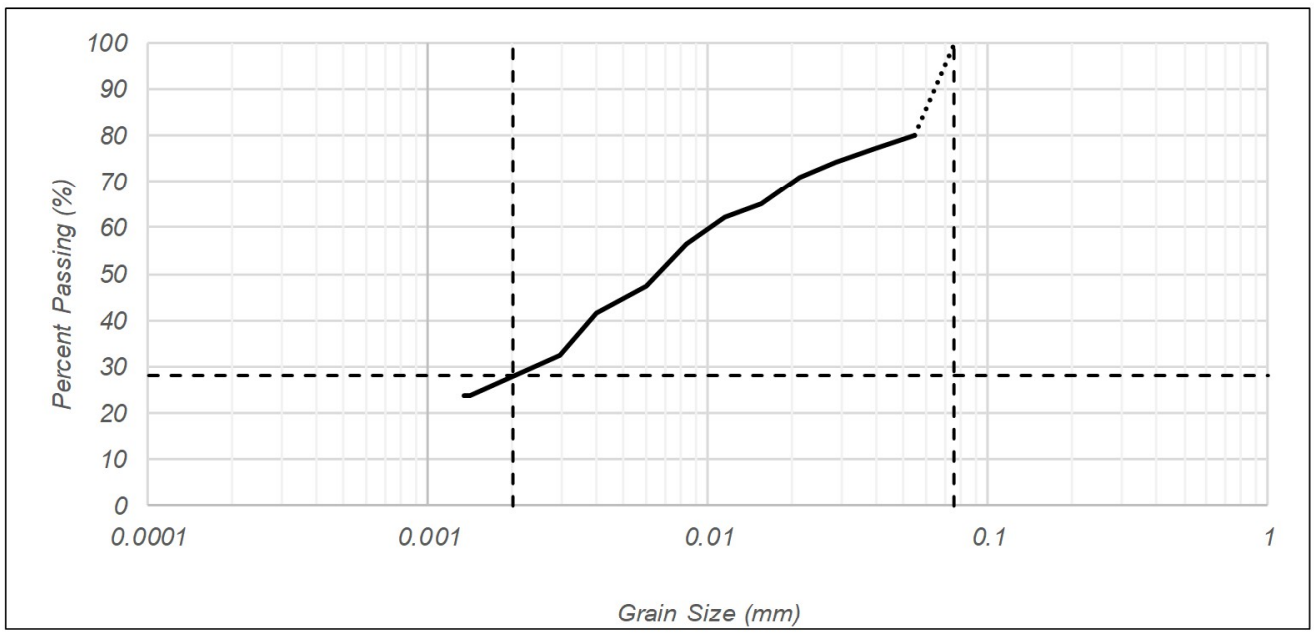

P200 Wash

Container ID: $\quad$ E1

Before - $M_{d}$ :

After - $M_{d}$ :

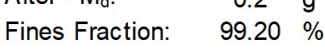

Coarse Fraction: $\quad 0.80 \quad \%$

Procedures Done By:
$24.9 \mathrm{~g}$

$0.2 \mathrm{~g}$

Kayla/Melissa P.

\begin{tabular}{clr} 
& & \multicolumn{1}{c}{ Soil Breakdown } \\
\cline { 2 - 3 } & Coarse: & $0.80 \%$ \\
& Silts: & $71.20 \%$ \\
From Hydrometer & Clays: & $28.00 \%$
\end{tabular}




\section{胥 Portland State}

Hydrometer Test

\begin{tabular}{|c|c|c|c|c|c|c|c|c|c|}
\hline \multirow[t]{2}{*}{ Boring: } & \multicolumn{2}{|c|}{ Su_Ha1 } & & \multicolumn{2}{|l|}{ Sample: } & \multirow{2}{*}{$\begin{array}{l}\text { Depth (ft-ft): } \\
\text { Ave. Depth (ft): }\end{array}$} & \multirow{2}{*}{$\begin{array}{ll}4.9 & 5.8 \\
& 5.4 \\
\end{array}$} & \multirow[t]{2}{*}{ Start Time: } & \multirow[t]{2}{*}{ 10:30:00 AM } \\
\hline & & & & & & & & & \\
\hline & \multicolumn{2}{|c|}{ Constants } & & & & & & & \\
\hline & $M_{s}(g)$ & 64.73 & & & & & & & \\
\hline & $\mathrm{G}_{\mathrm{s}}$ & 2.65 & & & & & & & \\
\hline & $\mathrm{G}_{\mathrm{w}}$ & 1 & & & & & & & \\
\hline & $\begin{array}{l}\text { Time } \\
(\mathrm{min})\end{array}$ & $\begin{array}{l}\text { Soil } \\
\text { (slurry) }\end{array}$ & Control & $\begin{array}{l}\text { Temp } \\
\left({ }^{\circ} \mathrm{C}\right)\end{array}$ & $\begin{array}{c}\text { Temp } \\
\text { Correction }(\mathrm{K})\end{array}$ & \begin{tabular}{|c|} 
Hydrometer \\
Correction \\
(R)
\end{tabular} & $\begin{array}{l}\text { Effective } \\
\text { Depth, L } \\
\quad(\mathrm{cm})\end{array}$ & Diameter $(\mathrm{mm})$ & $\begin{array}{c}\text { Percent } \\
\text { Passing (\%) }\end{array}$ \\
\hline & 0.5 & 1.037 & 1.004 & 19.5 & 0.013735 & 1.033 & 6.5 & 0.049522247 & 82 \\
\hline & 1 & 1.034 & 1.004 & 19.5 & 0.013735 & 1.030 & 7.3 & 0.037109926 & 74 \\
\hline & 2 & 1.030 & 1.004 & 19.5 & 0.013735 & 1.026 & 8.4 & 0.028148374 & 65 \\
\hline & 4 & 1.028 & 1.004 & 19.5 & 0.013735 & 1.024 & 8.9 & 0.020487722 & 60 \\
\hline & 8 & 1.024 & 1.004 & 19.5 & 0.013735 & 1.020 & 10.0 & 0.015356197 & 50 \\
\hline & 14 & 1.021 & 1.004 & 19.4 & 0.013752 & 1.017 & 10.7 & 0.012022471 & 42 \\
\hline & 30 & 1.018 & 1.004 & 19.3 & 0.013769 & 1.014 & 11.5 & 0.008524927 & 35 \\
\hline & 64 & 1.016 & 1.004 & 19.0 & 0.013820 & 1.012 & 12.1 & 0.006009118 & 30 \\
\hline & 159 & 1.014 & 1.004 & 17.0 & 0.014170 & 1.010 & 12.6 & 0.003988931 & 25 \\
\hline & 296 & 1.013 & 1.004 & 17.0 & 0.014170 & 1.009 & 12.9 & 0.002958141 & 22 \\
\hline & 1443 & 1.011 & 1.004 & 16.0 & 0.014350 & 1.007 & 13.4 & 0.001382837 & 17 \\
\hline & 1612 & 1.011 & 1.004 & 16.0 & 0.014350 & 1.007 & 13.4 & 0.001308344 & 17 \\
\hline
\end{tabular}

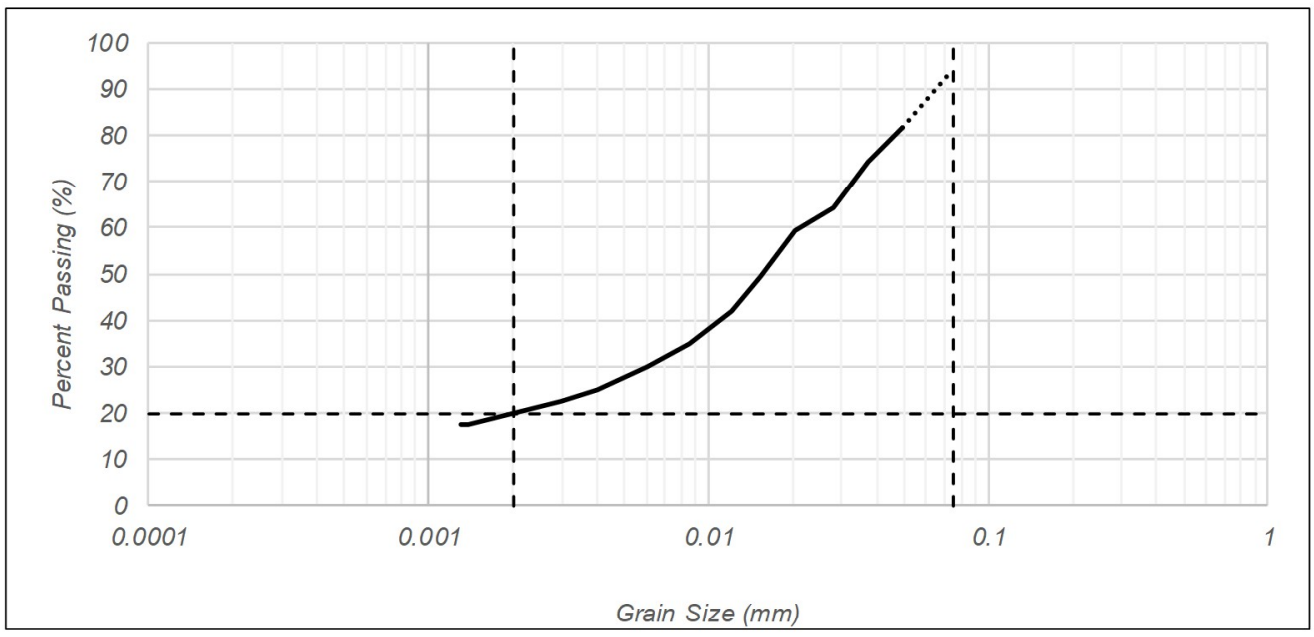

P200 Wash

Container ID: $\quad \mathrm{K} 1$

Before - $M_{d}$ :

After - $M_{\mathrm{d}}$ :

Fines Fraction: $\quad 94.18 \%$

Coarse Fraction: $5.82 \%$

Procedures Done By:
$29.2 \mathrm{~g}$

$1.7 \mathrm{~g}$

Kayla/Melissa P.

\begin{tabular}{llr} 
& & \multicolumn{1}{c}{ Soil Breakdown } \\
\cline { 2 - 3 } & Coarse: & $5.82 \%$ \\
& Silts: & $74.18 \%$ \\
From Hydrometer & Clays: & $20.00 \%$
\end{tabular}




\section{龟 Portland State}

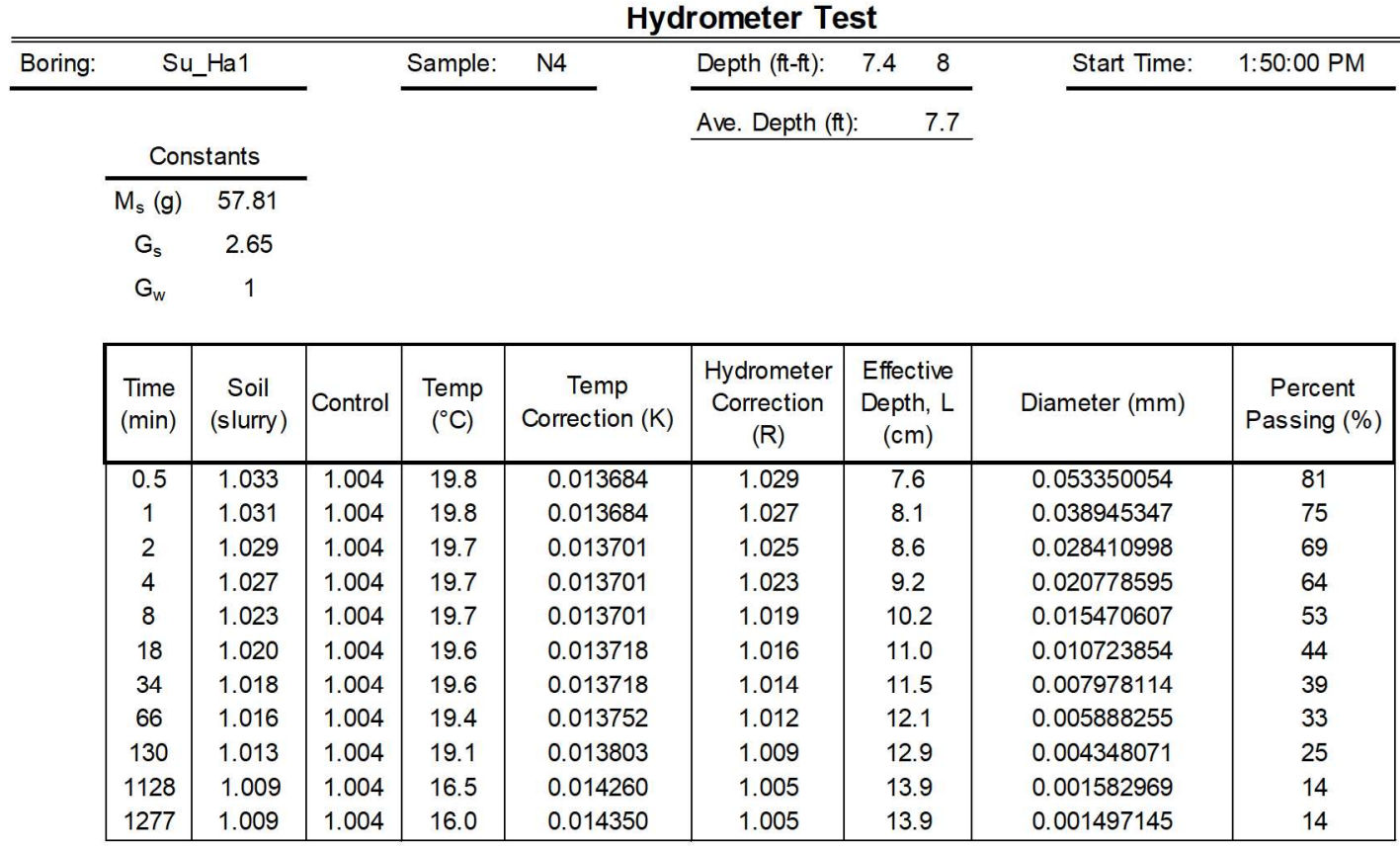

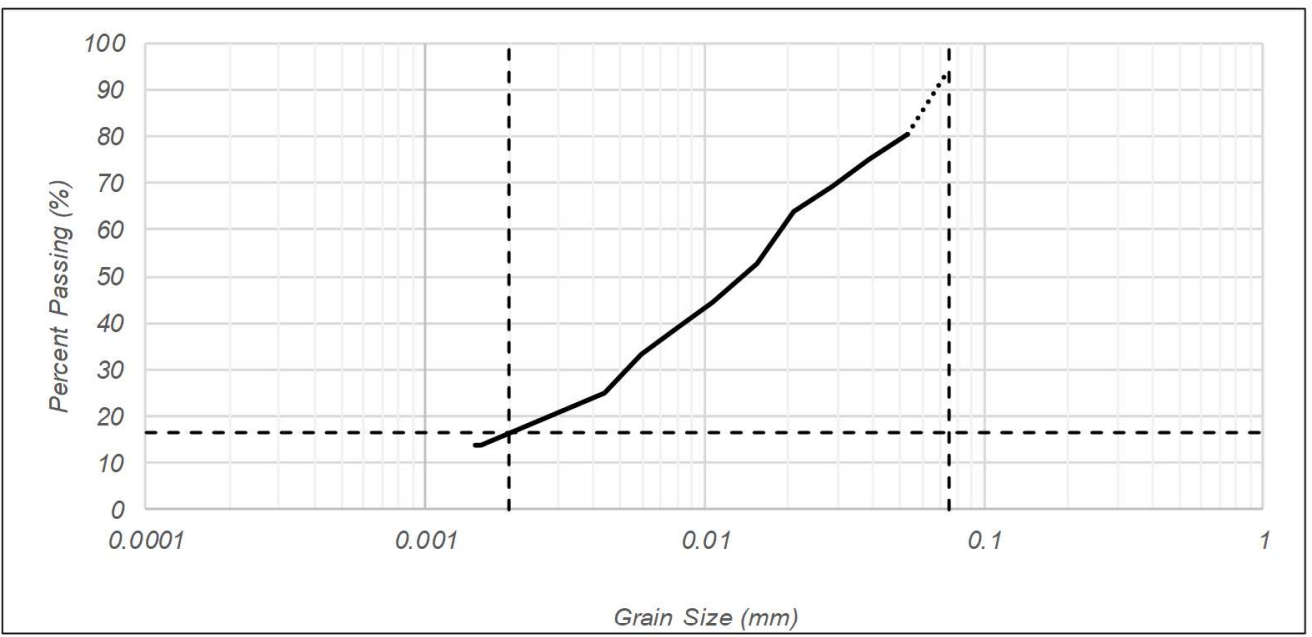

P200 W ash

$\begin{array}{lcc}\text { Container ID: } & \text { K1 } & \\ \text { Before - M }: & 29.2 & \mathrm{~g} \\ \text { After - M } & 1.7 & \mathrm{~g} \\ \text { Fines Fraction: } & 94.18 & \% \\ \text { Coarse Fraction: } & 5.82 & \%\end{array}$

\begin{tabular}{llr} 
& & Soil Breakdown \\
\cline { 2 - 3 } & Coarse: & $5.82 \%$ \\
& Silts: & $77.68 \%$ \\
From Hydrometer & Clays: & $16.50 \%$
\end{tabular}

Procedures Done By: Kayla/Melissa P. 


\section{胥 Portland State}

Hydrometer Test

\begin{tabular}{|c|c|c|c|c|c|c|c|c|c|}
\hline \multirow[t]{2}{*}{ Boring: } & \multicolumn{2}{|c|}{$\overline{\mathrm{S} \text { Su_Ha1 }}$} & & \multirow[t]{2}{*}{ Sample: } & \multirow[t]{2}{*}{ N5 } & Depth (ft-ft): & 9.3 & \multirow[t]{2}{*}{ Start Time: } & 1:52:00 PM \\
\hline & & & & & & Ave. Depth (tt) & 9.45 & & \\
\hline \multicolumn{10}{|c|}{ Constants } \\
\hline & $M_{s}(g)$ & 57.38 & & & & & & & \\
\hline & $\mathrm{G}_{\mathrm{s}}$ & 2.65 & & & & & & & \\
\hline & $\mathrm{G}_{\mathrm{w}}$ & 1 & & & & & & & \\
\hline & $\begin{array}{l}\text { Time } \\
(\mathrm{min})\end{array}$ & $\begin{array}{c}\text { Soil } \\
\text { (slurry) }\end{array}$ & Control & $\begin{array}{l}\text { Temp } \\
\left({ }^{\circ} \mathrm{C}\right)\end{array}$ & $\begin{array}{c}\text { Temp } \\
\text { Correction (K) }\end{array}$ & $\begin{array}{c}\text { Hydrometer } \\
\text { Correction } \\
(\mathrm{R})\end{array}$ & $\begin{array}{l}\text { Effective } \\
\text { Depth, L } \\
\text { (cm) }\end{array}$ & Diameter $(\mathrm{mm})$ & $\begin{array}{c}\text { Percent } \\
\text { Passing (\%) }\end{array}$ \\
\hline & 0.5 & 1.035 & 1.004 & 19.9 & 0.013667 & 1.031 & 7.0 & 0.051137232 & 87 \\
\hline & 1 & 1.033 & 1.004 & 19.9 & 0.013667 & 1.029 & 7.6 & 0.037677319 & 81 \\
\hline & 2 & 1.032 & 1.004 & 19.9 & 0.013667 & 1.028 & 7.8 & 0.026990162 & 78 \\
\hline & 4 & 1.030 & 1.004 & 19.9 & 0.013667 & 1.026 & 8.4 & 0.019805365 & 73 \\
\hline & 8 & 1.027 & 1.004 & 19.9 & 0.013667 & 1.023 & 9.2 & 0.014656225 & 64 \\
\hline & 16 & 1.024 & 1.004 & 19.7 & 0.013701 & 1.020 & 10.0 & 0.010831592 & 56 \\
\hline & 32 & 1.022 & 1.004 & 19.6 & 0.013718 & 1.018 & 10.5 & 0.007857972 & 50 \\
\hline & 64 & 1.019 & 1.004 & 19.4 & 0.013752 & 1.015 & 11.3 & 0.0057785 & 42 \\
\hline & 128 & 1.016 & 1.004 & 19.2 & 0.013786 & 1.012 & 12.1 & 0.004238635 & 34 \\
\hline & 1126 & 1.011 & 1.004 & 17.0 & 0.014170 & 1.007 & 13.4 & 0.001545799 & 20 \\
\hline & 1275 & 1.011 & 1.004 & 16.0 & 0.014350 & 1.007 & 13.4 & 0.001471124 & 20 \\
\hline
\end{tabular}

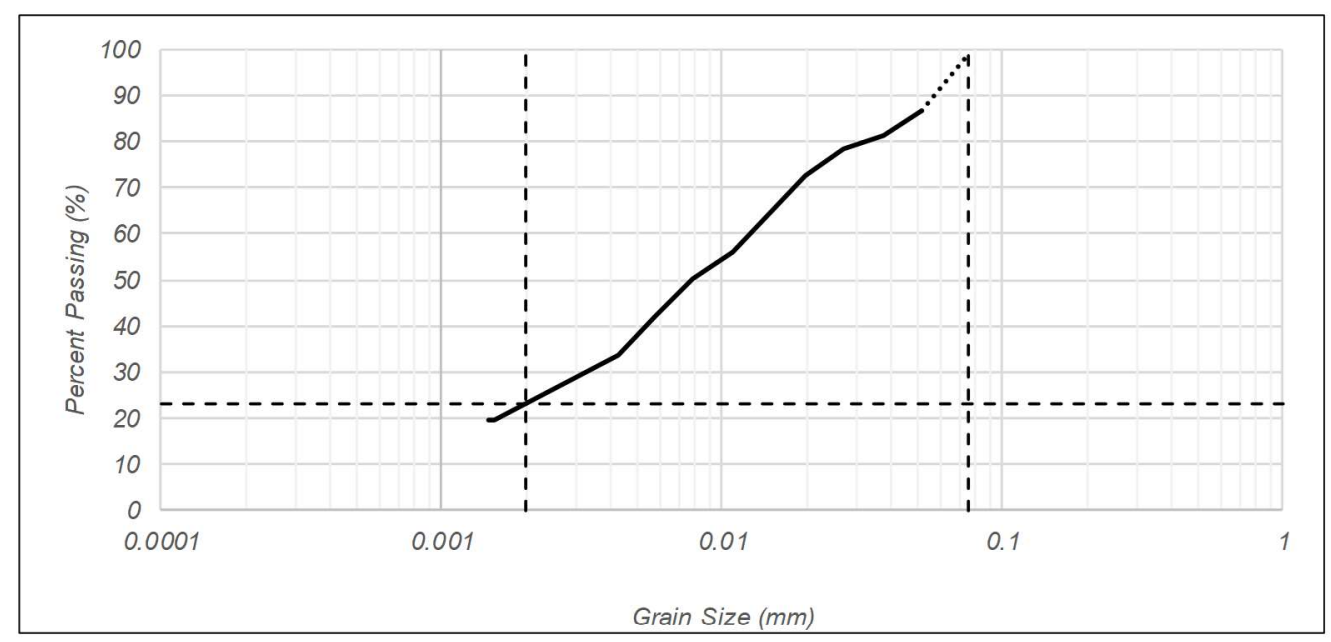

P200 Wash

Container ID:

Before $-M_{d}$ :

After - $M_{d}$ :

Fines Fraction: $\quad 98.89 \%$

Coarse Fraction: $1.11 \%$

\begin{tabular}{llr} 
& \multicolumn{2}{c}{ Soil Breakdown } \\
\cline { 2 - 3 } From Hydrometer & $1.11 \%$ \\
& Silts: & $75.89 \%$ \\
& Clays: & $23.00 \%$
\end{tabular}

$\underline{\text { Procedures Done By: Kayla/Melissa P. }}$ 


\section{S Portland State}

Hydrometer Test

\begin{tabular}{|c|c|c|c|c|c|c|c|c|c|}
\hline \multirow[t]{2}{*}{ Boring: } & \multicolumn{2}{|c|}{ Su_Ha1 } & & \multirow[t]{2}{*}{ Sample: } & N6 & Depth (ft-ft): & 12.8 & \multirow[t]{2}{*}{ Start Time: } & \multirow[t]{2}{*}{ 1:54:00 PM } \\
\hline & & & & & & Ave. Depth (ft) & 13.05 & & \\
\hline & \multicolumn{2}{|c|}{ Constants } & & & & & & & \\
\hline & $M_{s}(g)$ & 54.45 & & & & & & & \\
\hline & $\mathrm{G}_{\mathrm{s}}$ & 2.65 & & & & & & & \\
\hline & $\mathrm{G}_{\mathrm{w}}$ & 1 & & & & & & & \\
\hline & $\begin{array}{l}\text { Time } \\
(\mathrm{min})\end{array}$ & $\begin{array}{c}\text { Soil } \\
\text { (slurry) }\end{array}$ & Control & $\begin{array}{l}\text { Temp } \\
\left({ }^{\circ} \mathrm{C}\right)\end{array}$ & $\begin{array}{c}\text { Temp } \\
\text { Correction (K) }\end{array}$ & $\begin{array}{l}\text { Hydrometer } \\
\text { Correction } \\
\text { (R) }\end{array}$ & $\begin{array}{c}\text { Effective } \\
\text { Depth, L }(\mathrm{cm})\end{array}$ & Diameter $(\mathrm{mm})$ & $\begin{array}{c}\text { Percent } \\
\text { Passing (\%) }\end{array}$ \\
\hline & 0.5 & 1.032 & 1.004 & 20.2 & 0.013616 & 1.028 & 7.8 & 0.053778891 & 83 \\
\hline & 1 & 1.031 & 1.004 & 20.2 & 0.013616 & 1.027 & 8.1 & 0.038751815 & 80 \\
\hline & 2 & 1.026 & 1.004 & 20.2 & 0.013616 & 1.022 & 9.4 & 0.029518785 & 65 \\
\hline & 4 & 1.024 & 1.004 & 20.2 & 0.013616 & 1.020 & 10.0 & 0.021528786 & 59 \\
\hline & 8 & 1.020 & 1.004 & 20.1 & 0.013633 & 1.016 & 11.0 & 0.01598611 & 47 \\
\hline & 14 & 1.017 & 1.004 & 20.0 & 0.013650 & 1.013 & 11.8 & 0.01253169 & 38 \\
\hline & 30 & 1.015 & 1.004 & 19.9 & 0.013667 & 1.011 & 12.3 & 0.00875115 & 32 \\
\hline & 62 & 1.013 & 1.004 & 19.6 & 0.013718 & 1.009 & 12.9 & 0.006257341 & 27 \\
\hline & 126 & 1.011 & 1.004 & 19.2 & 0.013786 & 1.007 & 13.4 & 0.004495784 & 21 \\
\hline & 1124 & 1.008 & 1.004 & 16.0 & 0.014350 & 1.004 & 14.2 & 0.00161292 & 12 \\
\hline & 1273 & 1.008 & 1.004 & 16.0 & 0.014350 & 1.004 & 14.2 & 0.00151559 & 12 \\
\hline
\end{tabular}

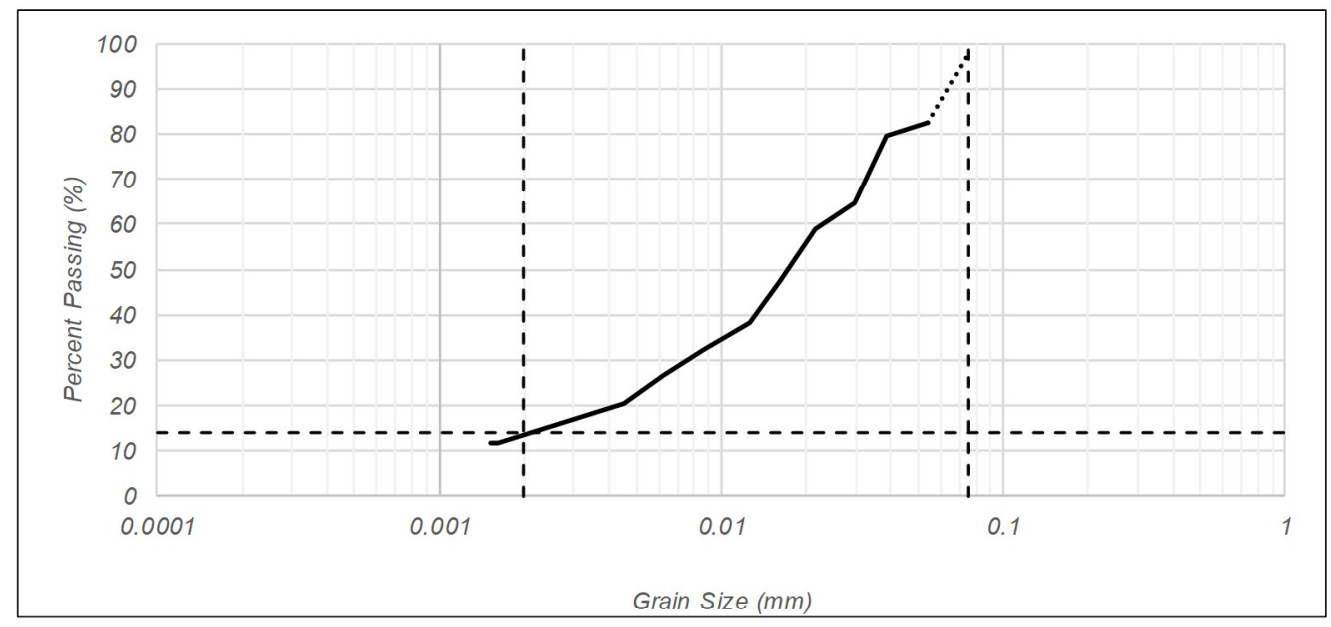

P200 Wash

$\begin{array}{lcc}\text { Container ID: } & \text { F1 } & \\ \text { Before }-\mathrm{M}_{\mathrm{d}}: & 27.5 & \mathrm{~g} \\ \text { After }-\mathrm{M}_{\mathrm{d}} \text { : } & 0.7 & \mathrm{~g} \\ \text { Fines Fraction: } & 97.45 \mathrm{\%} \\ \text { Coarse Fraction: } & 2.55 \%\end{array}$

\begin{tabular}{llr} 
& & \multicolumn{2}{c}{ Soil Breakdown } \\
\cline { 2 - 3 } & Coarse: & $2.55 \%$ \\
& Silts: & $83.45 \%$ \\
From Hydrometer & Clays: & $14.00 \%$
\end{tabular}

Procedures Done By:

Kayla/Melissa P. 


\section{胥 Portland State}

Hydrometer Test

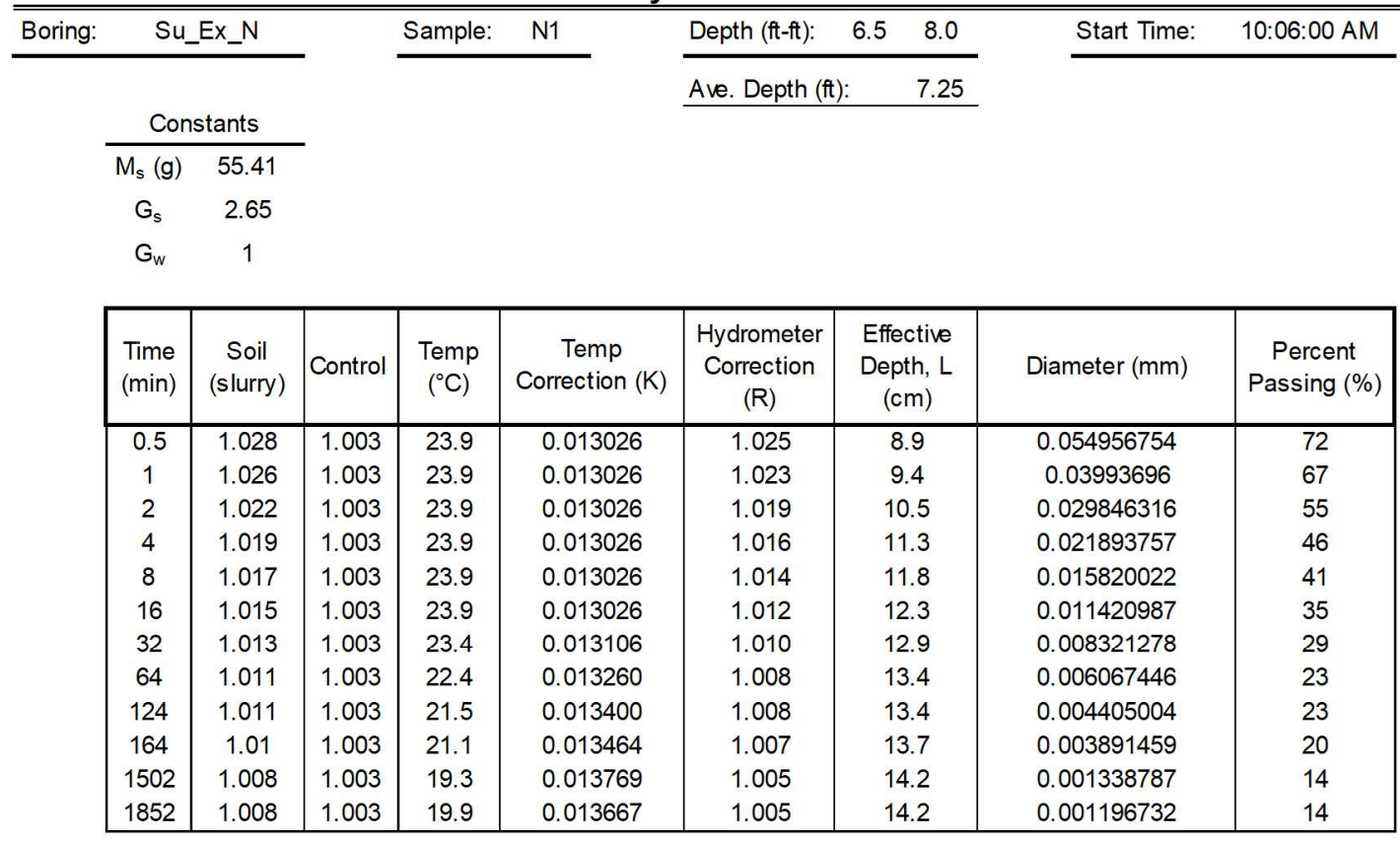

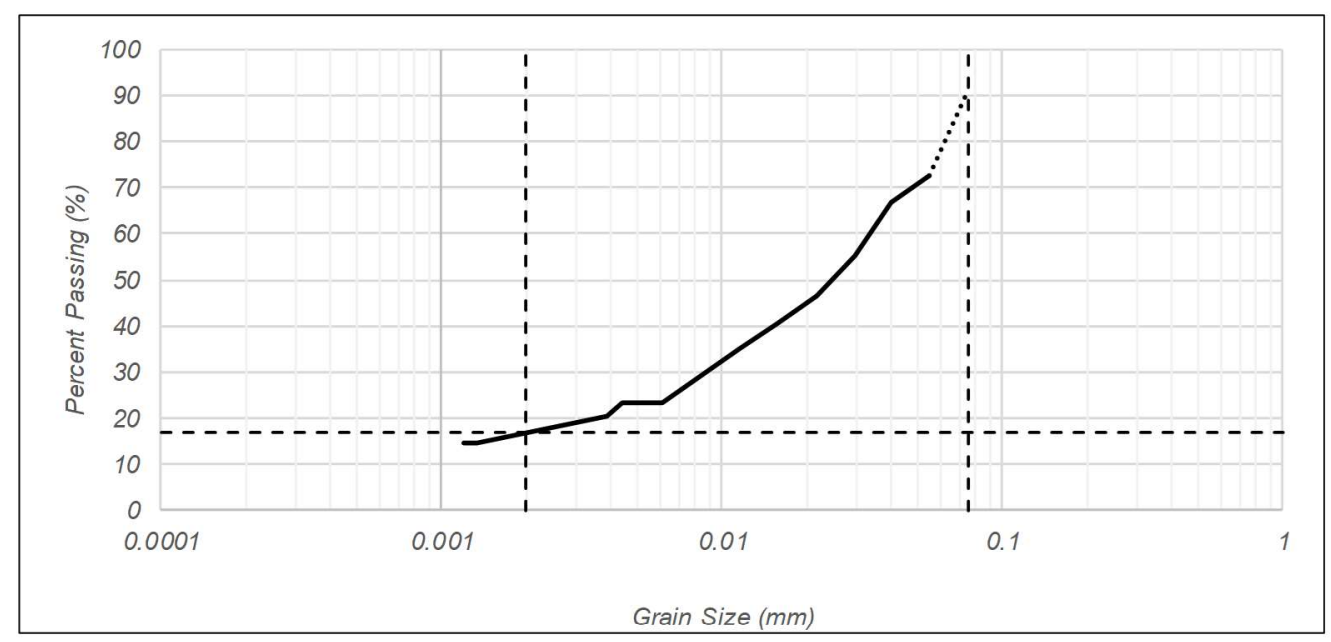

P200 Wash

$\begin{array}{lcc}\text { Container ID: } & \text { S1 } & \\ \text { Before }-\mathrm{M}_{\mathrm{d}}: & 35.5 & \mathrm{~g} \\ \text { After }-\mathrm{M}_{\mathrm{d}} \text { : } & 3.3 & \mathrm{~g} \\ \text { Fines Fraction: } & 90.70 \% \\ \text { Coarse Fraction: } & 9.30 & \%\end{array}$

\begin{tabular}{llr} 
& & \multicolumn{2}{c}{ Soil Breakdown } \\
\cline { 2 - 3 } & Coarse: & $9.30 \%$ \\
& Silts: & $73.70 \%$ \\
From Hydrometer & Clays: & $17.00 \%$
\end{tabular}

Procedures Done By: Kayla/Melissa P. 


\section{S Portland State}

Hydrometer Test

\begin{tabular}{|c|c|c|c|c|c|c|c|c|c|}
\hline \multirow[t]{2}{*}{ Boring: } & \multicolumn{2}{|c|}{ Su_Ex_N } & & \multirow[t]{2}{*}{ Sample: } & \multirow[t]{2}{*}{ N2 } & Depth (ft-ft): & 16.5 & \multirow[t]{2}{*}{ Start Time: } & \multirow[t]{2}{*}{ 10:09:00 AM } \\
\hline & & & & & & Ave. Depth (ft) & 17.25 & & \\
\hline & \multicolumn{2}{|c|}{ Constants } & & & & & & & \\
\hline & $M_{s}(g)$ & 52.16 & & & & & & & \\
\hline & $\mathrm{G}_{\mathrm{s}}$ & 2.65 & & & & & & & \\
\hline & $\mathrm{G}_{\mathrm{w}}$ & 1 & & & & & & & \\
\hline & $\begin{array}{l}\text { Time } \\
(\min )\end{array}$ & $\begin{array}{c}\text { Soil } \\
\text { (slurry) }\end{array}$ & Control & $\begin{array}{l}\text { Temp } \\
\left({ }^{\circ} \mathrm{C}\right)\end{array}$ & $\begin{array}{c}\text { Temp } \\
\text { Correction (K) }\end{array}$ & $\begin{array}{l}\text { Hydrometer } \\
\text { Correction } \\
\text { (R) }\end{array}$ & $\begin{array}{c}\text { Effective } \\
\text { Depth, L }(\mathrm{cm})\end{array}$ & Diameter (mm) & $\begin{array}{c}\text { Percent } \\
\text { Passing (\%) }\end{array}$ \\
\hline & 0.5 & 1.029 & 1.003 & 23.0 & 0.013170 & 1.026 & 8.6 & 0.054619787 & 80 \\
\hline & 1 & 1.027 & 1.003 & 23.0 & 0.013170 & 1.024 & 9.2 & 0.039946588 & 74 \\
\hline & 2 & 1.025 & 1.003 & 23.0 & 0.013170 & 1.022 & 9.7 & 0.029003916 & 68 \\
\hline & 4 & 1.023 & 1.003 & 23.0 & 0.013170 & 1.020 & 10.2 & 0.021030803 & 62 \\
\hline & 8 & 1.022 & 1.003 & 23.0 & 0.013170 & 1.019 & 10.5 & 0.01508813 & 59 \\
\hline & 16 & 1.019 & 1.003 & 23.0 & 0.013170 & 1.016 & 11.3 & 0.011067894 & 49 \\
\hline & 32 & 1.016 & 1.003 & 22.6 & 0.013230 & 1.013 & 12.1 & 0.008135374 & 40 \\
\hline & 64 & 1.014 & 1.003 & 21.9 & 0.013336 & 1.011 & 12.6 & 0.005917263 & 34 \\
\hline & 119 & 1.012 & 1.003 & 21.2 & 0.013448 & 1.009 & 13.1 & 0.0044619 & 28 \\
\hline & 164 & 1.012 & 1.003 & 21.0 & 0.013480 & 1.009 & 13.1 & 0.003809813 & 28 \\
\hline & 1502 & 1.008 & 1.003 & 19.3 & 0.013769 & 1.005 & 14.2 & 0.001338787 & 15 \\
\hline & 1852 & 1.008 & 1.003 & 19.3 & 0.013769 & 1.005 & 14.2 & 0.001205663 & 15 \\
\hline
\end{tabular}

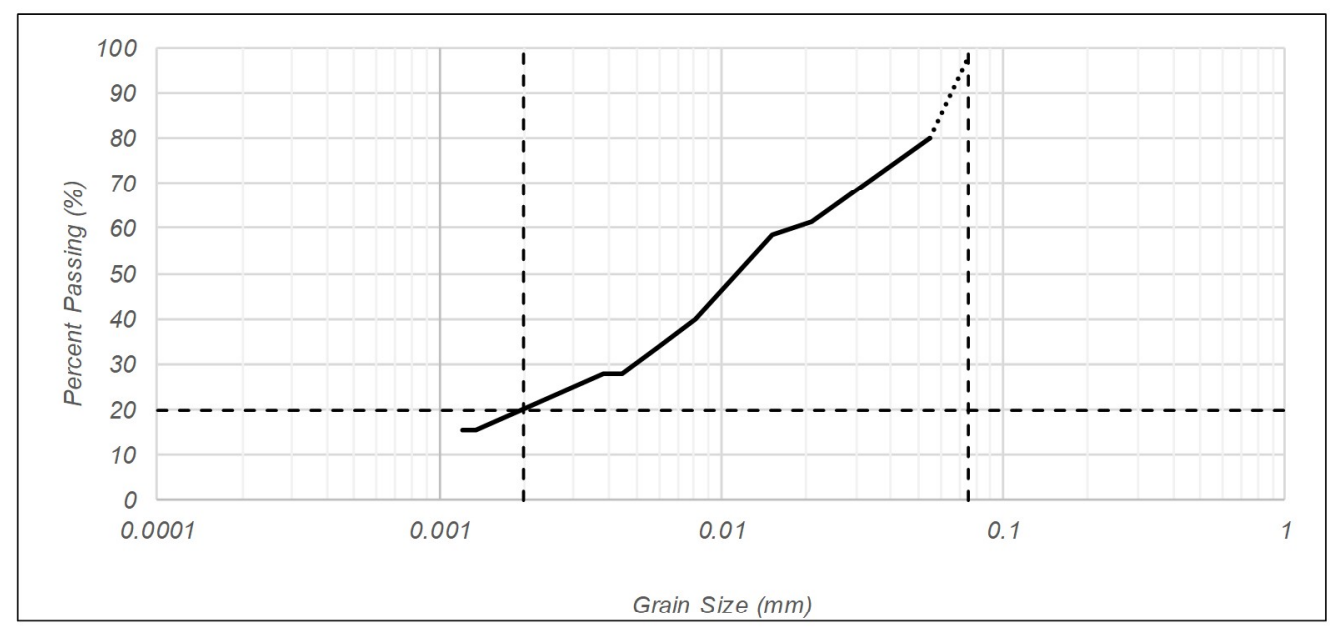

P200 Wash

$\begin{array}{lcc}\text { Container ID: } & \text { T1 } & \\ \text { Before - } \mathrm{M}_{\mathrm{d}} \text { : } & 29.2 & \mathrm{~g} \\ \text { After }-\mathrm{M}_{\mathrm{d}} \text { : } & 0.7 & \mathrm{~g} \\ \text { Fines Fraction: } & 97.60 & \% \\ \text { Coarse Fraction: } & 2.40 & \%\end{array}$

\begin{tabular}{llr} 
& & \multicolumn{1}{c}{ Soil Breakdown } \\
\cline { 2 - 3 } & Coarse: & $2.40 \%$ \\
& Silts: & $77.60 \%$ \\
From Hydrometer & Clays: & $20.00 \%$
\end{tabular}

Procedures Done By:

Kayla/Melissa P. 


\section{胥 Portland State}

Hydrometer Test

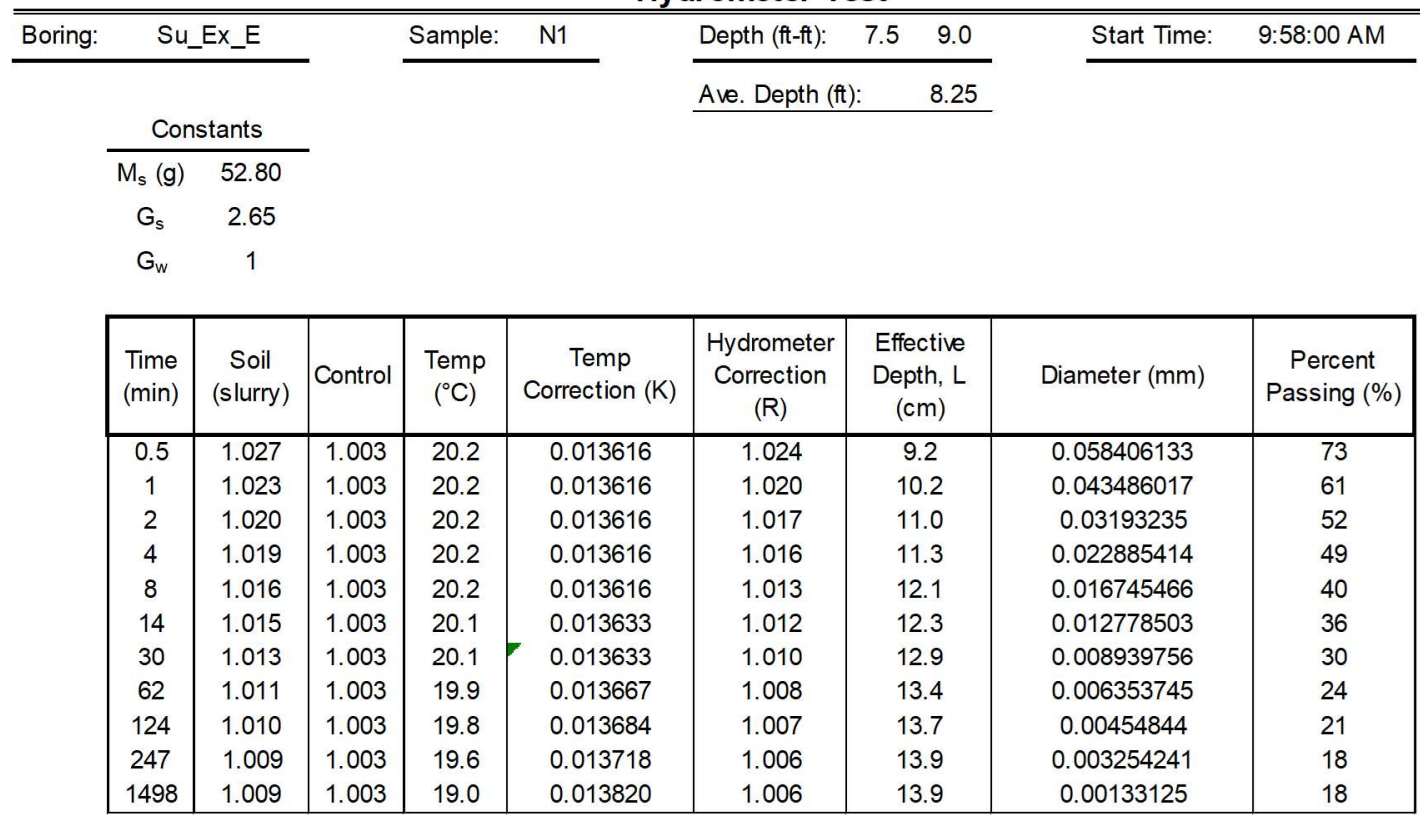

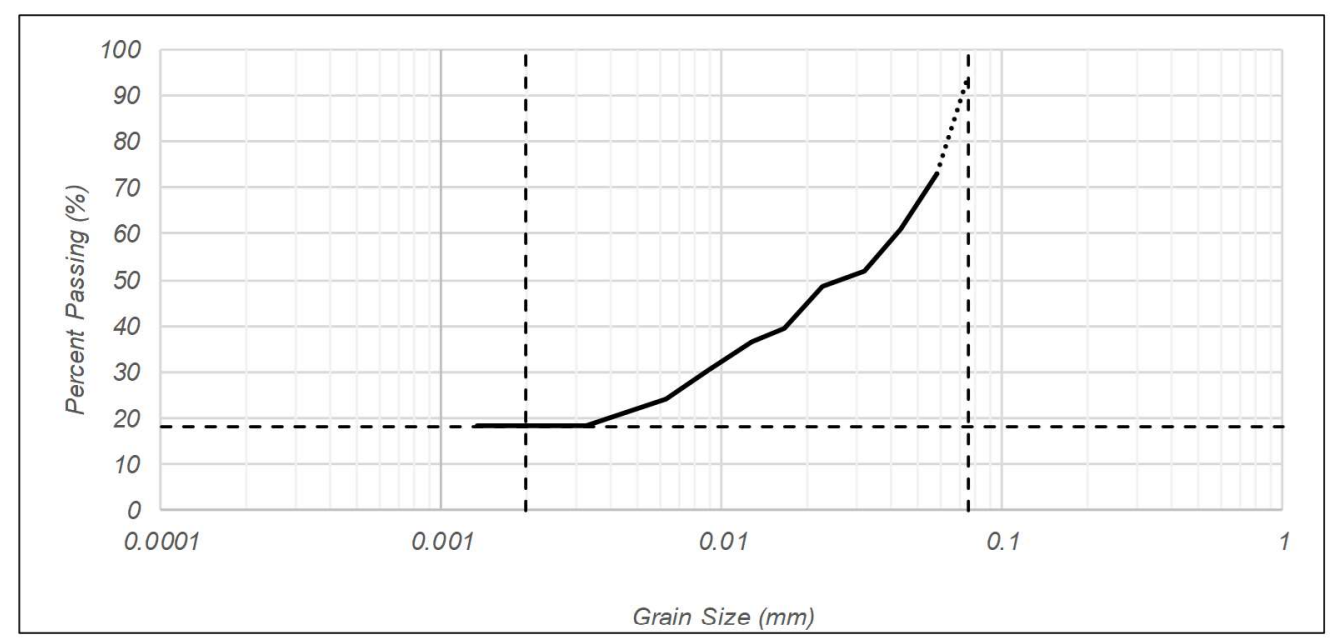

P200 Wash

$\begin{array}{lcc}\text { Container ID: } & \text { D1 } & \\ \text { Before }-\mathrm{M}_{\mathrm{d}}: & 36.1 & \mathrm{~g} \\ \text { After }-\mathrm{M}_{\mathrm{d}}: & 2.3 & \mathrm{~g} \\ \text { Fines Fraction: } & 93.63 \% \\ \text { Coarse Fraction: } & 6.37 \%\end{array}$

\begin{tabular}{llr} 
& & Soil Breakdown \\
\cline { 2 - 3 } & Coarse: & $6.37 \%$ \\
& Silts: & $75.63 \%$ \\
From Hydrometer & Clays: & $18.00 \%$
\end{tabular}

$\underline{\text { Procedures Done By: Kayla/Melissa P. }}$ 


\section{S Portland State}

Hydrometer Test

\begin{tabular}{|c|c|c|c|c|c|c|c|c|c|}
\hline \multirow[t]{2}{*}{ Boring: } & \multicolumn{2}{|c|}{ Su_Ex_E } & & \multirow[t]{2}{*}{ Sample: } & N2 & Depth (ft-ft): & 12.5 & \multirow[t]{2}{*}{ Start Time: } & \multirow[t]{2}{*}{ 10:03:00 AM } \\
\hline & & & & & & Ave. Depth (ft) & 13.25 & & \\
\hline & \multicolumn{2}{|c|}{ Constants } & & & & & & & \\
\hline & $M_{s}(g)$ & 52.80 & & & & & & & \\
\hline & $\mathrm{G}_{\mathrm{s}}$ & 2.65 & & & & & & & \\
\hline & $\mathrm{G}_{\mathrm{w}}$ & 1 & & & & & & & \\
\hline & $\begin{array}{l}\text { Time } \\
(\min )\end{array}$ & $\begin{array}{c}\text { Soil } \\
\text { (slurry) }\end{array}$ & Control & $\begin{array}{l}\text { Temp } \\
\left({ }^{\circ} \mathrm{C}\right)\end{array}$ & $\begin{array}{c}\text { Temp } \\
\text { Correction (K) }\end{array}$ & $\begin{array}{l}\text { Hydrometer } \\
\text { Correction } \\
\text { (R) }\end{array}$ & $\begin{array}{c}\text { Effective } \\
\text { Depth, L }(\mathrm{cm})\end{array}$ & Diameter $(\mathrm{mm})$ & $\begin{array}{c}\text { Percent } \\
\text { Passing (\%) }\end{array}$ \\
\hline & 0.5 & 1.030 & 1.003 & 19.8 & 0.013684 & 1.027 & 8.4 & 0.05608771 & 82 \\
\hline & 1 & 1.029 & 1.003 & 19.8 & 0.013684 & 1.026 & 8.6 & 0.040129365 & 79 \\
\hline & 2 & 1.028 & 1.003 & 19.8 & 0.013684 & 1.025 & 8.9 & 0.02886643 & 76 \\
\hline & 4 & 1.025 & 1.003 & 19.8 & 0.013684 & 1.022 & 9.7 & 0.021309288 & 67 \\
\hline & 8 & 1.023 & 1.003 & 19.8 & 0.013684 & 1.020 & 10.2 & 0.015451411 & 61 \\
\hline & 16 & 1.020 & 1.003 & 19.7 & 0.013701 & 1.017 & 11.0 & 0.011360269 & 52 \\
\hline & 34 & 1.018 & 1.003 & 19.7 & 0.013701 & 1.015 & 11.5 & 0.007968228 & 46 \\
\hline & 70 & 1.015 & 1.003 & 19.5 & 0.013735 & 1.012 & 12.3 & 0.005757477 & 36 \\
\hline & 130 & 1.012 & 1.003 & 19.5 & 0.013735 & 1.009 & 13.1 & 0.004360062 & 27 \\
\hline & 247 & 1.011 & 1.003 & 19.5 & 0.013735 & 1.008 & 13.4 & 0.003199135 & 24 \\
\hline & 347 & 1.010 & 1.003 & 19.4 & 0.013752 & 1.007 & 13.7 & 0.002732508 & 21 \\
\hline & 5787 & 1.007 & 1.003 & 16.5 & 0.014260 & 1.004 & 14.4 & 0.000711335 & 12 \\
\hline
\end{tabular}

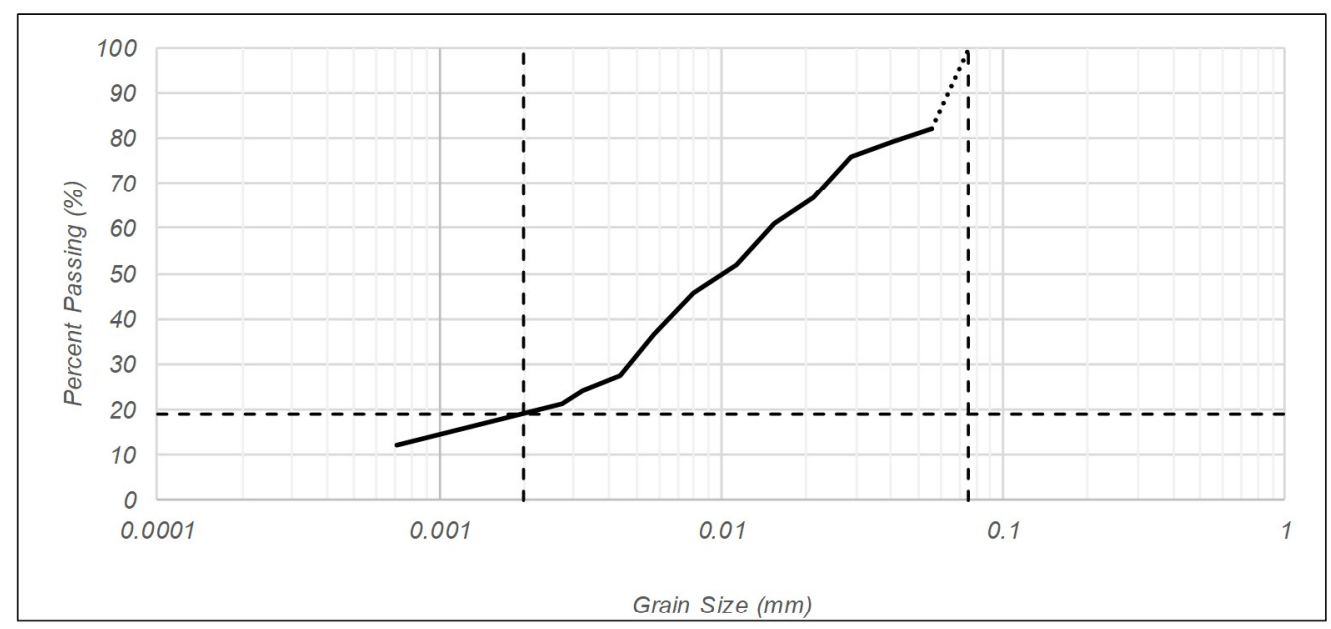

P200 Wash

$\begin{array}{lcc}\text { Container ID: } & \text { C1 } & \\ \text { Before - } \mathrm{M}_{\mathrm{d}} \text { : } & 28.5 & \mathrm{~g} \\ \text { After - } \mathrm{M}_{\mathrm{d}} \text { : } & 0.2 & \mathrm{~g} \\ \text { Fines Fraction: } & 99.30 & \% \\ \text { Coarse Fraction: } & 0.70 & \%\end{array}$

\begin{tabular}{llr} 
& & \multicolumn{2}{c}{ Soil Breakdown } \\
\cline { 2 - 3 } & Coarse: & $0.70 \%$ \\
& Silts: & $80.30 \%$ \\
From Hydrometer & Clays: & $19.00 \%$
\end{tabular}

Procedures Done By:

Kayla/Melissa P. 


\section{S Portland State}

Hydrometer Test

\begin{tabular}{|c|c|c|c|c|c|c|c|c|c|}
\hline \multirow[t]{2}{*}{ Boring: } & \multicolumn{2}{|c|}{ Su_Ex_E } & & \multirow[t]{2}{*}{ Sample: } & N3 & Depth (ft-ft): & 17.5 & \multirow[t]{2}{*}{ Start Time: } & \multirow[t]{2}{*}{ 10:05:00 AM } \\
\hline & & & & & & Ave. Depth (ft) & 18.25 & & \\
\hline & \multicolumn{2}{|c|}{ Constants } & & & & & & & \\
\hline & $M_{s}(g)$ & 55.43 & & & & & & & \\
\hline & $\mathrm{G}_{\mathrm{s}}$ & 2.65 & & & & & & & \\
\hline & $\mathrm{G}_{\mathrm{w}}$ & 1 & & & & & & & \\
\hline & $\begin{array}{l}\text { Time } \\
(\min )\end{array}$ & $\begin{array}{c}\text { Soil } \\
\text { (slurry) }\end{array}$ & Control & $\begin{array}{l}\text { Temp } \\
\left({ }^{\circ} \mathrm{C}\right)\end{array}$ & $\begin{array}{c}\text { Temp } \\
\text { Correction (K) }\end{array}$ & $\begin{array}{l}\text { Hydrometer } \\
\text { Correction } \\
\text { (R) }\end{array}$ & $\begin{array}{c}\text { Effective } \\
\text { Depth, L }(\mathrm{cm})\end{array}$ & Diameter $(\mathrm{mm})$ & $\begin{array}{c}\text { Percent } \\
\text { Passing (\%) }\end{array}$ \\
\hline & 0.5 & 1.033 & 1.003 & 19.9 & 0.013667 & 1.030 & 7.6 & 0.053283775 & 87 \\
\hline & 1 & 1.032 & 1.003 & 19.9 & 0.013667 & 1.029 & 7.8 & 0.038169854 & 84 \\
\hline & 2 & 1.029 & 1.003 & 19.9 & 0.013667 & 1.026 & 8.6 & 0.028340494 & 75 \\
\hline & 4 & 1.026 & 1.003 & 19.9 & 0.013667 & 1.023 & 9.4 & 0.020951114 & 67 \\
\hline & 8 & 1.023 & 1.003 & 19.9 & 0.013667 & 1.020 & 10.2 & 0.015432216 & 58 \\
\hline & 16 & 1.019 & 1.003 & 19.8 & 0.013684 & 1.016 & 11.3 & 0.011499853 & 46 \\
\hline & 32 & 1.017 & 1.003 & 19.7 & 0.013701 & 1.014 & 11.8 & 0.008319903 & 41 \\
\hline & 68 & 1.014 & 1.003 & 19.6 & 0.013718 & 1.011 & 12.6 & 0.005905023 & 32 \\
\hline & 128 & 1.013 & 1.003 & 19.6 & 0.013718 & 1.010 & 12.9 & 0.004354925 & 29 \\
\hline & 245 & 1.011 & 1.003 & 19.5 & 0.013735 & 1.008 & 13.4 & 0.003212166 & 23 \\
\hline & 345 & 1.011 & 1.003 & 19.4 & 0.013752 & 1.008 & 13.4 & 0.002710246 & 23 \\
\hline & 5785 & 1.008 & 1.003 & 16.5 & 0.014260 & 1.005 & 14.2 & 0.0007065 & 14 \\
\hline
\end{tabular}

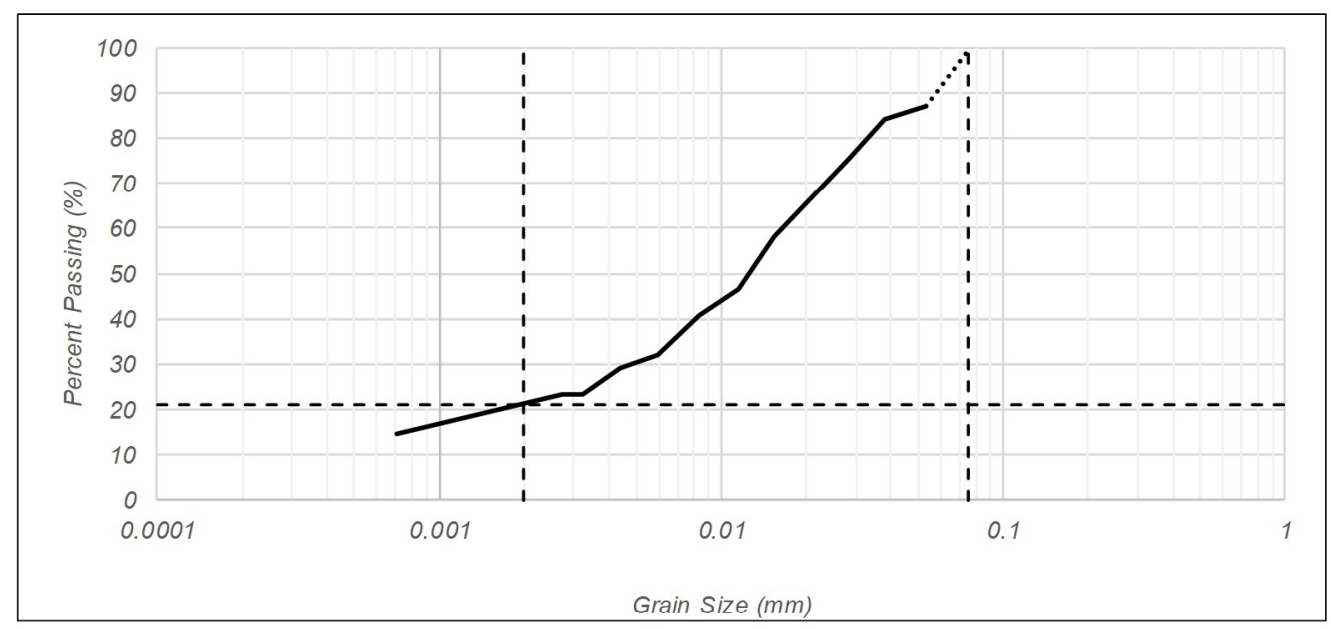

P200 Wash

$\begin{array}{lcl}\text { Container ID: } & \text { B1 } & \\ \text { Before }-\mathrm{M}_{\mathrm{d}}: & 31.2 & \mathrm{~g} \\ \text { After }-\mathrm{M}_{\mathrm{d}} \text { : } & 0.3 & \mathrm{~g} \\ \text { Fines Fraction: } & 99.04 & \% \\ \text { Coarse Fraction: } & 0.96 & \%\end{array}$

\begin{tabular}{llr} 
& & Soil Breakdown \\
\cline { 2 - 3 } & Coarse: & $0.96 \%$ \\
& Silts: & $78.04 \%$ \\
From Hydrometer & Clays: & $21.00 \%$
\end{tabular}

Procedures Done By

Kayla/Melissa P. 


\section{胥 Portland State}

Hydrometer Test

\begin{tabular}{|c|c|c|c|c|c|c|c|c|c|}
\hline \multirow[t]{2}{*}{ Boring: } & \multicolumn{2}{|c|}{ Su_Ex_E } & & \multirow[t]{2}{*}{ Sample: } & N4 & Depth (ft-ft): & $22.5 \quad 24.0$ & \multirow[t]{2}{*}{ Start Time: } & $10: 07: 00 \mathrm{AM}$ \\
\hline & & & & & & Ave. Depth (ft): & 23.25 & & \\
\hline & \multicolumn{2}{|c|}{ Constants } & & & & & & & \\
\hline & $M_{s}(g)$ & 53.40 & & & & & & & \\
\hline & $\mathrm{G}_{\mathrm{s}}$ & 2.65 & & & & & & & \\
\hline & $\mathrm{G}_{\mathrm{w}}$ & 1 & & & & & & & \\
\hline & $\begin{array}{c}\text { Time } \\
(\mathrm{min})\end{array}$ & $\begin{array}{c}\text { Soil } \\
\text { (slurry) }\end{array}$ & Control & $\begin{array}{l}\text { Temp } \\
\left({ }^{\circ} \mathrm{C}\right)\end{array}$ & $\begin{array}{c}\text { Temp } \\
\text { Correction }(\mathrm{K})\end{array}$ & $\begin{array}{l}\text { Hydrometer } \\
\text { Correction } \\
\text { (R) }\end{array}$ & $\begin{array}{c}\text { Effective } \\
\text { Depth, L }(\mathrm{cm})\end{array}$ & Diameter (mm) & $\begin{array}{c}\text { Percent } \\
\text { Passing (\%) }\end{array}$ \\
\hline & 0.5 & 1.026 & 1.003 & 20.0 & 0.013650 & 1.023 & 9.4 & 0.05918499 & 69 \\
\hline & 1 & 1.023 & 1.003 & 20.0 & 0.013650 & 1.020 & 10.2 & 0.043594604 & 60 \\
\hline & 2 & 1.022 & 1.003 & 20.0 & 0.013650 & 1.019 & 10.5 & 0.031276079 & 57 \\
\hline & 4 & 1.018 & 1.003 & 20.0 & 0.013650 & 1.015 & 11.5 & 0.023144701 & 45 \\
\hline & 8 & 1.015 & 1.003 & 20.0 & 0.013650 & 1.012 & 12.3 & 0.01692545 & 36 \\
\hline & 16 & 1.013 & 1.003 & 19.9 & 0.013667 & 1.010 & 12.9 & 0.012271794 & 30 \\
\hline & 30 & 1.011 & 1.003 & 19.7 & 0.013701 & 1.008 & 13.4 & 0.009156807 & 24 \\
\hline & 66 & 1.010 & 1.003 & 19.6 & 0.013718 & 1.007 & 13.7 & 0.006249987 & 21 \\
\hline & 126 & 1.009 & 1.003 & 19.5 & 0.013735 & 1.006 & 13.9 & 0.004561953 & 18 \\
\hline & 243 & 1.008 & 1.003 & 19.5 & 0.013735 & 1.005 & 14.2 & 0.003320242 & 15 \\
\hline & 343 & 1.008 & 1.003 & 19.4 & 0.013752 & 1.005 & 14.2 & 0.002798098 & 15 \\
\hline & 5783 & 1.006 & 1.003 & 16.0 & 0.014350 & 1.003 & 14.7 & 0.000723492 & 9 \\
\hline
\end{tabular}

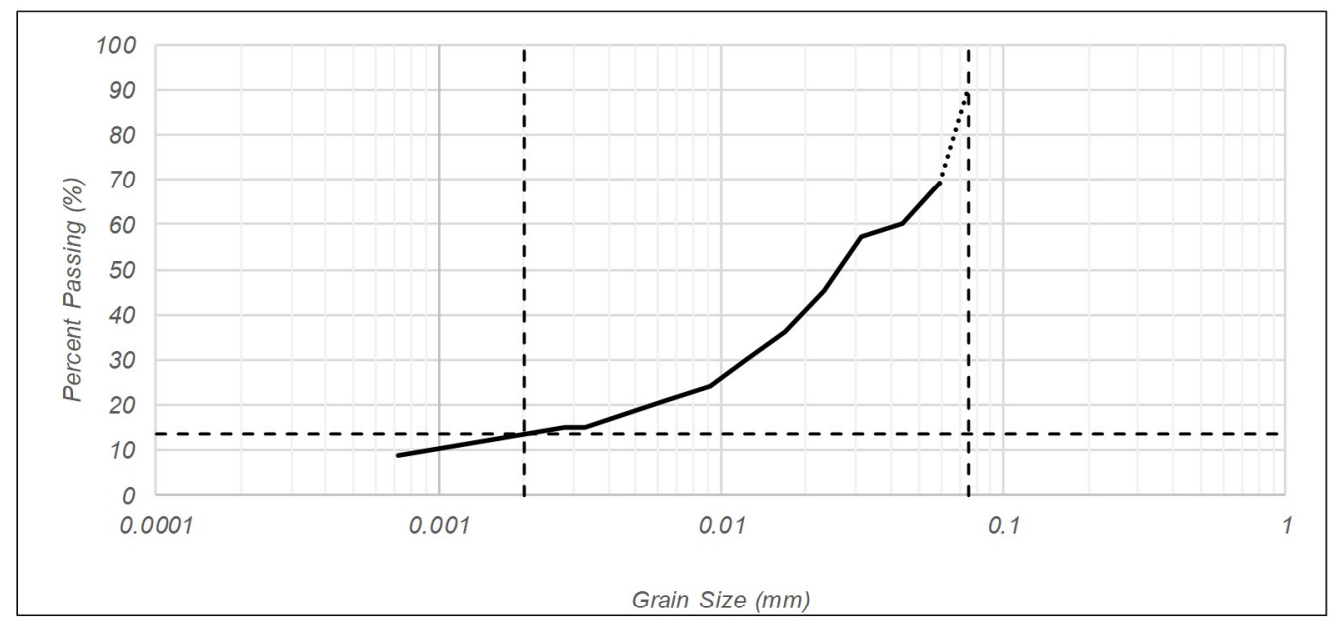

P200 Wash

$\begin{array}{lcc}\text { Container ID: } & \text { A1 } & \\ \text { Before }-\mathrm{M}_{\mathrm{d}}: & 23.2 & \mathrm{~g} \\ \text { After - } \mathrm{M}_{\mathrm{d}}: & 2.3 & \mathrm{~g} \\ \text { Fines Fraction: } & 90.09 & \% \\ \text { Coarse Fraction: } & 9.91 & \%\end{array}$

\begin{tabular}{llr} 
& & \multicolumn{2}{c}{ Soil Breakdown } \\
\cline { 2 - 3 } & Coarse: & $9.91 \%$ \\
& Silts: & $76.59 \%$ \\
From Hydrometer & Clays: & $13.50 \%$
\end{tabular}

$\underline{\text { Procedures Done By: Kayla/Melissa P. }}$ 


\section{胥 Portland State}

Hydrometer Test

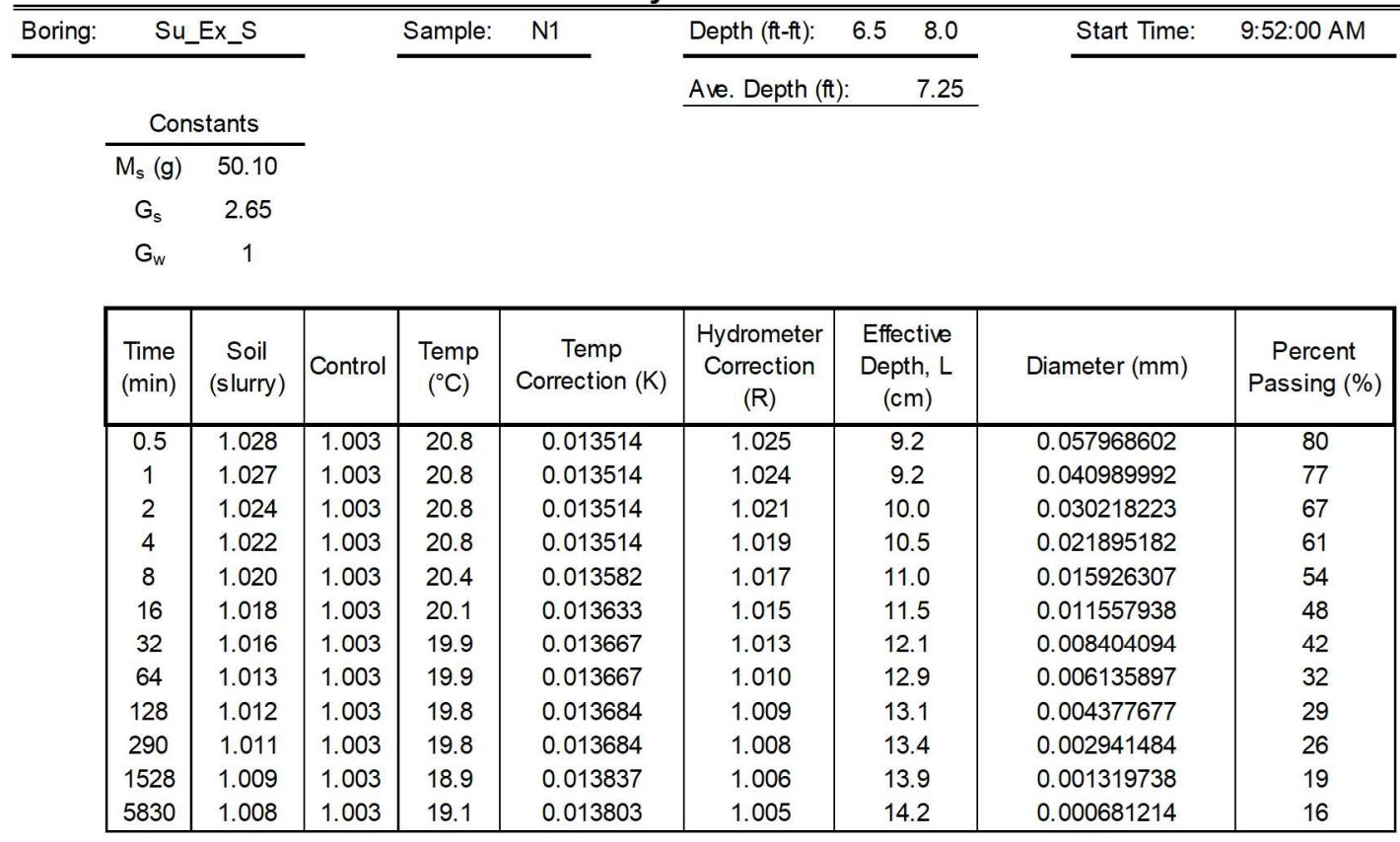

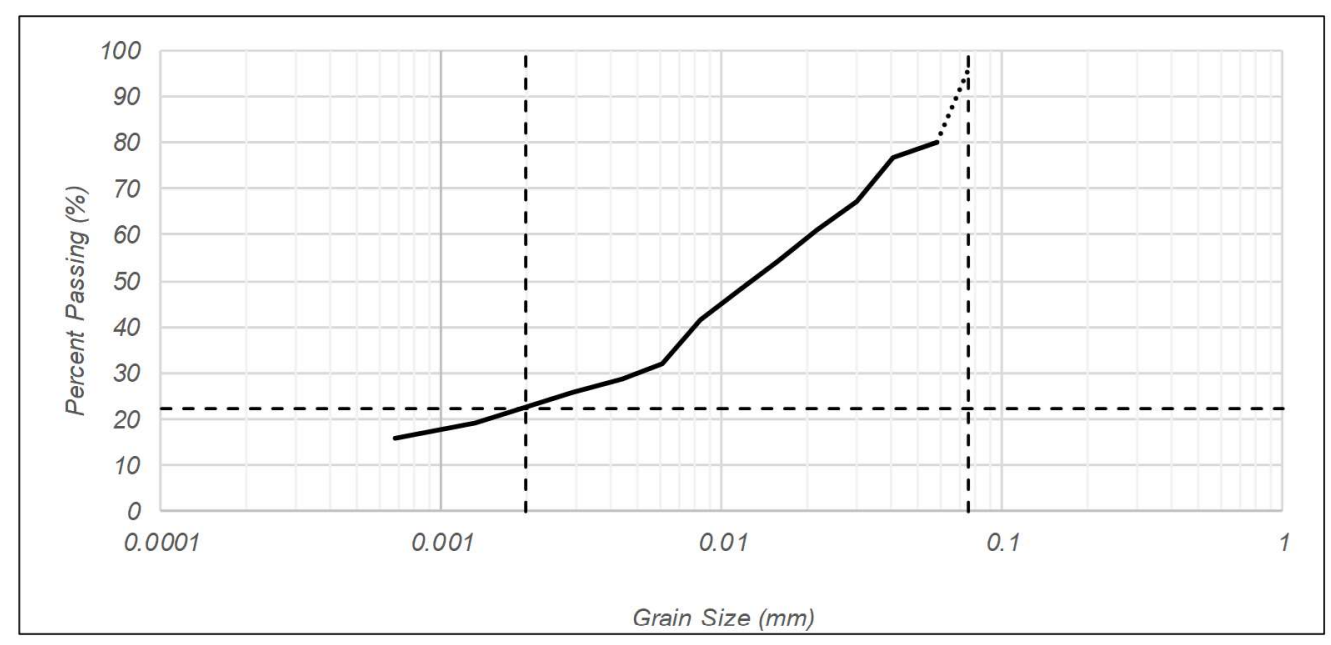

P200 Wash

$\begin{array}{lcc}\text { Container ID: } & \text { W1 } & \\ \text { Before }-\mathrm{M}_{\mathrm{d}}: & 31.3 & \mathrm{~g} \\ \text { After }-\mathrm{M}_{\mathrm{d}}: & 1.4 & \mathrm{~g} \\ \text { Fines Fraction: } & 95.53 \% \\ \text { Coarse Fraction: } & 4.47 \%\end{array}$

\begin{tabular}{llr} 
& & \multicolumn{1}{c}{ Soil Breakdown } \\
\cline { 2 - 3 } & Coarse: & $4.47 \%$ \\
& Silts: & $73.03 \%$ \\
From Hydrometer & Clays: & $22.50 \%$
\end{tabular}

$\underline{\text { Procedures Done By: Kayla/Melissa P. }}$ 


\section{S Portland State}

Hydrometer Test

\begin{tabular}{|c|c|c|c|c|c|c|c|c|c|}
\hline \multirow[t]{2}{*}{ Boring: } & \multicolumn{2}{|c|}{ Su_Ex_S } & & \multirow[t]{2}{*}{ Sample: } & N2 & Depth (ft-ft): & 11.5 & \multirow[t]{2}{*}{ Start Time: } & \multirow[t]{2}{*}{ 10:13:00 AM } \\
\hline & & & & & & Ave. Depth (ft) & 12.25 & & \\
\hline & \multicolumn{2}{|c|}{ Constants } & & & & & & & \\
\hline & $M_{s}(g)$ & 51.12 & & & & & & & \\
\hline & $\mathrm{G}_{\mathrm{s}}$ & 2.65 & & & & & & & \\
\hline & $\mathrm{G}_{\mathrm{w}}$ & 1 & & & & & & & \\
\hline & $\begin{array}{l}\text { Time } \\
(\min )\end{array}$ & $\begin{array}{c}\text { Soil } \\
\text { (slurry) }\end{array}$ & Control & $\begin{array}{l}\text { Temp } \\
\left({ }^{\circ} \mathrm{C}\right)\end{array}$ & $\begin{array}{c}\text { Temp } \\
\text { Correction (K) }\end{array}$ & $\begin{array}{l}\text { Hydrometer } \\
\text { Correction } \\
\text { (R) }\end{array}$ & $\begin{array}{c}\text { Effective } \\
\text { Depth, L }(\mathrm{cm})\end{array}$ & Diameter $(\mathrm{mm})$ & $\begin{array}{c}\text { Percent } \\
\text { Passing (\%) }\end{array}$ \\
\hline & 0.5 & 1.029 & 1.003 & 20.4 & 0.013582 & 1.026 & 8.6 & 0.056328469 & 82 \\
\hline & 1 & 1.028 & 1.003 & 20.4 & 0.013582 & 1.025 & 8.9 & 0.040519001 & 79 \\
\hline & 2 & 1.026 & 1.003 & 20.4 & 0.013582 & 1.023 & 9.4 & 0.029445074 & 72 \\
\hline & 4 & 1.023 & 1.003 & 19.9 & 0.013667 & 1.020 & 10.2 & 0.021824449 & 63 \\
\hline & 8 & 1.021 & 1.003 & 19.9 & 0.013667 & 1.018 & 10.7 & 0.015805931 & 57 \\
\hline & 16 & 1.018 & 1.003 & 19.8 & 0.013684 & 1.015 & 11.5 & 0.011601175 & 47 \\
\hline & 32 & 1.016 & 1.003 & 19.9 & 0.013667 & 1.013 & 12.1 & 0.008404094 & 41 \\
\hline & 64 & 1.014 & 1.003 & 19.9 & 0.013667 & 1.011 & 12.6 & 0.00606413 & 35 \\
\hline & 128 & 1.013 & 1.003 & 19.8 & 0.013684 & 1.010 & 12.9 & 0.004344131 & 31 \\
\hline & 269 & 1.011 & 1.003 & 19.8 & 0.013684 & 1.008 & 13.4 & 0.003054143 & 25 \\
\hline & 1507 & 1.009 & 1.003 & 18.9 & 0.013837 & 1.006 & 13.9 & 0.001328901 & 19 \\
\hline & 5809 & 1.008 & 1.003 & 19.1 & 0.013803 & 1.005 & 14.2 & 0.000682444 & 16 \\
\hline
\end{tabular}

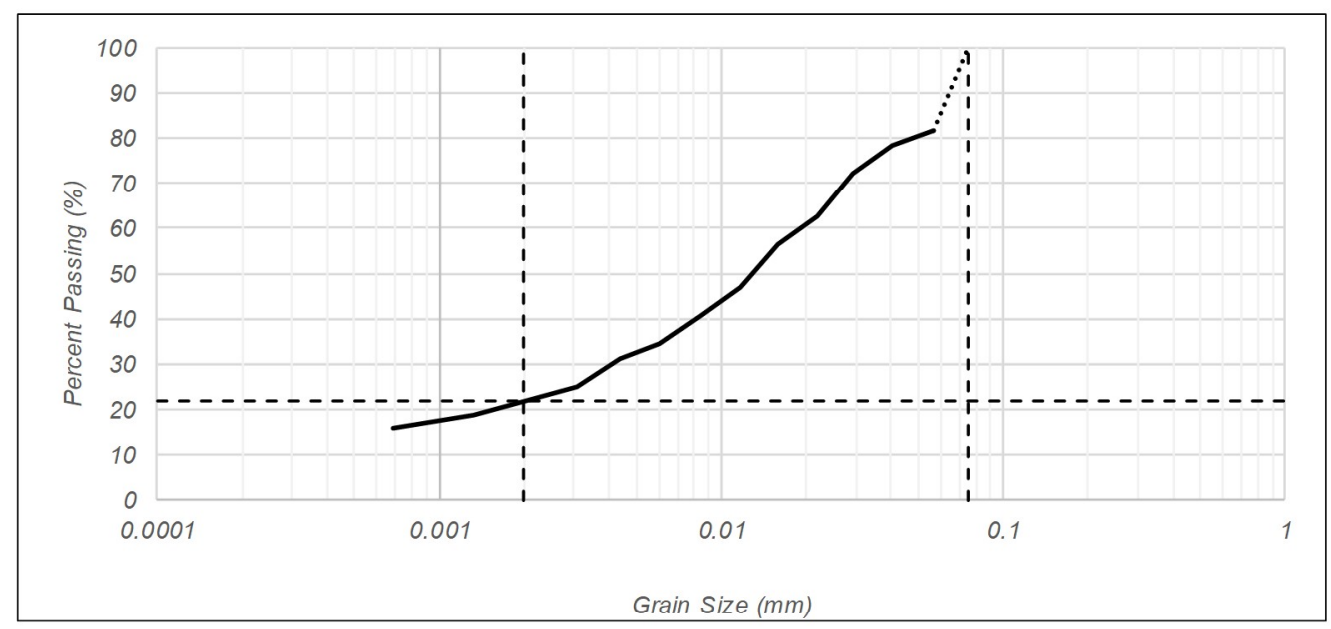

P200 Wash

$\begin{array}{lcc}\text { Container ID: } & 01 & \\ \text { Before }-\mathrm{M}_{\mathrm{d}}: & 34.8 \mathrm{~g} \\ \text { After }-\mathrm{M}_{\mathrm{d}} \text { : } & 0.2 & \mathrm{~g} \\ \text { Fines Fraction: } & 99.43 \mathrm{\%} \\ \text { Coarse Fraction: } & 0.57 \quad \%\end{array}$

\begin{tabular}{llr} 
& & \multicolumn{2}{c}{ Soil Breakdown } \\
\cline { 2 - 3 } & Coarse: & $0.57 \%$ \\
& Silts: & $77.43 \%$ \\
From Hydrometer & Clays: & $22.00 \%$
\end{tabular}

Procedures Done By:

Kayla/Melissa P. 


\section{S P Portland State}

Hydrometer Test

\begin{tabular}{|c|c|c|c|c|c|c|c|c|c|}
\hline \multirow[t]{2}{*}{ Boring: } & \multicolumn{2}{|c|}{ Su_Ex_S } & & \multirow[t]{2}{*}{ Sample: } & N3 & Depth (ft-ft): & 16.5 & \multirow[t]{2}{*}{ Start Time: } & \multirow[t]{2}{*}{ 10:13:00 AM } \\
\hline & & & & & & Ave. Depth (ft) & 17.25 & & \\
\hline & \multicolumn{2}{|c|}{ Constants } & & & & & & & \\
\hline & $M_{s}(g)$ & 50.98 & & & & & & & \\
\hline & $\mathrm{G}_{\mathrm{s}}$ & 2.65 & & & & & & & \\
\hline & $\mathrm{G}_{\mathrm{w}}$ & 1 & & & & & & & \\
\hline & $\begin{array}{l}\text { Time } \\
(\min )\end{array}$ & $\begin{array}{c}\text { Soil } \\
\text { (slurry) }\end{array}$ & Control & $\begin{array}{l}\text { Temp } \\
\left({ }^{\circ} \mathrm{C}\right)\end{array}$ & $\begin{array}{c}\text { Temp } \\
\text { Correction (K) }\end{array}$ & $\begin{array}{l}\text { Hydrometer } \\
\text { Correction } \\
\text { (R) }\end{array}$ & $\begin{array}{c}\text { Effective } \\
\text { Depth, L }(\mathrm{cm})\end{array}$ & Diameter $(\mathrm{mm})$ & $\begin{array}{c}\text { Percent } \\
\text { Passing (\%) }\end{array}$ \\
\hline & 0.5 & 1.026 & 1.003 & 19.8 & 0.013684 & 1.023 & 8.6 & 0.056751493 & 72 \\
\hline & 1 & 1.025 & 1.003 & 19.8 & 0.013684 & 1.022 & 8.9 & 0.040823296 & 69 \\
\hline & 2 & 1.023 & 1.003 & 19.8 & 0.013684 & 1.020 & 9.4 & 0.029666205 & 63 \\
\hline & 4 & 1.021 & 1.003 & 19.8 & 0.013684 & 1.018 & 10.2 & 0.021851596 & 57 \\
\hline & 8 & 1.019 & 1.003 & 19.9 & 0.013667 & 1.016 & 10.7 & 0.015805931 & 50 \\
\hline & 16 & 1.017 & 1.003 & 19.8 & 0.013684 & 1.014 & 11.5 & 0.011601175 & 44 \\
\hline & 32 & 1.014 & 1.003 & 19.9 & 0.013667 & 1.011 & 12.1 & 0.008404094 & 35 \\
\hline & 64 & 1.013 & 1.003 & 19.9 & 0.013667 & 1.010 & 12.6 & 0.00606413 & 32 \\
\hline & 128 & 1.011 & 1.003 & 19.8 & 0.013684 & 1.008 & 12.9 & 0.004344131 & 25 \\
\hline & 253 & 1.01 & 1.003 & 19.8 & 0.013684 & 1.007 & 13.4 & 0.003149236 & 22 \\
\hline & 1491 & 1.007 & 1.003 & 18.9 & 0.013837 & 1.004 & 13.9 & 0.001336012 & 13 \\
\hline & 5793 & 1.006 & 1.003 & 19.1 & 0.013803 & 1.003 & 14.2 & 0.000683386 & 9 \\
\hline
\end{tabular}

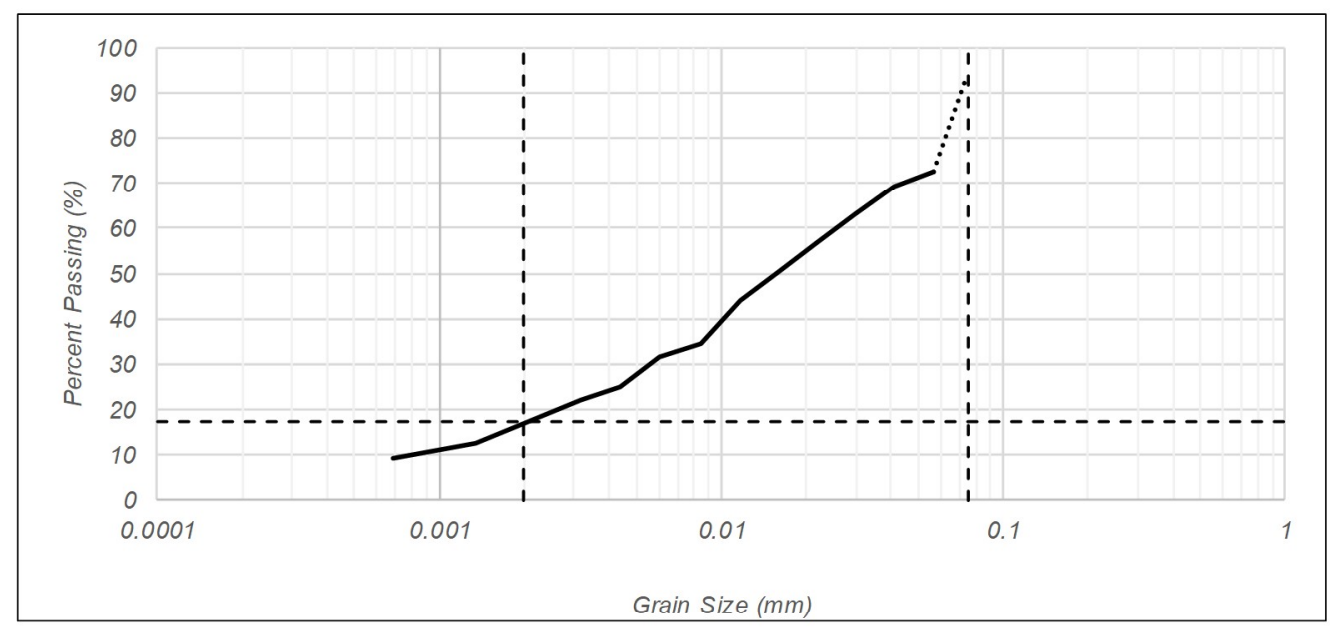

P200 Wash

$\begin{array}{lcc}\text { Container ID: } & \text { P1 } & \\ \text { Before }-\mathrm{M}_{\mathrm{d}}: & 26.2 & \mathrm{~g} \\ \text { After }-\mathrm{M}_{\mathrm{d}} \text { : } & 1.5 & \mathrm{~g} \\ \text { Fines Fraction: } & 94.27 & \% \\ \text { Coarse Fraction: } & 5.73 & \%\end{array}$

\begin{tabular}{llr} 
& & \multicolumn{2}{c}{ Soil Breakdown } \\
\cline { 2 - 3 } & Coarse: & $5.73 \%$ \\
& Silts: & $77.02 \%$ \\
From Hydrometer & Clays: & $17.25 \%$
\end{tabular}

Procedures Done By:

Kayla/Melissa P. 


\section{S Portland State}

Hydrometer Test

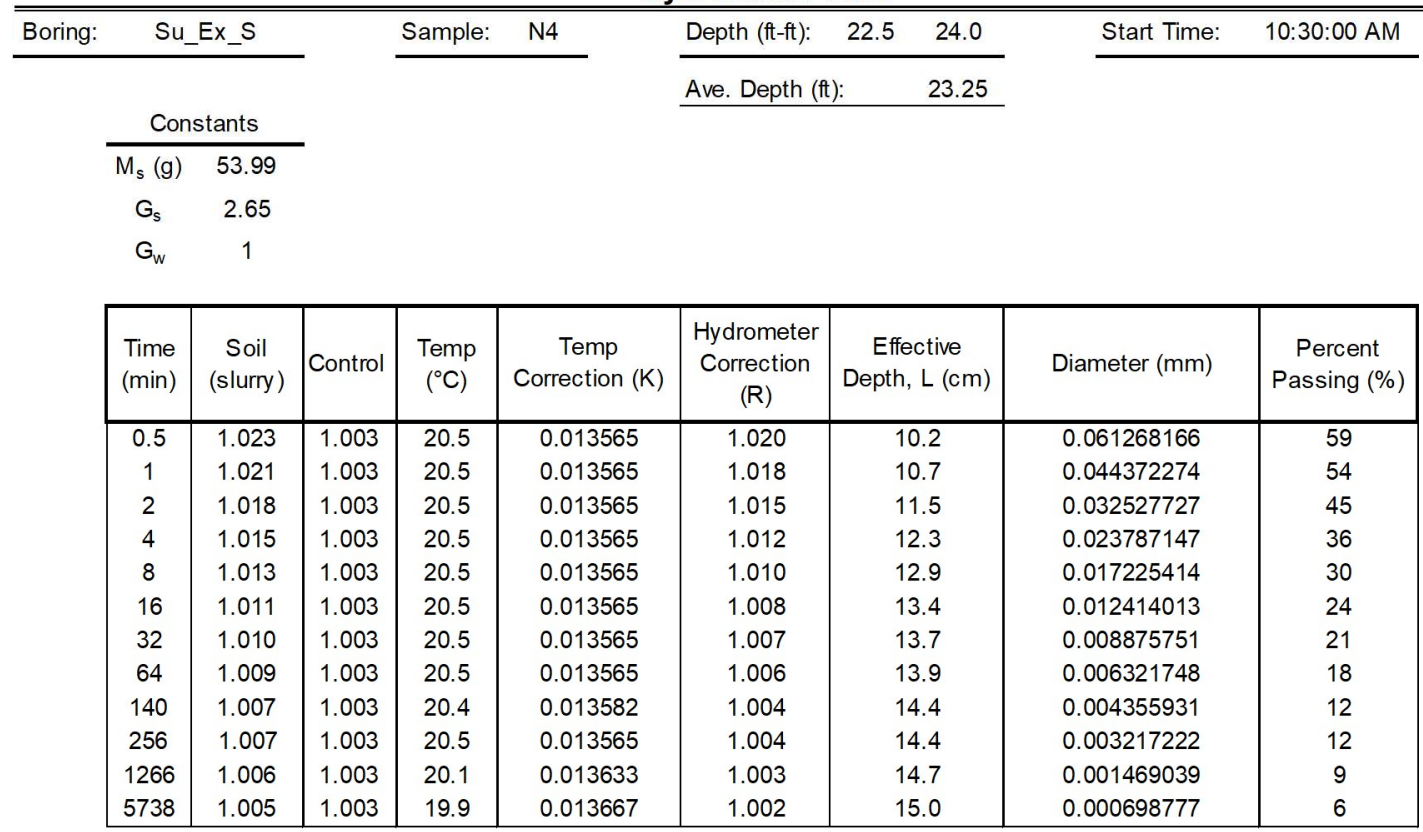

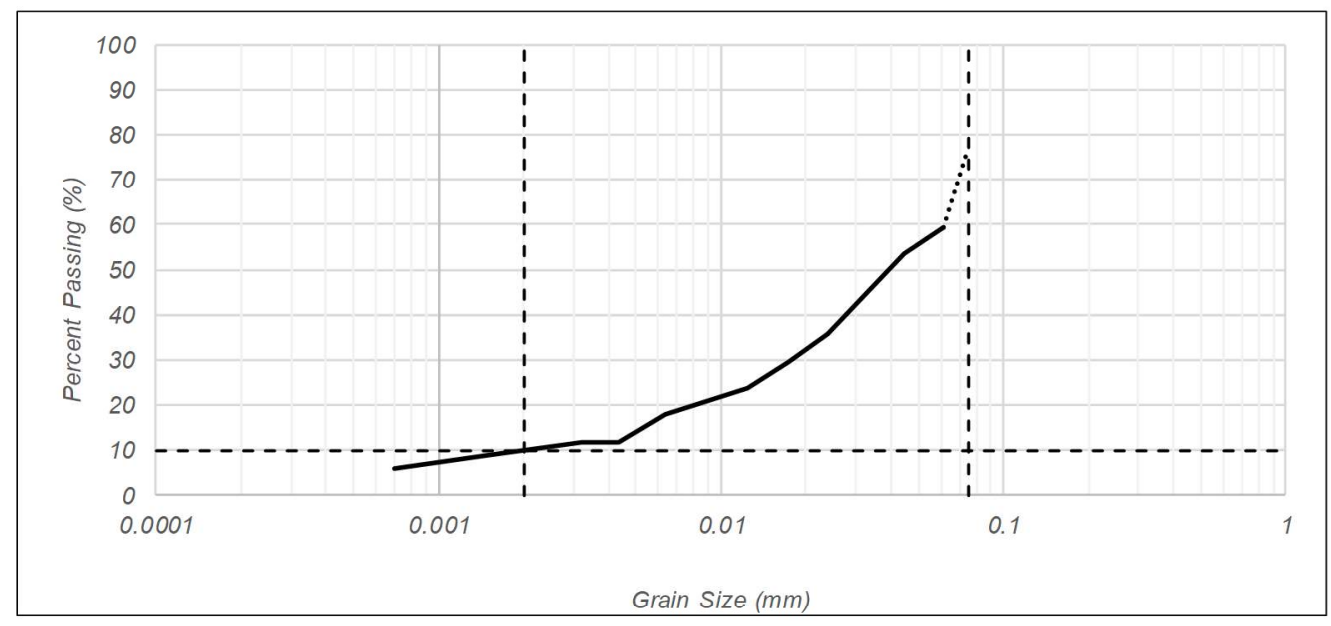

P200 Wash

$\begin{array}{lcl}\text { Container ID: } & \text { M1 } & \\ \text { Before }-\mathrm{M}_{\mathrm{d}}: & 34.2 & \mathrm{~g} \\ \text { After }-\mathrm{M}_{\mathrm{d}}: & 7.9 & \mathrm{~g} \\ \text { Fines Fraction: } & 76.90 & \% \\ \text { Coarse Fraction: } & 23.10 \%\end{array}$

\begin{tabular}{llc} 
& \multicolumn{2}{c}{ Soil Breakdown } \\
\cline { 2 - 3 } & Coarse: & $23.10 \%$ \\
From Hydrometer & Silts: & $66.90 \%$ \\
& Clays: & $10.00 \%$
\end{tabular}

$\underline{\text { Procedures Done By: Kayla/Melissa P. }}$ 


\section{胥 Portland State}

Hydrometer Test

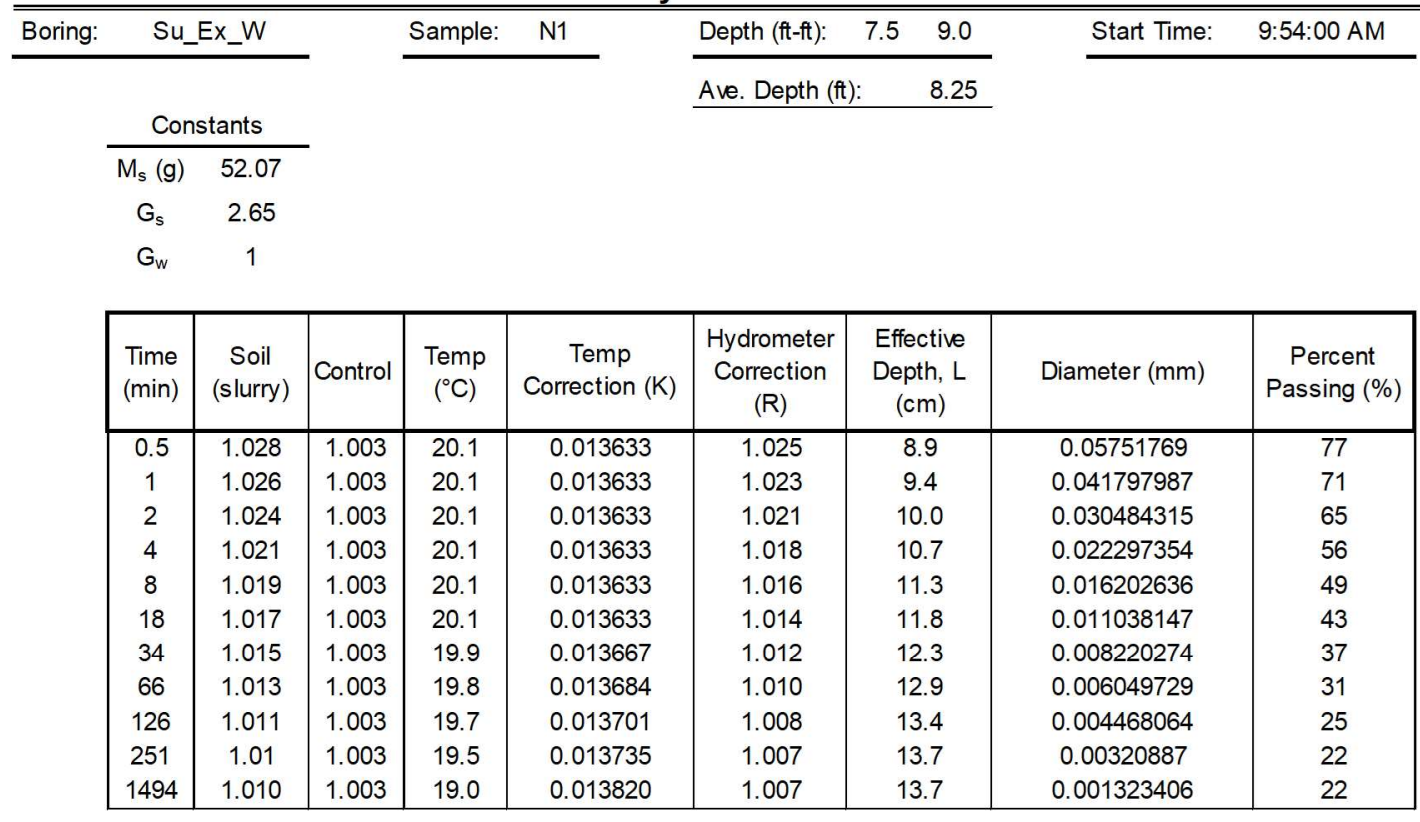

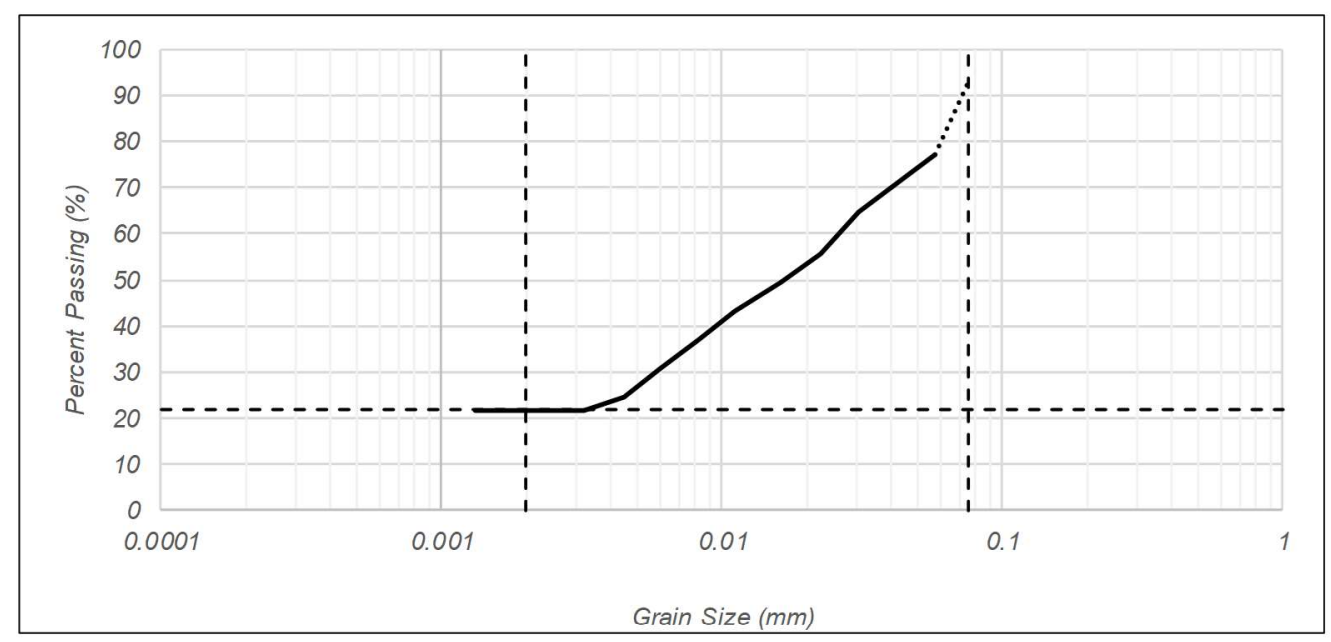

P200 Wash

$\begin{array}{lcc}\text { Container ID: } & \mathrm{J} 1 & \\ \text { Before }-\mathrm{M}_{\mathrm{d}}: & 26.4 & \mathrm{~g} \\ \text { After }-\mathrm{M}_{\mathrm{d}}: & 2.0 & \mathrm{~g} \\ \text { Fines Fraction: } & 92.42 & \% \\ \text { Coarse Fraction: } & 7.58 & \%\end{array}$

\begin{tabular}{llr} 
& & \multicolumn{2}{c}{ Soil Breakdown } \\
\cline { 2 - 3 } & Coarse: & $7.58 \%$ \\
& Silts: & $70.42 \%$ \\
From Hydrometer & Clays: & $22.00 \%$
\end{tabular}

$\underline{\text { Procedures Done By: Kayla/Melissa P. }}$ 


\section{S Portland State}

Hydrometer Test

\begin{tabular}{|c|c|c|c|c|c|c|c|c|c|}
\hline \multirow[t]{2}{*}{ Boring: } & \multicolumn{2}{|c|}{ Su_Ex_W } & & \multirow[t]{2}{*}{ Sample: } & \multirow[t]{2}{*}{ N2 } & Depth (ft-ft): & 16.5 & \multirow[t]{2}{*}{ Start Time: } & \multirow[t]{2}{*}{ 9:56:00 AM } \\
\hline & & & & & & Ave. Depth (ft) & 17.25 & & \\
\hline & \multicolumn{2}{|c|}{ Constants } & & & & & & & \\
\hline & $M_{s}(g)$ & 50.86 & & & & & & & \\
\hline & $\mathrm{G}_{\mathrm{s}}$ & 2.65 & & & & & & & \\
\hline & $\mathrm{G}_{\mathrm{w}}$ & 1 & & & & & & & \\
\hline & $\begin{array}{l}\text { Time } \\
(\mathrm{min})\end{array}$ & $\begin{array}{c}\text { Soil } \\
\text { (slurry) }\end{array}$ & Control & $\begin{array}{l}\text { Temp } \\
\left({ }^{\circ} \mathrm{C}\right)\end{array}$ & $\begin{array}{c}\text { Temp } \\
\text { Correction (K) }\end{array}$ & $\begin{array}{l}\text { Hydrometer } \\
\text { Correction } \\
\text { (R) }\end{array}$ & $\begin{array}{c}\text { Effective } \\
\text { Depth, L }(\mathrm{cm})\end{array}$ & Diameter $(\mathrm{mm})$ & $\begin{array}{c}\text { Percent } \\
\text { Passing (\%) }\end{array}$ \\
\hline & 0.5 & 1.026 & 1.003 & 20.2 & 0.013616 & 1.023 & 9.4 & 0.059037569 & 73 \\
\hline & 1 & 1.024 & 1.003 & 20.2 & 0.013616 & 1.021 & 10.0 & 0.043057573 & 66 \\
\hline & 2 & 1.022 & 1.003 & 20.2 & 0.013616 & 1.019 & 10.5 & 0.031198175 & 60 \\
\hline & 4 & 1.021 & 1.003 & 20.2 & 0.013616 & 1.018 & 10.7 & 0.02226955 & 57 \\
\hline & 8 & 1.018 & 1.003 & 20.2 & 0.013616 & 1.015 & 11.5 & 0.016325011 & 47 \\
\hline & 16 & 1.016 & 1.003 & 20.1 & 0.013633 & 1.013 & 12.1 & 0.011855616 & 41 \\
\hline & 32 & 1.014 & 1.003 & 20.1 & 0.013633 & 1.011 & 12.6 & 0.00855464 & 35 \\
\hline & 64 & 1.012 & 1.003 & 20.0 & 0.013650 & 1.009 & 13.1 & 0.006175588 & 28 \\
\hline & 124 & 1.011 & 1.003 & 19.8 & 0.013684 & 1.008 & 13.4 & 0.004498364 & 25 \\
\hline & 249 & 1.009 & 1.003 & 19.6 & 0.013718 & 1.006 & 13.9 & 0.003241145 & 19 \\
\hline & 1496 & 1.008 & 1.003 & 19.0 & 0.013820 & 1.005 & 14.2 & 0.001346438 & 16 \\
\hline
\end{tabular}

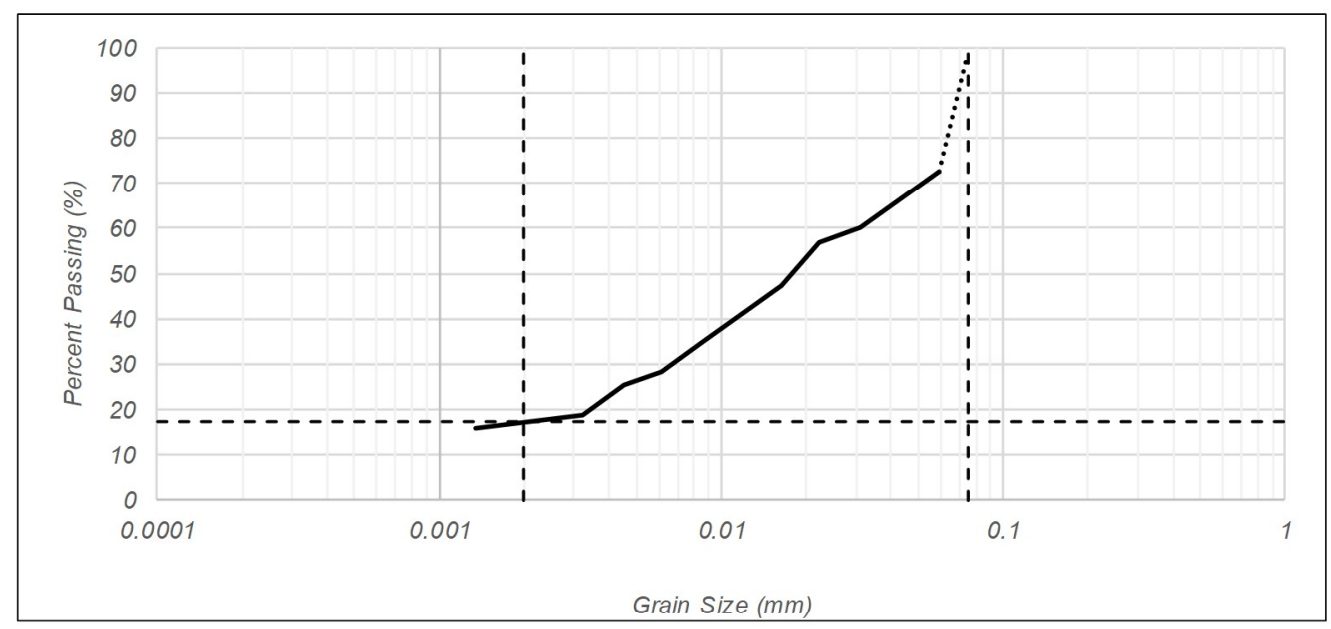

P200 Wash

$\begin{array}{lcc}\text { Container ID: } & \mathrm{H} 1 & \\ \text { Before }-\mathrm{M}_{\mathrm{d}}: & 23.5 & \mathrm{~g} \\ \text { After }-\mathrm{M}_{\mathrm{d}} \text { : } & 0.4 & \mathrm{~g} \\ \text { Fines Fraction: } & 98.30 & \% \\ \text { Coarse Fraction: } & 1.70 & \%\end{array}$

\begin{tabular}{llr} 
& & Soil Breakdown \\
\cline { 2 - 3 } & Coarse: & $1.70 \%$ \\
& Silts: & $80.80 \%$ \\
From Hydrometer & Clays: & $17.50 \%$
\end{tabular}

Procedures Done By:

Kayla/Melissa P. 


\section{胥 Portland State}

Hydrometer Test

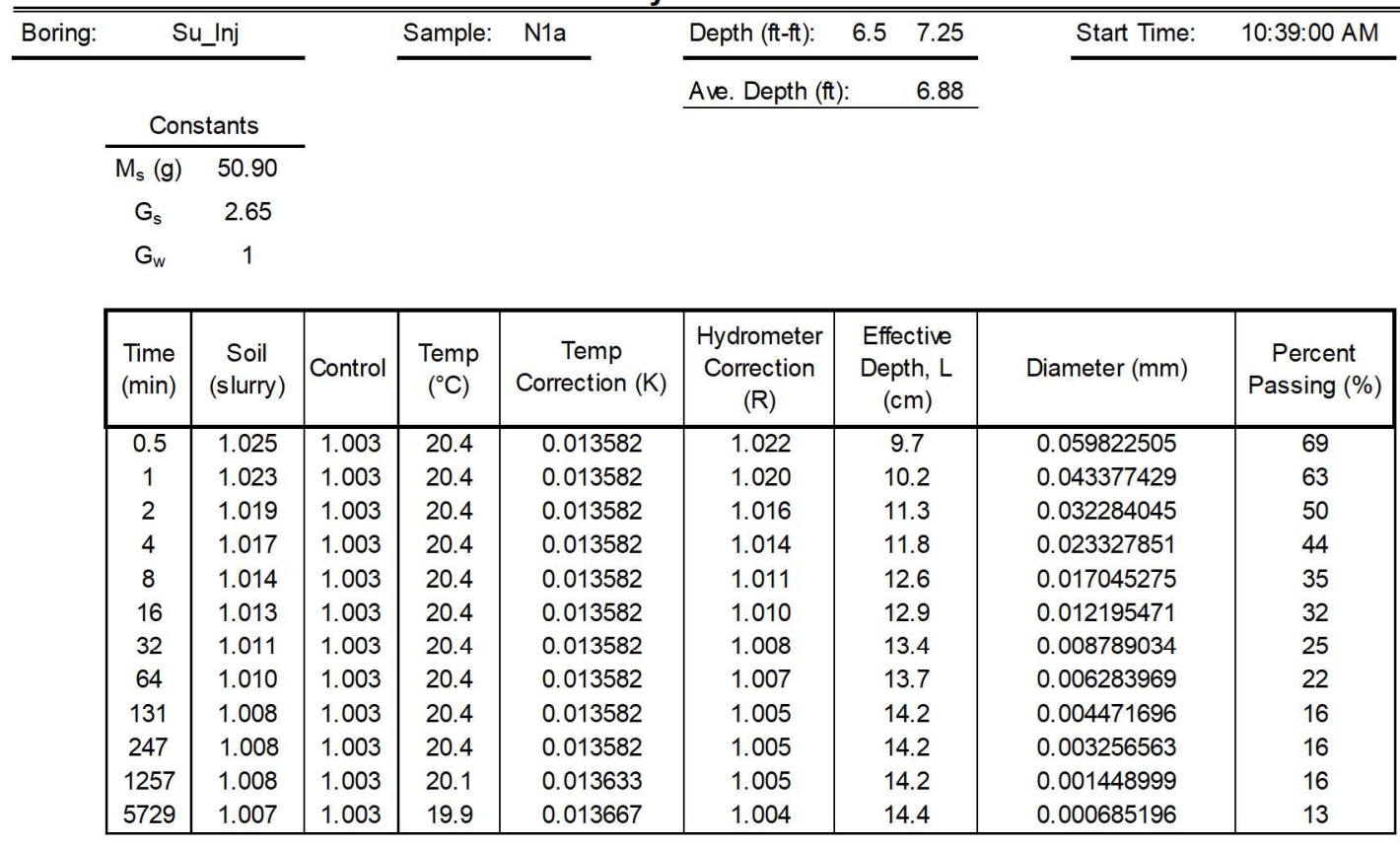

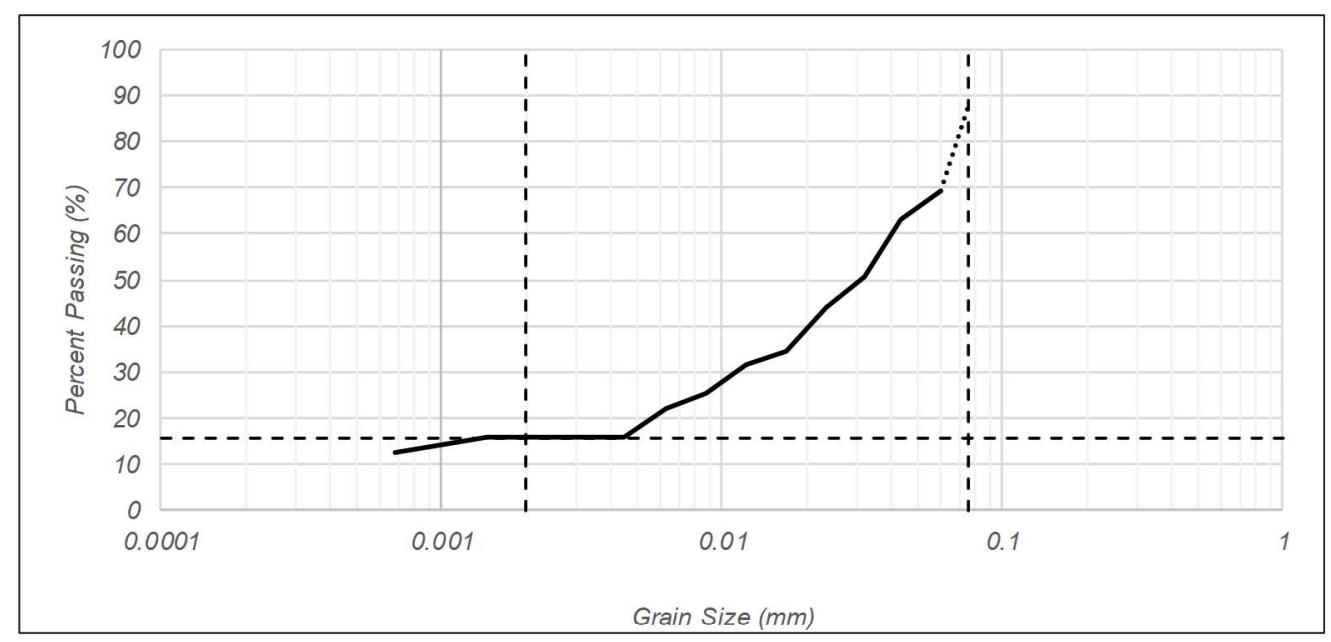

P200 Wash

\begin{tabular}{|c|c|}
\hline Container ID: & U1 \\
\hline Before - $M_{d}$ : & 24.7 \\
\hline After - $M_{d}$ : & 3.2 \\
\hline Fines Fraction: & 87.04 \\
\hline Coarse Fraction: & 12.96 \\
\hline
\end{tabular}

\begin{tabular}{llr} 
& & Soil Breakdown \\
\cline { 2 - 3 } & Coarse: & $12.96 \%$ \\
& Silts: & $71.54 \%$ \\
From Hydrometer & Clays: & $15.50 \%$
\end{tabular}

$\underline{\text { Procedures Done By: Kayla/Melissa P. }}$ 


\section{胥 Portland State}

Hydrometer Test

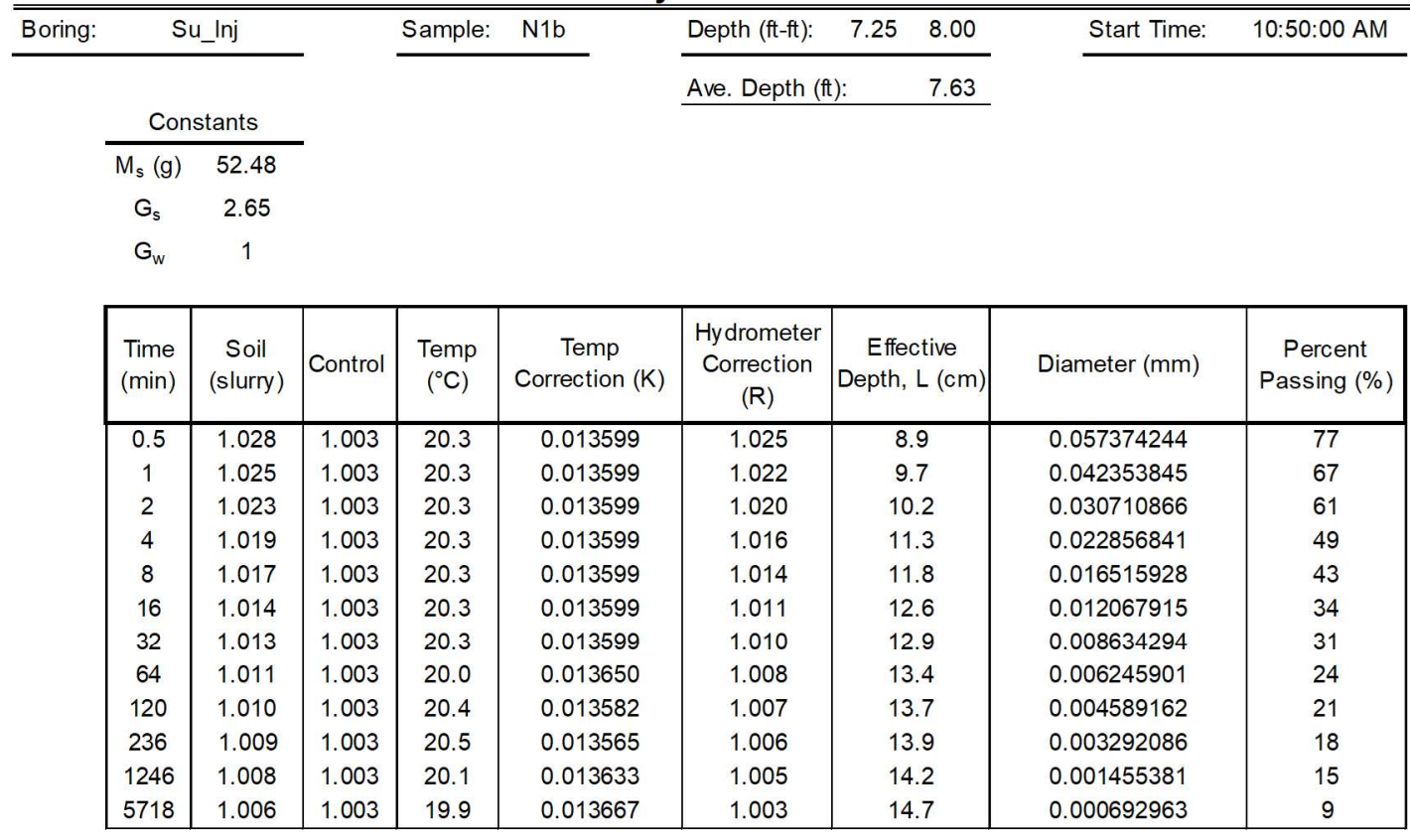

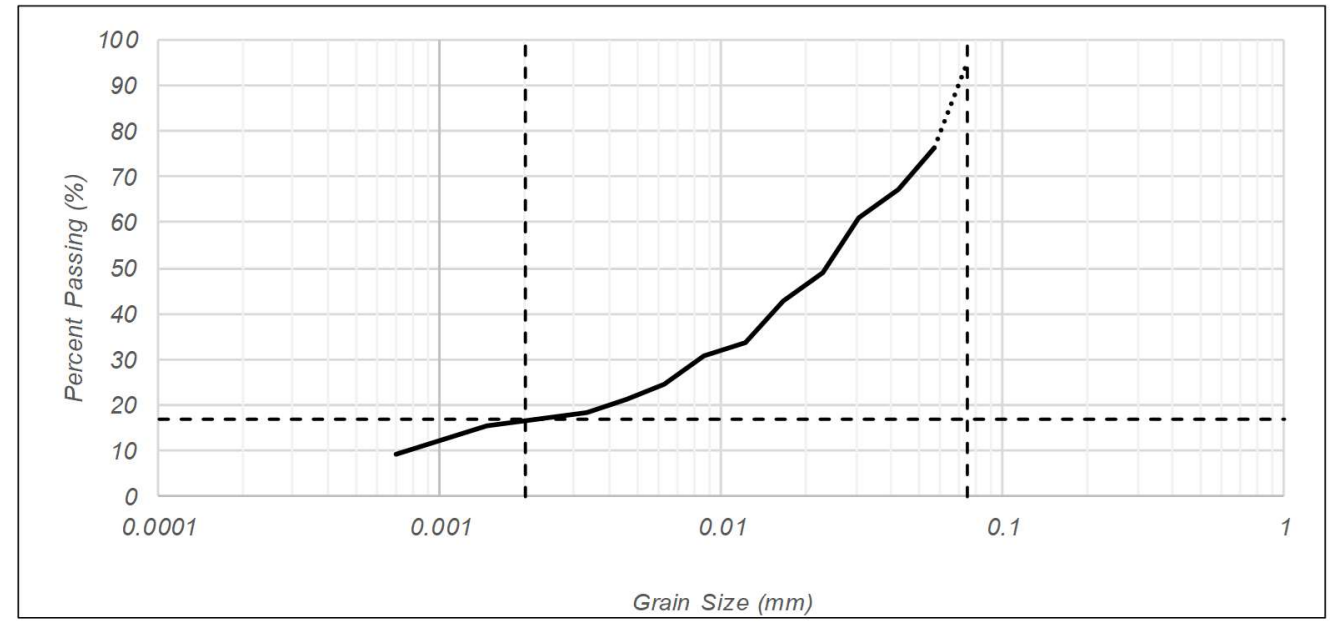

P200 Wash

$\begin{array}{lcc}\text { Container ID: } & \mathrm{V} 1 & \\ \text { Before }-\mathrm{M}_{\mathrm{d}} \text { : } & 31.6 & \mathrm{~g} \\ \text { After }-\mathrm{M}_{\mathrm{d}} \text { : } & 1.7 & \mathrm{~g} \\ \text { Fines Fraction: } & 94.62 & \% \\ \text { Coarse Fraction: } & 5.38 & \%\end{array}$

\begin{tabular}{llr} 
& & \multicolumn{2}{c}{ Soil Breakdown } \\
\cline { 2 - 3 } & Coarse: & $5.38 \%$ \\
& Silts: & $77.87 \%$ \\
From Hydrometer & Clays: & $16.75 \%$
\end{tabular}

$\underline{\text { Procedures Done By: } \quad \text { Kayla/Melissa P. }}$ 


\section{S Portland State}

Hydrometer Test

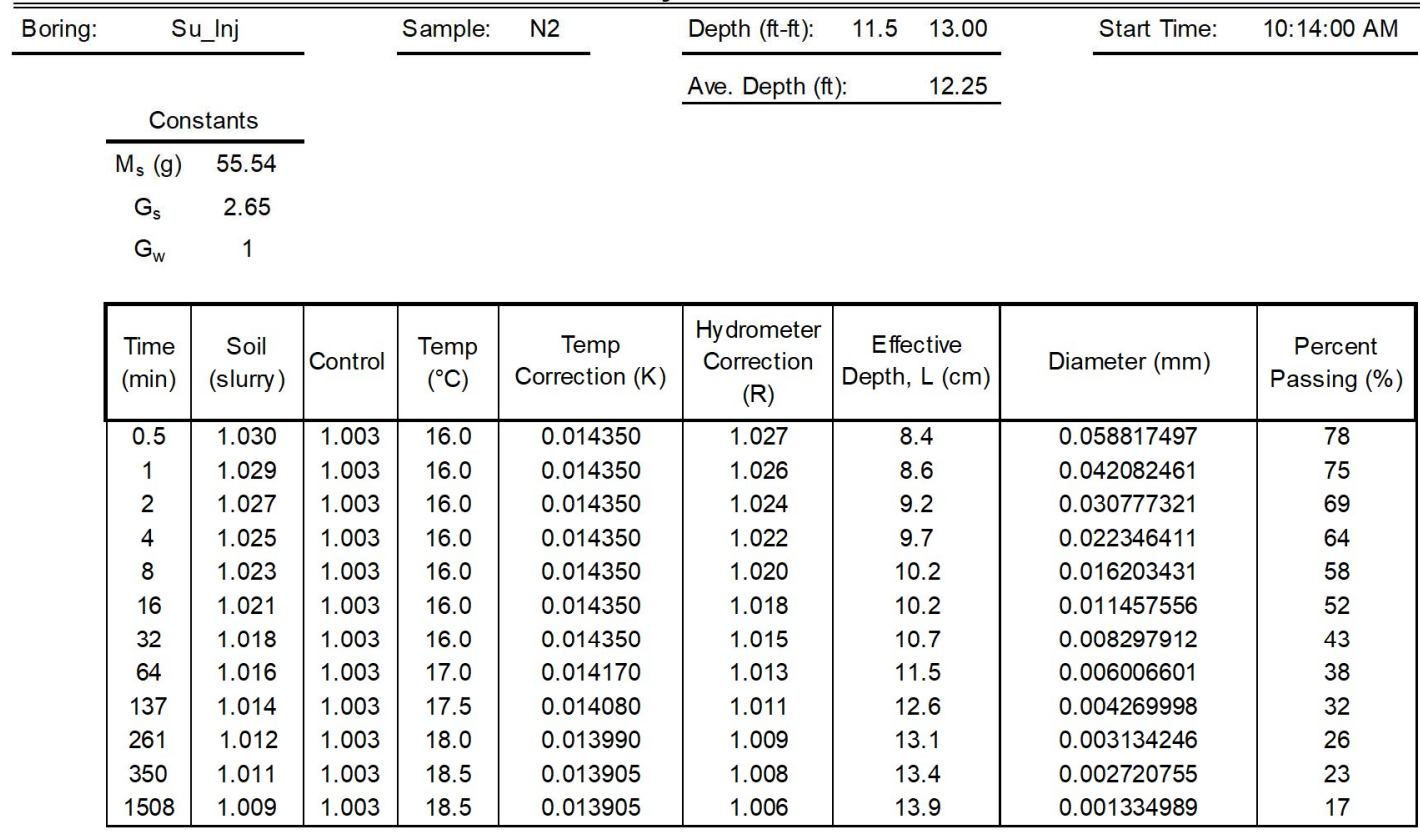

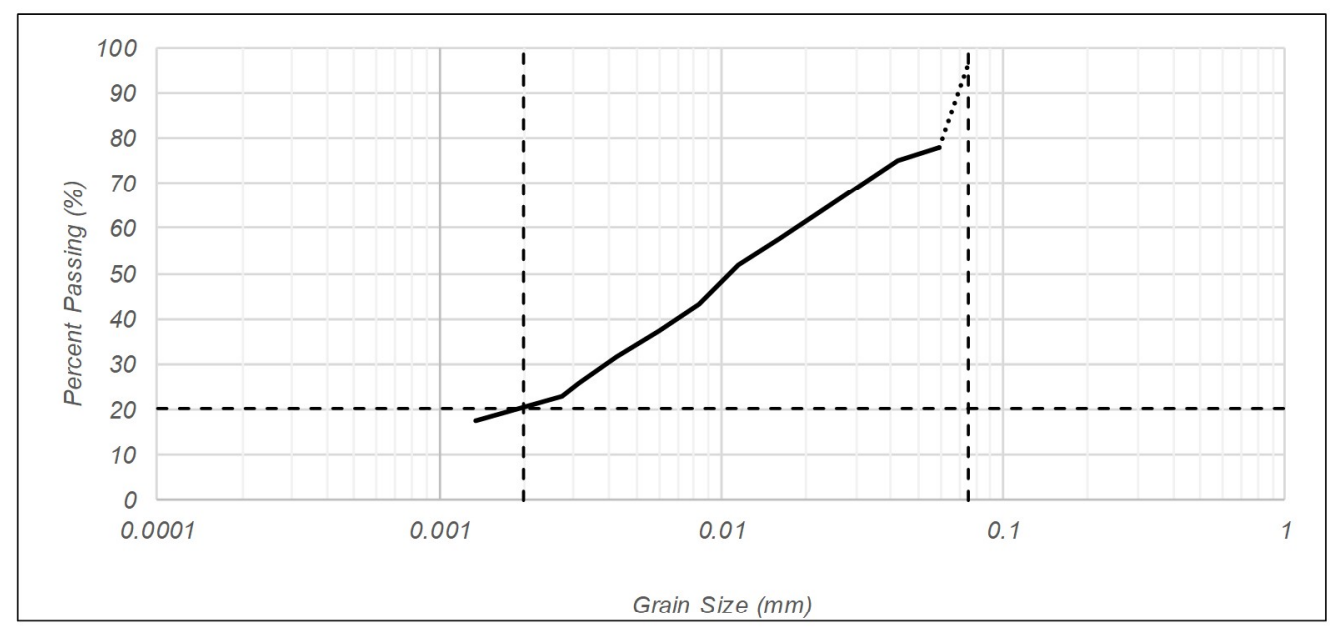

P200 Wash

$\begin{array}{lcc}\text { Container ID: } & \text { Q1 } & \\ \text { Before - } \mathrm{M}_{\mathrm{d}}: & 27.4 & \mathrm{~g} \\ \text { After - } \mathrm{M}_{\mathrm{d}} \text { : } & 1.2 & \mathrm{~g} \\ \text { Fines Fraction: } & 95.62 & \% \\ \text { Coarse Fraction: } & 4.38 & \%\end{array}$

\begin{tabular}{llr} 
& & Soil Breakdown \\
\cline { 2 - 3 } & Coarse: & $4.38 \%$ \\
& Silts: & $75.37 \%$ \\
From Hydrometer & Clays: & $20.25 \%$
\end{tabular}

Procedures Done By

Kayla/Melissa P. 


\section{S Portland State}

Hydrometer Test

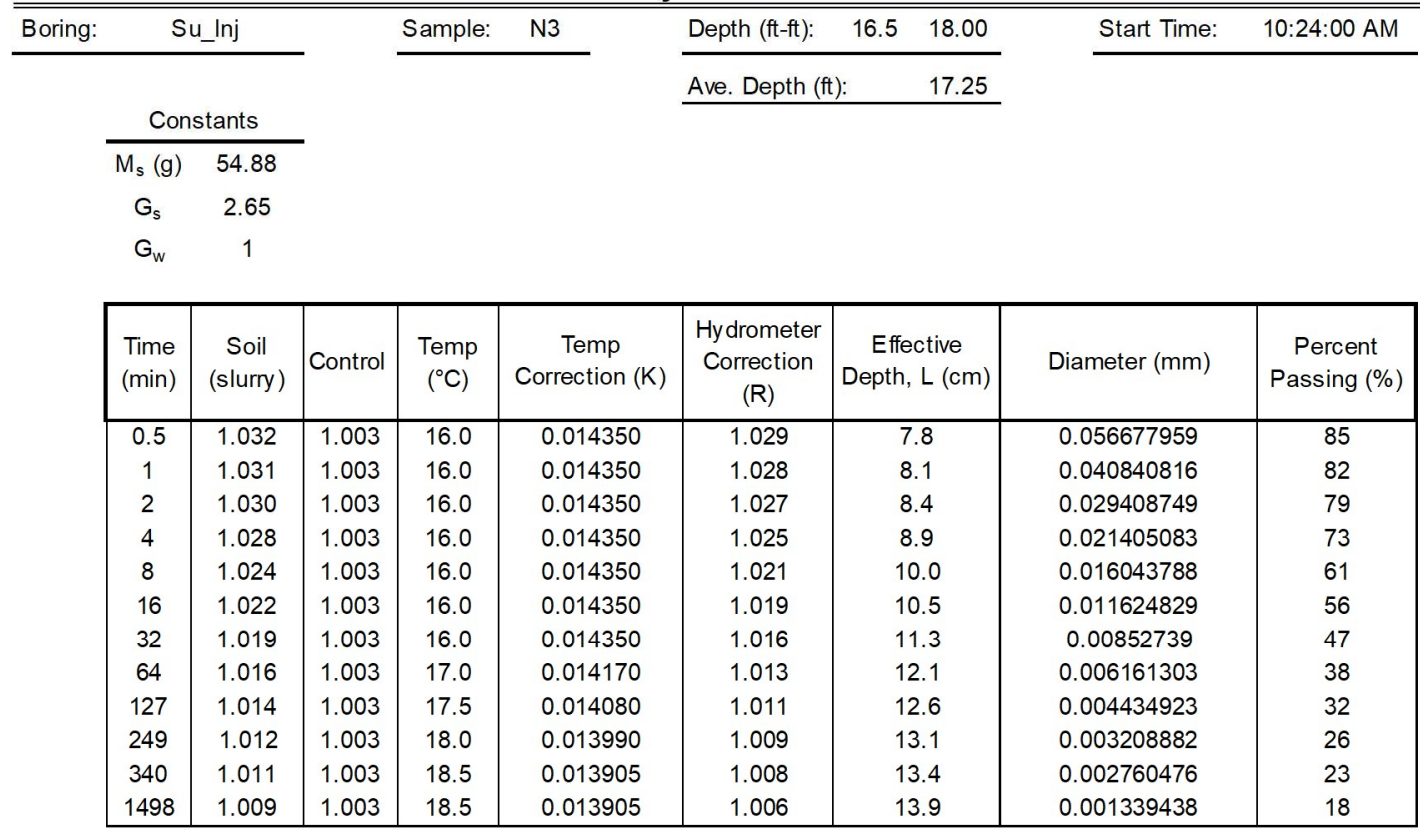

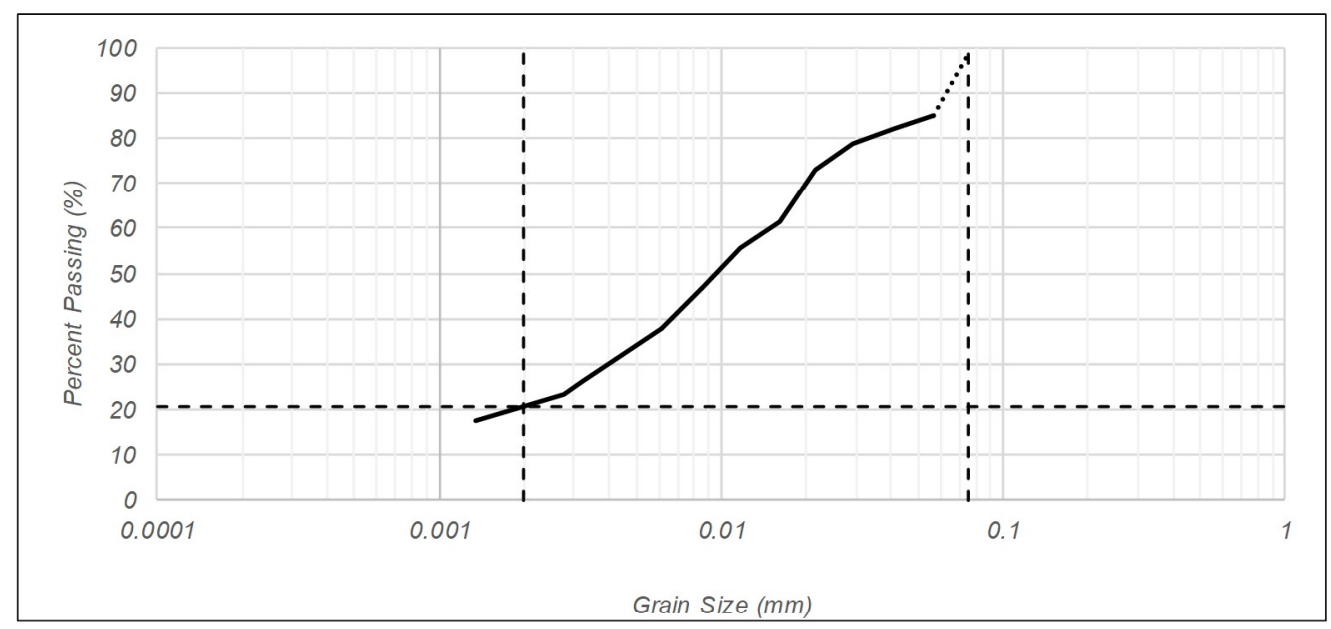

P200 Wash

$\begin{array}{lcc}\text { Container ID: } & \text { N1 } & \\ \text { Before }-\mathrm{M}_{\mathrm{d}}: & 30.3 \mathrm{~g} \\ \text { After }-\mathrm{M}_{\mathrm{d}} \text { : } & 0.5 & \mathrm{~g} \\ \text { Fines Fraction: } & 98.35 \% \\ \text { Coarse Fraction: } & 1.65 \%\end{array}$

\begin{tabular}{llr} 
& & Soil Breakdown \\
\cline { 2 - 3 } & Coarse: & $1.65 \%$ \\
& Silts: & $77.85 \%$ \\
From Hydrometer & Clays: & $20.50 \%$
\end{tabular}

Procedures Done By:

Kayla/Melissa P. 


\section{S Portland State}

Hydrometer Test

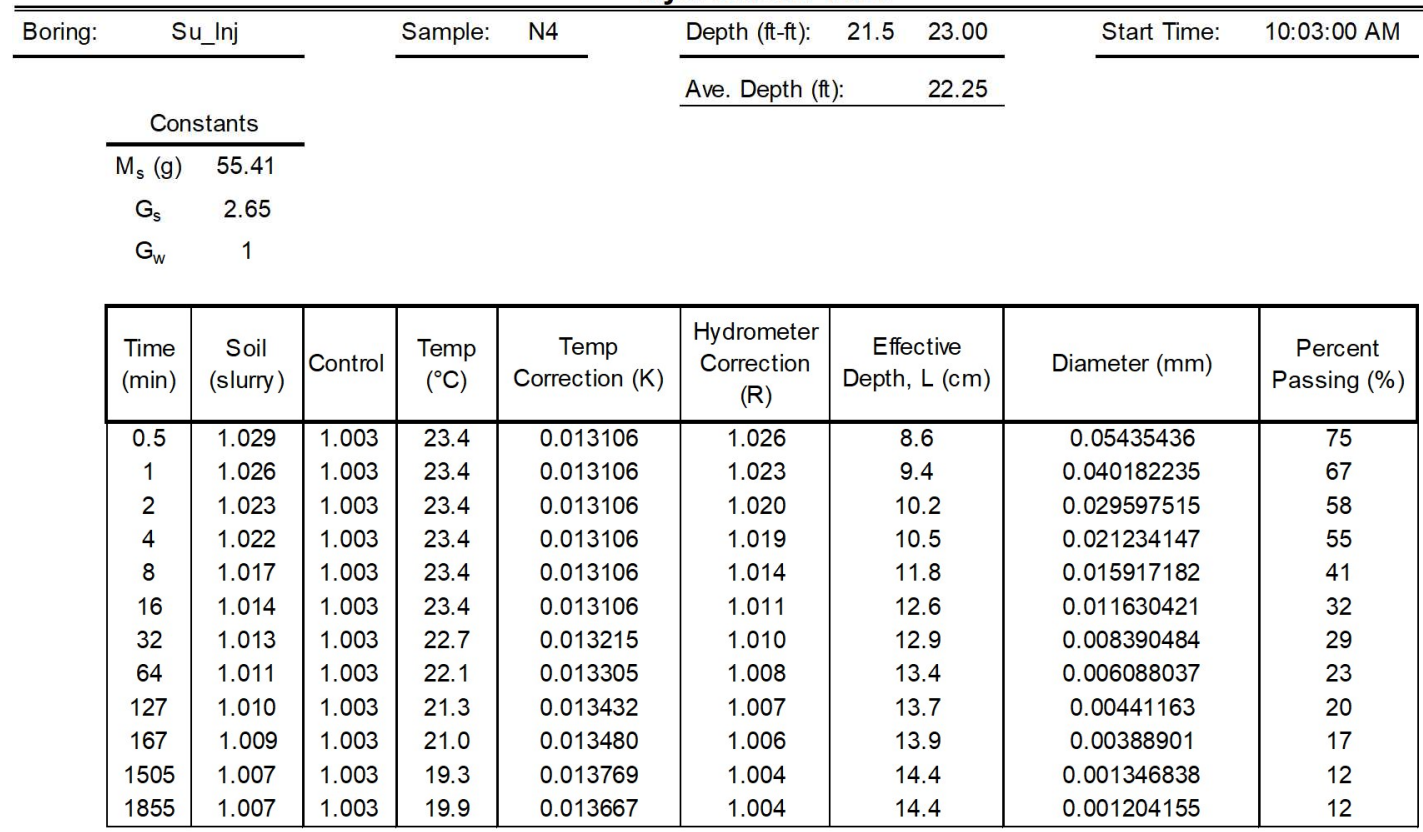

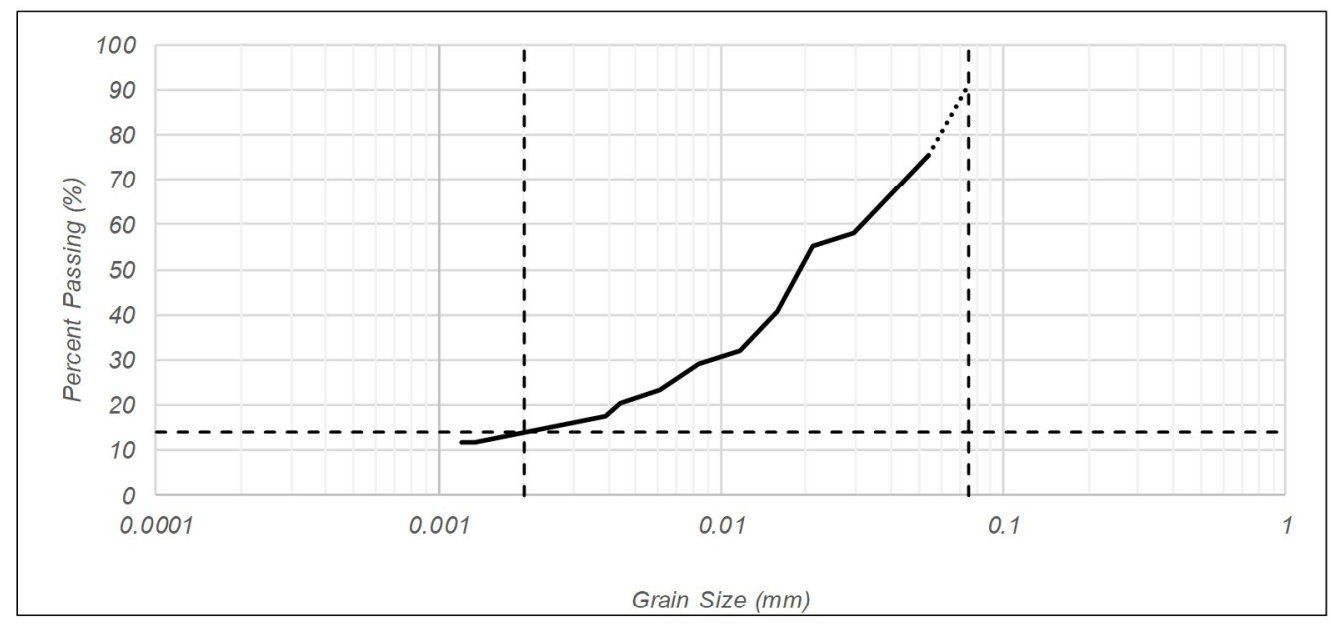

P200 Wash

$\begin{array}{lcl}\text { Container ID: } & \text { R1 } & \\ \text { Before }-\mathrm{M}_{\mathrm{d}}: & 25.7 & \mathrm{~g} \\ \text { After }-\mathrm{M}_{\mathrm{d}}: & 2.4 & \mathrm{~g} \\ \text { Fines Fraction: } & 90.66 & \% \\ \text { Coarse Fraction: } & 9.34 & \%\end{array}$

\begin{tabular}{llr} 
& \multicolumn{2}{c}{ Soil Breakdown } \\
\cline { 2 - 3 } & Coarse: & $9.34 \%$ \\
From Hydrometer & Silts: & $76.66 \%$ \\
& Clays: & $14.00 \%$
\end{tabular}

$\underline{\text { Procedures Done By: Kayla/Melissa P. }}$ 


\section{蛋 Portland $\underset{\text { SNIVESTIYY }}{\text { State }}$}

Hydrometer Test

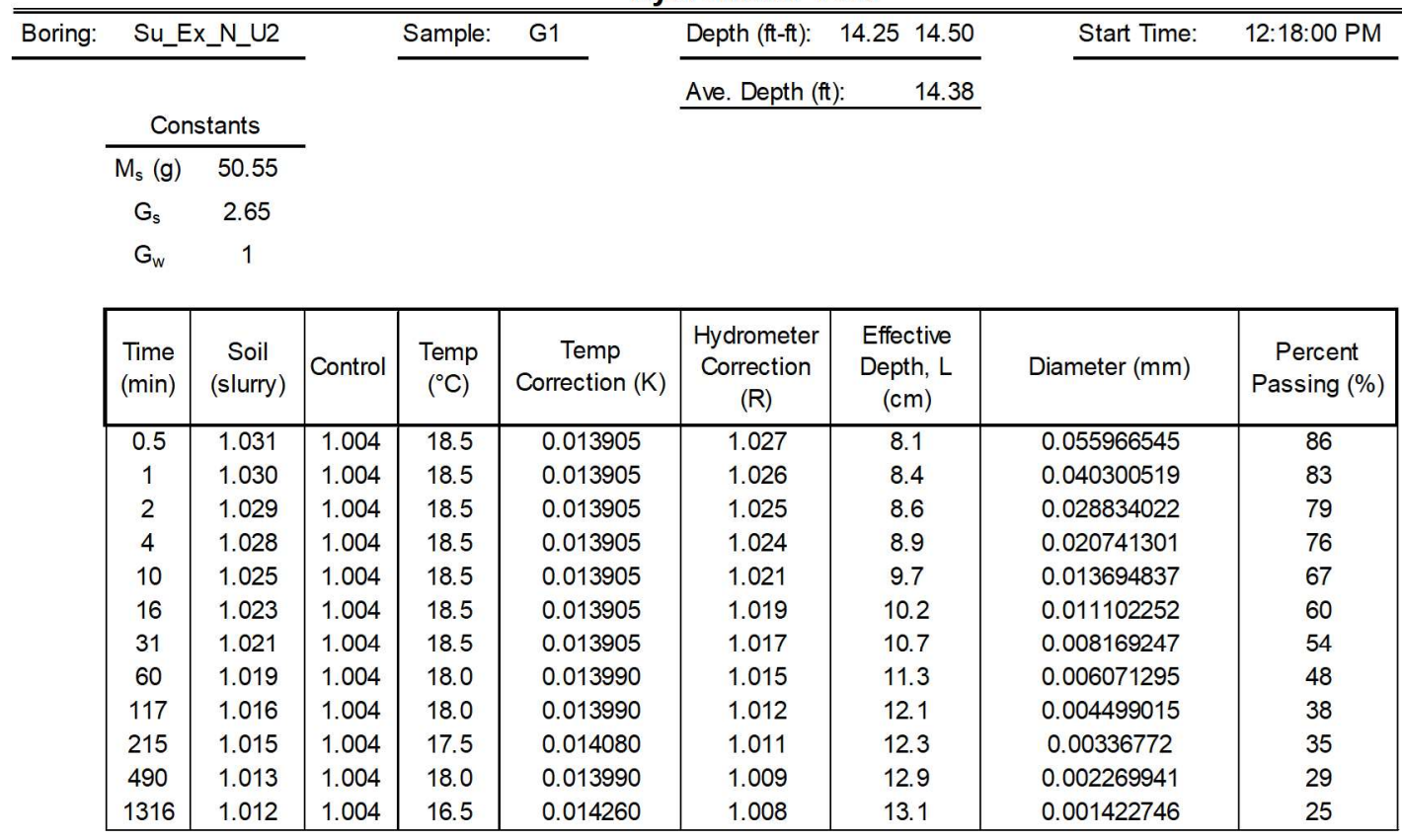

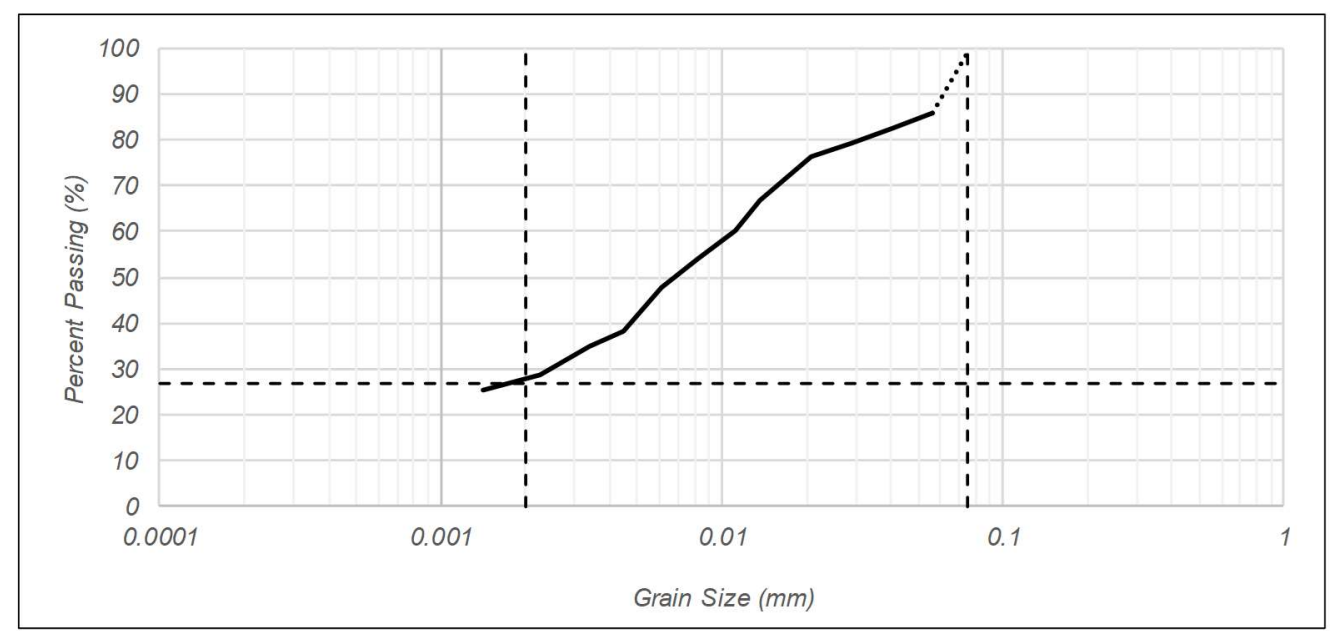

P200 Wash

$\begin{array}{lcc}\text { Container ID: } & \text { F1 } & \\ \text { Before }-\mathrm{M}_{\mathrm{d}}: & 6.8 & \mathrm{~g} \\ \text { After }-\mathrm{M}_{\mathrm{d}} \text { : } & 0.1 & \mathrm{~g} \\ \text { Fines Fraction: } & 98.53 & \% \\ \text { Coarse Fraction: } & 1.47 & \%\end{array}$

\begin{tabular}{llr} 
& & \multicolumn{1}{c}{ Soil Breakdown } \\
\cline { 2 - 3 } & Coarse: & $1.47 \%$ \\
& Silts: & $71.53 \%$ \\
From Hydrometer & Clays: & $27.00 \%$
\end{tabular}

Procedures Done By: Melissa B. 


\section{蛋 Portland $\underset{\text { SNIVEASITY }}{\text { State }}$}

Hydrometer Test

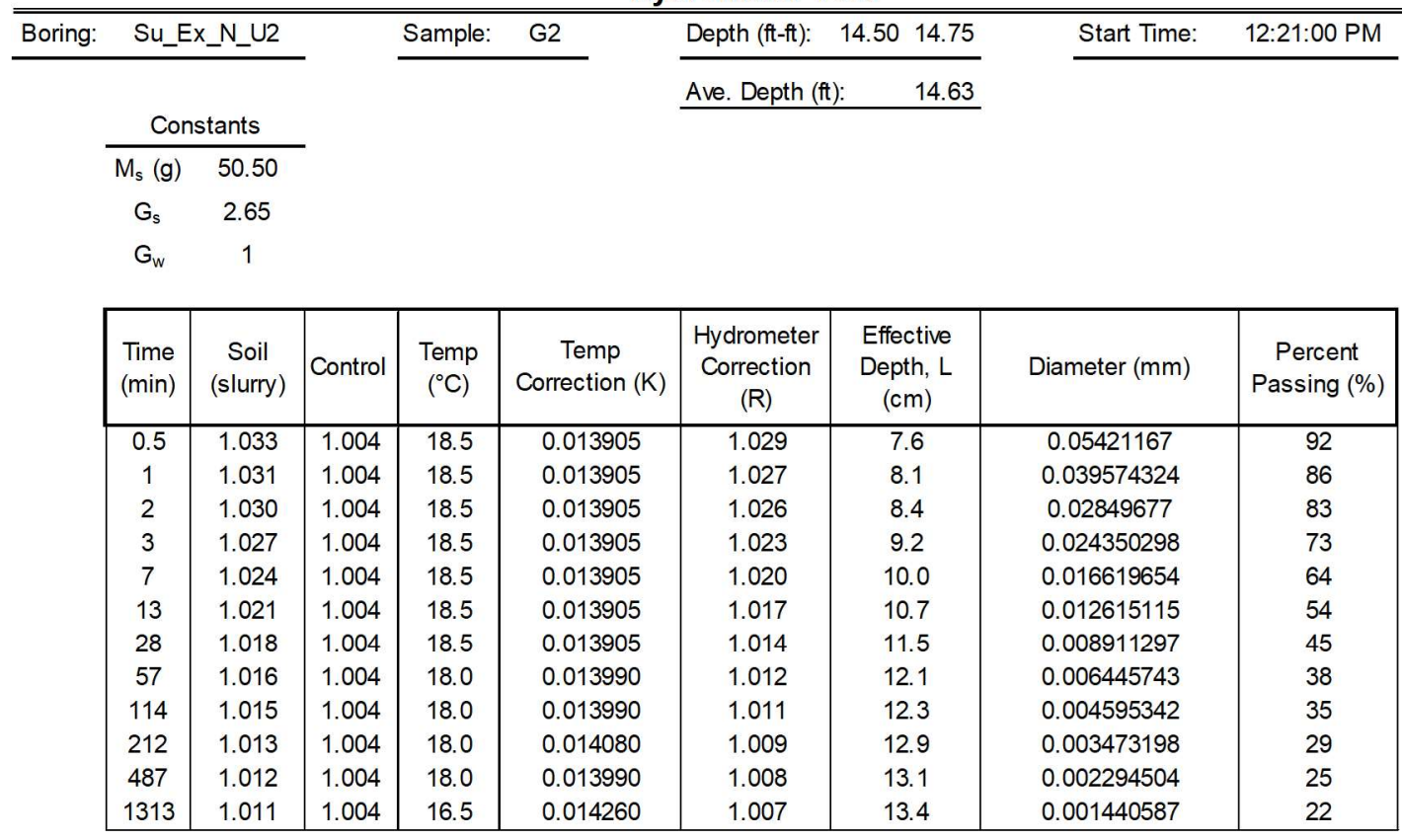

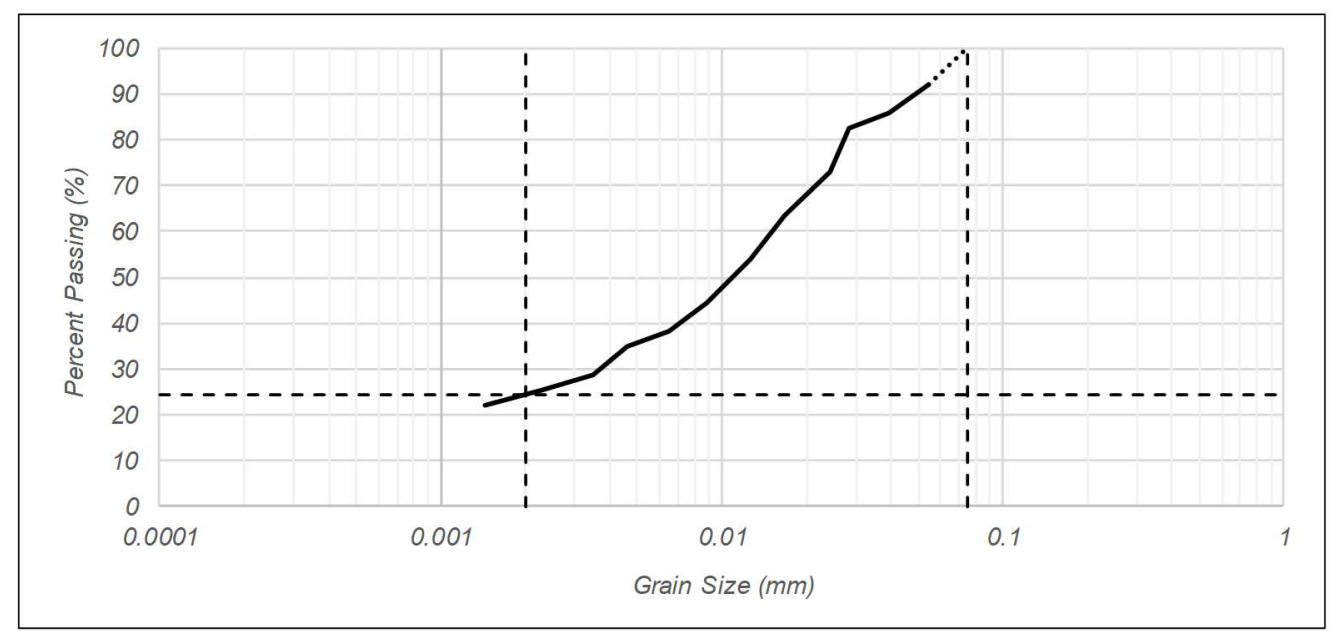

P200 Wash

$\begin{array}{lcc}\text { Container ID: } & \mathrm{K} 1 & \\ \text { Before }-\mathrm{M}_{\mathrm{d}}: & 15.9 \mathrm{~g} \\ \text { After }-\mathrm{M}_{\mathrm{d}}: & 0 & \mathrm{~g} \\ \text { Fines Fraction: } & 100.00 \% \\ \text { Coarse Fraction: } & 0.00 \%\end{array}$

\begin{tabular}{llr} 
& & Soil Breakdown \\
\cline { 2 - 3 } & Coarse: & $0.00 \%$ \\
From Hydrometer & Silts: & $75.50 \%$ \\
& Clays: & $24.50 \%$
\end{tabular}

Procedures Done By: Melissa B. 


\section{蛋 Portland $\underset{\text { UNIVESAITY }}{\text { State }}$}

Hydrometer Test

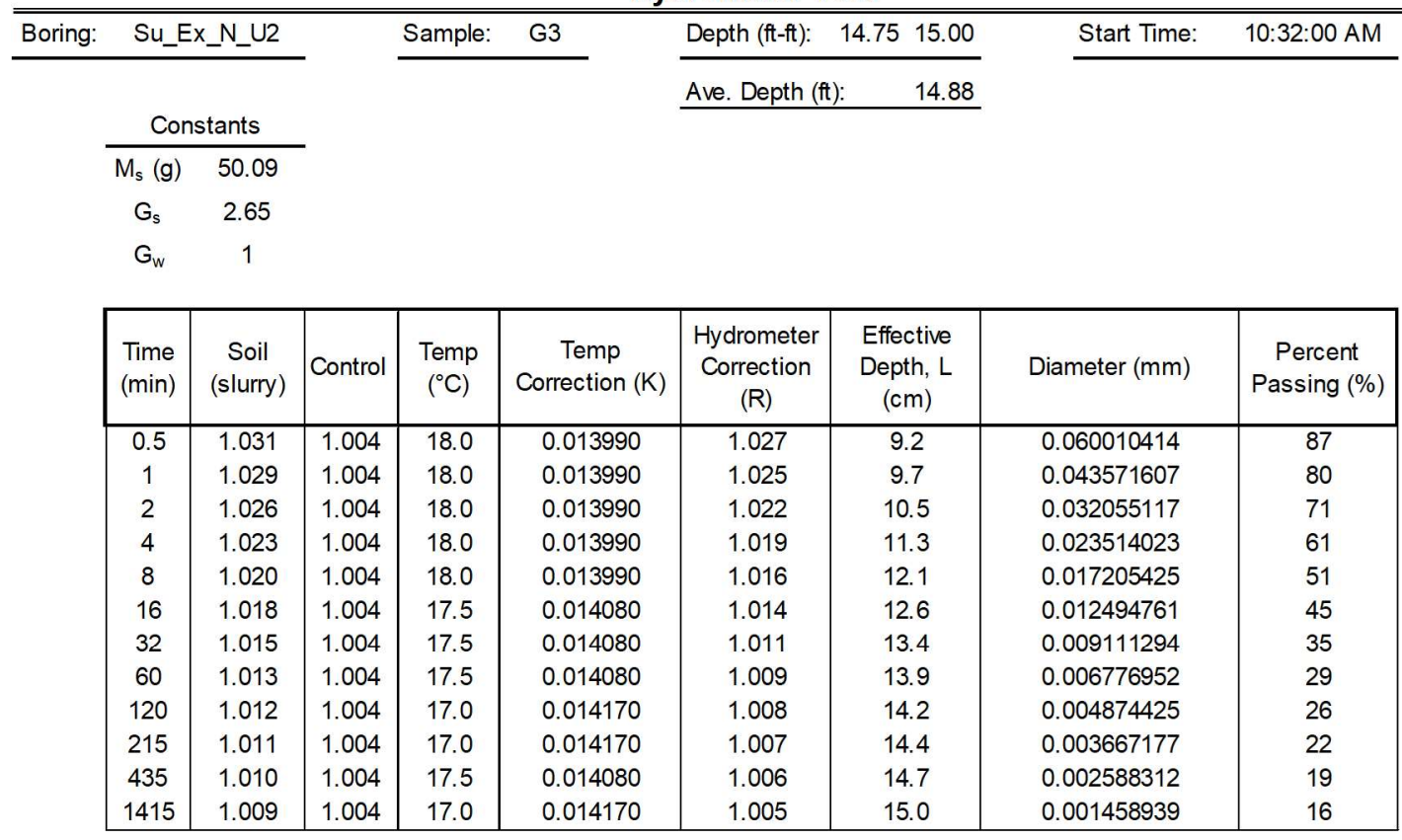

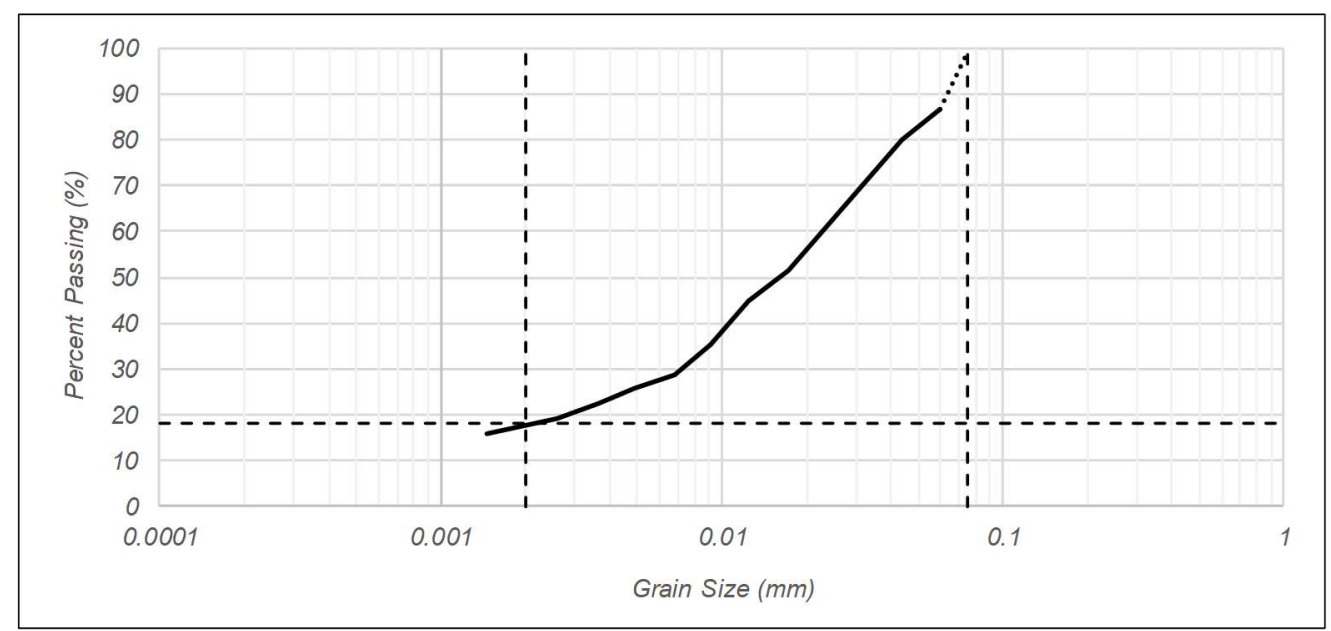

P200 Wash

$\begin{array}{lcc}\text { Container ID: } & \mathrm{V} 1 & \\ \text { Before }-\mathrm{M}_{\mathrm{d}} \text { : } & 9.0 & \mathrm{~g} \\ \text { After }-\mathrm{M}_{\mathrm{d}} \text { : } & 0.1 & \mathrm{~g} \\ \text { Fines Fraction: } & 98.89 & \% \\ \text { Coarse Fraction: } & 1.11 & \%\end{array}$

\begin{tabular}{llr} 
& & Soil Breakdown \\
\cline { 2 - 3 } & Coarse: & $1.11 \%$ \\
& Silts: & $80.89 \%$ \\
From Hydrometer & Clays: & $18.00 \%$
\end{tabular}

Procedures Done By: Melissa B. 


\section{蛋 Portland $\underset{\text { SNIVESTIYY }}{\text { State }}$}

Hydrometer Test

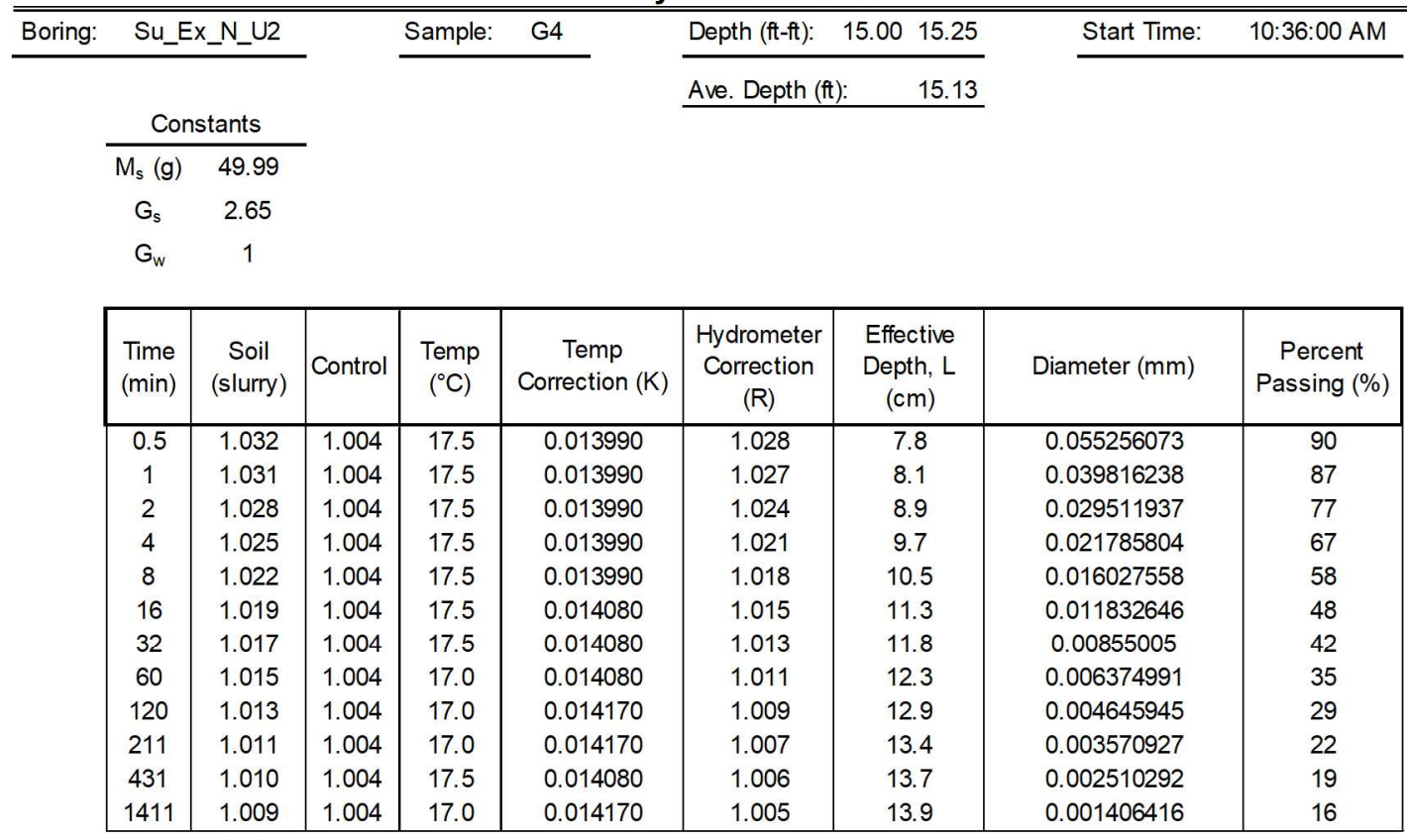

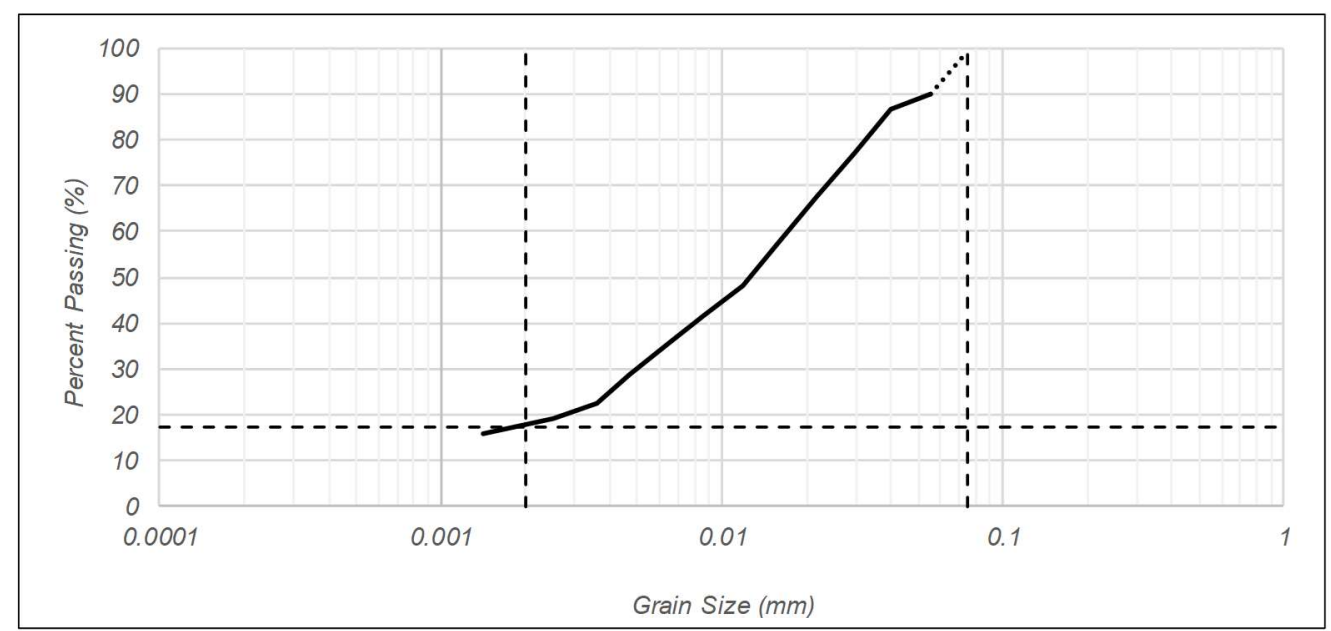

P200 Wash

$\begin{array}{lcc}\text { Container ID: } & \text { D1 } & \\ \text { Before }-\mathrm{M}_{\mathrm{d}}: & 12.2 & \mathrm{~g} \\ \text { After }-\mathrm{M}_{\mathrm{d}} \text { : } & 0.1 & \mathrm{~g} \\ \text { Fines Fraction: } & 99.18 \% \\ \text { Coarse Fraction: } & 0.82 \%\end{array}$

\begin{tabular}{llr} 
& & Soil Breakdown \\
\cline { 2 - 3 } & Coarse: & $0.82 \%$ \\
& Silts: & $81.68 \%$ \\
From Hydrometer & Clays: & $17.50 \%$
\end{tabular}

Procedures Done By: Melissa B. 


\section{蛋 Portland $\underset{\text { SNIVESTIYY }}{\text { State }}$}

Hydrometer Test

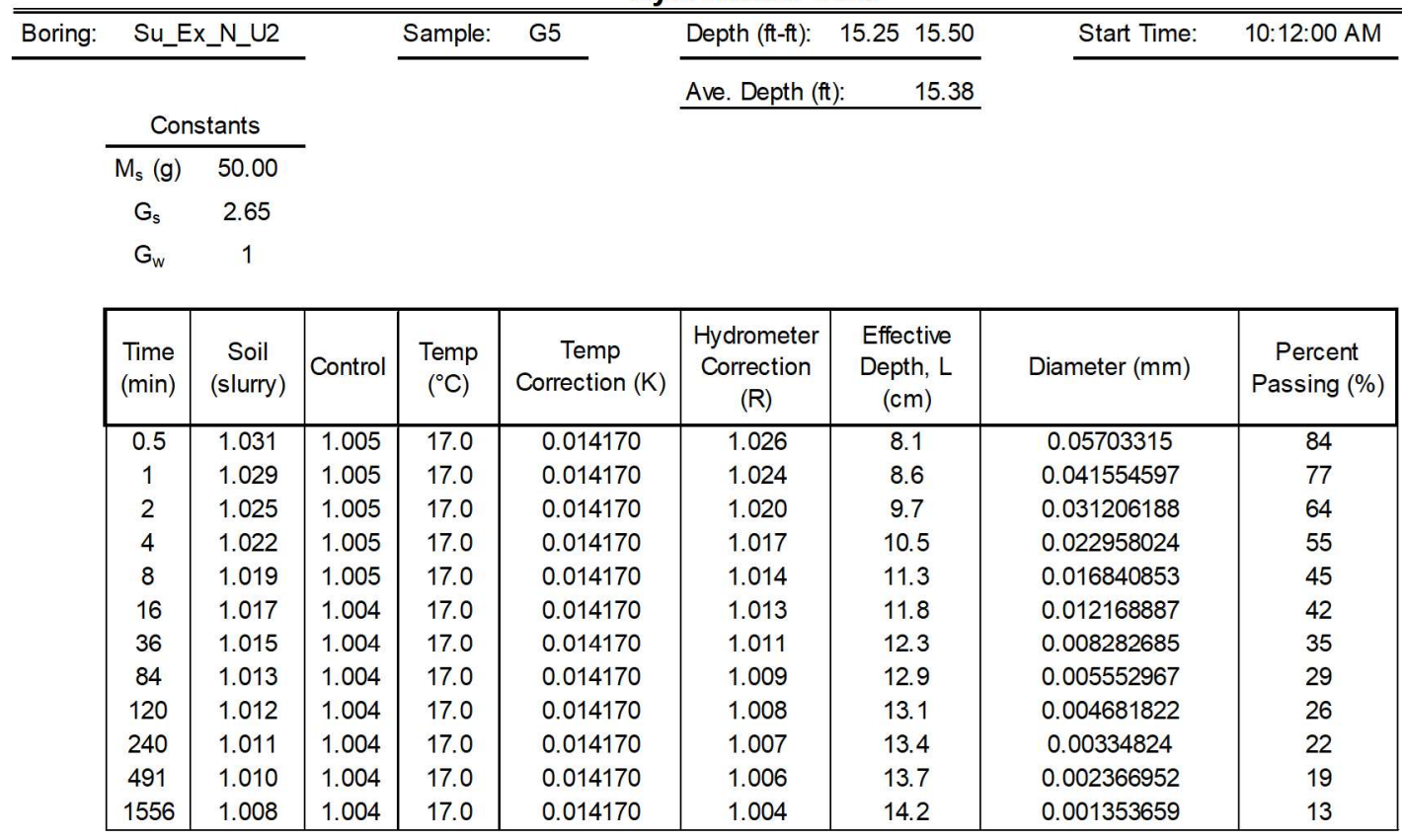

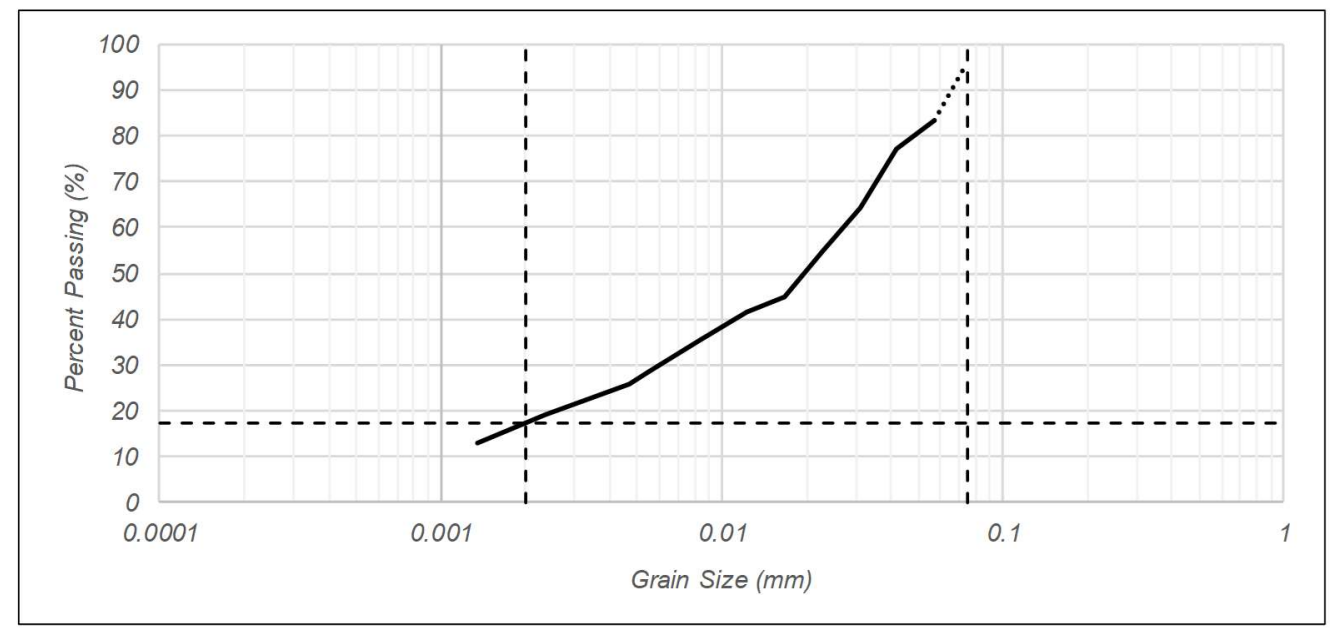

P200 Wash

$\begin{array}{lcc}\text { Container ID: } & \text { N1 } & \\ \text { Before }-\mathrm{M}_{\mathrm{d}}: & 7.4 & \mathrm{~g} \\ \text { After }-\mathrm{M}_{\mathrm{d}}: & 0.3 & \mathrm{~g} \\ \text { Fines Fraction: } & 95.95 \% \\ \text { Coarse Fraction: } & 4.05 \%\end{array}$

\begin{tabular}{llr} 
& & \multicolumn{2}{c}{ Soil Breakdown } \\
\cline { 2 - 3 } & Coarse: & $4.05 \%$ \\
& Silts: & $78.45 \%$ \\
From Hydrometer & Clays: & $17.50 \%$
\end{tabular}

Procedures Done By: $\quad$ Melissa B. 


\section{蛋 Portland $\underset{\text { UNIVESAITY }}{\text { State }}$}

Hydrometer Test

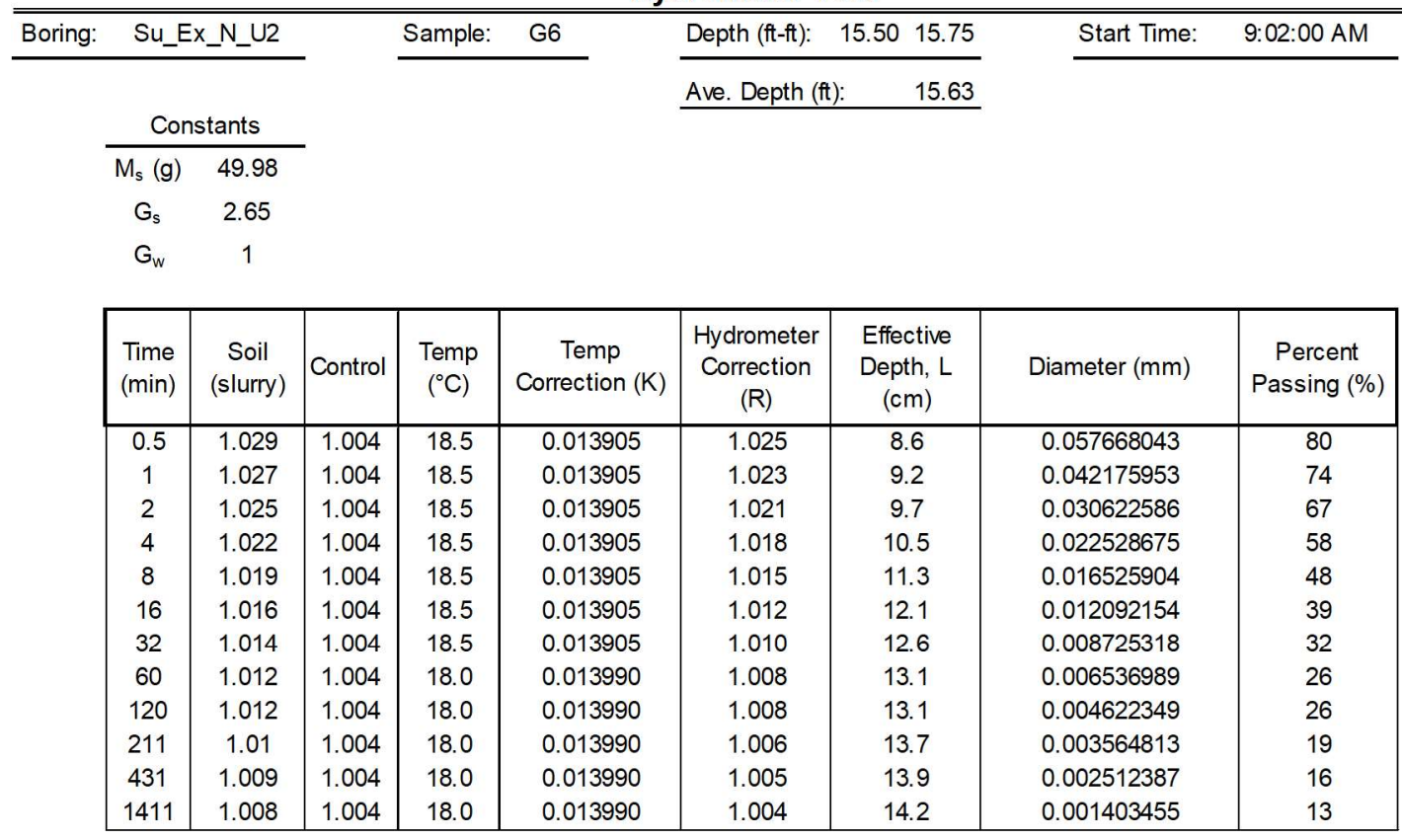

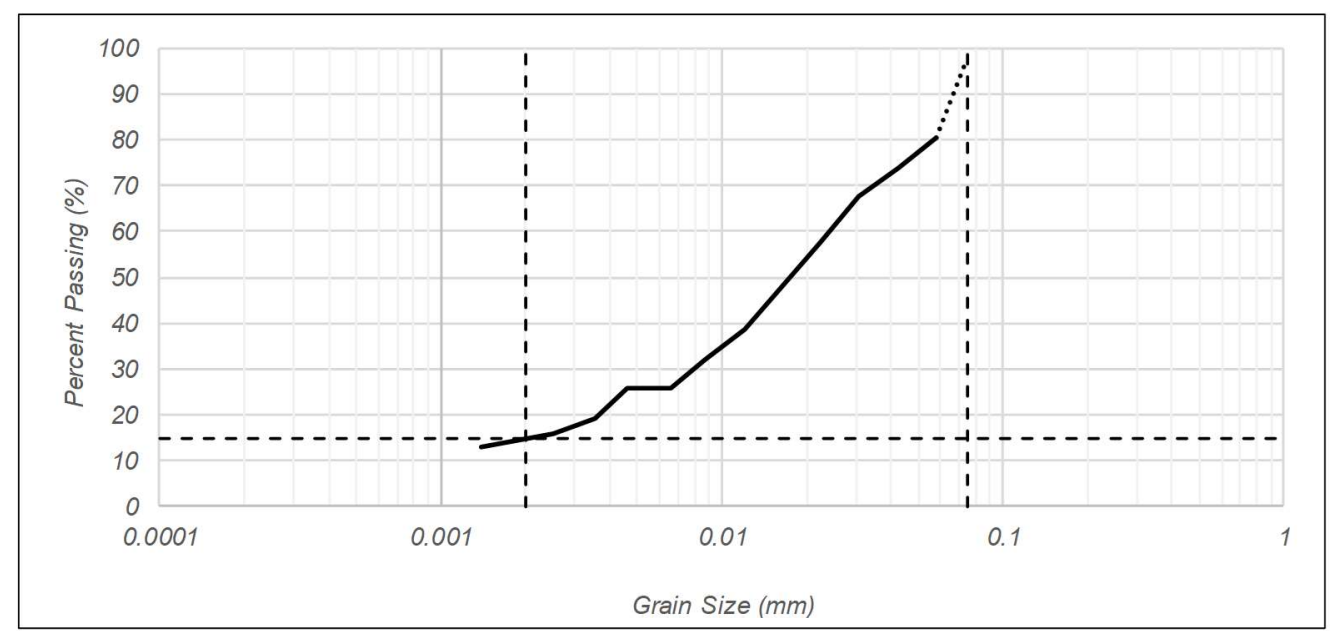

P200 Wash

$\begin{array}{lcc}\text { Container ID: } & \mathrm{J} 1 & \\ \text { Before }-\mathrm{M}_{\mathrm{d}} \text { : } & 7.4 & \mathrm{~g} \\ \text { After }-\mathrm{M}_{\mathrm{d}} \text { : } & 0.2 & \mathrm{~g} \\ \text { Fines Fraction: } & 97.30 & \% \\ \text { Coarse Fraction: } & 2.70 & \%\end{array}$

\begin{tabular}{llr} 
& & Soil Breakdown \\
\cline { 2 - 3 } & Coarse: & $2.70 \%$ \\
& Silts: & $82.30 \%$ \\
From Hydrometer & Clays: & $15.00 \%$
\end{tabular}

Procedures Done By: Melissa B. 


\section{蛋 Portland Stative StIYY}

Hydrometer Test

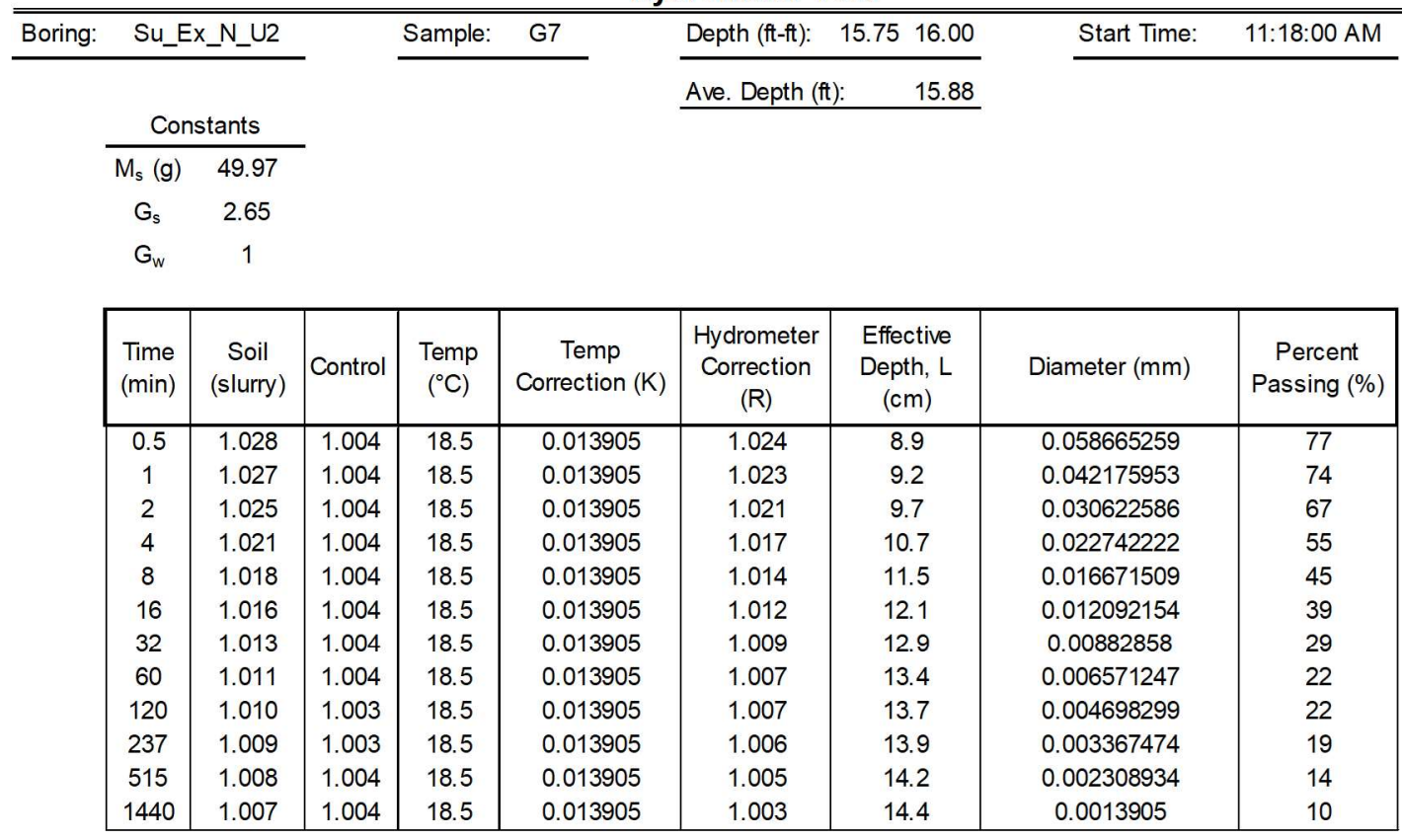

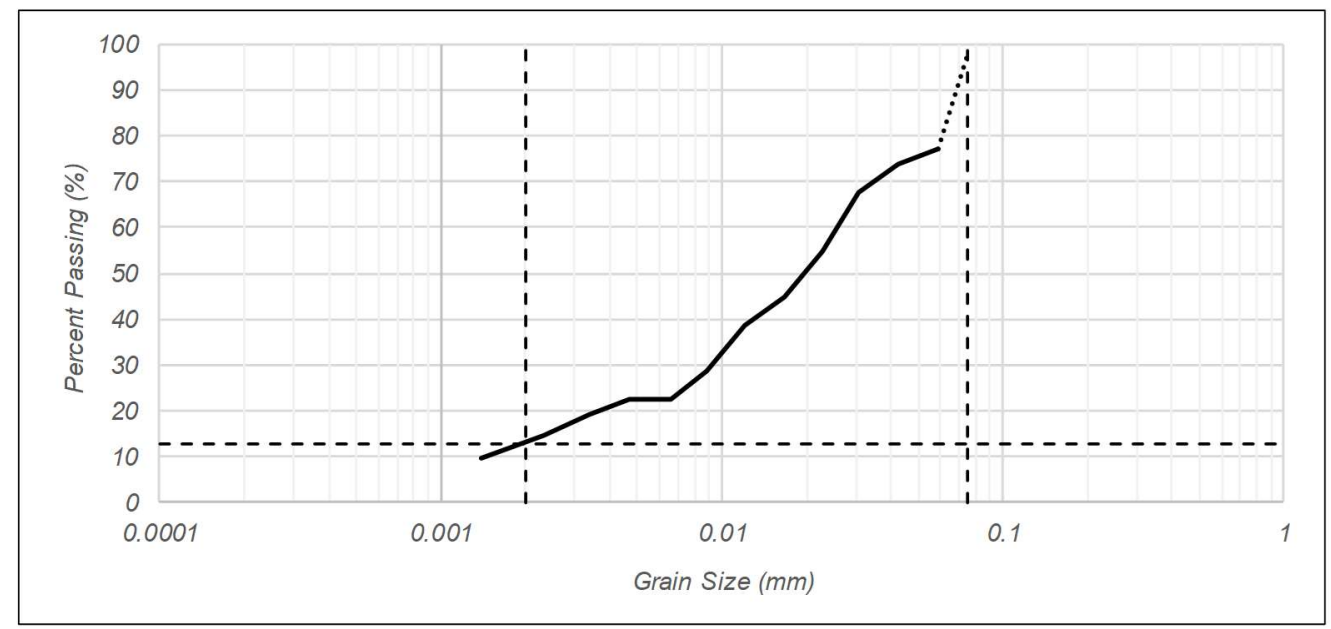

P200 Wash

$\begin{array}{lcc}\text { Container ID: } & \text { X1 } & \\ \text { Before }-\mathrm{M}_{\mathrm{d}}: & 6.8 & \mathrm{~g} \\ \text { After }-\mathrm{M}_{\mathrm{d}} \text { : } & 0.2 & \mathrm{~g} \\ \text { Fines Fraction: } & 97.06 & \% \\ \text { Coarse Fraction: } & 2.94 & \%\end{array}$

\begin{tabular}{llr} 
& & Soil Breakdown \\
\cline { 2 - 3 } & Coarse: & $2.94 \%$ \\
& Silts: & $84.06 \%$ \\
From Hydrometer & Clays: & $13.00 \%$
\end{tabular}

Procedures Done By: Melissa B. 


\section{蛋 Portland $\underset{\text { SNIVEASITY }}{\text { State }}$}

Hydrometer Test

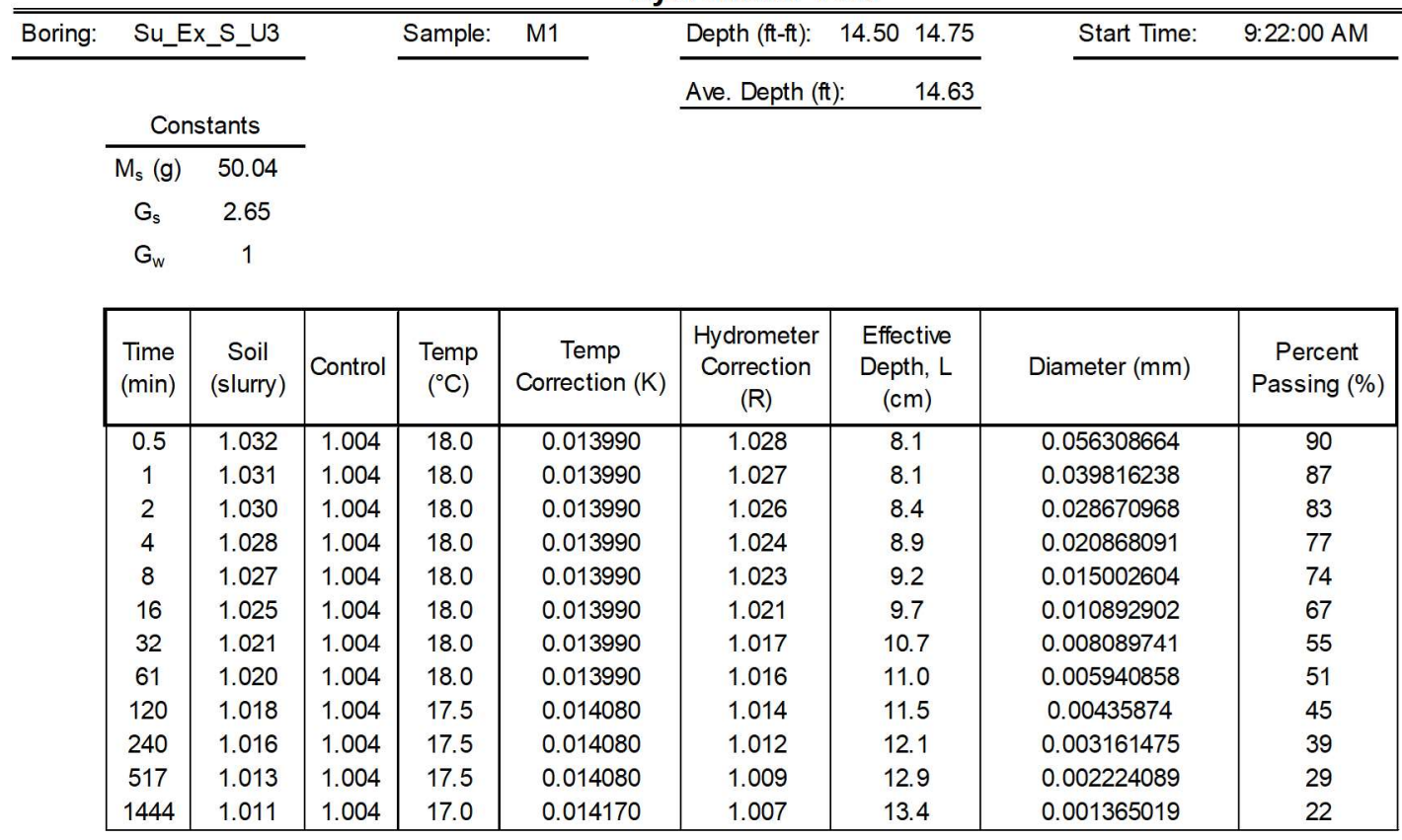

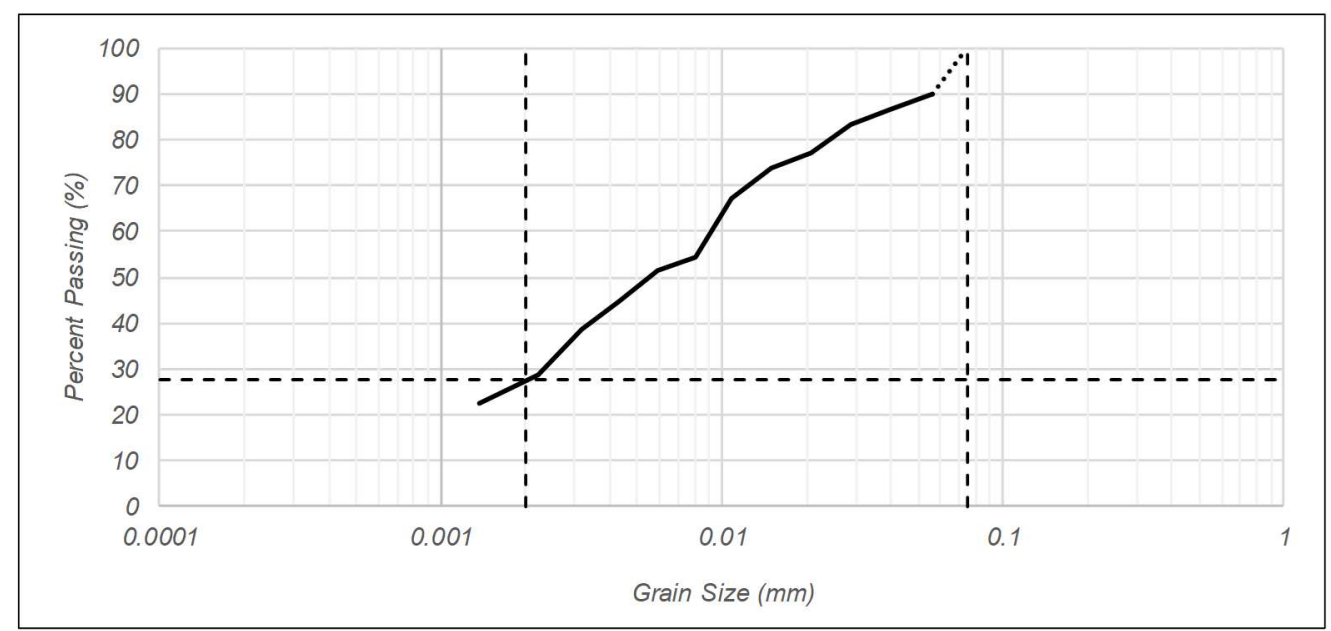

P200 Wash

Container ID: $\quad$ D1

Before - $\mathrm{M}_{\mathrm{d}}$ : $\quad 4.5 \mathrm{~g}$

After $-\mathrm{M}_{\mathrm{d}}$ : $\quad 0 \mathrm{~g}$

Fines Fraction: $\quad 100.00 \%$

Coarse Fraction: $\quad 0.00 \%$

$\begin{array}{llr} & & \\ & & \\ & \text { Coarse: } & \text { Soil Breakdown } \\ & \text { Silts: } & 72.00 \% \\ \text { From Hydrometer } & \text { Clays: } & 27.50 \%\end{array}$

Procedures Done By: Melissa B. 


\section{蛋 Portland $\underset{\text { SNIVESITY }}{\text { State }}$}

Hydrometer Test

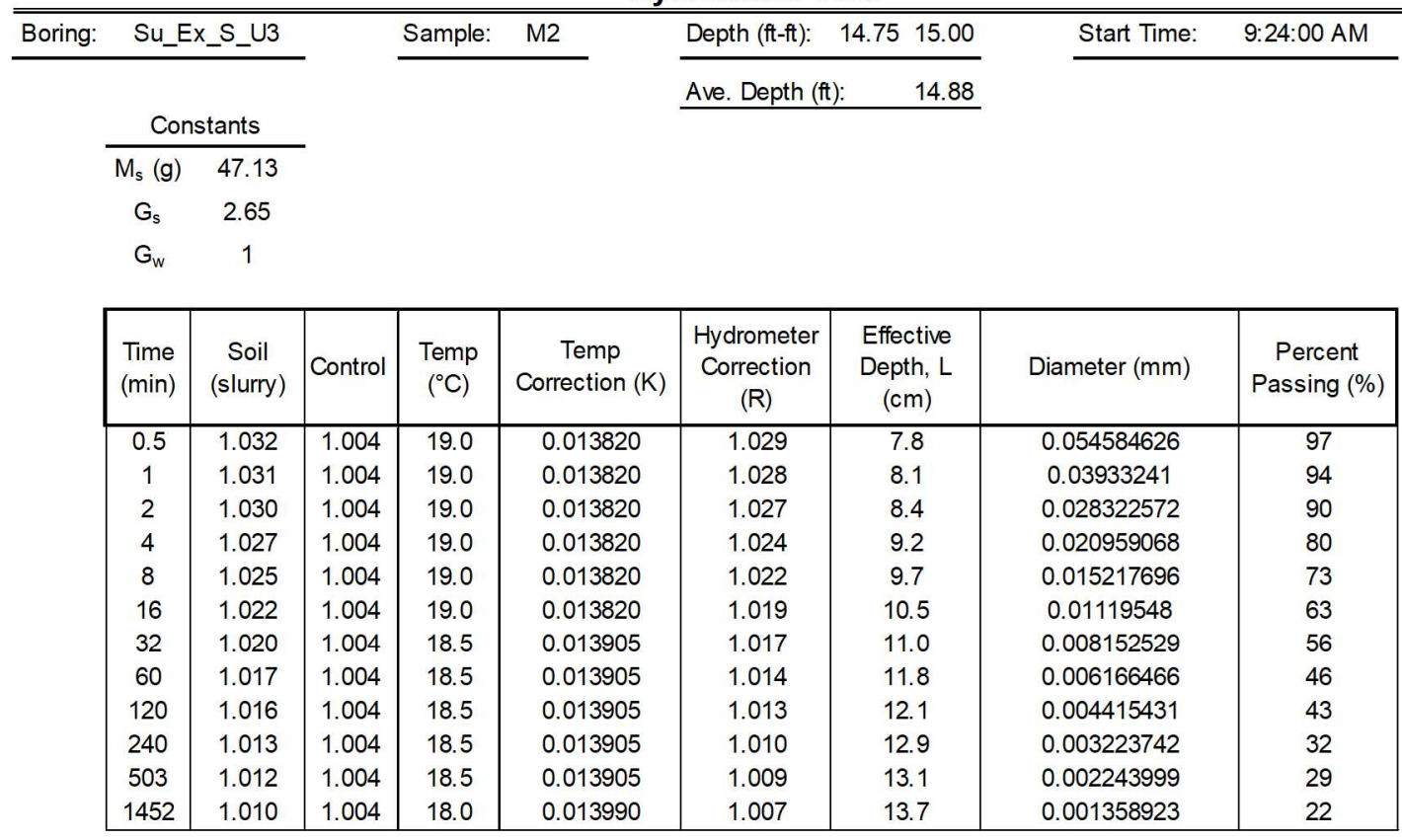

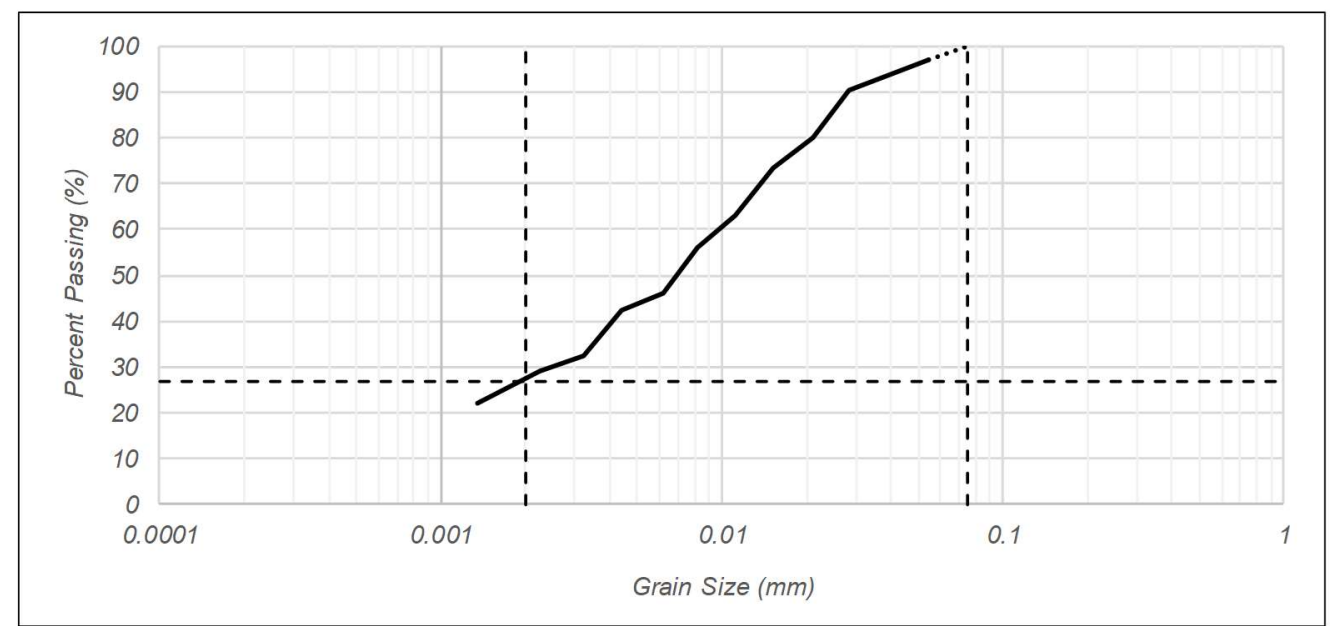

P200 Wash

Before - $M_{d}$ : $\quad 16.3 \mathrm{~g}$

After $-M_{d}: \quad 0 \mathrm{~g}$

Fines Fraction: $\quad 100.00 \%$

Coarse Fraction: $\quad 0.00 \%$

\begin{tabular}{llr} 
& & Soil Breakdown \\
\cline { 2 - 3 } & Coarse: & $0.00 \%$ \\
& Silts: & $73.00 \%$ \\
From Hydrometer & Clays: & $27.00 \%$
\end{tabular}

Procedures Done By: Melissa B. 


\section{蛋 Portland $\underset{\text { UNIVESAITY }}{\text { State }}$}

Hydrometer Test

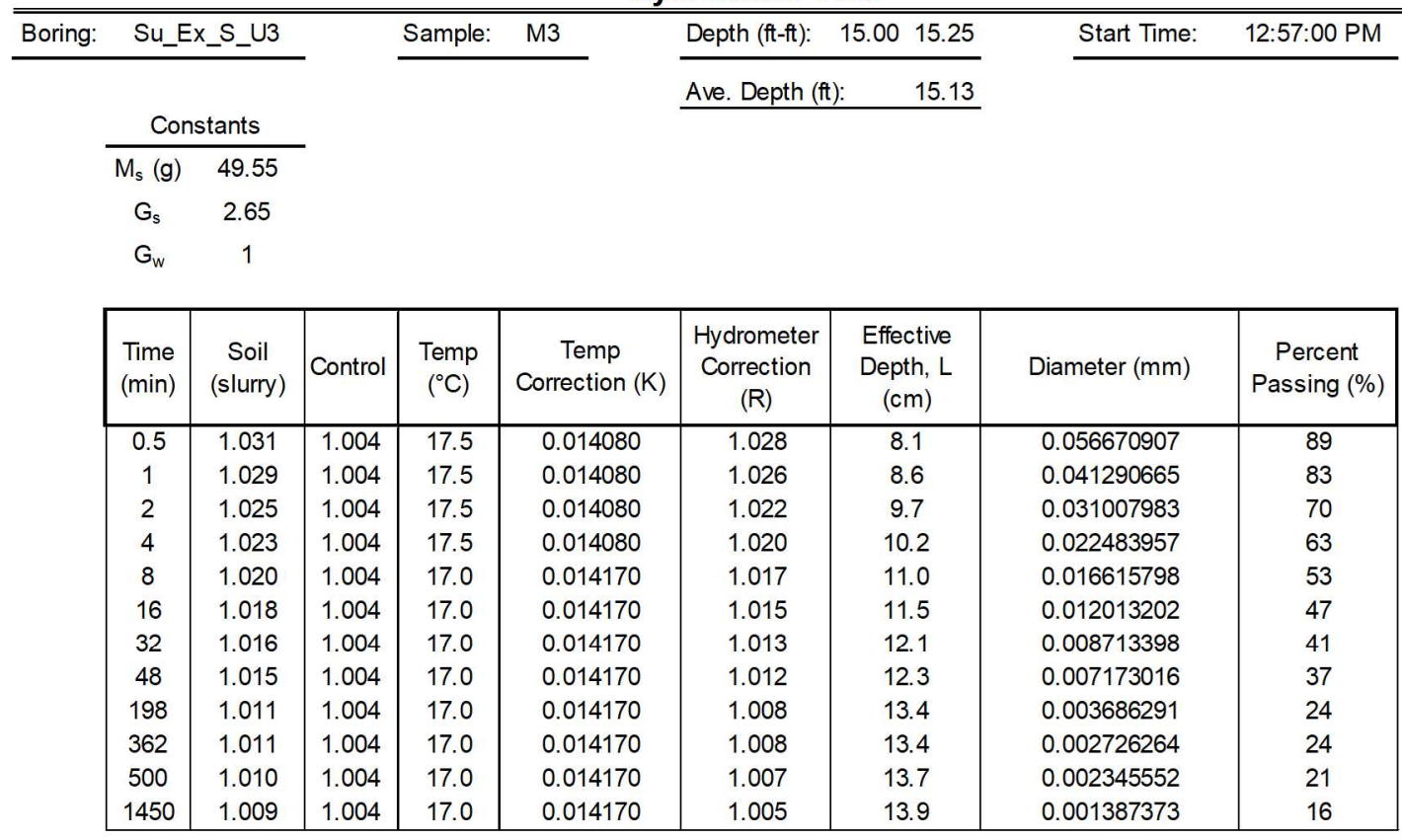

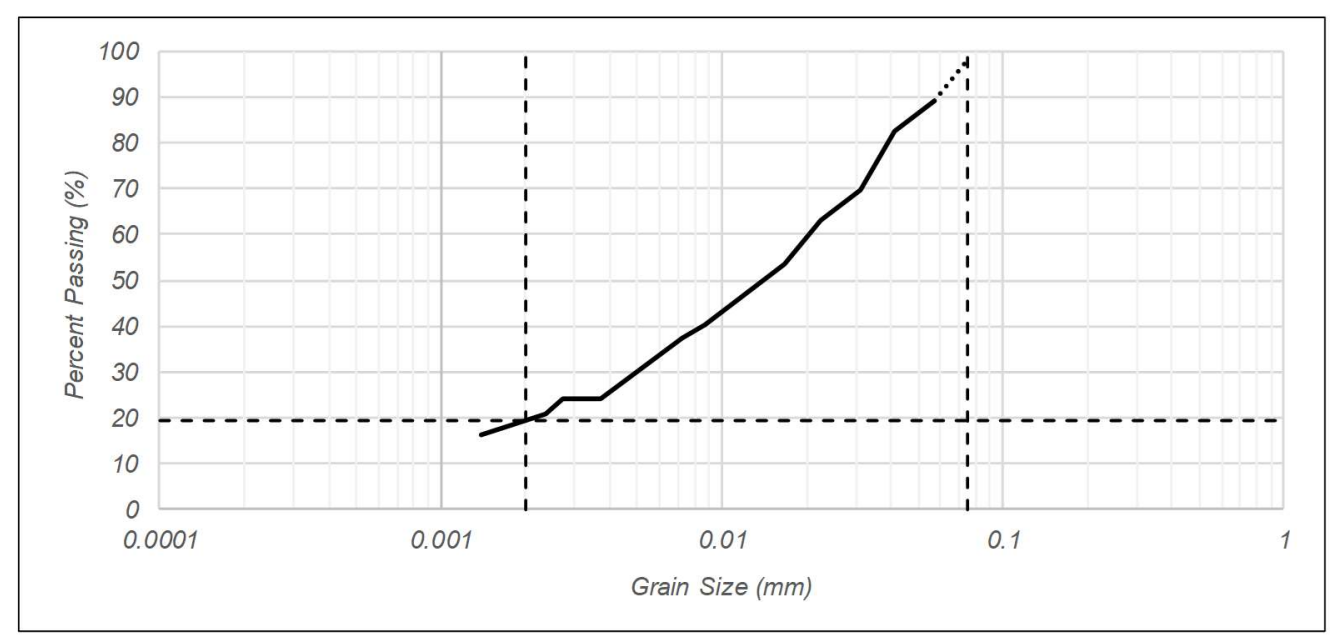

P200 Wash

$\begin{array}{lcc}\text { Container ID: } & \text { A1 } & \\ \text { Before }-\mathrm{M}_{\mathrm{d}}: & 20.5 & \mathrm{~g} \\ \text { After }-\mathrm{M}_{\mathrm{d}} \text { : } & 0.4 & \mathrm{~g} \\ \text { Fines Fraction: } & 98.05 \% \\ \text { Coarse Fraction: } & 1.95 \%\end{array}$

\begin{tabular}{llr} 
& & Soil Breakdown \\
\cline { 2 - 3 } & Coarse: & $1.95 \%$ \\
& Silts: & $78.55 \%$ \\
From Hydrometer & Clays: & $19.50 \%$
\end{tabular}

Procedures Done By: $\quad$ Melissa B. 


\section{蛋 Portland $\underset{\text { SNIVESAITY }}{\text { State }}$}

Hydrometer Test

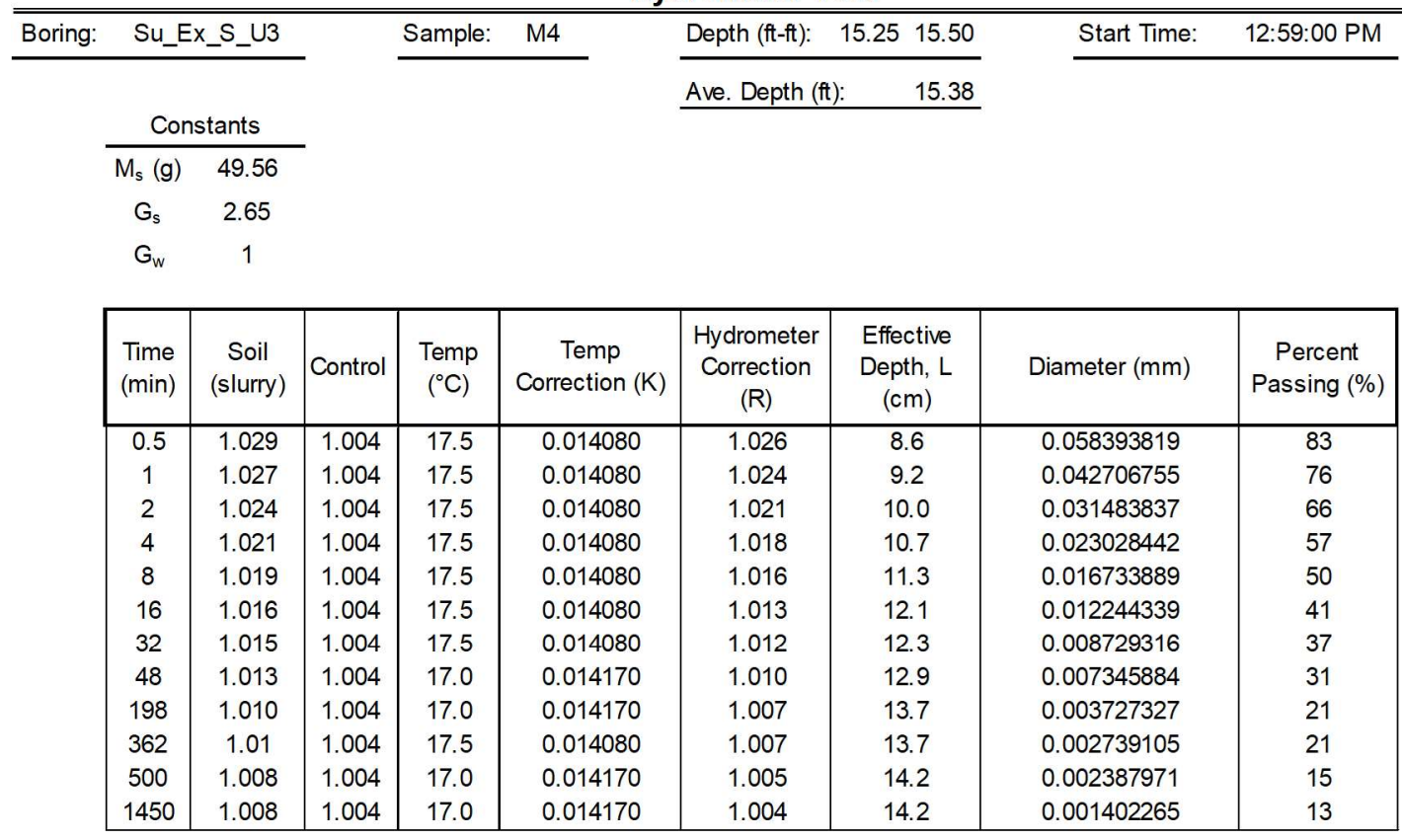

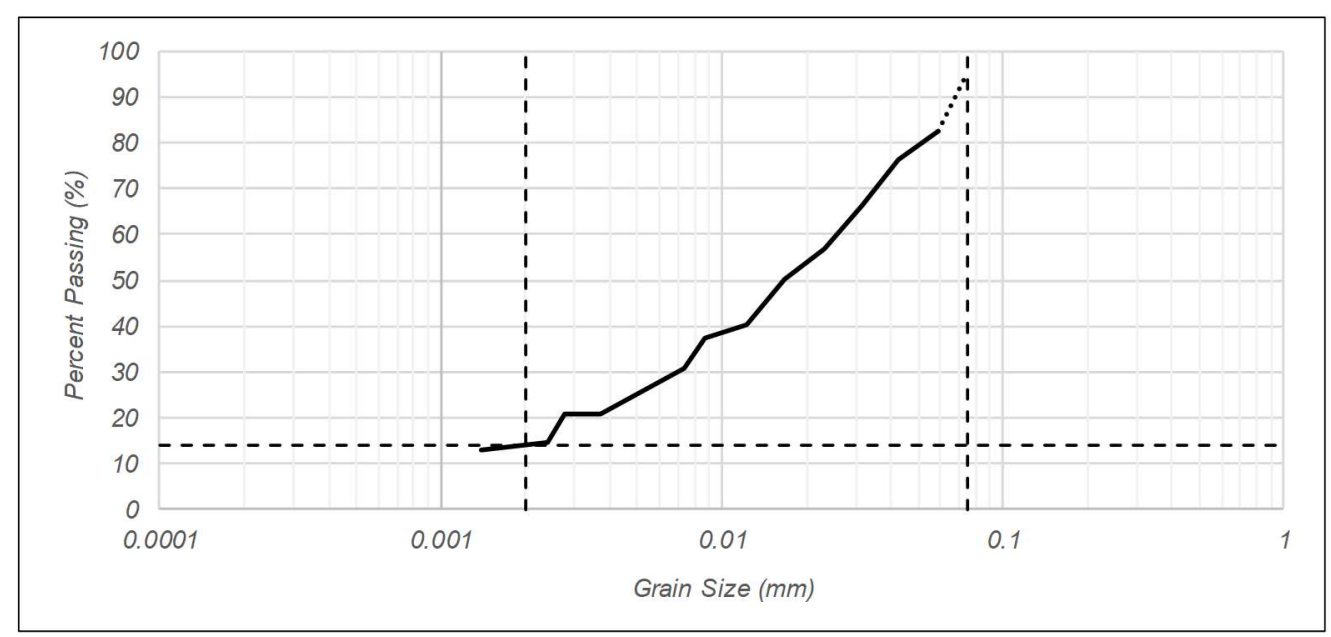

P200 Wash

$\begin{array}{lcc}\text { Container ID: } & \text { C1 } & \\ \text { Before }-\mathrm{M}_{\mathrm{d}}: & 17.2 & \mathrm{~g} \\ \text { After }-\mathrm{M}_{\mathrm{d}} \text { : } & 0.9 & \mathrm{~g} \\ \text { Fines Fraction: } & 94.77 & \% \\ \text { Coarse Fraction: } & 5.23 & \%\end{array}$

\begin{tabular}{llr} 
& & Soil Breakdown \\
\cline { 2 - 3 } & Coarse: & $5.23 \%$ \\
& Silts: & $80.77 \%$ \\
From Hydrometer & Clays: & $14.00 \%$
\end{tabular}

Procedures Done By: Melissa B. 


\section{蛋 Portland $\underset{\text { SNIVESITY }}{\text { State }}$}

Hydrometer Test

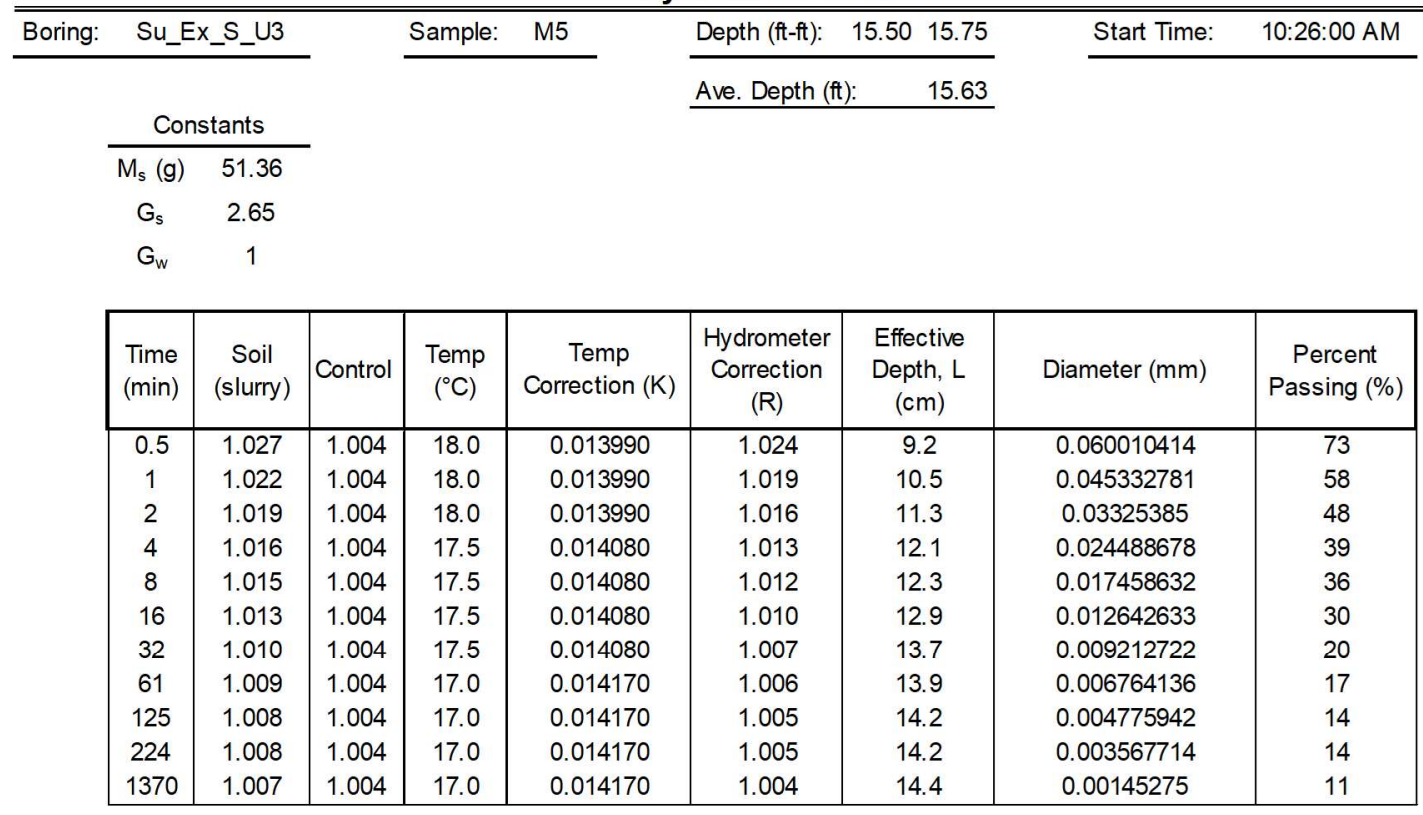

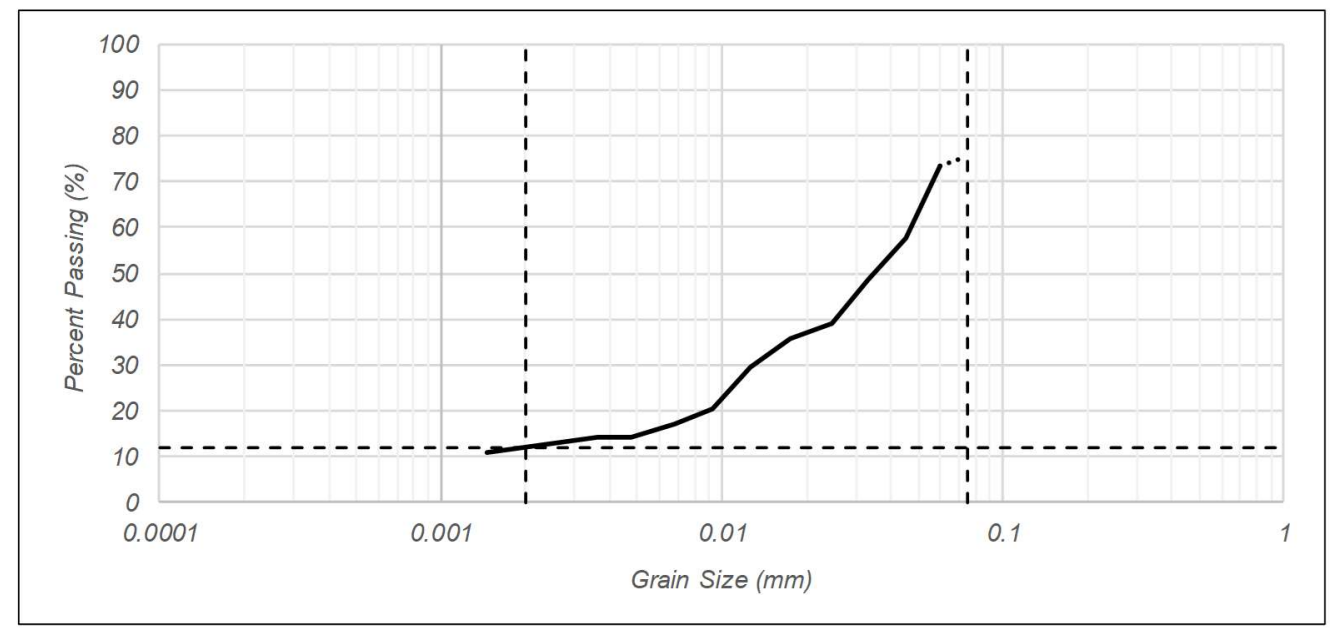

P200 Wash

Before - $M_{d}$ : $\quad 15.4 \mathrm{~g}$

After $-\mathrm{M}_{\mathrm{d}}$ : $\quad 3.8 \mathrm{~g}$

Fines Fraction: $\quad 75.32 \%$

Coarse Fraction: $\quad 24.68 \%$

\begin{tabular}{llr} 
& & Soil Breakdown \\
\cline { 2 - 3 } & Coarse: & $24.68 \%$ \\
& Silts: & $63.32 \%$ \\
From Hydrometer & Clays: & $12.00 \%$
\end{tabular}

Procedures Done By: Melissa B. 


\section{Appendix E: Field Logs of Well Installation - Harborton}

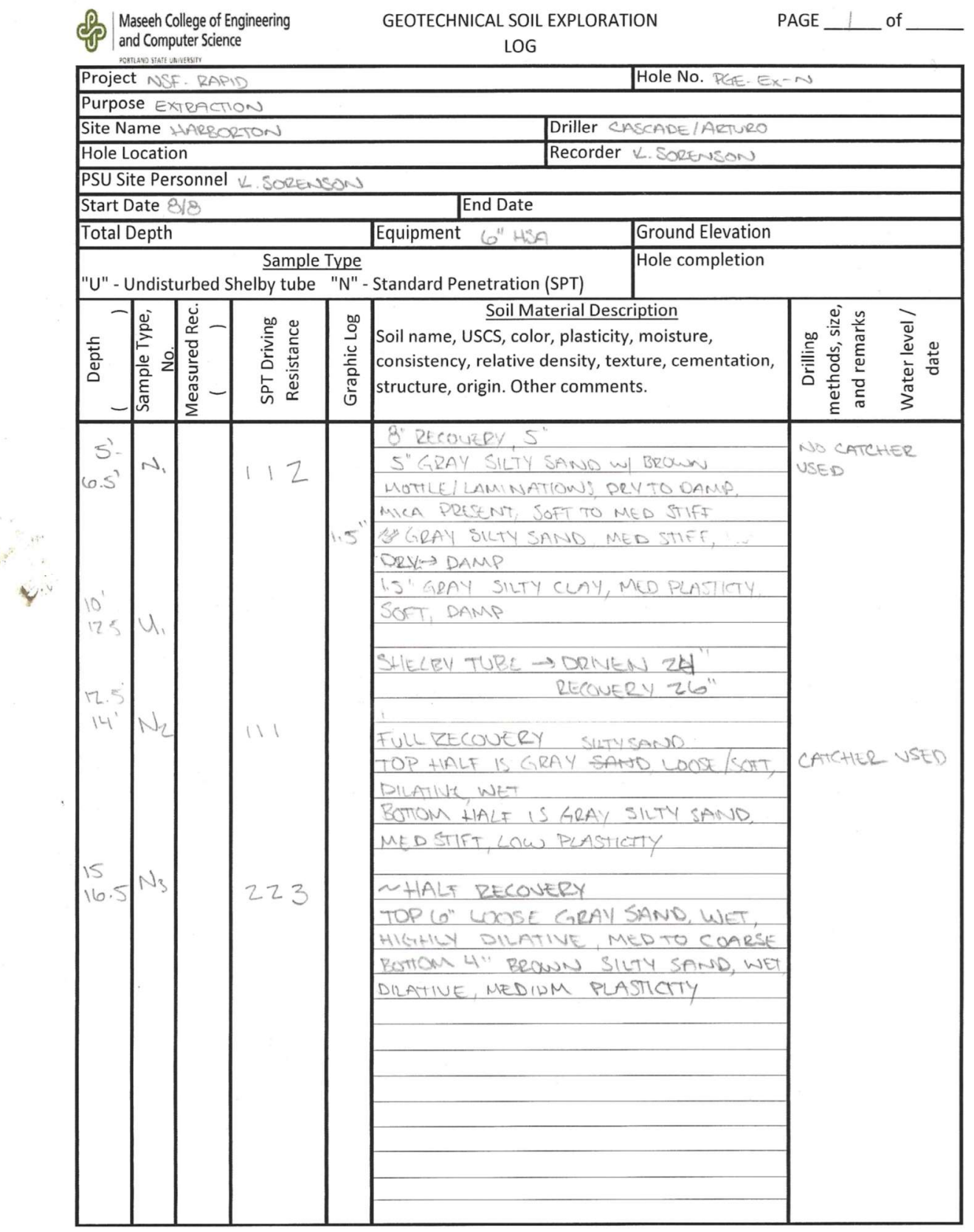




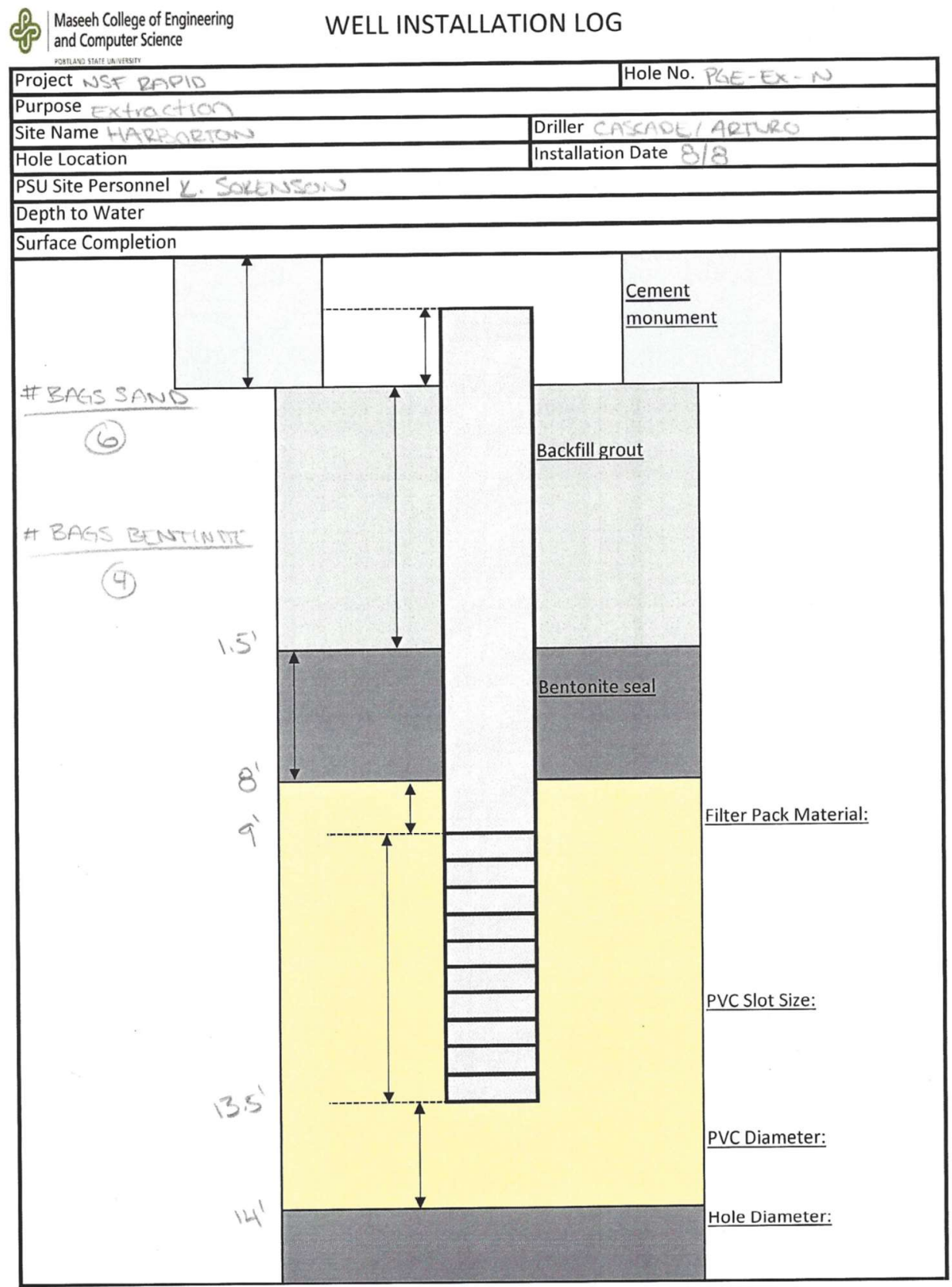




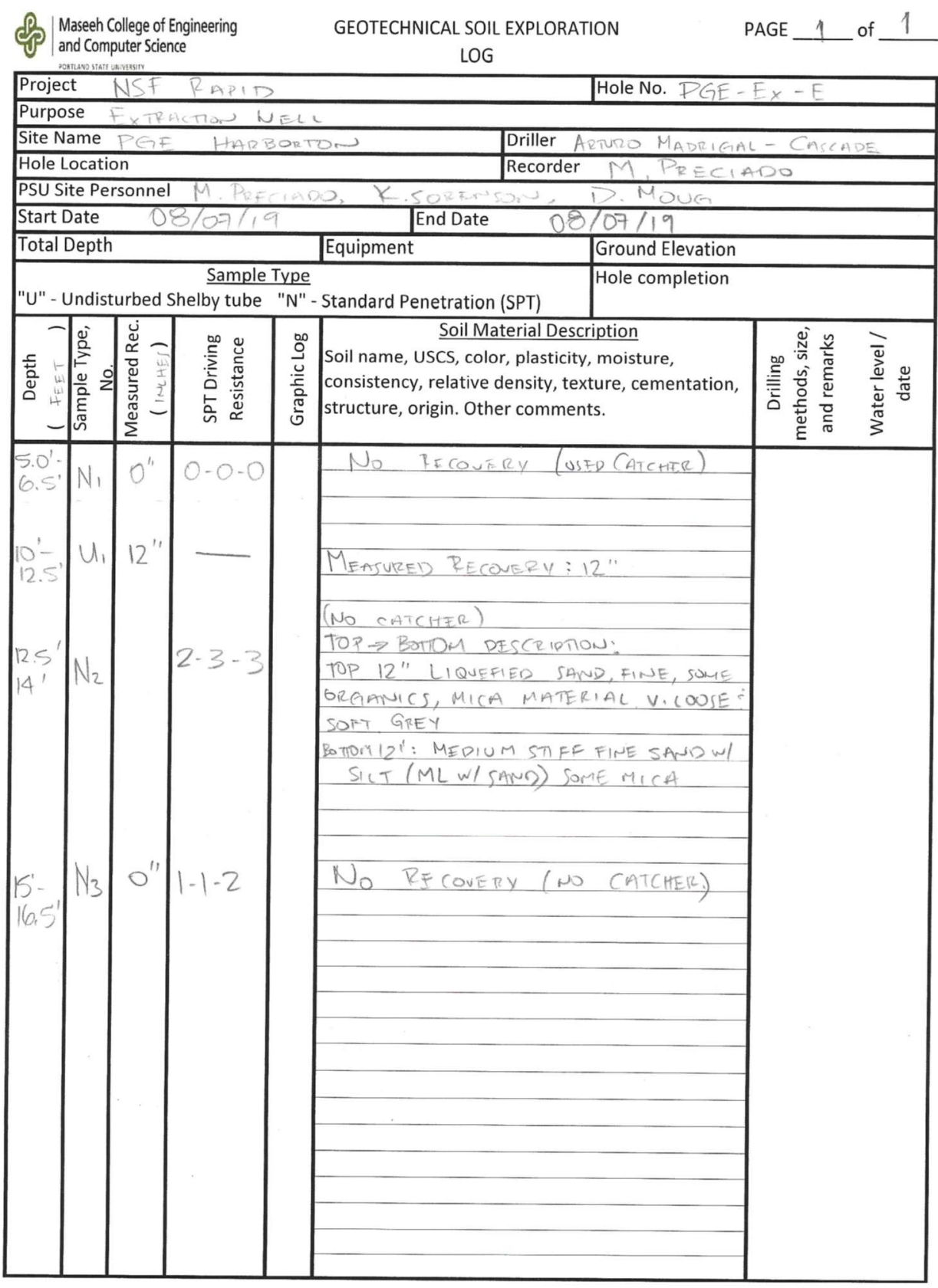




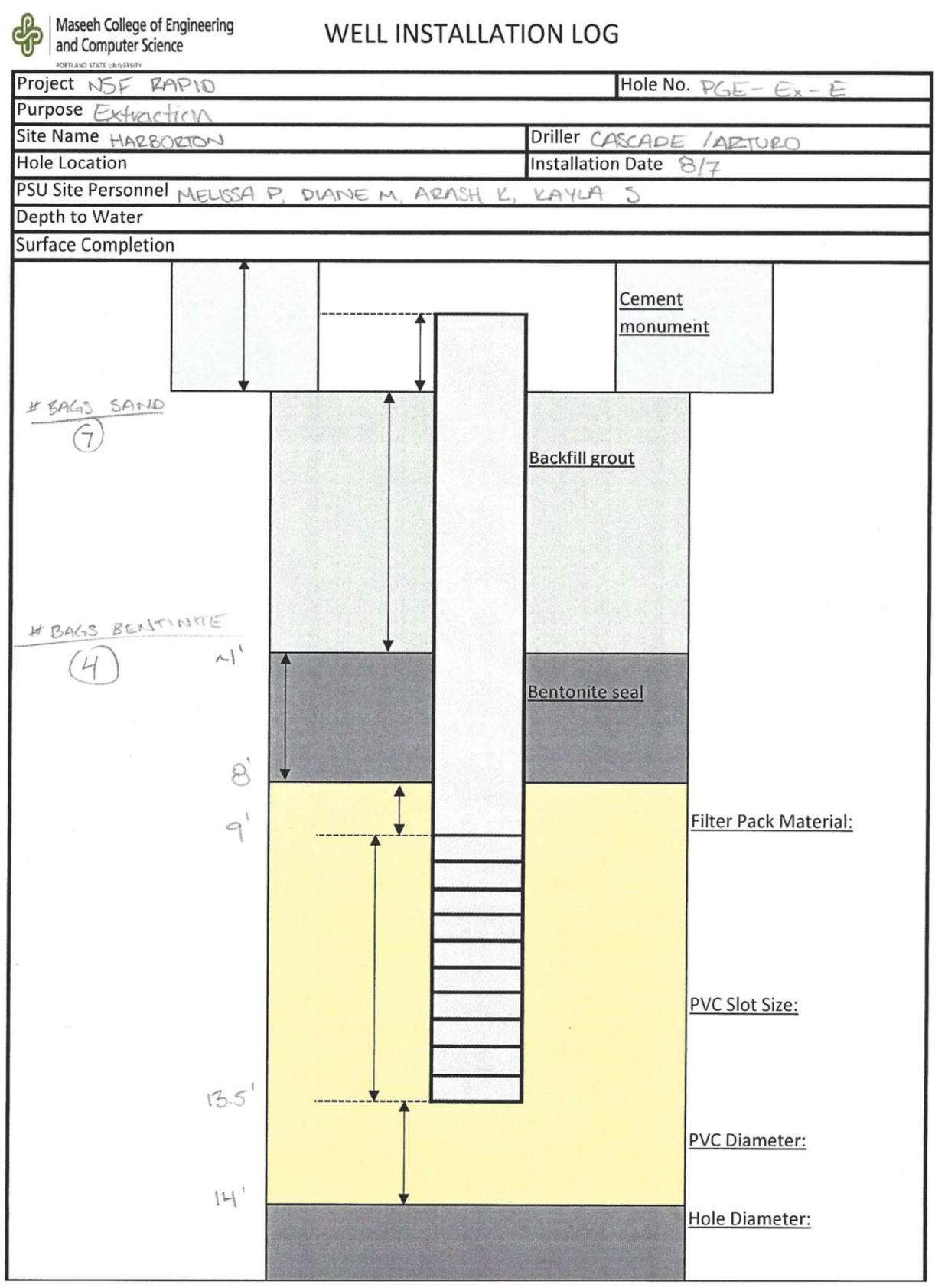




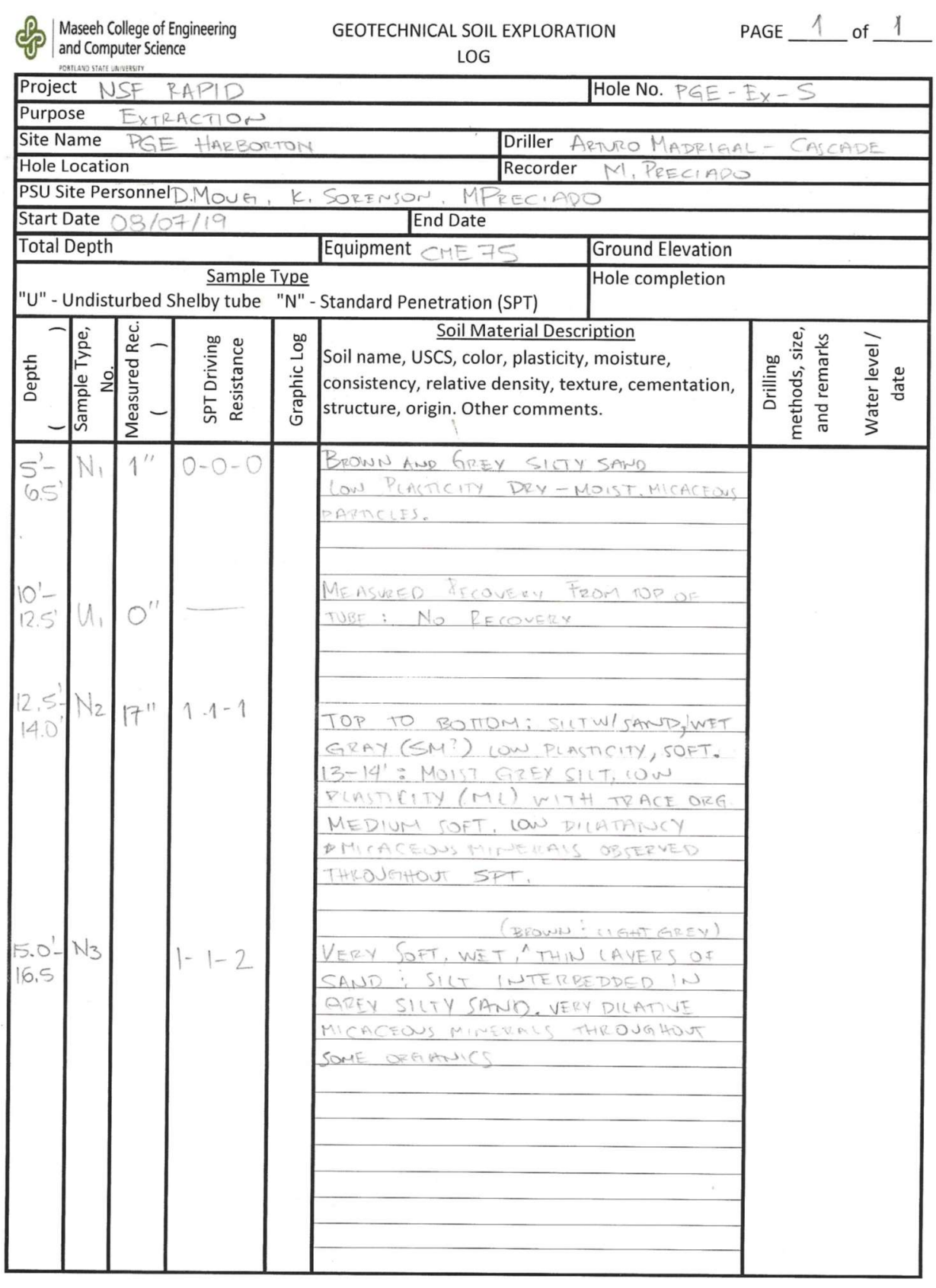




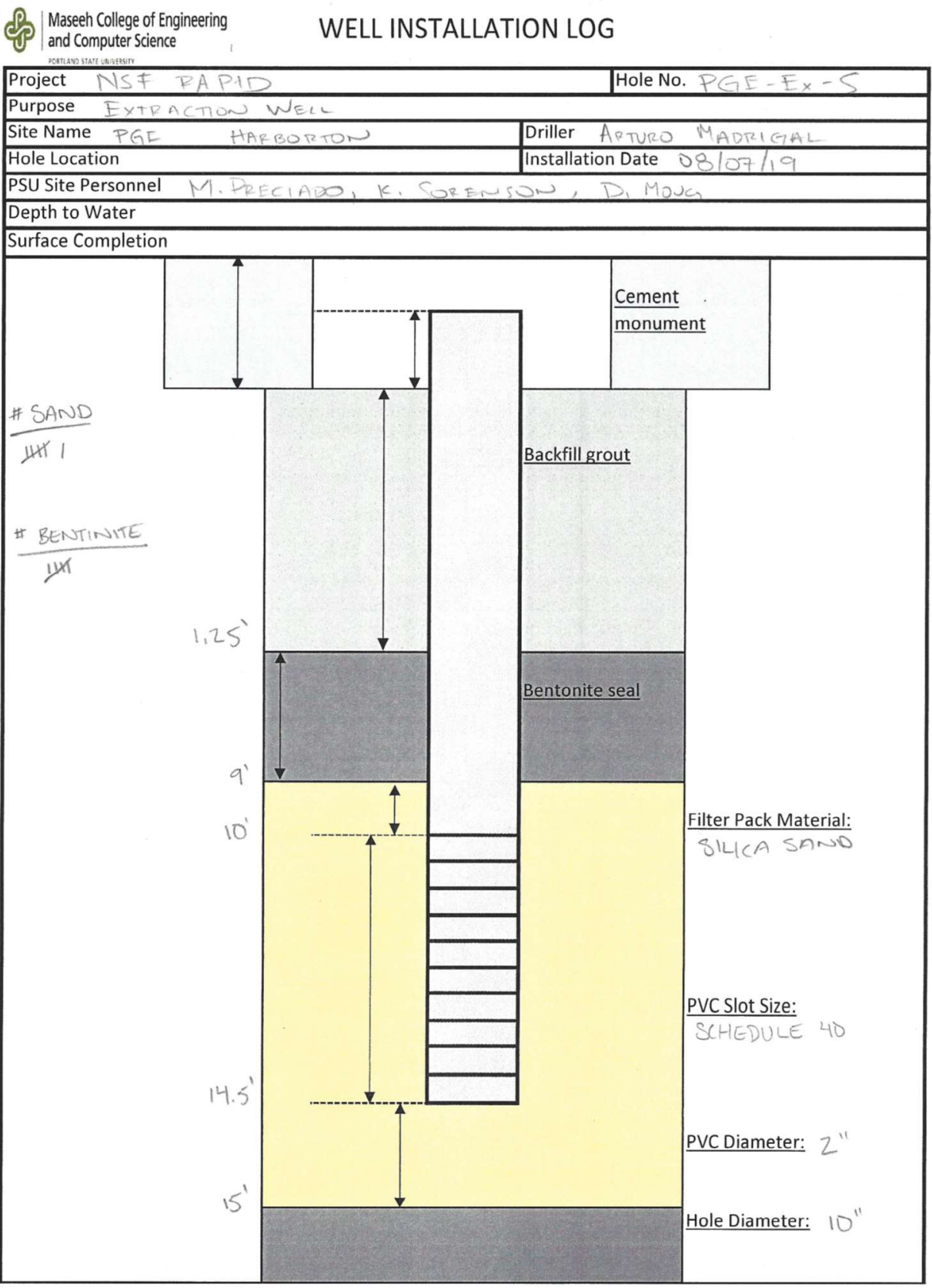




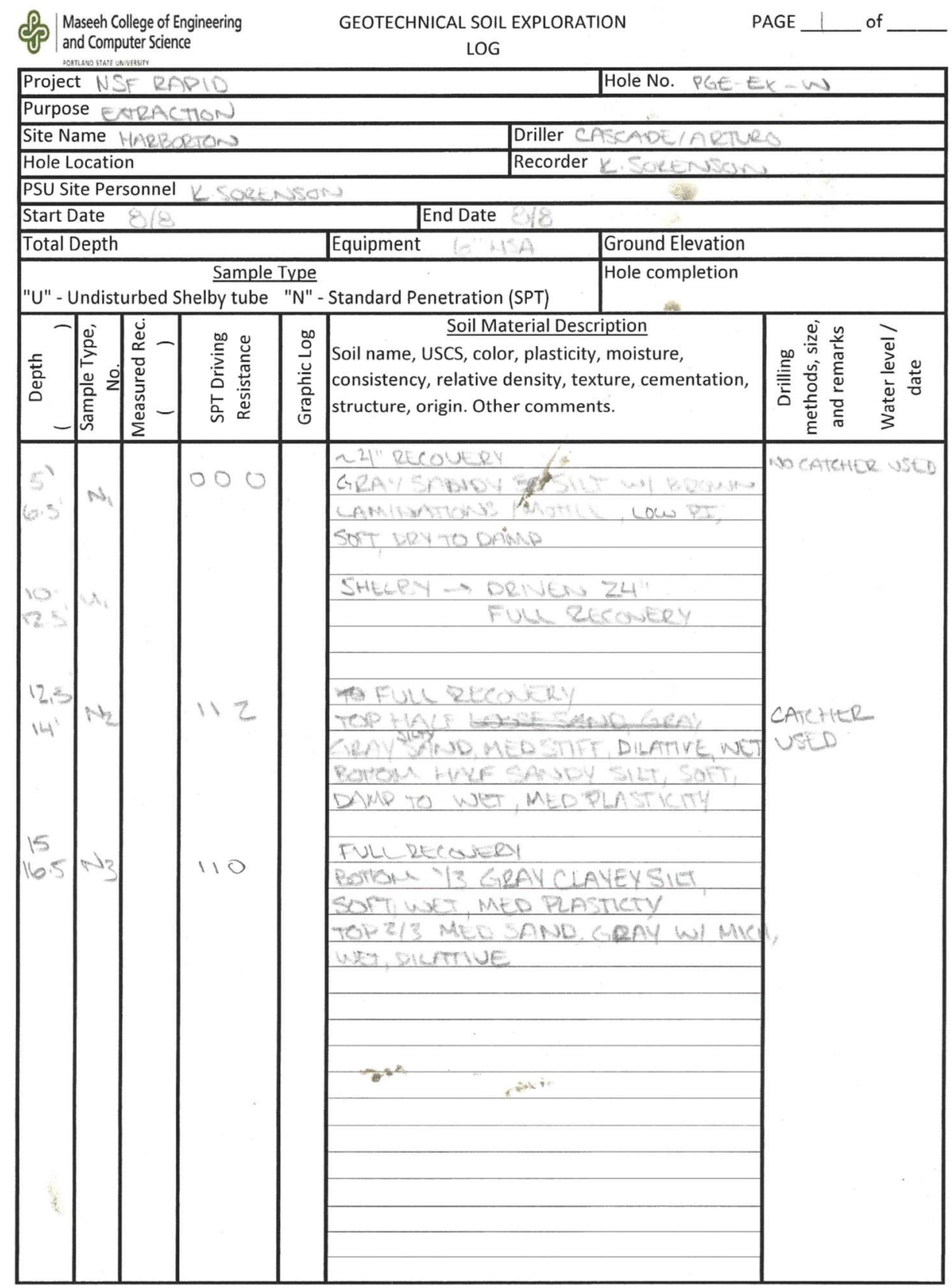




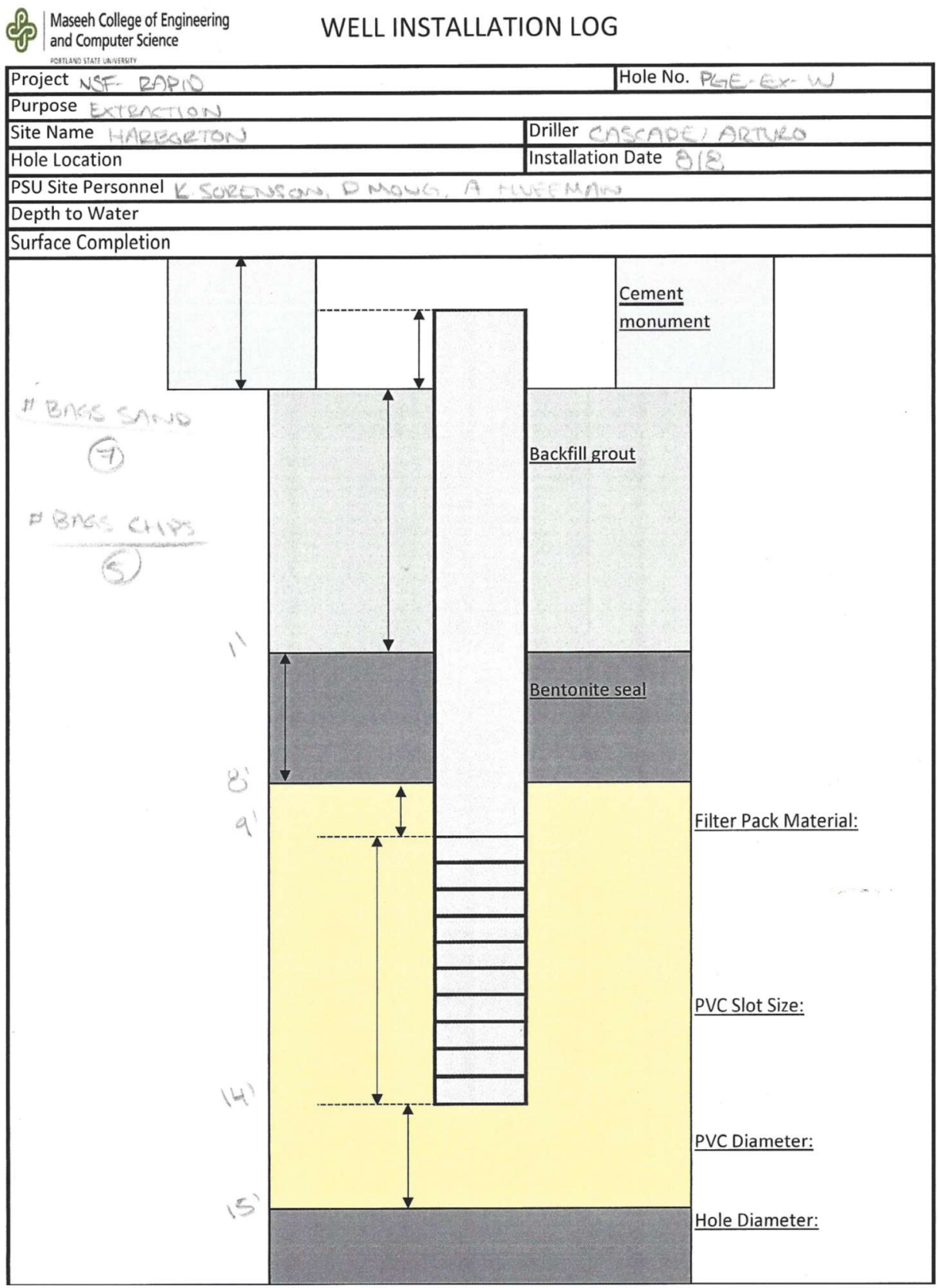




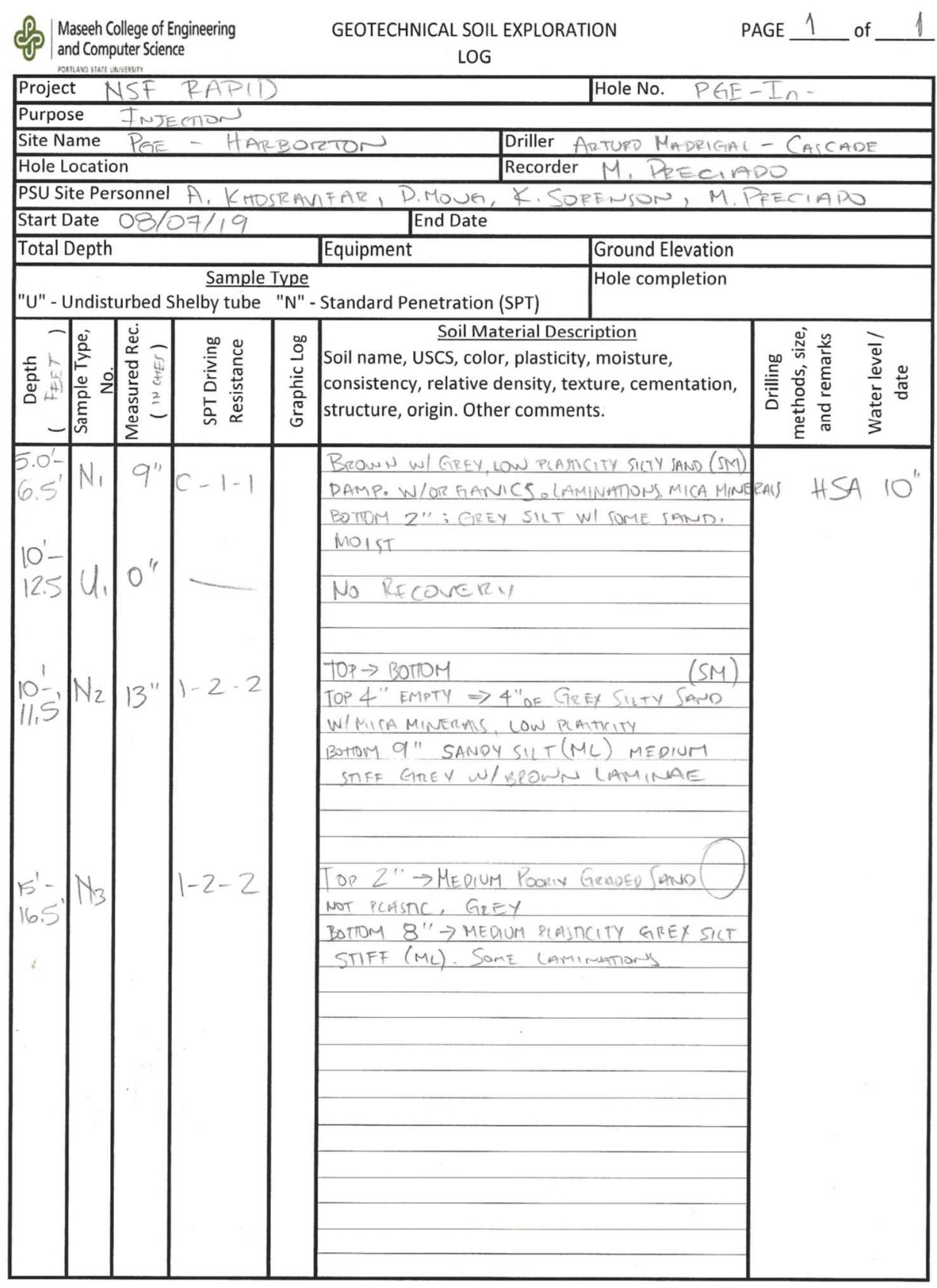




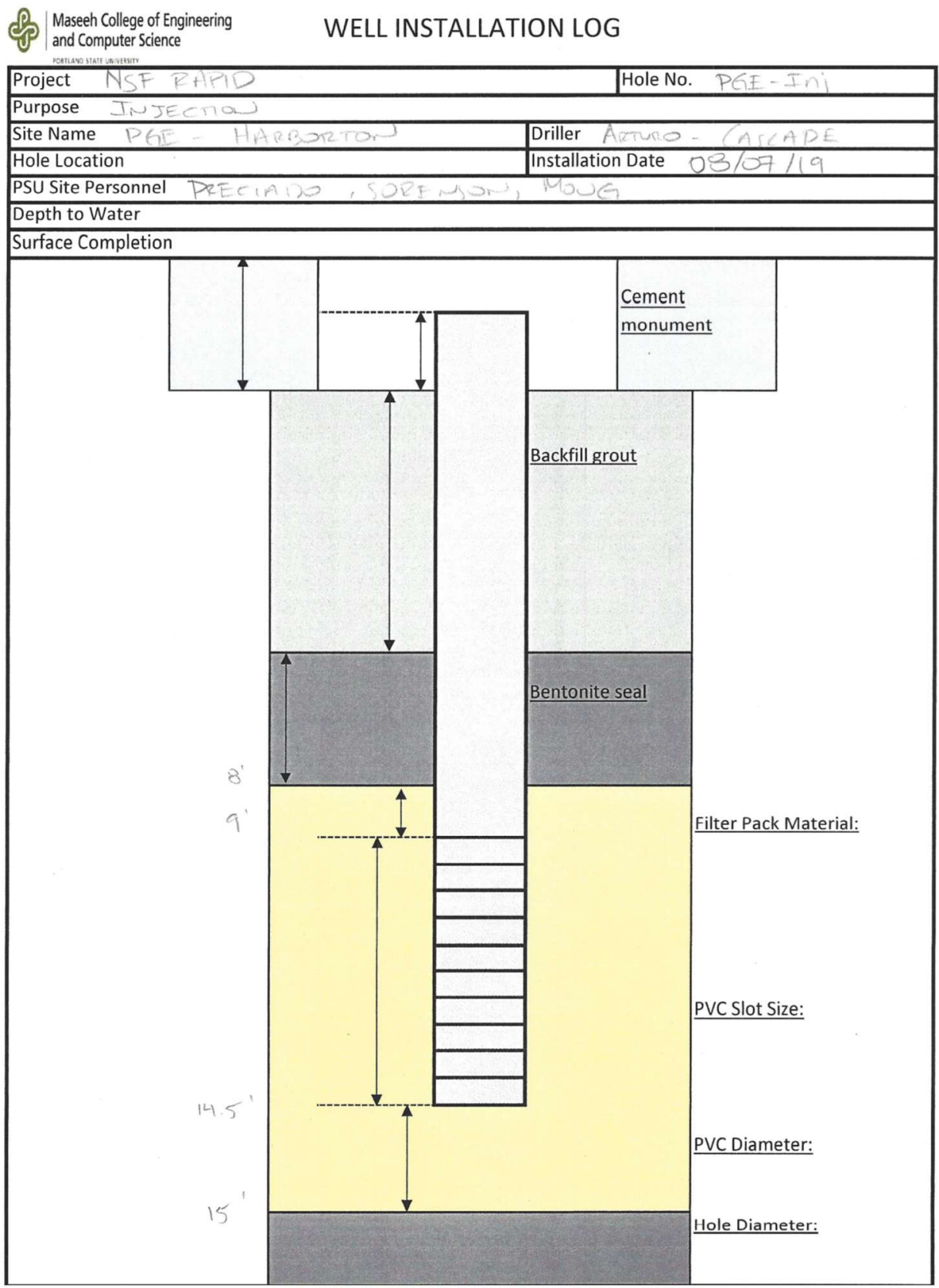




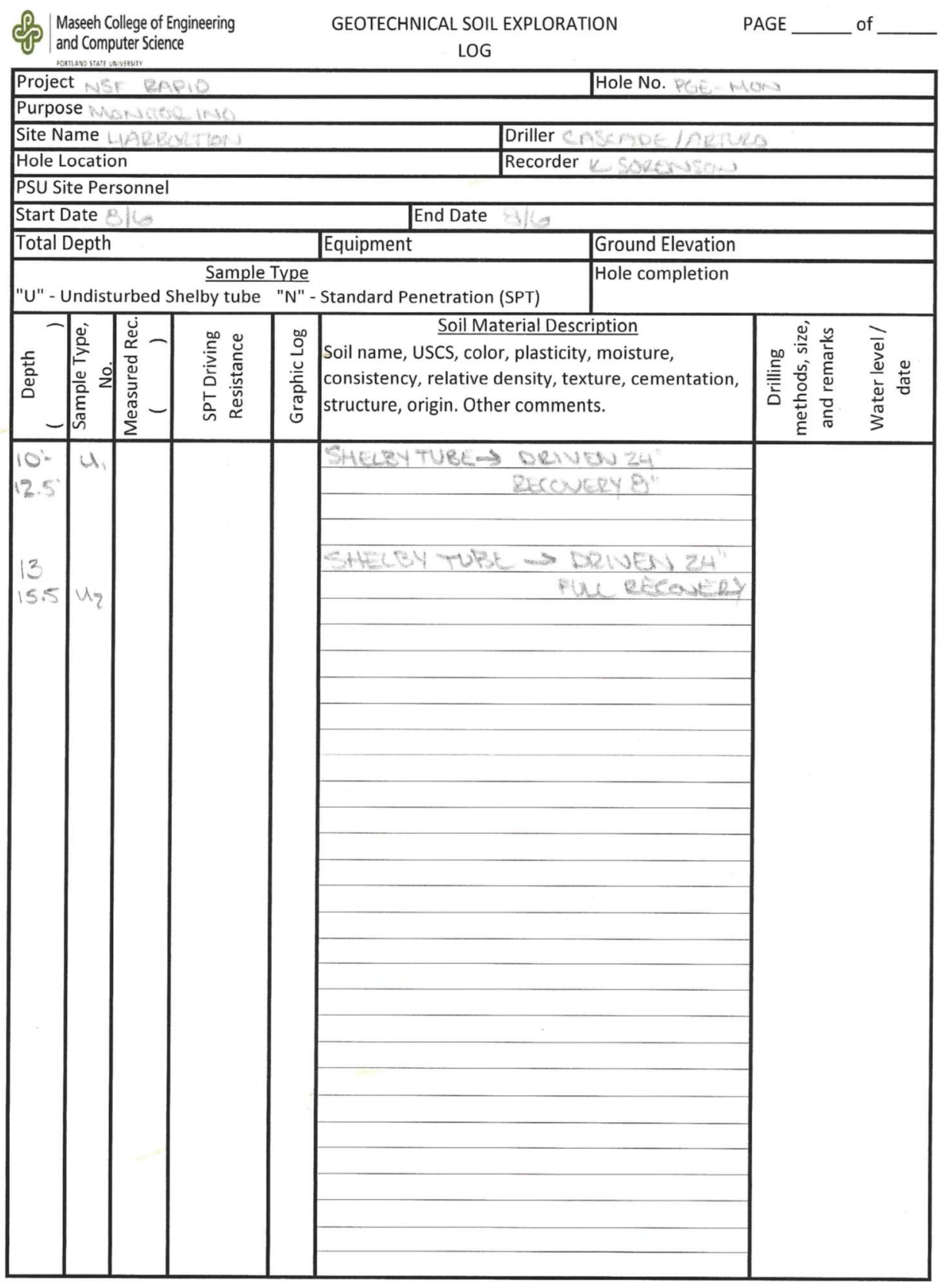




\section{Appendix F: Finished Boring Logs - Harborton}

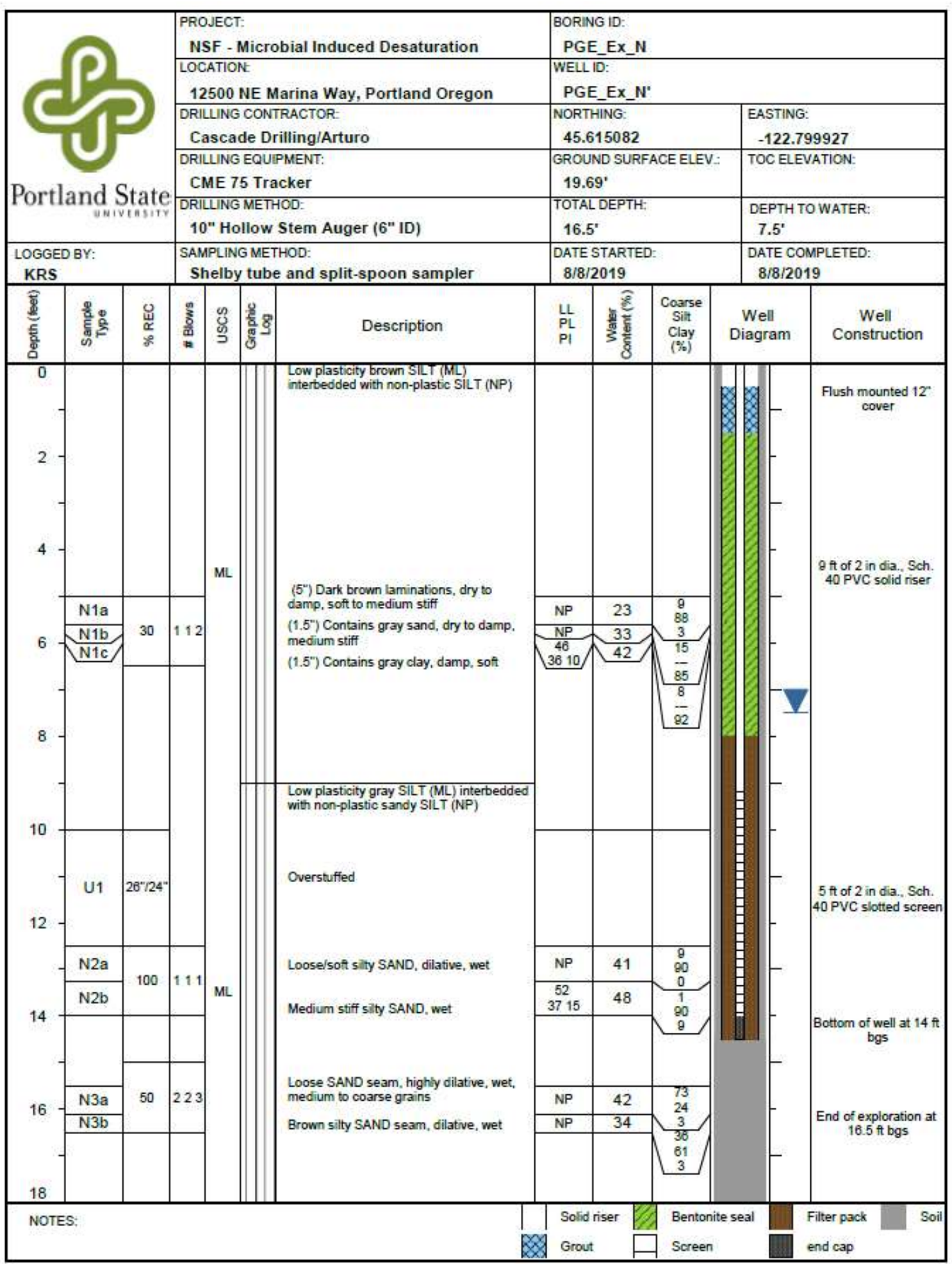




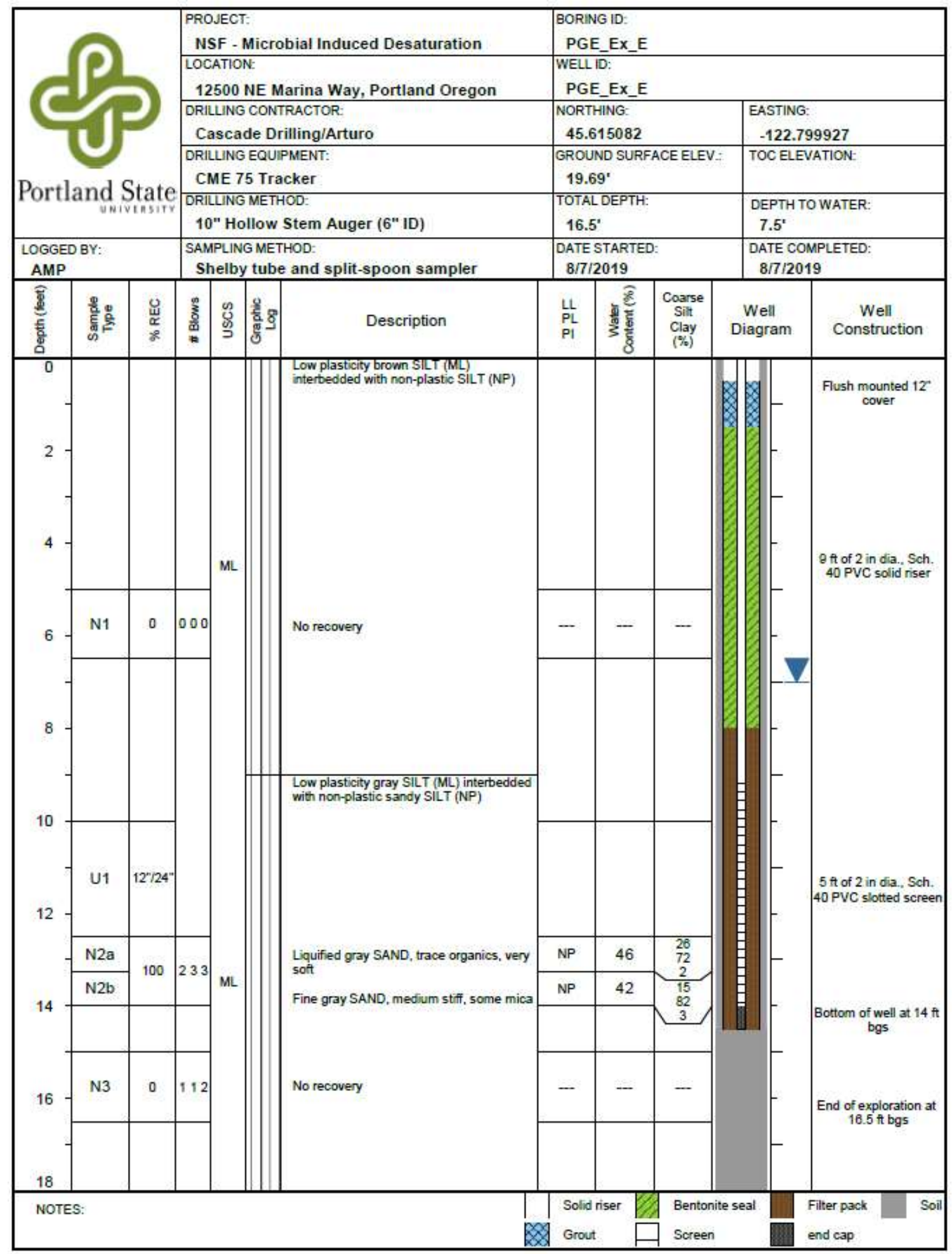




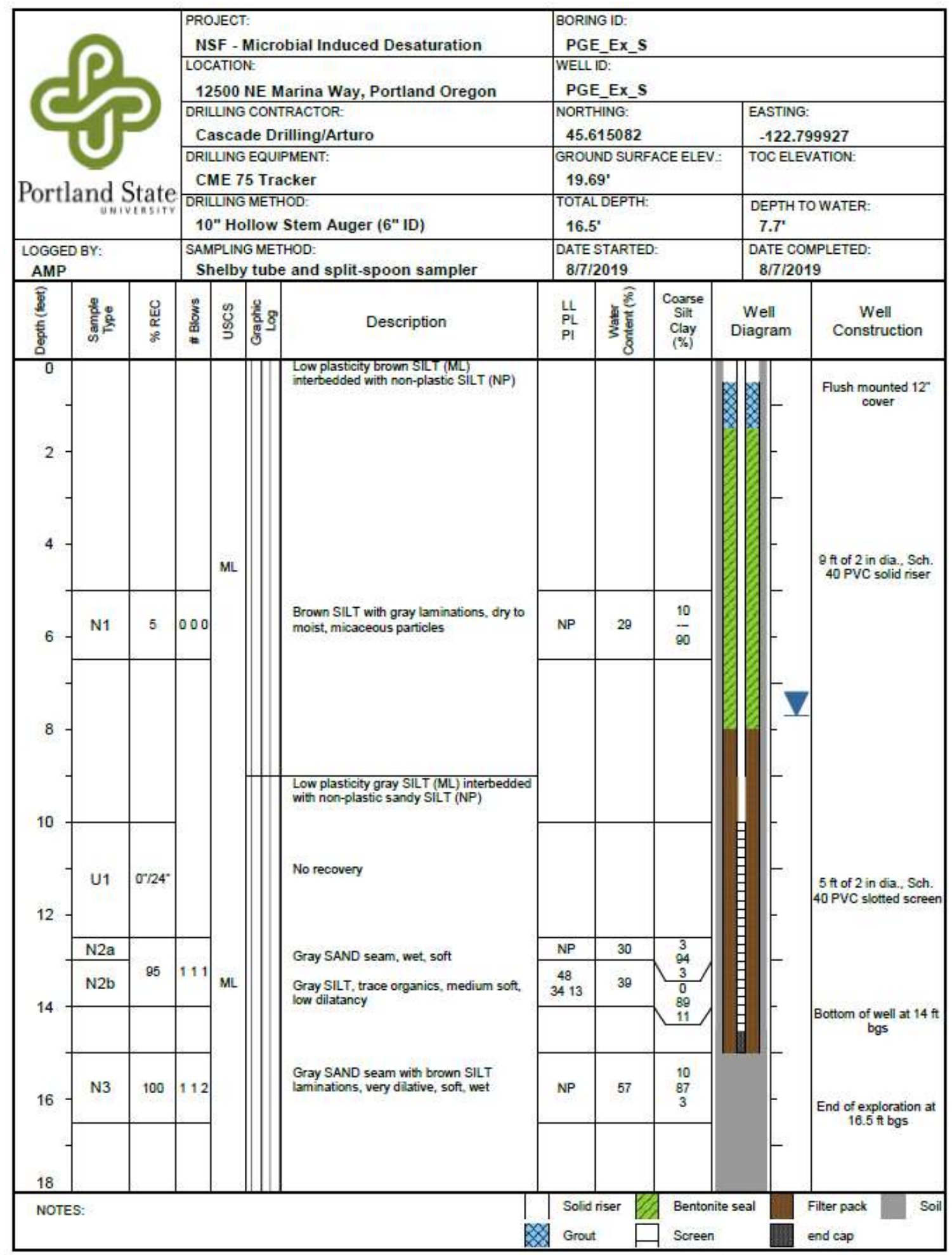




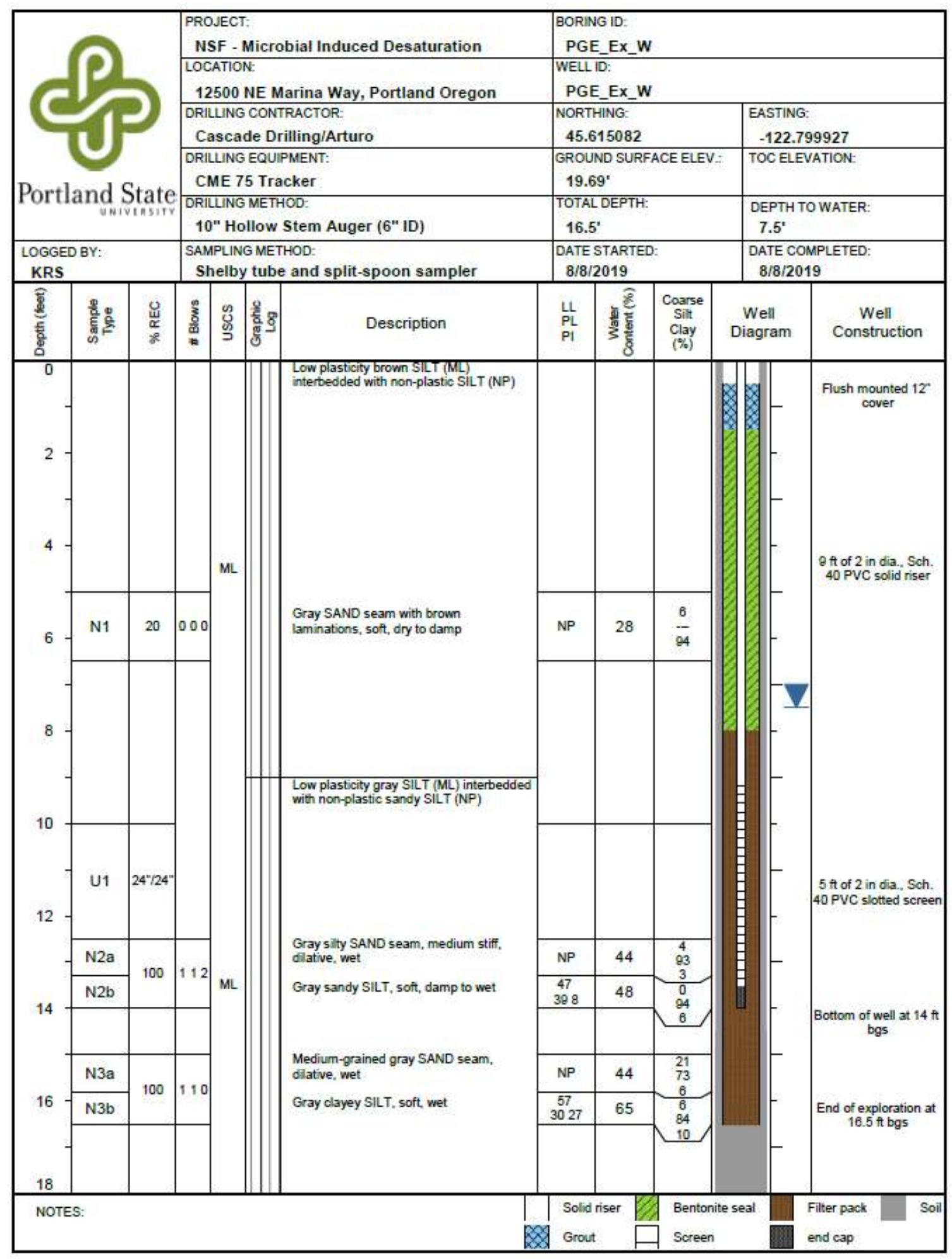




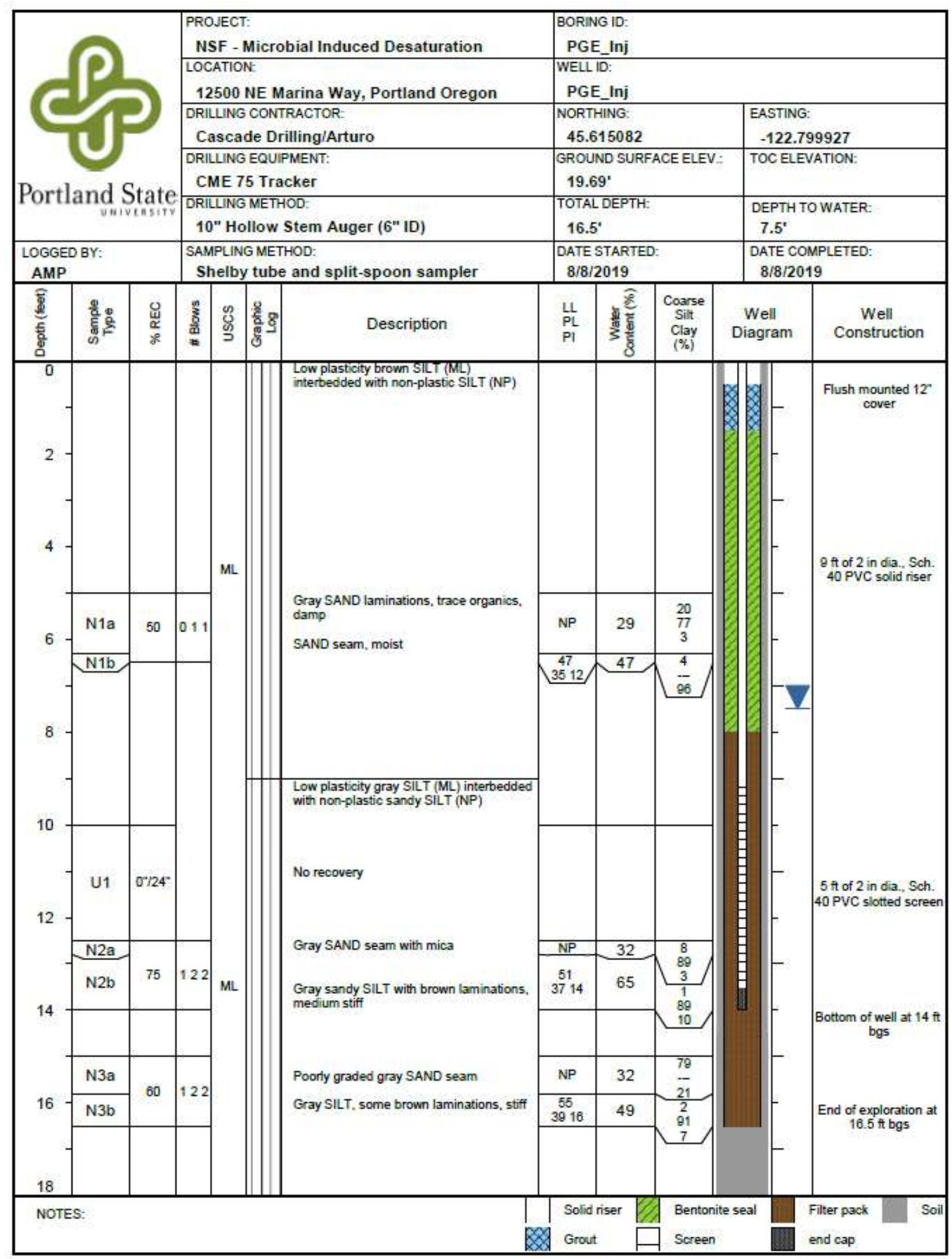




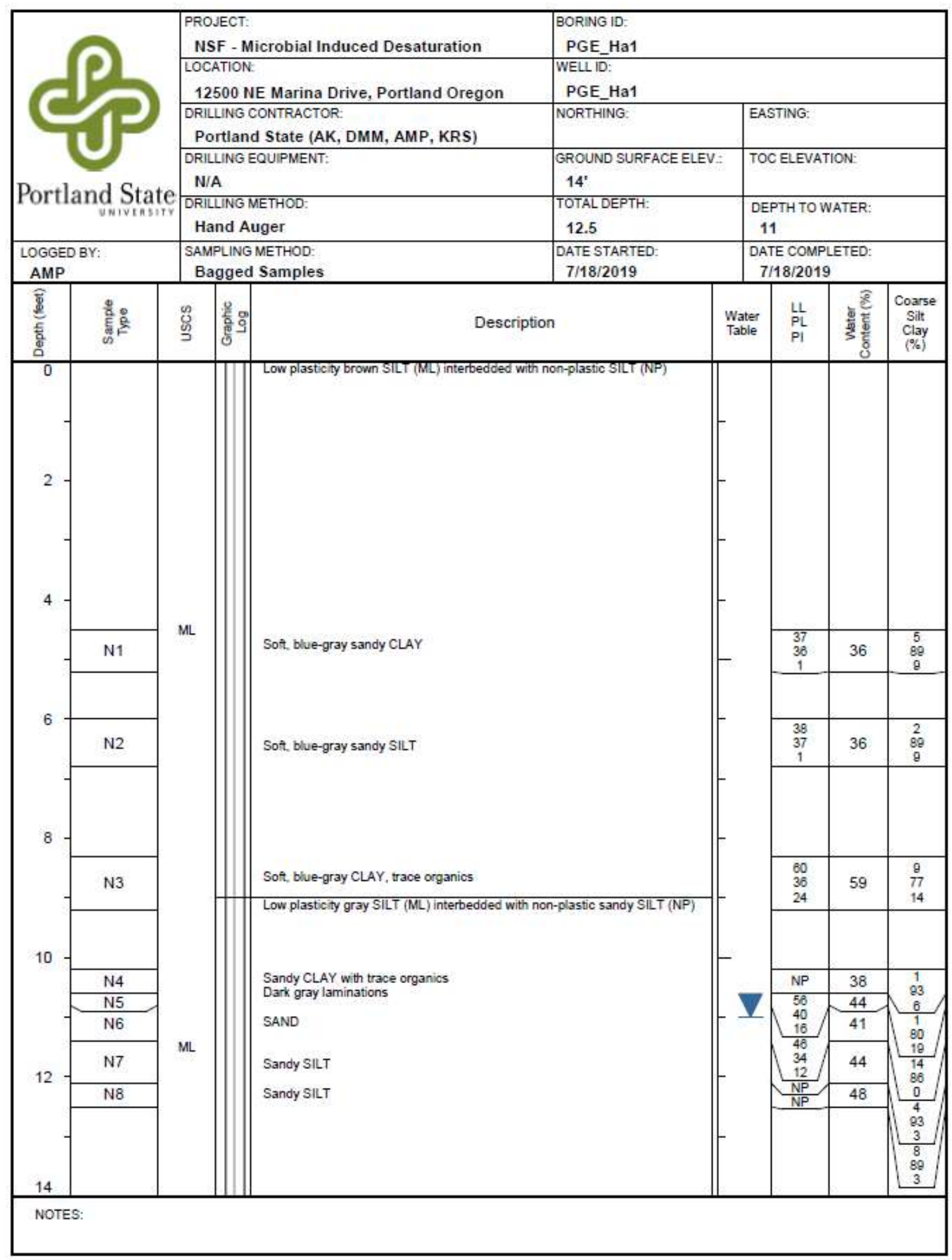




\section{Appendix G: Atterberg Limits - Harborton}

\section{S Portland State}

\begin{tabular}{|c|c|c|c|c|c|c|c|c|c|c|}
\hline \multicolumn{2}{|c|}{ Project: } & \multicolumn{9}{|c|}{ NSF - Microbial Induced Desaturation } \\
\hline \multicolumn{2}{|c|}{ Location: } & \multicolumn{9}{|c|}{ Harborton } \\
\hline \multicolumn{2}{|c|}{ Borehole: } & \multicolumn{2}{|c|}{ PGE_Ha1 } & \multicolumn{3}{|c|}{ Sample Number: } & \multicolumn{2}{|l|}{ N1 } & \multicolumn{2}{|c|}{ Depth (ft-ft): } \\
\hline \multicolumn{6}{|c|}{ Liquid Limit } & \multicolumn{5}{|c|}{ Plastic Limit } \\
\hline 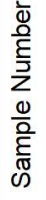 & 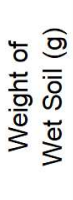 & 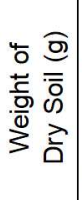 & 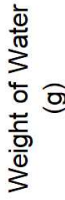 & $\begin{array}{l}\frac{\pi}{0} \\
\frac{0}{ \pm} \\
0 \\
0 \\
0 \\
\frac{1}{0} \\
\frac{ \pm}{\pi} \\
3\end{array}$ & 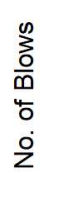 & 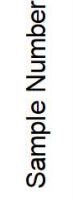 & 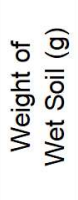 & 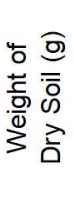 & 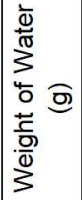 & 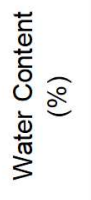 \\
\hline 1 & 3.4 & 2.5 & 0.5 & 36.0 & 39 & 1 & 7.6 & 5.6 & 2 & 35.7 \\
\hline 2 & 1.5 & 1.1 & 0.2 & 36.4 & 31 & 2 & 9.8 & 7.2 & 2.6 & 36.1 \\
\hline 3 & 2.2 & 1.6 & 0.6 & 37.5 & 18 & & & & & \\
\hline 4 & 3.1 & 2.1 & 1 & 47.6 & 6 & & & & & \\
\hline & & & & & & & & & & \\
\hline & & & & & & & & & & \\
\hline
\end{tabular}
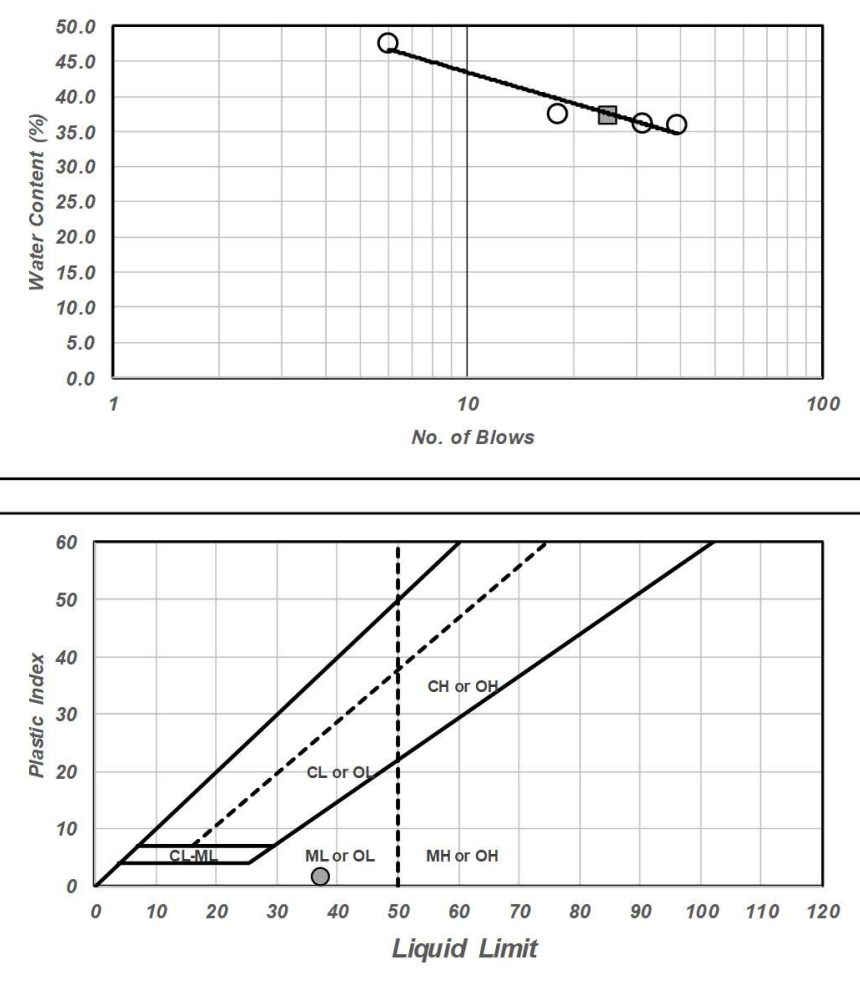

Date Collected: July 18, 2019

\begin{tabular}{ll|ll}
4.5 & 5.2 & Average Depth (ft): 4.85
\end{tabular}

\begin{tabular}{lc} 
Liquid Limit: & 37 \\
Plastic Limit: & 36 \\
Plasticity Index: & 1 \\
\hline
\end{tabular}

Total amount of materials passing sieve No. 40 : $100 \%$
Fines

Classification: $\mathrm{ML}$

Procedure Done By: Kayla 


\section{S Portland State}

\begin{tabular}{|c|c|c|c|c|c|c|}
\hline Project: & ISF - Micr & ration & & & & \\
\hline Location: & Harborton & & & Date Collected & July 18, 2019 & \\
\hline Borehole: & PGE_Ha1 & Sample Number: & Depth (ft-ft): & 6.8 & Average Depth $(\mathrm{ft})$ : & 6.40 \\
\hline
\end{tabular}

\begin{tabular}{|c|c|c|c|c|c|c|c|c|c|c|}
\hline \multicolumn{6}{|c|}{ Liquid Limit } & \multicolumn{5}{|c|}{ Plastic Limit } \\
\hline 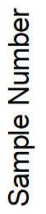 & 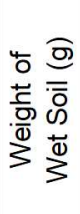 & 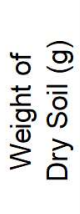 & 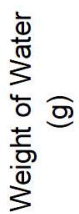 & 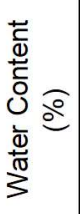 & $\begin{array}{l}00 \\
\sum^{0} \\
0 \\
{ }^{4} \\
0 \\
\vdots\end{array}$ & 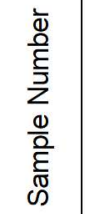 & 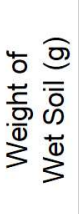 & 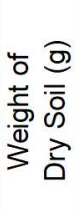 & 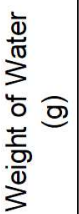 & 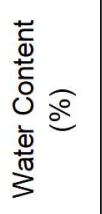 \\
\hline 1 & 1.9 & 1.4 & 0.5 & 35.7 & 34 & 1 & 10.9 & 8.0 & 2.9 & 36.3 \\
\hline 2 & 2.5 & 1.8 & 0.7 & 38.9 & 22 & 2 & 11.1 & 8.1 & 3 & 37.0 \\
\hline 3 & 2.1 & 1.5 & 0.6 & 40.0 & 13 & & & & & \\
\hline & & & & & & & & & & \\
\hline & & & & & & & & & & \\
\hline & & & & & & & & & & \\
\hline
\end{tabular}
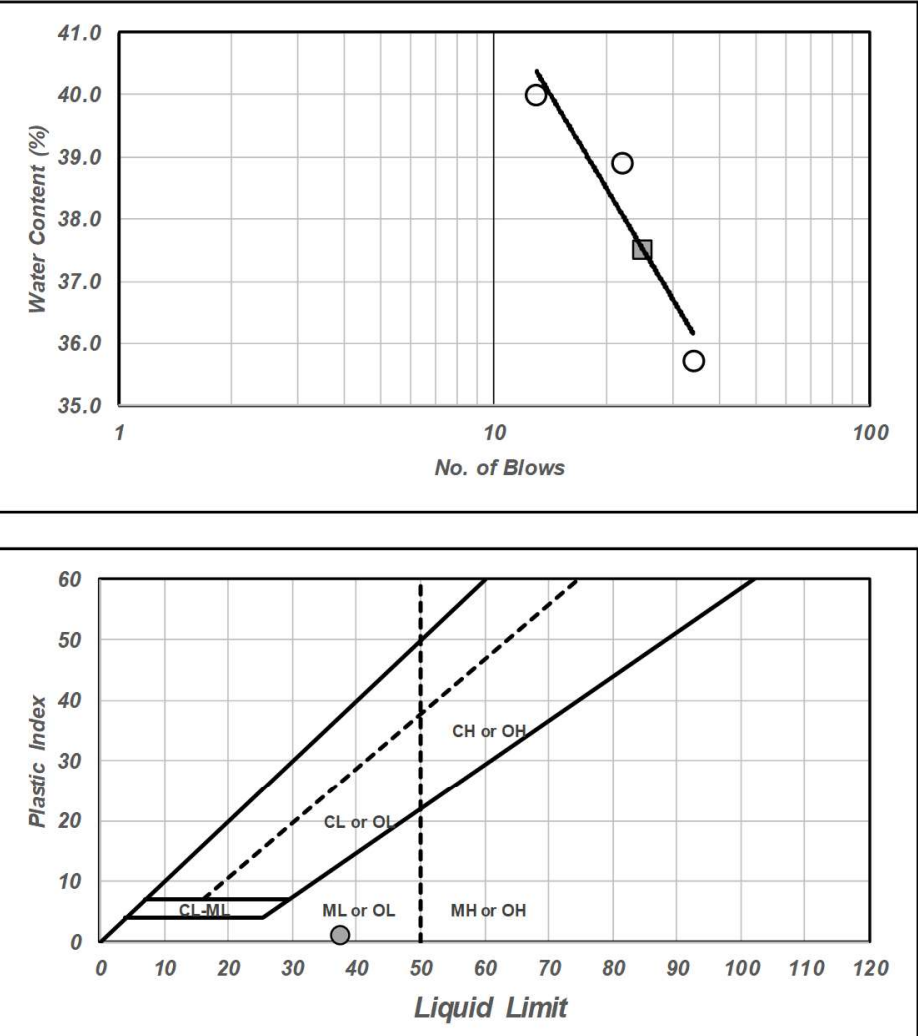

Procedure Done By: Kayla \begin{tabular}{lc} 
Liquid Limit: & 38 \\
Plastic Limit: & $\frac{37}{3}$ \\
Plasticity Index: & $\quad 1$ \\
\hline
\end{tabular}

Total amount of materials passing sieve No. 40: $100 \%$
Fines

Classification: $\mathrm{ML}$ 


\section{S Portland State}

\begin{tabular}{|c|c|c|c|c|c|c|}
\hline Project: & $\mathrm{SF}-\mathrm{Micr}$ & ration & & & & \\
\hline Location: & Harborton & & & Date Collected & July 18, 2019 & \\
\hline Borehole: & PGE_Ha1 & Sample Number: & Depth (ft-ft): & 9.2 & Average Depth $(\mathrm{ft})$ : & 8.75 \\
\hline
\end{tabular}

\begin{tabular}{|c|c|c|c|c|c|c|c|c|c|c|}
\hline \multicolumn{6}{|c|}{ Liquid Limit } & \multicolumn{5}{|c|}{ Plastic Limit } \\
\hline 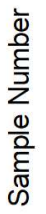 & 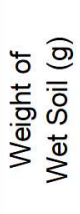 & 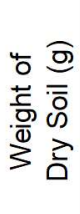 & 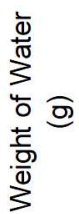 & 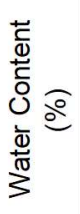 & $\begin{array}{l}00 \\
\sum^{0} \\
0 \\
{ }^{4} \\
0 \\
\vdots\end{array}$ & 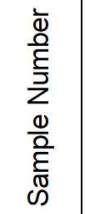 & 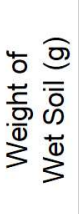 & 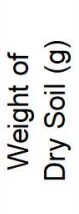 & 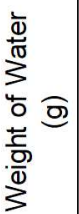 & 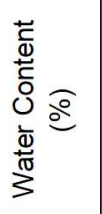 \\
\hline 1 & 3.5 & 2.2 & 1.3 & 59.1 & 37 & 1 & 9.3 & 6.8 & 2.5 & 36.8 \\
\hline 2 & 2.9 & 1.8 & 1.1 & 61.1 & 21 & 2 & 10.0 & 7.4 & 2.6 & 35.1 \\
\hline 3 & 4.7 & 2.9 & 1.8 & 62.1 & 12 & & & & & \\
\hline & & & & & & & & & & \\
\hline & & & & & & & & & & \\
\hline & & & & & & & & & & \\
\hline
\end{tabular}
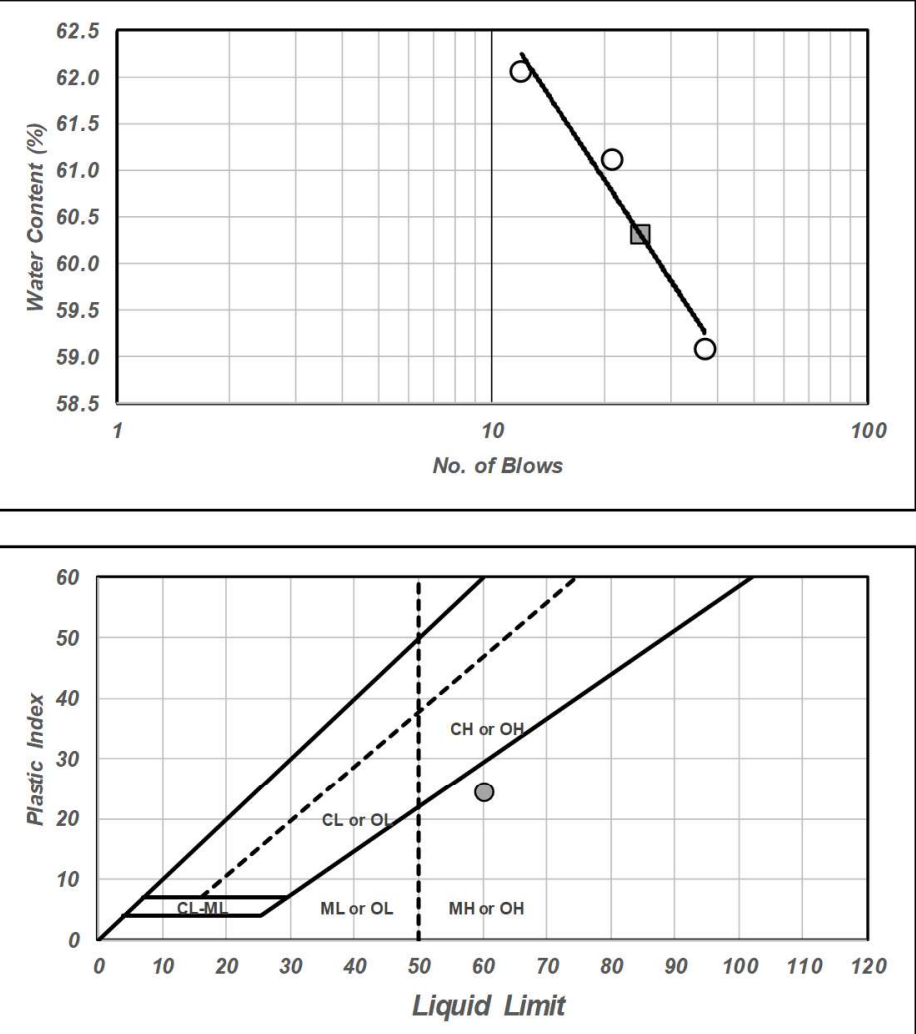

Procedure Done By: Kayla

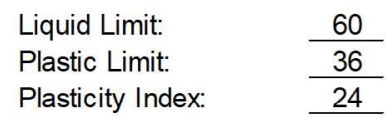

Total amount of materials passing sieve No. 40: $100 \%$
Fines

Classification: $\mathrm{MH}$ 


\section{S Portland State}

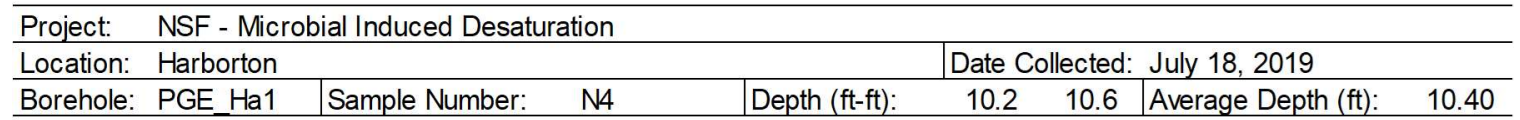

\begin{tabular}{|c|c|c|c|c|c|c|c|c|c|c|}
\hline \multicolumn{6}{|c|}{ Liquid Limit } & \multicolumn{5}{|c|}{ Plastic Limit } \\
\hline 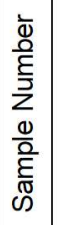 & 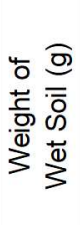 & 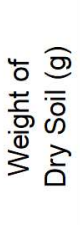 & 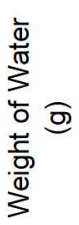 & 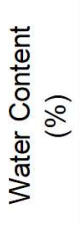 & 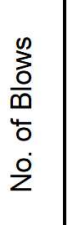 & 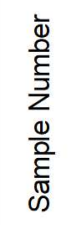 & 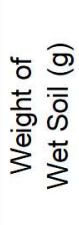 & 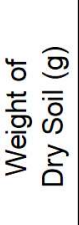 & 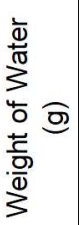 & 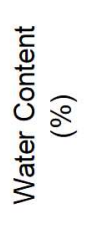 \\
\hline 1 & 8.0 & 5.8 & 2.2 & 37.9 & 14 & & & & & \\
\hline 2 & 6.3 & 4.7 & 1.6 & 34.0 & 1 & & & & & \\
\hline 3 & 9.8 & 6.8 & 3 & 44.1 & 18 & & & & & \\
\hline 4 & 11.2 & 7.4 & 3.8 & 51.4 & 4 & & & & & \\
\hline & & & & & & & & & & \\
\hline & & & & & & & & & & \\
\hline
\end{tabular}

Liquid Limit:

Plastic Limit:

Plasticity Index:

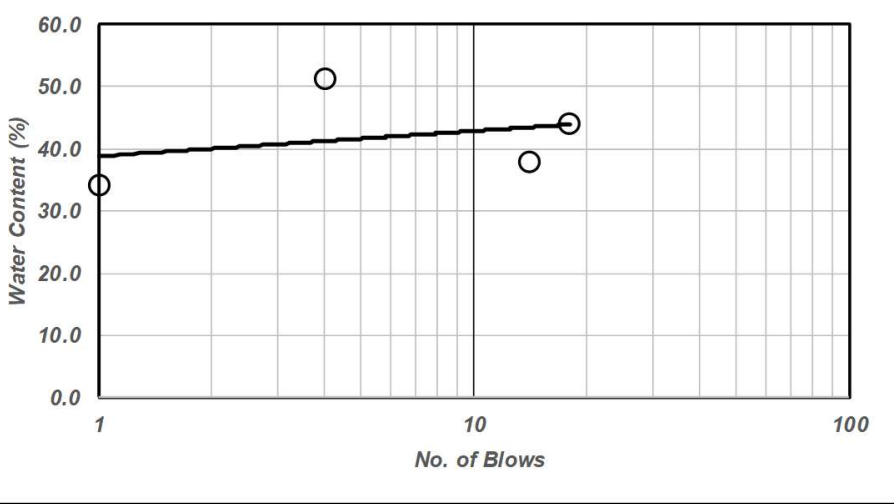

Total amount of materials passing sieve No. 40: $100 \%$

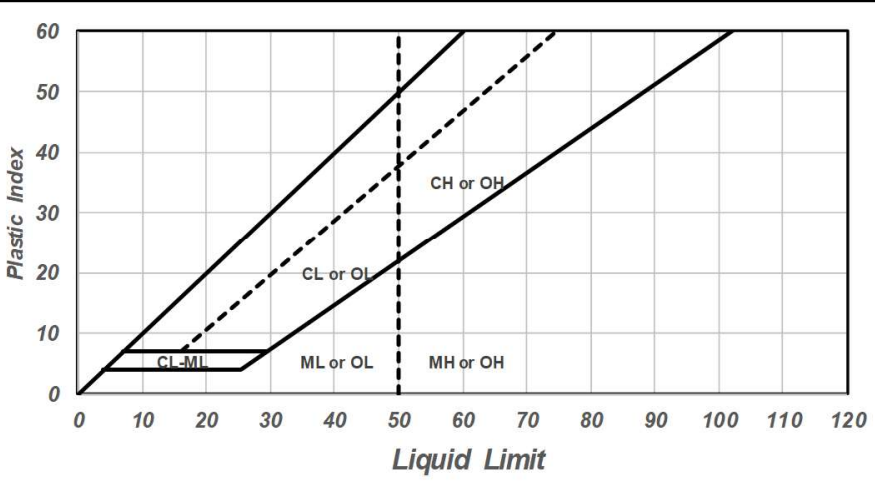

Procedure Done By: Kayla
Fines

Classification: NP 


\section{S Portland State}

\begin{tabular}{|c|c|c|c|c|c|c|}
\hline oject: & NSF - Micr & I Induced Desaturation & & & & \\
\hline Location: & Harborton & & & Date Collected & July 18,2019 & \\
\hline Borehole: & PGE Ha1 & Sample Number: & Depth (ft-ft): & 10.6 & Average Depth $(\mathrm{ft})$ : & 10.70 \\
\hline
\end{tabular}

\begin{tabular}{|c|c|c|c|c|c|c|c|c|c|c|}
\hline \multicolumn{6}{|c|}{ Liquid Limit } & \multicolumn{5}{|c|}{ Plastic Limit } \\
\hline 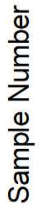 & 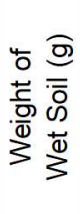 & 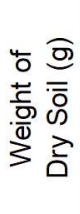 & 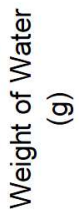 & 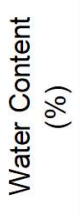 & \begin{tabular}{l}
00 \\
3 \\
0 \\
0 \\
4 \\
0 \\
0 \\
\hdashline
\end{tabular} & 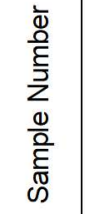 & 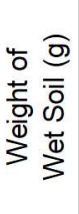 & 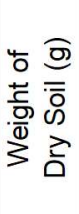 & 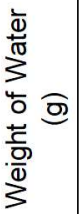 & 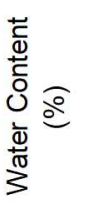 \\
\hline 1 & 14.3 & 9.9 & 4.4 & 44.4 & 166 & 1 & 6.7 & 4.8 & 1.9 & 39.6 \\
\hline 2 & 11.0 & 7.0 & 4 & 57.1 & 33 & 2 & 7.0 & 5.0 & 2 & 40.0 \\
\hline 3 & 10.8 & 6.6 & 4.2 & 63.6 & 7 & & & & & \\
\hline 4 & 15.5 & 9.7 & 5.8 & 59.8 & 14 & & & & & \\
\hline & & & & & & & & & & \\
\hline & & & & & & & & & & \\
\hline
\end{tabular}

$\begin{array}{ll}\text { Liquid Limit: } & \frac{57}{40} \\ \text { Plastic Limit: } & \quad \\ \text { Plasticity Index: } & \end{array}$

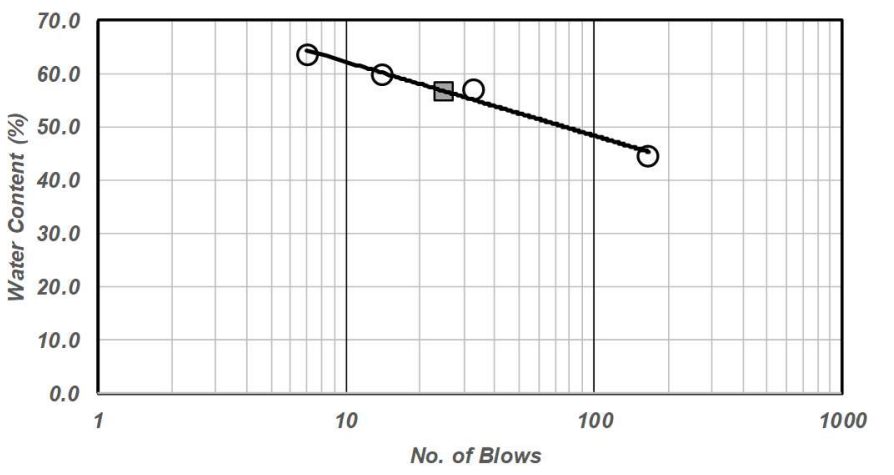

Total amount of materials passing sieve No. 40: $100 \%$

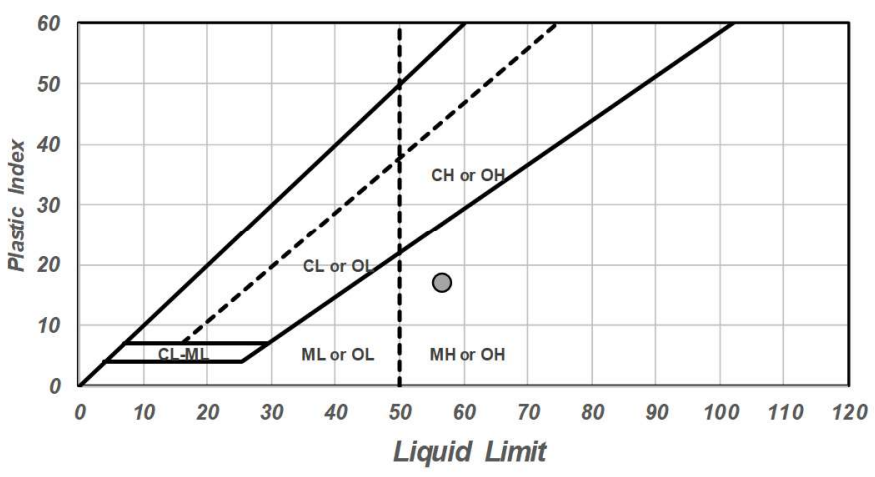

Fines

Classification: $\mathrm{MH}$

Procedure Done By: Kayla 


\section{S Portland State}

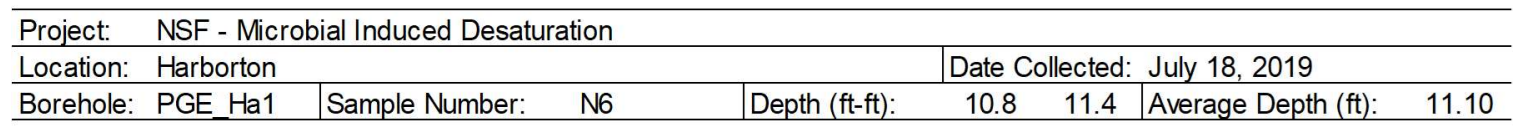

\begin{tabular}{|c|c|c|c|c|c|c|c|c|c|c|}
\hline \multicolumn{6}{|c|}{ Liquid Limit } & \multicolumn{5}{|c|}{ Plastic Limit } \\
\hline 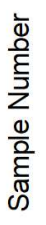 & 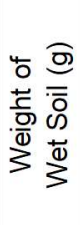 & 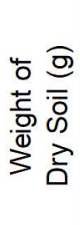 & 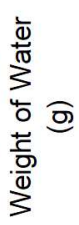 & 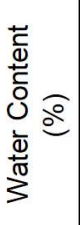 & $\begin{array}{l}00 \\
\sum^{0} \\
0 \\
{ }^{4} \\
0 \\
\vdots\end{array}$ & 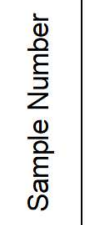 & 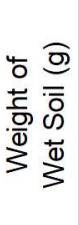 & 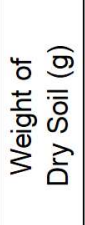 & 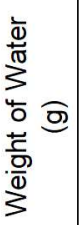 & 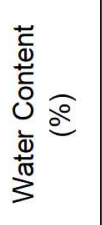 \\
\hline 1 & 7.9 & 5.6 & 2.3 & 41.1 & 44 & 1 & 8.5 & 6.3 & 2.2 & 34.9 \\
\hline 2 & 7.2 & 4.9 & 2.3 & 46.9 & 27 & 2 & 8.4 & 6.3 & 2.1 & 33.3 \\
\hline 3 & 6.3 & 4.2 & 2.1 & 50.0 & 11 & & & & & \\
\hline 4 & 9.3 & 6.0 & 3.3 & 55.0 & 6 & & & & & \\
\hline & & & & & & & & & & \\
\hline & & & & & & & & & & \\
\hline
\end{tabular}
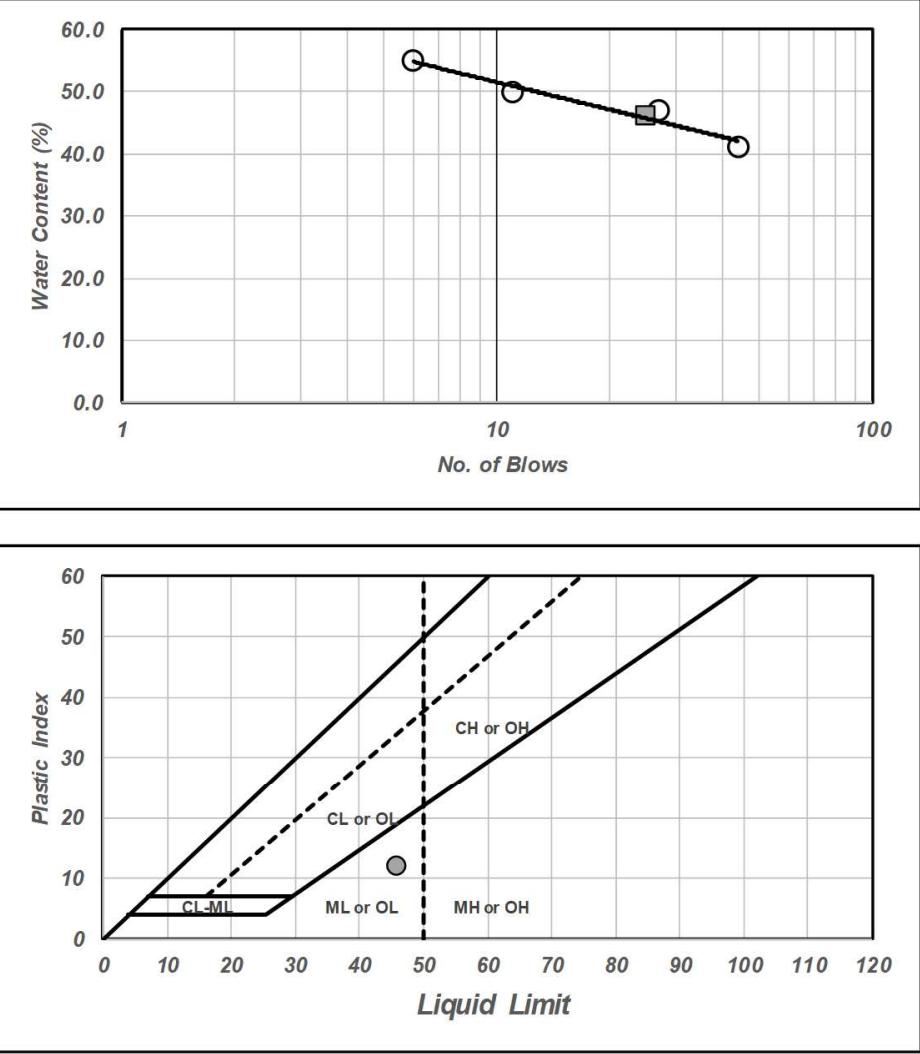

Liquid Limit:

Plastic Limit:

Plasticity Index:
Total amount of materials passing sieve No. 40: $100 \%$

Procedure Done By: Ariadna

Fines

Classification: ML 


\section{S Portland State}

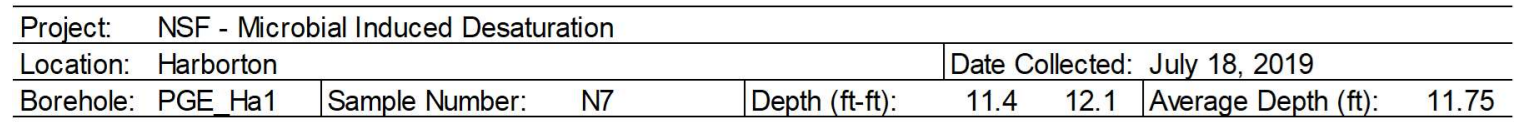

\begin{tabular}{|c|c|c|c|c|c|c|c|c|c|c|}
\hline \multicolumn{6}{|c|}{ Liquid Limit } & \multicolumn{5}{|c|}{ Plastic Limit } \\
\hline $\begin{array}{l}\frac{1}{\mathbf{D}} \\
\frac{O}{E} \\
\bar{\Xi} \\
\Sigma \\
\frac{\omega}{O} \\
\frac{O}{E} \\
\tilde{D}\end{array}$ & 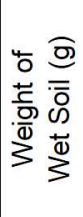 & 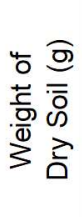 & 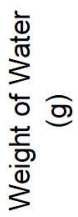 & 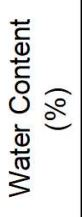 & 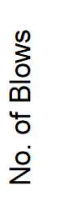 & 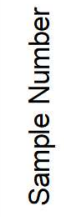 & 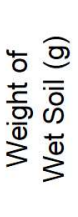 & 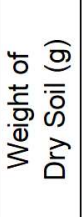 & 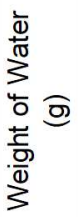 & 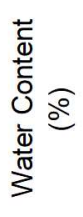 \\
\hline 1 & 43.8 & 30.5 & 13.3 & 43.6 & 1 & & & & & \\
\hline 2 & 7.1 & 5.1 & 2 & 39.2 & 4 & & & & & \\
\hline 3 & 11.5 & 8.4 & 3.1 & 36.9 & 10 & & & & & \\
\hline 4 & 19.7 & 15.1 & 4.6 & 30.5 & 1 & & & & & \\
\hline & & & & & & & & & & \\
\hline & & & & & & & & & & \\
\hline
\end{tabular}

Liquid Limit:

Plastic Limit:

Plasticity Index:

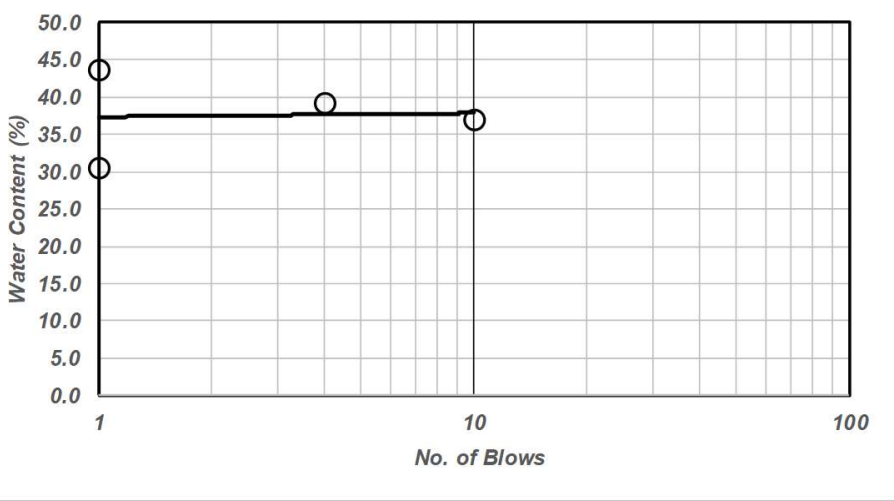

Total amount of materials passing sieve No. 40 : $100 \%$

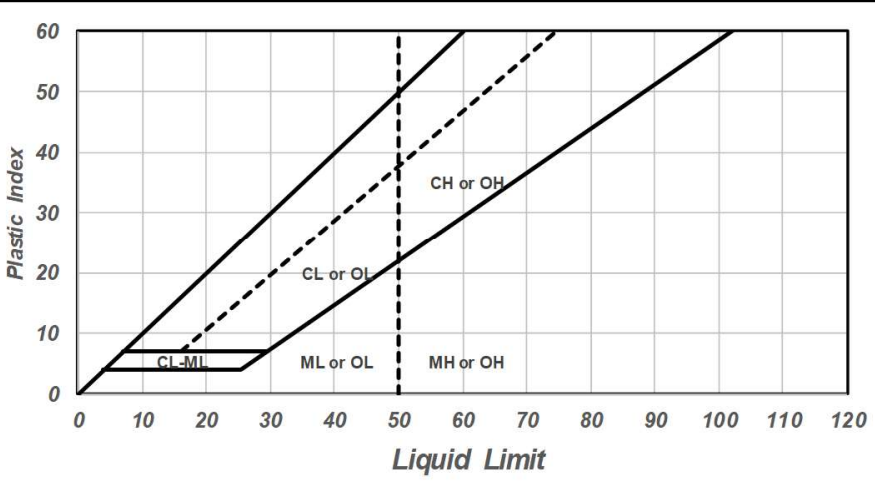

Procedure Done By: Kayla
Fines

Classification: NP 


\section{S Portland State}

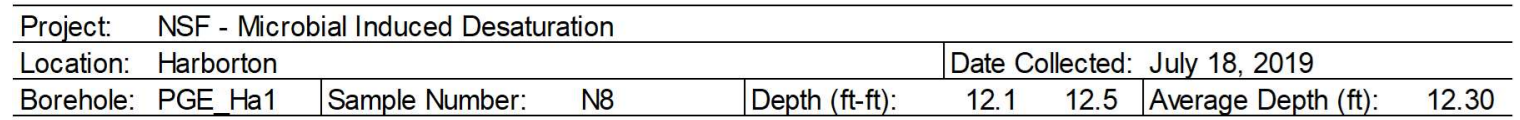

\begin{tabular}{|c|c|c|c|c|c|c|c|c|c|c|}
\hline \multicolumn{6}{|c|}{ Liquid Limit } & \multicolumn{5}{|c|}{ Plastic Limit } \\
\hline $\begin{array}{l}\frac{1}{\mathbf{D}} \\
\frac{O}{E} \\
\bar{\Xi} \\
\Sigma \\
\frac{\omega}{O} \\
\frac{O}{E} \\
\tilde{D}\end{array}$ & 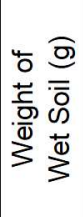 & 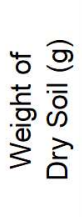 & 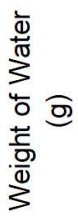 & 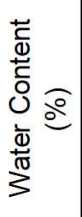 & 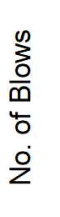 & 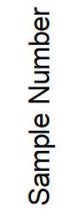 & 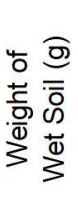 & 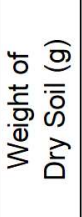 & 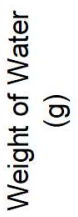 & 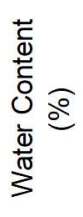 \\
\hline 1 & 47.2 & 31.9 & 15.3 & 48.0 & 1 & & & & & \\
\hline 2 & 12.5 & 9.0 & 3.5 & 38.9 & 9 & & & & & \\
\hline 3 & 11.0 & 8.1 & 2.9 & 35.8 & 15 & & & & & \\
\hline 4 & 19.2 & 14.7 & 4.5 & 30.6 & 1 & & & & & \\
\hline & & & & & & & & & & \\
\hline & & & & & & & & & & \\
\hline
\end{tabular}

Liquid Limit:

Plastic Limit:

Plasticity Index:

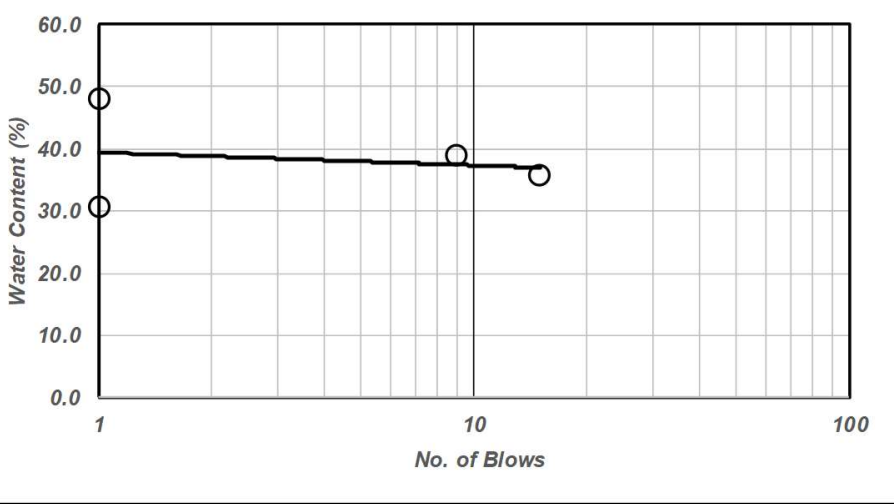

Total amount of materials passing sieve No. 40: $100 \%$

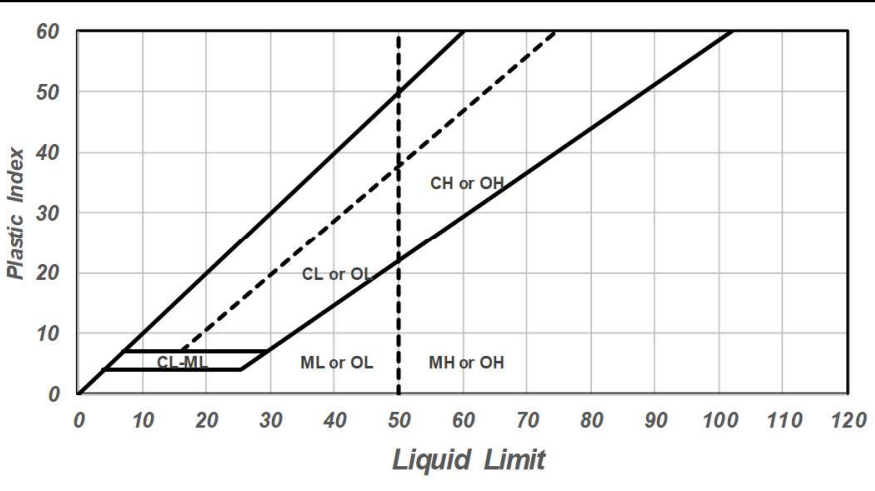

Procedure Done By: Kayla
Fines

Classification: NP 


\section{S Portland State}

\begin{tabular}{|c|c|c|c|c|c|c|}
\hline Project: & NSF - Mic & n & & & & \\
\hline Location: & Harborton & & & Date Collected: & August 8, 2019 & \\
\hline Borehole: & PGE_Ex_N & Sample Number: & Depth (ft-ft): & 5.6 & Average Depth $(\mathrm{ft})$ : & 5.30 \\
\hline
\end{tabular}

\begin{tabular}{|c|c|c|c|c|c|c|c|c|c|c|}
\hline \multicolumn{6}{|c|}{ Liquid Limit } & \multicolumn{5}{|c|}{ Plastic Limit } \\
\hline 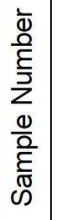 & 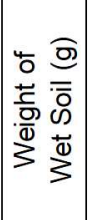 & 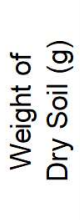 & 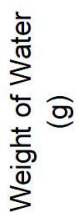 & 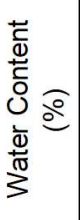 & 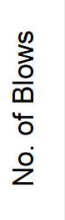 & 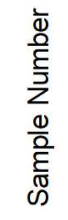 & 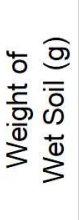 & 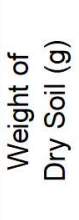 & 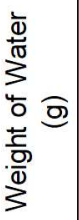 & 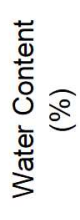 \\
\hline 1 & 5.3 & 4.3 & 1 & 23.3 & 1 & & & & & \\
\hline 2 & 12.5 & 9.8 & 2.7 & 27.6 & 1 & & & & & \\
\hline 3 & 9.8 & 7.4 & 2.4 & 32.4 & 18 & & & & & \\
\hline 4 & 14.4 & 10.7 & 3.7 & 34.6 & 7 & & & & & \\
\hline & & & & & & & & & & \\
\hline & & & & & & & & & & \\
\hline
\end{tabular}
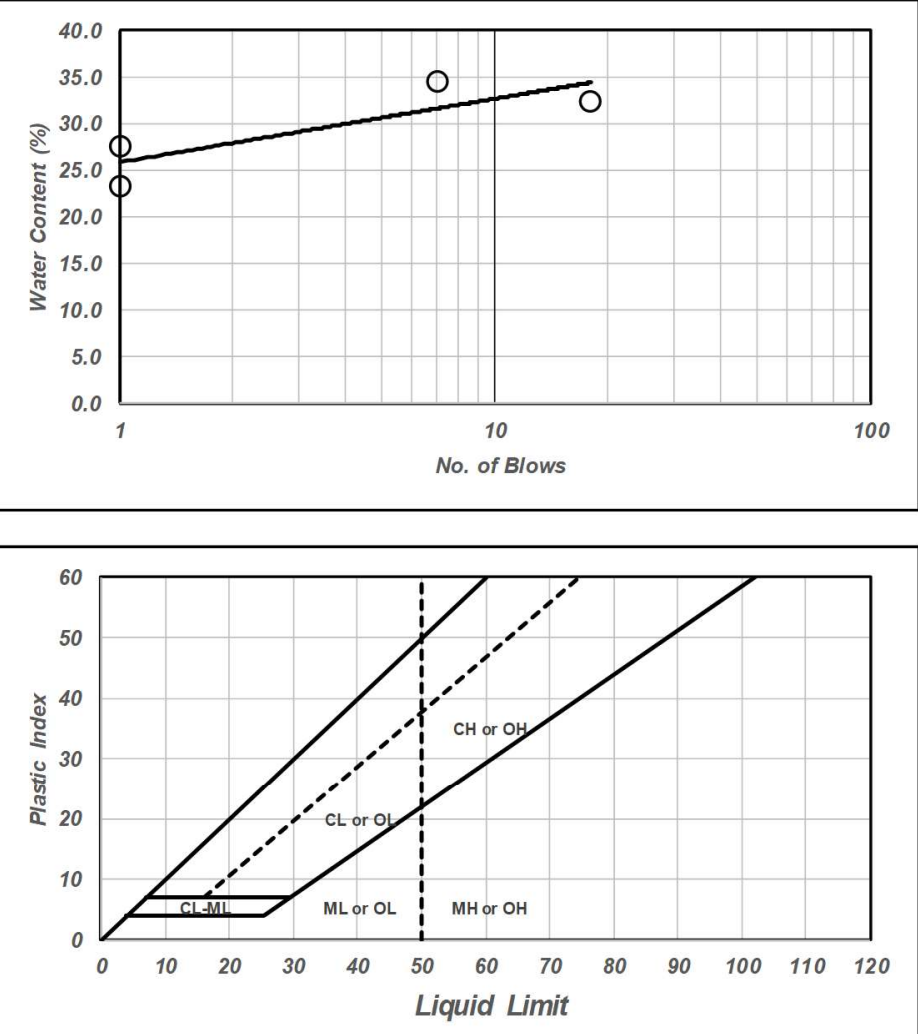

Liquid Limit:

Plastic Limit:

Plasticity Index:
Total amount of materials passing sieve No. 40: $100 \%$
Fines

Classification: NP

Procedure Done By: Kayla 


\section{S Portland State}

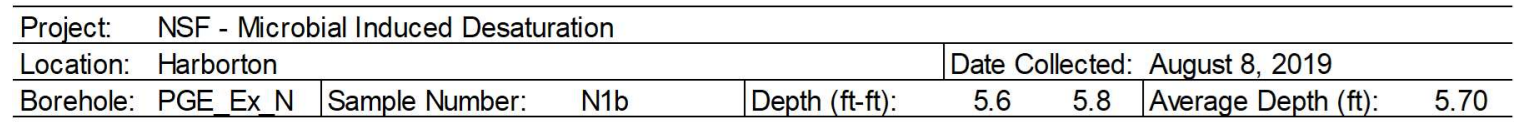

\begin{tabular}{|c|c|c|c|c|c|c|c|c|c|c|}
\hline \multicolumn{6}{|c|}{ Liquid Limit } & \multicolumn{5}{|c|}{ Plastic Limit } \\
\hline 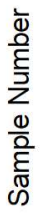 & 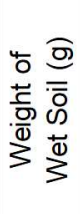 & 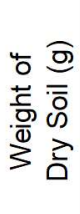 & 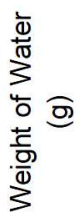 & 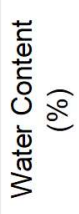 & 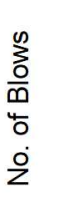 & 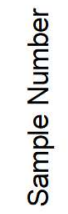 & 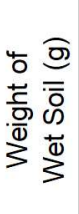 & 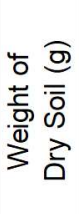 & 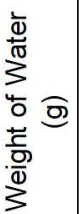 & 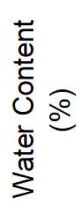 \\
\hline 1 & 5.7 & 4.3 & 1.4 & 32.6 & 1 & & & & & \\
\hline 2 & 4.9 & 3.4 & 1.5 & 44.1 & 5 & & & & & \\
\hline 3 & 6.9 & 4.4 & 2.5 & 56.8 & 1 & & & & & \\
\hline & & & & & & & & & & \\
\hline & & & & & & & & & & \\
\hline & & & & & & & & & & \\
\hline
\end{tabular}
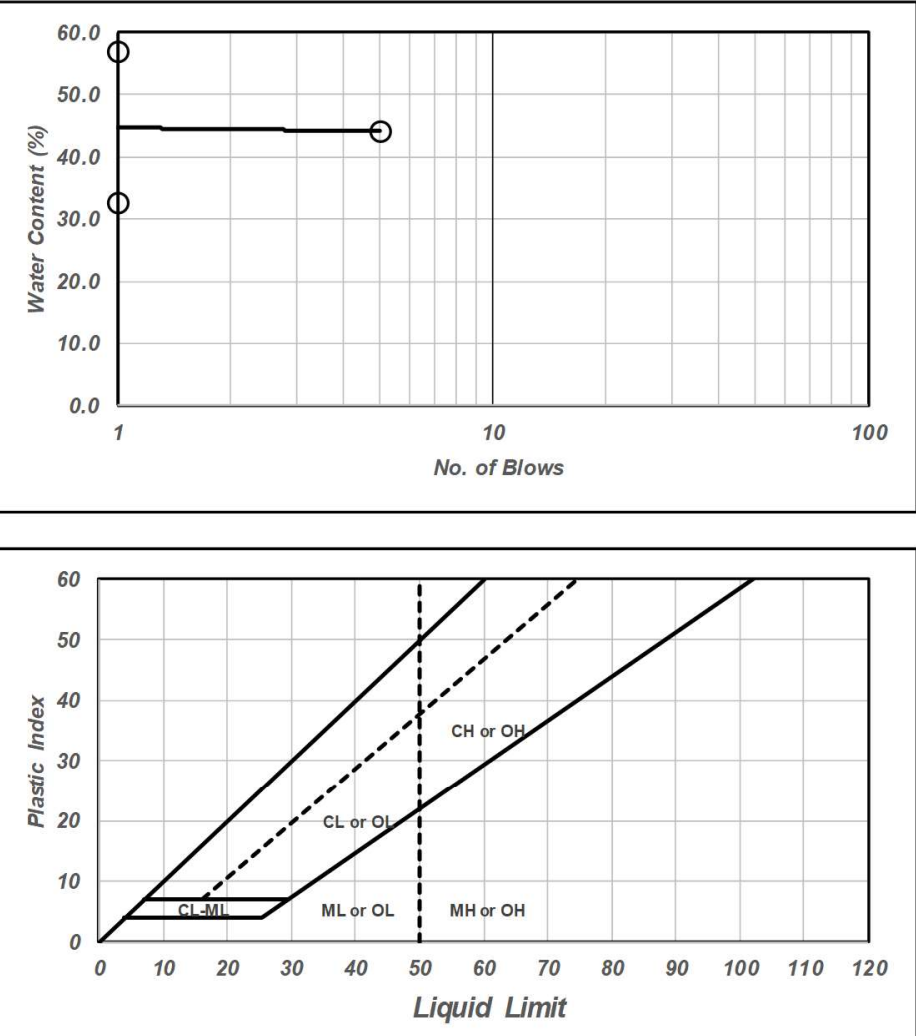

Liquid Limit:

Plastic Limit:

Plasticity Index:
Total amount of materials passing sieve No. 40 : $100 \%$

Procedure Done By: Kayla 


\section{\& Portland State}

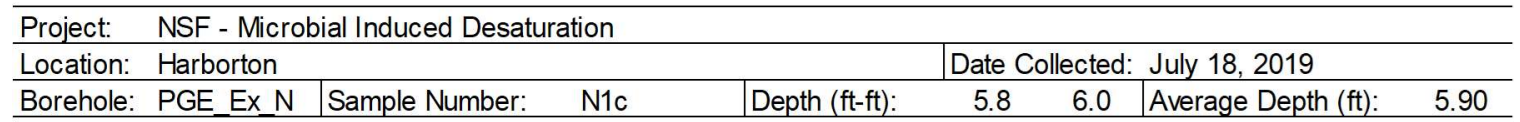

\begin{tabular}{|c|c|c|c|c|c|c|c|c|c|c|}
\hline \multicolumn{6}{|c|}{ Liquid Limit } & \multicolumn{5}{|c|}{ Plastic Limit } \\
\hline 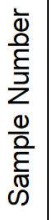 & 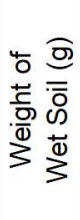 & 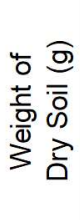 & 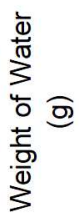 & 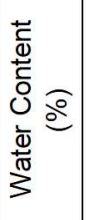 & $\begin{array}{l}0 \\
\sum_{0}^{0} \\
\frac{0}{0} \\
40 \\
\dot{0} \\
\dot{0}\end{array}$ & 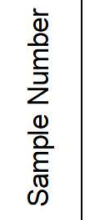 & 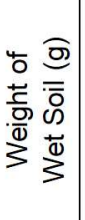 & 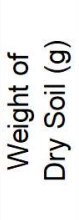 & 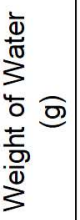 & 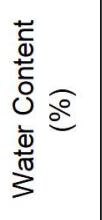 \\
\hline 1 & 3.4 & 2.4 & 1.0 & 41.7 & & 1 & 6.5 & 4.8 & 1.7 & 35.4 \\
\hline 2 & 3.0 & 2.1 & 0.95 & 46.3 & 18 & 2 & 6.3 & 4.6 & 1.7 & 37.0 \\
\hline 3 & 4.1 & 2.9 & 1.2 & 41.4 & 47 & & & & & \\
\hline 4 & 5.4 & 3.6 & 1.8 & 50.0 & 28 & & & & & \\
\hline 5 & 4.6 & 3.1 & 1.5 & 48.4 & 6 & & & & & \\
\hline & & & & & & & & & & \\
\hline
\end{tabular}
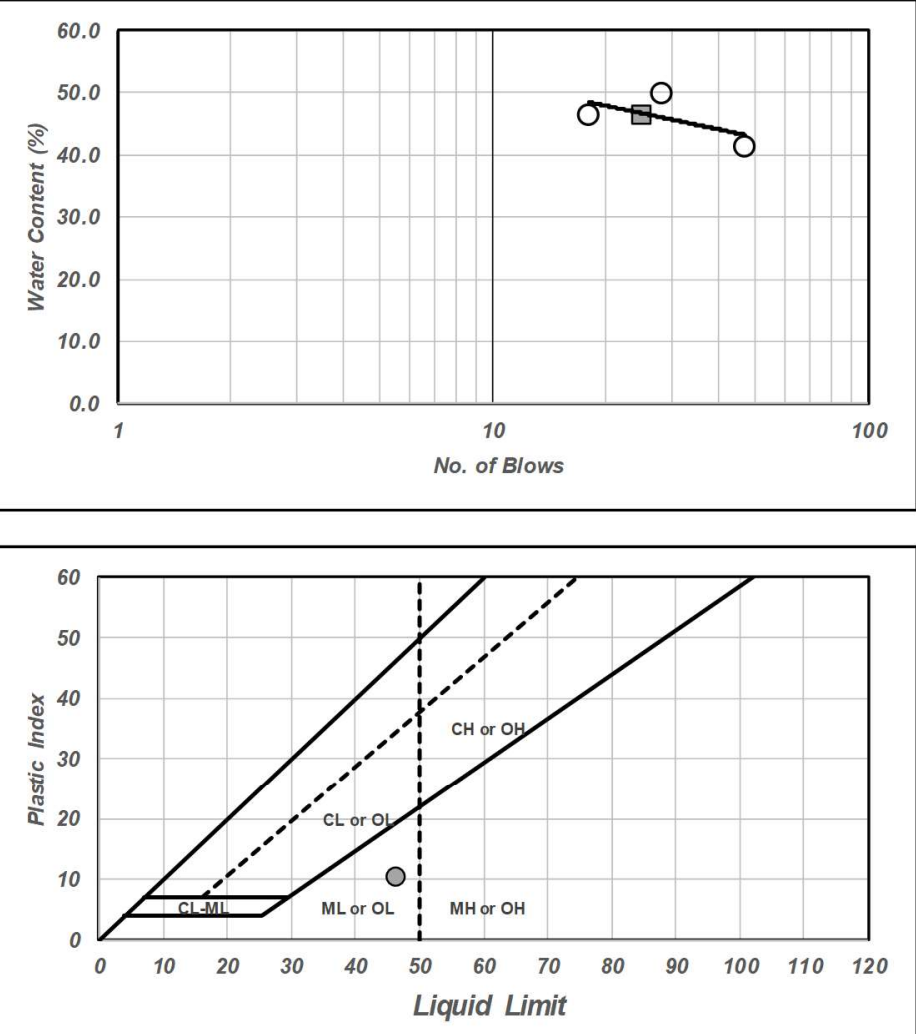

Procedure Done By: Kayla
Liquid Limit:

Plastic Limit:

Plasticity Index:
Total amount of materials passing sieve No. 40: $100 \%$
Fines

Classification: $\mathrm{ML}$ 


\section{S Portland State}

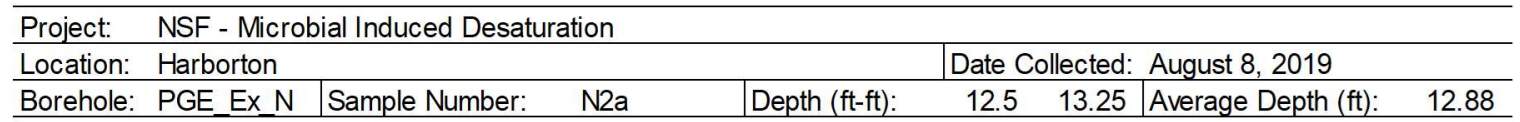

\begin{tabular}{|c|c|c|c|c|c|c|c|c|c|c|}
\hline \multicolumn{6}{|c|}{ Liquid Limit } & \multicolumn{5}{|c|}{ Plastic Limit } \\
\hline 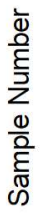 & 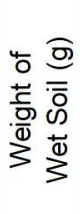 & 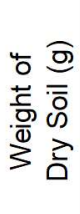 & 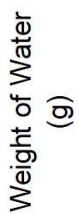 & 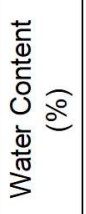 & 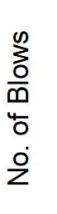 & 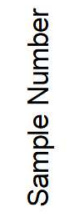 & 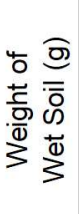 & 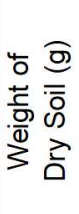 & 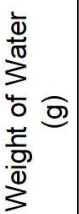 & 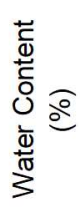 \\
\hline 1 & 3.1 & 2.2 & 0.9 & 40.9 & 3 & & & & & \\
\hline 2 & 4.3 & 3.4 & 0.9 & 26.5 & 1 & & & & & \\
\hline 3 & 3.8 & 2.8 & 1.0 & 35.7 & 3 & & & & & \\
\hline 4 & 5.9 & 4.0 & 1.9 & 47.5 & 1 & & & & & \\
\hline & & & & & & & & & & \\
\hline & & & & & & & & & & \\
\hline
\end{tabular}
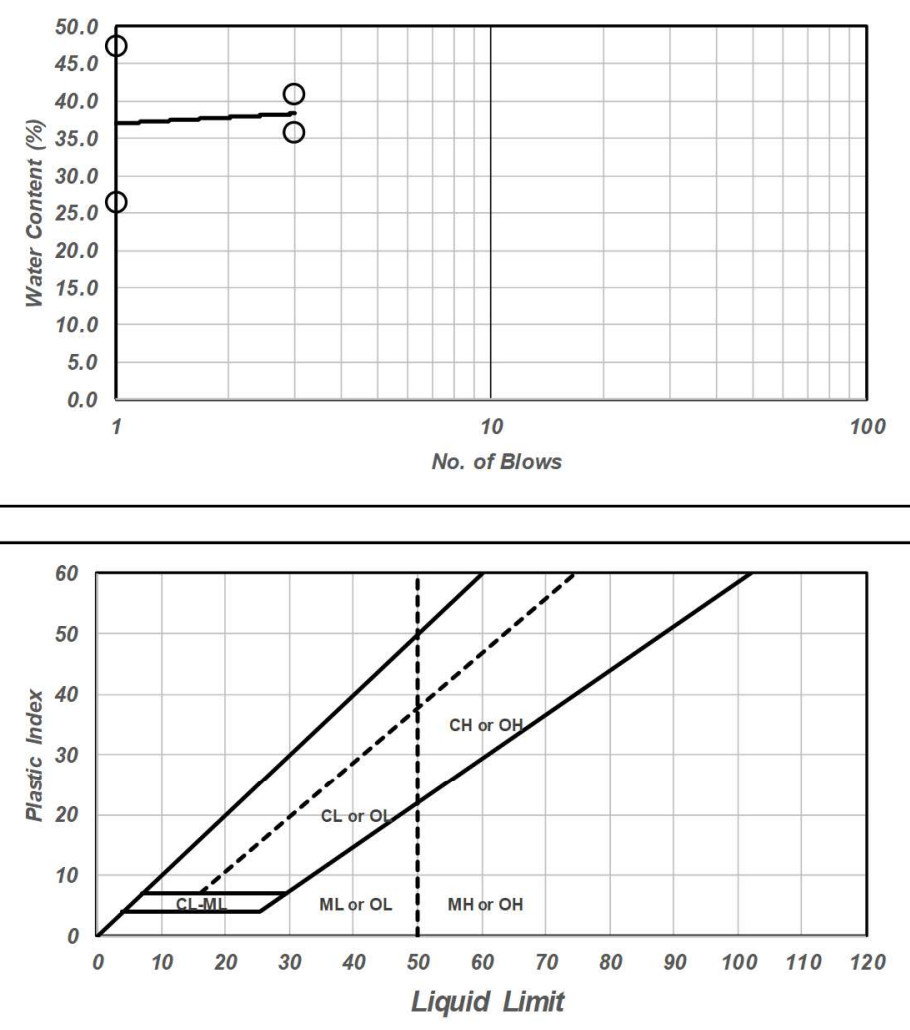

Liquid Limit:

Plastic Limit:

Plasticity Index:
Total amount of materials passing sieve No. 40: $100 \%$
Fines

Classification: NP

Procedure Done By: Kayla 


\section{S Portland State}

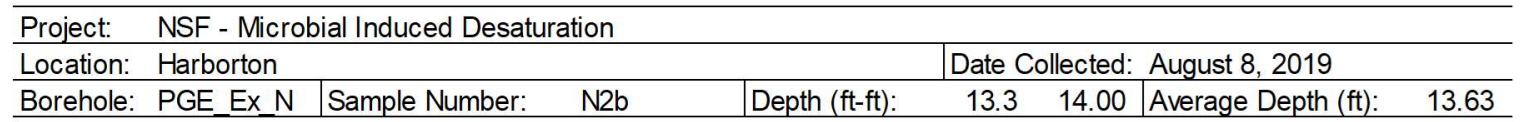

\begin{tabular}{|c|c|c|c|c|c|c|c|c|c|c|}
\hline \multicolumn{6}{|c|}{ Liquid Limit } & \multicolumn{5}{|c|}{ Plastic Limit } \\
\hline 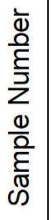 & 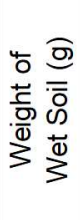 & 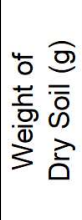 & 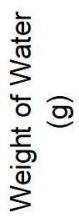 & 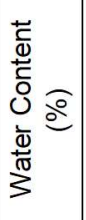 & $\begin{array}{l}0 \\
\sum_{0}^{0} \\
\frac{0}{0} \\
40 \\
\dot{0} \\
\dot{0}\end{array}$ & 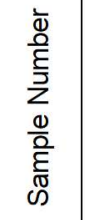 & 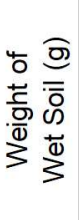 & 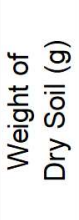 & 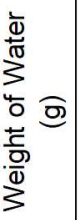 & 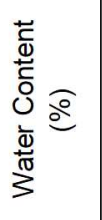 \\
\hline 1 & 4.0 & 2.7 & 1.3 & 48.1 & 45 & 1 & 9.2 & 6.7 & 2.5 & 37.3 \\
\hline 2 & 4.3 & 2.8 & 1.5 & 53.6 & 14 & 2 & 7.8 & 5.7 & 2.1 & 36.8 \\
\hline 3 & 3.1 & 2.1 & 1.0 & 47.6 & 85 & & & & & \\
\hline 4 & 4.9 & 3.3 & 1.6 & 48.5 & 40 & & & & & \\
\hline 5 & 3.9 & 2.5 & 1.4 & 56.0 & 16 & & & & & \\
\hline & & & & & & & & & & \\
\hline
\end{tabular}
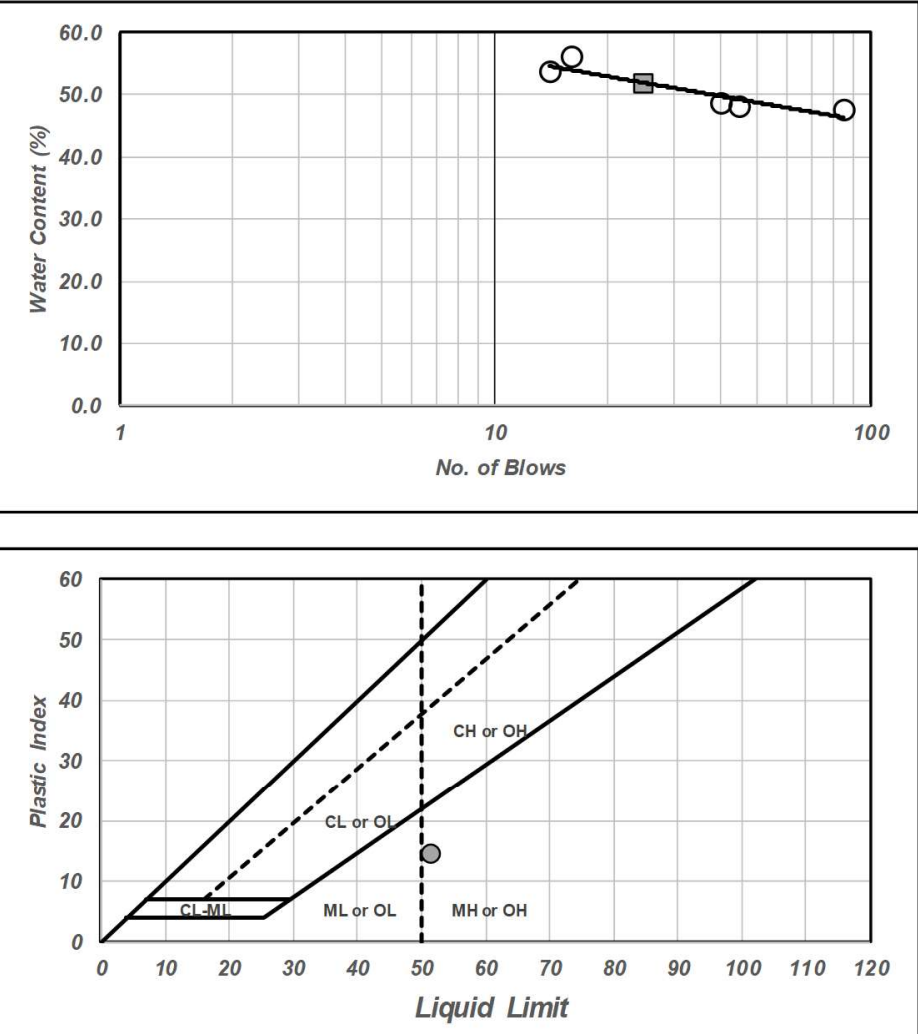

Procedure Done By: Kayla \begin{tabular}{ll} 
Liquid Limit: & $\frac{52}{37}$ \\
Plastic Limit: & $\quad$ \\
Plasticity Index: & \\
\hline
\end{tabular}

Total amount of materials passing sieve No. 40: $100 \%$
Fines

Classification: $\mathrm{MH}$ 


\section{S Portland State}

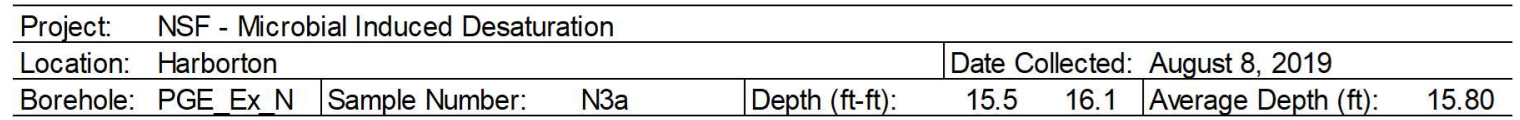

\begin{tabular}{|c|c|c|c|c|c|c|c|c|c|c|}
\hline \multicolumn{6}{|c|}{ Liquid Limit } & \multicolumn{5}{|c|}{ Plastic Limit } \\
\hline $\begin{array}{l}\frac{1}{\mathbf{D}} \\
\frac{O}{E} \\
\bar{\Xi} \\
\Sigma \\
\frac{\omega}{O} \\
\frac{O}{E} \\
\tilde{D}\end{array}$ & 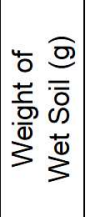 & 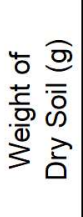 & 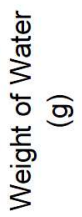 & 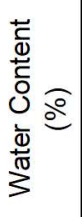 & 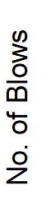 & 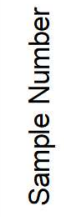 & 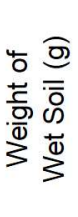 & 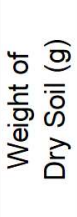 & 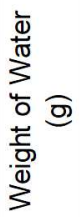 & 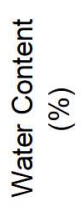 \\
\hline 1 & 14.6 & 10.3 & 4.3 & 41.7 & 1 & & & & & \\
\hline 2 & 11.7 & 9.0 & 2.7 & 30.0 & 4 & & & & & \\
\hline 3 & 9.6 & 7.9 & 1.7 & 21.5 & 1 & & & & & \\
\hline & & & & & & & & & & \\
\hline & & & & & & & & & & \\
\hline & & & & & & & & & & \\
\hline
\end{tabular}

Liquid Limit:

Plastic Limit:

Plasticity Index:

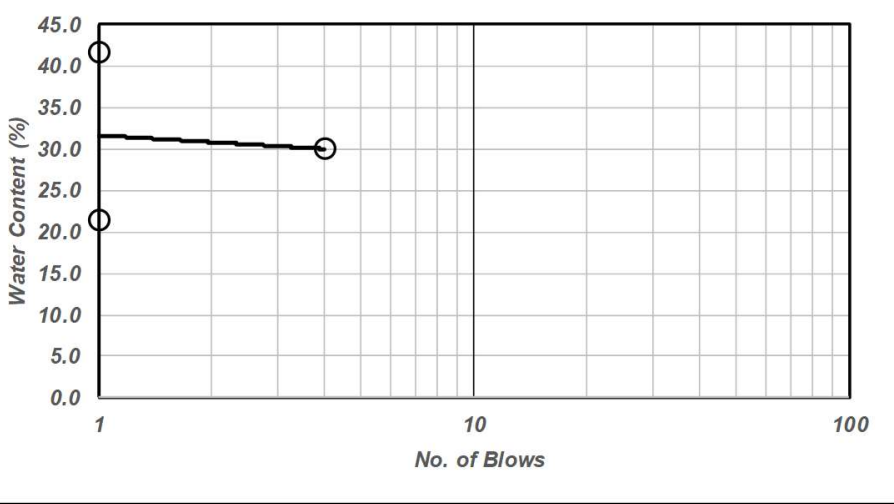

Total amount of materials passing sieve No. 40: $100 \%$

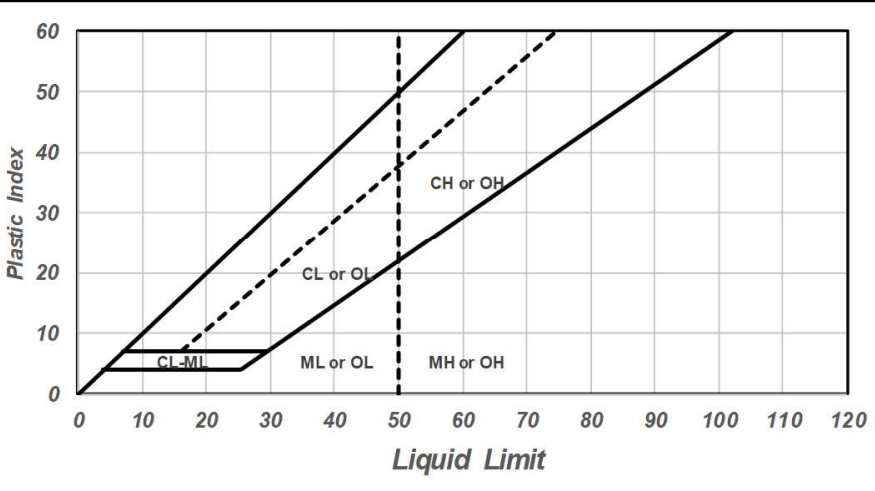

Procedure Done By: Kayla
Fines

Classification: NP 


\section{S Portland State}

\begin{tabular}{|c|c|c|c|c|c|c|c|}
\hline oject: & ISF - Micr & nduced Des & tion & & & & \\
\hline Location: & Harborton & & & & Date Collected: & August 8, 2019 & \\
\hline Borehole: & PGE Ex N & Sample Number: & N3b & Depth (ft-ft): & $16.1 \quad 16.5$ & Average Depth $(\mathrm{ft})$ : & 16.30 \\
\hline
\end{tabular}

\begin{tabular}{|c|c|c|c|c|c|c|c|c|c|c|}
\hline \multicolumn{6}{|c|}{ Liquid Limit } & \multicolumn{5}{|c|}{ Plastic Limit } \\
\hline 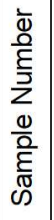 & 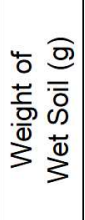 & 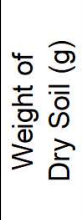 & 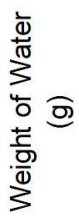 & 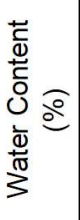 & 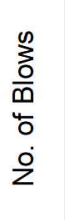 & 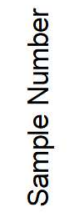 & 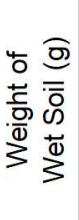 & 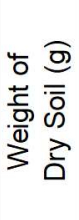 & 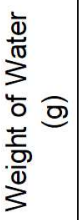 & 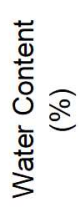 \\
\hline 1 & 8.6 & 6.4 & 2.2 & 34.4 & 6 & & & & & \\
\hline 2 & 5.5 & 4.0 & 1.5 & 37.5 & 3 & & & & & \\
\hline 3 & 6.4 & 4.8 & 1.6 & 33.3 & 15 & & & & & \\
\hline 4 & 5.2 & 3.7 & 1.5 & 40.5 & 3 & & & & & \\
\hline & & & & & & & & & & \\
\hline & & & & & & & & & & \\
\hline
\end{tabular}
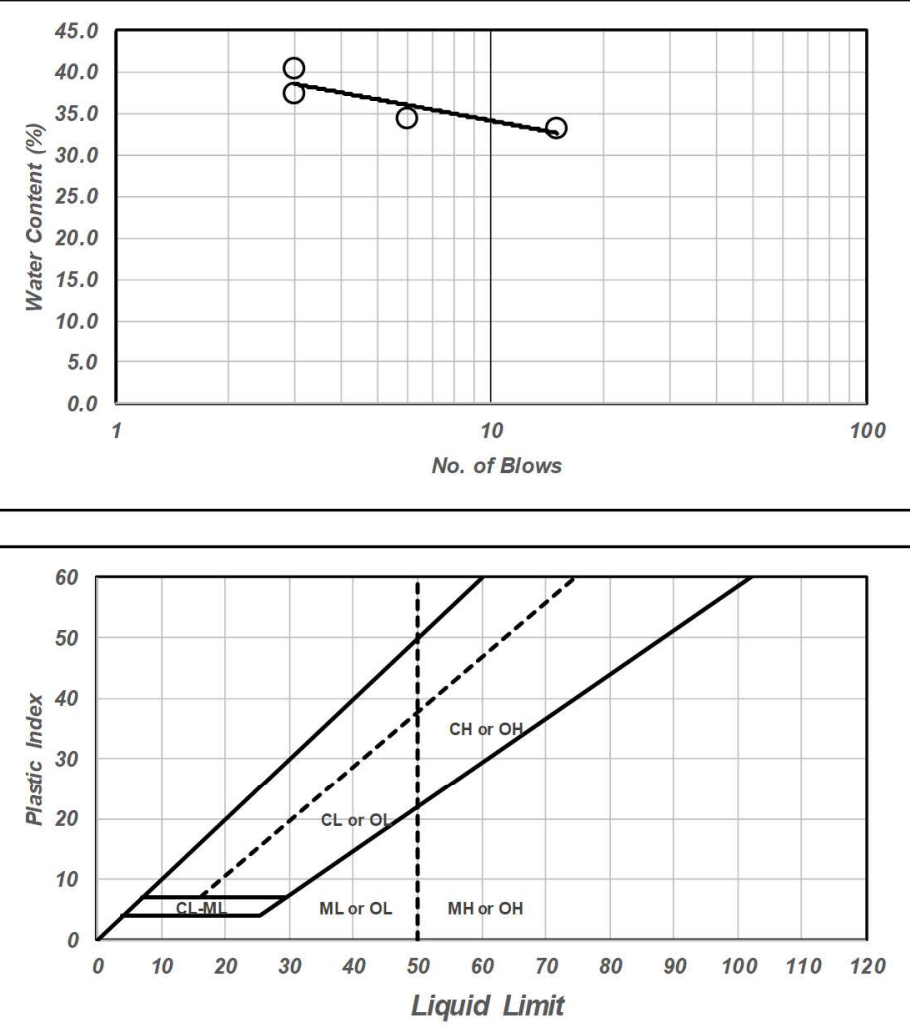

Liquid Limit:

Plastic Limit:

Plasticity Index:

Total amount of materials passing sieve No. 40: $100 \%$

Procedure Done By: Kayla

Fines

Classification: NP 


\section{S Portland State}

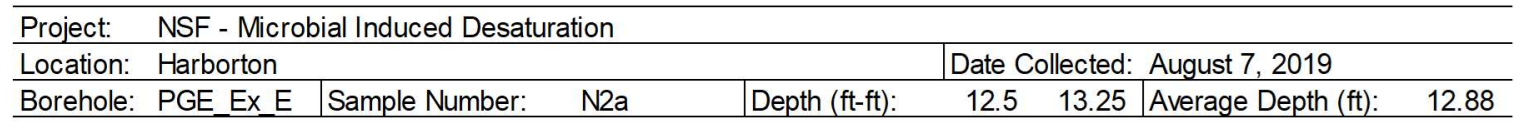

\begin{tabular}{|c|c|c|c|c|c|c|c|c|c|c|}
\hline \multicolumn{6}{|c|}{ Liquid Limit } & \multicolumn{5}{|c|}{ Plastic Limit } \\
\hline 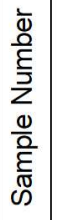 & 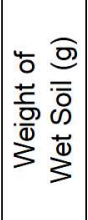 & 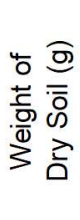 & 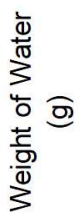 & 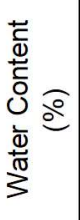 & $\begin{array}{l}0 \\
\sum_{0} \\
\frac{0}{0} \\
4 \\
0 \\
\dot{0} \\
\dot{2}\end{array}$ & 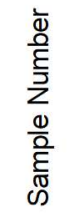 & 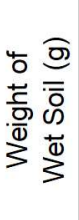 & 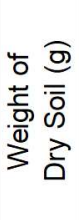 & 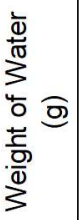 & 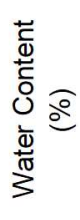 \\
\hline 1 & 12.4 & 8.5 & 3.9 & 45.9 & 2 & & & & & \\
\hline 2 & 12.0 & 8.8 & 3.2 & 36.4 & 5 & & & & & \\
\hline 3 & 8.3 & 6.3 & 2 & 31.7 & 3 & & & & & \\
\hline 4 & 10.0 & 8.5 & 1.5 & 17.6 & 1 & & & & & \\
\hline & & & & & & & & & & \\
\hline & & & & & & & & & & \\
\hline
\end{tabular}
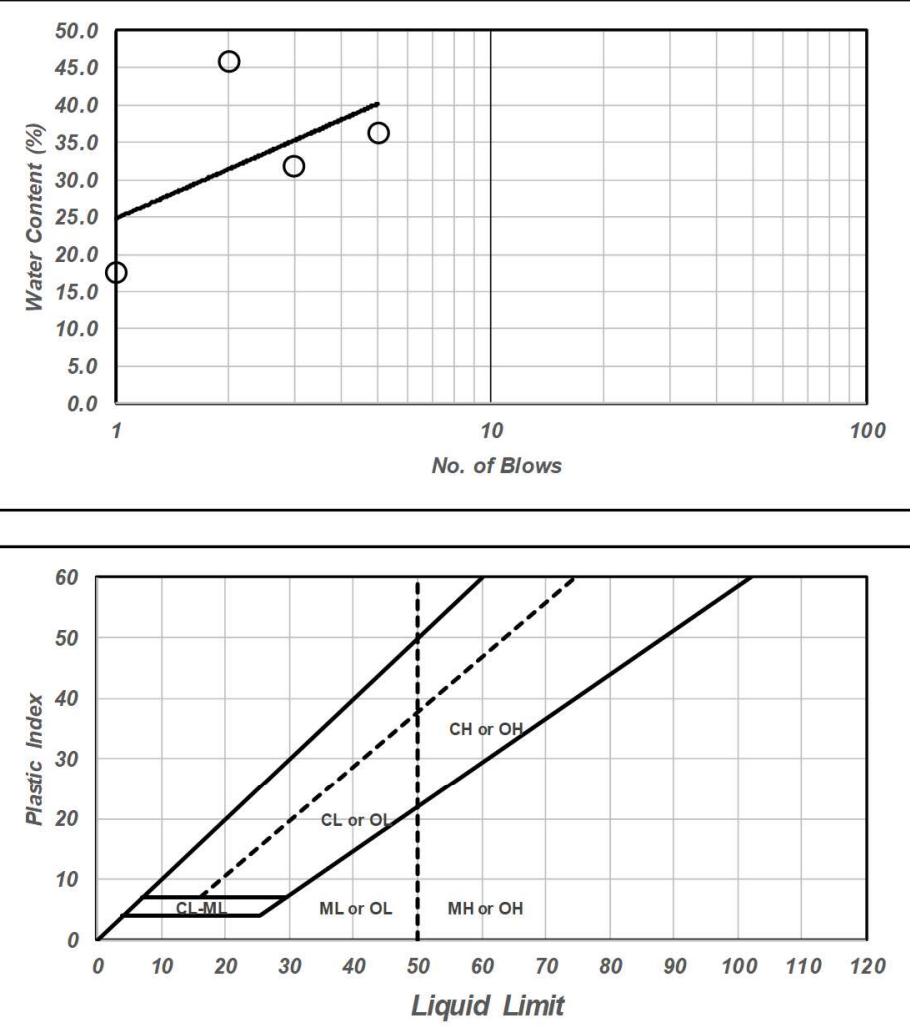

Liquid Limit:

Plastic Limit:

Plasticity Index:
Total amount of materials passing sieve No. 40: $100 \%$
Fines

Classification: NP

Procedure Done By: Melissa P. 


\section{S Portland State}

\begin{tabular}{|c|c|c|c|c|c|c|c|}
\hline oject: & ISF - Mic & nduced Des & tion & & & & \\
\hline Location: & Harborton & & & & Date Collected: & August 7, 2019 & \\
\hline Borehole: & PGE Ex E & Sample Number: & $\mathrm{N} 2 \mathrm{~b}$ & Depth (ft-ft): & 13.2514 .0 & Average Depth (ft): & 13.63 \\
\hline
\end{tabular}

\begin{tabular}{|c|c|c|c|c|c|c|c|c|c|c|}
\hline \multicolumn{6}{|c|}{ Liquid Limit } & \multicolumn{5}{|c|}{ Plastic Limit } \\
\hline 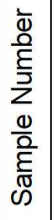 & 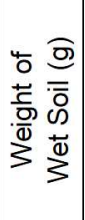 & 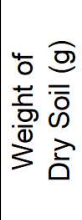 & 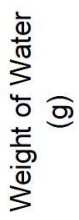 & 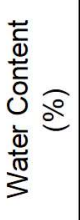 & 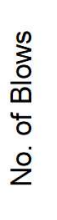 & 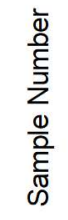 & 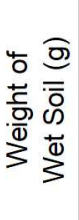 & 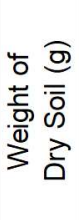 & 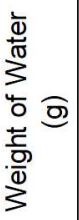 & 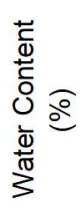 \\
\hline 1 & 10.2 & 7.2 & 3 & 41.7 & 1 & & & & & \\
\hline 2 & 7.7 & 5.4 & 2.3 & 42.6 & 4 & & & & & \\
\hline 3 & 7.5 & 5.2 & 2.3 & 44.2 & 3 & & & & & \\
\hline 4 & 5.4 & 3.6 & 1.8 & 50.0 & 1 & & & & & \\
\hline & & & & & & & & & & \\
\hline & & & & & & & & & & \\
\hline
\end{tabular}
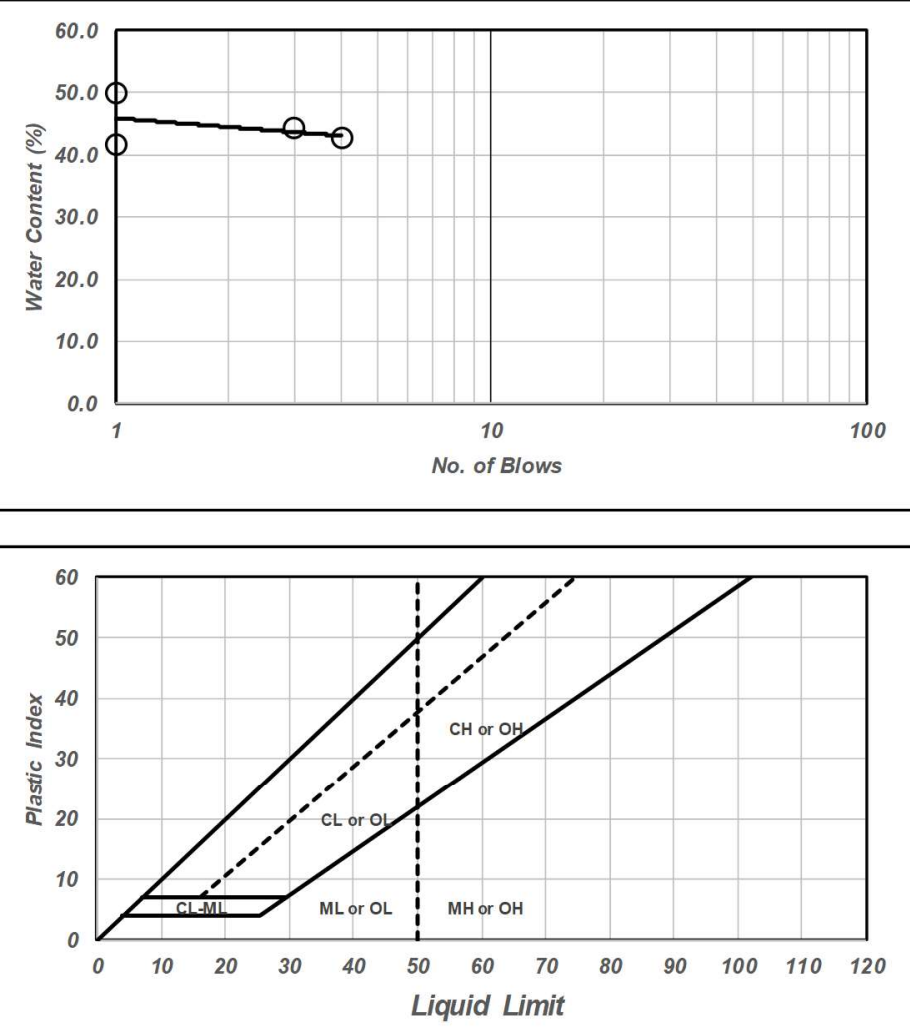

Procedure Done By: Kayla
Liquid Limit:

Plastic Limit:

Plasticity Index:
Total amount of materials passing sieve No. 40: $100 \%$
Fines

Classification: NP 


\section{S Portland State}

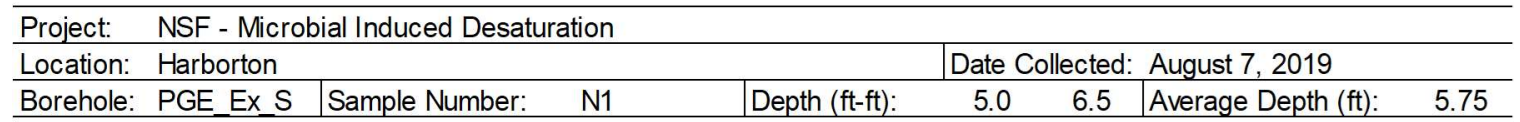

\begin{tabular}{|c|c|c|c|c|c|c|c|c|c|c|}
\hline \multicolumn{6}{|c|}{ Liquid Limit } & \multicolumn{5}{|c|}{ Plastic Limit } \\
\hline 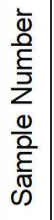 & 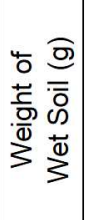 & 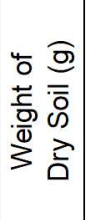 & 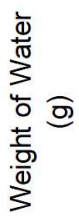 & 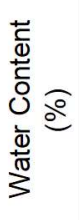 & 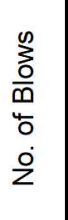 & 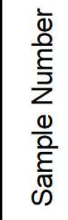 & 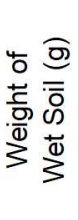 & 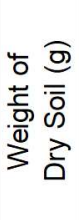 & 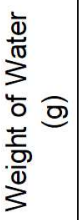 & 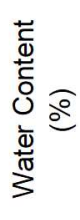 \\
\hline 1 & 12.1 & 9.4 & 2.7 & 28.7 & 1 & & & & & \\
\hline & & & & & & & & & & \\
\hline & & & & & & & & & & \\
\hline & & & & & & & & & & \\
\hline & & & & & & & & & & \\
\hline & & & & & & & & & & \\
\hline
\end{tabular}
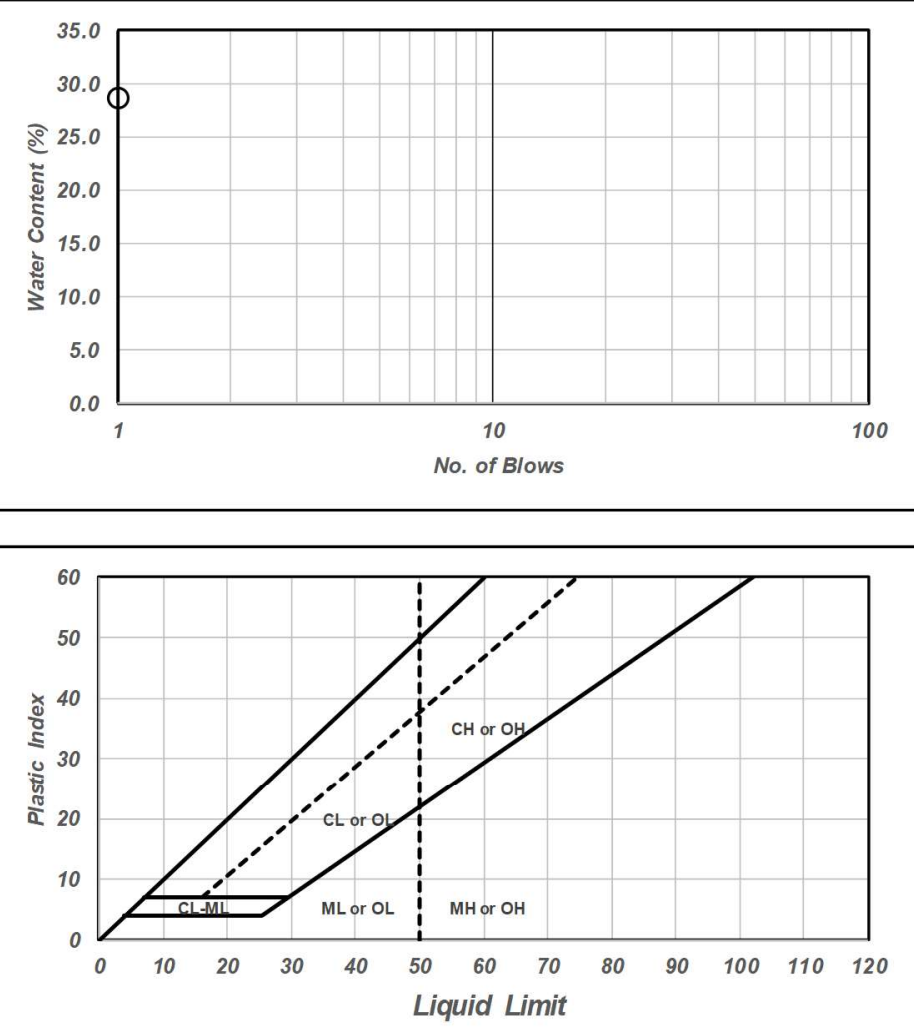

Liquid Limit:

Plastic Limit:

Plasticity Index:
Total amount of materials passing sieve No. 40 : $100 \%$
Fines NP by

Classification: observation

Procedure Done By: Kayla 


\section{S Portland State}

\begin{tabular}{|c|c|c|c|c|c|c|}
\hline Project: & ISF - Mic & on & & & & \\
\hline Location: & Harborton & & & Date Collected: & August 7, 2019 & \\
\hline Borehole: & PGE_Ex S & Sample Number: & Depth (ft-ft): & 13.0 & Average Depth $(\mathrm{ft})$ : & 12.75 \\
\hline
\end{tabular}

\begin{tabular}{|c|c|c|c|c|c|c|c|c|c|c|}
\hline \multicolumn{6}{|c|}{ Liquid Limit } & \multicolumn{5}{|c|}{ Plastic Limit } \\
\hline 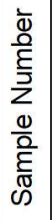 & 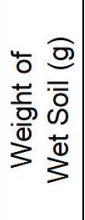 & 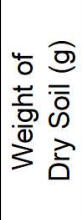 & 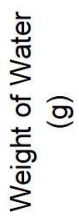 & 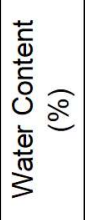 & 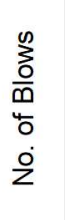 & 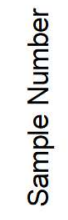 & 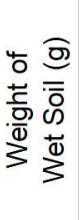 & 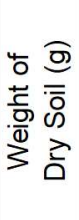 & 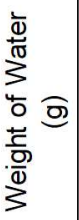 & 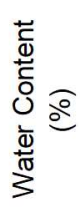 \\
\hline 1 & 4.3 & 3.3 & 1.0 & 30.3 & 10 & & & & & \\
\hline 2 & 4.2 & 2.9 & 1.3 & 44.8 & 2 & & & & & \\
\hline 3 & 3.4 & 2.5 & 0.9 & 36.0 & 1 & & & & & \\
\hline 4 & 4.5 & 3.2 & 1.3 & 40.6 & 6 & & & & & \\
\hline & & & & & & & & & & \\
\hline & & & & & & & & & & \\
\hline
\end{tabular}
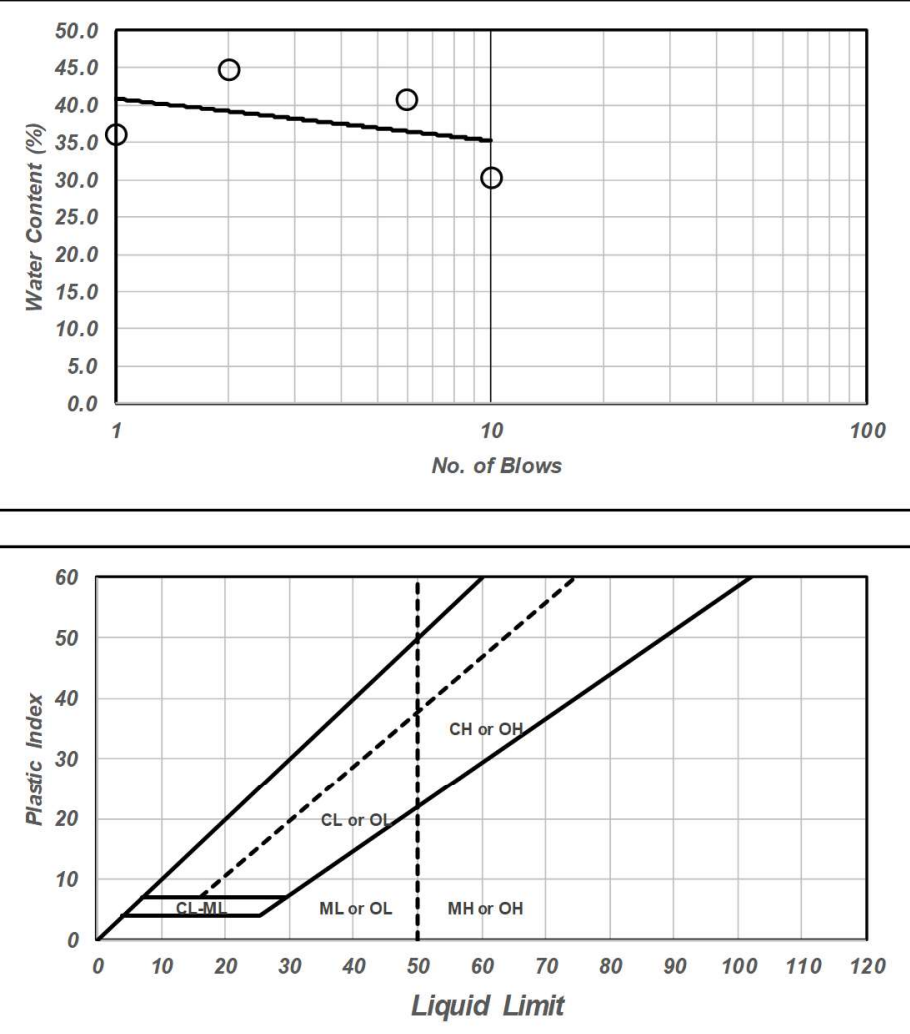

Procedure Done By: Kayla
Liquid Limit:

Plastic Limit:

Plasticity Index:
Total amount of materials passing sieve No. 40: $100 \%$
Fines

Classification: NP 


\section{S Portland State}

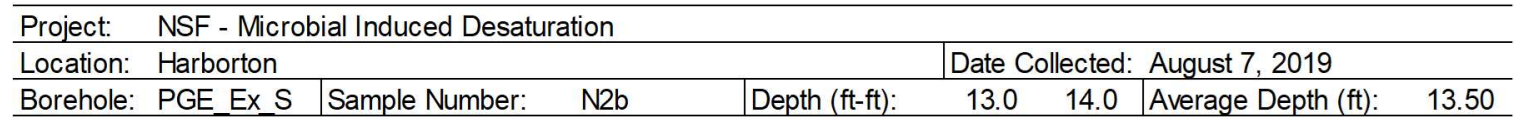

\begin{tabular}{|c|c|c|c|c|c|c|c|c|c|c|}
\hline \multicolumn{6}{|c|}{ Liquid Limit } & \multicolumn{5}{|c|}{ Plastic Limit } \\
\hline 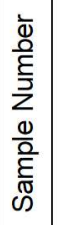 & 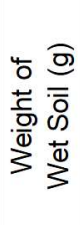 & 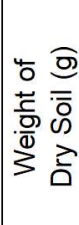 & 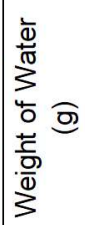 & 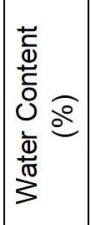 & $\begin{array}{l}00 \\
\sum_{0}^{0} \\
0 \\
40 \\
\dot{0} \\
\dot{0}\end{array}$ & 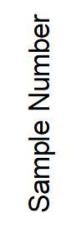 & 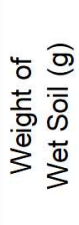 & 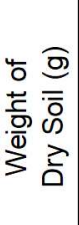 & 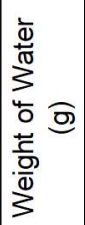 & 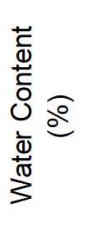 \\
\hline 1 & 3.2 & 2.3 & 0.9 & 39.1 & & 1 & 9.0 & 6.7 & 2.3 & 34.3 \\
\hline 2 & 4.0 & 2.7 & 1.3 & 48.1 & 23 & 2 & 8.7 & 6.5 & 2.2 & 33.8 \\
\hline 3 & 6.8 & 4.5 & 2.3 & 51.1 & 21 & & & & & \\
\hline 4 & 4.0 & 2.8 & 1.2 & 42.9 & 31 & & & & & \\
\hline 5 & 3.8 & 2.7 & 1.1 & 40.7 & 69 & & & & & \\
\hline & & & & & & & & & & \\
\hline
\end{tabular}

Liquid Limit:

Plastic Limit:

Plasticity Index:

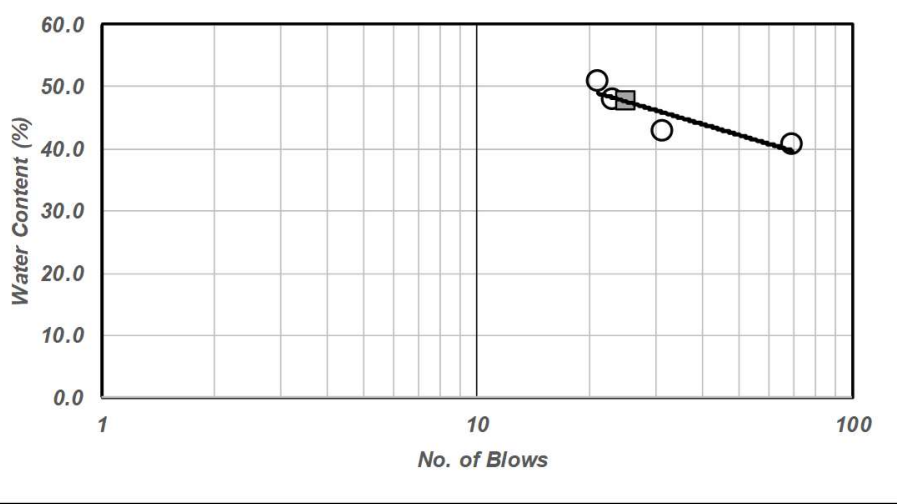

Total amount of materials passing sieve No. 40: $100 \%$

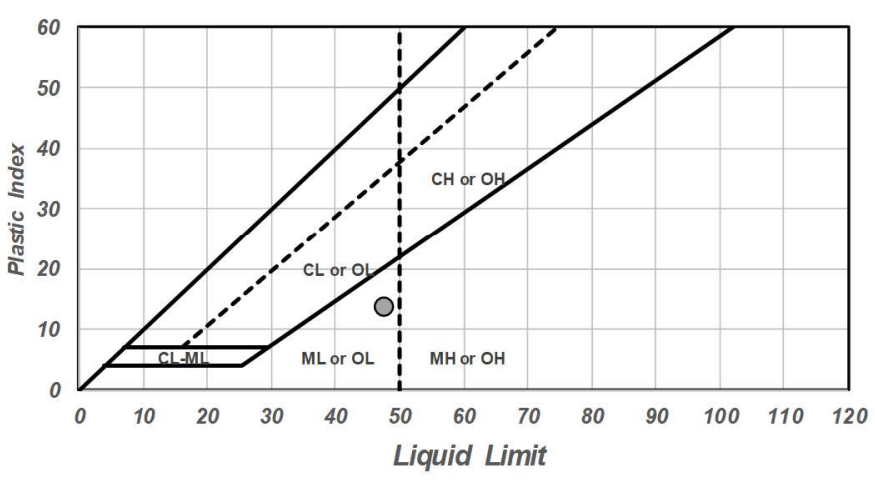

Procedure Done By:
Rawan
Fines

Classification: $\mathrm{ML}$ 


\section{S Portland State}

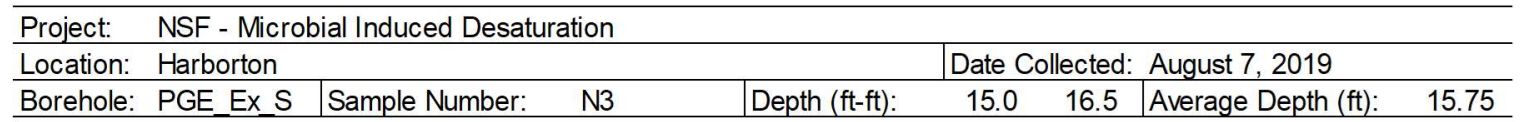

\begin{tabular}{|c|c|c|c|c|c|c|c|c|c|c|}
\hline \multicolumn{6}{|c|}{ Liquid Limit } & \multicolumn{5}{|c|}{ Plastic Limit } \\
\hline $\begin{array}{l}\frac{1}{\mathbf{D}} \\
\frac{O}{E} \\
\bar{\Xi} \\
\Sigma \\
\frac{\omega}{O} \\
\frac{O}{E} \\
\tilde{D}\end{array}$ & 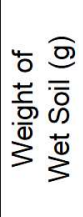 & 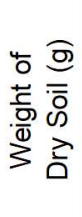 & 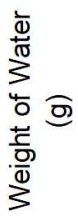 & 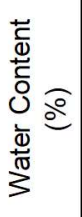 & 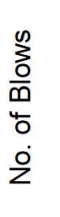 & 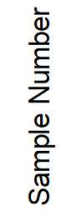 & 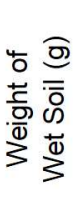 & 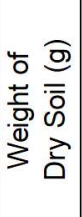 & 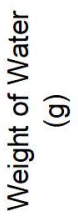 & 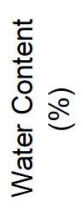 \\
\hline 1 & 26.9 & 17.1 & 9.8 & 57.3 & 1 & & & & & \\
\hline 2 & 15.7 & 11.2 & 4.5 & 40.2 & 22 & & & & & \\
\hline 3 & 12.5 & 8.7 & 3.8 & 43.7 & 14 & & & & & \\
\hline 4 & 15.7 & 10.7 & 5.0 & 46.7 & 7 & & & & & \\
\hline & & & & & & & & & & \\
\hline & & & & & & & & & & \\
\hline
\end{tabular}

Liquid Limit:

Plastic Limit:

Plasticity Index:

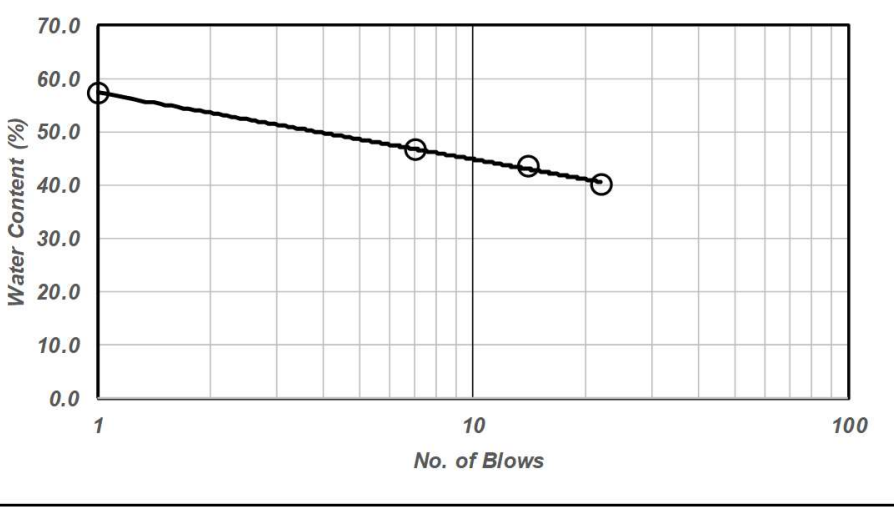

Total amount of materials passing sieve No. 40: $100 \%$

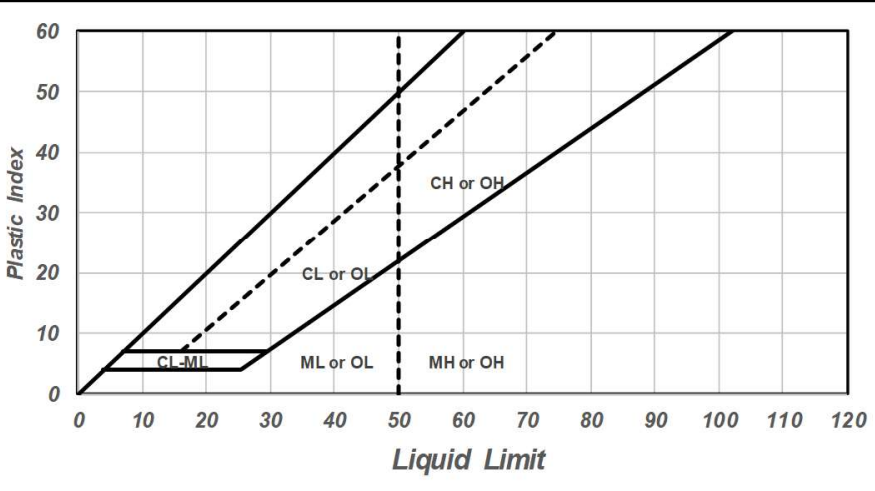

Procedure Done By: Kayla
Fines

Classification: NP 


\section{S Portland State}

\begin{tabular}{|c|c|c|c|c|c|c|}
\hline \multirow{2}{*}{$\begin{array}{l}\text { Project: } \\
\text { Location: }\end{array}$} & \multicolumn{6}{|c|}{ NSF - Microbial Induced Desaturation } \\
\hline & Harborton & & & & Date & ected \\
\hline Borehole: & PGE_Ex_W & Sample Number: & $\mathrm{N} 1$ & Depth (ft-ft): & 5.0 & 6.5 \\
\hline
\end{tabular}

\begin{tabular}{|c|c|c|c|c|c|c|c|c|c|c|}
\hline \multicolumn{6}{|c|}{ Liquid Limit } & \multicolumn{5}{|c|}{ Plastic Limit } \\
\hline 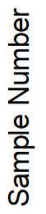 & 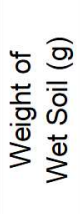 & 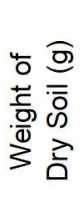 & 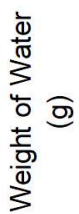 & 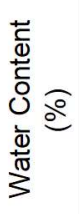 & 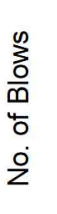 & 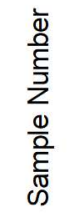 & 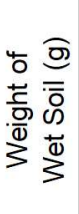 & 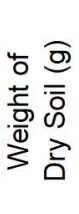 & 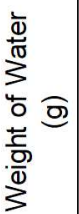 & 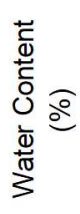 \\
\hline 1 & 5.1 & 4 & 1.1 & 27.5 & 1 & & & & & \\
\hline & & & & & & & & & & \\
\hline & & & & & & & & & & \\
\hline & & & & & & & & & & \\
\hline & & & & & & & & & & \\
\hline & & & & & & & & & & \\
\hline
\end{tabular}
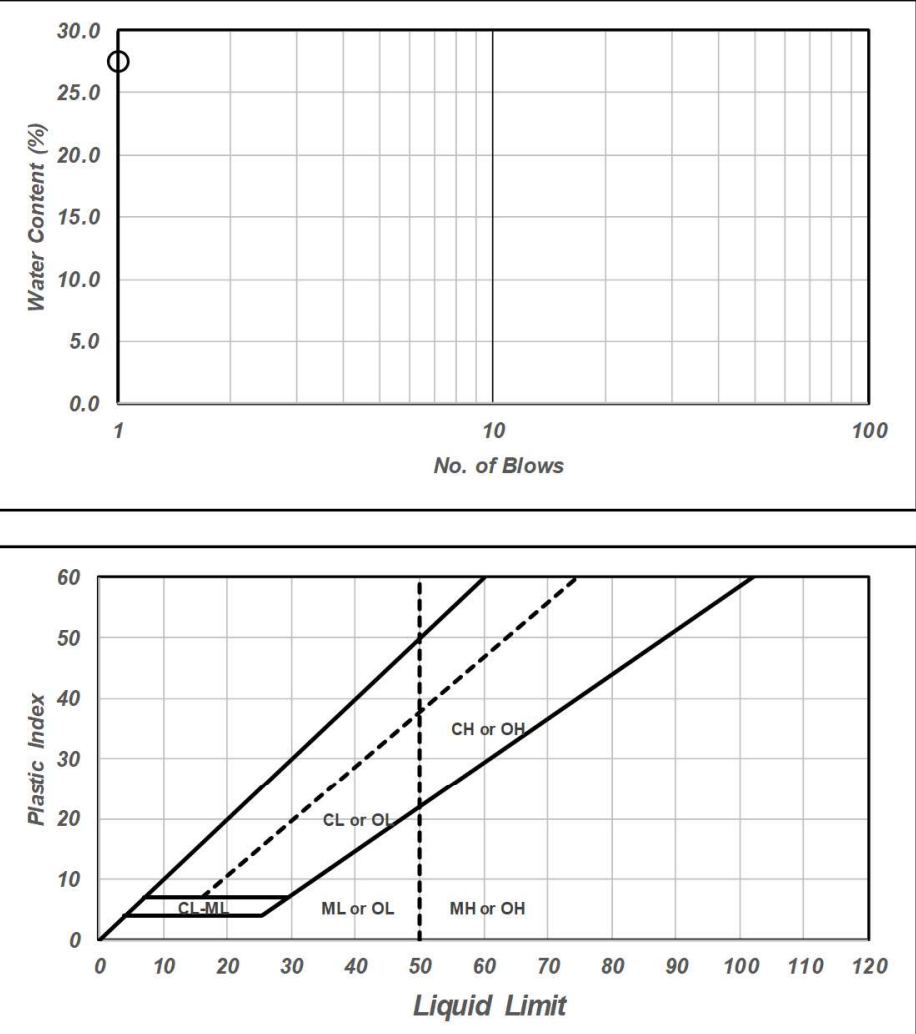

Liquid Limit:

Plastic Limit:

Plasticity Index:
Total amount of materials passing sieve No. 40 : $100 \%$
Fines NP by

Classification: observation

Procedure Done By:

Nathan 


\section{S Portland State}

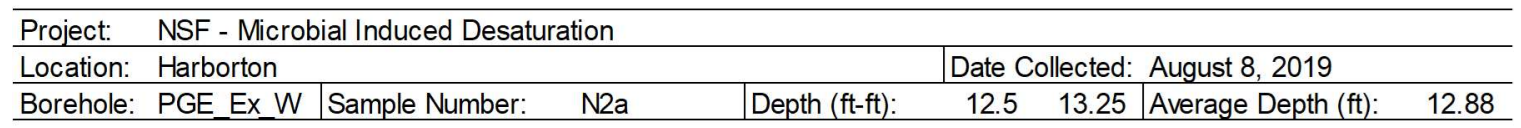

\begin{tabular}{|c|c|c|c|c|c|c|c|c|c|c|}
\hline \multicolumn{6}{|c|}{ Liquid Limit } & \multicolumn{5}{|c|}{ Plastic Limit } \\
\hline 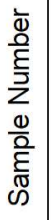 & 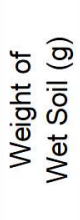 & 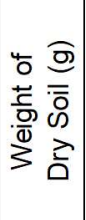 & 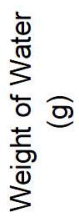 & 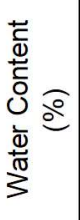 & $\begin{array}{l}0 \\
\sum_{0} \\
\frac{0}{0} \\
4 \\
0 \\
\dot{0} \\
\dot{2}\end{array}$ & 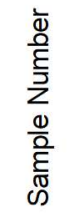 & 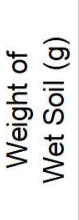 & 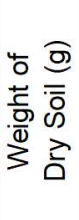 & 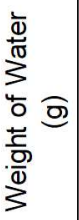 & 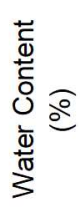 \\
\hline 1 & 8.2 & 5.7 & 2.5 & 43.9 & 1 & & & & & \\
\hline & & & & & & & & & & \\
\hline & & & & & & & & & & \\
\hline & & & & & & & & & & \\
\hline & & & & & & & & & & \\
\hline & & & & & & & & & & \\
\hline
\end{tabular}
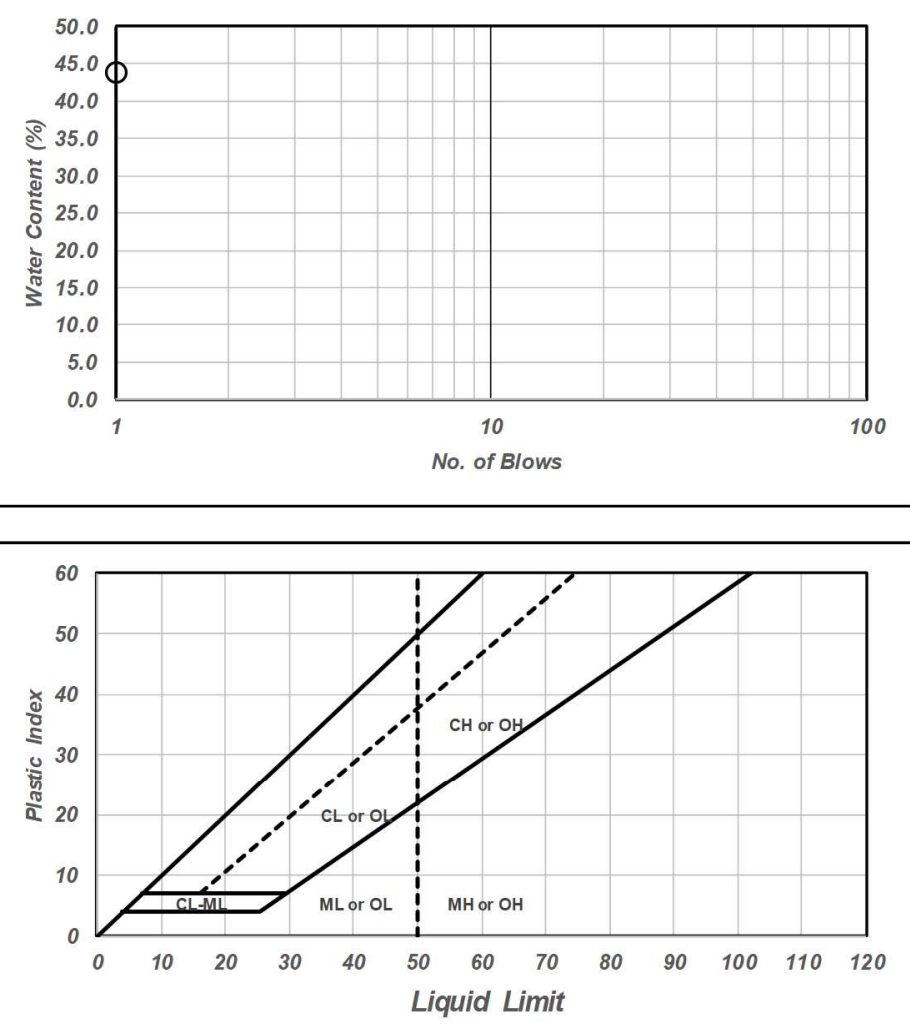

Liquid Limit:

Plastic Limit:

Plasticity Index:
Total amount of materials passing sieve No. 40: $100 \%$
Fines NP by

Classification: observation

Procedure Done By:

Nathan 


\section{S Portland State}

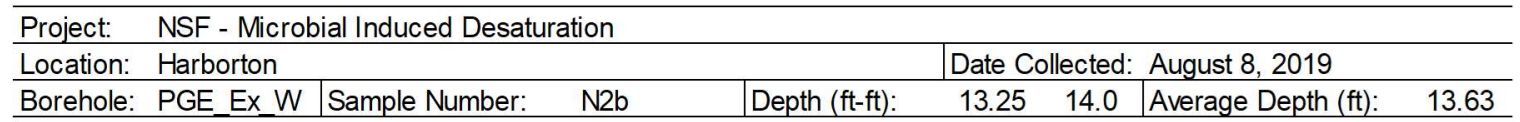

\begin{tabular}{|c|c|c|c|c|c|c|c|c|c|c|}
\hline \multicolumn{6}{|c|}{ Liquid Limit } & \multicolumn{5}{|c|}{ Plastic Limit } \\
\hline 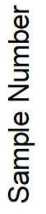 & 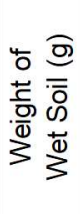 & 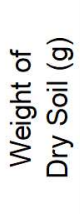 & 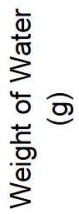 & 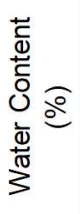 & \begin{tabular}{l}
00 \\
3 \\
0 \\
0 \\
4 \\
0 \\
0 \\
\hdashline
\end{tabular} & 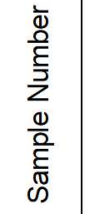 & 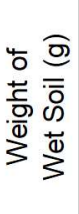 & 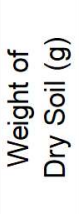 & 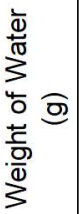 & 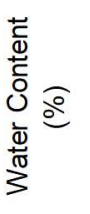 \\
\hline 1 & 8.3 & 5.6 & 2.7 & 48.2 & & 1 & 8.3 & 6.0 & 2.3 & 38.3 \\
\hline 2 & 14.1 & 9.8 & 4.3 & 43.9 & 30 & 2 & 8.7 & 6.2 & 2.5 & 40.3 \\
\hline 3 & 13.4 & 9.1 & 4.3 & 47.3 & 24 & & & & & \\
\hline 4 & 13.6 & 8.8 & 4.8 & 54.5 & 17 & & & & & \\
\hline & & & & & & & & & & \\
\hline & & & & & & & & & & \\
\hline
\end{tabular}

Liquid Limit:

Plastic Limit:

Plasticity Index: $\frac{47}{39}$
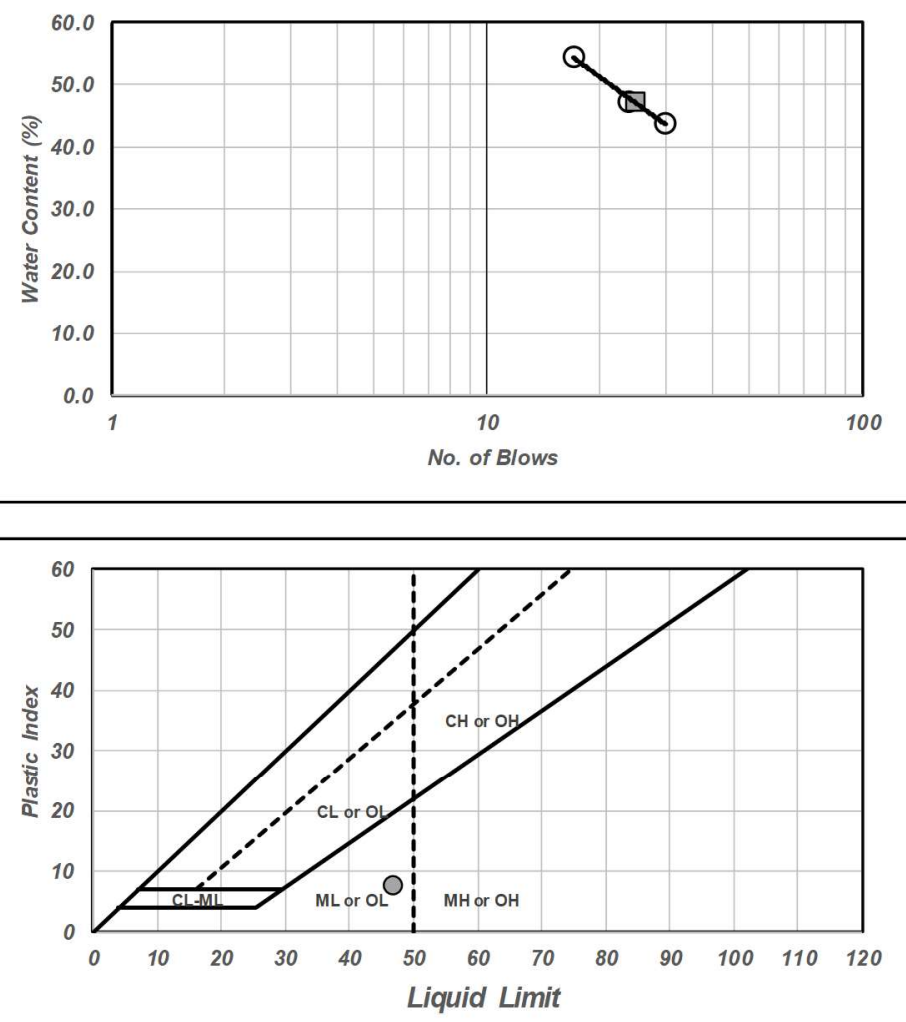

Total amount of materials passing sieve No. 40: $100 \%$
Fines

Classification: ML

Procedure Done By:

Nathan 


\section{S Portland State}

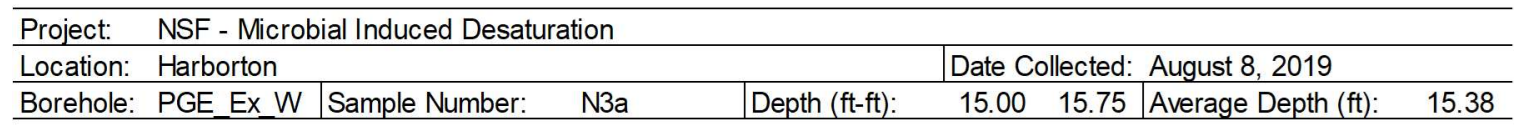

\begin{tabular}{|c|c|c|c|c|c|c|c|c|c|c|}
\hline \multicolumn{6}{|c|}{ Liquid Limit } & \multicolumn{5}{|c|}{ Plastic Limit } \\
\hline 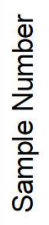 & 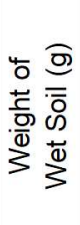 & 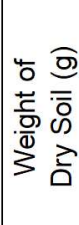 & 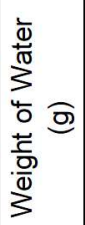 & 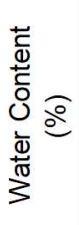 & 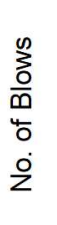 & 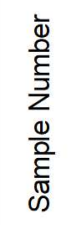 & 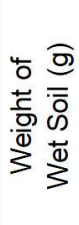 & 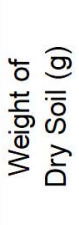 & 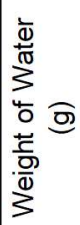 & 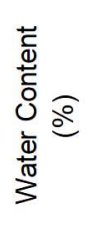 \\
\hline 1 & 16.1 & 11.2 & 4.9 & 43.8 & 1 & & & & & \\
\hline & & & & & & & & & & \\
\hline & & & & & & & & & & \\
\hline & & & & & & & & & & \\
\hline & & & & & & & & & & \\
\hline & & & & & & & & & & \\
\hline
\end{tabular}
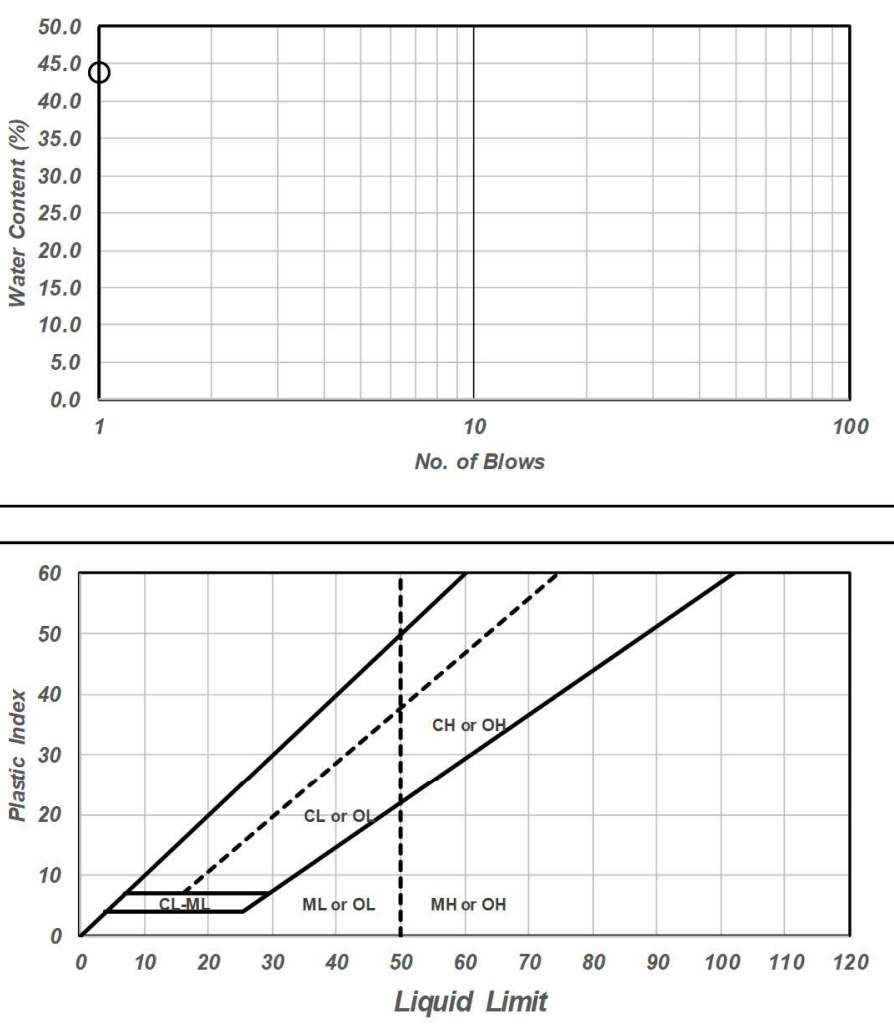

Liquid Limit:

Plastic Limit:

Plasticity Index:
Total amount of materials passing sieve No. 40:
Fines NP by

Classification: observation

Procedure Done By:

Nathan 


\section{S Portland State}

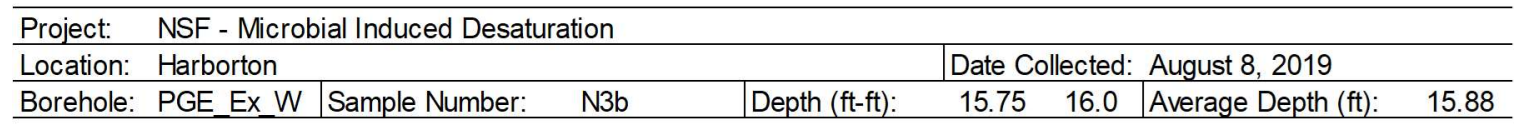

\begin{tabular}{|c|c|c|c|c|c|c|c|c|c|c|}
\hline \multicolumn{6}{|c|}{ Liquid Limit } & \multicolumn{5}{|c|}{ Plastic Limit } \\
\hline 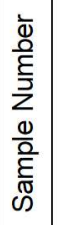 & 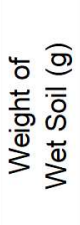 & 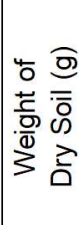 & 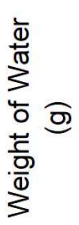 & 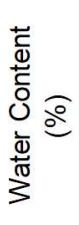 & $\begin{array}{l}00 \\
\sum_{0}^{0} \\
0 \\
40 \\
\dot{0} \\
\dot{0}\end{array}$ & 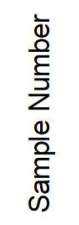 & 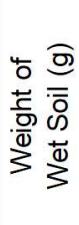 & 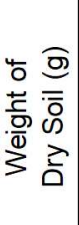 & 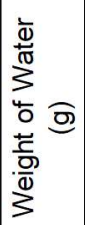 & 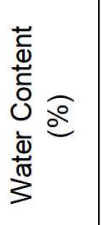 \\
\hline 1 & 12.4 & 7.5 & 4.9 & 65.3 & & 1 & 6.5 & 5.0 & 1.5 & 30.0 \\
\hline 2 & 14.5 & 9.2 & 5.3 & 57.6 & 20 & 2 & 7.1 & 5.3 & 1.8 & 34.0 \\
\hline 3 & 17.1 & 10.8 & 6.3 & 58.3 & 15 & & & & & \\
\hline 4 & 14.4 & 9.0 & 5.4 & 60.0 & 11 & & & & & \\
\hline & & & & & & & & & & \\
\hline & & & & & & & & & & \\
\hline
\end{tabular}

$\begin{array}{ll}\text { Liquid Limit: } & \frac{57}{32} \\ \text { Plastic Limit: } & \underline{25} \\ \text { Plasticity Index: } & \end{array}$

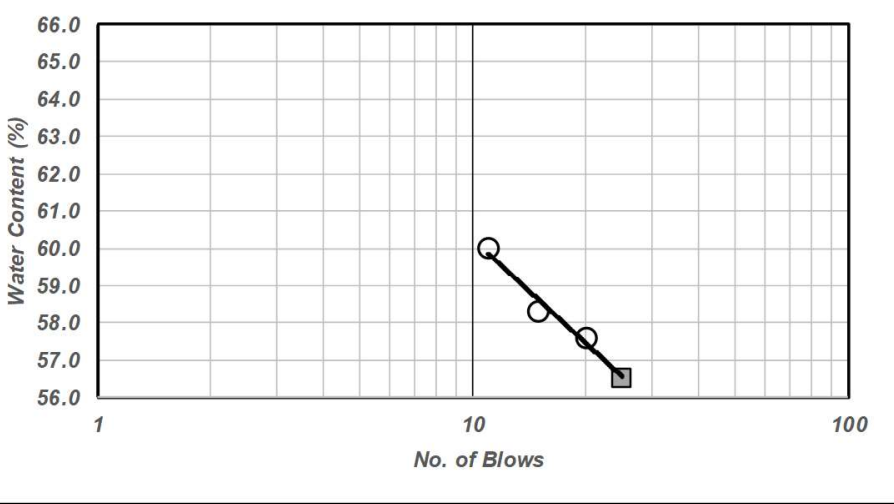

Total amount of materials passing sieve No. 40: $100 \%$

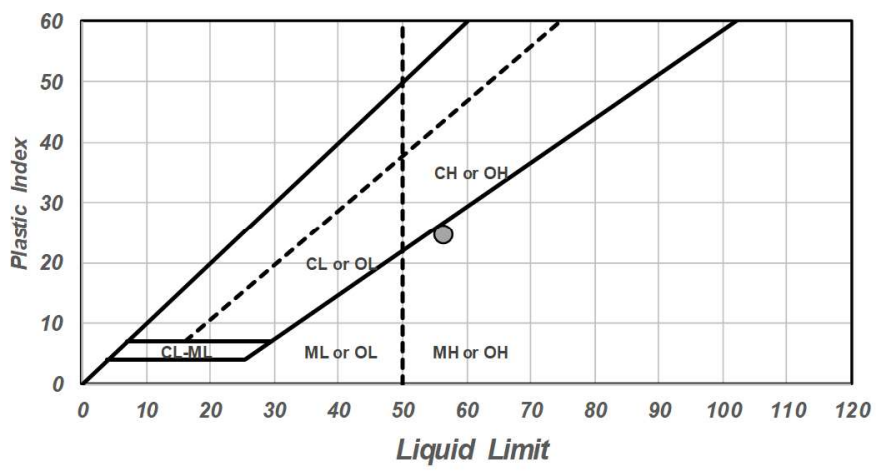

Fines

Classification: $\mathrm{MH}$

Procedure Done By:

Nathan 


\section{S Portland State}

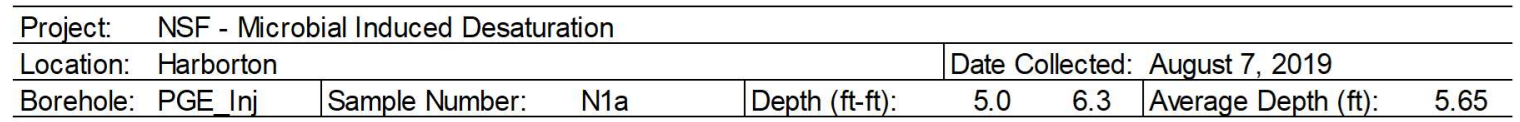

\begin{tabular}{|c|c|c|c|c|c|c|c|c|c|c|}
\hline \multicolumn{6}{|c|}{ Liquid Limit } & \multicolumn{5}{|c|}{ Plastic Limit } \\
\hline 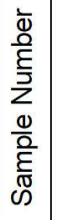 & 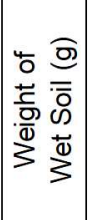 & 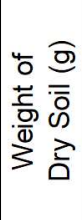 & 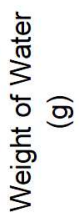 & 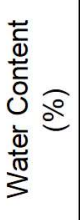 & 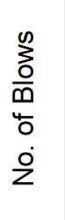 & 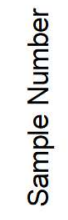 & 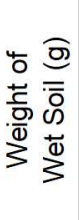 & 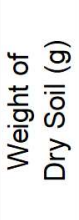 & 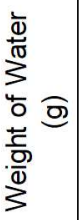 & 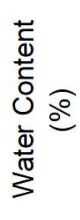 \\
\hline 1 & 8 & 6.2 & 1.8 & 29.0 & 1 & & & & & \\
\hline 2 & 5.5 & 4.1 & 1.4 & 34.1 & 8 & & & & & \\
\hline 3 & 4.9 & 3.5 & 1.4 & 40.0 & 2 & & & & & \\
\hline 4 & 7.4 & 5.3 & 2.1 & 39.6 & 1 & & & & & \\
\hline & & & & & & & & & & \\
\hline & & & & & & & & & & \\
\hline
\end{tabular}
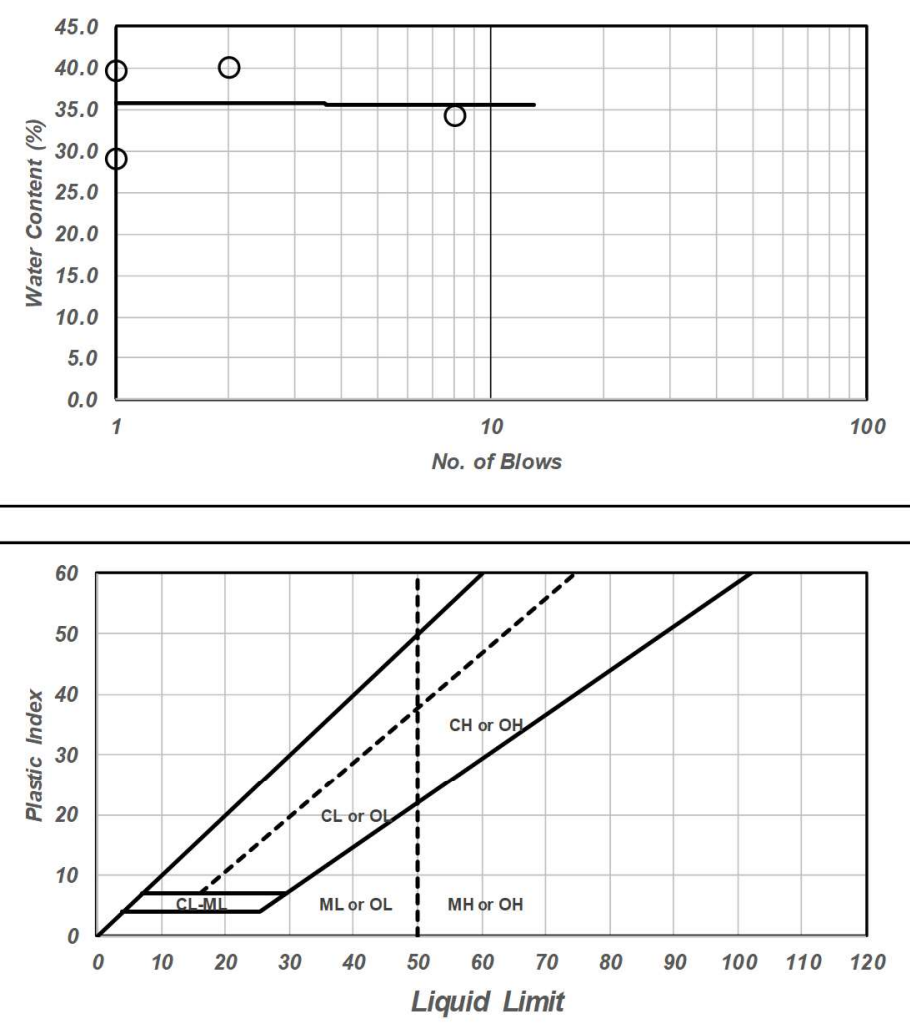

Procedure Done By: Kayla
Liquid Limit:

Plastic Limit:

Plasticity Index:
Total amount of materials passing sieve No. 40: $100 \%$
Fines

Classification: NP 


\section{S Portland State}

\begin{tabular}{|c|c|c|c|c|c|c|}
\hline Project: & SF - Mi & on & & & & \\
\hline Location: & Harborton & & & Date Collected: & August 7, 2019 & \\
\hline Borehole: & PGE Inj & Sample Number: & Depth (ft-ft): & 6.3 & Average Depth (ft): & 6.40 \\
\hline
\end{tabular}

\begin{tabular}{|c|c|c|c|c|c|c|c|c|c|c|}
\hline \multicolumn{6}{|c|}{ Liquid Limit } & \multicolumn{5}{|c|}{ Plastic Limit } \\
\hline 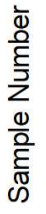 & 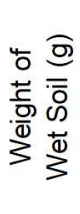 & 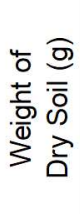 & 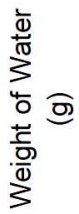 & 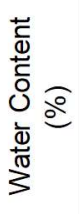 & \begin{tabular}{l}
00 \\
3 \\
0 \\
0 \\
4 \\
0 \\
0 \\
\hdashline
\end{tabular} & 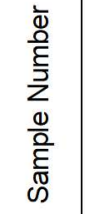 & 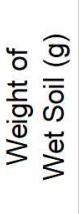 & 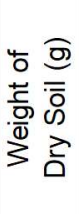 & 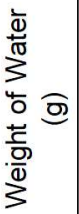 & 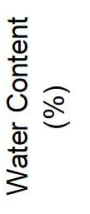 \\
\hline 1 & 8.1 & 5.5 & 2.6 & 47.3 & 20 & 1 & 9.5 & 7.0 & 2.5 & 35.7 \\
\hline 2 & 7.1 & 4.9 & 2.2 & 44.9 & 49 & 2 & 9.4 & 7.0 & 2.4 & 34.3 \\
\hline 3 & 14.0 & 9.7 & 4.3 & 44.3 & 63 & & & & & \\
\hline 4 & 13.5 & 8.9 & 4.6 & 51.7 & 5 & & & & & \\
\hline & & & & & & & & & & \\
\hline & & & & & & & & & & \\
\hline
\end{tabular}

\begin{tabular}{ll} 
Liquid Limit: & $\frac{47}{35}$ \\
Plastic Limit: & $\quad$ \\
Plasticity Index: & \\
\hline
\end{tabular}

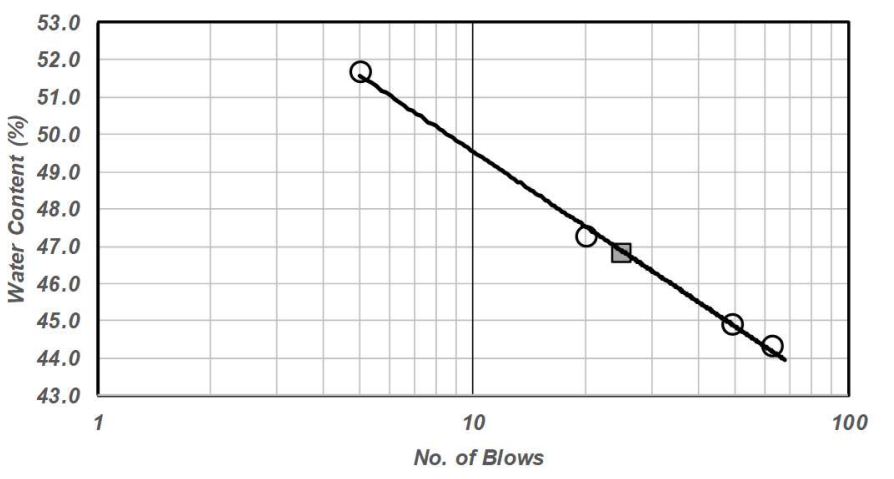

Total amount of materials passing sieve No. 40: $100 \%$

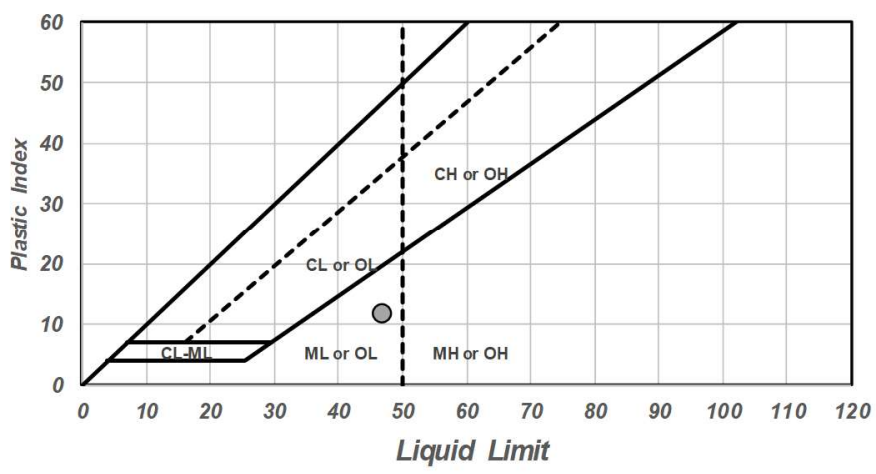

Fines

Classification: $\mathrm{ML}$

Procedure Done By: Melissa P./Kayla 


\section{S Portland State}

\begin{tabular}{|c|c|c|c|c|c|c|}
\hline oject: & NSF - Micr & nduced Desaturation & & & & \\
\hline Location: & Harborton & & & Date Collected: & August 7, 2019 & \\
\hline Borehole: & PGE Inj & Sample Number: & Depth (ft-ft): & $10.0 \quad 10.25$ & Average Depth (ft): & 10.13 \\
\hline
\end{tabular}

\begin{tabular}{|c|c|c|c|c|c|c|c|c|c|c|}
\hline \multicolumn{6}{|c|}{ Liquid Limit } & \multicolumn{5}{|c|}{ Plastic Limit } \\
\hline 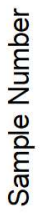 & 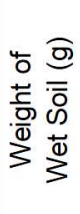 & 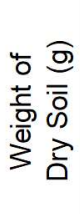 & 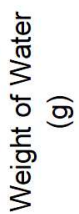 & 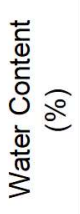 & 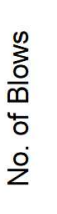 & 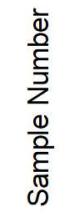 & 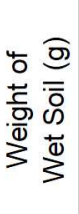 & 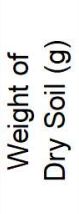 & 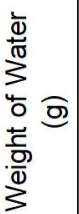 & 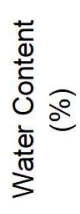 \\
\hline 1 & 8.7 & 6.6 & 2.1 & 31.8 & 1 & & & & & \\
\hline 2 & 8.9 & 6.7 & 2.2 & 32.8 & 2 & & & & & \\
\hline 3 & 12.4 & 8.3 & 4.1 & 49.4 & 1 & & & & & \\
\hline & & & & & & & & & & \\
\hline & & & & & & & & & & \\
\hline & & & & & & & & & & \\
\hline
\end{tabular}
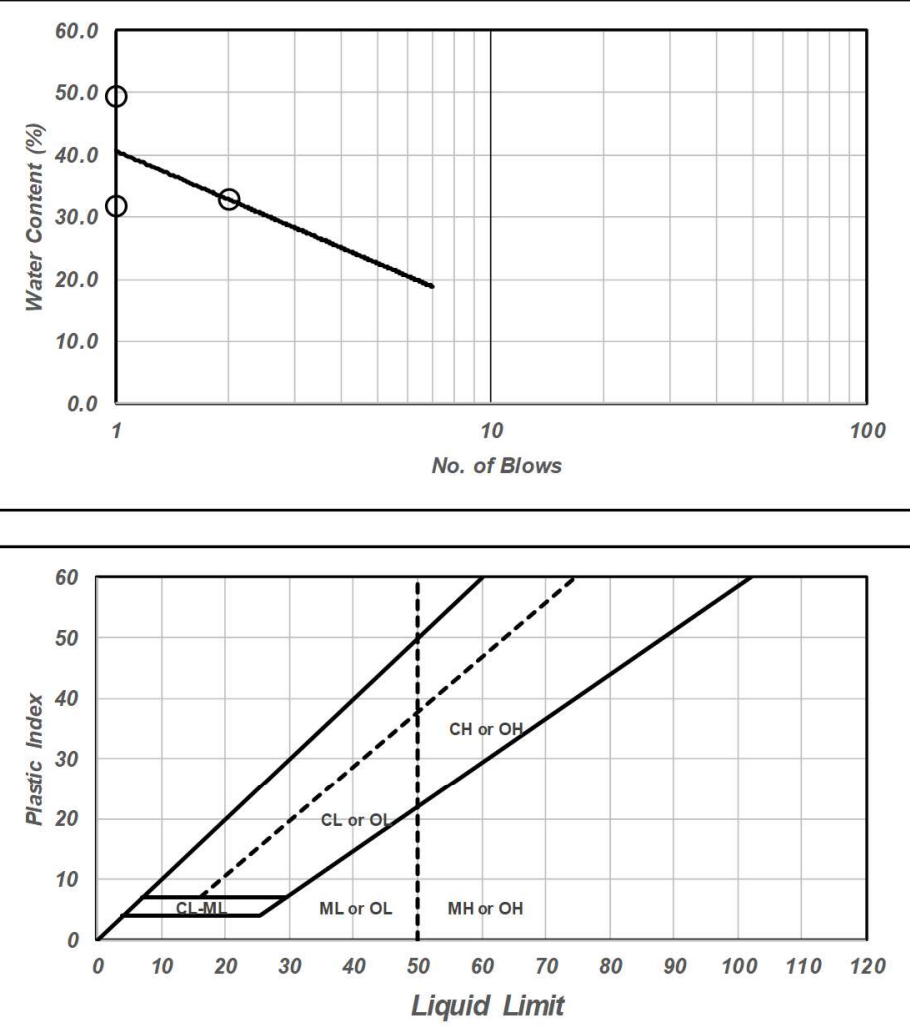

Liquid Limit:

Plastic Limit:

Plasticity Index:
Total amount of materials passing sieve No. 40: $100 \%$

Procedure Done By: Kayla 


\section{S Portland State}

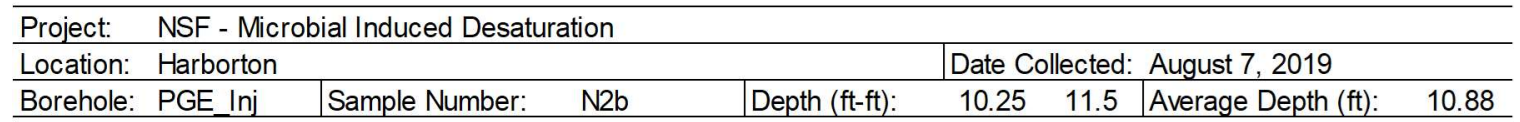

\begin{tabular}{|c|c|c|c|c|c|c|c|c|c|c|}
\hline \multicolumn{6}{|c|}{ Liquid Limit } & \multicolumn{5}{|c|}{ Plastic Limit } \\
\hline 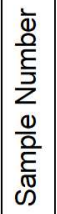 & 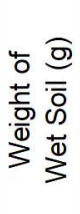 & 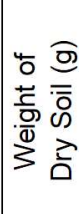 & 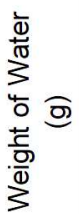 & 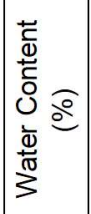 & 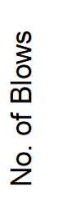 & 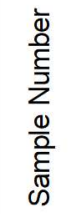 & 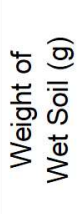 & 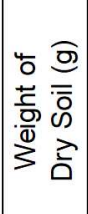 & 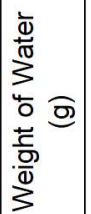 & 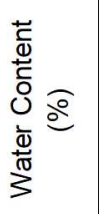 \\
\hline 1 & 11.4 & 6.9 & 4.5 & 65.2 & 12 & 1 & 5.5 & 4.0 & 1.5 & 37.5 \\
\hline 2 & 12.1 & 7.8 & 4.3 & 55.1 & 20 & 2 & 6.0 & 4.4 & 1.6 & 36.4 \\
\hline 3 & 11.1 & 7.2 & 3.9 & 54.2 & 25 & & & & & \\
\hline 4 & 7.4 & 5.2 & 2.2 & 42.3 & 36 & & & & & \\
\hline & & & & & & & & & & \\
\hline & & & & & & & & & & \\
\hline
\end{tabular}

\begin{tabular}{ll} 
Liquid Limit: & $\frac{51}{37}$ \\
Plastic Limit: & $\quad$ \\
Plasticity Index: & \\
\hline
\end{tabular}

Total amount of materials passing sieve No. 40: $100 \%$

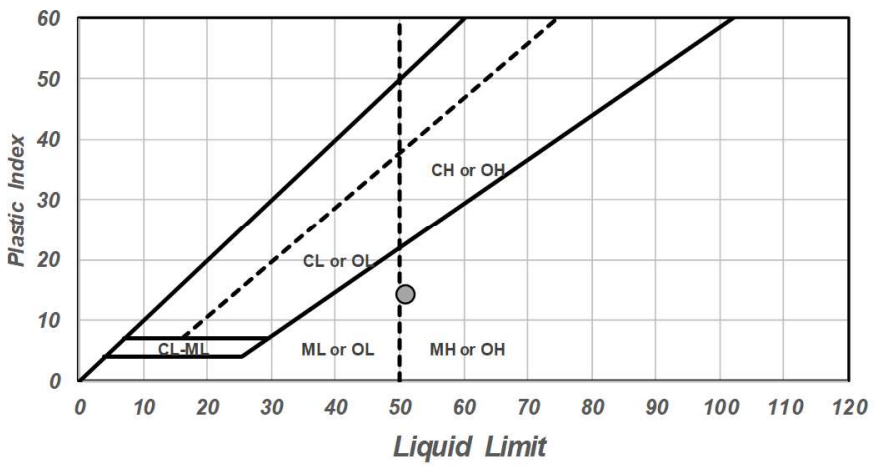

Procedure Done By
Fines

Classification: $\mathrm{MH}$ 


\section{S Portland State}

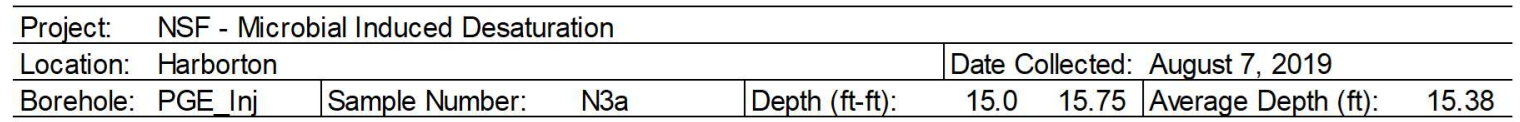

\begin{tabular}{|c|c|c|c|c|c|c|c|c|c|c|}
\hline \multicolumn{6}{|c|}{ Liquid Limit } & \multicolumn{5}{|c|}{ Plastic Limit } \\
\hline 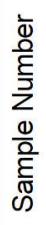 & 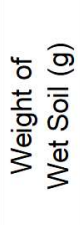 & 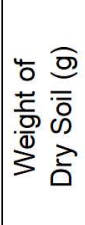 & 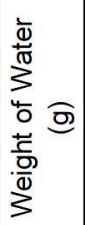 & 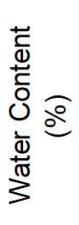 & 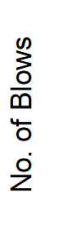 & 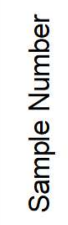 & 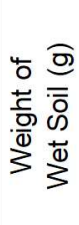 & 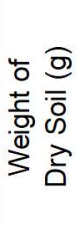 & 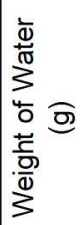 & 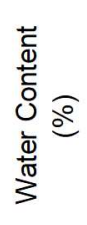 \\
\hline 1 & 9.4 & 7.1 & 2.3 & 32.4 & 1 & & & & & \\
\hline & & & & & & & & & & \\
\hline & & & & & & & & & & \\
\hline & & & & & & & & & & \\
\hline & & & & & & & & & & \\
\hline & & & & & & & & & & \\
\hline
\end{tabular}
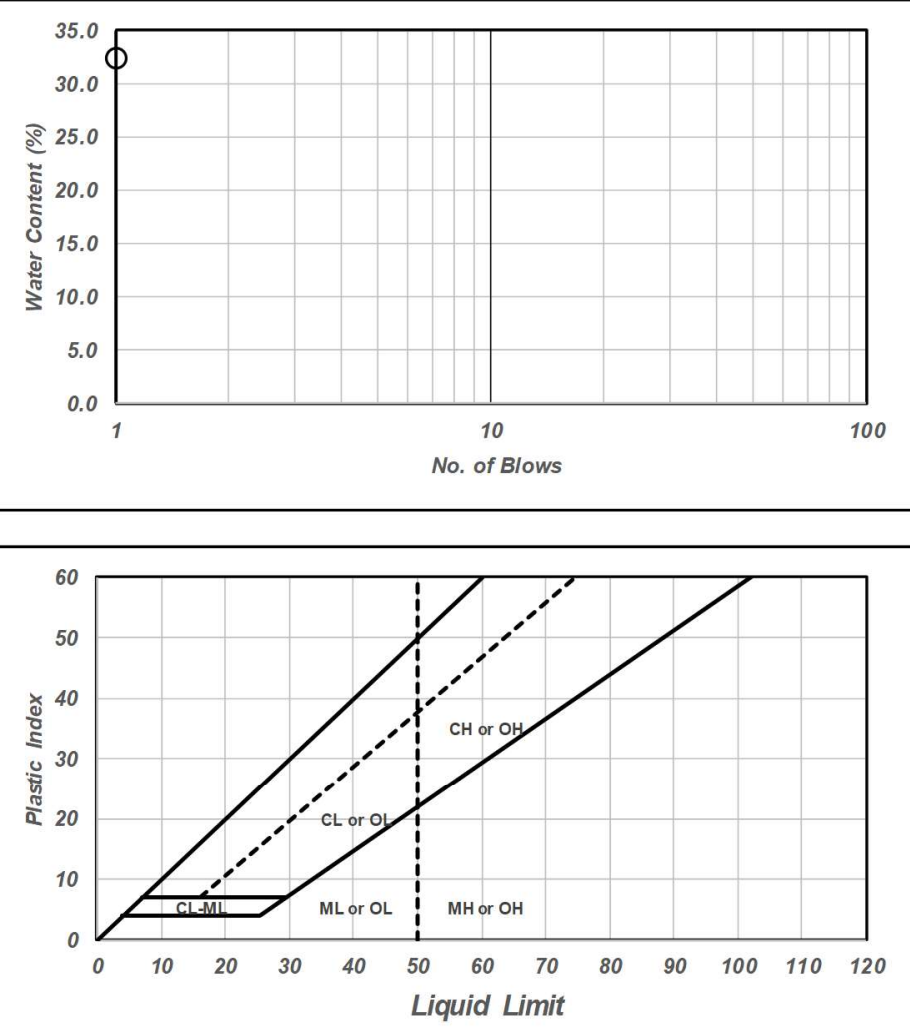

Liquid Limit:

Plastic Limit:

Plasticity Index:
Total amount of materials passing sieve No. 40: $100 \%$
Fines NP by

Classification: observation

Procedure Done By: Kayla 


\section{S Portland State}

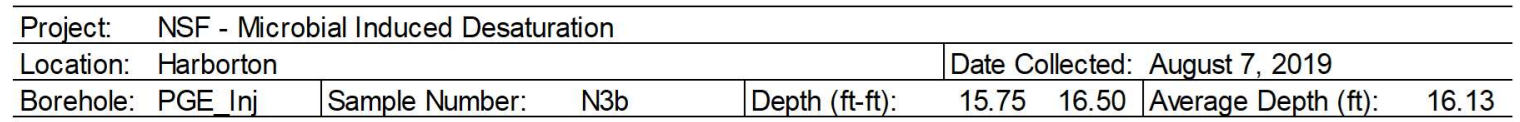

\begin{tabular}{|c|c|c|c|c|c|c|c|c|c|c|}
\hline \multicolumn{6}{|c|}{ Liquid Limit } & \multicolumn{5}{|c|}{ Plastic Limit } \\
\hline 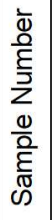 & 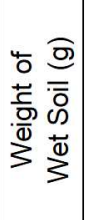 & 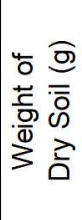 & 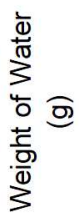 & 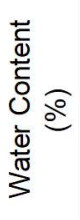 & $\begin{array}{l}0 \\
\sum_{0}^{0} \\
\frac{0}{0} \\
40 \\
\dot{0} \\
\dot{0}\end{array}$ & 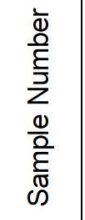 & 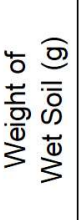 & 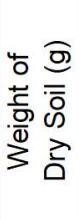 & 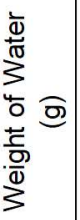 & 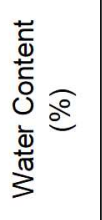 \\
\hline 1 & 8.8 & 5.9 & 2.9 & 49.2 & 85 & 1 & 6.8 & 4.9 & 1.9 & 38.8 \\
\hline 2 & 6.8 & 4.4 & 2.4 & 54.5 & 26 & 2 & 7.1 & 5.1 & 2.0 & 39.2 \\
\hline 3 & 9.0 & 5.6 & 3.4 & 60.7 & 7 & & & & & \\
\hline 4 & 8.6 & 5.2 & 3.4 & 65.4 & 4 & & & & & \\
\hline & & & & & & & & & & \\
\hline & & & & & & & & & & \\
\hline
\end{tabular}
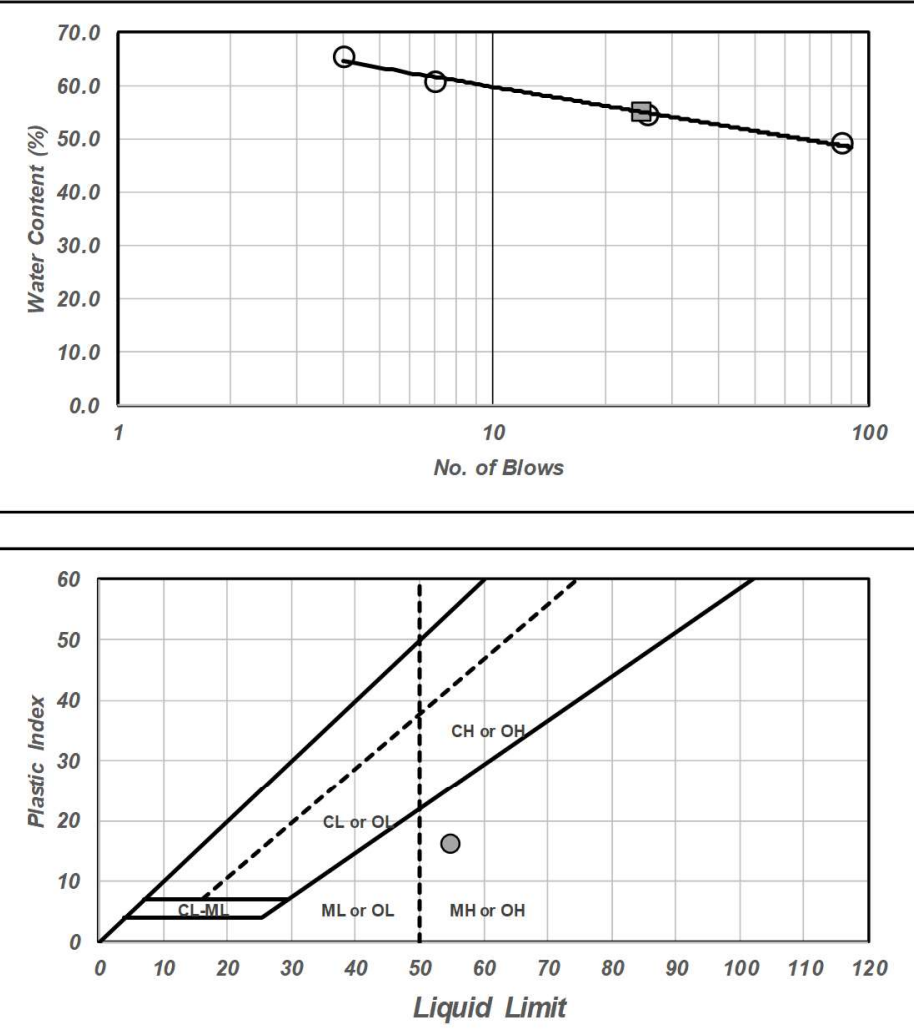

Procedure Done By: Kayla \begin{tabular}{ll} 
Liquid Limit: & 55 \\
Plastic Limit: & $\quad 39$ \\
Plasticity Index: & $\quad 16$ \\
\hline
\end{tabular}

Total amount of materials passing sieve No. 40: $100 \%$
Fines

Classification: $\mathrm{MH}$ 


\section{Appendix H: Hydrometer and P200 Washes - Harborton}

\section{胥 Portland State}

\begin{tabular}{|c|c|c|c|c|c|c|c|c|c|}
\hline & \multicolumn{9}{|c|}{ Hydrometer Test } \\
\hline \multirow[t]{2}{*}{ Boring: } & \multicolumn{2}{|c|}{ PGE_Ha1 } & & \multirow[t]{2}{*}{ Sample: } & \multirow[t]{2}{*}{ N1 } & \multirow{2}{*}{$\begin{array}{l}\text { Depth (ft-ft): } \\
\text { Ave. Depth (ft) }\end{array}$} & \multirow{2}{*}{$\begin{array}{ll}4.5 & 5.2 \\
& 4.85 \\
\end{array}$} & \multirow[t]{2}{*}{ Start Time: } & \multirow[t]{2}{*}{ 10:37:00 AM } \\
\hline & & & & & & & & & \\
\hline & \multicolumn{2}{|c|}{ Constants } & & & & & & & \\
\hline & $\overline{M_{s}(g)}$ & 55.21 & & & & & & & \\
\hline & $\mathrm{G}_{\mathrm{s}}$ & 2.65 & & & & & & & \\
\hline & $\mathrm{G}_{\mathrm{w}}$ & 1 & & & & & & & \\
\hline & $\begin{array}{l}\text { Time } \\
(\mathrm{min})\end{array}$ & $\begin{array}{l}\text { Soil } \\
\text { (slurry) }\end{array}$ & Control & $\begin{array}{l}\text { Temp } \\
\left({ }^{\circ} \mathrm{C}\right)\end{array}$ & $\begin{array}{c}\text { Temp } \\
\text { Correction (K) }\end{array}$ & $\begin{array}{c}\text { Hydrometer } \\
\text { Correction } \\
\text { (R) }\end{array}$ & $\begin{array}{c}\text { Effective } \\
\text { Depth, L } \\
\text { (cm) }\end{array}$ & Diameter (mm) & $\begin{array}{c}\text { Percent } \\
\text { Passing (\%) }\end{array}$ \\
\hline & 0.5 & 1.031 & 1.004 & 16.0 & 0.014350 & 1.027 & 8.1 & 0.057757636 & 79 \\
\hline & 1 & 1.029 & 1.004 & 16.0 & 0.014350 & 1.025 & 8.6 & 0.042082461 & 73 \\
\hline & 2 & 1.026 & 1.004 & 16.0 & 0.014350 & 1.022 & 10.5 & 0.032879981 & 64 \\
\hline & 4 & 1.023 & 1.004 & 16.0 & 0.014350 & 1.019 & 10.2 & 0.022915112 & 55 \\
\hline & 8 & 1.019 & 1.004 & 16.0 & 0.014350 & 1.015 & 11.3 & 0.01705478 & 44 \\
\hline & 18 & 1.015 & 1.004 & 16.0 & 0.014350 & 1.011 & 12.3 & 0.011862281 & 32 \\
\hline & 34 & 1.012 & 1.004 & 16.0 & 0.014350 & 1.008 & 13.1 & 0.008907341 & 23 \\
\hline & 66 & 1.010 & 1.004 & 16.0 & 0.014350 & 1.006 & 13.7 & 0.006537929 & 17 \\
\hline & 145 & 1.009 & 1.004 & 15.5 & 0.014435 & 1.005 & 13.9 & 0.004469307 & 15 \\
\hline & 274 & 1.008 & 1.004 & 15.5 & 0.014435 & 1.004 & 14.2 & 0.003286137 & 12 \\
\hline & 366 & 1.007 & 1.004 & 15.5 & 0.014435 & 1.003 & 14.4 & 0.002863238 & 9 \\
\hline & 1421 & 1.007 & 1.004 & 15.5 & 0.014435 & 1.003 & 14.4 & 0.001453118 & 9 \\
\hline
\end{tabular}

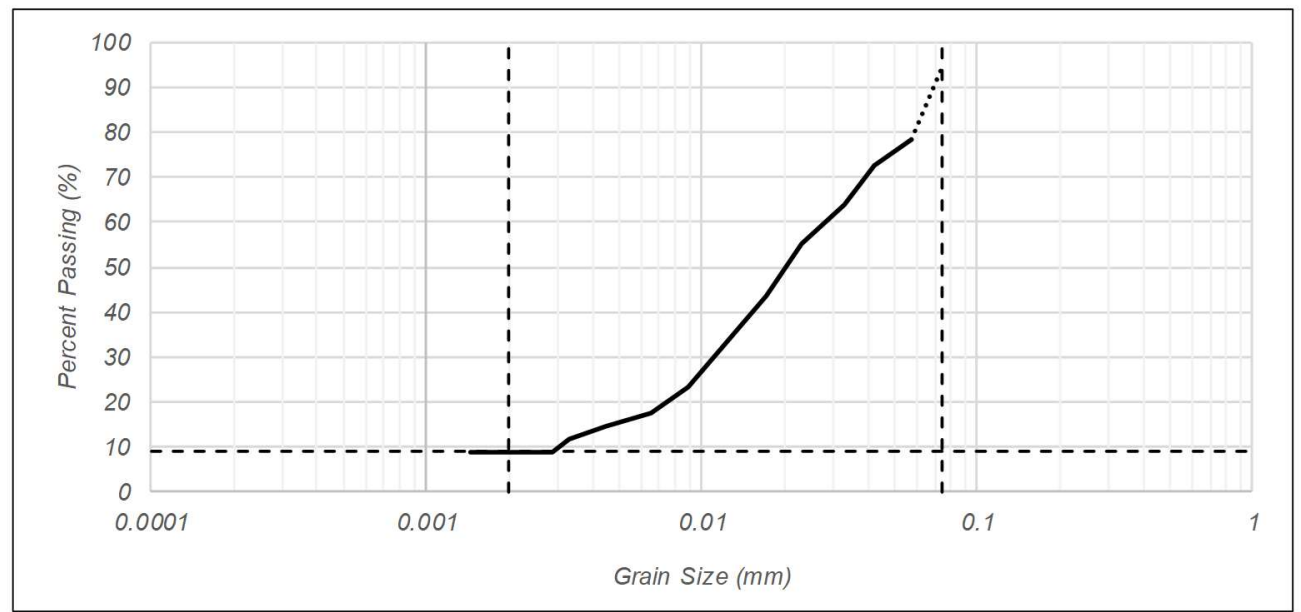

P200 Wash

$\begin{array}{lcc}\text { Container ID: } & \text { R1 } & \\ \text { Before - } \mathrm{M}_{\mathrm{d}}: & 20.6 & \mathrm{~g} \\ \text { After - } \mathrm{M}_{\mathrm{d}}: & 1 & \mathrm{~g} \\ \text { Fines Fraction: } & 95.15 & \% \\ \text { Coarse Fraction: } & 4.85 & \%\end{array}$

\begin{tabular}{llr} 
& \multicolumn{2}{c}{ Soil Breakdown } \\
\cline { 2 - 3 } & Coarse: & $4.85 \%$ \\
& Silts: & $86.15 \%$ \\
From Hydrometer & Clays: & $9.00 \%$
\end{tabular}

Procedures Done By:

Kayla/Melissa P. 


\section{S Portland State}

Hydrometer Test

\begin{tabular}{|c|c|c|c|c|c|c|c|c|c|}
\hline \multirow[t]{2}{*}{ Boring: } & \multicolumn{2}{|c|}{ PGE_Ha1 } & & \multirow[t]{2}{*}{ Sample: } & \multirow[t]{2}{*}{ N2 } & Depth (ft-ft): & 6.8 & \multirow[t]{2}{*}{ Start Time: } & \multirow[t]{2}{*}{ 10:39:00 AM } \\
\hline & & & & & & Ave. Depth (ft & 6.4 & & \\
\hline & \multicolumn{2}{|c|}{ Constants } & & & & & & & \\
\hline & $\overline{M_{s}(g)}$ & 54.33 & & & & & & & \\
\hline & $\mathrm{G}_{\mathrm{s}}$ & 2.65 & & & & & & & \\
\hline & $\mathrm{G}_{\mathrm{w}}$ & 1 & & & & & & & \\
\hline & $\begin{array}{l}\text { Time } \\
(\min )\end{array}$ & $\begin{array}{l}\text { Soil } \\
\text { (slurry) }\end{array}$ & Control & $\begin{array}{l}\text { Temp } \\
\left({ }^{\circ} \mathrm{C}\right)\end{array}$ & $\begin{array}{c}\text { Temp } \\
\text { Correction }(\mathrm{K})\end{array}$ & $\begin{array}{l}\text { Hydrometer } \\
\text { Correction } \\
\text { (R) }\end{array}$ & $\begin{array}{l}\text { Effective } \\
\text { Depth, L } \\
\text { (cm) }\end{array}$ & Diameter (mm) & $\begin{array}{c}\text { Percent } \\
\text { Passing (\%) }\end{array}$ \\
\hline & 0.5 & 1.033 & 1.004 & 16.5 & 0.014260 & 1.029 & 7.6 & 0.055595715 & 86 \\
\hline & 1 & 1.031 & 1.004 & 16.5 & 0.014260 & 1.027 & 8.1 & 0.040584671 & 80 \\
\hline & 2 & 1.027 & 1.004 & 16.5 & 0.014260 & 1.023 & 9.2 & 0.030584293 & 68 \\
\hline & 4 & 1.024 & 1.004 & 16.5 & 0.014260 & 1.020 & 10.0 & 0.02254704 & 59 \\
\hline & 8 & 1.020 & 1.004 & 16.5 & 0.014260 & 1.016 & 11.0 & 0.016721332 & 47 \\
\hline & 16 & 1.016 & 1.004 & 16.5 & 0.014260 & 1.012 & 12.1 & 0.012400872 & 35 \\
\hline & 32 & 1.013 & 1.004 & 16.5 & 0.014260 & 1.009 & 12.9 & 0.009053977 & 27 \\
\hline & 64 & 1.011 & 1.004 & 16.0 & 0.014350 & 1.007 & 13.4 & 0.006566203 & 21 \\
\hline & 143 & 1.009 & 1.004 & 16.0 & 0.014350 & 1.005 & 13.9 & 0.004473952 & 15 \\
\hline & 272 & 1.008 & 1.004 & 15.5 & 0.014435 & 1.004 & 14.2 & 0.003298196 & 12 \\
\hline & 364 & 1.007 & 1.004 & 15.5 & 0.014435 & 1.003 & 14.4 & 0.002871094 & 9 \\
\hline & 1419 & 1.007 & 1.004 & 15.0 & 0.014435 & 1.003 & 14.4 & 0.001454142 & 9 \\
\hline
\end{tabular}

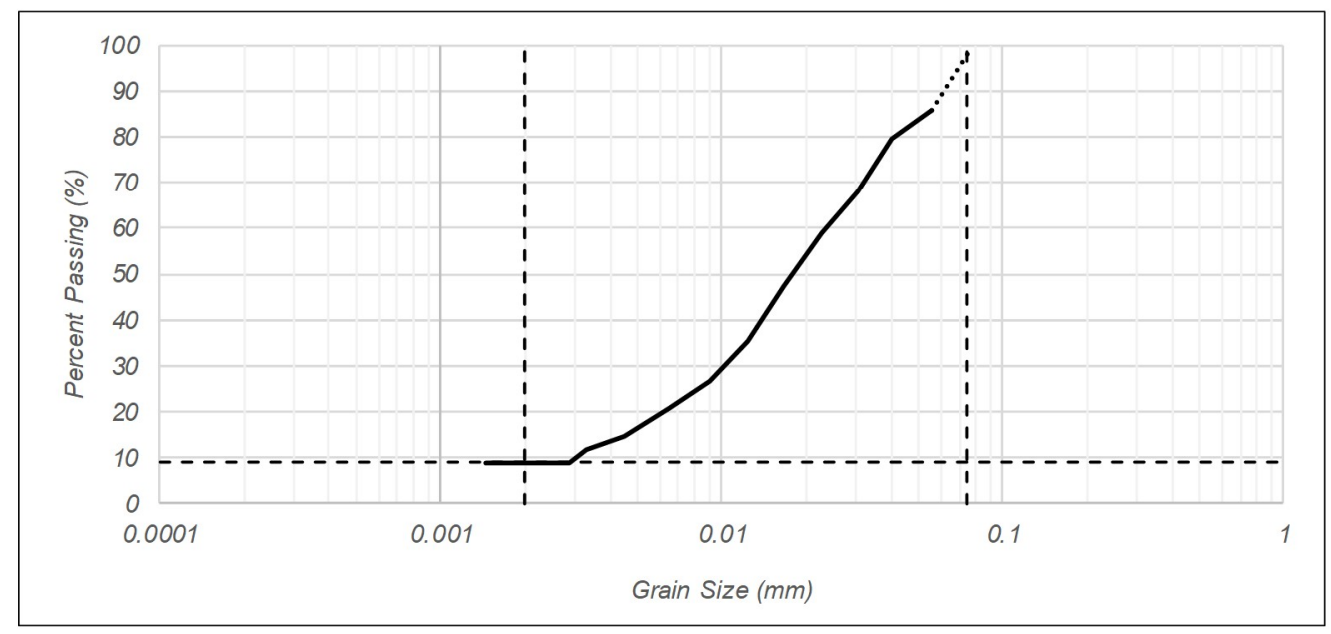

P200 Wash

$\begin{array}{lcc}\text { Container ID: } & \text { M1 } & \\ \text { Before }-\mathrm{M}_{\mathrm{d}}: & 23 & \mathrm{~g} \\ \text { After }-\mathrm{M}_{\mathrm{d}}: & 0.4 & \mathrm{~g} \\ \text { Fines Fraction: } & 98.26 & \% \\ \text { Coarse Fraction: } & 1.74 & \%\end{array}$

Procedures Done By: Kayla/Melissa P 


\section{S Portland State}

Hydrometer Test

\begin{tabular}{|c|c|c|c|c|c|c|c|c|c|}
\hline \multirow[t]{2}{*}{ Boring: } & \multicolumn{2}{|c|}{ PGE_Ha1 } & & \multirow[t]{2}{*}{ Sample: } & \multirow[t]{2}{*}{ N3 } & Depth (ft-ft): & 9.2 & \multirow[t]{2}{*}{ Start Time: } & \multirow[t]{2}{*}{ 10:41:00 AM } \\
\hline & & & & & & Ave. Depth $(\mathrm{ft})$ & 8.75 & & \\
\hline & \multicolumn{2}{|c|}{ Constants } & & & & & & & \\
\hline & $M_{s}(g)$ & 57.27 & & & & & & & \\
\hline & $\mathrm{G}_{\mathrm{s}}$ & 2.65 & & & & & & & \\
\hline & $\mathrm{G}_{w}$ & 1 & & & & & & & \\
\hline & $\begin{array}{l}\text { Time } \\
\text { (min) }\end{array}$ & $\begin{array}{c}\text { Soil } \\
\text { (slurry) }\end{array}$ & Control & $\begin{array}{l}\text { Temp } \\
\left({ }^{\circ} \mathrm{C}\right)\end{array}$ & $\begin{array}{c}\text { Temp } \\
\text { Correction }(\mathrm{K})\end{array}$ & $\begin{array}{c}\text { Hydrometer } \\
\text { Correction } \\
(R)\end{array}$ & $\begin{array}{l}\text { Effective } \\
\text { Depth, L } \\
\text { (cm) }\end{array}$ & Diameter (mm) & $\begin{array}{c}\text { Percent } \\
\text { Passing (\%) }\end{array}$ \\
\hline & 0.5 & 1.033 & 1.004 & 16.0 & 0.014350 & 1.029 & 7.6 & 0.0559466 & 81 \\
\hline & 1 & 1.032 & 1.004 & 16.0 & 0.014350 & 1.028 & 7.8 & 0.040077369 & 79 \\
\hline & 2 & 1.029 & 1.004 & 16.0 & 0.014350 & 1.025 & 8.6 & 0.029756793 & 70 \\
\hline & 4 & 1.027 & 1.004 & 16.0 & 0.014350 & 1.023 & 9.2 & 0.021762853 & 64 \\
\hline & 8 & 1.024 & 1.004 & 16.0 & 0.014260 & 1.020 & 10.0 & 0.015943165 & 56 \\
\hline & 14 & 1.022 & 1.004 & 16.0 & 0.014350 & 1.018 & 10.5 & 0.012427465 & 50 \\
\hline & 30 & 1.019 & 1.004 & 16.0 & 0.014350 & 1.015 & 11.3 & 0.008807051 & 42 \\
\hline & 62 & 1.016 & 1.004 & 16.0 & 0.014350 & 1.012 & 12.1 & 0.006339409 & 34 \\
\hline & 141 & 1.012 & 1.004 & 16.0 & 0.014350 & 1.008 & 13.1 & 0.004373992 & 22 \\
\hline & 270 & 1.011 & 1.004 & 15.5 & 0.014435 & 1.007 & 13.4 & 0.003215787 & 20 \\
\hline & 362 & 1.010 & 1.004 & 15.5 & 0.014435 & 1.006 & 13.7 & 0.002808166 & 17 \\
\hline & 1417 & 1.008 & 1.004 & 15.0 & 0.014520 & 1.004 & 14.2 & 0.001453536 & 11 \\
\hline
\end{tabular}

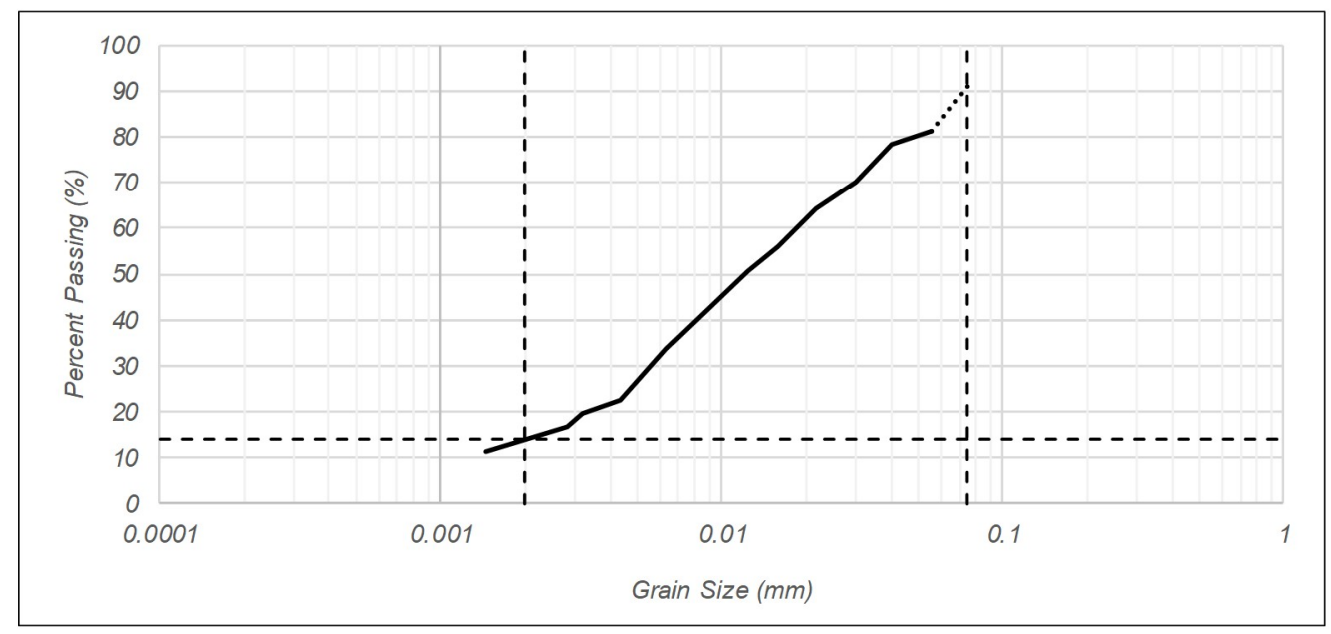

P200 Wash

$\begin{array}{lcc}\text { Container ID: } & \text { U1 } & \\ \text { Before }-\mathrm{M}_{\mathrm{d}} \text { : } & 20.9 & \mathrm{~g} \\ \text { After }-\mathrm{M}_{\mathrm{d}} \text { : } & 1.8 & \mathrm{~g} \\ \text { Fines Fraction: } & 91.39 & \% \\ \text { Coarse Fraction: } & 8.61 & \%\end{array}$

Procedures Done By: Kayla/Melissa P. 


\section{S Portland State}

Hydrometer Test

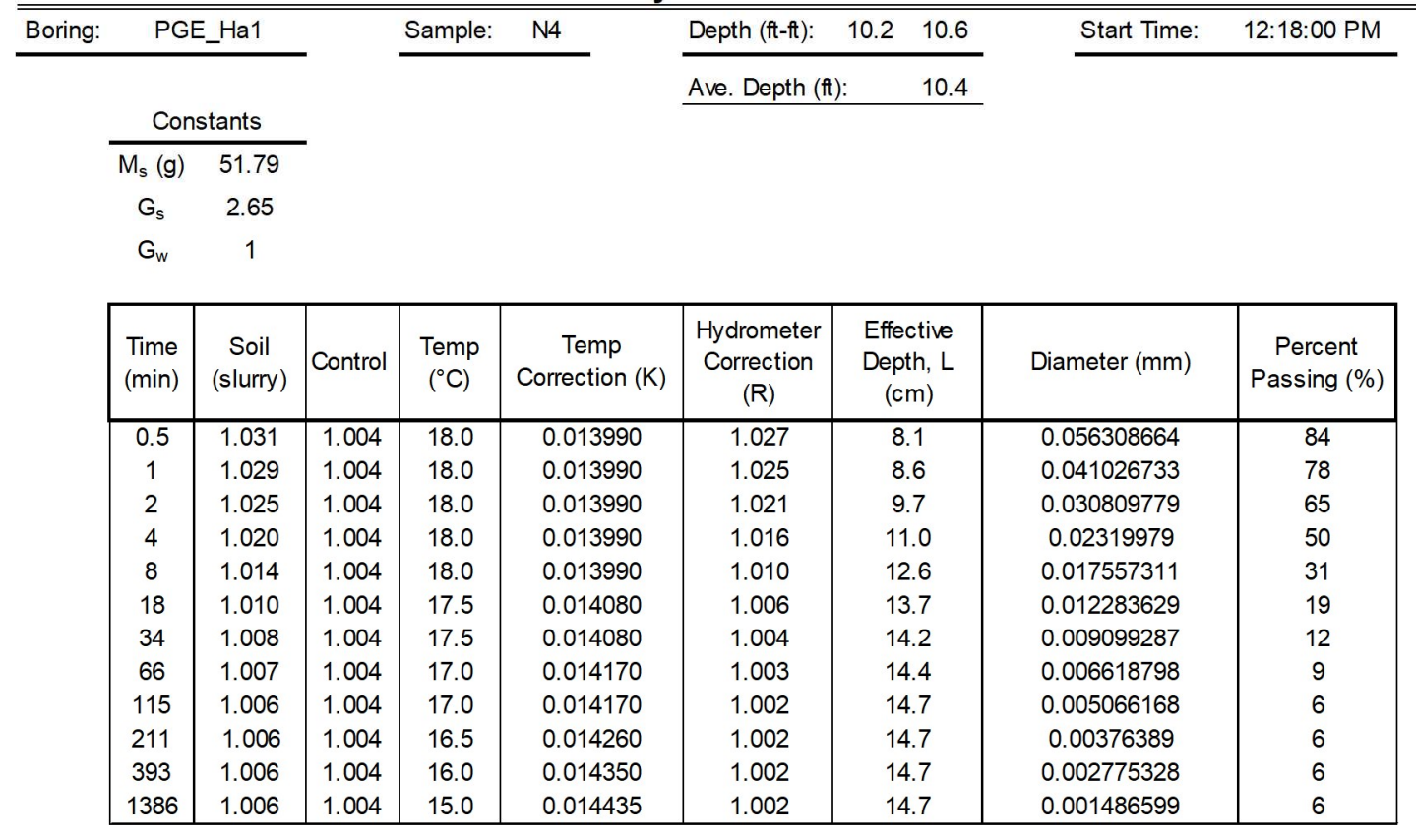

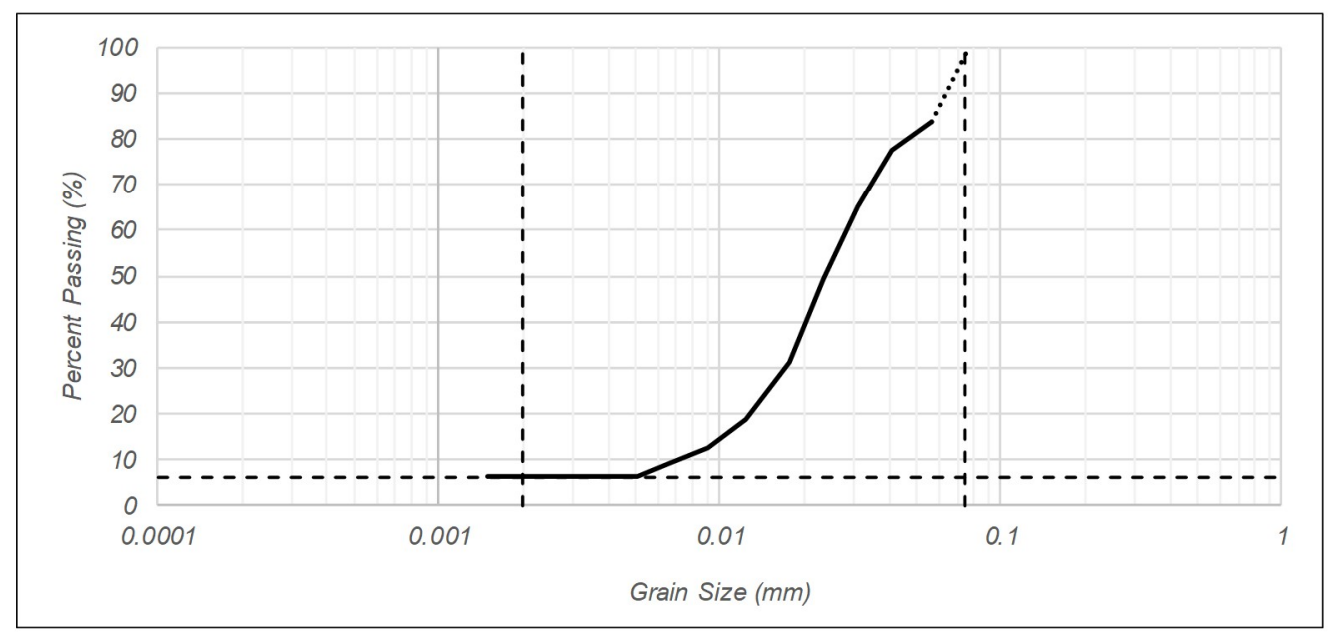

P200 Wash

$\begin{array}{lcc}\text { Container ID: } & \text { P1 } & \\ \text { Before }-\mathrm{M}_{\mathrm{d}}: & 22.7 & \mathrm{~g} \\ \text { After }-\mathrm{M}_{\mathrm{d}}: & 0.3 & \mathrm{~g} \\ \text { Fines Fraction: } & 98.68 & \% \\ \text { Coarse Fraction: } & 1.32 & \%\end{array}$

Procedures Done By: Kayla/Melissa B. 


\section{S Portland State}

Hydrometer Test

\begin{tabular}{|c|c|c|c|c|c|c|c|c|c|}
\hline \multirow[t]{2}{*}{ Boring: } & \multicolumn{2}{|c|}{ PGE_Ha1 } & & \multirow[t]{2}{*}{ Sample: } & \multirow[t]{2}{*}{ N5 } & \multirow{2}{*}{\multicolumn{2}{|c|}{$\begin{array}{lll}\text { Depth }(\mathrm{ft}-\mathrm{ft}): & 10.6 & 10.8 \\
\text { Ave. Depth }(\mathrm{ft}): & 10.7 \\
\end{array}$}} & \multirow[t]{2}{*}{ Start Time: } & \multirow[t]{2}{*}{ 12:20:00 PM } \\
\hline & & & & & & & & & \\
\hline & \multicolumn{2}{|c|}{ Constants } & & & & & & & \\
\hline & $M_{s}(g)$ & 54.40 & & & & & & & \\
\hline & $\mathrm{G}_{\mathrm{s}}$ & 2.65 & & & & & & & \\
\hline & $\mathrm{G}_{w}$ & 1 & & & & & & & \\
\hline & $\begin{array}{l}\text { Time } \\
\text { (min) }\end{array}$ & $\begin{array}{c}\text { Soil } \\
\text { (slurry) }\end{array}$ & Control & $\begin{array}{l}\text { Temp } \\
\left({ }^{\circ} \mathrm{C}\right)\end{array}$ & $\begin{array}{c}\text { Temp } \\
\text { Correction }(\mathrm{K})\end{array}$ & \begin{tabular}{|c|} 
Hydrometer \\
Correction \\
$(\mathrm{R})$
\end{tabular} & $\begin{array}{l}\text { Effective } \\
\text { Depth, L } \\
\text { (cm) }\end{array}$ & Diameter (mm) & $\begin{array}{c}\text { Percent } \\
\text { Passing (\%) }\end{array}$ \\
\hline & 0.5 & 1.036 & 1.004 & 17.0 & 0.014170 & 1.032 & 6.8 & 0.052256378 & 94 \\
\hline & 1 & 1.035 & 1.004 & 17.0 & 0.014170 & 1.031 & 7.0 & 0.037490296 & 92 \\
\hline & 2 & 1.034 & 1.004 & 17.0 & 0.014170 & 1.030 & 7.3 & 0.027071747 & 89 \\
\hline & 4 & 1.030 & 1.004 & 17.0 & 0.014170 & 1.026 & 8.4 & 0.020534281 & 77 \\
\hline & 8 & 1.028 & 1.004 & 17.0 & 0.014170 & 1.024 & 8.9 & 0.014945824 & 71 \\
\hline & 16 & 1.024 & 1.004 & 16.5 & 0.014260 & 1.020 & 10.0 & 0.01127352 & 59 \\
\hline & 32 & 1.019 & 1.004 & 16.5 & 0.014260 & 1.015 & 11.3 & 0.008473908 & 44 \\
\hline & 64 & 1.016 & 1.004 & 16.5 & 0.014260 & 1.012 & 12.1 & 0.006200436 & 35 \\
\hline & 113 & 1.014 & 1.004 & 16.5 & 0.014260 & 1.010 & 12.6 & 0.004761739 & 30 \\
\hline & 209 & 1.012 & 1.004 & 16.0 & 0.014350 & 1.008 & 13.1 & 0.003592646 & 24 \\
\hline & 391 & 1.011 & 1.004 & 16.0 & 0.014350 & 1.007 & 13.4 & 0.002656537 & 21 \\
\hline & 1384 & 1.010 & 1.004 & 15.5 & 0.014435 & 1.006 & 13.7 & 0.001436181 & 18 \\
\hline
\end{tabular}

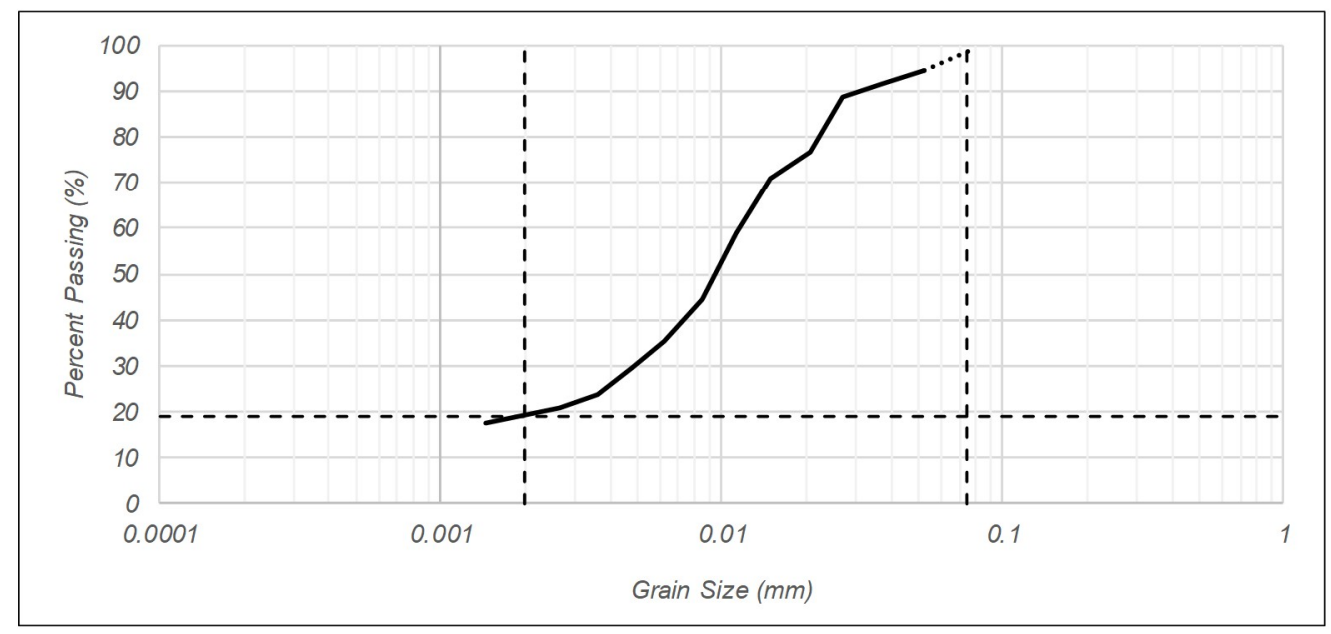

P200 Wash

$\begin{array}{lcc}\text { Container ID: } & \text { F1 } & \\ \text { Before }-\mathrm{M}_{\mathrm{d}}: & 15.2 & \mathrm{~g} \\ \text { After }-\mathrm{M}_{\mathrm{d}}: & 0.2 & \mathrm{~g} \\ \text { Fines Fraction: } & 98.68 & \% \\ \text { Coarse Fraction: } & 1.32 & \%\end{array}$

Procedures Done By: Kayla/Melissa B. 


\section{S Portland State}

Hydrometer Test

\begin{tabular}{|c|c|c|c|c|c|c|c|c|c|}
\hline \multirow[t]{2}{*}{ Boring: } & \multicolumn{2}{|c|}{ PGE_Ha1 } & & \multirow[t]{2}{*}{ Sample: } & \multirow[t]{2}{*}{ N6 } & \multirow{2}{*}{\multicolumn{2}{|c|}{$\begin{array}{lll}\text { Depth }(\mathrm{ft}-\mathrm{ft}): & 10.8 & 11.4 \\
\text { Ave. Depth }(\mathrm{ft}): & 11.1 \\
\end{array}$}} & \multirow[t]{2}{*}{ Start Time: } & \multirow[t]{2}{*}{$8: 14: 00$ AM } \\
\hline & & & & & & & & & \\
\hline & \multicolumn{2}{|c|}{ Constants } & & & & & & & \\
\hline & $M_{s}(g)$ & 49.82 & & & & & & & \\
\hline & $\mathrm{G}_{\mathrm{s}}$ & 2.65 & & & & & & & \\
\hline & $\mathrm{G}_{w}$ & 1 & & & & & & & \\
\hline & $\begin{array}{l}\text { Time } \\
\text { (min) }\end{array}$ & $\begin{array}{c}\text { Soil } \\
\text { (slurry) }\end{array}$ & Control & $\begin{array}{l}\text { Temp } \\
\left({ }^{\circ} \mathrm{C}\right)\end{array}$ & $\begin{array}{c}\text { Temp } \\
\text { Correction }(\mathrm{K})\end{array}$ & $\begin{array}{c}\text { Hydrometer } \\
\text { Correction } \\
(R)\end{array}$ & $\begin{array}{l}\text { Effective } \\
\text { Depth, L } \\
\text { (cm) }\end{array}$ & Diameter (mm) & $\begin{array}{c}\text { Percent } \\
\text { Passing (\%) }\end{array}$ \\
\hline & 0.5 & 1.028 & 1.004 & 17.5 & 0.014080 & 1.024 & 8.9 & 0.059403585 & 77 \\
\hline & 1 & 1.027 & 1.004 & 17.5 & 0.014080 & 1.023 & 9.2 & 0.042706755 & 74 \\
\hline & 2 & 1.025 & 1.004 & 17.5 & 0.014080 & 1.021 & 9.7 & 0.031007983 & 68 \\
\hline & 4 & 1.022 & 1.004 & 18.0 & 0.013990 & 1.018 & 10.5 & 0.022666391 & 58 \\
\hline & 8 & 1.018 & 1.004 & 18.0 & 0.013990 & 1.014 & 11.5 & 0.016773421 & 45 \\
\hline & 16 & 1.015 & 1.004 & 18.0 & 0.013990 & 1.011 & 12.3 & 0.012266207 & 35 \\
\hline & 30 & 1.013 & 1.004 & 17.5 & 0.014080 & 1.009 & 12.9 & 0.009232873 & 29 \\
\hline & 69 & 1.010 & 1.004 & 17.5 & 0.014080 & 1.006 & 13.7 & 0.006273912 & 19 \\
\hline & 108 & 1.009 & 1.004 & 17.5 & 0.014080 & 1.005 & 13.9 & 0.005051242 & 16 \\
\hline & 213 & 1.008 & 1.004 & 17.5 & 0.014080 & 1.004 & 14.2 & 0.00363544 & 13 \\
\hline & 545 & 1.007 & 1.004 & 17.5 & 0.014080 & 1.003 & 14.4 & 0.002288683 & 10 \\
\hline & 1535 & 1.007 & 1.004 & 17.5 & 0.014080 & 1.003 & 14.4 & 0.001363734 & 10 \\
\hline
\end{tabular}

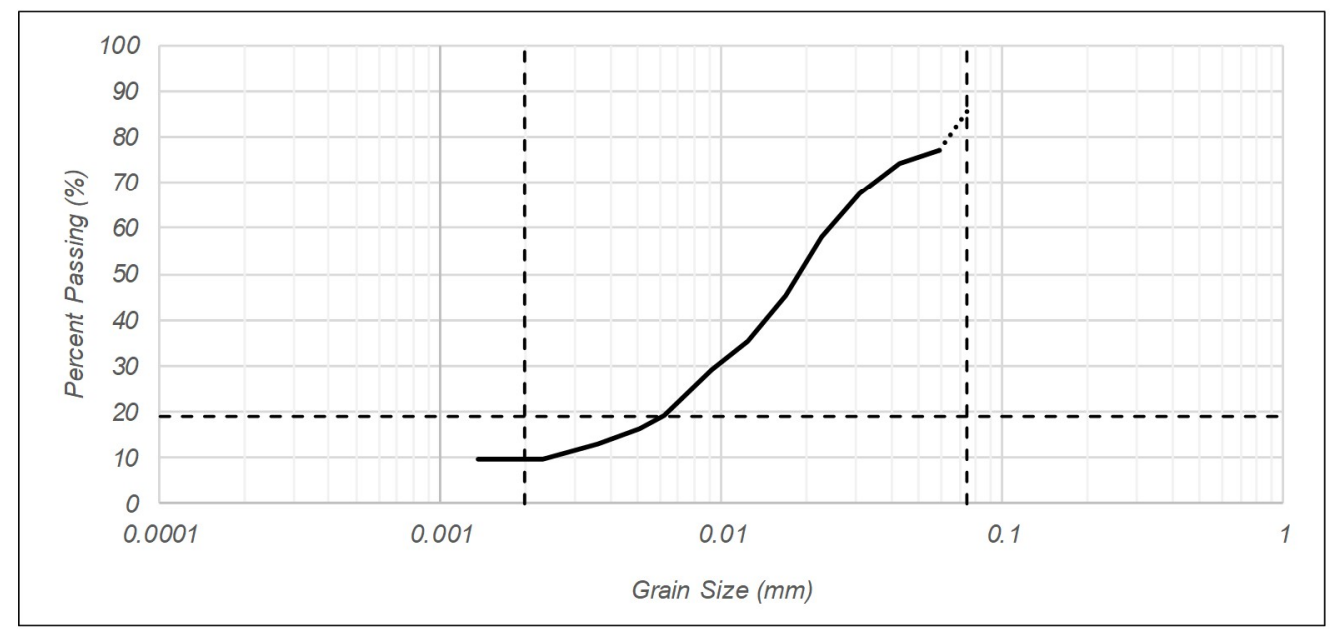

P200 Wash

$\begin{array}{lcc}\text { Container ID: } & \text { P1 } & \\ \text { Before }-\mathrm{M}_{\mathrm{d}}: & 27.5 & \mathrm{~g} \\ \text { After }-\mathrm{M}_{\mathrm{d}} \text { : } & 3.9 & \mathrm{~g} \\ \text { Fines Fraction: } & 85.82 & \% \\ \text { Coarse Fraction: } & 14.18 & \%\end{array}$

Procedures Done By: Kayla/Melissa B. 


\section{S Portland State}

Hydrometer Test

\begin{tabular}{|c|c|c|c|c|c|c|c|c|c|}
\hline \multirow[t]{2}{*}{ Boring: } & \multicolumn{2}{|c|}{ PGE_Ha1 } & & \multirow[t]{2}{*}{ Sample: } & \multirow[t]{2}{*}{ N7 } & \multirow{2}{*}{\multicolumn{2}{|c|}{$\begin{array}{lll}\text { Depth }(\mathrm{ft}-\mathrm{ft}): & 11.4 & 12.1 \\
\text { Ave. Depth }(\mathrm{ft}): & 11.75 \\
\end{array}$}} & \multirow[t]{2}{*}{ Start Time: } & \multirow[t]{2}{*}{ 12:22:00 PM } \\
\hline & & & & & & & & & \\
\hline & \multicolumn{2}{|c|}{ Constants } & & & & & & & \\
\hline & $M_{s}(g)$ & 57.24 & & & & & & & \\
\hline & $\mathrm{G}_{\mathrm{s}}$ & 2.65 & & & & & & & \\
\hline & $\mathrm{G}_{w}$ & 1 & & & & & & & \\
\hline & $\begin{array}{l}\text { Time } \\
\text { (min) }\end{array}$ & $\begin{array}{c}\text { Soil } \\
\text { (slurry) }\end{array}$ & Control & $\begin{array}{l}\text { Temp } \\
\left({ }^{\circ} \mathrm{C}\right)\end{array}$ & $\begin{array}{c}\text { Temp } \\
\text { Correction }(\mathrm{K})\end{array}$ & $\begin{array}{c}\text { Hydrometer } \\
\text { Correction } \\
(R)\end{array}$ & $\begin{array}{l}\text { Effective } \\
\text { Depth, L } \\
\text { (cm) }\end{array}$ & Diameter (mm) & $\begin{array}{c}\text { Percent } \\
\text { Passing (\%) }\end{array}$ \\
\hline & 0.5 & 1.030 & 1.004 & 17.0 & 0.014170 & 1.026 & 8.4 & 0.058079717 & 73 \\
\hline & 1 & 1.023 & 1.004 & 17.0 & 0.014170 & 1.019 & 10.2 & 0.045255351 & 53 \\
\hline & 2 & 1.019 & 1.004 & 17.0 & 0.014170 & 1.015 & 11.3 & 0.033681705 & 42 \\
\hline & 4 & 1.014 & 1.004 & 17.0 & 0.014170 & 1.010 & 12.6 & 0.025149255 & 28 \\
\hline & 8 & 1.009 & 1.004 & 17.0 & 0.014170 & 1.005 & 13.9 & 0.018678081 & 14 \\
\hline & 14 & 1.007 & 1.004 & 17.5 & 0.014080 & 1.003 & 14.4 & 0.014279726 & 8 \\
\hline & 30 & 1.006 & 1.004 & 17.5 & 0.014080 & 1.002 & 14.7 & 0.009856 & 6 \\
\hline & 62 & 1.006 & 1.004 & 17.0 & 0.014170 & 1.002 & 14.7 & 0.006899739 & 6 \\
\hline & 111 & 1.005 & 1.004 & 17.0 & 0.014170 & 1.001 & 15.0 & 0.005208996 & 3 \\
\hline & 207 & 1.005 & 1.004 & 16.5 & 0.014260 & 1.001 & 15.0 & 0.003838663 & 3 \\
\hline & 389 & 1.005 & 1.004 & 16.0 & 0.014350 & 1.001 & 15.0 & 0.002817882 & 3 \\
\hline & 1382 & 1.005 & 1.004 & 15.5 & 0.014435 & 1.001 & 15.0 & 0.001503863 & 3 \\
\hline
\end{tabular}

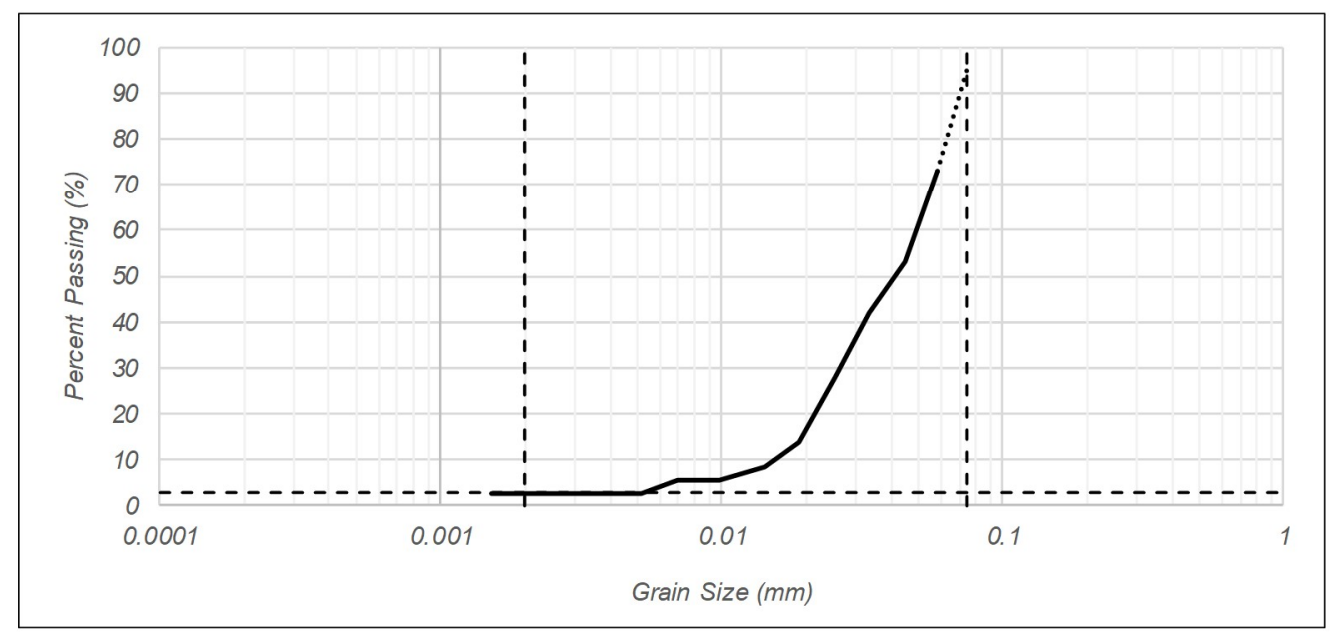

P200 Wash

$\begin{array}{lcc}\text { Container ID: } & \mathrm{H} 1 & \\ \text { Before }-\mathrm{M}_{\mathrm{d}}: & 26.2 & \mathrm{~g} \\ \text { After }-\mathrm{M}_{\mathrm{d}} \text { : } & 1.1 & \mathrm{~g} \\ \text { Fines Fraction: } & 95.80 & \% \\ \text { Coarse Fraction: } & 4.20 & \%\end{array}$

Procedures Done By: Kayla/Melissa B. 


\section{S Portland State}

\begin{tabular}{|c|c|c|c|c|c|c|c|c|c|}
\hline \multirow{2}{*}{ Boring: } & \multicolumn{9}{|c|}{ Hydrometer Test } \\
\hline & \multicolumn{2}{|c|}{ PGE_Ha1 } & & Sample: & N8 & \multirow{2}{*}{\multicolumn{2}{|c|}{$\begin{array}{lll}\text { Depth }(\mathrm{ft}-\mathrm{ft}): & 12.1 & 12.5 \\
\text { Ave. Depth }(\mathrm{ft}): & 12.3\end{array}$}} & \multirow[t]{2}{*}{ Start Time: } & \multirow[t]{2}{*}{ 12:17:00 PM } \\
\hline & & & & & & & & & \\
\hline & \multicolumn{2}{|c|}{ Constants } & & & & & & & \\
\hline & $M_{s}(g)$ & 50.99 & & & & & & & \\
\hline & $\mathrm{G}_{\mathrm{s}}$ & 2.65 & & & & & & & \\
\hline & $\mathrm{G}_{w}$ & 1 & & & & & & & \\
\hline & $\begin{array}{l}\text { Time } \\
\text { (min) }\end{array}$ & $\begin{array}{c}\text { Soil } \\
\text { (slurry) }\end{array}$ & Control & $\begin{array}{l}\text { Temp } \\
\left({ }^{\circ} \mathrm{C}\right)\end{array}$ & $\begin{array}{c}\text { Temp } \\
\text { Correction }(\mathrm{K})\end{array}$ & $\begin{array}{l}\text { Hydrometer } \\
\text { Correction } \\
(R)\end{array}$ & $\begin{array}{l}\text { Effective } \\
\text { Depth, L } \\
\text { (cm) }\end{array}$ & Diameter (mm) & $\begin{array}{c}\text { Percent } \\
\text { Passing (\%) }\end{array}$ \\
\hline & 0.5 & 1.023 & 1.004 & 16.5 & 0.014260 & 1.019 & 10.2 & 0.064407228 & 60 \\
\hline & 1 & 1.021 & 1.004 & 16.5 & 0.014260 & 1.017 & 10.7 & 0.046645678 & 54 \\
\hline & 2 & 1.017 & 1.004 & 16.5 & 0.014260 & 1.013 & 11.8 & 0.03463742 & 41 \\
\hline & 4 & 1.014 & 1.004 & 16.5 & 0.014260 & 1.010 & 12.6 & 0.025308989 & 31 \\
\hline & 8 & 1.010 & 1.004 & 16.5 & 0.014260 & 1.006 & 13.7 & 0.018660996 & 19 \\
\hline & 16 & 1.007 & 1.004 & 16.5 & 0.014260 & 1.003 & 14.4 & 0.013528224 & 9 \\
\hline & 32 & 1.006 & 1.004 & 16.5 & 0.014260 & 1.002 & 14.7 & 0.00966503 & 6 \\
\hline & 60 & 1.006 & 1.004 & 16.5 & 0.014260 & 1.002 & 14.7 & 0.00705834 & 6 \\
\hline & 120 & 1.006 & 1.004 & 16.5 & 0.014260 & 1.002 & 14.7 & 0.004991 & 6 \\
\hline & 227 & 1.005 & 1.004 & 16.5 & 0.014260 & 1.001 & 15.0 & 0.00366566 & 3 \\
\hline & 422 & 1.005 & 1.004 & 16.5 & 0.014260 & 1.001 & 15.0 & 0.002688493 & 3 \\
\hline & 1384 & 1.005 & 1.004 & 16.5 & 0.014260 & 1.001 & 15.0 & 0.001484558 & 3 \\
\hline
\end{tabular}

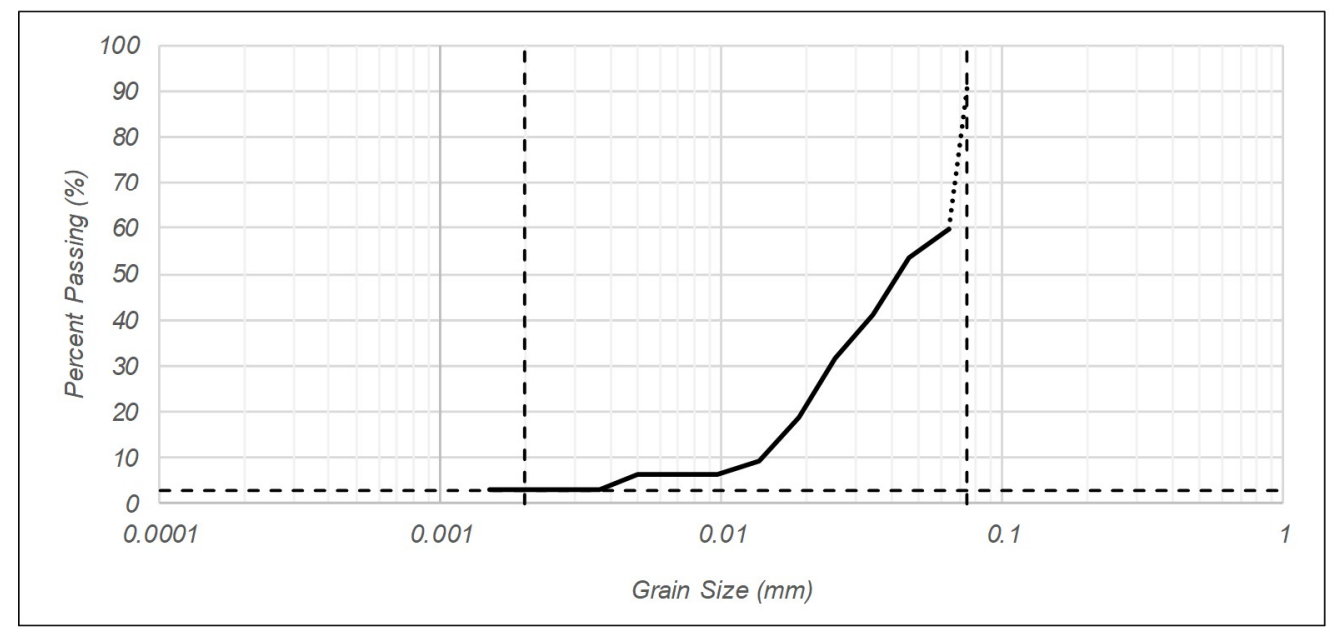

P200 Wash

$\begin{array}{lcc}\text { Container ID: } & \text { Q1 } & \\ \text { Before }-\mathrm{M}_{\mathrm{d}} \text { : } & 25.4 & \mathrm{~g} \\ \text { After }-\mathrm{M}_{\mathrm{d}} \text { : } & 2.0 & \mathrm{~g} \\ \text { Fines Fraction: } & 92.13 & \% \\ \text { Coarse Fraction: } & 7.87 & \%\end{array}$

Procedures Done By: Kayla/Melissa B. 


\section{S Portland State}

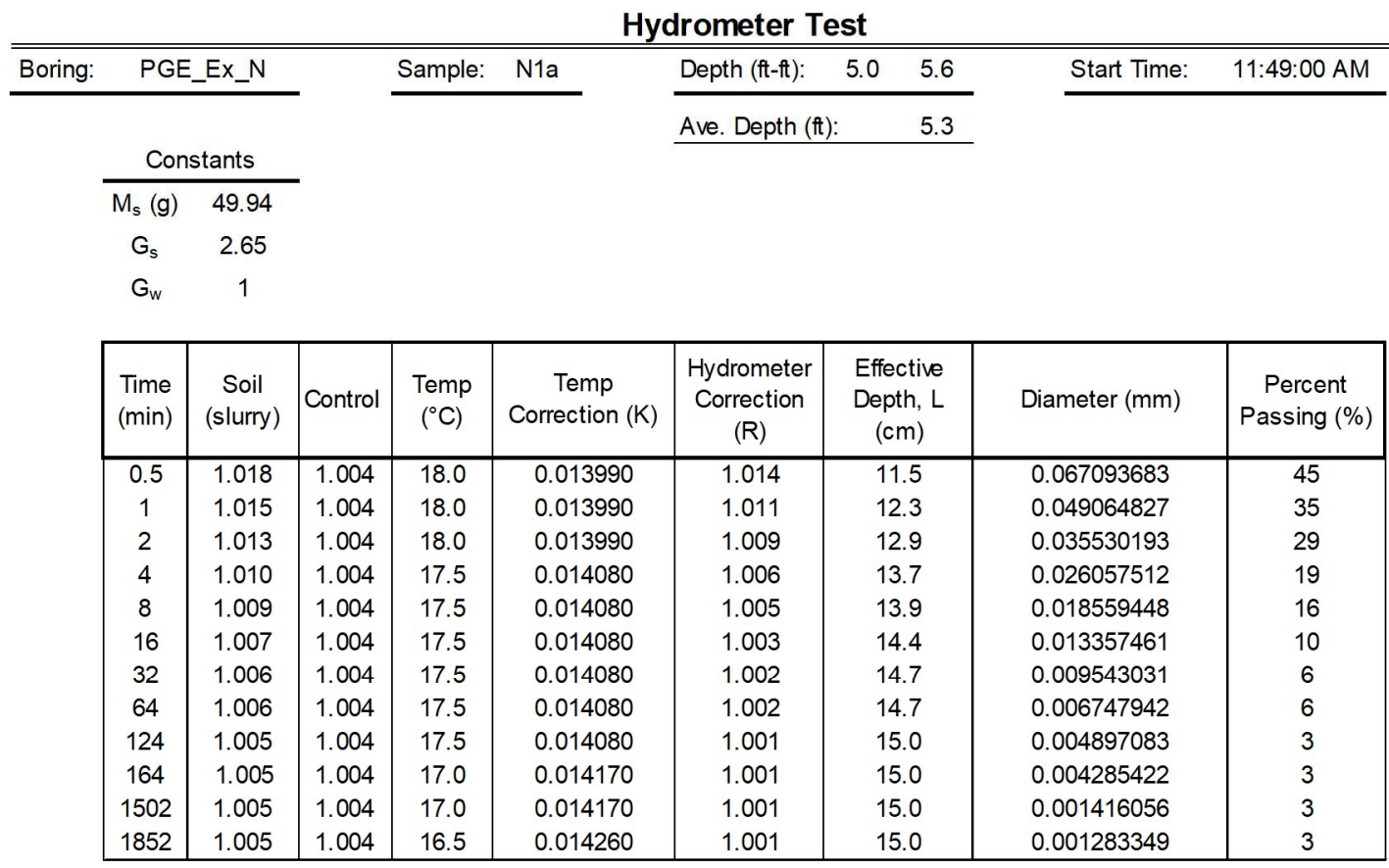

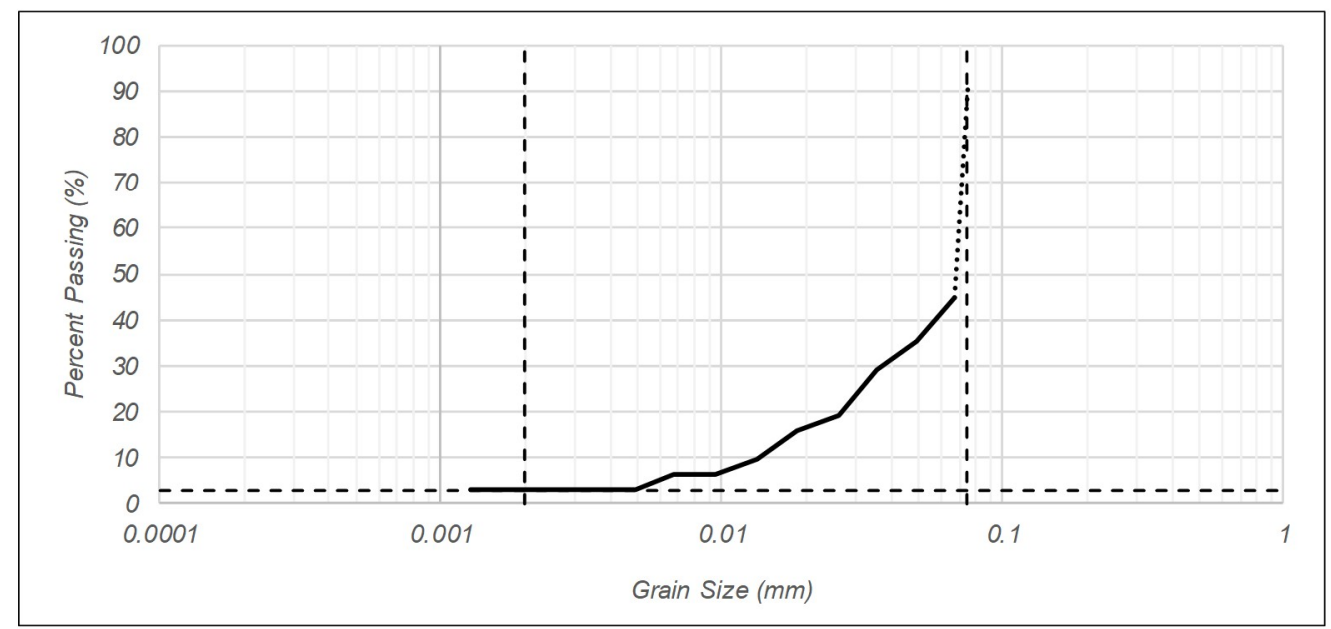

P200 Wash

$\begin{array}{lcc}\text { Container ID: } & \text { W1 } & \\ \text { Before }-\mathrm{M}_{\mathrm{d}}: & 24.3 & \mathrm{~g} \\ \text { After }-\mathrm{M}_{\mathrm{d}} \text { : } & 2.2 & \mathrm{~g} \\ \text { Fines Fraction: } & 90.95 & \% \\ \text { Coarse Fraction: } & 9.05 & \%\end{array}$

Procedures Done By: Kayla/Melissa P. 


\section{蛋 Portland $\underset{\text { SNIVESITYY }}{\text { State }}$}

Hydrometer Test

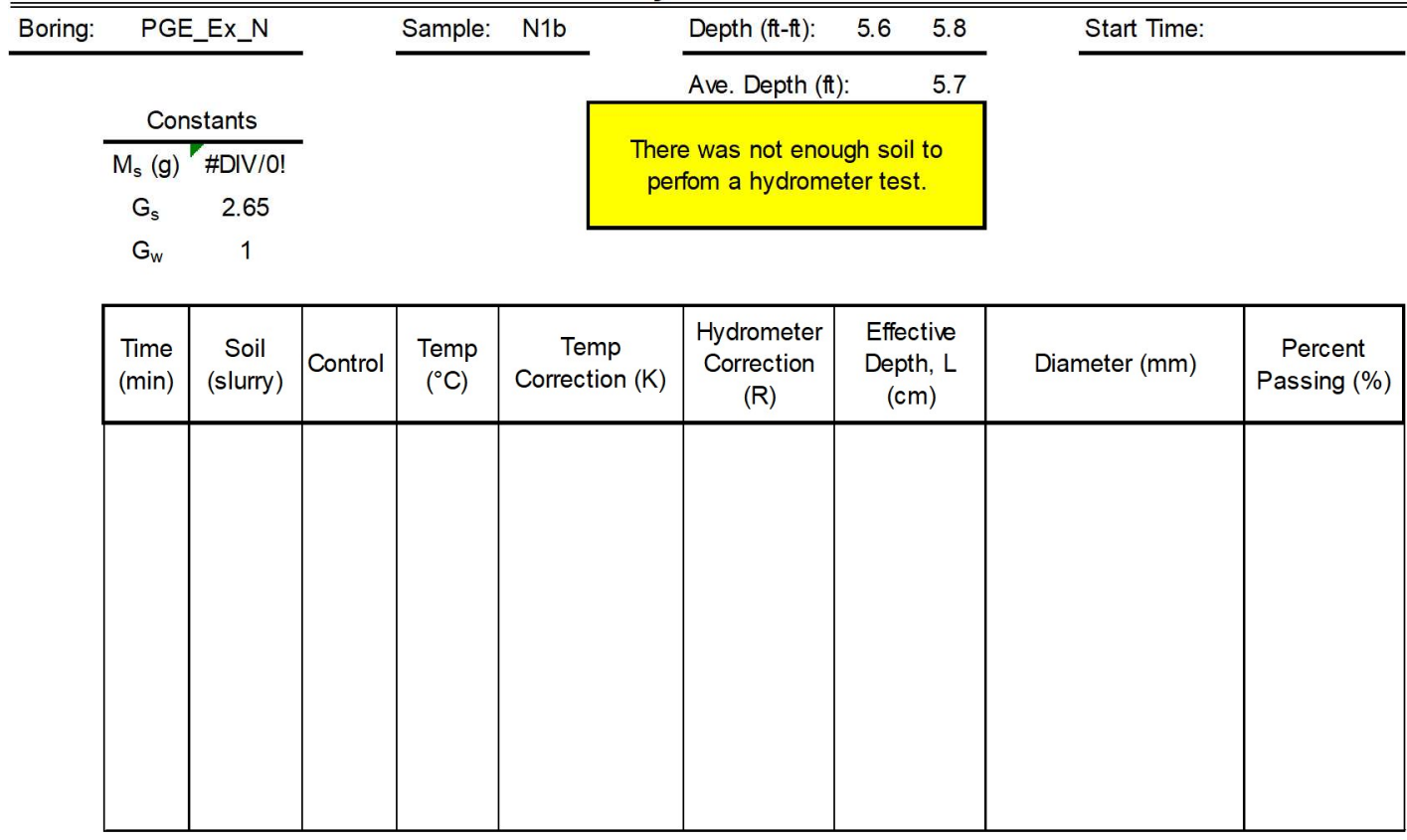

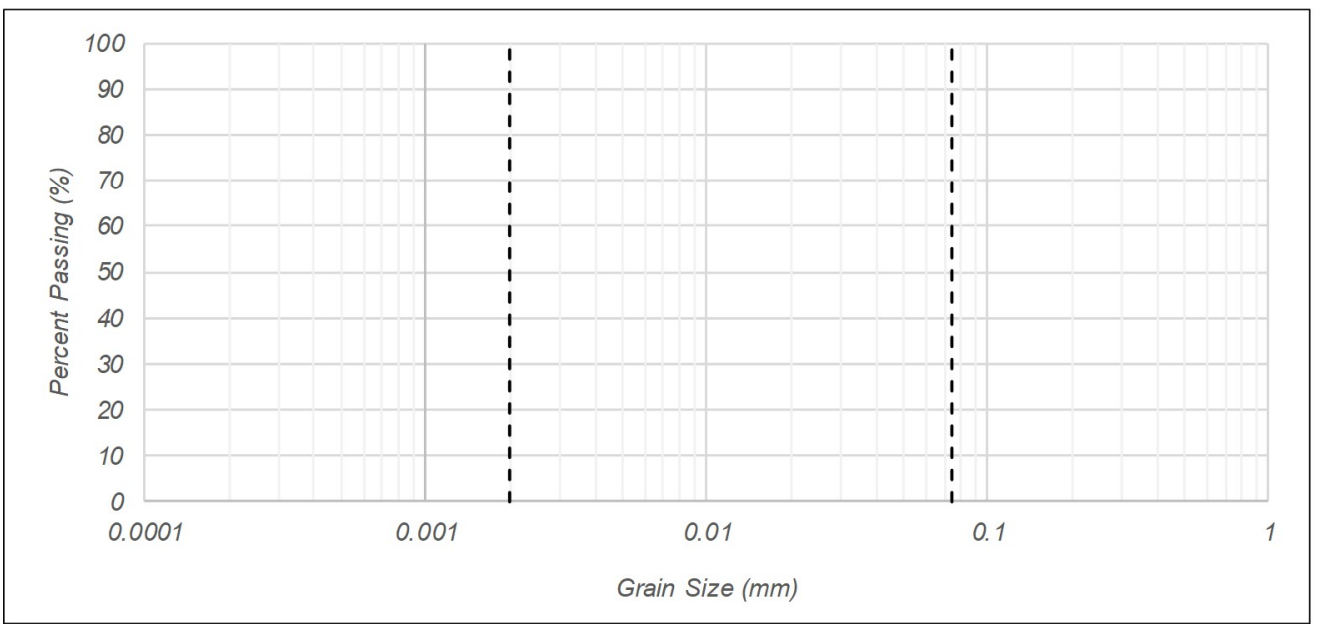

P200 Wash

$\begin{array}{lcc}\text { Container ID: } & \text { G1, L1 } & \\ \text { Before }-\mathrm{M}_{\mathrm{d}}: & 25.7 & \mathrm{~g} \\ \text { After }-\mathrm{M}_{\mathrm{d}} \text { : } & 3.9 & \mathrm{~g} \\ \text { Fines Fraction: } & 84.82 & \% \\ \text { Coarse Fraction: } & 15.18 & \%\end{array}$

Procedures Done By:

Kayla/Melissa P. 


\section{蛋 Portland $\underset{\text { SNIVESITYY }}{\text { State }}$}

Hydrometer Test

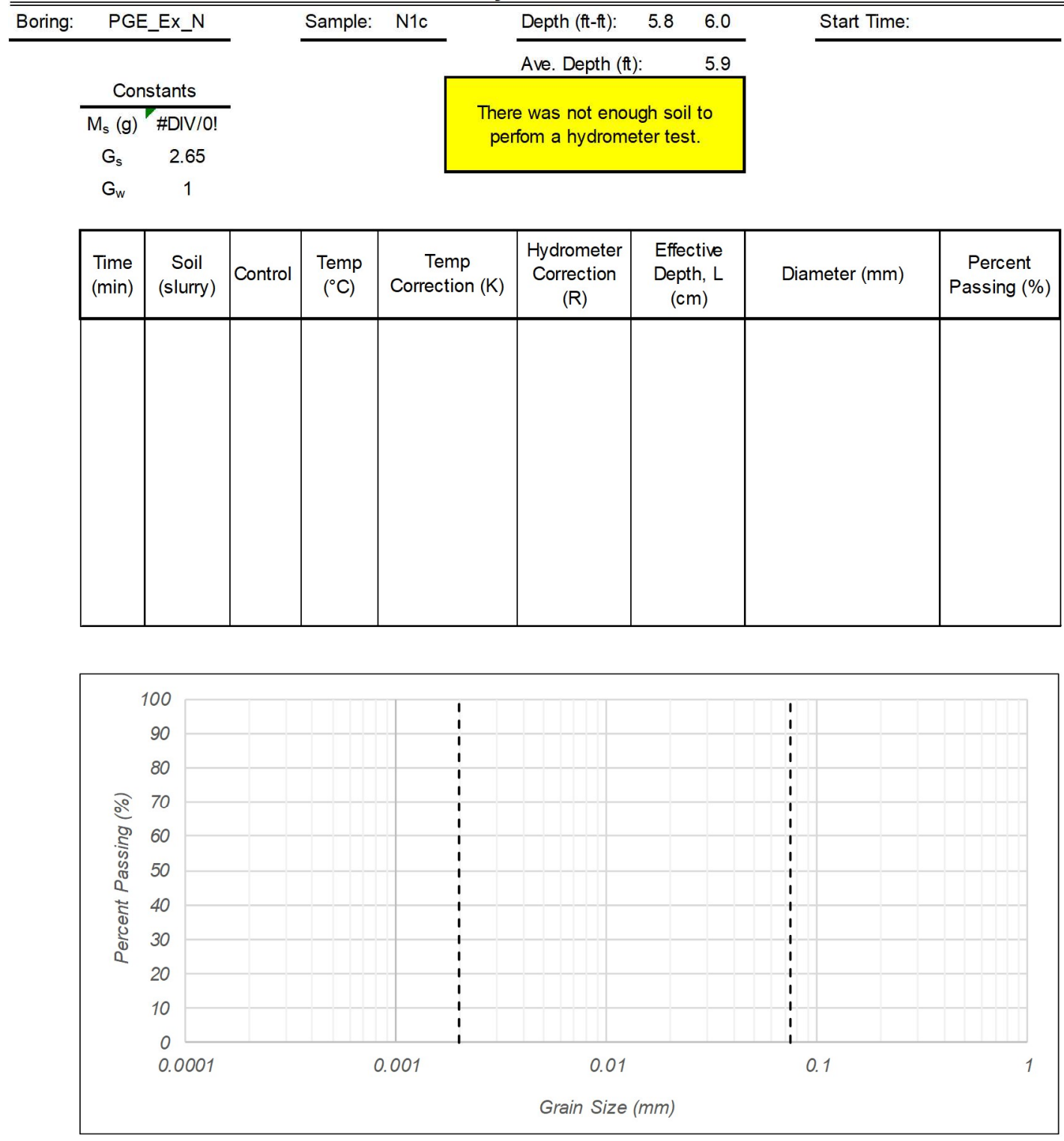

P200 Wash

$\begin{array}{lcc}\text { Container ID: } & \mathrm{S} 1 & \\ \text { Before }-\mathrm{M}_{\mathrm{d}} \text { : } & 30.3 \mathrm{~g} \\ \text { After }-\mathrm{M}_{\mathrm{d}} \text { : } & 2.4 & \mathrm{~g} \\ \text { Fines Fraction: } & 92.08 & \% \\ \text { Coarse Fraction: } & 7.92 & \%\end{array}$

Procedures Done By:

Kayla/Melissa P. 


\section{S Portland State}

Hydrometer Test

\begin{tabular}{|c|c|c|c|c|c|c|c|c|c|}
\hline \multirow[t]{2}{*}{ Boring: } & \multicolumn{2}{|c|}{ PGE_Ex_N } & & \multirow[t]{2}{*}{ Sample: } & \multirow[t]{2}{*}{$\mathrm{N} 2 \mathrm{a}$} & \multirow{2}{*}{\multicolumn{2}{|c|}{$\begin{array}{lll}\text { Depth }(\mathrm{ft}-\mathrm{ft}): & 12.5 & 13.3 \\
\text { Ave. Depth }(\mathrm{ft}): & 12.9 \\
\end{array}$}} & \multirow[t]{2}{*}{ Start Time: } & \multirow[t]{2}{*}{ 8:16:00 AM } \\
\hline & & & & & & & & & \\
\hline & \multicolumn{2}{|c|}{ Constants } & & & & & & & \\
\hline & $M_{s}(g)$ & 49.76 & & & & & & & \\
\hline & $\mathrm{G}_{\mathrm{s}}$ & 2.65 & & & & & & & \\
\hline & $\mathrm{G}_{w}$ & 1 & & & & & & & \\
\hline & $\begin{array}{l}\text { Time } \\
\text { (min) }\end{array}$ & $\begin{array}{c}\text { Soil } \\
\text { (slurry) }\end{array}$ & Control & $\begin{array}{l}\text { Temp } \\
\left({ }^{\circ} \mathrm{C}\right)\end{array}$ & $\begin{array}{c}\text { Temp } \\
\text { Correction }(\mathrm{K})\end{array}$ & $\begin{array}{c}\text { Hydrometer } \\
\text { Correction } \\
(R)\end{array}$ & $\begin{array}{l}\text { Effective } \\
\text { Depth, L } \\
\text { (cm) }\end{array}$ & Diameter (mm) & $\begin{array}{c}\text { Percent } \\
\text { Passing (\%) }\end{array}$ \\
\hline & 0.5 & 1.027 & 1.004 & 18.0 & 0.013990 & 1.023 & $\overline{9.2}$ & 0.060010414 & 74 \\
\hline & 1 & 1.023 & 1.004 & 18.0 & 0.013990 & 1.019 & 10.2 & 0.044680477 & 61 \\
\hline & 2 & 1.015 & 1.004 & 18.0 & 0.013990 & 1.011 & 12.3 & 0.034694072 & 36 \\
\hline & 4 & 1.012 & 1.004 & 18.0 & 0.013990 & 1.008 & 13.1 & 0.025317649 & 26 \\
\hline & 8 & 1.009 & 1.004 & 18.0 & 0.013990 & 1.005 & 13.9 & 0.018440815 & 16 \\
\hline & 16 & 1.007 & 1.004 & 18.0 & 0.013990 & 1.003 & 14.4 & 0.013272079 & 10 \\
\hline & 30 & 1.006 & 1.004 & 18.0 & 0.013990 & 1.002 & 14.7 & 0.009793 & 6 \\
\hline & 67 & 1.005 & 1.004 & 18.0 & 0.013990 & 1.001 & 15.0 & 0.006619512 & 3 \\
\hline & 106 & 1.005 & 1.004 & 17.5 & 0.014080 & 1.001 & 15.0 & 0.005296578 & 3 \\
\hline & 211 & 1.005 & 1.004 & 17.5 & 0.014080 & 1.001 & 15.0 & 0.003754111 & 3 \\
\hline & 543 & 1.004 & 1.004 & 17.5 & 0.014080 & 1.000 & 15.2 & 0.002355725 & 0 \\
\hline & 1533 & 1.004 & 1.004 & 17.5 & 0.014080 & 1.000 & 15.2 & 0.001402017 & 0 \\
\hline
\end{tabular}

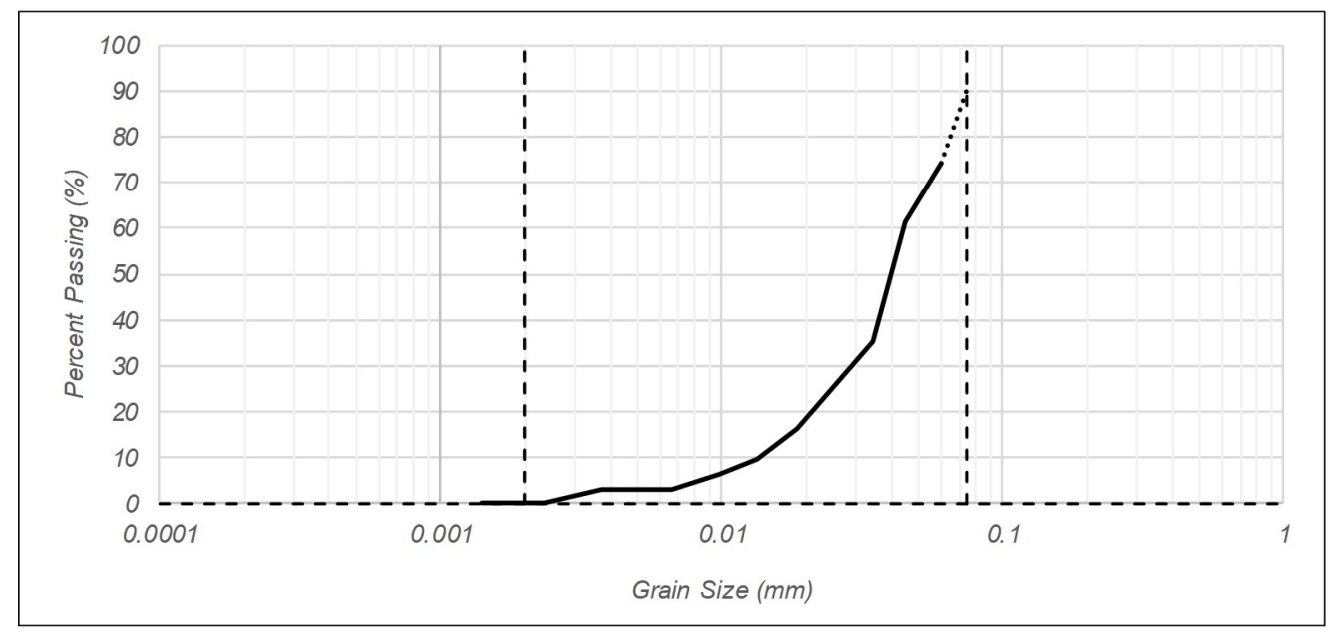

P200 Wash

$\begin{array}{lcc}\text { Container ID: } & \text { W1 } & \\ \text { Before }-\mathrm{M}_{\mathrm{d}} \text { : } & 24.3 & \mathrm{~g} \\ \text { After }-\mathrm{M}_{\mathrm{d}} \text { : } & 2.2 & \mathrm{~g} \\ \text { Fines Fraction: } & 90.95 & \% \\ \text { Coarse Fraction: } & 9.05 & \%\end{array}$

Procedures Done By: Kayla/Melissa P 


\section{胥 Portland State}

Hydrometer Test

\begin{tabular}{|c|c|c|c|c|c|c|c|c|c|}
\hline \multirow[t]{2}{*}{ Boring: } & \multicolumn{2}{|c|}{ PGE_Ex_N } & & \multirow[t]{2}{*}{ Sample: } & $\mathrm{N} 2 \mathrm{~b}$ & Depth (ft-ft): & 13.2514 .0 & \multirow[t]{2}{*}{ Start Time: } & $8: 42: 00$ AM \\
\hline & & & & & & Ave. Depth (ft & 13.63 & & \\
\hline \multicolumn{10}{|c|}{ Constants } \\
\hline & $M_{s}(g)$ & 50.05 & & & & & & & \\
\hline & $\mathrm{G}_{\mathrm{s}}$ & 2.65 & & & & & & & \\
\hline & $\mathrm{G}_{w}$ & 1 & & & & & & & \\
\hline & $\begin{array}{r}\text { Time } \\
(\min )\end{array}$ & $\begin{array}{c}\text { Soil } \\
\text { (slurry) }\end{array}$ & Control & $\begin{array}{l}\text { Temp } \\
\left({ }^{\circ} \mathrm{C}\right)\end{array}$ & $\begin{array}{c}\text { Temp } \\
\text { Correction }(\mathrm{K})\end{array}$ & $\begin{array}{c}\text { Hydrometer } \\
\text { Correction } \\
(\mathrm{R})\end{array}$ & $\begin{array}{c}\text { Effective } \\
\text { Depth, L (cm) }\end{array}$ & Diameter (mm) & $\begin{array}{c}\text { Percent } \\
\text { Passing (\%) }\end{array}$ \\
\hline & 0.5 & 1.032 & 1.004 & 18.5 & 0.013990 & 1.028 & 7.8 & 0.055256073 & 90 \\
\hline & 1 & 1.030 & 1.004 & 18.5 & 0.013990 & 1.026 & 8.4 & 0.040546872 & 83 \\
\hline & 2 & 1.026 & 1.004 & 18.0 & 0.013990 & 1.022 & 9.4 & 0.030329597 & 71 \\
\hline & 4 & 1.024 & 1.004 & 18.5 & 0.013990 & 1.020 & 10.0 & 0.022120132 & 64 \\
\hline & 8 & 1.019 & 1.004 & 18.5 & 0.013990 & 1.015 & 11.3 & 0.016626925 & 48 \\
\hline & 16 & 1.015 & 1.004 & 18.0 & 0.013990 & 1.011 & 12.3 & 0.012266207 & 35 \\
\hline & 41 & 1.011 & 1.004 & 18.0 & 0.013990 & 1.007 & 13.4 & 0.007997941 & 22 \\
\hline & 77 & 1.010 & 1.004 & 18.0 & 0.013990 & 1.006 & 13.7 & 0.005901095 & 19 \\
\hline & 134 & 1.009 & 1.004 & 17.5 & 0.014080 & 1.005 & 13.9 & 0.004534795 & 16 \\
\hline & 242 & 1.008 & 1.004 & 17.5 & 0.014080 & 1.004 & 14.2 & 0.003410666 & 13 \\
\hline & 1036 & 1.006 & 1.004 & 18.0 & 0.014080 & 1.002 & 14.7 & 0.001677187 & 6 \\
\hline
\end{tabular}

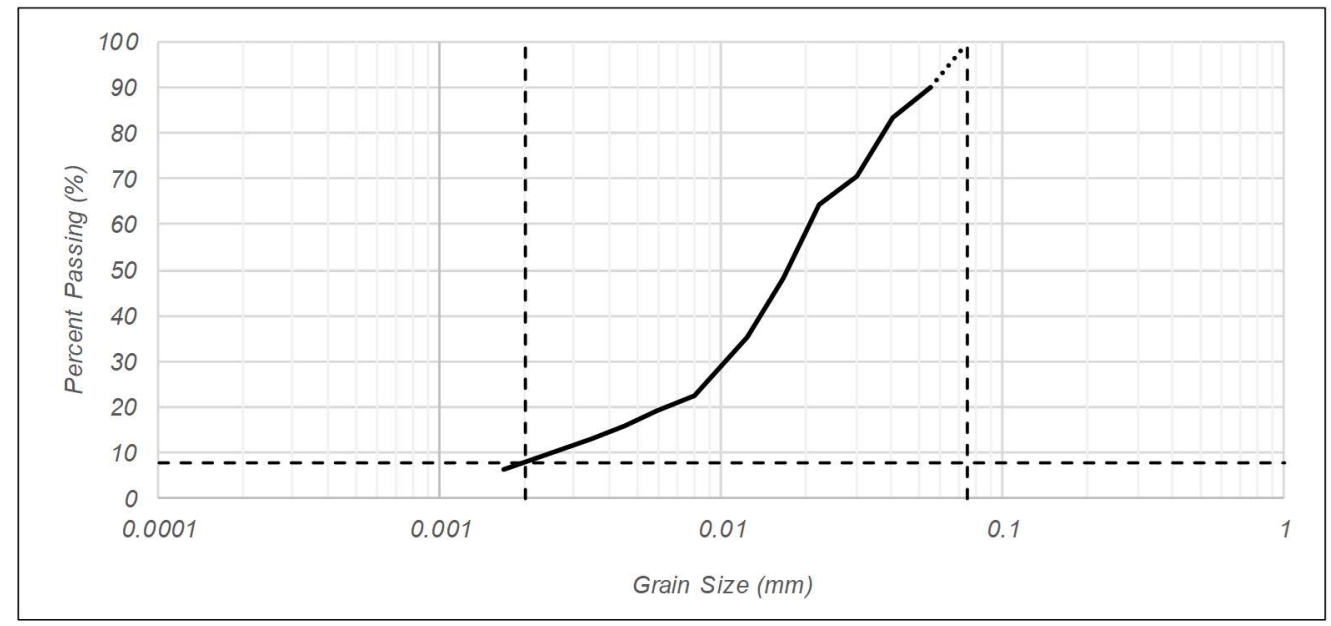

P200 Wash

\begin{tabular}{|c|c|c|c|c|c|}
\hline Container ID: & E1 & & & & kdown \\
\hline Before - $M_{d}$ : & 31 & g & & Coarse: & $0.65 \%$ \\
\hline After $-M_{d}$ : & 0.2 & $g$ & & Silts: & $91.35 \%$ \\
\hline Fines Fraction: & 99.35 & $\%$ & From Hydrometer & Clays: & $8.00 \%$ \\
\hline Coarse Fraction: & 0.65 & $\%$ & & & \\
\hline Procedures Done & By: & Kayla/Melissa P. & & & \\
\hline
\end{tabular}




\section{胥 Portland State}

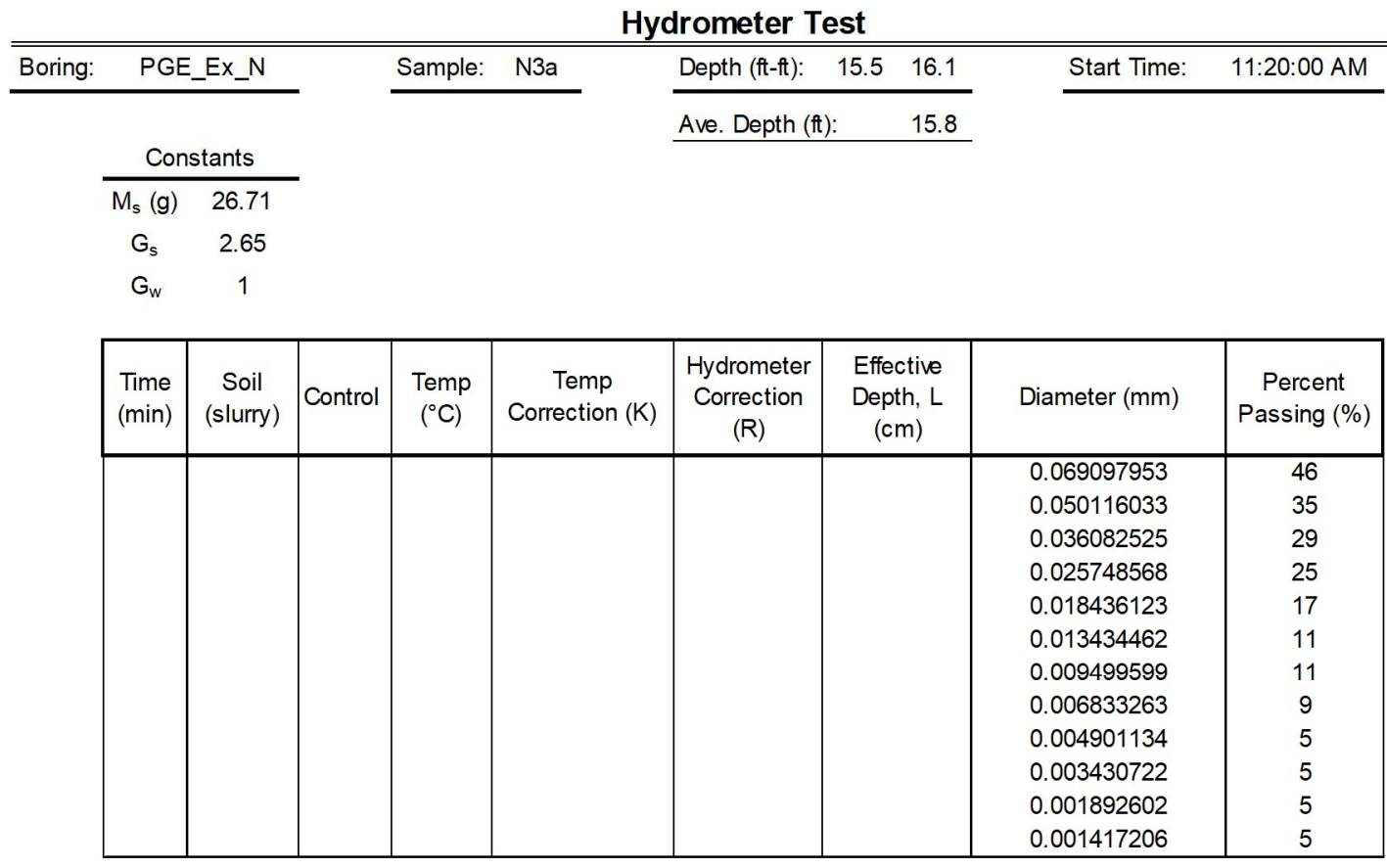

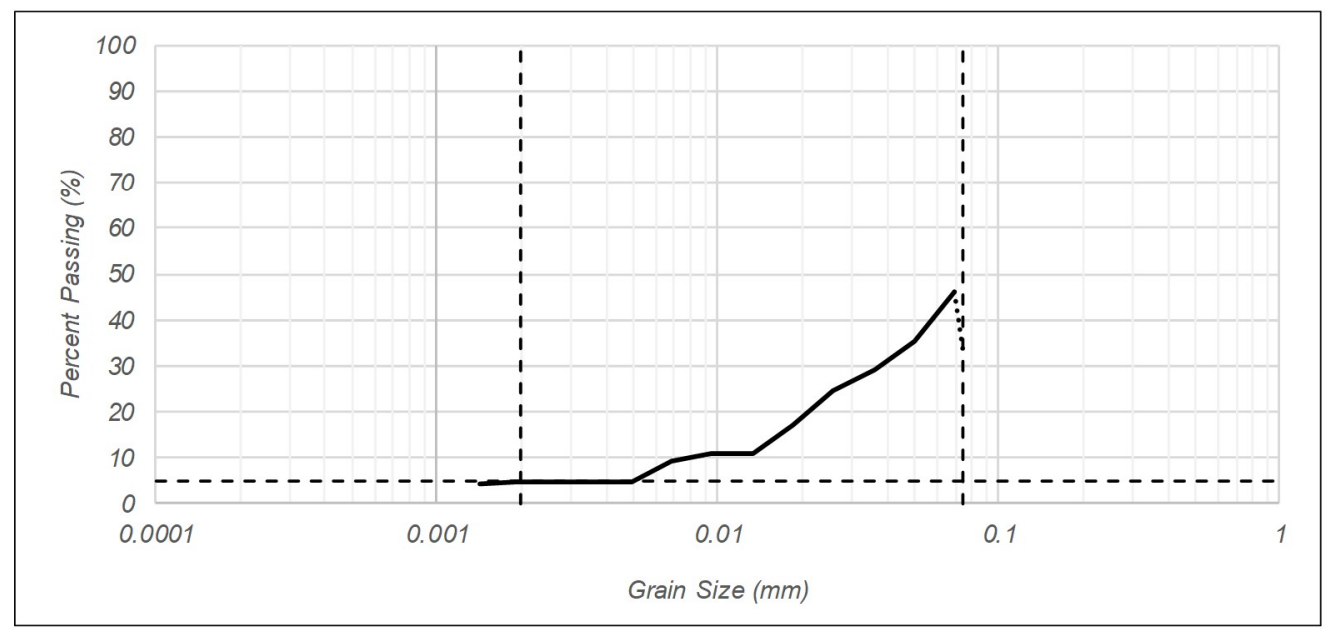

P200 Wash

Container ID:

Before $-M_{d}$ :

After $-M_{d}$ :

Fines Fraction: $\quad 31.98 \%$

Coarse Fraction: $68.02 \%$

Procedures Done By:

Kayla/Melissa B.
Soil Breakdown

\begin{tabular}{lr}
\hline Coarse: & $68.02 \%$ \\
Silts: & $26.98 \%$ \\
Clays: & $5.00 \%$
\end{tabular}




\section{S Portland State}

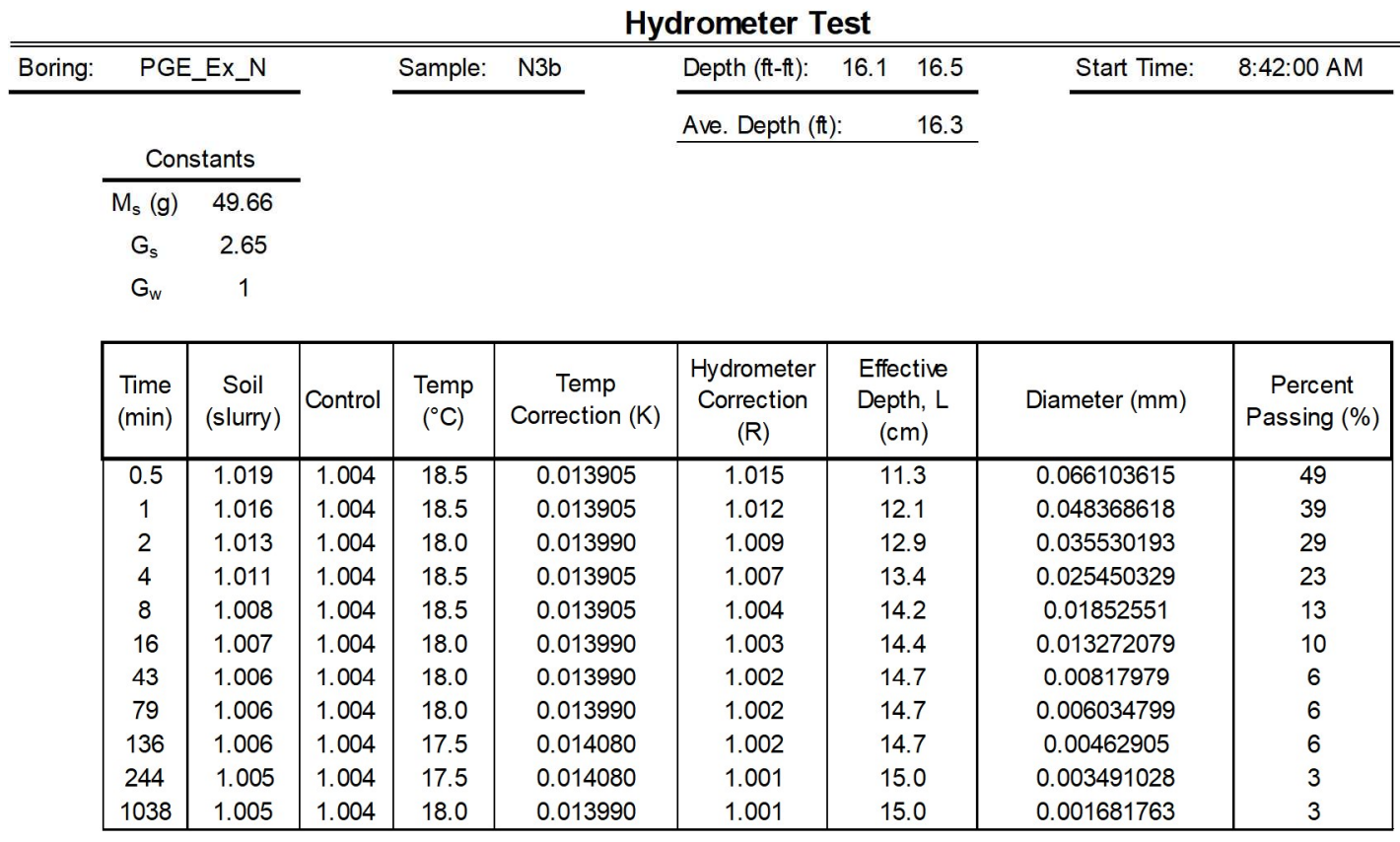

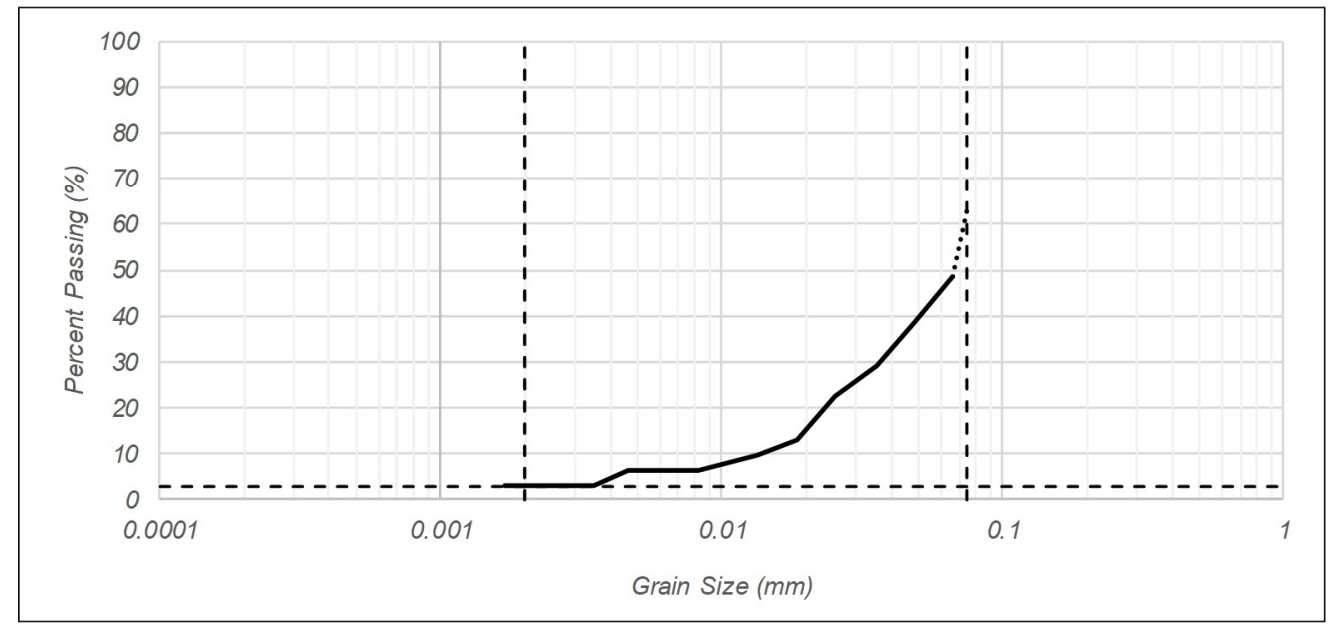

P200 Wash

$\begin{array}{lcc}\text { Container ID: } & \text { F1 } & \\ \text { Before }-\mathrm{M}_{\mathrm{d}}: & 38.4 & \mathrm{~g} \\ \text { After }-\mathrm{M}_{\mathrm{d}} \text { : } & 13.9 & \mathrm{~g} \\ \text { Fines Fraction: } & 63.80 & \% \\ \text { Coarse Fraction: } & 36.20 & \%\end{array}$

Procedures Done By: Kayla/Melissa B. 


\section{S Portland State}

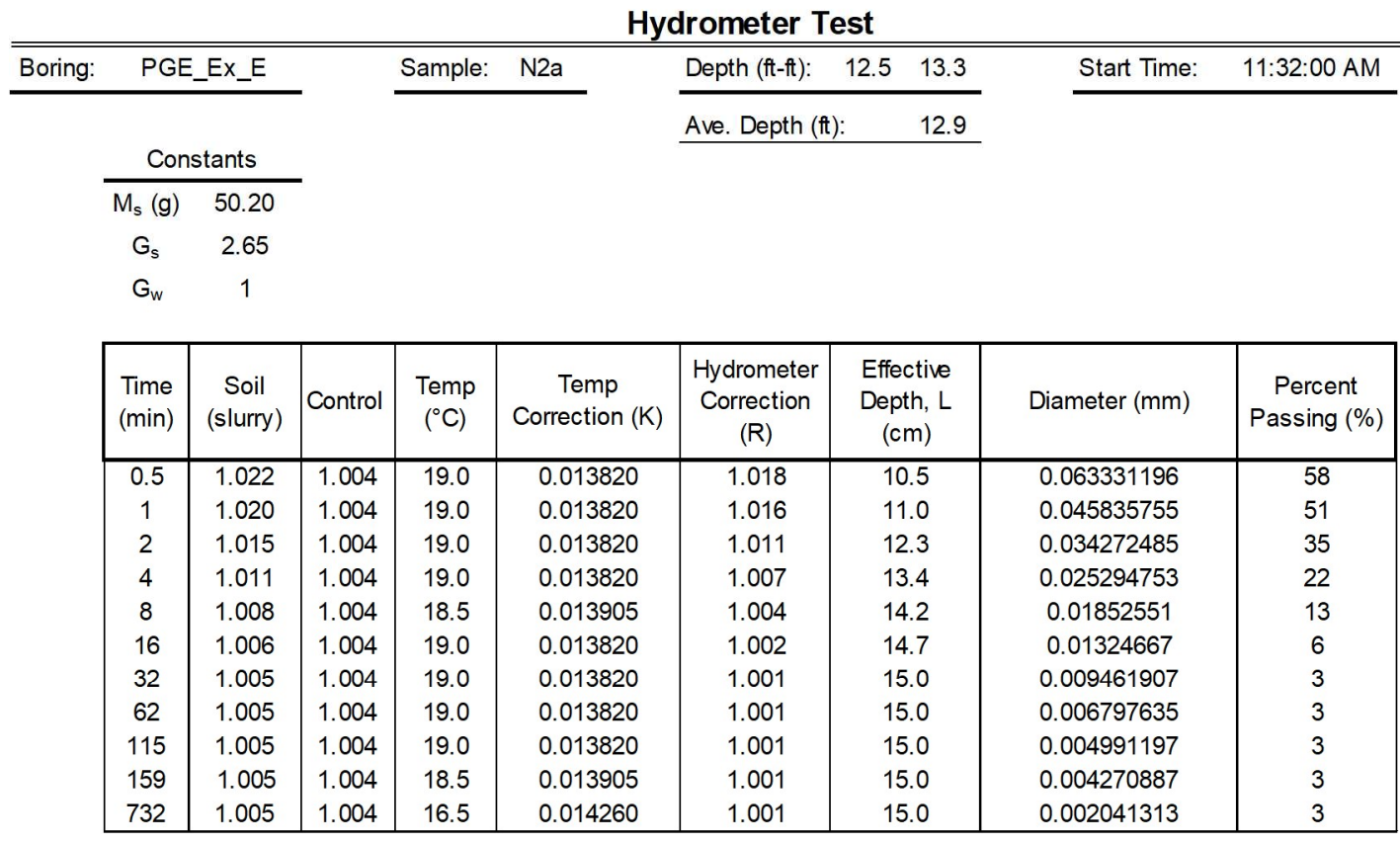

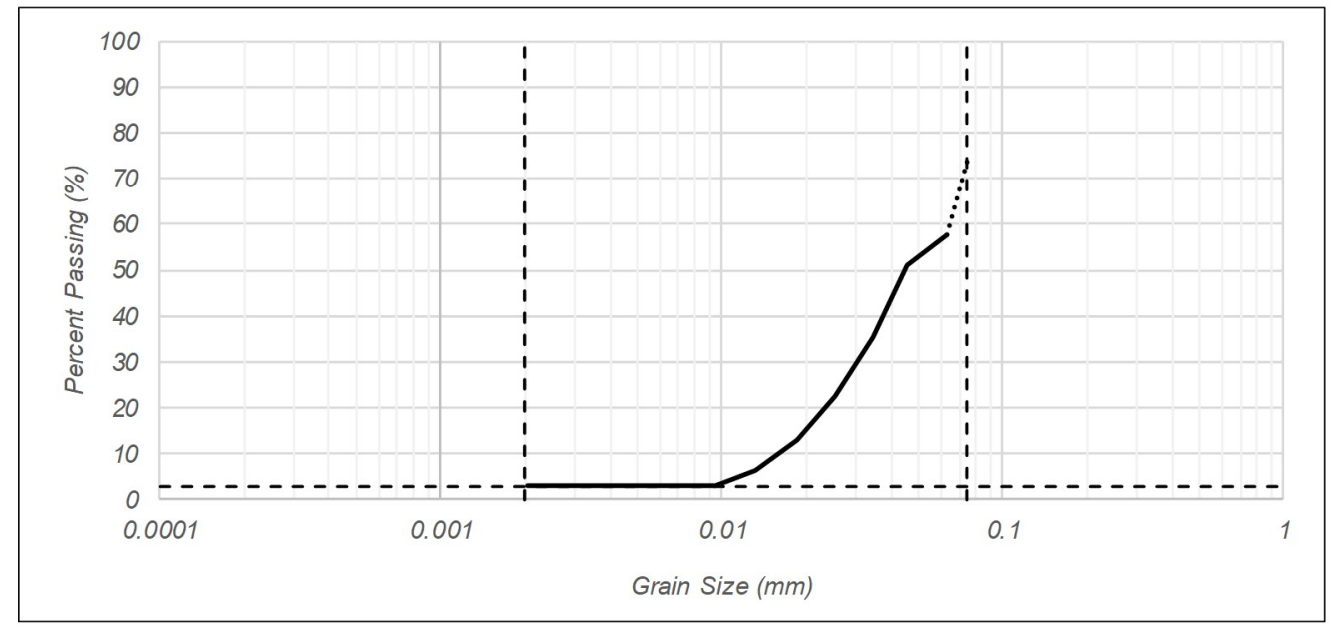

P200 Wash

$\begin{array}{lcc}\text { Container ID: } & \text { K1 } & \\ \text { Before }-\mathrm{M}_{\mathrm{d}} \text { : } & 24.5 & \mathrm{~g} \\ \text { After }-\mathrm{M}_{\mathrm{d}} \text { : } & 6.3 & \mathrm{~g} \\ \text { Fines Fraction: } & 74.29 & \% \\ \text { Coarse Fraction: } & 25.71 & \%\end{array}$

Procedures Done By: Kayla/Melissa B. 


\section{S Portland State}

Hydrometer Test

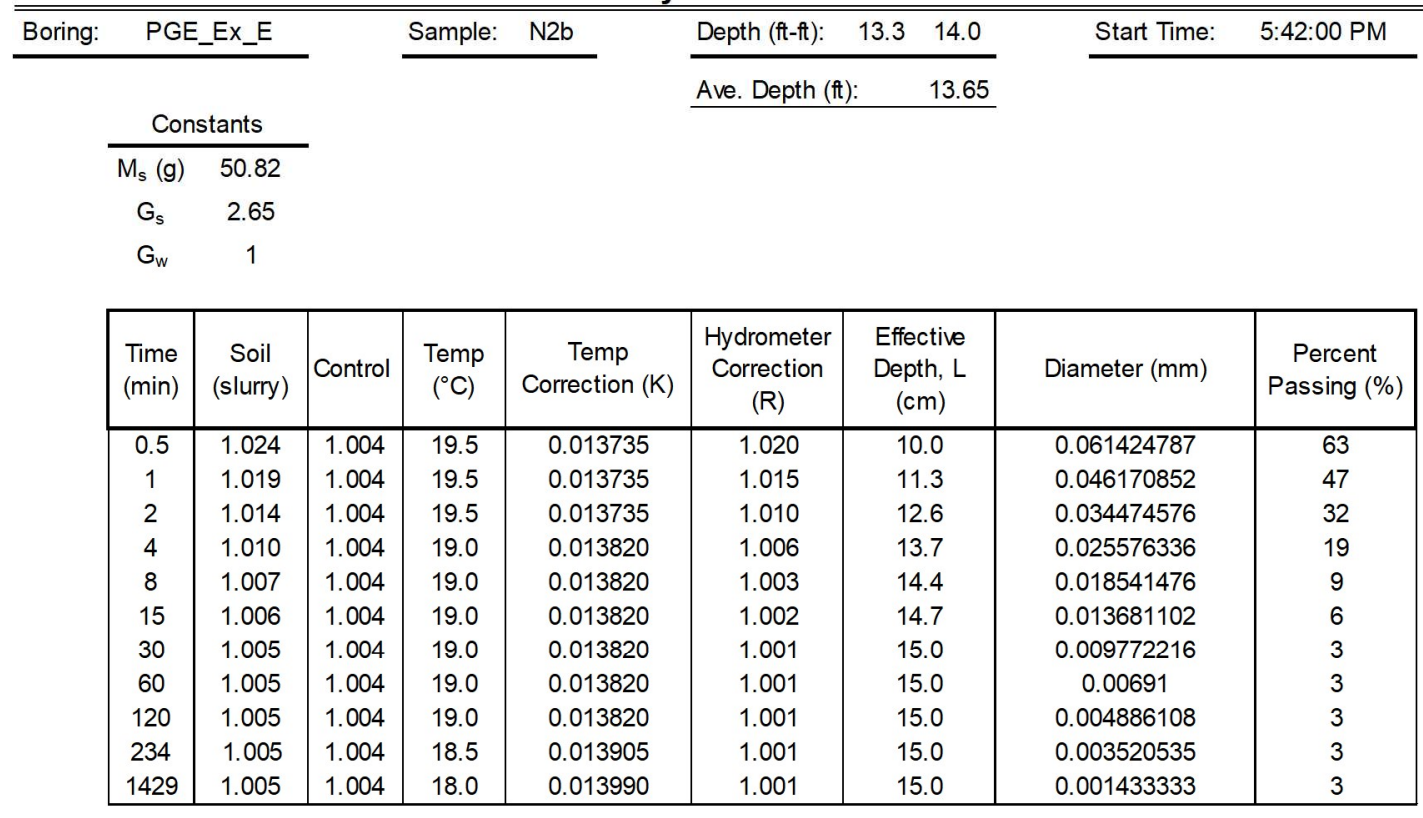

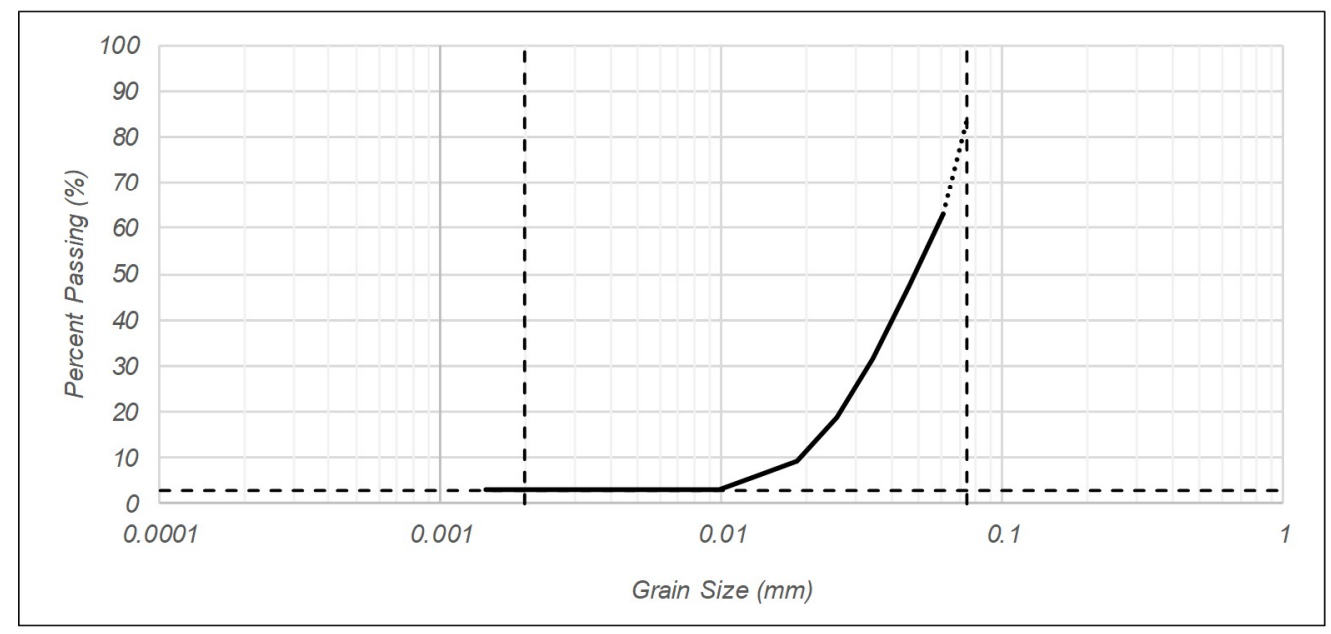

P200 Wash

$\begin{array}{lcc}\text { Container ID: } & \text { I1 } & \\ \text { Before }-\mathrm{M}_{\mathrm{d}} \text { : } & 26.2 & \mathrm{~g} \\ \text { After - } \mathrm{M}_{\mathrm{d}} \text { : } & 4 & \mathrm{~g} \\ \text { Fines Fraction: } & 84.73 & \% \\ \text { Coarse Fraction: } & 15.27 & \%\end{array}$

Procedures Done By: Kayla/Melissa P. 
胥 Portland $\underset{\text { UNIVES StITY }}{\text { State }}$

Hydrometer Test

\begin{tabular}{|c|c|c|c|c|c|c|c|c|c|}
\hline Boring: & PGE & Ex_S & & Sample: & N1 & Depth (ft-ft): & $5.0 \quad 5.25$ & Start Time & \\
\hline & & & & & & Ave. Depth (ft & 5.125 & & \\
\hline & Cor & tants & & & & & & & \\
\hline & $M_{s}(g)$ & \#DIV/O! & & & Ther & Was not enol & $\begin{array}{l}\text { h soil to } \\
\text { er test }\end{array}$ & & \\
\hline & $\mathrm{G}_{\mathrm{s}}$ & 2.65 & & & & & & & \\
\hline & $\mathrm{G}_{\mathrm{w}}$ & 1 & & & & & & & \\
\hline & $\begin{array}{l}\text { Time } \\
\text { (min) }\end{array}$ & $\begin{array}{l}\text { Soil } \\
\text { (slurry) }\end{array}$ & Control & $\begin{array}{l}\text { Temp } \\
\left({ }^{\circ} \mathrm{C}\right)\end{array}$ & $\begin{array}{c}\text { Temp } \\
\text { Correction }(\mathrm{K})\end{array}$ & $\begin{array}{c}\text { Hydrometer } \\
\text { Correction } \\
(R)\end{array}$ & $\begin{array}{l}\text { Effective } \\
\text { Depth, L } \\
\text { (cm) }\end{array}$ & Diameter $(\mathrm{mm})$ & $\begin{array}{c}\text { Percent } \\
\text { Passing (\%) }\end{array}$ \\
\hline & & & & & & & & & \\
\hline & & & & & & & & & \\
\hline & & & & & & & & & \\
\hline & & & & & & & & & \\
\hline & & & & & & & & & \\
\hline & & & & & & & & & \\
\hline
\end{tabular}

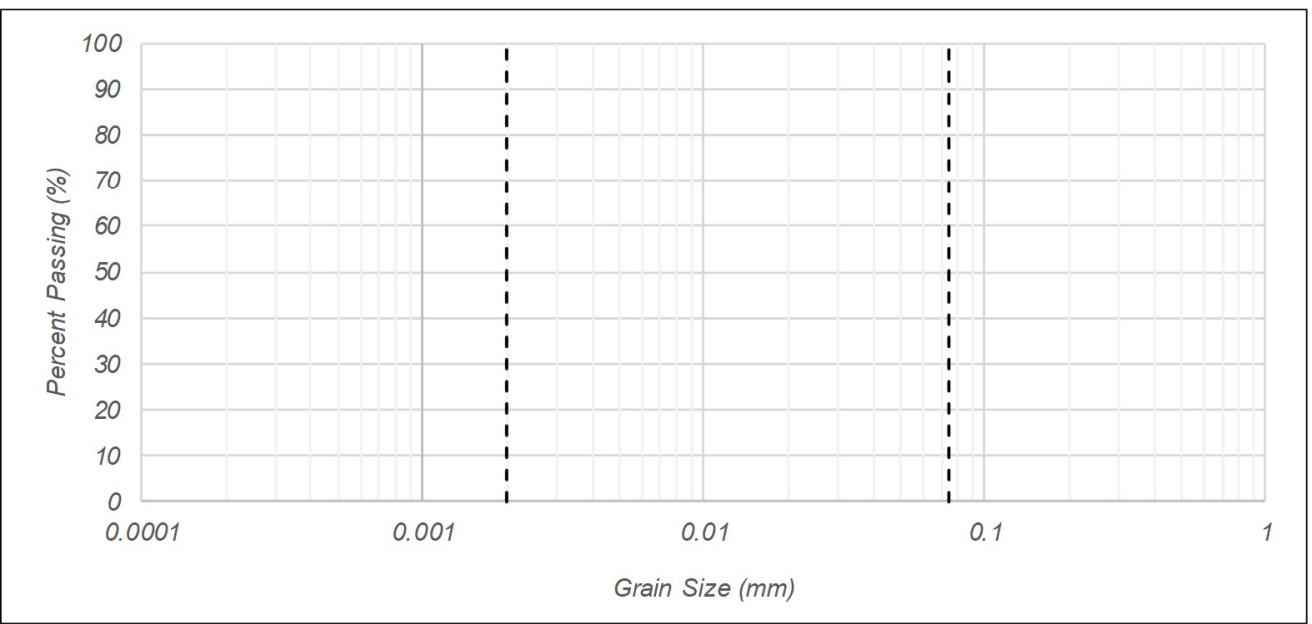

P200 Wash

$\begin{array}{lcc}\text { Container ID: } & \text { K1 } & \\ \text { Before }-\mathrm{M}_{\mathrm{d}}: & 33.4 \mathrm{~g} \\ \text { After }-\mathrm{M}_{\mathrm{d}} \text { : } & 3.2 \mathrm{~g} \\ \text { Fines Fraction: } & 90.42 \% \\ \text { Coarse Fraction: } & 9.58 \%\end{array}$

Procedures Done By:

Melissa B 


\section{S Portland State}

Hydrometer Test

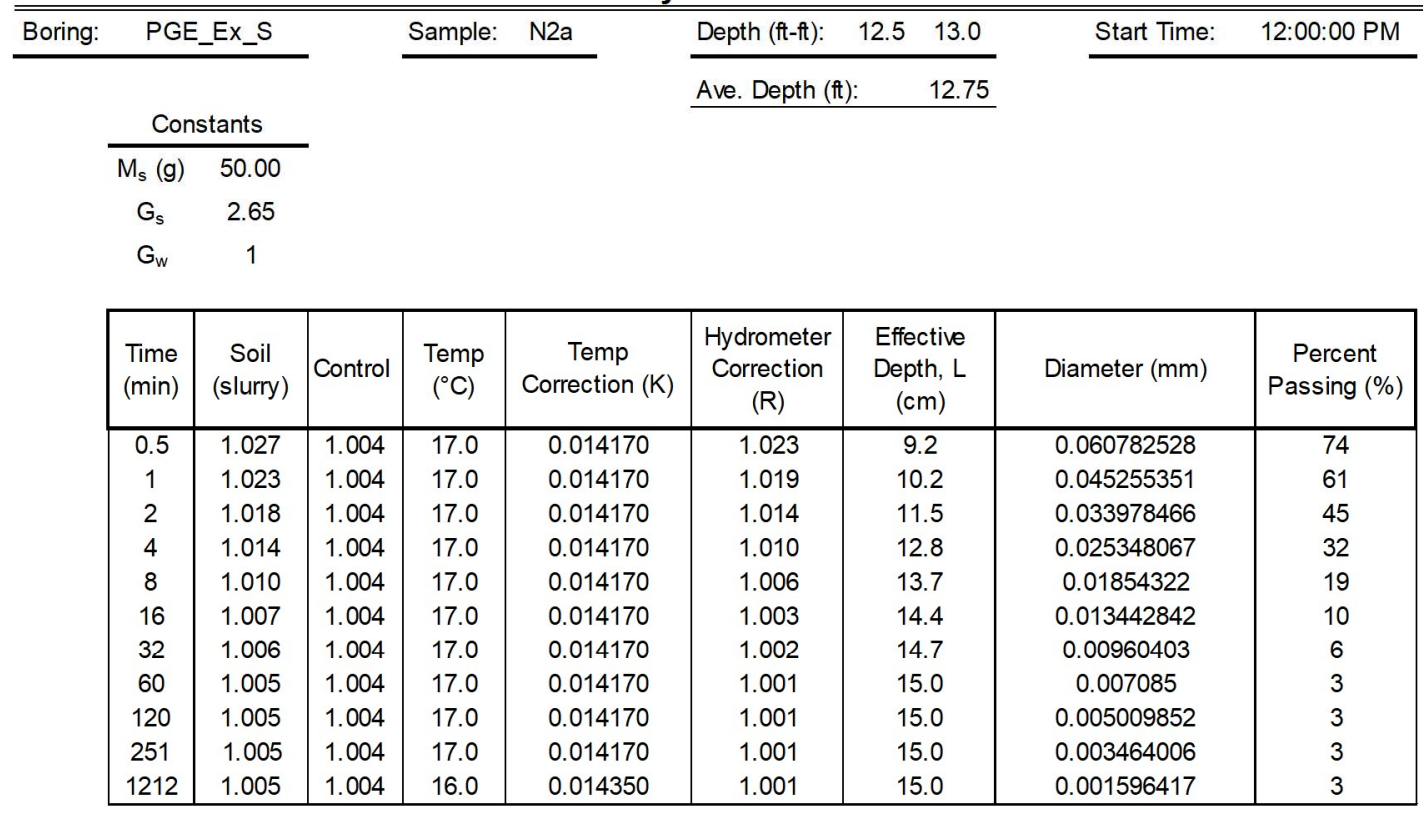

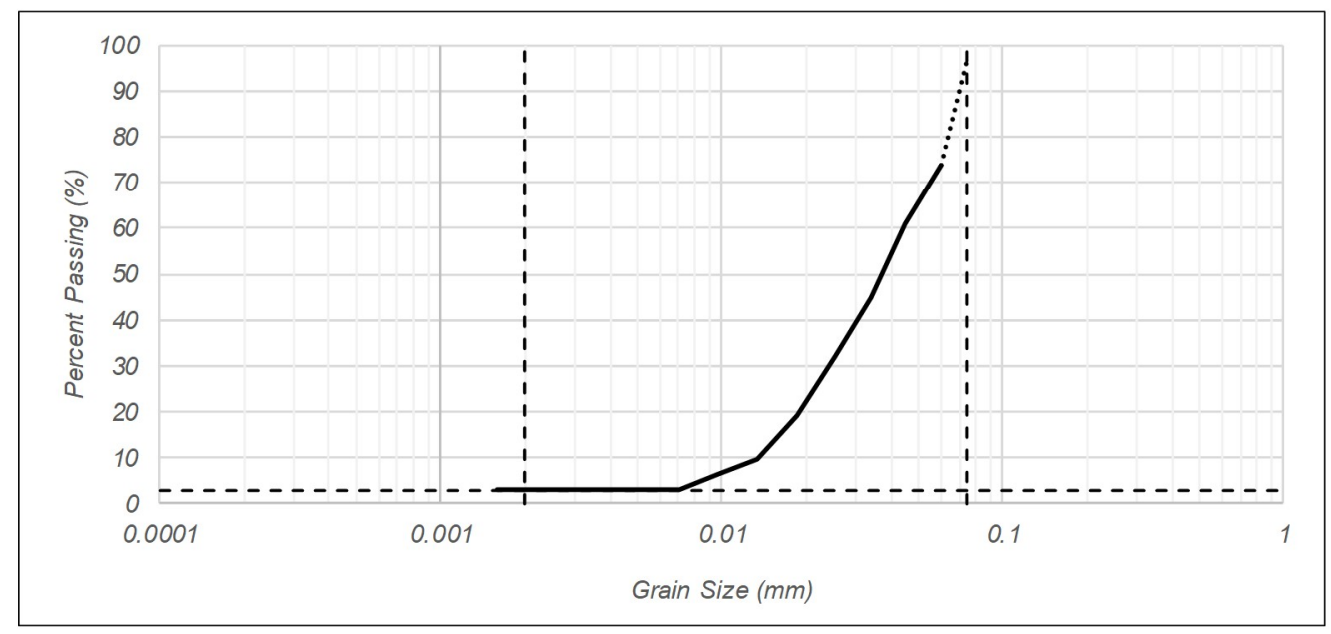

P200 Wash

$\begin{array}{lcc}\text { Container ID: } & \text { E1 } & \\ \text { Before }-\mathrm{M}_{\mathrm{d}}: & 22.2 & \mathrm{~g} \\ \text { After }-\mathrm{M}_{\mathrm{d}} \text { : } & 0.6 & \mathrm{~g} \\ \text { Fines Fraction: } & 97.30 & \% \\ \text { Coarse Fraction: } & 2.70 & \%\end{array}$

Procedures Done By: Kayla/Melissa P. 


\section{S Portland State}

\begin{tabular}{|c|c|c|c|c|c|c|c|c|c|}
\hline \multirow{2}{*}{ Boring: } & \multicolumn{9}{|c|}{ Hydrometer Test } \\
\hline & \multicolumn{2}{|c|}{ PGE_Ex_S } & & Sample: & $\mathrm{N} 2 \mathrm{~b}$ & \multirow{2}{*}{\multicolumn{2}{|c|}{$\begin{array}{lll}\text { Depth }(\mathrm{ft}-\mathrm{ft}): & 13.0 & 14.0 \\
\text { Ave. Depth }(\mathrm{ft}): & 13.5\end{array}$}} & \multirow[t]{2}{*}{ Start Time: } & \multirow[t]{2}{*}{ 10:32:00 AM } \\
\hline & & & & & & & & & \\
\hline & \multicolumn{2}{|c|}{ Constants } & & & & & & & \\
\hline & $M_{s}(g)$ & 49.97 & & & & & & & \\
\hline & $\mathrm{G}_{\mathrm{s}}$ & 2.65 & & & & & & & \\
\hline & $\mathrm{G}_{\mathrm{w}}$ & 1 & & & & & & & \\
\hline & $\begin{array}{l}\text { Time } \\
\text { (min) }\end{array}$ & $\begin{array}{c}\text { Soil } \\
\text { (slurry) }\end{array}$ & Control & $\begin{array}{l}\text { Temp } \\
\left({ }^{\circ} \mathrm{C}\right)\end{array}$ & $\begin{array}{c}\text { Temp } \\
\text { Correction }(\mathrm{K})\end{array}$ & $\begin{array}{l}\text { Hydrometer } \\
\text { Correction } \\
(R)\end{array}$ & $\begin{array}{l}\text { Effective } \\
\text { Depth, L } \\
\text { (cm) }\end{array}$ & Diameter (mm) & $\begin{array}{c}\text { Percent } \\
\text { Passing (\%) }\end{array}$ \\
\hline & 0.5 & 1.033 & 1.004 & 17.0 & 0.014170 & 1.029 & 7.6 & 0.05524483 & 93 \\
\hline & 1 & 1.030 & 1.004 & 17.0 & 0.014170 & 1.026 & 8.4 & 0.041068562 & 84 \\
\hline & 2 & 1.027 & 1.004 & 17.0 & 0.014170 & 1.023 & 9.2 & 0.030391264 & 74 \\
\hline & 4 & 1.023 & 1.004 & 17.0 & 0.014170 & 1.019 & 10.2 & 0.022627675 & 61 \\
\hline & 8 & 1.020 & 1.004 & 17.0 & 0.014170 & 1.016 & 11.0 & 0.016615798 & 51 \\
\hline & 16 & 1.017 & 1.004 & 17.0 & 0.014170 & 1.013 & 11.8 & 0.012168887 & 42 \\
\hline & 32 & 1.013 & 1.004 & 17.0 & 0.014170 & 1.009 & 12.9 & 0.008996834 & 29 \\
\hline & 65 & 1.011 & 1.004 & 16.5 & 0.014260 & 1.007 & 13.4 & 0.006474634 & 22 \\
\hline & 135 & 1.009 & 1.004 & 16.5 & 0.014260 & 1.005 & 13.9 & 0.004575726 & 16 \\
\hline & 242 & 1.008 & 1.004 & 16.5 & 0.014260 & 1.004 & 14.2 & 0.003454268 & 13 \\
\hline & 1469 & 1.007 & 1.004 & 16.0 & 0.014260 & 1.003 & 14.4 & 0.001411854 & 10 \\
\hline
\end{tabular}

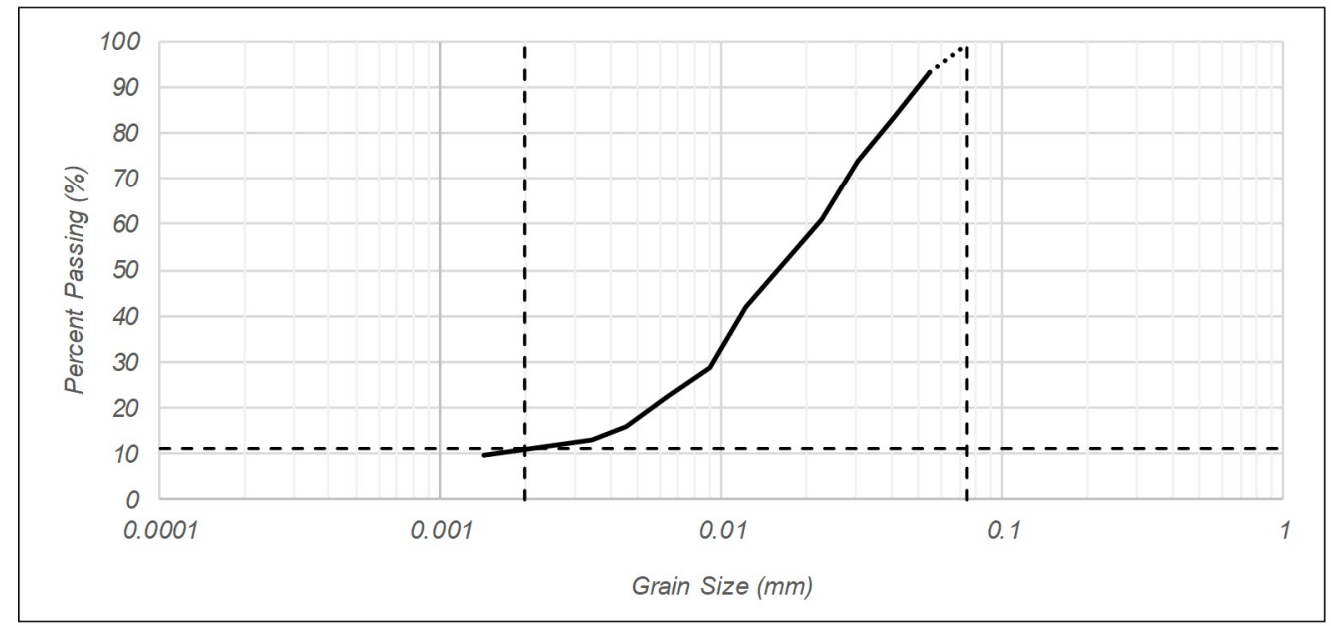

P200 Wash

$\begin{array}{lcc}\text { Container ID: } & \text { I1 } & \\ \text { Before }-\mathrm{M}_{\mathrm{d}} \text { : } & 30.6 & \mathrm{~g} \\ \text { After }-\mathrm{M}_{\mathrm{d}} \text { : } & 0.1 & \mathrm{~g} \\ \text { Fines Fraction: } & 99.67 & \% \\ \text { Coarse Fraction: } & 0.33 & \%\end{array}$

Procedures Done By: Kayla/Melissa B. 


\section{S Portland State}

Hydrometer Test

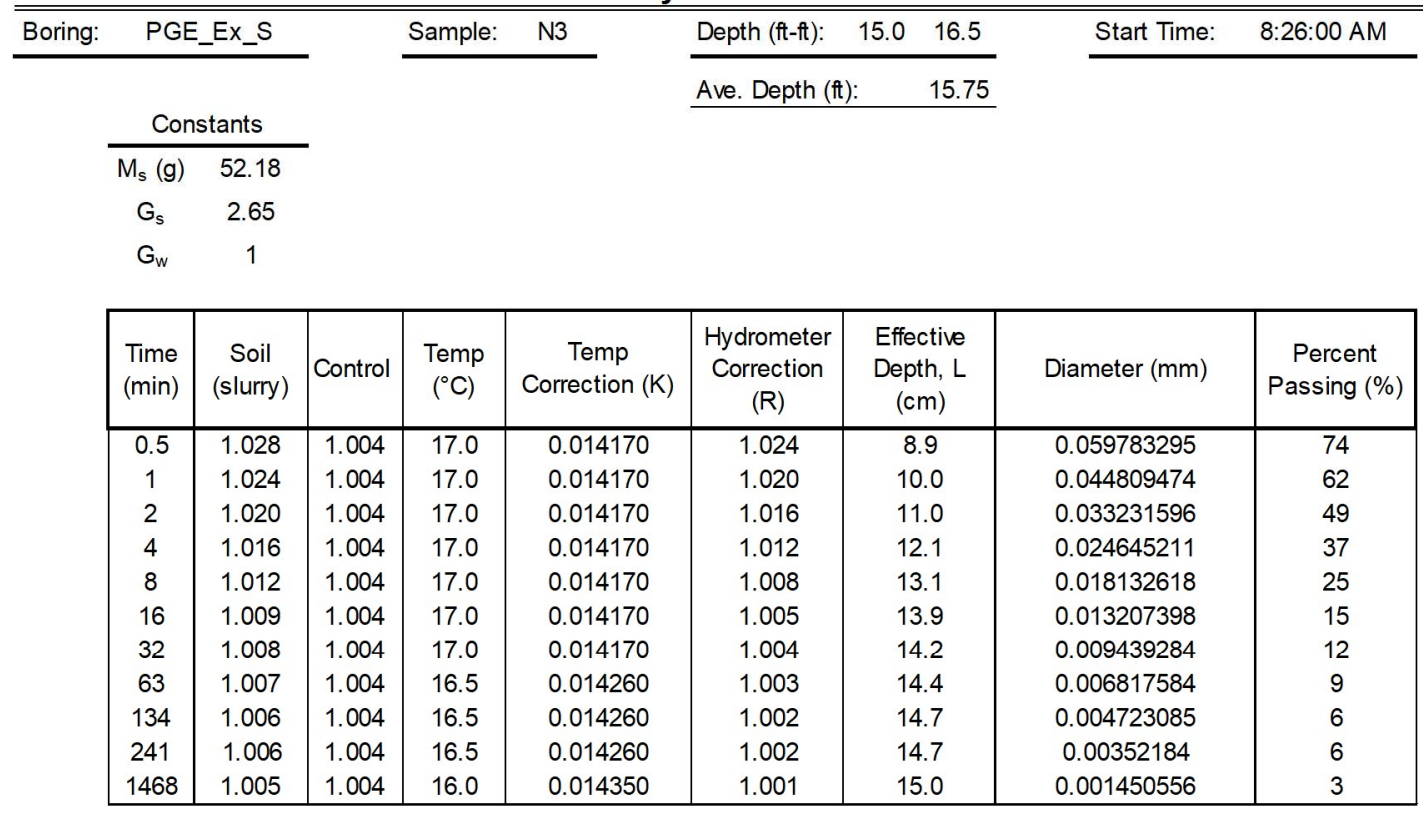

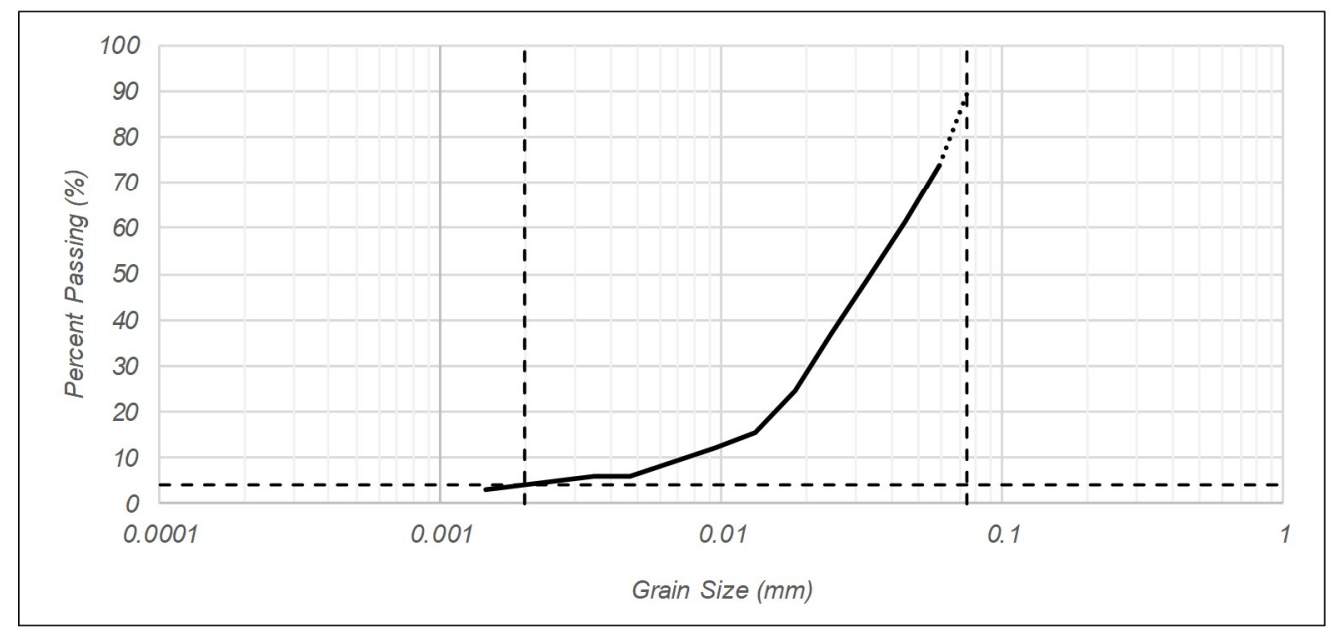

P200 Wash

$\begin{array}{lcc}\text { Container ID: } & \text { M1 } & \\ \text { Before }-\mathrm{M}_{\mathrm{d}} \text { : } & 17.9 & \mathrm{~g} \\ \text { After }-\mathrm{M}_{\mathrm{d}} \text { : } & 1.8 & \mathrm{~g} \\ \text { Fines Fraction: } & 89.94 & \% \\ \text { Coarse Fraction: } & 10.06 \%\end{array}$

Procedures Done By: Kayla/Melissa B. 


\section{蛋 Portland $\underset{\text { SNIVESITY }}{\text { State }}$}

Hydrometer Test

\begin{tabular}{|c|c|c|c|c|c|c|c|c|c|}
\hline Boring: & PGE & Ex_W & & Sample: & N1 & Depth (ft-ft): & $5.0 \quad 5.5$ & Start Time: & \\
\hline & & & & & & Ave. Depth (ft & 5.25 & & \\
\hline & Con & tants & & & & & 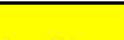 & & \\
\hline & $\overline{M_{s}(g)}$ & \#DIV/0! & & & $\begin{array}{r}\text { Ther } \\
\text { pe }\end{array}$ & $\begin{array}{l}\text { was not eno } \\
\text { fom a hydrom }\end{array}$ & $\begin{array}{l}\text { h soil to } \\
\text { er test. }\end{array}$ & & \\
\hline & $\mathrm{G}_{\mathrm{s}}$ & 2.65 & & & & & & & \\
\hline & $\mathrm{G}_{\mathrm{w}}$ & 1 & & & & & & & \\
\hline & $\begin{array}{l}\text { Time } \\
(\min )\end{array}$ & $\begin{array}{c}\text { Soil } \\
\text { (slurry) }\end{array}$ & Control & $\begin{array}{l}\text { Temp } \\
\left({ }^{\circ} \mathrm{C}\right)\end{array}$ & $\begin{array}{c}\text { Temp } \\
\text { Correction }(K)\end{array}$ & $\begin{array}{l}\text { Hydrometer } \\
\text { Correction } \\
\text { (R) }\end{array}$ & $\begin{array}{l}\text { Effective } \\
\text { Depth, L } \\
\text { (cm) }\end{array}$ & Diameter $(\mathrm{mm})$ & $\begin{array}{c}\text { Percent } \\
\text { Passing (\%) }\end{array}$ \\
\hline & & & & & & & & & \\
\hline & & & & & & & & & \\
\hline & & & & & & & & & \\
\hline & & & & & & & & & \\
\hline & & & & & & & & & \\
\hline & & & & & & & & & \\
\hline
\end{tabular}

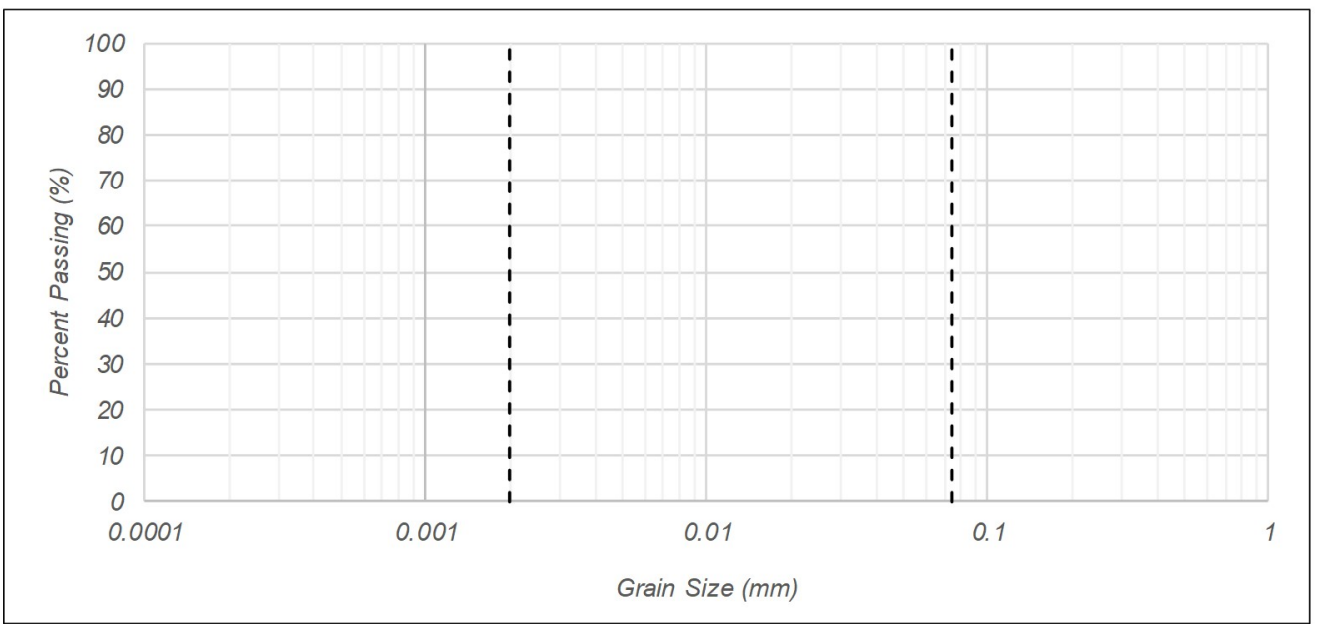

P200 Wash

$\begin{array}{lcc}\text { Container ID: } & \text { U1 } & \\ \text { Before - } \mathrm{M}_{\mathrm{d}}: & 19.3 \mathrm{~g} \\ \text { After }-\mathrm{M}_{\mathrm{d}}: & 1.1 \mathrm{~g} \\ \text { Fines Fraction: } & 94.30 \% \\ \text { Coarse Fraction: } & 5.70 \%\end{array}$

Procedures Done By:

Melissa P 


\section{胥 Portland $\operatorname{State}_{U \text { NIV }}$}

Hydrometer Test

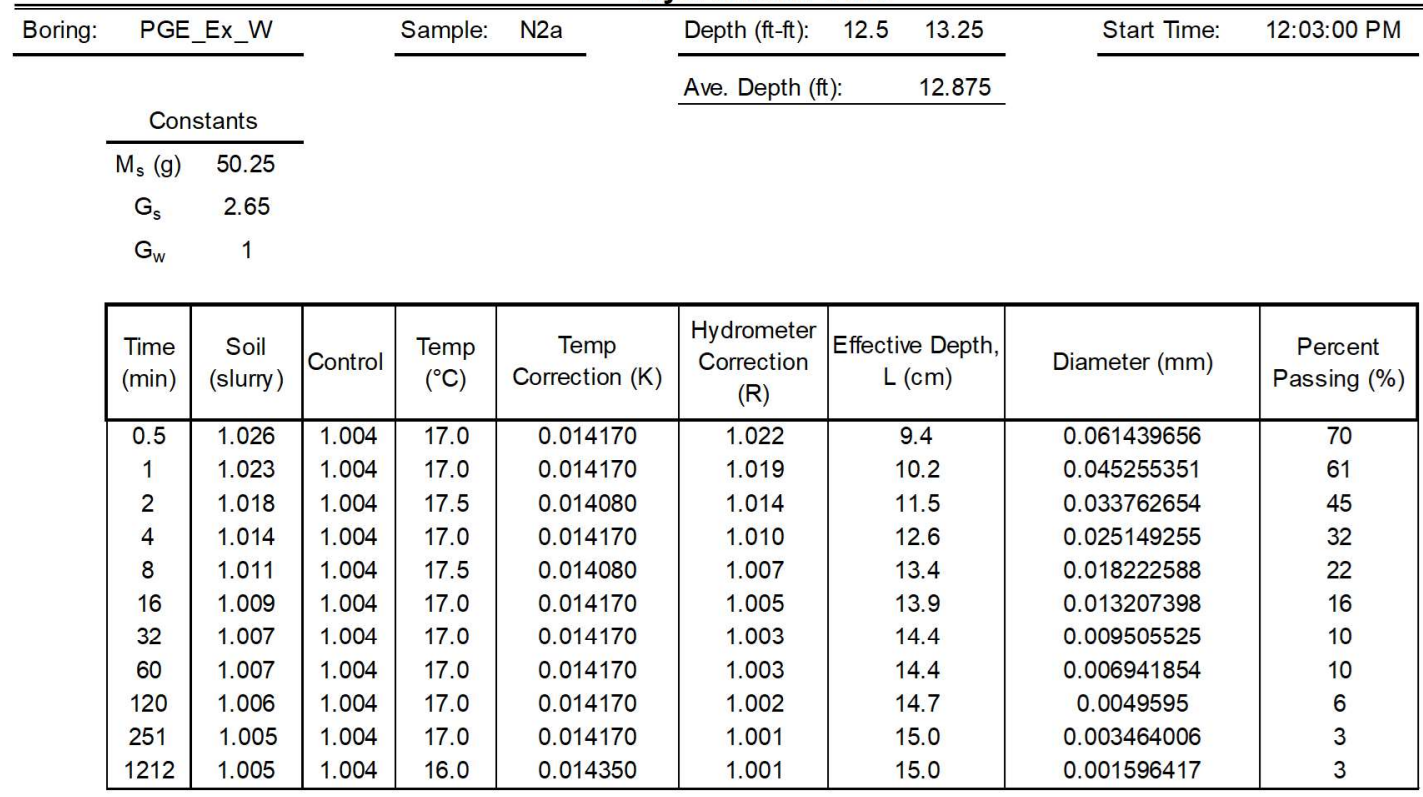

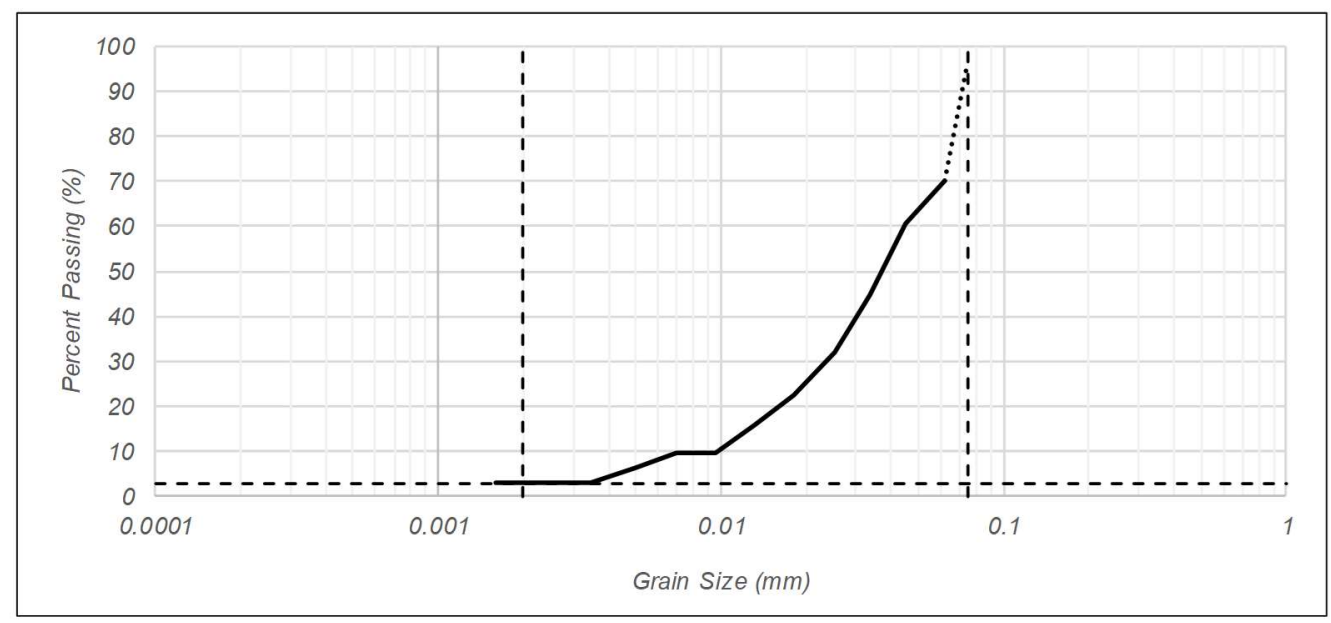

P200 Wash

Container ID: $\quad \mathrm{S} 1$

Before - $M_{d}$ : $\quad 23.7 \mathrm{~g}$

$\begin{array}{lcc}\text { After }-\mathrm{M}_{\mathrm{d}}: & 1 & \mathrm{~g} \\ \text { Fines Fraction: } & 95.78 \%\end{array}$

Coarse Fraction: $4.22 \%$

Procedures Done By:
Soil Breakdown

\begin{tabular}{lr}
\hline Coarse: & $4.22 \%$ \\
Silts: & $92.78 \%$ \\
Clays: & $3.00 \%$
\end{tabular}




\section{胥 Portland State}

Hydrometer Test

\begin{tabular}{|c|c|c|c|c|c|c|c|c|c|}
\hline \multirow[t]{2}{*}{ Boring: } & \multicolumn{2}{|c|}{ PGE_Ex_W } & & \multicolumn{2}{|c|}{ Sample: $\quad \mathrm{N} 2 \mathrm{~b}$} & \multirow{2}{*}{\multicolumn{2}{|c|}{$\begin{array}{lll}\text { Depth (ft-ft): } & 13.25 & 14.0 \\
\text { Ave. Depth (ft): } & 13.63 \\
\end{array}$}} & \multirow[t]{3}{*}{ Start Time: } & \multirow[t]{2}{*}{ 11:30:00 AM } \\
\hline & & & & & & & & & \\
\hline & \multicolumn{2}{|c|}{ Constants } & & & & & & & \\
\hline & $M_{s}(g)$ & 50.13 & & & & & & & \\
\hline & $\mathrm{G}_{\mathrm{s}}$ & 2.65 & & & & & & & \\
\hline & $\mathrm{G}_{\mathrm{w}}$ & 1 & & & & & & & \\
\hline & $\begin{array}{r}\text { Time } \\
(\mathrm{min})\end{array}$ & $\begin{array}{l}\text { Soil } \\
\text { (slurry) }\end{array}$ & Control & $\begin{array}{l}\text { Temp } \\
\left({ }^{\circ} \mathrm{C}\right)\end{array}$ & $\begin{array}{c}\text { Temp } \\
\text { Correction }(\mathrm{K})\end{array}$ & \begin{tabular}{|c|} 
Hydrometer \\
Correction \\
$(R)$
\end{tabular} & $\begin{array}{c}\text { Effective } \\
\text { Depth, L (cm) }\end{array}$ & Diameter (mm) & $\begin{array}{c}\text { Percent } \\
\text { Passing (\%) }\end{array}$ \\
\hline & $\overline{0.5}$ & 1.031 & 1.004 & 19.0 & 0.013820 & 1.027 & $\overline{8.1}$ & 0.055624427 & 87 \\
\hline & 1 & 1.030 & 1.004 & 19.0 & 0.013820 & 1.026 & 8.4 & 0.040054165 & 83 \\
\hline & 2 & 1.028 & 1.004 & 19.0 & 0.013820 & 1.024 & 8.9 & 0.029153322 & 77 \\
\hline & 4 & 1.024 & 1.004 & 19.0 & 0.013820 & 1.020 & 10.0 & 0.021851339 & 64 \\
\hline & 8 & 1.019 & 1.004 & 19.0 & 0.013820 & 1.015 & 11.3 & 0.016424882 & 48 \\
\hline & 16 & 1.014 & 1.004 & 19.0 & 0.013820 & 1.010 & 12.6 & 0.012264033 & 32 \\
\hline & 32 & 1.011 & 1.004 & 19.0 & 0.013820 & 1.007 & 13.4 & 0.008943046 & 22 \\
\hline & 60 & 1.010 & 1.004 & 18.5 & 0.013905 & 1.006 & 13.7 & 0.006644398 & 19 \\
\hline & 113 & 1.008 & 1.004 & 18.5 & 0.013905 & 1.004 & 14.2 & 0.004929194 & 13 \\
\hline & 157 & 1.007 & 1.004 & 19.0 & 0.013820 & 1.003 & 14.4 & 0.004185424 & 10 \\
\hline & 730 & 1.006 & 1.004 & 16.5 & 0.014260 & 1.002 & 14.7 & 0.002023563 & 6 \\
\hline
\end{tabular}

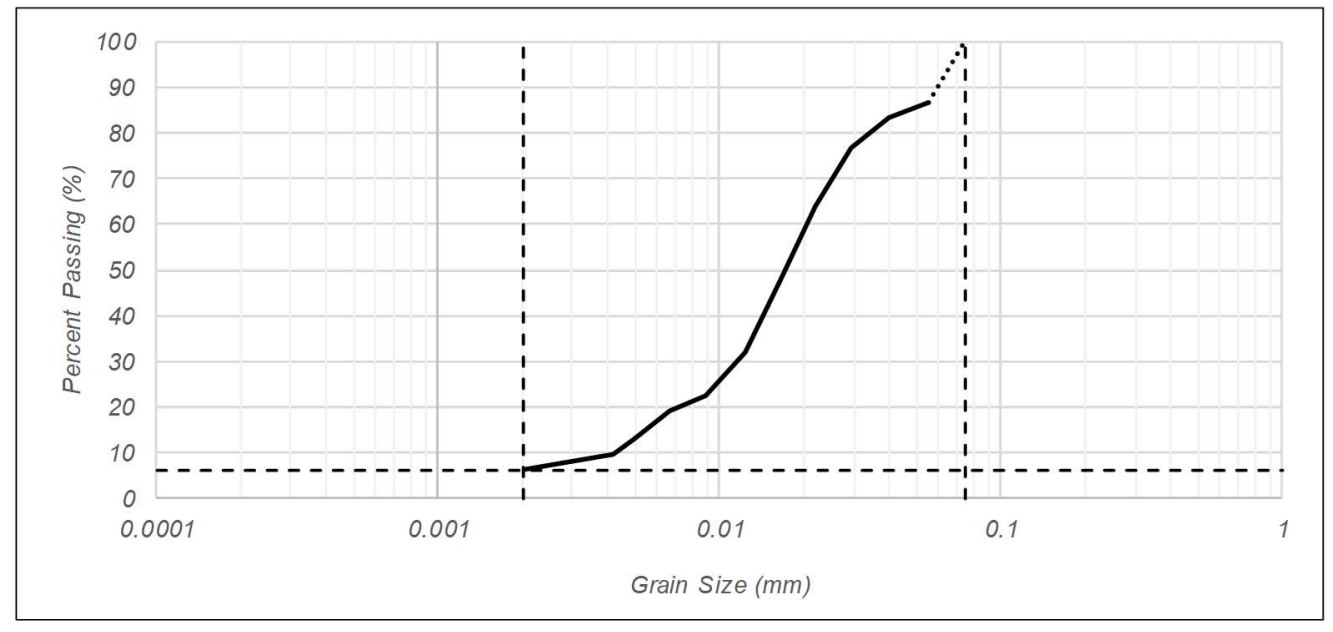

P200 Wash

$\begin{array}{lcc}\text { Container ID: } & \mathrm{J} 1 & \\ \text { Before }-\mathrm{M}_{\mathrm{d}}: & 13.6 & \mathrm{~g} \\ \text { After }-\mathrm{M}_{\mathrm{d}} \text { : } & 0.0 & \mathrm{~g} \\ \text { Fines Fraction: } & 100.00 \% \\ \text { Coarse Fraction: } & 0.00 \%\end{array}$

\begin{tabular}{llr} 
& & Soil Breakdown \\
\cline { 2 - 3 } & Coarse: & $0.00 \%$ \\
& Silts: & $94.00 \%$ \\
From Hydrometer & Clays: & $6.00 \%$
\end{tabular}

Procedures Done By: Melissa B. 


\section{胥 Portland State}

Hydrometer Test

\begin{tabular}{|c|c|c|c|c|c|c|c|c|c|}
\hline \multirow[t]{2}{*}{ Boring: } & \multicolumn{2}{|c|}{ PGE_Ex_W } & & \multicolumn{2}{|c|}{ Sample: N3a } & \multirow{2}{*}{\multicolumn{2}{|c|}{$\begin{array}{lll}\text { Depth (ft-ft): } & 15.00 & 15.8 \\
\text { Ave. Depth (ft): } & 15.38 \\
\end{array}$}} & \multirow[t]{3}{*}{ Start Time: } & \multirow[t]{2}{*}{ 4:53:00 AM } \\
\hline & & & & & & & & & \\
\hline & \multicolumn{2}{|c|}{ Constants } & & & & & & & \\
\hline & $M_{s}(g)$ & 49.87 & & & & & & & \\
\hline & $\mathrm{G}_{\mathrm{s}}$ & 2.65 & & & & & & & \\
\hline & $\mathrm{G}_{\mathrm{w}}$ & 1 & & & & & & & \\
\hline & $\begin{array}{r}\text { Time } \\
(\mathrm{min})\end{array}$ & $\begin{array}{l}\text { Soil } \\
\text { (slurry) }\end{array}$ & Control & $\begin{array}{c}\text { Temp } \\
\left({ }^{\circ} \mathrm{C}\right)\end{array}$ & $\begin{array}{c}\text { Temp } \\
\text { Correction }(\mathrm{K})\end{array}$ & \begin{tabular}{|c|} 
Hydrometer \\
Correction \\
$(R)$
\end{tabular} & $\begin{array}{c}\text { Effective } \\
\text { Depth, L (cm) }\end{array}$ & Diameter (mm) & $\begin{array}{c}\text { Percent } \\
\text { Passing (\%) }\end{array}$ \\
\hline & $\overline{0.5}$ & 1.016 & 1.004 & $\overline{19.5}$ & 0.013735 & 1.012 & $\overline{12.1}$ & 0.067567266 & 39 \\
\hline & 1 & 1.013 & 1.004 & 19.5 & 0.013735 & 1.009 & 12.9 & 0.049331409 & 29 \\
\hline & 2 & 1.012 & 1.004 & 19.5 & 0.013735 & 1.008 & 13.1 & 0.035151941 & 26 \\
\hline & 4 & 1.011 & 1.004 & 19.5 & 0.013735 & 1.007 & 13.4 & 0.025139178 & 23 \\
\hline & 8 & 1.010 & 1.004 & 19.0 & 0.013820 & 1.006 & 13.7 & 0.018085201 & 19 \\
\hline & 16 & 1.008 & 1.004 & 19.5 & 0.013735 & 1.004 & 14.2 & 0.012939361 & 13 \\
\hline & 32 & 1.007 & 1.004 & 19.0 & 0.013820 & 1.003 & 14.4 & 0.009270738 & 10 \\
\hline & 62 & 1.006 & 1.004 & 19.0 & 0.013820 & 1.002 & 14.7 & 0.006729315 & 6 \\
\hline & 313 & 1.006 & 1.004 & 19.0 & 0.013820 & 1.002 & 14.7 & 0.002994984 & 6 \\
\hline & 278 & 1.006 & 1.004 & 19.0 & 0.013820 & 1.002 & 14.7 & 0.00317793 & 6 \\
\hline & 1101 & 1.006 & 1.004 & 18.0 & 0.013990 & 1.002 & 14.7 & 0.001616526 & 6 \\
\hline
\end{tabular}

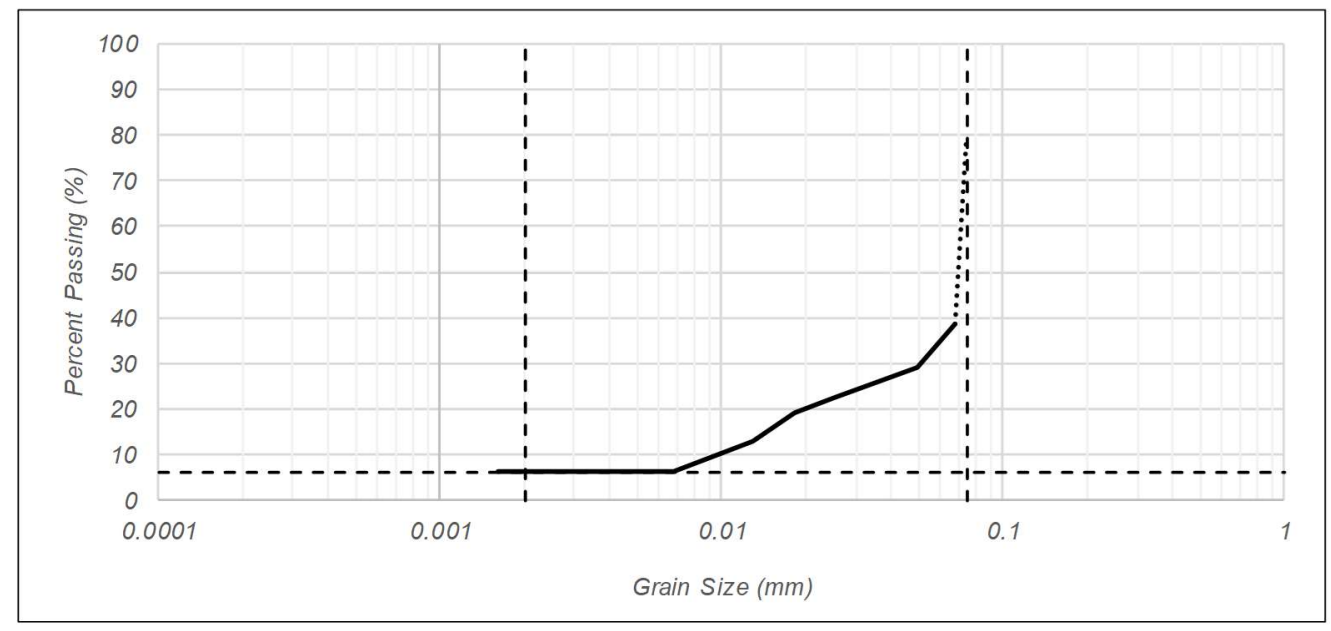

P200 Wash

$\begin{array}{lcc}\text { Container ID: } & \text { W1 } & \\ \text { Before }-\mathrm{M}_{\mathrm{d}} \text { : } & 25.9 & \mathrm{~g} \\ \text { After }-\mathrm{M}_{\mathrm{d}} \text { : } & 5.4 & \mathrm{~g} \\ \text { Fines Fraction: } & 79.15 & \% \\ \text { Coarse Fraction: } & 20.85 & \%\end{array}$

Procedures Done By: Melissa B. 


\section{胥 Portland State}

Hydrometer Test

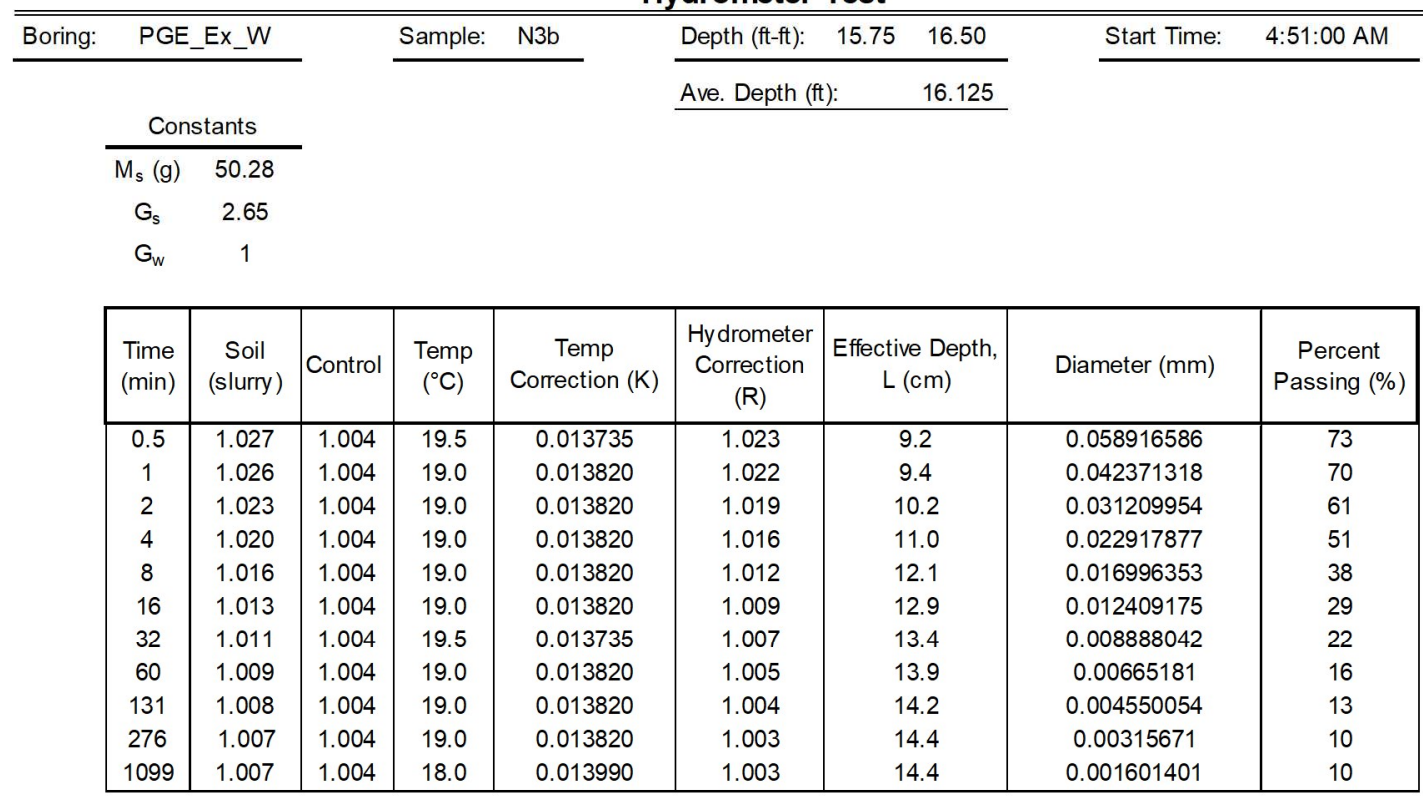

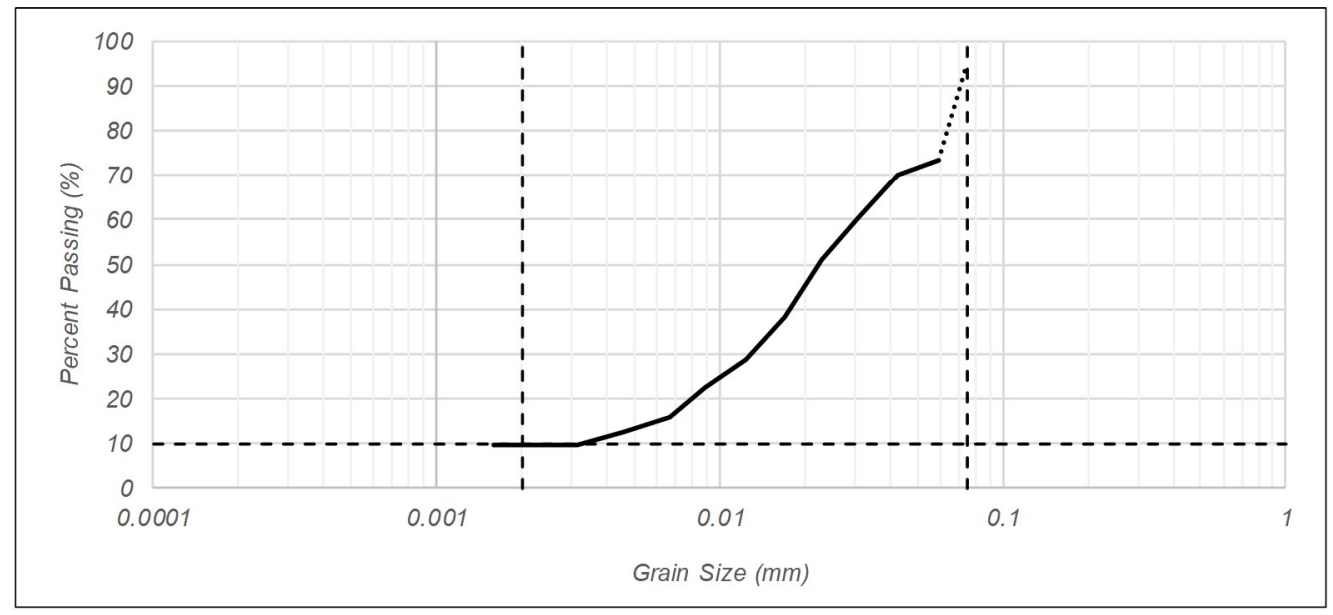

P200 Wash

Container ID: $\quad$ R1

Before - $\mathrm{M}_{\mathrm{d}}$ : $\quad 10.7 \mathrm{~g}$

After - $\mathrm{M}_{\mathrm{d}}: \quad 0.6 \mathrm{~g}$

Fines Fraction: $\quad 94.39 \%$

Coarse Fraction: $5.61 \%$

Procedures Done By:

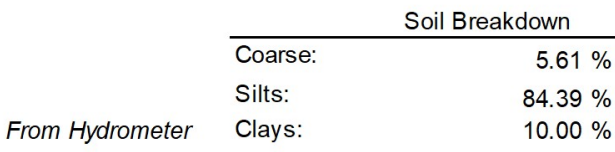

Melissa B. 


\section{S Portland State}

\begin{tabular}{|c|c|c|c|c|c|c|c|c|c|}
\hline & \multicolumn{9}{|c|}{ Hydrometer Test } \\
\hline \multirow[t]{2}{*}{ Boring: } & \multicolumn{2}{|c|}{ PGE_Inj } & & \multirow[t]{2}{*}{ Sample: } & \multirow[t]{2}{*}{$\mathrm{N} 1 \mathrm{a}$} & \multirow{2}{*}{\multicolumn{2}{|c|}{ 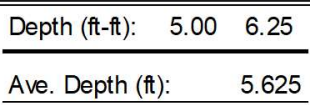 }} & \multirow[t]{2}{*}{ Start Time: } & \multirow[t]{2}{*}{ 10:49:00 AM } \\
\hline & & & & & & & & & \\
\hline \multicolumn{10}{|c|}{ Constants } \\
\hline & $M_{s}(g)$ & 52.24 & & & & & & & \\
\hline & $\mathrm{G}_{\mathrm{s}}$ & 2.65 & & & & & & & \\
\hline & $\mathrm{G}_{w}$ & 1 & & & & & & & \\
\hline & $\begin{array}{l}\text { Time } \\
(\min )\end{array}$ & $\begin{array}{c}\text { Soil } \\
\text { (slurry) }\end{array}$ & Control & $\begin{array}{c}\text { Temp } \\
\left({ }^{\circ} \mathrm{C}\right)\end{array}$ & $\begin{array}{c}\text { Temp } \\
\text { Correction }(K)\end{array}$ & $\begin{array}{l}\text { Hydrometer } \\
\text { Correction } \\
(R)\end{array}$ & $\begin{array}{l}\text { Effective } \\
\text { Depth, L } \\
\text { (cm) }\end{array}$ & Diameter (mm) & $\begin{array}{c}\text { Percent } \\
\text { Passing (\%) }\end{array}$ \\
\hline & 0.5 & 1.021 & 1.003 & 17.0 & 0.014170 & 1.018 & 10.7 & 0.06555061 & 55 \\
\hline & 1 & 1.017 & 1.003 & 17.0 & 0.014170 & 1.014 & 11.8 & 0.048675548 & 43 \\
\hline & 2 & 1.013 & 1.003 & 17.0 & 0.014170 & 1.010 & 12.9 & 0.035987337 & 31 \\
\hline & 4 & 1.009 & 1.003 & 17.0 & 0.014170 & 1.006 & 13.9 & 0.026414796 & 18 \\
\hline & 8 & 1.007 & 1.003 & 17.0 & 0.014170 & 1.004 & 14.4 & 0.01901105 & 12 \\
\hline & 16 & 1.006 & 1.003 & 17.0 & 0.014170 & 1.003 & 14.7 & 0.01358215 & 9 \\
\hline & 36 & 1.005 & 1.003 & 17.0 & 0.014170 & 1.002 & 15.0 & 0.009146696 & 6 \\
\hline & 68 & 1.004 & 1.003 & 17.0 & 0.014170 & 1.001 & 15.2 & 0.00669942 & 3 \\
\hline & 127 & 1.004 & 1.003 & 17.0 & 0.014170 & 1.001 & 15.2 & 0.004902186 & 3 \\
\hline & 236 & 1.004 & 1.003 & 17.0 & 0.014170 & 1.001 & 15.2 & 0.003596132 & 3 \\
\hline & 1449 & 1.004 & 1.003 & 16.0 & 0.014350 & 1.001 & 15.2 & 0.001469737 & 3 \\
\hline
\end{tabular}

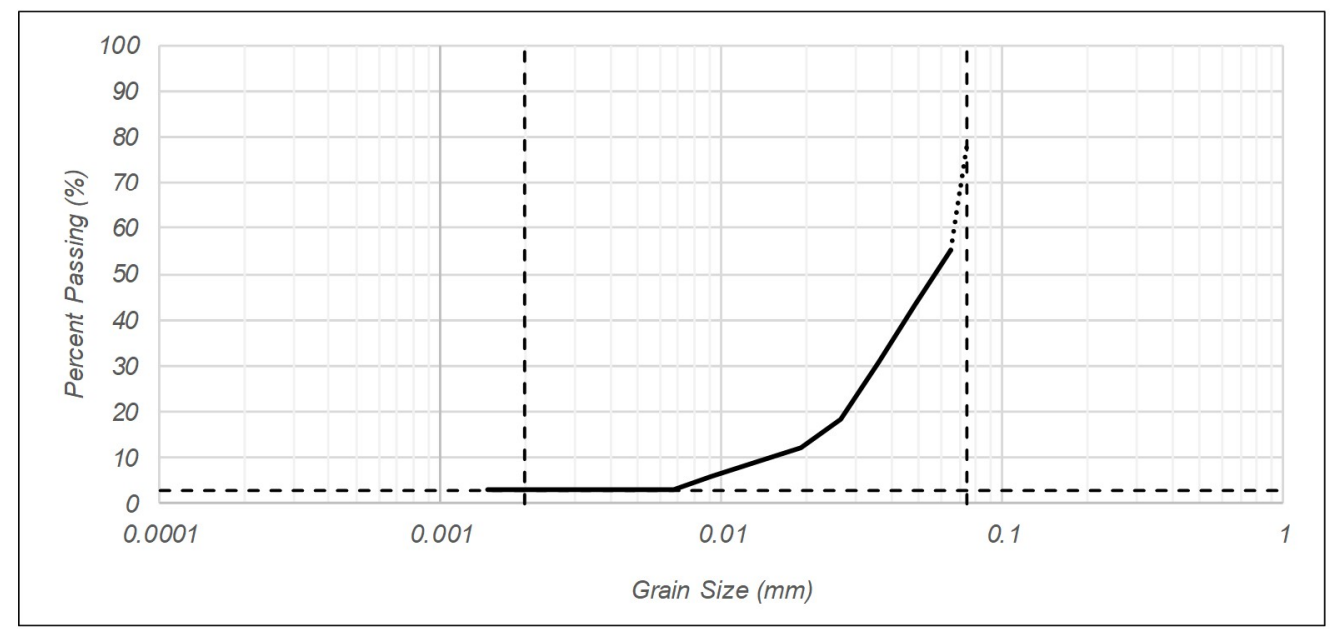

P200 Wash

$\begin{array}{lcc}\text { Container ID: } & \text { Z1 } & \\ \text { Before }-\mathrm{M}_{\mathrm{d}}: & 55.5 & \mathrm{~g} \\ \text { After }-\mathrm{M}_{\mathrm{d}} \text { : } & 11.3 & \mathrm{~g} \\ \text { Fines Fraction: } & 79.64 & \% \\ \text { Coarse Fraction: } & 20.36 & \%\end{array}$

Procedures Done By: Kayla/Melissa P. 
胥 Portland $\underset{\text { UNIVES StITY }}{\text { State }}$

Hydrometer Test

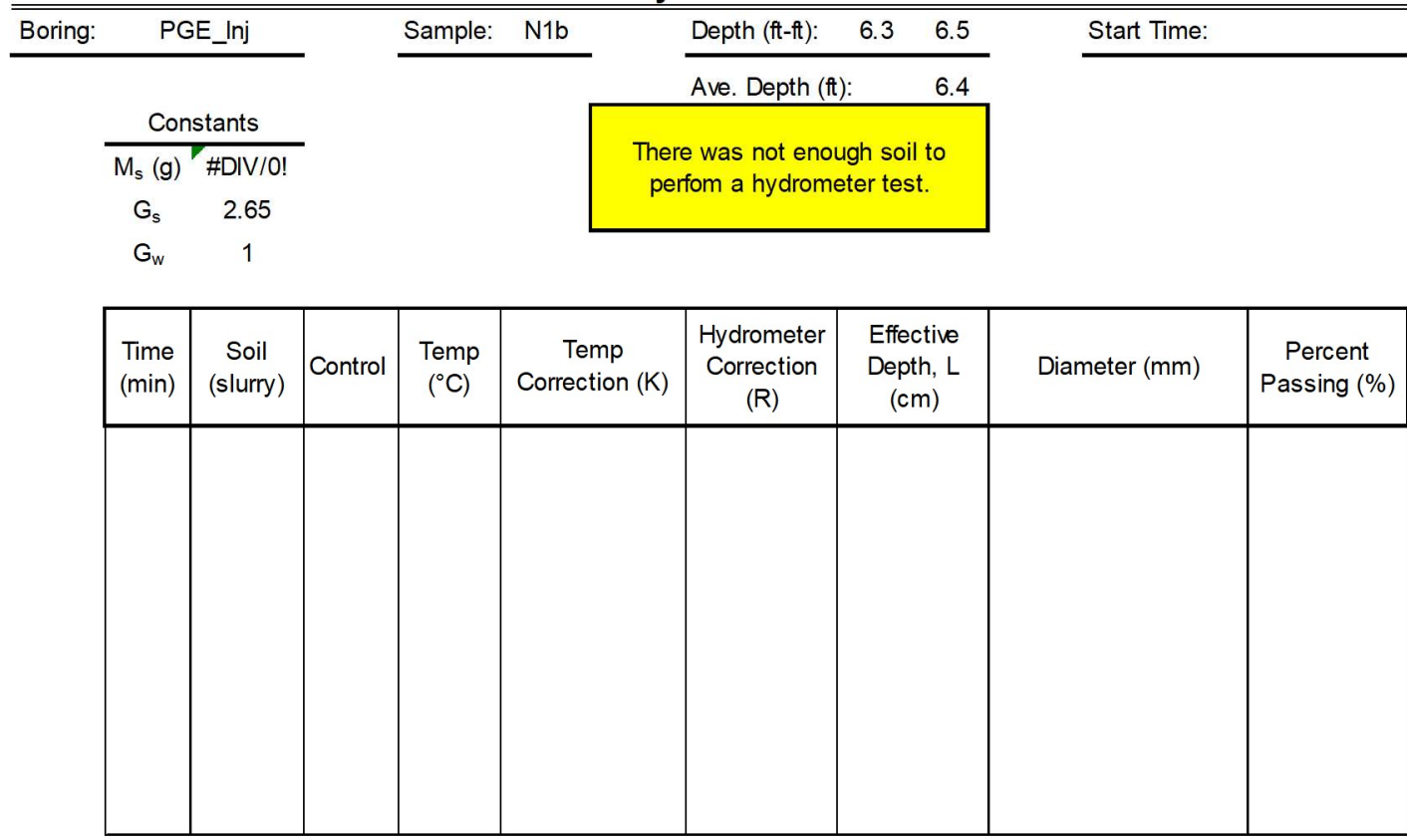

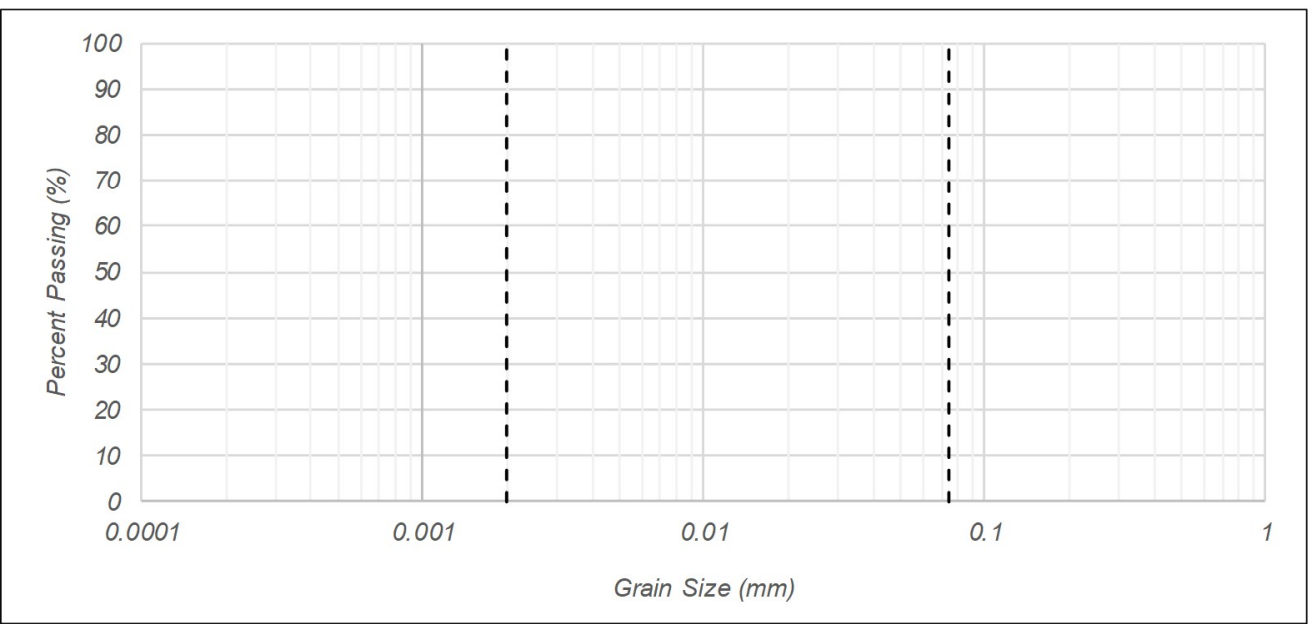

P200 Wash

$\begin{array}{lcc}\text { Container ID: } & \text { X1 } & \\ \text { Before }-\mathrm{M}_{\mathrm{d}}: & 30.7 \mathrm{~g} \\ \text { After }-\mathrm{M}_{\mathrm{d}} \text { : } & 1.1 \mathrm{~g} \\ \text { Fines Fraction: } & 96.42 & \% \\ \text { Coarse Fraction: } & 3.58 & \%\end{array}$

Procedures Done By:

Melissa P 


\section{S马 Portland State}

Hydrometer Test

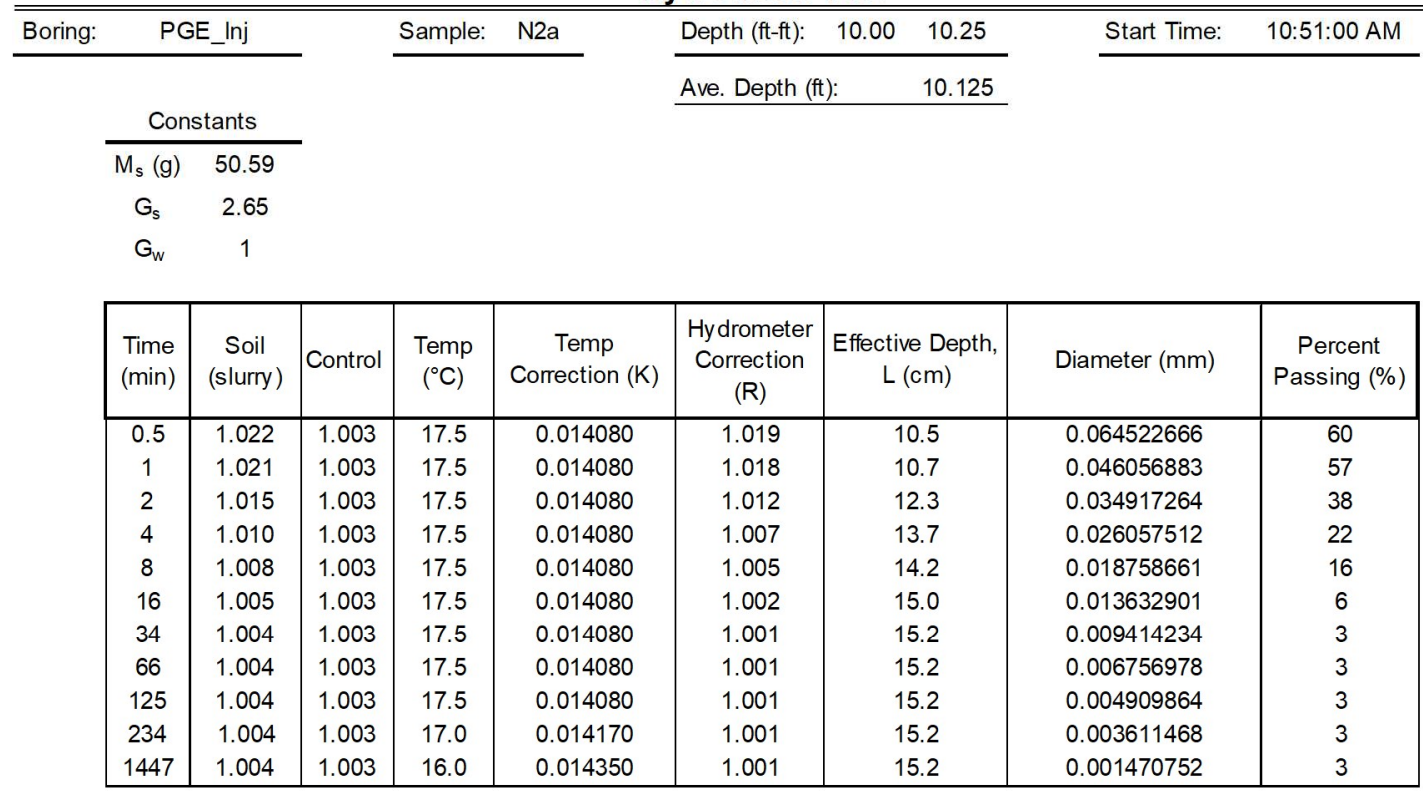

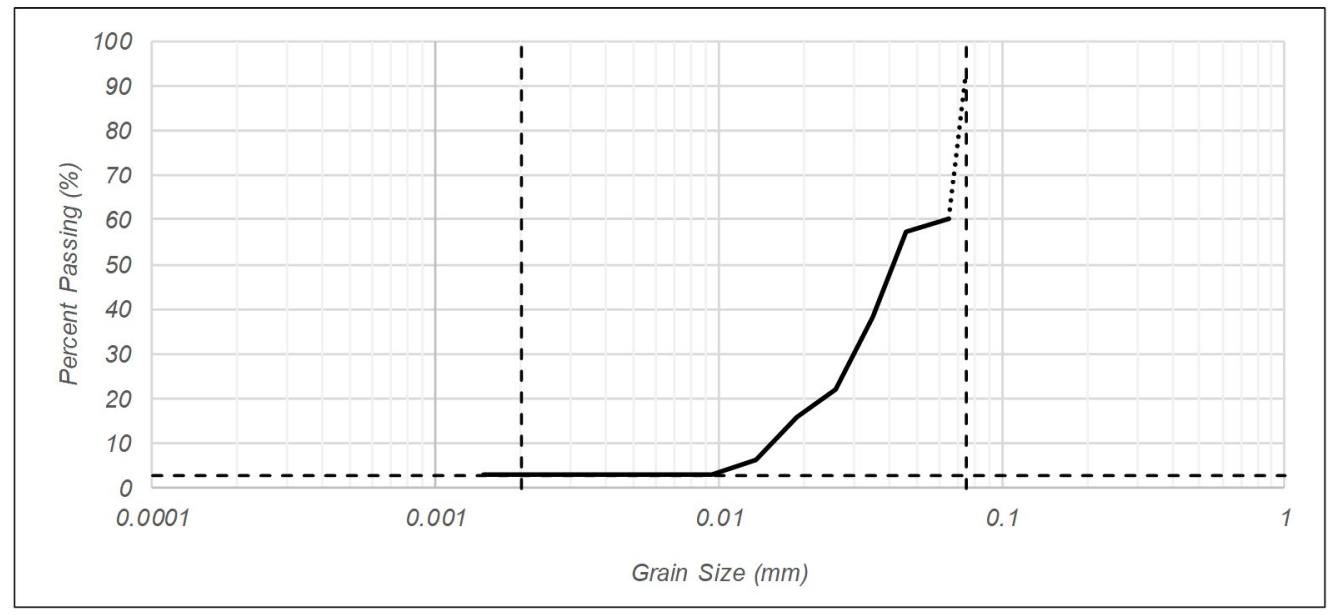

P200 Wash

Container ID: $\quad$ D1

Before - $\mathrm{M}_{\mathrm{d}}$ : $\quad 27.6 \mathrm{~g}$

After - $M_{d}: \quad 2.2 \mathrm{~g}$

Fines Fraction: $\quad 92.03 \%$

Coarse Fraction: $\quad 7.97 \%$

Procedures Done By:

Kayla/Melissa P. 


\section{胥 Portland State}

Hydrometer Test

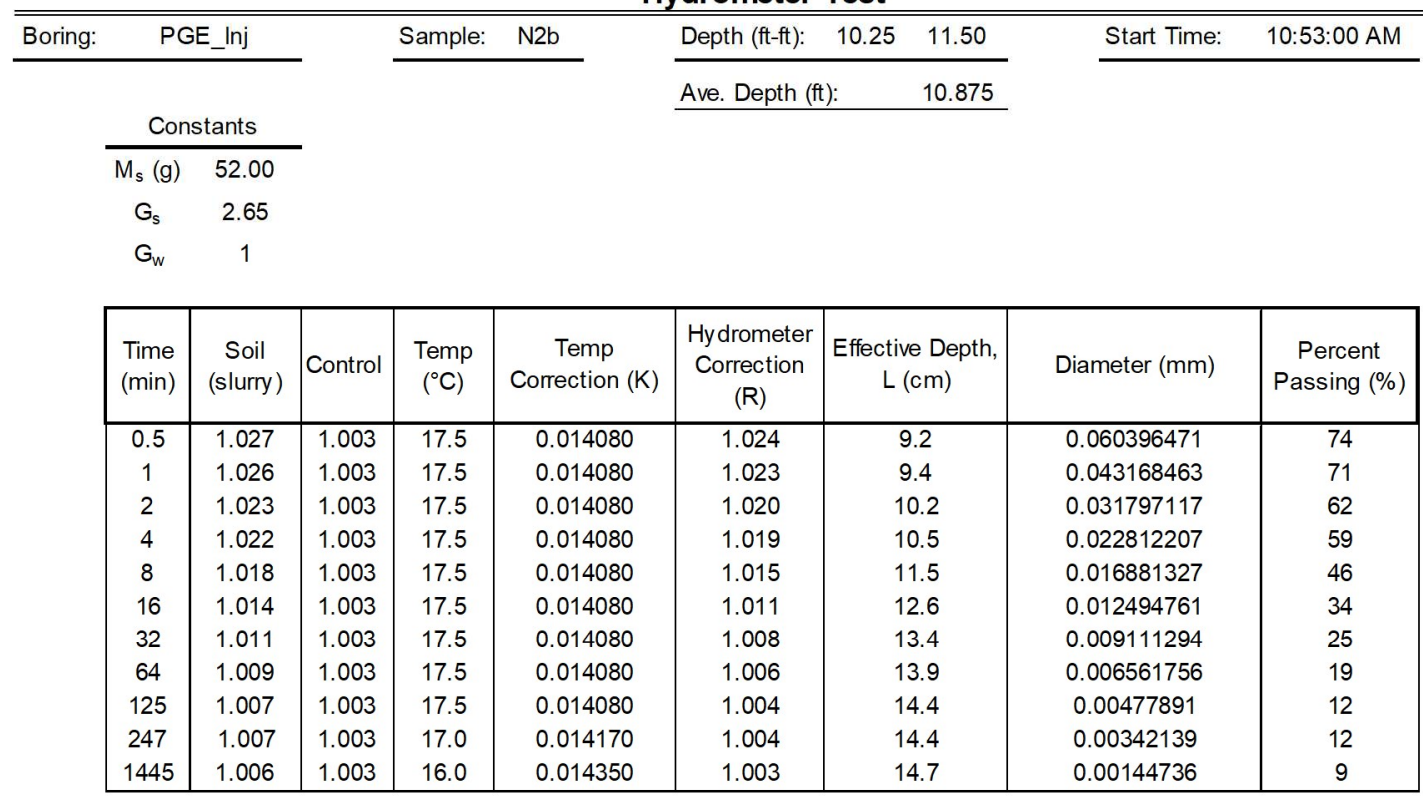

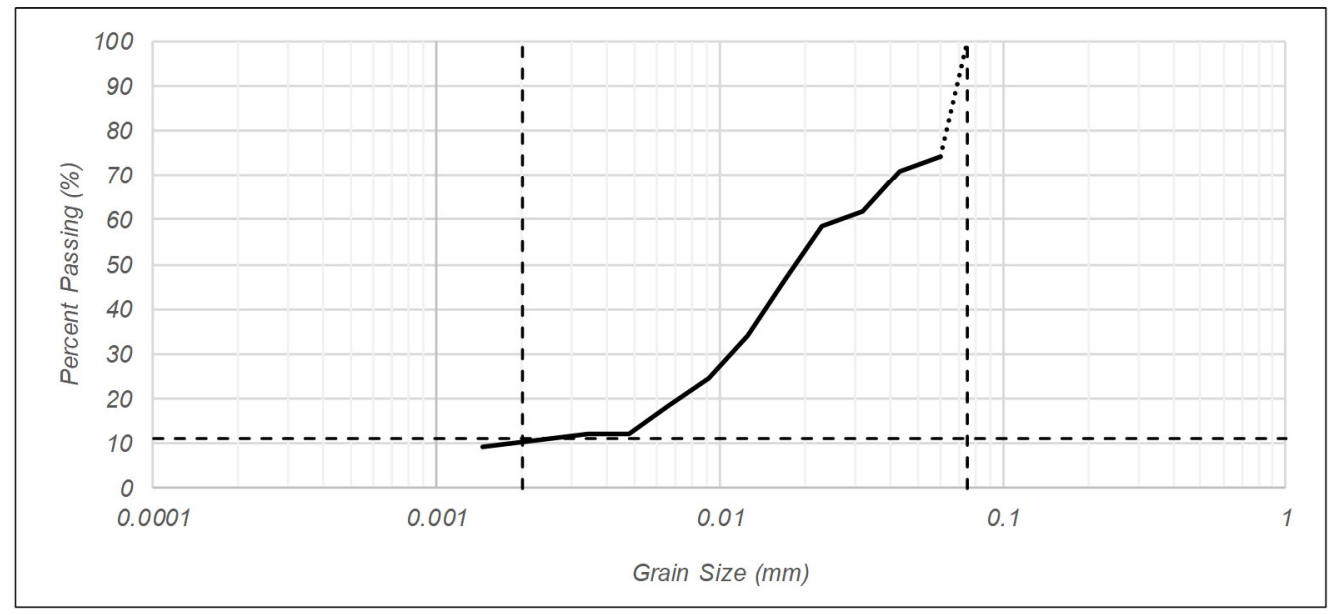

P200 Wash

$\begin{array}{lcc}\text { Container ID: } & \mathrm{A} 1 & \\ \text { Before }-\mathrm{M}_{\mathrm{d}} \text { : } & 37.6 & \mathrm{~g} \\ \text { After }-\mathrm{M}_{\mathrm{d}} \text { : } & 0.3 & \mathrm{~g} \\ \text { Fines Fraction: } & 99.20 & \% \\ \text { Coarse Fraction: } & 0.80 & \%\end{array}$

\begin{tabular}{llr} 
& & \multicolumn{2}{c}{ Soil Breakdown } \\
\cline { 2 - 3 } & Coarse: & $0.80 \%$ \\
& Silts: & $88.20 \%$ \\
From Hydrometer & Clays: & $11.00 \%$
\end{tabular}

Procedures Done By:

Kayla/Melissa $\mathrm{P}$. 
胥 Portland Stativensiry

Hydrometer Test

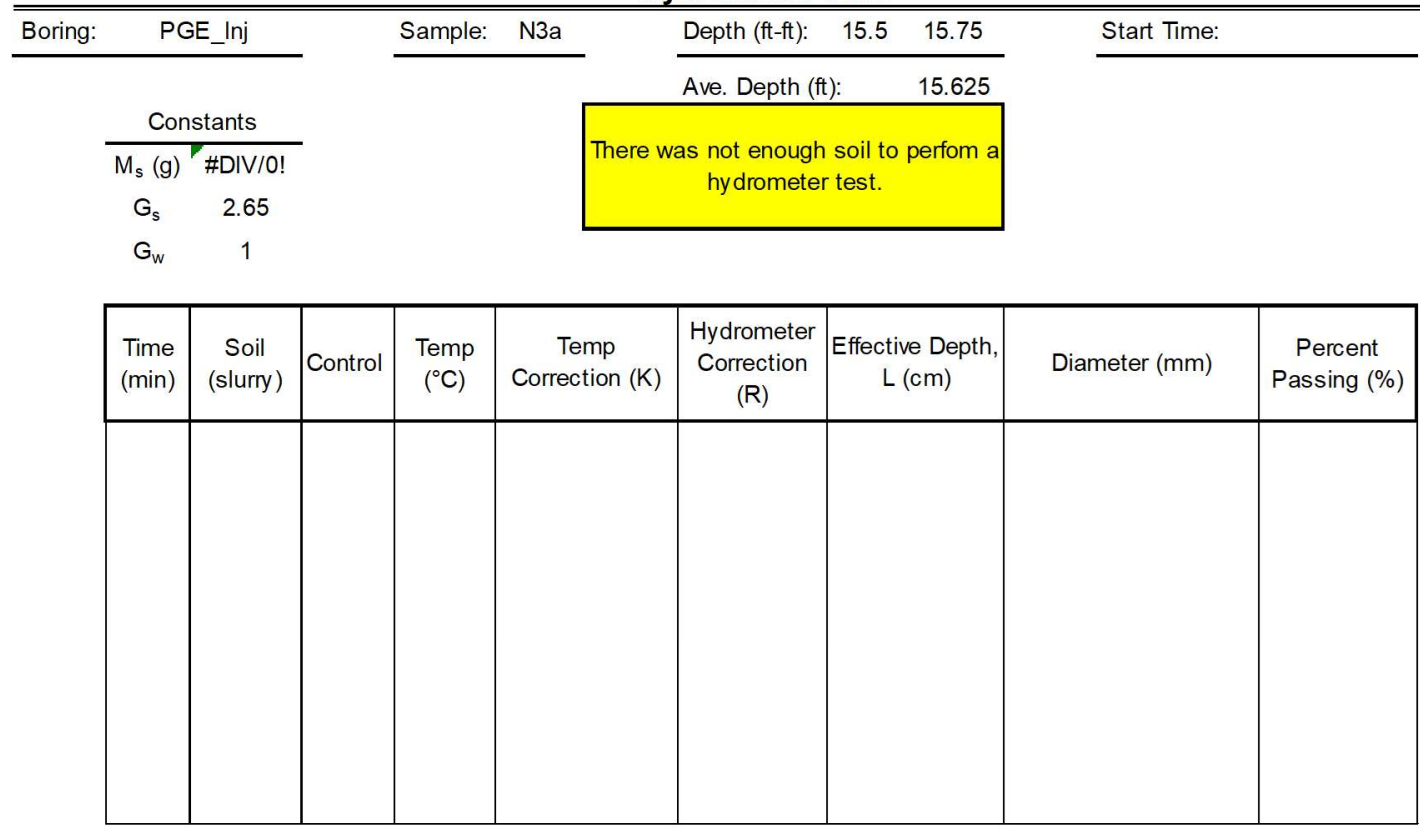

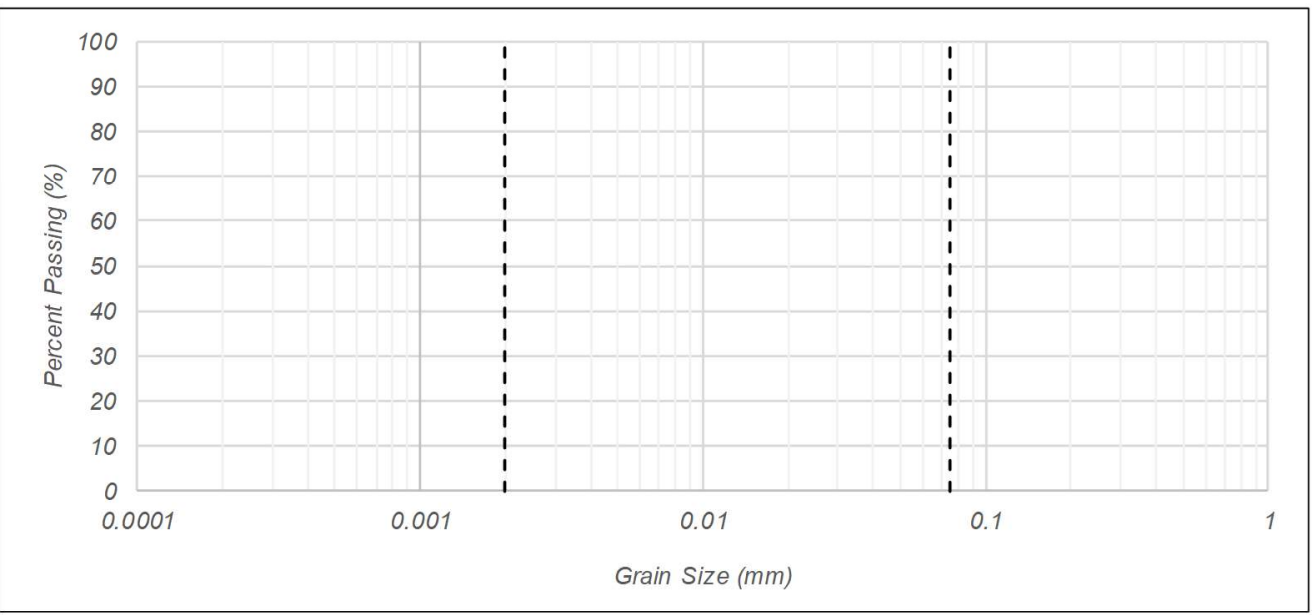

P200 Wash

Container ID

Before $-M_{d}$ :

Fines Fraction: $\quad 21.22 \%$

Coarse Fraction: $78.78 \%$

Procedures Done By:

Melissa B. 


\section{胥 Portland State}

Hydrometer Test

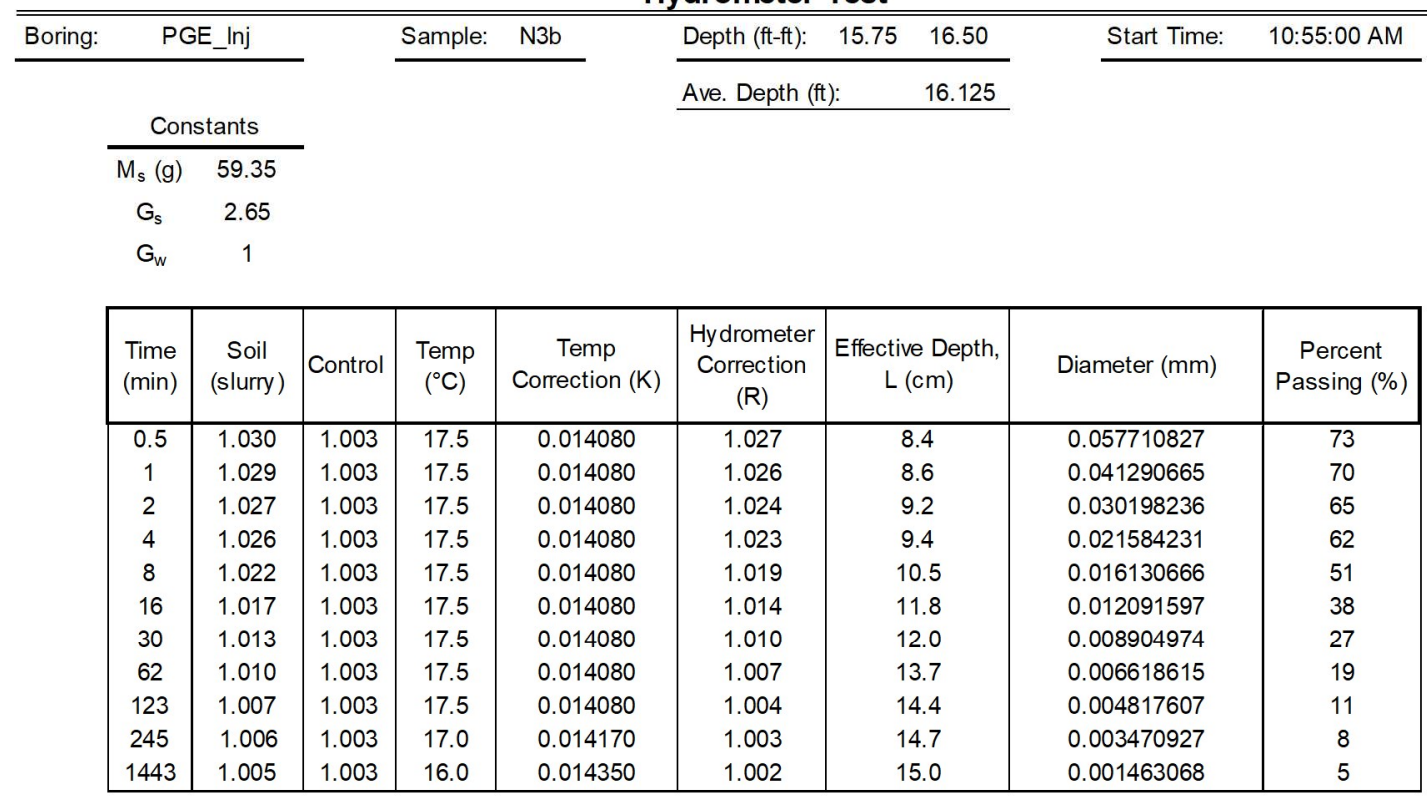

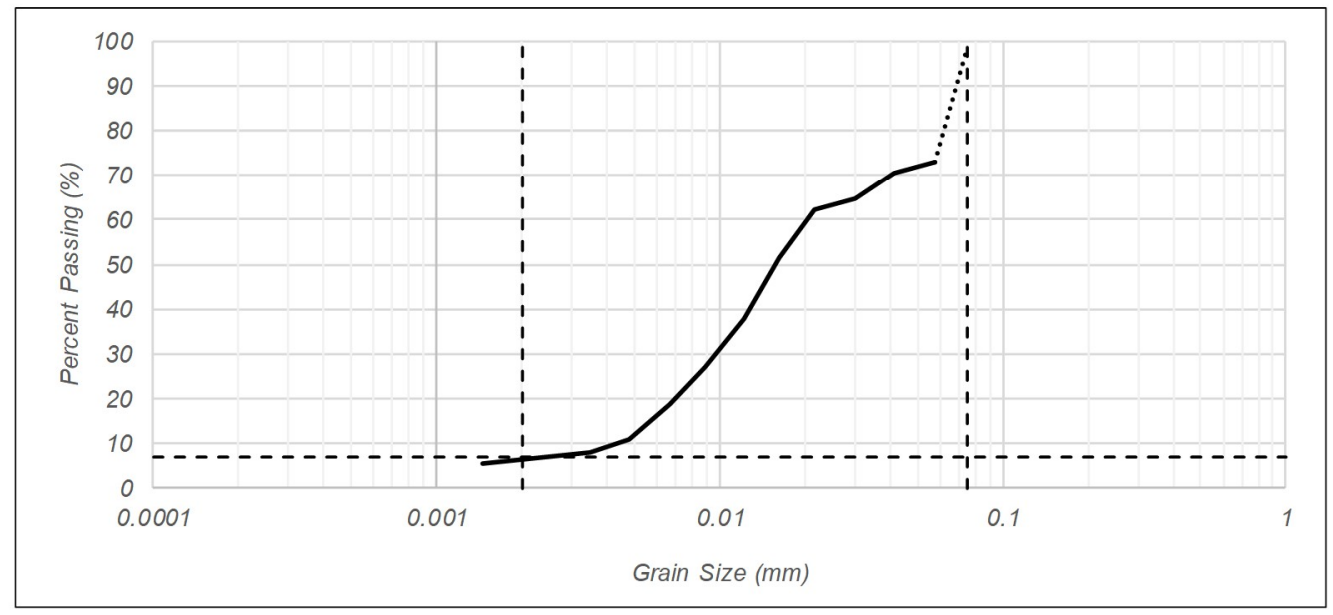

P200 Wash

$\begin{array}{lcc}\text { Container ID: } & \text { C1 } & \\ \text { Before }-\mathrm{M}_{\mathrm{d}} \text { : } & 27.5 & \mathrm{~g} \\ \text { After }-\mathrm{M}_{\mathrm{d}} \text { : } & 0.6 & \mathrm{~g} \\ \text { Fines Fraction: } & 97.82 & \% \\ \text { Coarse Fraction: } & 2.18 & \%\end{array}$

\begin{tabular}{llr} 
& & \multicolumn{2}{c}{ Soil Breakdown } \\
\cline { 2 - 3 } & Coarse: & $2.18 \%$ \\
& Silts: & $90.82 \%$ \\
From Hydrometer & Clays: & $7.00 \%$
\end{tabular}

Procedures Done By:

Kayla/Melissa $\mathrm{P}$. 


\section{Appendix I: $V_{\mathrm{s}} / \mathbf{V}_{\mathrm{p}}$ Acquisition Software Installation Instructions}

1. Download the file 'TREX_Software' onto your personal computer.

2. Open the 'Driver_CD' folder.

3. Open the application 'setup', date modified 10/12/2010.

Allow the program access to make changes to your computer.

4. Click 'Driver Installation'.

5. Choose 'USBDAQ'.

6. Click 'Driver Installation'.

Go through the Setup Wizard.

7. Exit the Setup Wizard. The software is now installed. 
Appendix J: $V_{s} / V_{p}$ Field Instrumentation Schematic

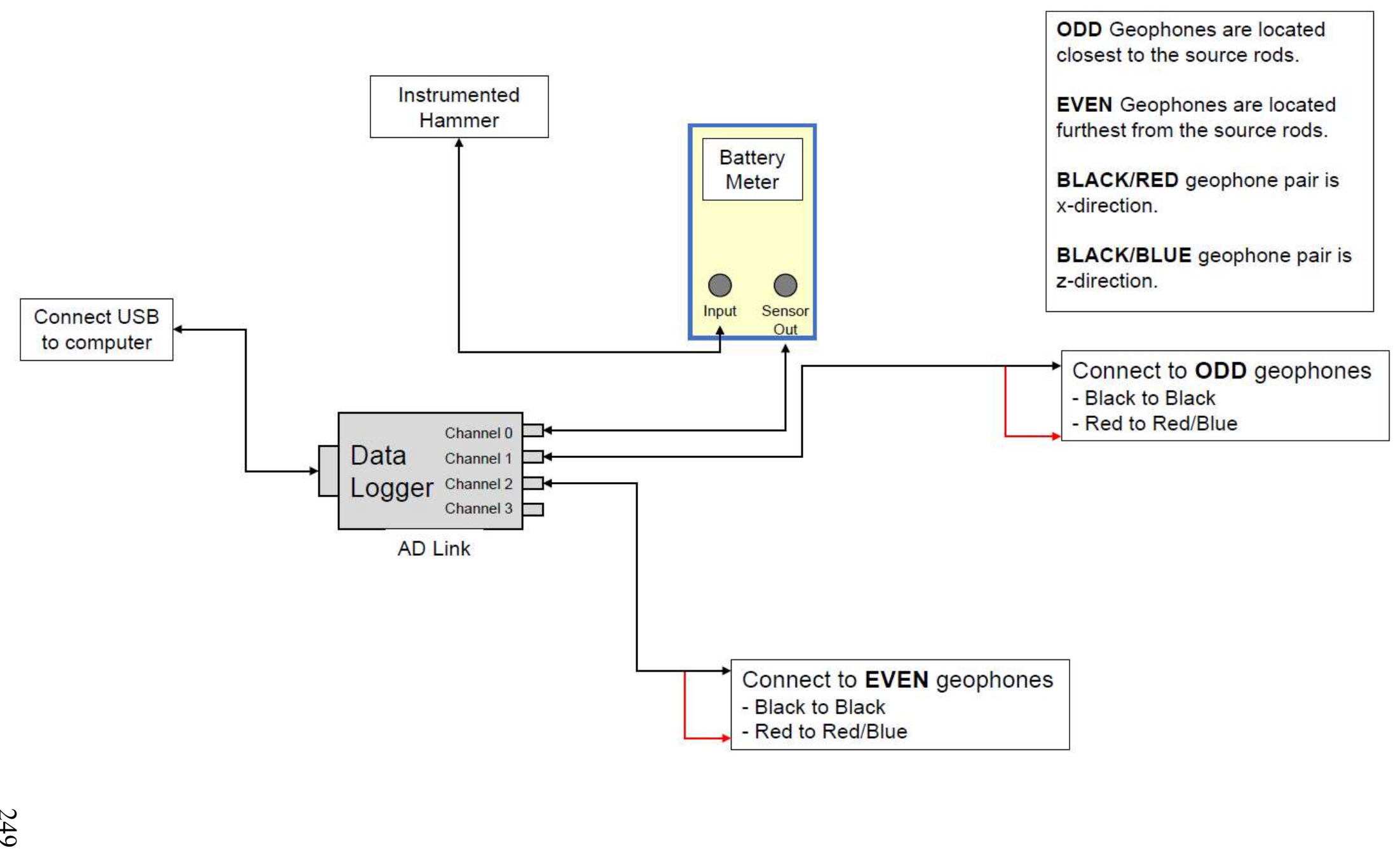




\section{Appendix K: $V_{s} / V_{p}$ Data Collection Instructions}

1. Setup the equipment.

2. Open the 'VB_ADLink' application, located within the TREX_Software folder. NOTE: This will only open if the Data Logger is connected to the laptop with the software already installed.

3. Click the 'Open Excel' button. There are 5 sheets in this workbook.

- The first sheet is Setup. We use this sheet to change the name of the files for the data acquired.

- The second sheet is AveragedRecord. We do not use this sheet during data acquisition.

- The third sheet is Sheet1. This sheet displays the data received from the hammer.

NOTE: This is the sheet that is looked at while collecting the data, to make sure the average is representative of the set of 10 hammer hits.

It also displays the maximum values of Sheet 2 and Sheet 3 to make sure that the data gathered is good quality (these values should be in the $10^{-4}$ to $10^{-2}$ magnitude range, and both values should have close to the same order or magnitude).

- The fourth sheet is Sheet2. This sheet displays the data captured from the nearest geophone.

- The fifth sheet is Sheet3. This sheet displays the data captured from the furthest geophone.

3. Go back to the AD_Link software window. Click on the Start button to start collecting data. 
4. Look at the excel sheet Sheet1 while gently tap the hammer 10 times on the designated rod. Check the quality of the hammer hits and the maximum values recorded for the geophones.

NOTE: If the average line on the graph is representative of all 10 hammer hits, and the maximums for Sheet2 and Sheet3 are acceptable, go to the Setup sheet and save the file. IMPORTANT: Save the data in the same format to keep the file names consistent. First is the hitting direction, then the set of geophones, then the direction data is being collected from, e.g., Up 1\&2 X or Down 5\&6 Z.

5. Repeat step 4 until you have a set a data for both up hits and down hits for each direction $(\mathrm{X}$ and $\mathrm{Z})$ for each set of geophones. You should have a total of 16 folders when done with the data collection. 


\section{Appendix $L: V_{s} / V_{p}$ Data Filtering Instructions}

1. Go to the 'Crosshole Data' folder on the stash. Create a folder and name it with the date that the data was collected in the format year_month_day (i.e. 2019_11_07).

2. Upload the collected data to the stash. If there are 2 data sets, name the folders 'Data_Set_1' and 'Data_Set_2'.

3. Open the Anaconda prompt (part of Python) and type 'Jupyter Notebook' in the command screen. This will open up your default browser.

4. Navigate through the files displayed until you find the filtering code, then double-click. This will open a new webpage with the code displayed.

5. Change the file paths for where the data is located that you want to filter (Step 2), and where you want the filtered data to be saved to (this can be the same folder if desired). These paths are displayed in red text within the code.

6. Run the code. 


\section{Appendix M: $V_{\mathrm{s}} / V_{\mathrm{p}}$ Data Formatting Instructions}

1. Go to the 'Crosshole Data' folder on the stash and choose the date of the data that needs to be filtered.

\section{2.}

3. Open a folder from a previous date, and transfer the excel workbook (YYYY_MM_DD_Filtered) into the new folder with the new data. Rename the workbook with the date of the new data.

NOTE: This workbook has 8 sheets. The sheets are named with the source rod name and the data set, i.e. Rod 3_2 is the data for source rod 3, from the second data set. The headers describe which geophone the data is from, as well as the type of hammer hit and the type of wave captured. I.e. Sheet3UZ is data from the furthest geophone, up hits, and the shear wave is what was captured. The workbook tab indicates which rod the data is from.

4. Open the folder 'Data_Set_1'. Open the folder 'Down 1\&2 X'. Open the workbook 'AveragedRecord_Filtered'. This is the data source rod 1, hitting downwards, and capturing the X-directional wave (pressure wave). Ch 0 is the average of the hammer hits, Ch 1 is the average of the nearest geophone recordings, and $\mathrm{CH} 2$ is the average of the furthest geophone recordings.

5. Copy the data from columns labeled ' $\mathrm{Ch} 1$ ' and ' $\mathrm{Ch} 2$ ' and paste the data into the workbook with 8 tabs, named with the collection date. Paste the data in the sheet label 'Rod 1_1' under the headings 'Sheet2DX' and 'Sheet3DX'. This is the averaged data from downhits on source rod 1 for both the nearest and furthest geophones in the X direction. 
6. Close the 'AveragedRecord_Filtered' workbook. Go back into the 'Data_Set_1' folder and open the folder 'Down $1 \& 2 \mathrm{Z}$ '. Repeat step 5, but place the copied data into the columns labeled 'Sheet2DZ' and 'Sheet3DZ'. This is the averaged data from downhits on source rod 1 for both the nearest and furthest geophone in the $\mathrm{Z}$ direction.

7. Close the 'AveragedRecord_Filtered' workbook. Go back into the 'Data_Set_1' folder and open the folder 'Up $1 \& 2 \mathrm{X}$ '. Repeat step 5, placing the copied data into the columns labeled 'Sheet2UX' and 'Sheet3UX'.

8. Close the 'AveragedRecord_Filtered' workbook. Go back into the 'Data_Set_1' folder and open the folder 'Up $1 \& 2 Z$ '. Repeat step 5, placing the copied data into the columns labeled 'Sheet2UZ' and 'Sheet3UZ'.

9. All the data from source rod 1 for the first data set is now in the workbook. Move to the tab labeled 'Rod 2_1' and repeat steps 5-8 for the folders labeled 'Down 3\&4 X', 'Down 3\&4 Z', 'Up 3\&4 X', and 'Up 3\&4 Z'.

10. All the data from source rod 2 for the first data set is now in the workbook. Move to the tab labeled 'Rod 3_1' and repeat steps 5-8 for the folders labeled 'Down 5\&6 X', 'Down 5\&6 Z', 'Up 5\&6 X', and 'Up 5\&6 Z'.

11. All the data from source rod 3 for the first data set is now in the workbook. Move to the tab labeled 'Rod 4_1' and repeat steps 5-8 for the folders labeled 'Down 7\&8 X', 'Down 7\&8 Z', 'Up 7\&8 X', and 'Up 7\&8 Z'.

12. All the data from the first data set is now in the workbook. Repeat steps 4-11 for the data in the folder 'Data_Set_2'.

13. All data from both data sets are now in the workbook. Make sure this workbook is saved on the stash. 


\section{Appendix N: Analyzing the $V_{s} / V_{p}$ Data with Matlab}

\section{Open Matlab}

2. Run the script 'Pick_Arrivals.m'. This script in located on the stash in the 'Matlab' folder.

3. Make sure the excel named with date the data was taken is copied into the 'Matlab' folder on the stash. Make sure that Matlab is reading from this folder.

4. Click on the 'Import Data' button within Matlab.

5. Choose the workbook with the data you want to analyze. Click Open.

6. A pop-up window will open. Change the output type to 'Numeric Matrix'.

7. Click 'Import Selection'. Repeat this step for each of the 8 tabs.

8. Close the pop-up window.

9. In the workspace window of Matlab, you will now have 8 files. The order they were imported were 'Untitled', 'S1', 'S2', etc. In the order they were imported, rename the matrices as the next set of run numbers (i.e. Run00124, Run00125, etc.).

NOTE: The files were imported in the order of: $(\operatorname{Rod} 1$, data set 1$),(\operatorname{Rod} 1$, data set 2$)$, $(\operatorname{Rod} 2$, data set 1$),(\operatorname{Rod} 2$, data set 2$)$, etc.

10. Right click on each of these newly-renamed matrices. Save each one as the same run number. This will save the .mat file with the run number. This is what is called within the script.

11. Within the 'Pick_Arrivals.m' script, change the inputs on lines

$5,6,7,8,9,10,12,13,14,15,16$, and 17 to the run that you want to analyze. All inputs are the same run number.

12. Click 'Run' on the top ribbon under the 'Editor' tab. 
13. A pop-up window appears with 4 plots. Go to Edit --> copy figure.

14. Open the powerpoint file named 'Graphs_After_Treatment'. Paste this figure onto a new slide with the data set number, the date the data was collected, the run number, the rod the data was collected from, and whether it is from 'Data_Set_1' or 'Data_Set_2'. 15. In Matlab, under Tools, choose 'Data Tips'. Pick the arrival times for each graph. The top two graphs are the p-wave, the bottom two are the s-wave.

NOTE: You may need to use the 'Zoom In' function under Tools to accurately choose the arrival point. You can hold the 'Shift' key to choose multiple data points at once.

16. Once all data points are chosen, copy the graph. Paste the graph in the 'Graphs_After_Treatment' powerpoint on a new slide immediately following the slide with the information listen in step 14.

17. Open the excel file 'Summary_Velocity'. Create a new tab and name it with the date the data was taken and the data set number.

18. Insert the $\mathrm{X}$ coordinate values from the Matlab graphs into their respective cells. The velocity will auto-populate.

19. Repeat steps 11-18 for each run. There should be 8 runs for 2 data sets, 4 runs for 1 data set.

20. On the 'Data Summary' tab of the 'Summary_Velocity' excel, copy/paste the velocity values for the average $\mathrm{p}$-wave, $\mathrm{p}$-wave from data set 1 , $\mathrm{p}$-wave from data set 2 , and the average s-wave. The graphs up top should auto-populate. If not, include the new cells in the data being called for the graph. 
Appendix O: Before treatment: Crosshole geophone voltage time series for $V_{s}$ and $V_{p}$ arrival selection.
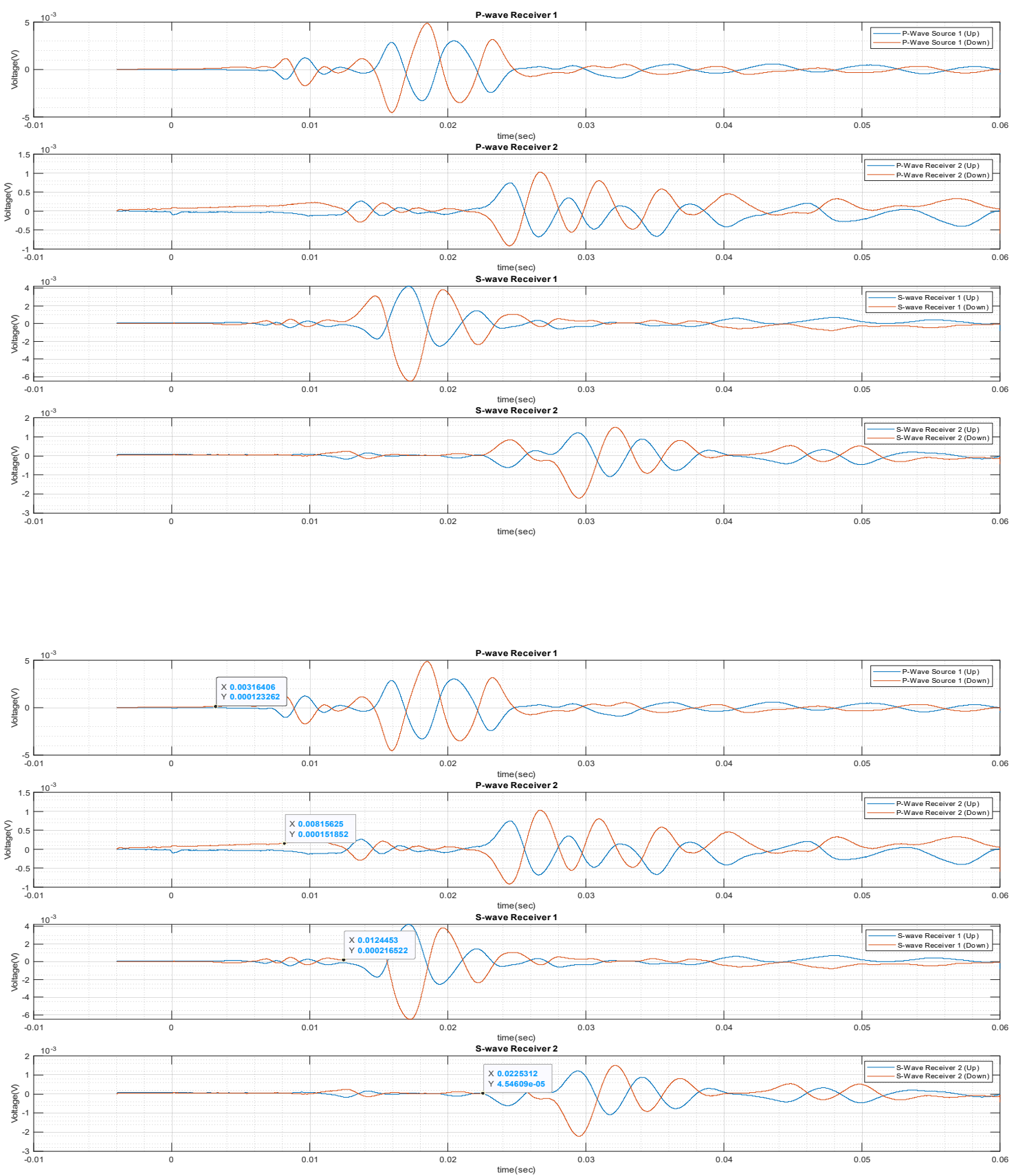

Data Set $2(8.9 .2019)$

Run00001f

R1 1 

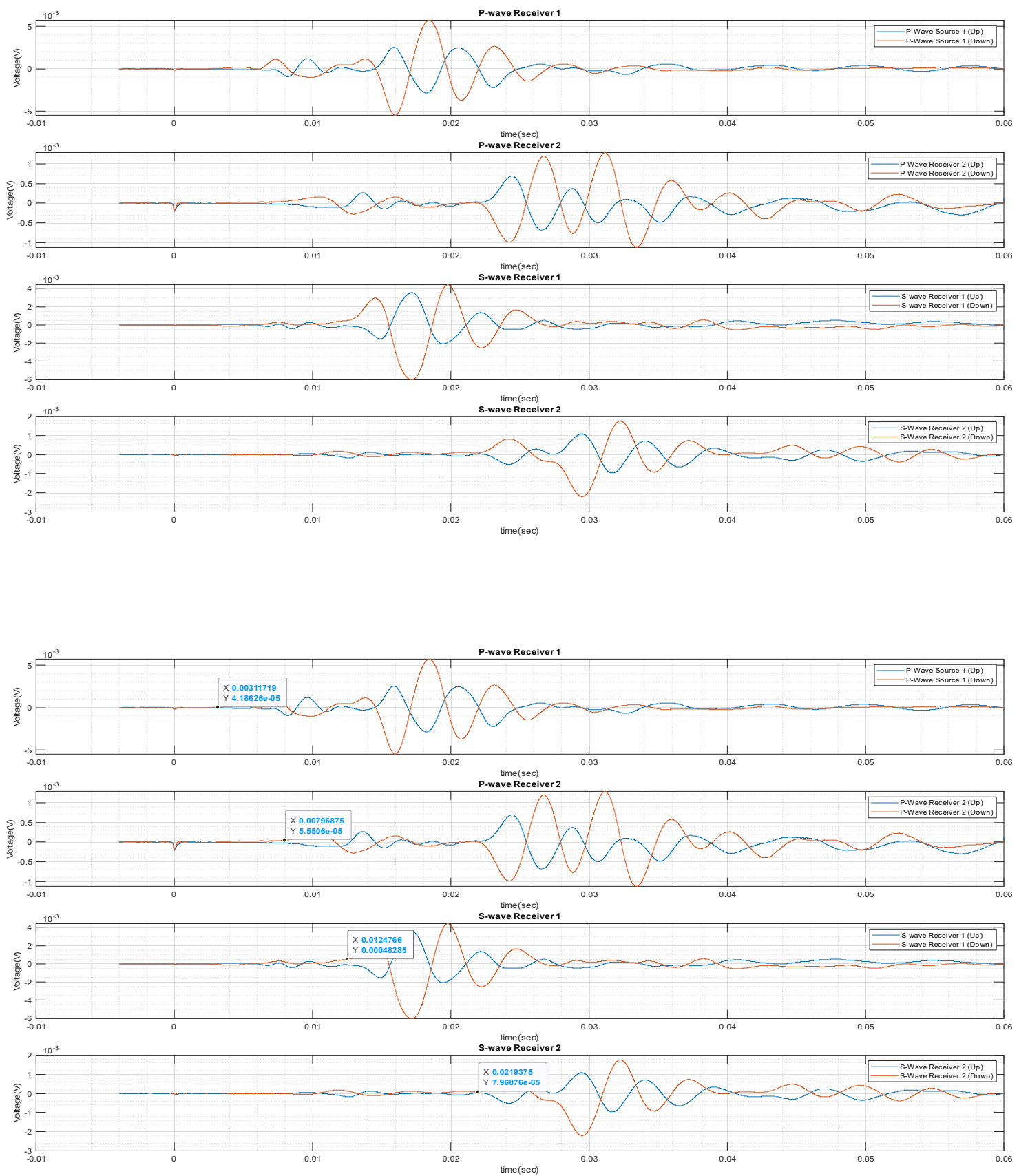

Data Set 2 (8.9.2019)

Run00002f

R1_2 

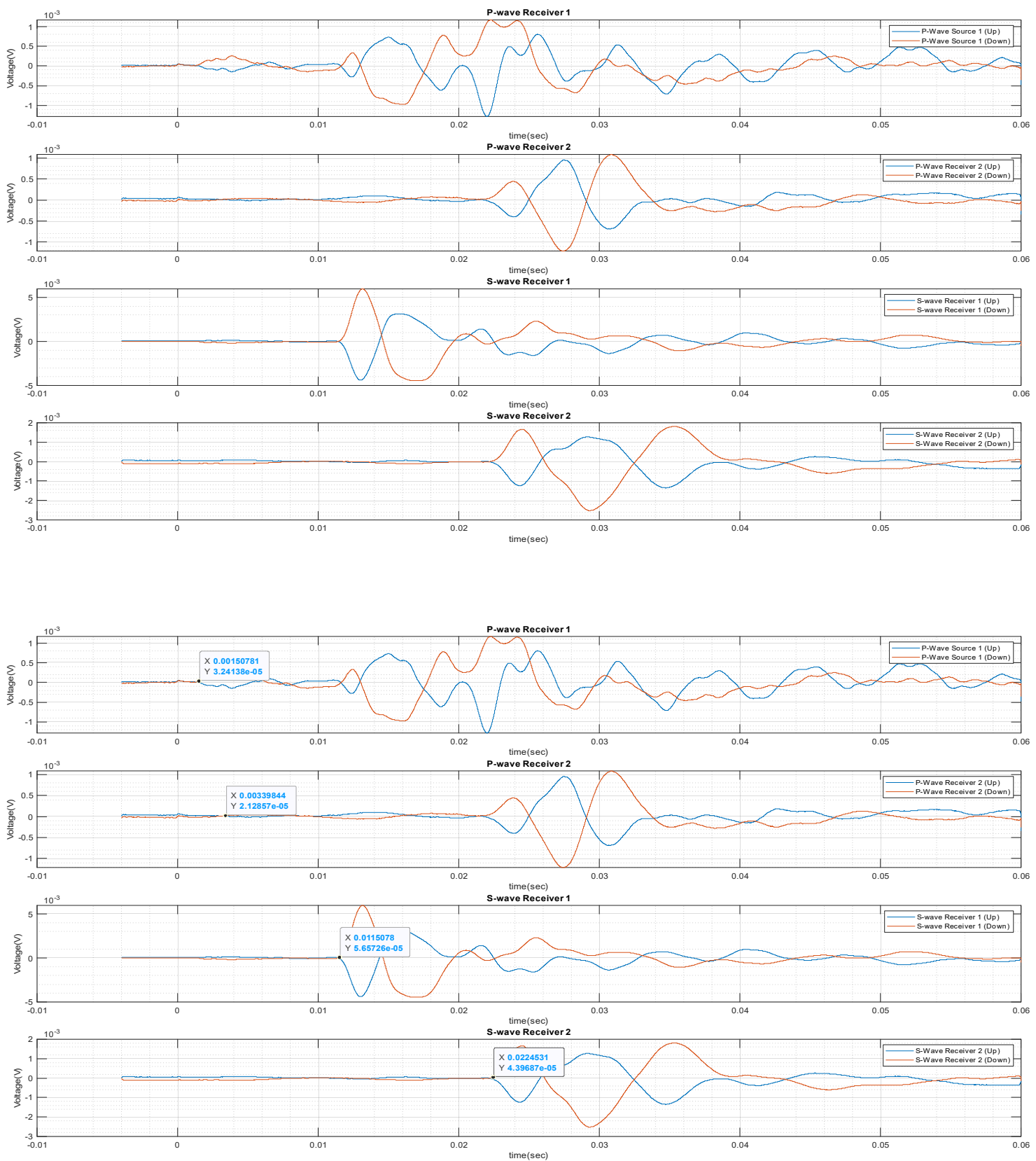

Data Set 2 (8.9.2019)

\section{Run00003f}

R2_1 

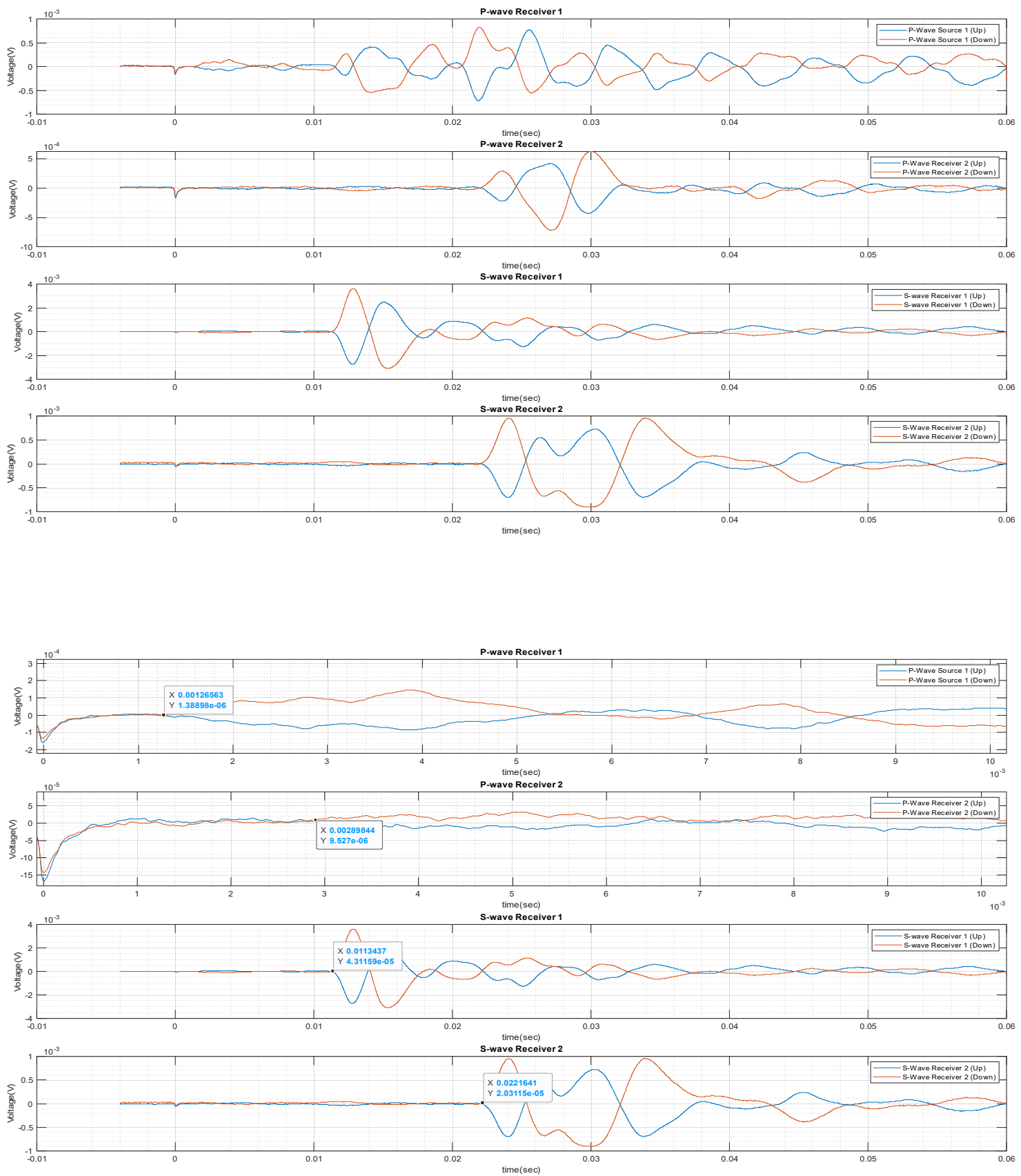

Data Set 2 (8.9.2019)

Run00004f

R2_2 

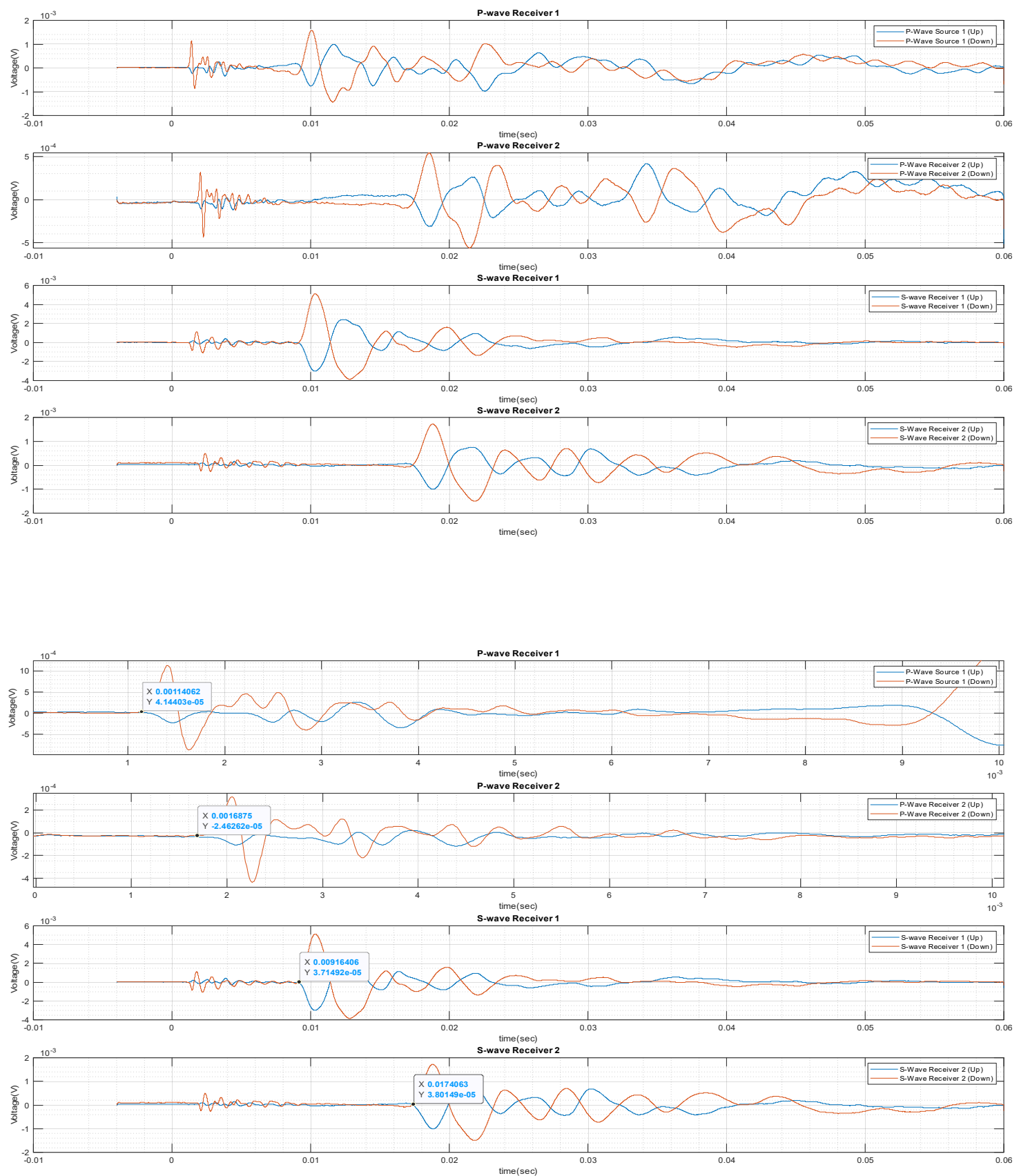

Data Set 2 (8.9.2019)

Run00005f

R3_1 

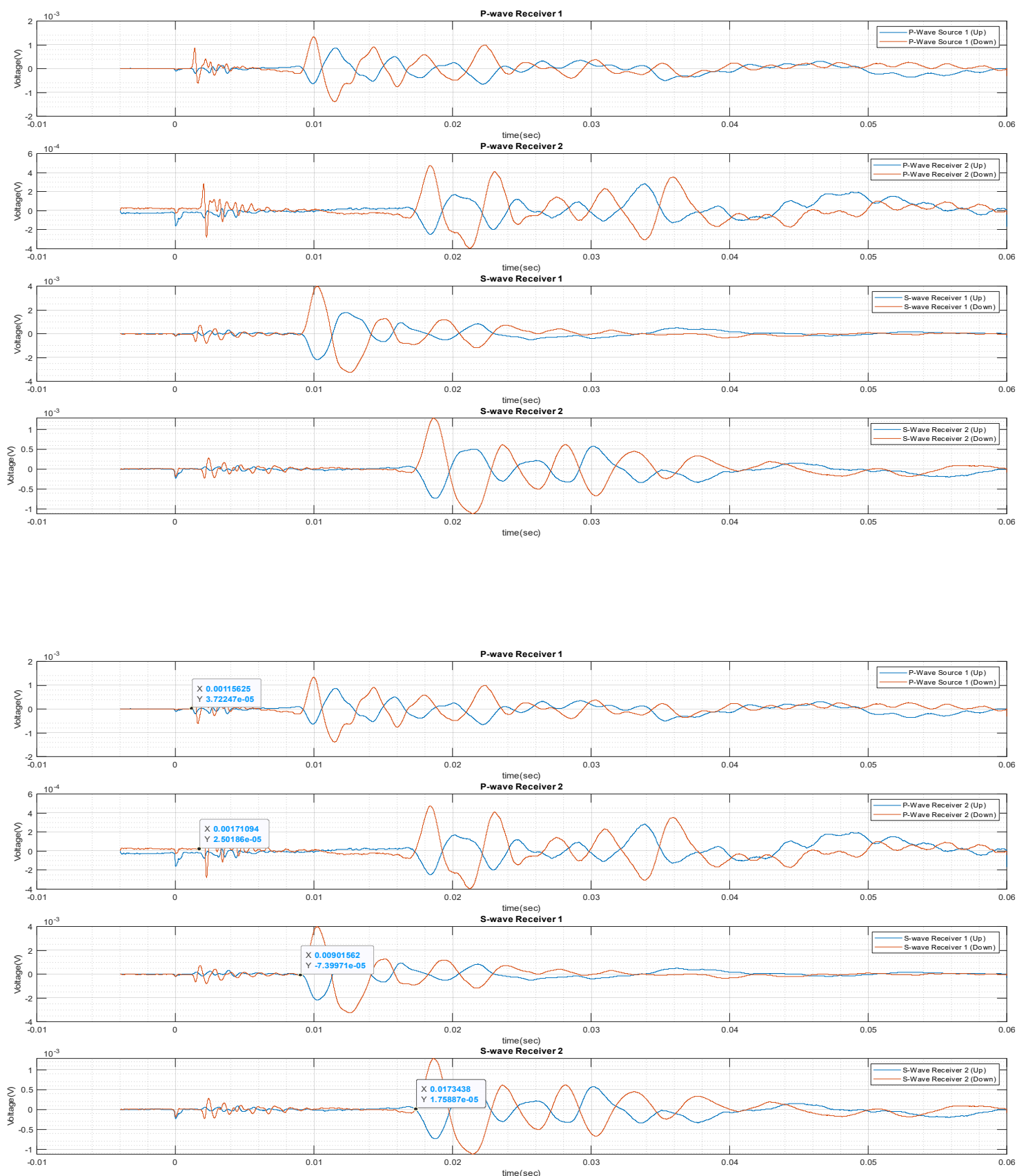

Data Set 2 (8.9.2019)

\section{Run00006f}

R3_2 

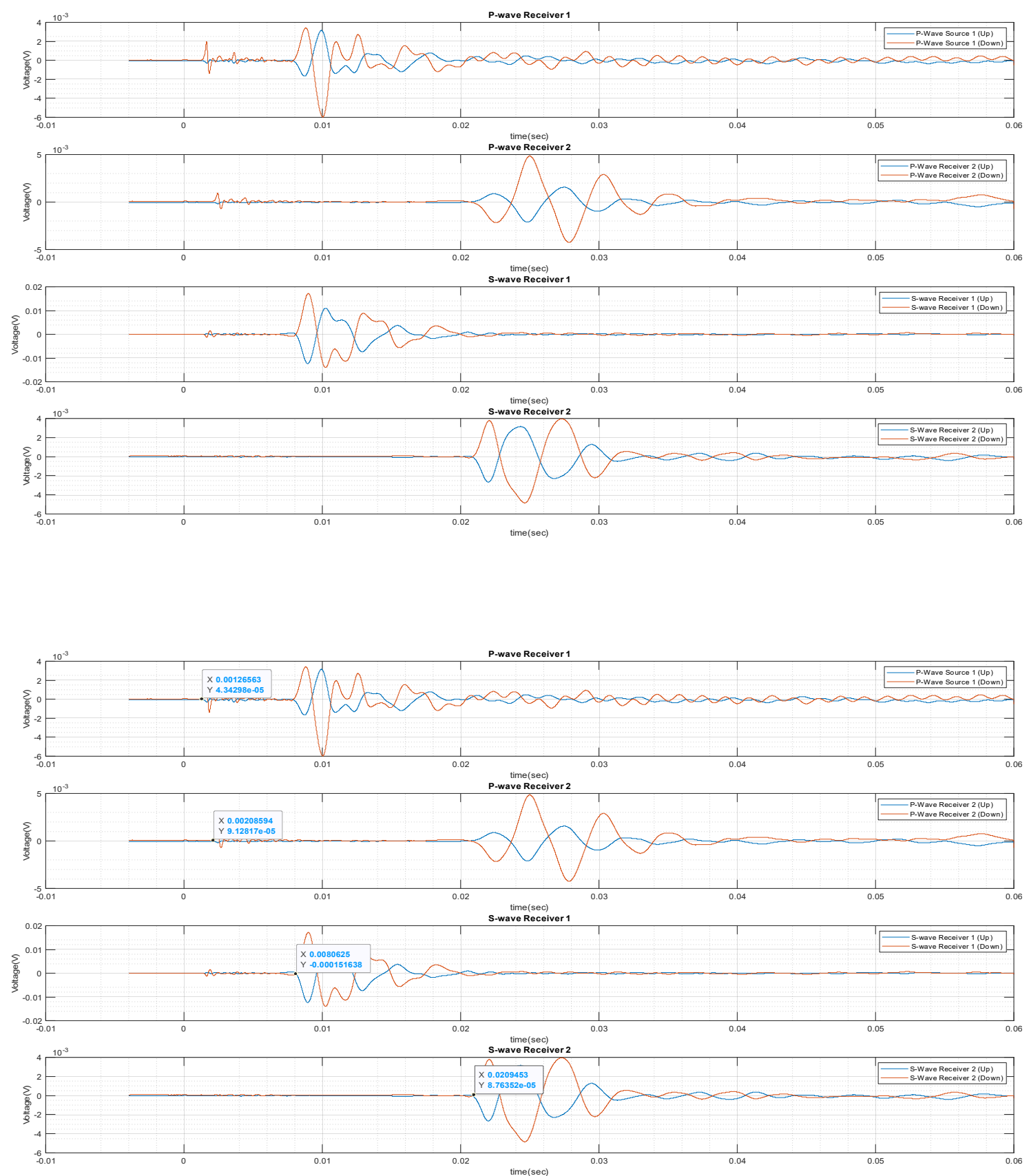

Data Set 2 (8.9.2019)

\section{Run00007f}

\section{R4_1}



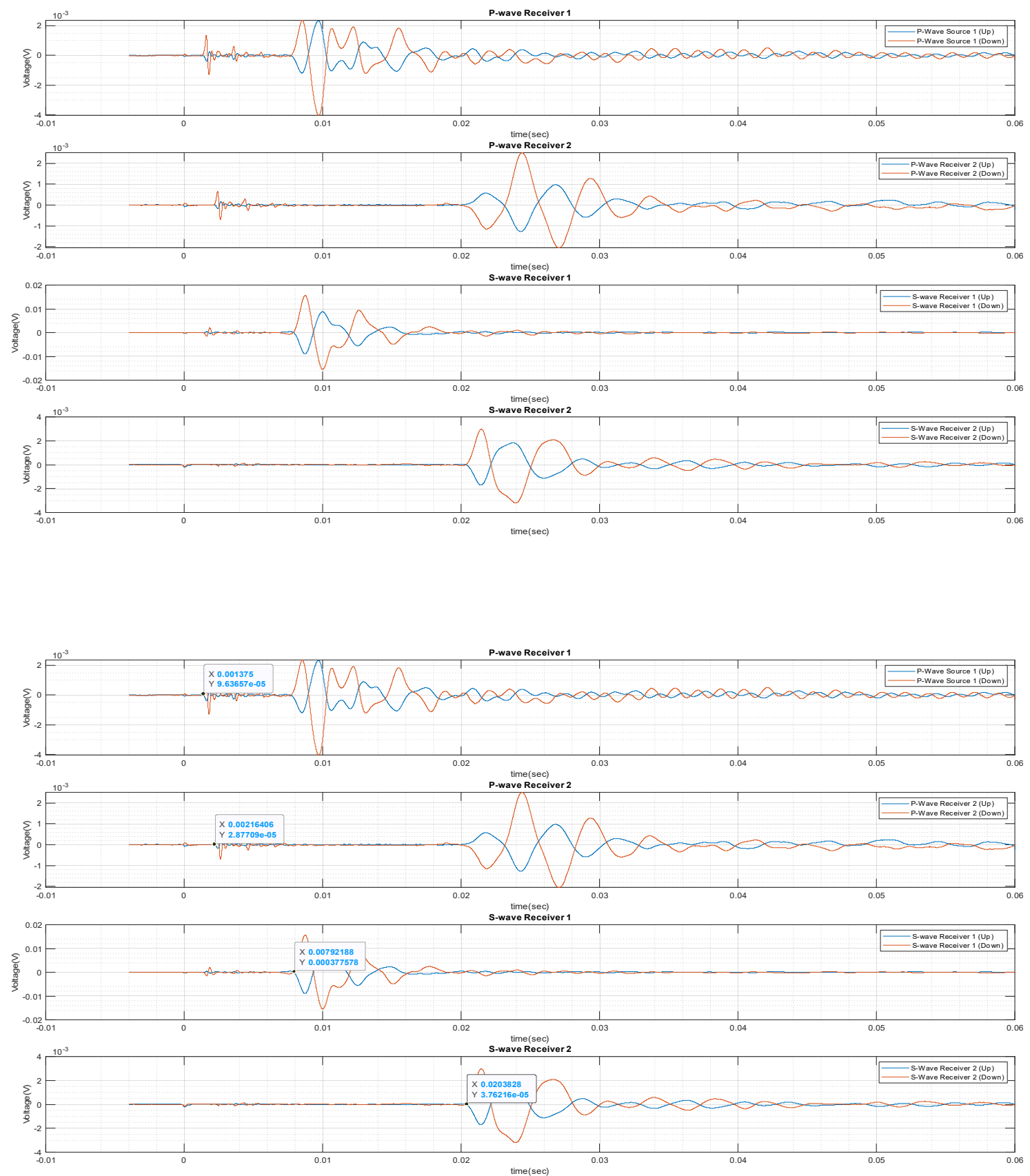

Data Set 2 (8.9.2019)

\section{Run00008f}

\section{R4 2}



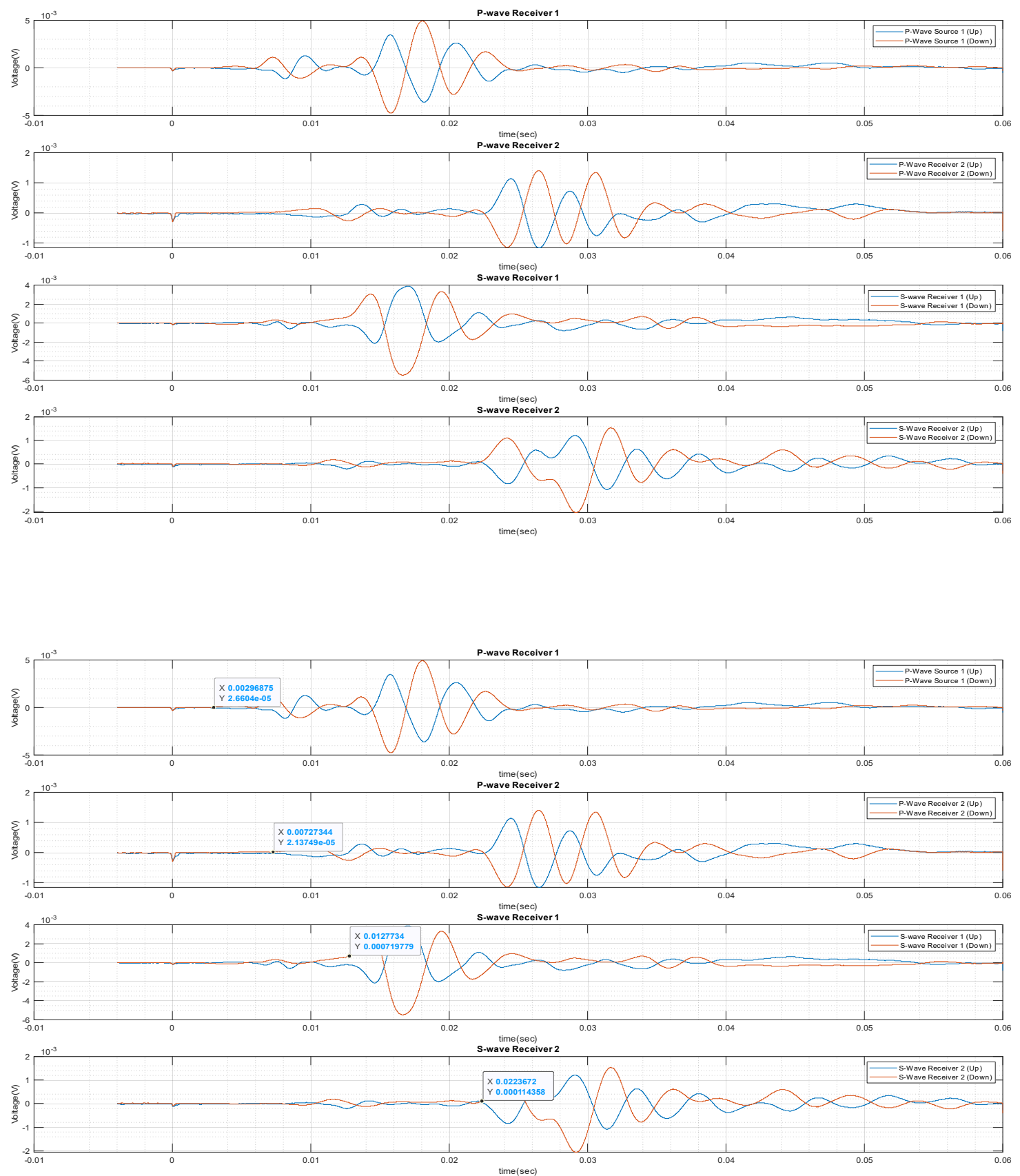

Data Set 3 (8.12.2019)

Run00009f

R1_1 

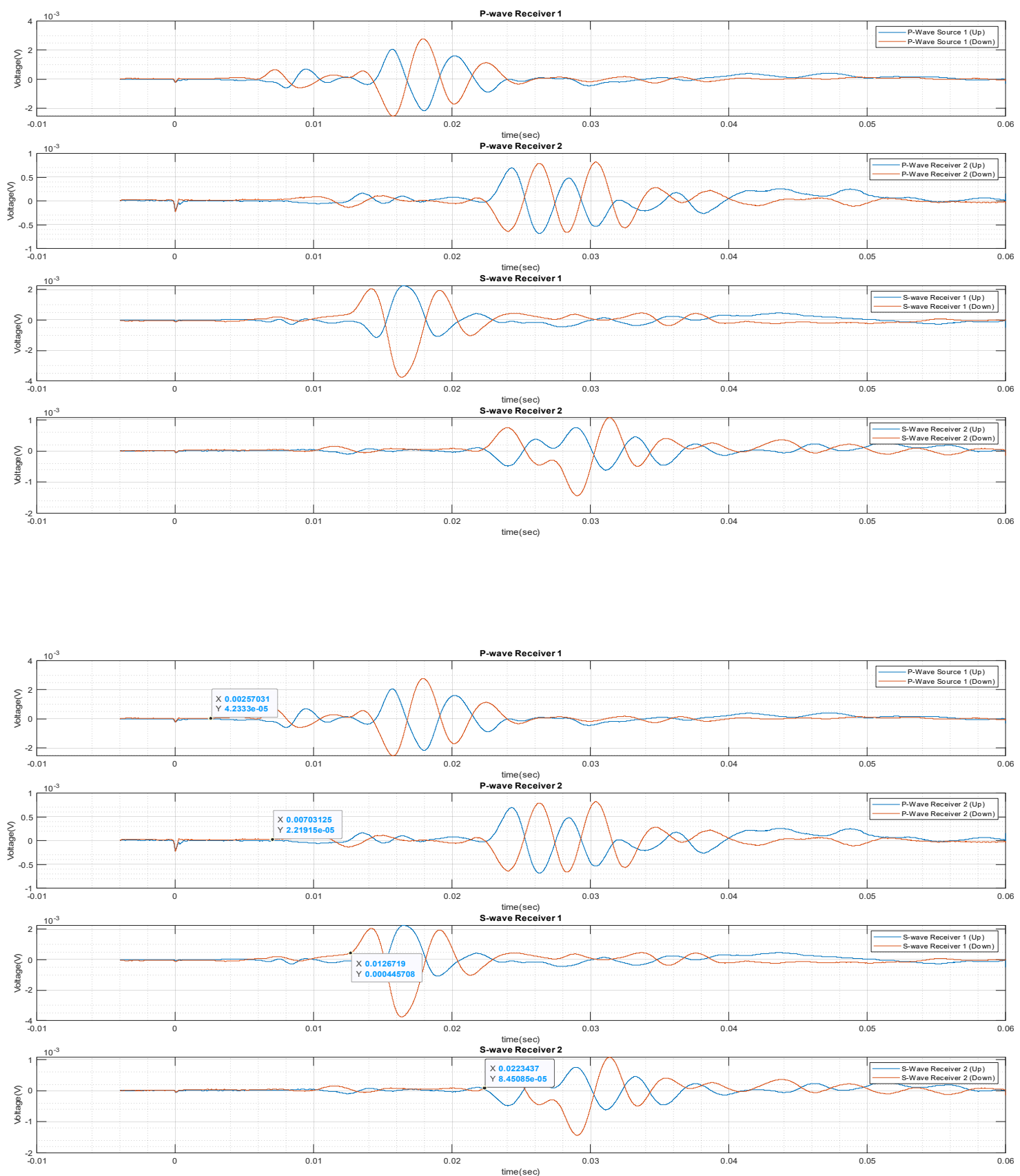

Data Set 3 (8.12.2019)

Run000010f

R1_2 

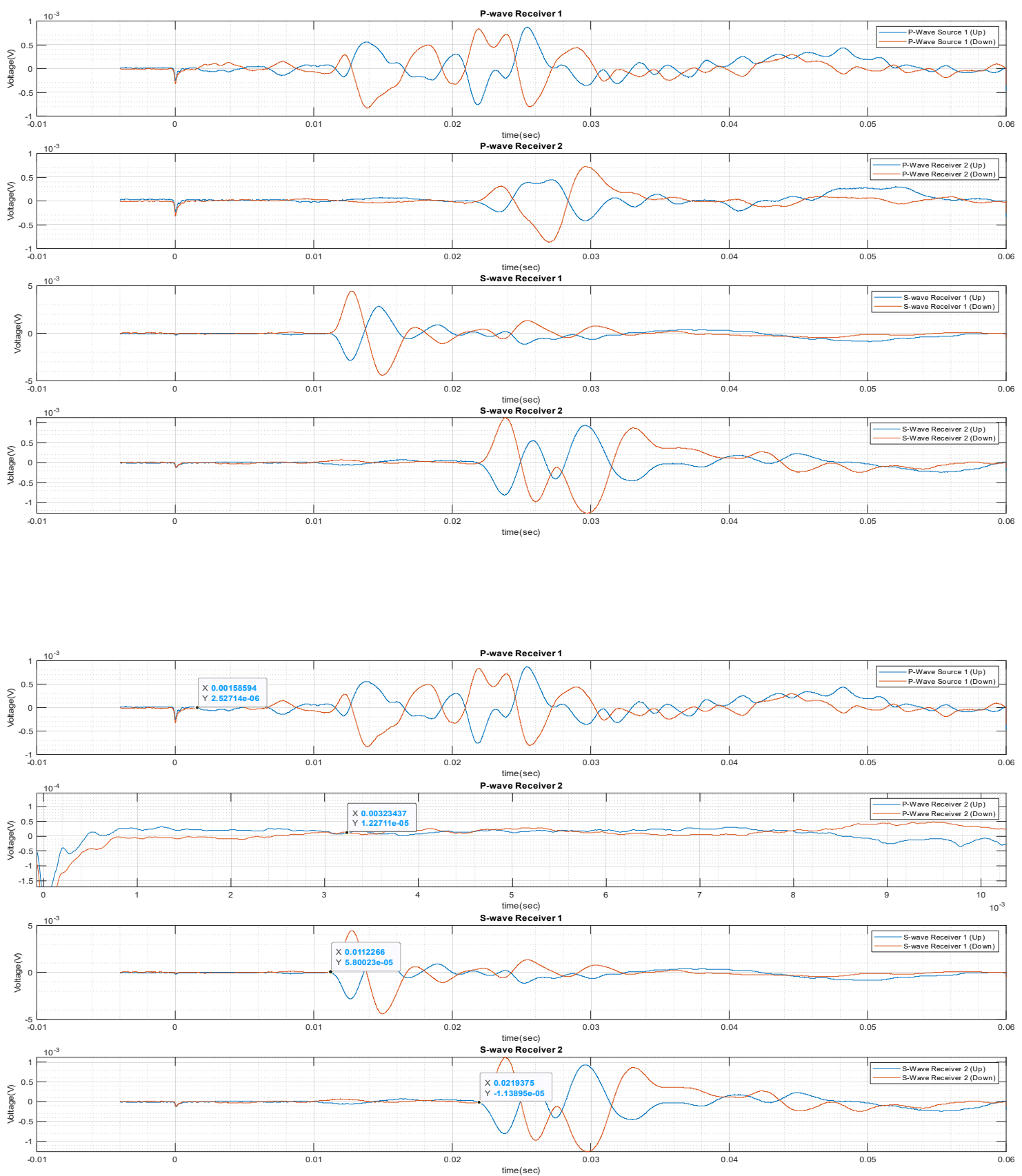

Data Set 3 (8.12.2019)

Run00011f

R2_1 

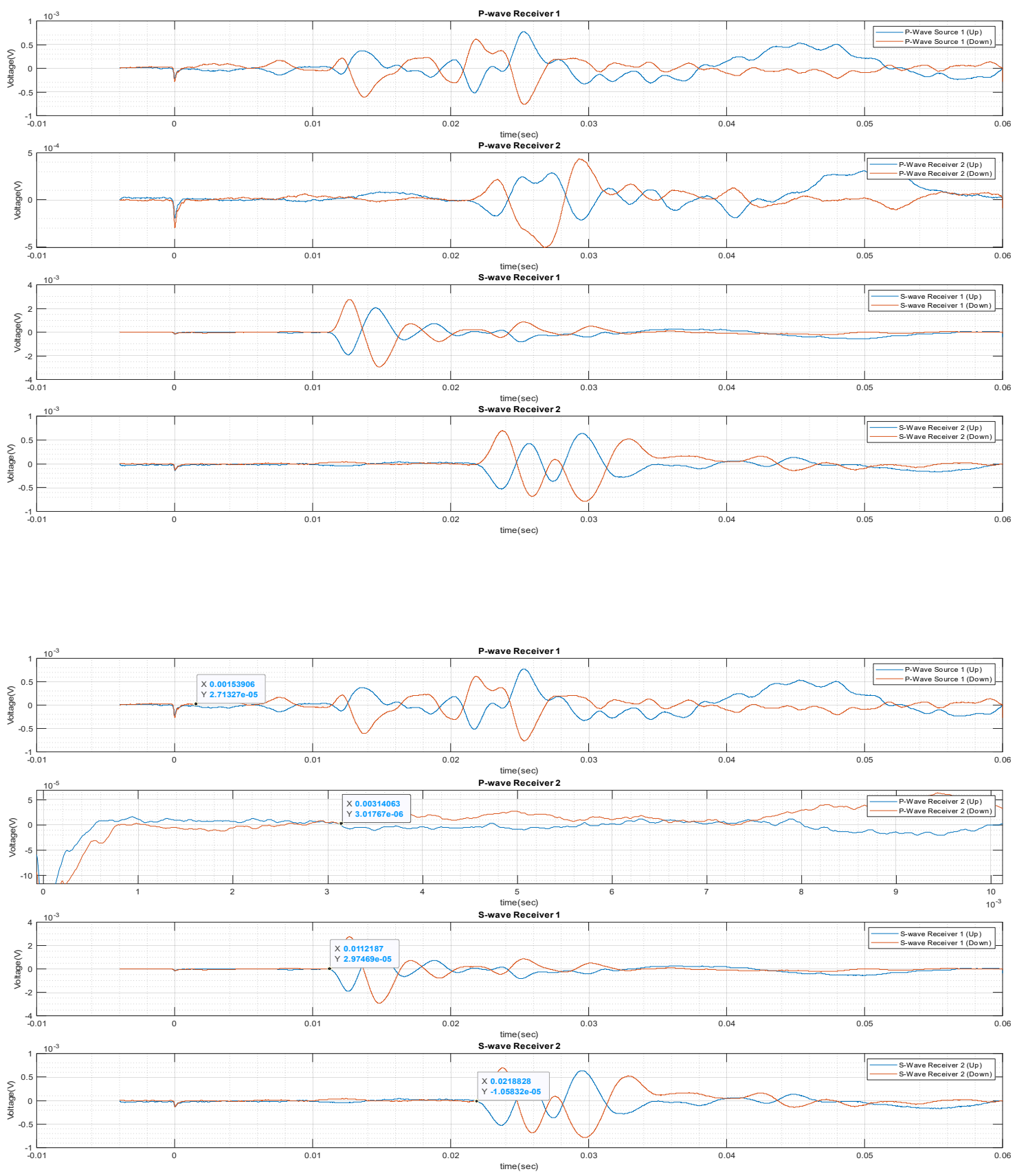

Data Set 3 (8.12.2019)

Run00012f

R2_2 

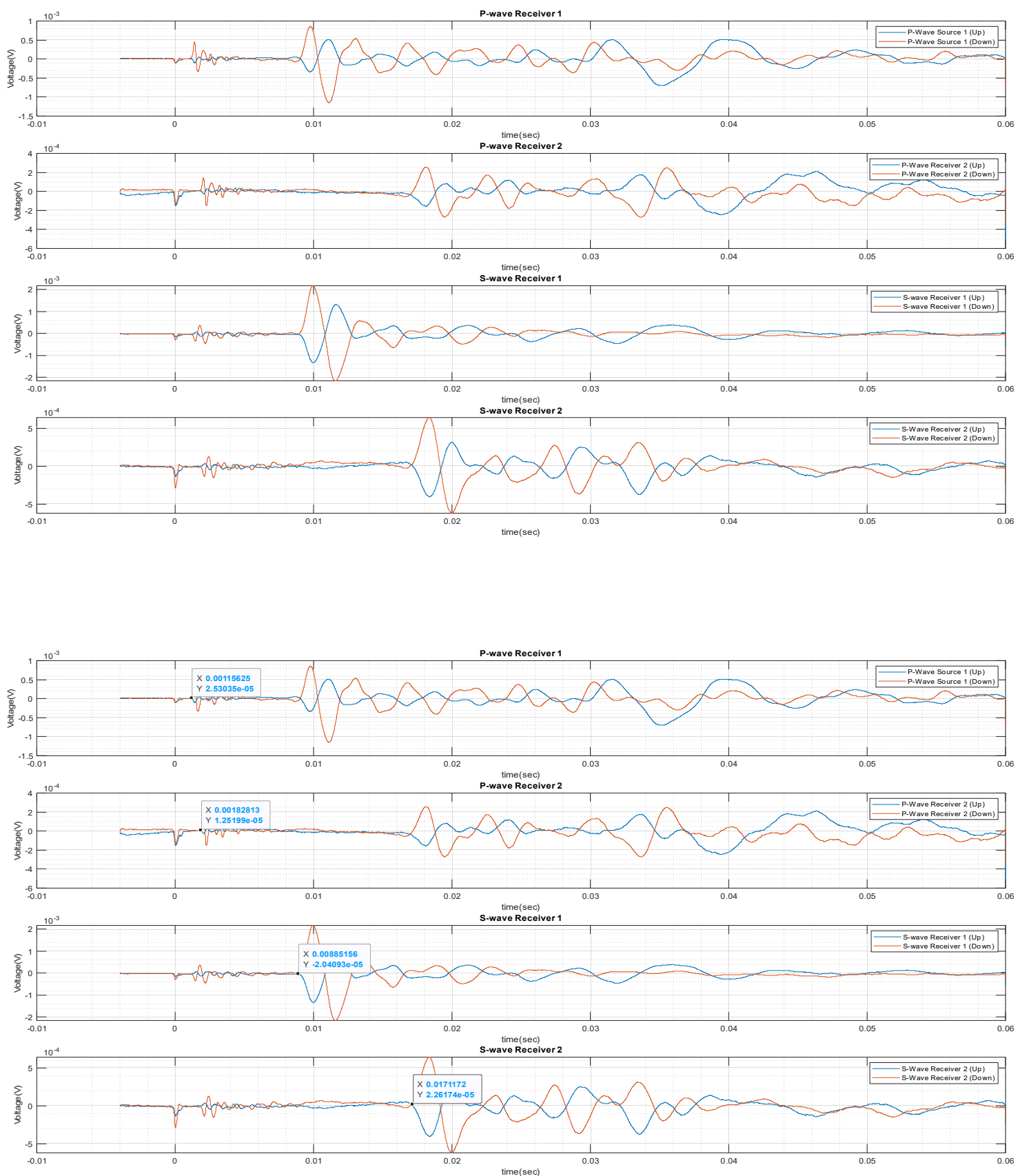

Data Set 3 (8.12.2019)

Run00013f

R3_1 

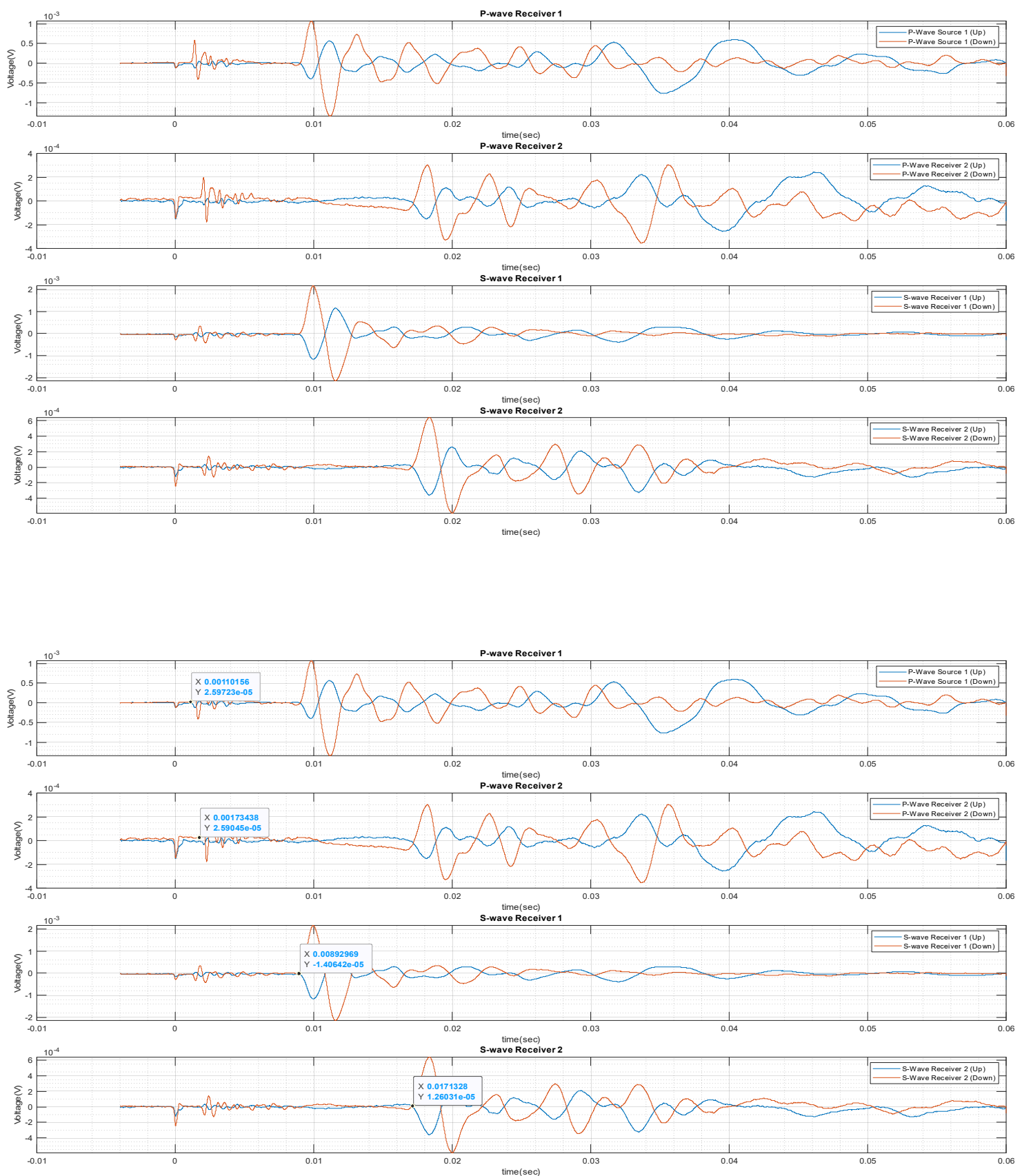

Data Set 3 (8.12.2019)

Run00014f

R3_2 

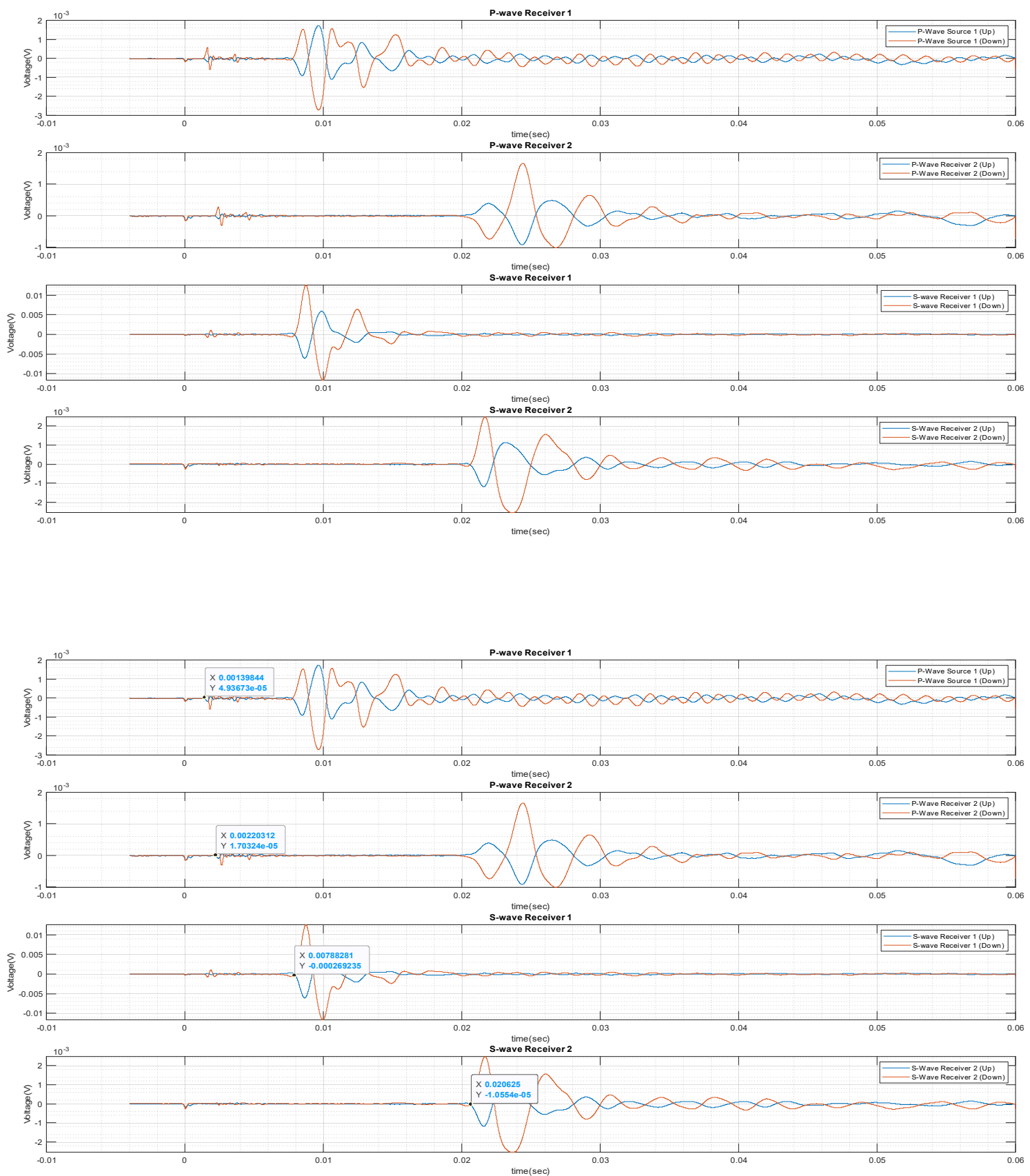

Data Set 3 (8.12.2019)

Run00015f

R4_1 

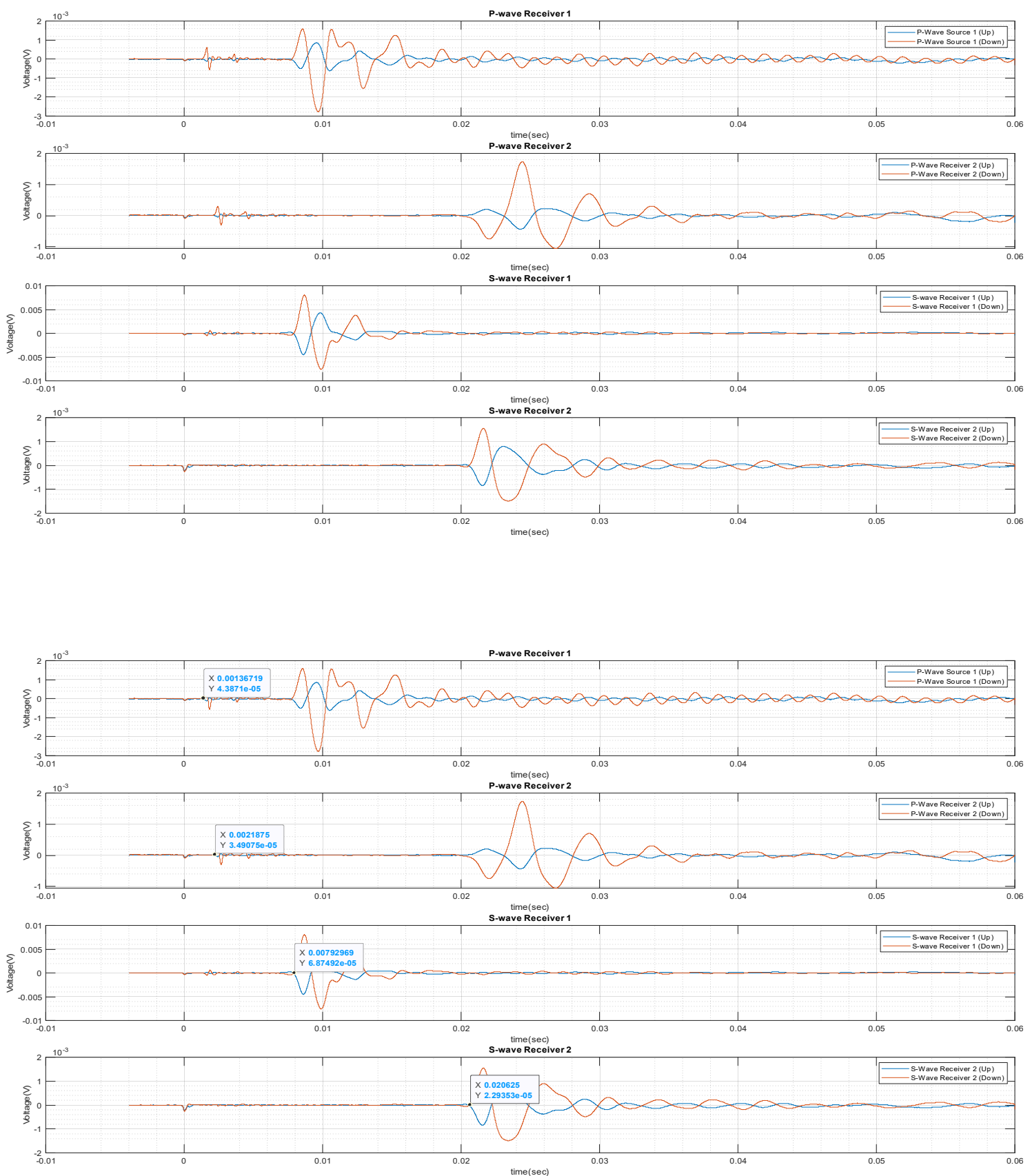

Data Set 3 (8.12.2019)

Run00016f

R4_2 

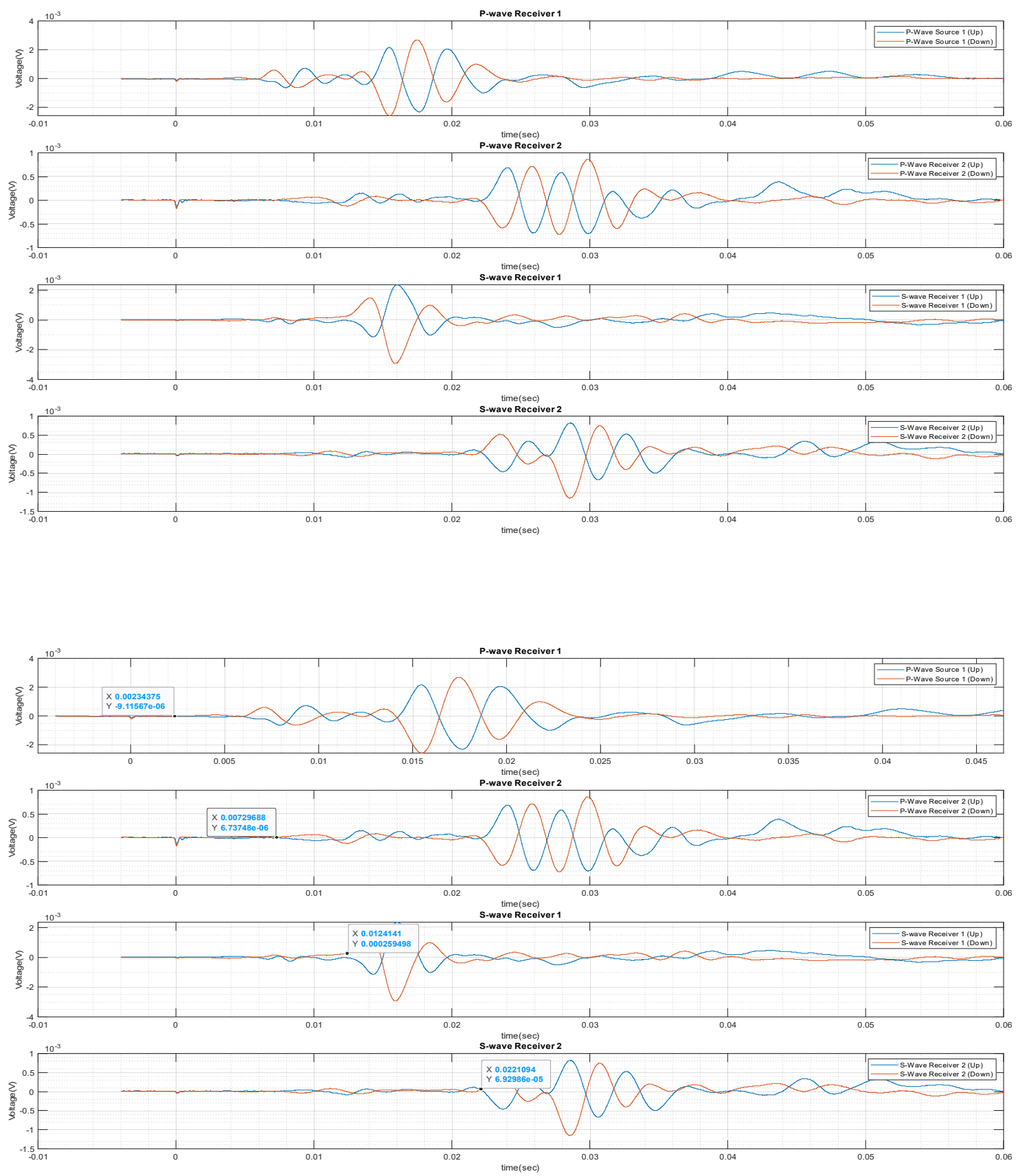

Data Set 4 (8.14.2019)

Run00017f

R1_1 

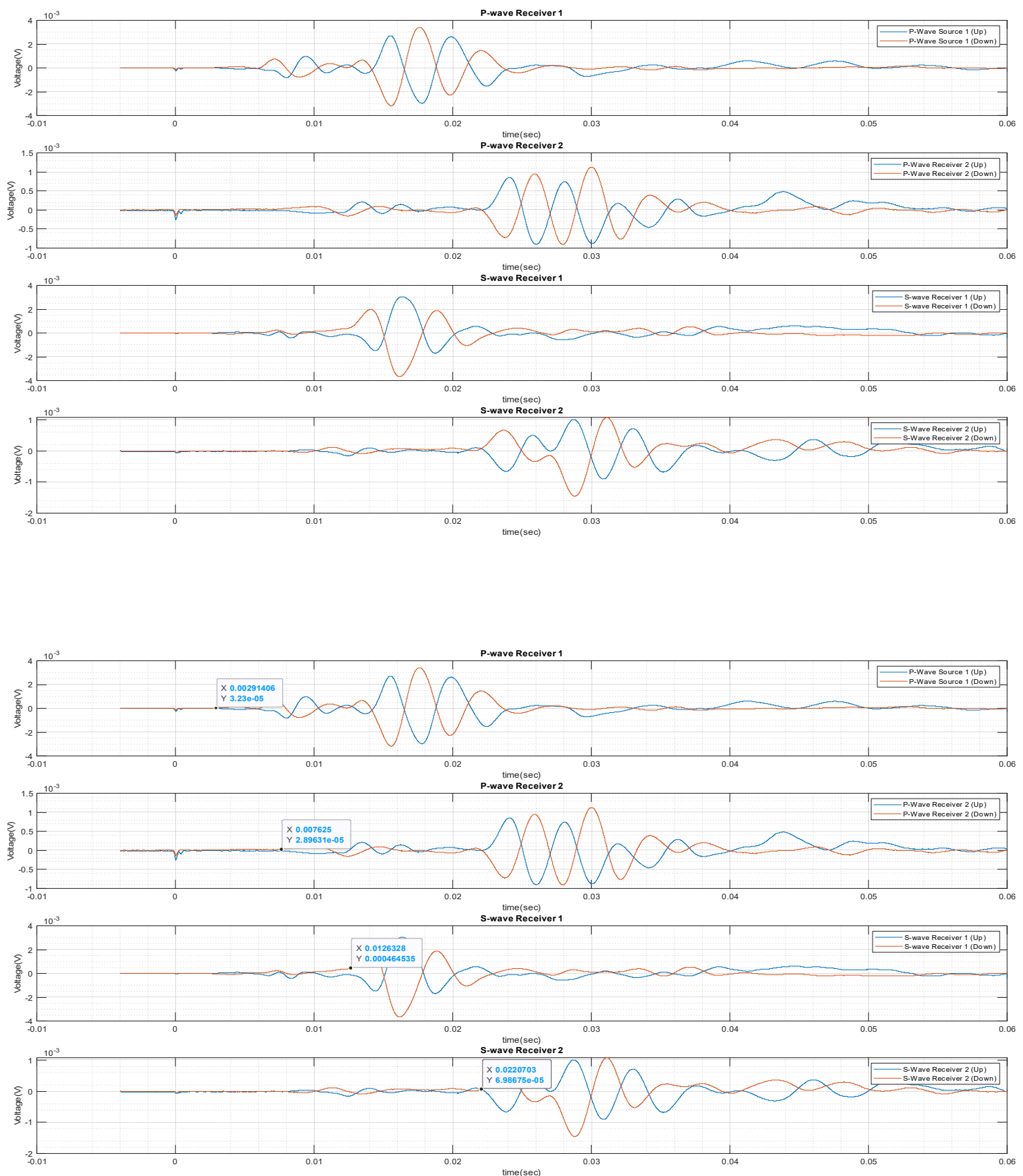

Data Set 4 (8.14.2019)

Run00018f

R1_2 

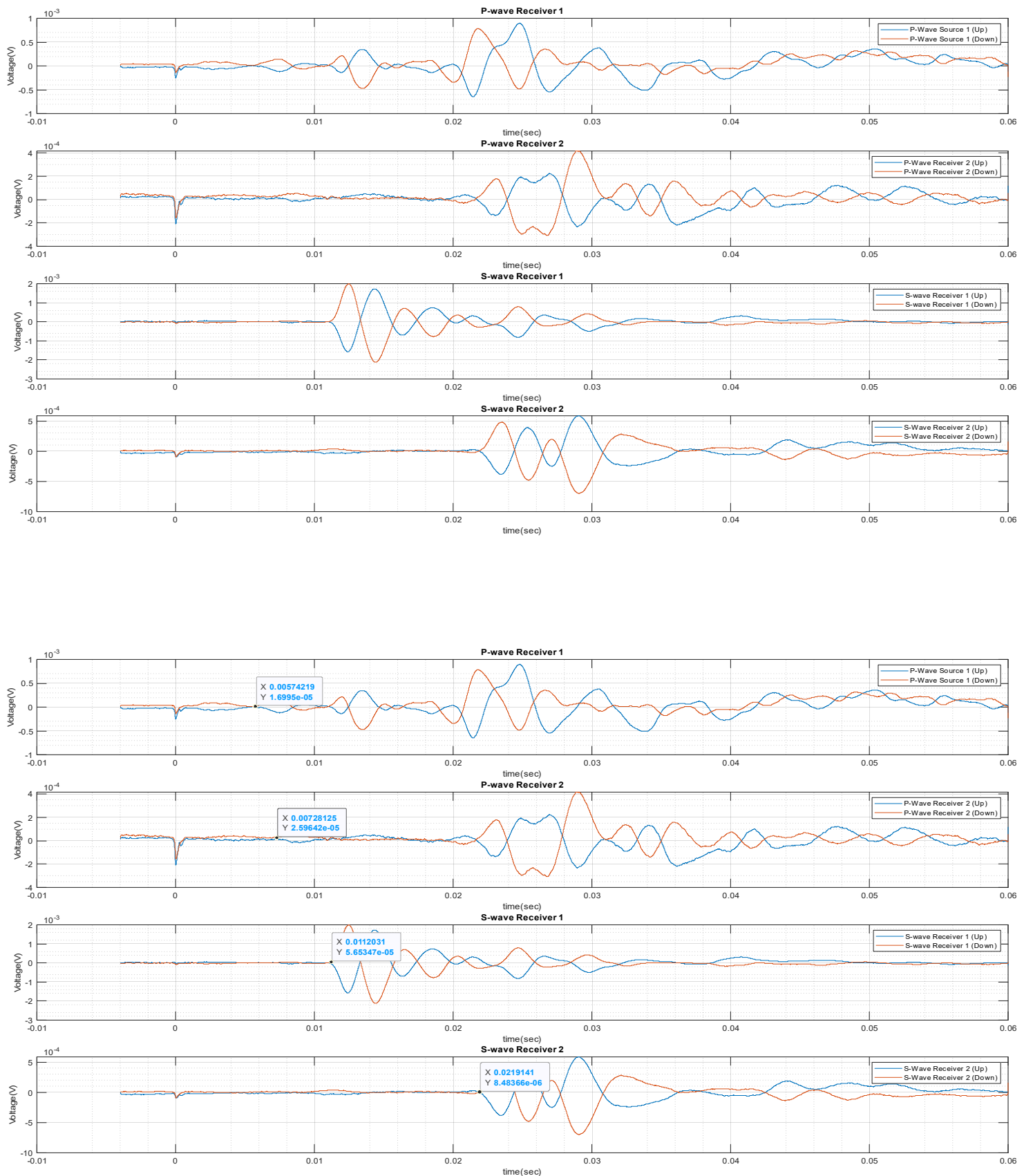

Data Set 4 (8.14.2019)

Run00019f

R2_1 

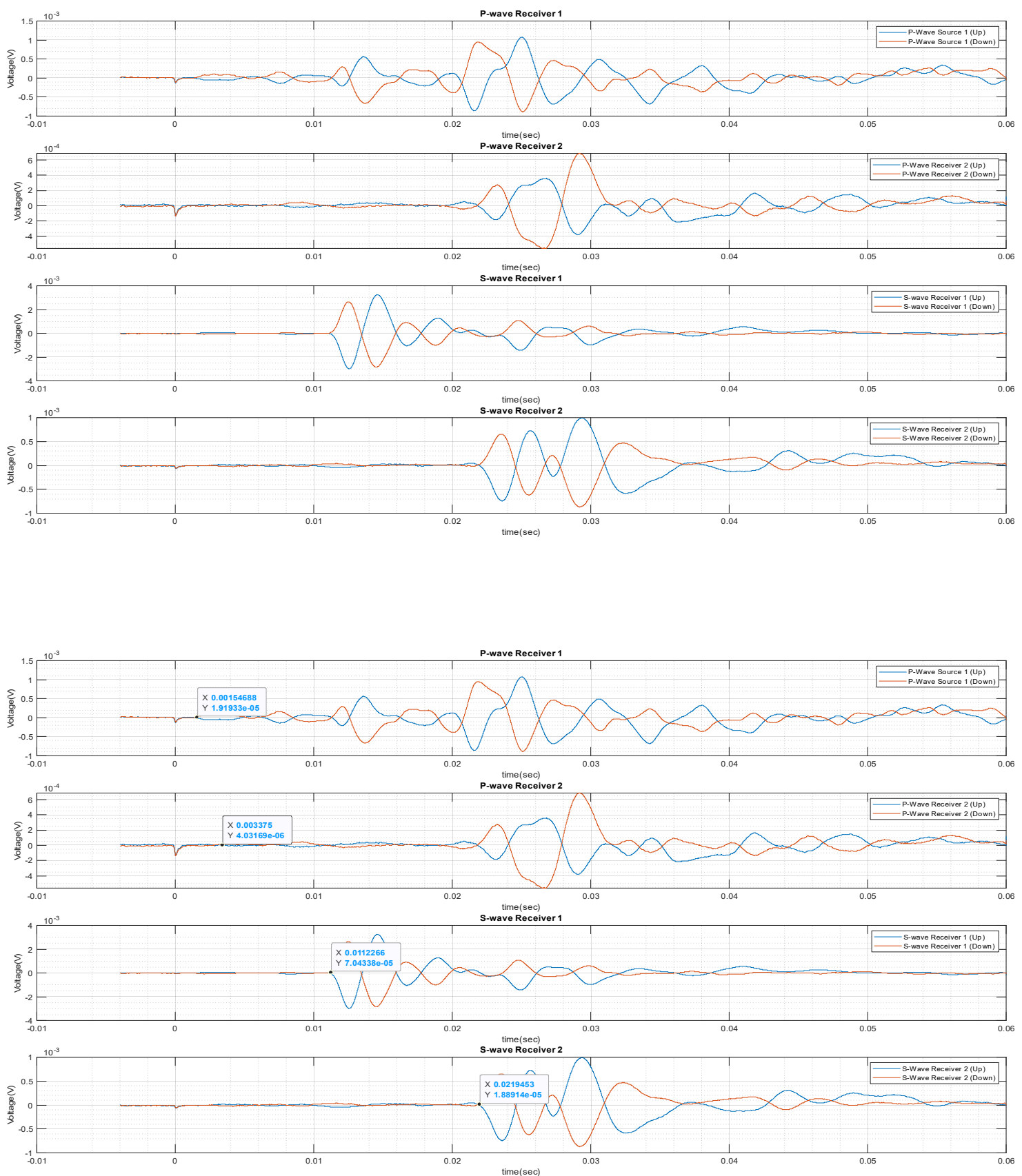

Data Set 4 (8.14.2019)

Run00020f

R2_2 

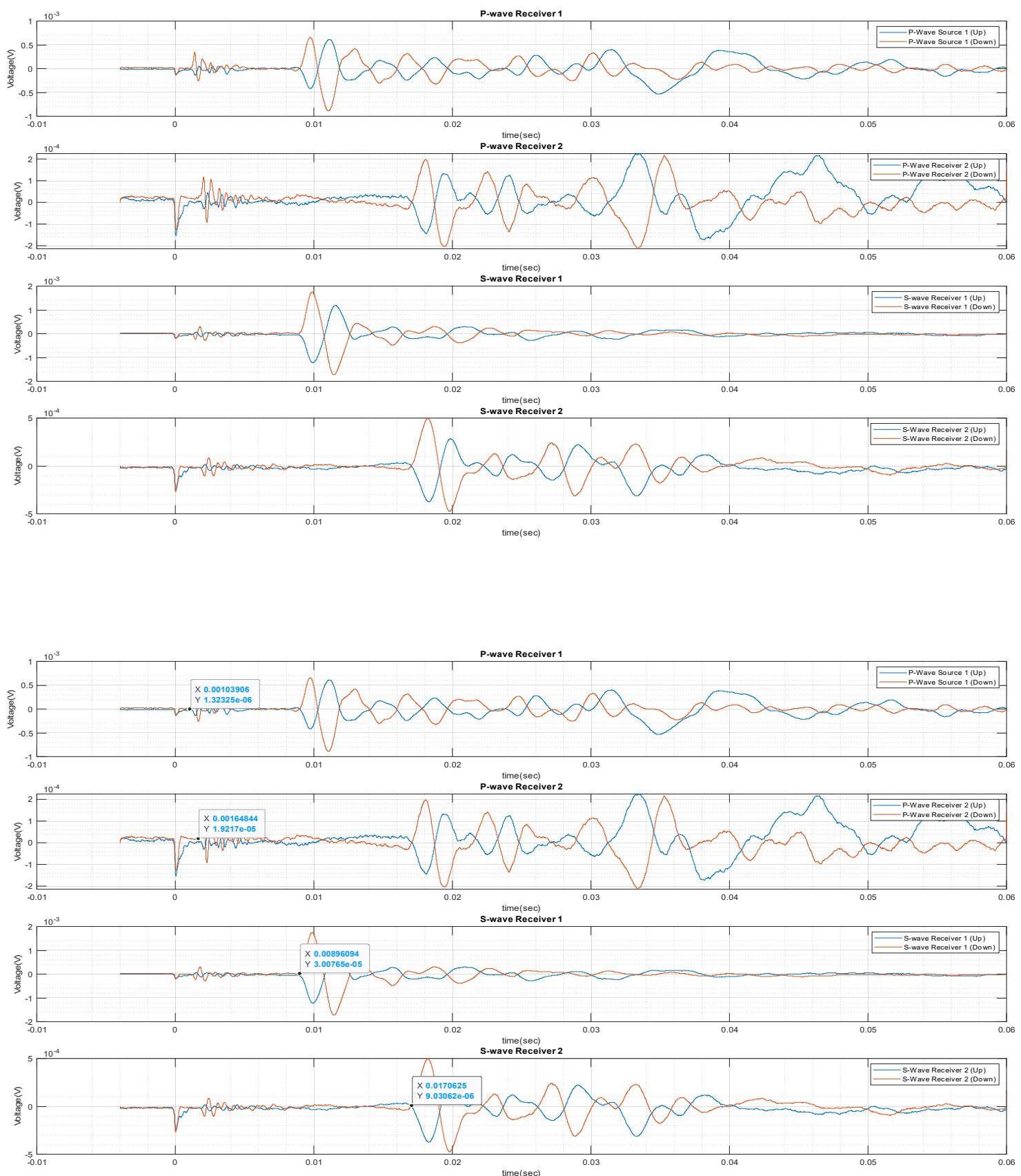

Data Set 4 (8.14.2019)

Run00021f

R3_1 

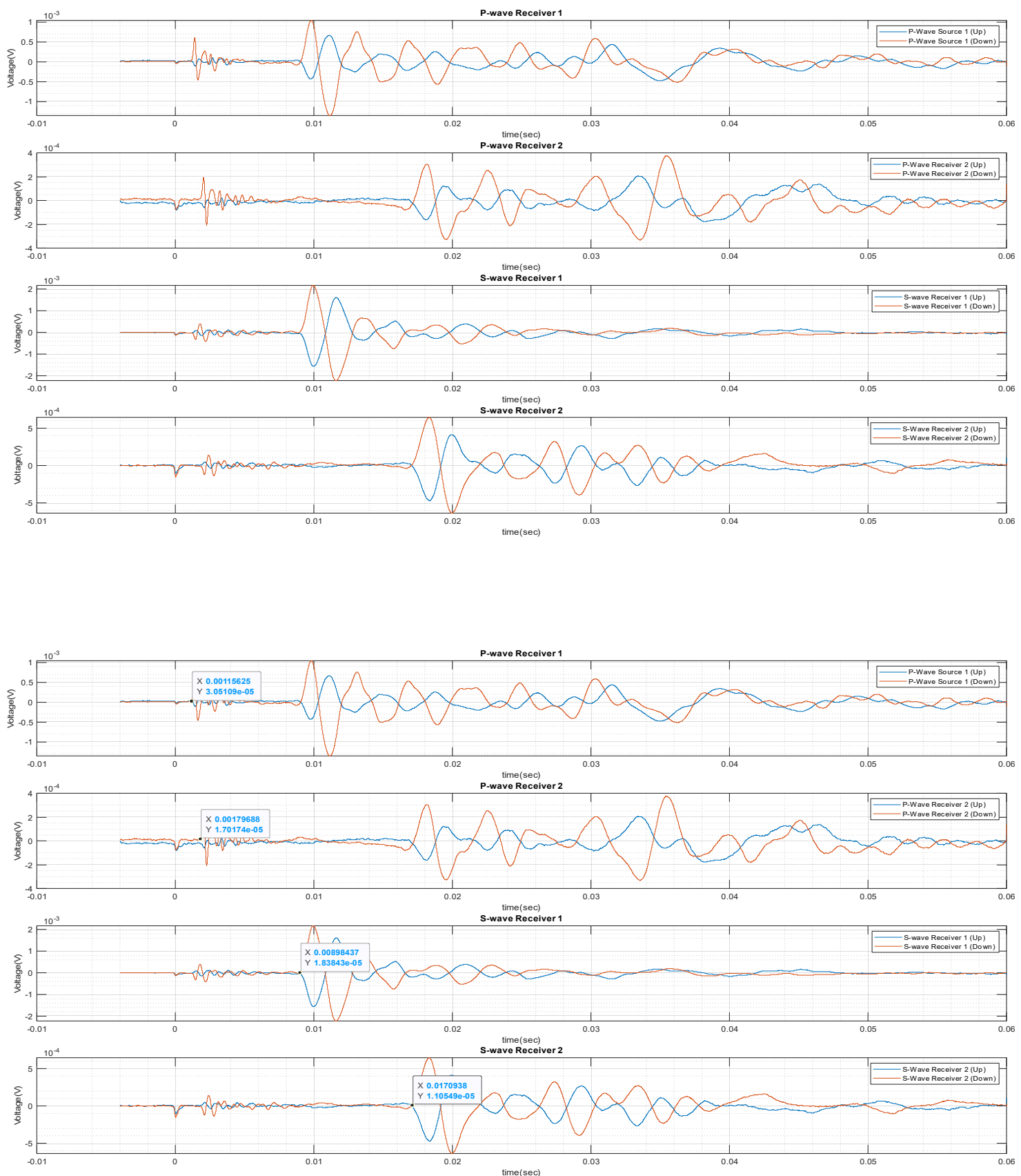

Data Set 4 (8.14.2019)

Run00022f

R3_2 

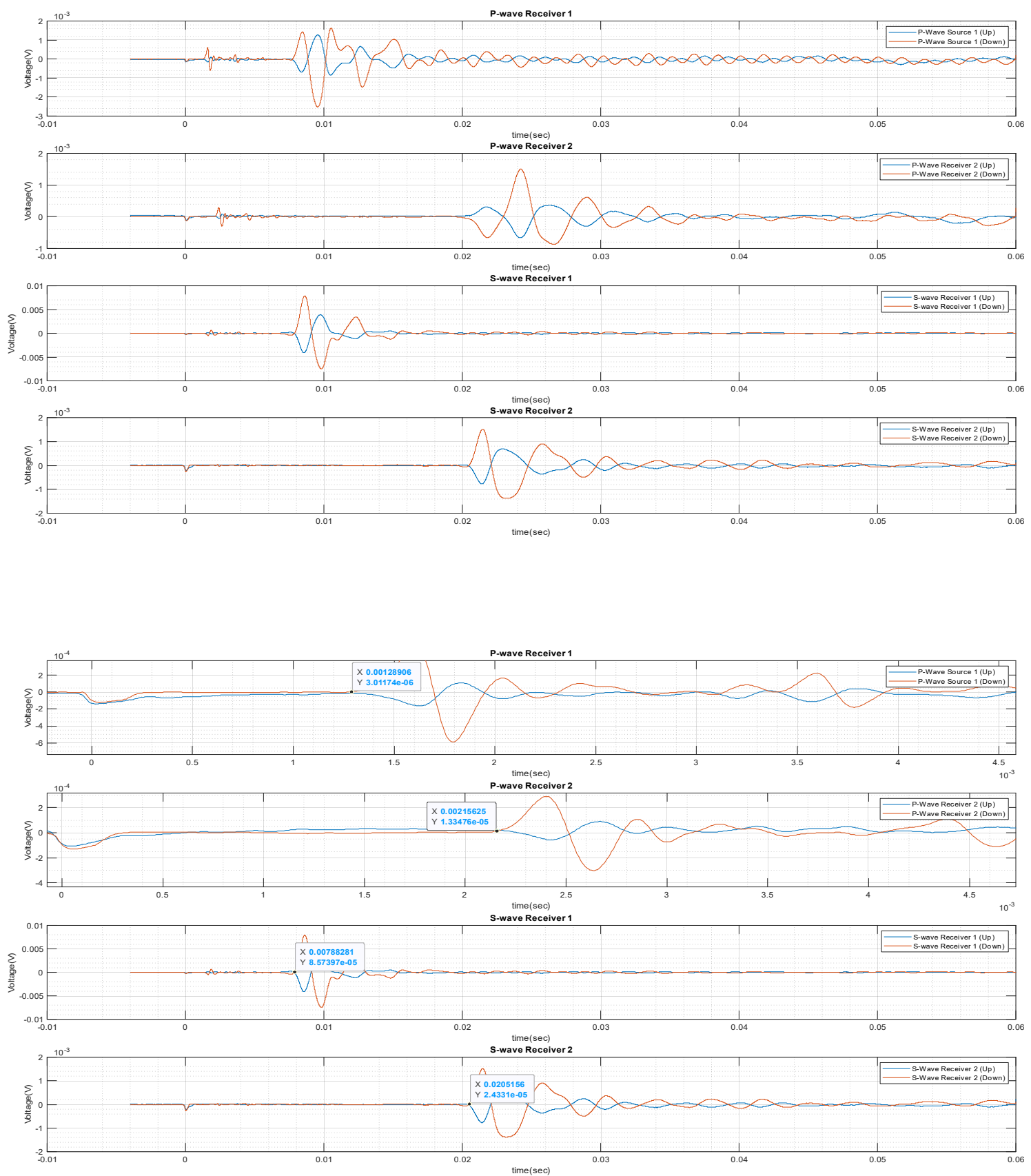

Data Set 4 (8.14.2019)

Run00023f

R4_1 

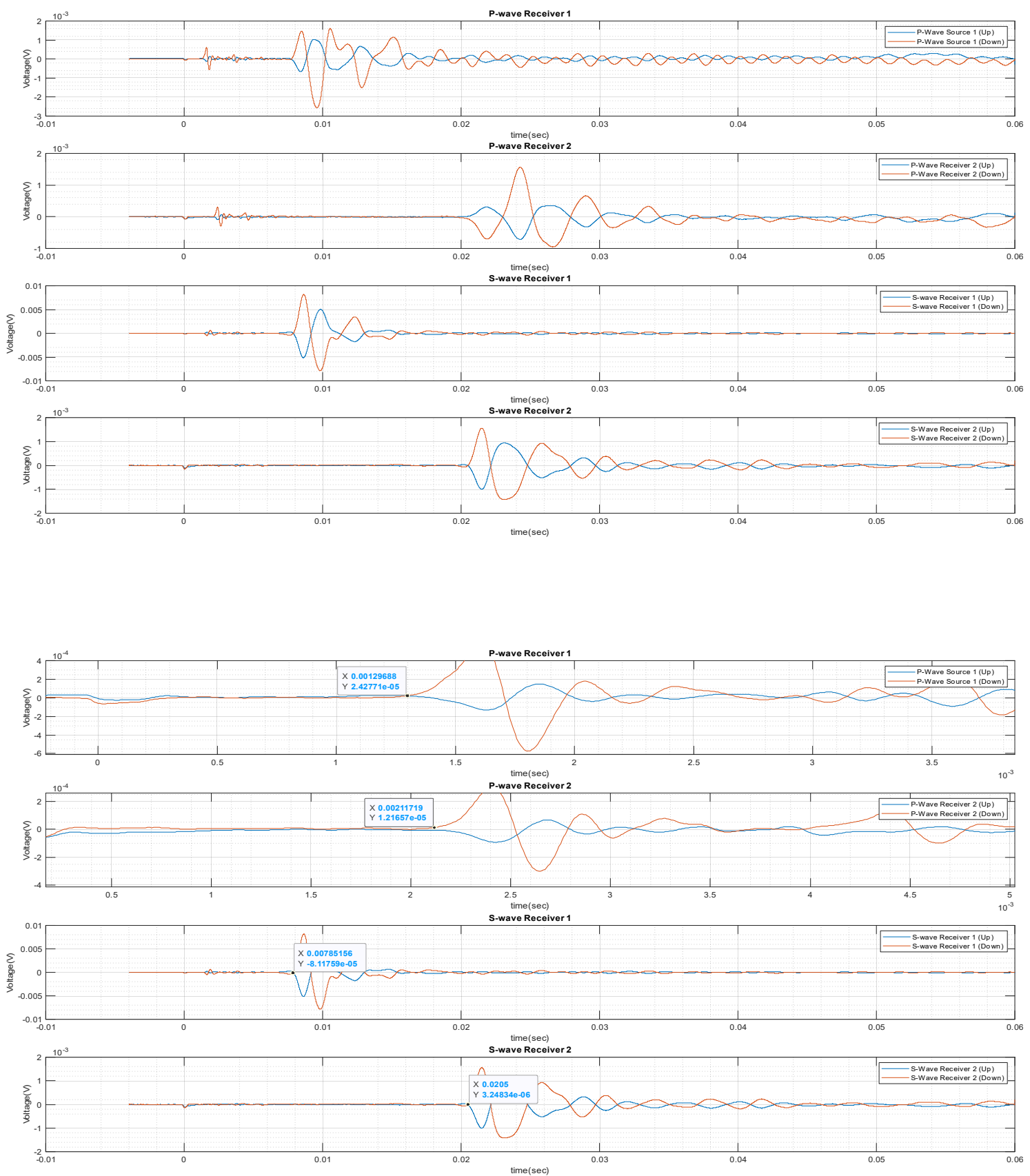

Data Set 4 (8.14.2019)

Run00024f

R4 2 
Appendix P: During treatment: Crosshole geophone voltage time series for $V_{s}$ and

\section{$V_{p}$ arrival selection.}
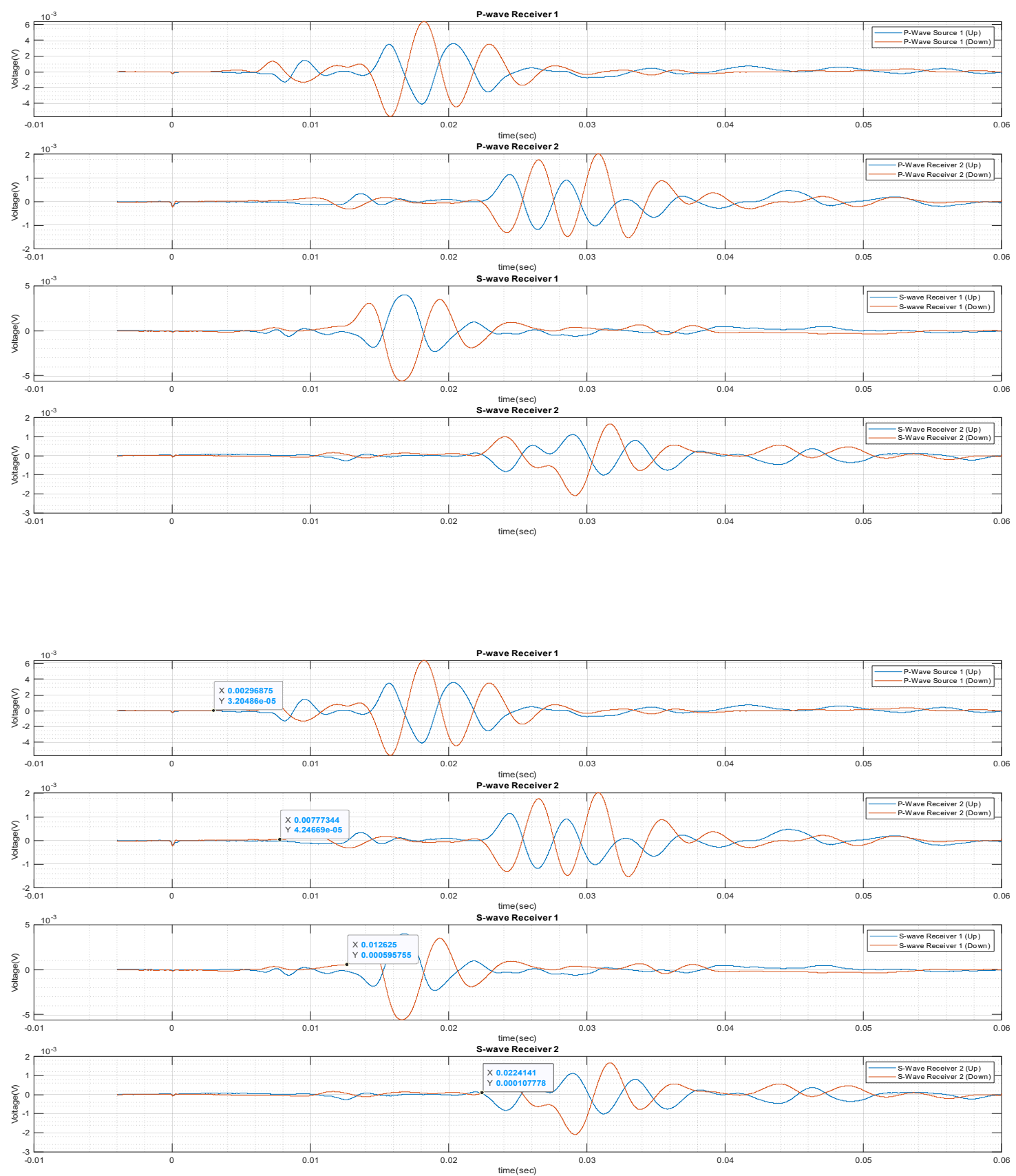

Data Set $5(8.15 .2019)$

Run00025f

R1_1 

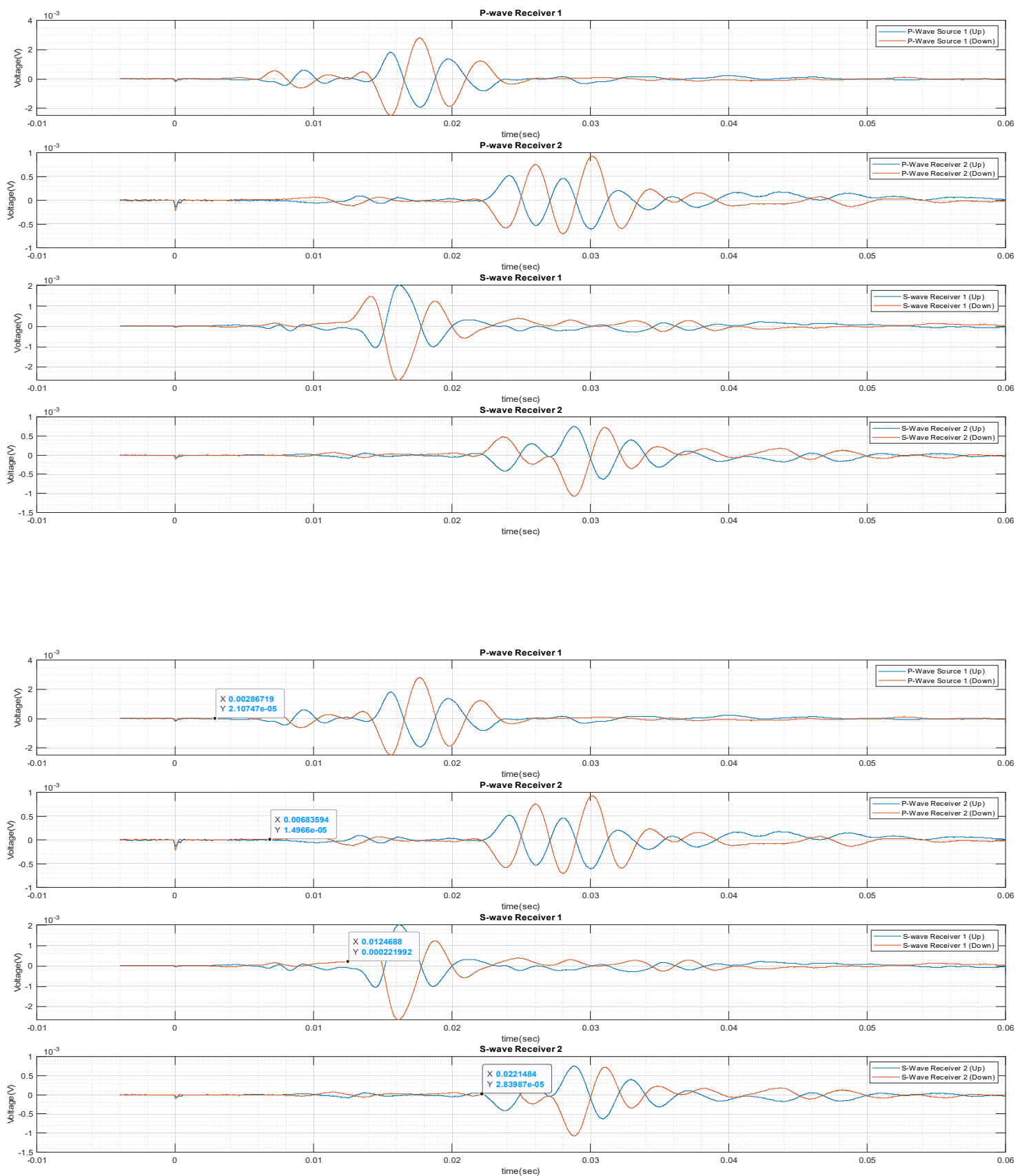

Data Set 5 (8.15.2019)

Run00026f

R1_2 

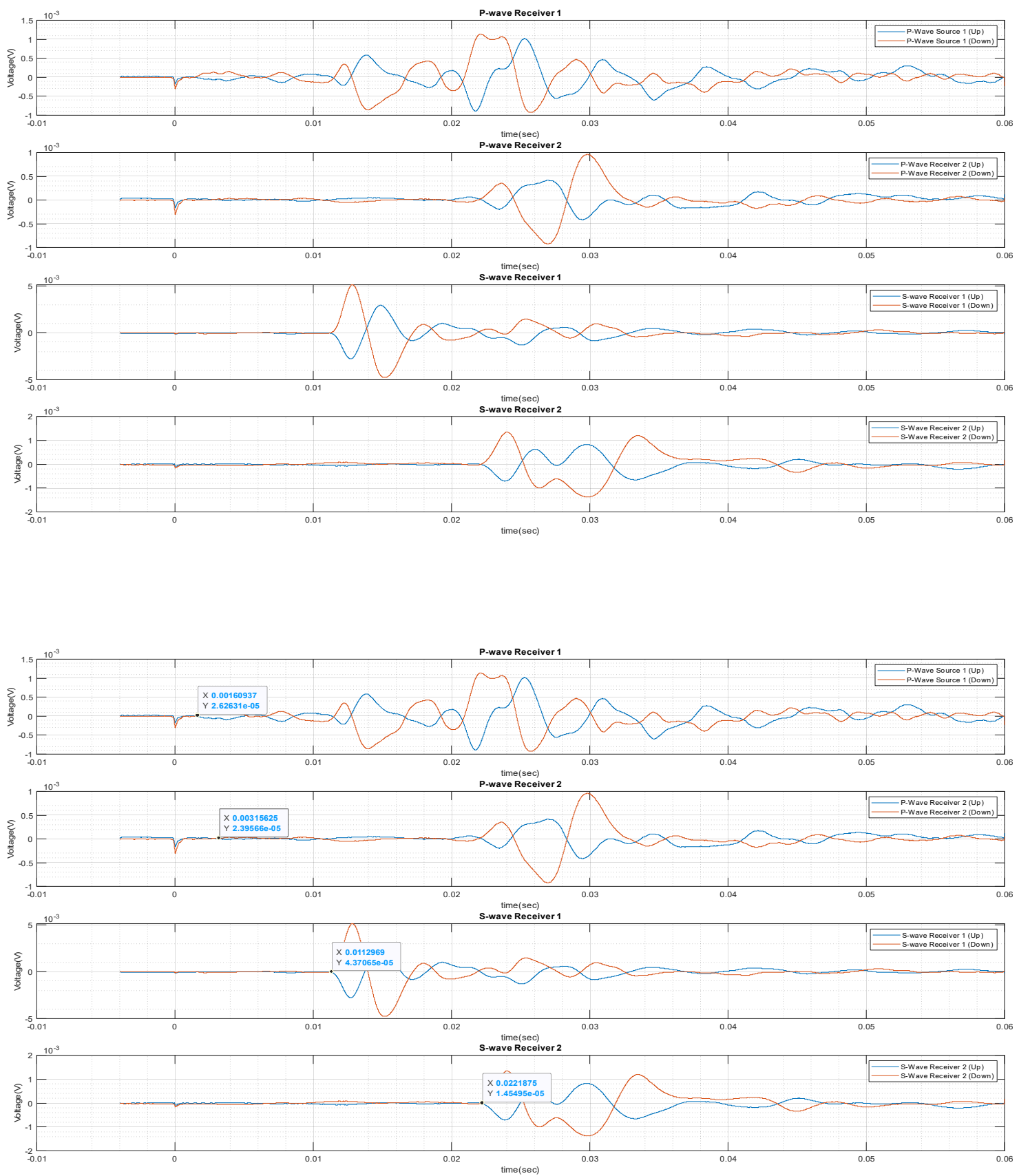

Data Set 5 (8.15.2019)

Run00027f

R2_1 

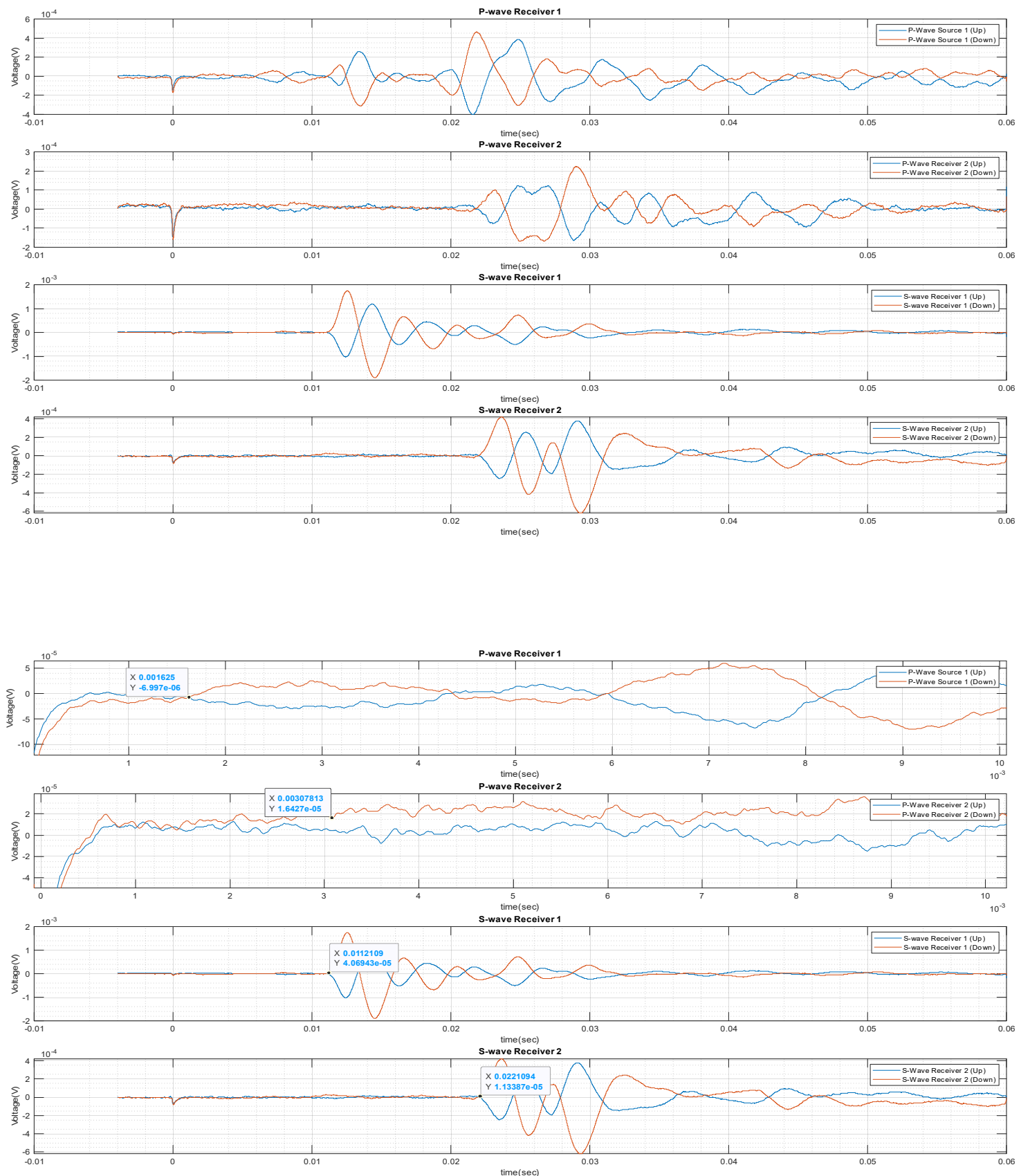

Data Set 5 (8.15.2019)

Run00028f

R2_2 

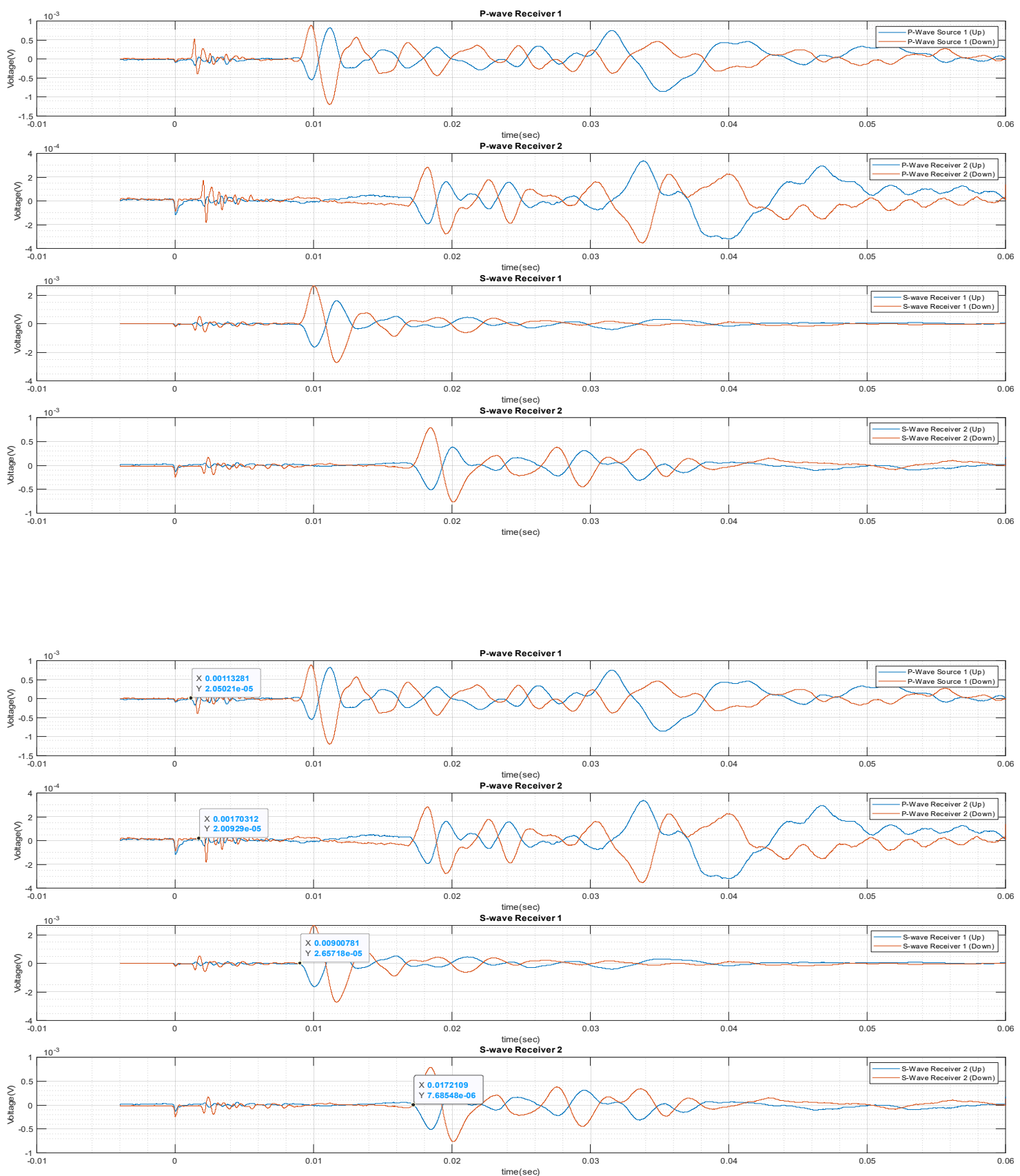

Data Set 5 (8.15.2019)

Run00029f

R3_1 

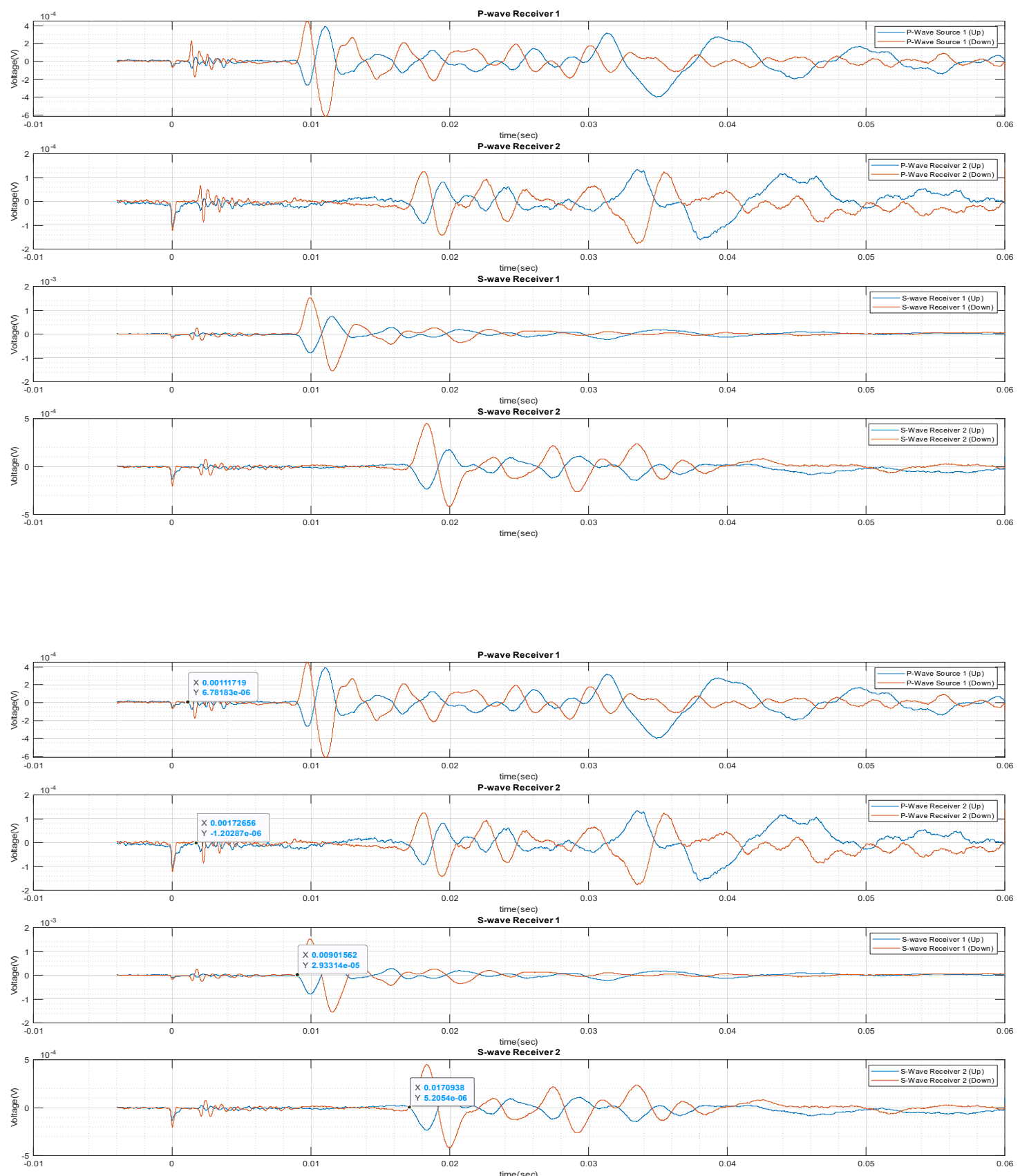

Data Set 5 (8.15.2019)

Run00030f

R3_2 

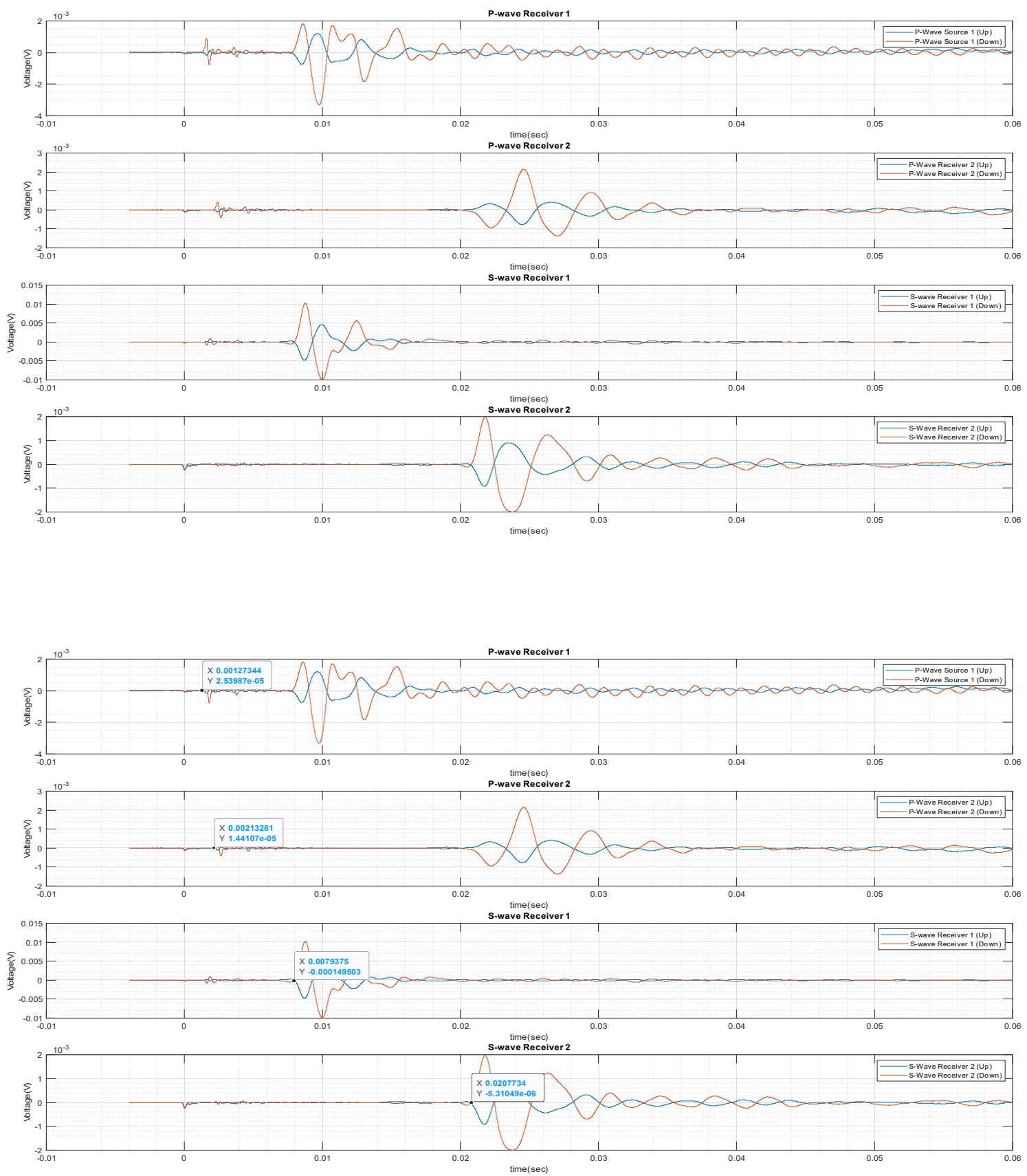

Data Set 5 (8.15.2019)

Run00031f

R4_1 

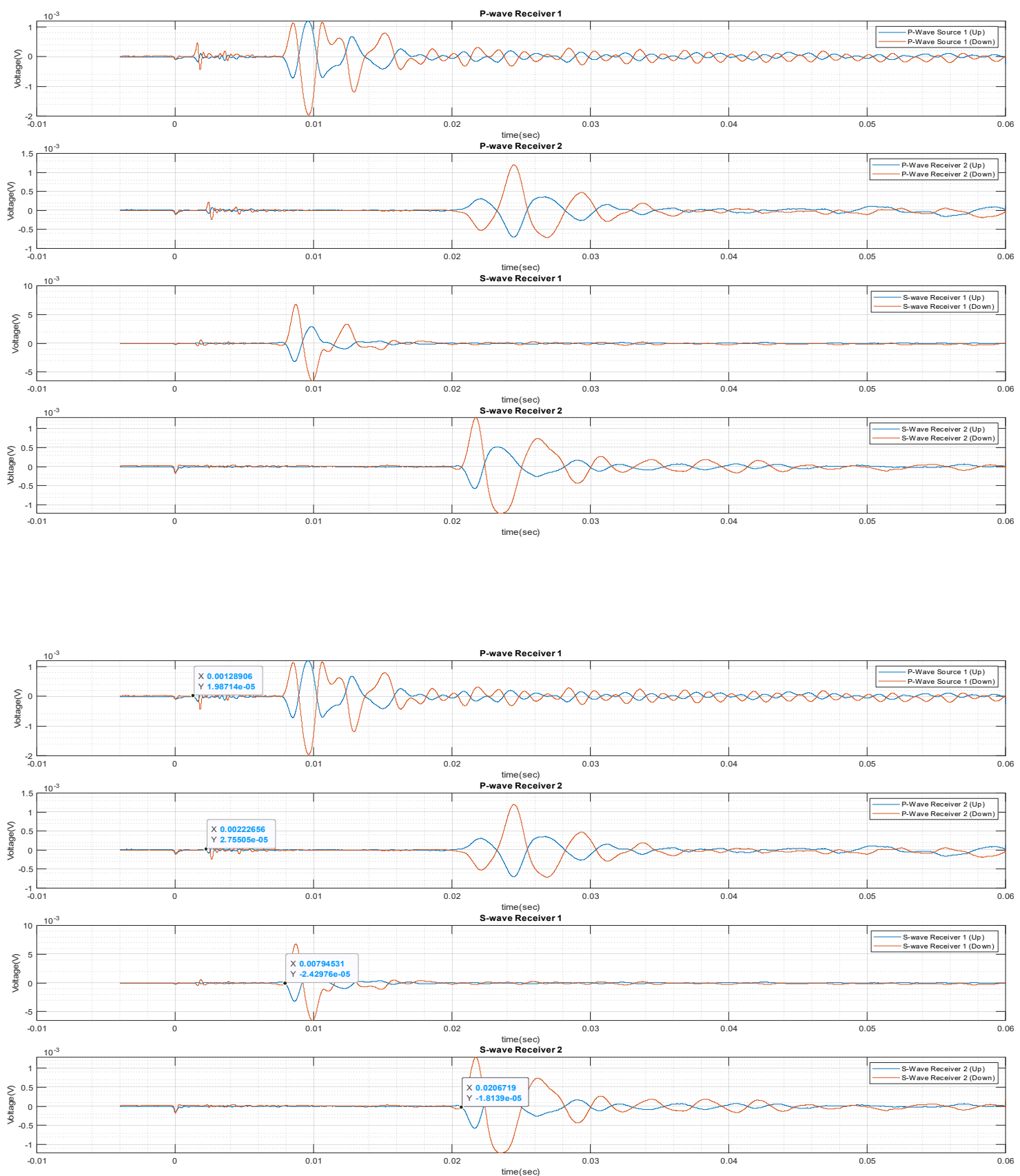

Data Set 5 (8.15.2019)

Run00032f

R4_2 

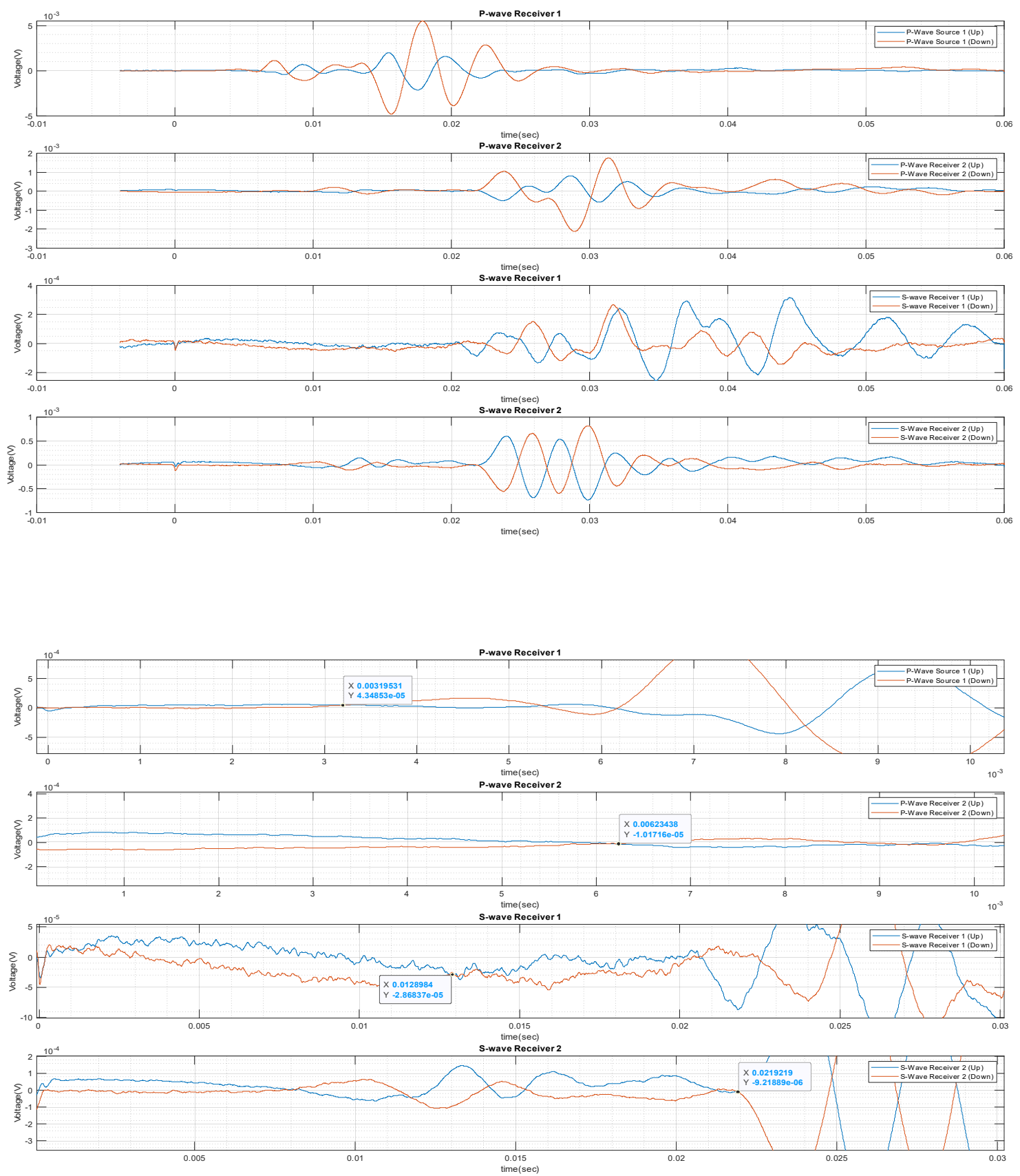

Data Set $6(8.16 .2019)$

Run00033f

R1_1 

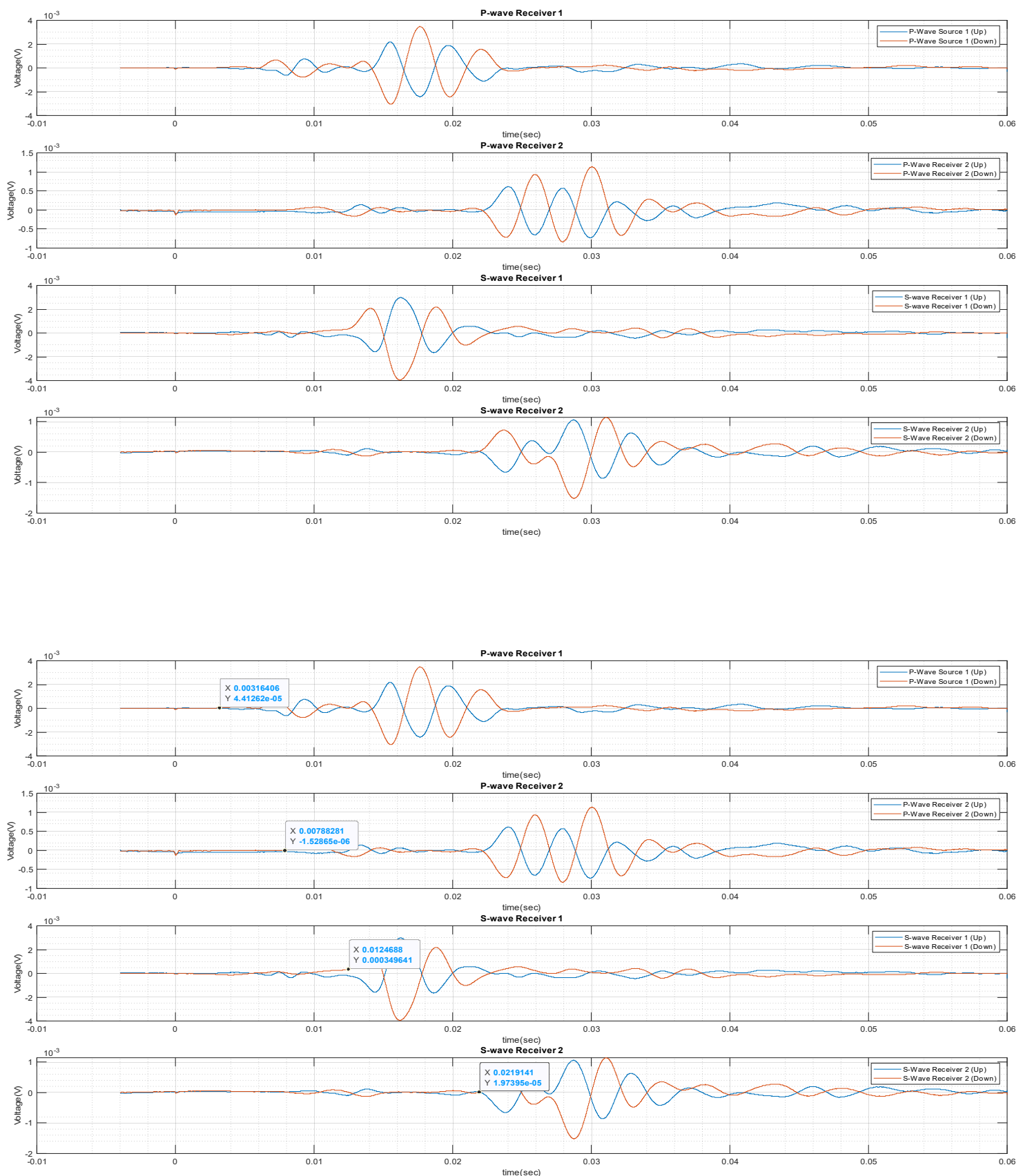

Data Set 6 (8.16.2019)

Run00034f

R1_2 

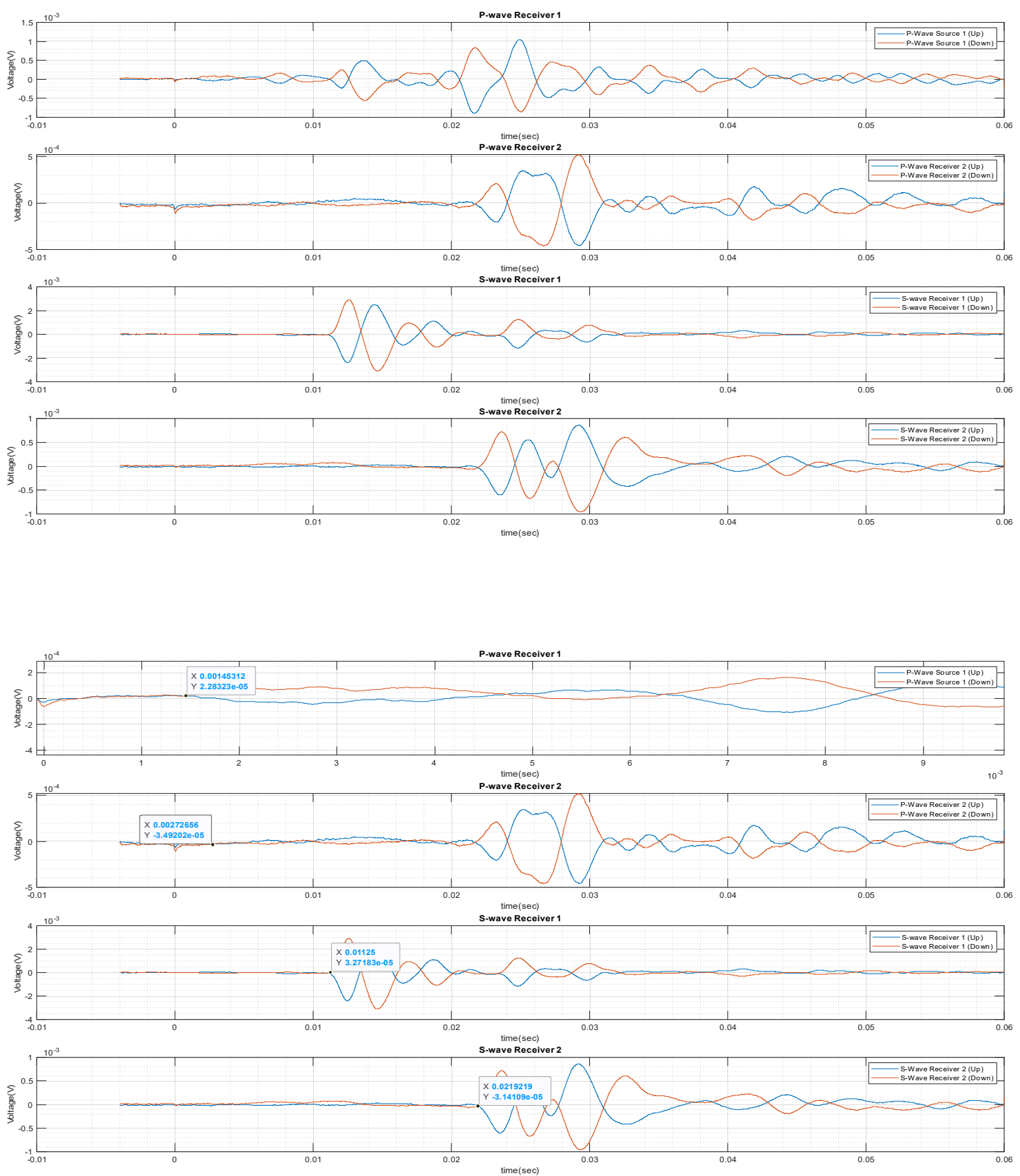

Data Set 6 (8.16.2019)

Run00035f

R2_1 

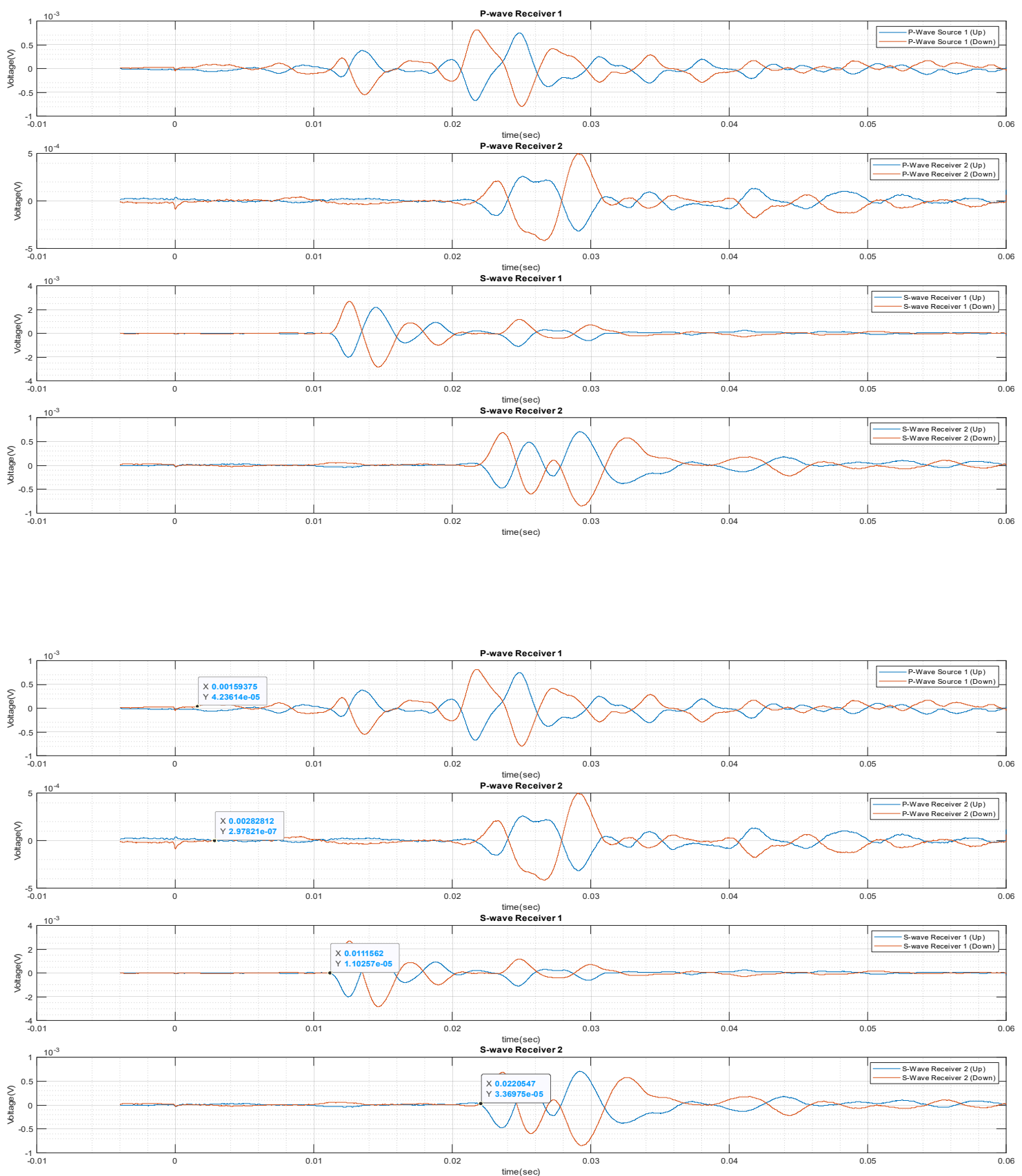

Data Set 6 (8.16.2019)

Run00036f

R2_2 

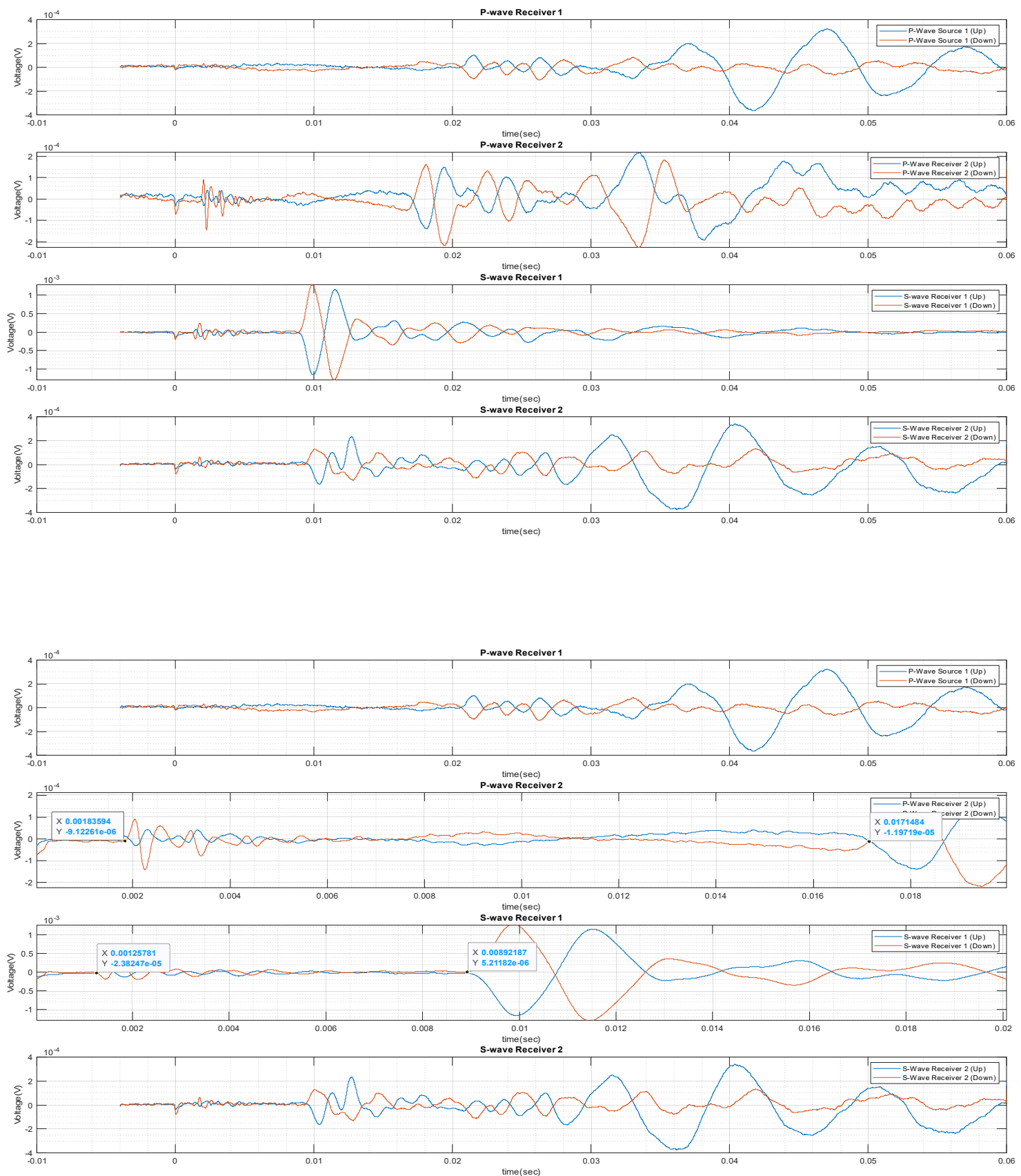

Data Set 6 (8.16.2019)

Run00037f

R3_1 

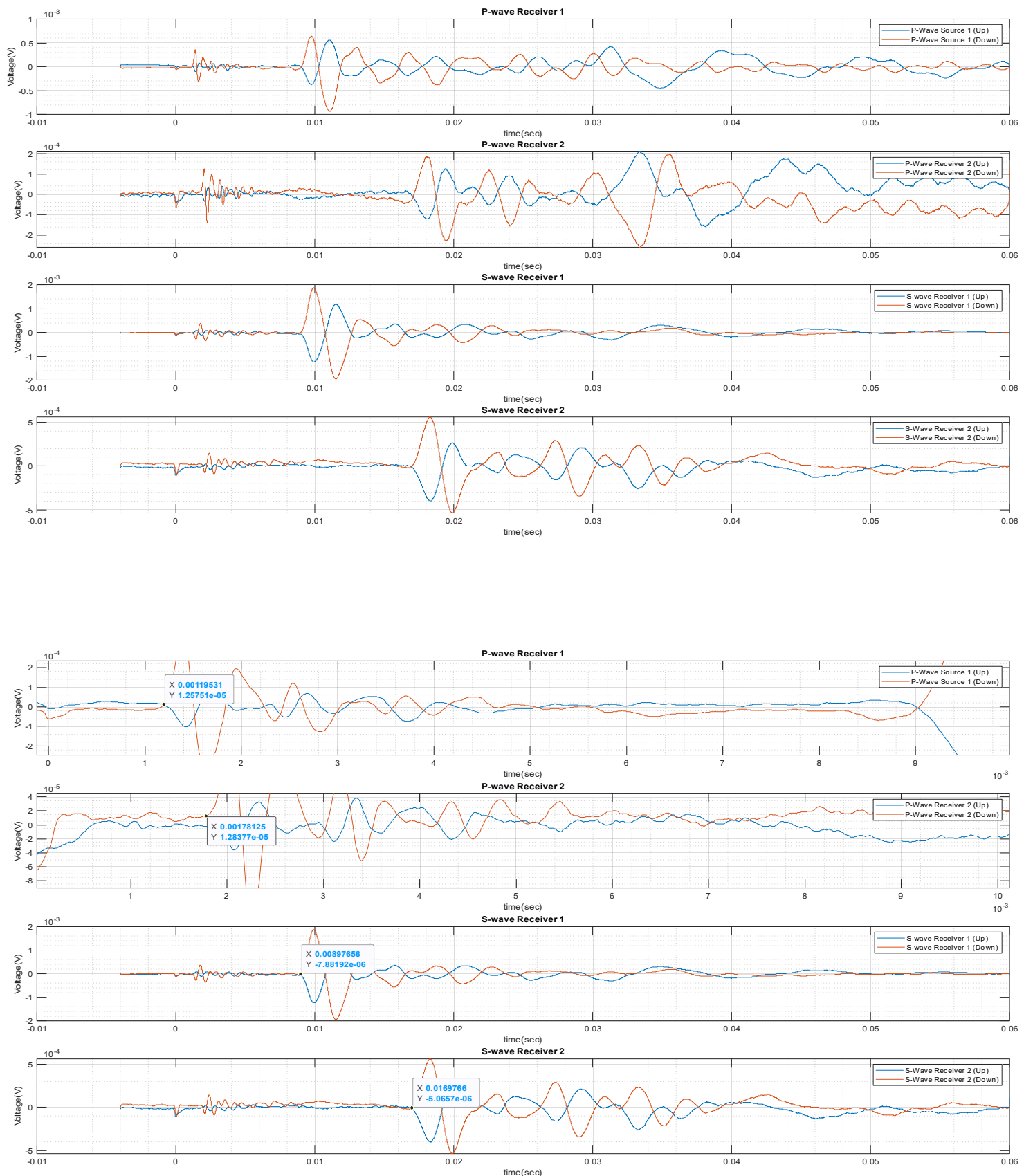

Data Set 6 (8.16.2019)

Run00038f

R3_2 

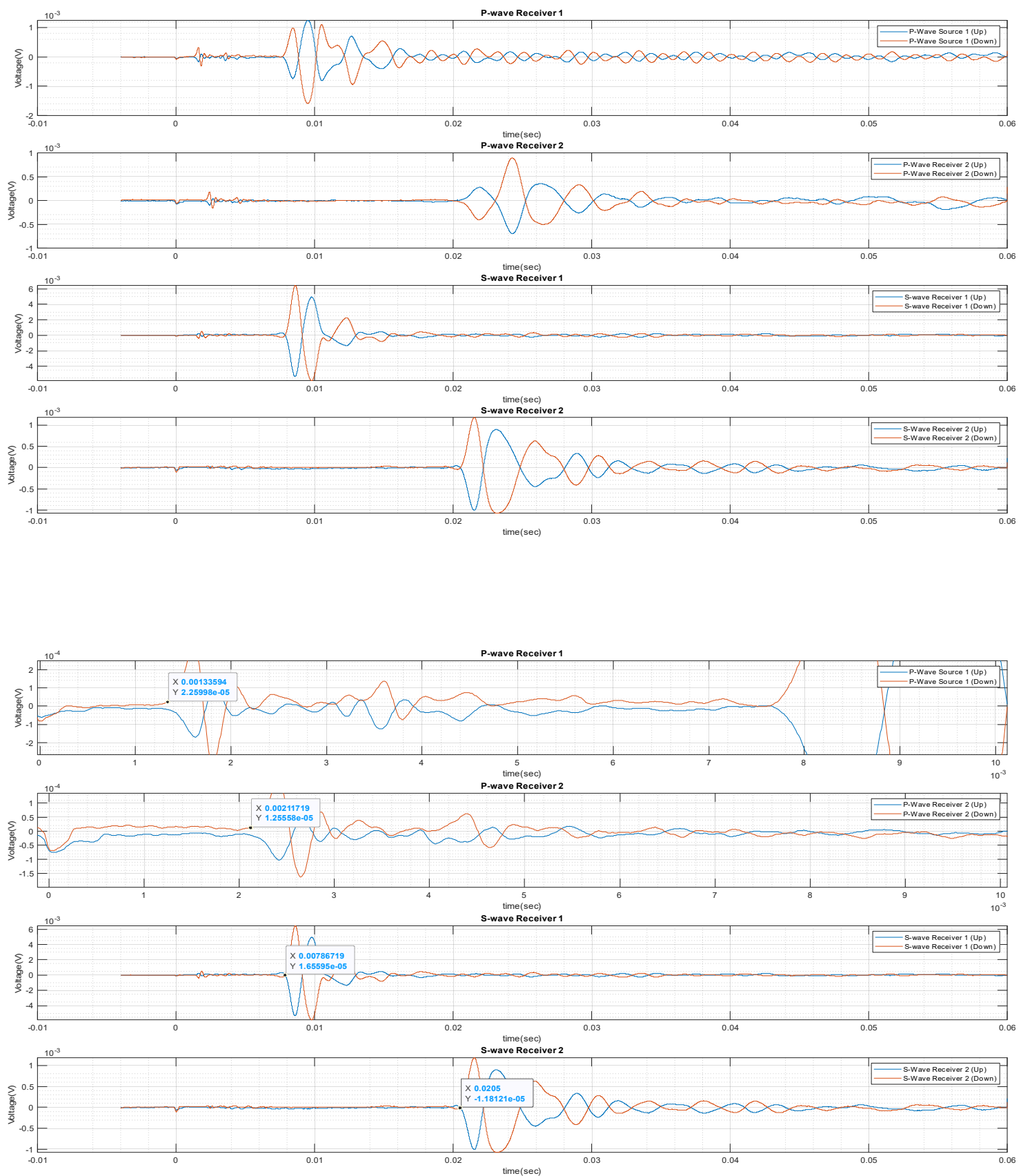

Data Set 6 (8.16.2019)

Run00039f

R4_1 

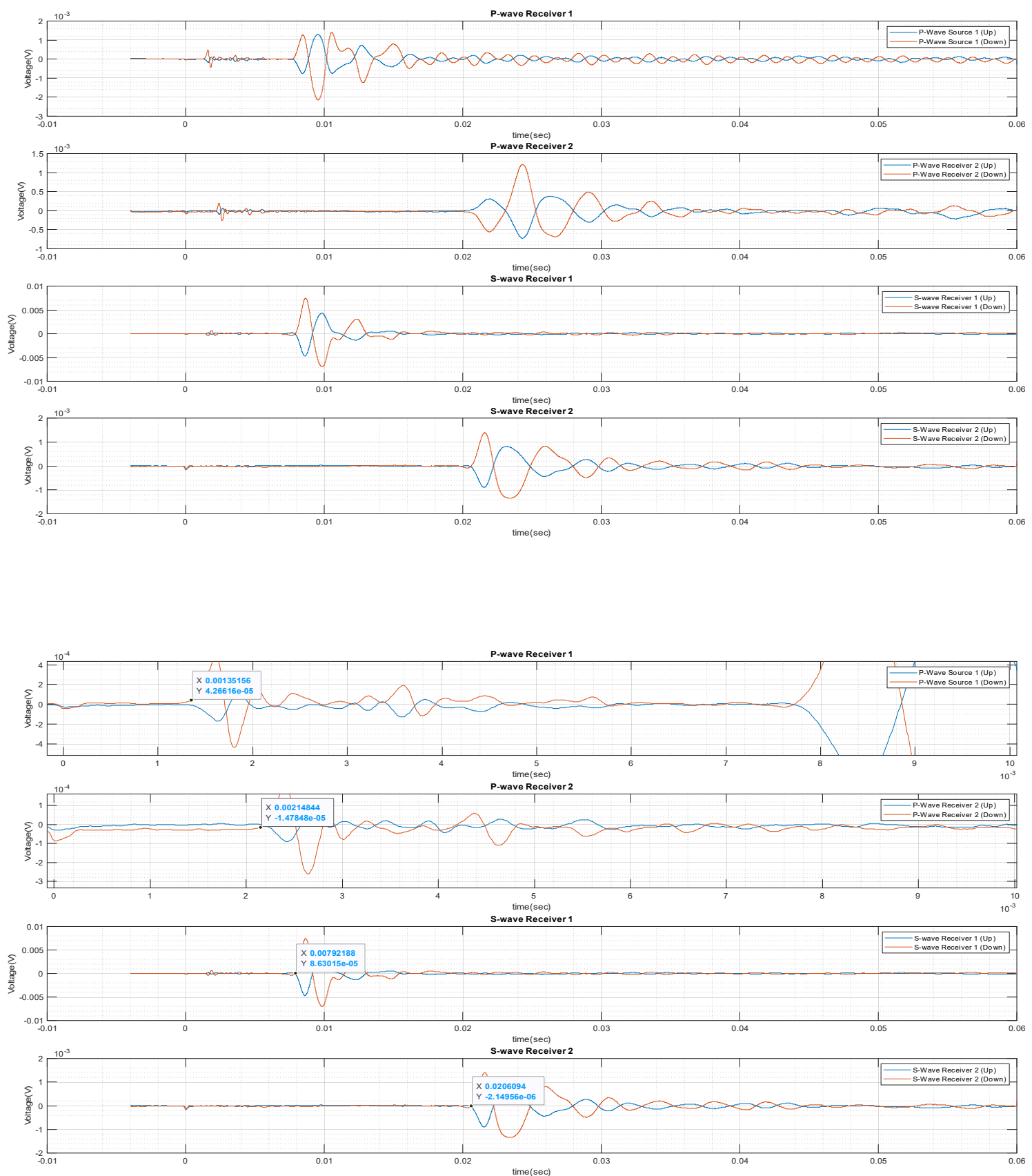

Data Set 6 (8.16.2019)

Run00040f

R4 2 

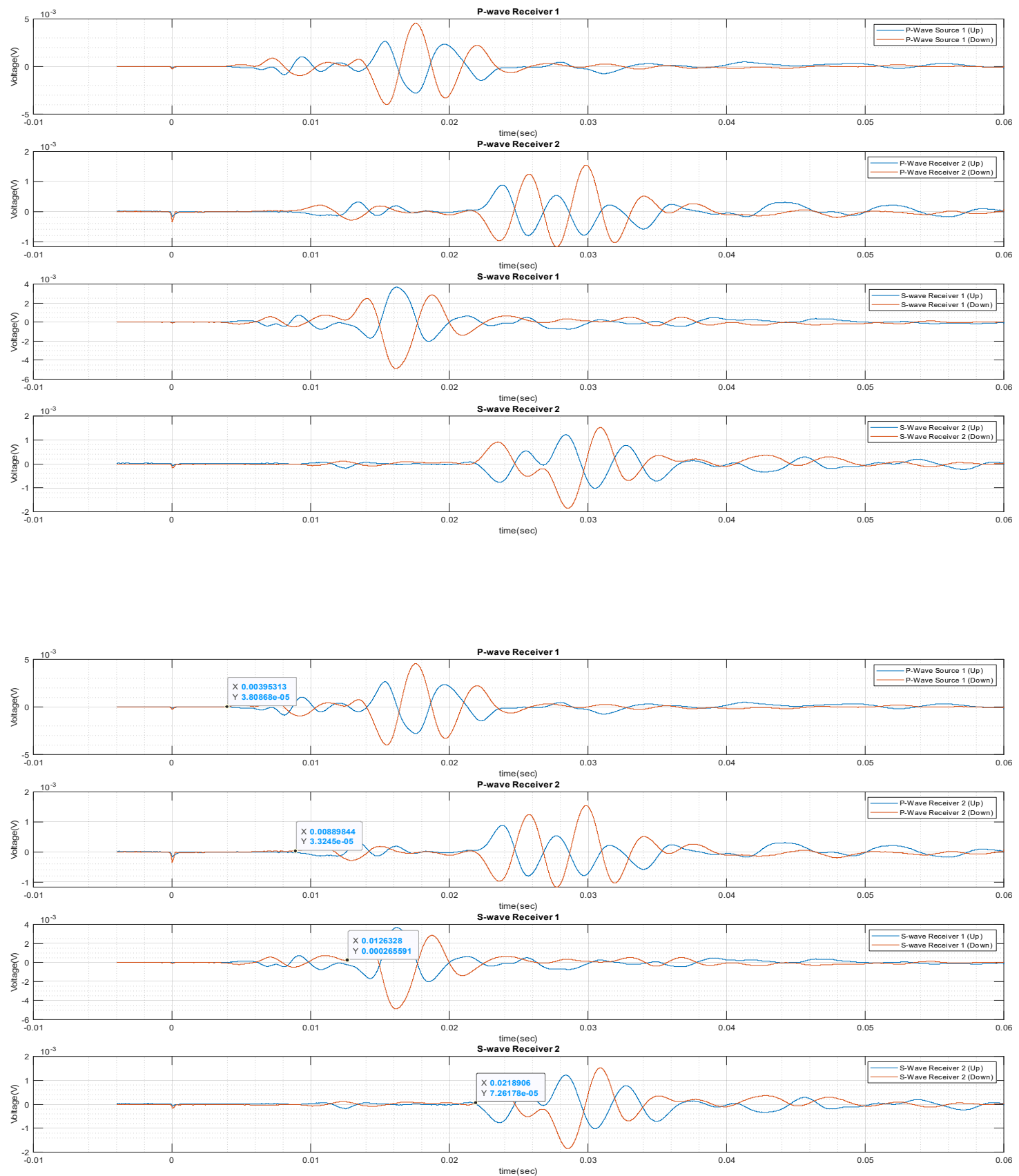

Data Set 7 (8.19.2019)

Run00041f

R1_1 

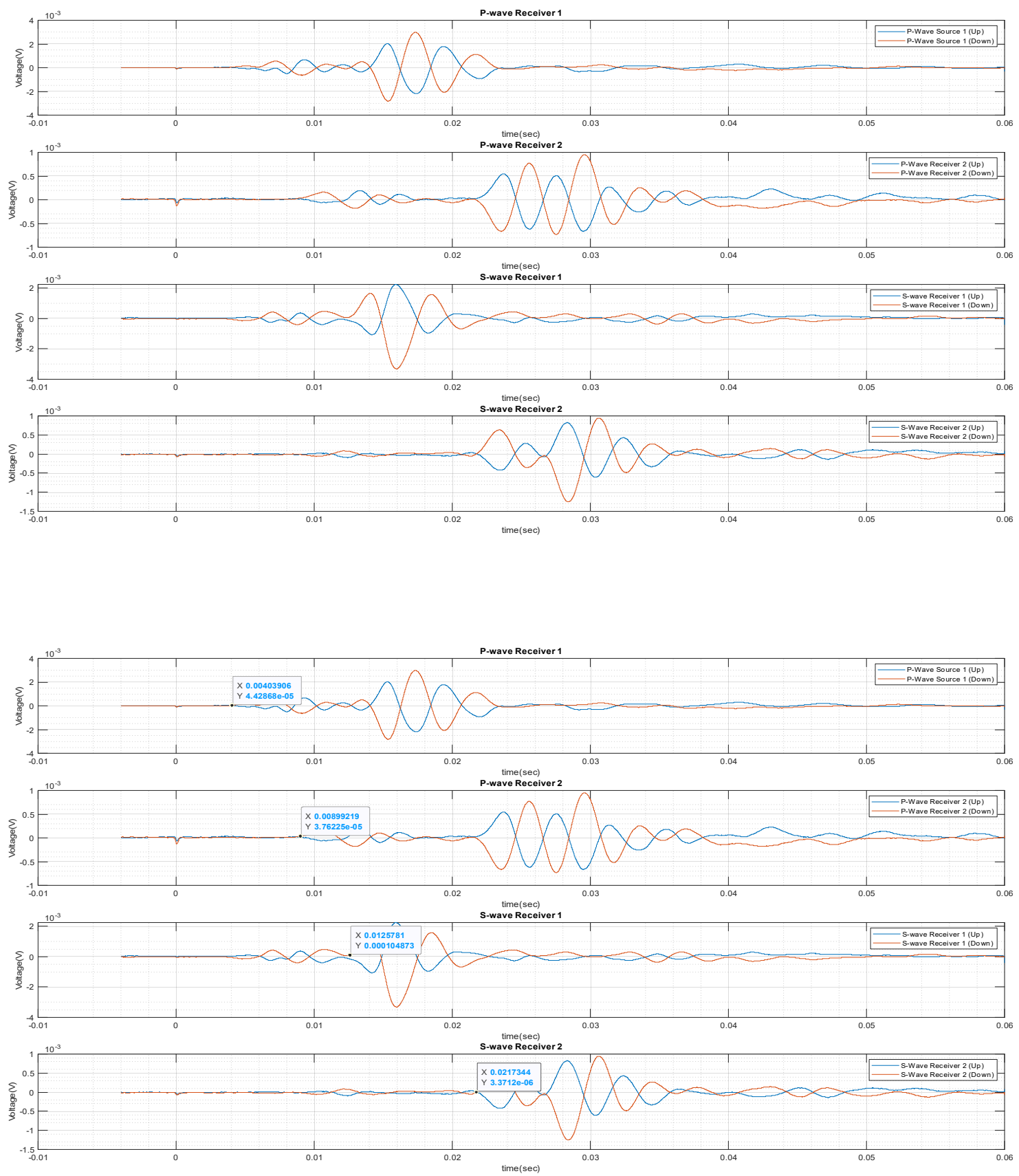

Data Set 7 (8.19.2019)

Run00042f

R1_2 

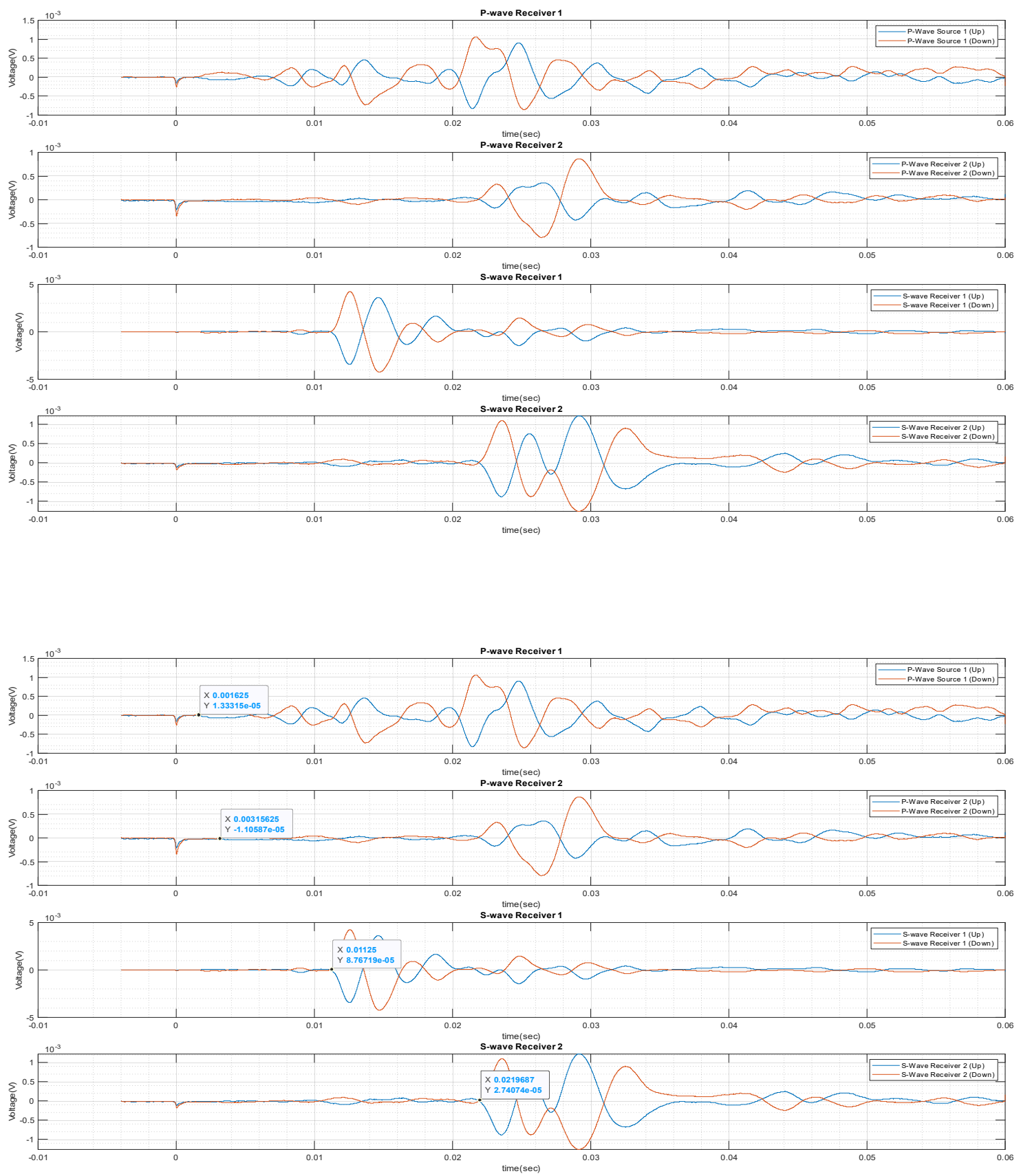

Data Set 7 (8.19.2019)

Run00043f

R2_1 

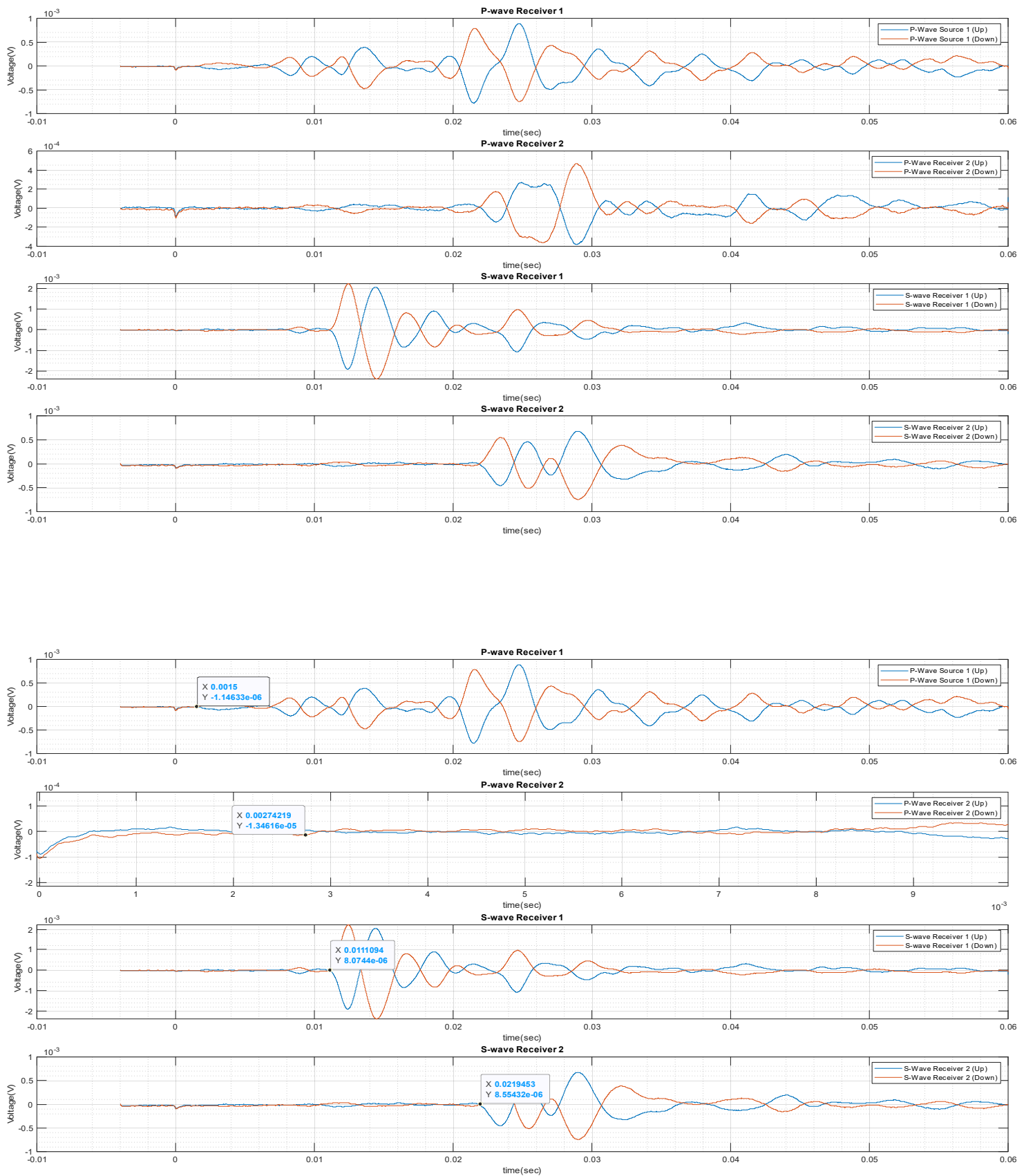

Data Set 7 (8.19.2019)

Run00044f

R2_2 

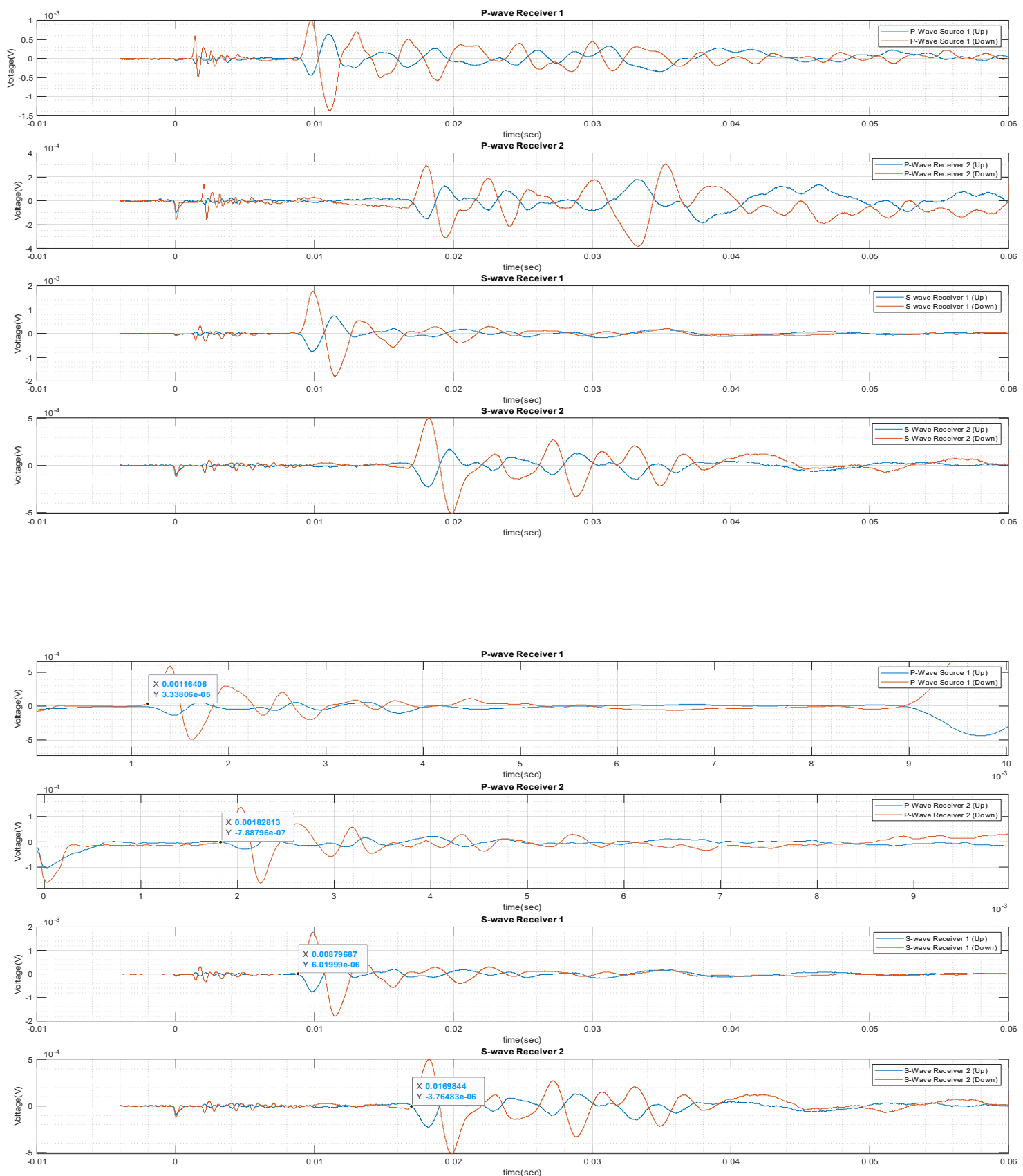

Data Set 7 (8.19.2019)

Run00045f

R3_1 

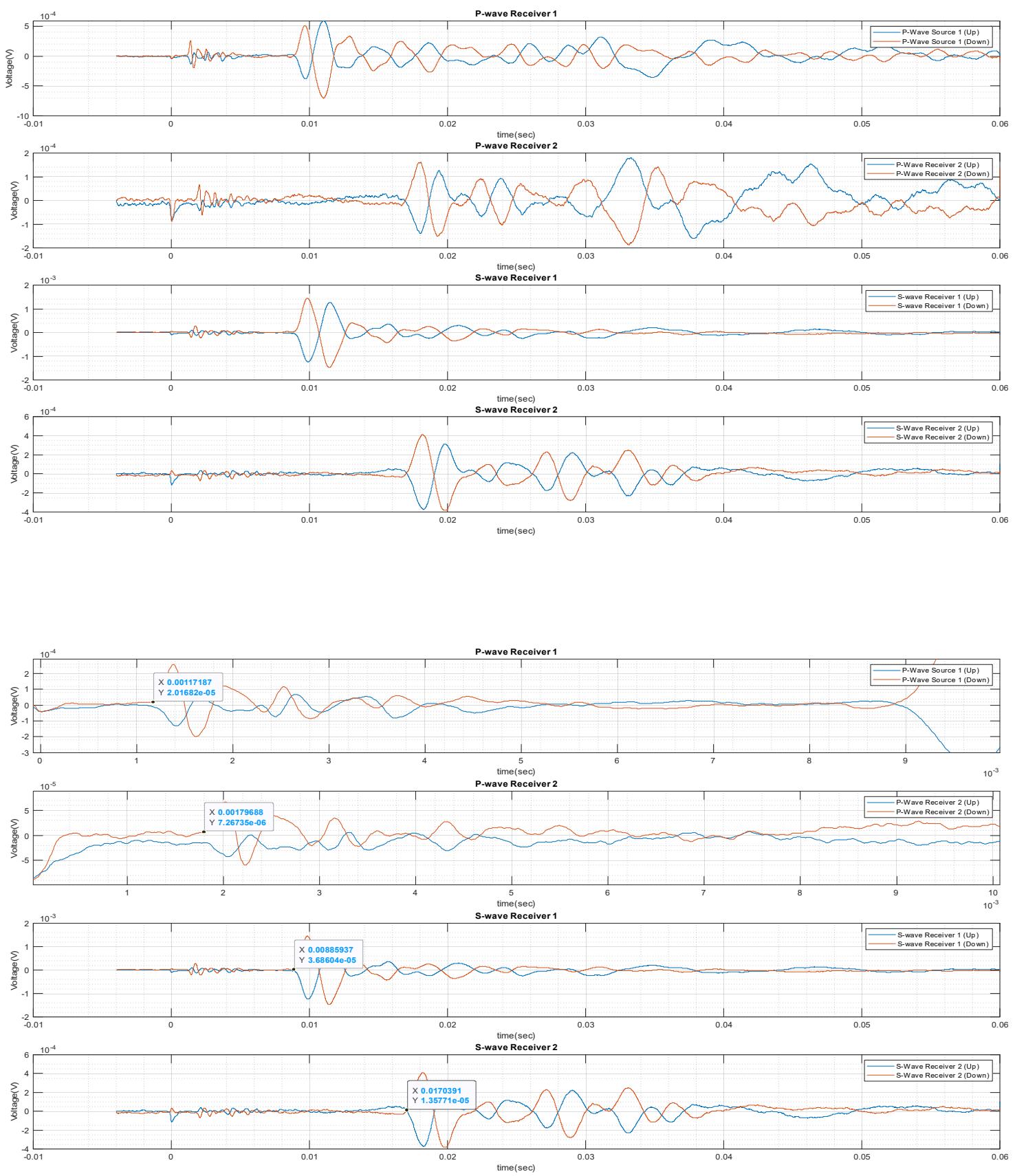

Data Set 7 (8.19.2019)

Run00046f

R3_2 

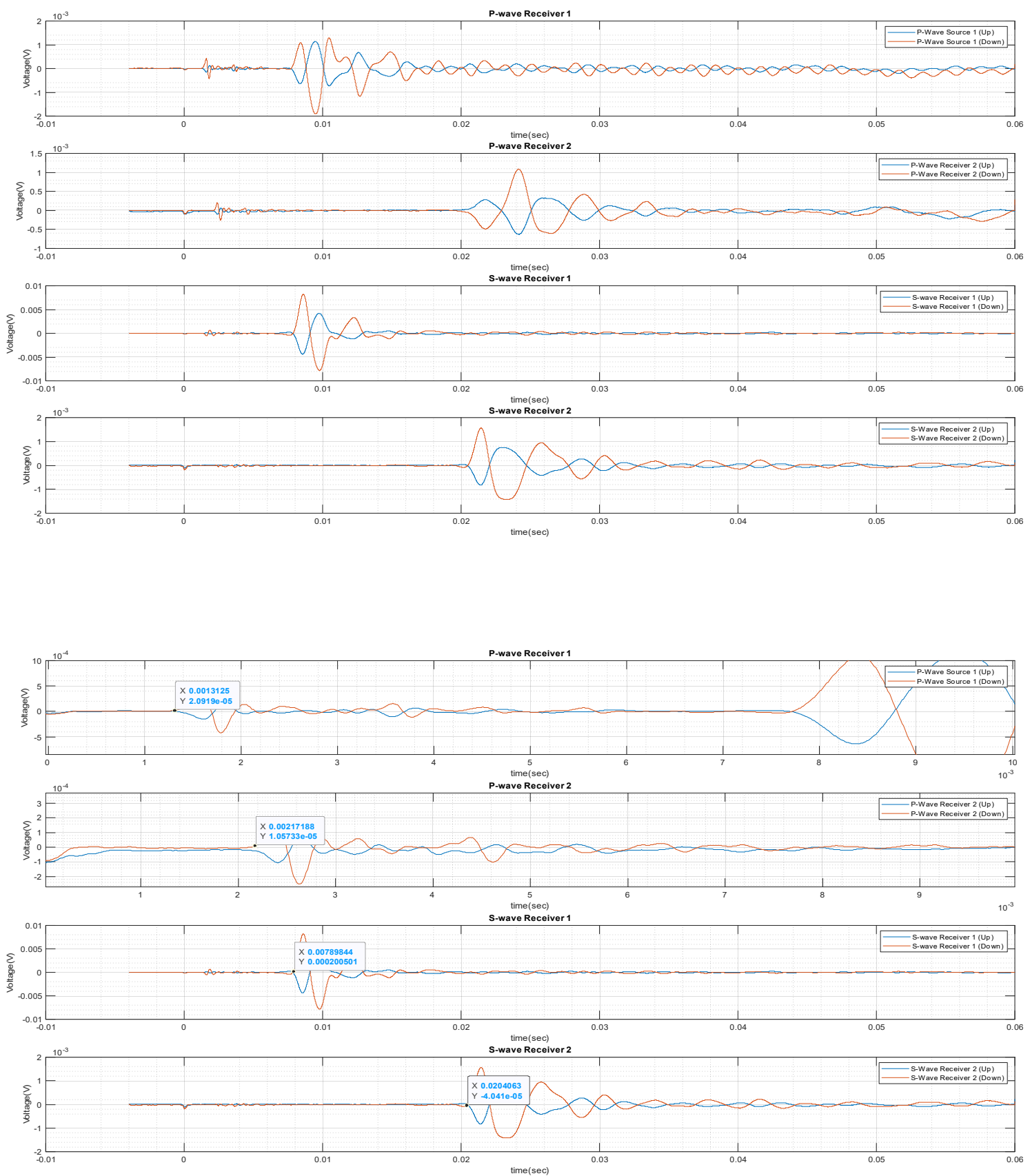

Data Set 7 (8.19.2019)

Run00047f

R4_1 

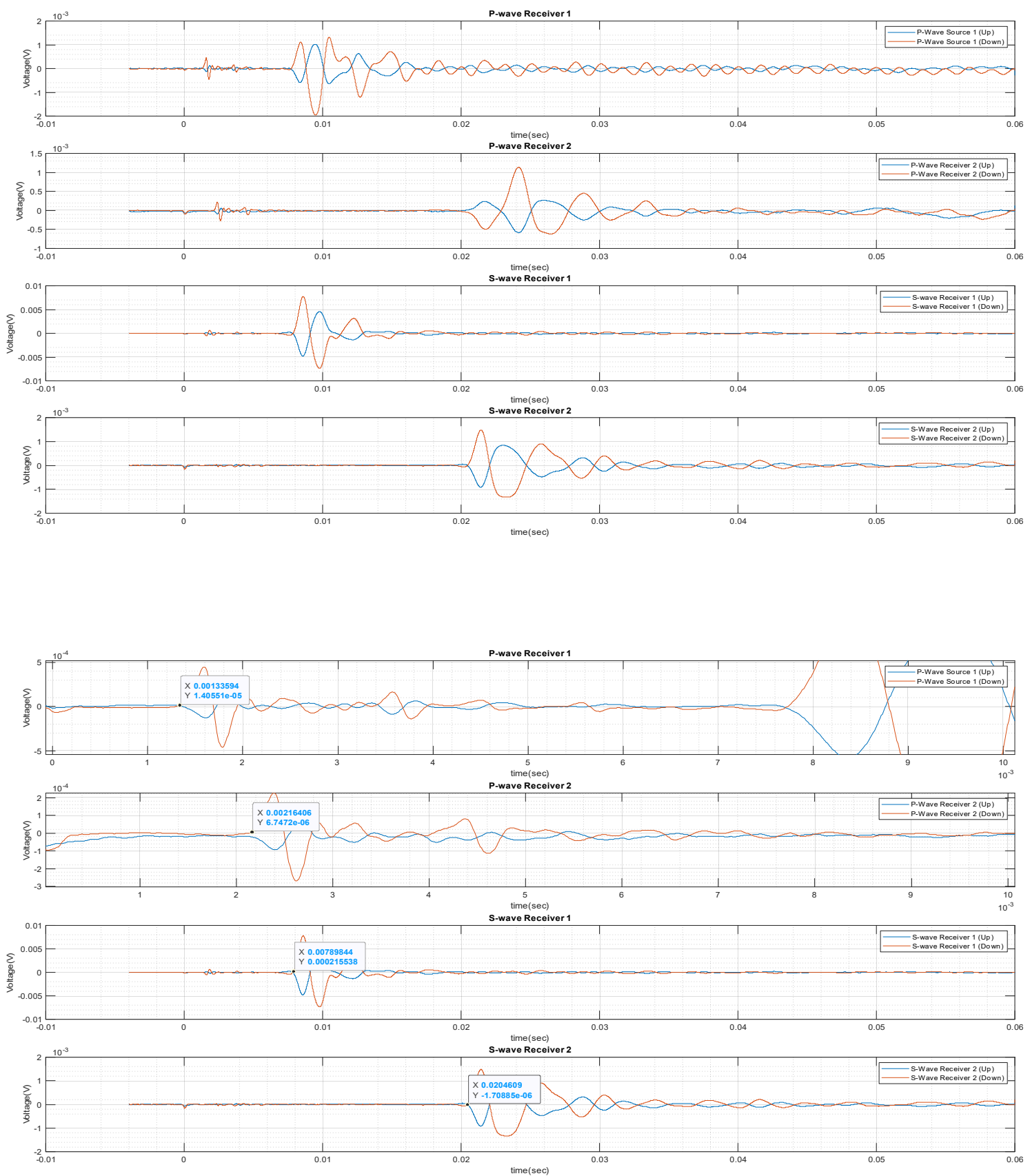

Data Set 7 (8.19.2019)

Run00048f

R4_2 

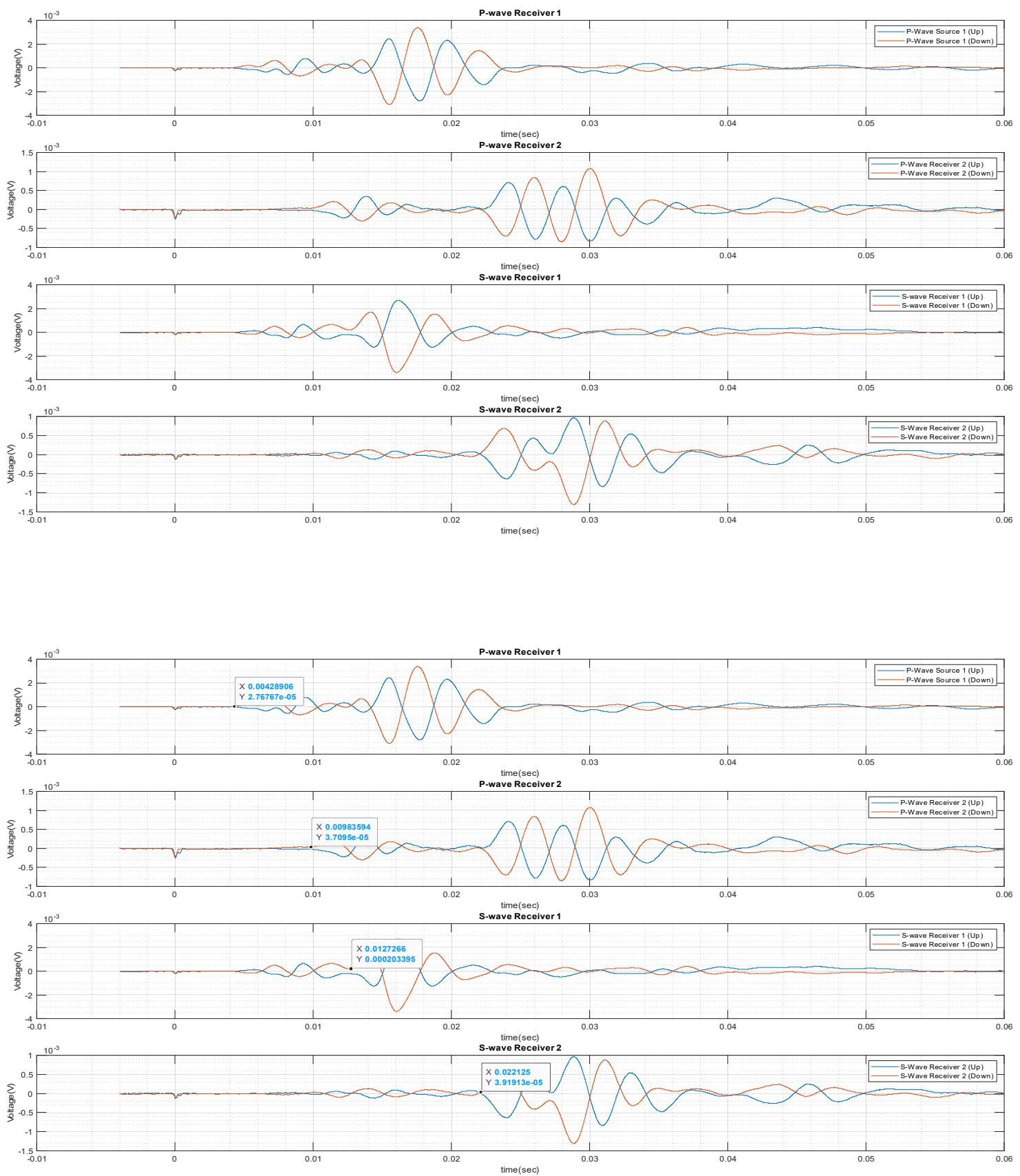

Data Set 8 (8.20.2019)

Run00049f

R1_1 

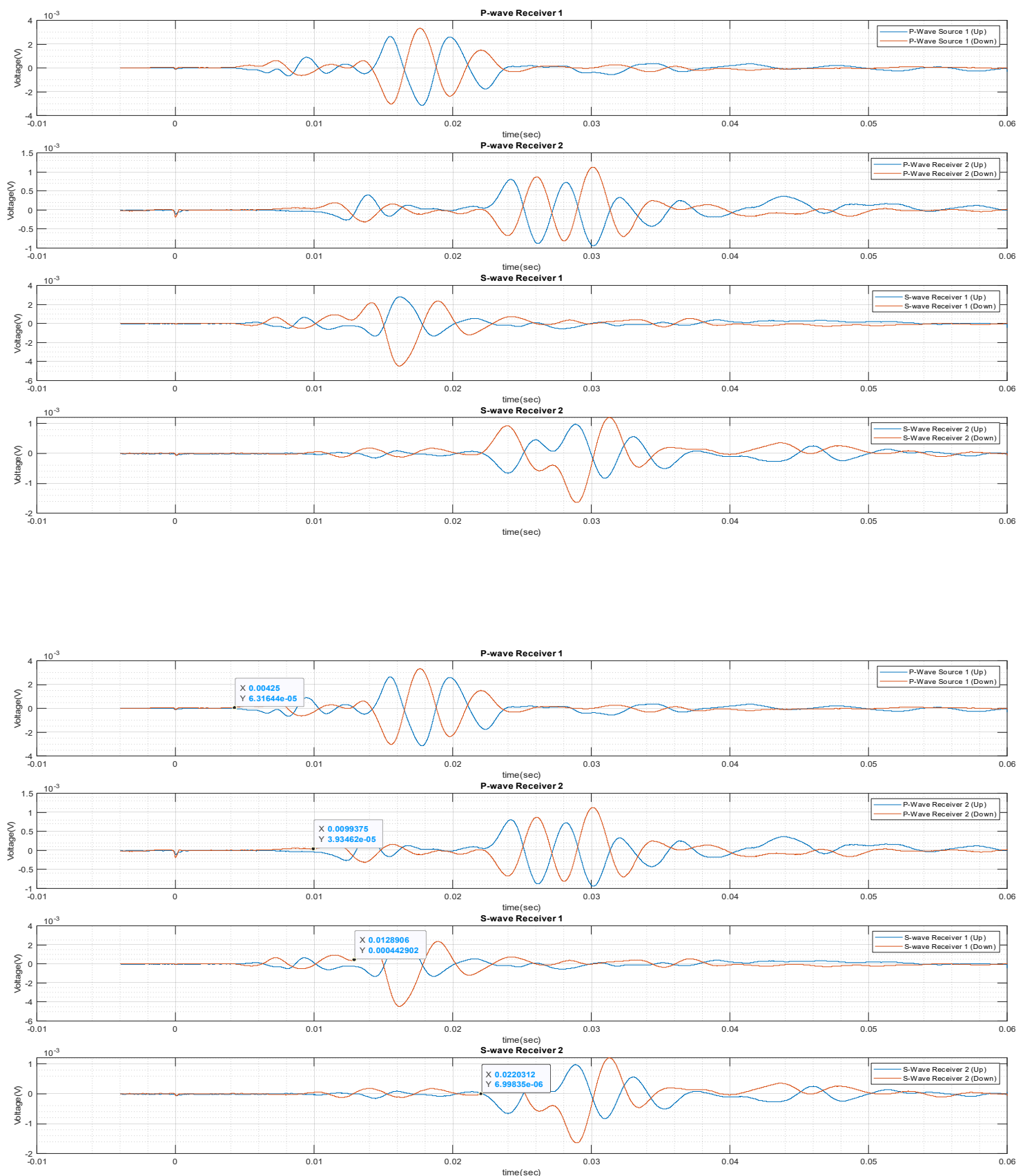

Data Set 8 (8.20.2019)

Run00050f

R1_2 

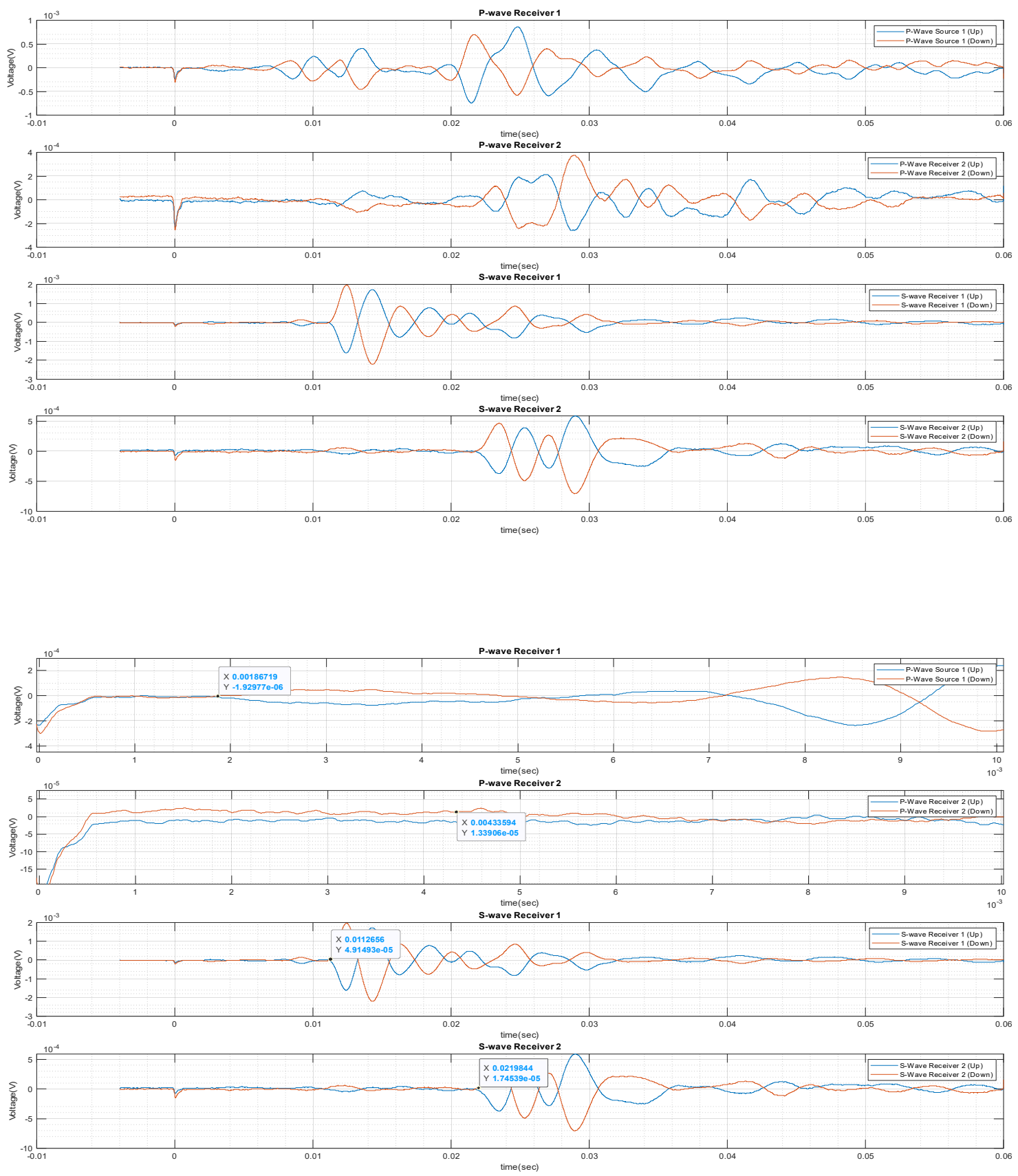

Data Set 8 (8.20.2019)

Run00051f

R2_1 

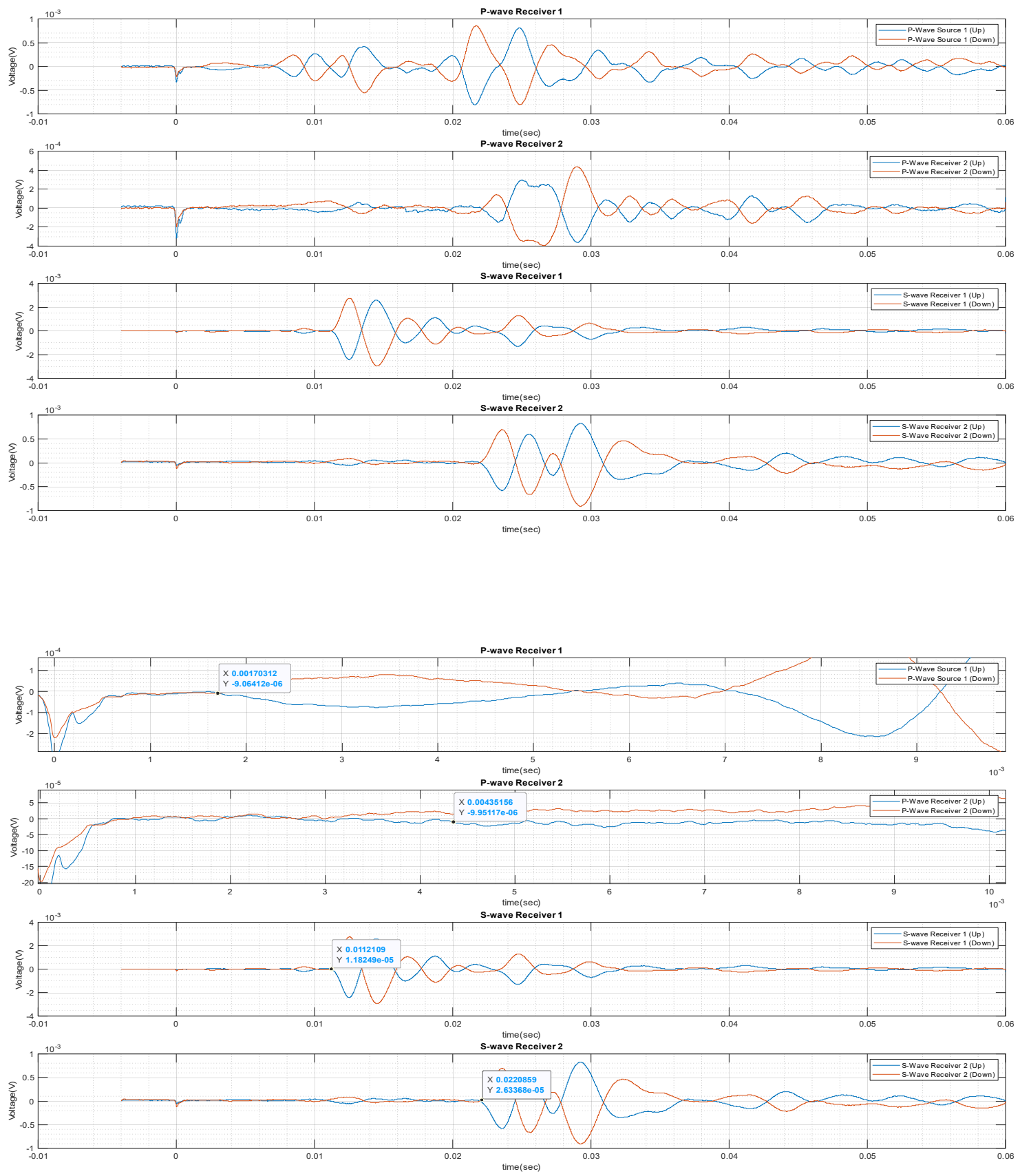

Data Set 8 (8.20.2019)

Run00052f

R2_2 

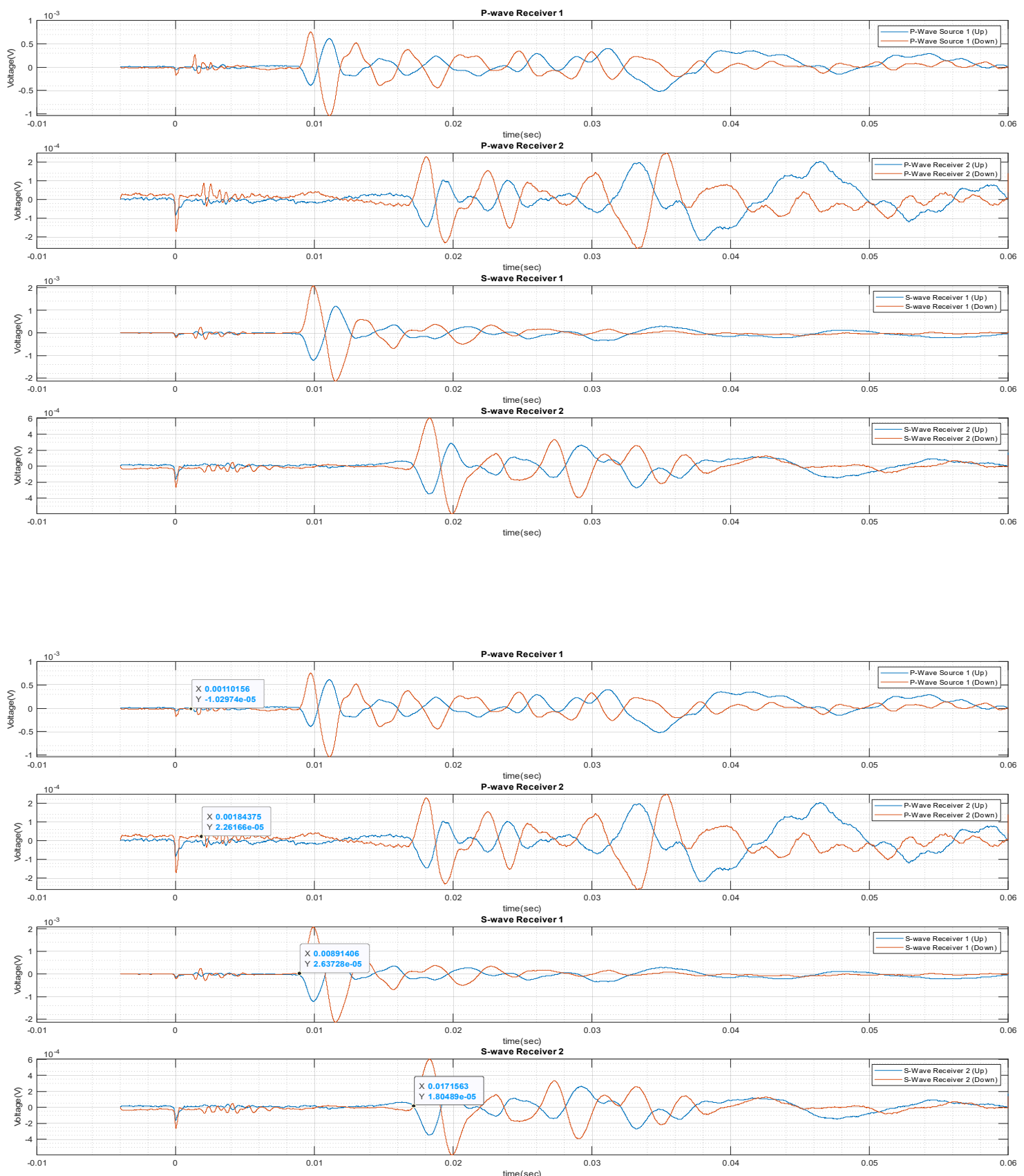

Data Set 8 (8.20.2019)

Run00053f

R3_1 

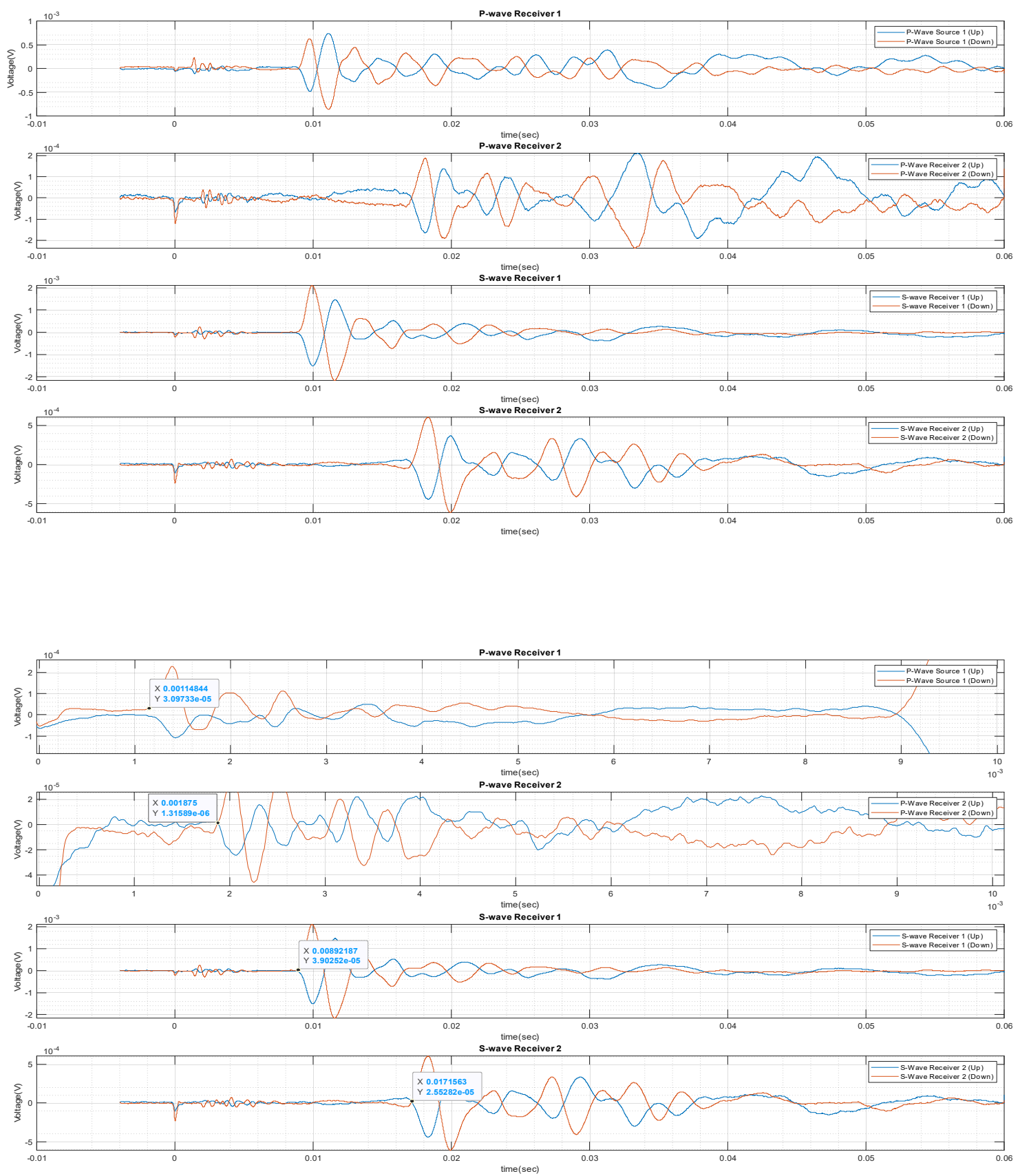

Data Set 8 (8.20.2019)

Run00054f

R3_2 

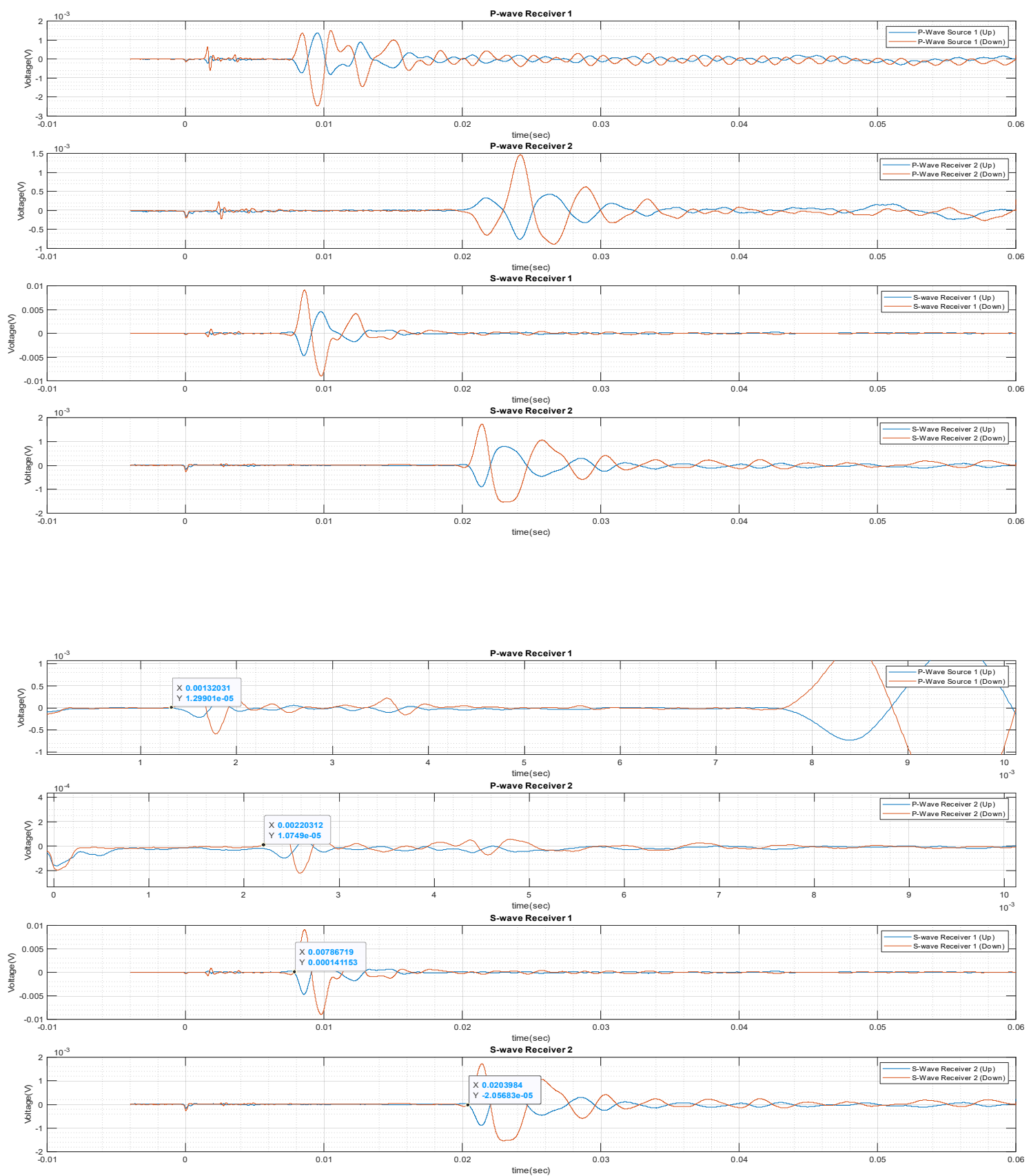

Data Set 8 (8.20.2019)

Run00055f

R4_1 

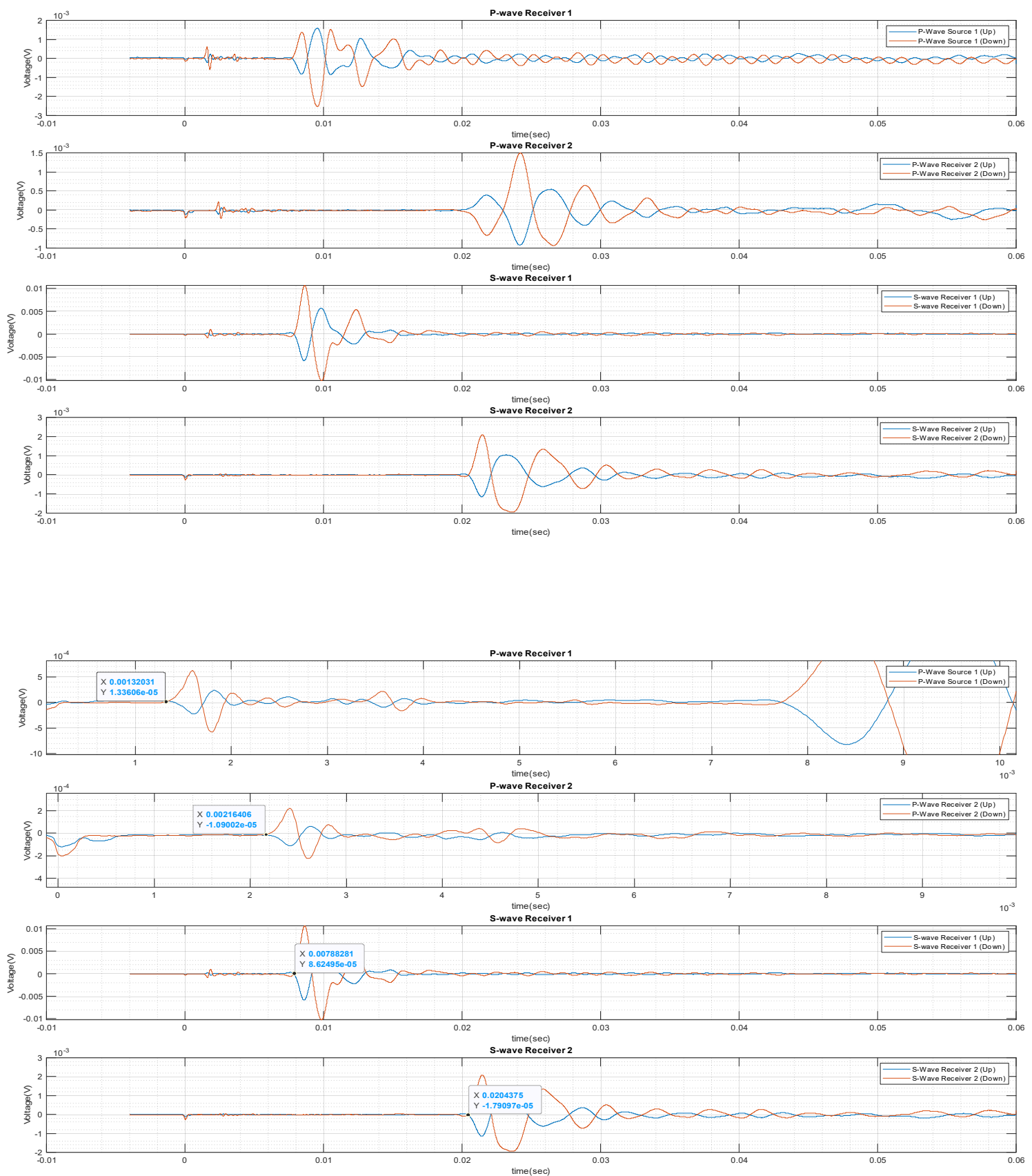

Data Set 8 (8.20.2019)

Run00056f

R4_2 

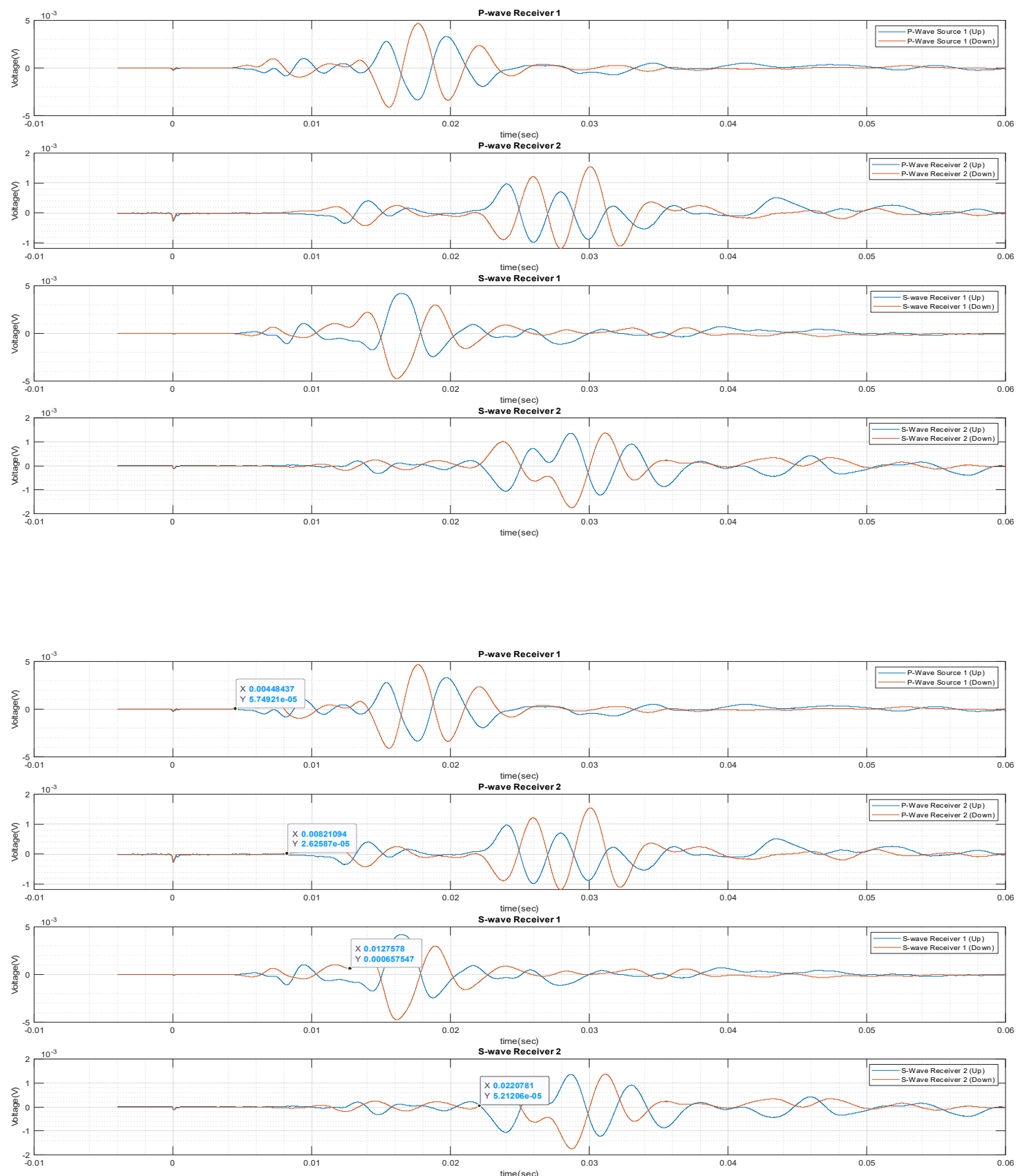

Data Set 9 (8.21.2019)

Run00057f

R1_1 

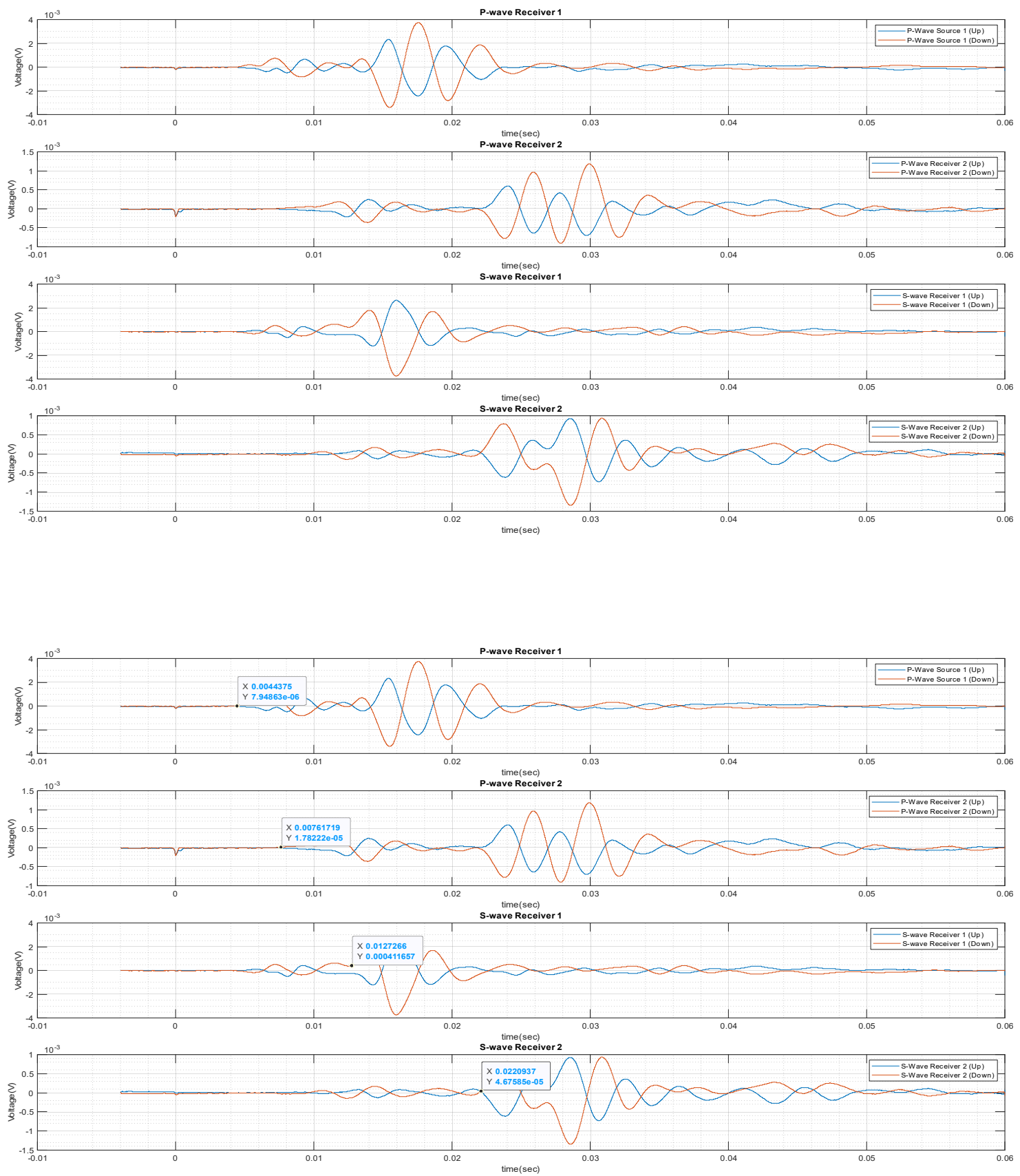

Data Set 9 (8.21.2019)

Run00058f

R1_2 

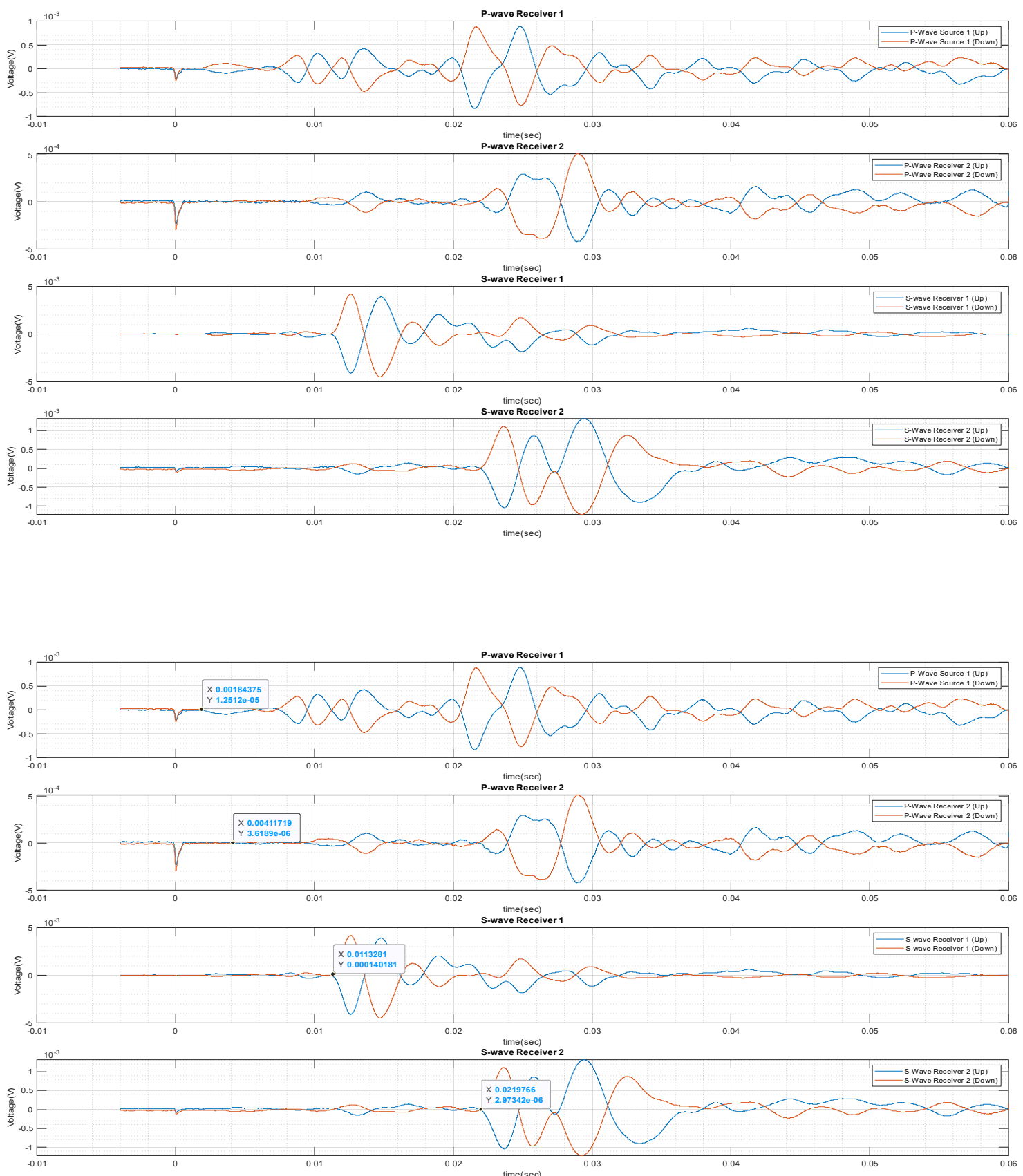

Data Set 9 (8.21.2019)

Run00059f

R2_1 

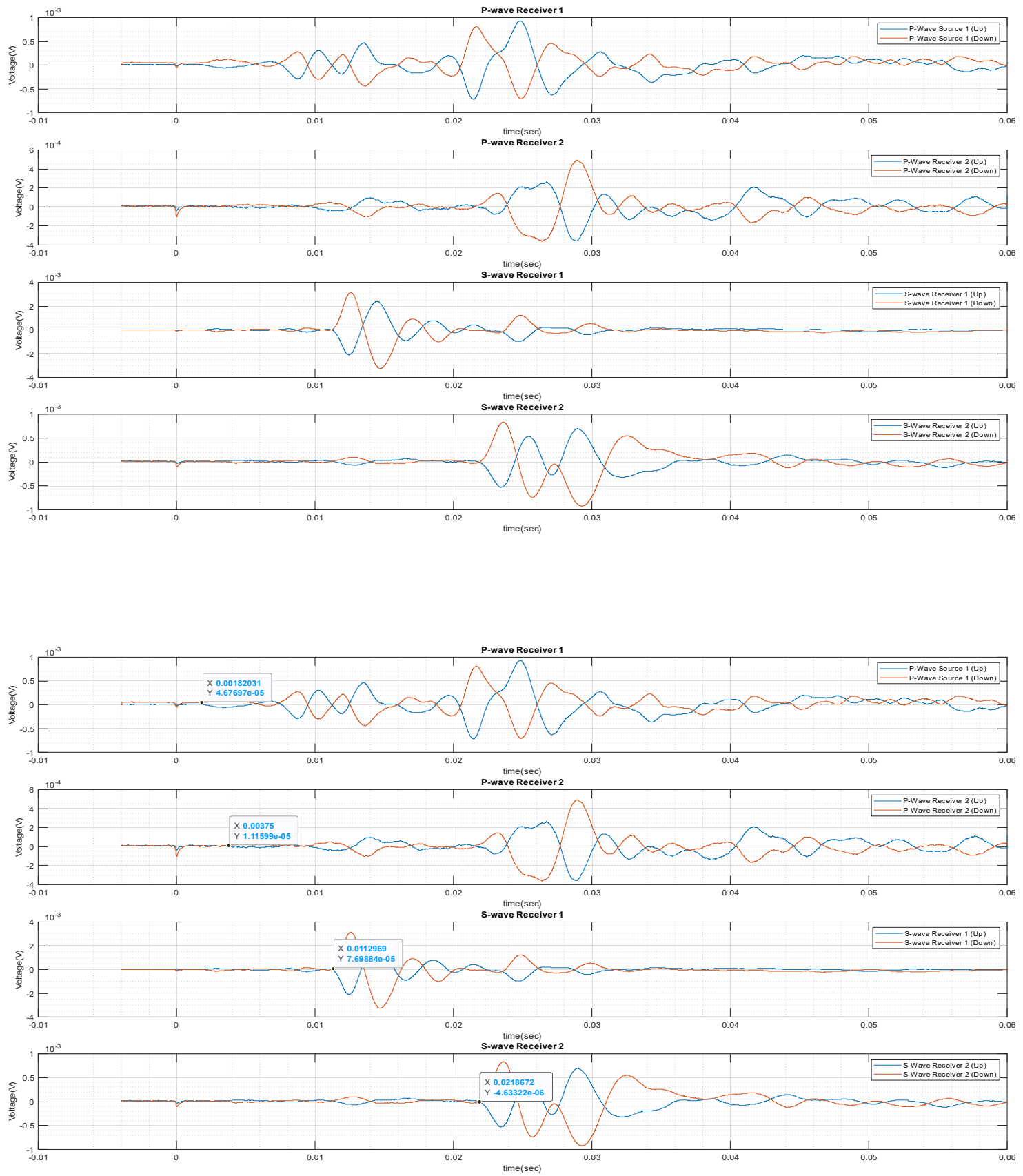

Data Set 9 (8.21.2019)

Run00060f

R2_2 

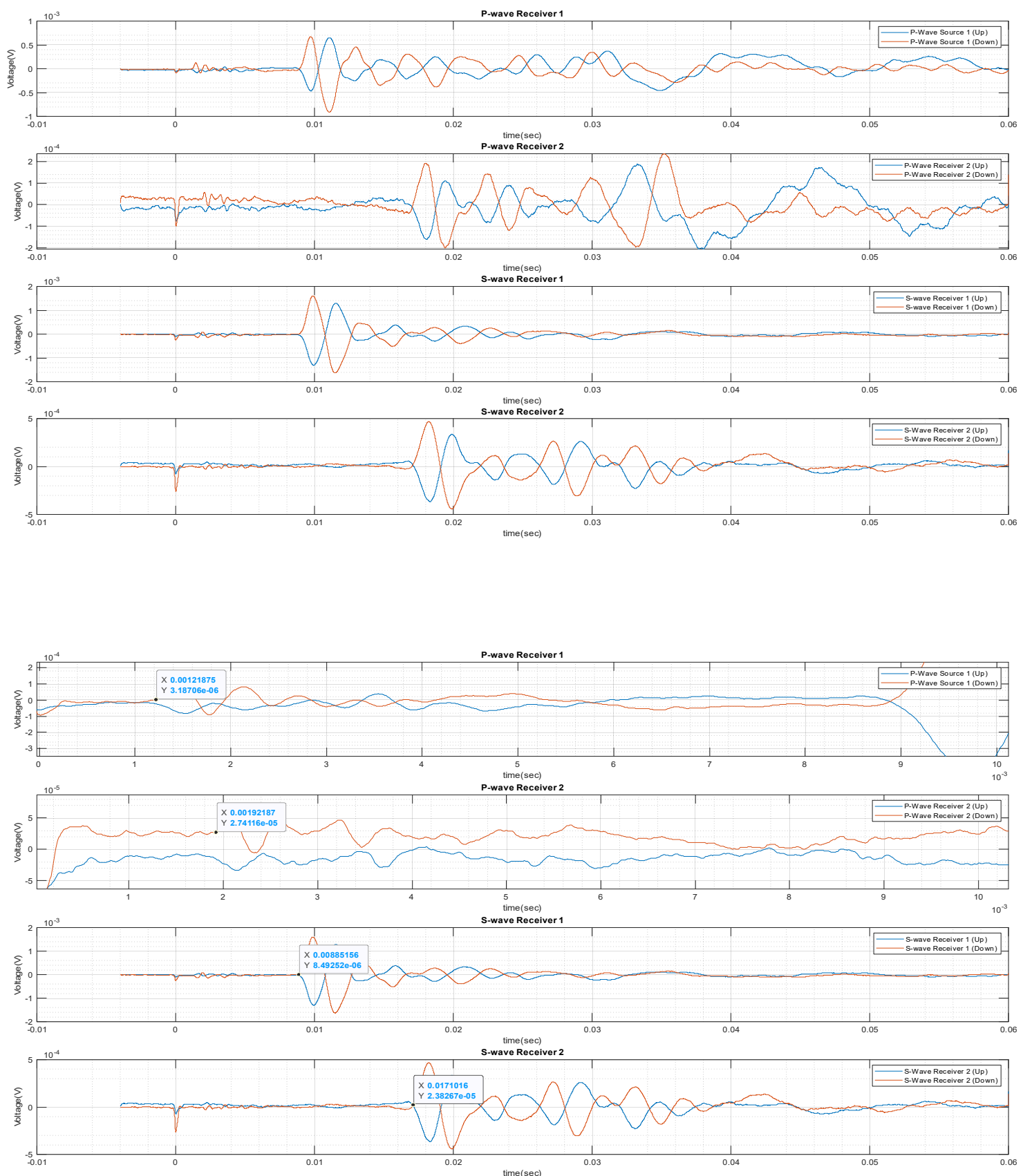

Data Set 9 (8.21.2019)

Run00061f

R3_1 

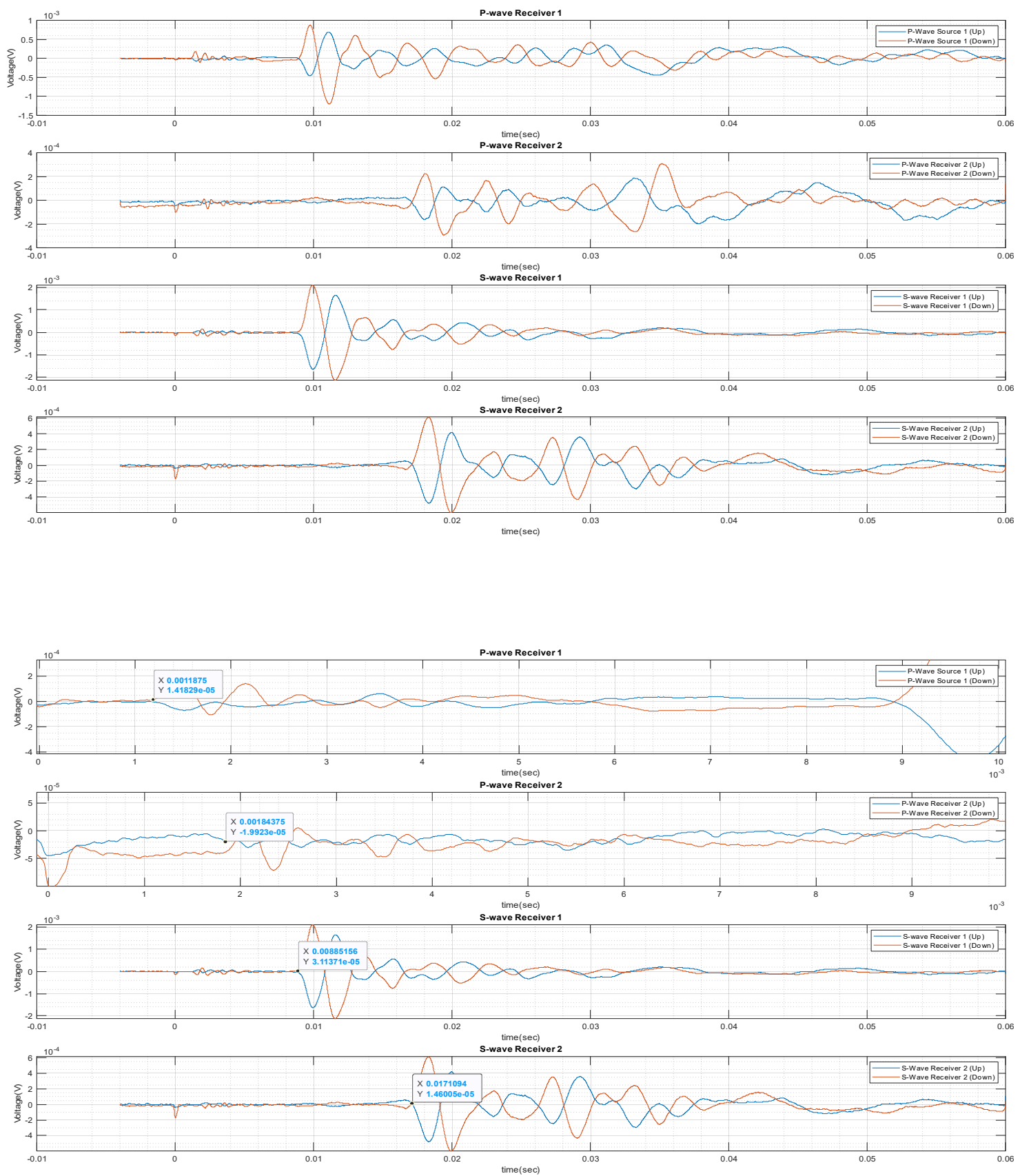

Data Set 9 (8.21.2019)

Run00062f

R3_2 

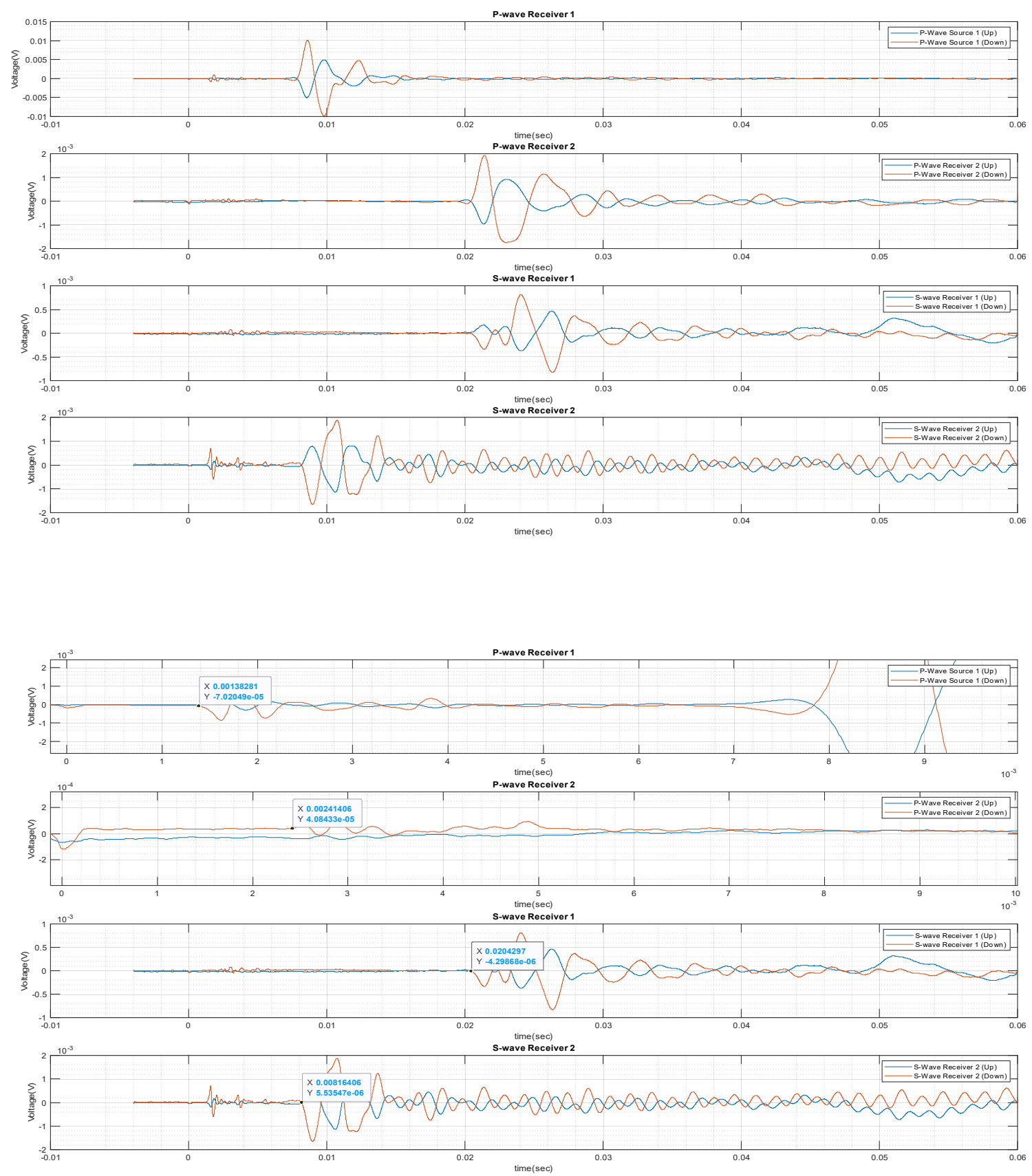

Data Set 9 (8.21.2019)

Run00063f

R4_1 

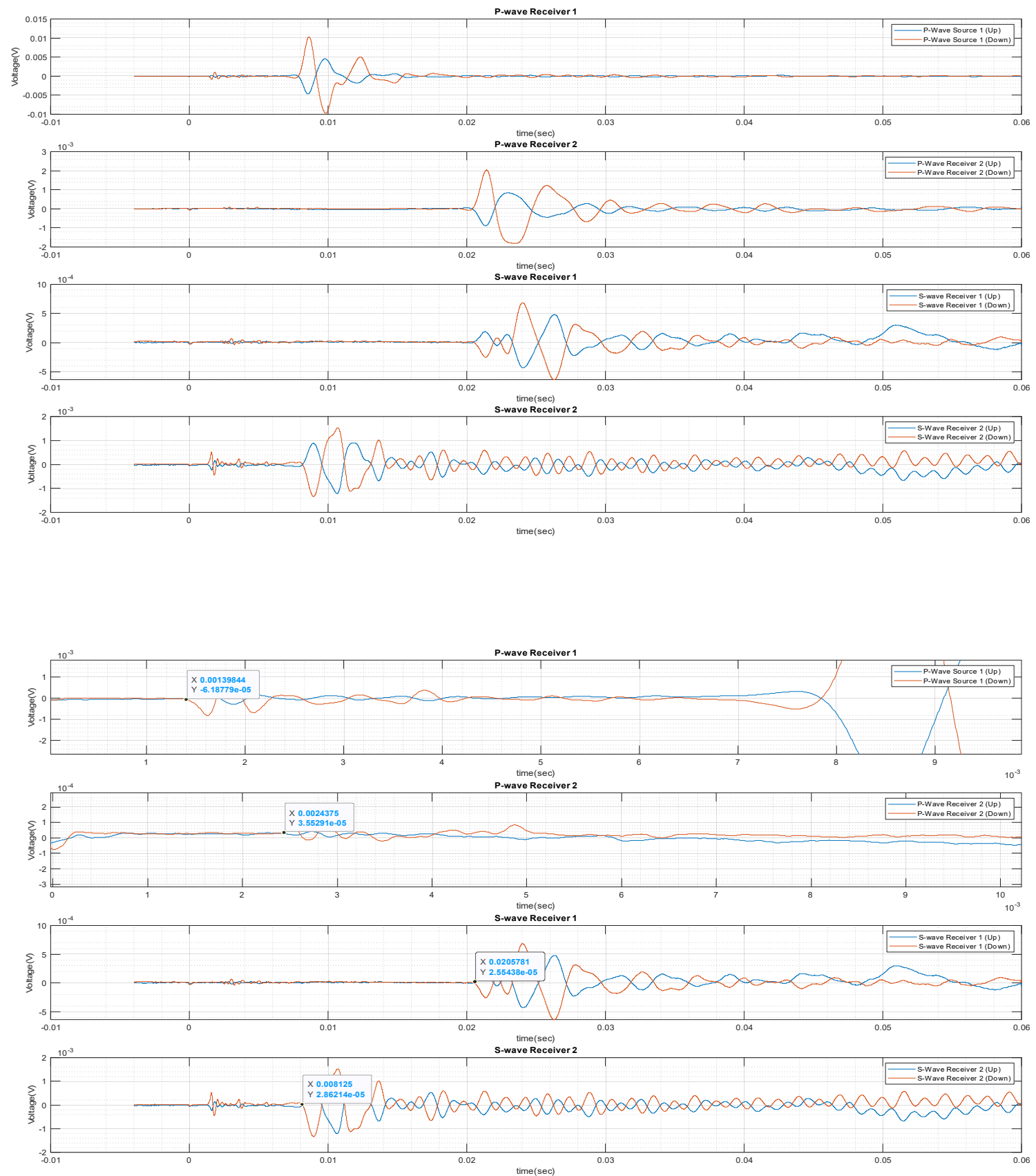

Data Set 9 (8.21.2019)

Run00064f

R4_2 

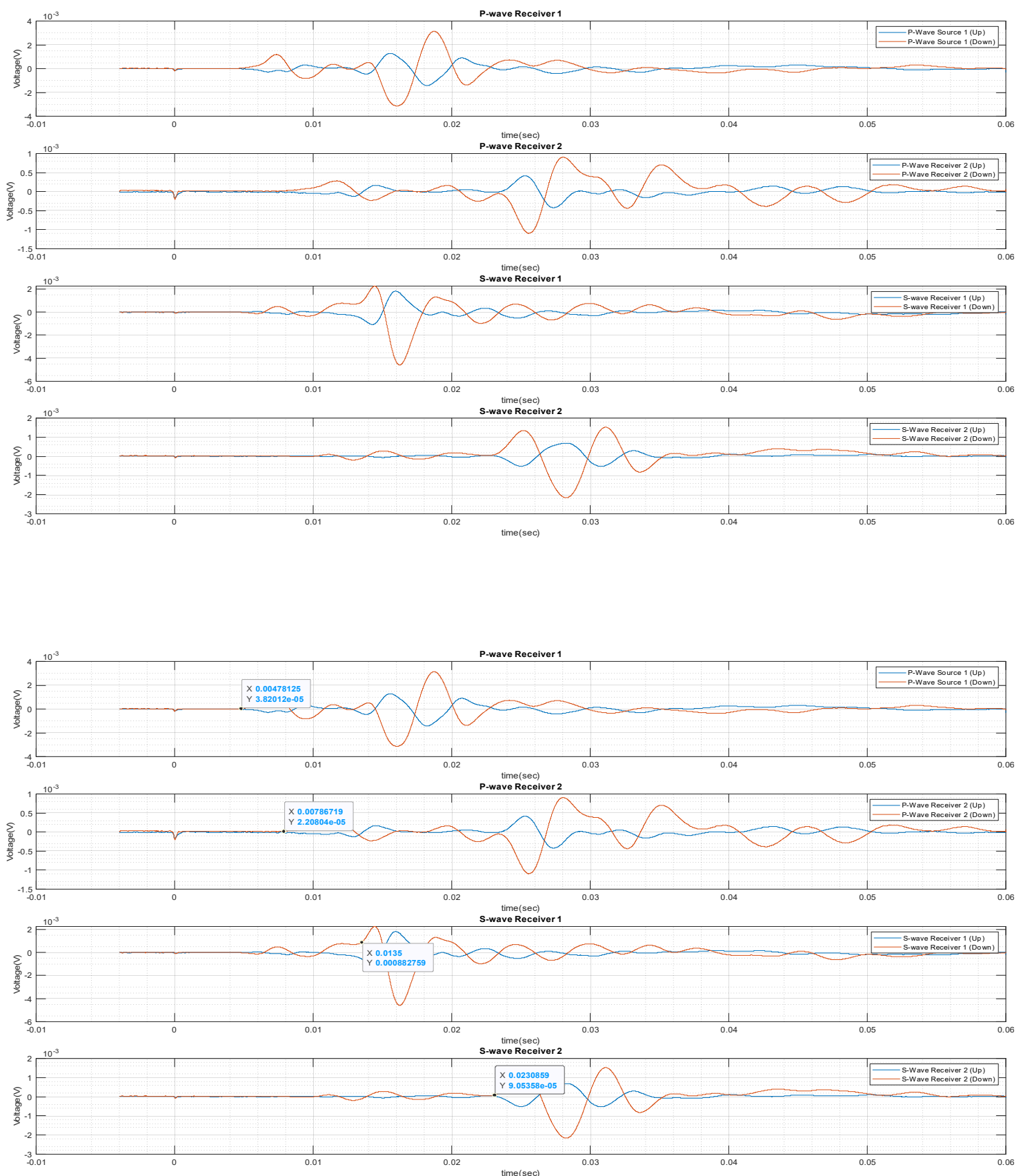

Data Set $10(8.22 .2019)$

Run00065f

R1_1 

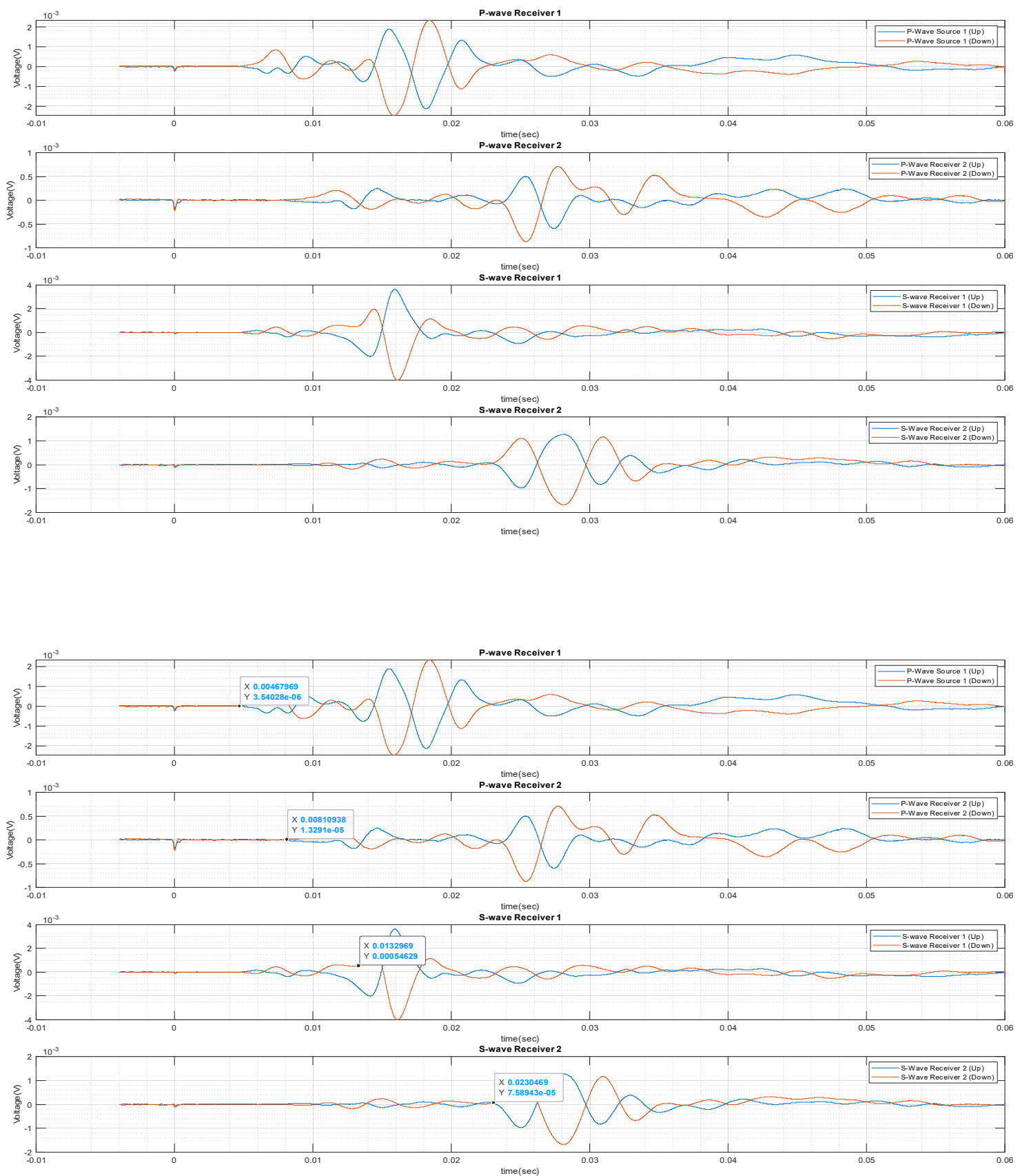

Data Set $10(8.22 .2019)$

Run00066f

R1_2 

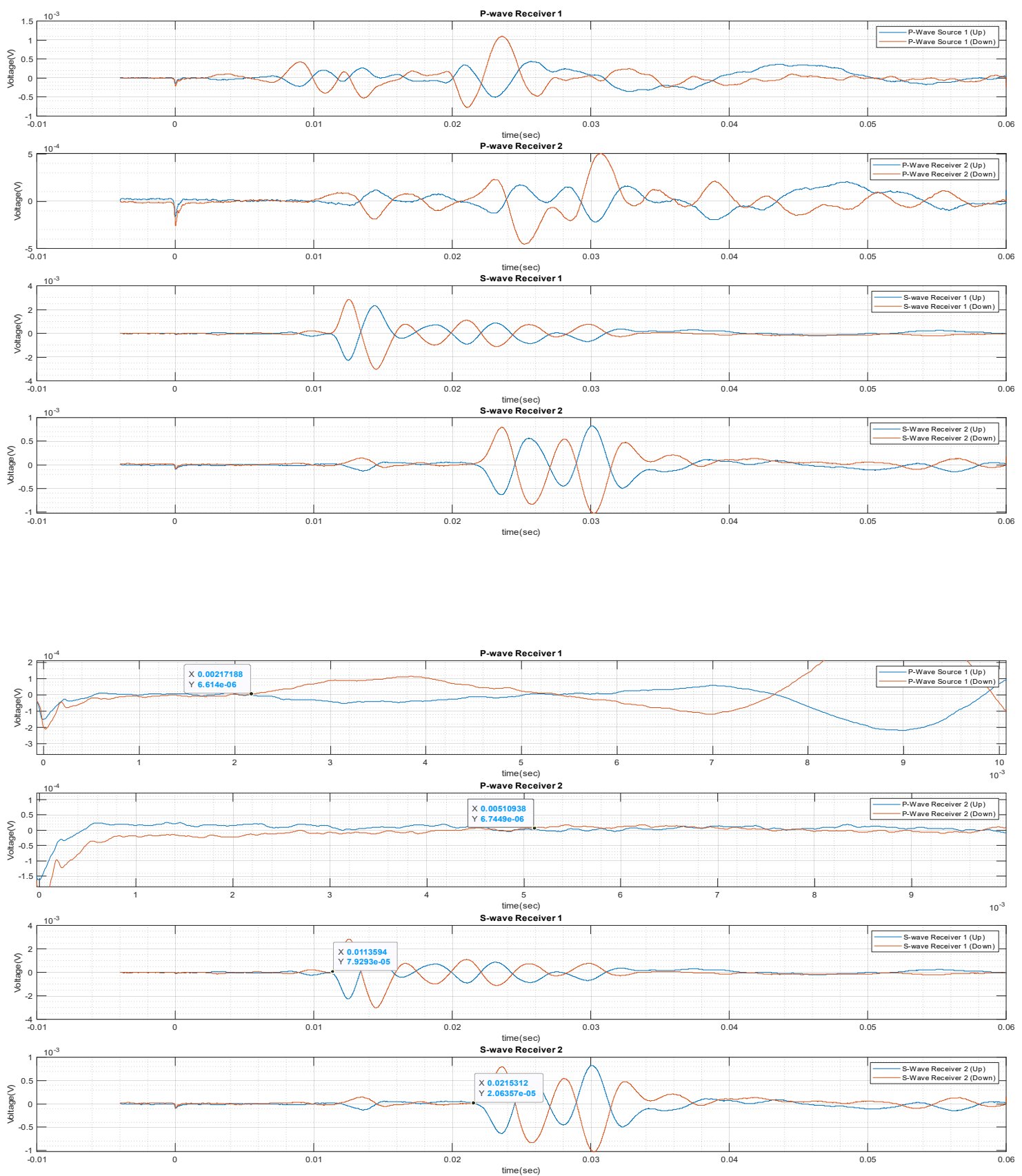

Data Set $10(8.22 .2019)$

Run00067f

R2_1 

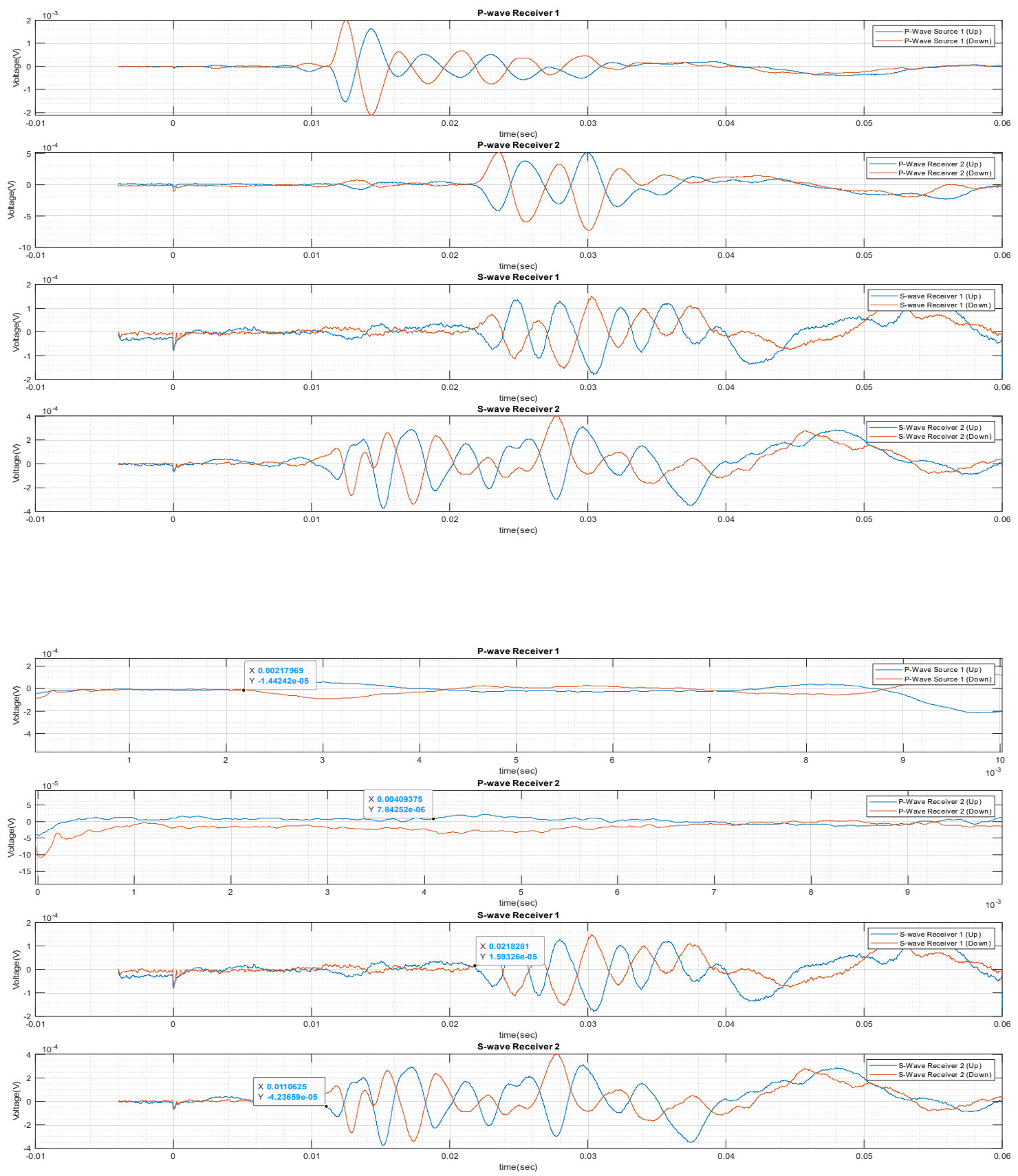

Data Set $10(8.22 .2019)$

Run00068f

R2_2 

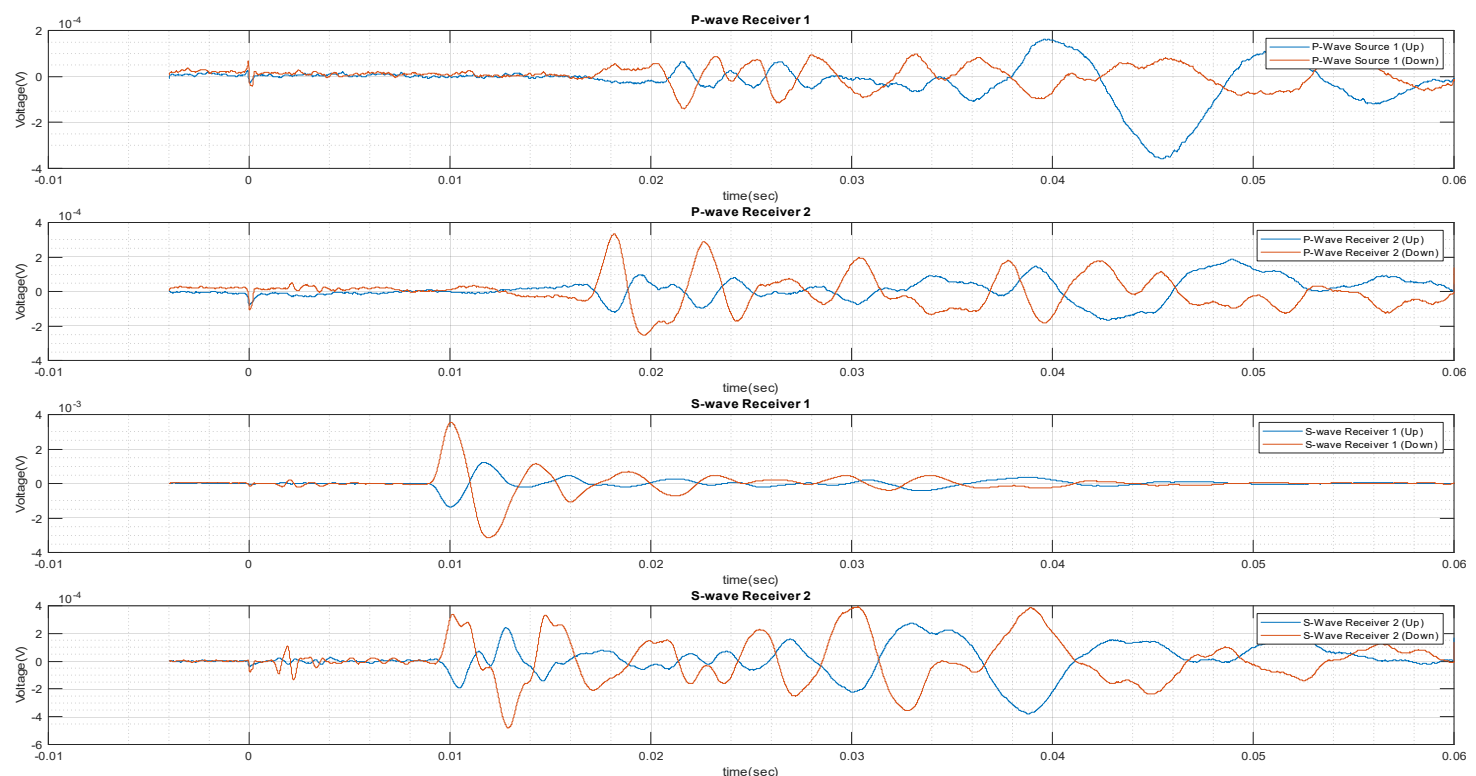

Cannot determine clear arrival times.

Data Set $10(8.22 .2019)$

Run00069f

R3 1 

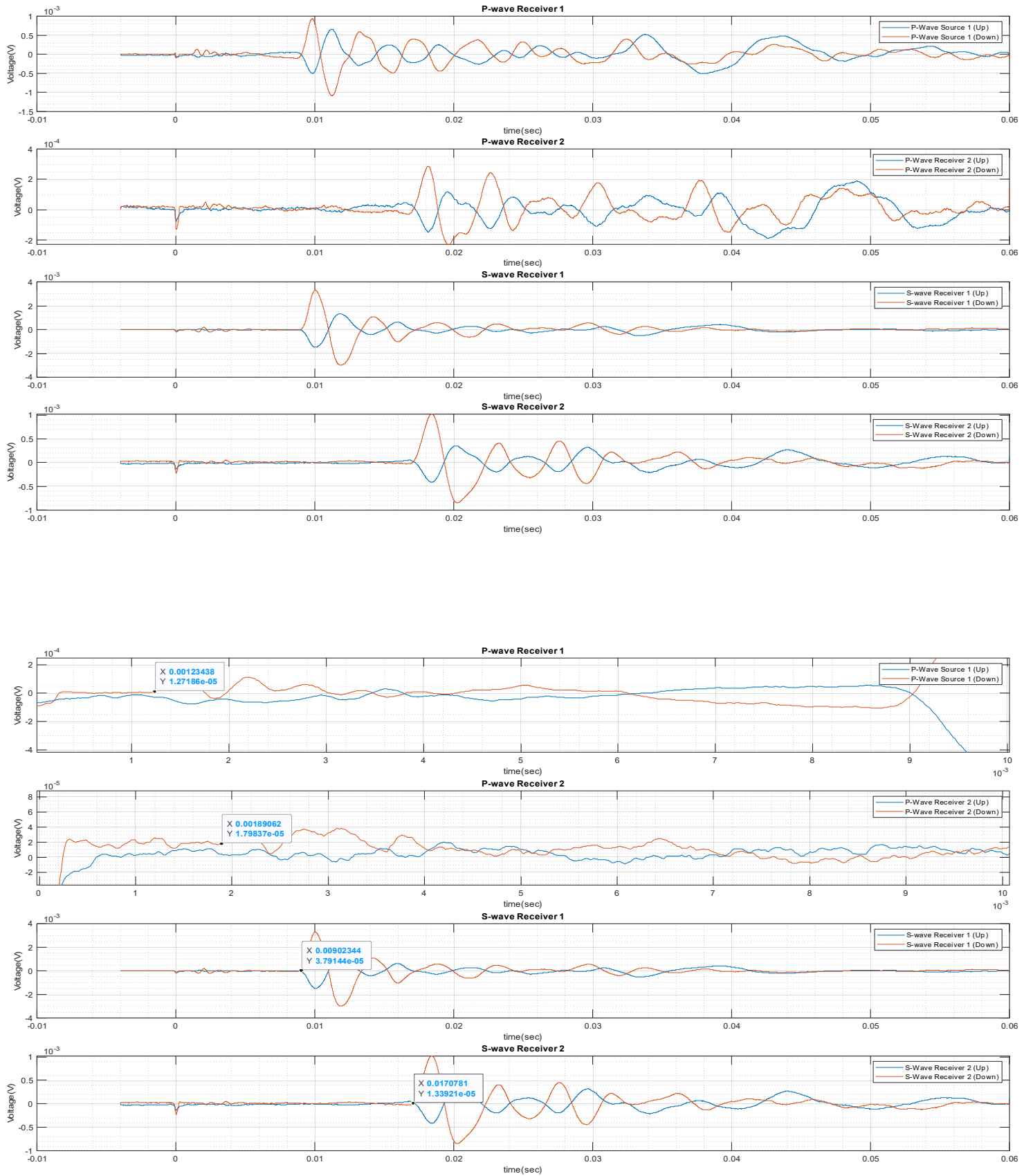

Data Set 10 (8.22.2019)

Run00070f

R3_2 

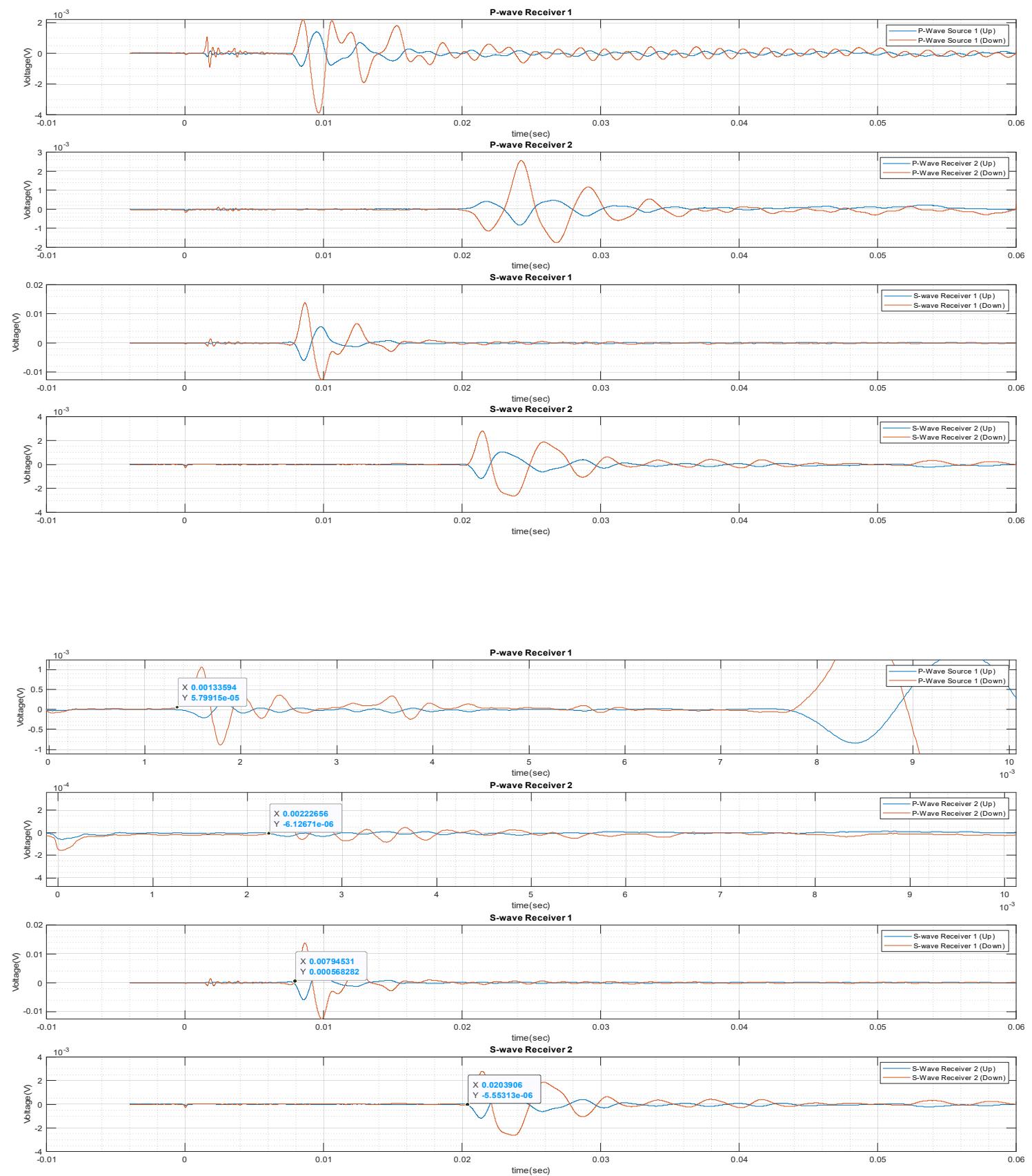

Data Set 10 (8.22.2019)

\section{Run00071f}

R4_1 

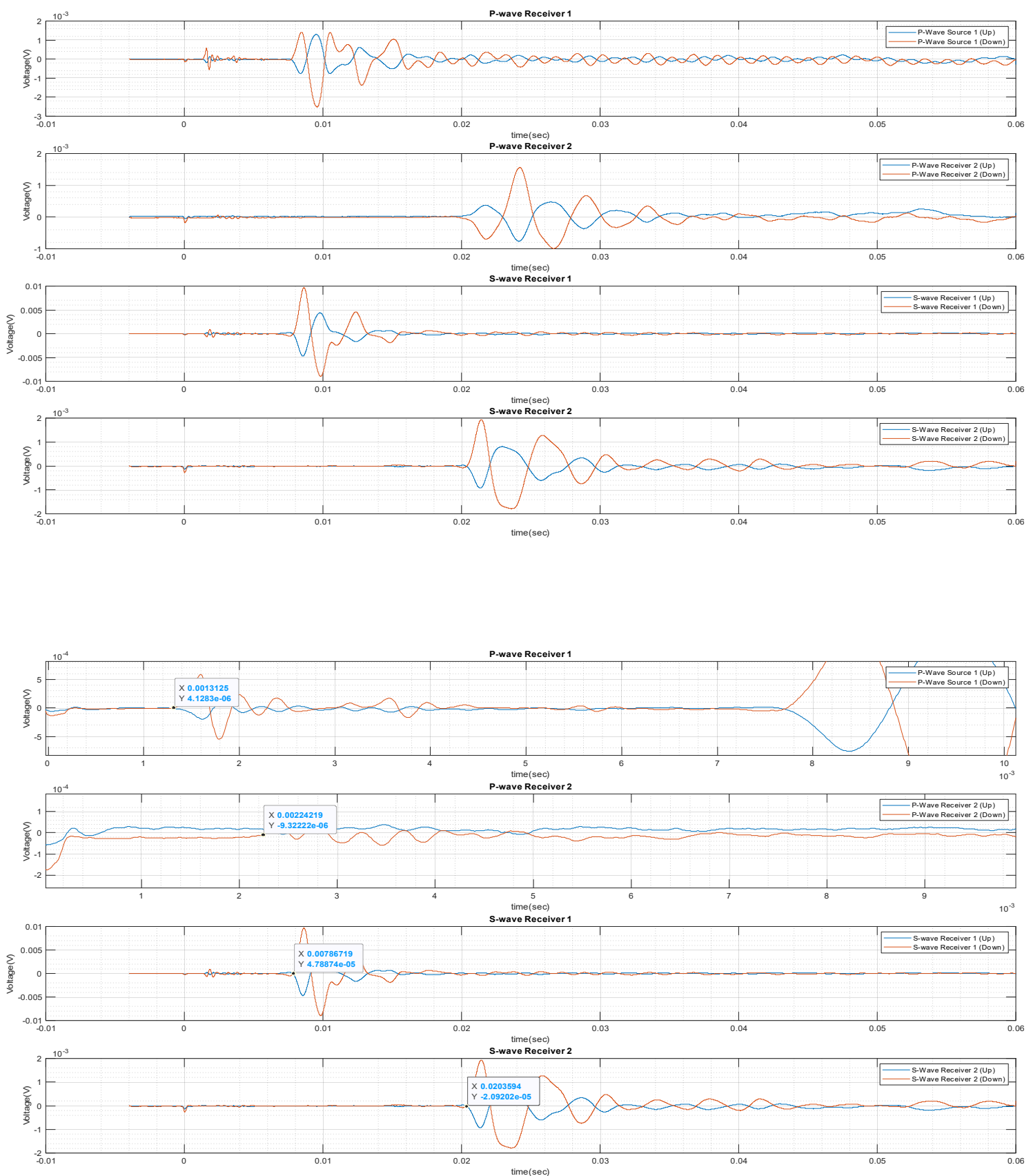

Data Set $10(8.22 .2019)$

Run00072f

R4_2 

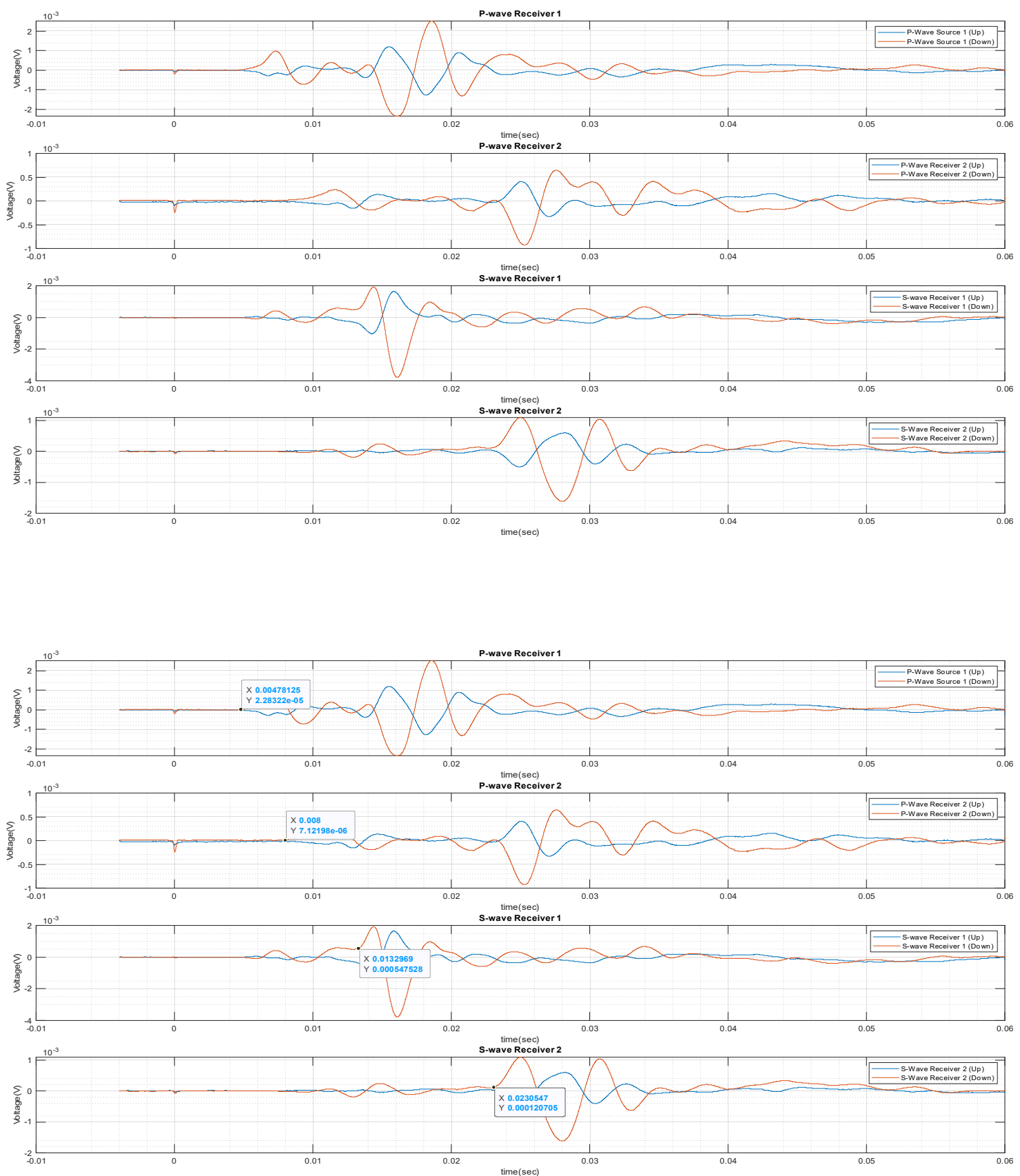

Data Set $11(8.23 .2019)$

Run00073f

R1_1 

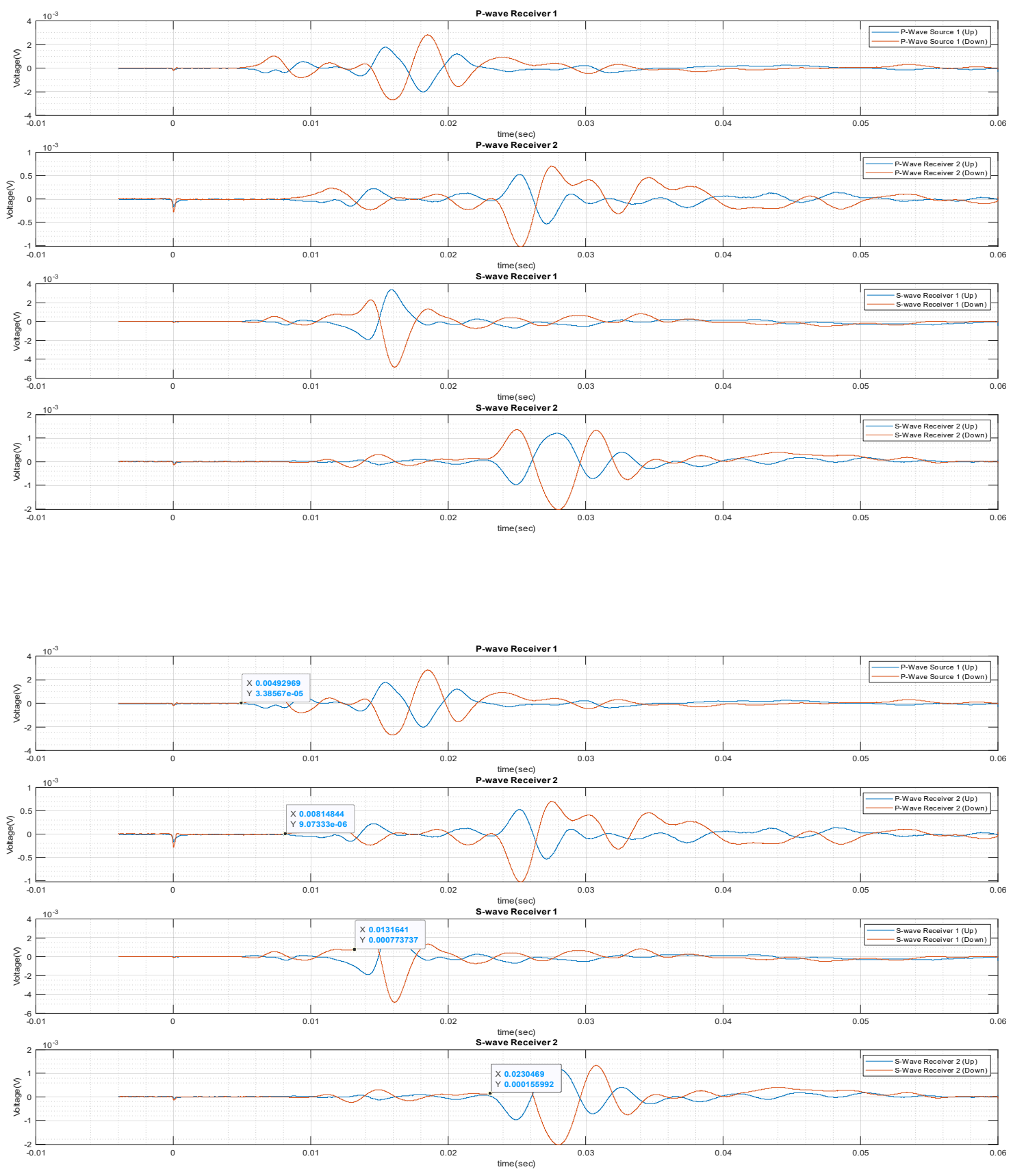

Data Set $11(8.23 .2019)$

Run00074f

R1_2 

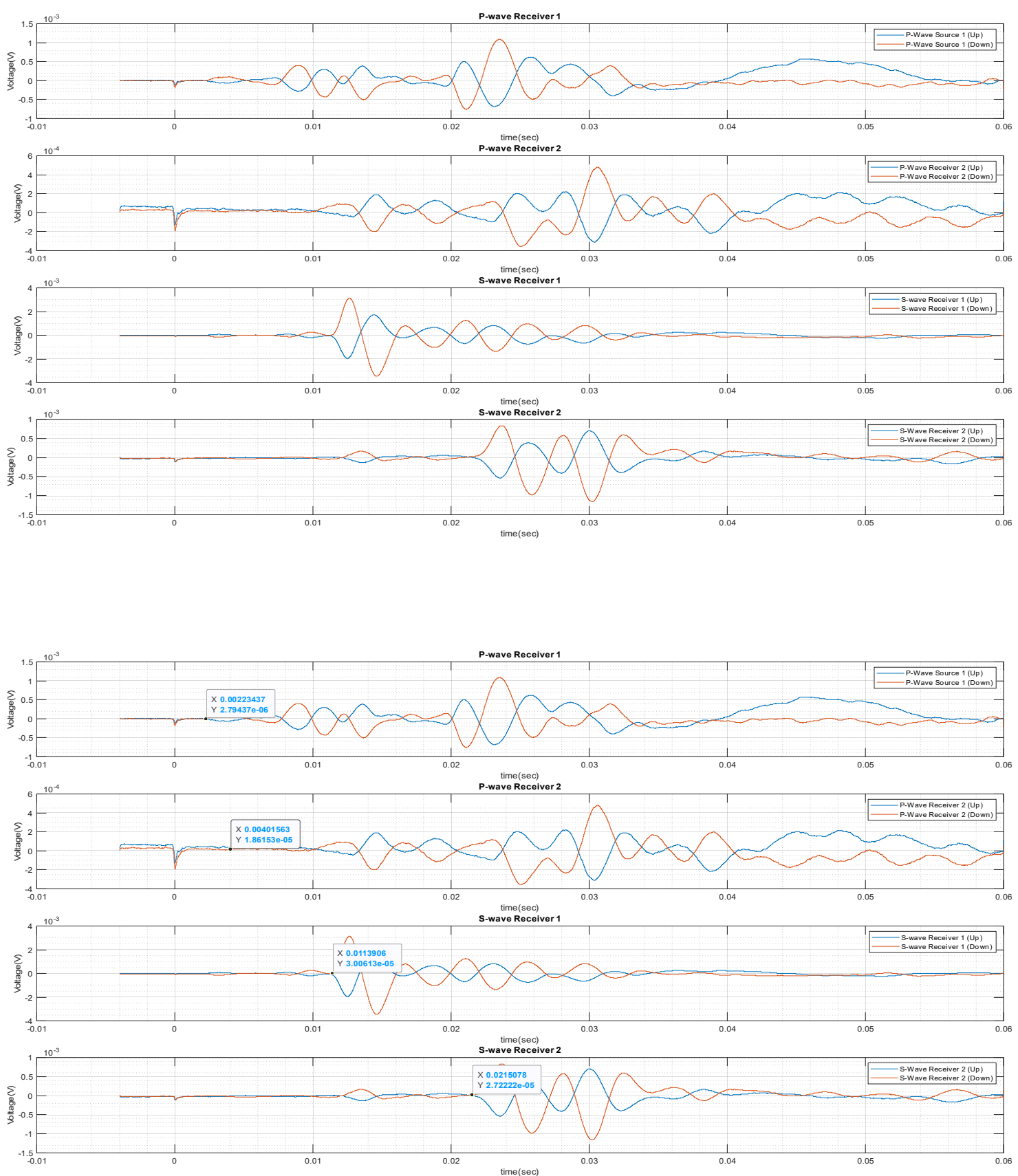

Data Set $11(8.23 .2019)$

Run00075f

R2_1 

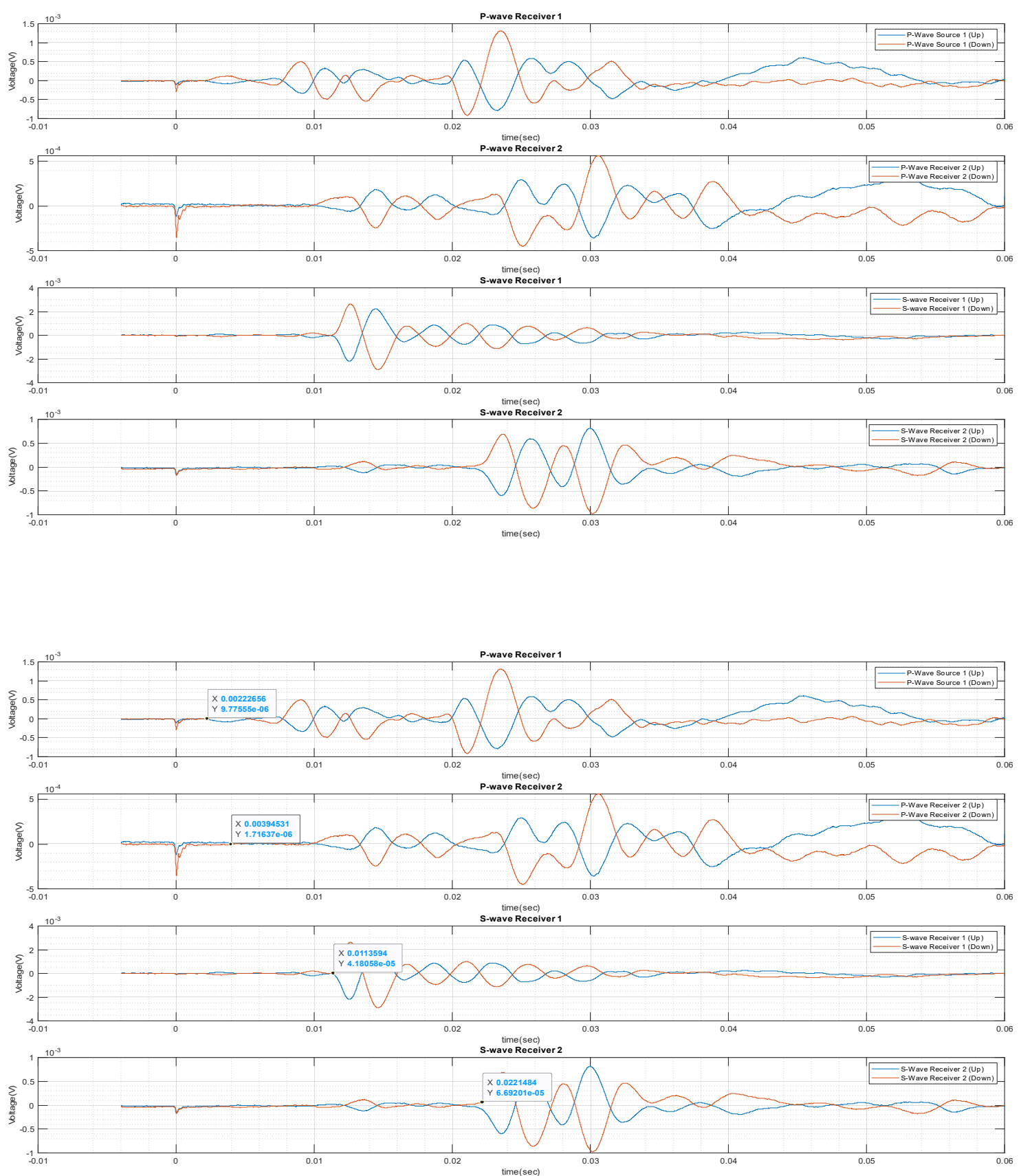

Data Set $11(8.23 .2019)$

Run00076f

R2_2 

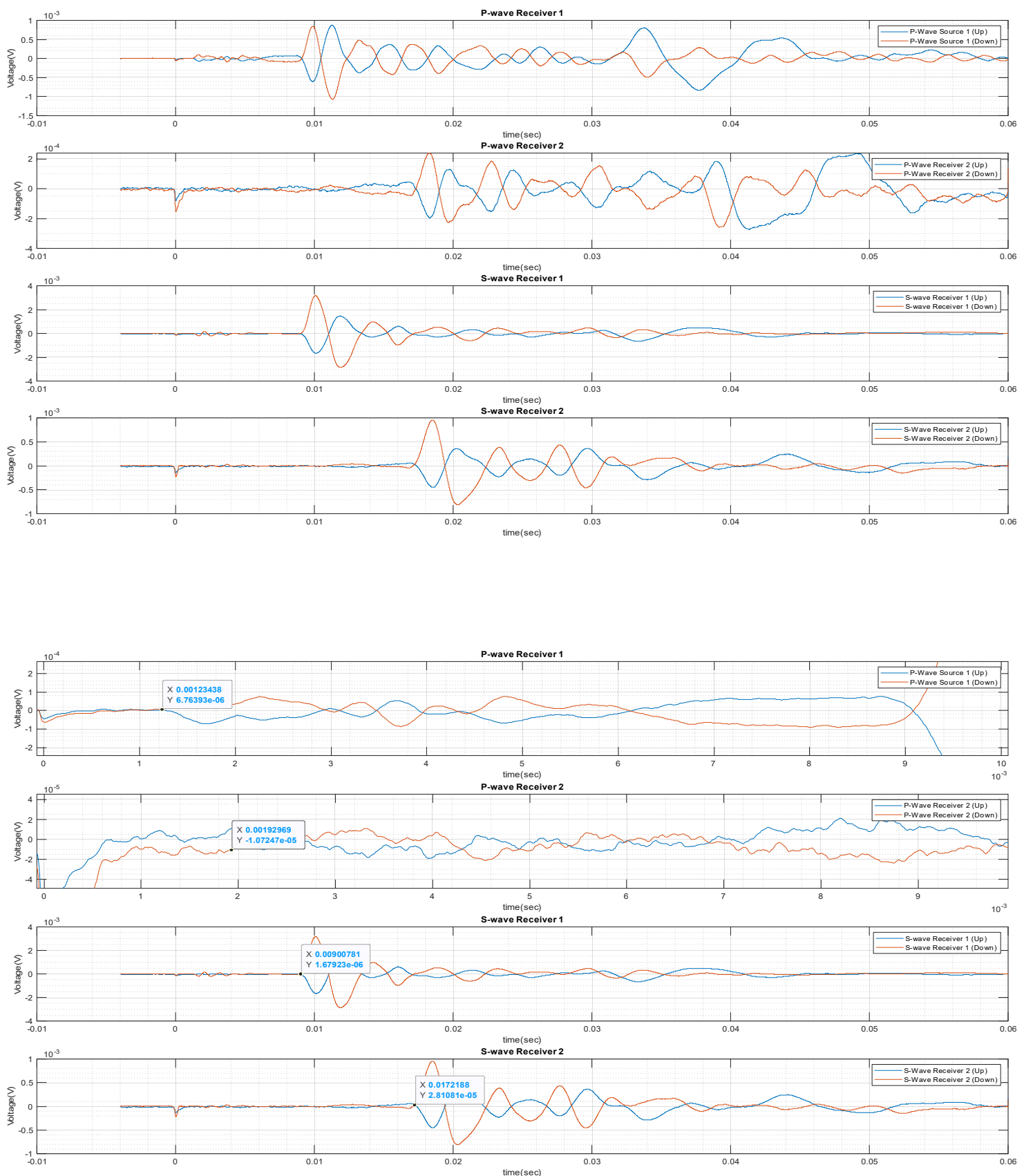

Data Set $11(8.23 .2019)$

Run00077f

R3_1 

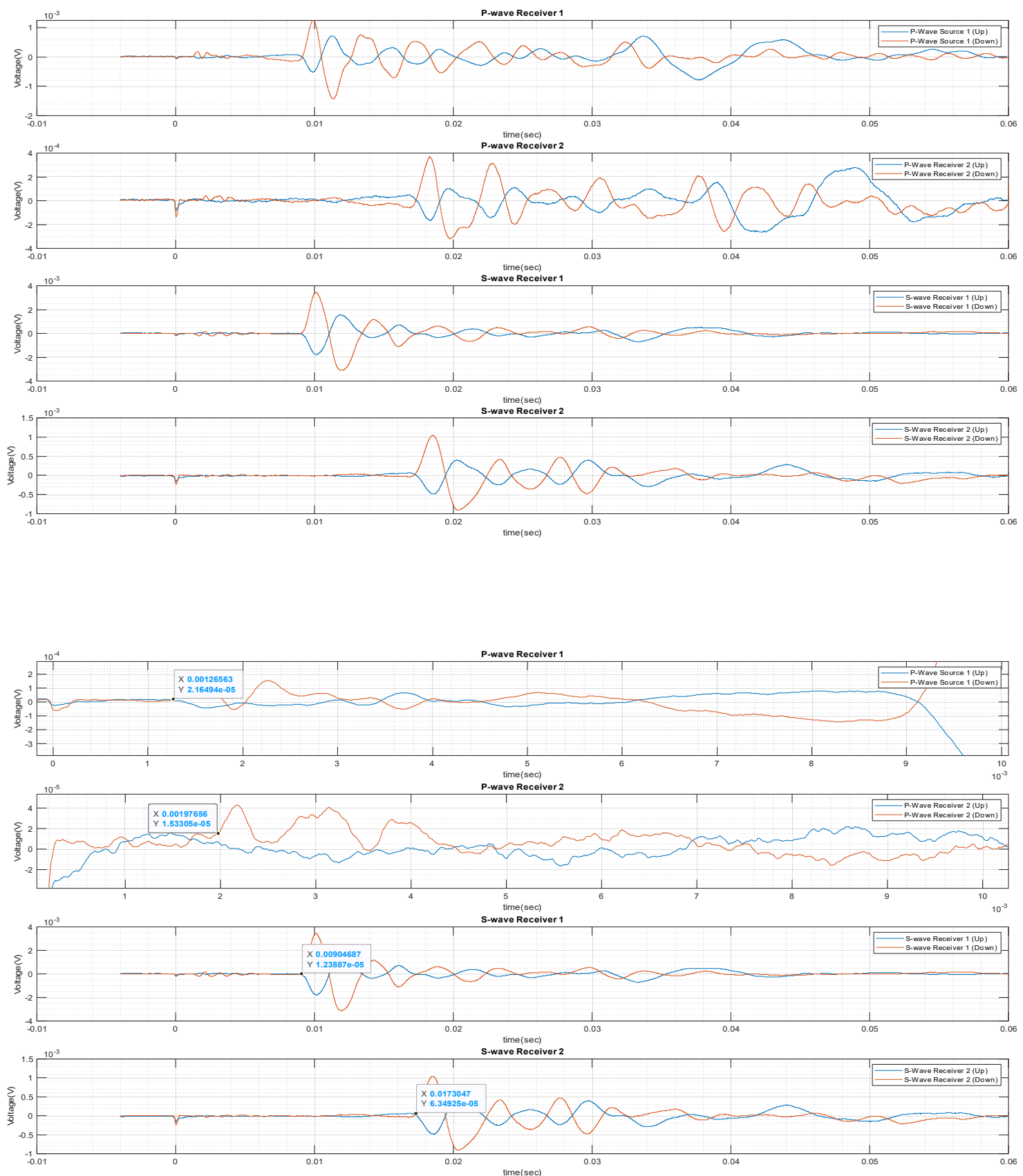

Data Set $11(8.23 .2019)$

Run00078f

R3_2 

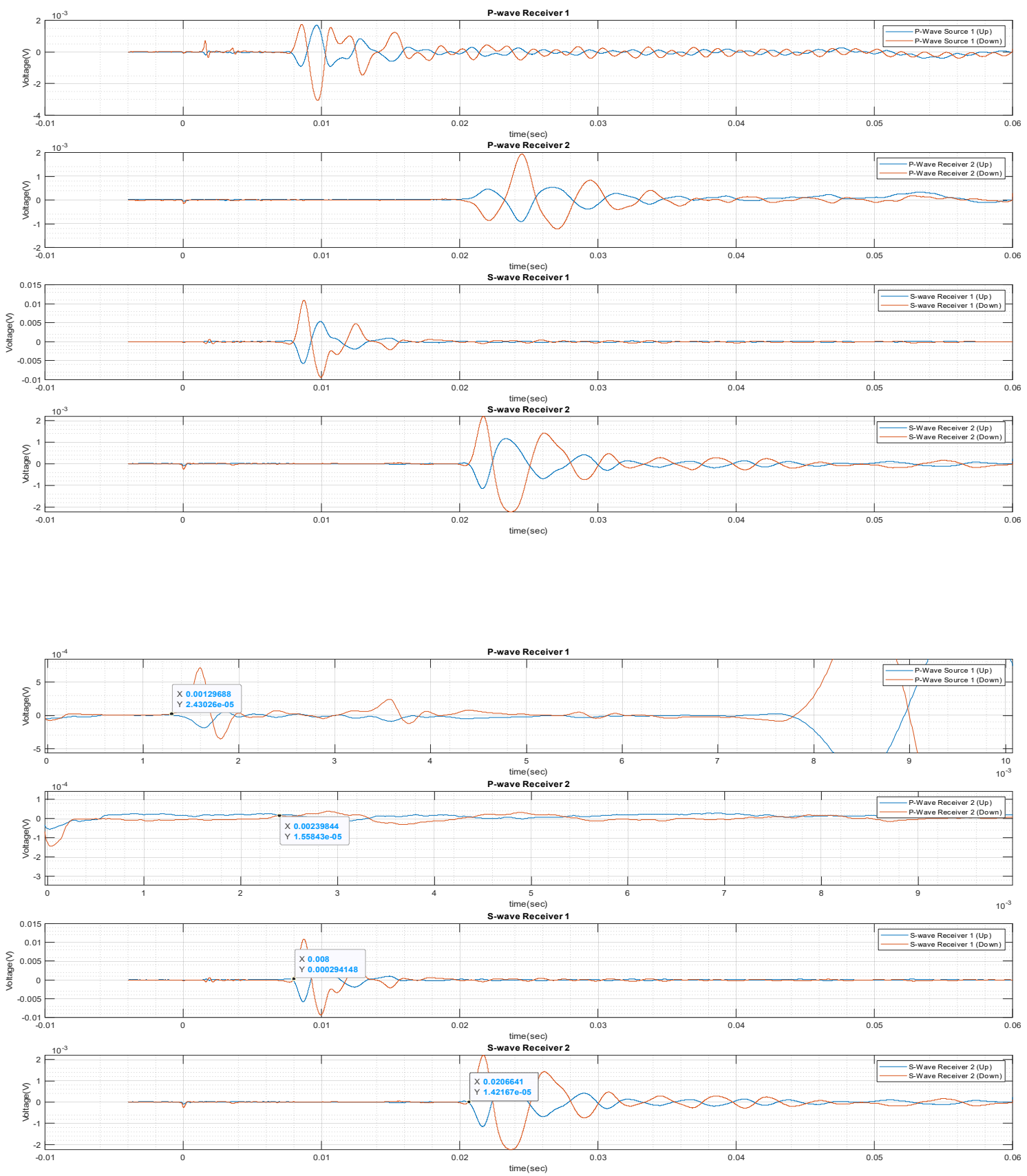

Data Set $11(8.23 .2019)$

Run00079f

R4_1 

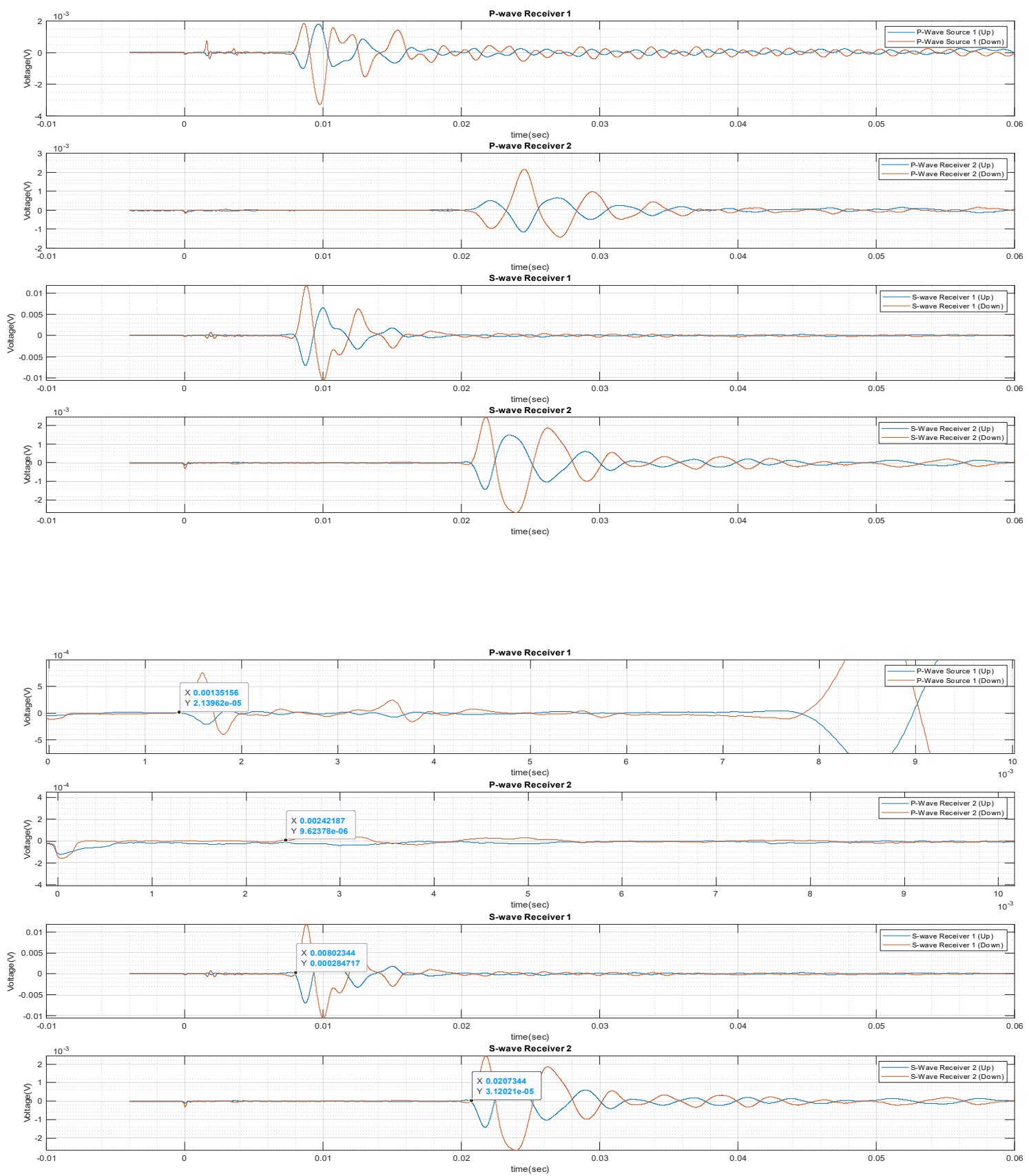

Data Set $11(8.23 .2019)$

Run00080f

R4_2 

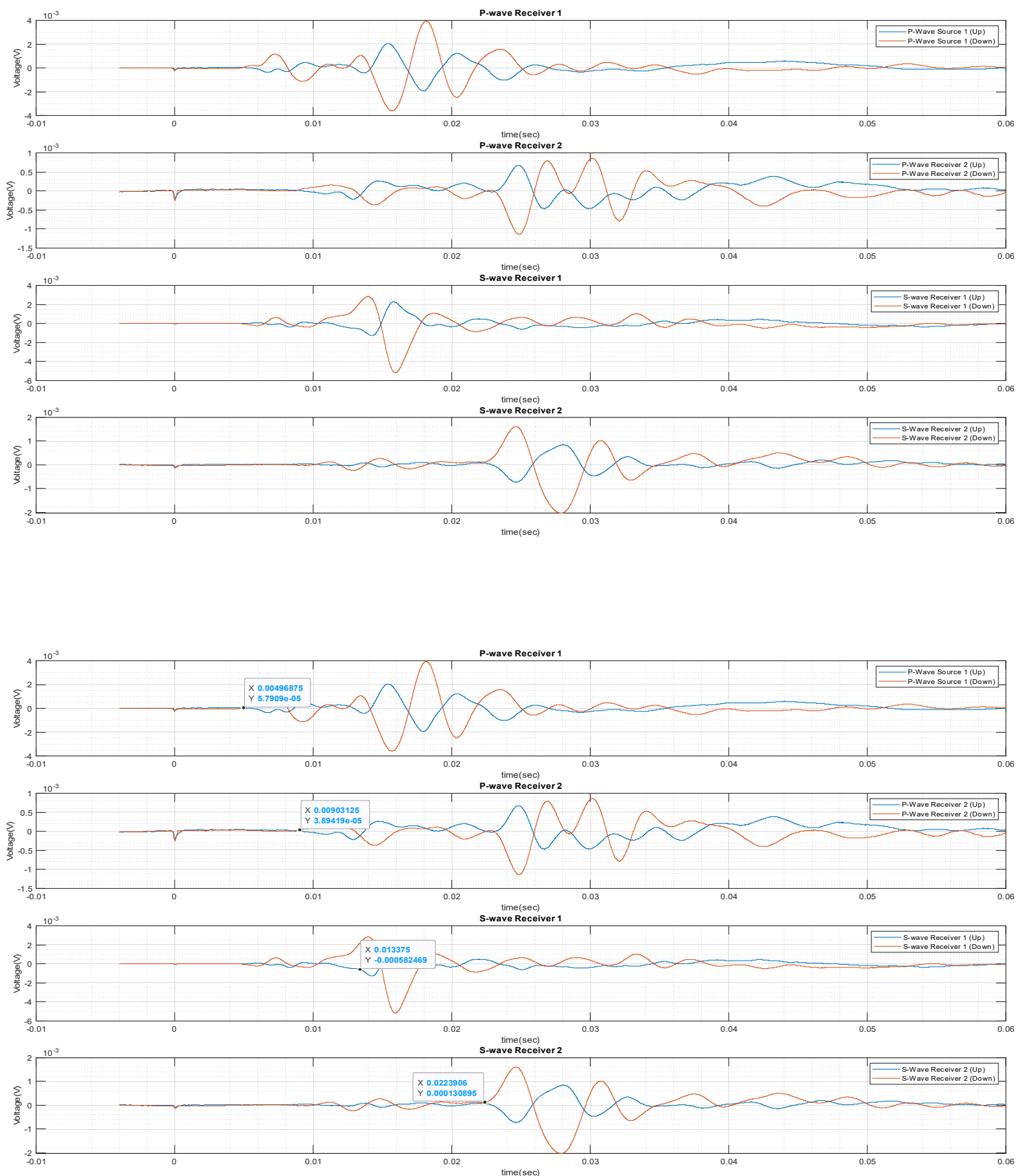

Data Set $12(8.26 .2019)$

Run00081f

R1_1 

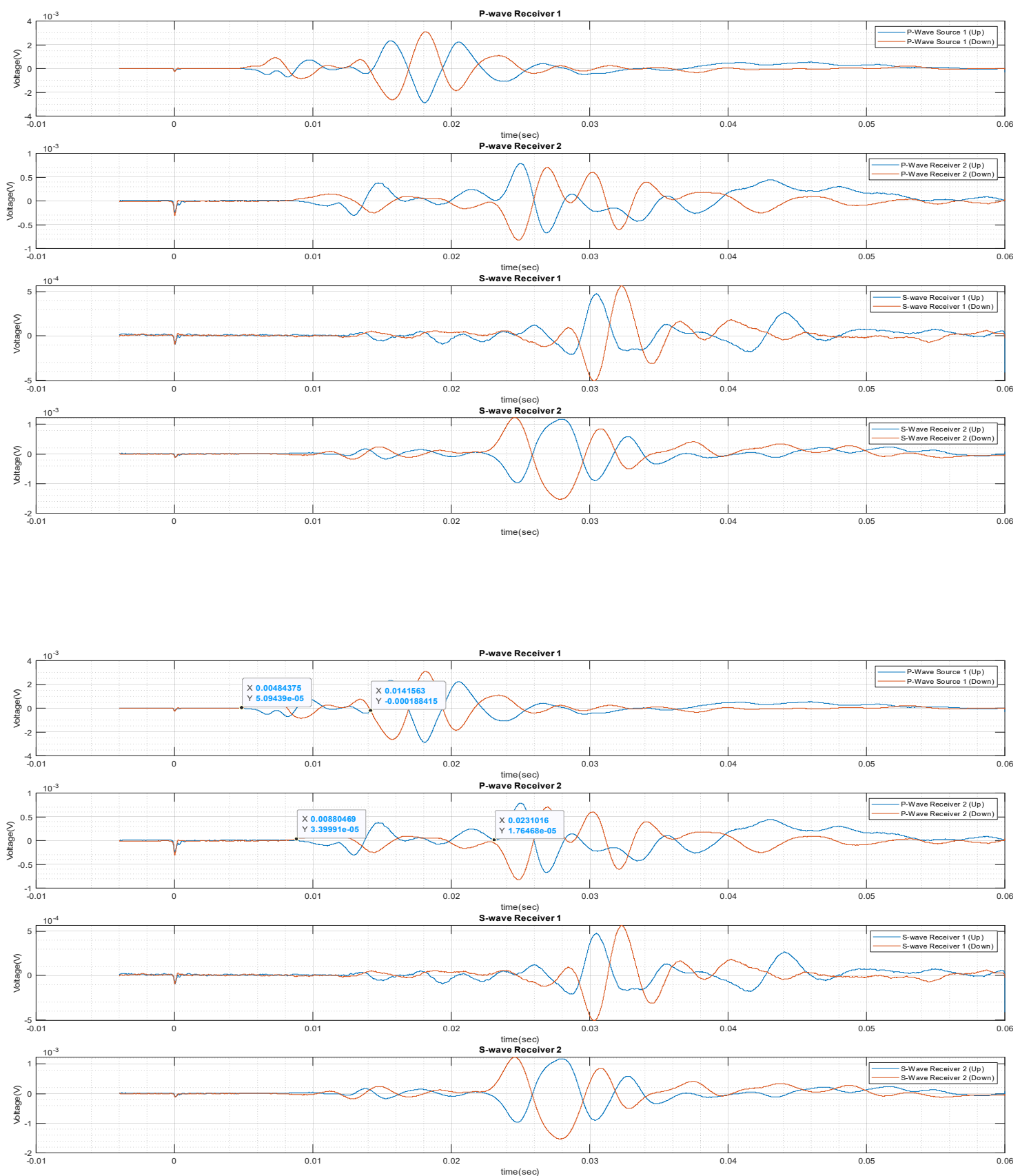

Data Set $12(8.26 .2019)$

Run00082f

R1_2 

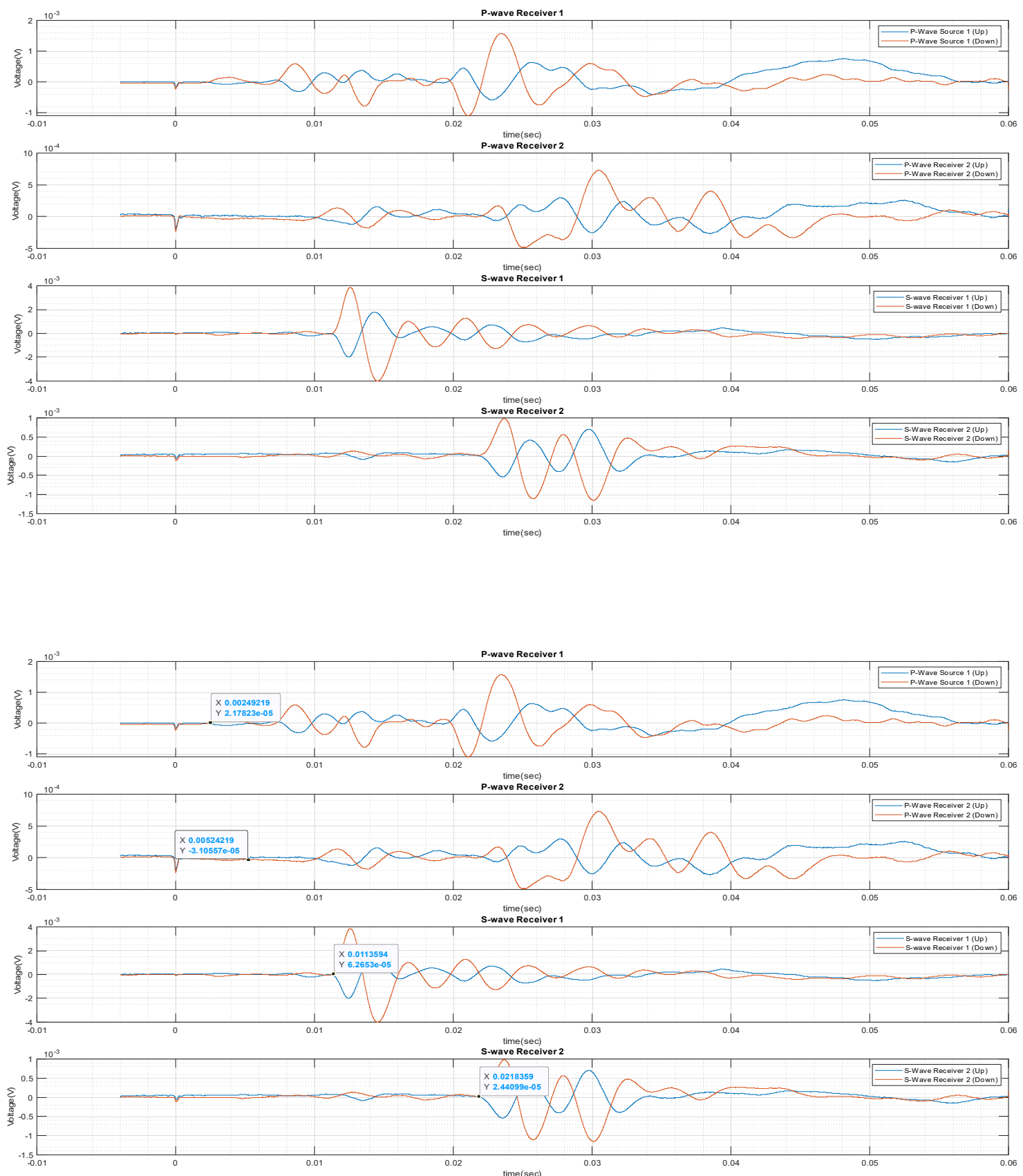

Data Set $12(8.26 .2019)$

Run00083f

R2_1 

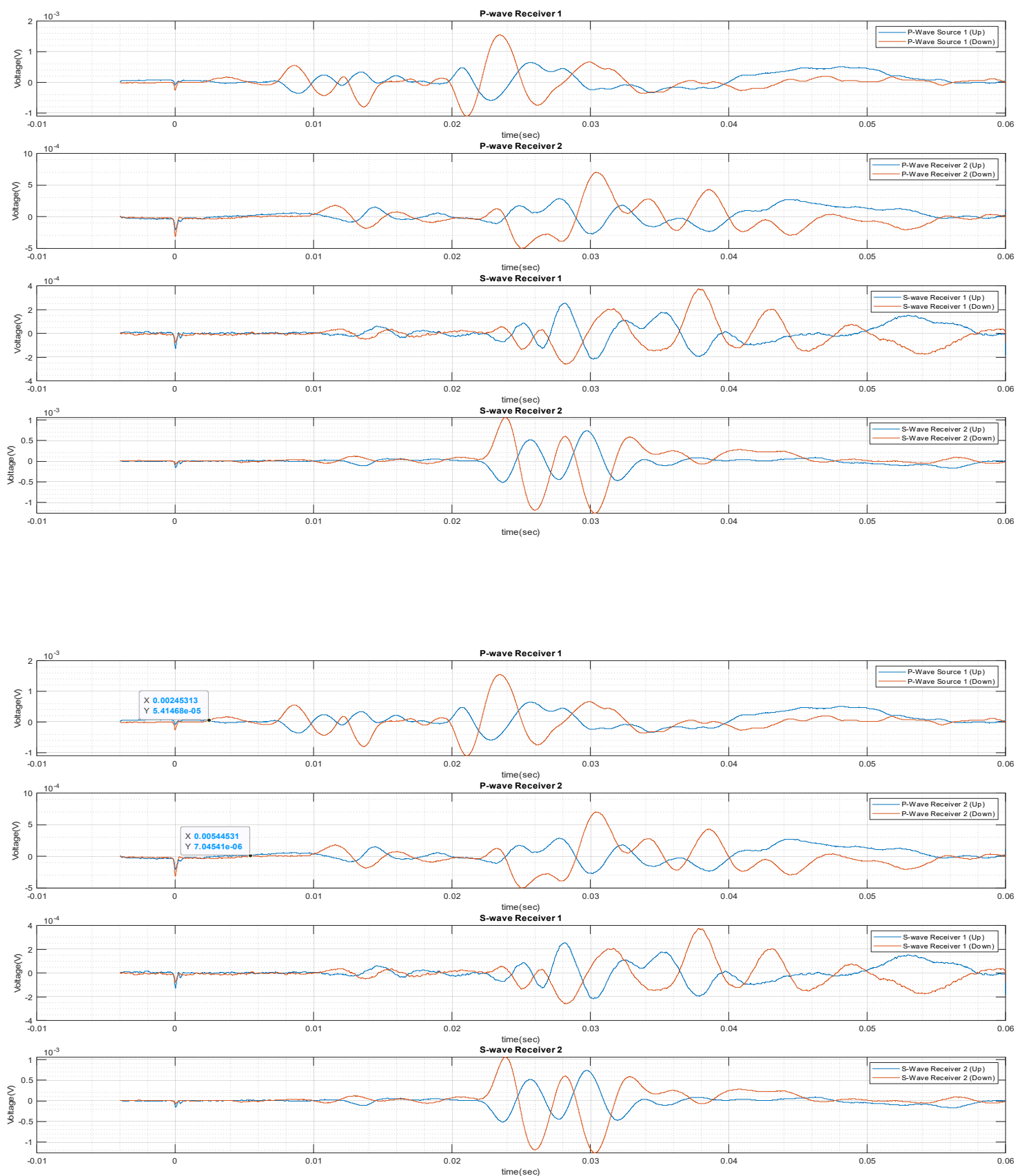

Data Set $12(8.26 .2019)$

Run00084f

R2_2 

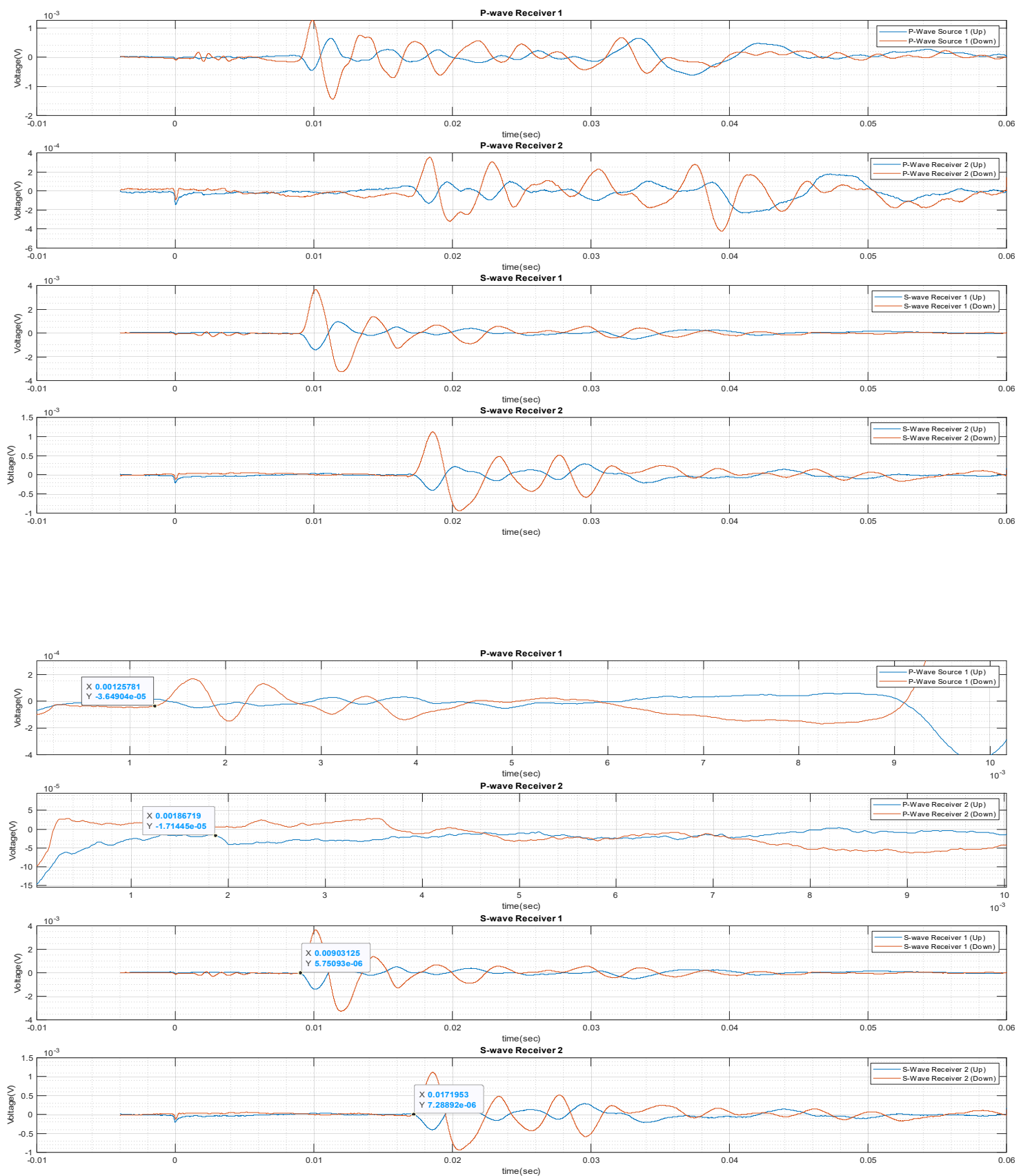

Data Set $12(8.26 .2019)$

Run00085f

R3_1 

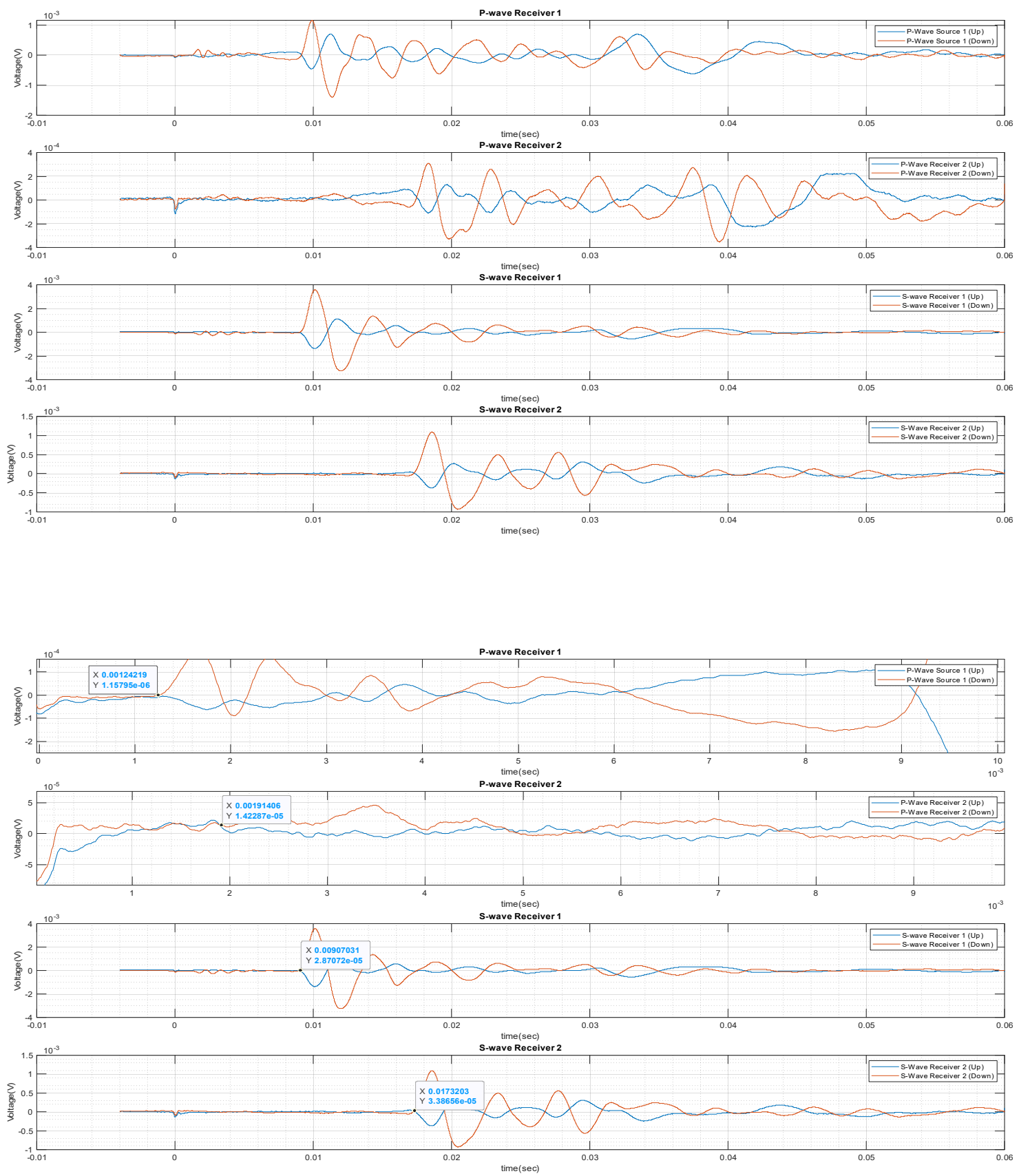

Data Set $12(8.26 .2019)$

Run00086f

R3_2 

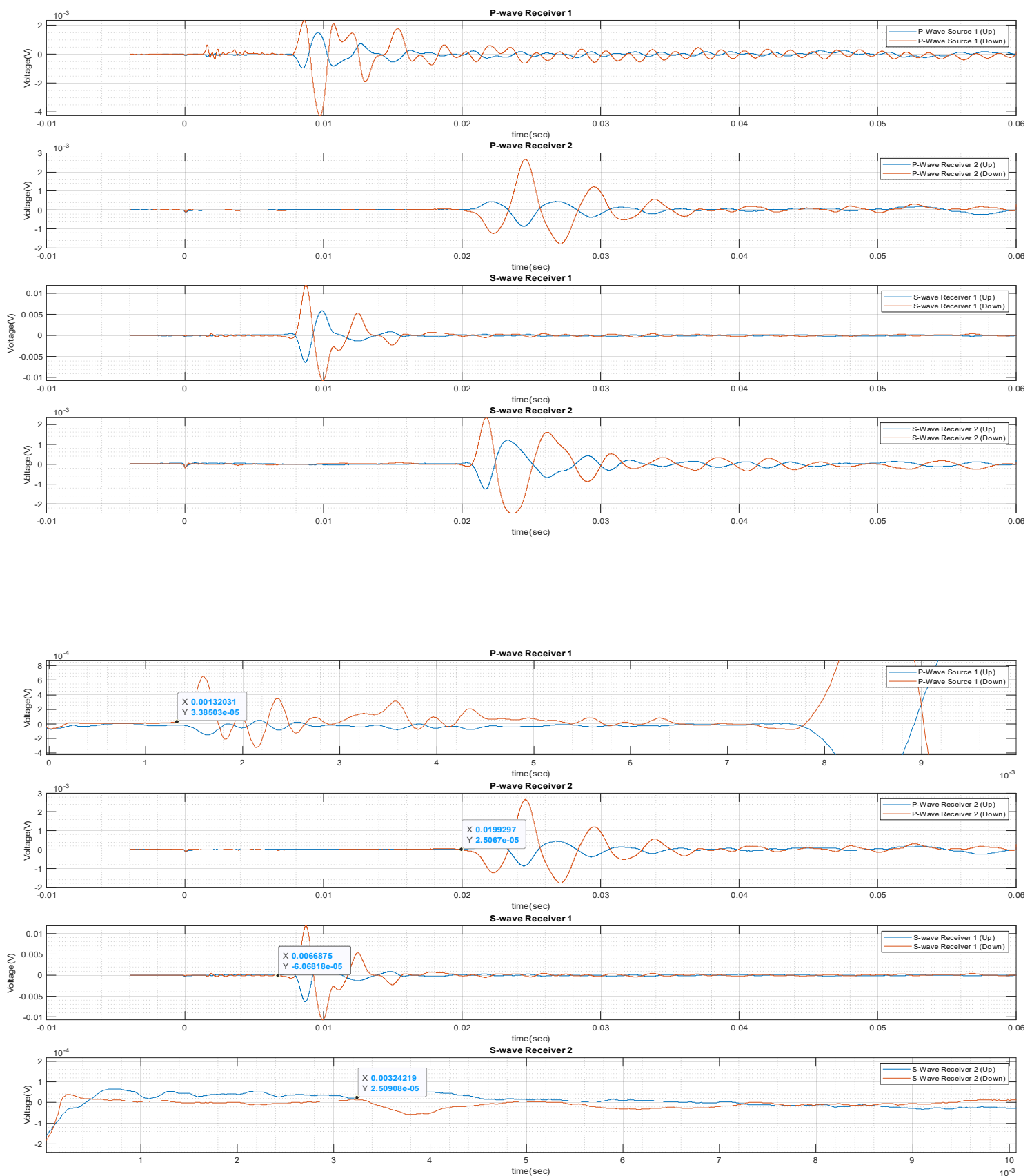

Data Set $12(8.26 .2019)$

Run00087f

R4_1 

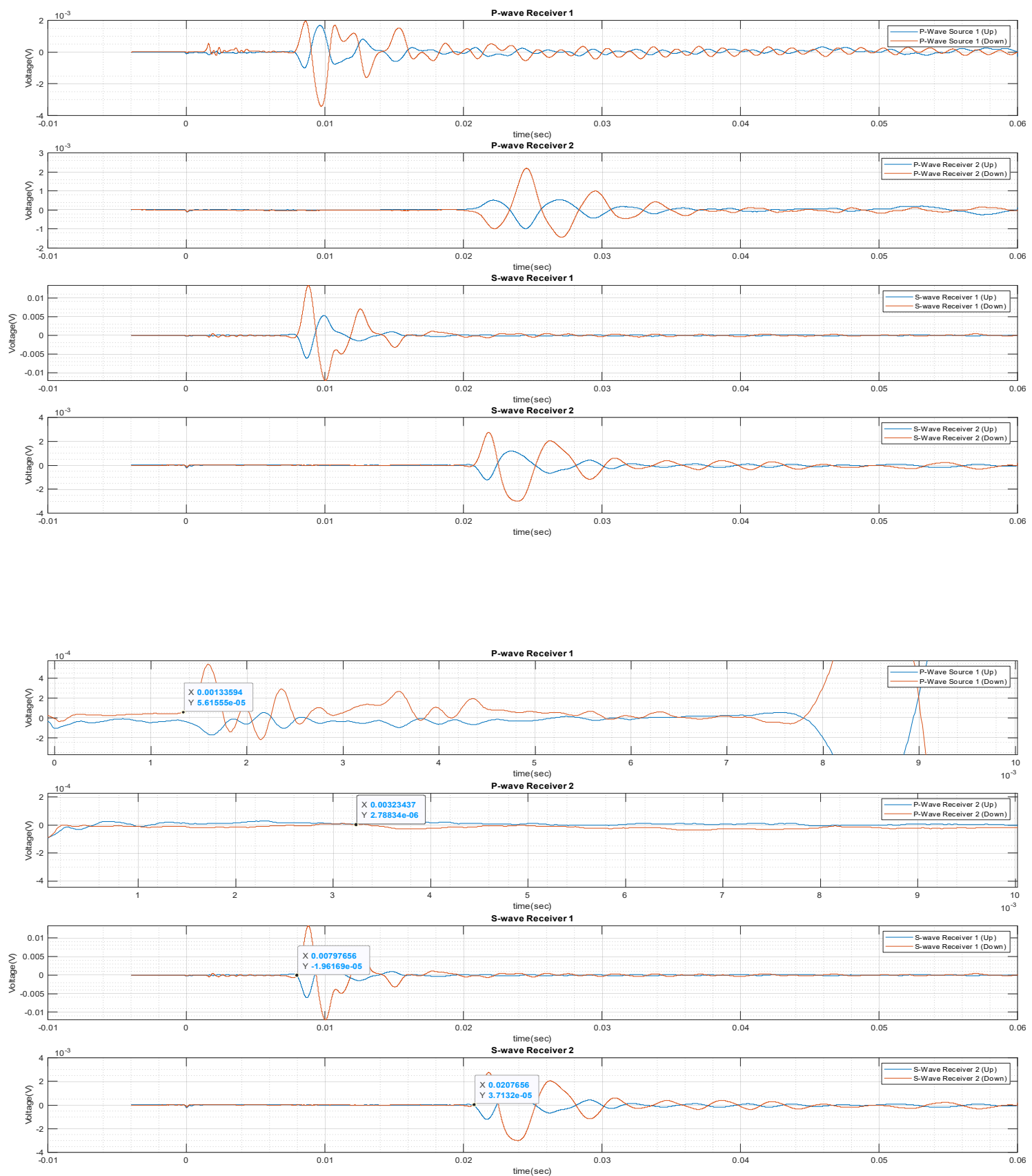

Data Set $12(8.26 .2019)$

Run00088f

R4_2 

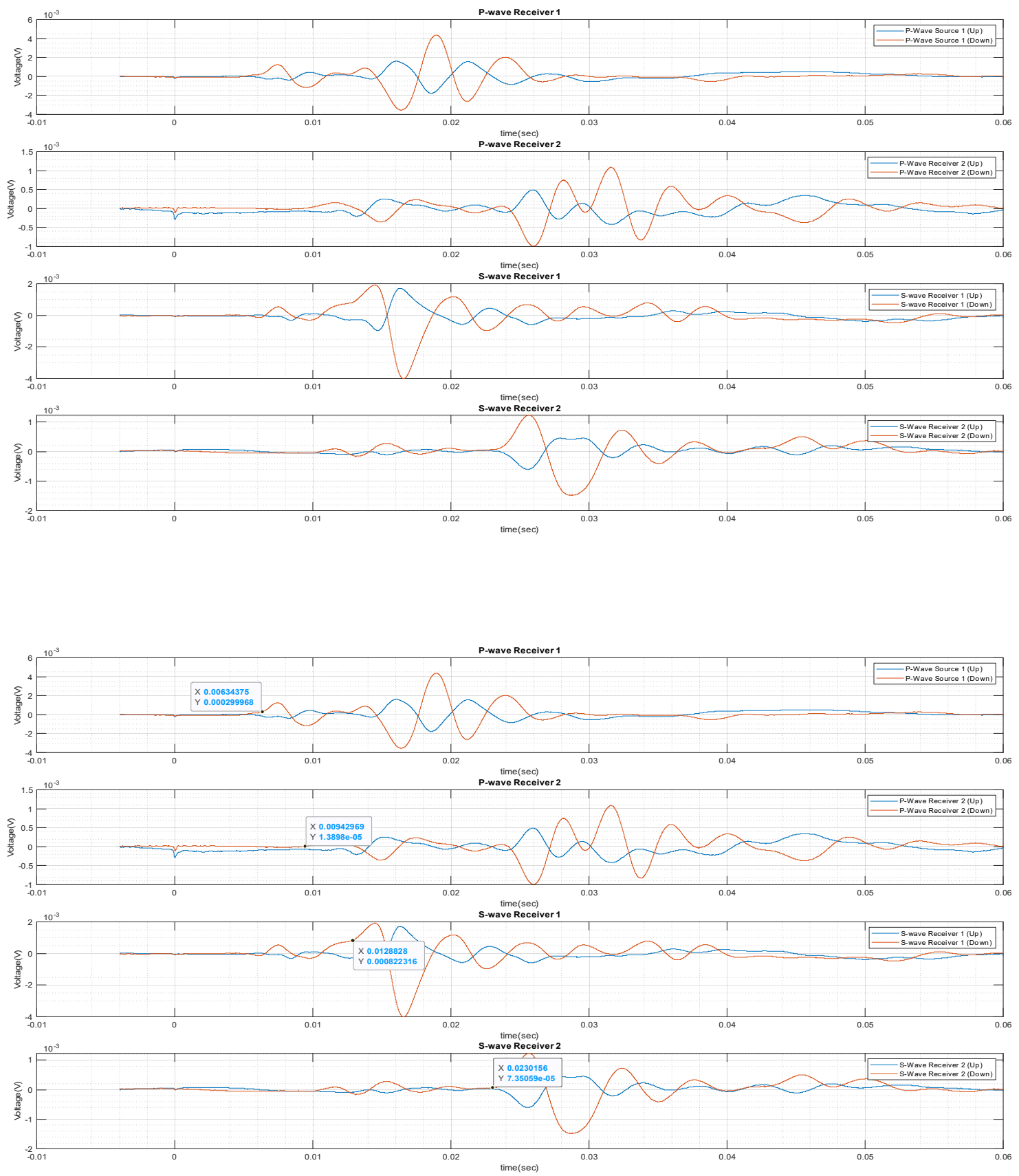

Data Set 13 (8.27.2019)

Run00089f

R1_1 

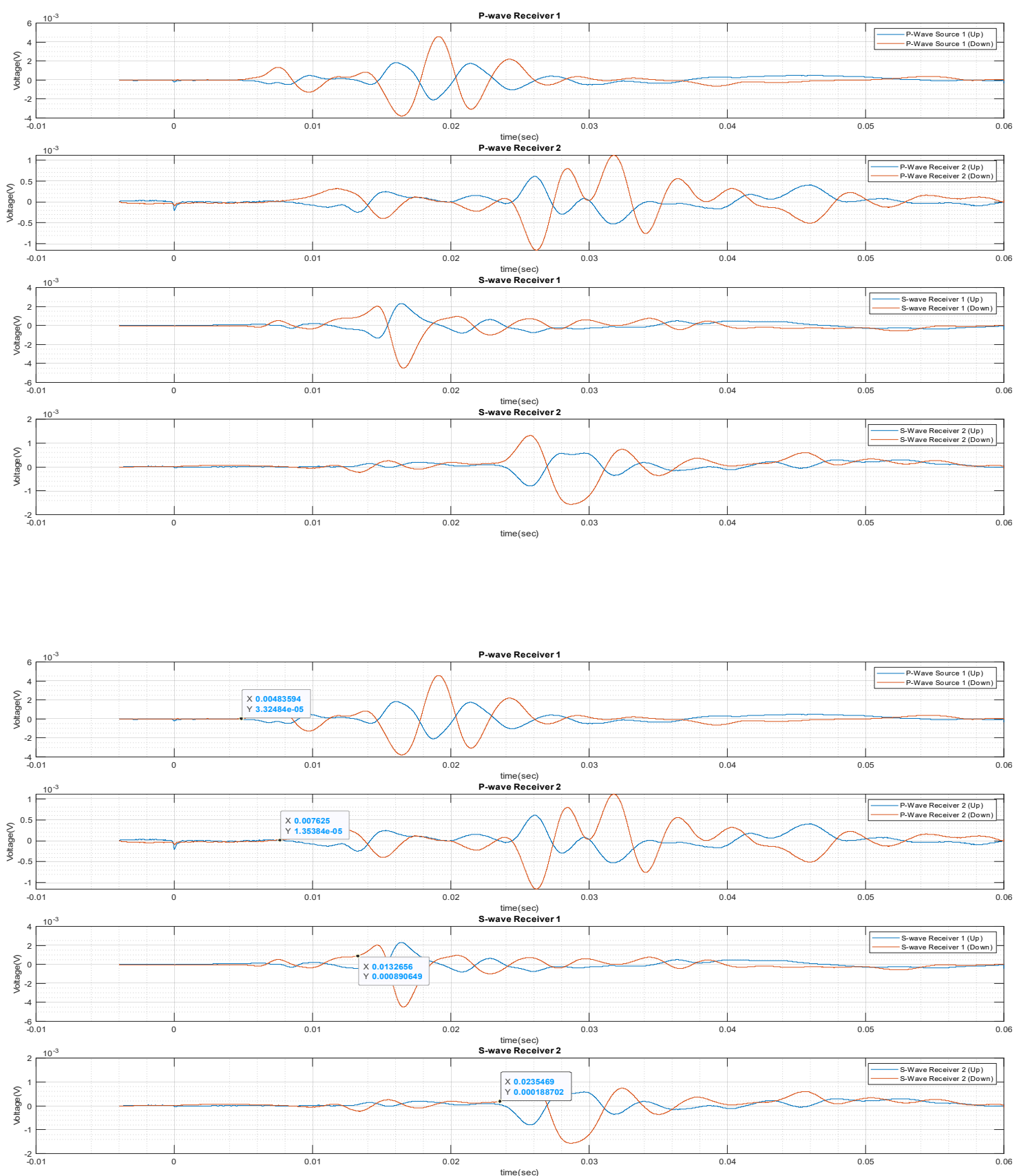

Data Set 13 (8.27.2019)

Run00090f

R1_2 

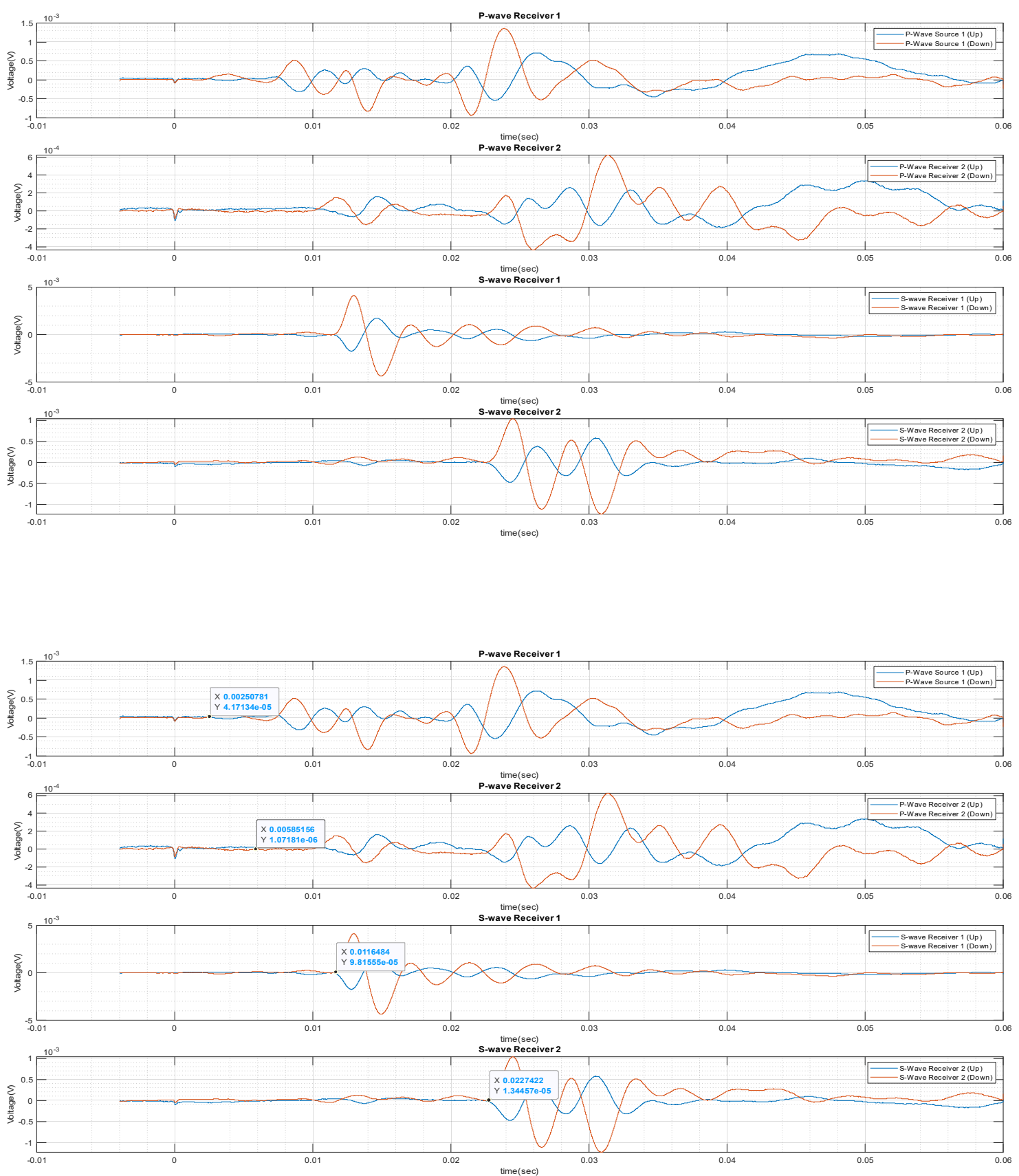

Data Set 13 (8.27.2019)

Run00091f

R2_1 

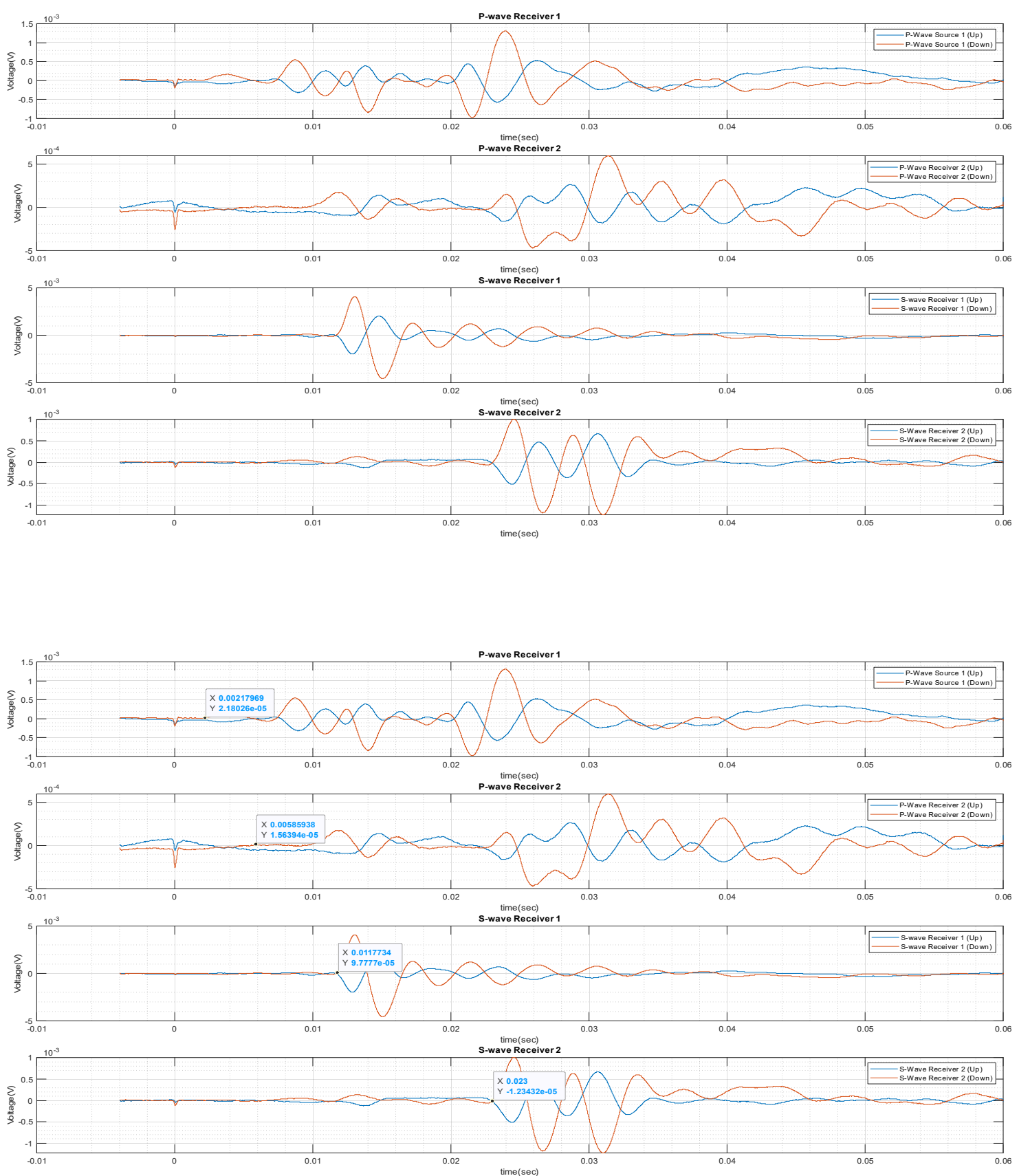

Data Set 13 (8.27.2019)

Run00092f

R2_2 

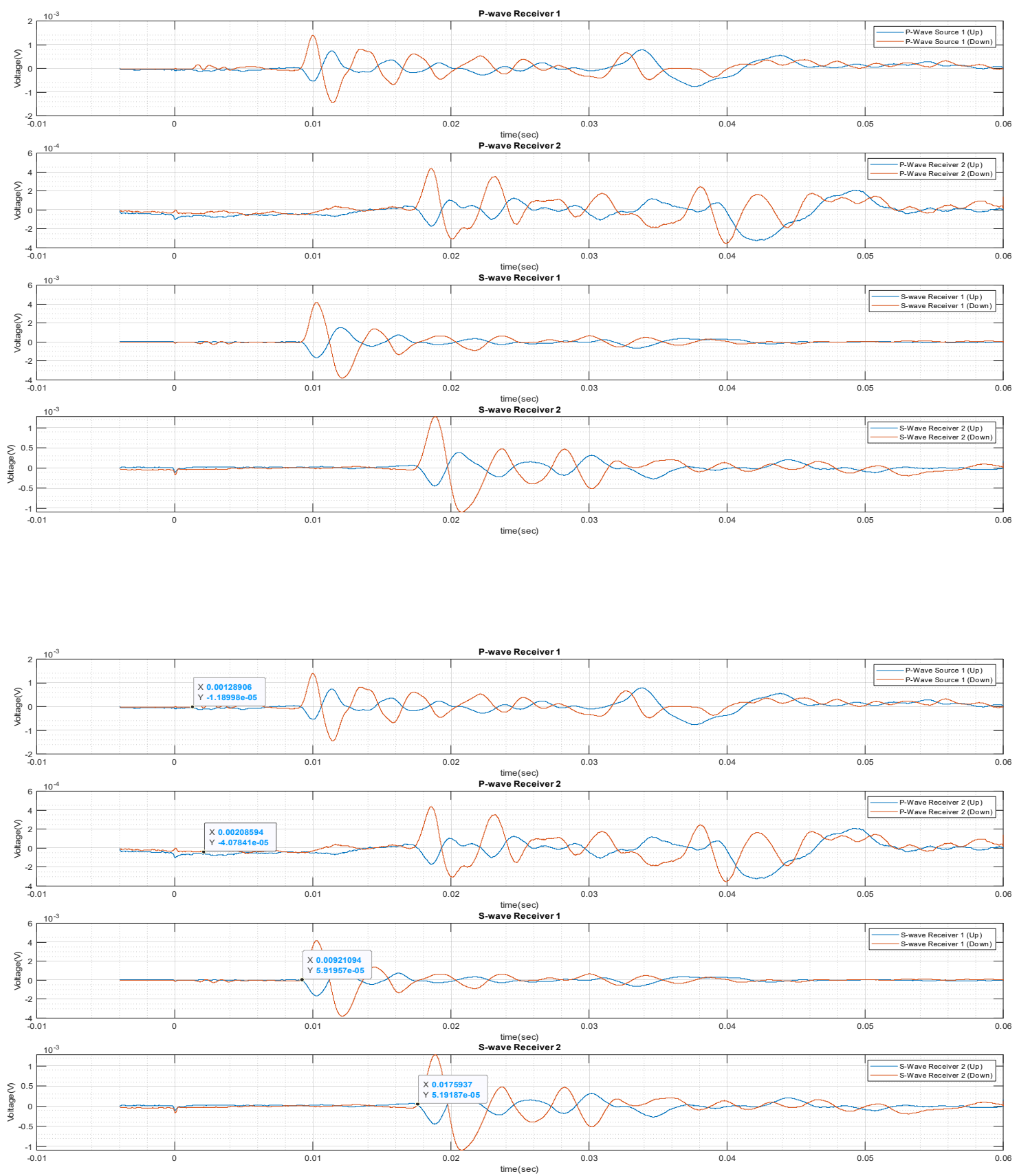

Data Set 13 (8.27.2019)

Run00093f

R3_1 

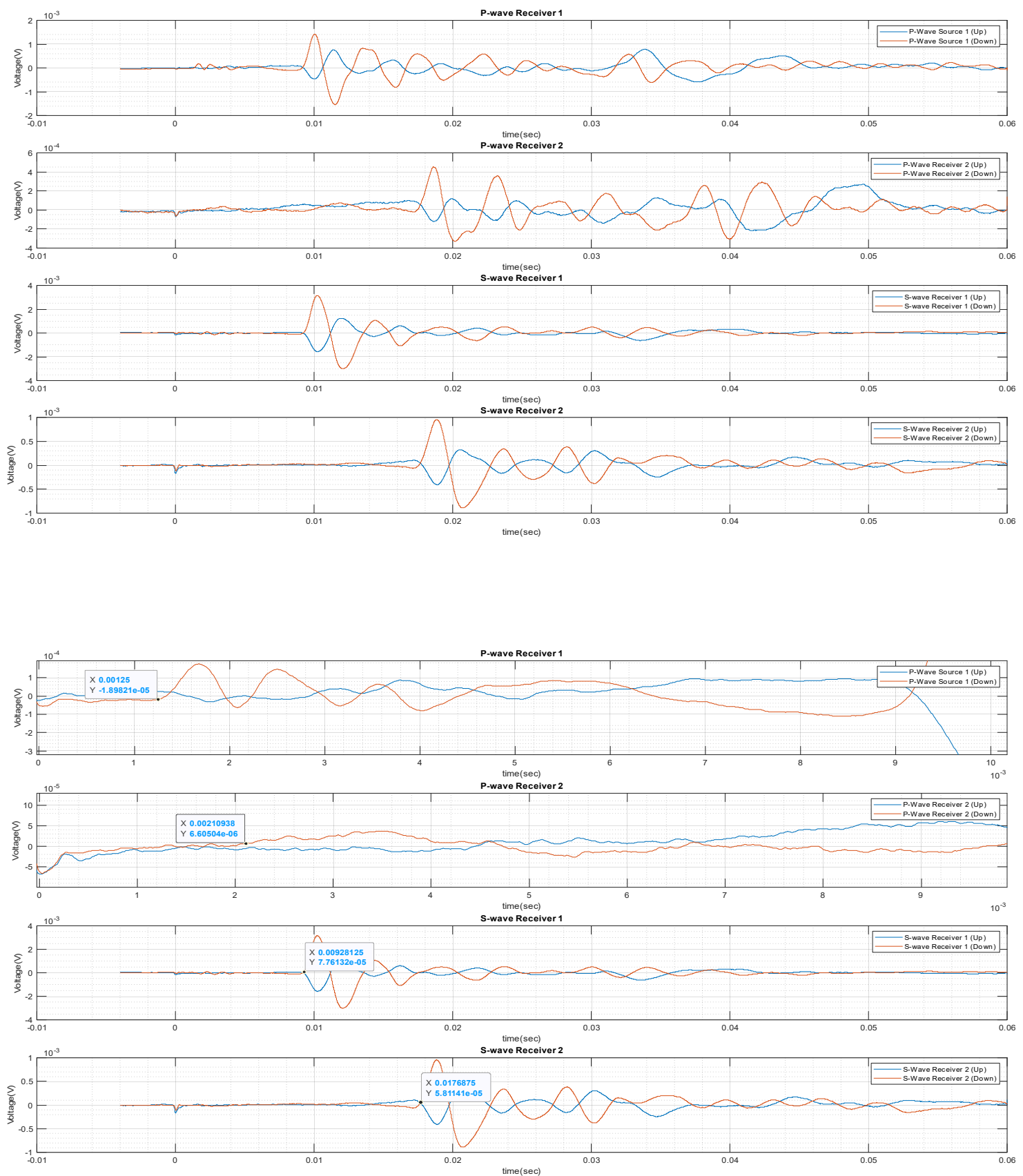

Data Set 13 (8.27.2019)

Run00094f

R3_2 

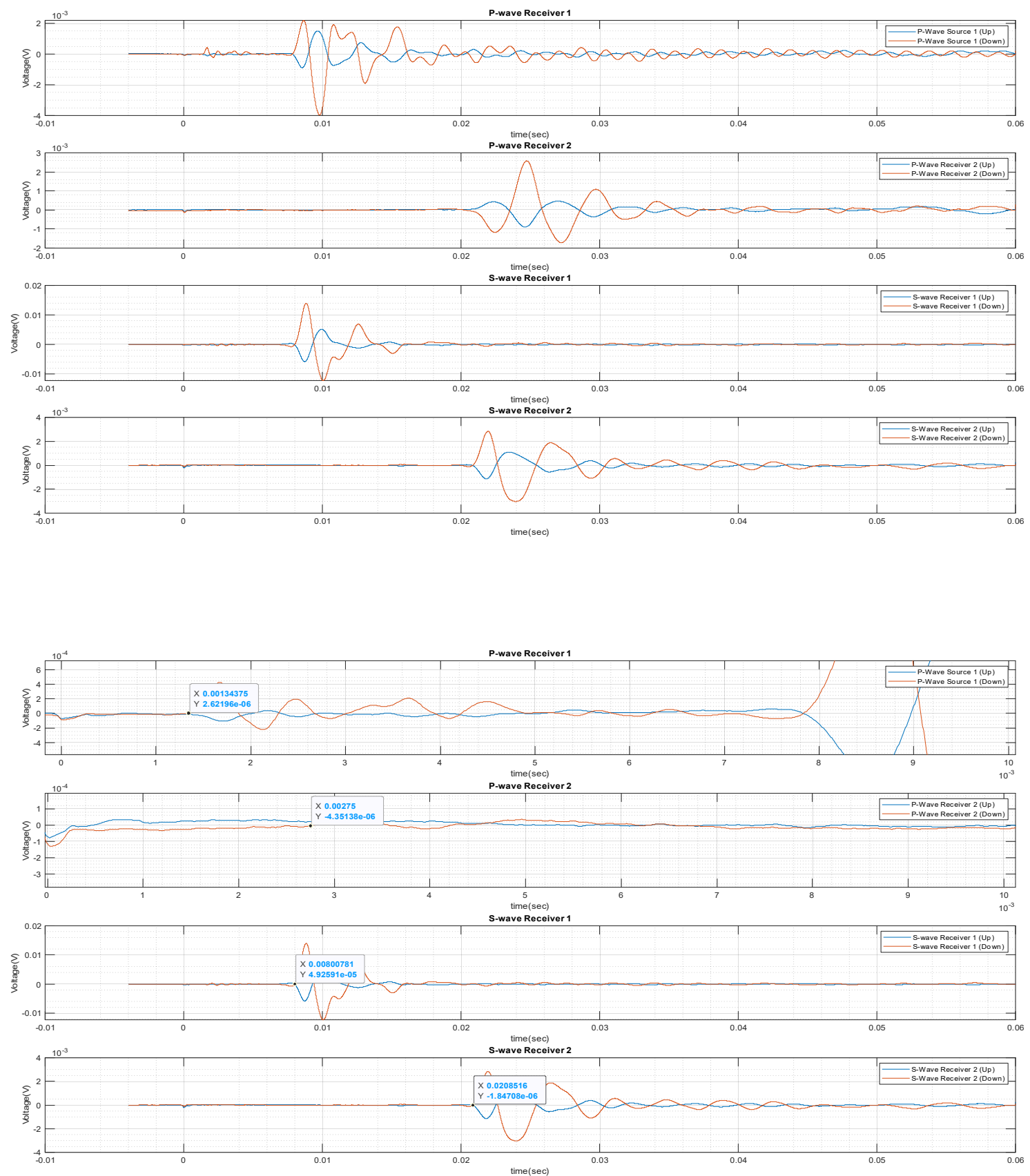

Data Set $13(8.27 .2019)$

Run00095f

R4_1 

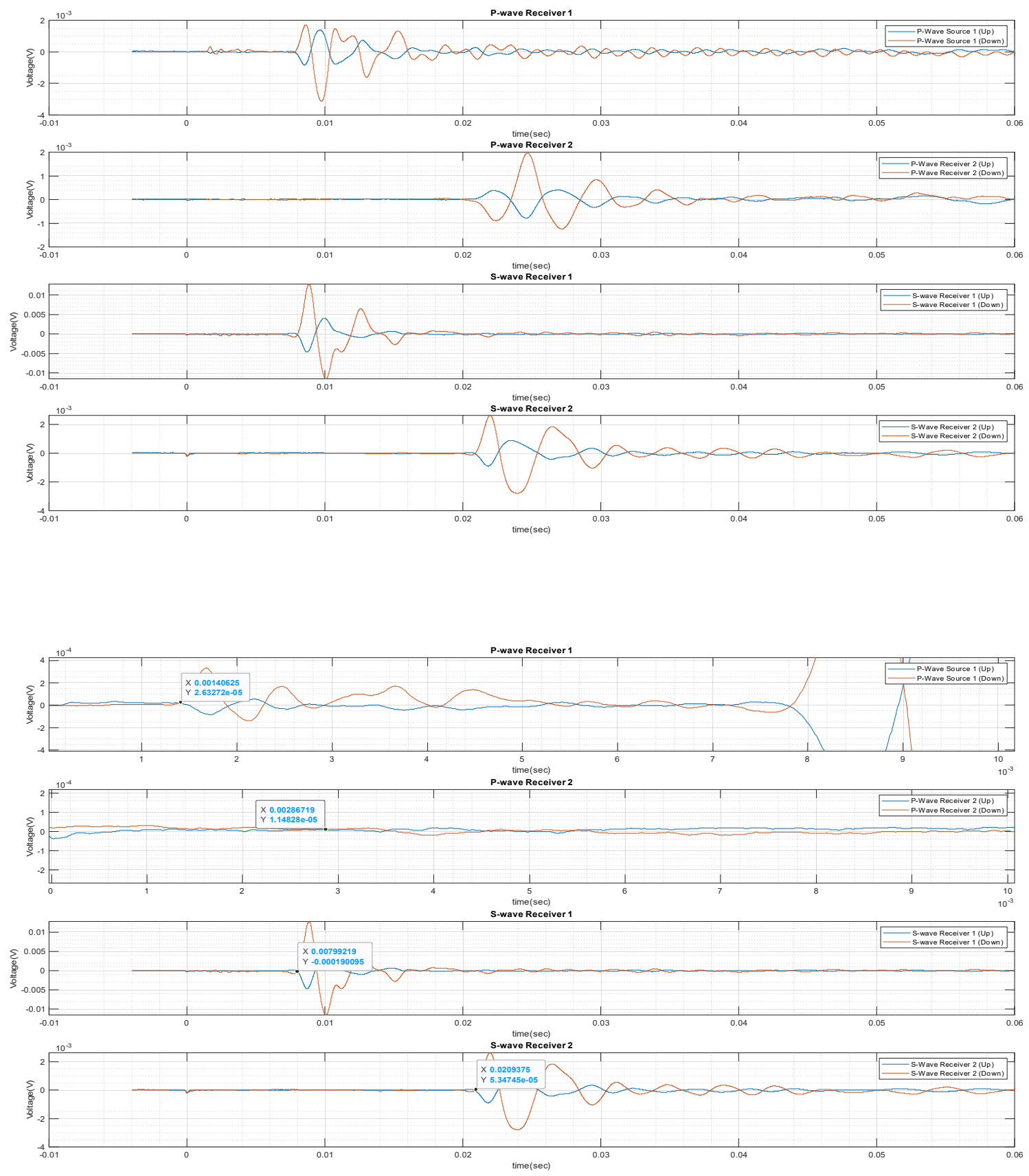

Data Set 13 (8.27.2019)

Run00096f

R4_2 

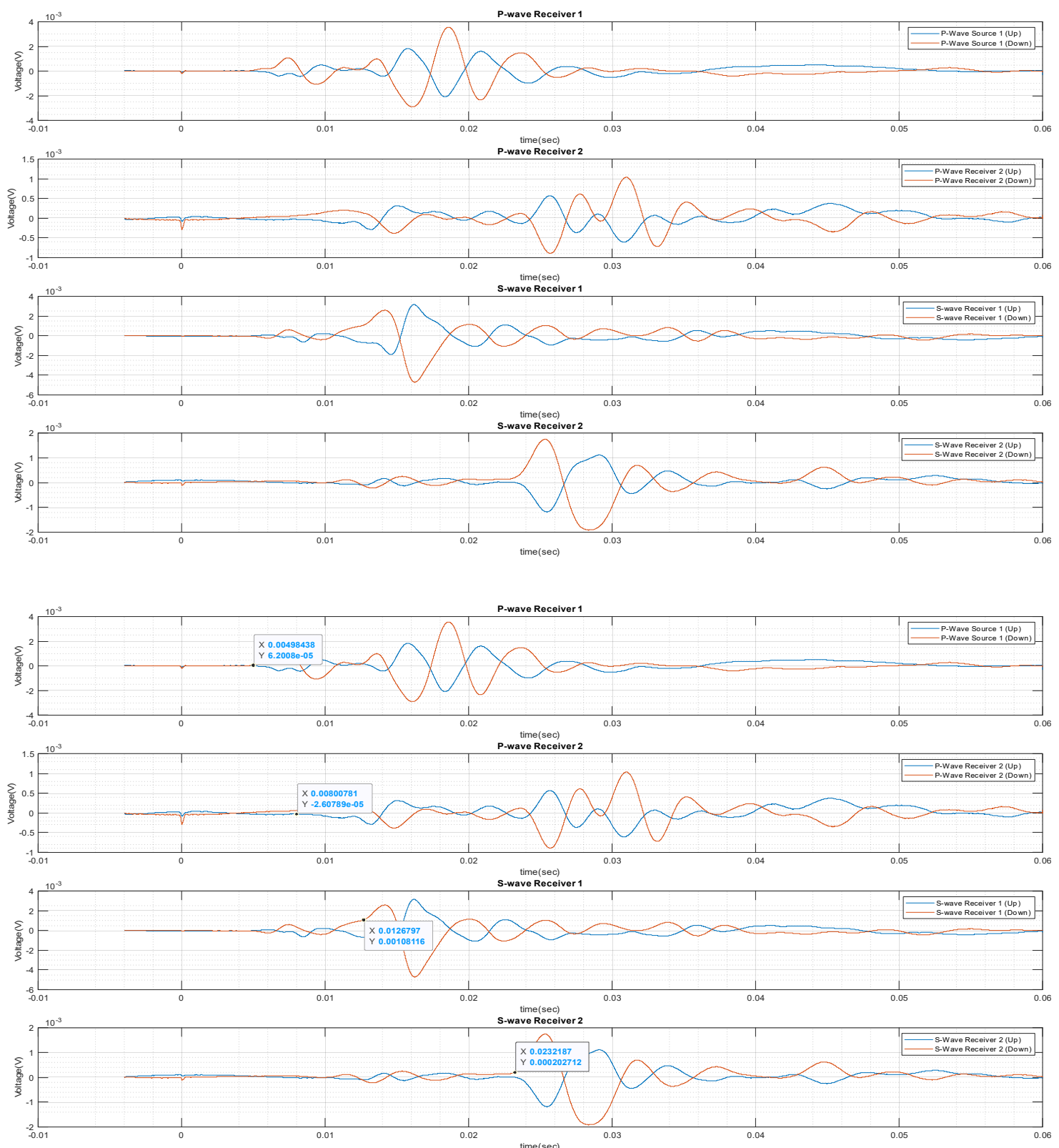

Data Set 14 (8.28.2019)

Run00097f

R1_1 

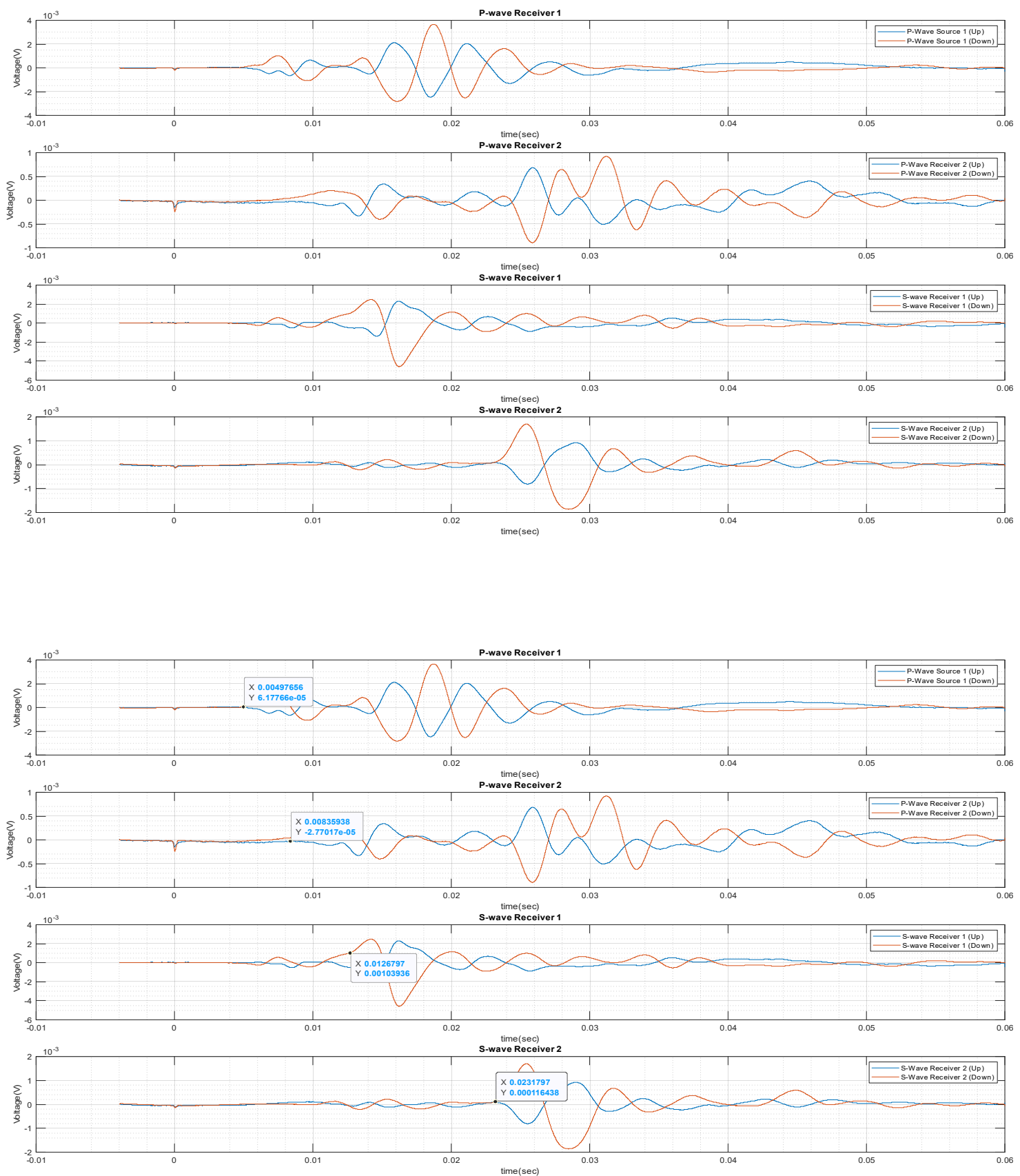

Data Set $14(8.28 .2019)$

Run00098f

R1_2 

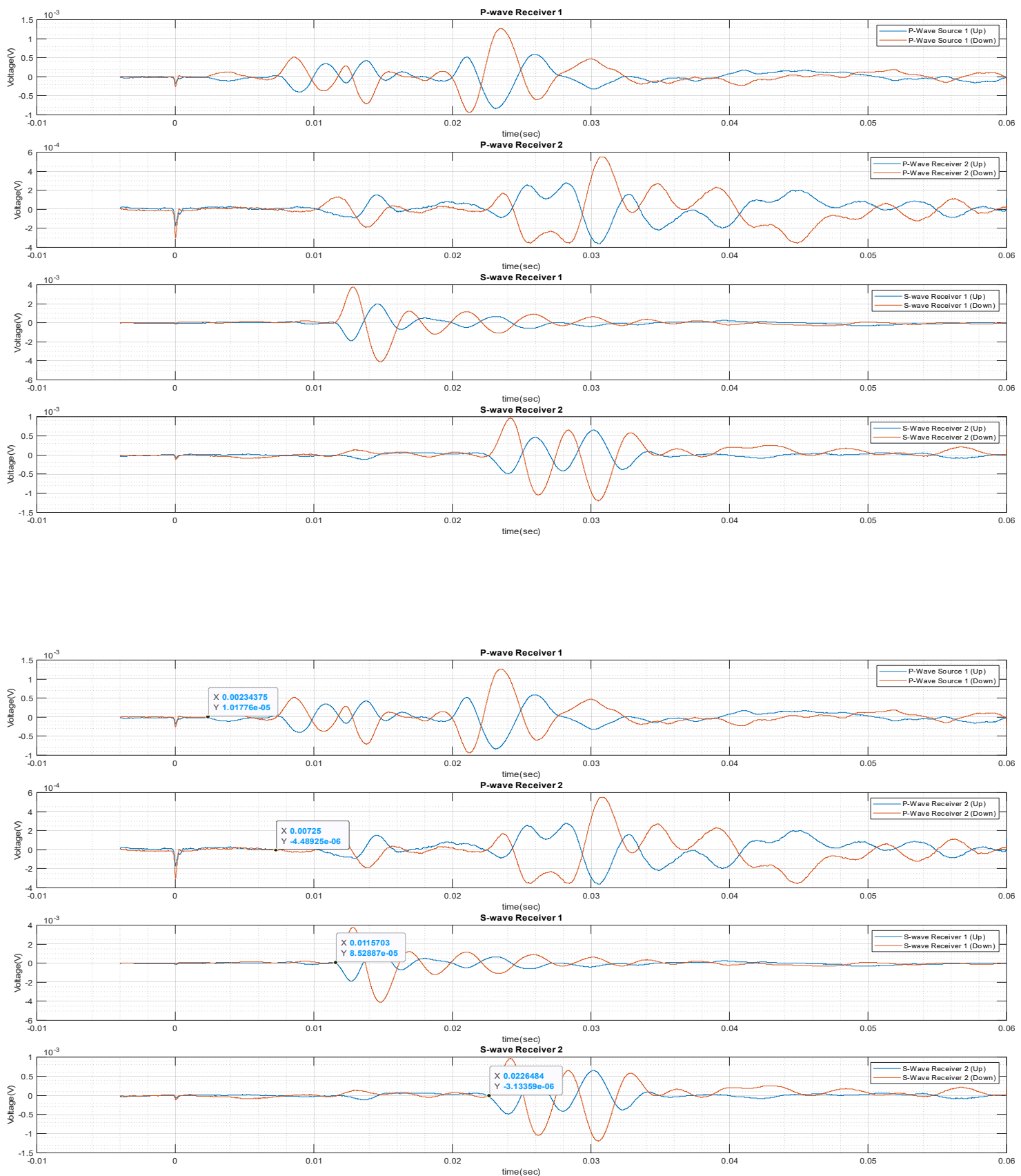

Data Set $14(8.28 .2019)$

Run00090f

R2_1 

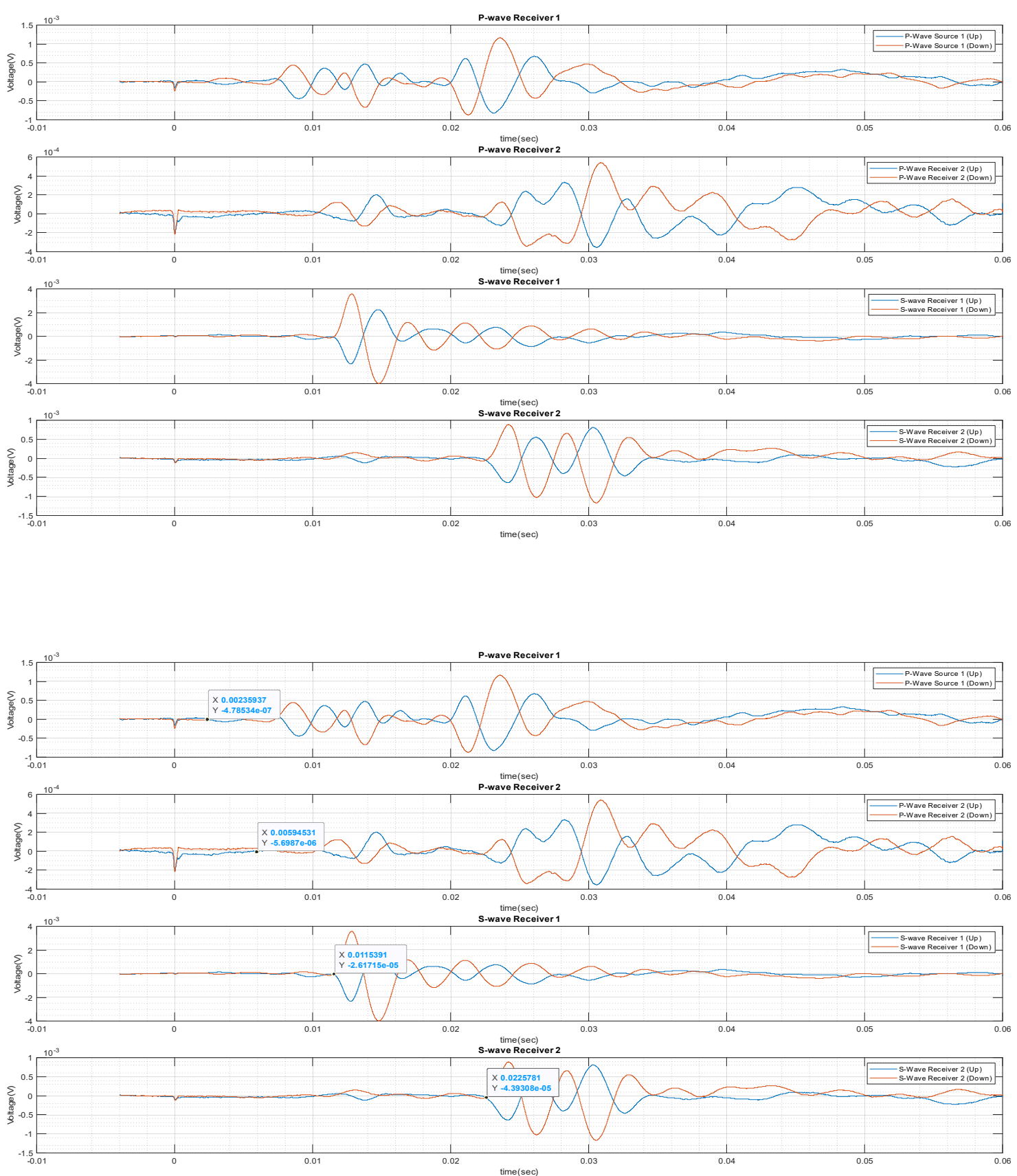

Data Set $14(8.28 .2019)$

Run00100f

R2_2 

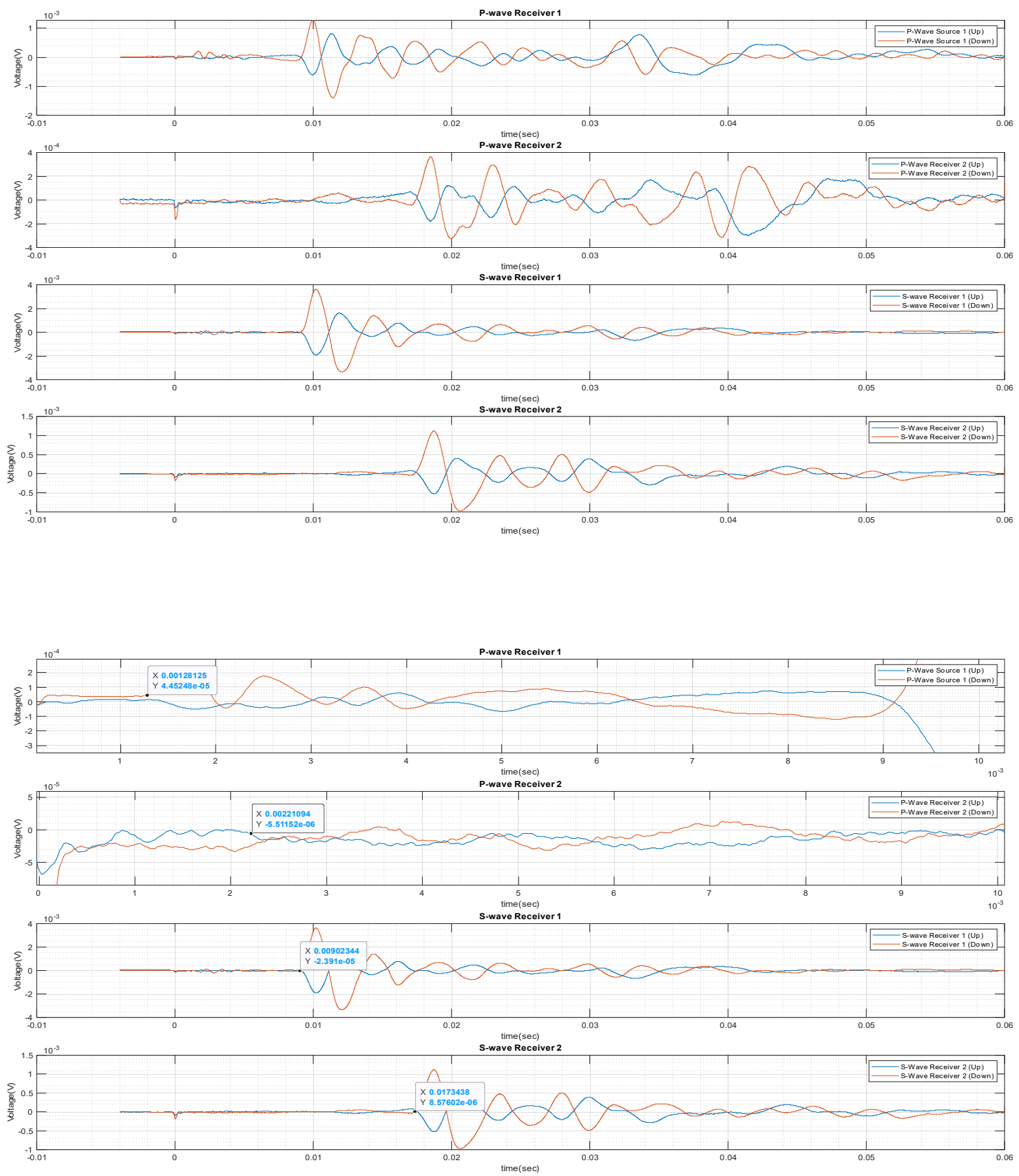

Data Set 14 (8.28.2019)

Run00101f

R3_1 

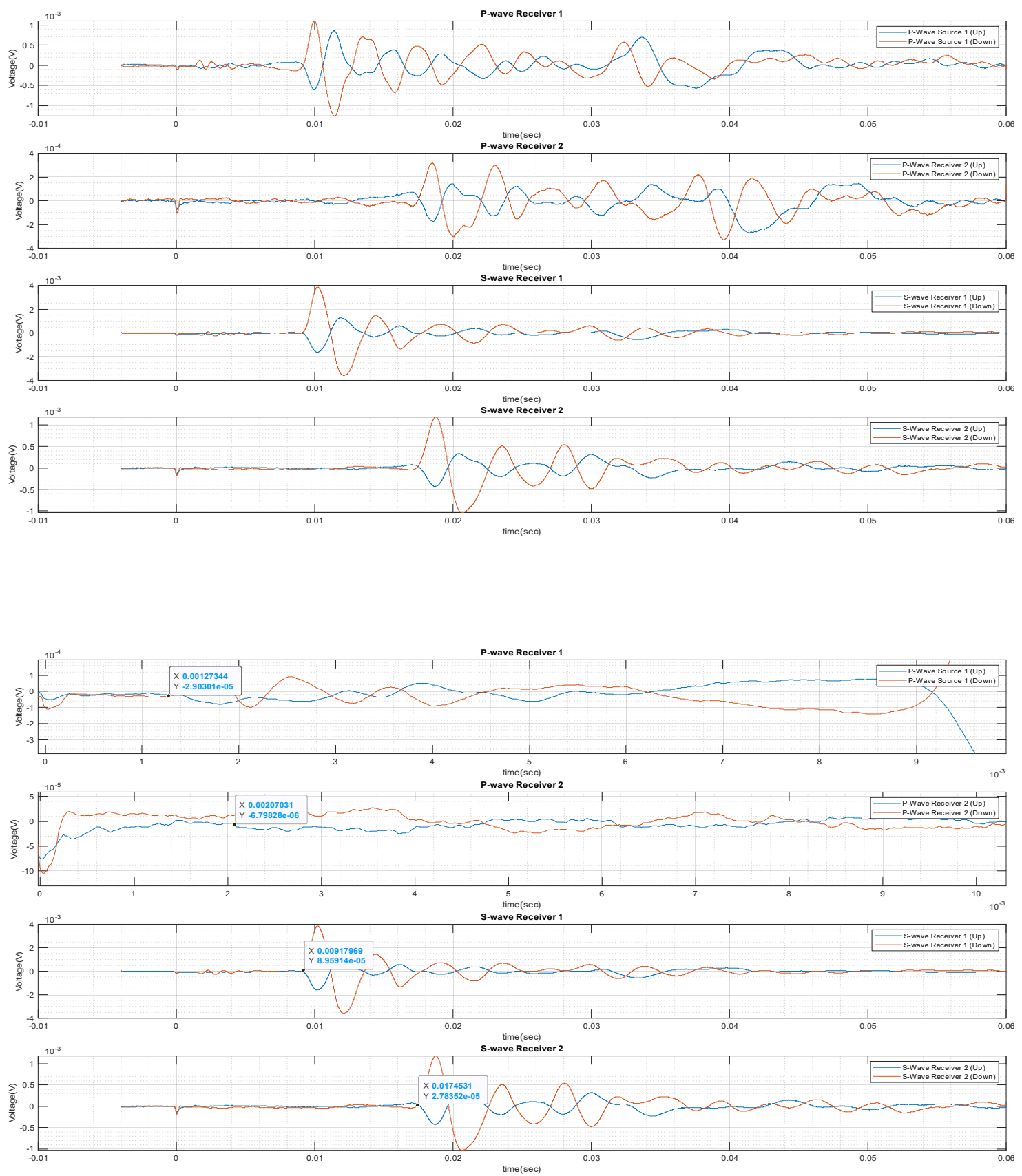

Data Set $14(8.28 .2019)$

Run00102f

R3_2 

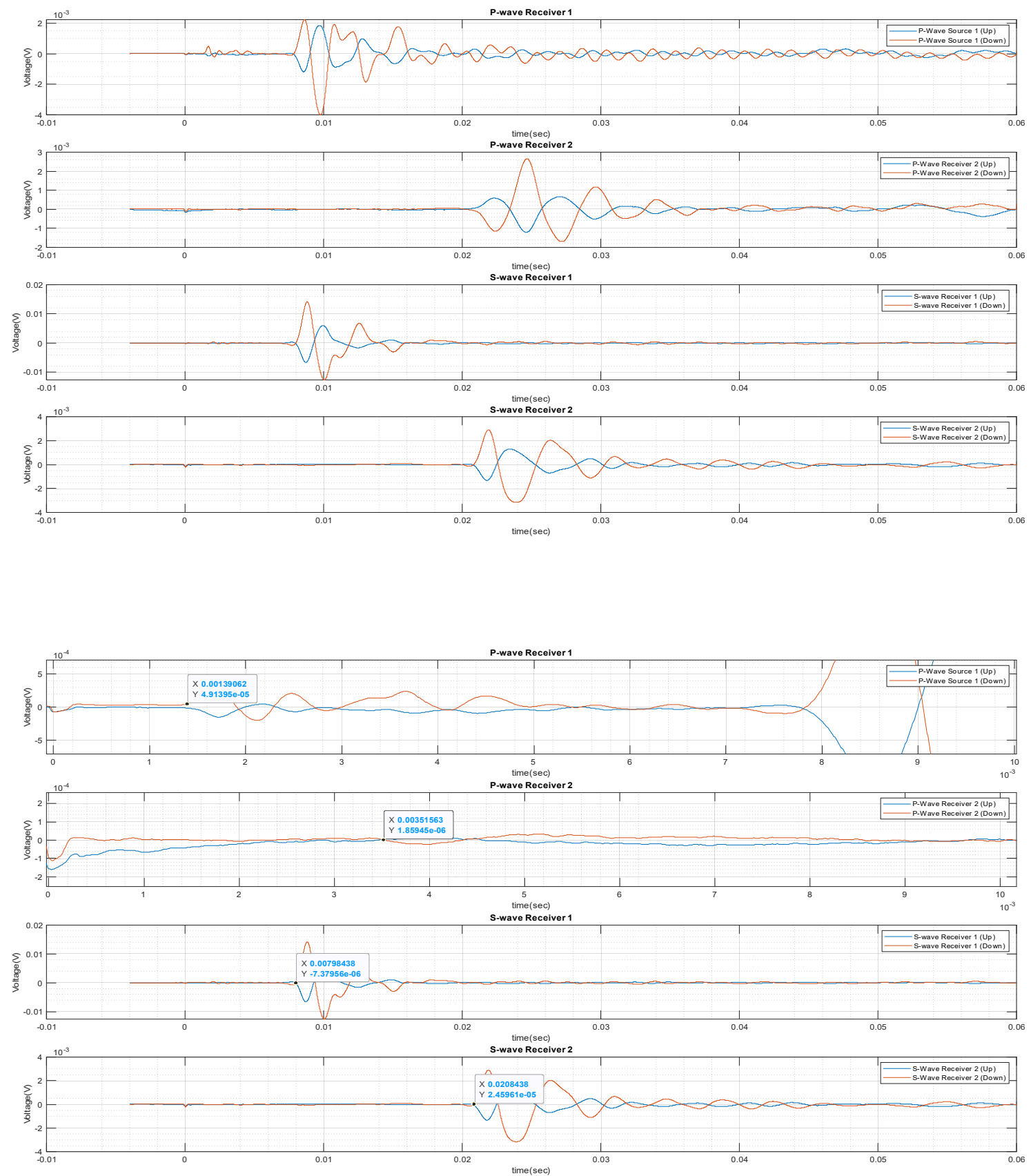

Data Set $14(8.28 .2019)$

Run00103f

R4_1 

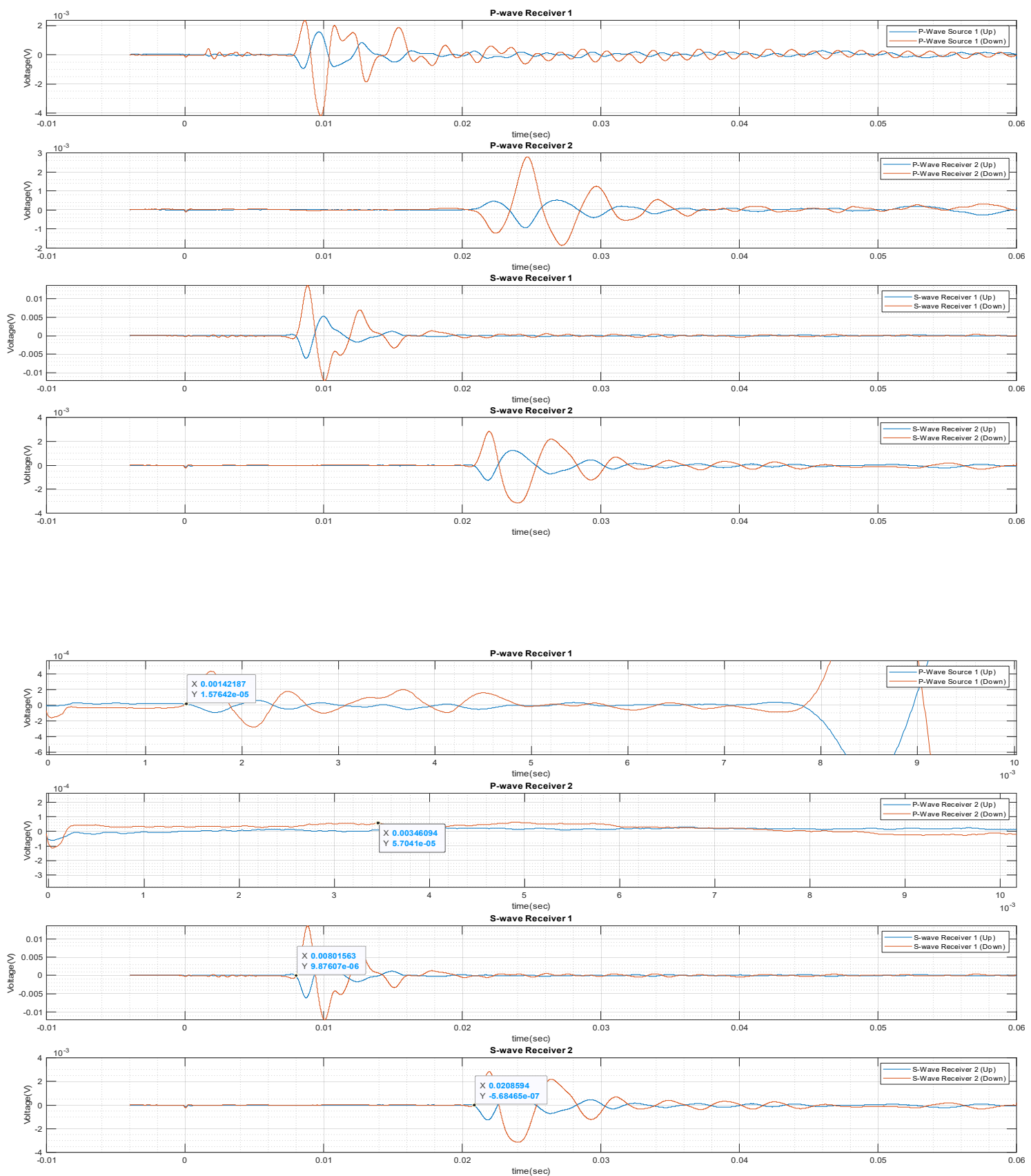

Data Set $14(8.28 .2019)$

Run00104f

R4_2 

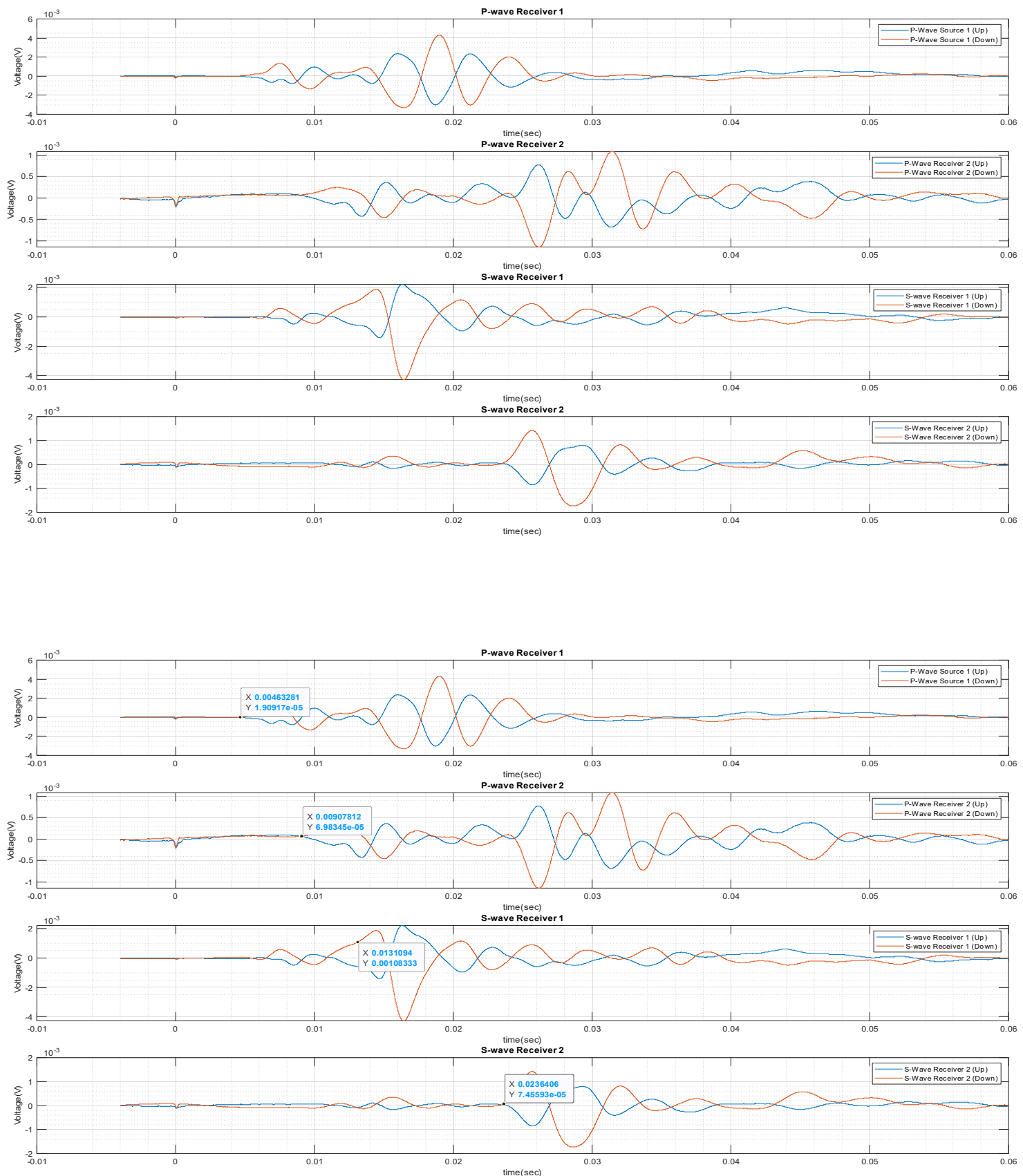

Data Set 15 (8.29.2019)

Run00105f

R1_1 

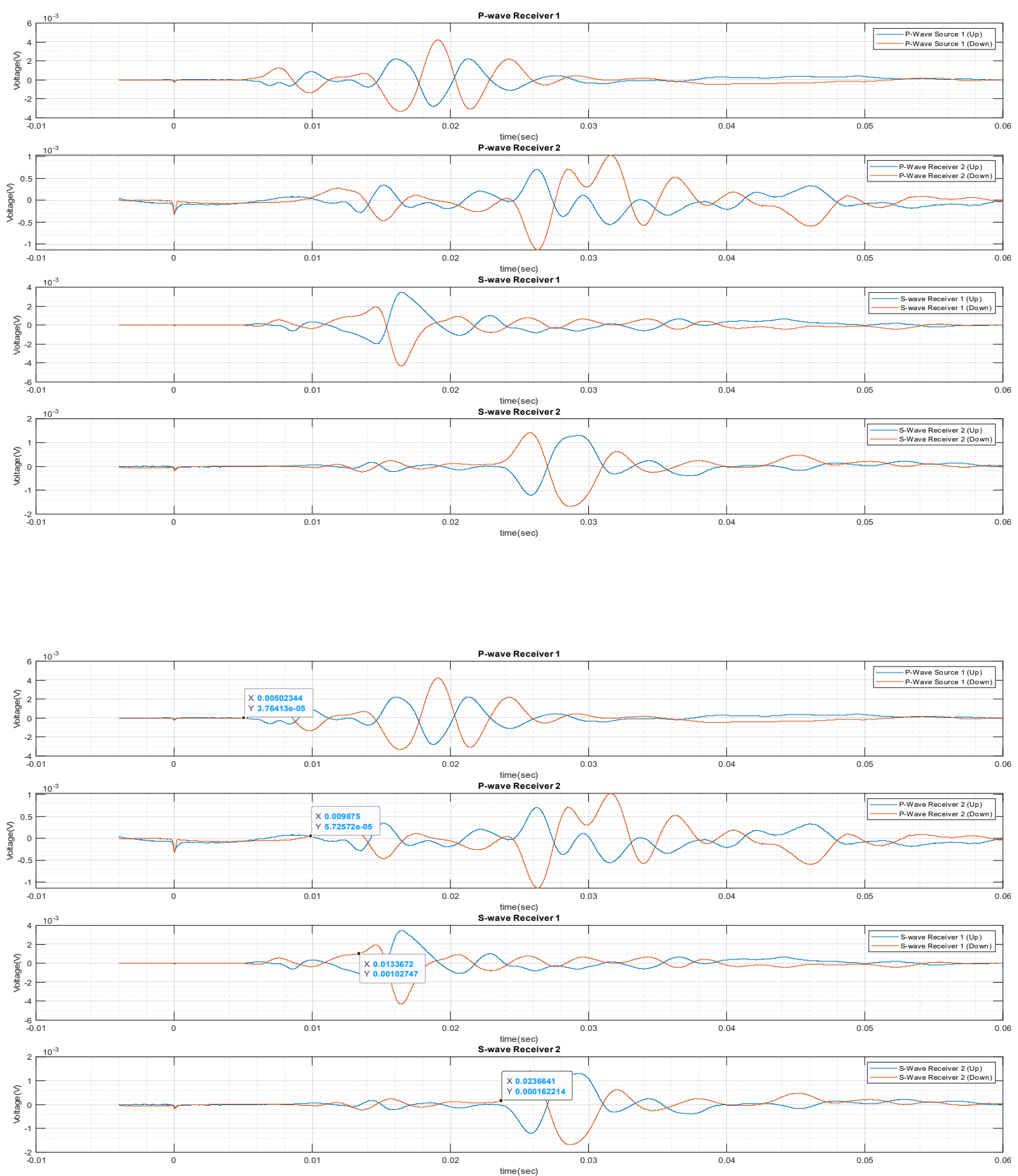

Data Set 15 (8.29.2019)

Run00106f

R1_2 

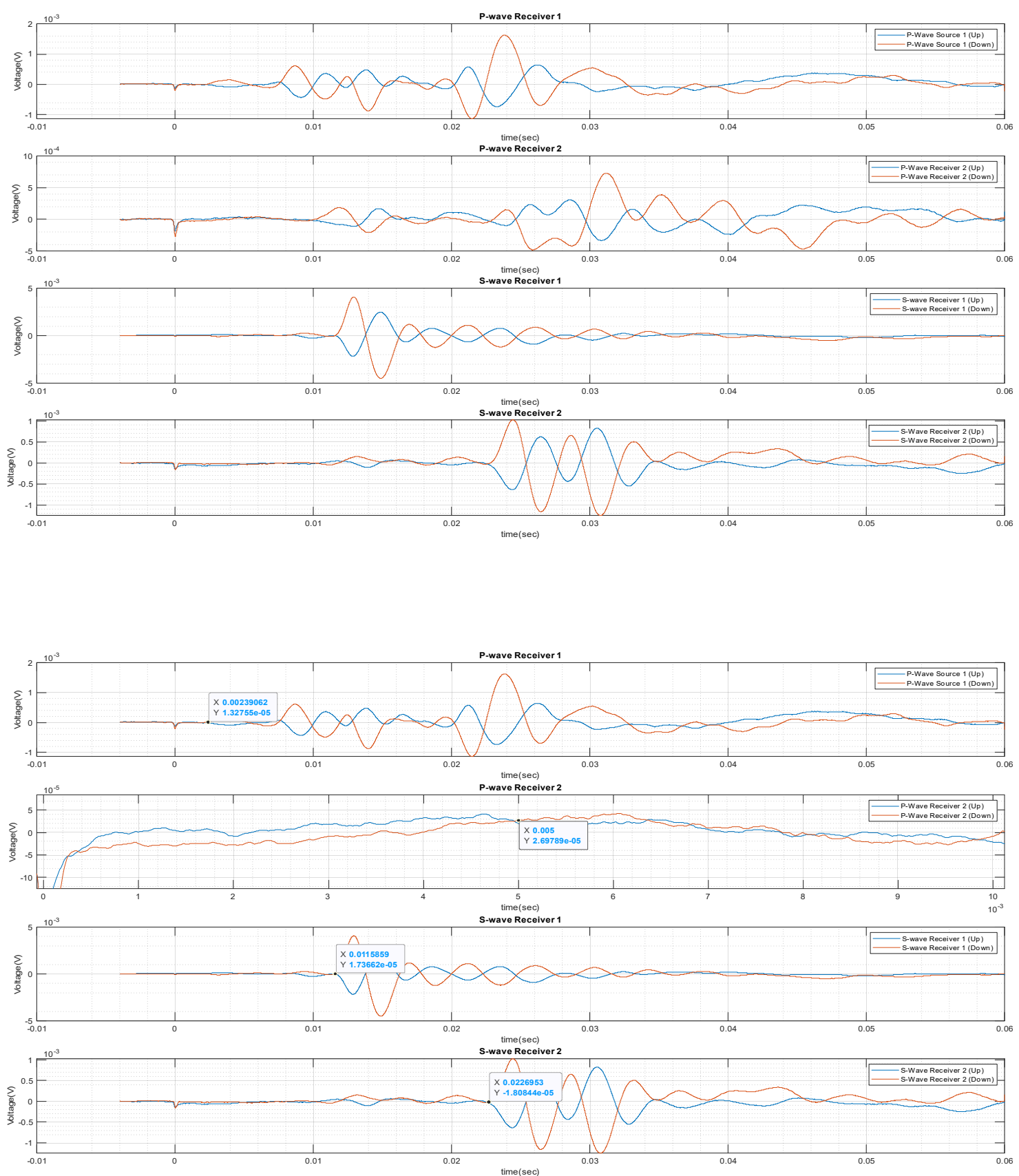

Data Set 15 (8.29.2019)

Run00107f

R2_1 

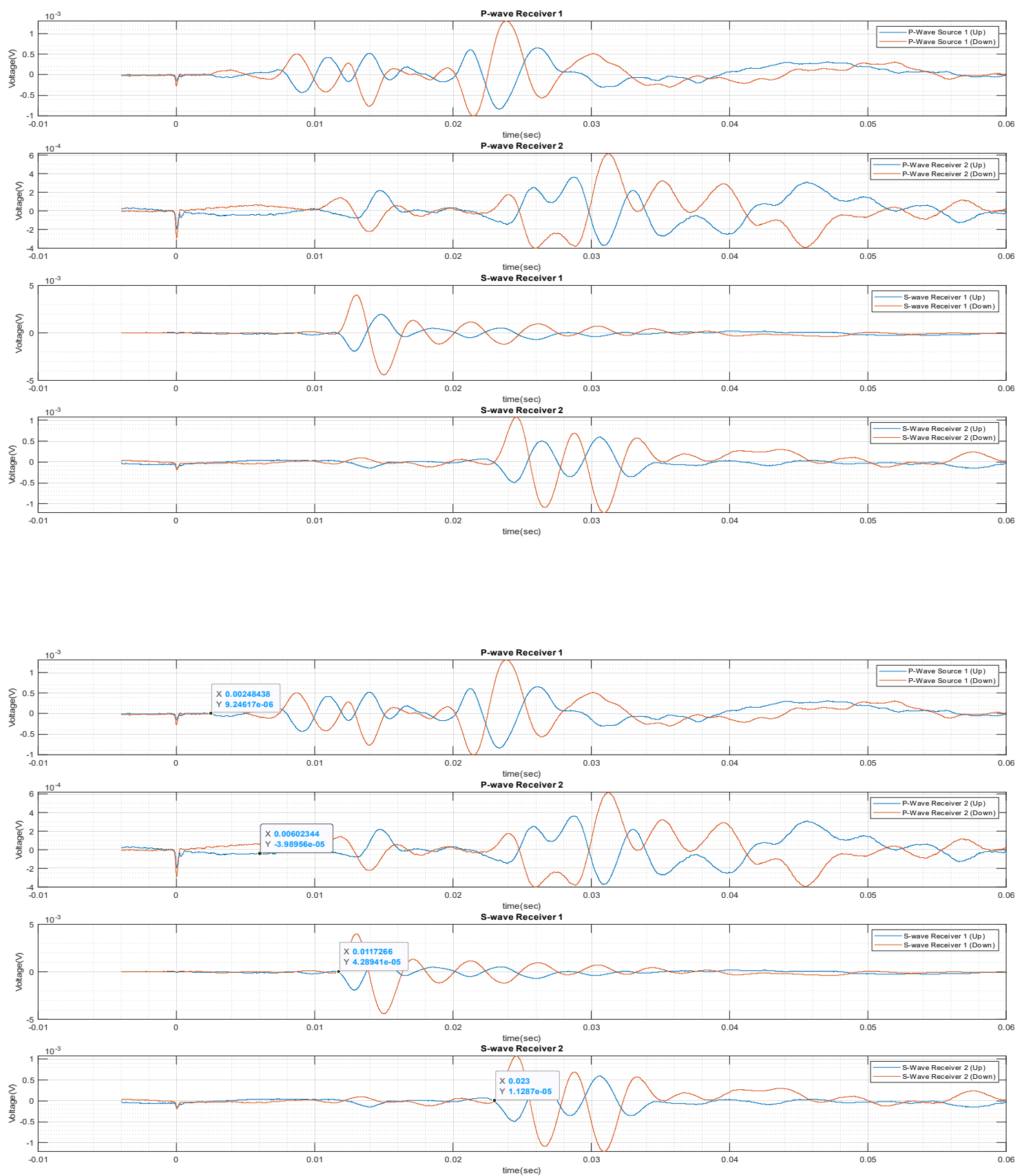

Data Set 15 (8.29.2019)

Run00108f

R2_2 

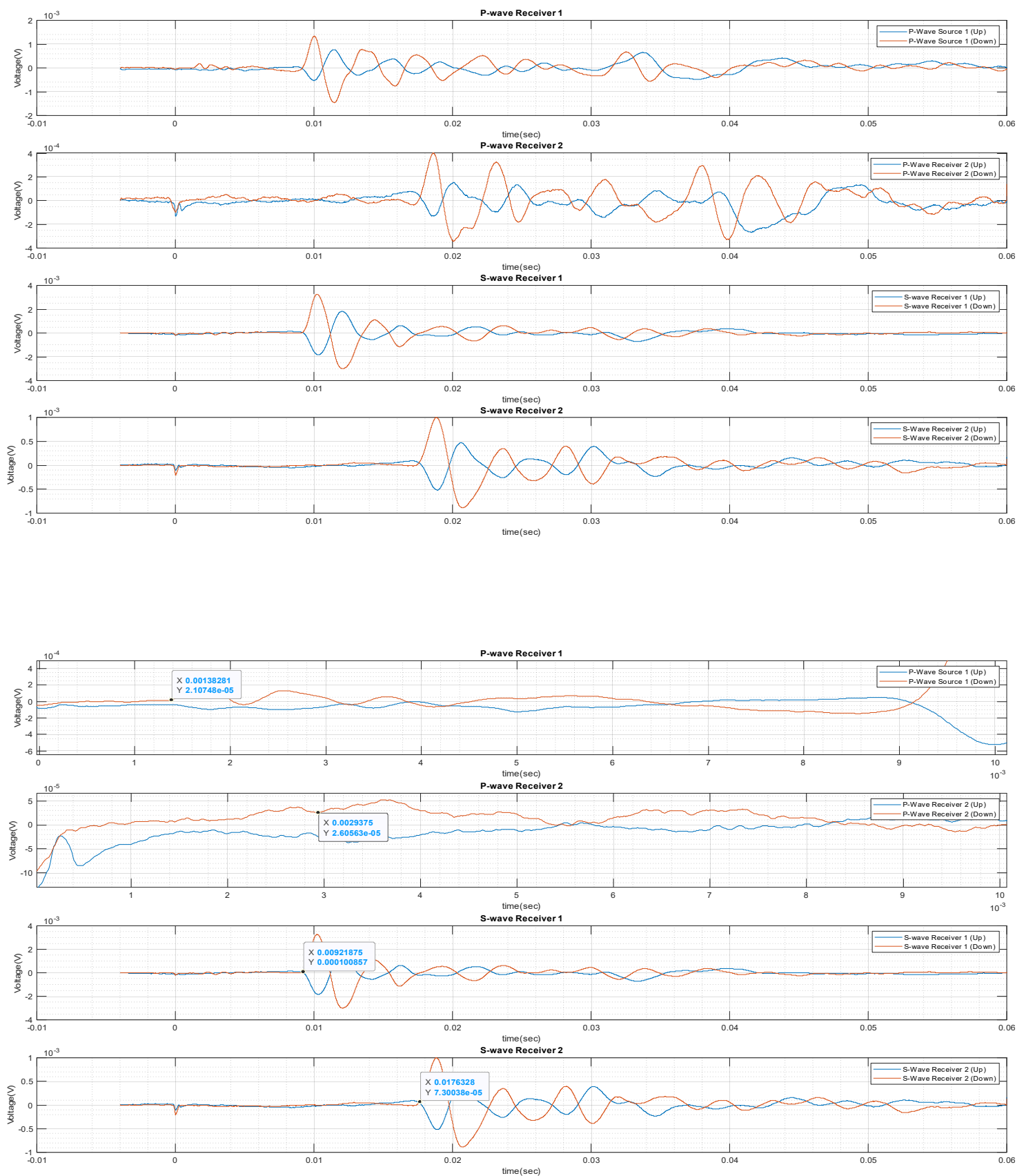

Data Set 15 (8.29.2019)

Run00109f

R3_1 

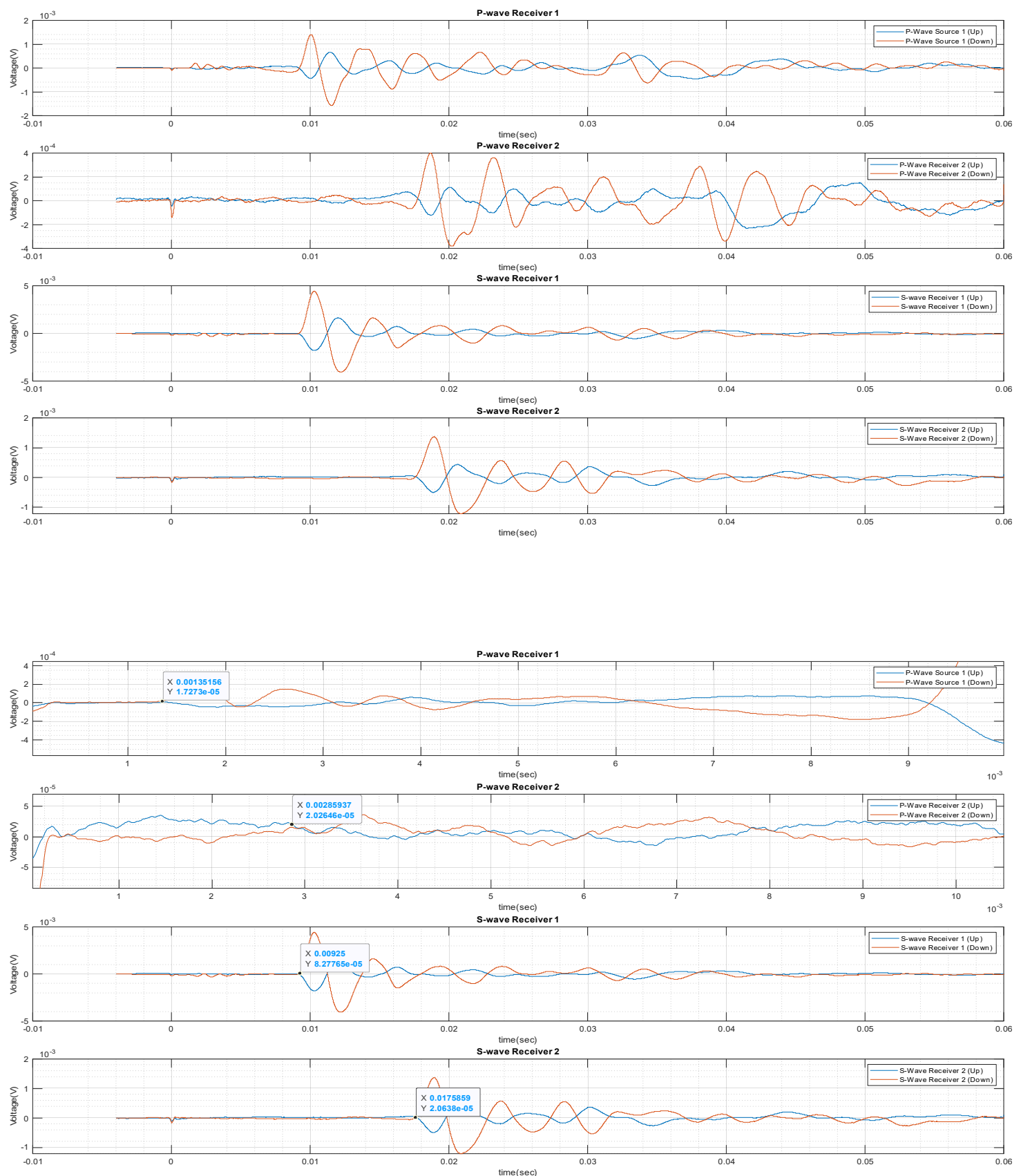

Data Set 15 (8.29.2019)

Run00110f

R3_2 

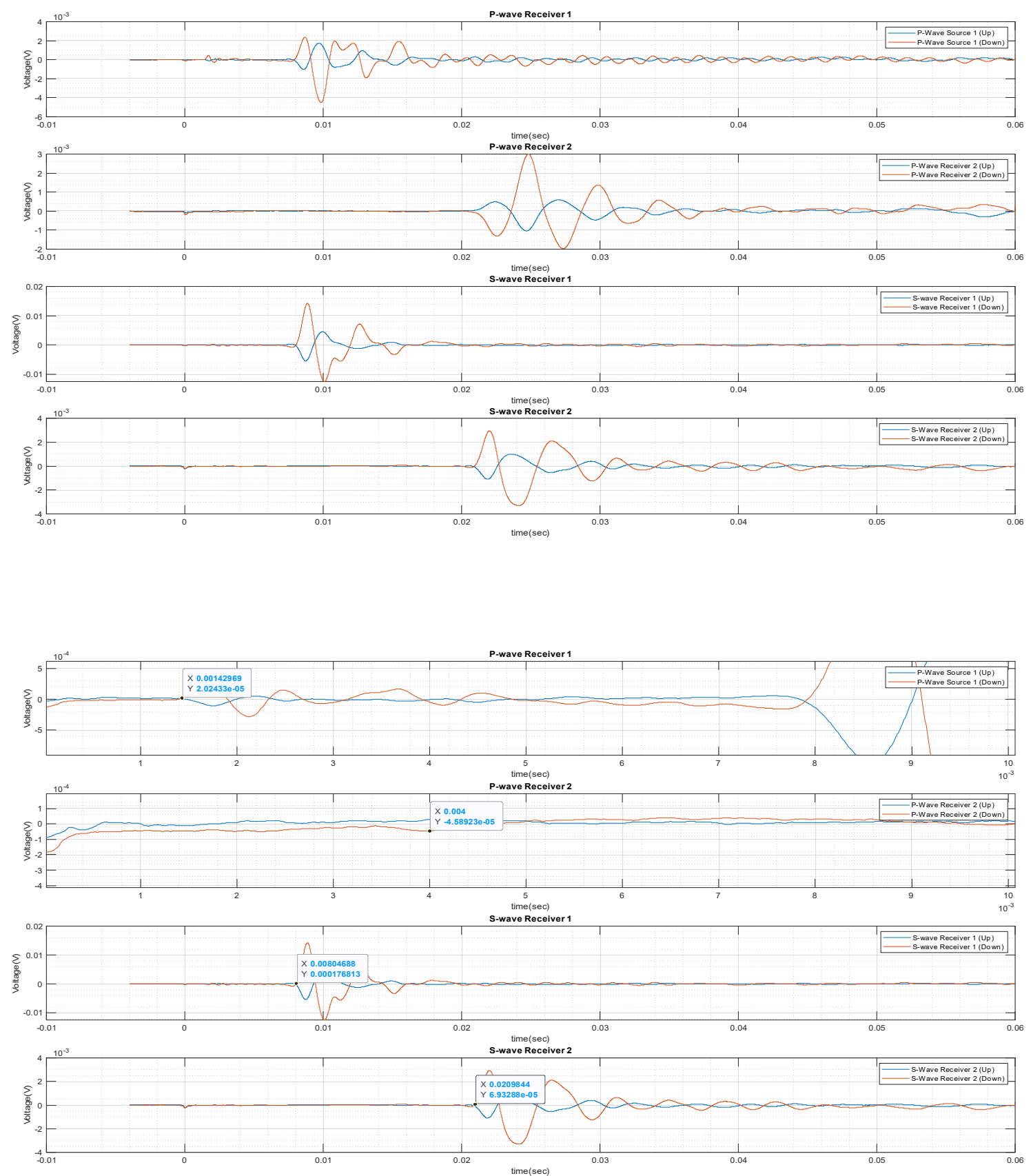

Data Set 15 (8.29.2019)

Run00111f

R4_1 

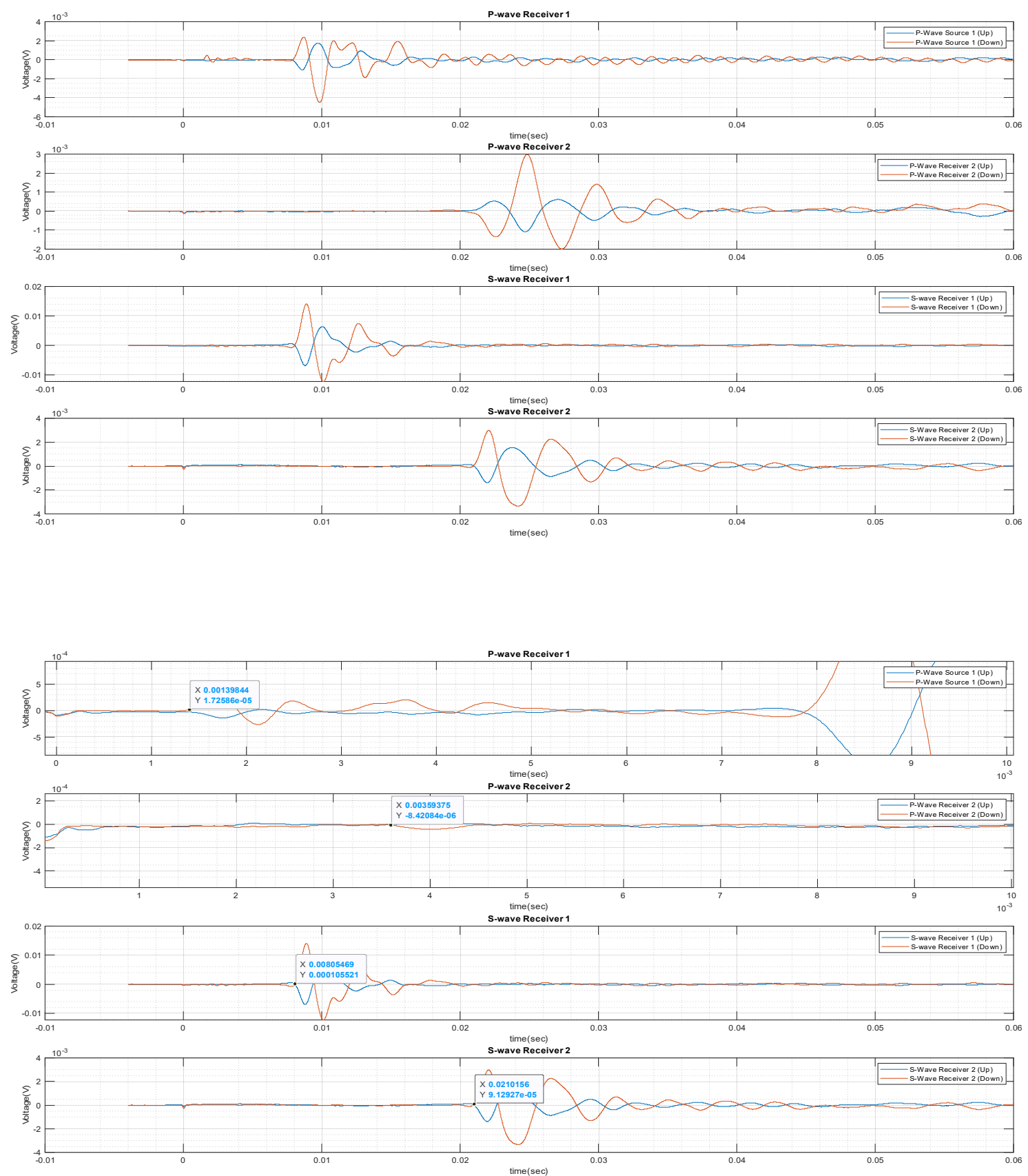

Data Set 15 (8.29.2019)

Run00112f

R4_2 

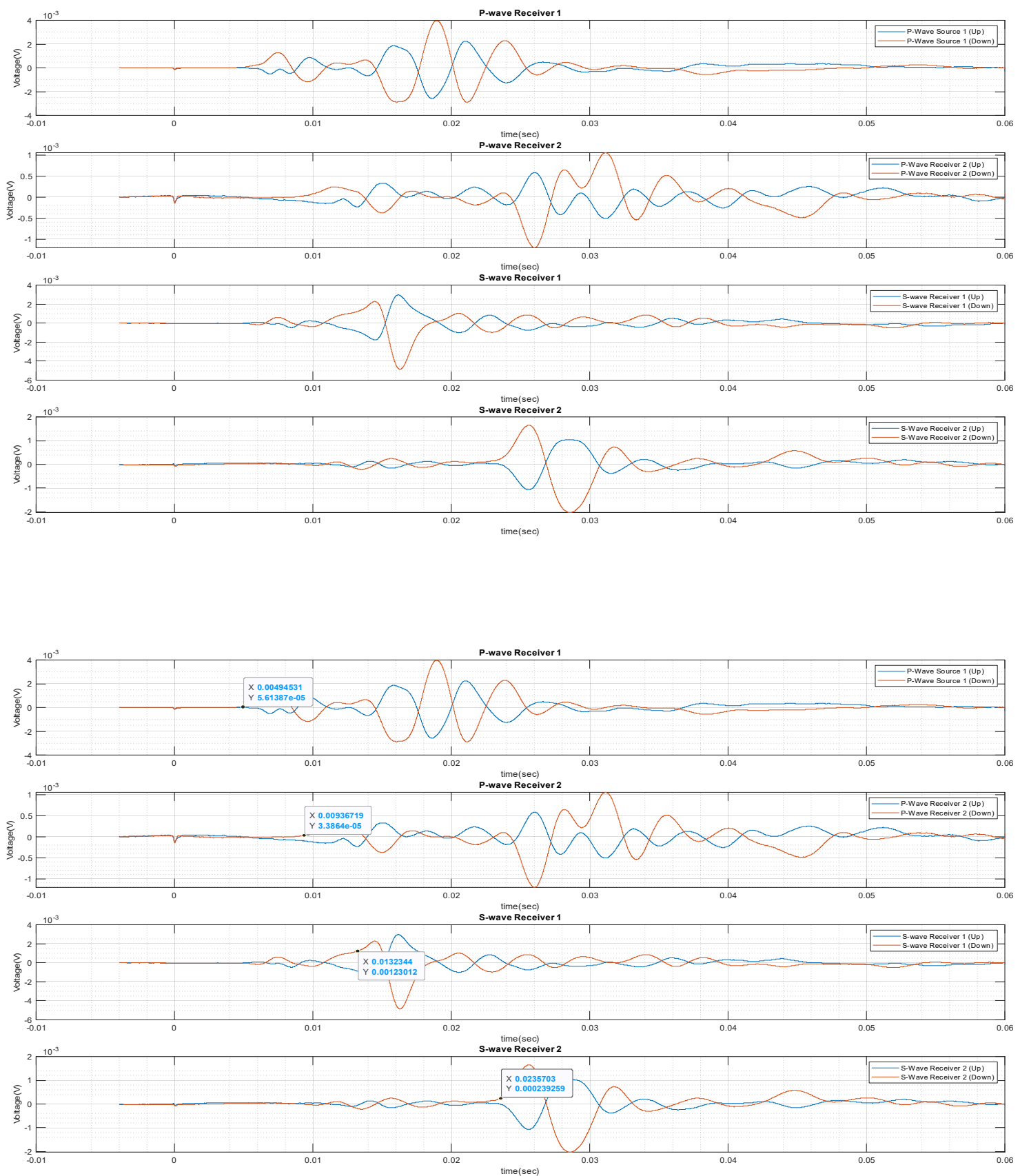

Data Set $16(8.30 .2019)$

Run00113f

R1_1 

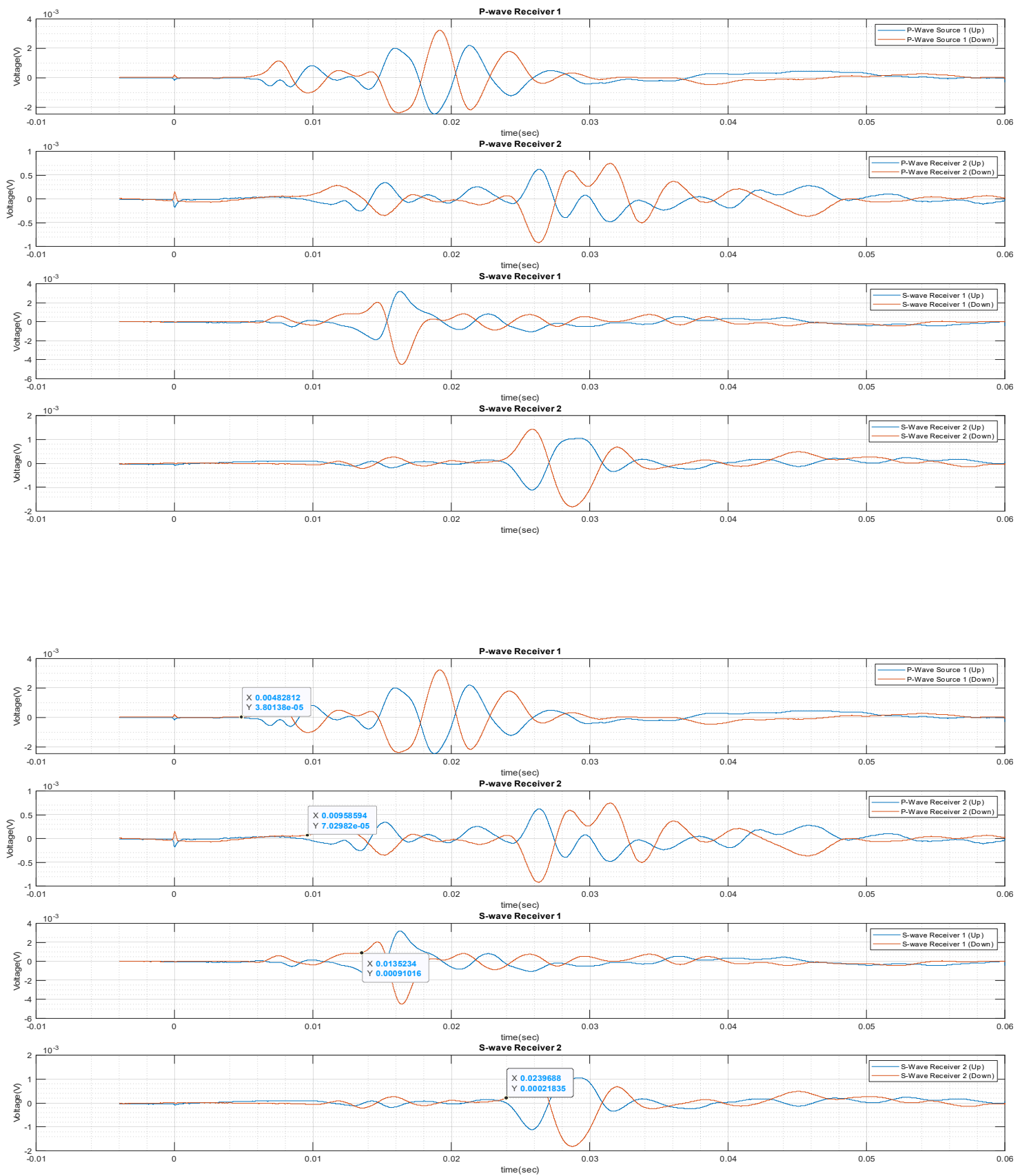

Data Set $16(8.30 .2019)$

Run00114f

R1_2 

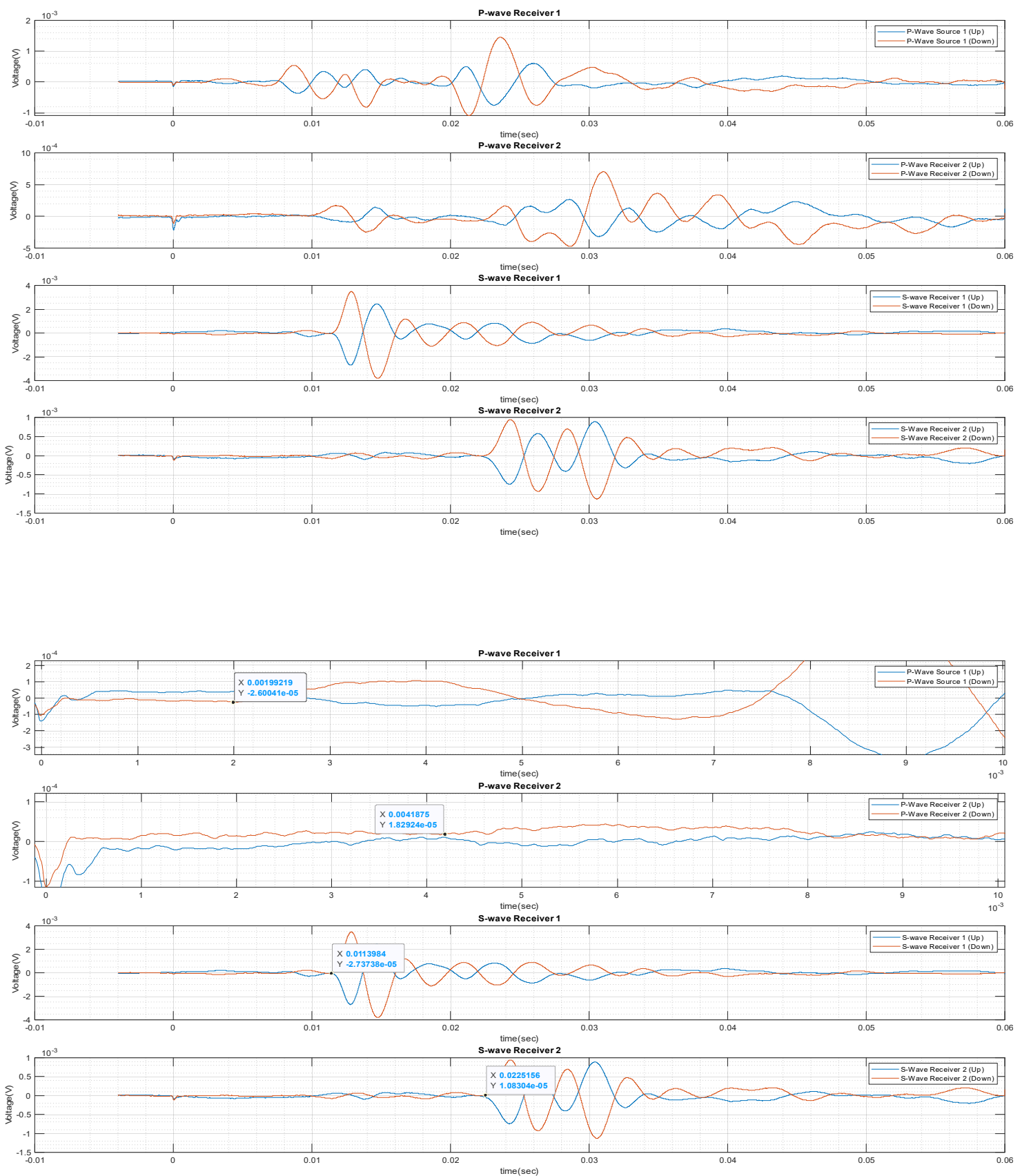

Data Set $16(8.30 .2019)$

Run00115f

R2_1 

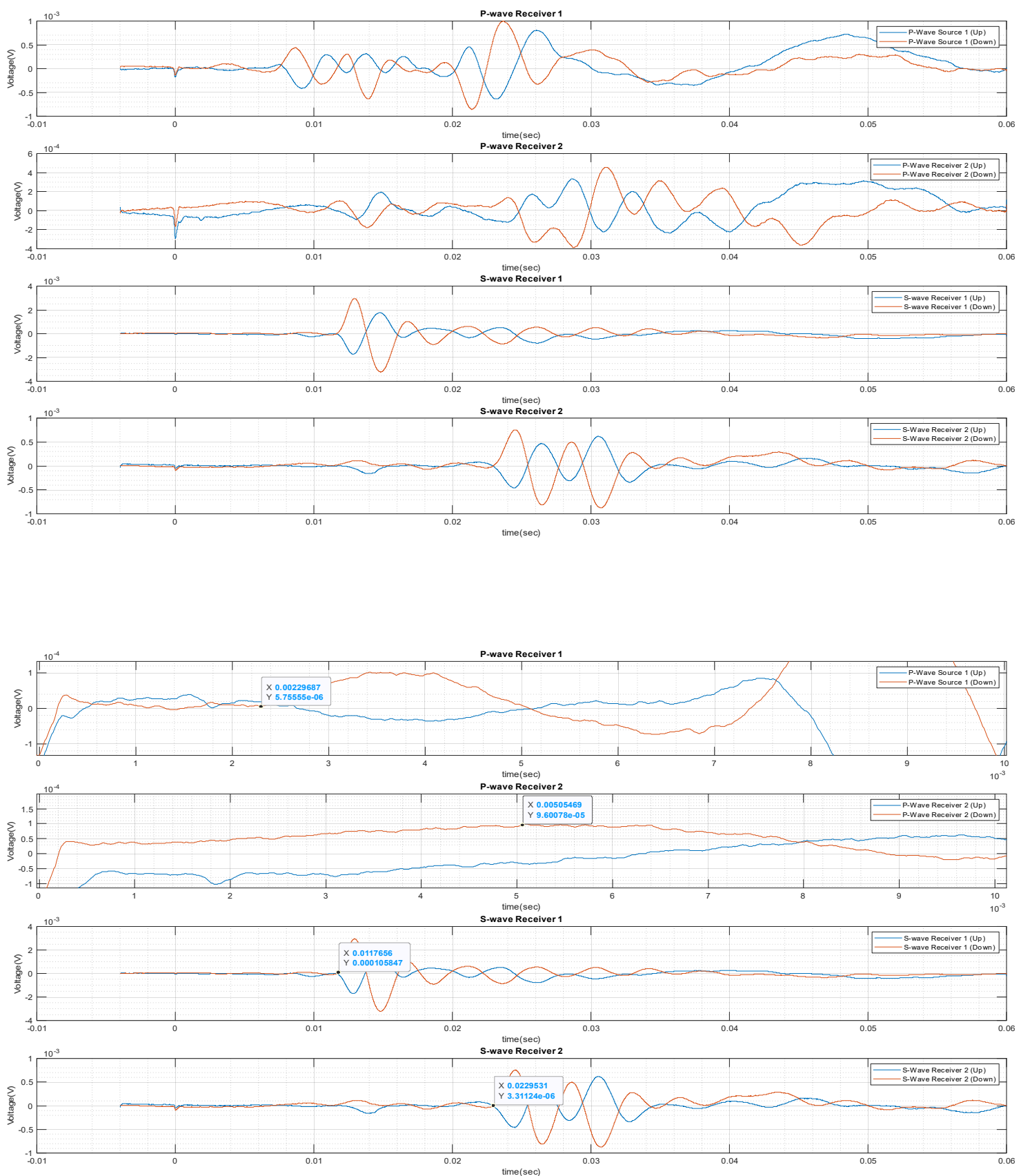

Data Set $16(8.30 .2019)$

Run00116f

R2_2 

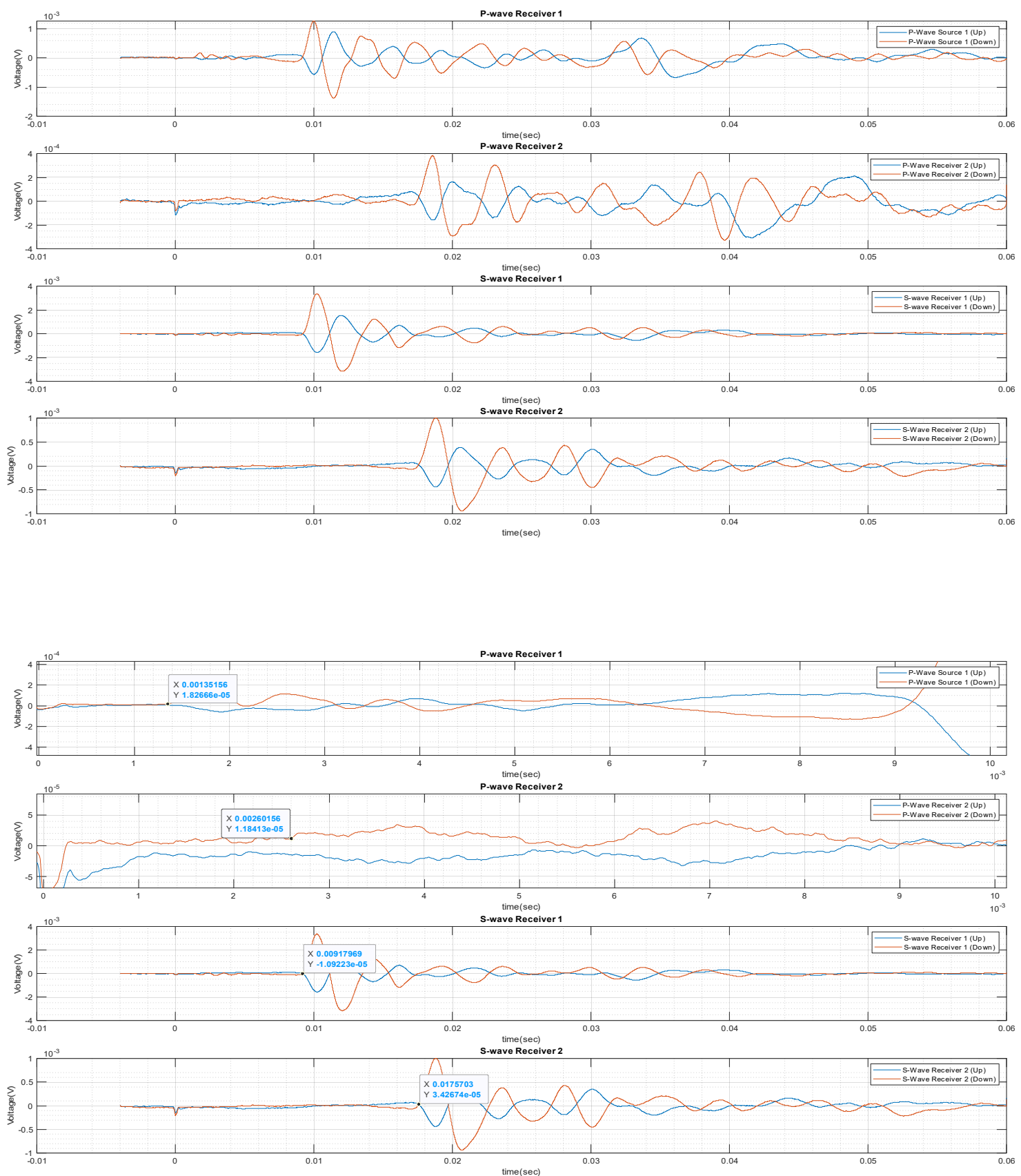

Data Set $16(8.30 .2019)$

Run00117f

R3_1 

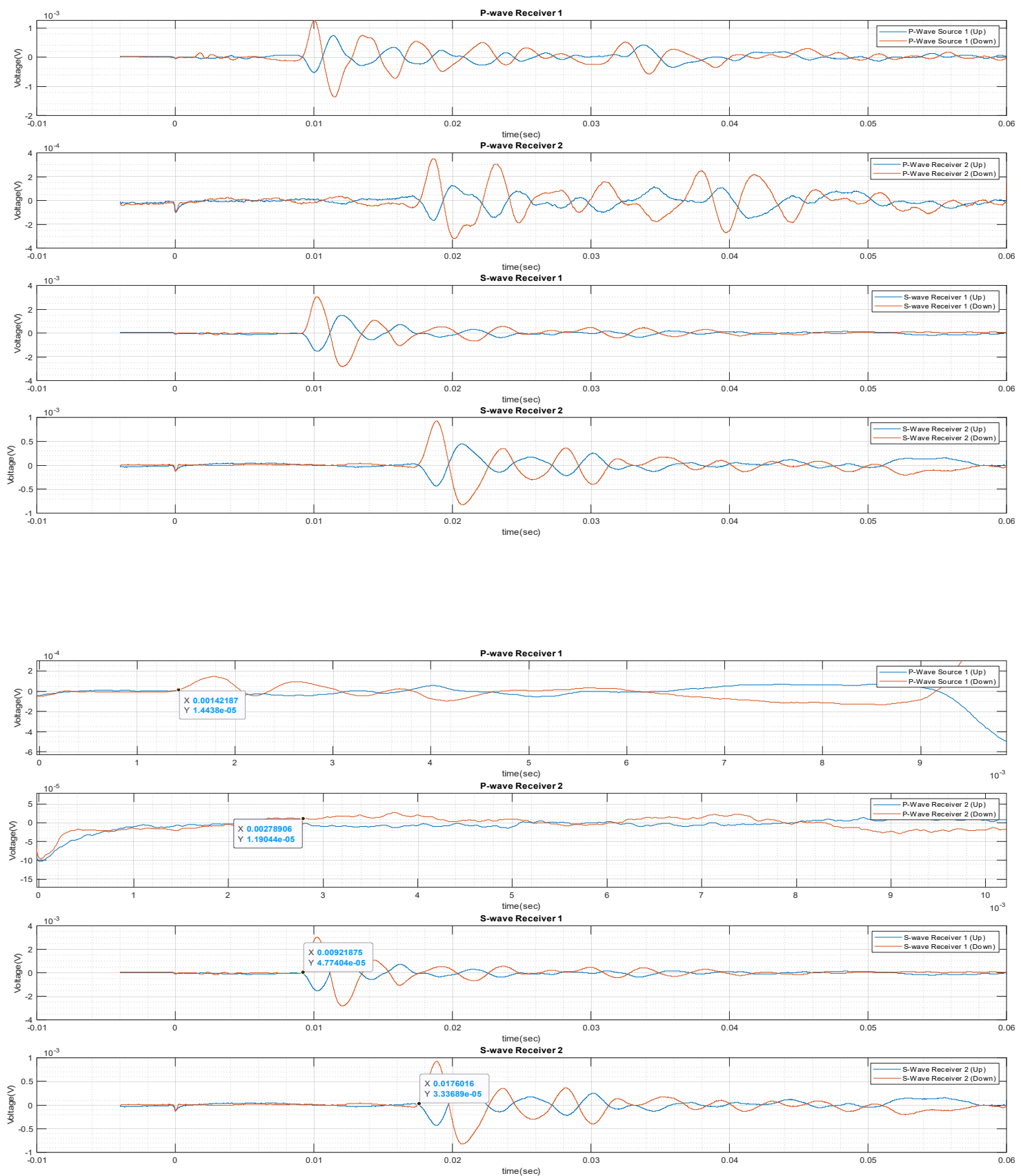

Data Set $16(8.30 .2019)$

Run00118f

R3_2 

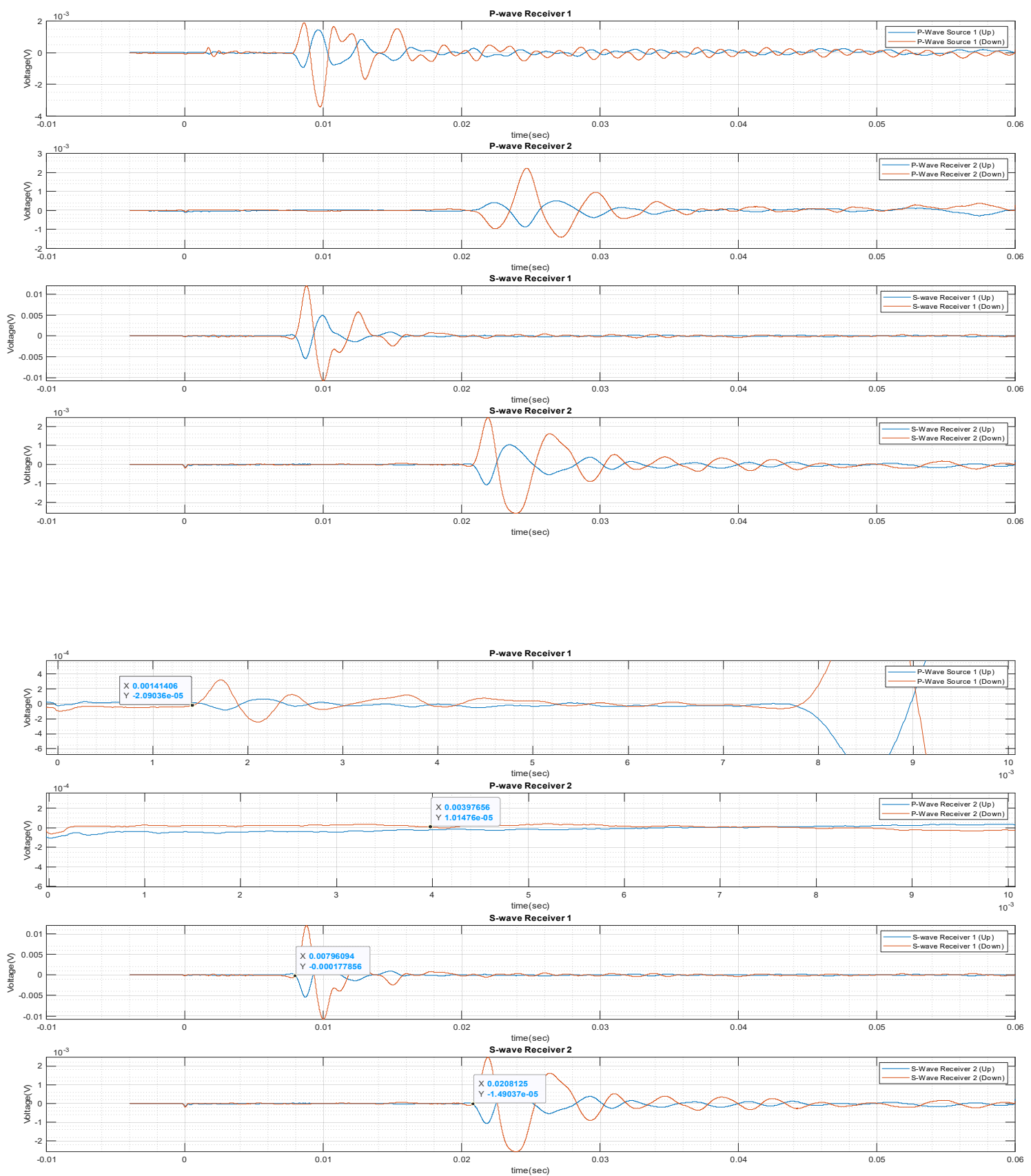

Data Set $16(8.30 .2019)$

Run00119f

R4_1 

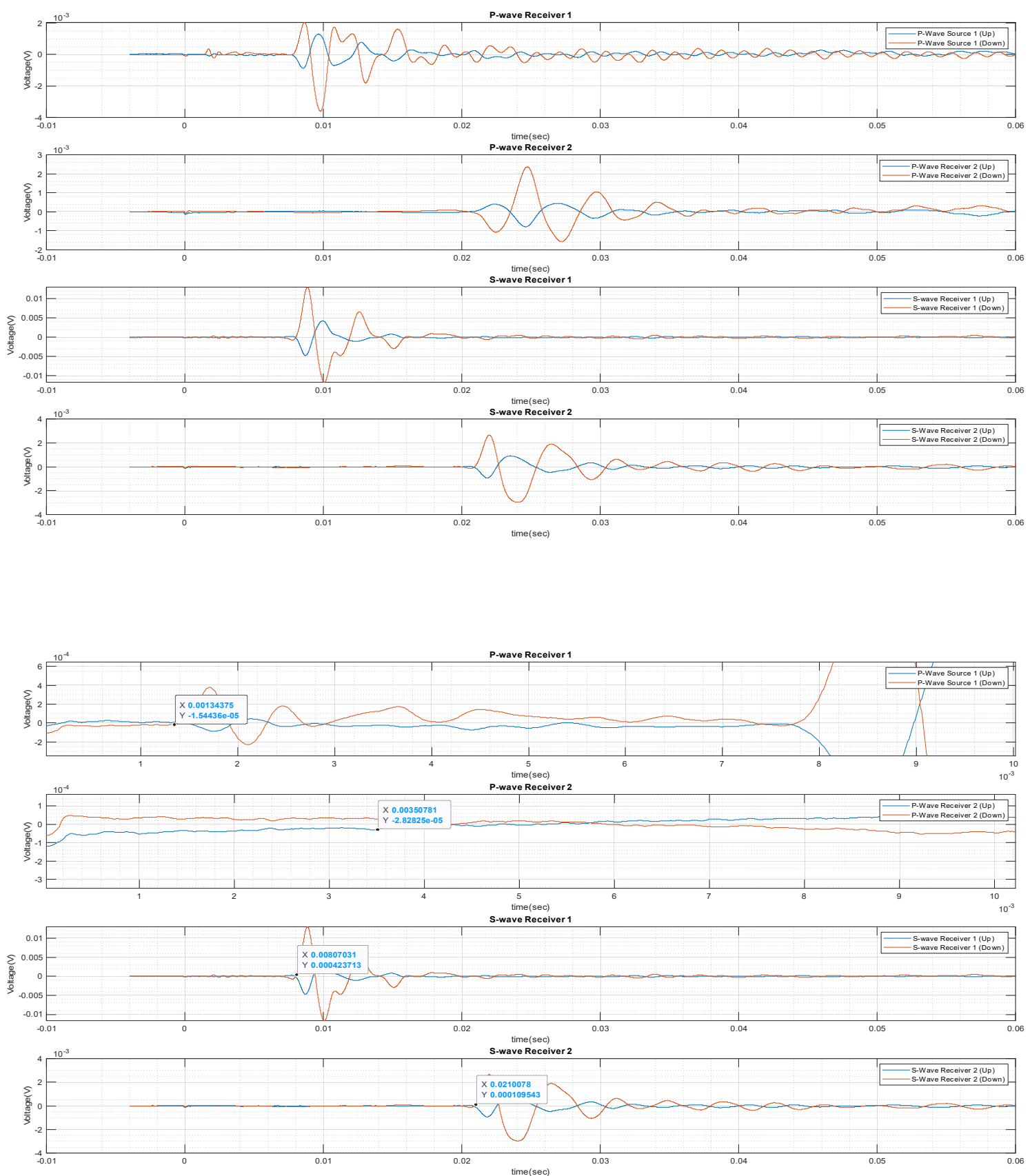

Data Set $16(8.30 .2019)$

Run00120f

R4_2 

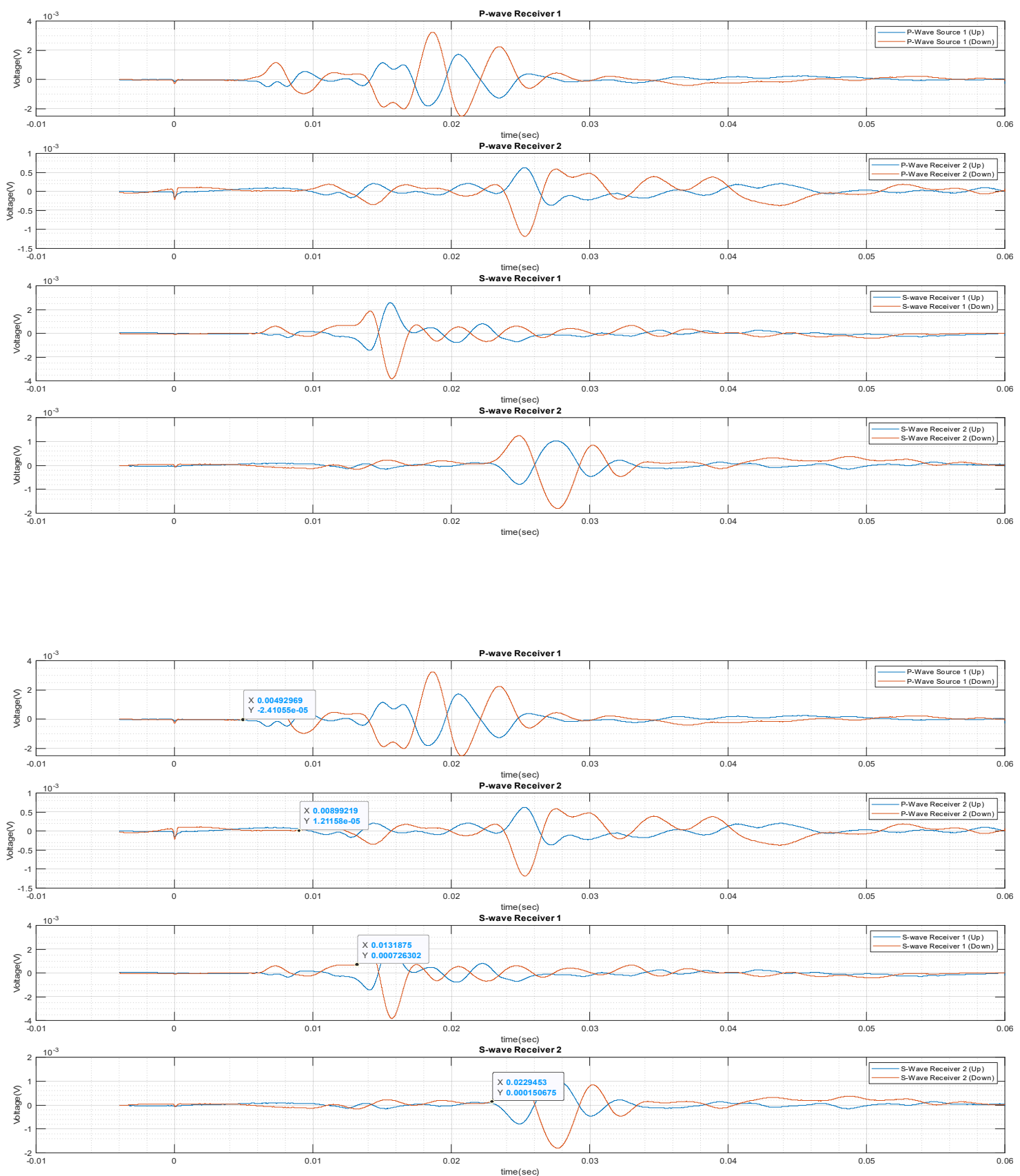

Data Set 17 (9.2.2019)

Run00121f

R1_1 

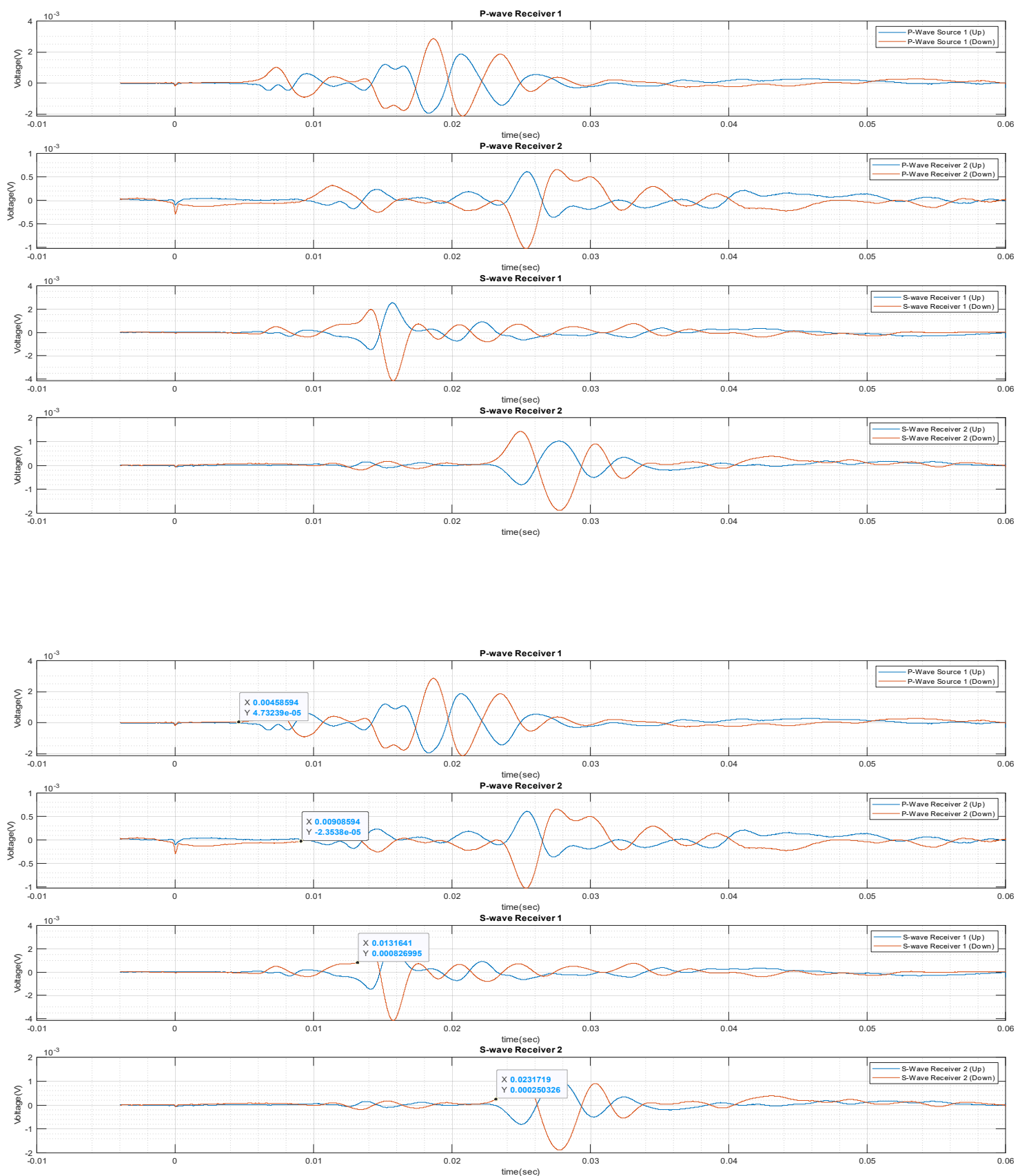

Data Set 17 (9.2.2019)

Run00122f

R1_2 

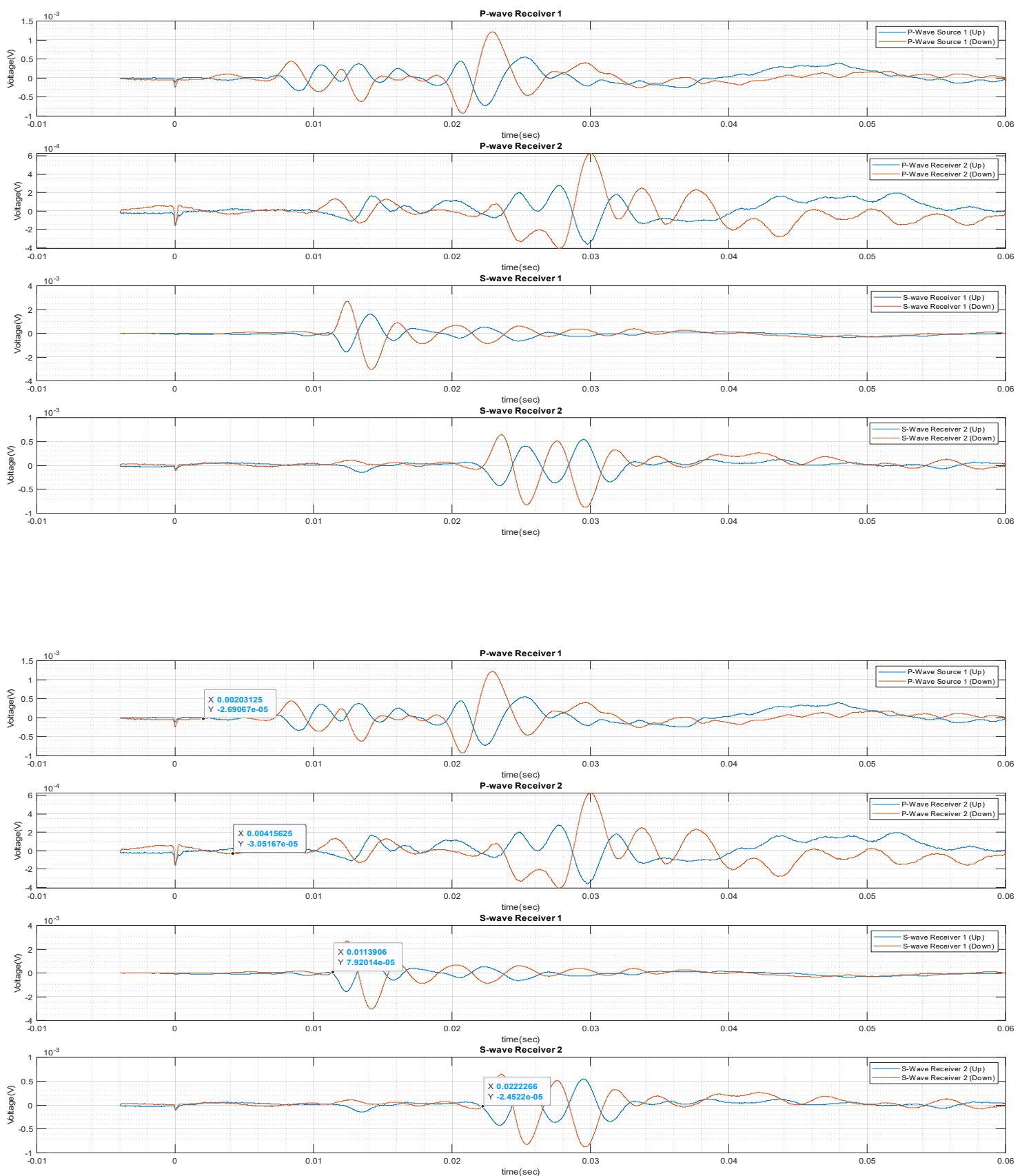

Data Set 17 (9.2.2019)

Run00123f

R2_1 

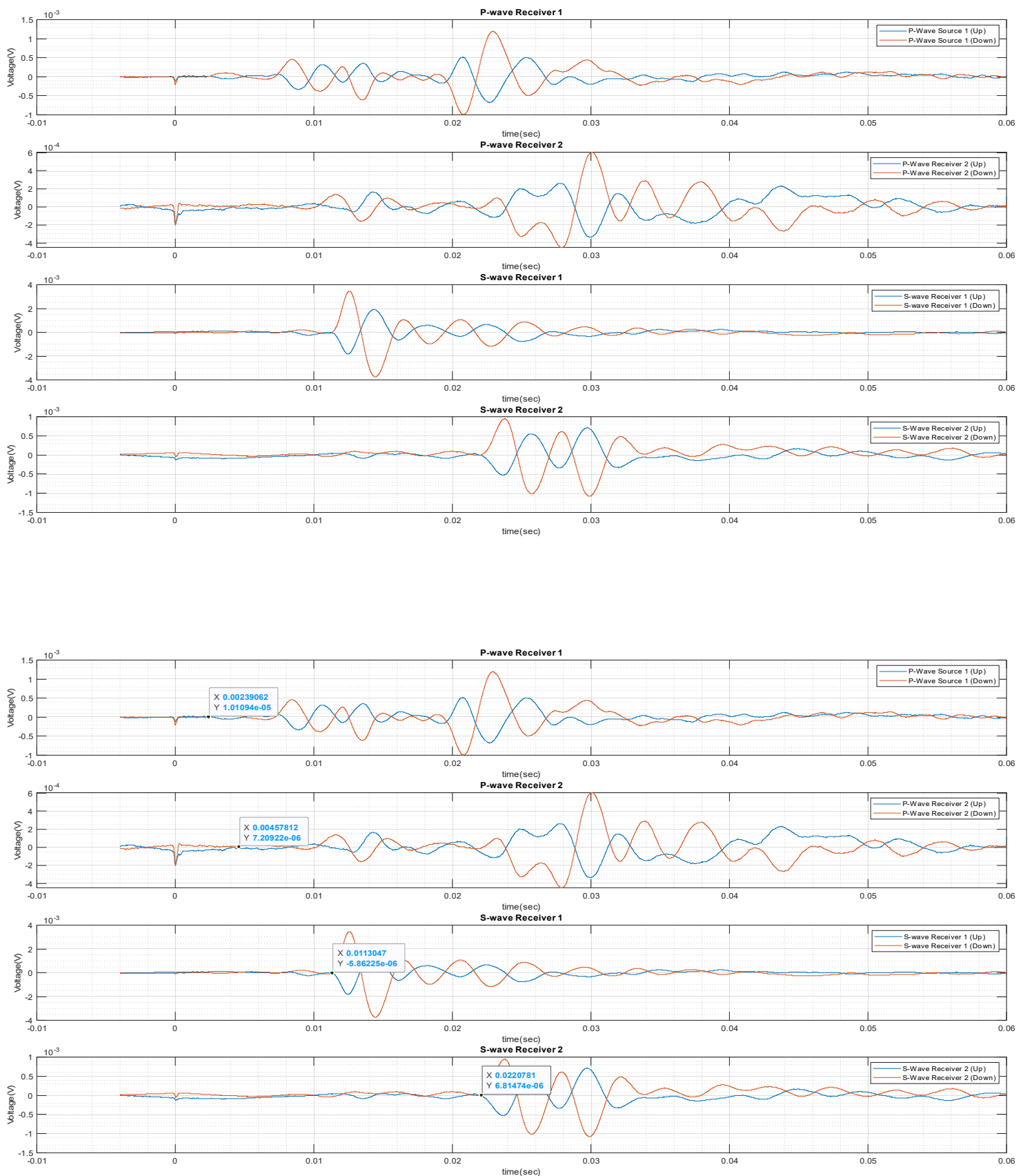

Data Set 17 (9.2.2019)

Run00124f

R2_2 

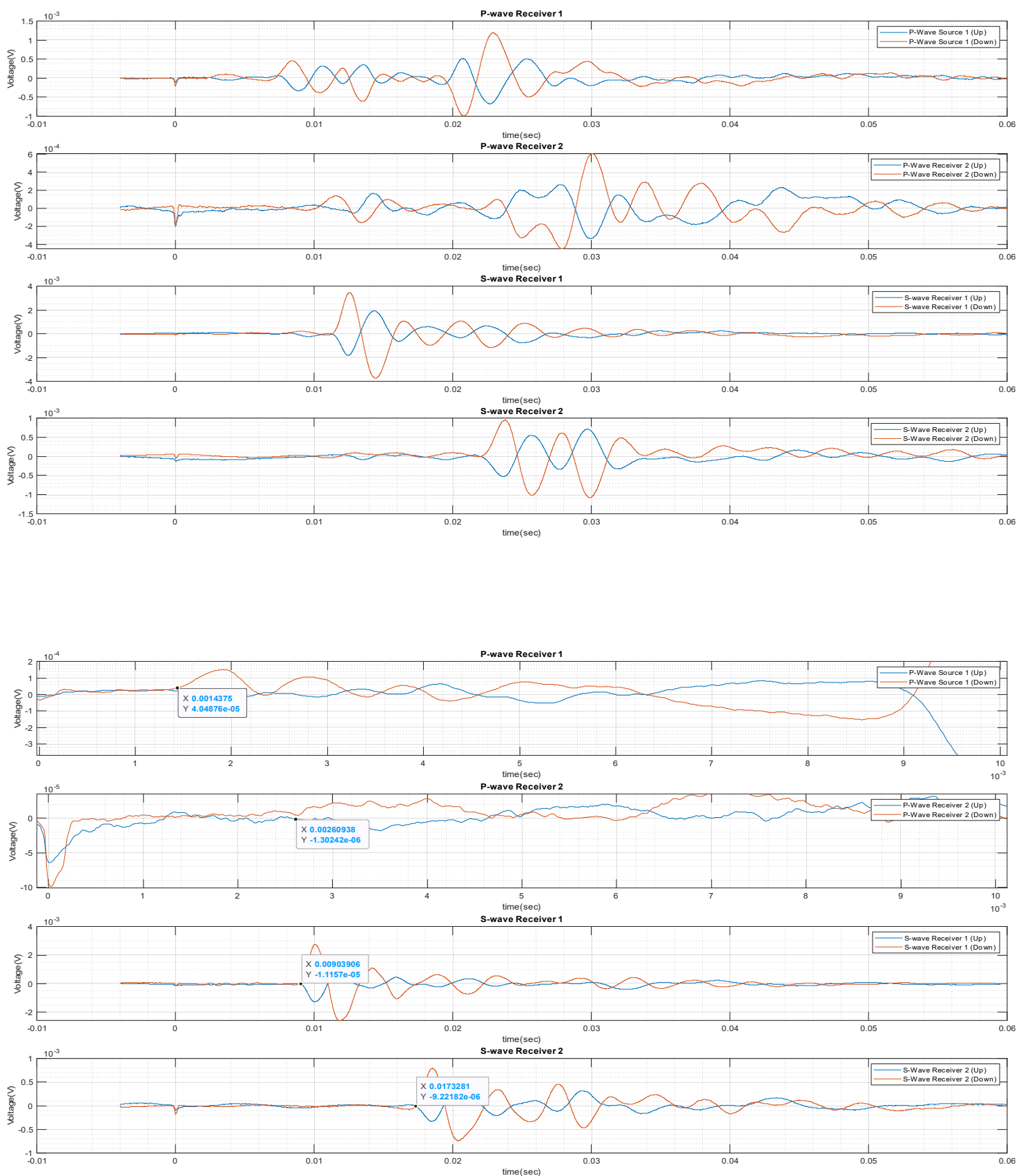

Data Set 17 (9.2.2019)

Run00125f

R3 1 

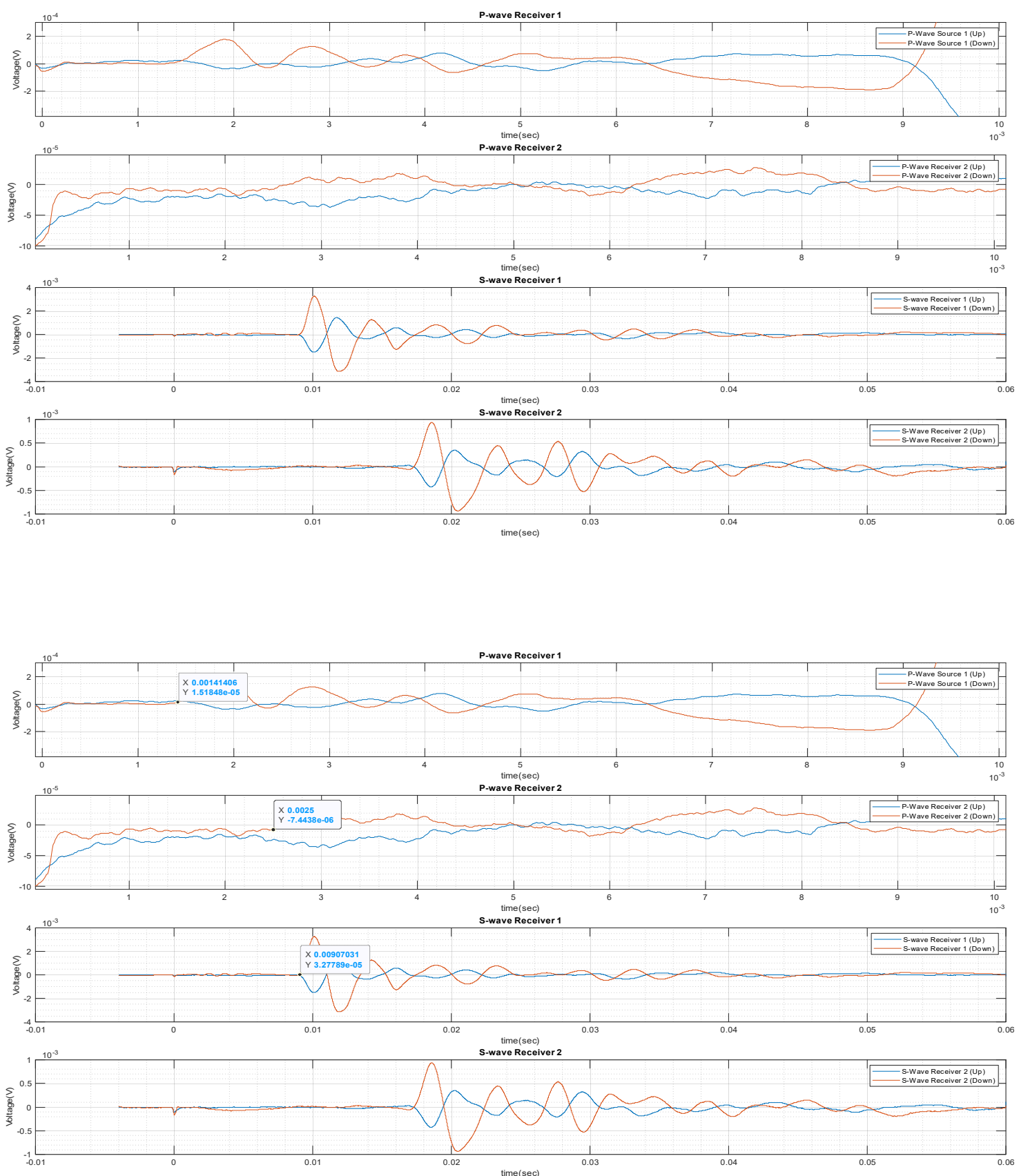

Data Set 17 (9.2.2019)

Run00126f

R3_2 

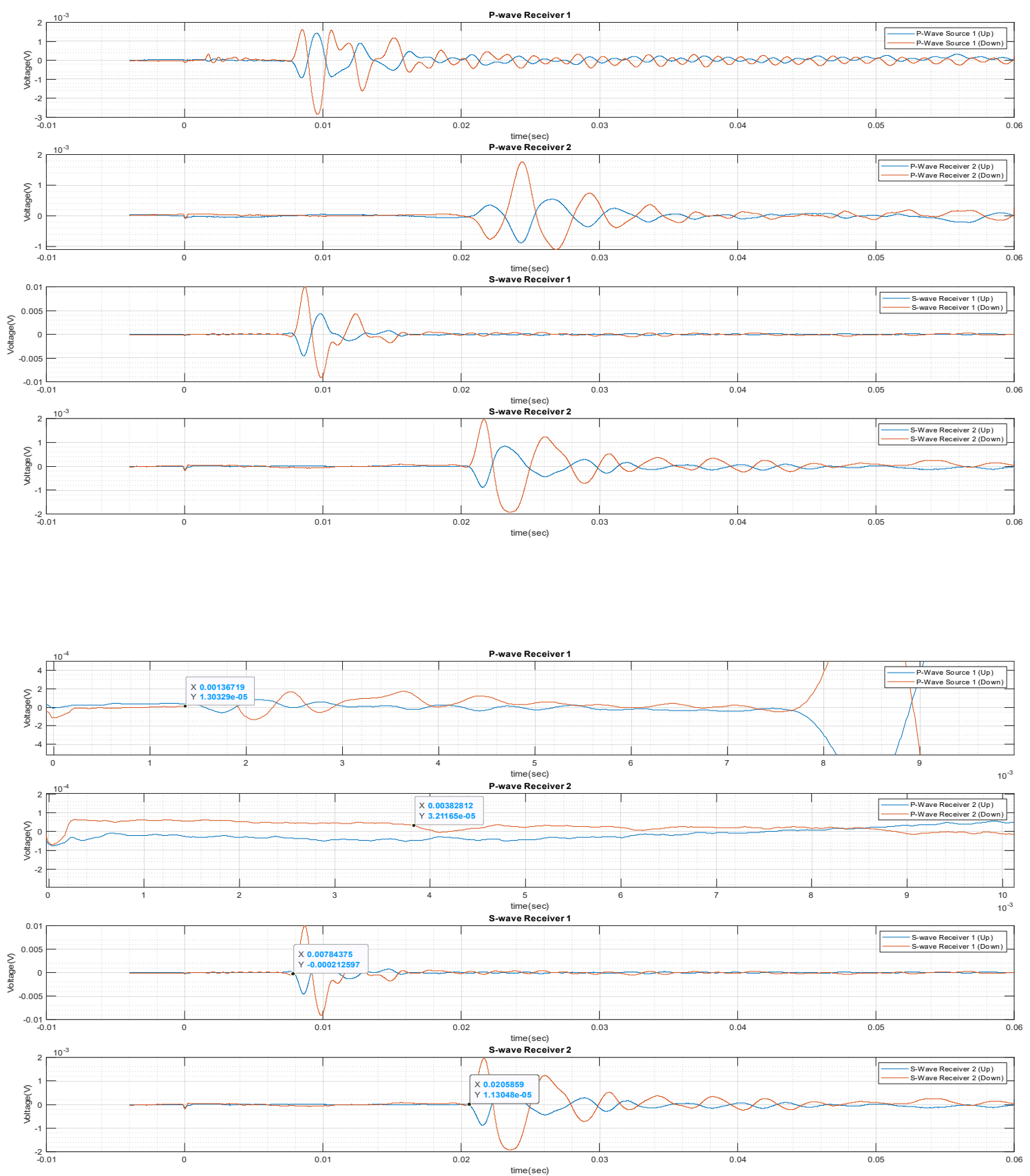

Data Set 17 (9.2.2019)

Run00127f

R4_1 

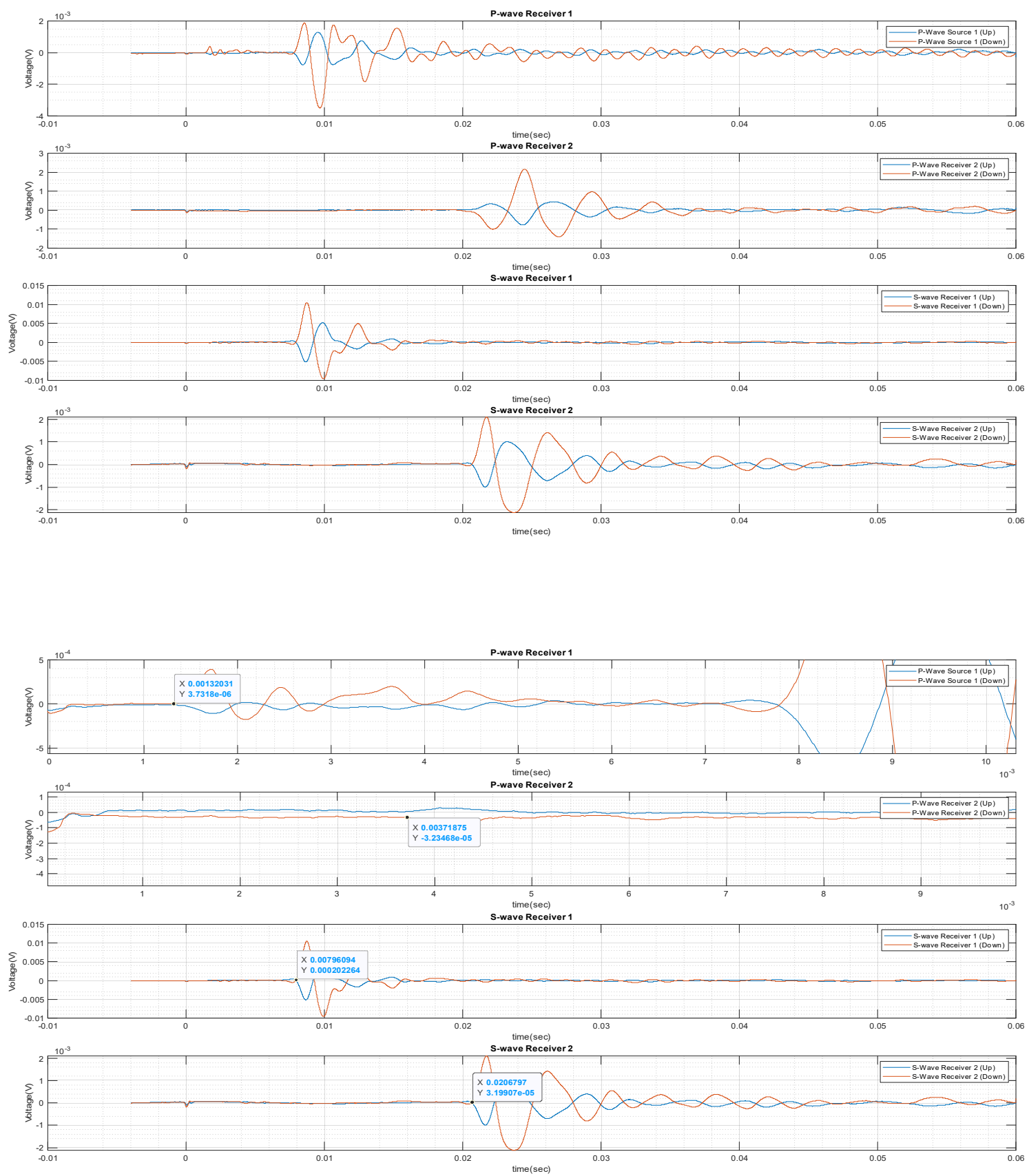

Data Set 17 (9.2.2019)

Run00128f

R4_2 

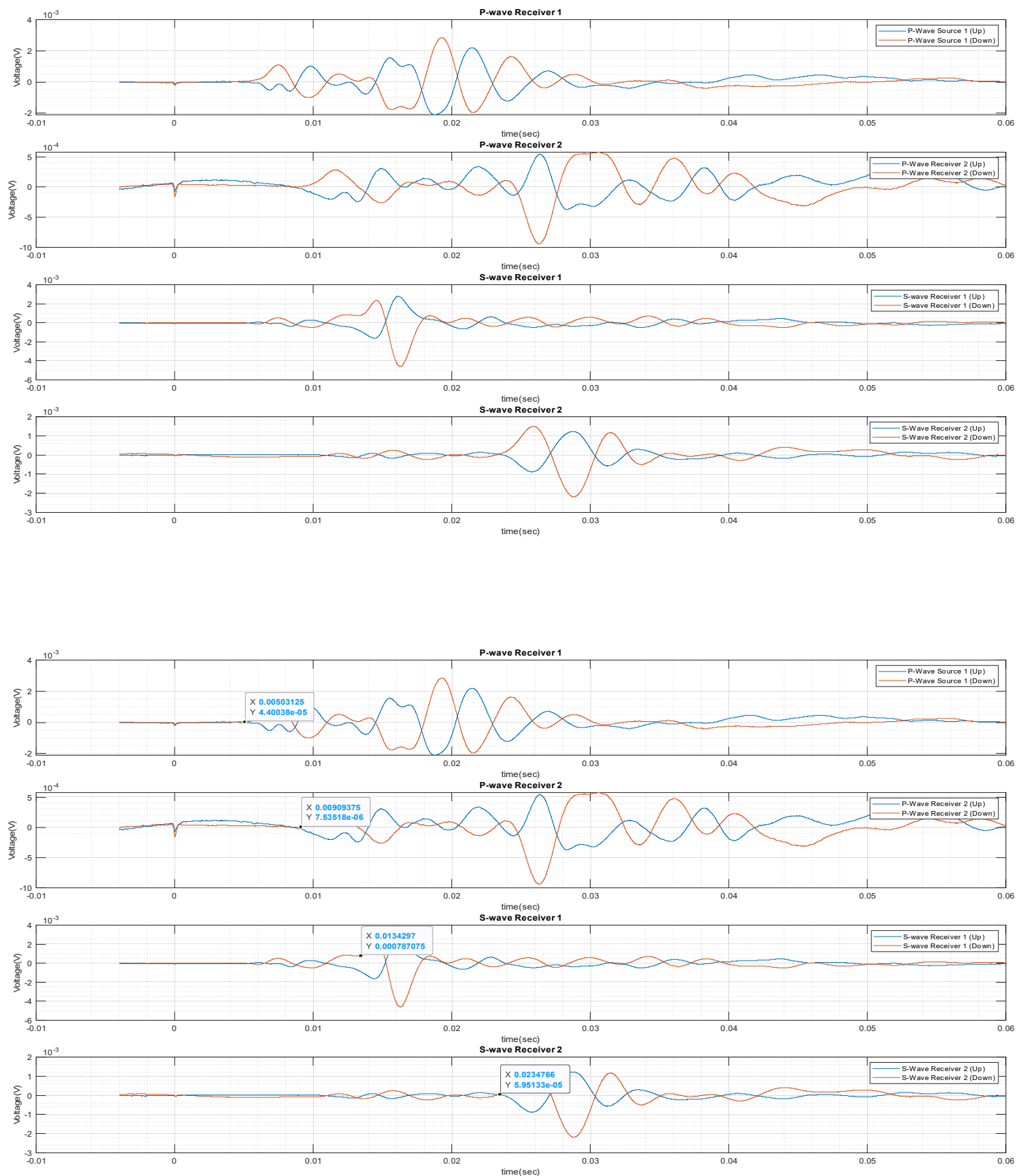

Data Set 18 (9.3.2019)

Run00129f

R1_1 

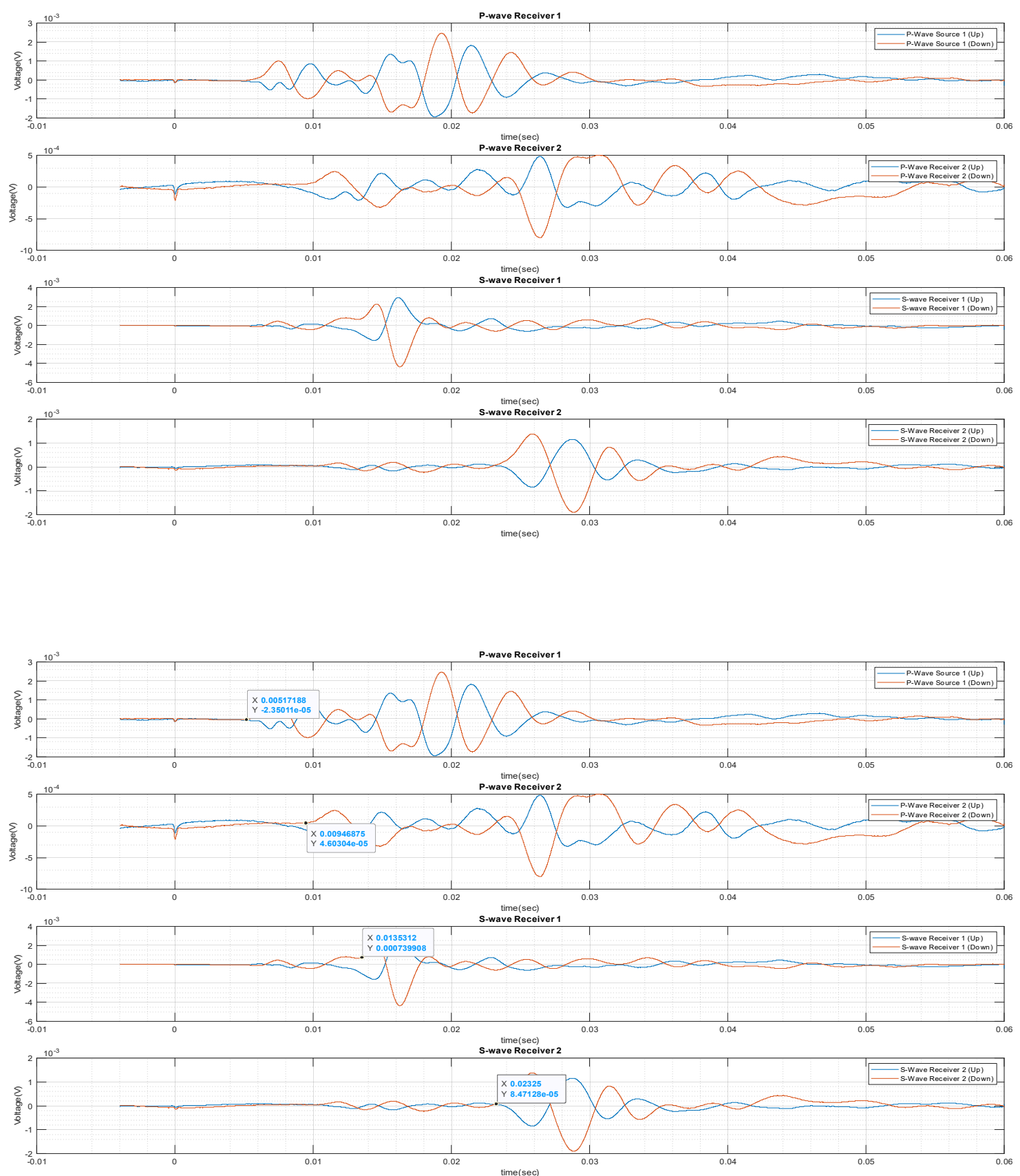

Data Set 18 (9.3.2019)

Run00130f

R1_2 

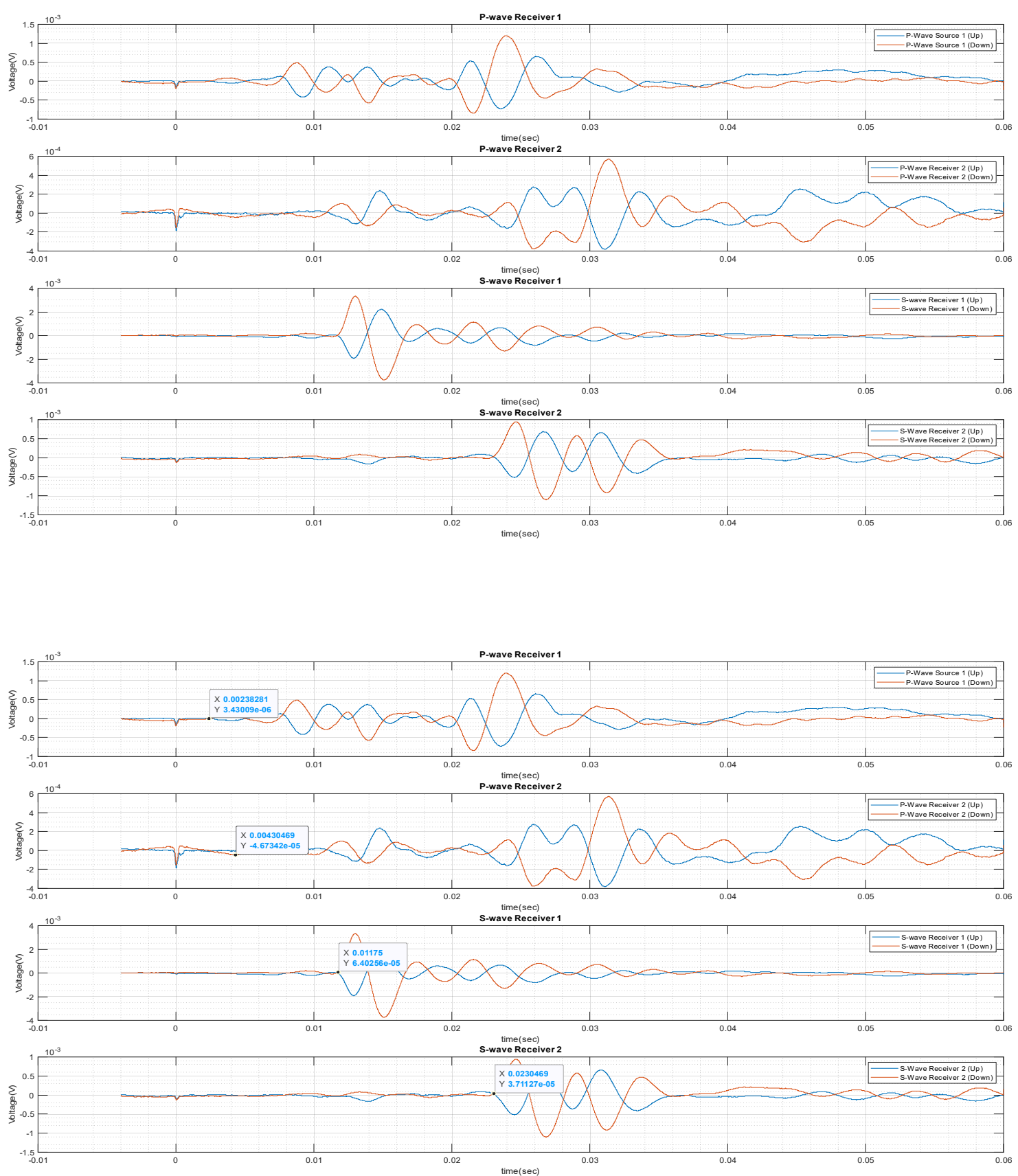

Data Set 18 (9.3.2019)

Run00131f

R2_1 

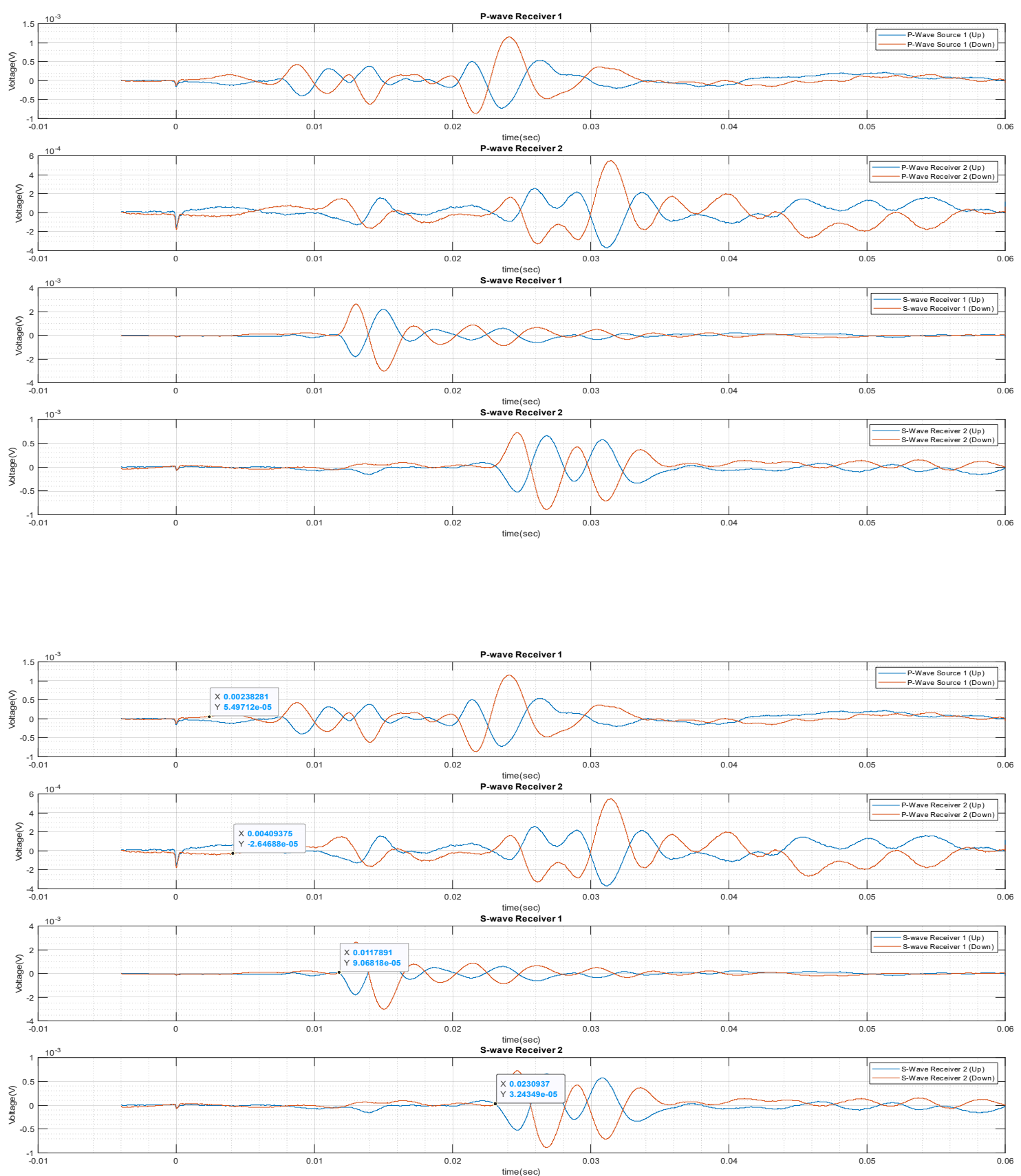

Data Set 18 (9.3.2019)

Run00132f

R2_2 

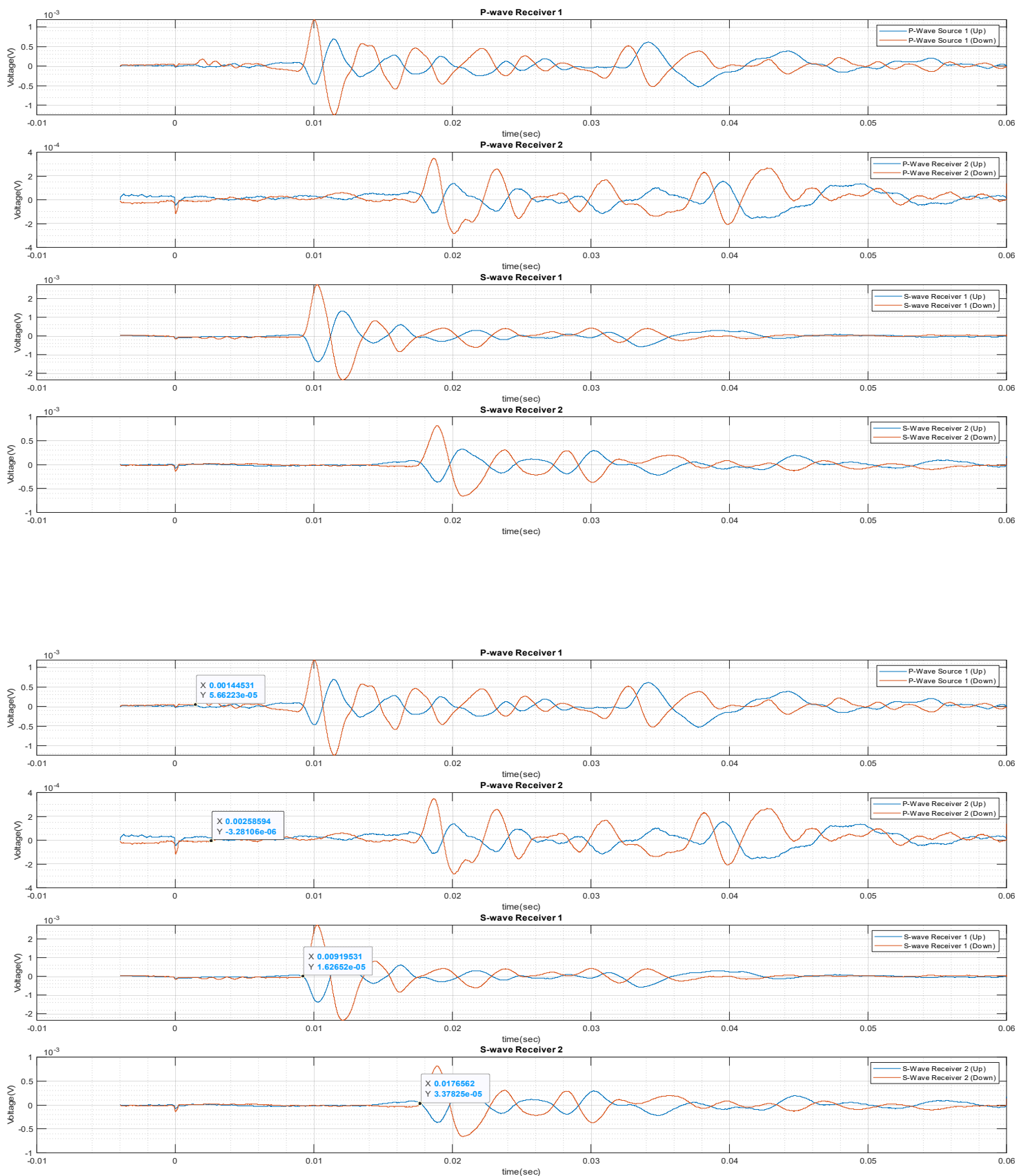

Data Set 18 (9.3.2019)

Run00133f

R3_1 

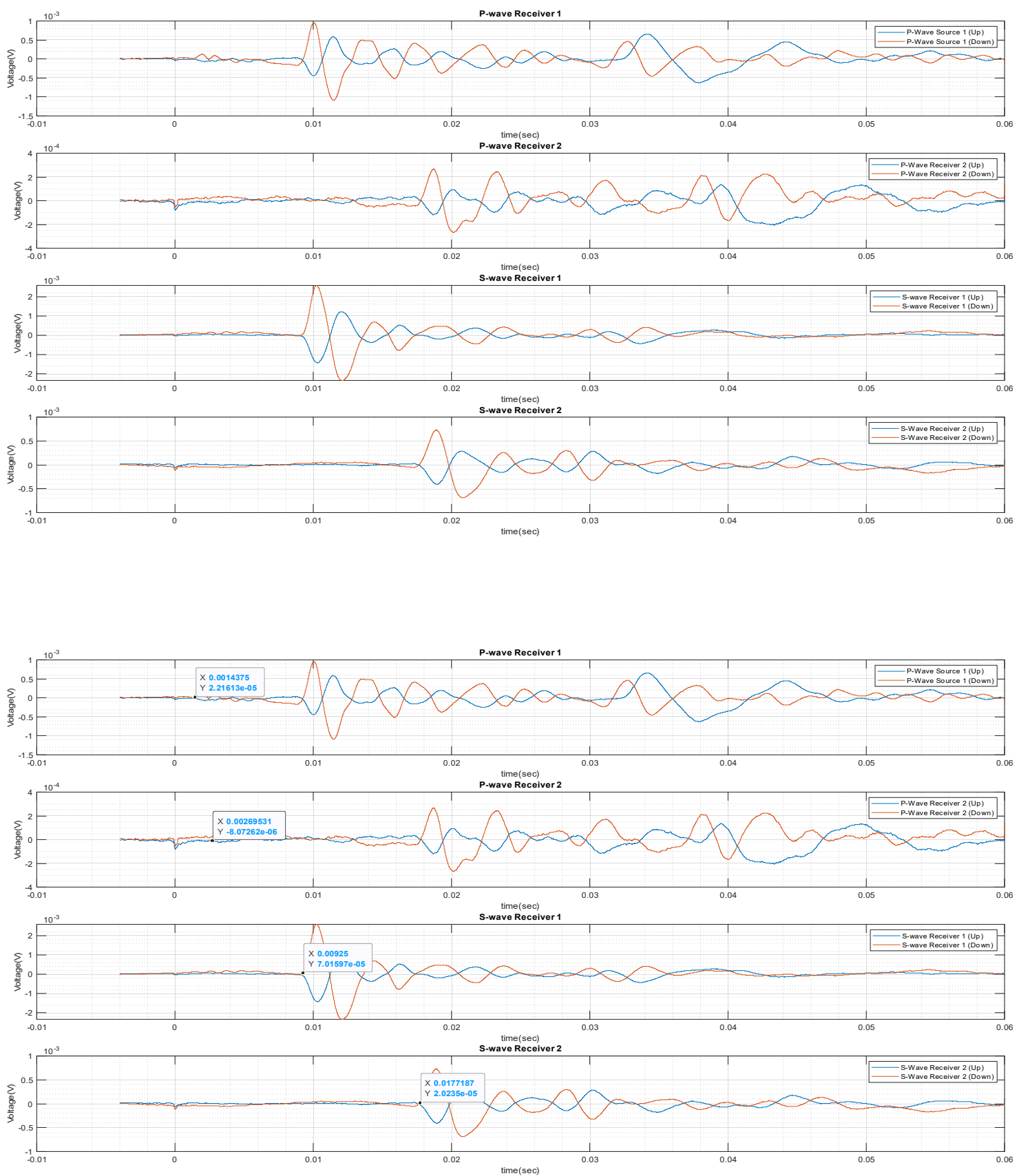

Data Set 18 (9.3.2019)

Run00134f

R3_2 

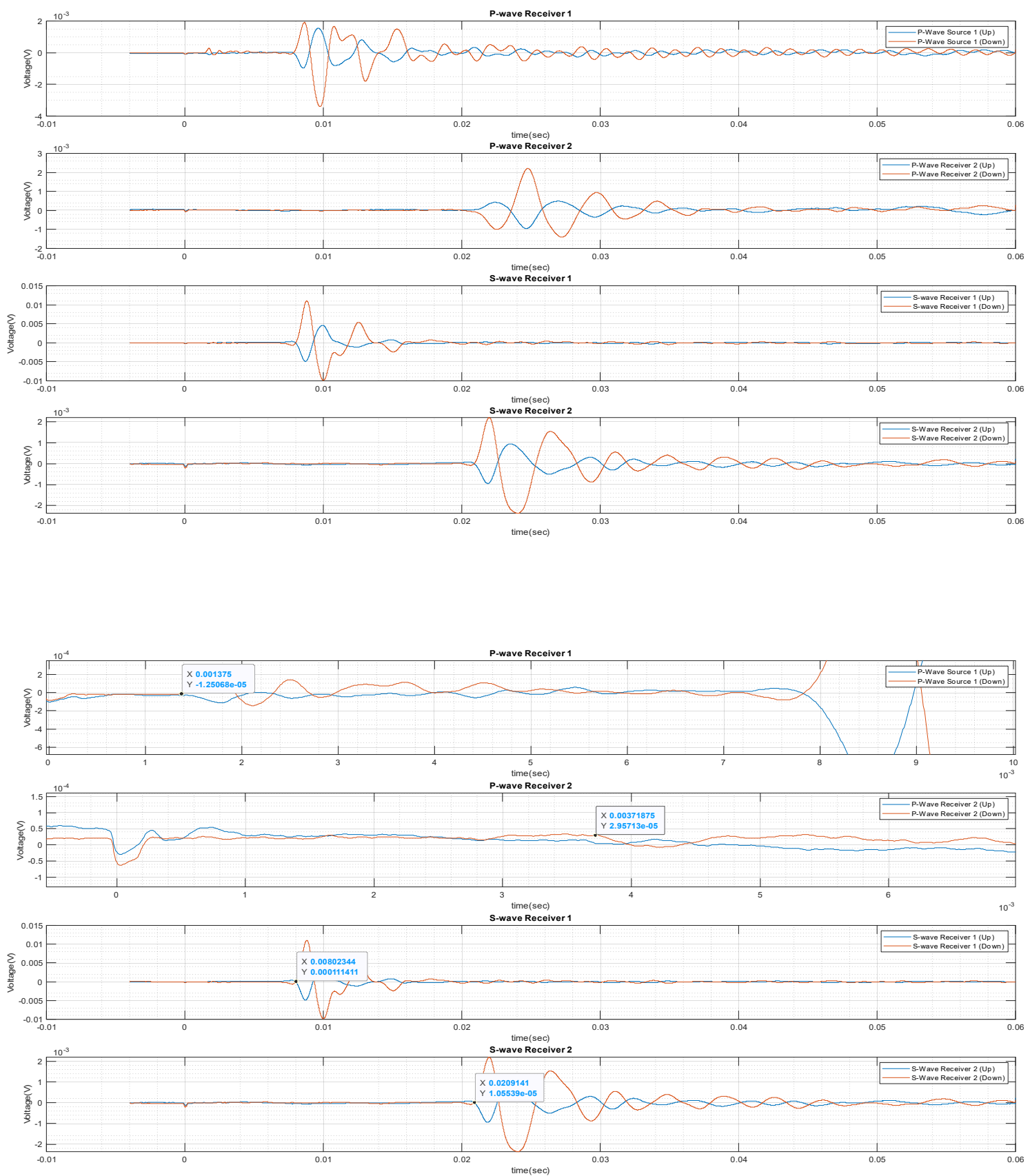

Data Set 18 (9.3.2019)

Run00135f

R4_1 

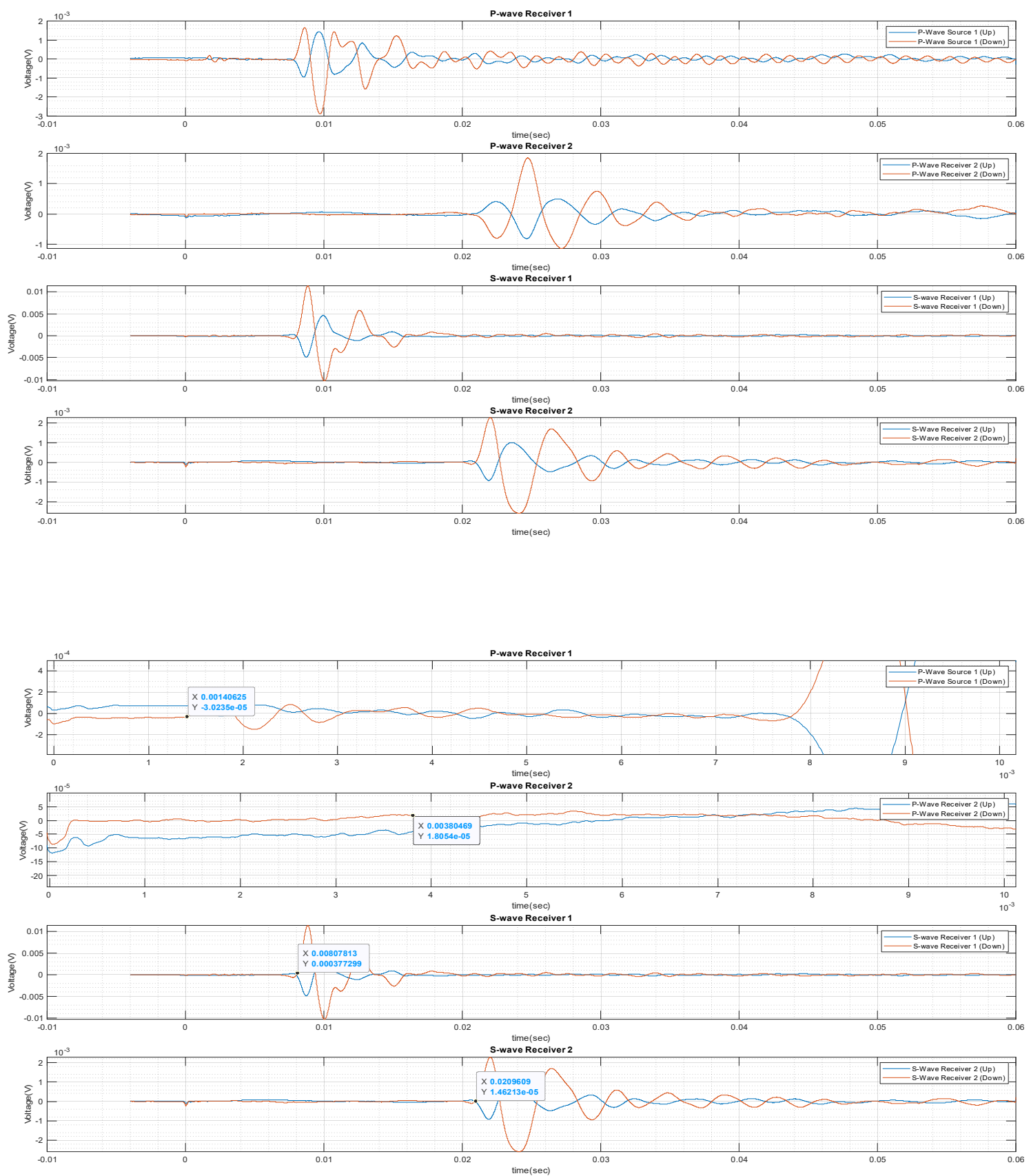

Data Set 18 (9.3.2019)

Run00136f

R4_2 

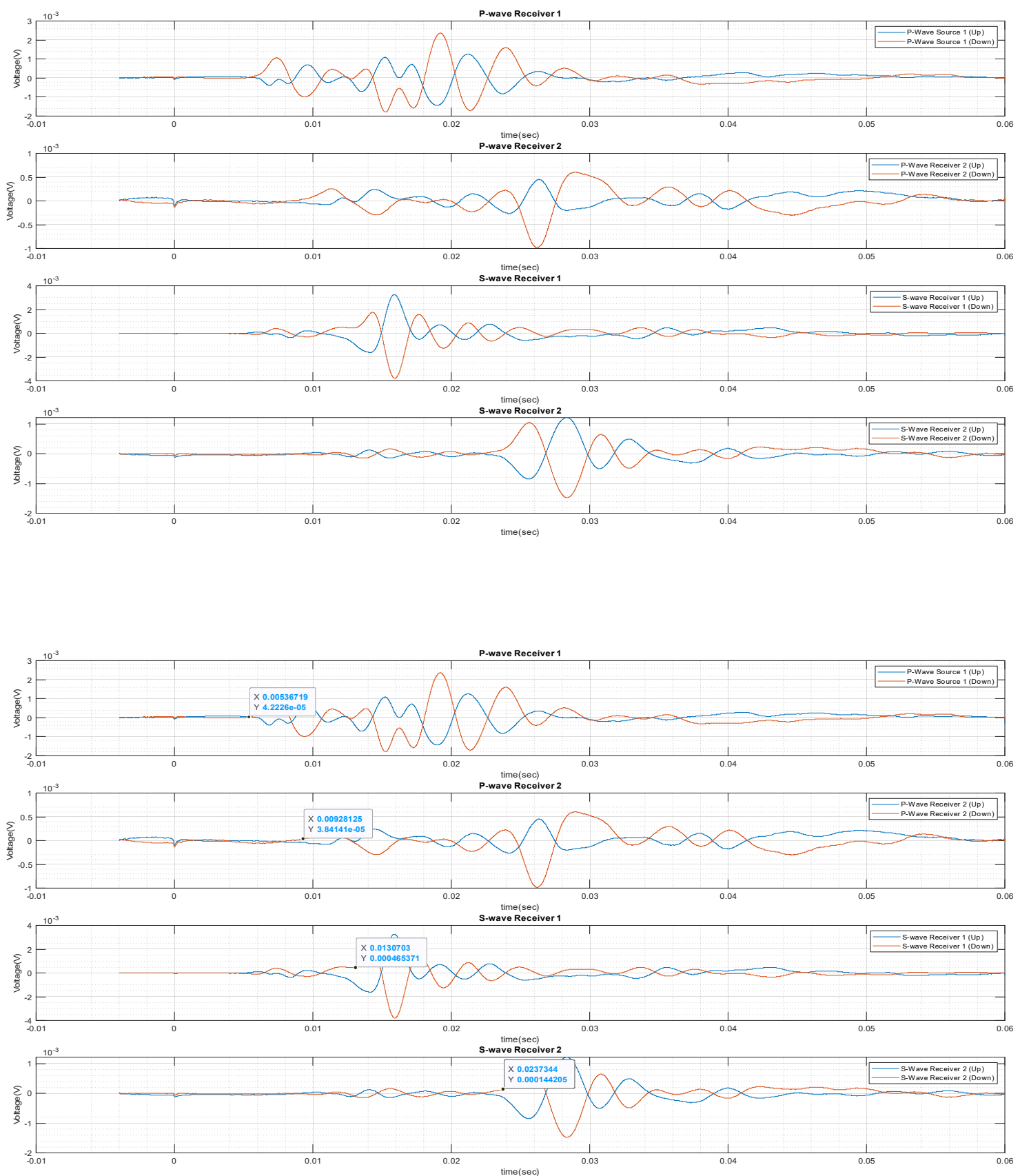

Data Set 19 (9.4.2019)

Run00137f

R1_1 

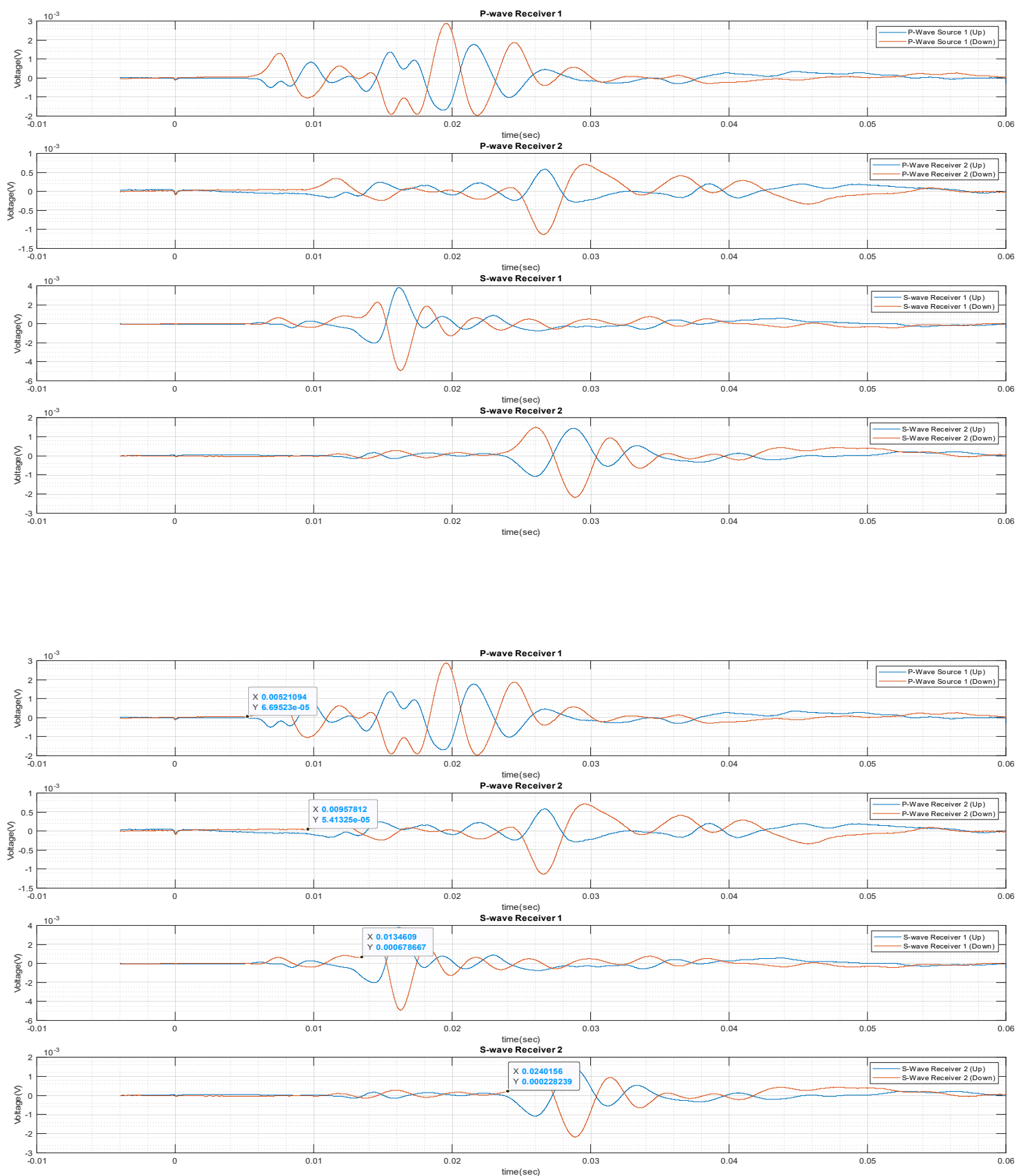

Data Set 19 (9.4.2019)

Run00138f

R1_2 

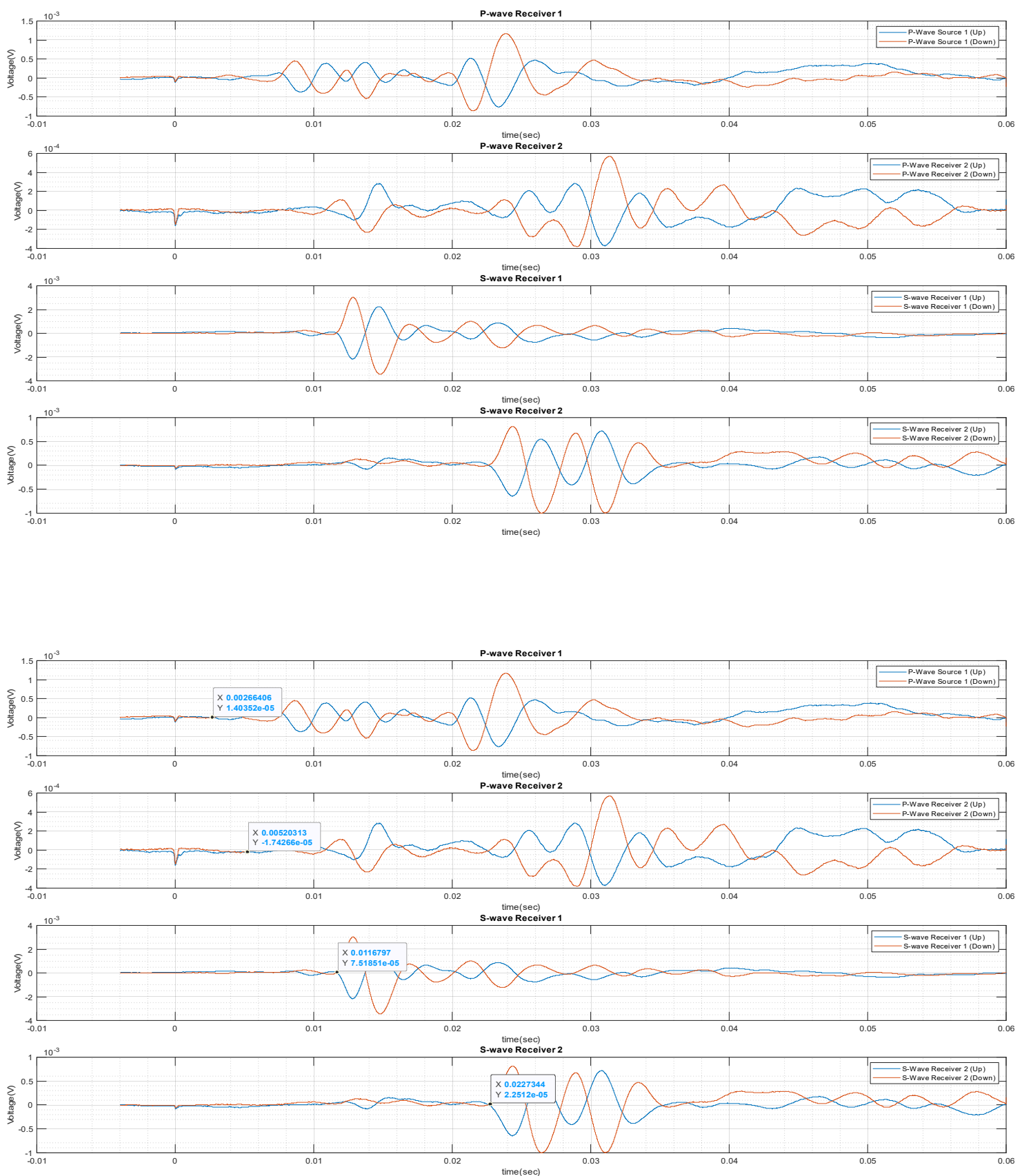

Data Set 19 (9.4.2019)

Run00139f

R2_1 

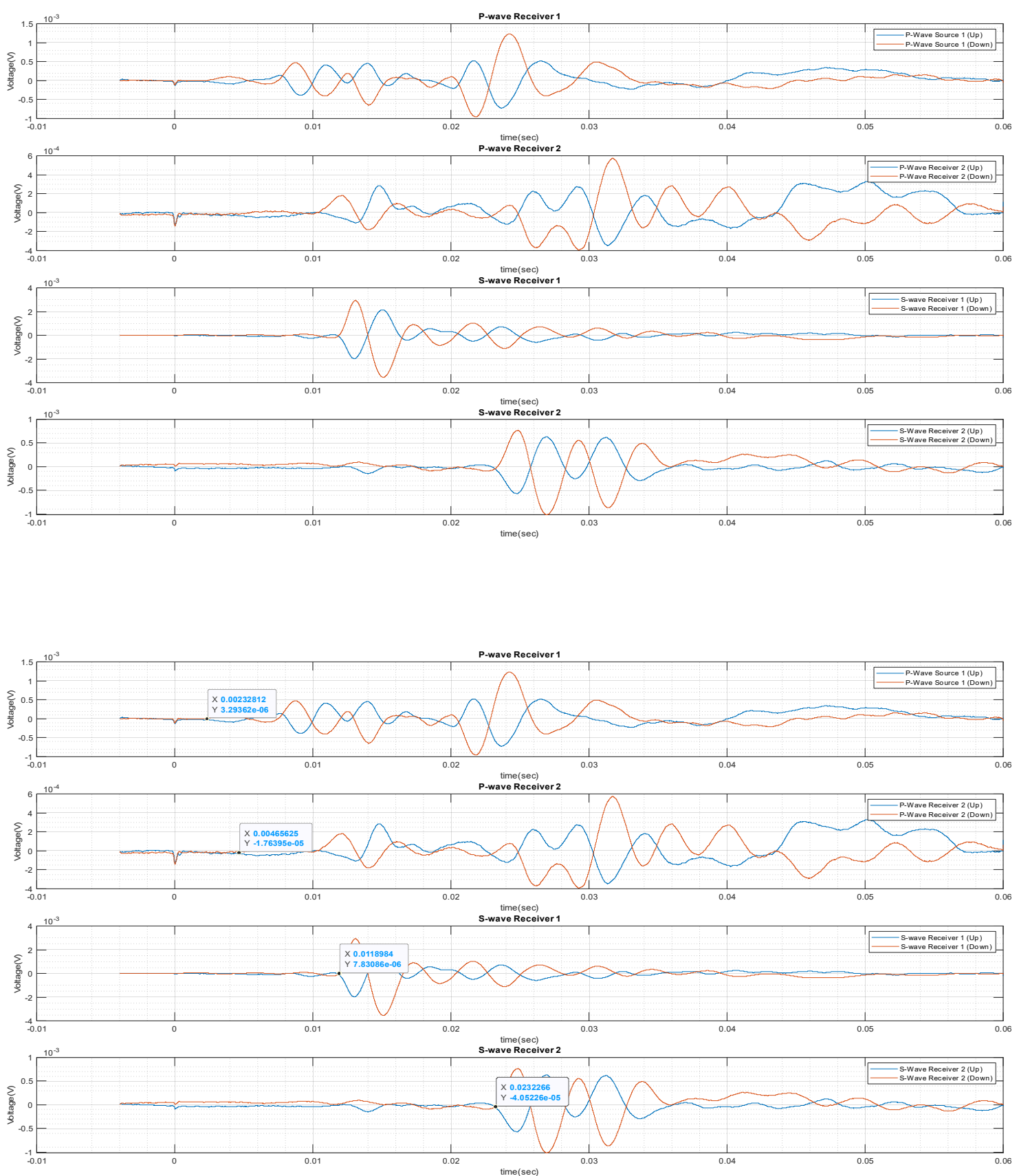

Data Set 19 (9.4.2019)

Run00140f

R2_2 

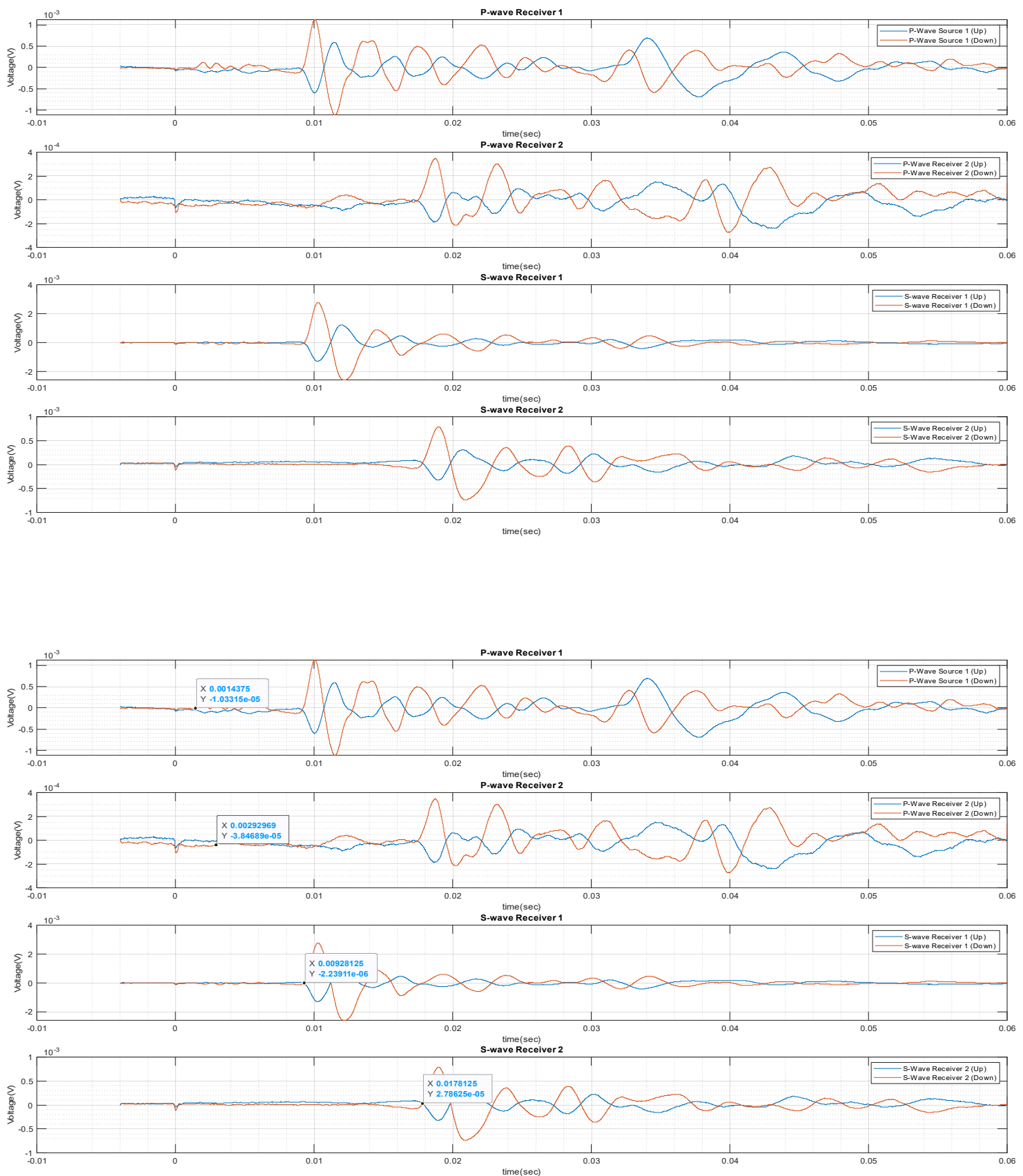

Data Set 19 (9.4.2019)

Run00141f

R3_1 

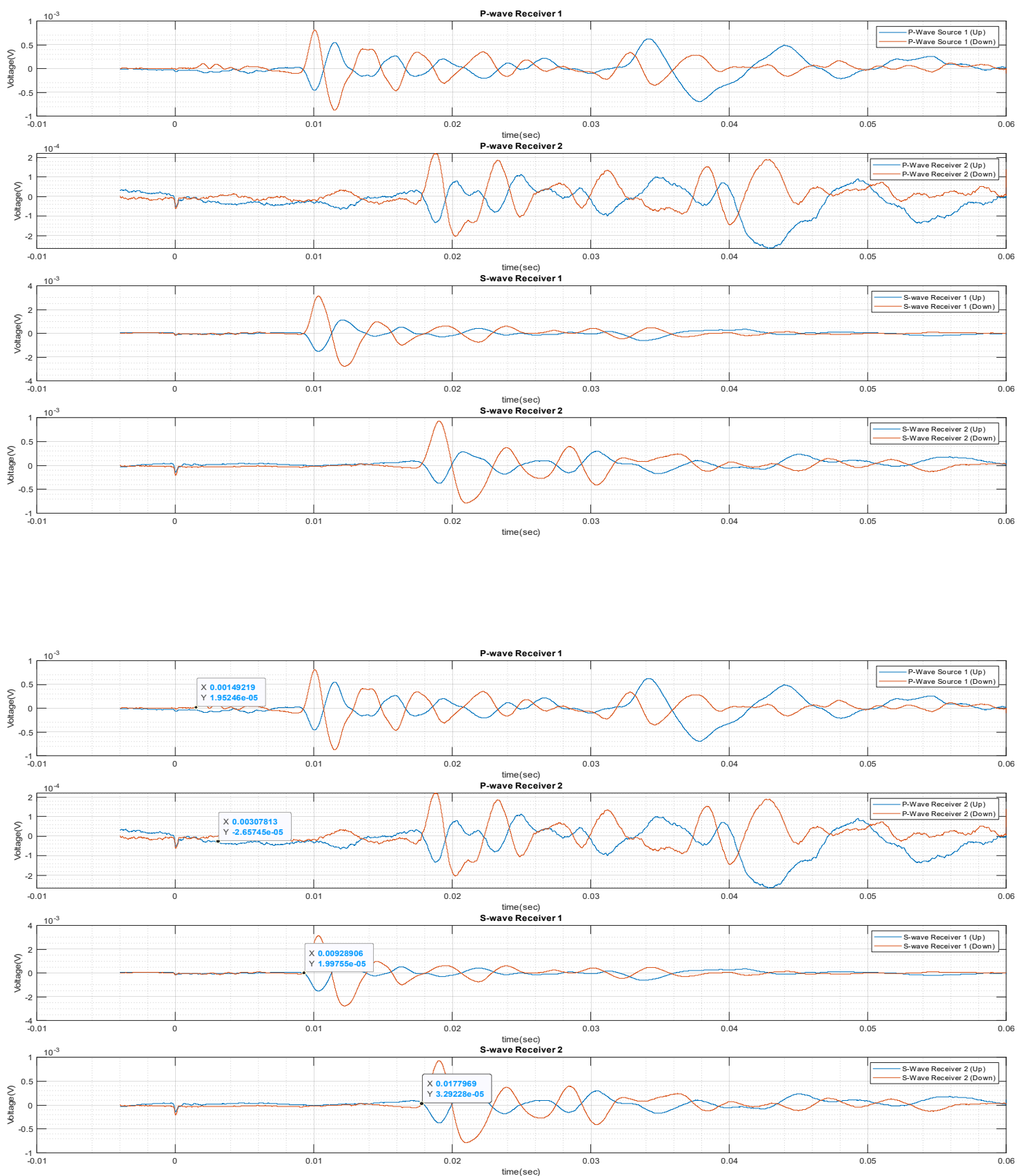

Data Set 19 (9.4.2019)

Run00142f

R3_2 

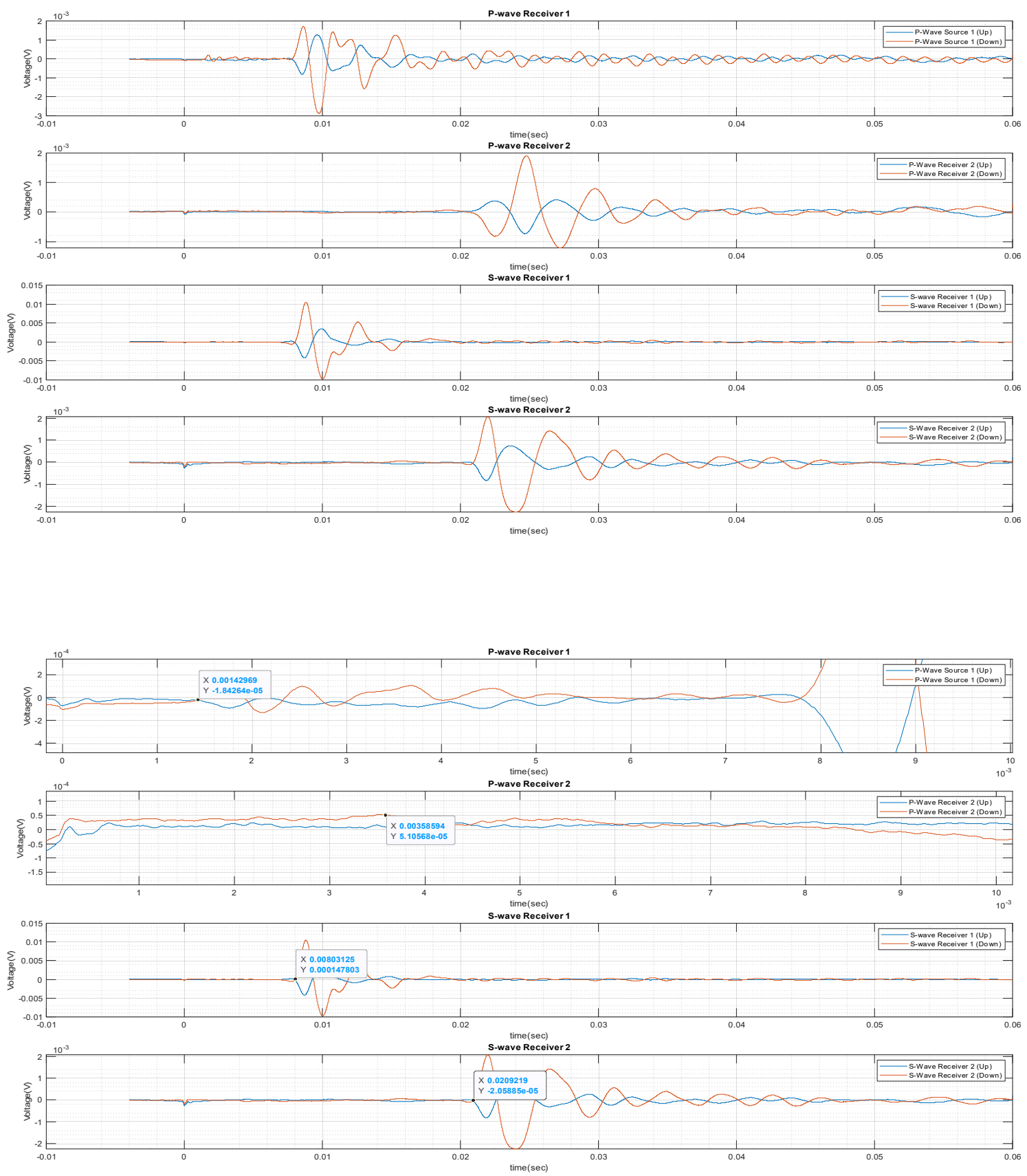

Data Set 19 (9.4.2019)

Run00143f

R4_1 

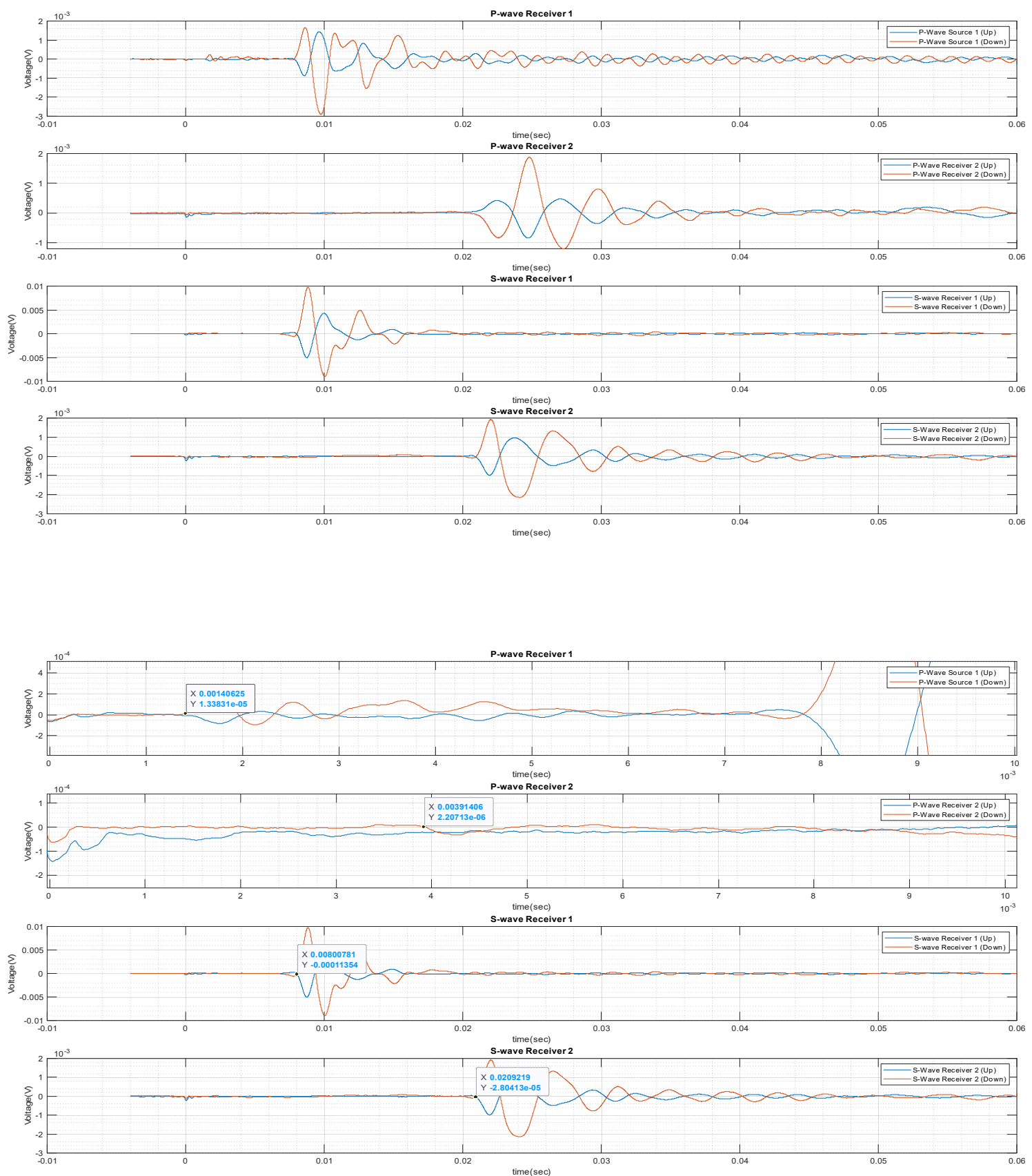

Data Set 19 (9.4.2019)

Run00144f

R4 2 

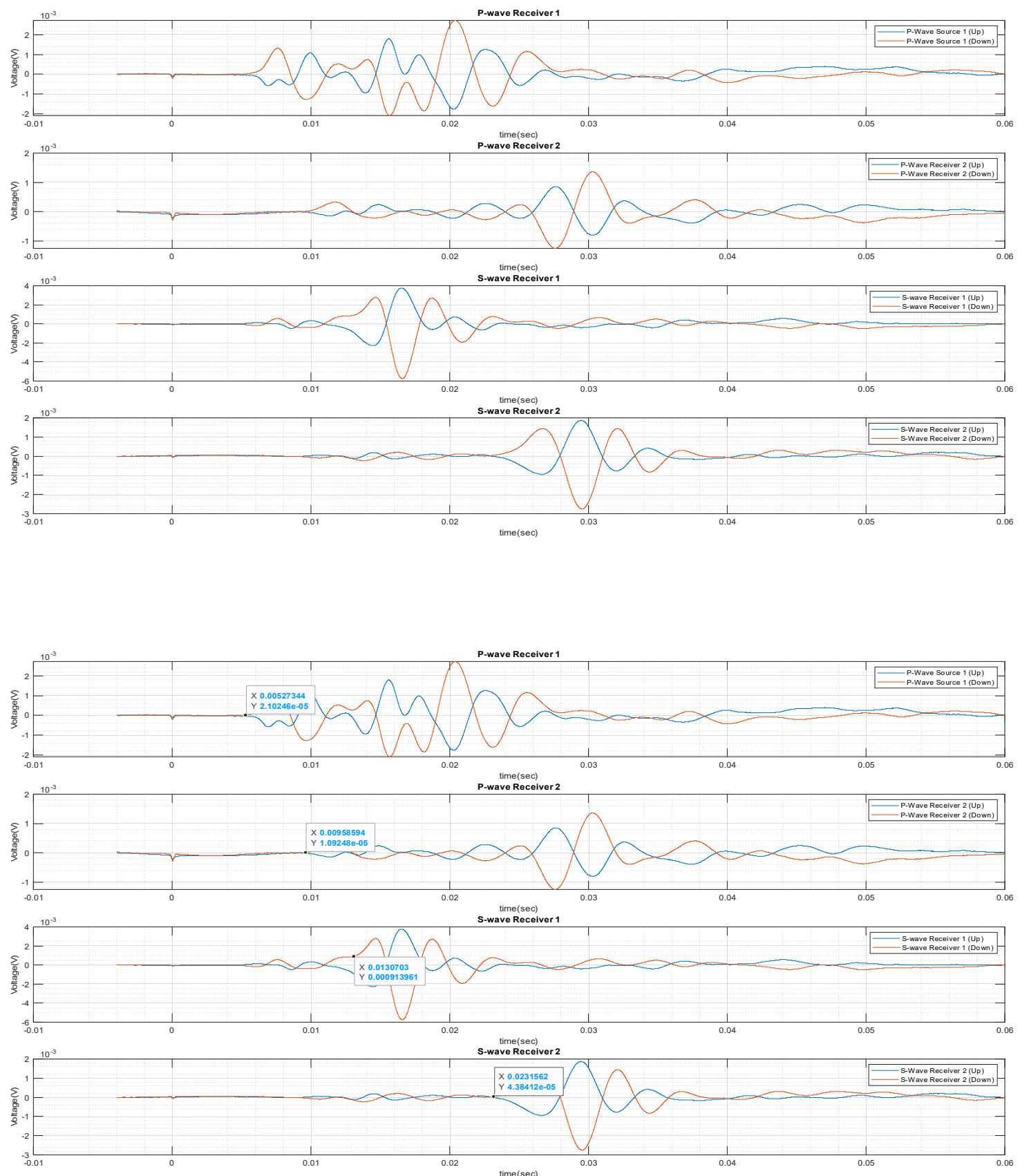

Data Set 20 (9.5.2019)

Run00145f

R1_1 

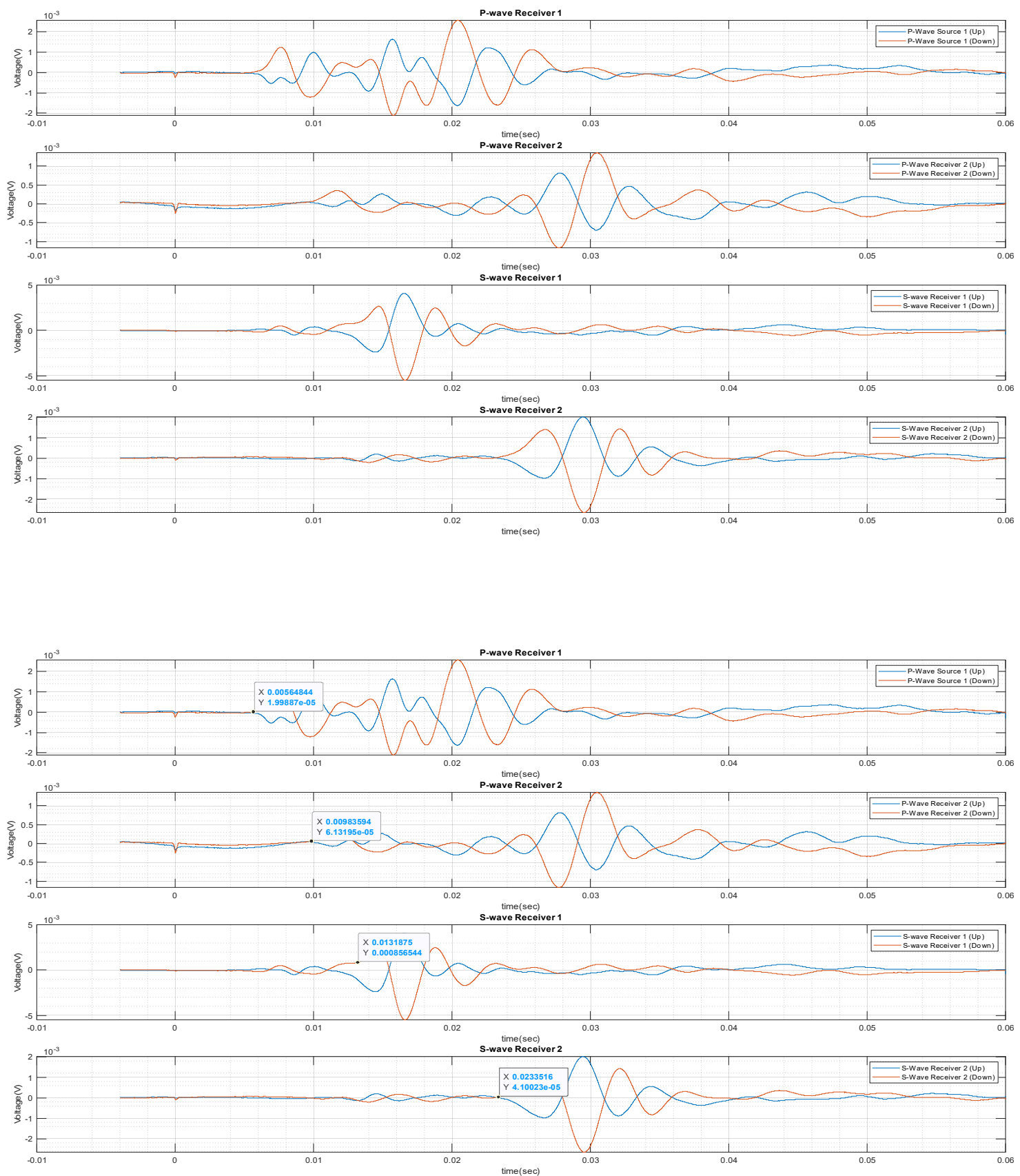

Data Set 20 (9.5.2019)

Run00146f

R1_2 

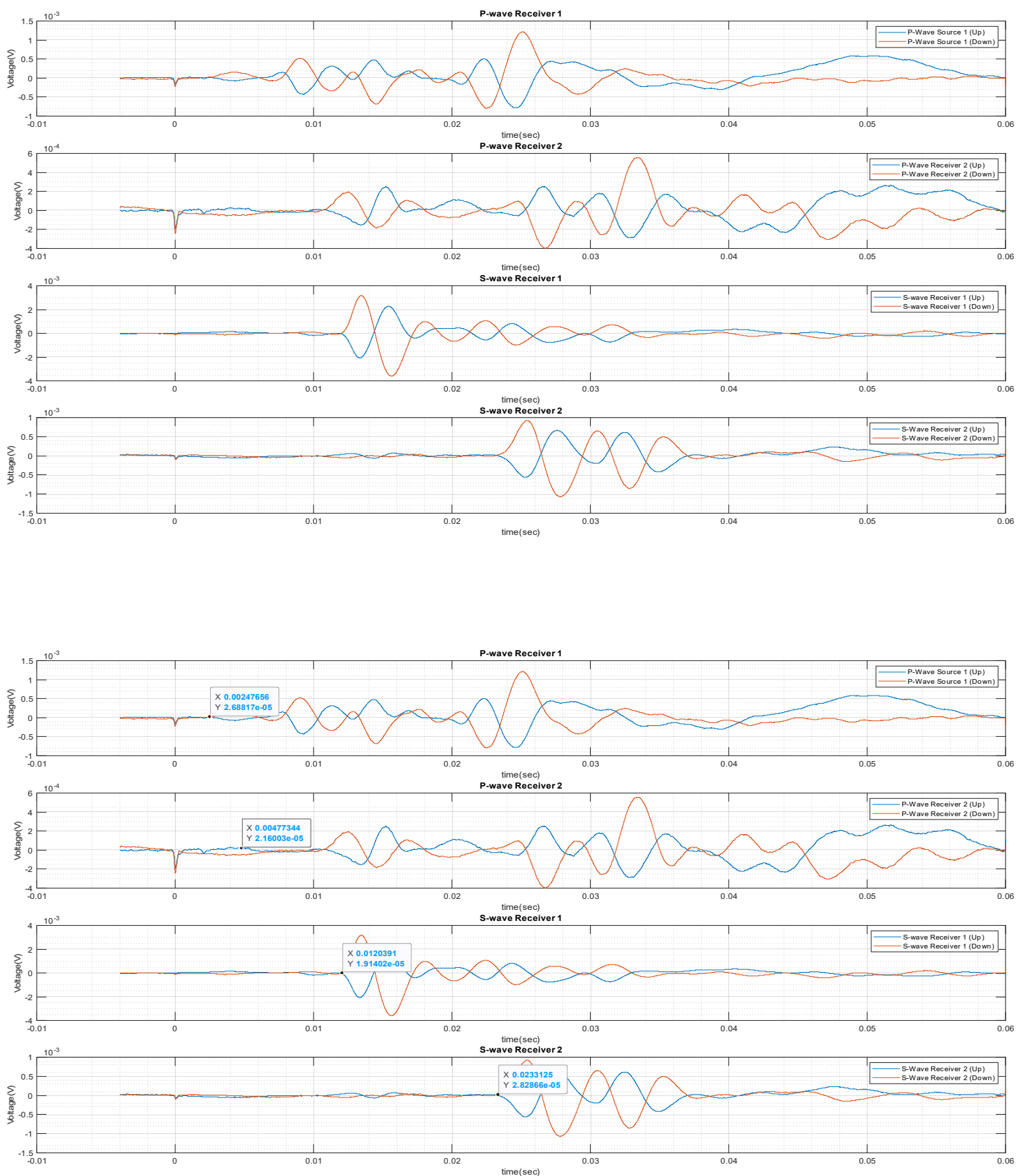

Data Set 20 (9.5.2019)

Run00147f

R2_1 

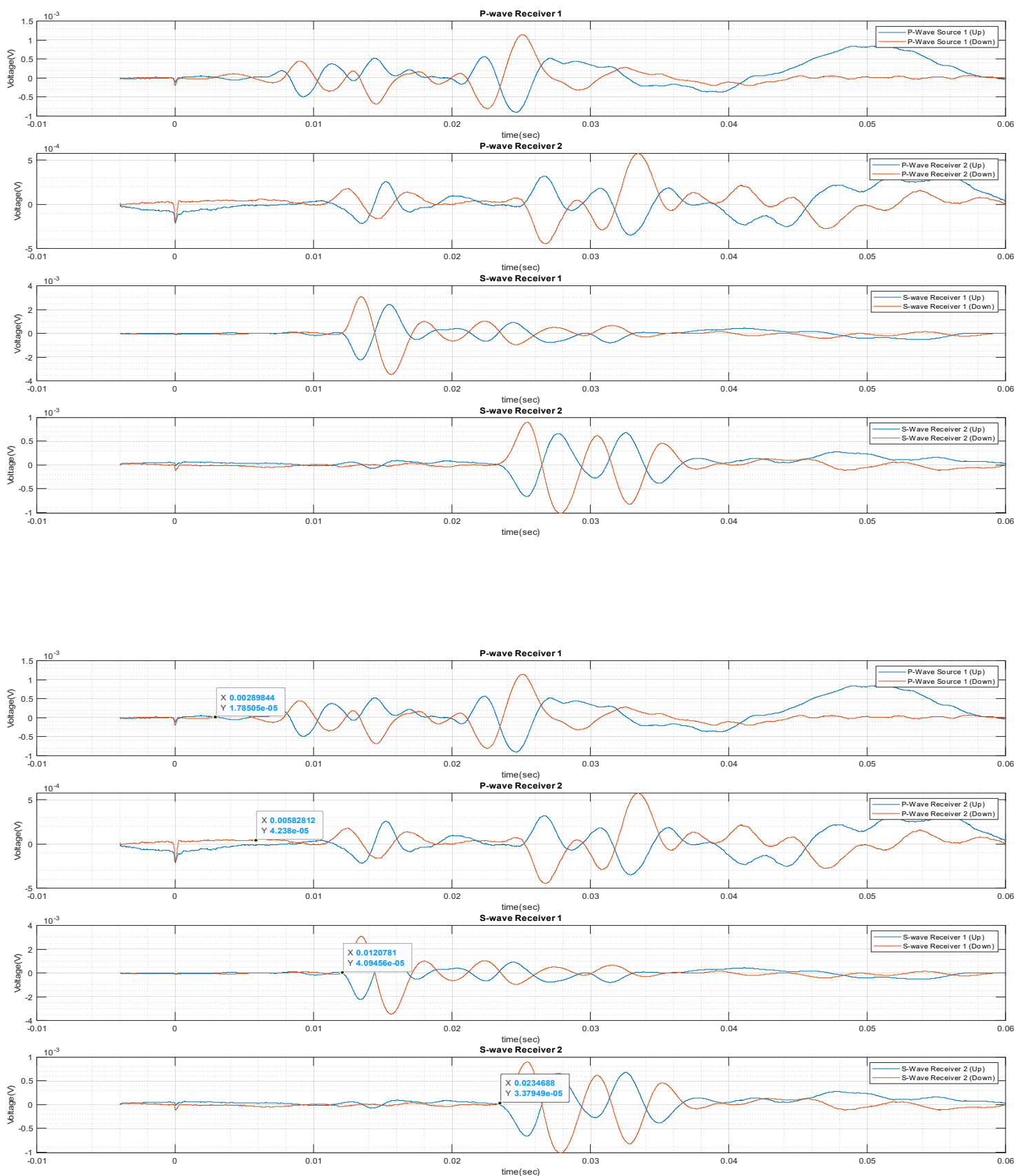

Data Set 20 (9.5.2019)

Run00148f

R2_2 

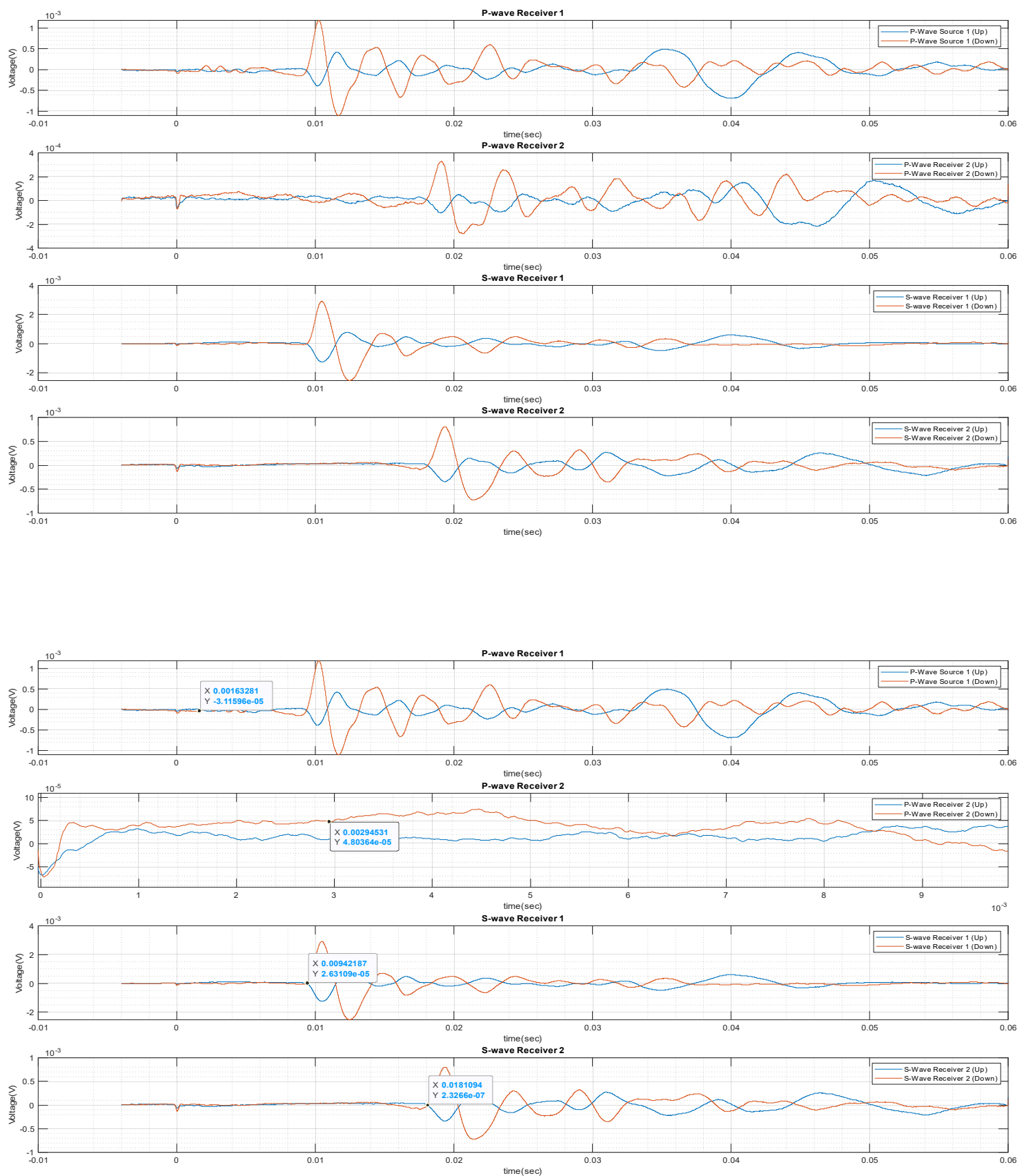

Data Set 20 (9.5.2019)

Run00149f

R3_1 

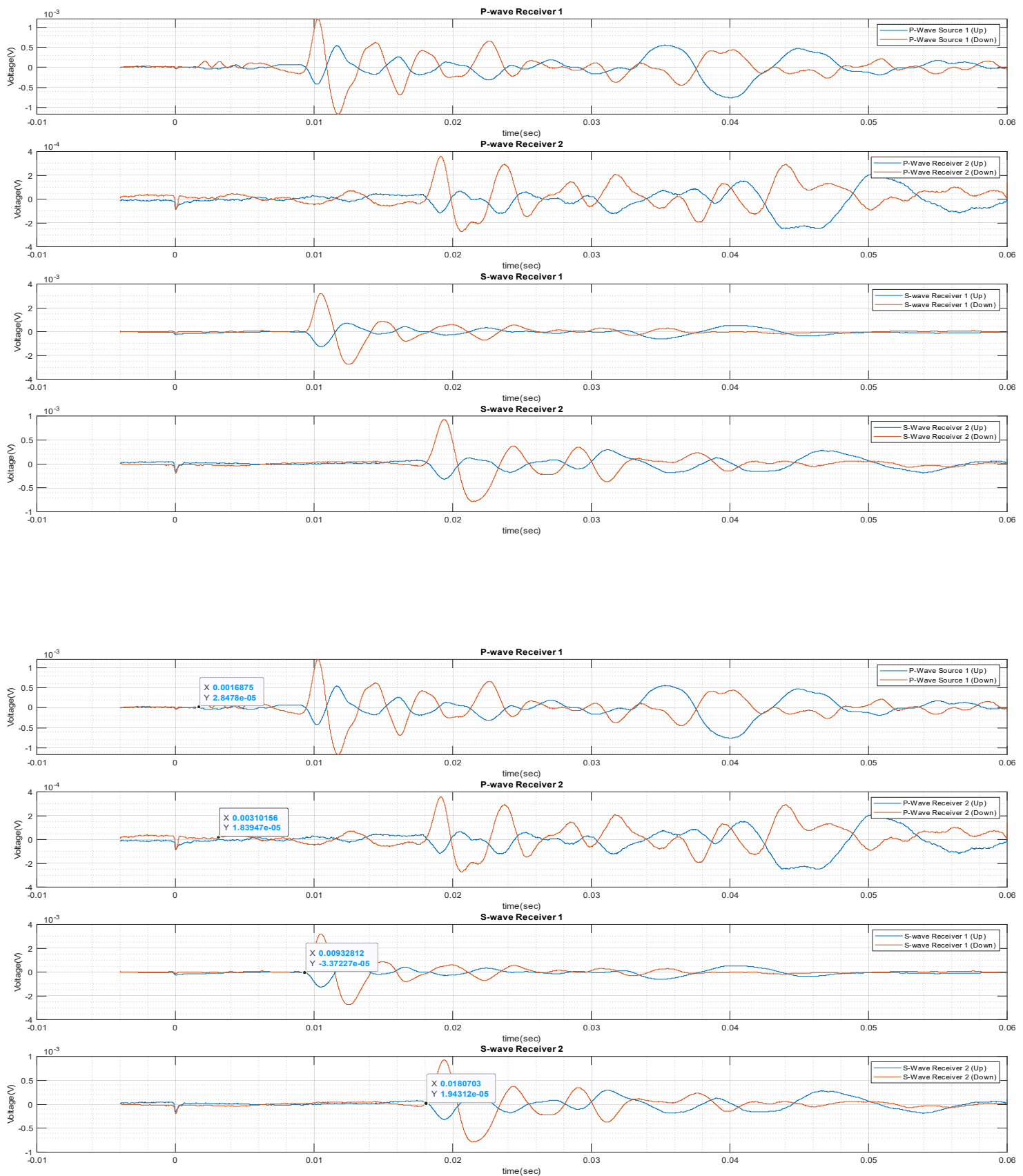

Data Set 20 (9.5.2019)

Run00150f

R3_2 

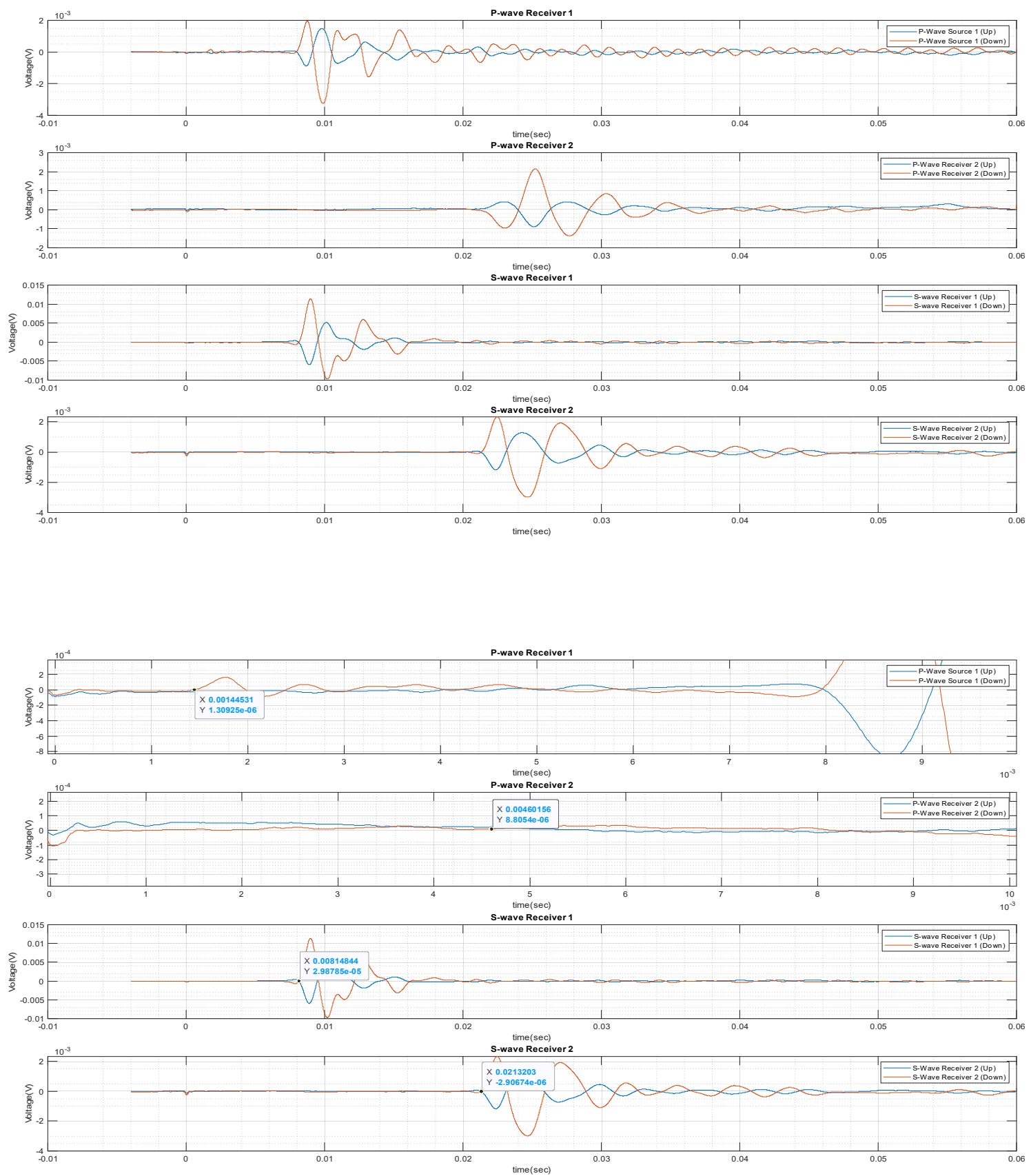

Data Set 20 (9.5.2019)

Run00151f

R4_1 

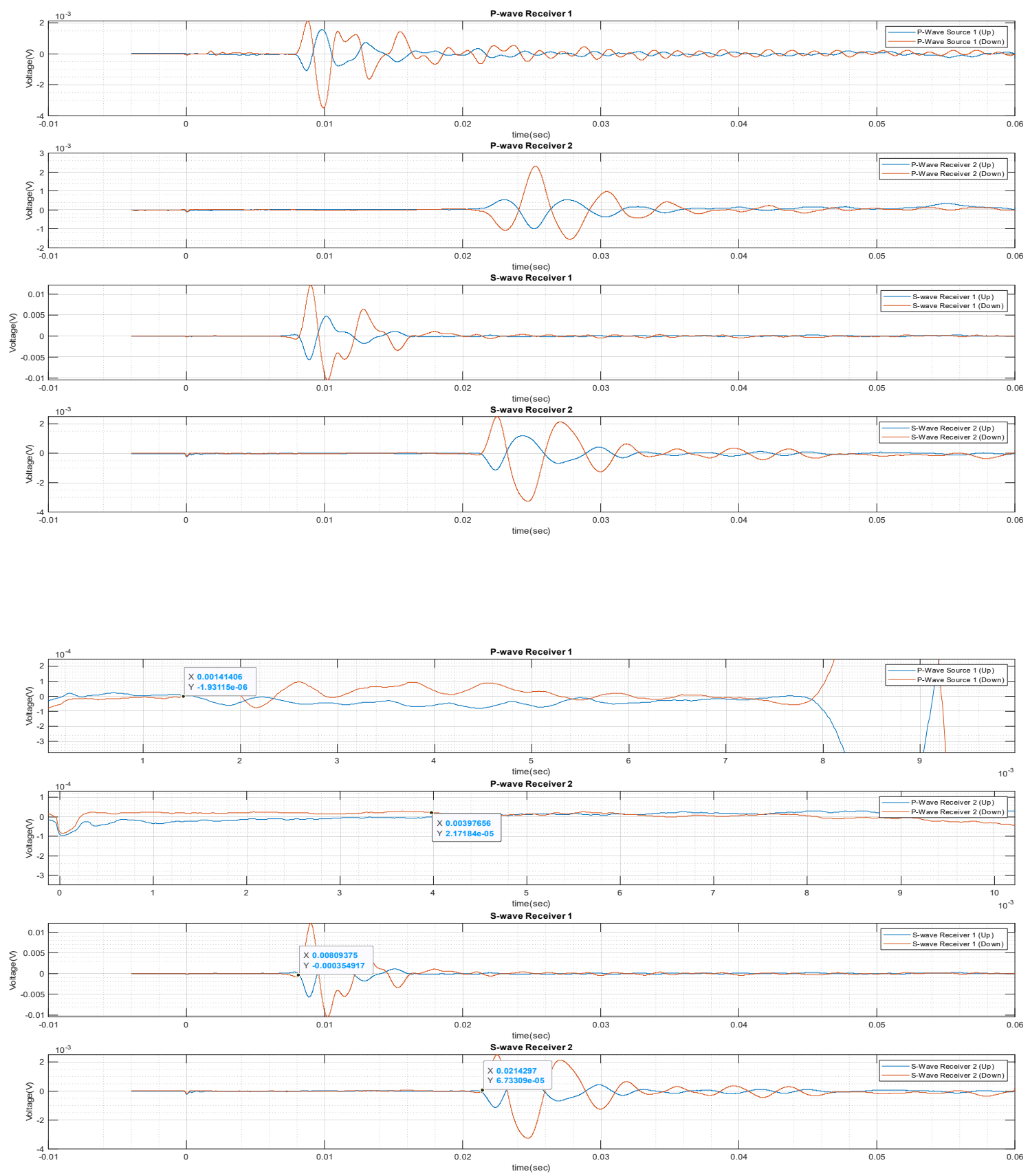

Data Set 20 (9.5.2019)

Run00152f

R4_2 

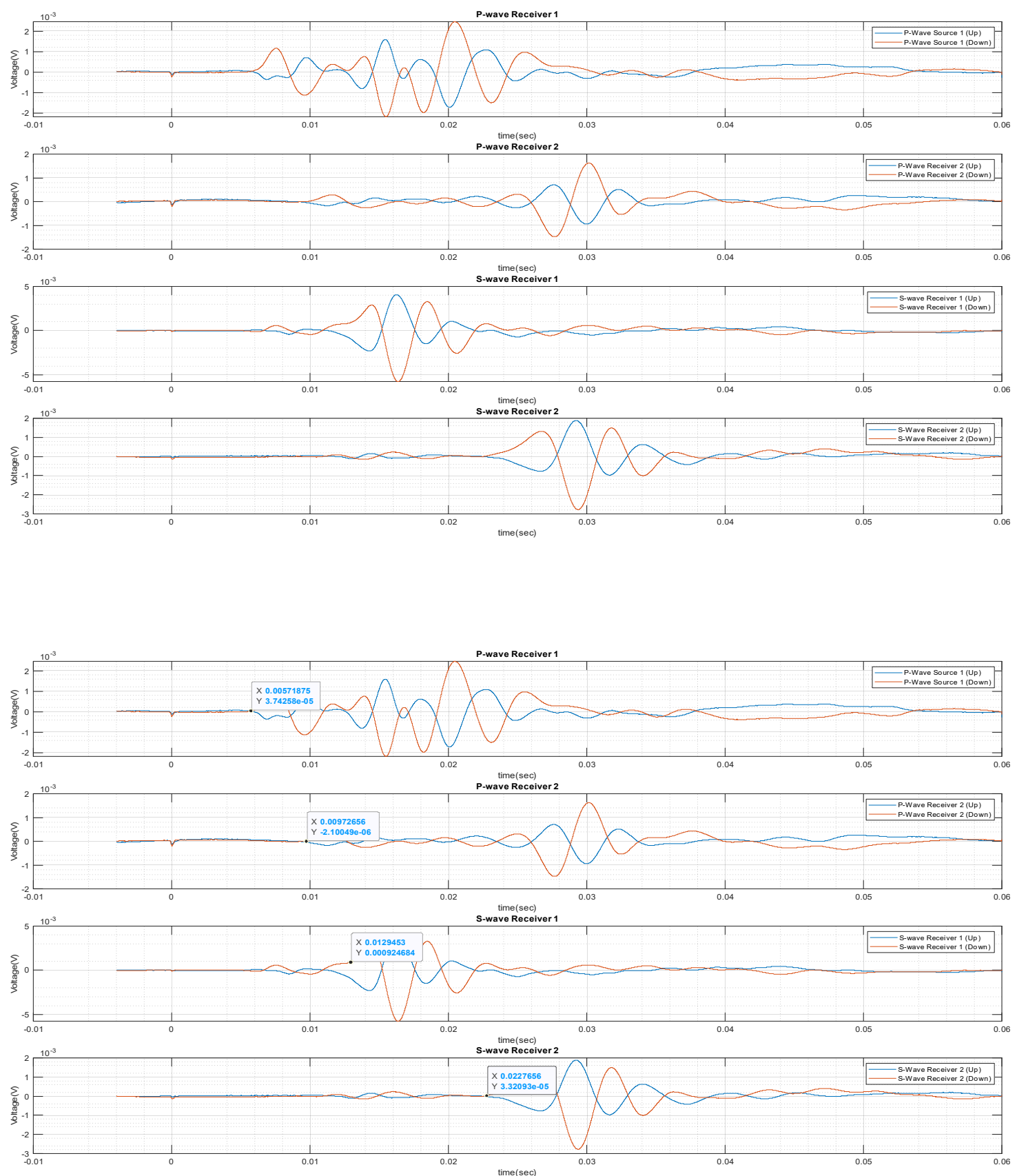

Data Set 21 (9.6.2019)

Run00153f

R1_1 

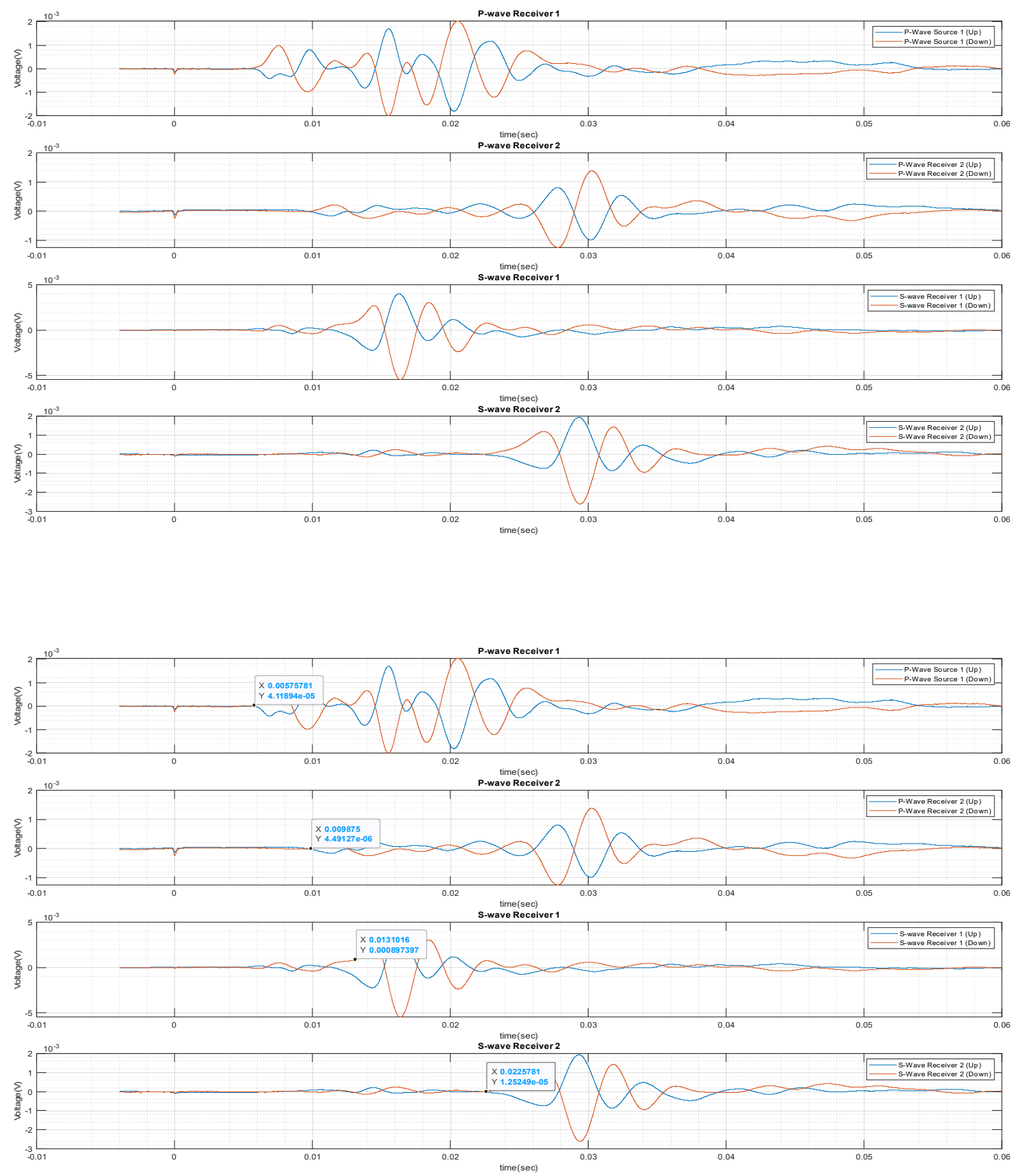

Data Set 21 (9.6.2019)

Run00154f

R1_2 

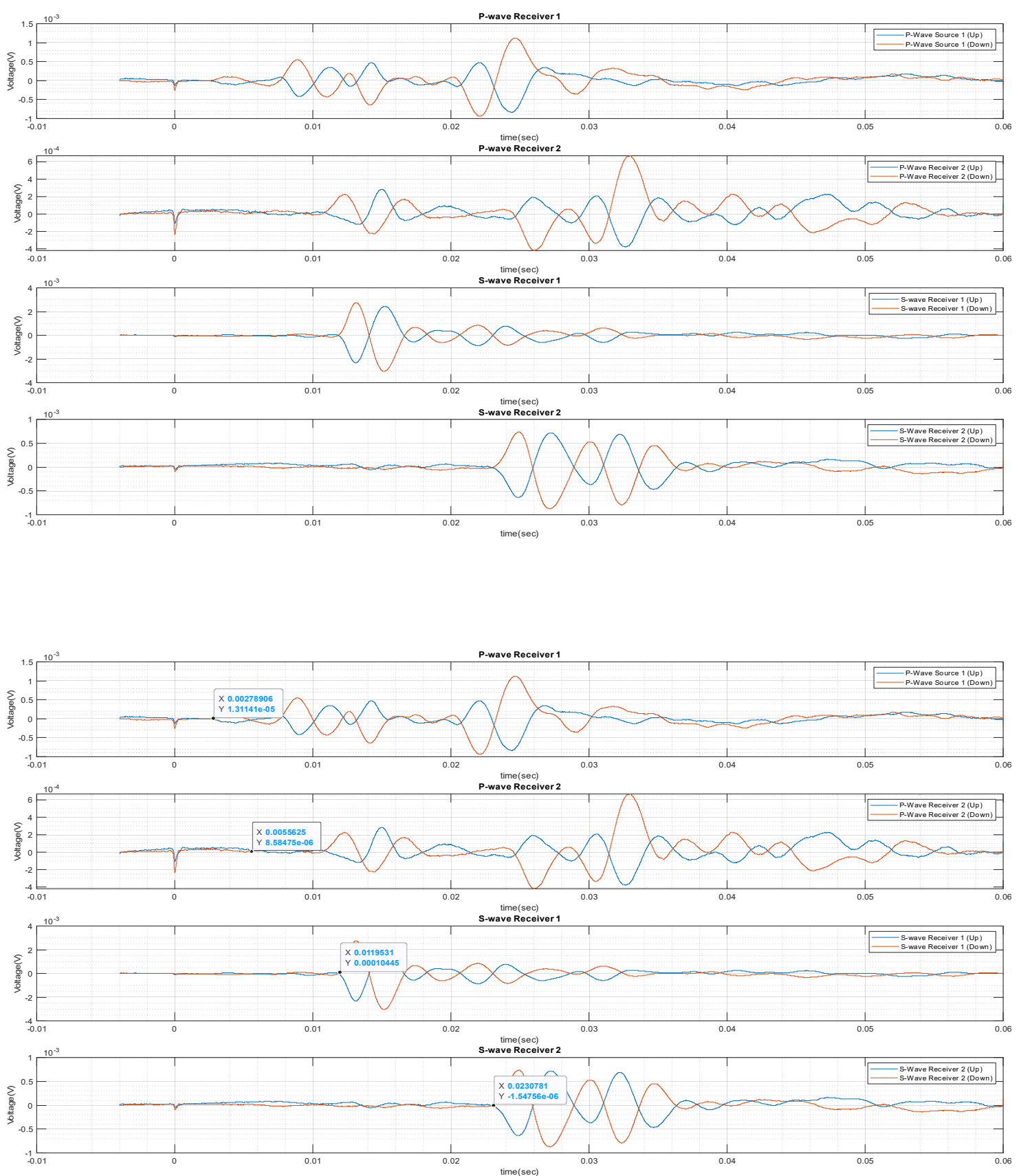

Data Set 21 (9.6.2019)

Run00155f

R2_1 

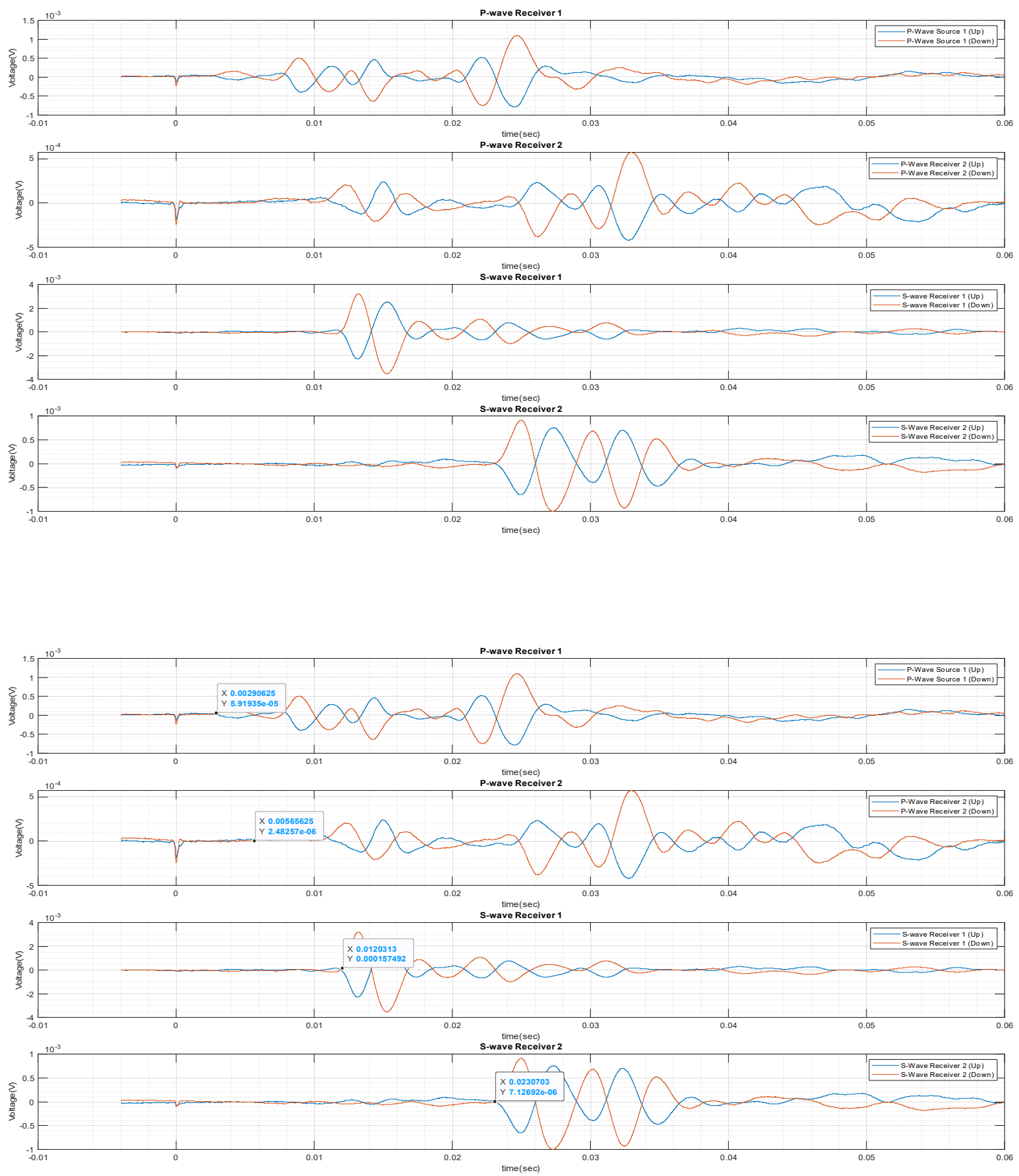

Data Set 21 (9.6.2019)

Run00156f

R2_2 

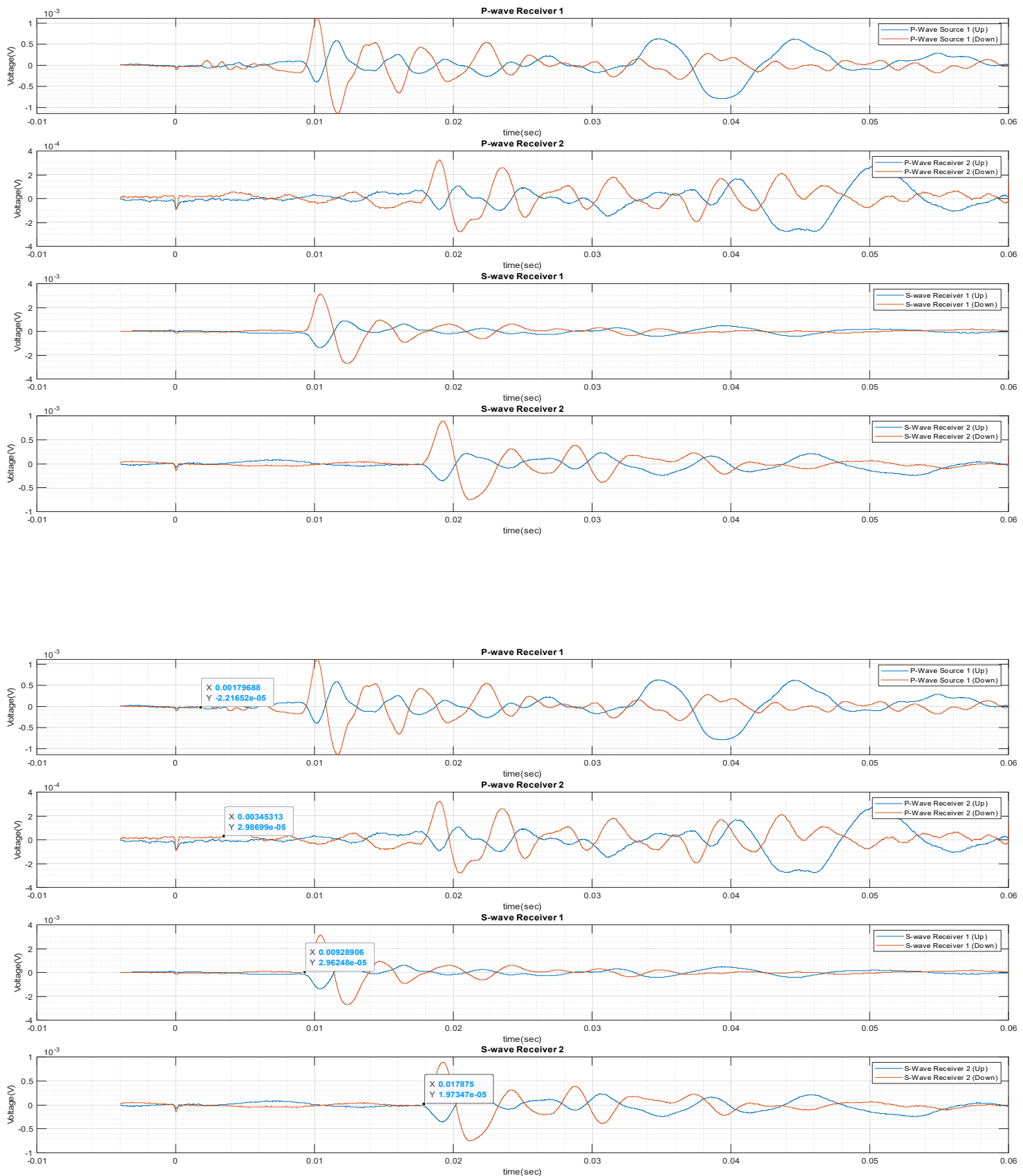

Data Set 21 (9.6.2019)

Run00157f

R3_1 

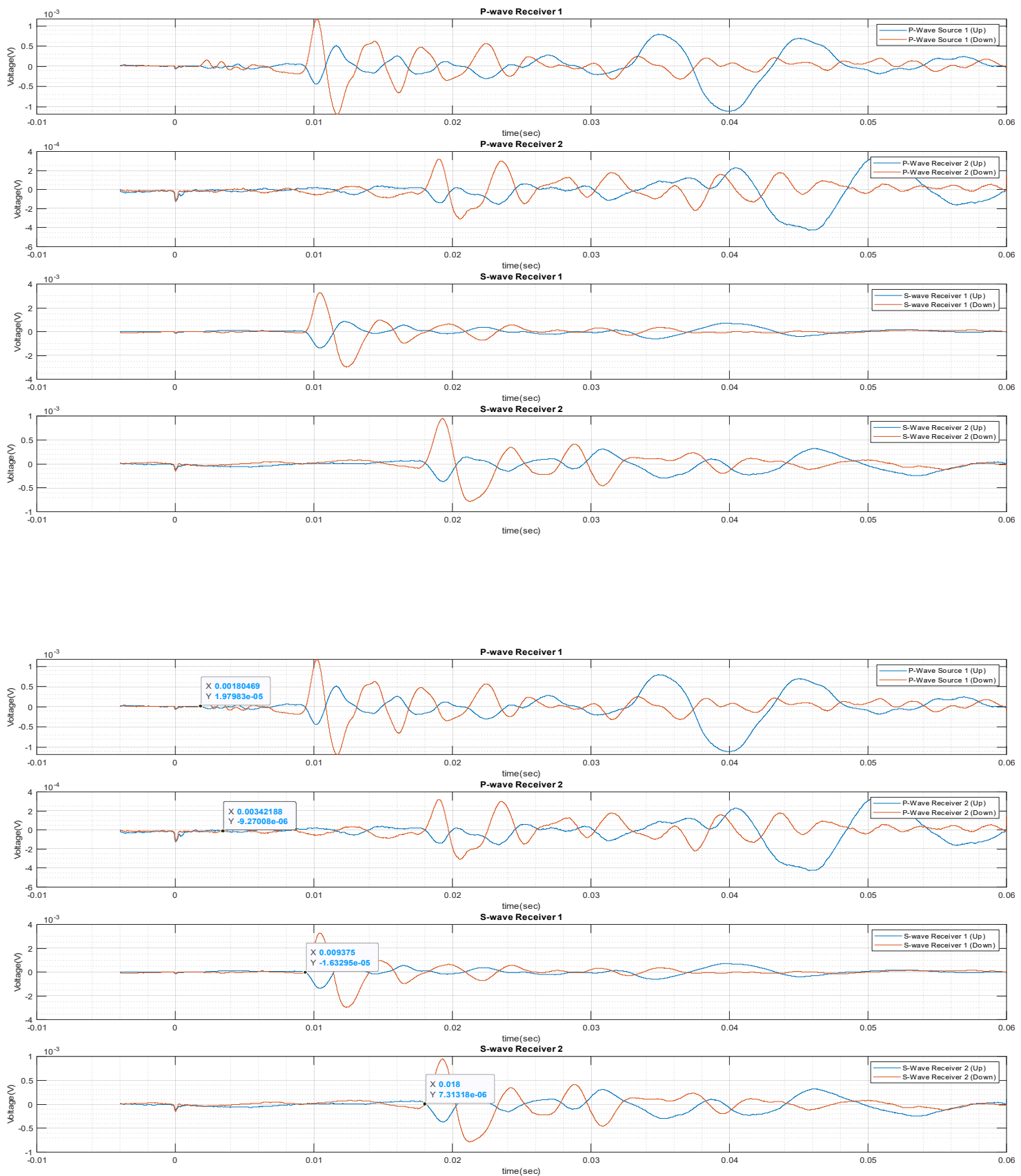

Data Set 21 (9.6.2019)

Run00158f

R3_2 

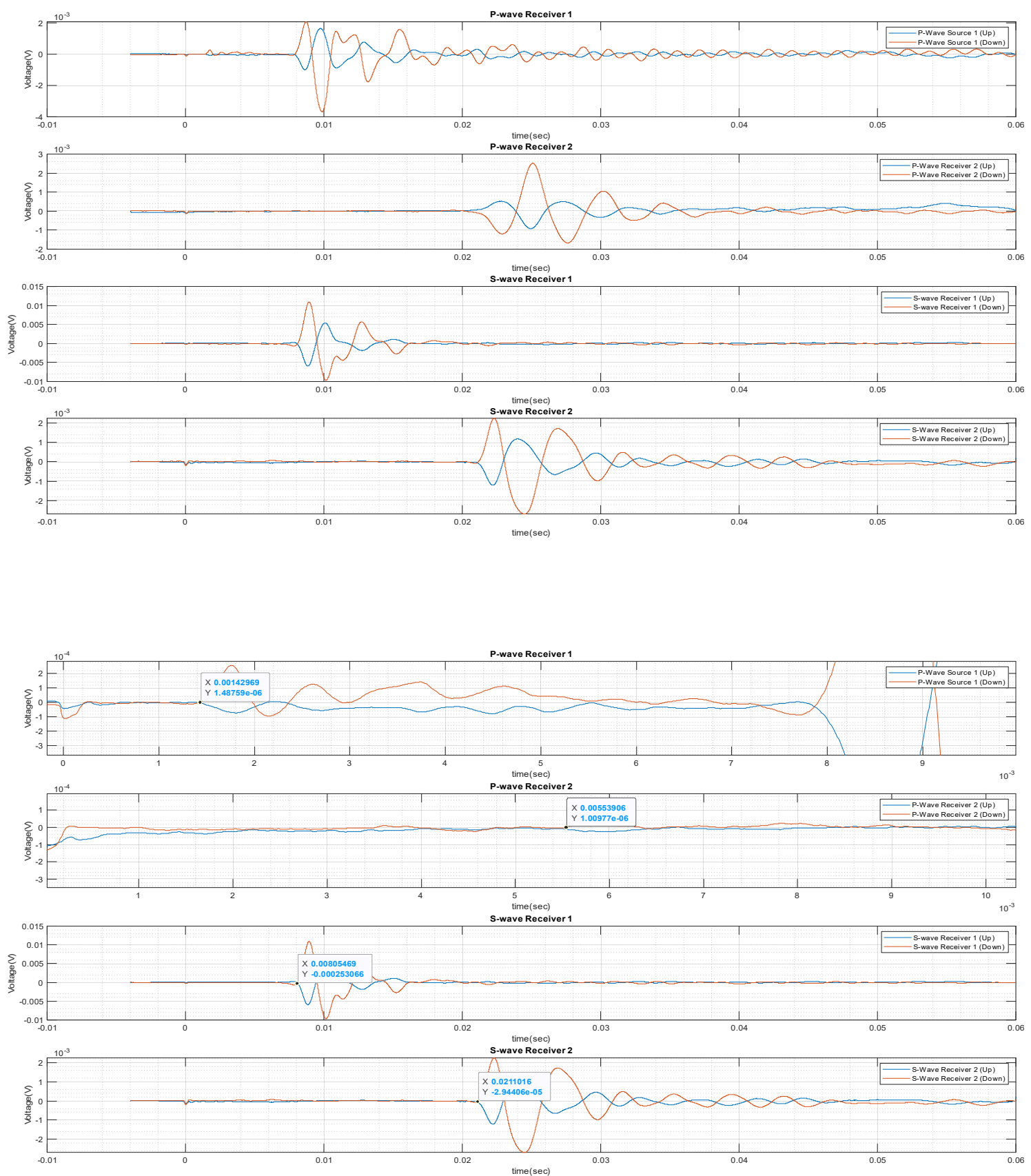

Data Set 21 (9.6.2019)

Run00159f

R4_1 

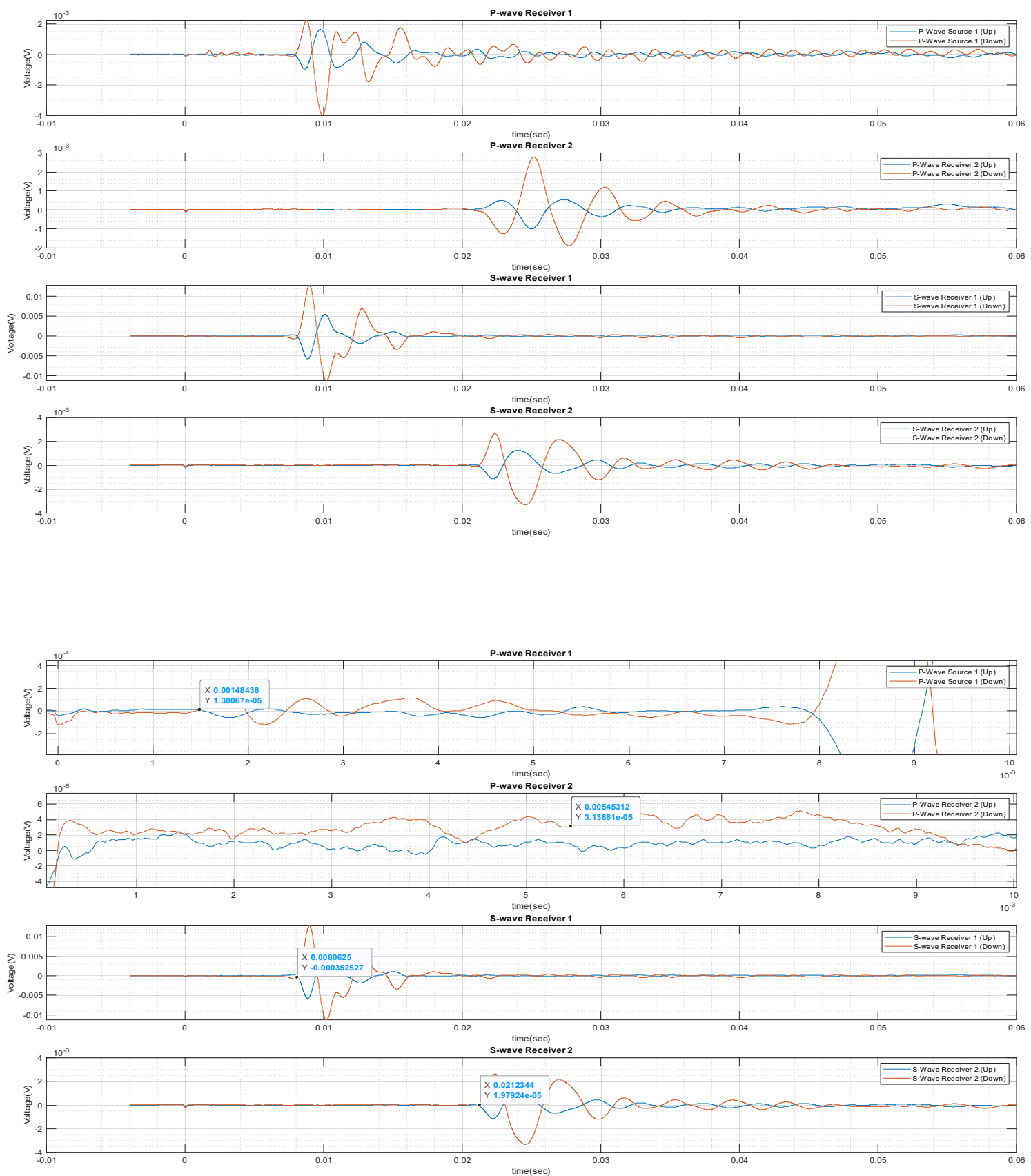

Data Set 21 (9.6.2019)

Run00160f

R4_2 

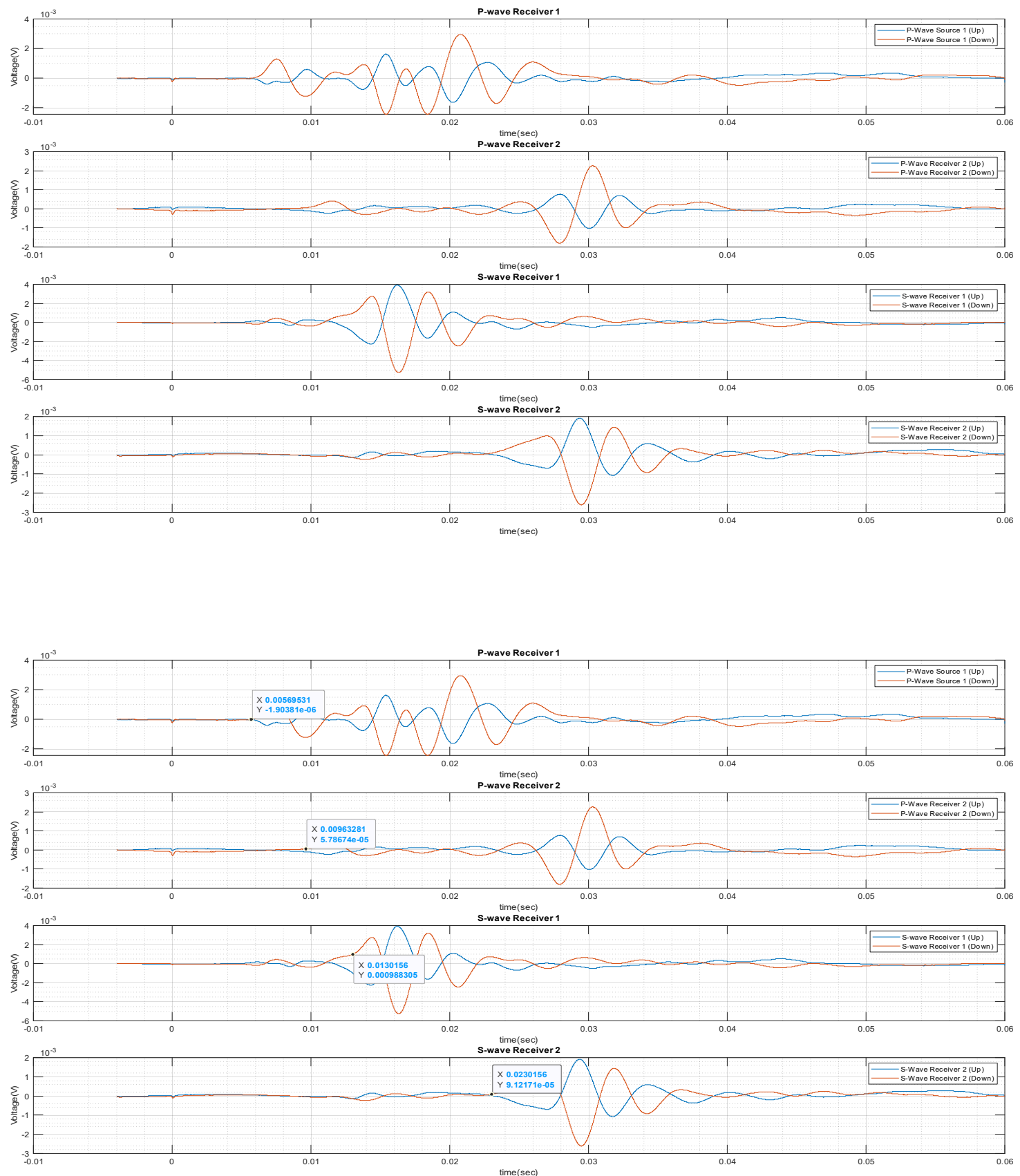

Data Set 22 (9.7.2019)

Run00161f

R1_1 

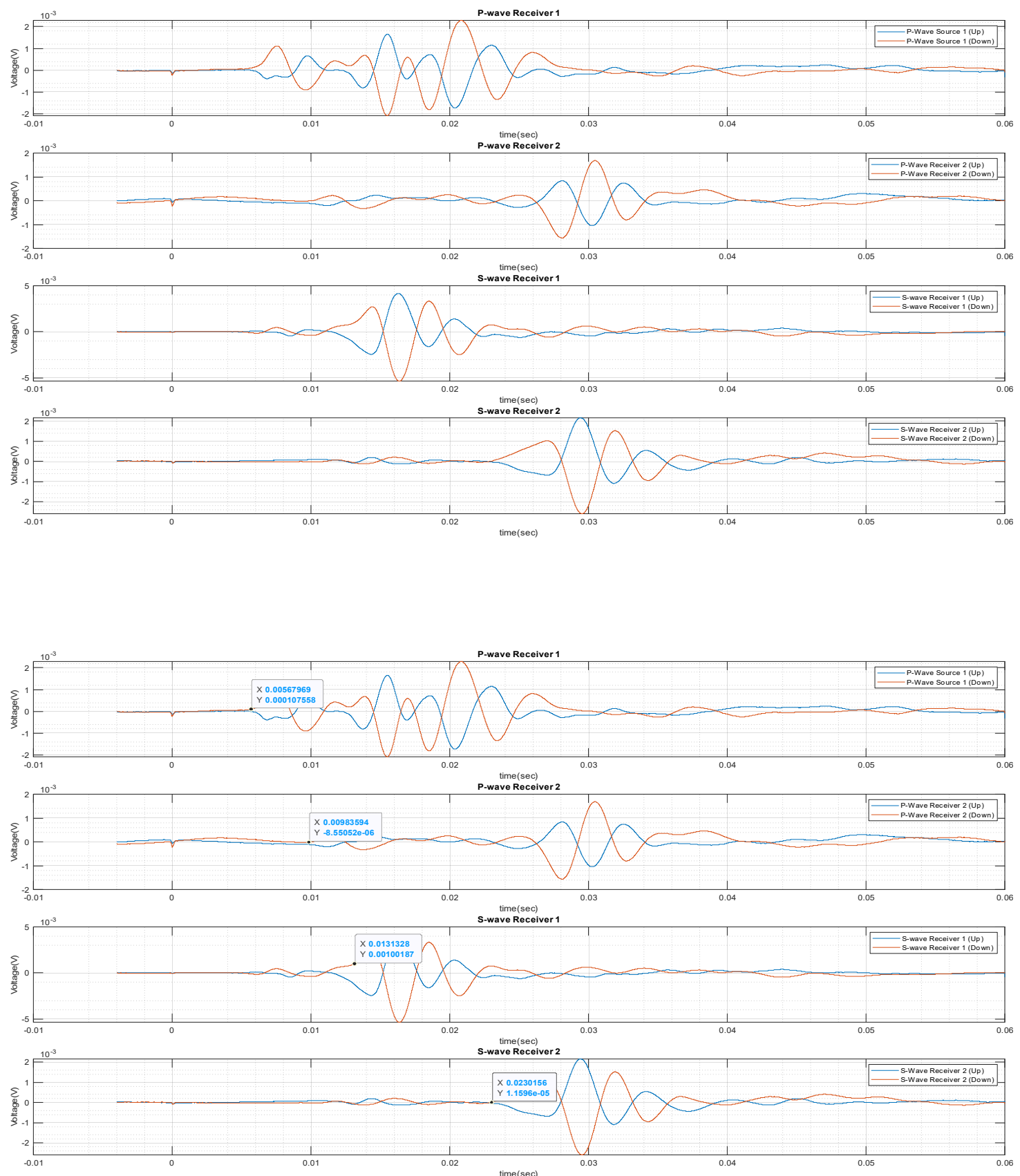

Data Set 22 (9.7.2019)

Run00162f

R1_2 

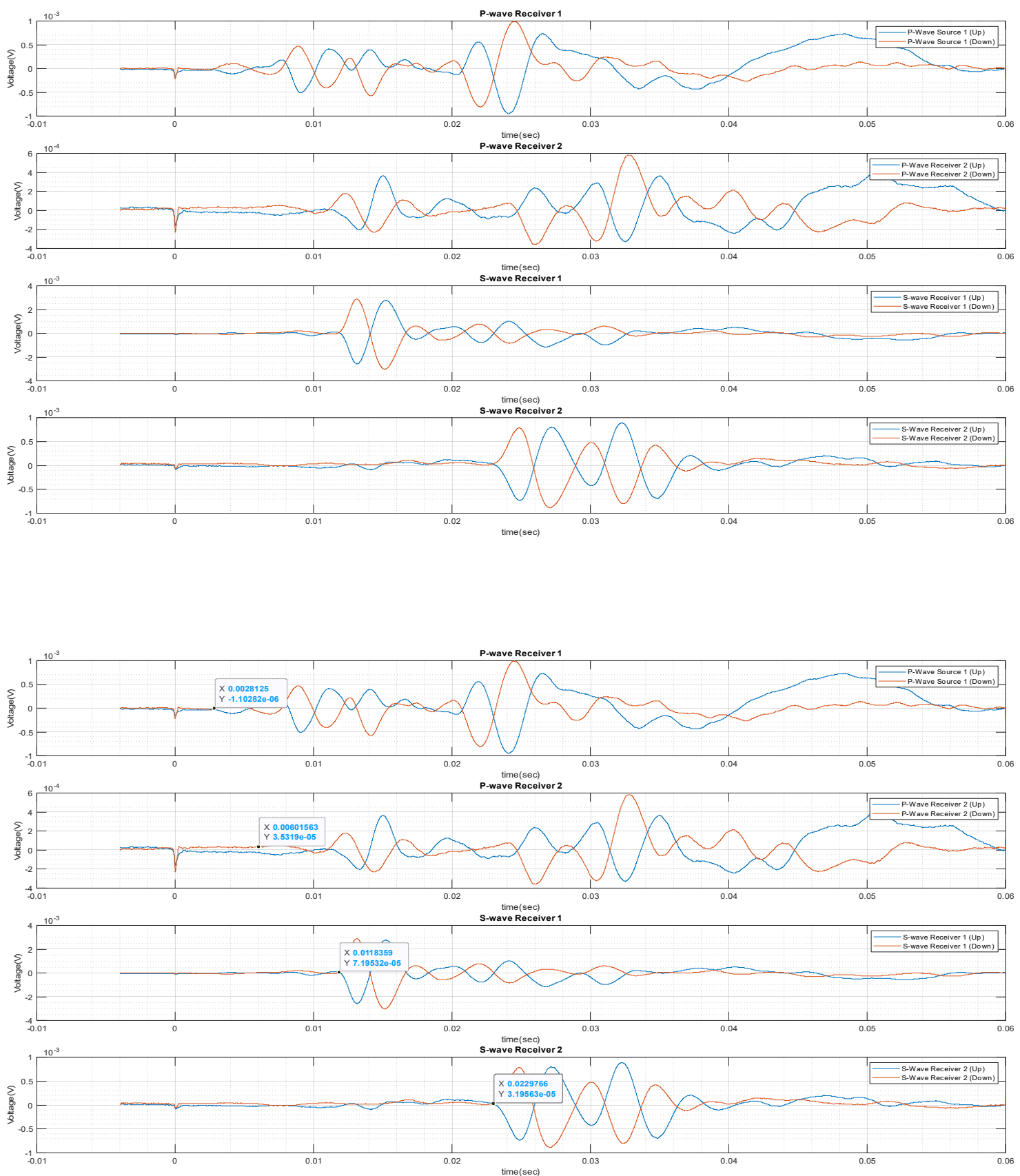

Data Set 22 (9.7.2019)

Run00163f

R2_1 

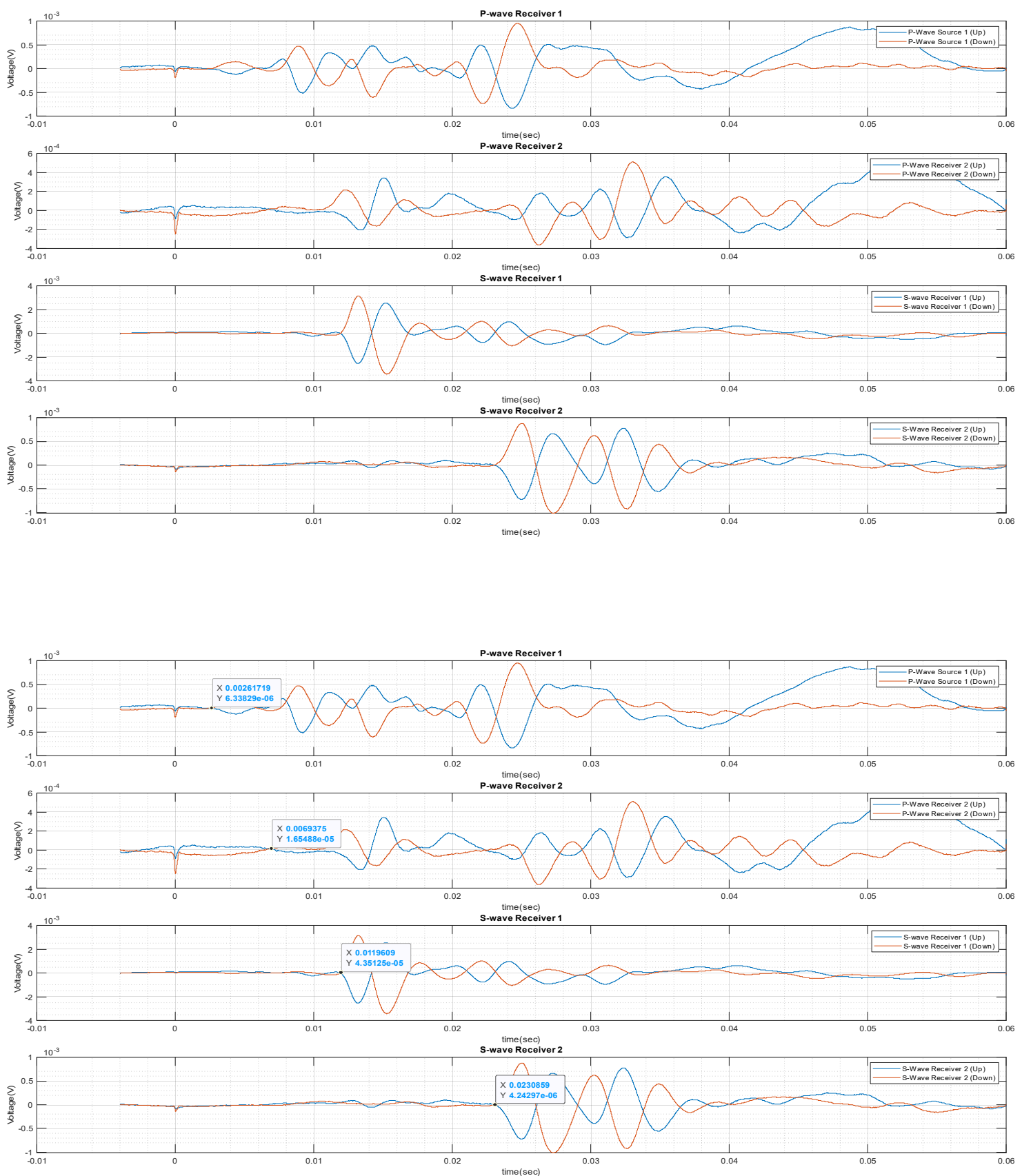

Data Set 22 (9.7.2019)

Run00164f

R2_2 

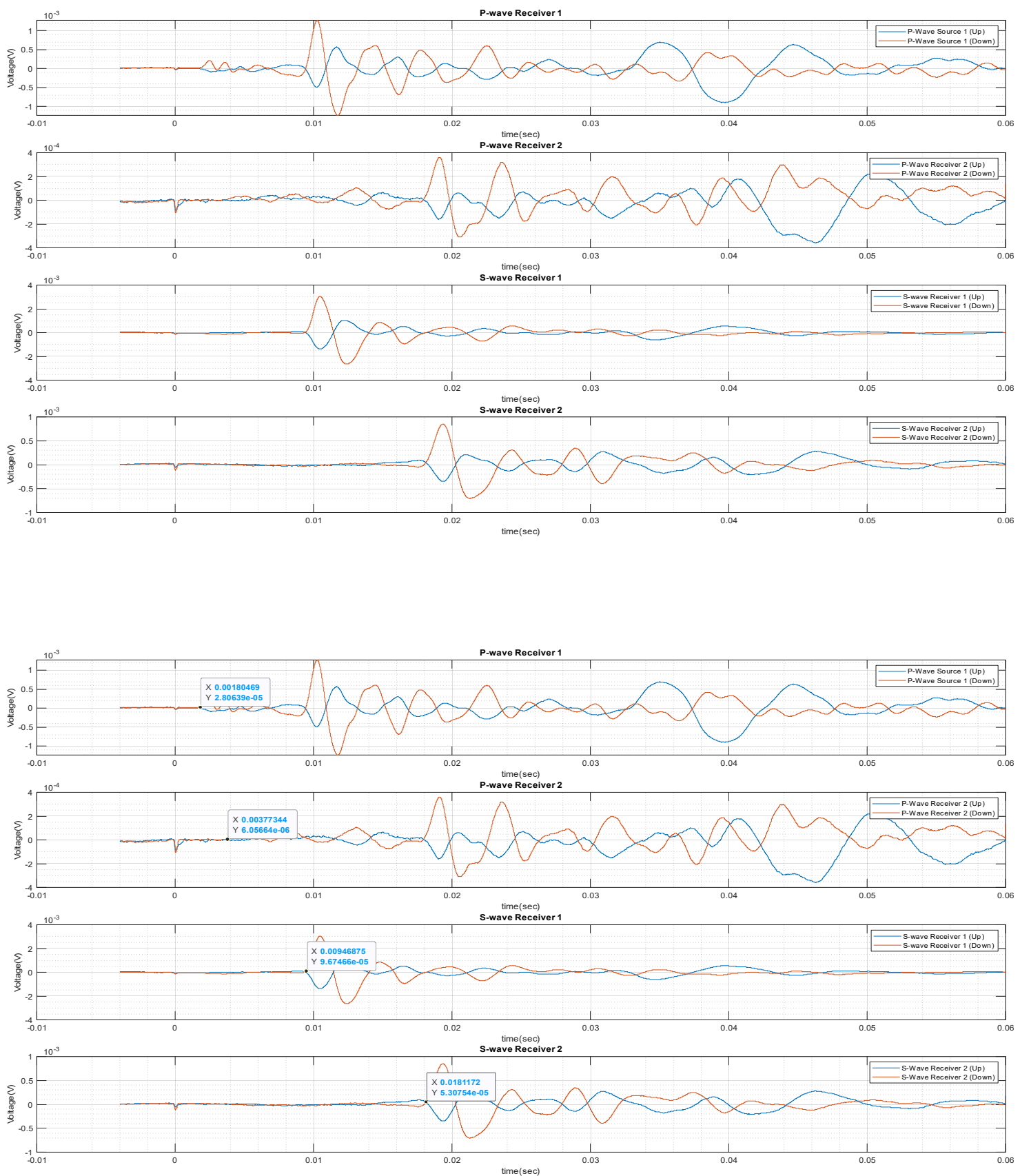

Data Set 22 (9.7.2019)

Run00165f

R3_1 

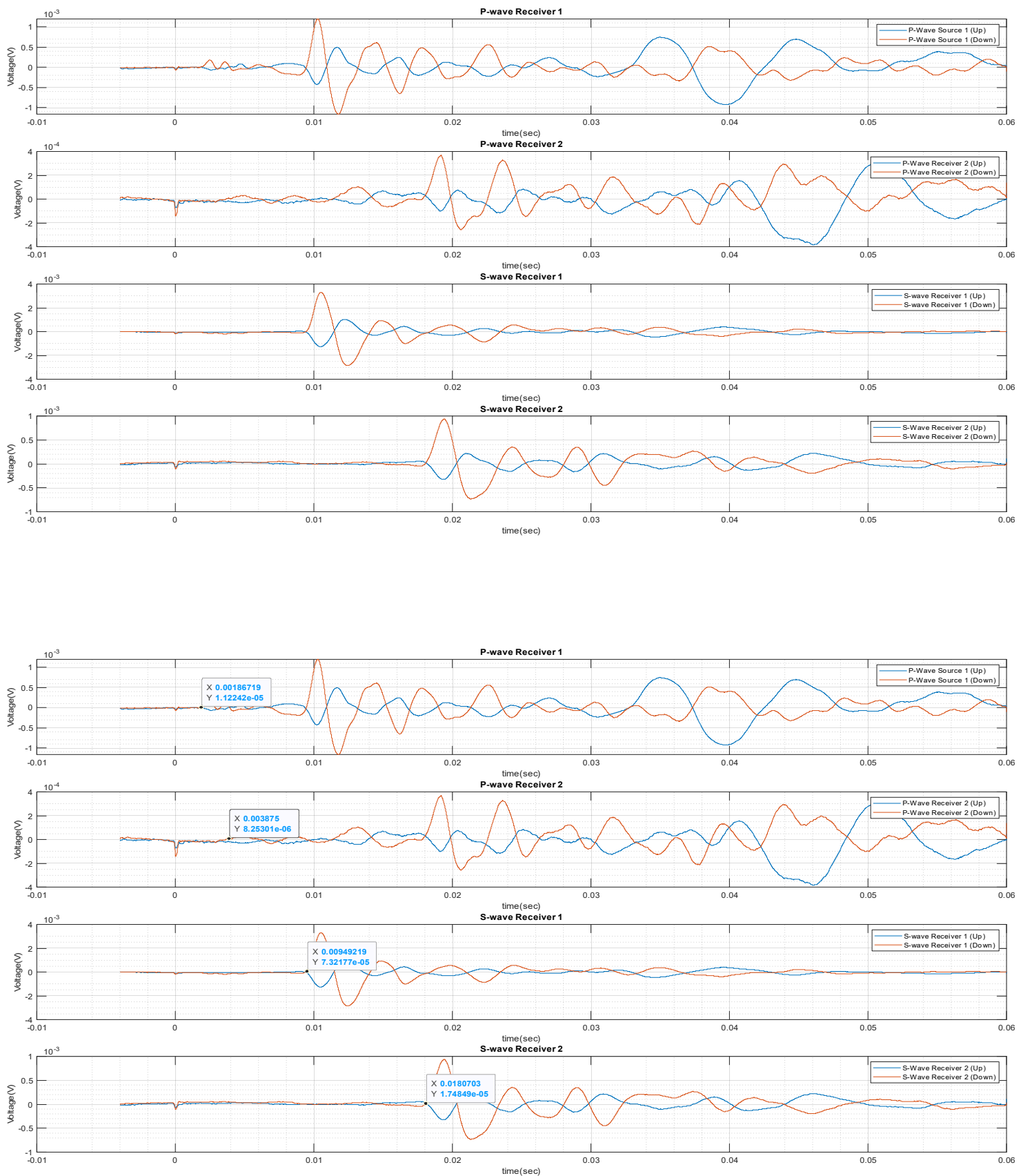

Data Set 22 (9.7.2019)

Run00166f

R3_2 

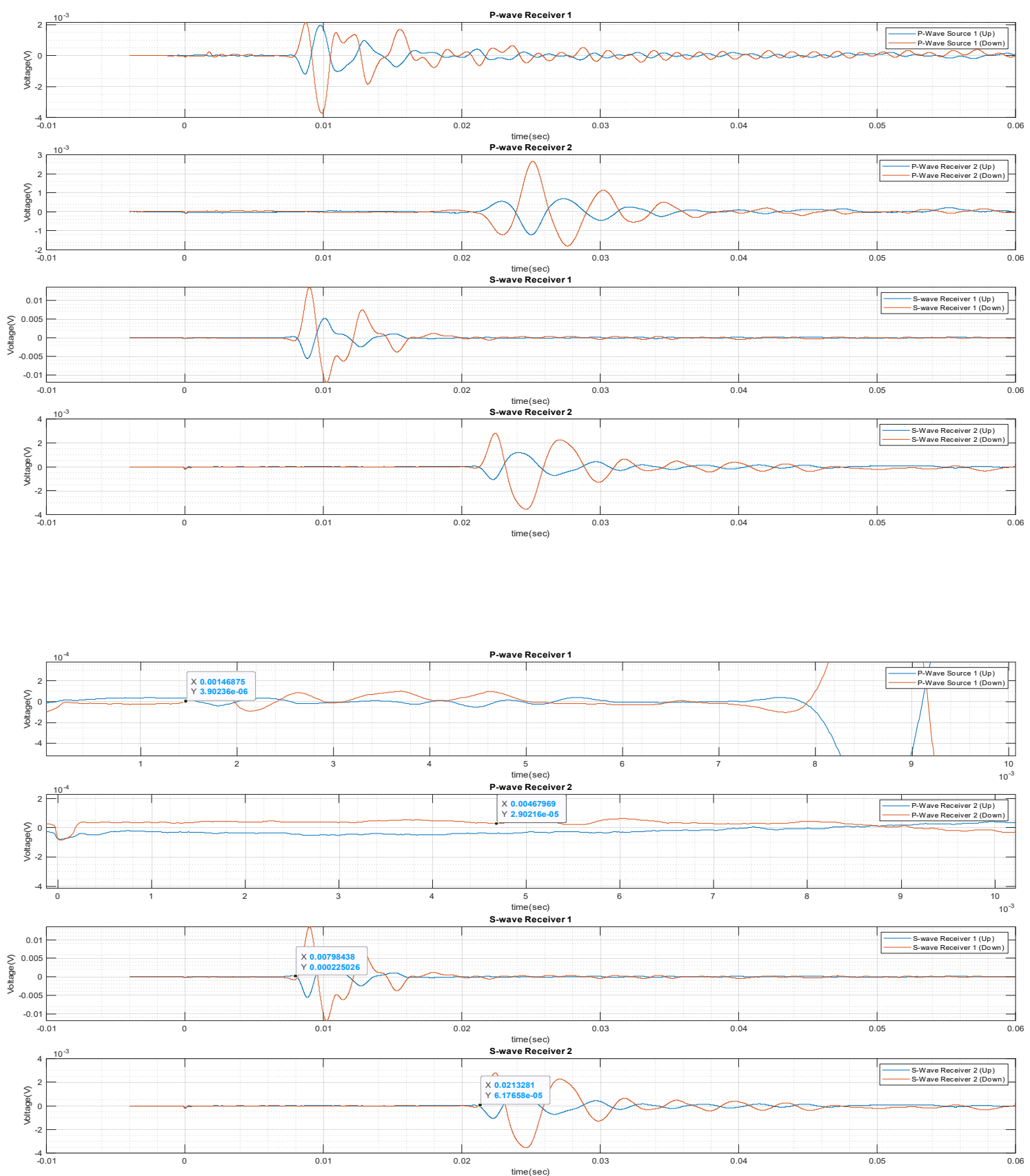

Data Set 22 (9.7.2019)

Run00167f

R4_1 

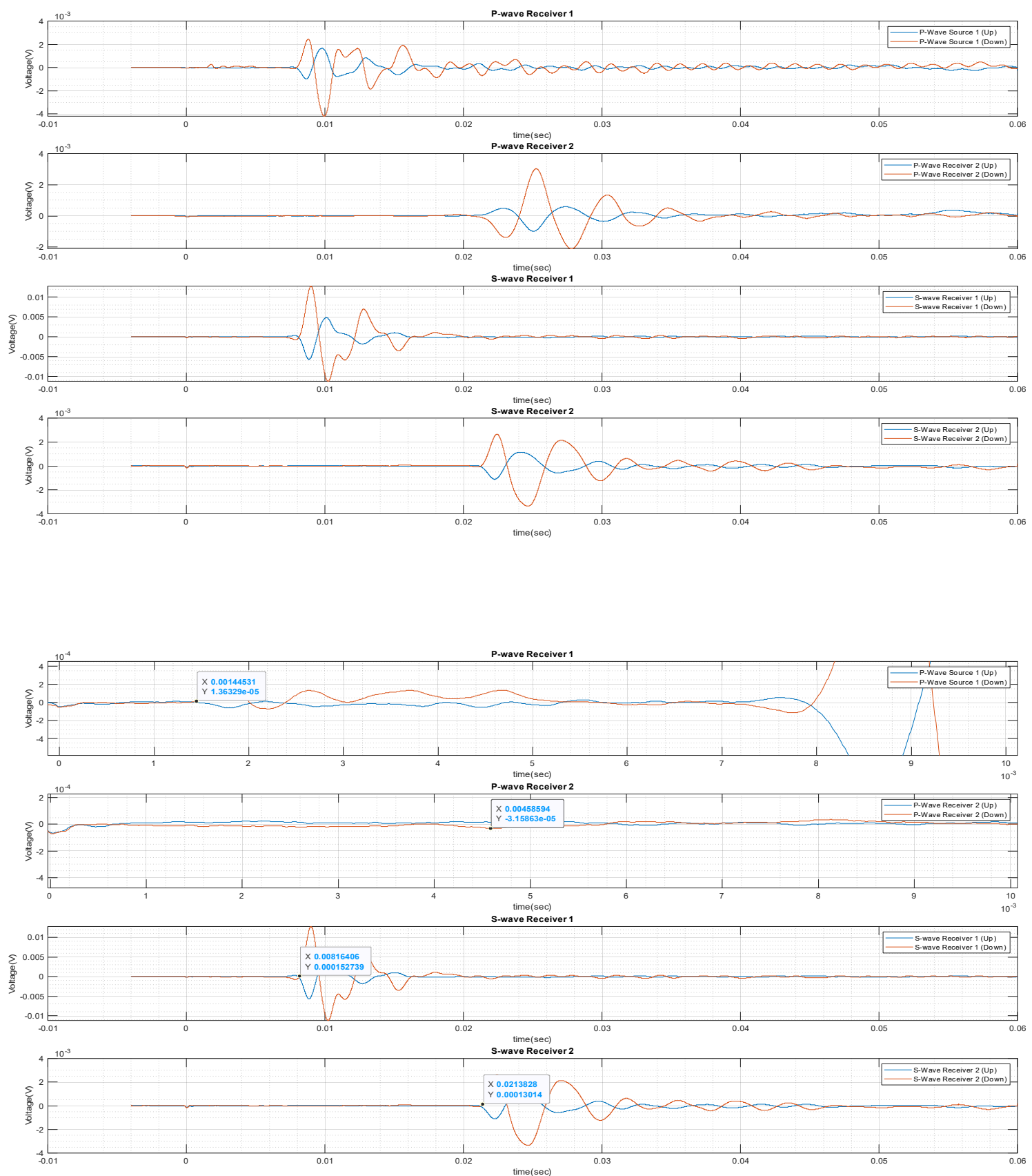

Data Set 22 (9.7.2019)

Run00168f

R4_2 

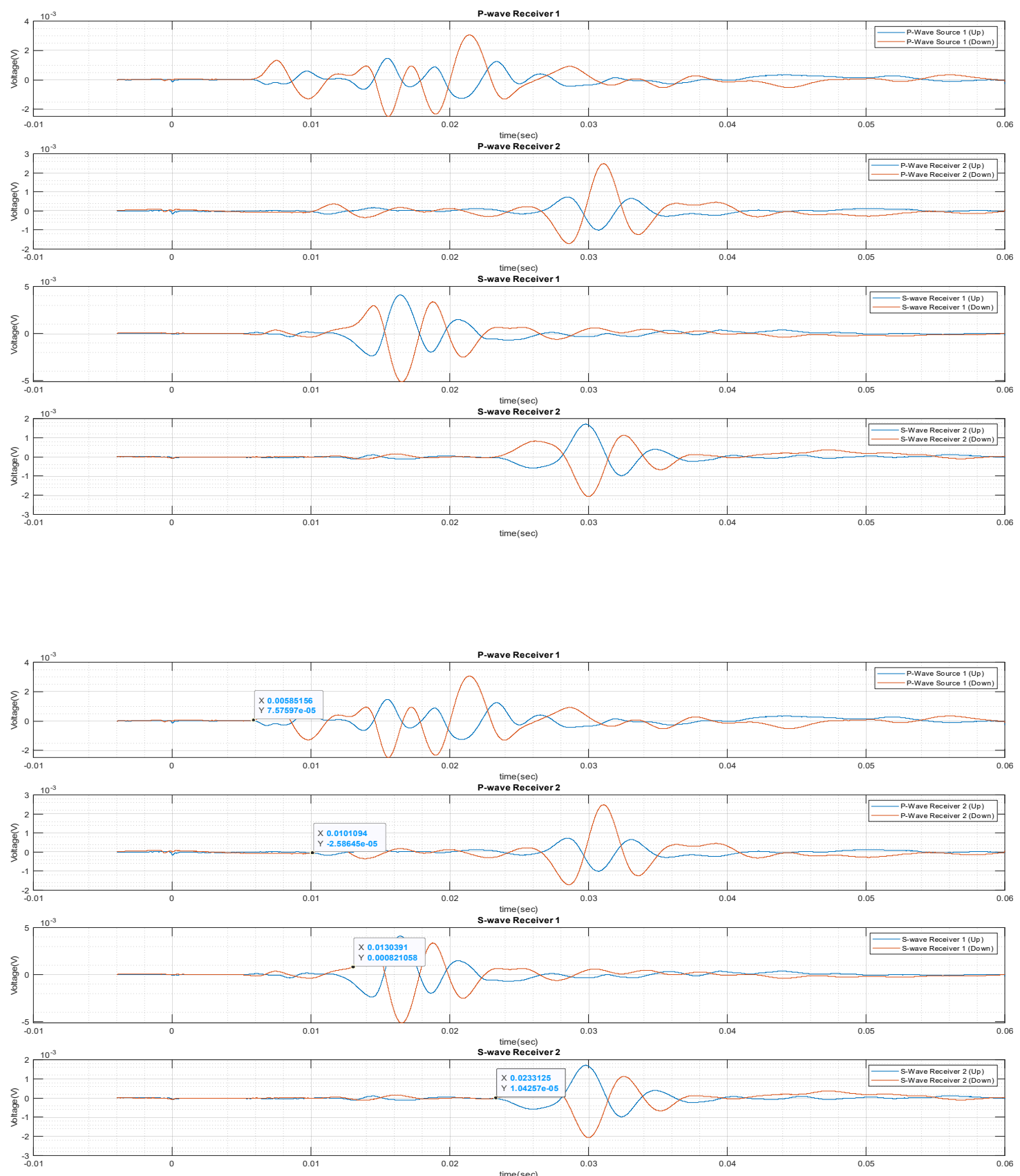

Data Set 23 (9.9.2019)

Run00169f

R1_1 

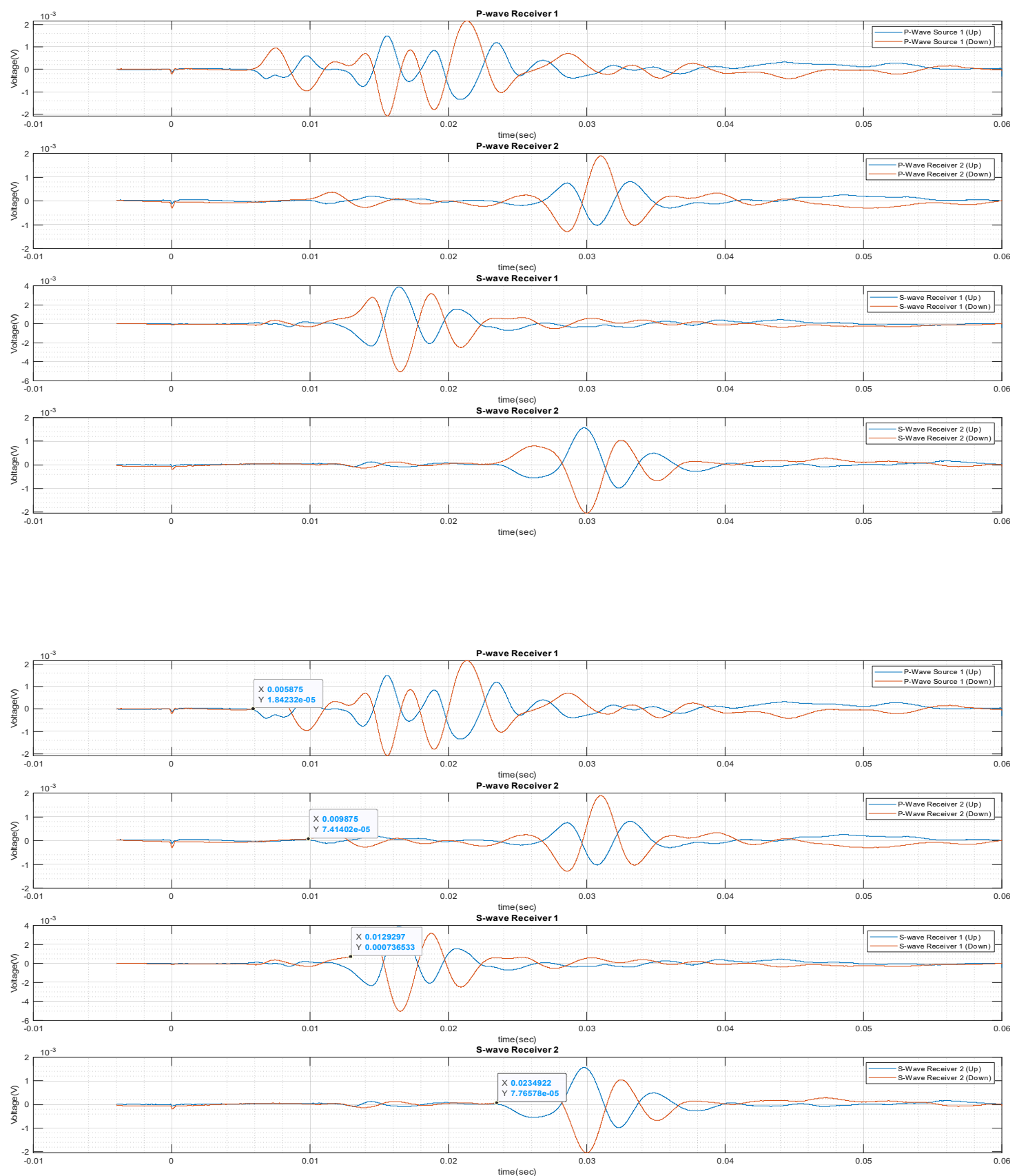

Data Set 23 (9.9.2019)

Run00170f

R1_2 

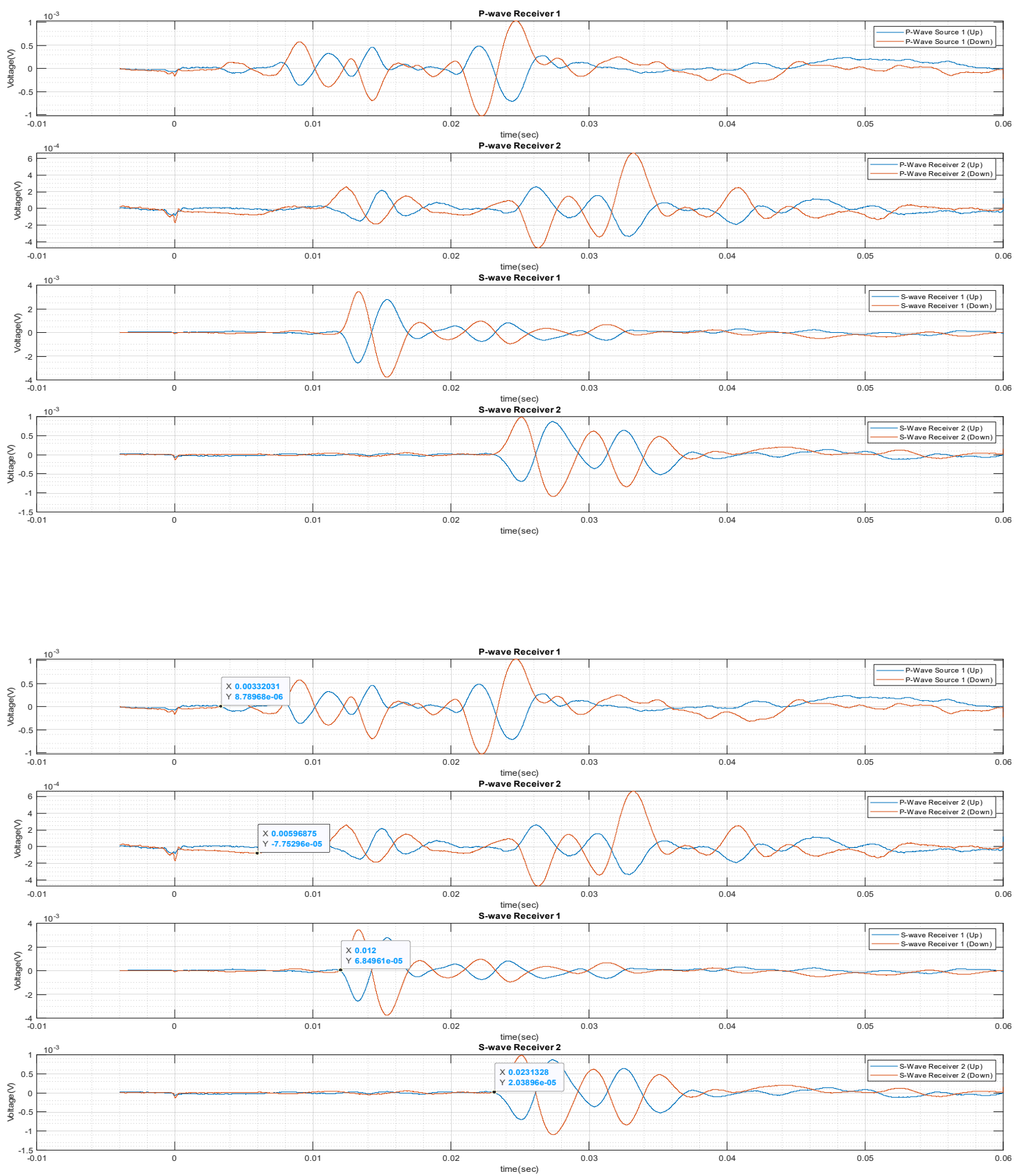

Data Set 23 (9.9.2019)

Run00171f

R2_1 

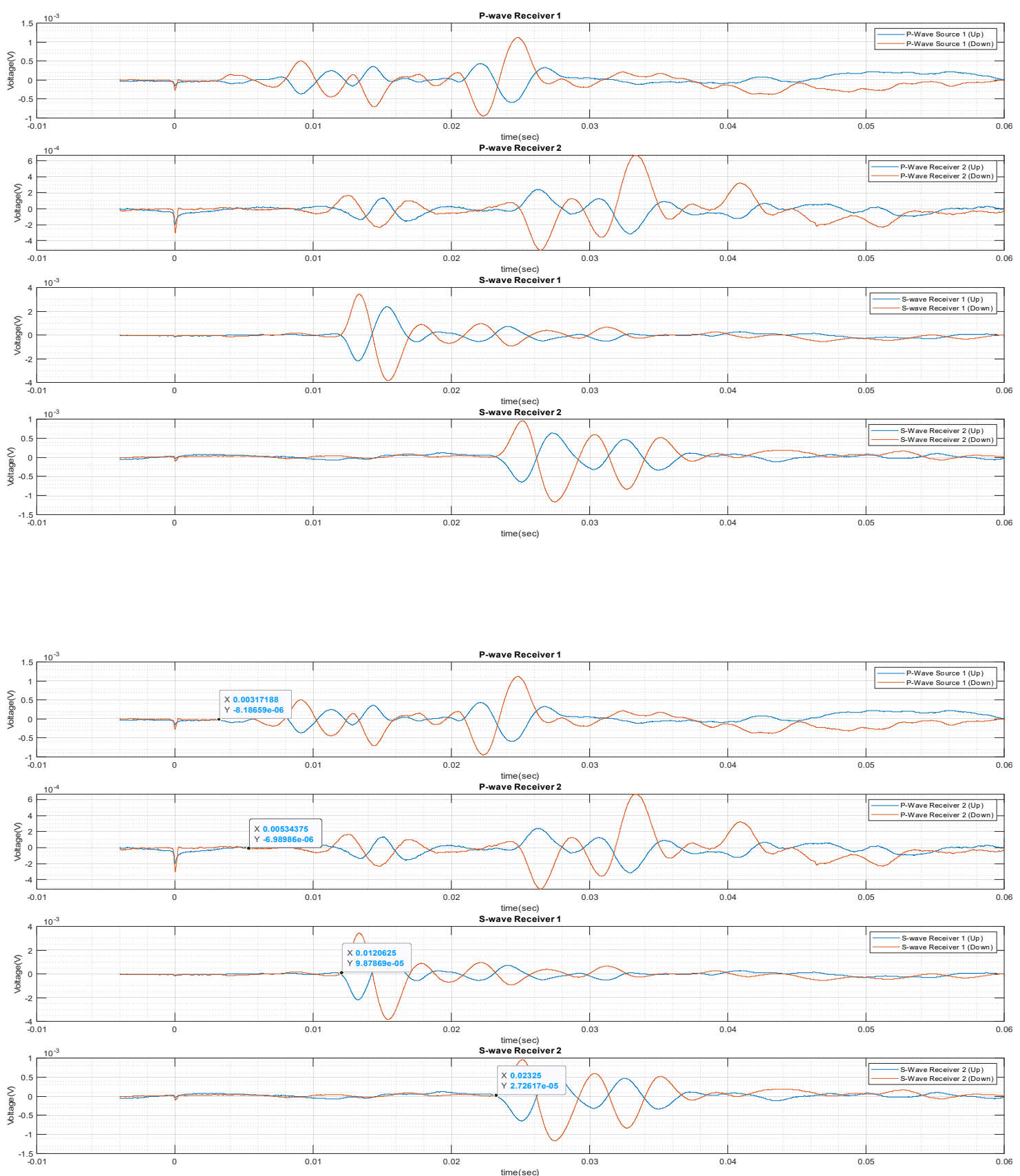

Data Set 23 (9.9.2019)

Run00172f

R2_2 

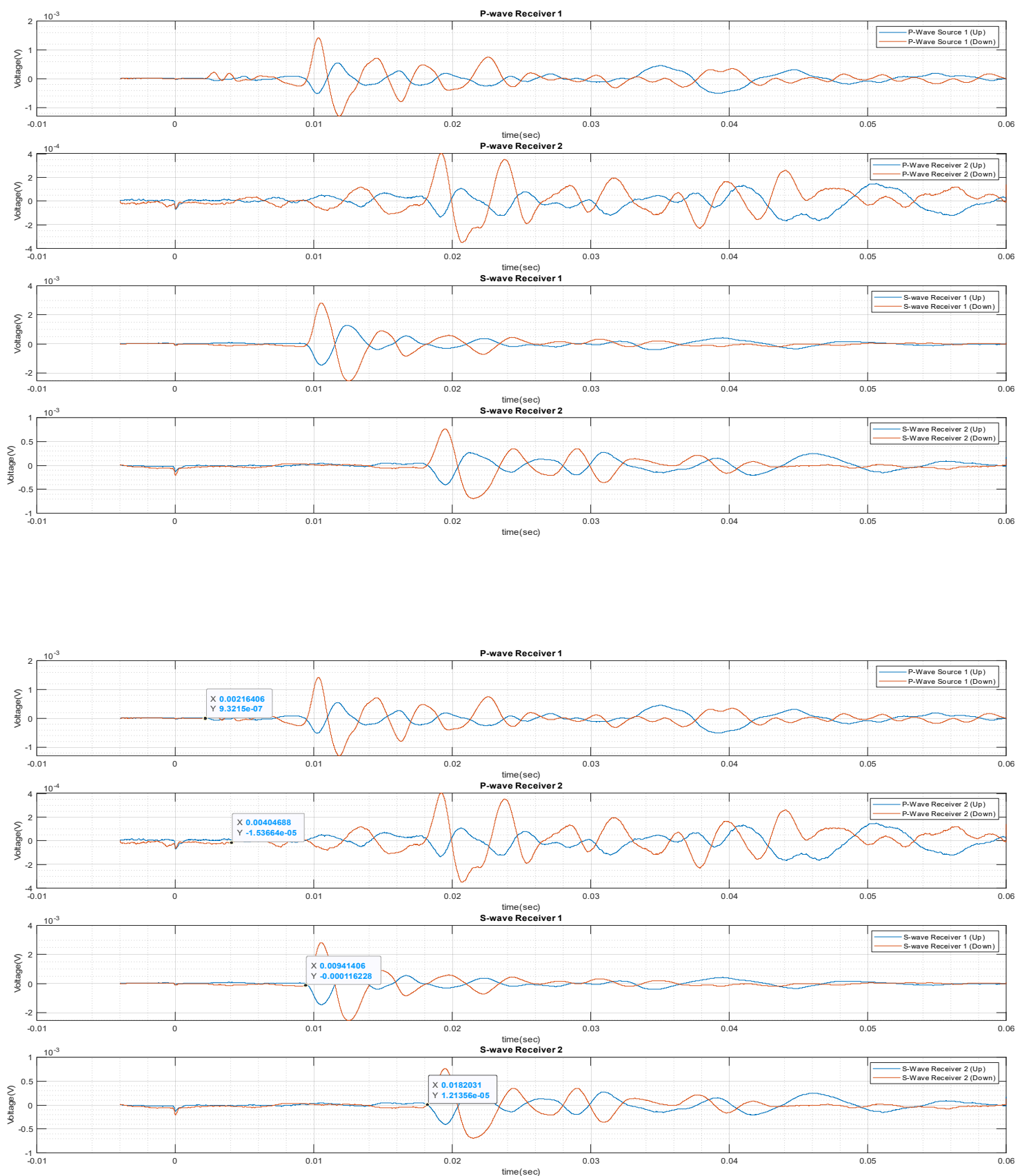

Data Set 23 (9.9.2019)

Run00173f

R3_1 

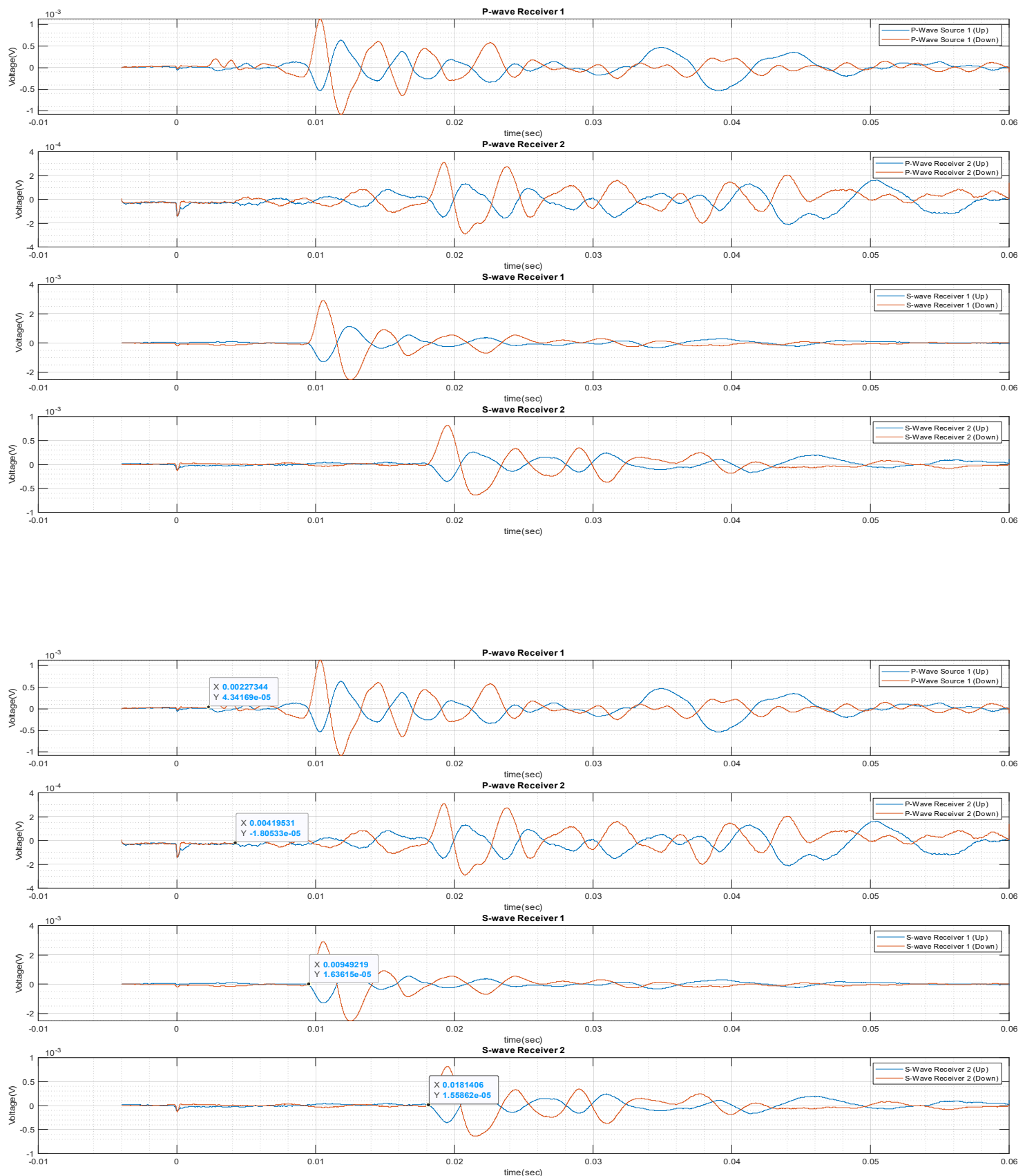

Data Set 23 (9.9.2019)

Run00174f

R3_2 

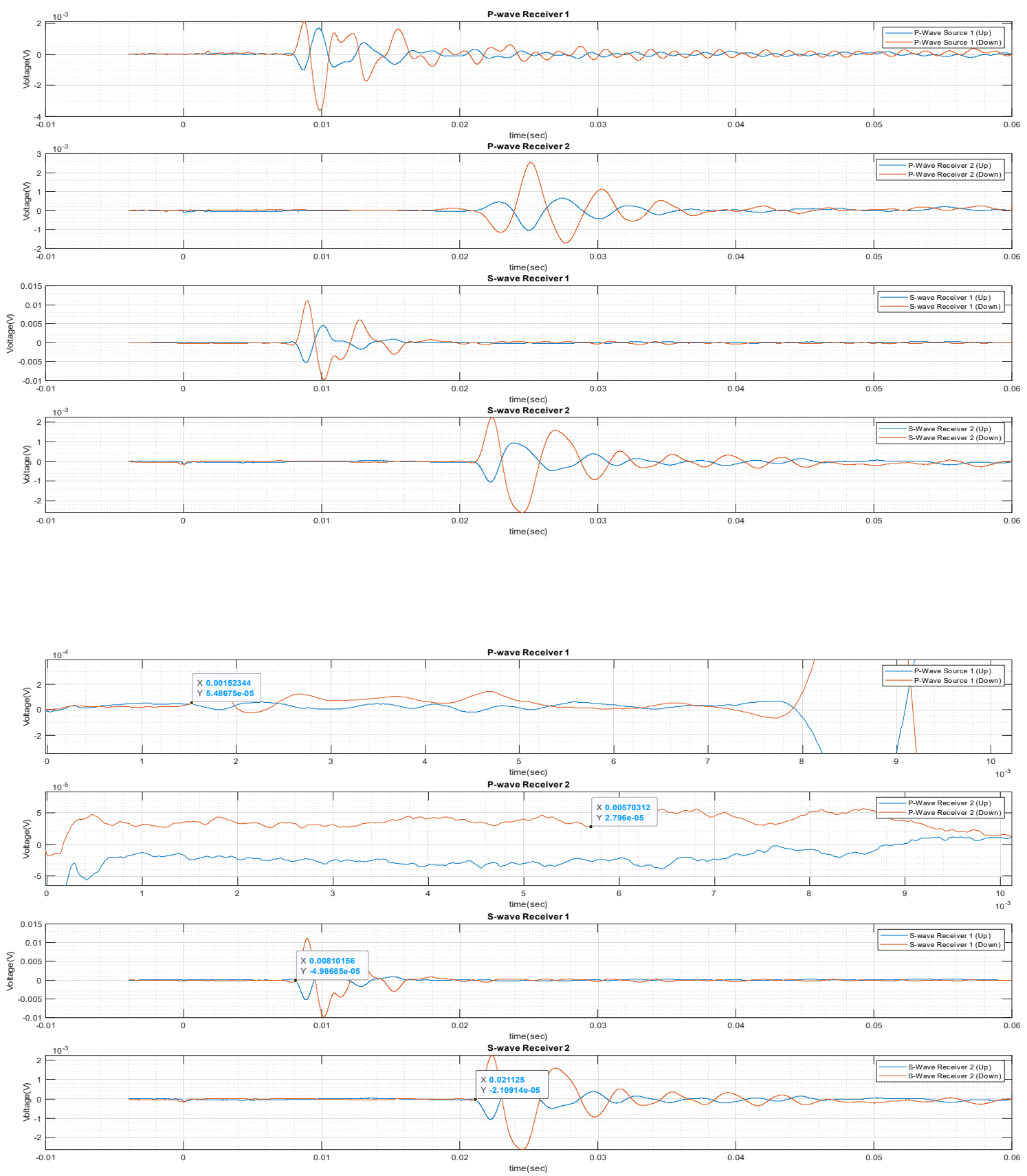

Data Set 23 (9.9.2019)

Run00175f

R4_1 

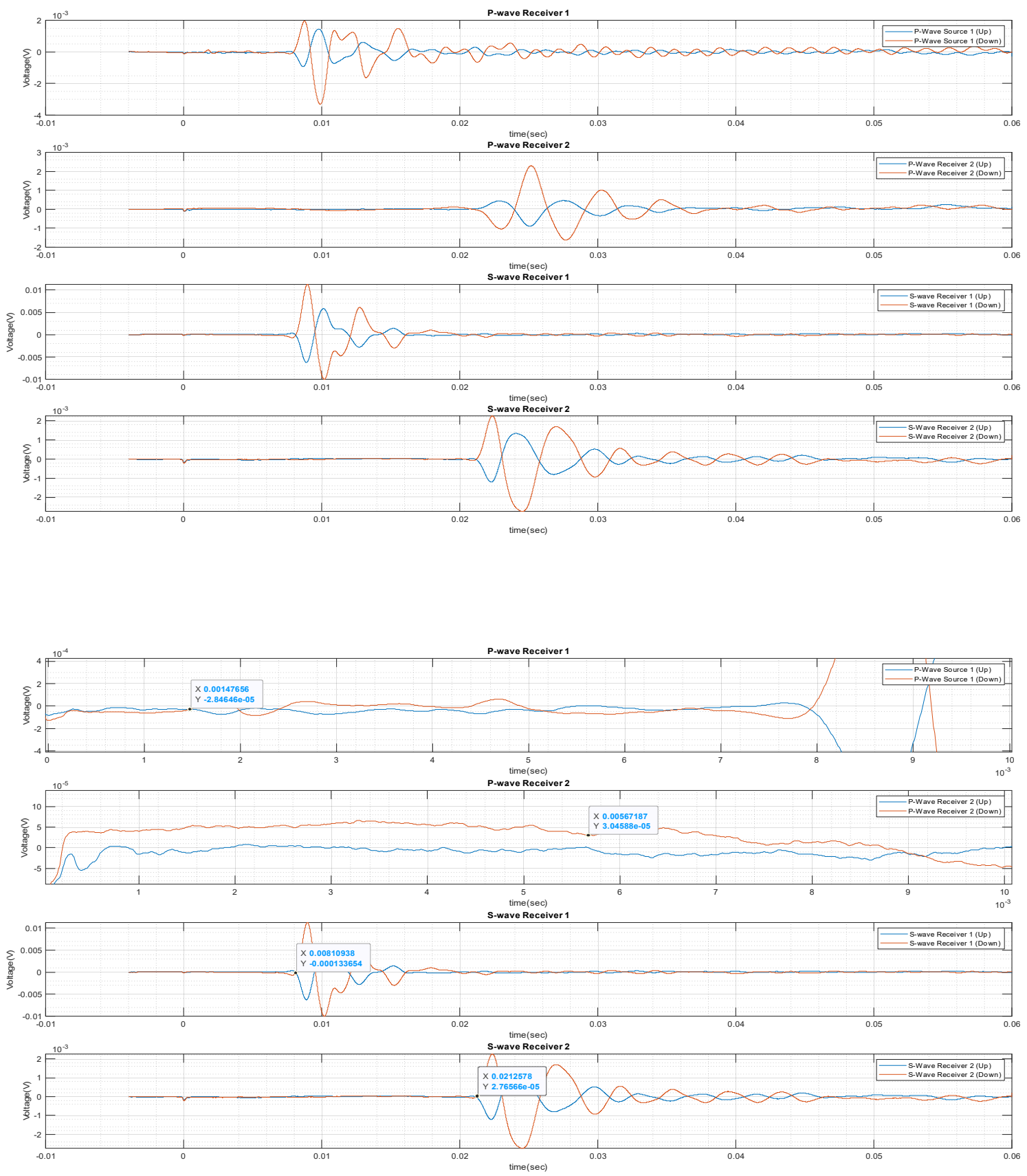

Data Set 23 (9.9.2019)

Run00176f

R4_2 

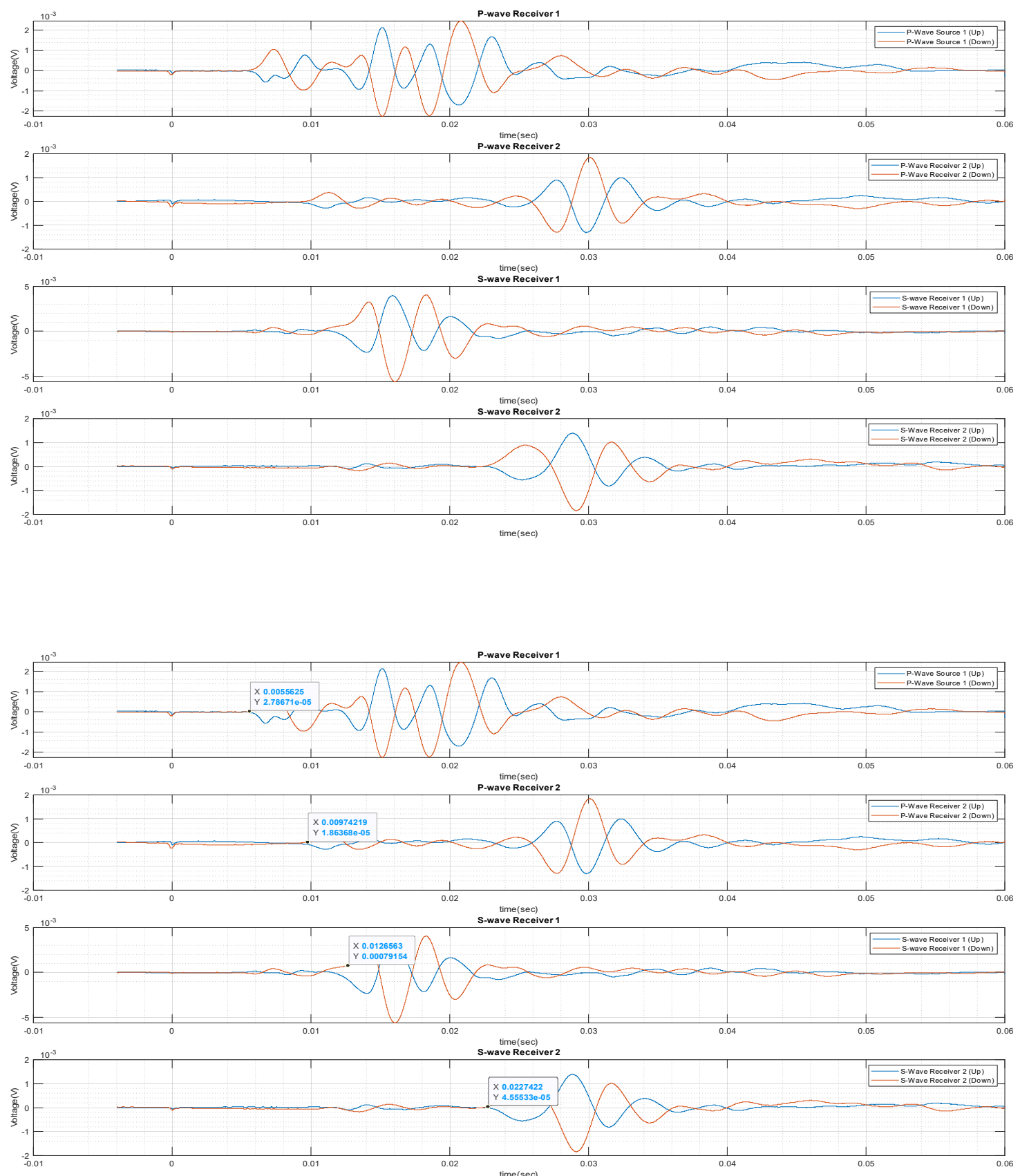

Data Set $24(9.10 .2019)$

Run00177f

R1_1 

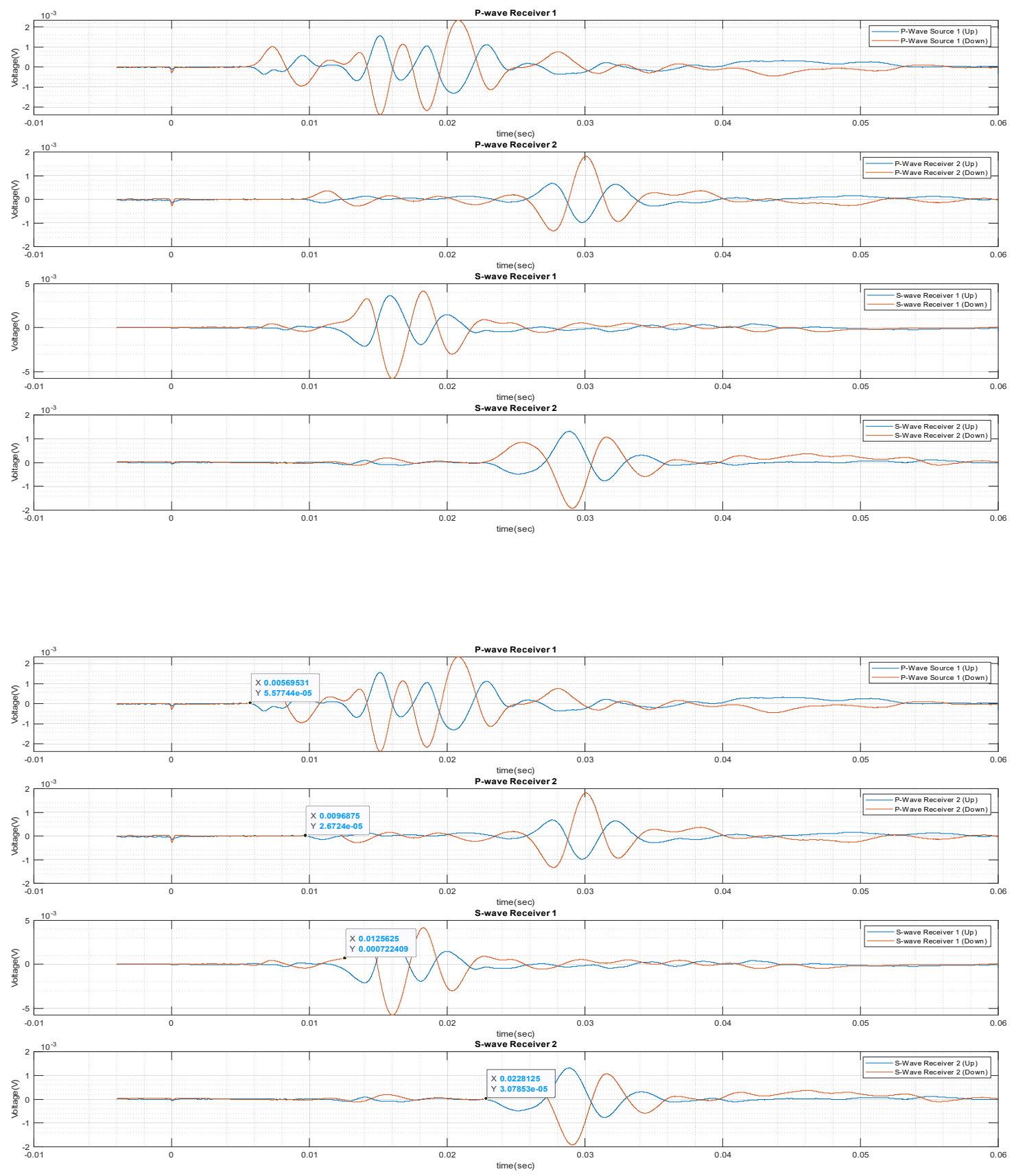

Data Set $24(9.10 .2019)$

Run00178f

R1_2 

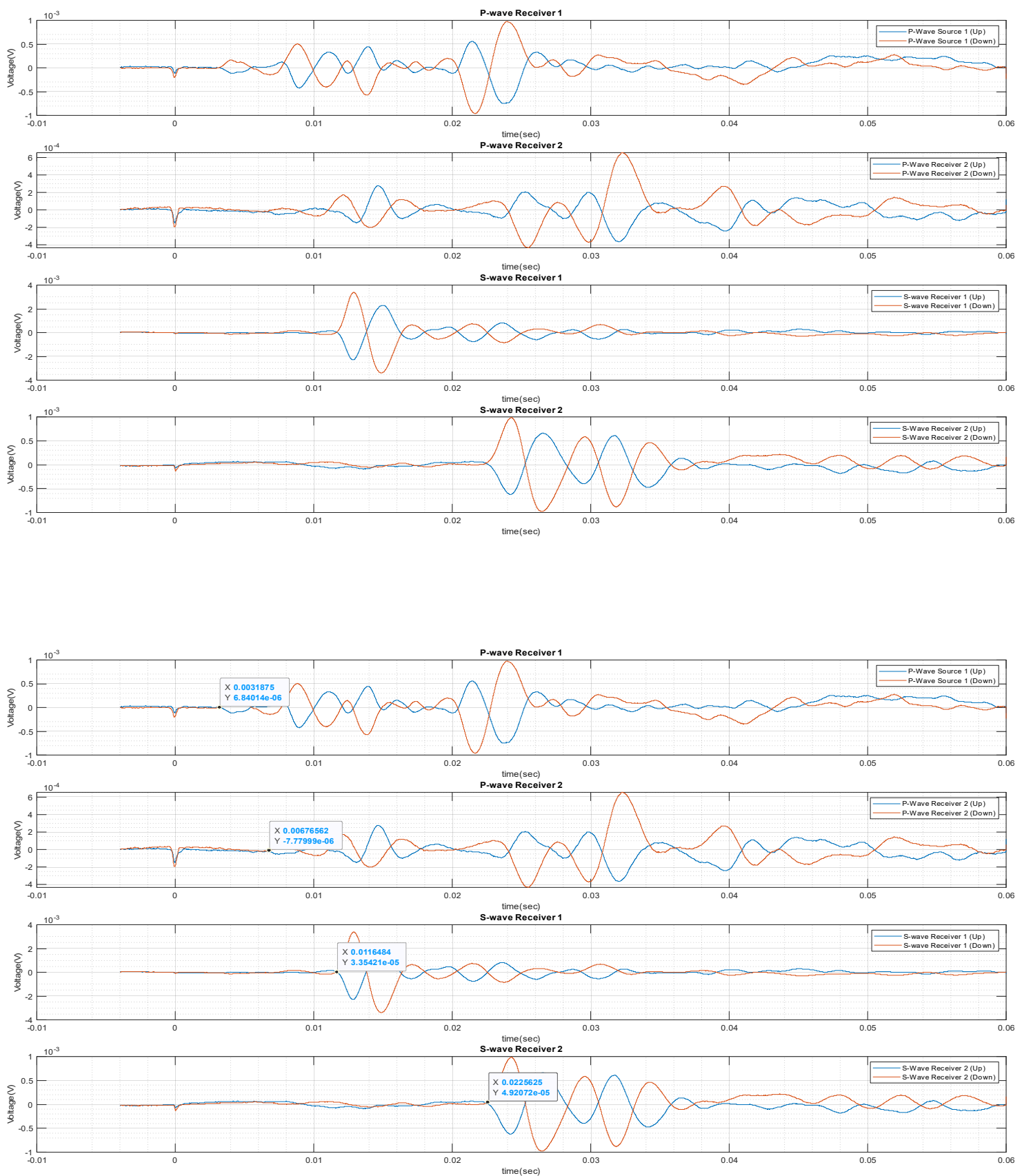

Data Set $24(9.10 .2019)$

Run00179f

R2_1 

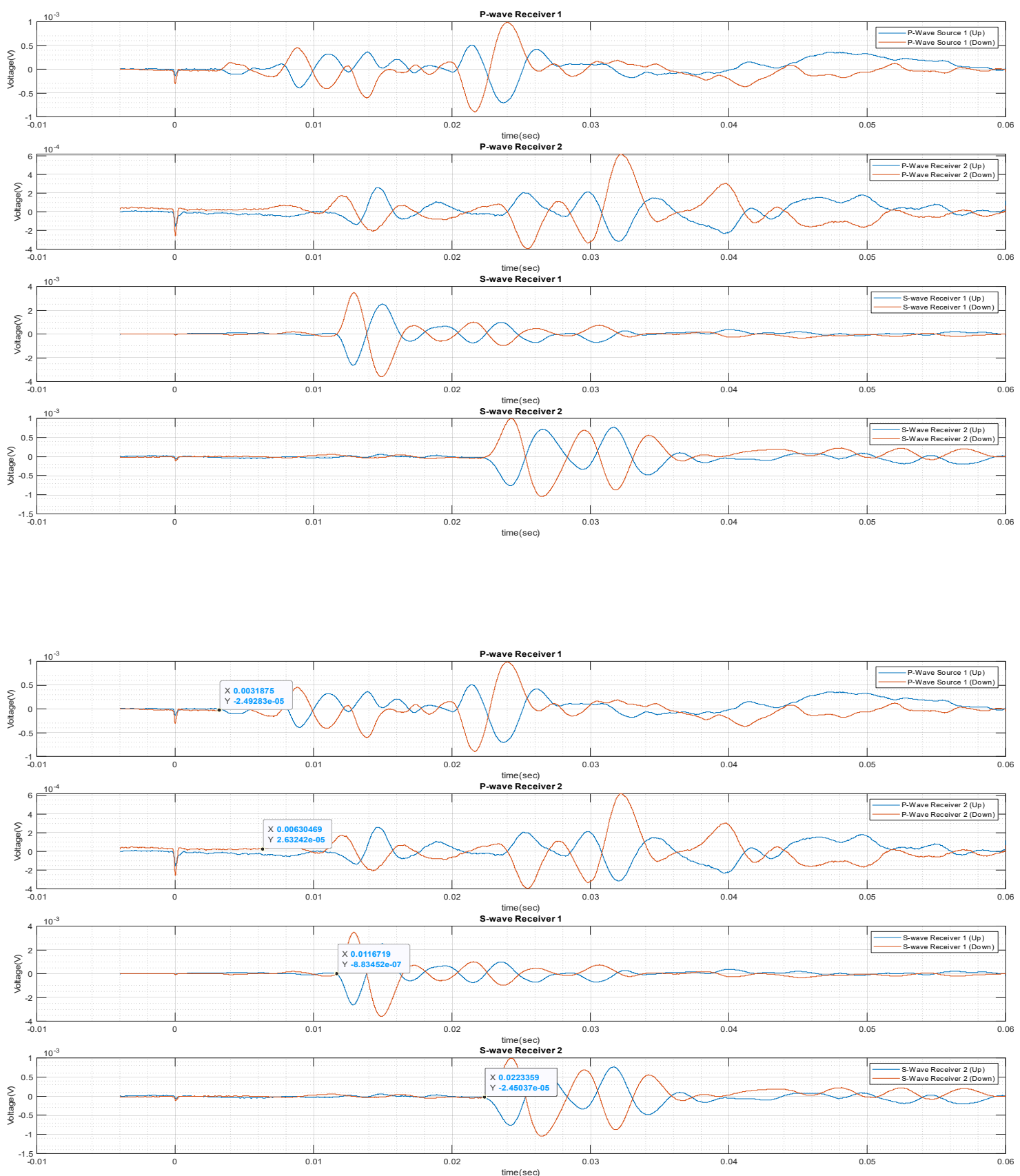

Data Set $24(9.10 .2019)$

Run00180f

R2_2 

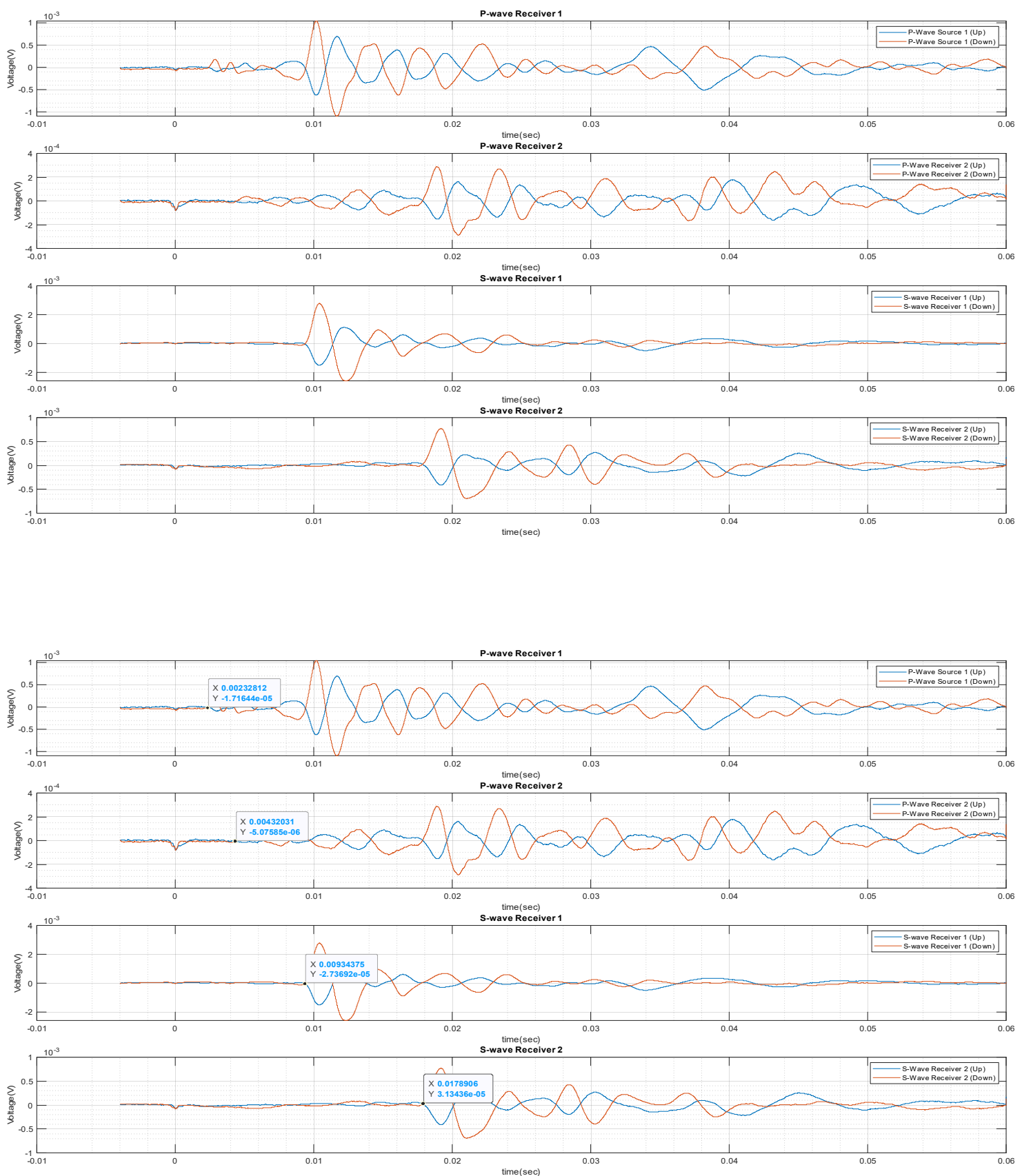

Data Set $24(9.10 .2019)$

Run00181f

R3_1 

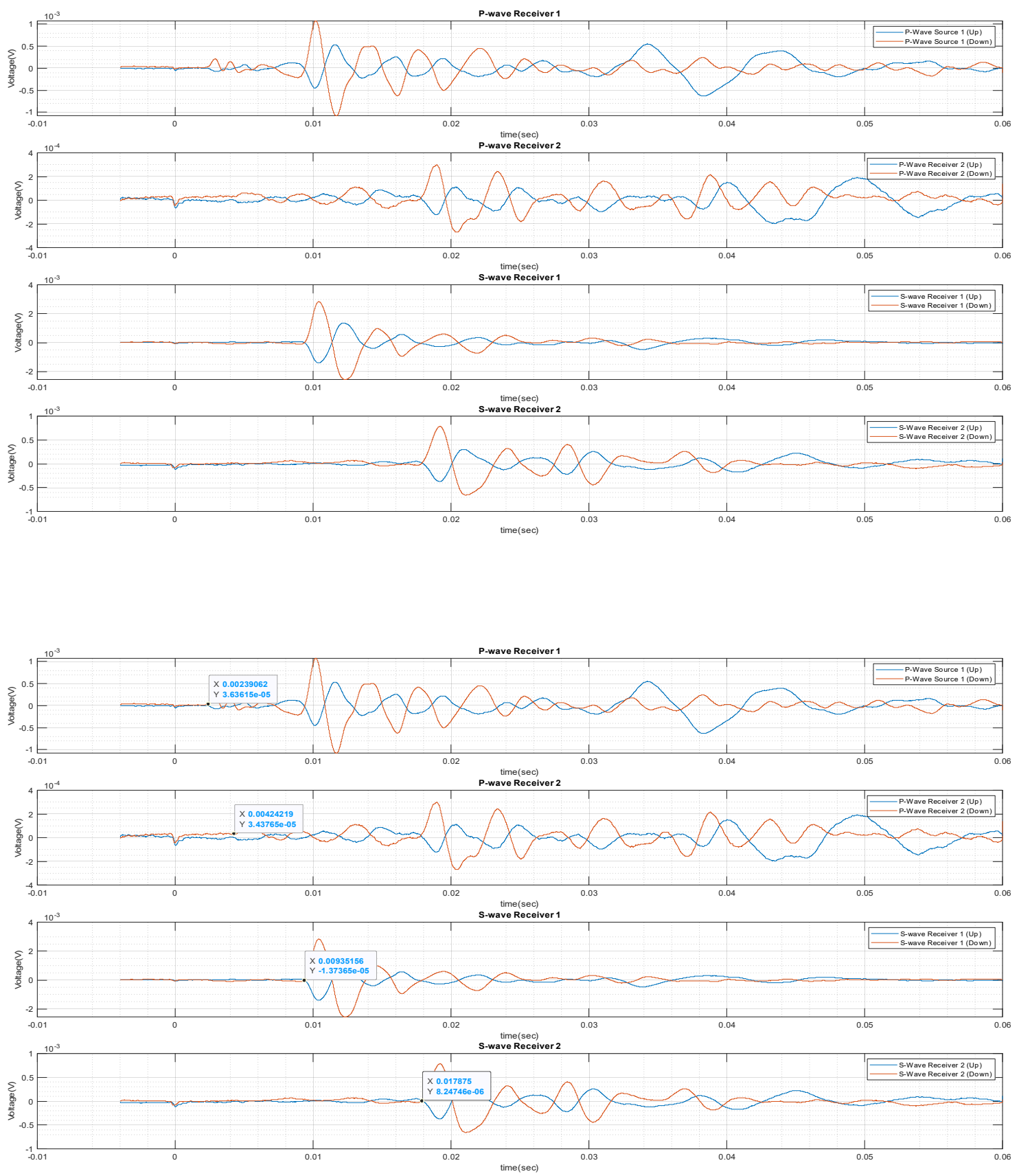

Data Set $24(9.10 .2019)$

\section{Run00182f}

\section{R3_2}



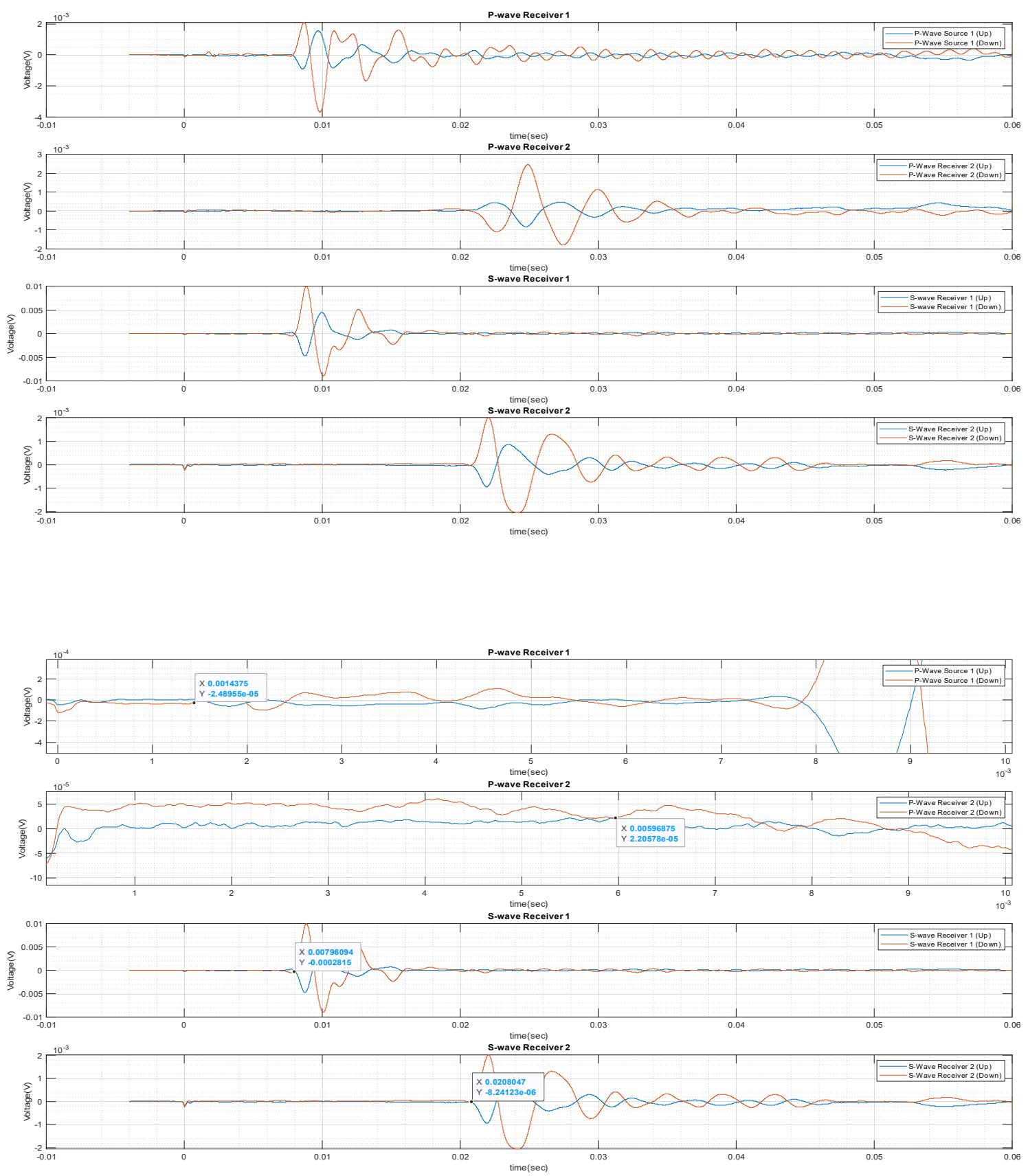

Data Set $24(9.10 .2019)$

Run00183f

R4_1 

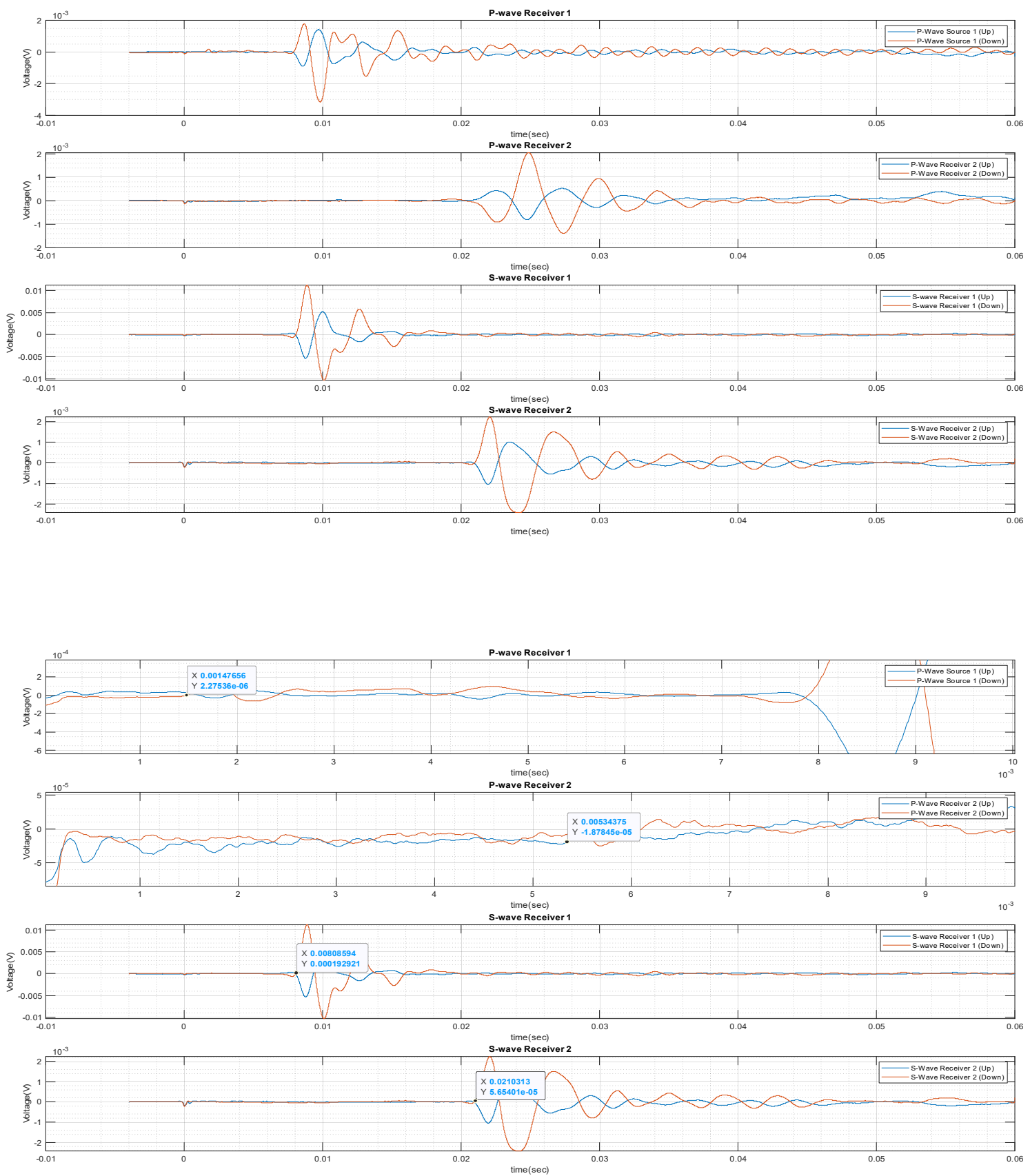

Data Set $24(9.10 .2019)$

Run00184f

R4_2 
Appendix Q: After treatment: Crosshole geophone voltage time series for $V_{s}$ and $V_{p}$ arrival selection.
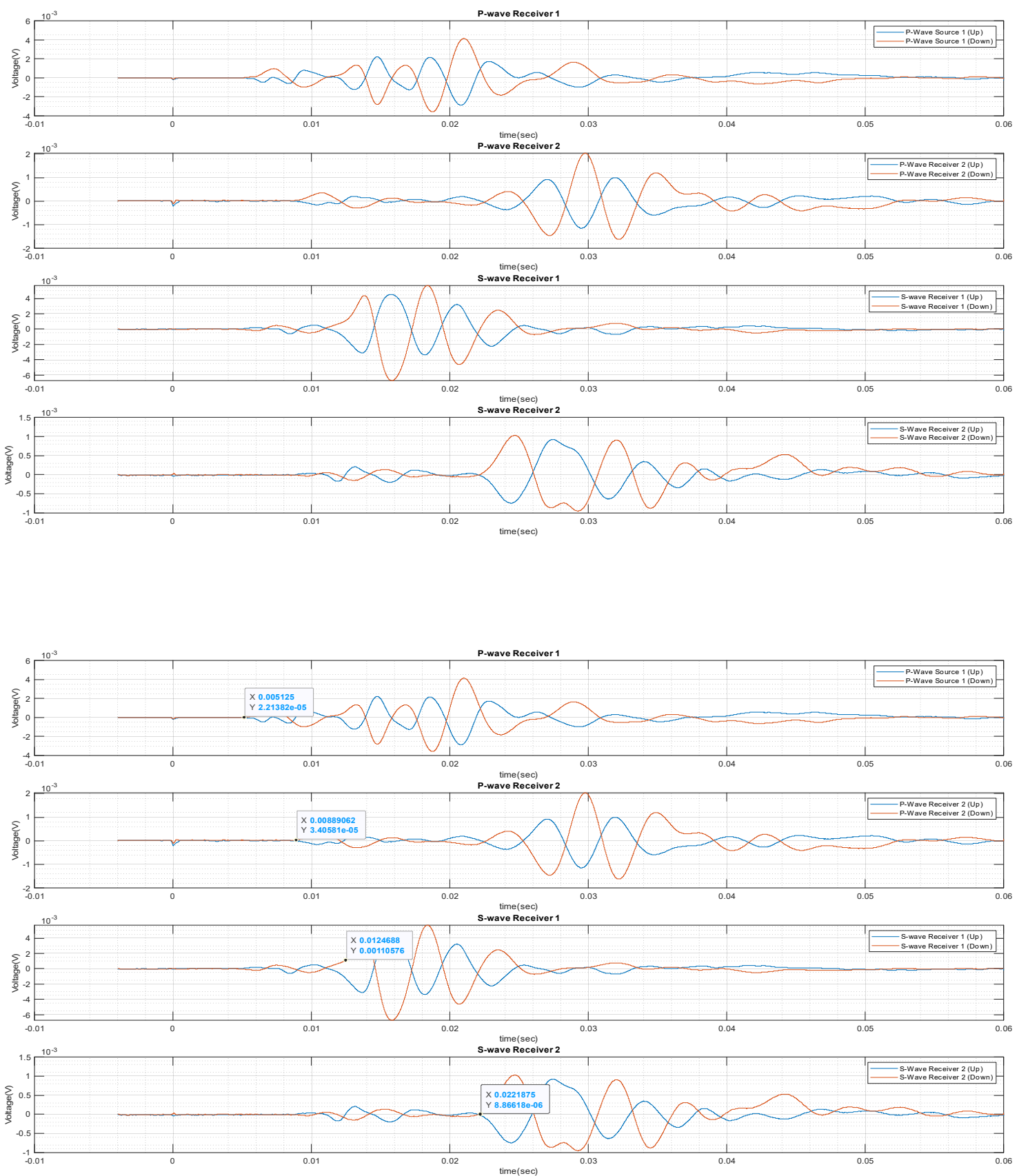

Data Set $25(9.16 .2019)$

Run00185f

R1_1 

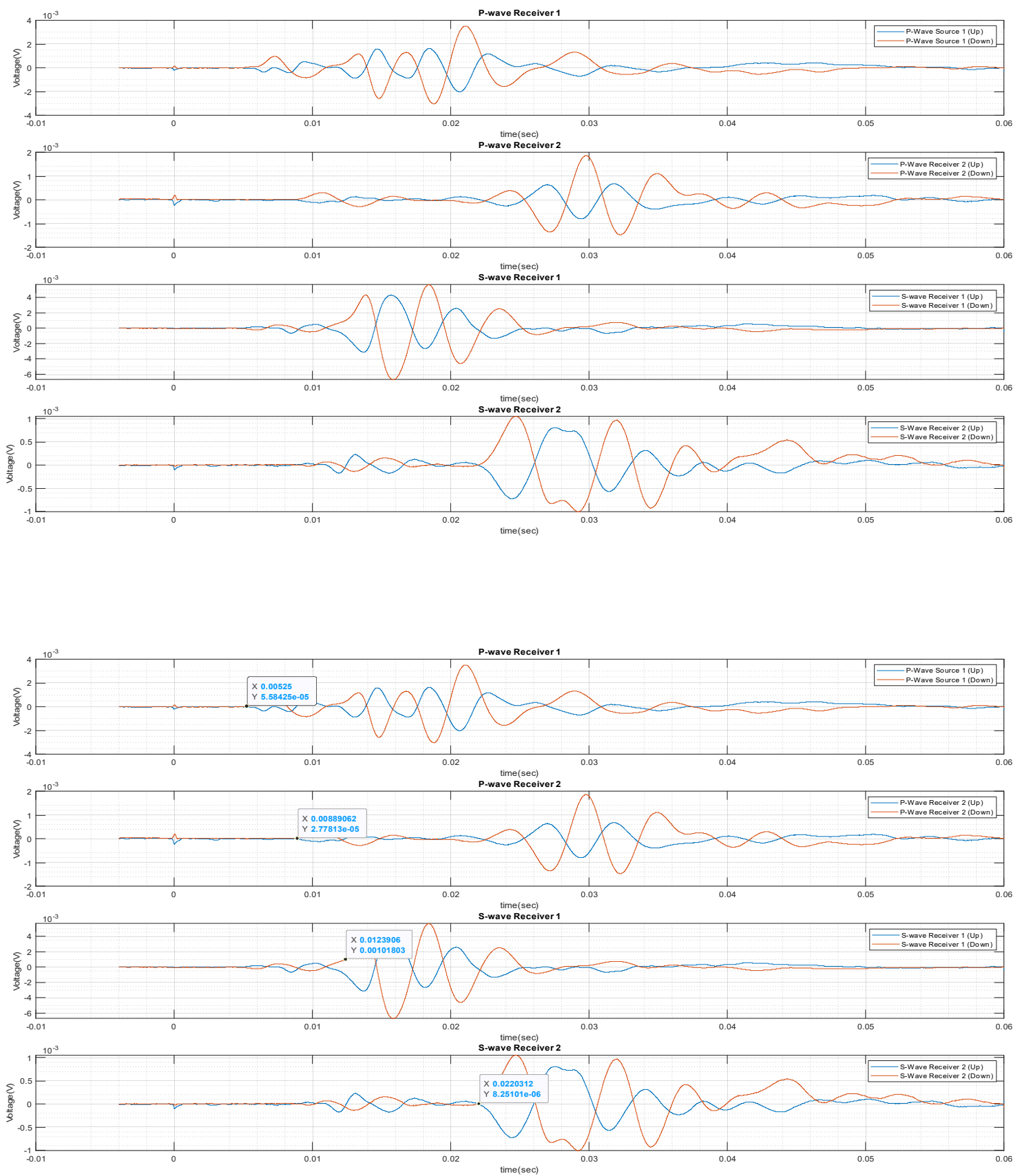

Data Set $25(9.16 .2019)$

Run00186f

R1_2 

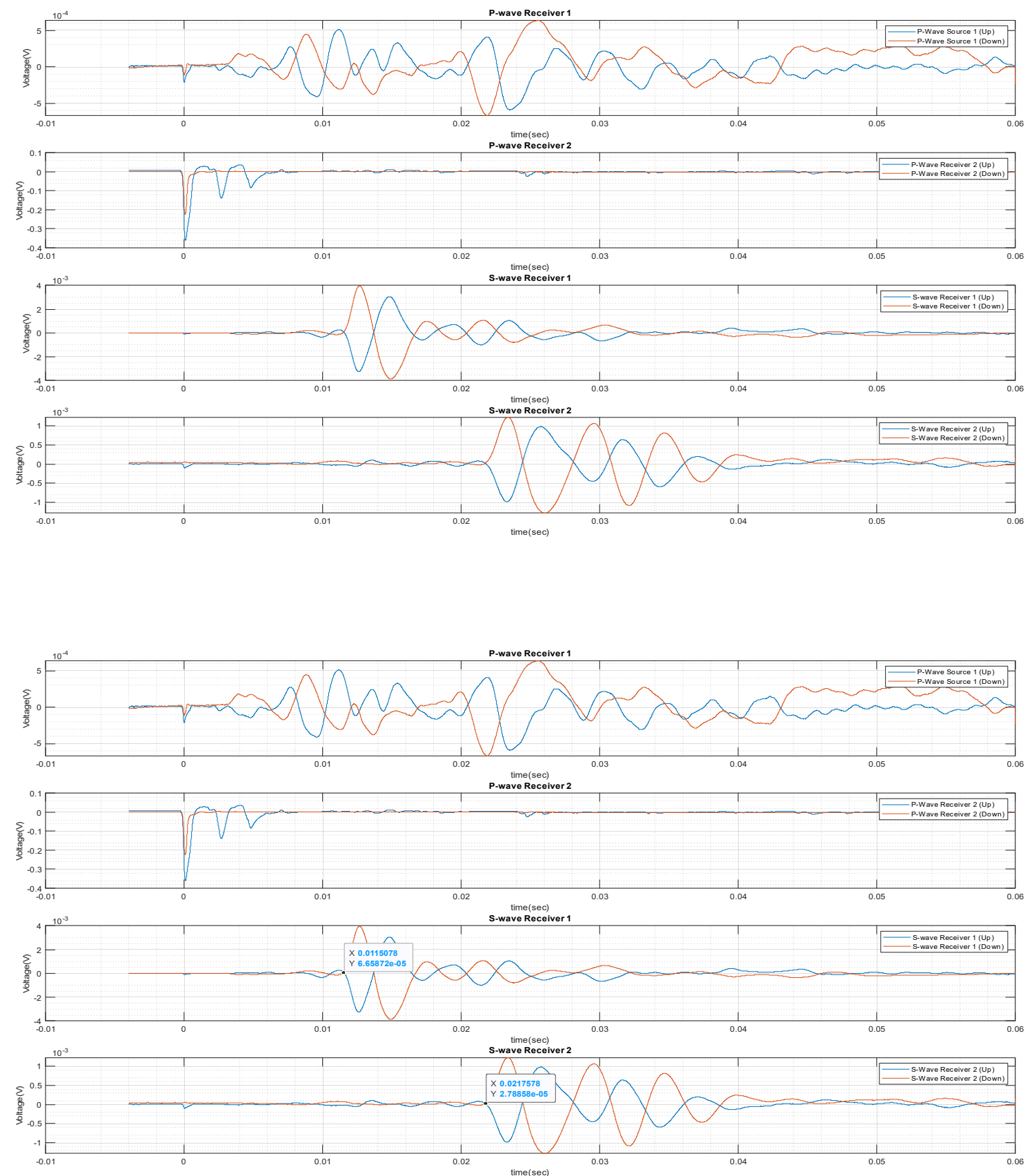

Data Set $25(9.16 .2019)$

Run00187f

R2_1 

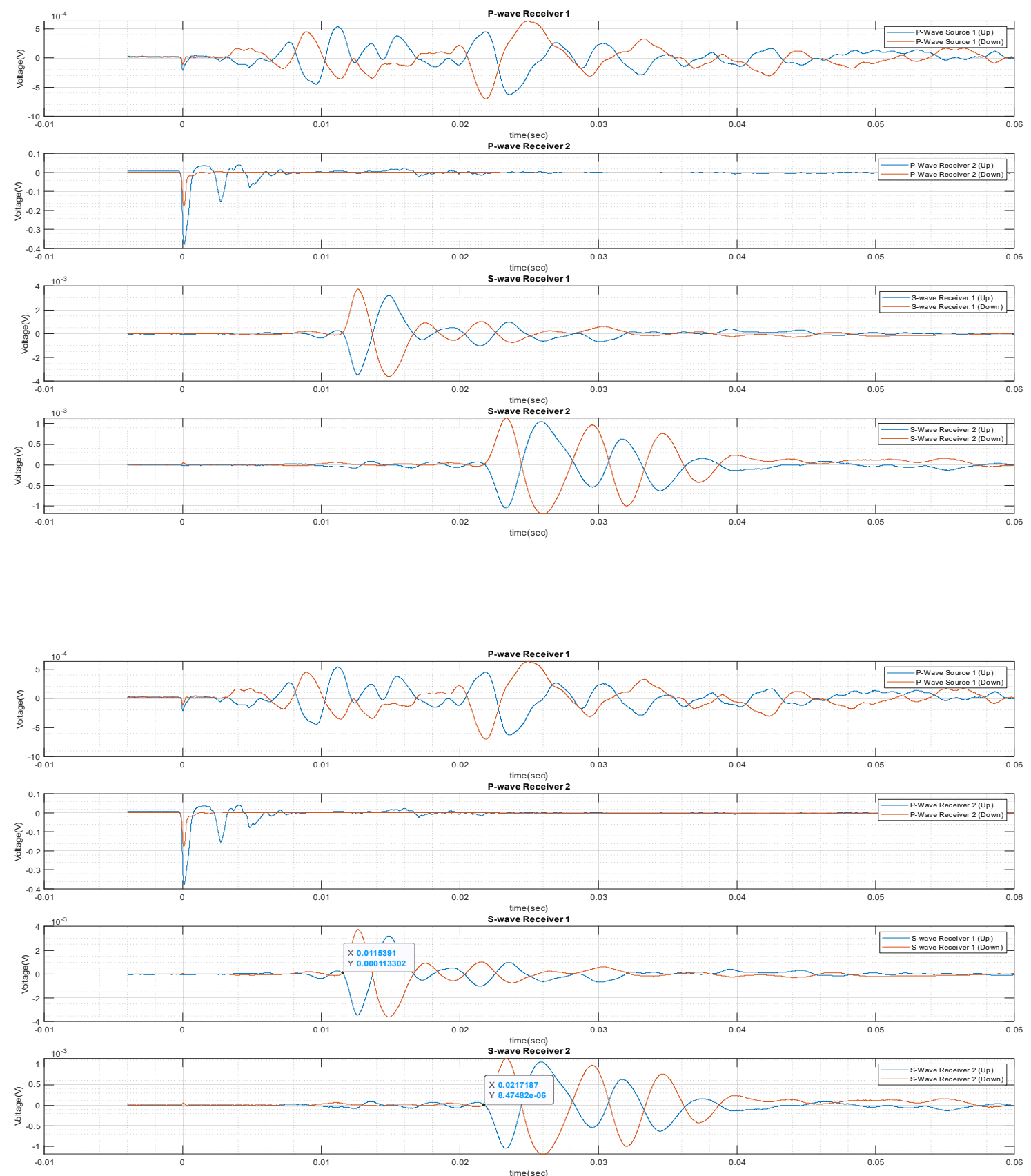

Data Set $25(9.16 .2019)$

Run00188f

R2_2 

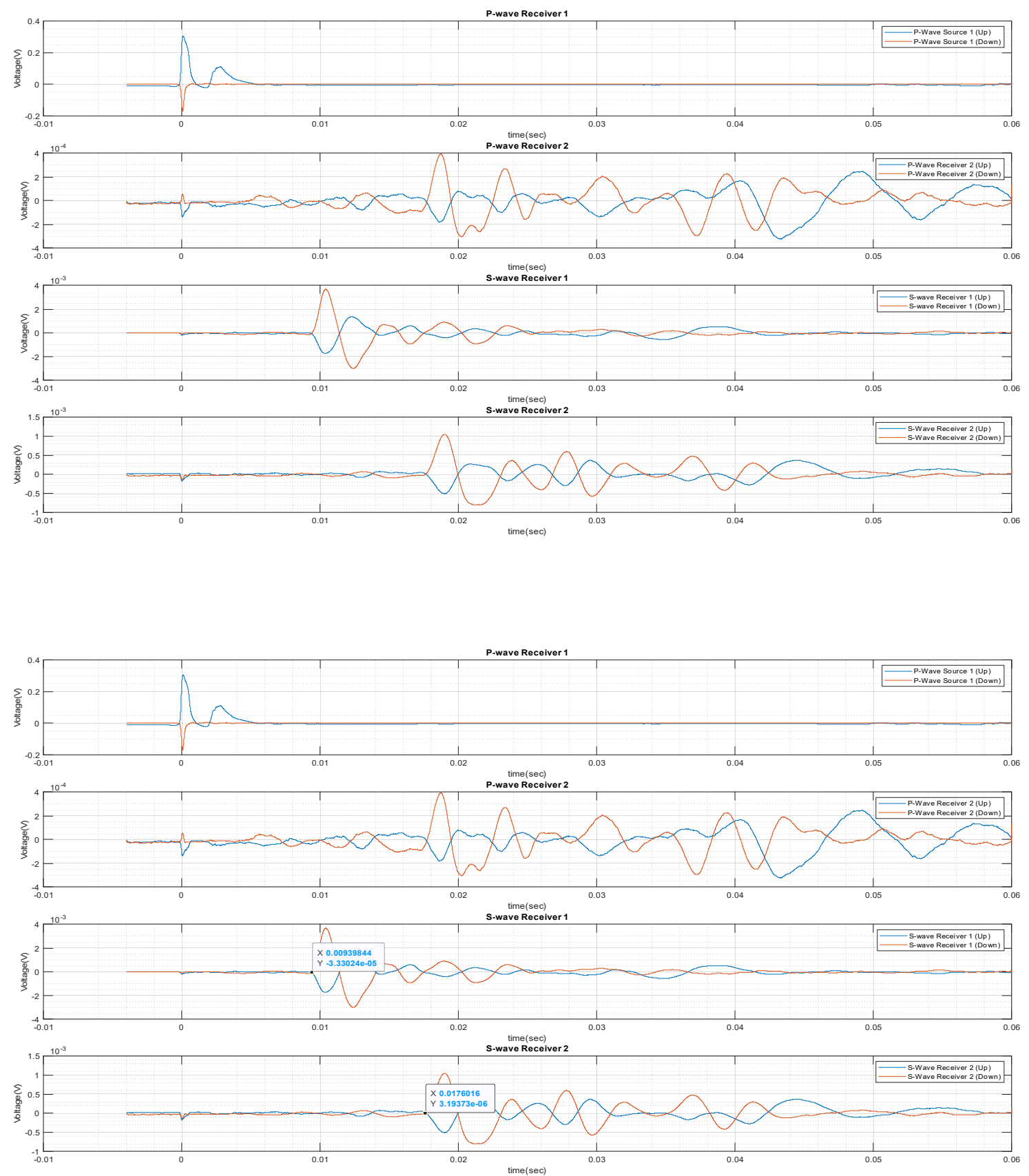

Data Set $25(9.16 .2019)$

Run00189f

R3_1 

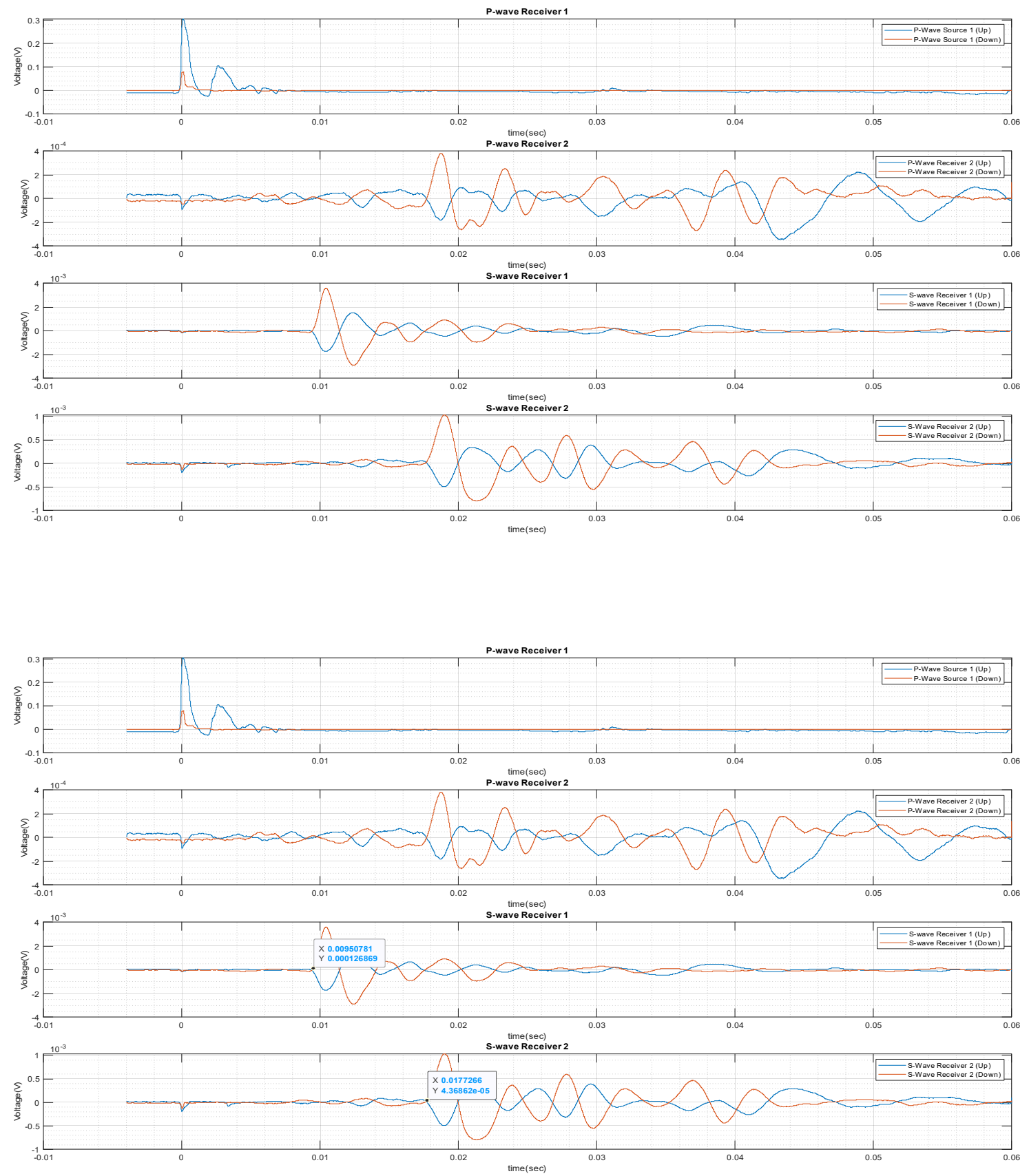

Data Set $25(9.16 .2019)$

Run00190f

R3_2 

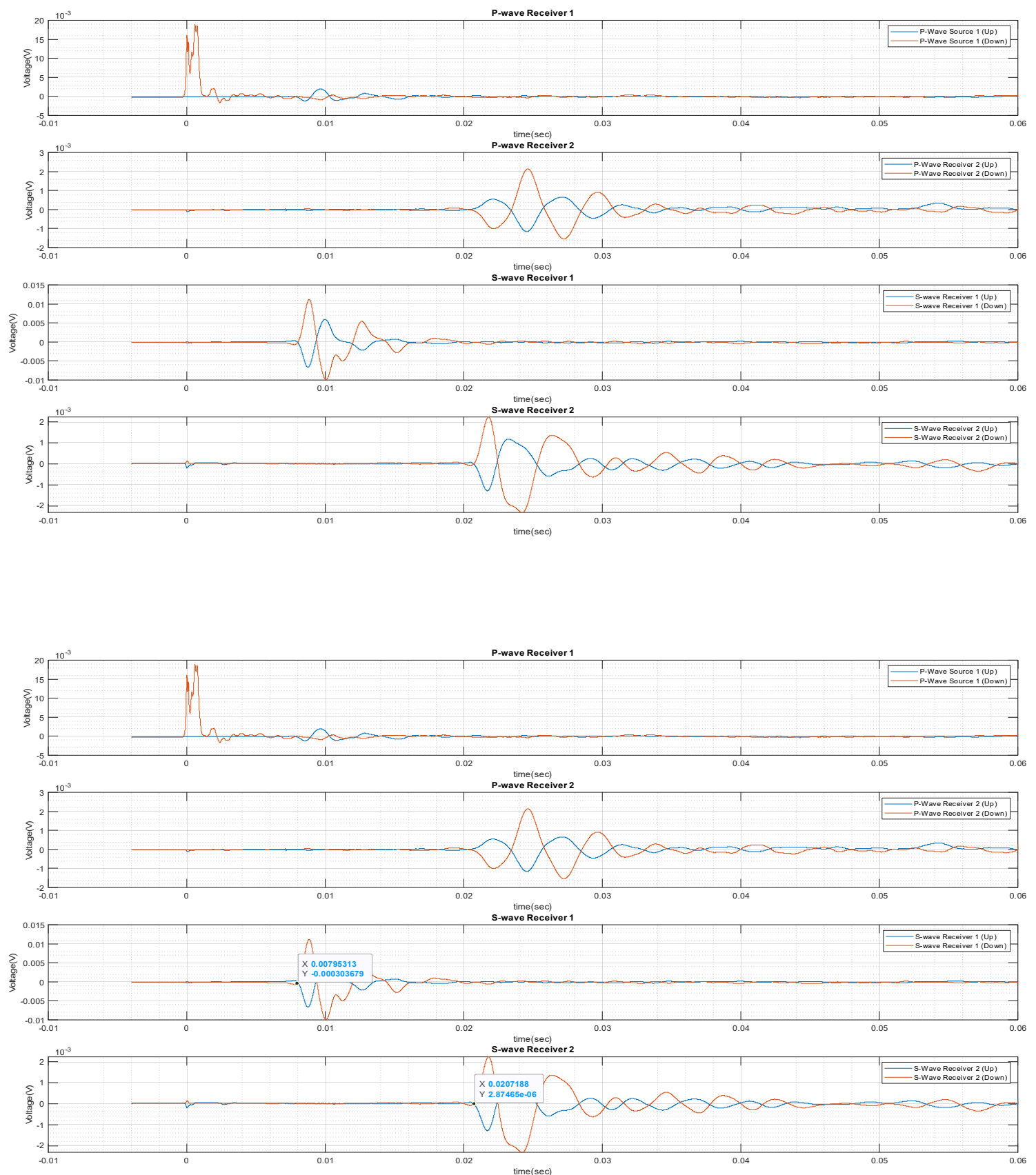

Data Set $25(9.16 .2019)$

Run00191f

R4_1 

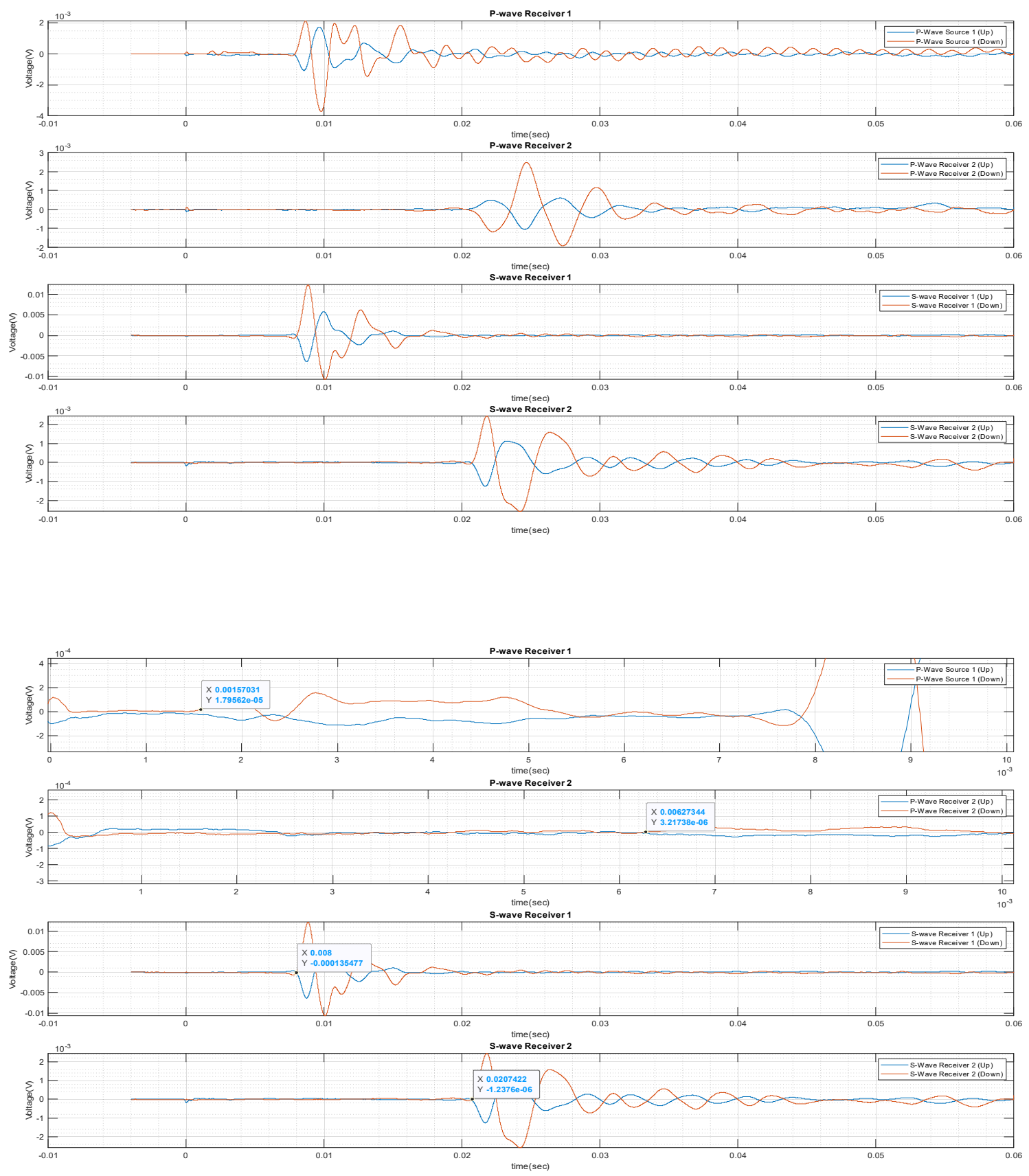

Data Set $25(9.16 .2019)$

Run00192f

R4_2 

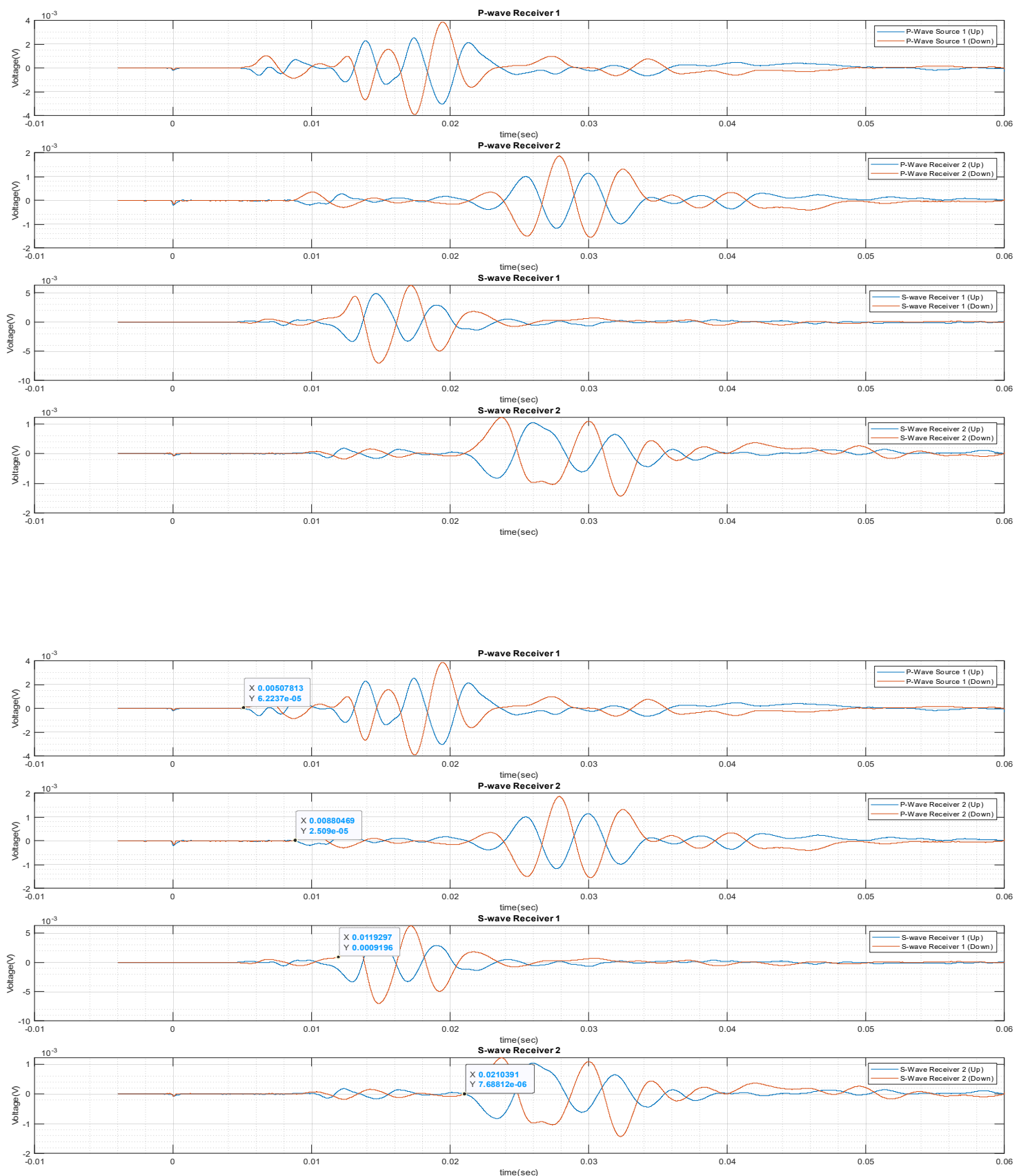

Data Set $26(9.26 .2019)$

Run00193f

R1_1 

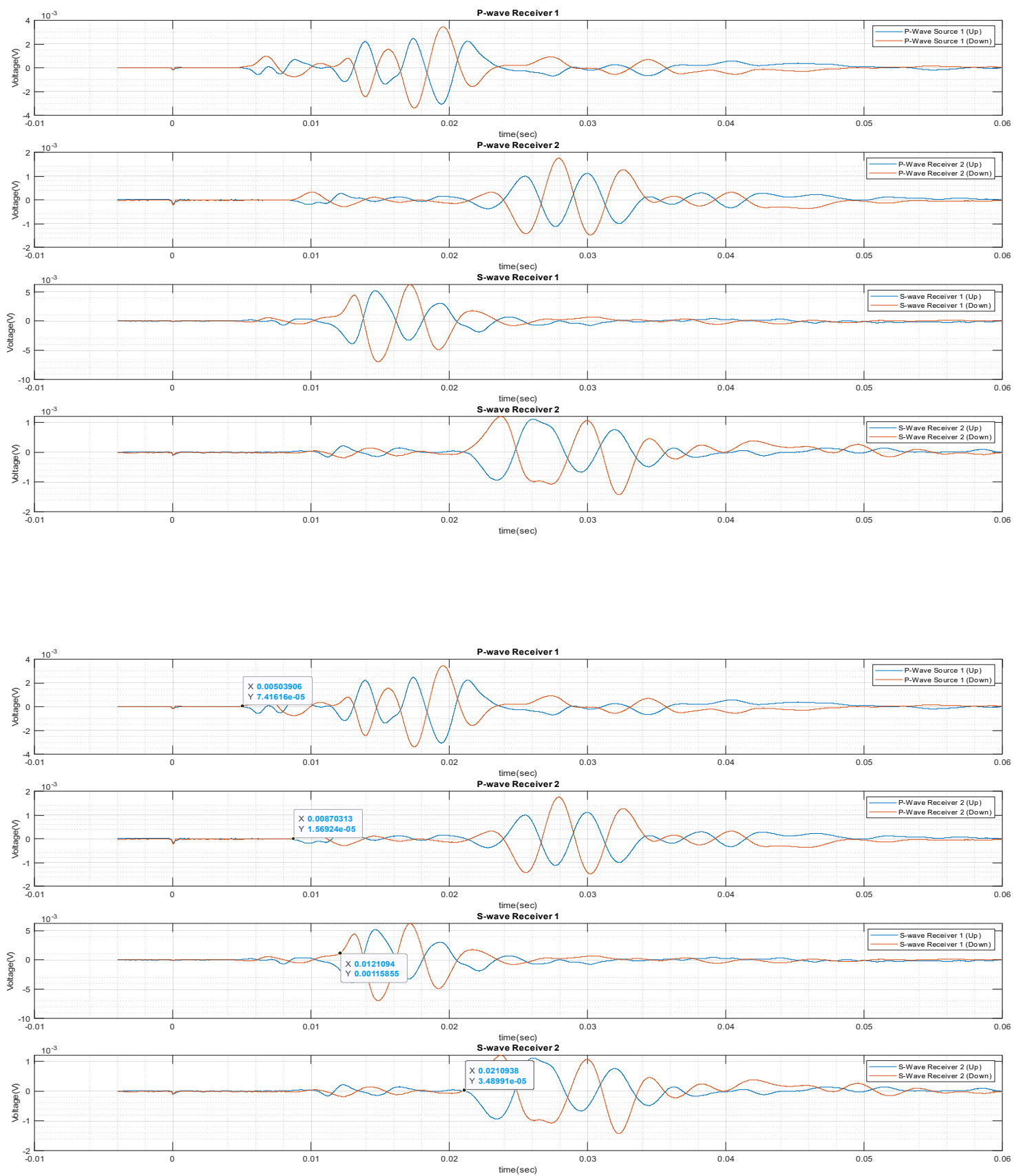

Data Set $26(9.26 .2019)$

Run00194f

R1_2 

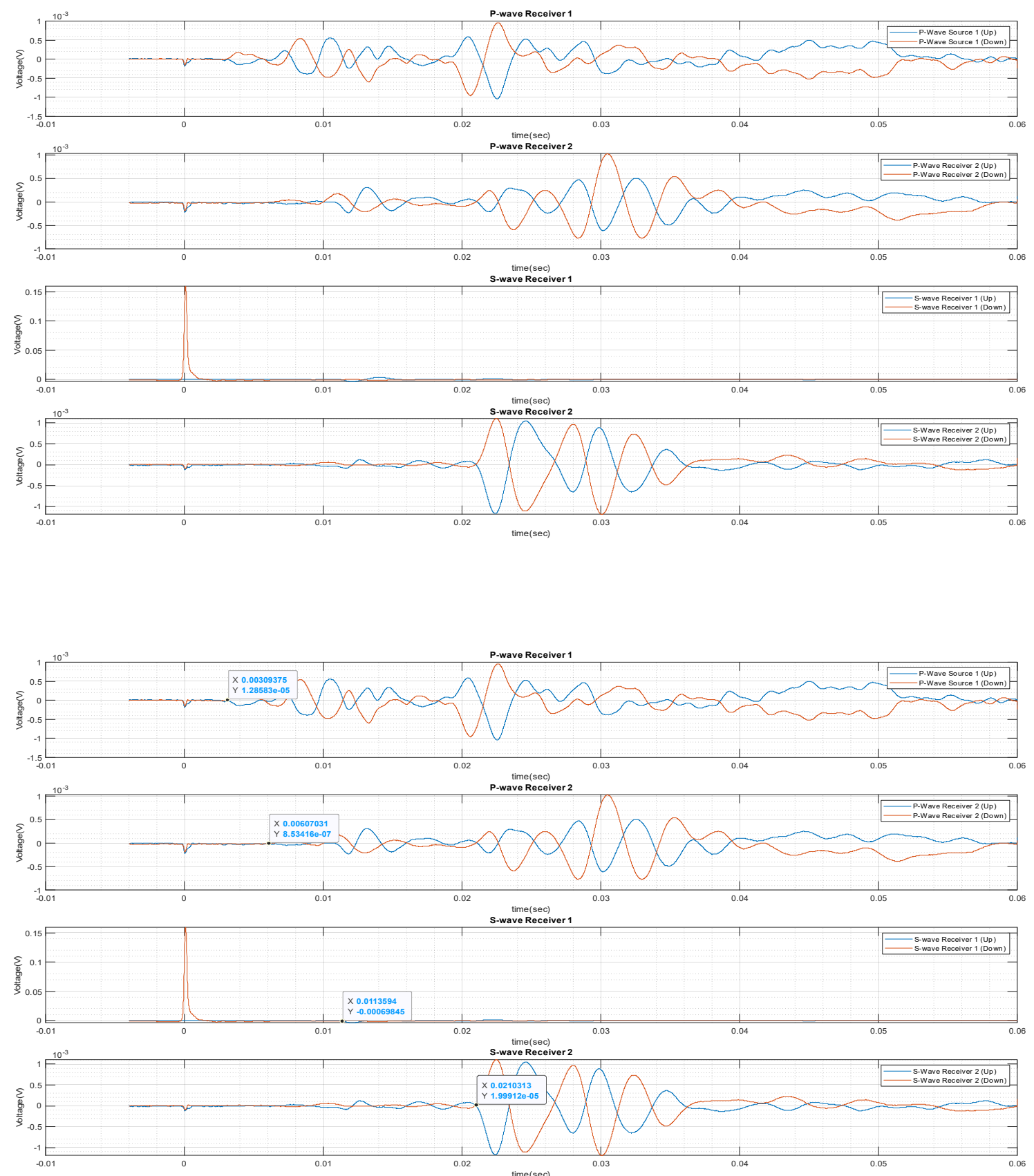

Data Set $26(9.26 .2019)$

Run00195f

R2_1 

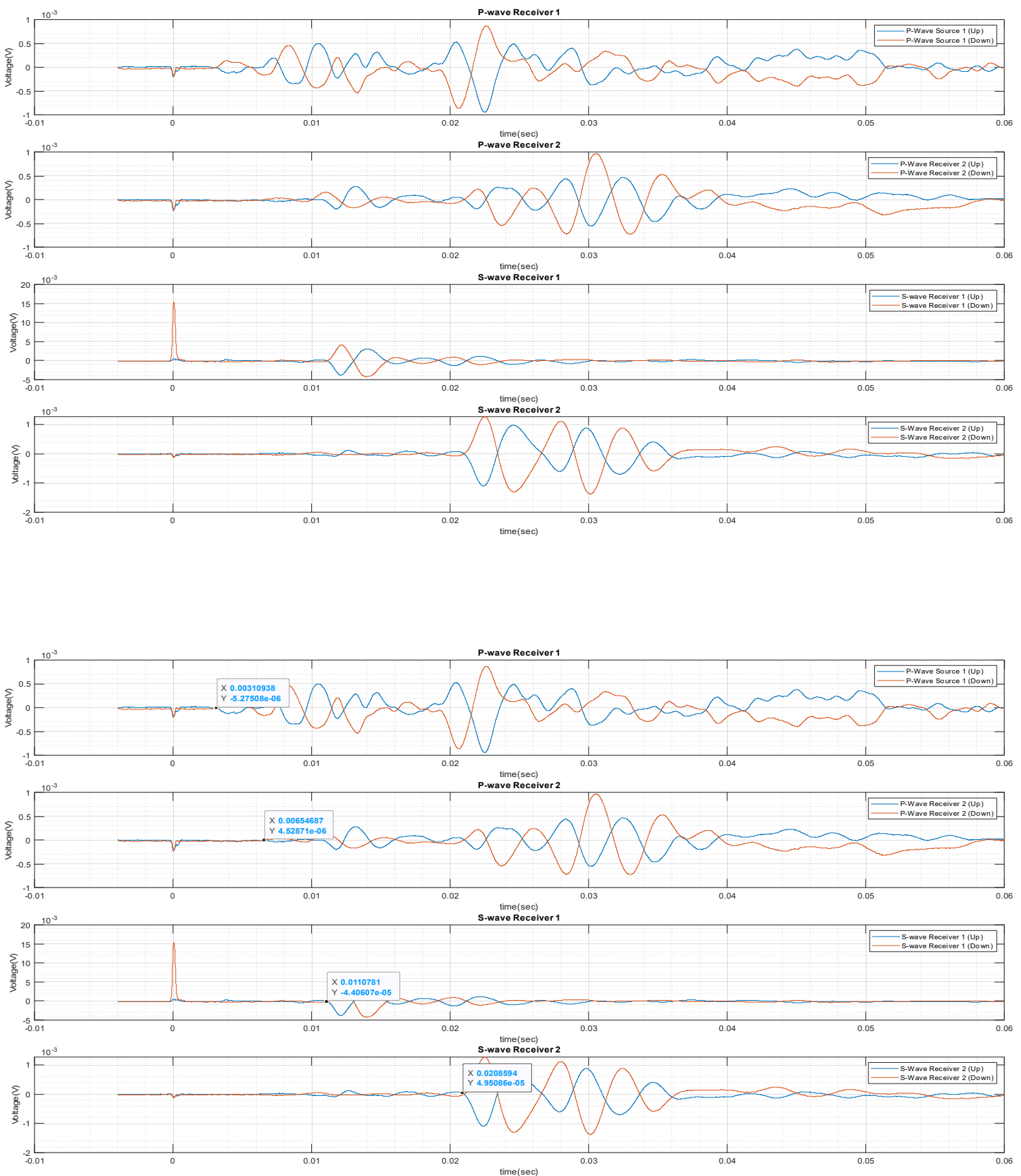

Data Set $26(9.26 .2019)$

Run00196f

R2_2 

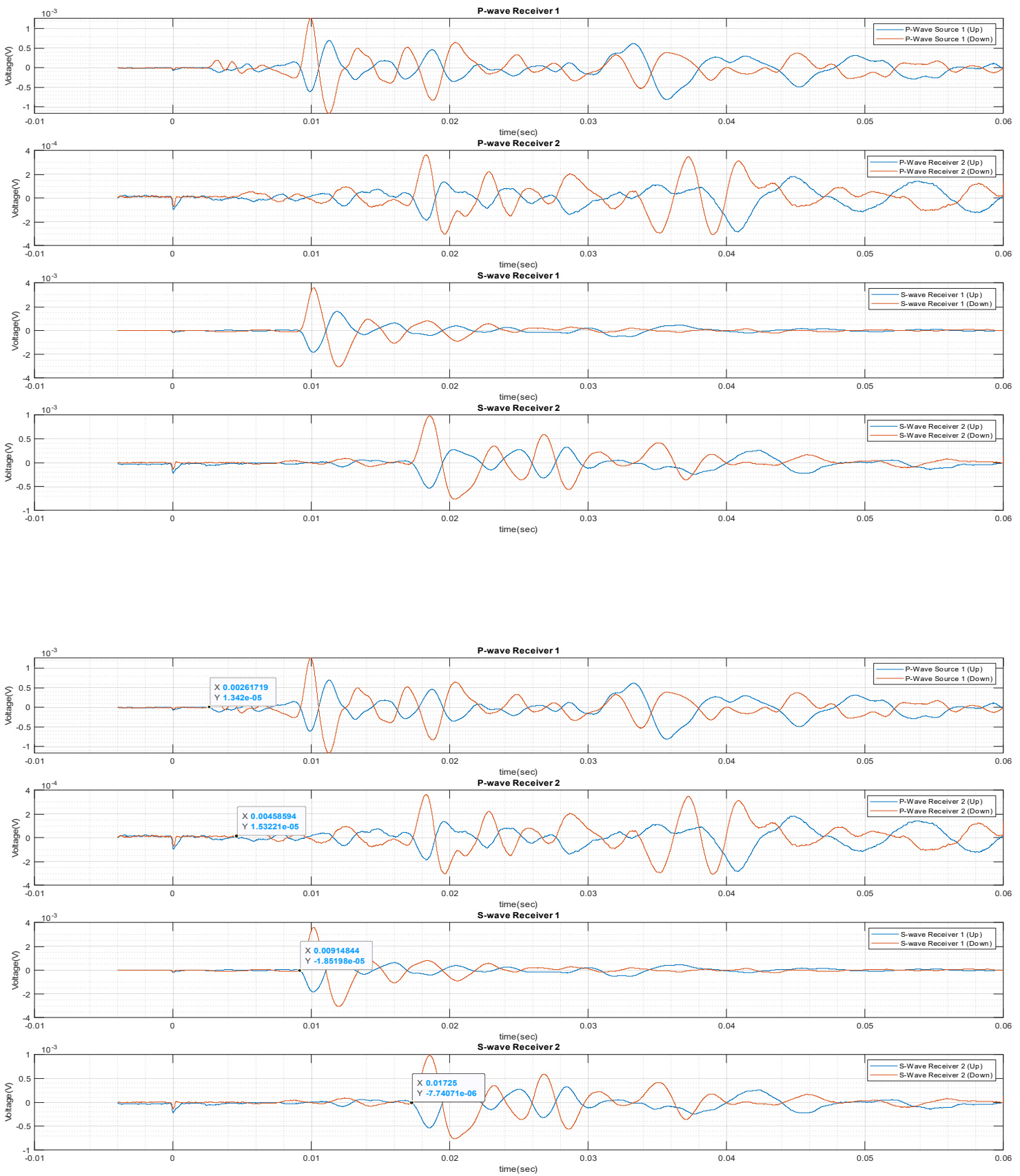

Data Set $26(9.26 .2019)$

Run00197f

R3_1 

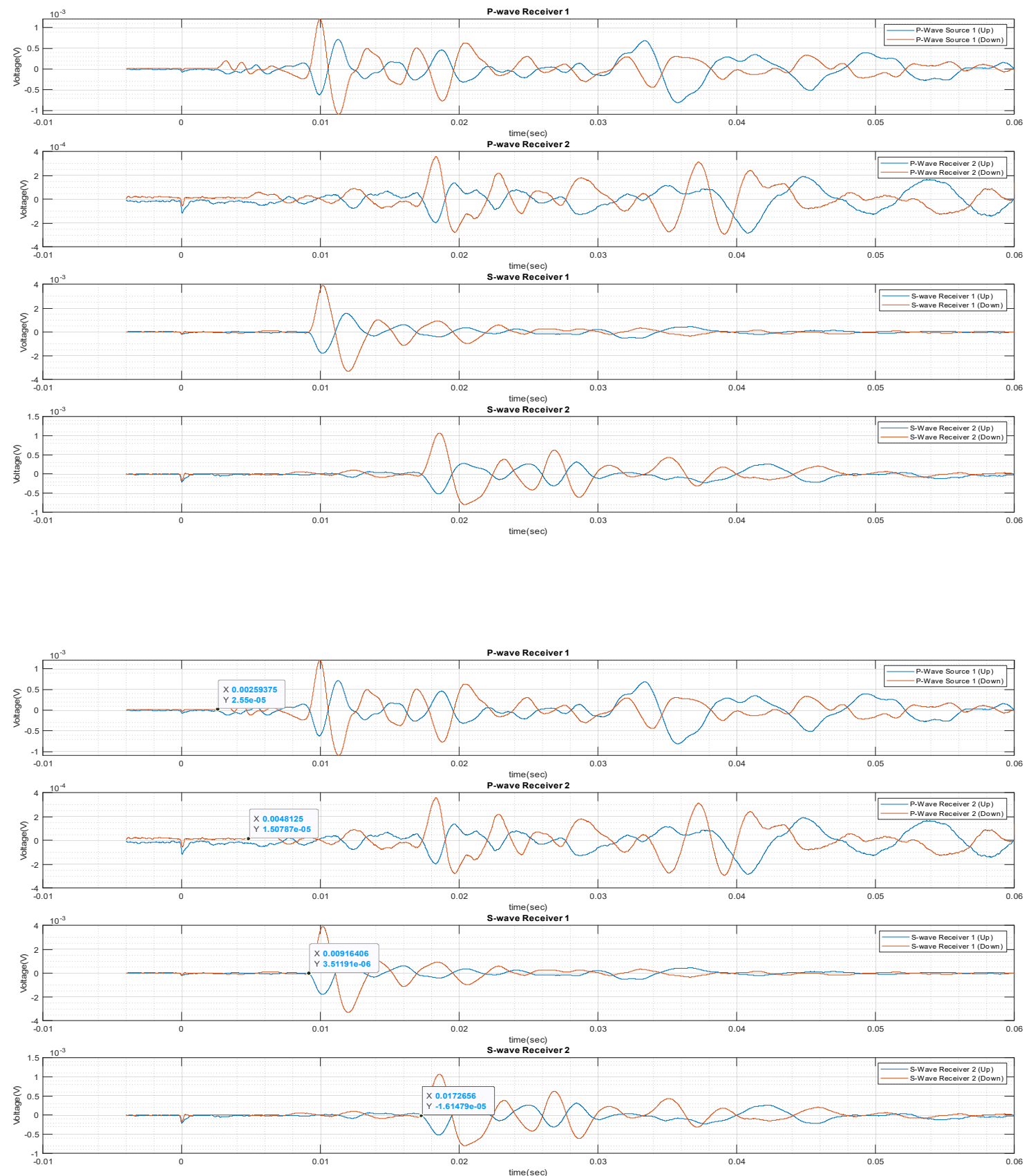

Data Set $26(9.26 .2019)$

Run00198f

R3_2 

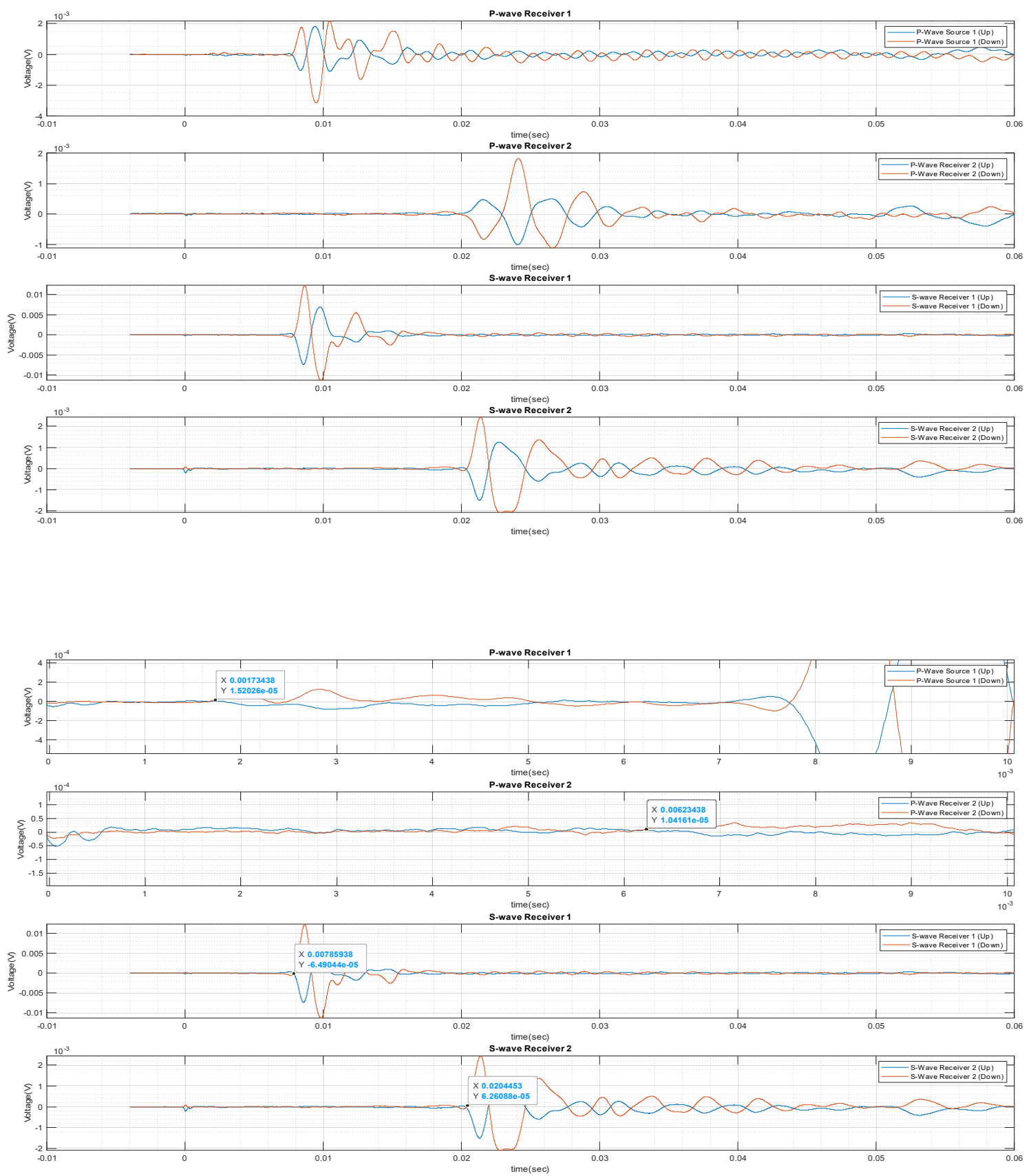

Data Set $26(9.26 .2019)$

Run00199f

R4_1 

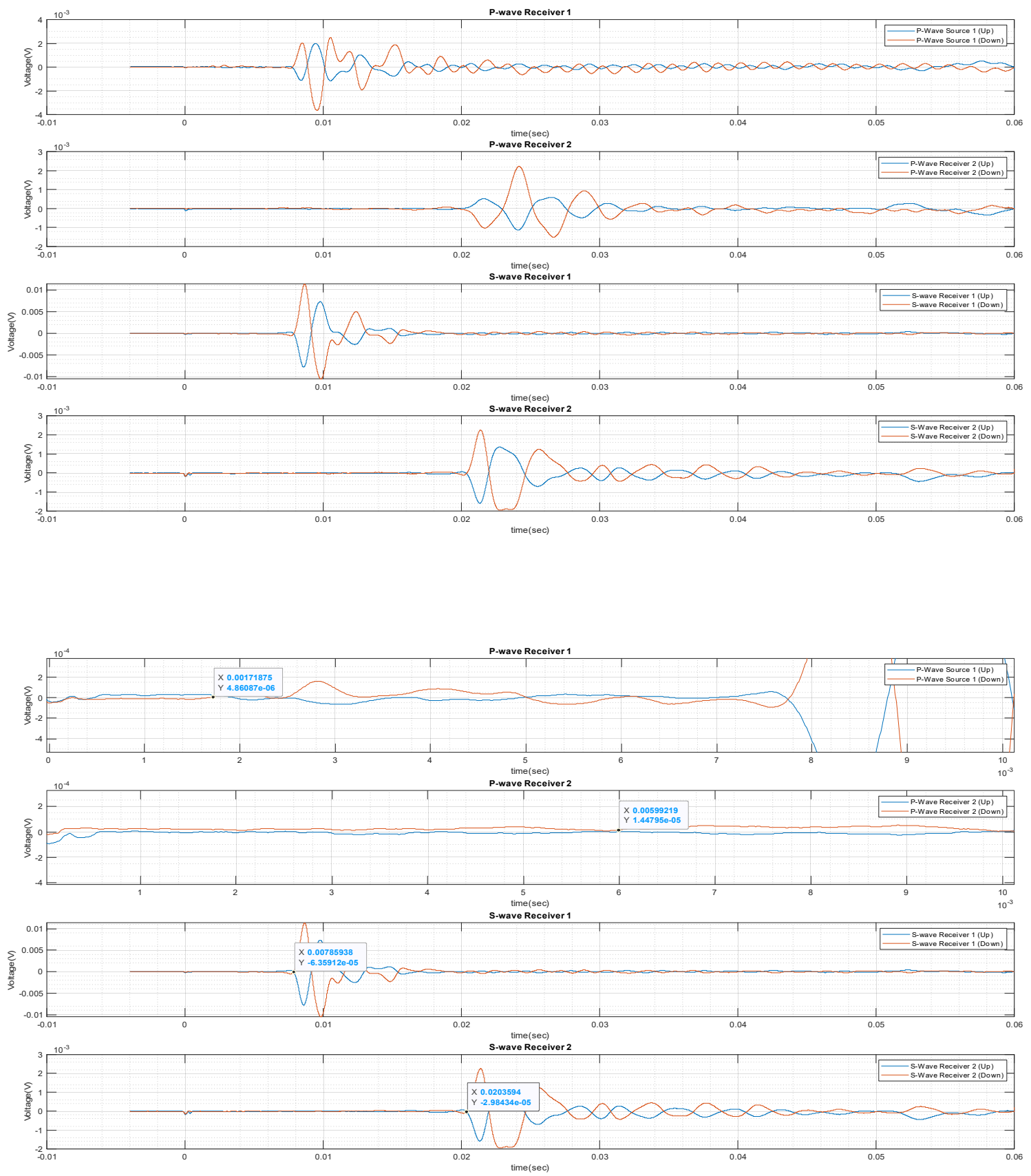

Data Set $26(9.26 .2019)$

Run00200f

R4_2 

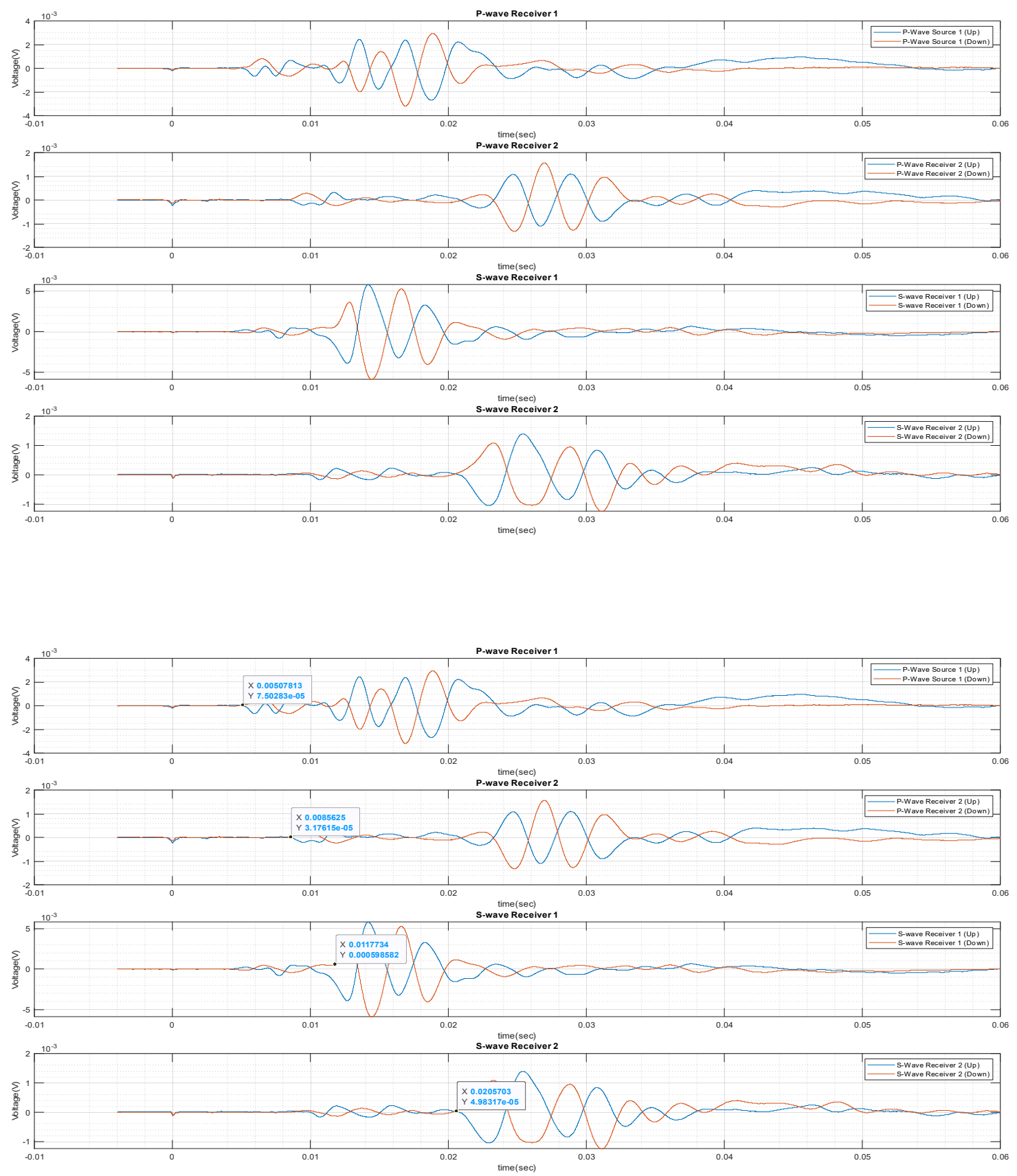

Data Set 27 (10.7.2019)

Run00201f

R1_1 

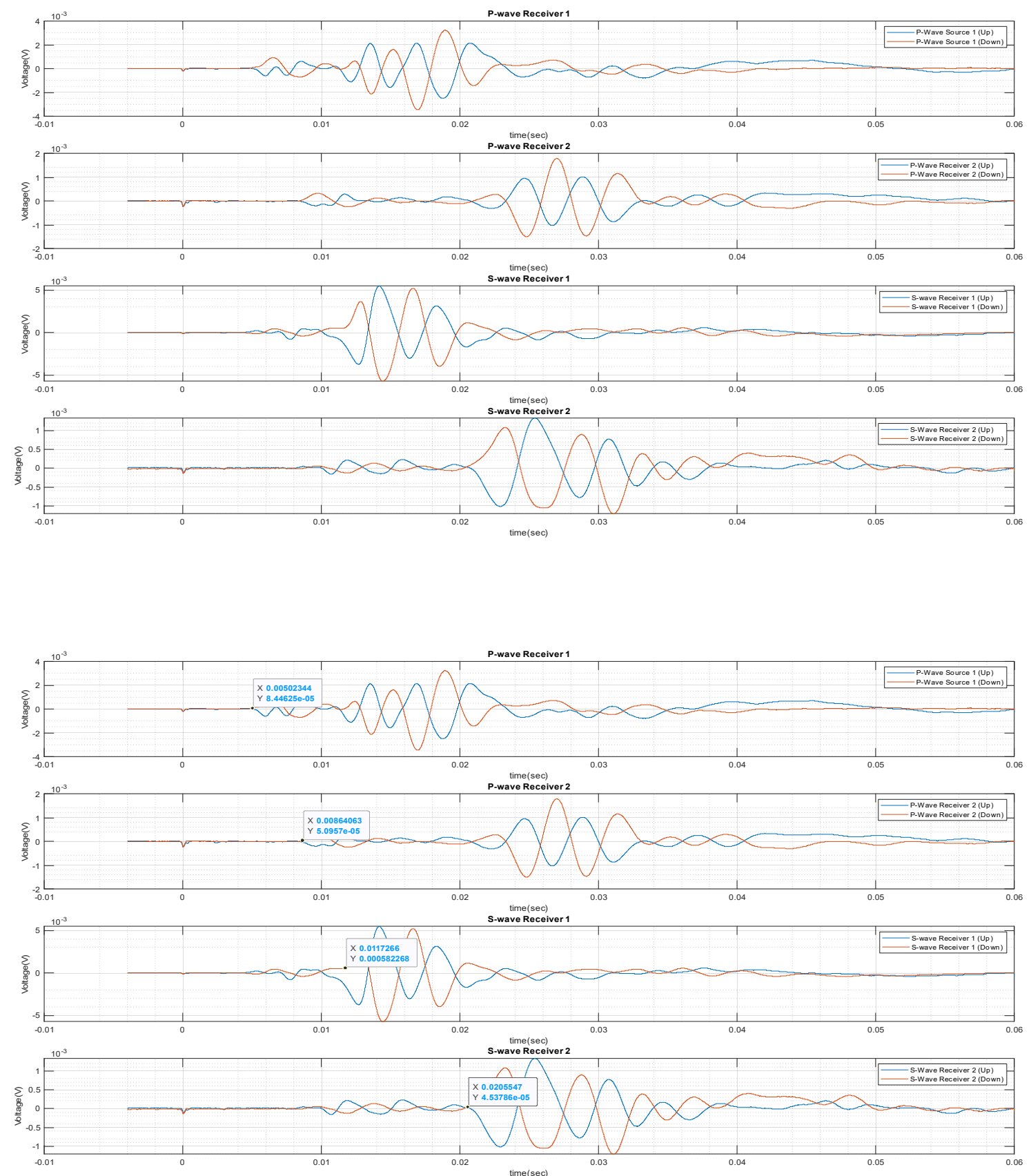

Data Set $27(10.7 .2019)$

Run00202f

R1_2 

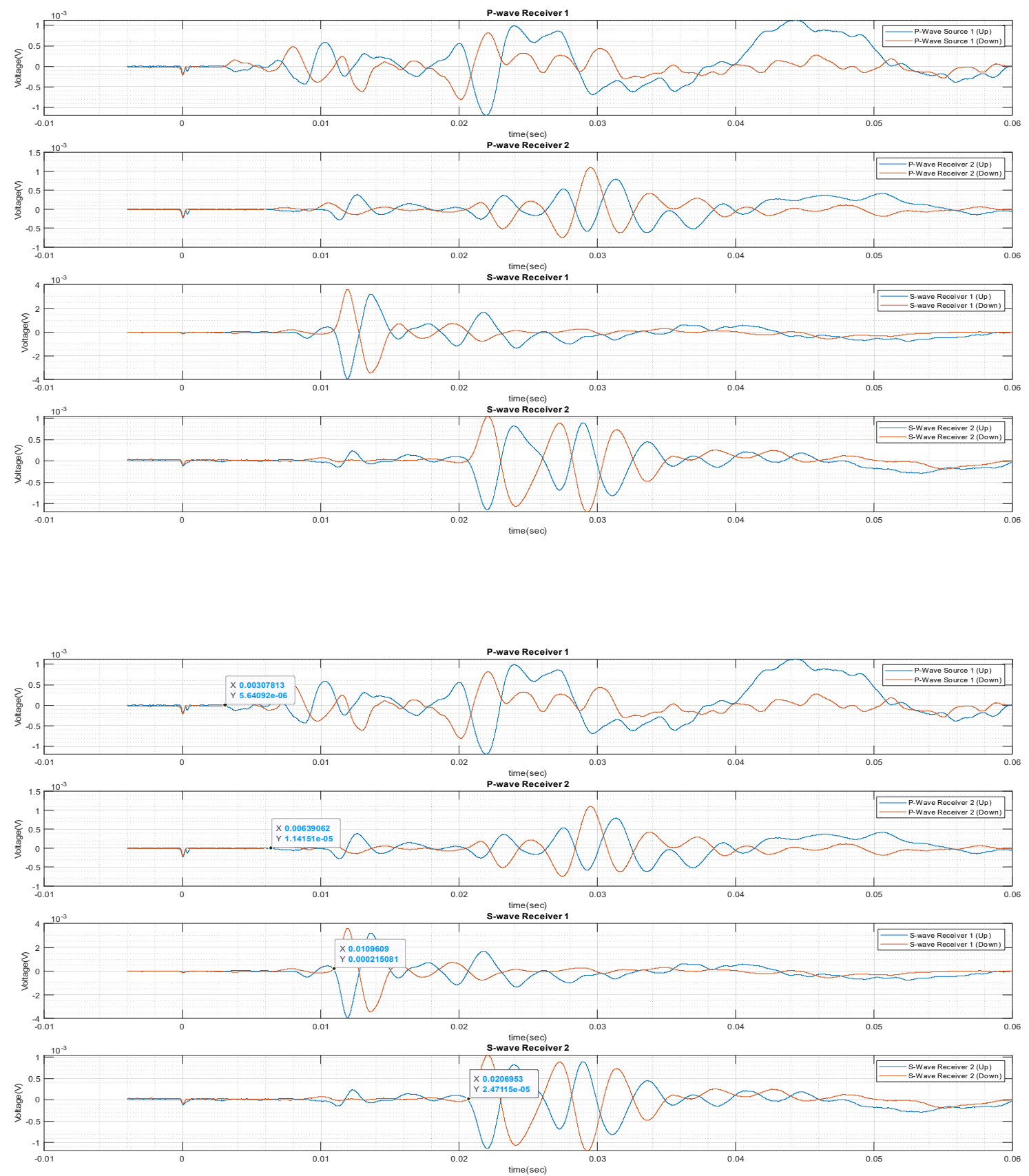

Data Set $27(10.7 .2019)$

Run00203f

R2_1 

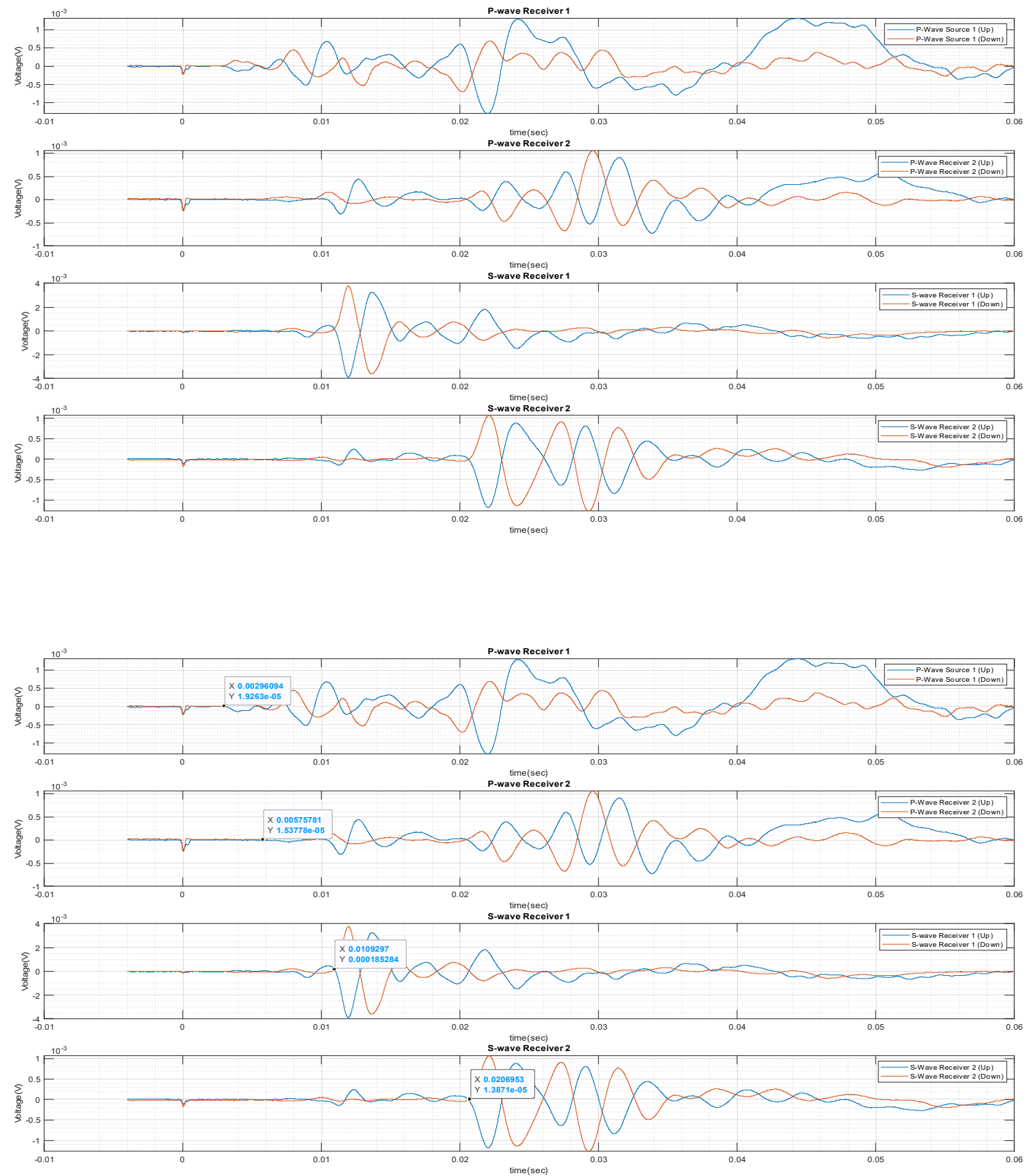

Data Set 27 (10.7.2019)

Run00204f

R2_2 

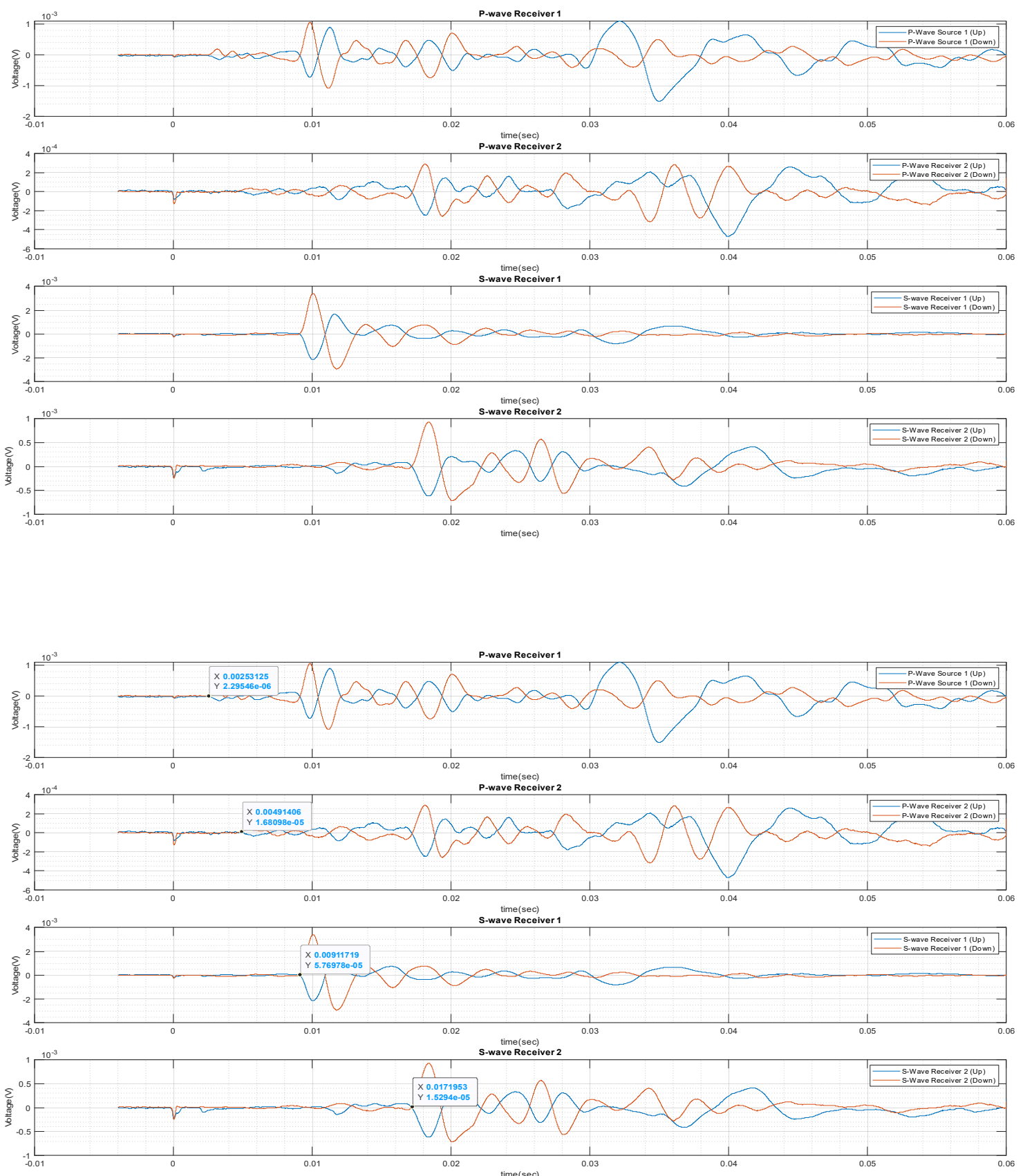

Data Set $27(10.7 .2019)$

Run00205f

R3_1 

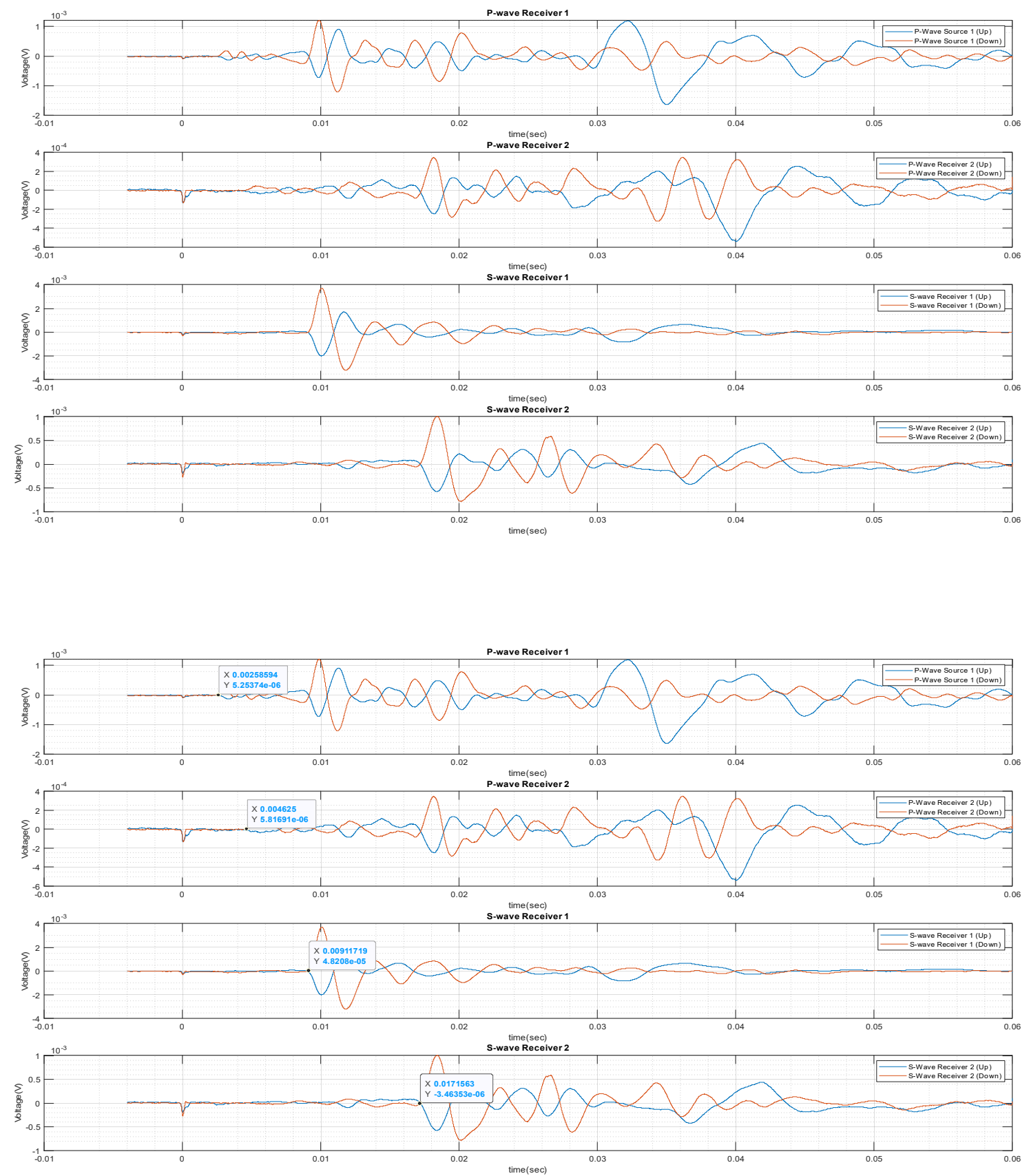

Data Set $27(10.7 .2019)$

Run00206f

R3_2 

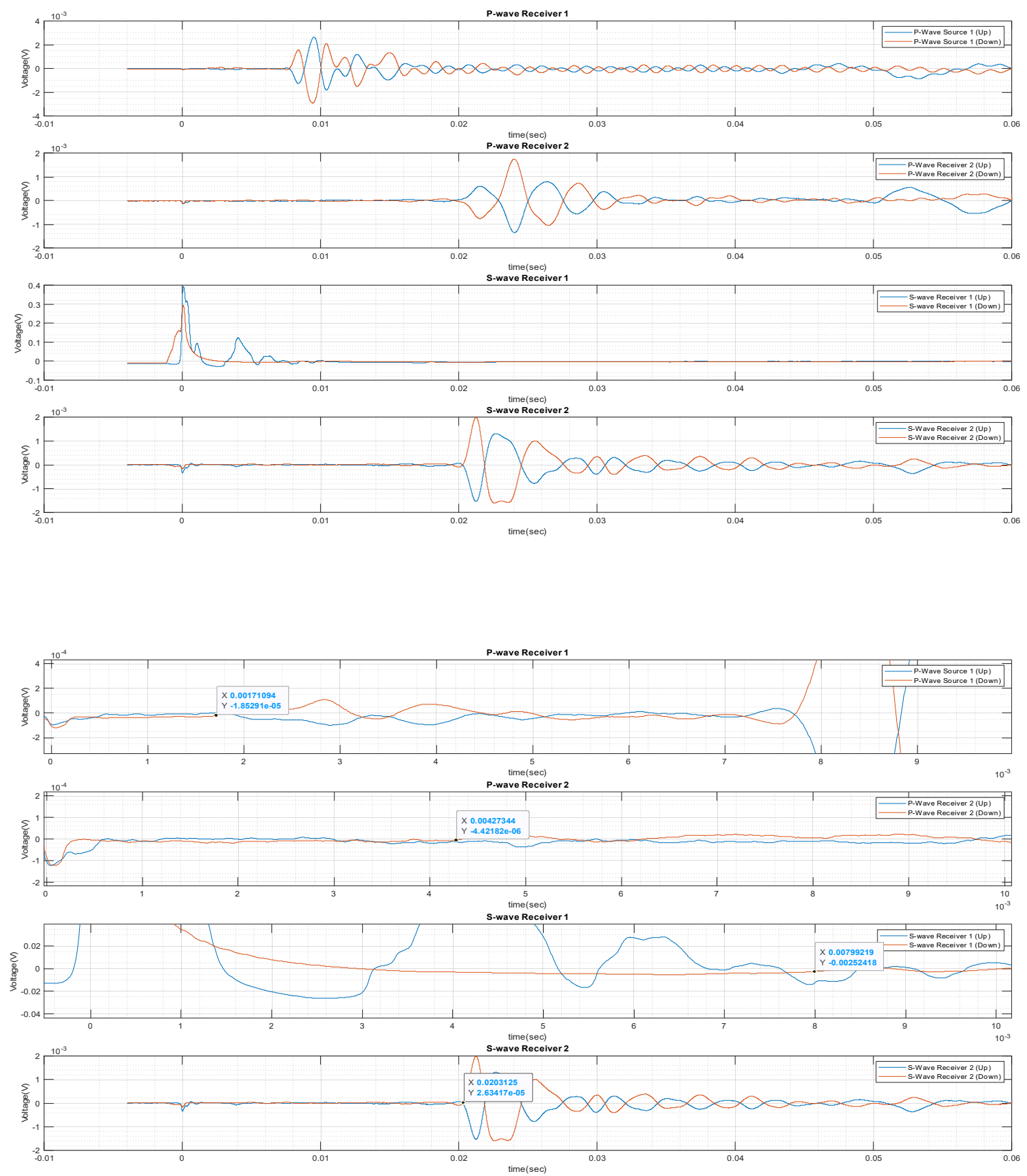

Data Set 27 (10.7.2019)

Run00207f

R4_1 

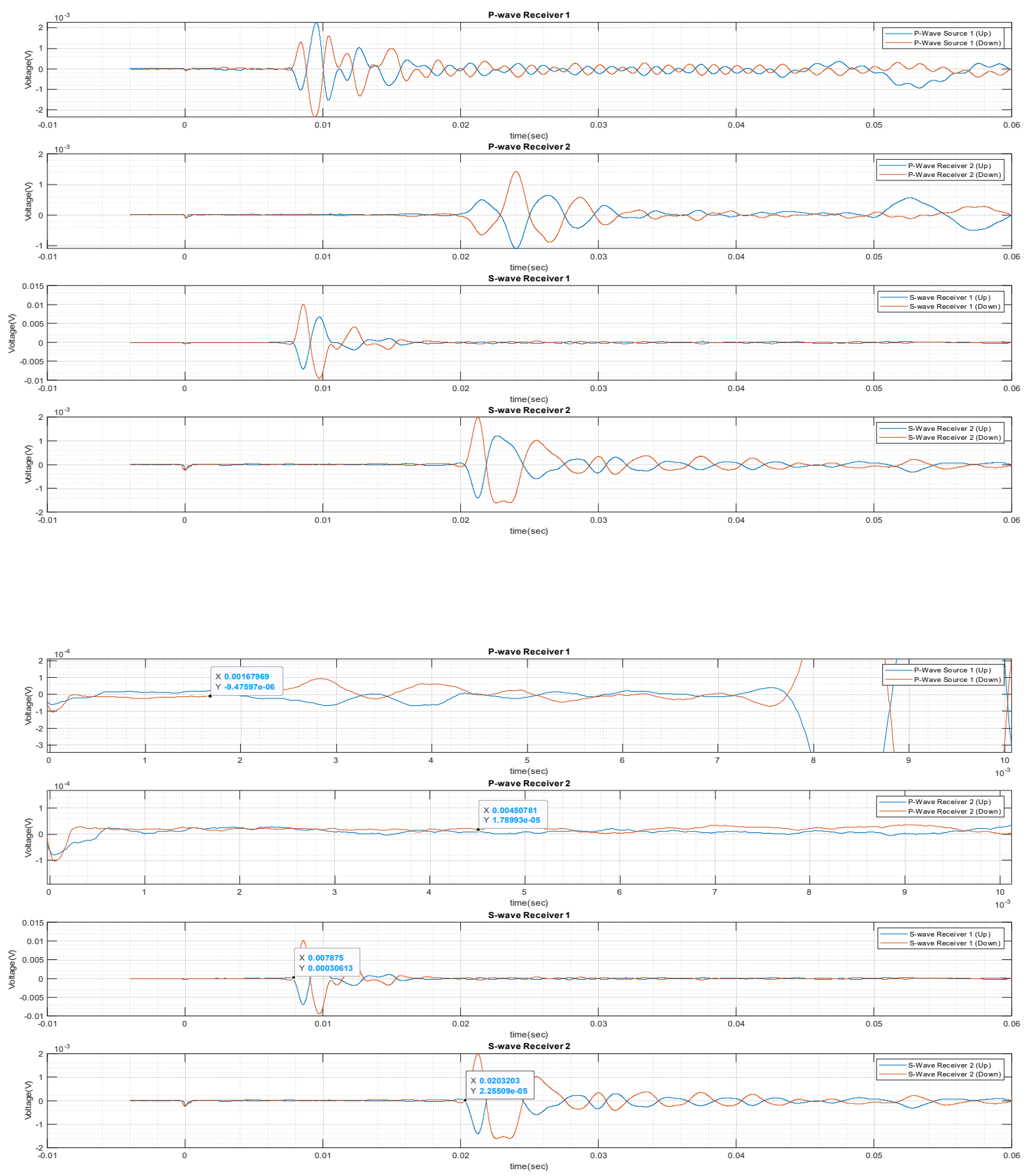

Data Set 27 (10.7.2019)

Run00208f

R4_2 

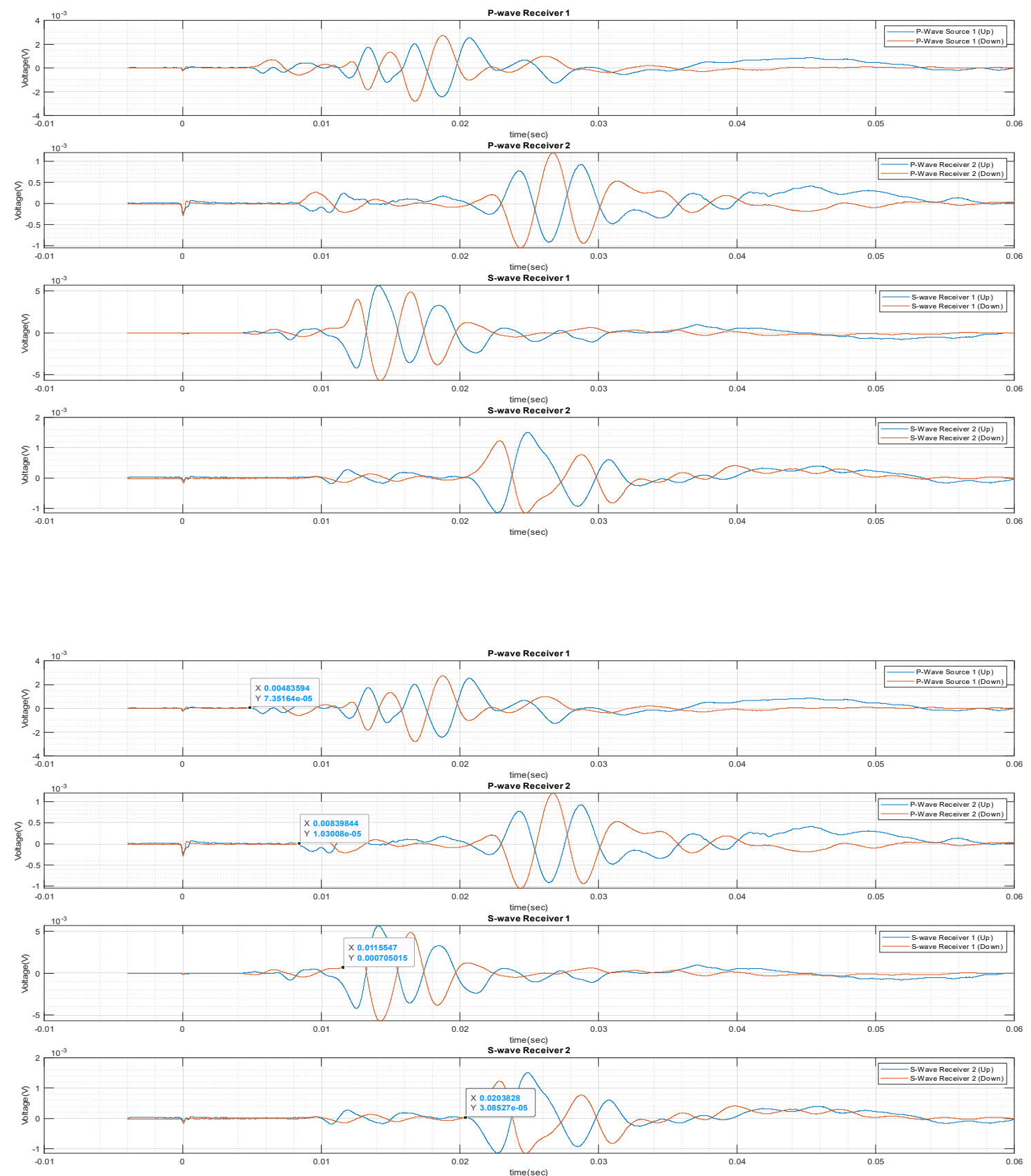

Data Set 28 (10.18.2019)

Run00209f

R1_1 

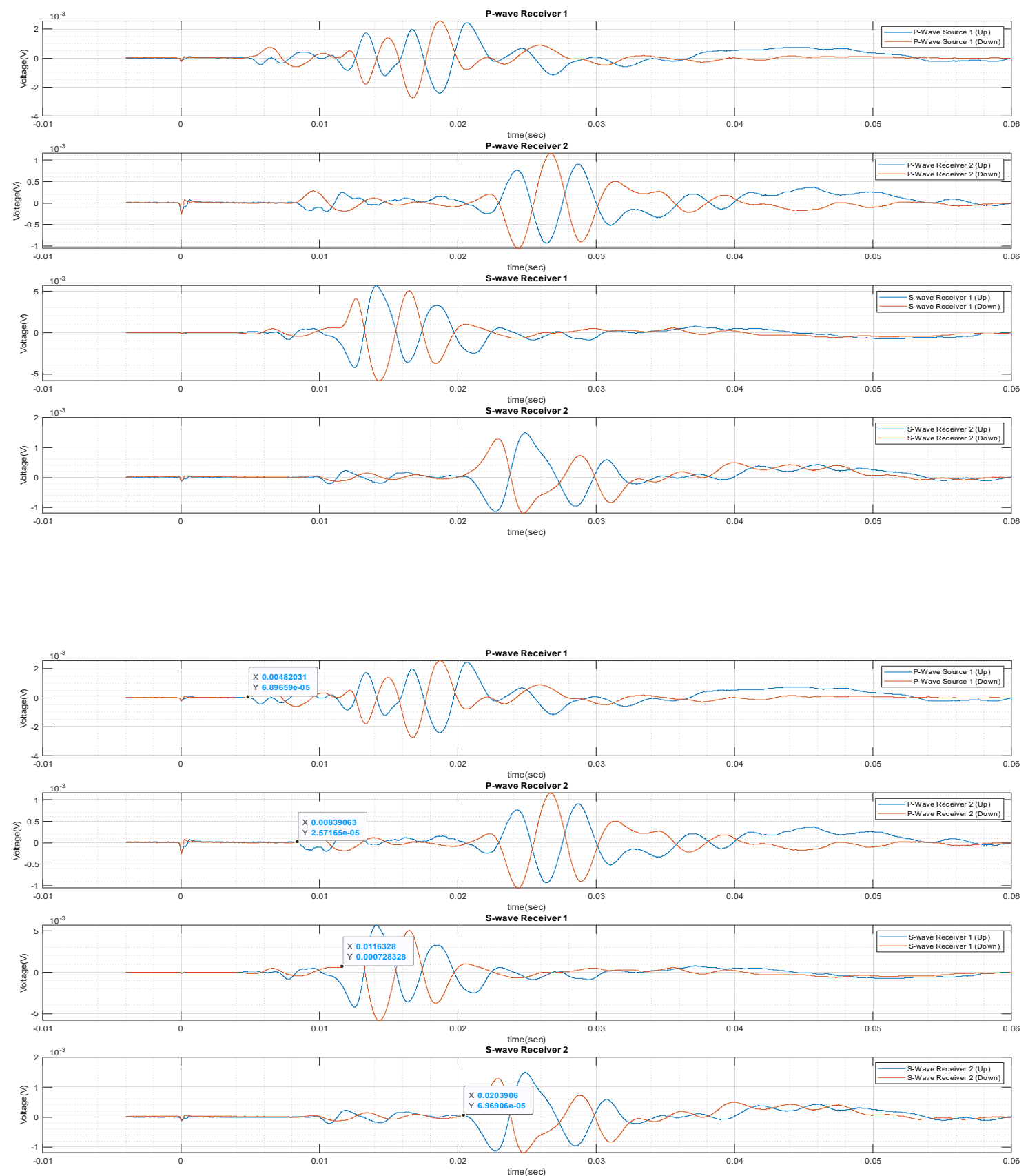

Data Set 28 (10.18.2019)

\section{Run00210f}

\section{R1_2}



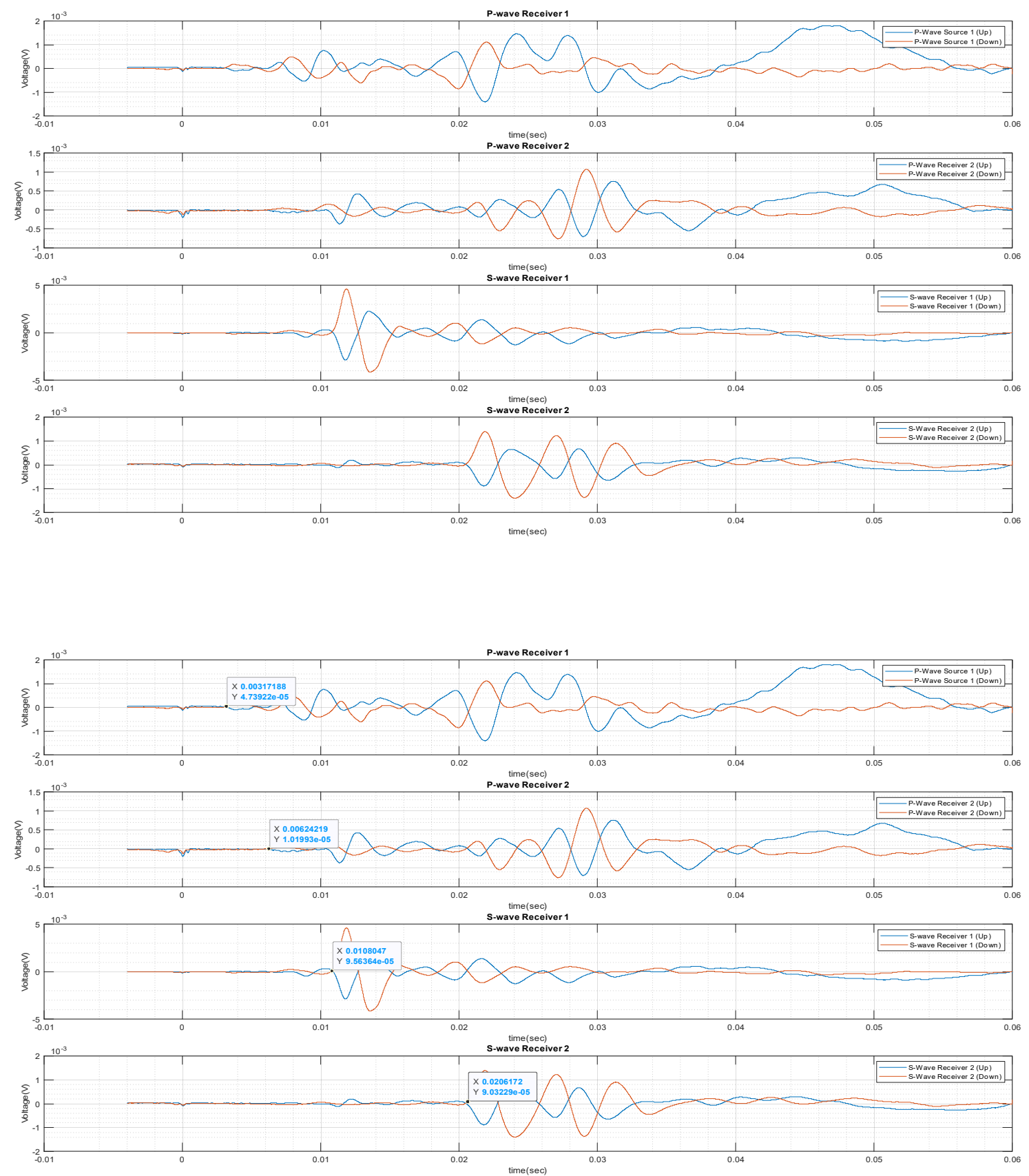

Data Set 28 (10.18.2019)

Run00211f

R2_1 

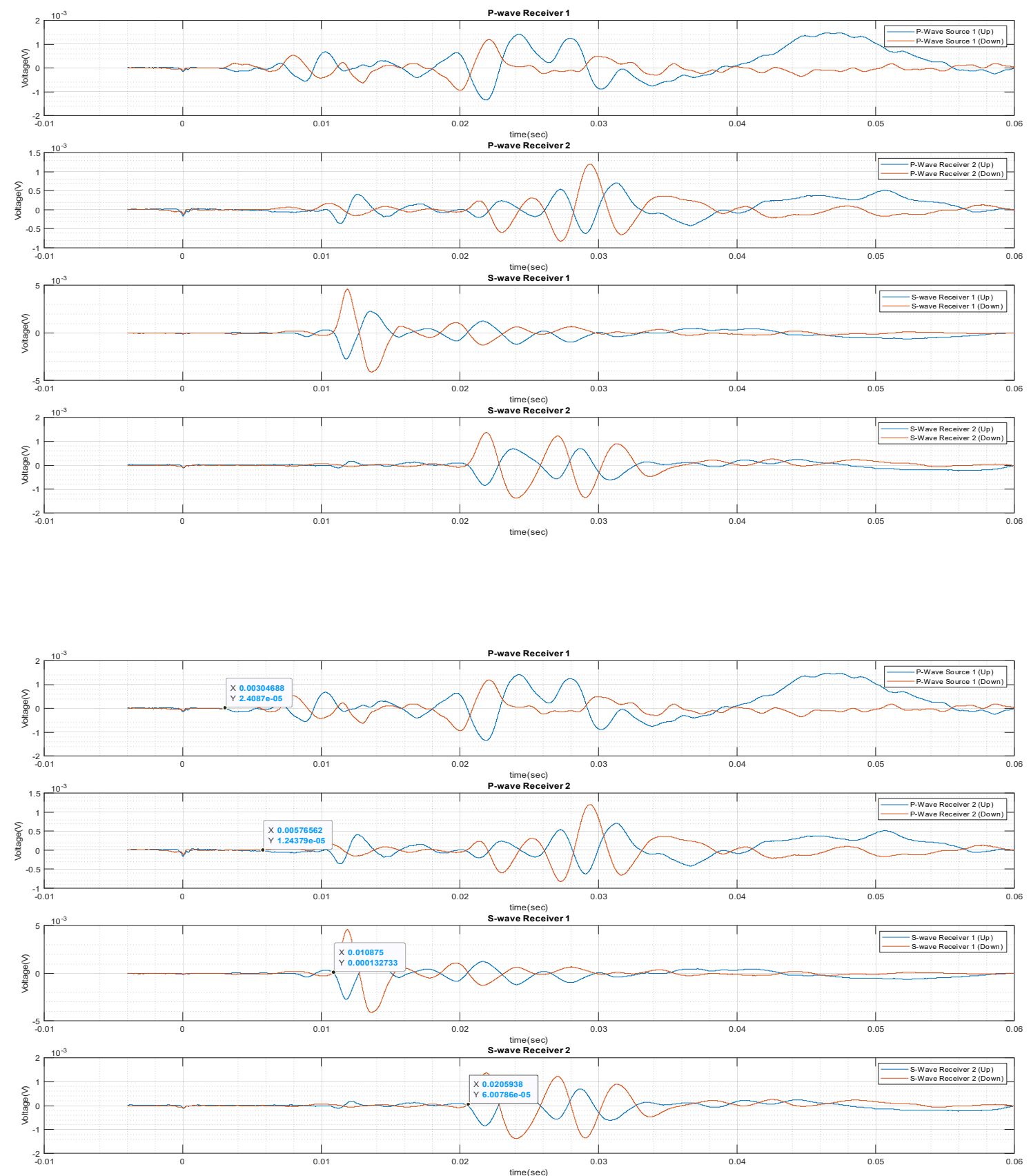

Data Set 28 (10.18.2019)

Run00212f

R2_2 

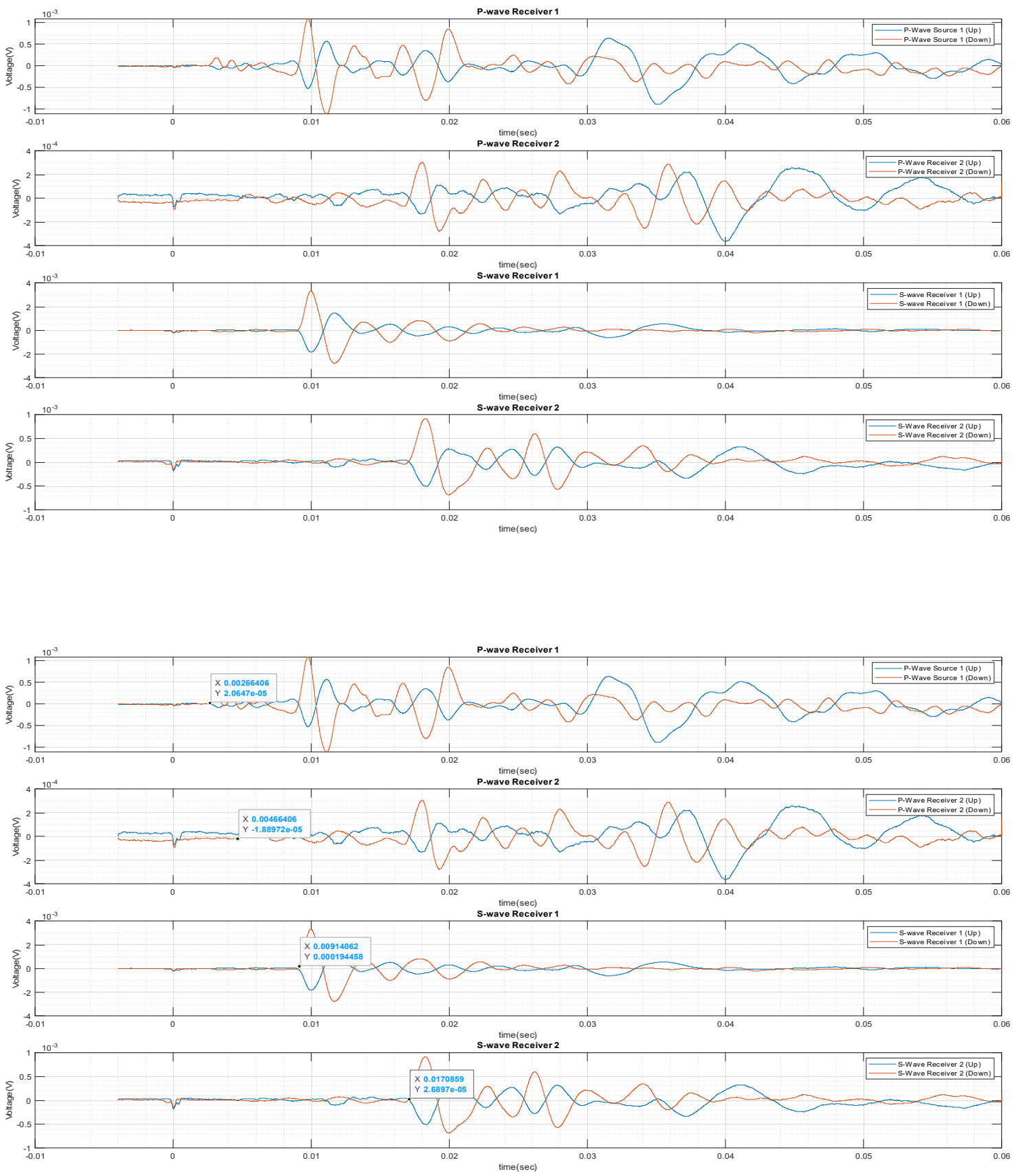

Data Set 28 (10.18.2019)

Run00213f

R3_1 

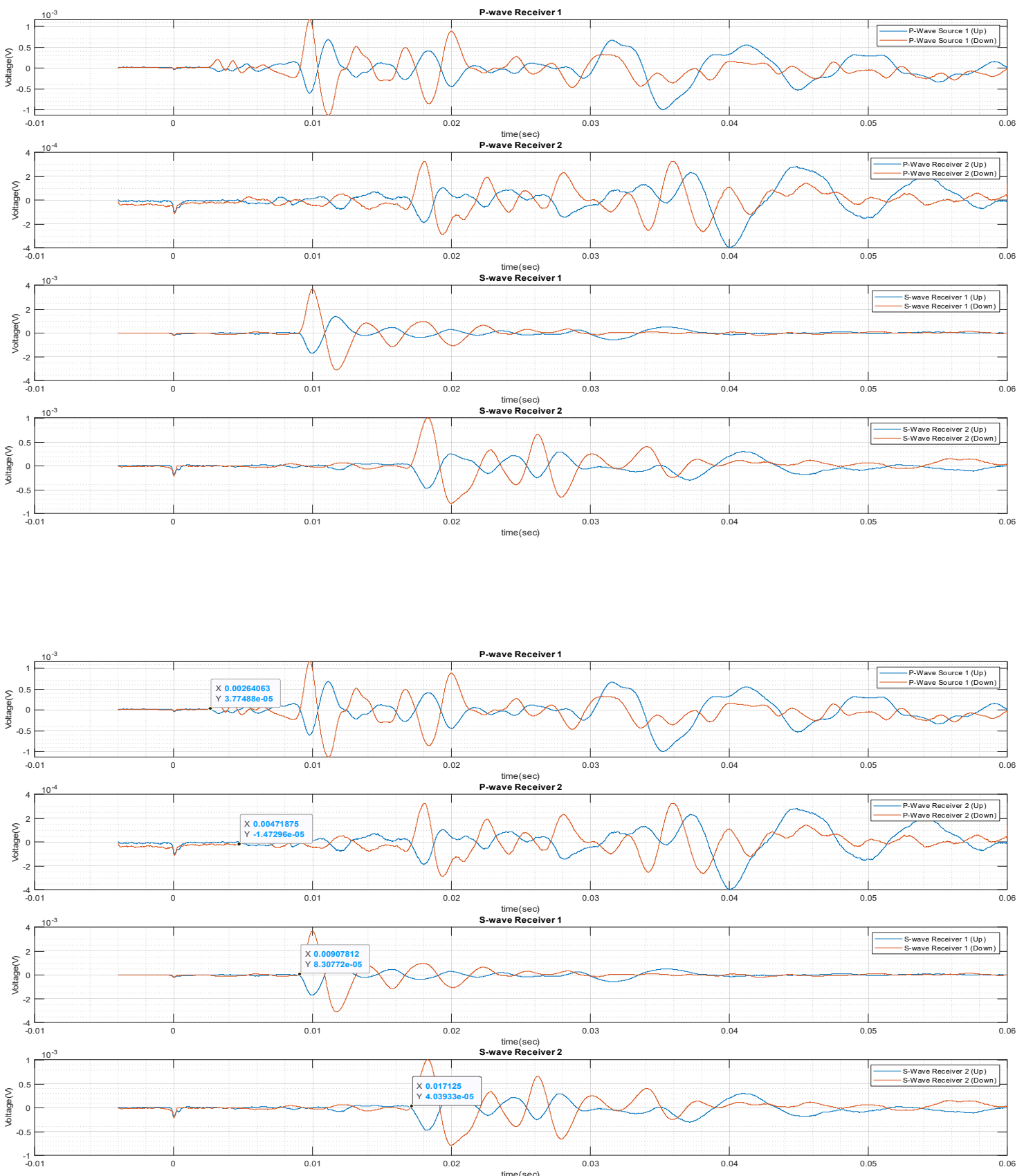

Data Set 28 (10.18.2019)

Run00214f

R3_2 

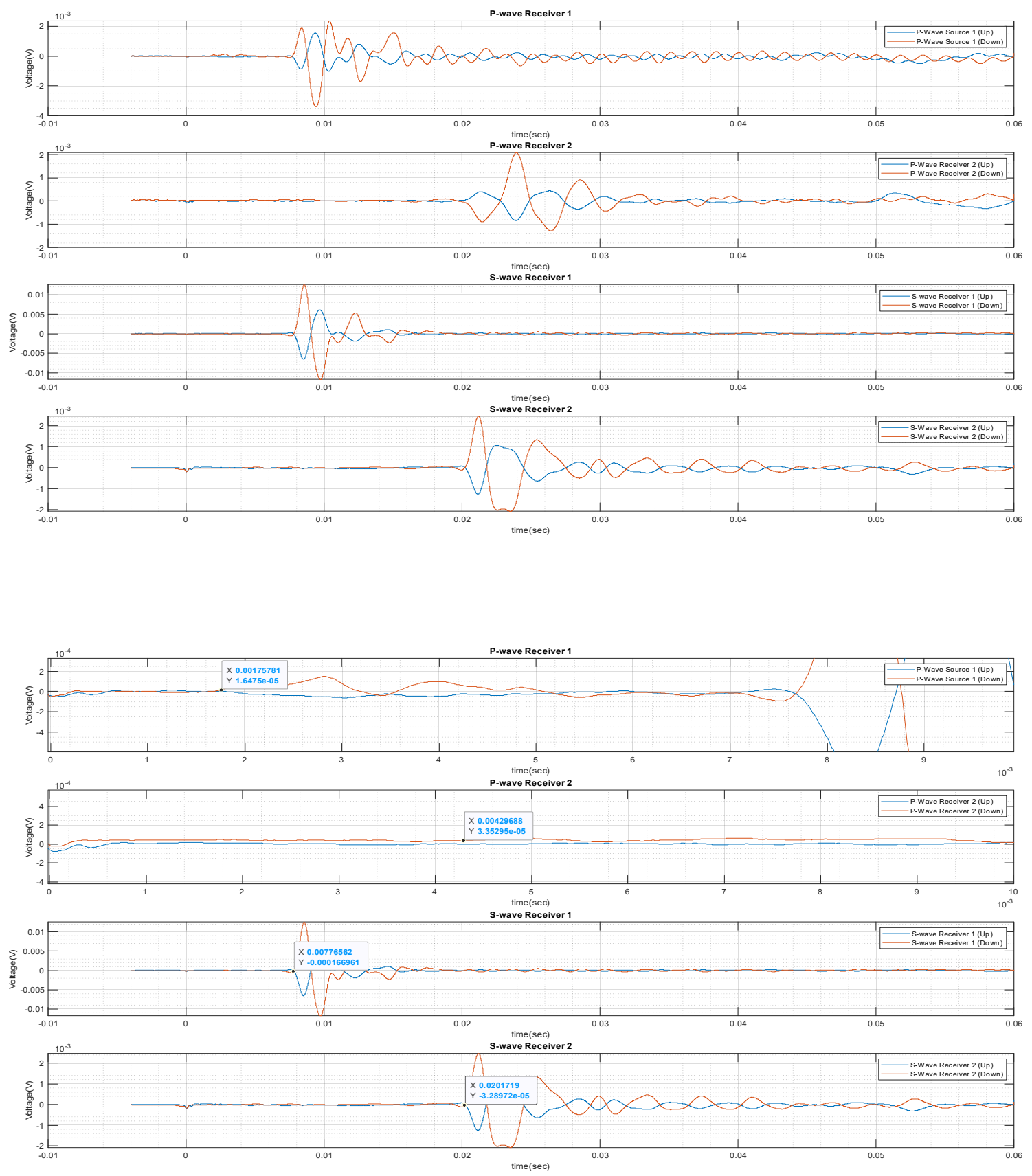

Data Set 28 (10.18.2019)

Run00215f

R4_1 

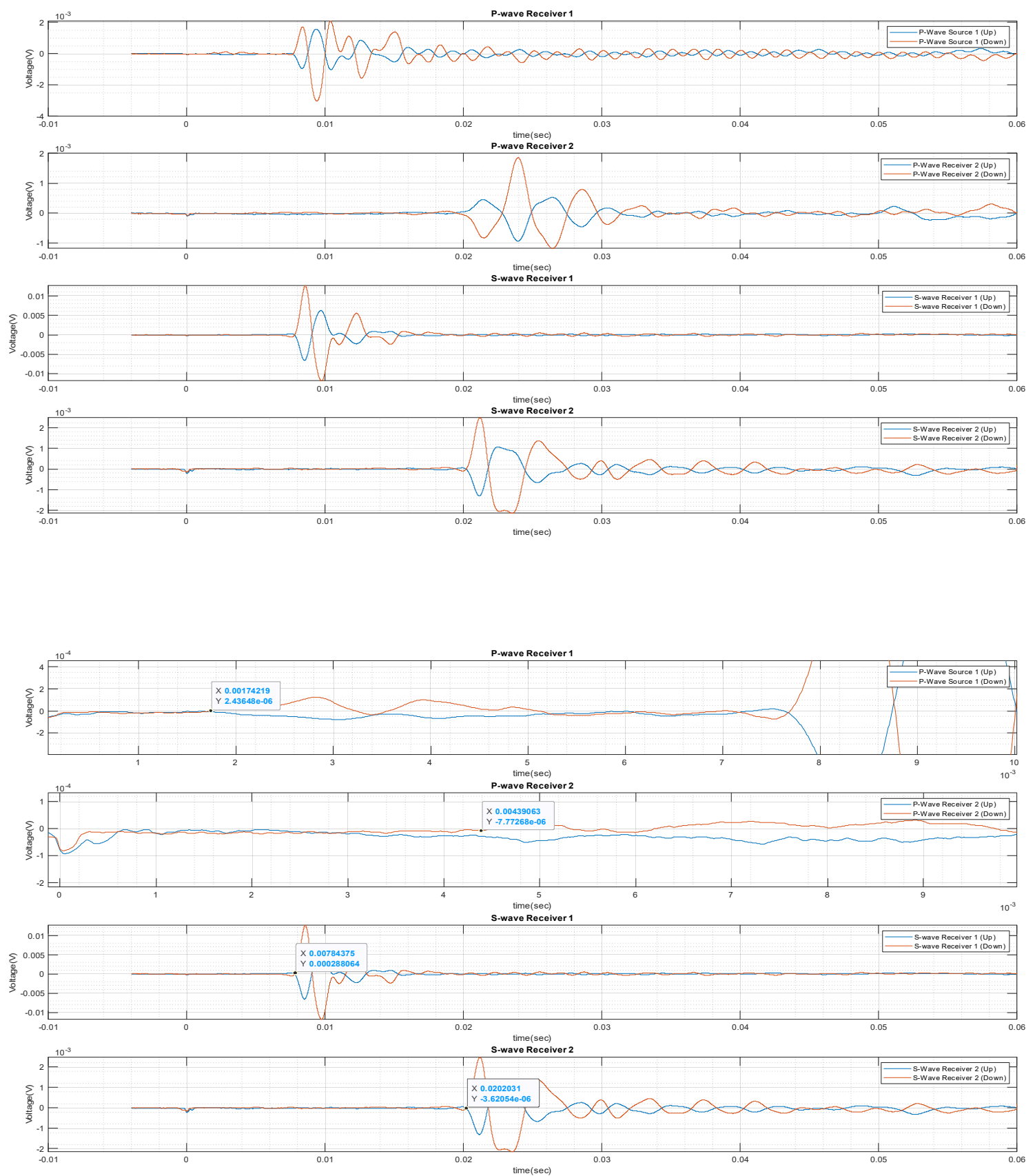

Data Set 28 (10.18.2019)

Run00216f

R4_2 

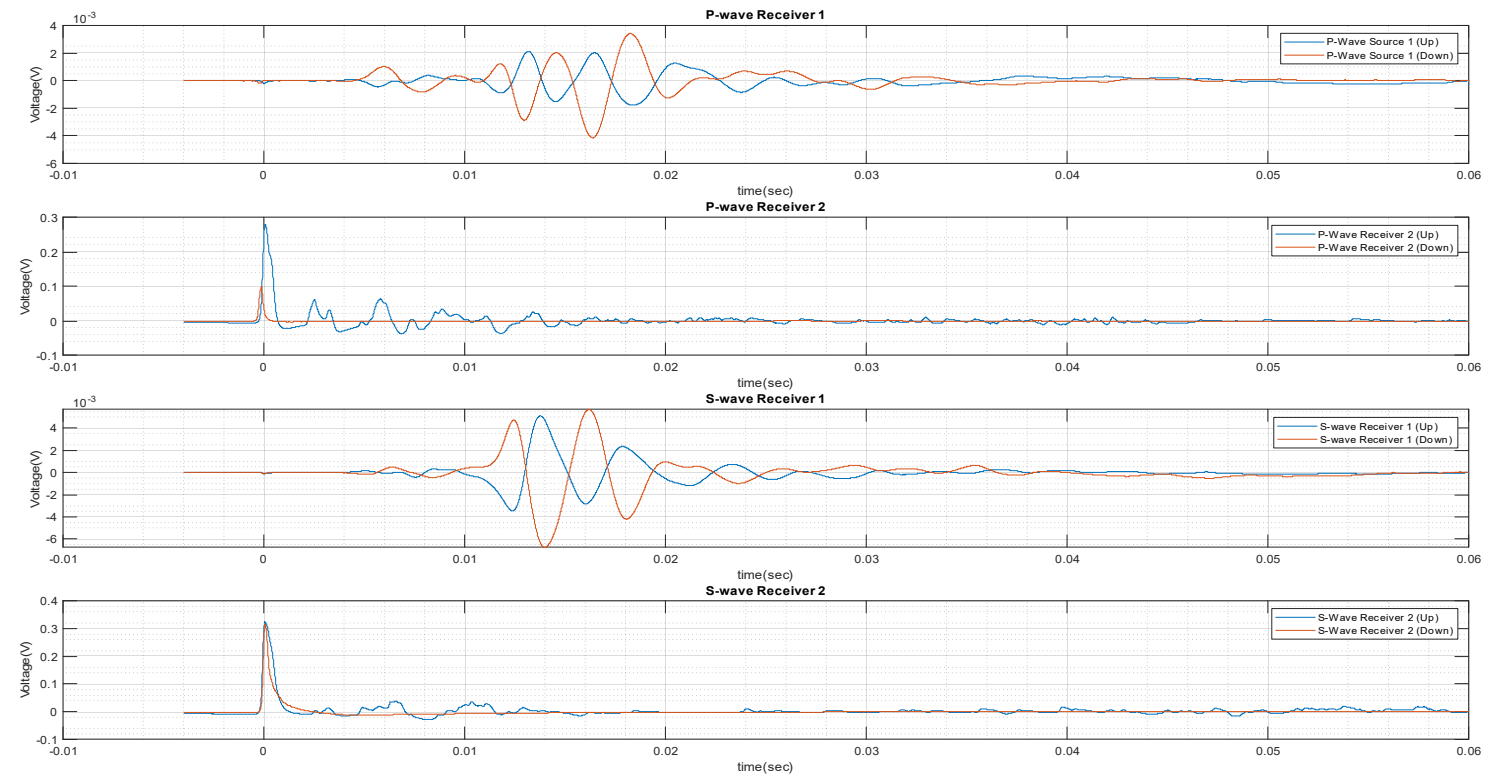

Cannot determine clear arrival times.

Data Set 29 (11.1.2019)

\section{Run00217f}

R1_1 

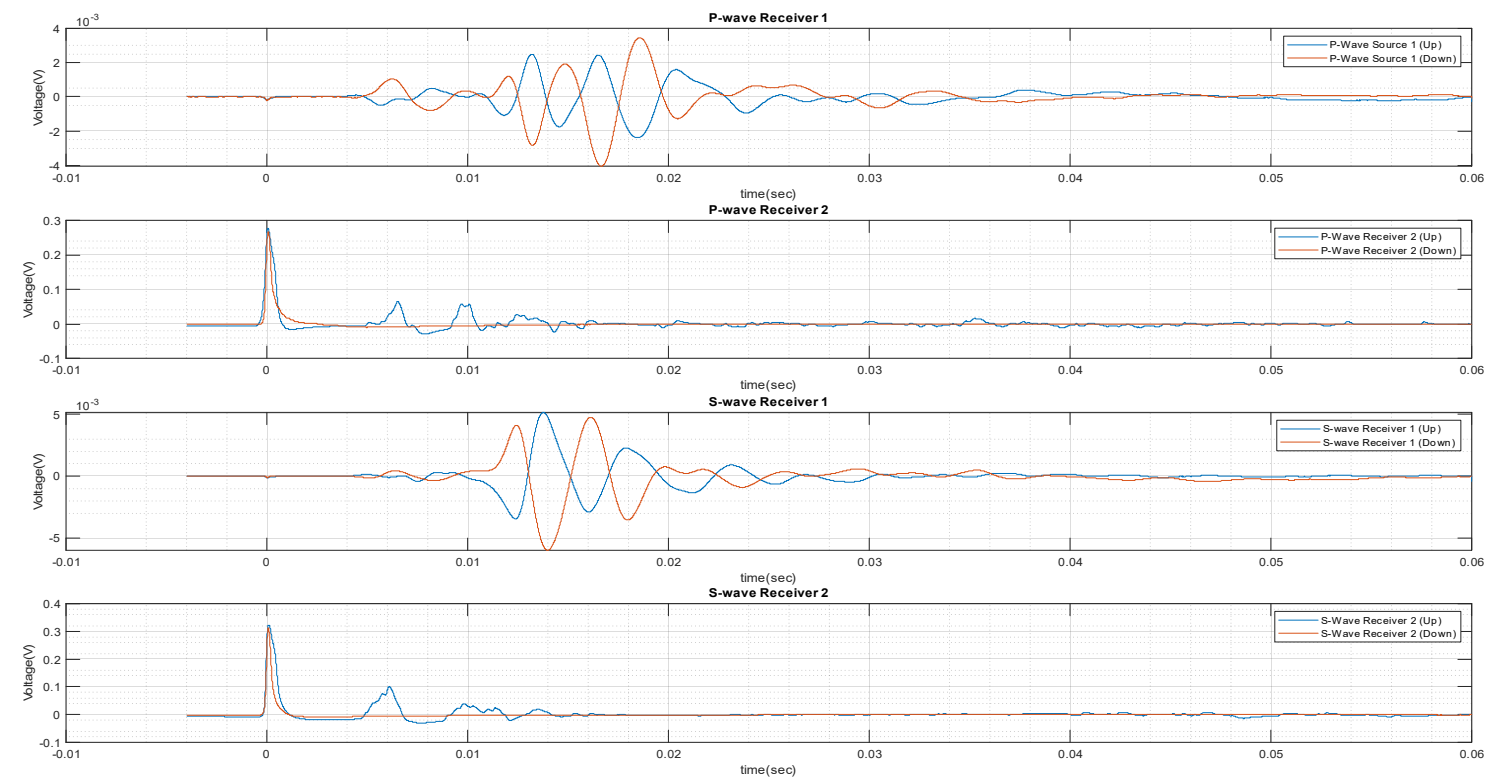

Cannot determine clear arrival times.

Data Set 29 (11.1.2019)

Run00218f

R1_2 

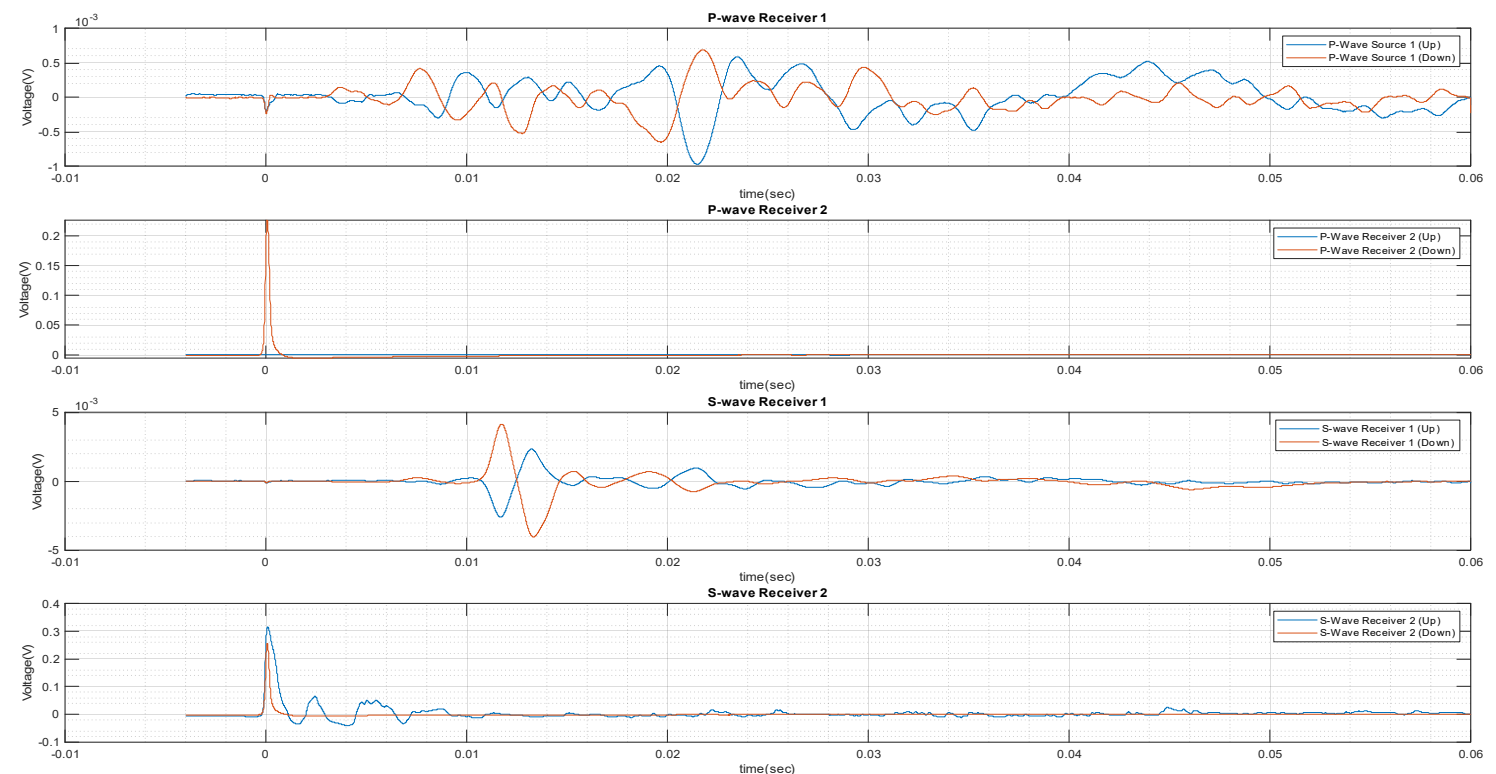

Cannot determine clear arrival times.

Data Set $29(11.1 .2019)$

Run00219f 
R2_1
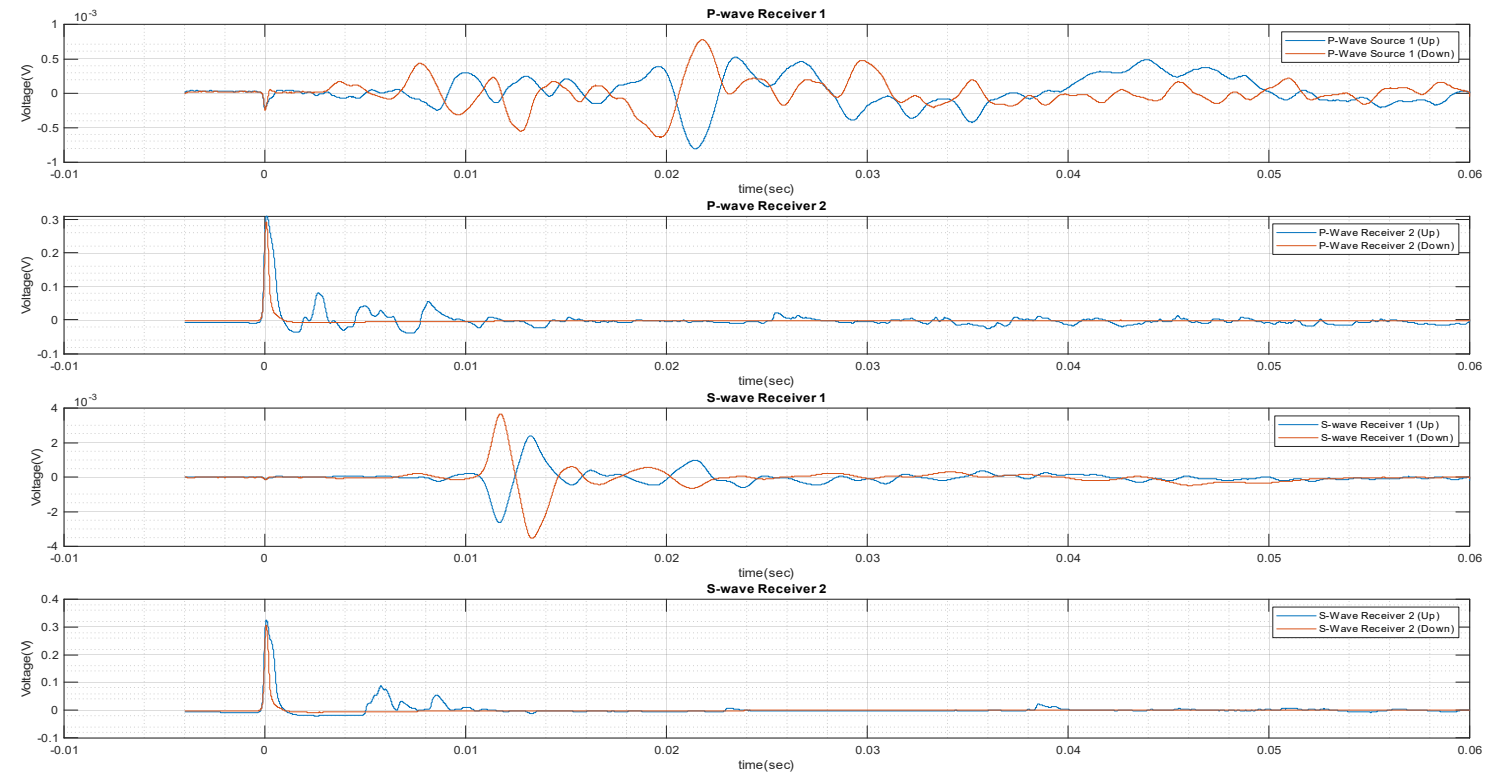

Cannot determine clear arrival times.

Data Set $29(11.1 .2019)$ 


\section{Run00220f}

R2_2
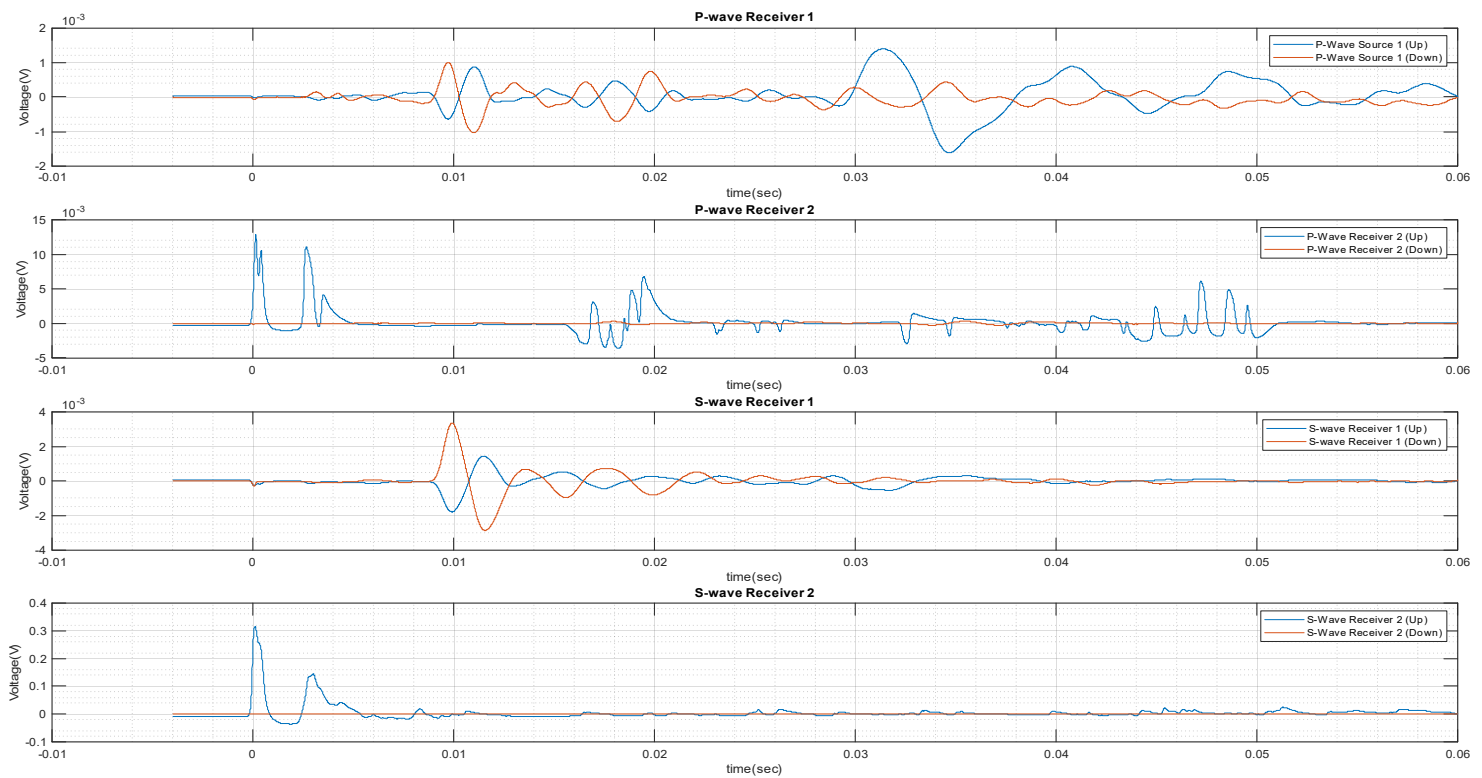

Cannot determine clear arrival times. 
Data Set 29 (11.1.2019)

Run00221f

R3_1
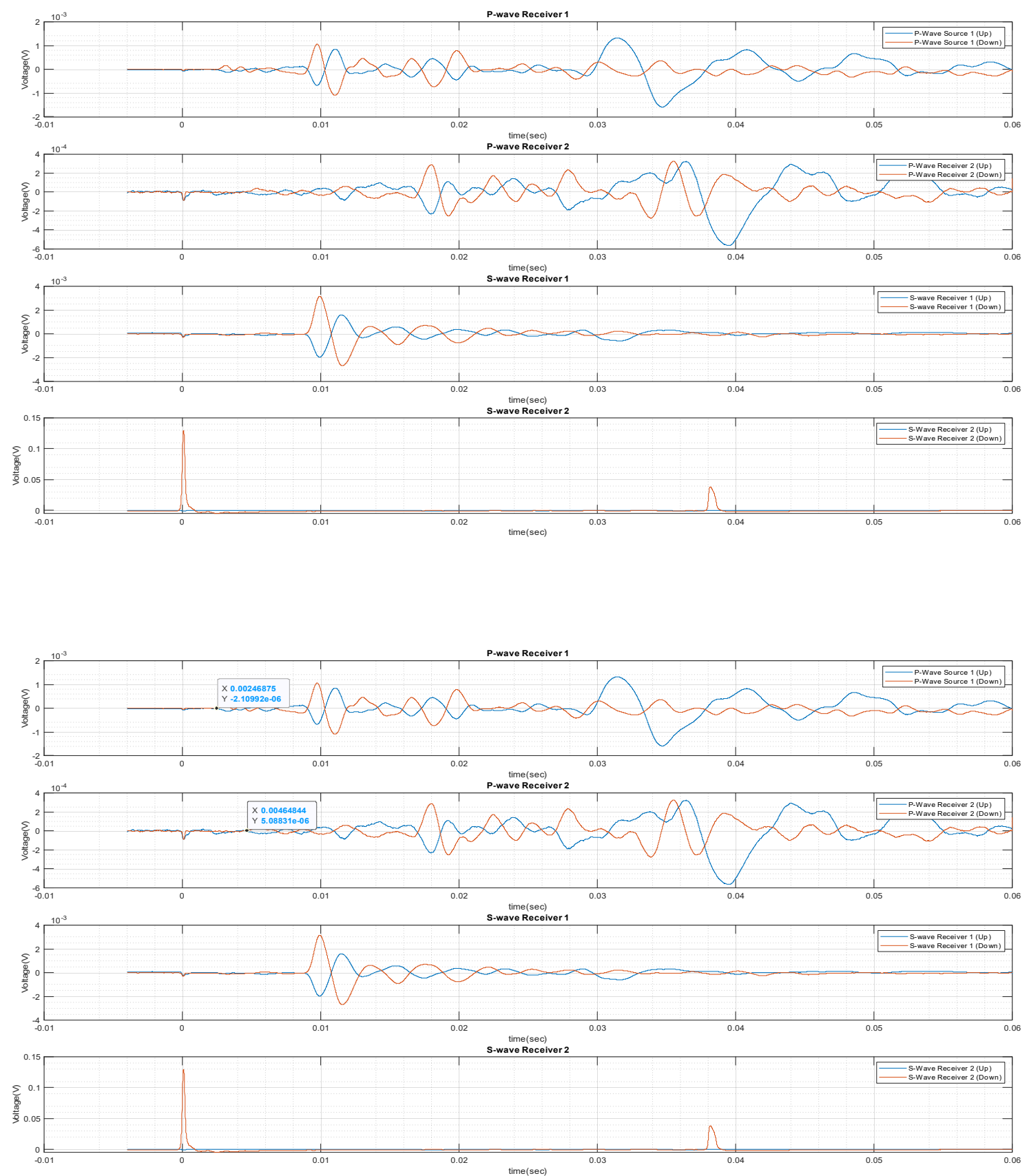


\section{Data Set 29 (11.1.2019)}

Run00222f

\section{R3 2}
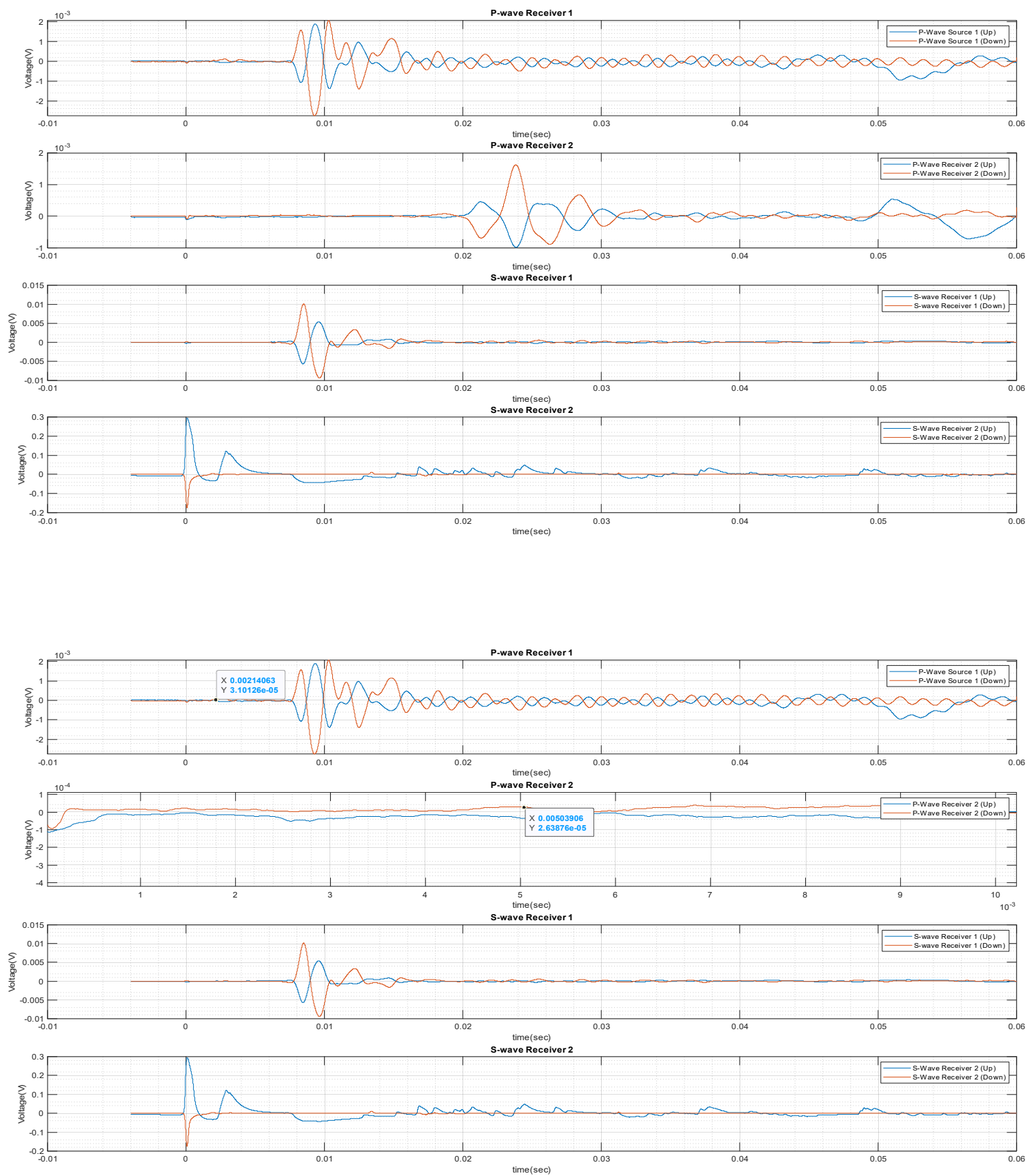


\section{Data Set 29 (11.1.2019.2019)}

Run00223f

R4_1
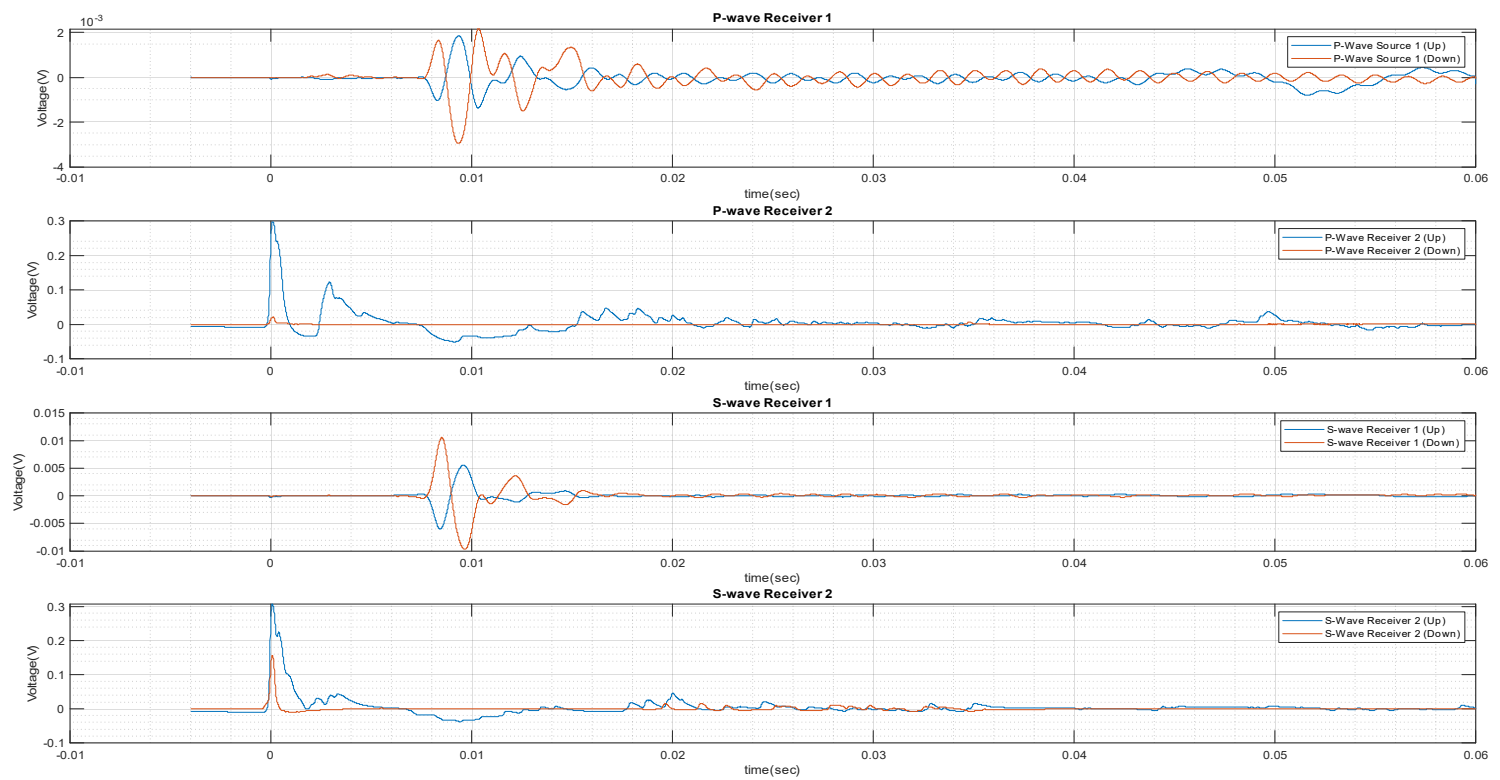

Cannot determine clear arrival times. 


\section{Data Set 29 (11.1.2019)}

Run00224f

R4_2
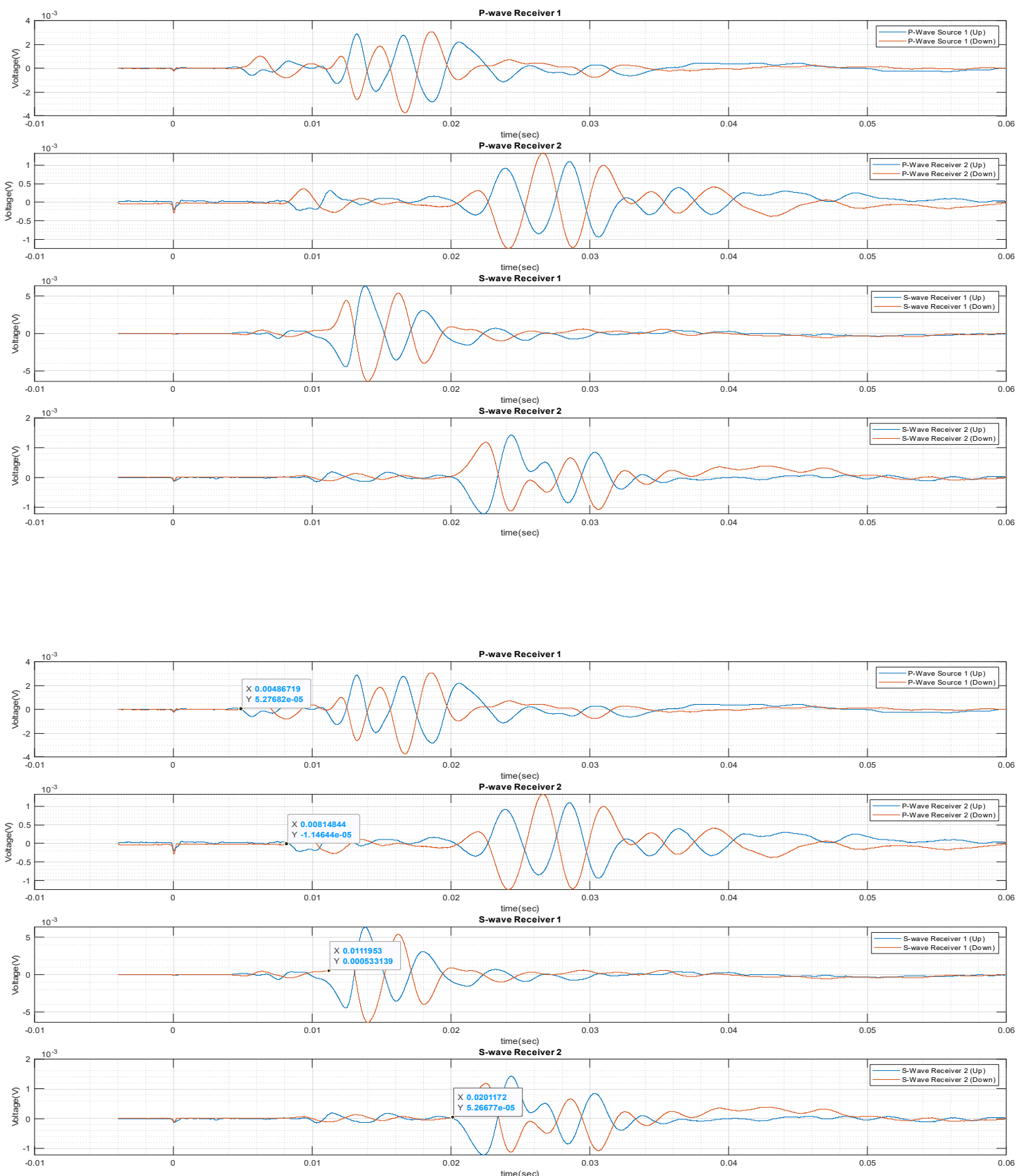
Data Set 30 (11.2.2019)

Run00225f

R1_1
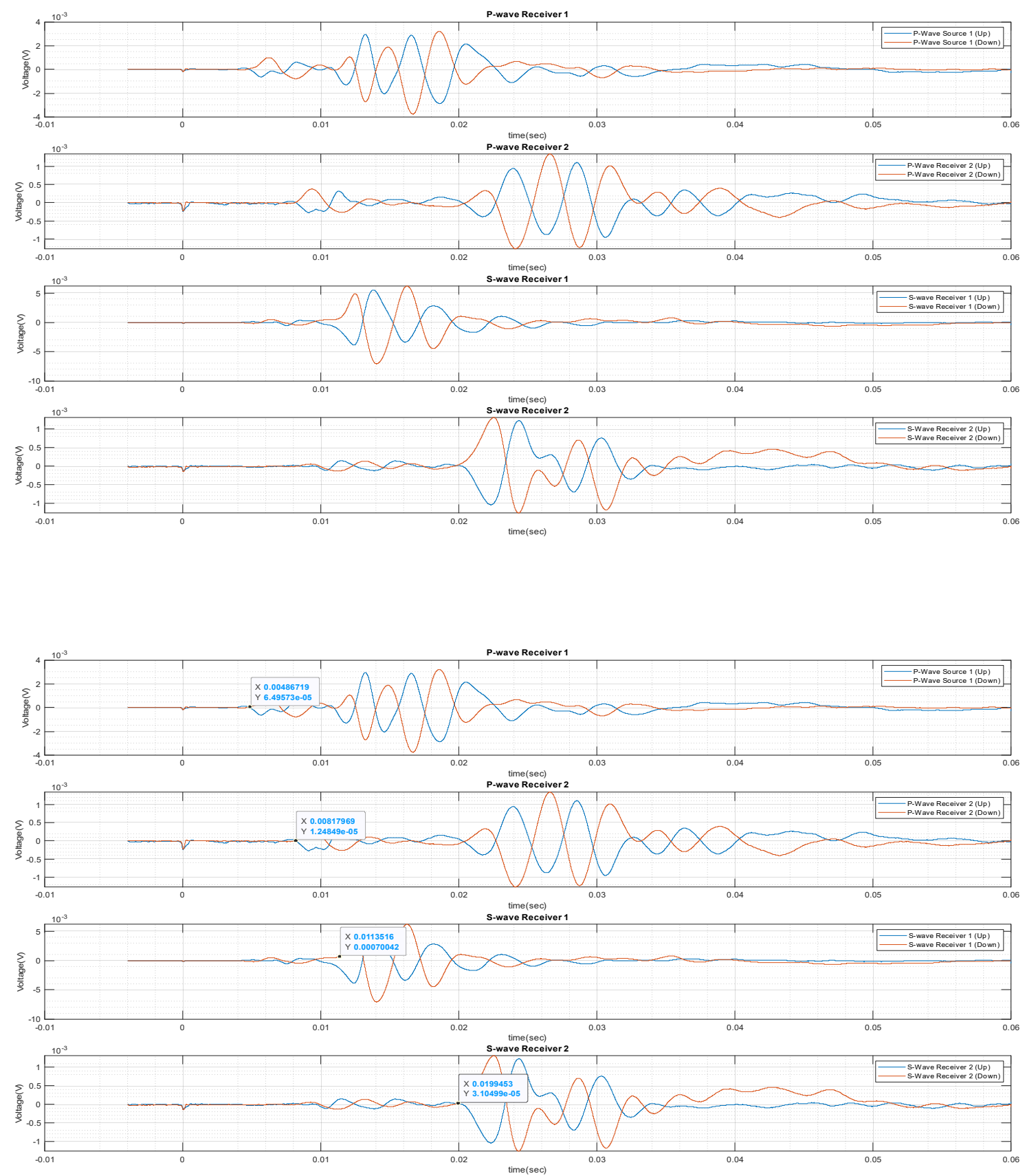

Data Set 30 (11.2.2019) 


\section{Run00226f}

R1_2

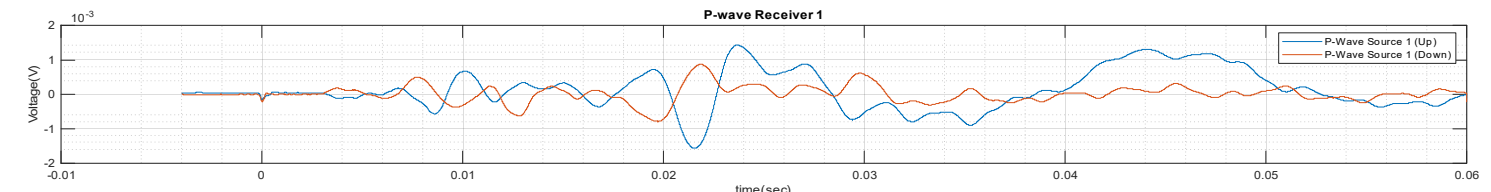

time(sec)
P-wave Receiver
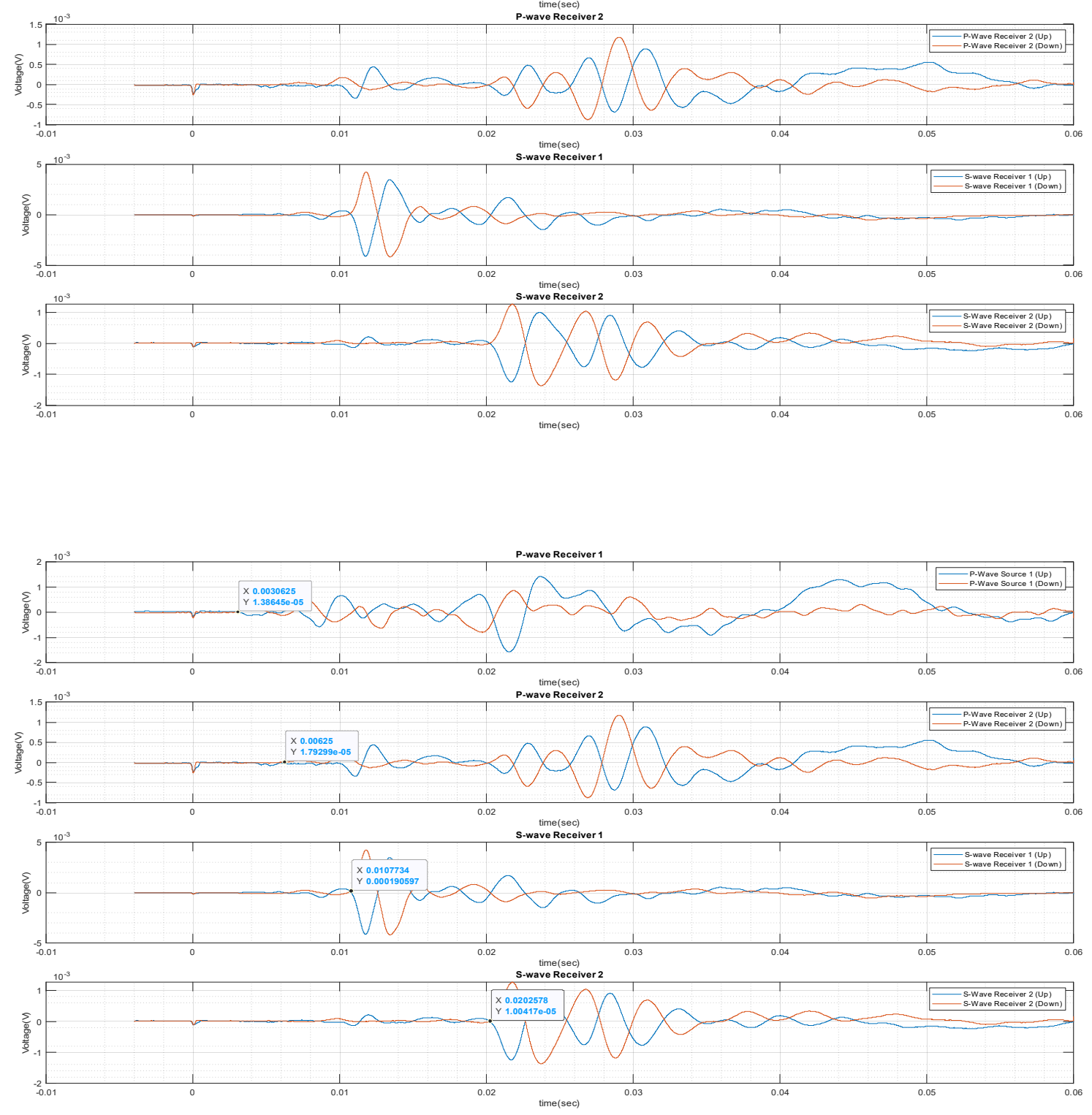

Data Set 30 (11.2.2019)

Run00227f 

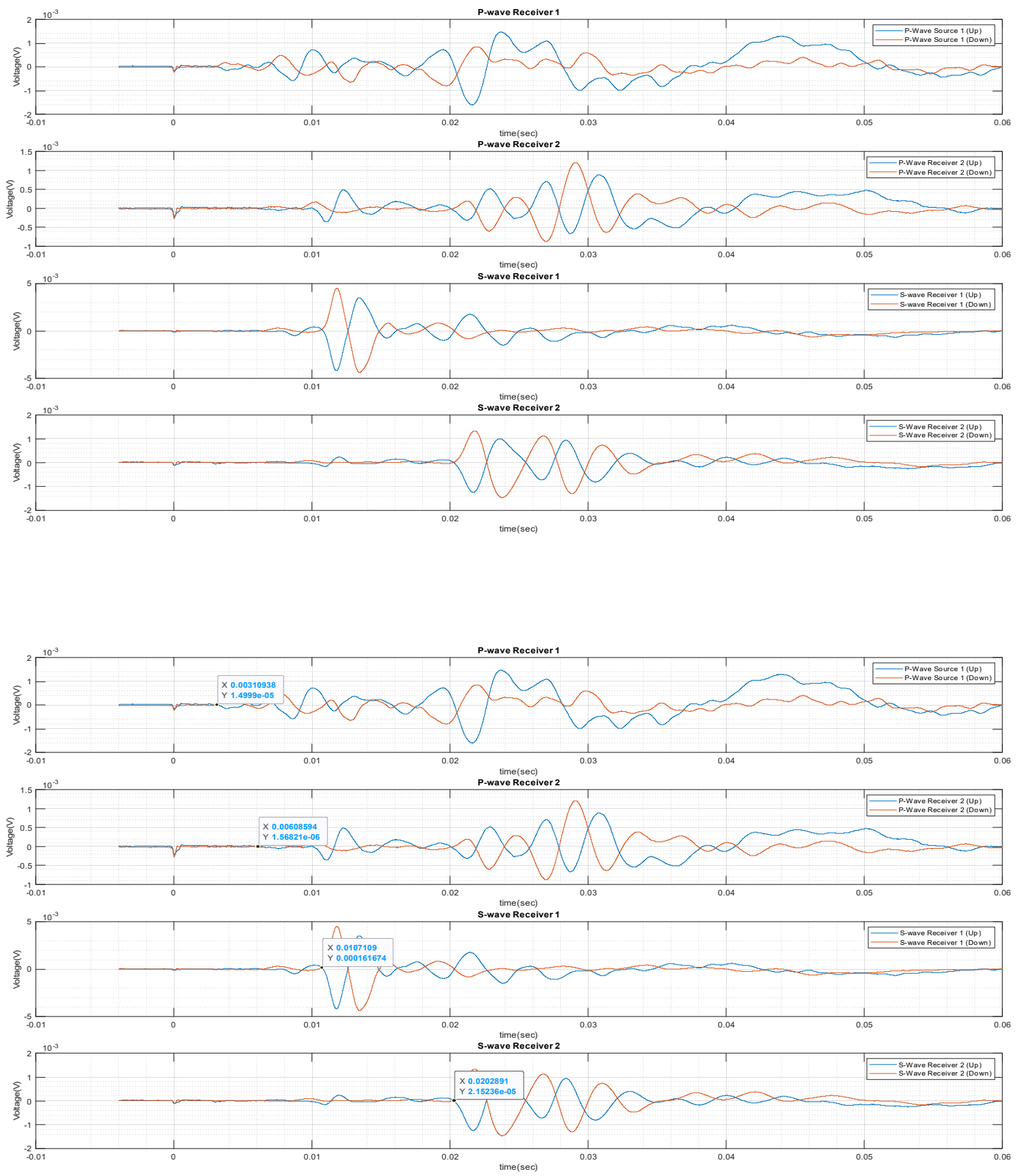

Data Set 30 (11.2.2019)

Run00228f

R2_2 

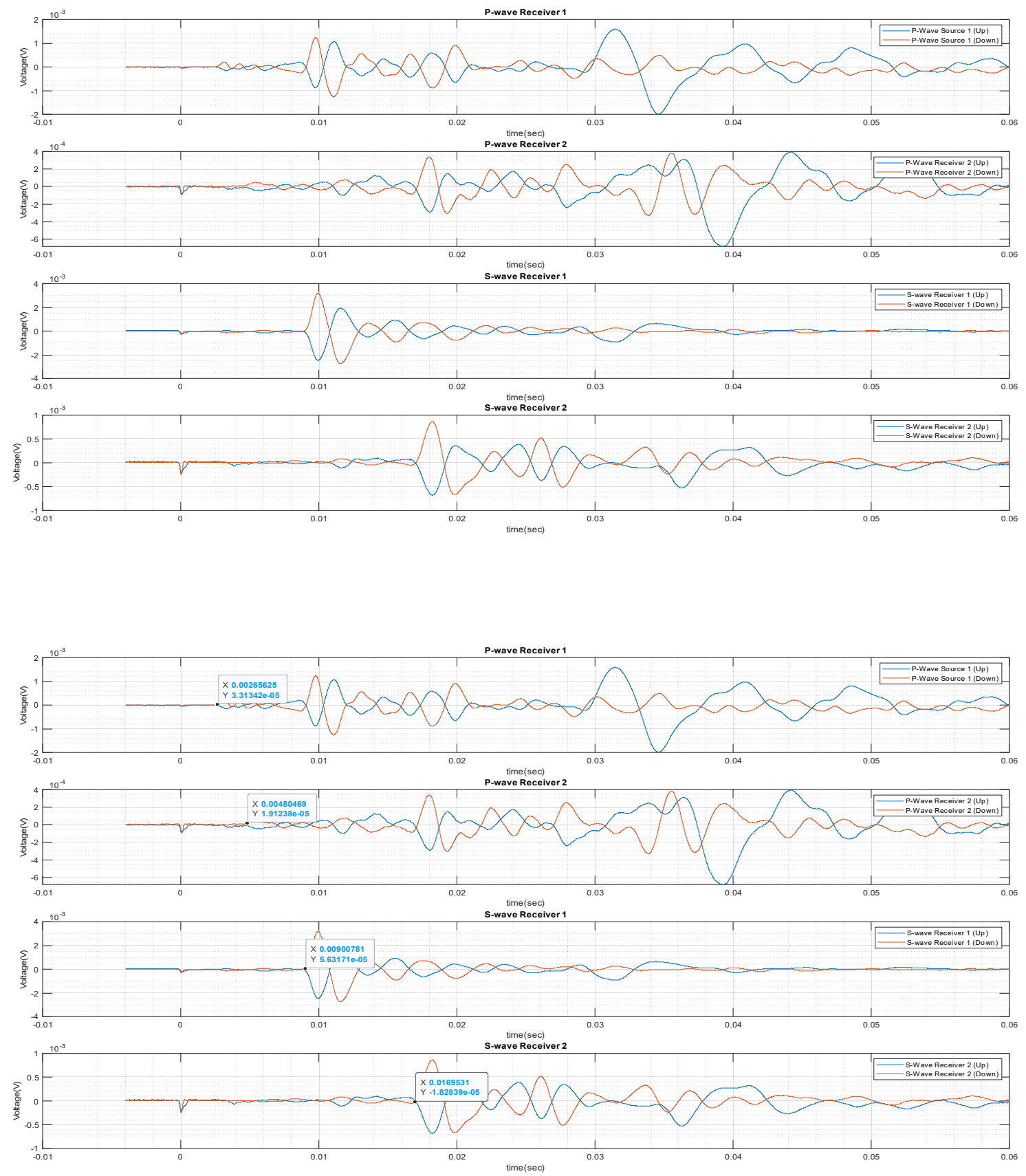

Data Set 30 (11.2.2019)

Run00229f

R3_1 

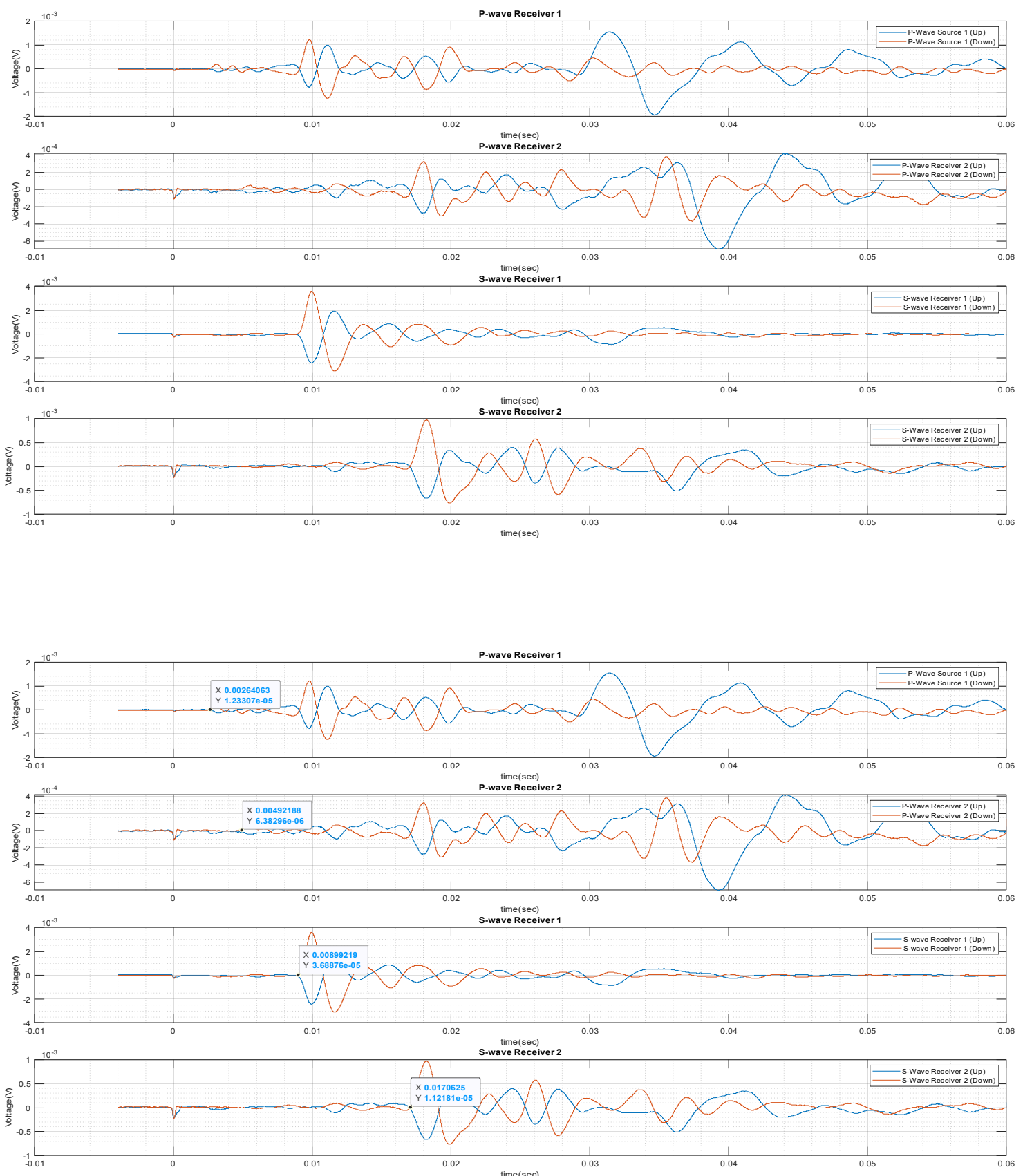

Data Set 30 (11.2.2019)

Run00230f

R3_2 

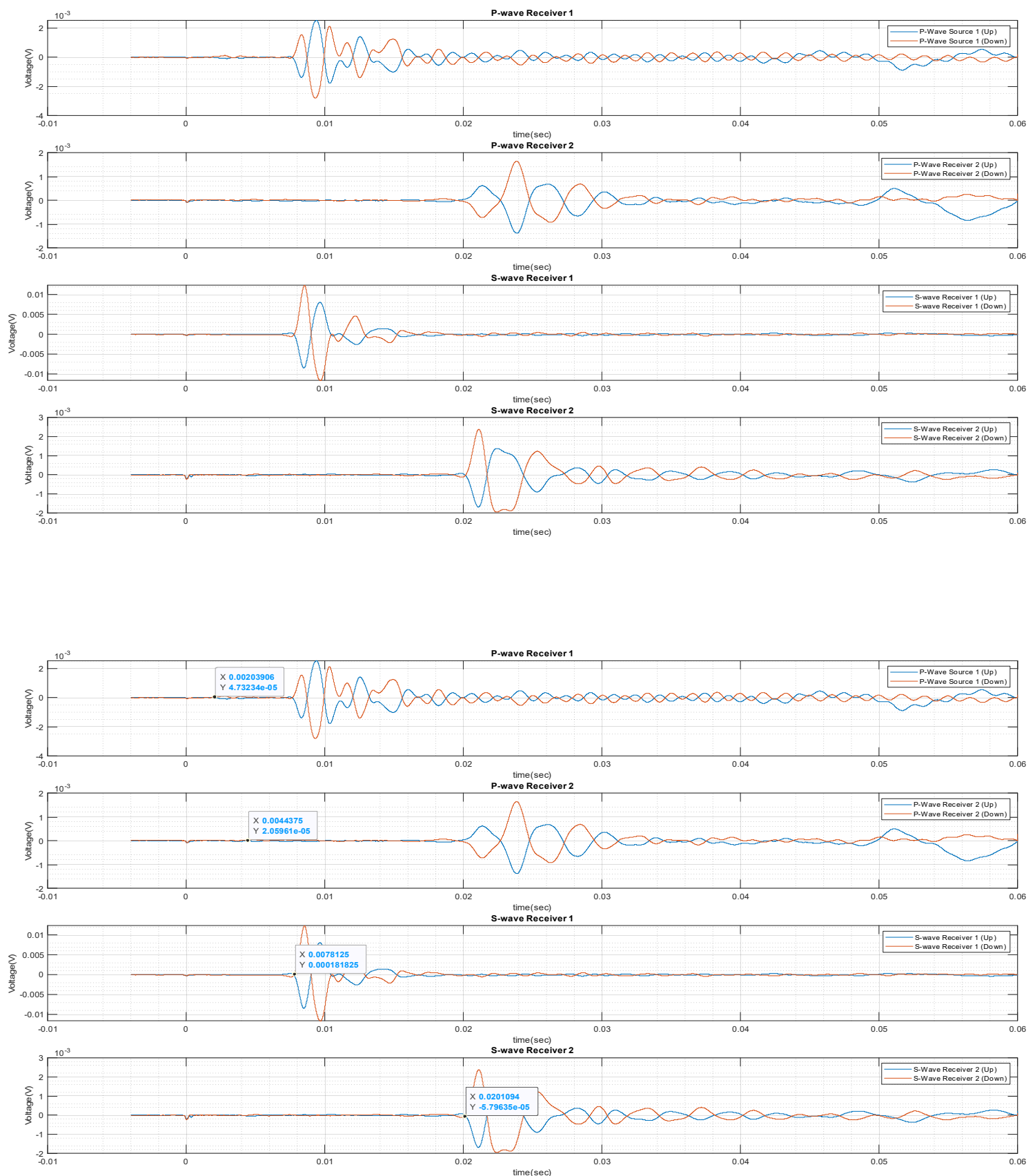

Data Set 30 (11.2.2019)

Run00231f

R4_1 

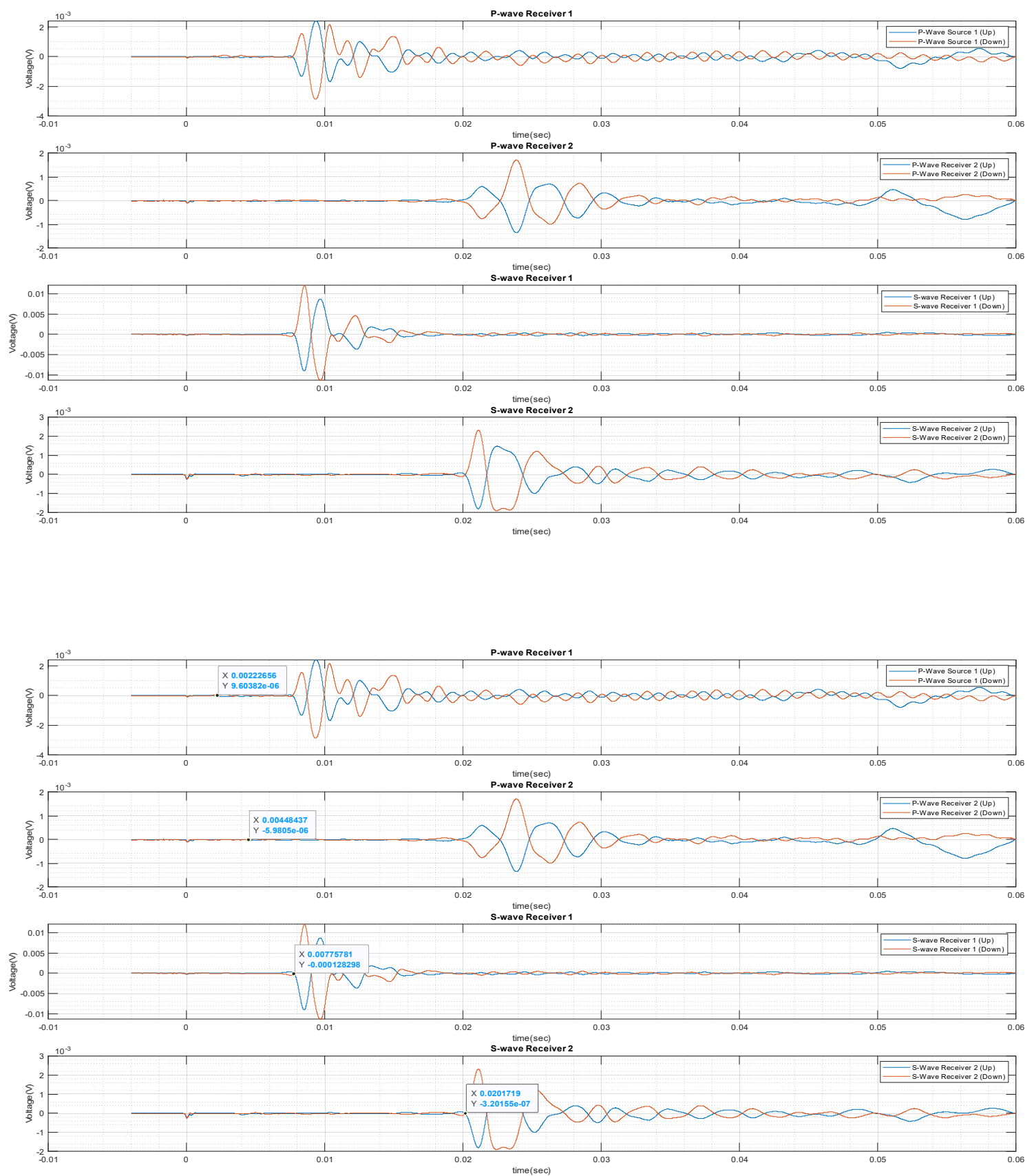

Data Set 30 (11.2.2019)

Run00232f

R4_2 

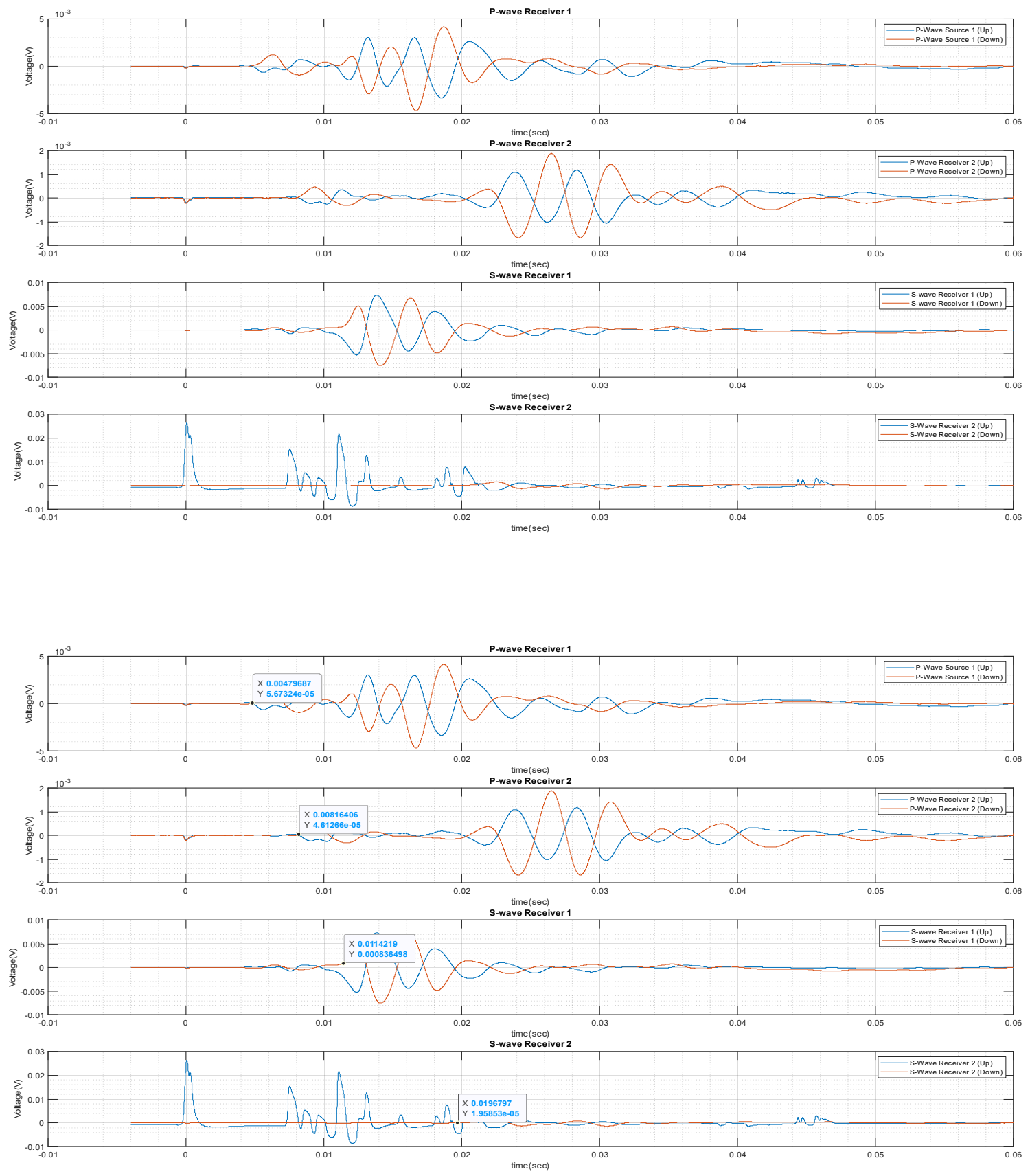

Data Set 31 (11.7.2019)

Run00233f

R1_1 

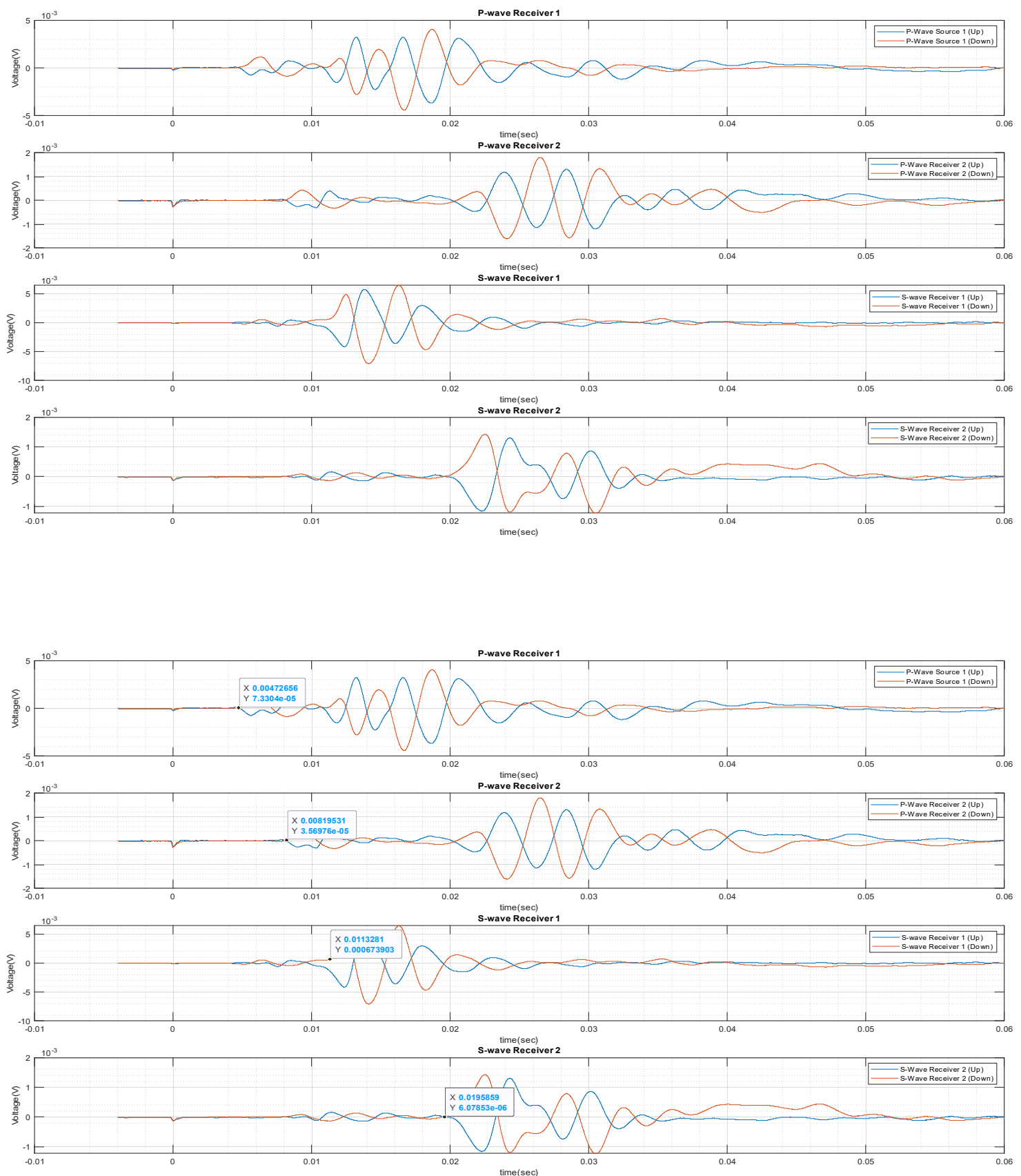

Data Set 31 (11.7.2019)

Run00234f

R1_2 

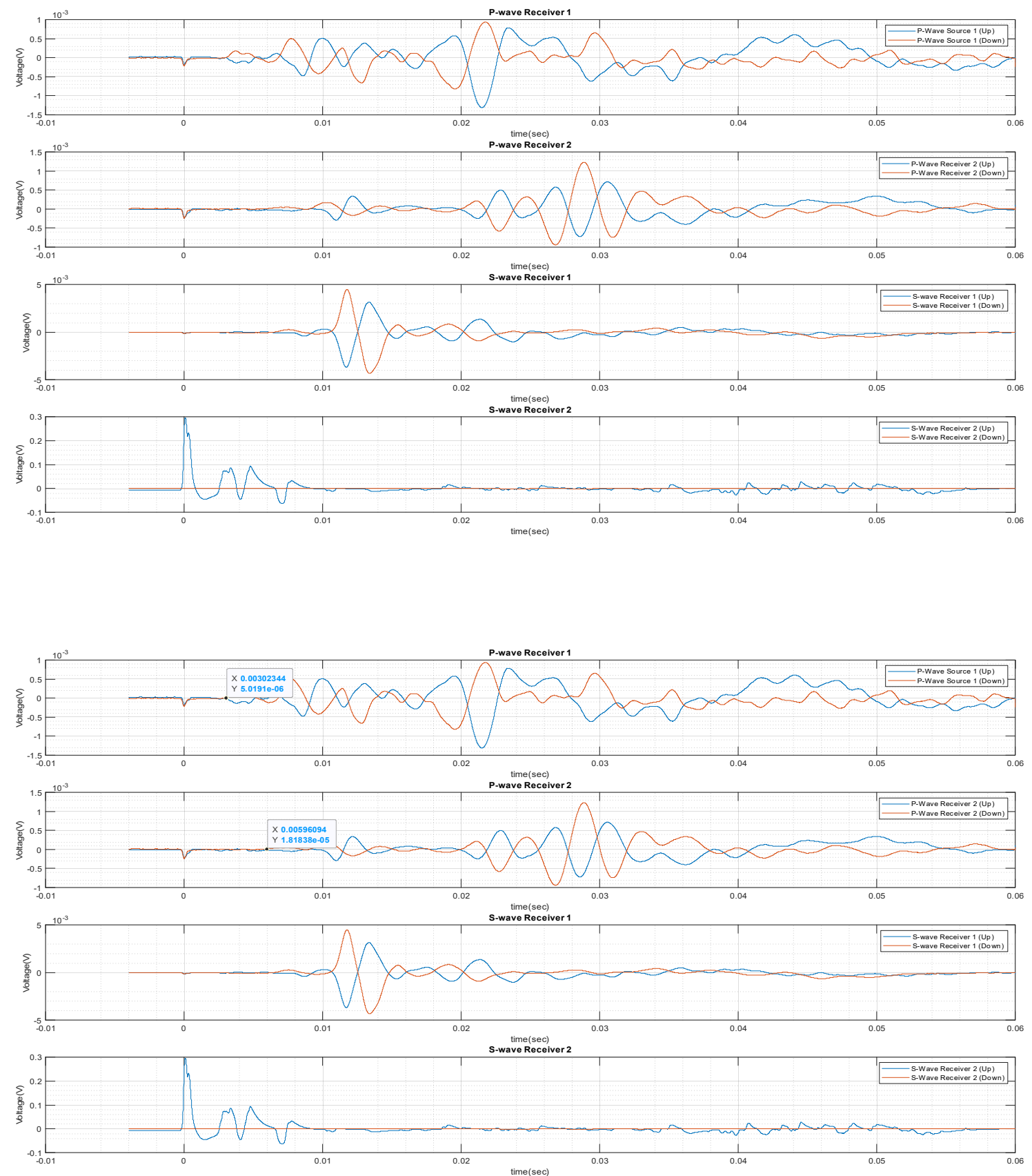

Data Set 31 (11.7.2019)

Run00235f

R2_1 

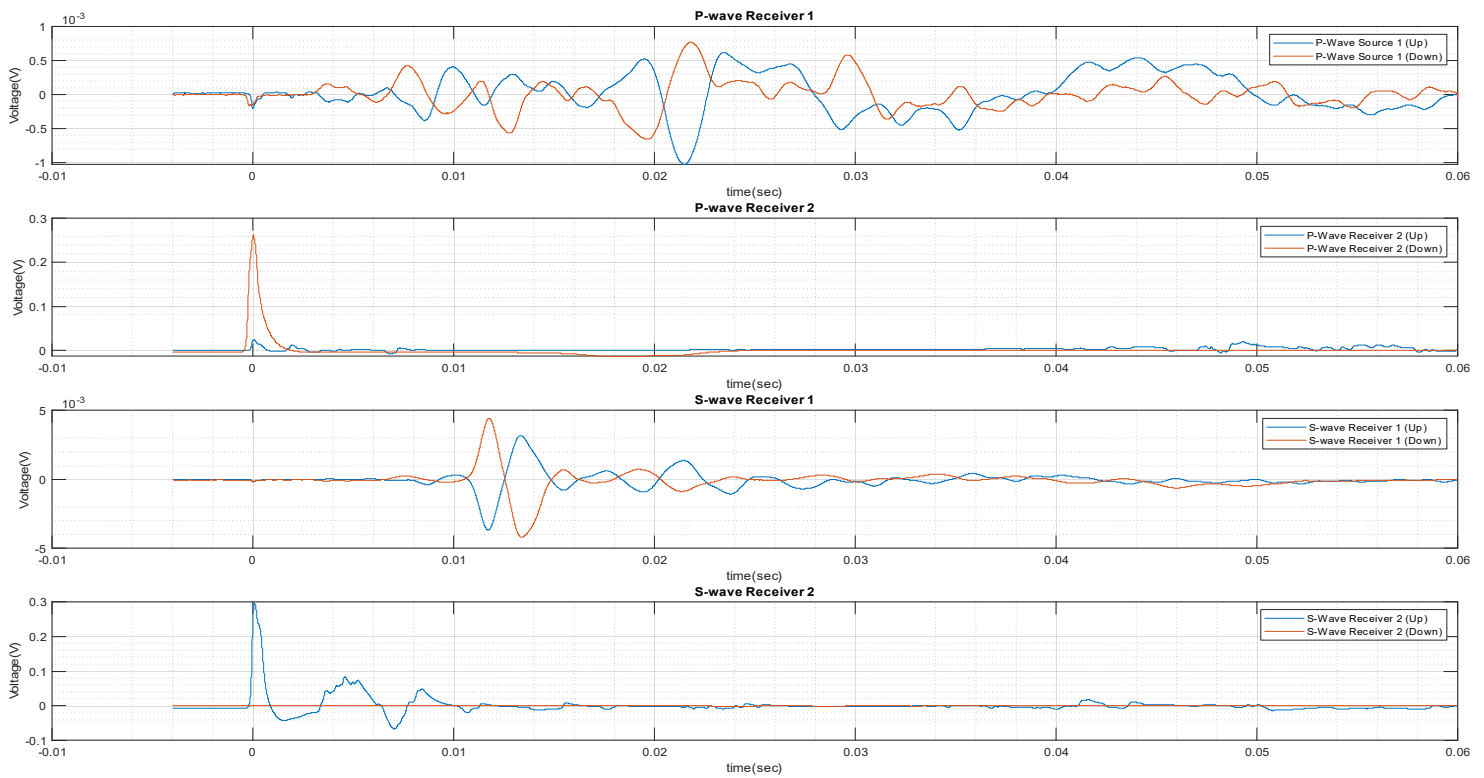

Cannot determine clear arrival times.

Data Set $31(11.7 .2019)$

Run00236f

R2 2 

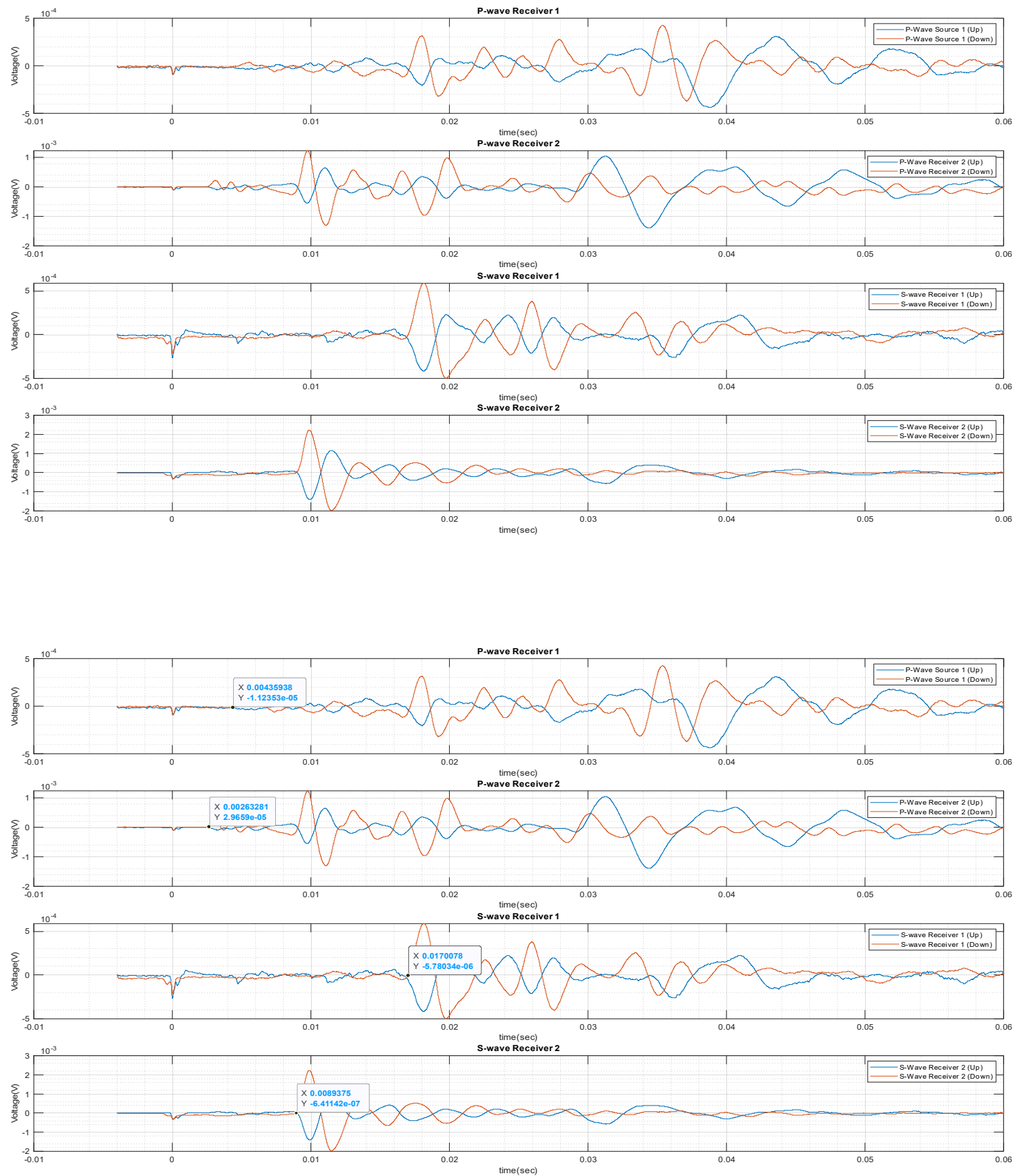

Data Set 31 (11.7.2019)

Run00237f

R3_1 

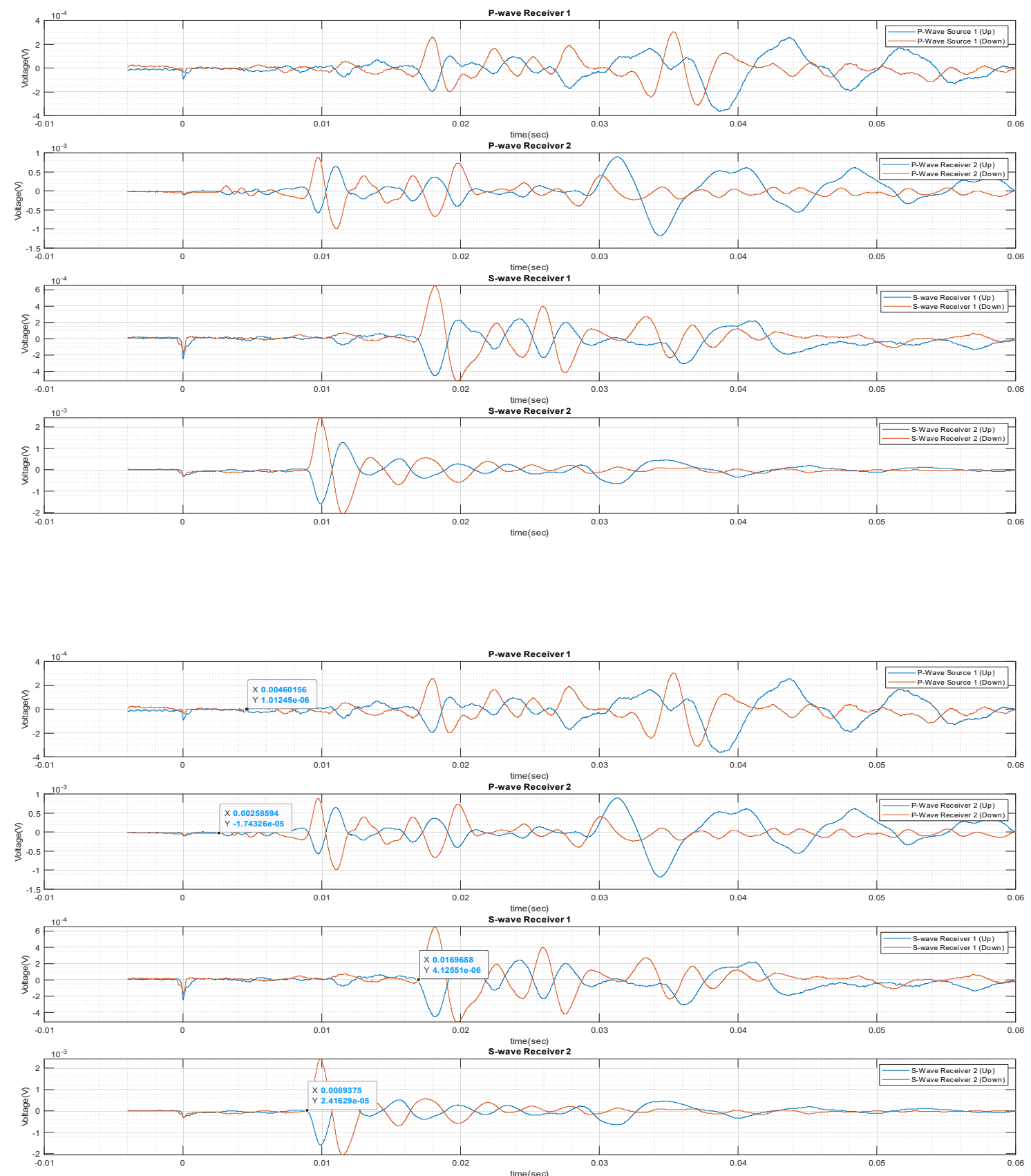

Data Set 31 (11.7.2019)

Run00238f

R3_2 

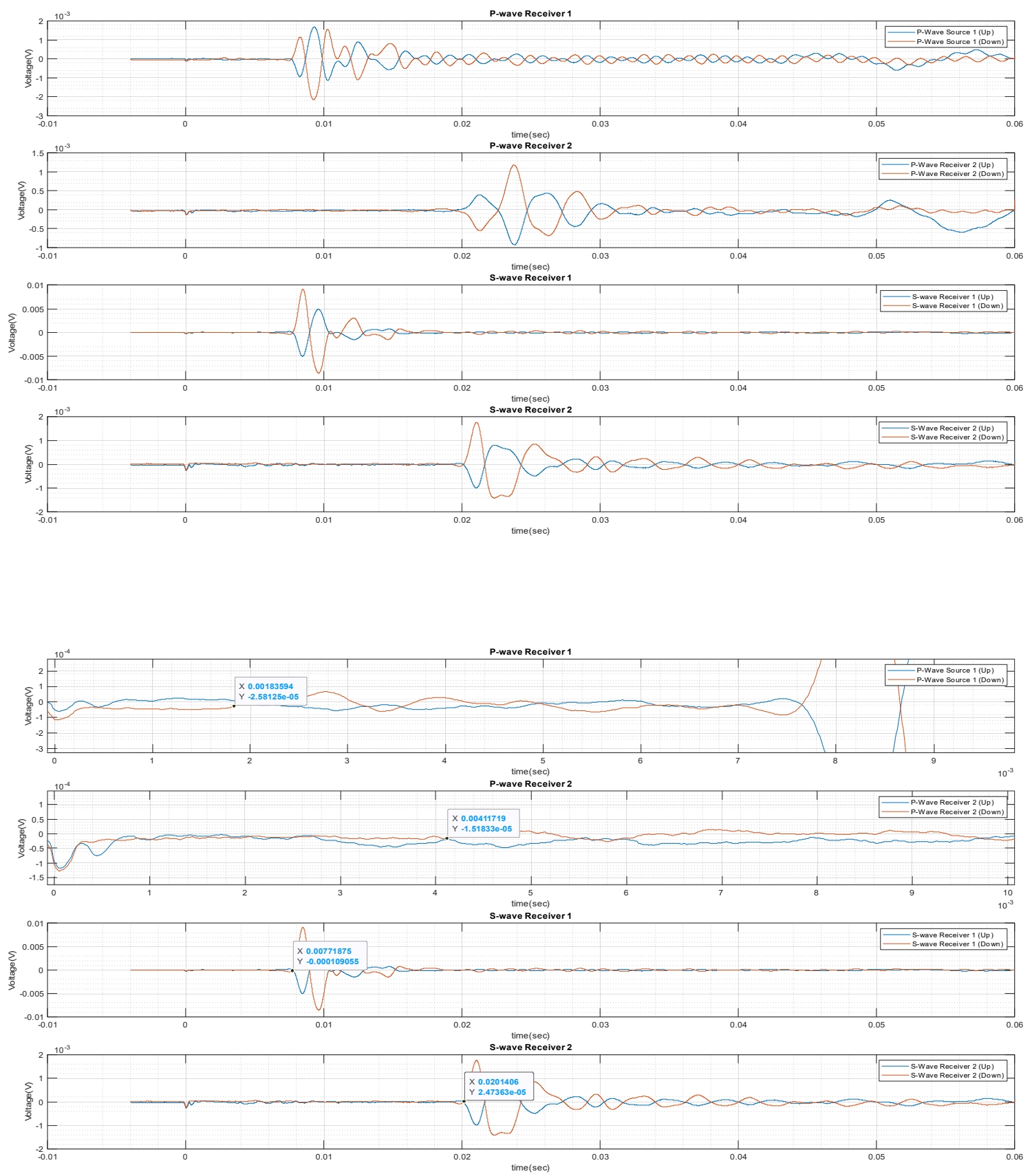

Data Set 31 (11.7.2019)

Run00239f

R4_1 

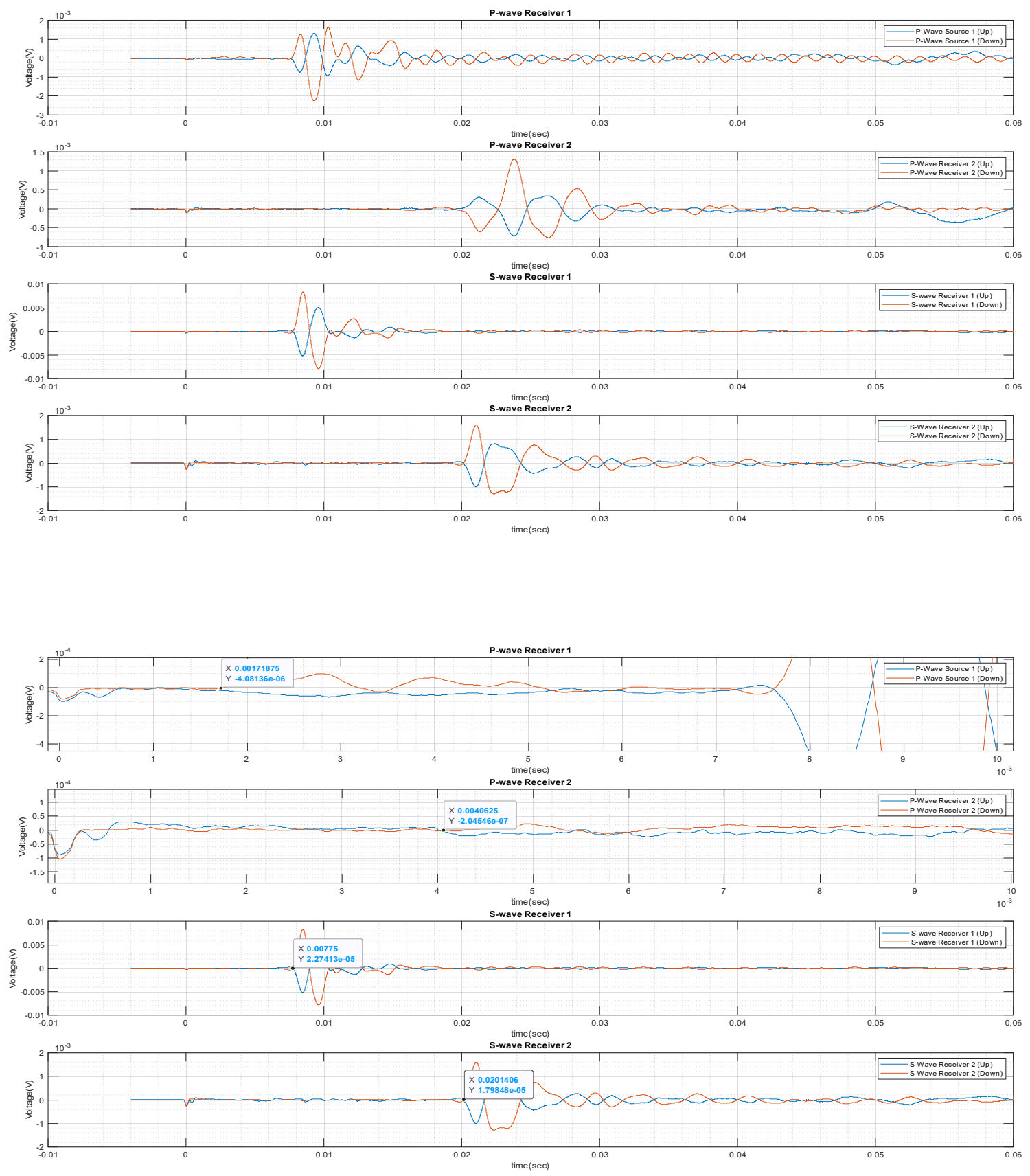

Data Set 31 (11.7.2019)

Run00240f

R4_2 

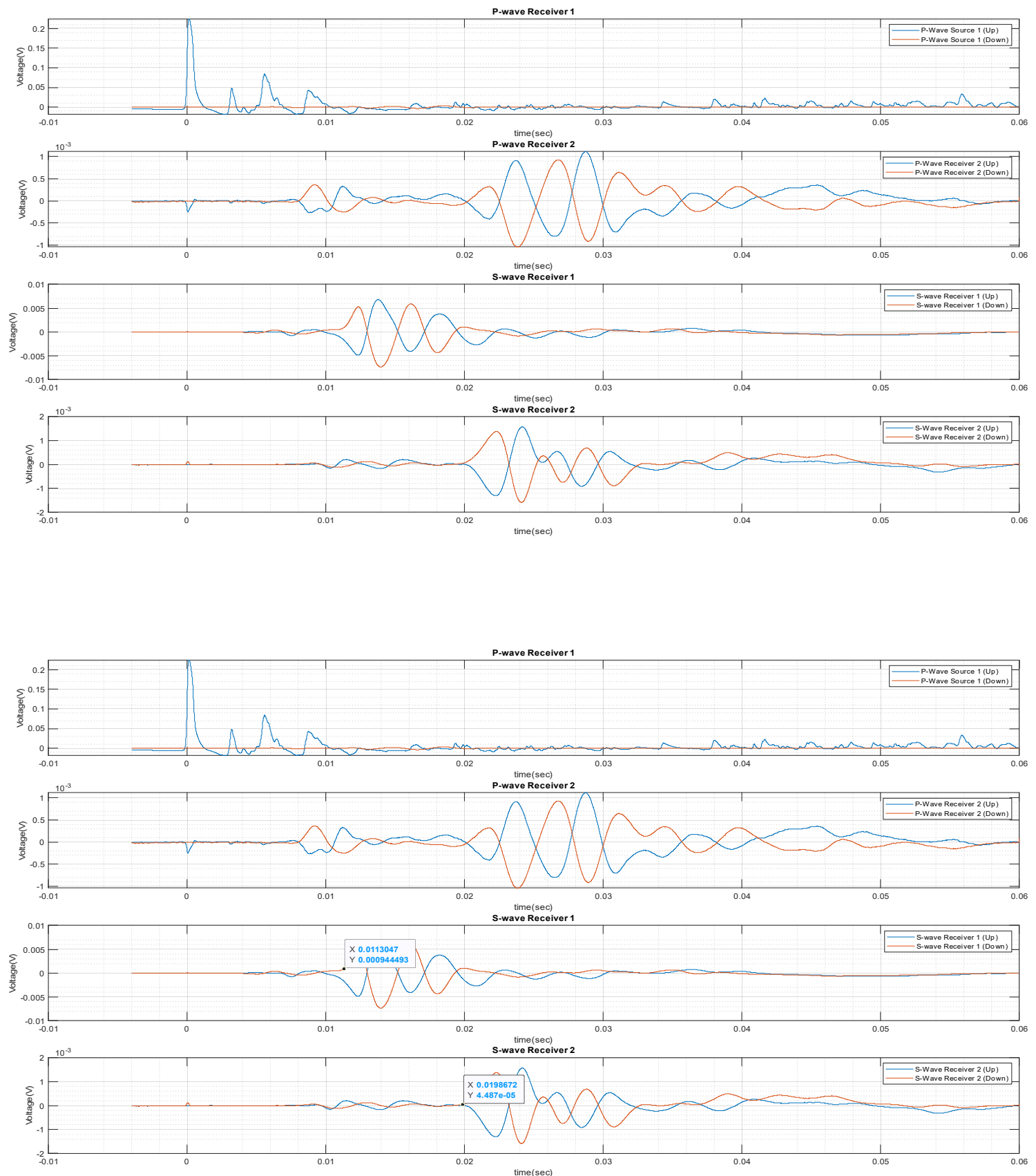

Data Set 32 (11.22.2019)

Run00241f

R1_1 

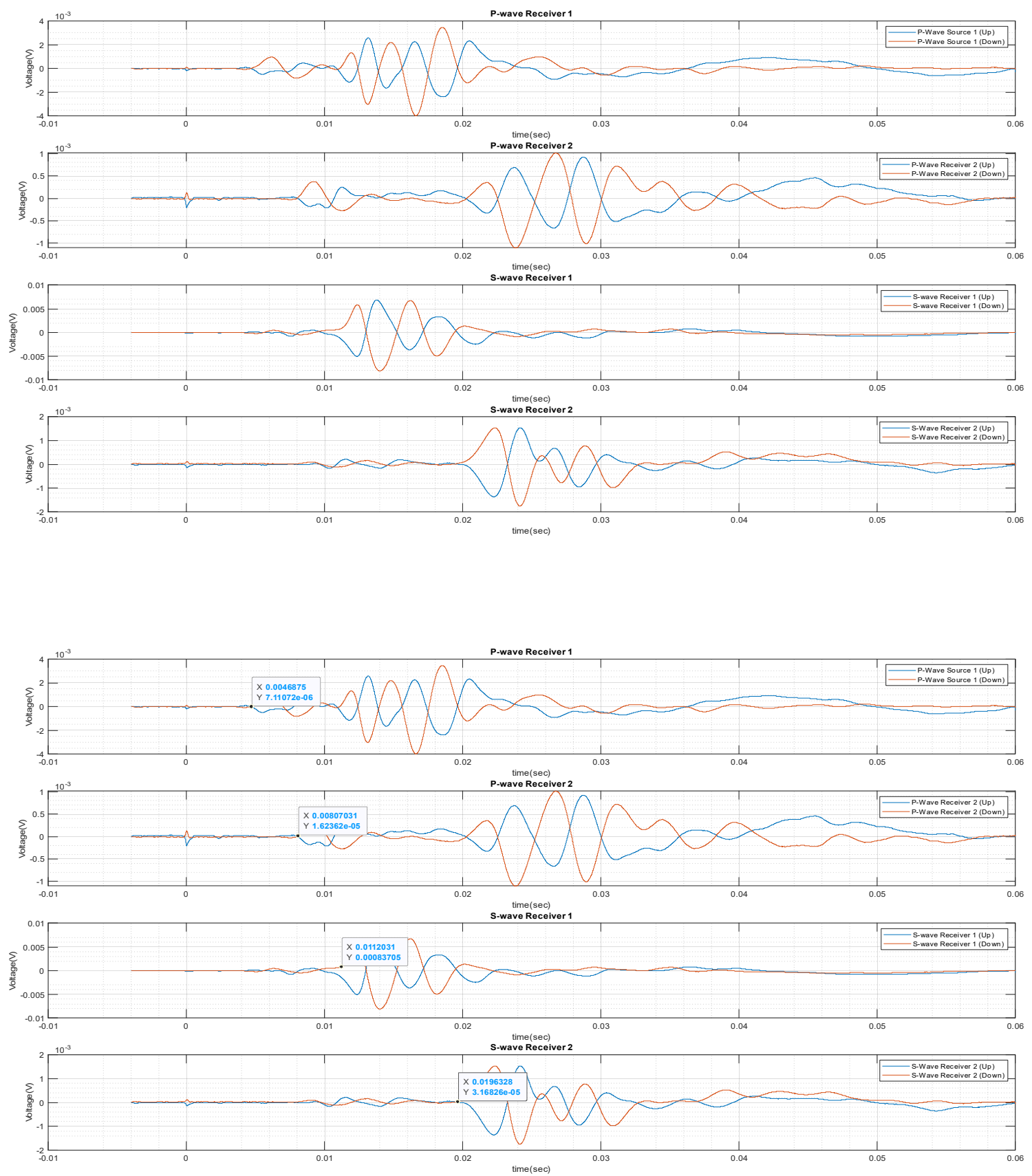

Data Set 32 (11.22.2019)

Run00242f

R1_2 

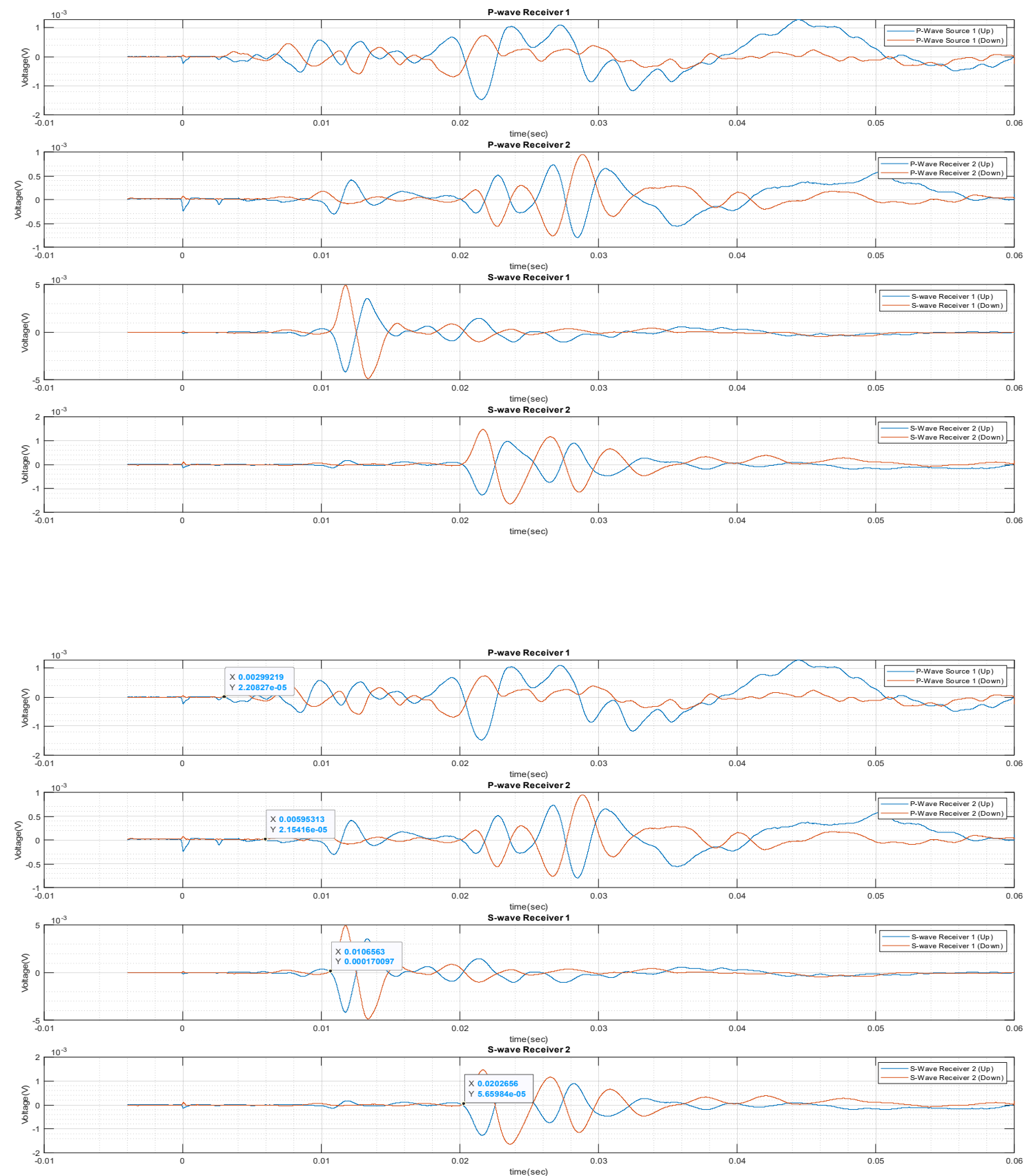

Data Set 32 (11.22.2019)

Run00243f

R2_1 

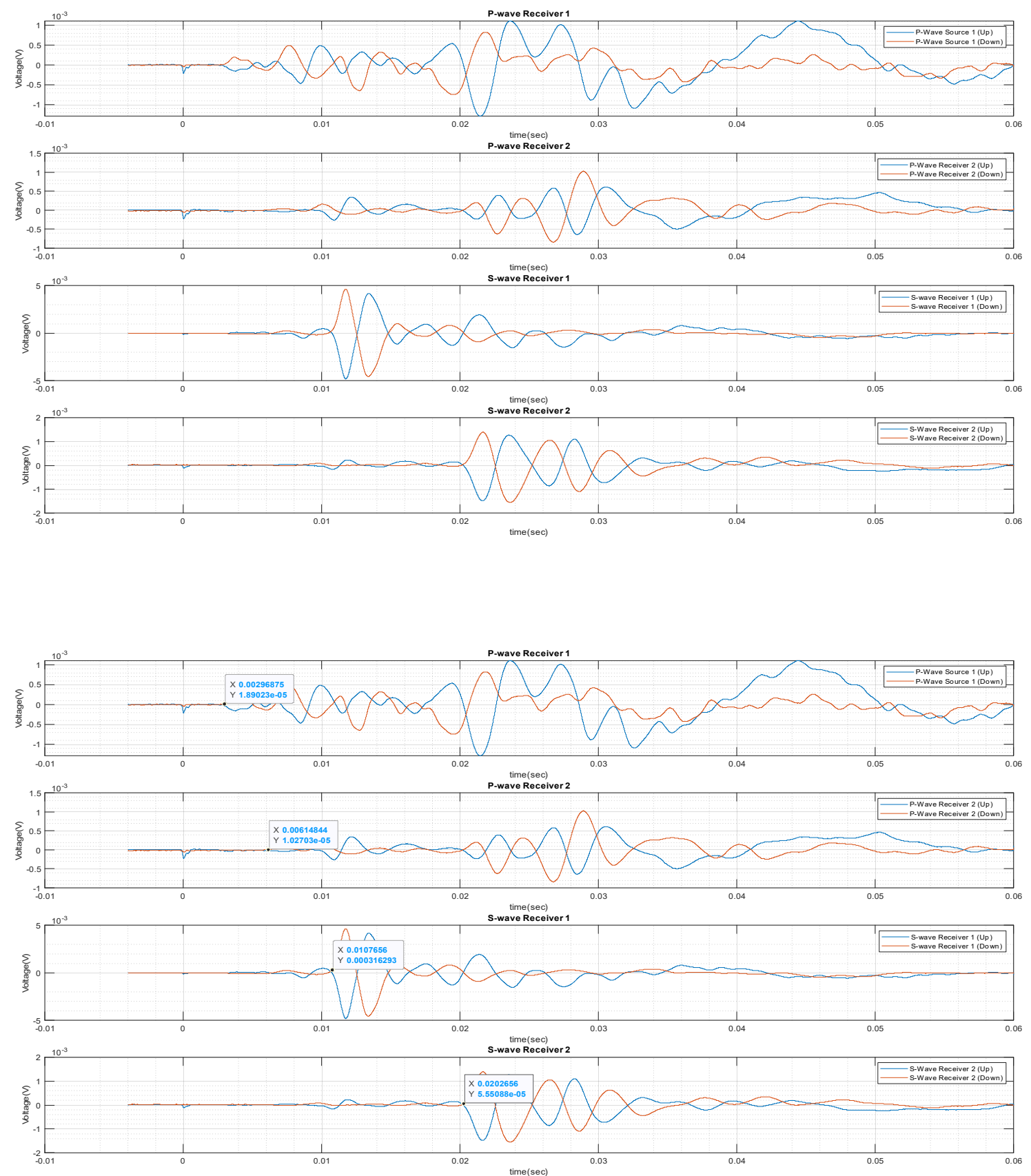

Data Set 32 (11.22.2019)

Run00244f

R2_2 

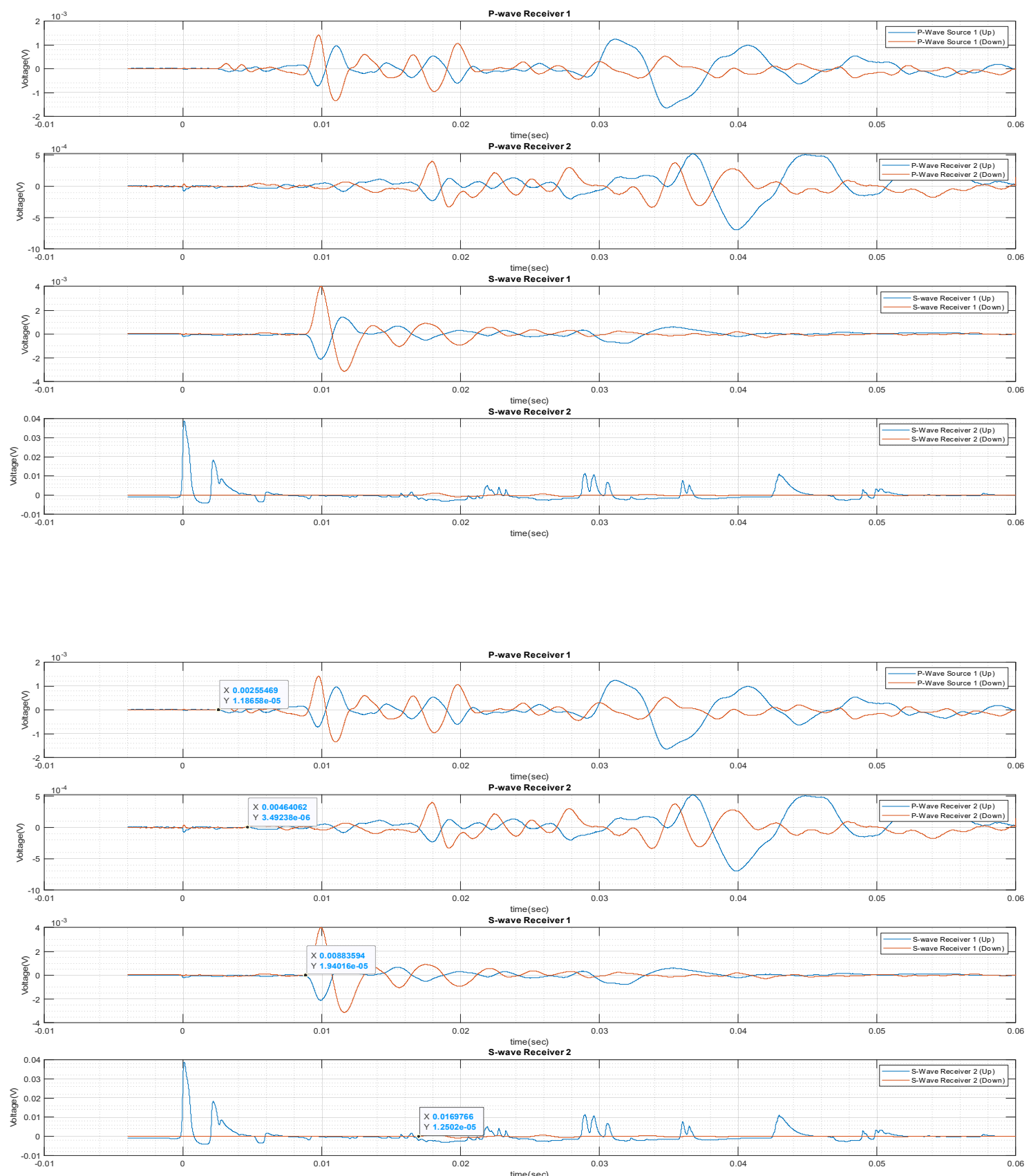

Data Set $32(11.22 .2019)$

Run00245f

R3_1 

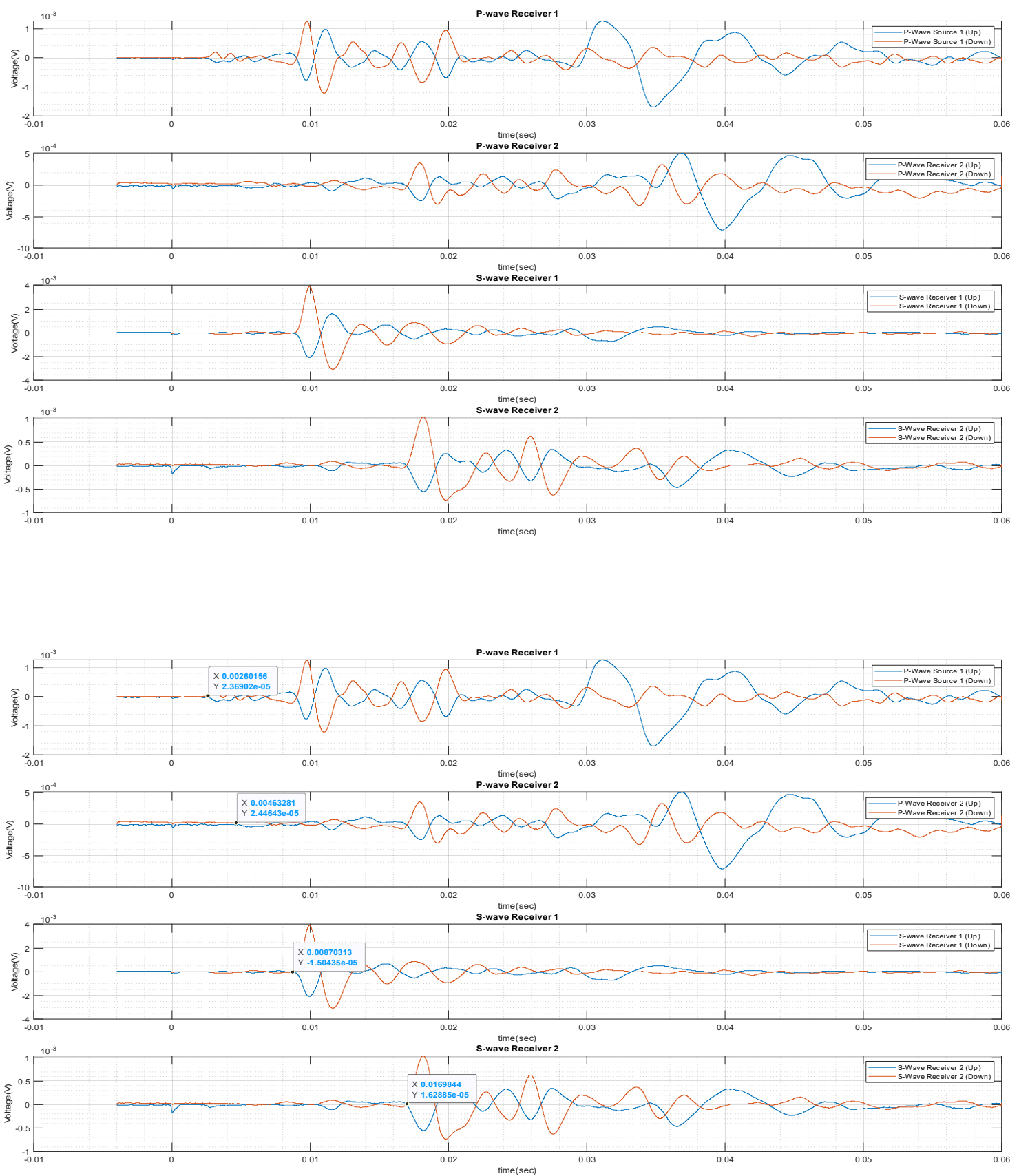

Data Set 32 (11.22.2019)

Run00246f

R3_2 

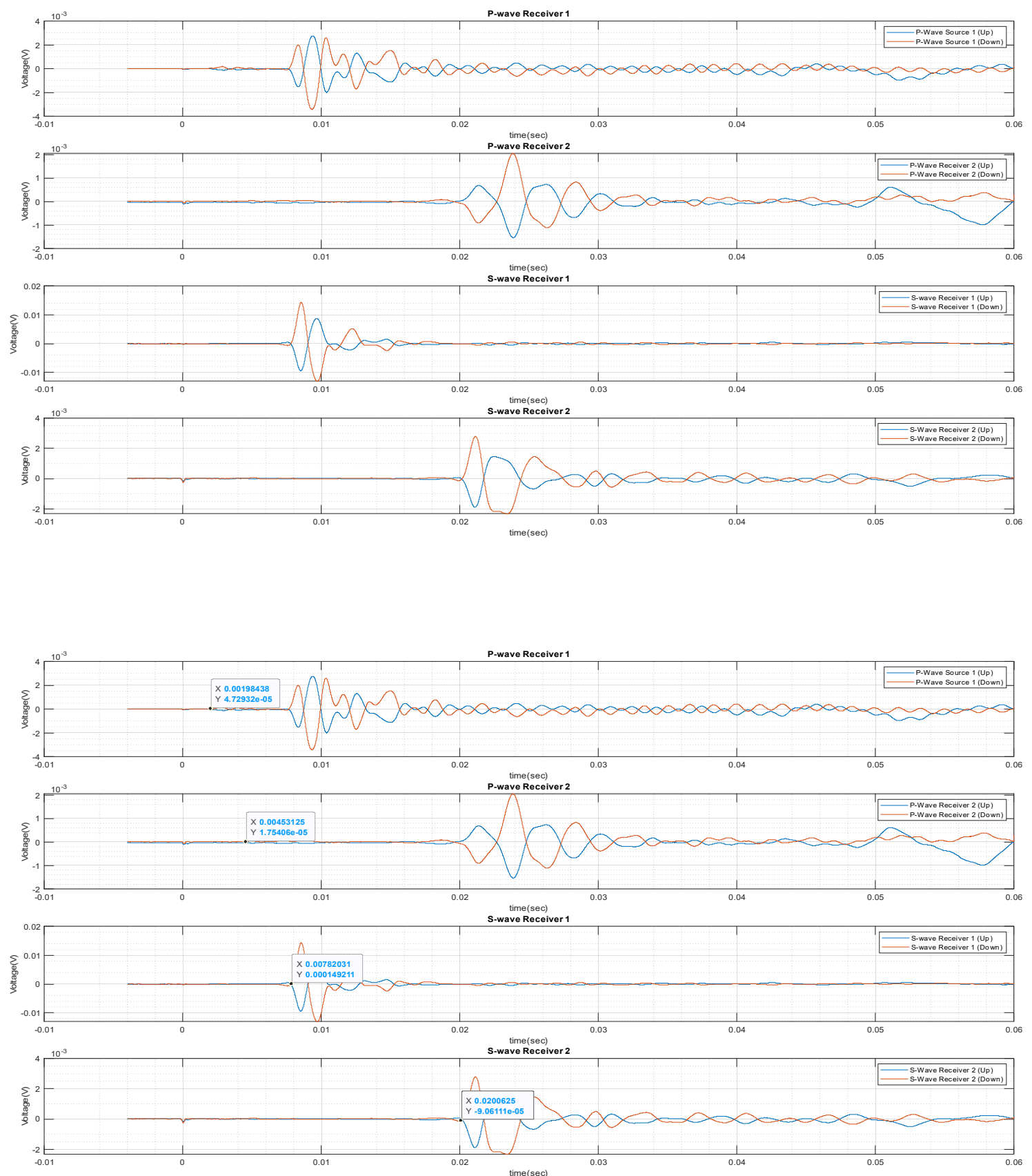

Data Set 32 (11.22.2019)

Run00247f

R4_1 

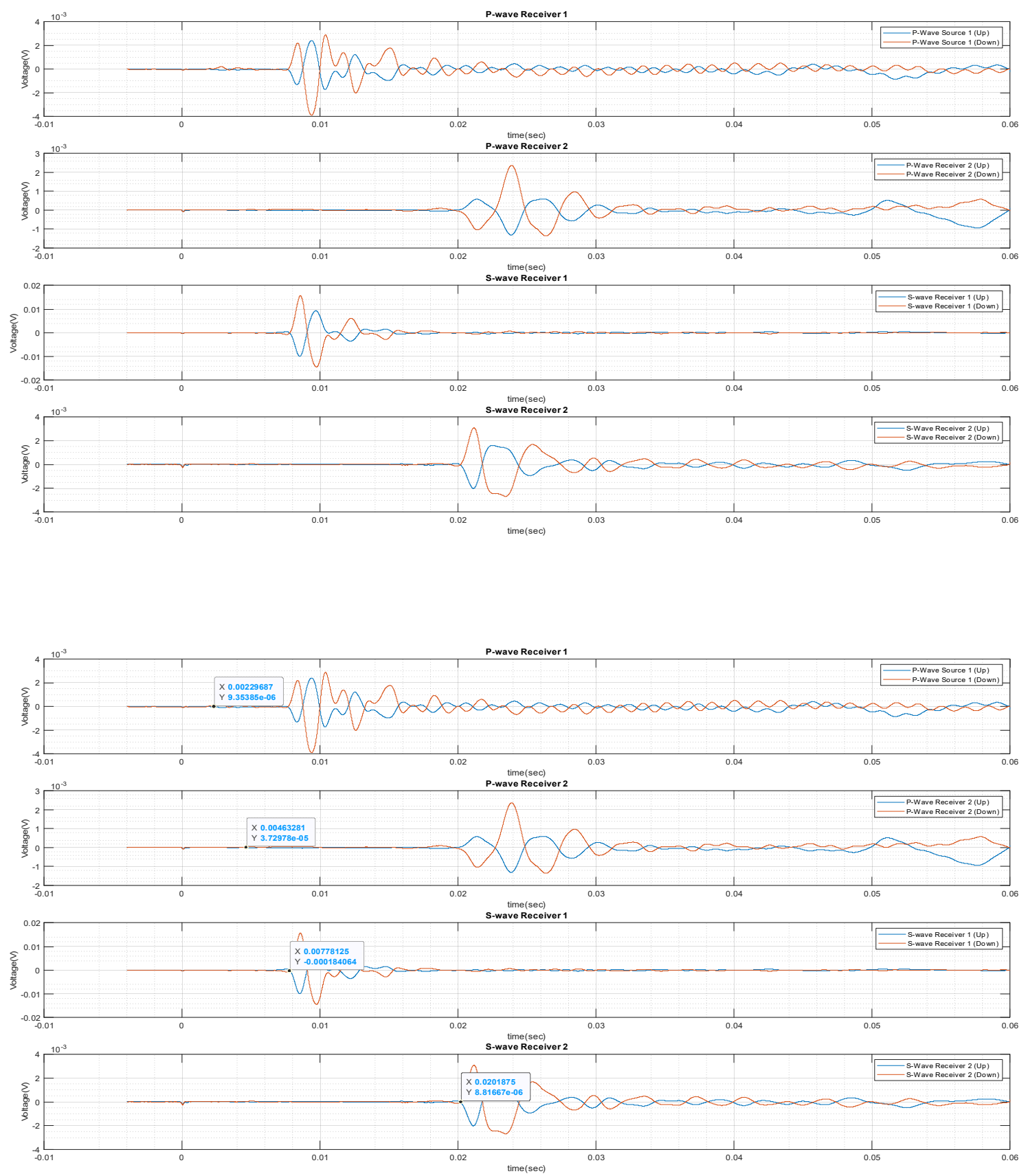

Data Set 32 (11.22.2019)

Run00248f

R4_2 

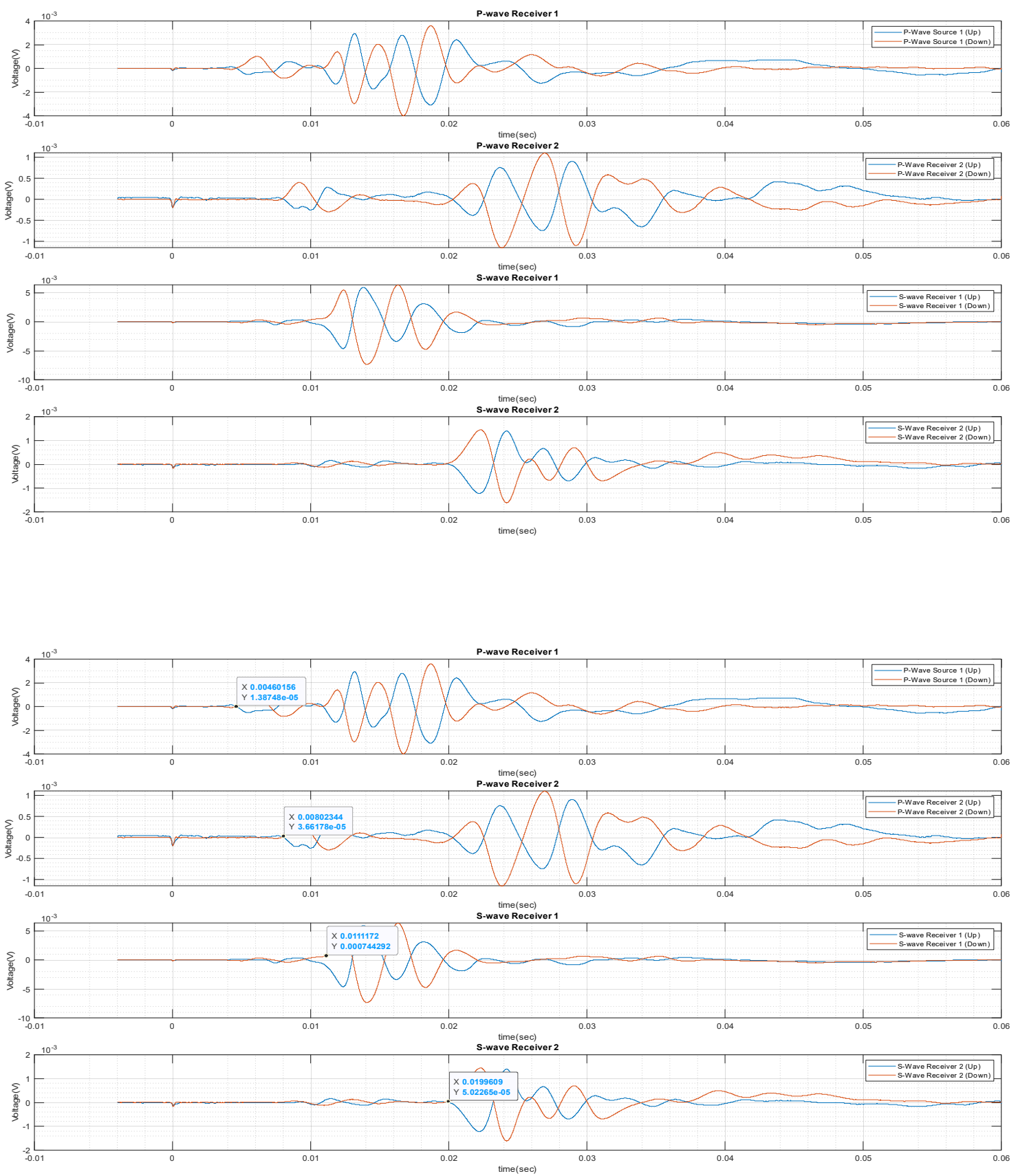

Data Set 33 (12.8.2019)

Run00249f

R1_1 

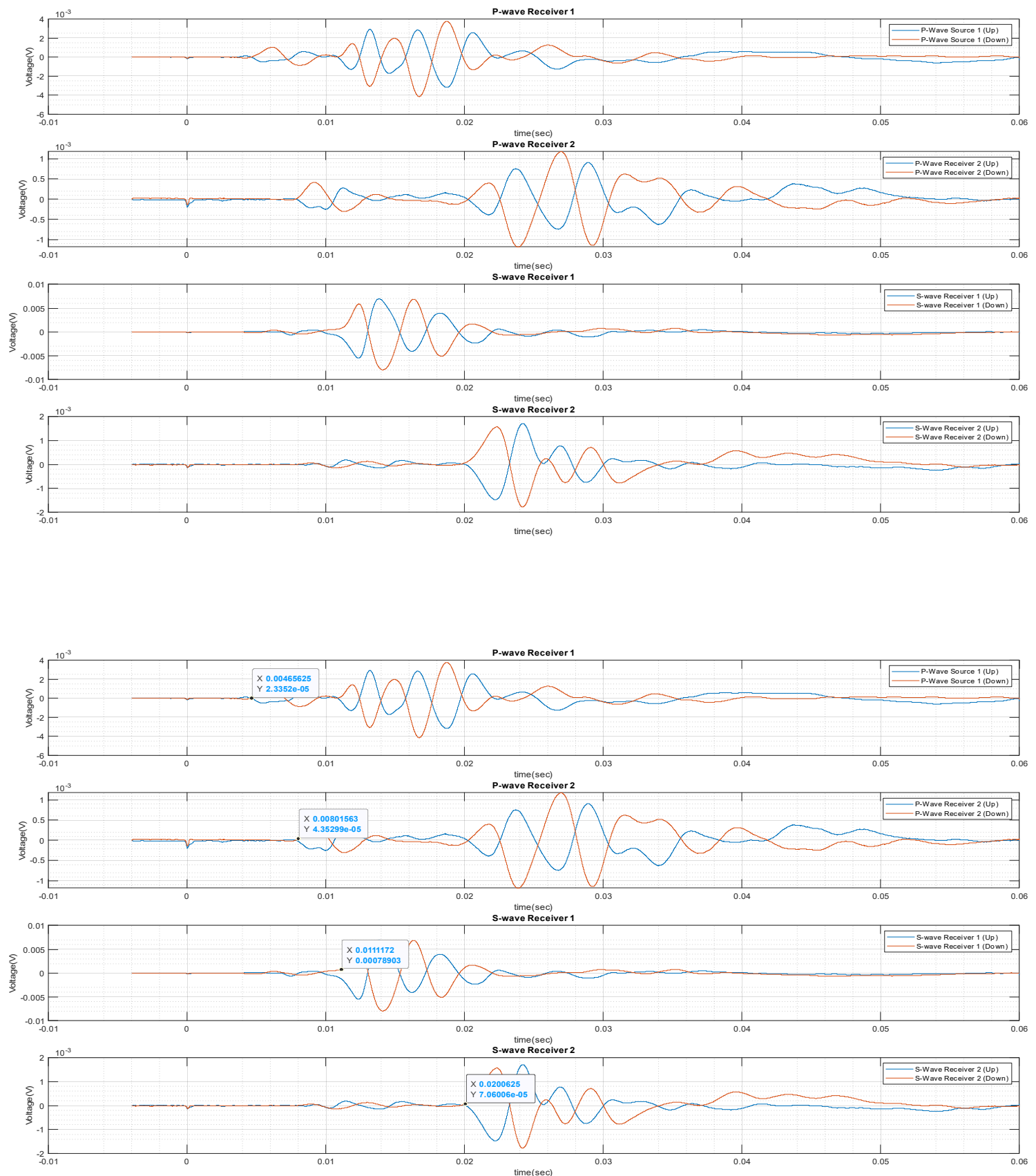

Data Set 33 (12.8.2019)

Run00250f

R1_2 

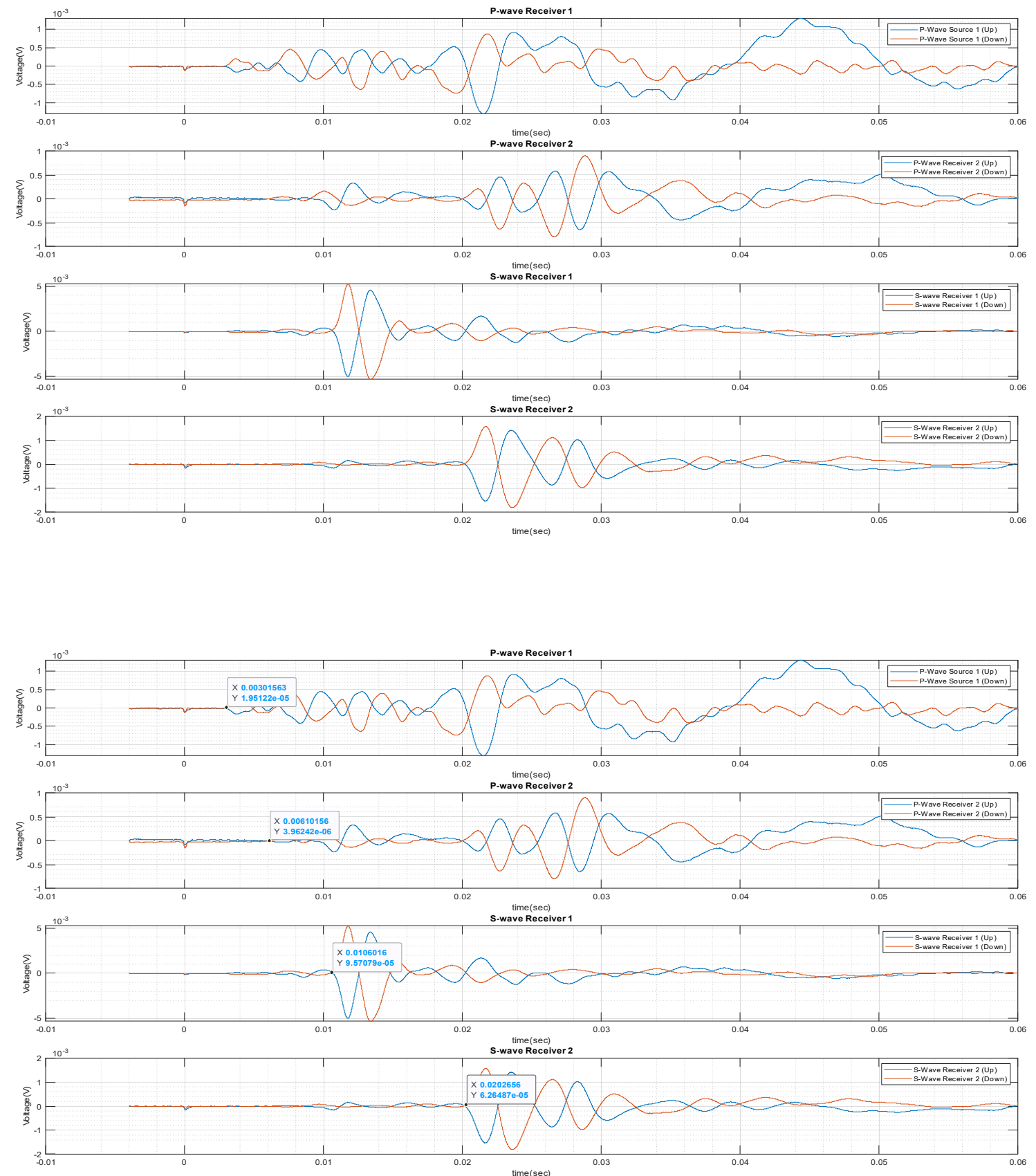

Data Set 33 (12.8.2019)

Run00251f

R2_1 

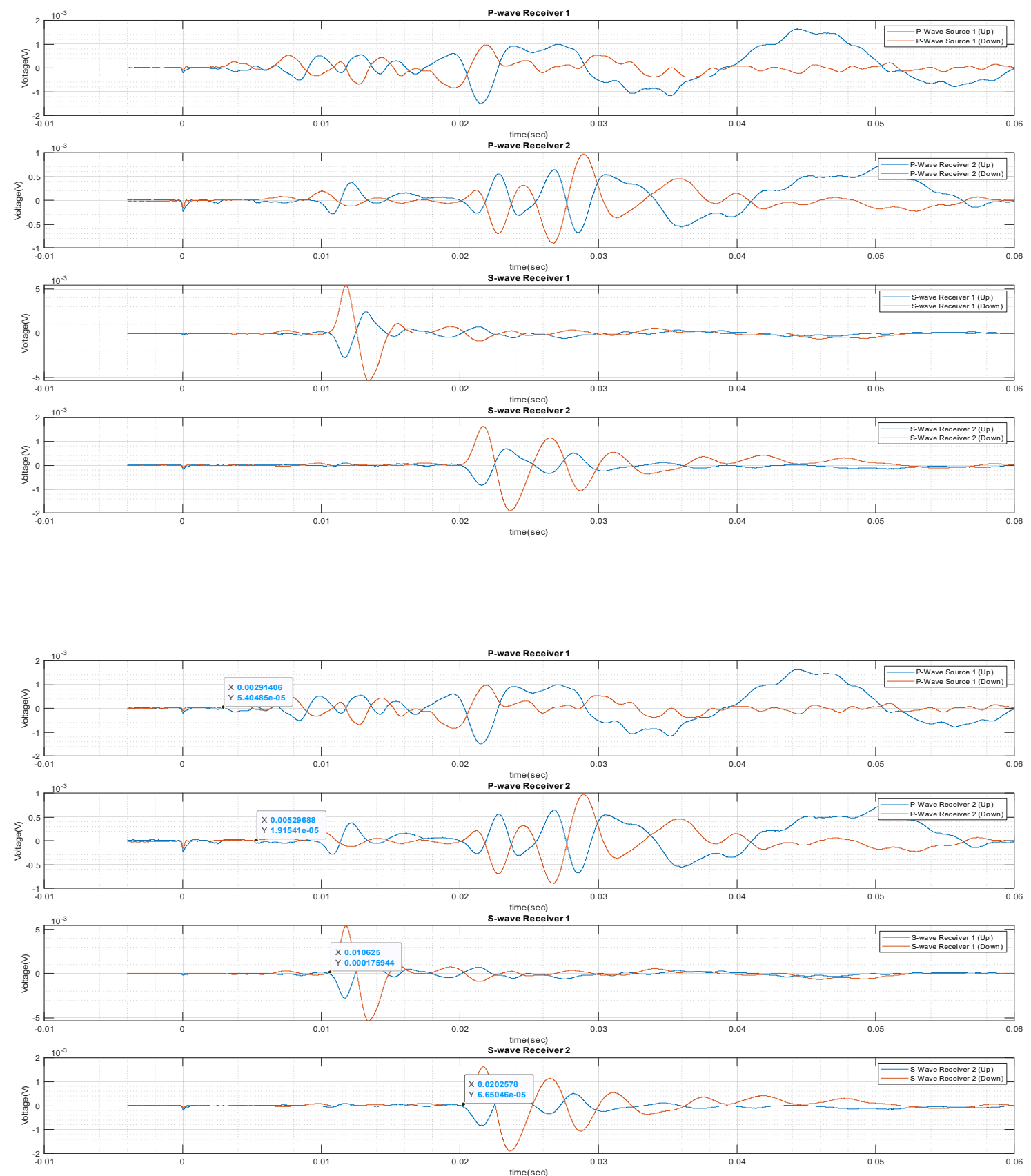

Data Set 33 (12.8.2019)

Run00252f

R2_2 

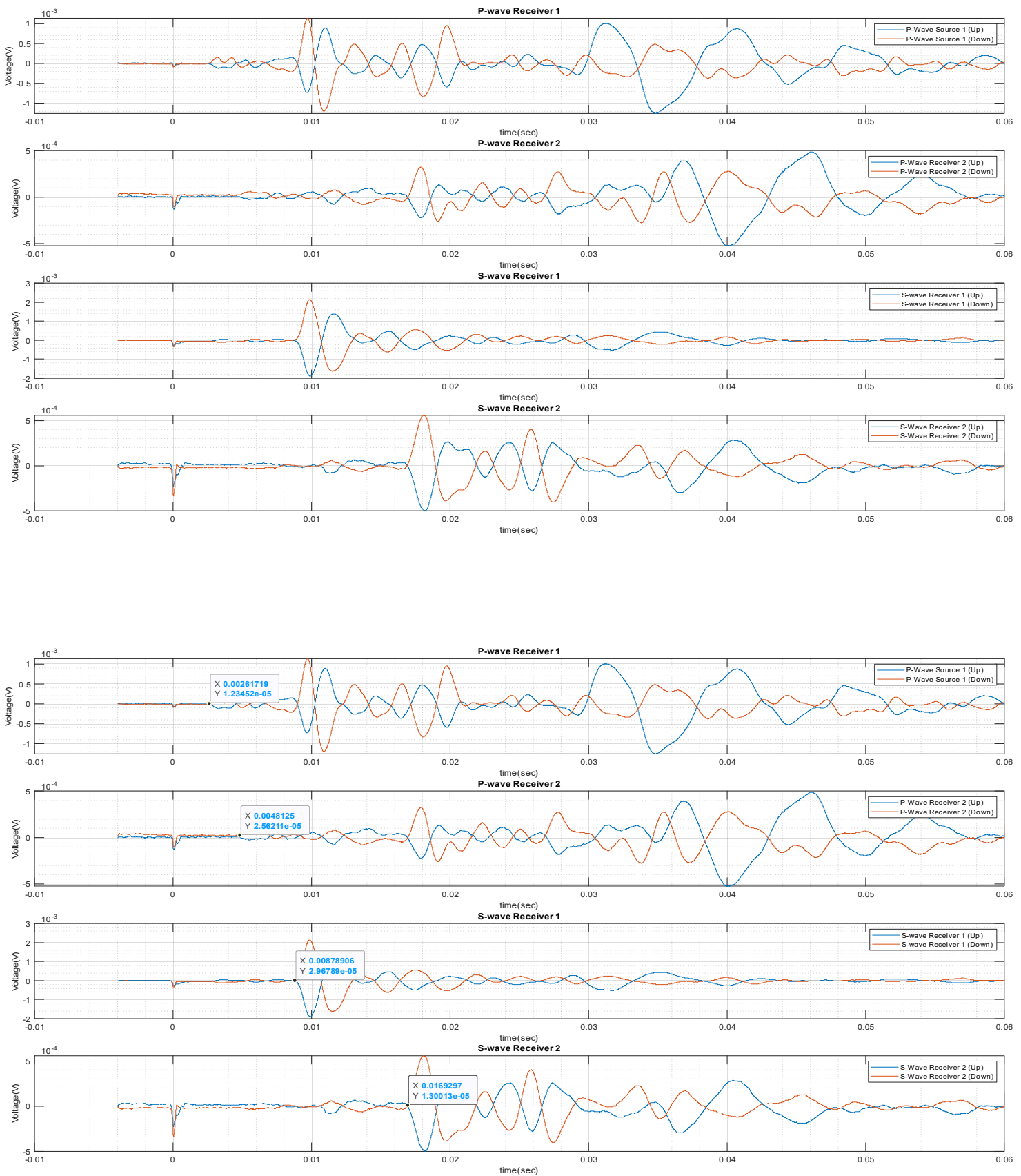

Data Set 33 (12.8.2019)

Run00253f

R3_1 

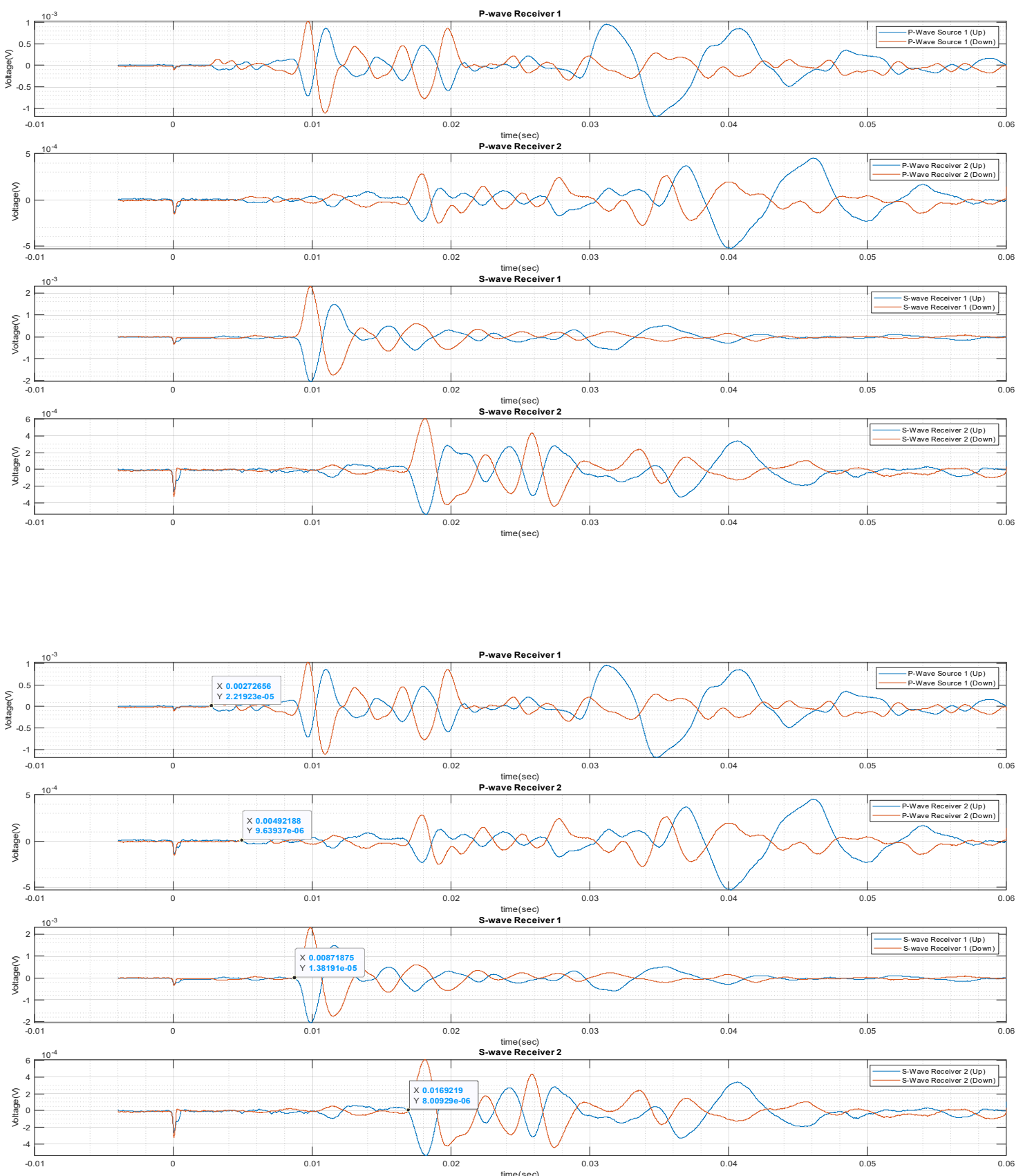

Data Set 33 (12.8.2019)

Run00254f

R3_2 

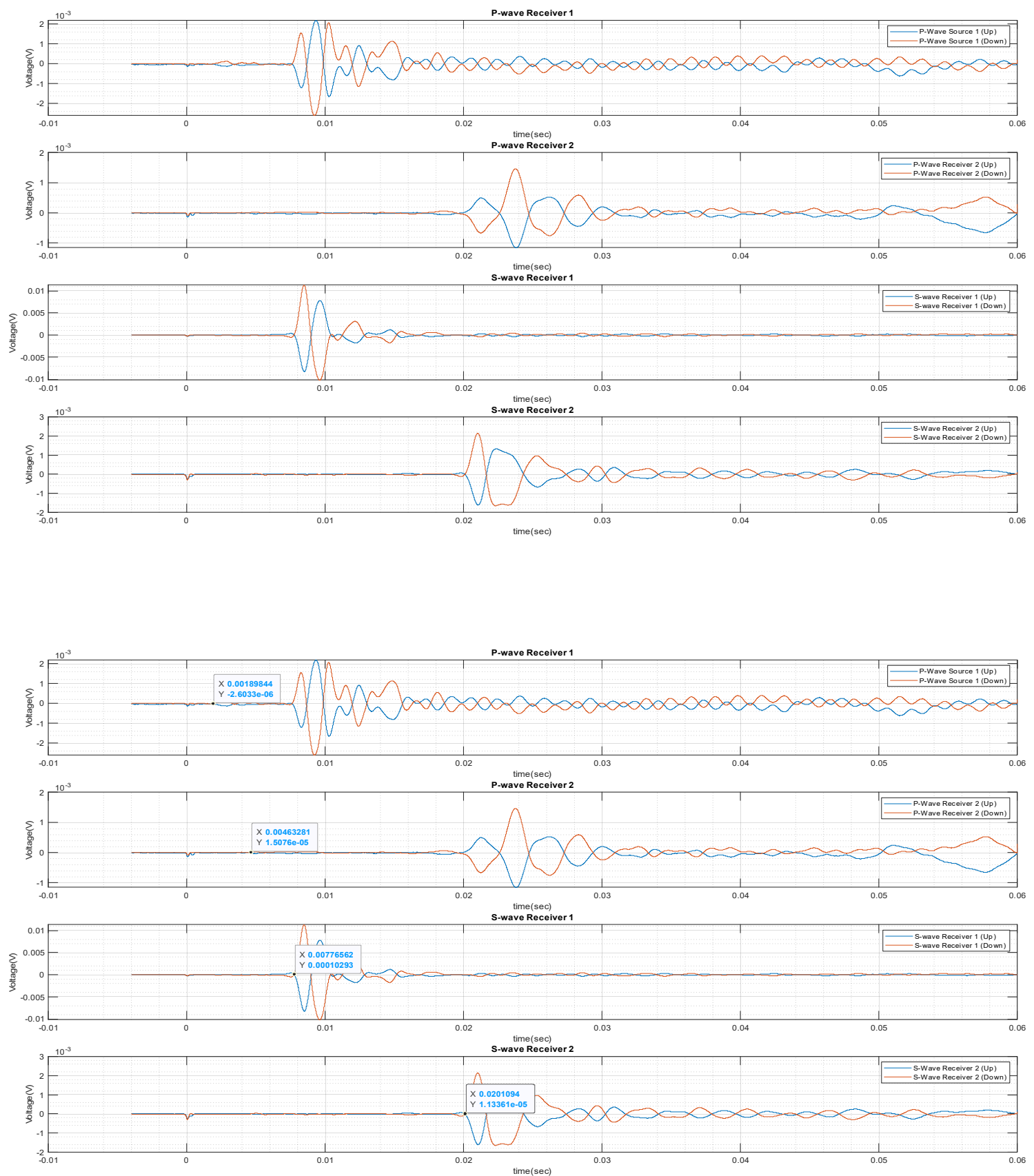

Data Set 33 (12.8.2019)

Run00255f

R4_1 

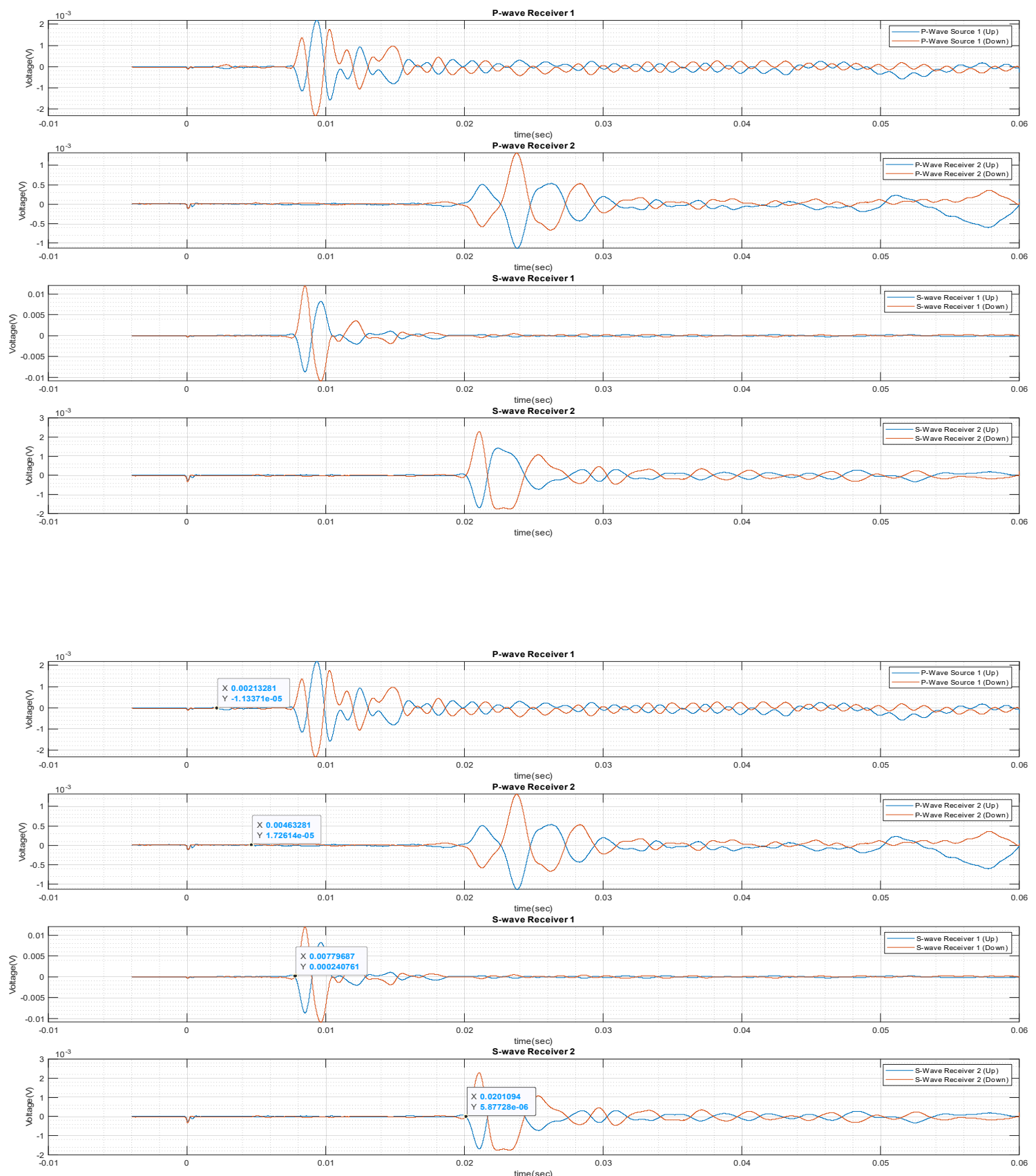

Data Set 33 (12.8.2019)

Run00256f

R4_2 

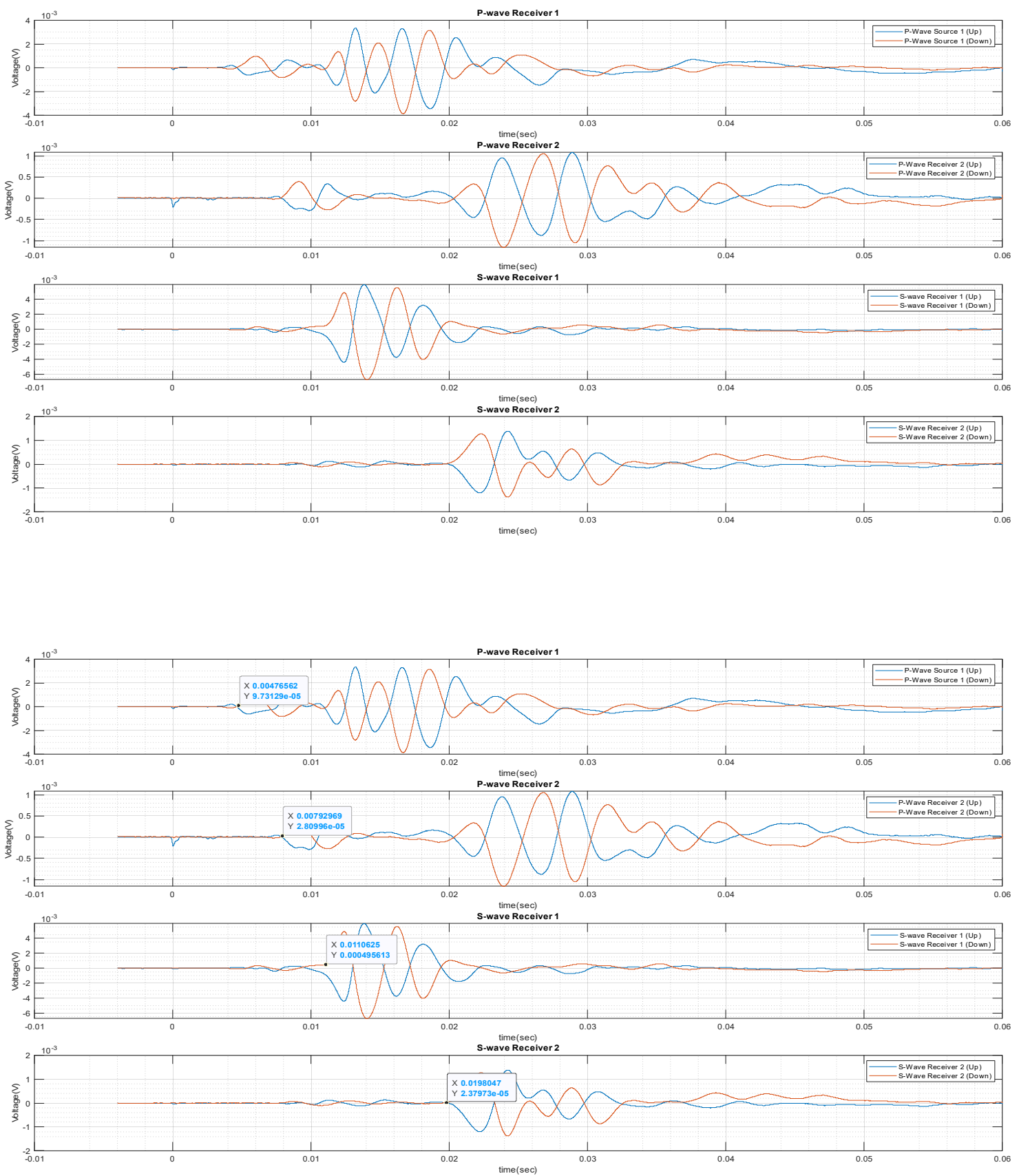

Data Set 34 (12.18.2019)

Run00257f

R1_1 

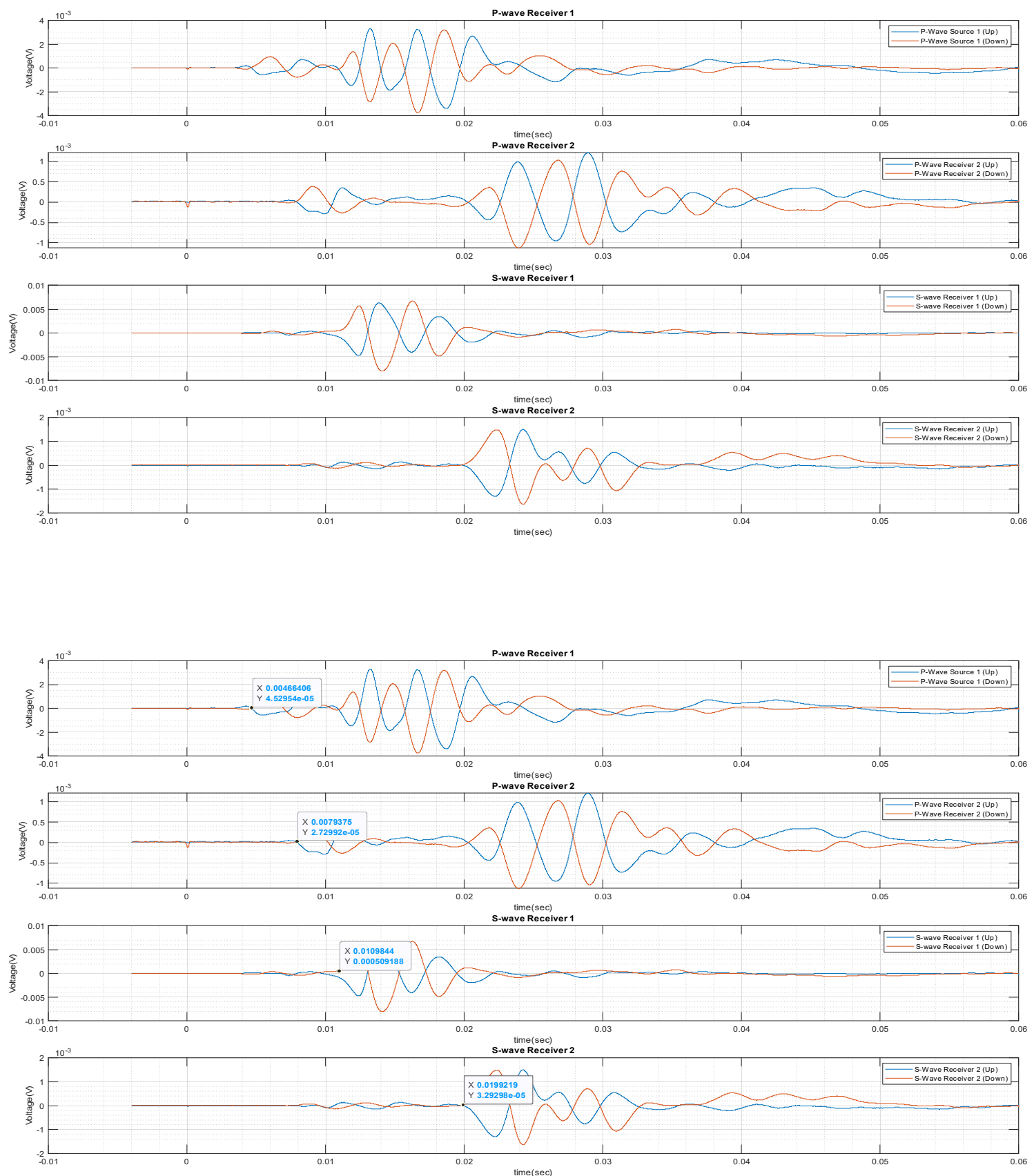

Data Set 34 (12.18.2019)

Run00258f

R1_2 

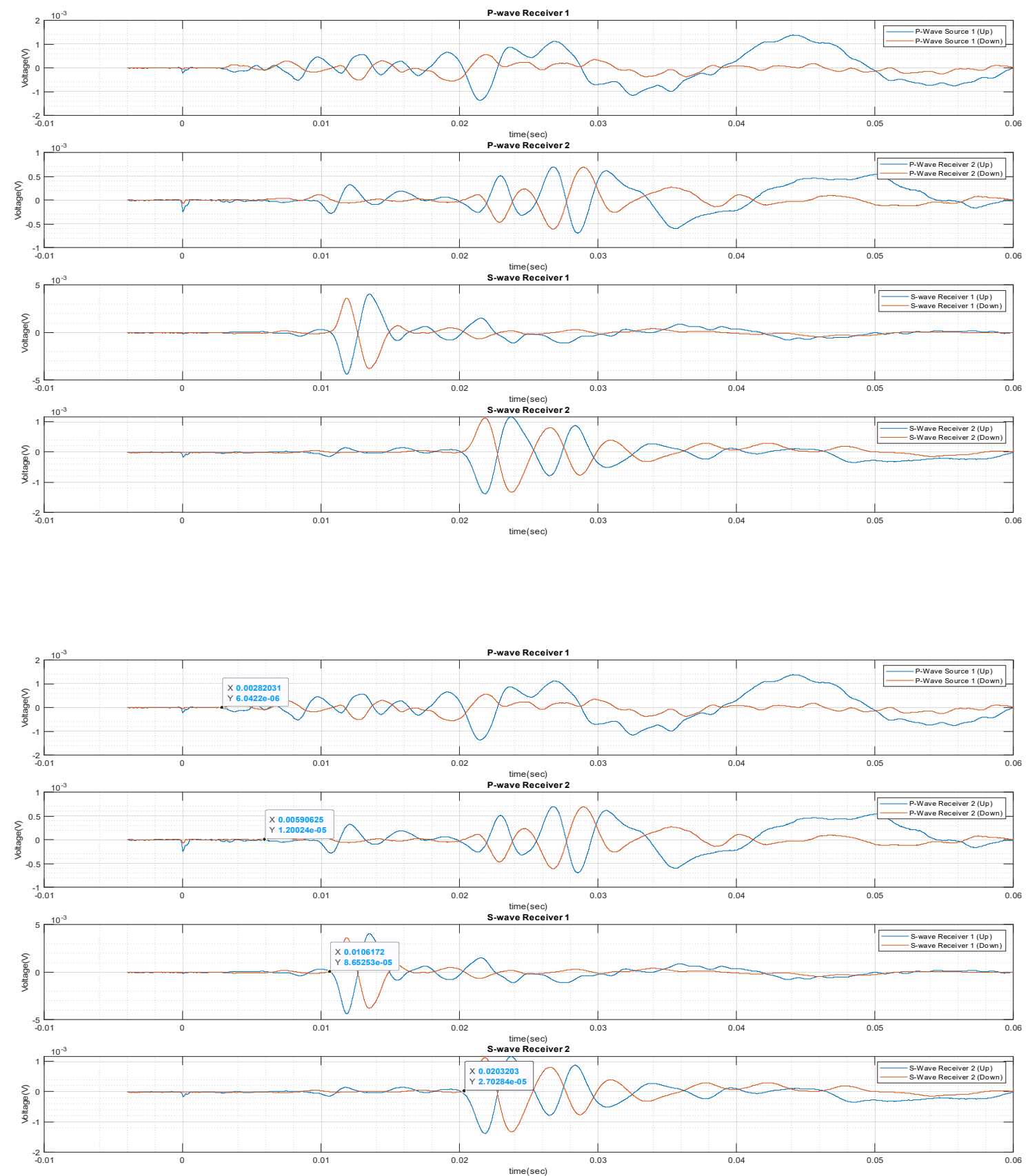

Data Set 34 (12.18.2019)

Run00259f

R2_1 

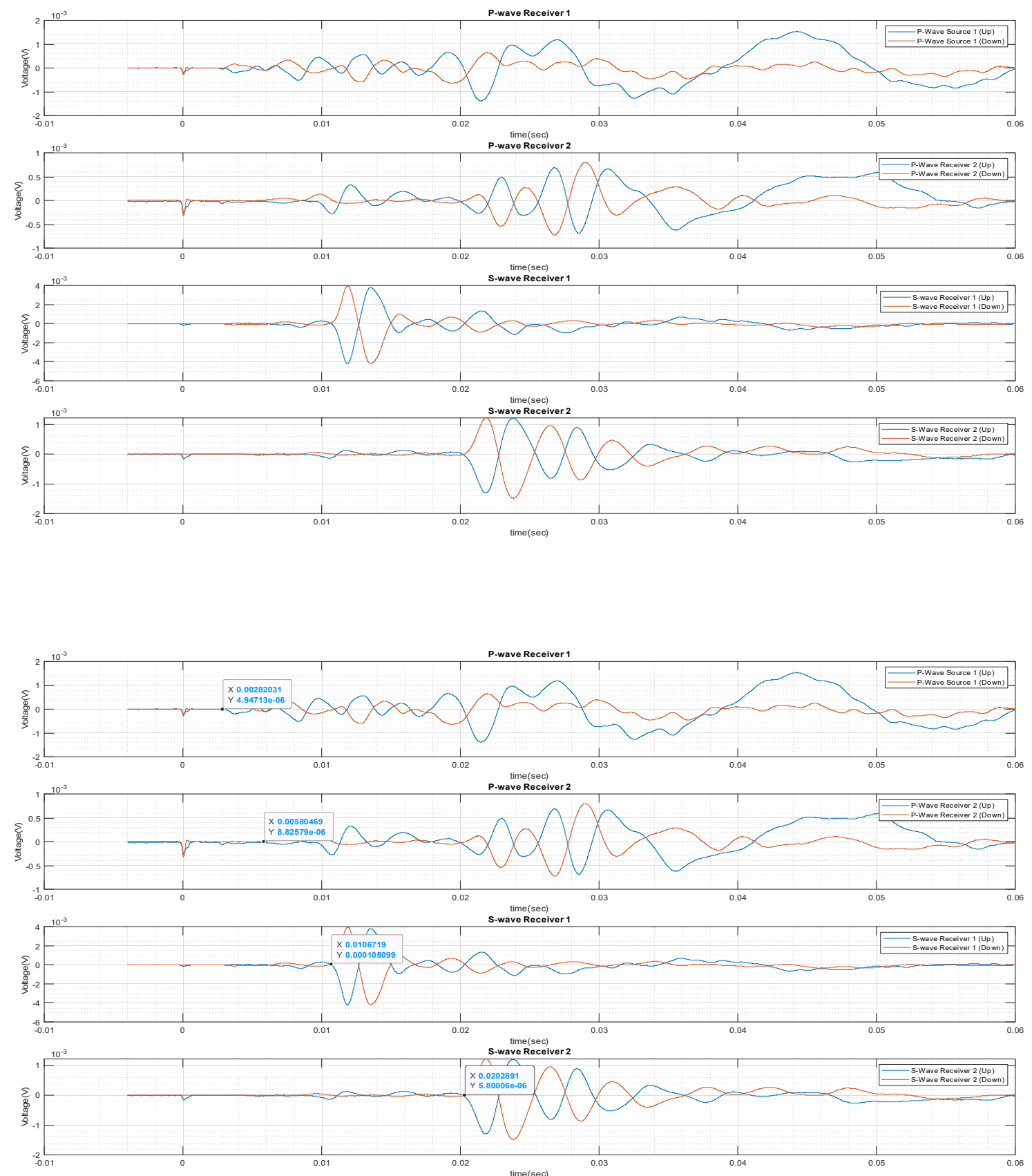

Data Set 34 (12.18.2019)

Run00260f

R2_2 

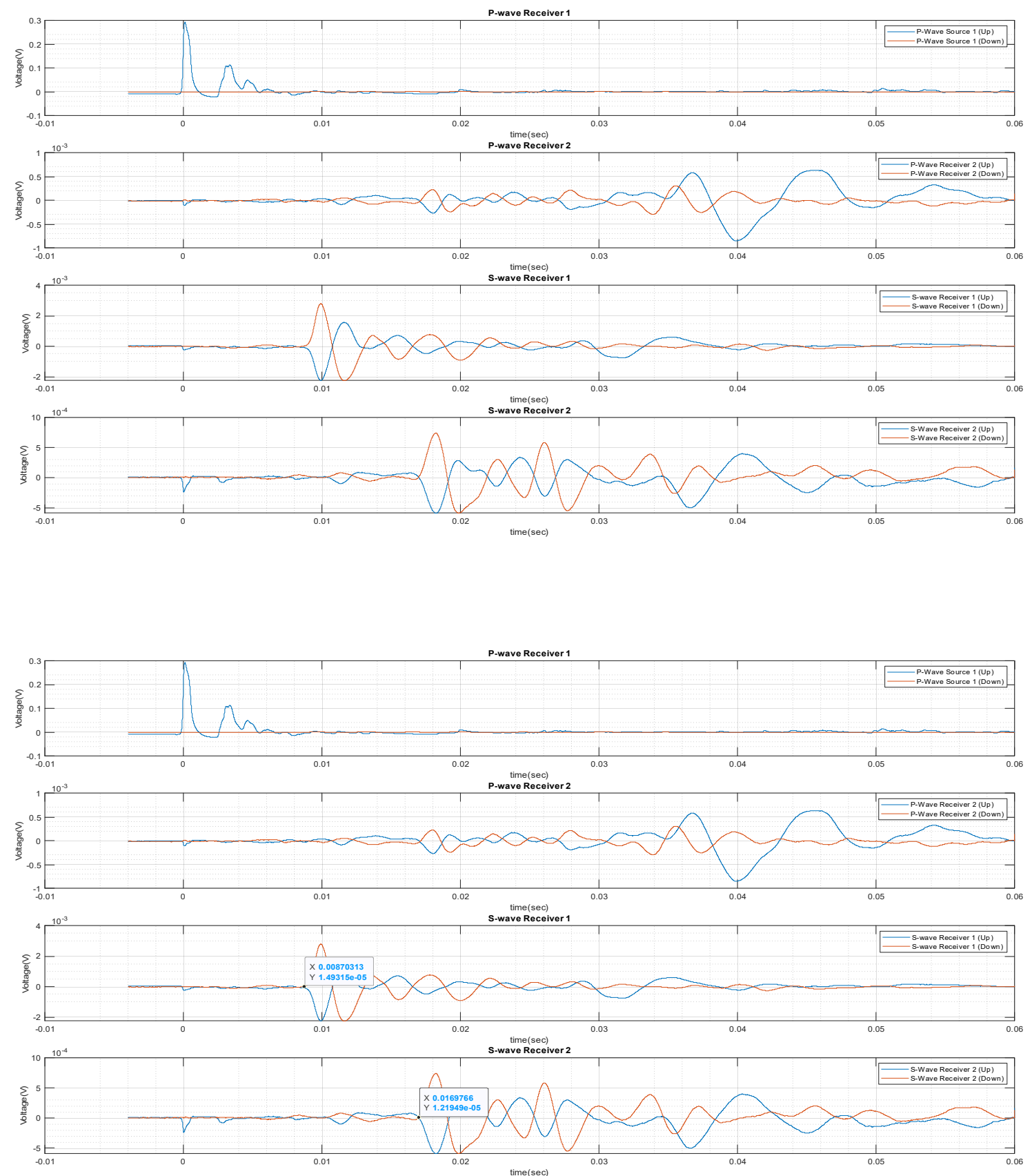

Data Set 34 (12.18.2019)

Run00261f

R3_1 

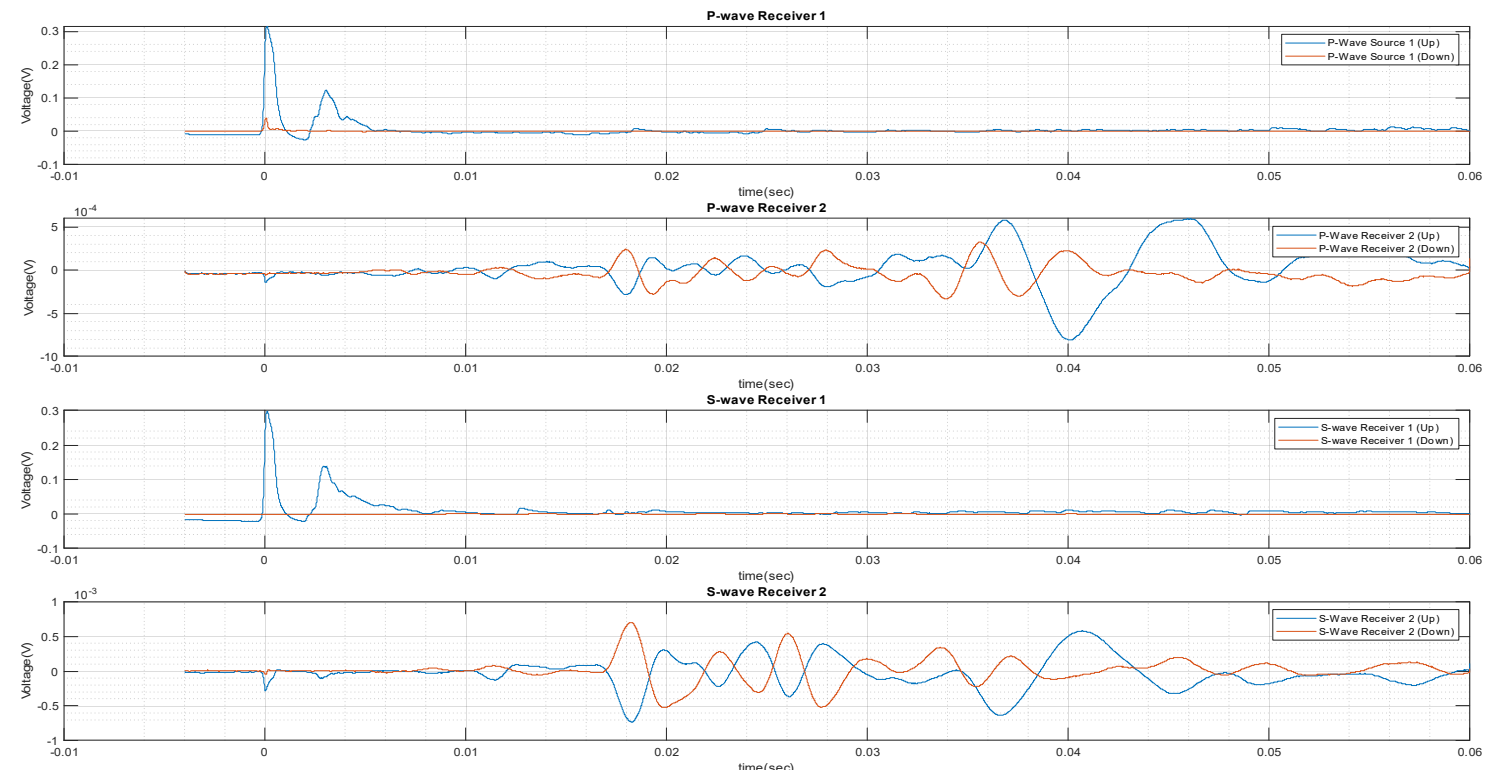

Cannot determine clear arrival times.

Data Set 34 (12.18.2019)

Run00262f

R3_2 

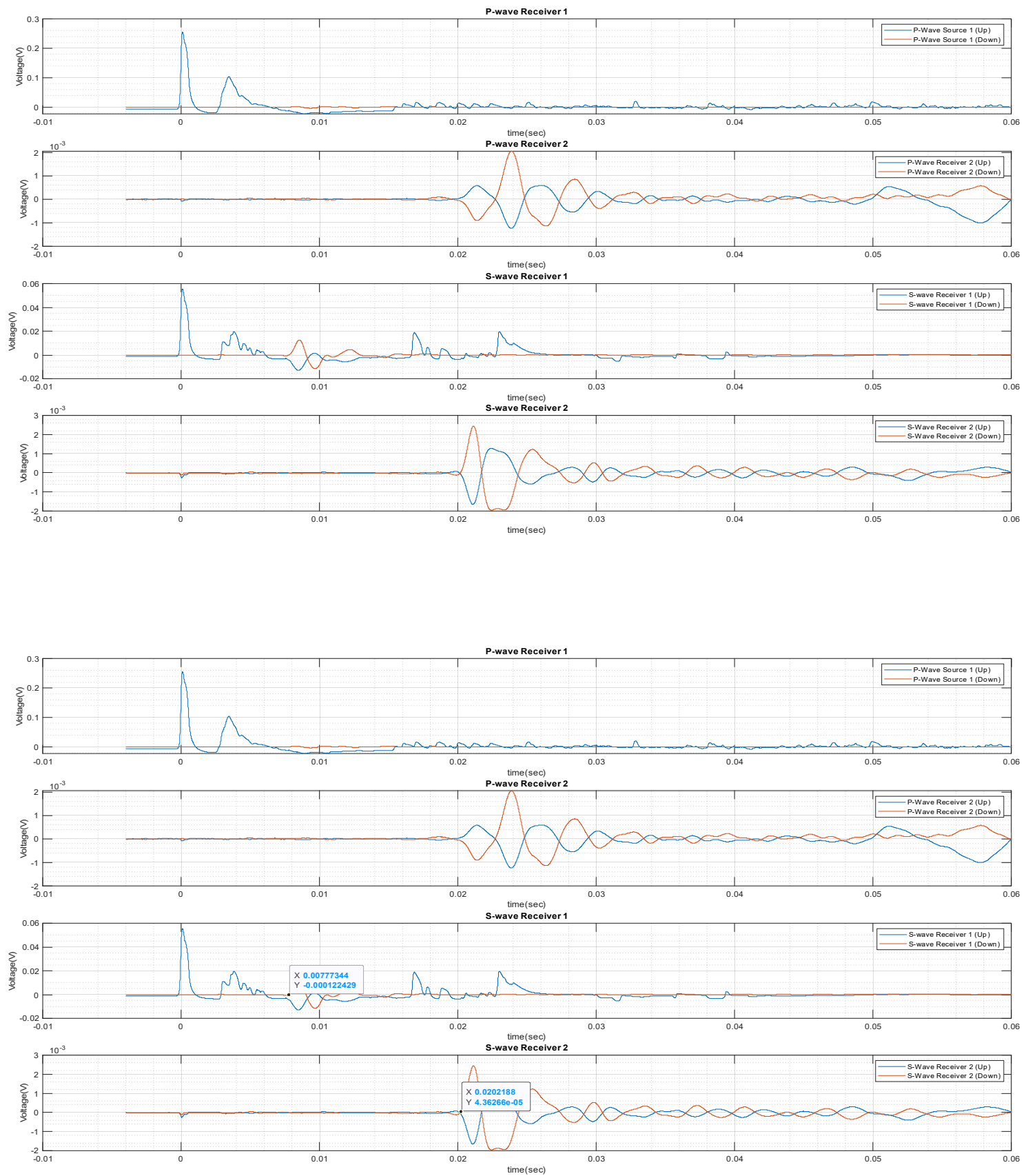

Data Set 34 (12.18.2019)

\section{Run00263f}

R4_1 

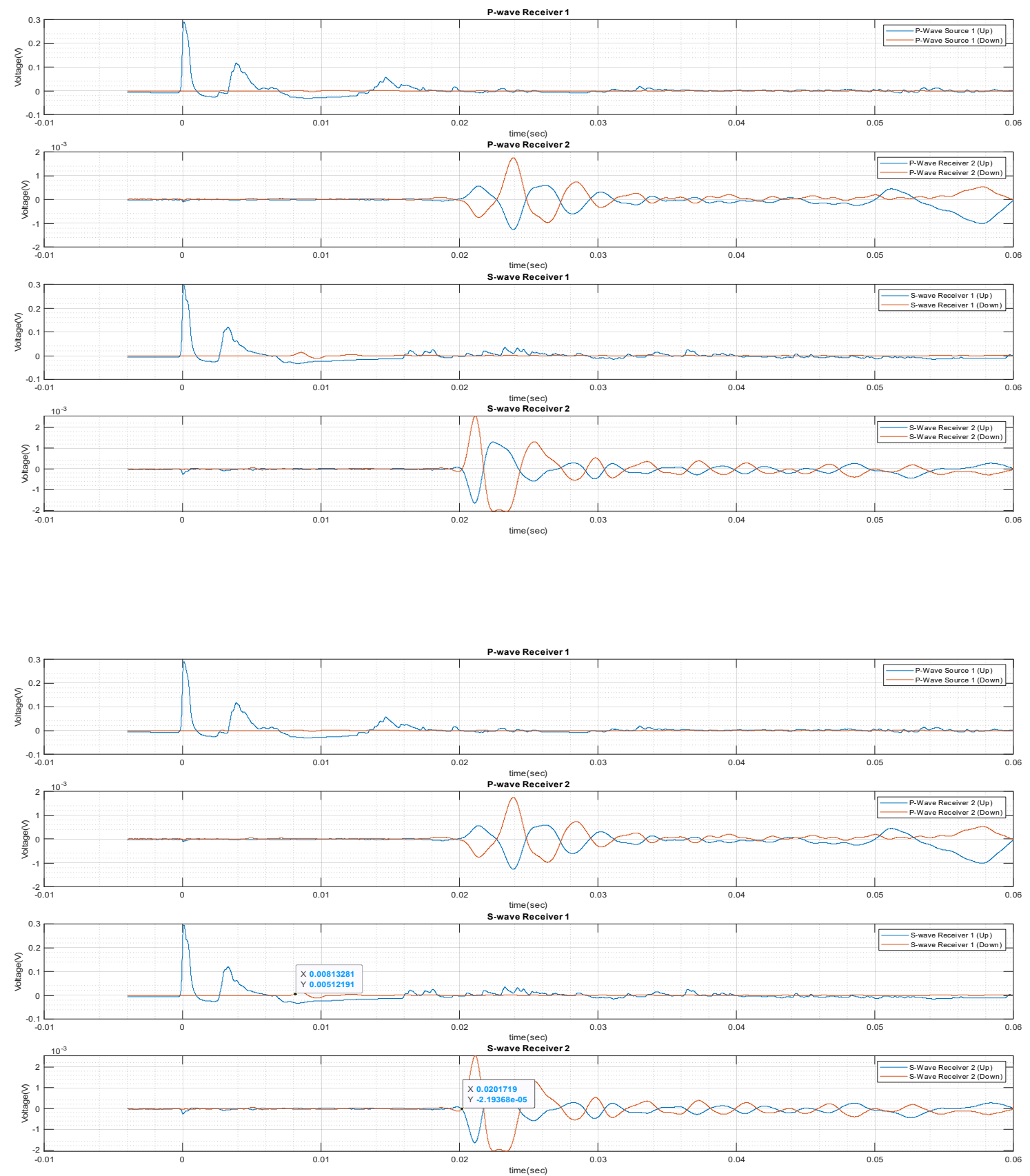

Data Set 34 (12.18.2019)

\section{Run00264f}

R4 2 

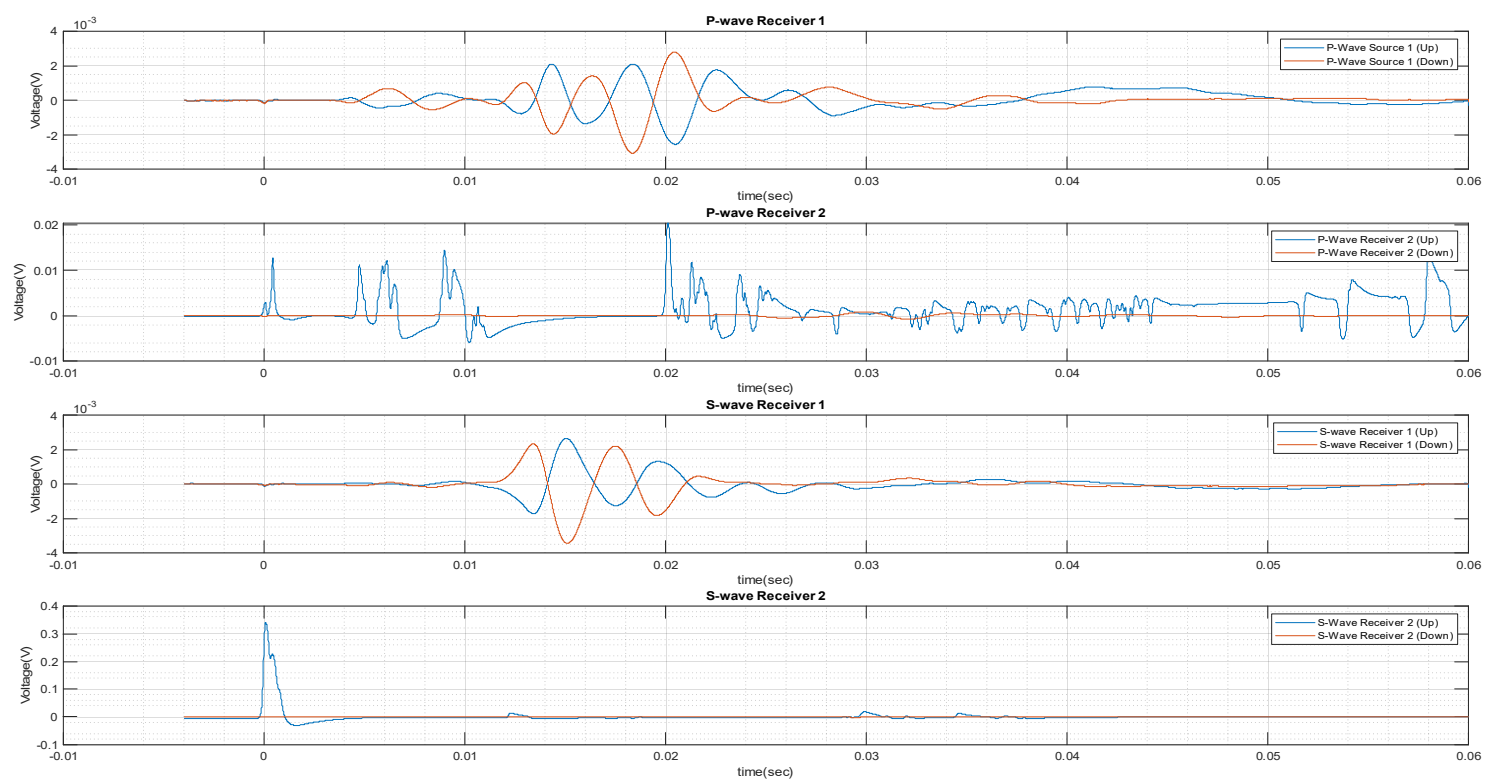

Cannot determine clear arrival times

Data Set $35(1.17 .2020)$

Run00265f

R1_1 

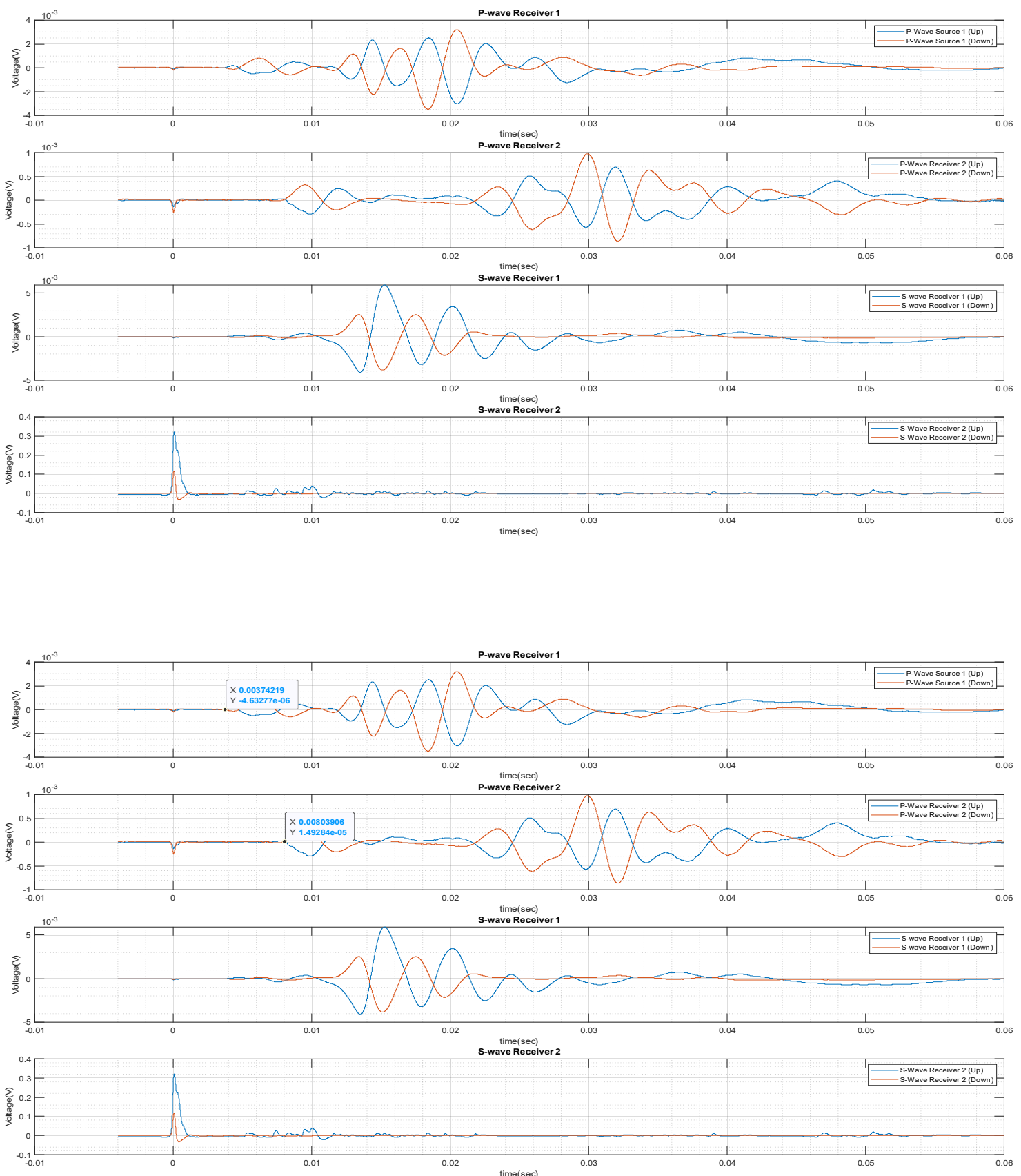

Data Set 35 (1.17.2020)

Run00266f

R1_2 

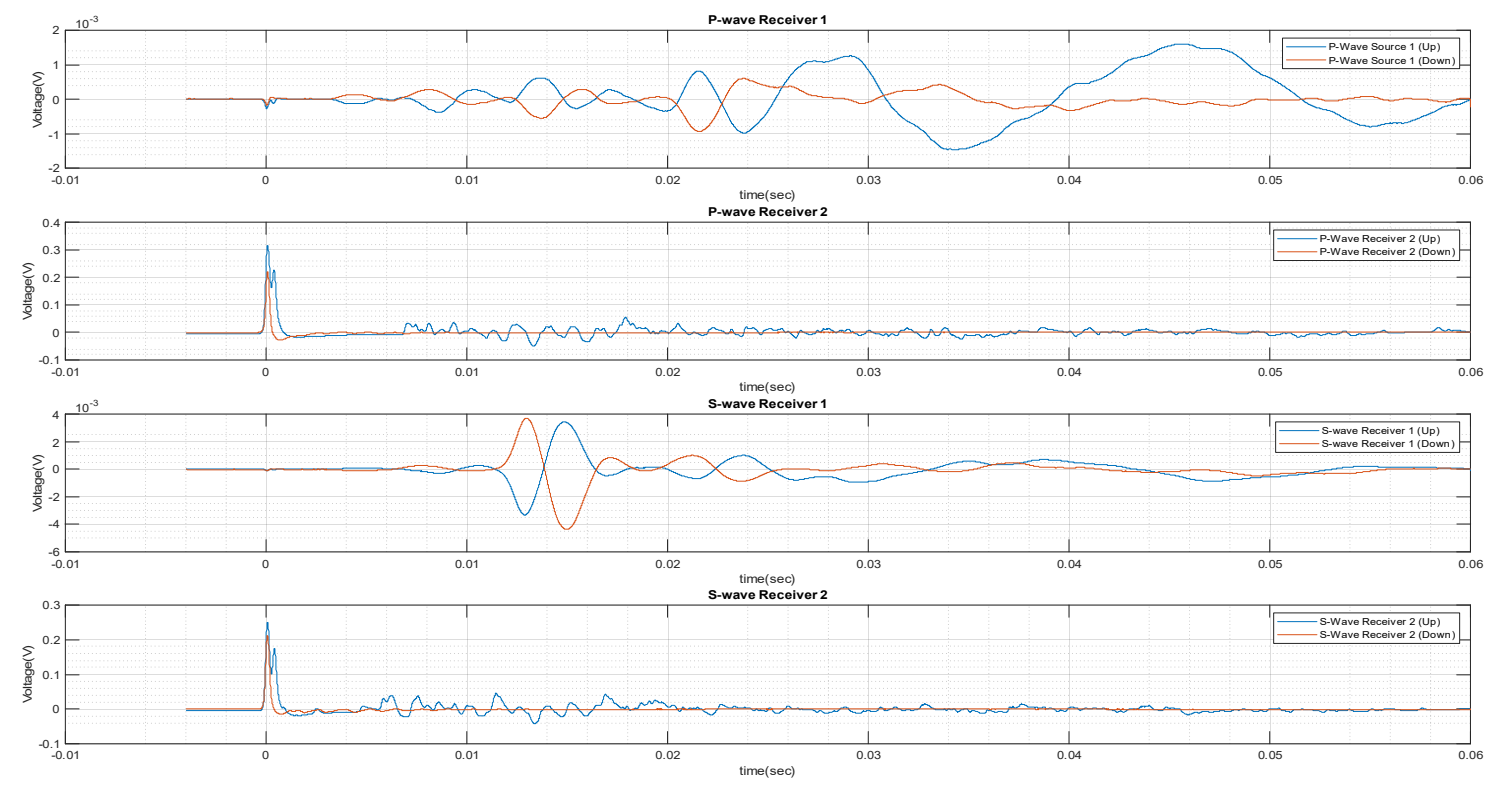

Cannot determine clear arrival times

Data Set $35(1.17 .2020)$

Run00267f

R2_1 

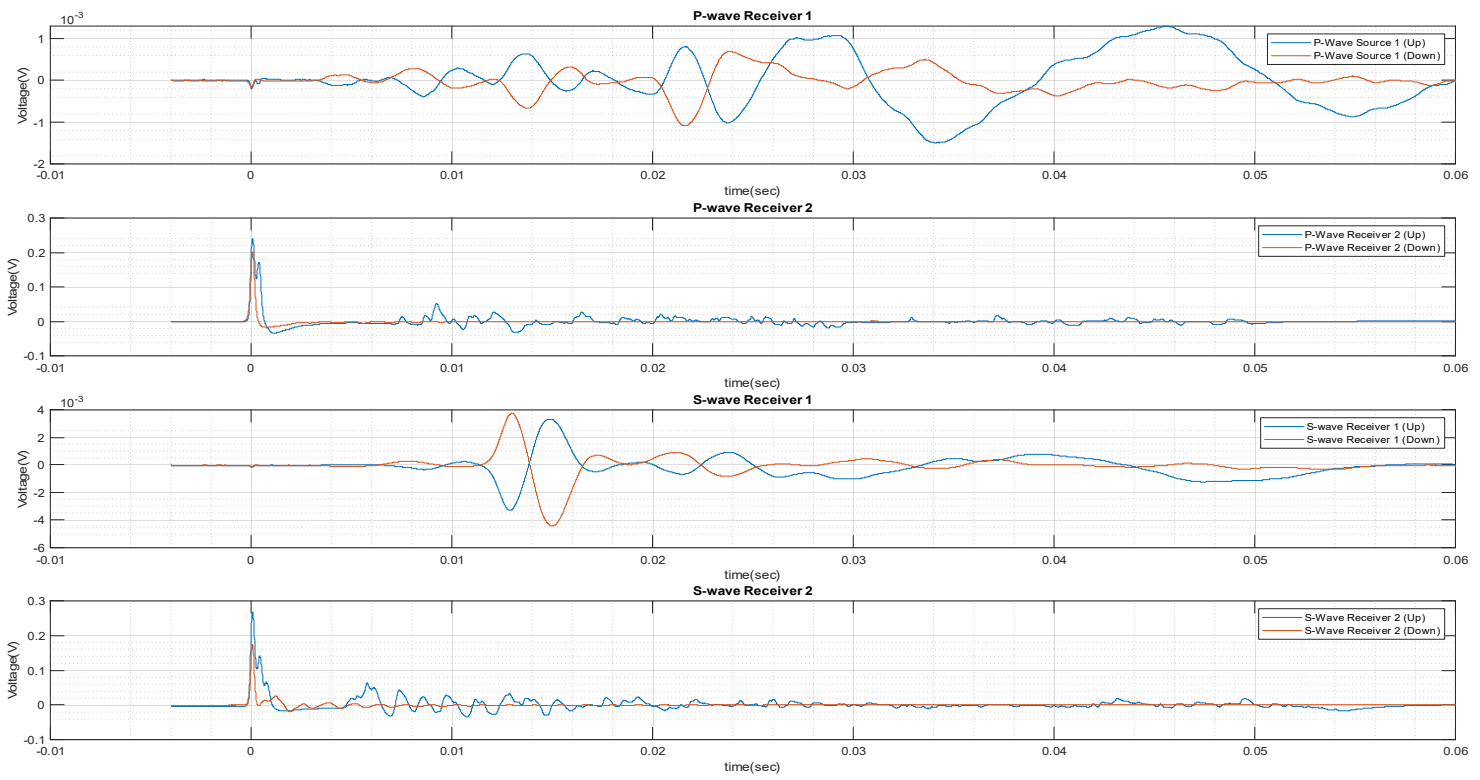

Cannot determine clear arrival times

Data Set $35(1.17 .2020)$

Run00268f 
R2_2
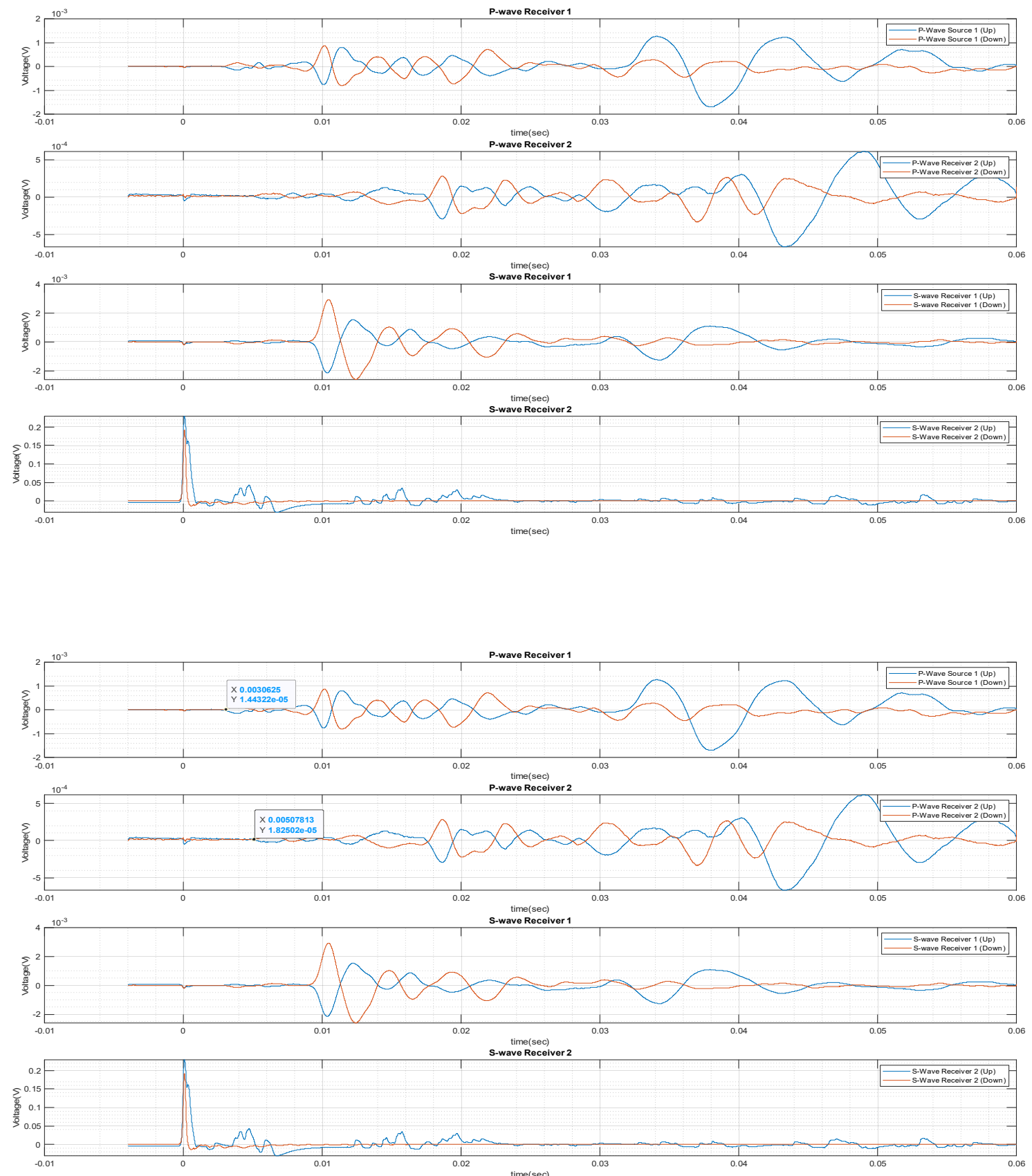

Data Set 35 (1.17.2020)

Run00269f 
R3_1
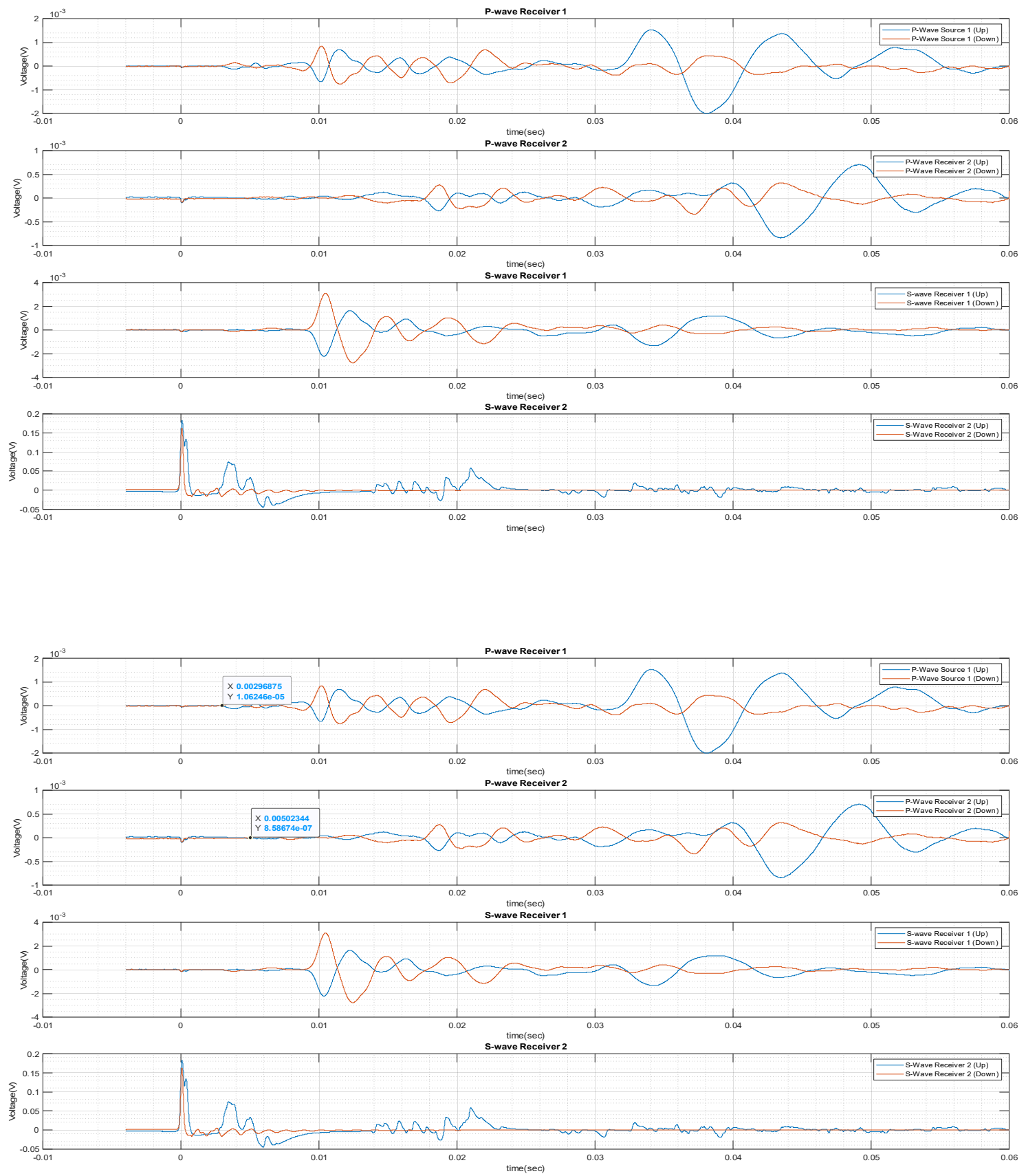

Data Set 35 (1.17.2020)

Run00270f

R3 2 

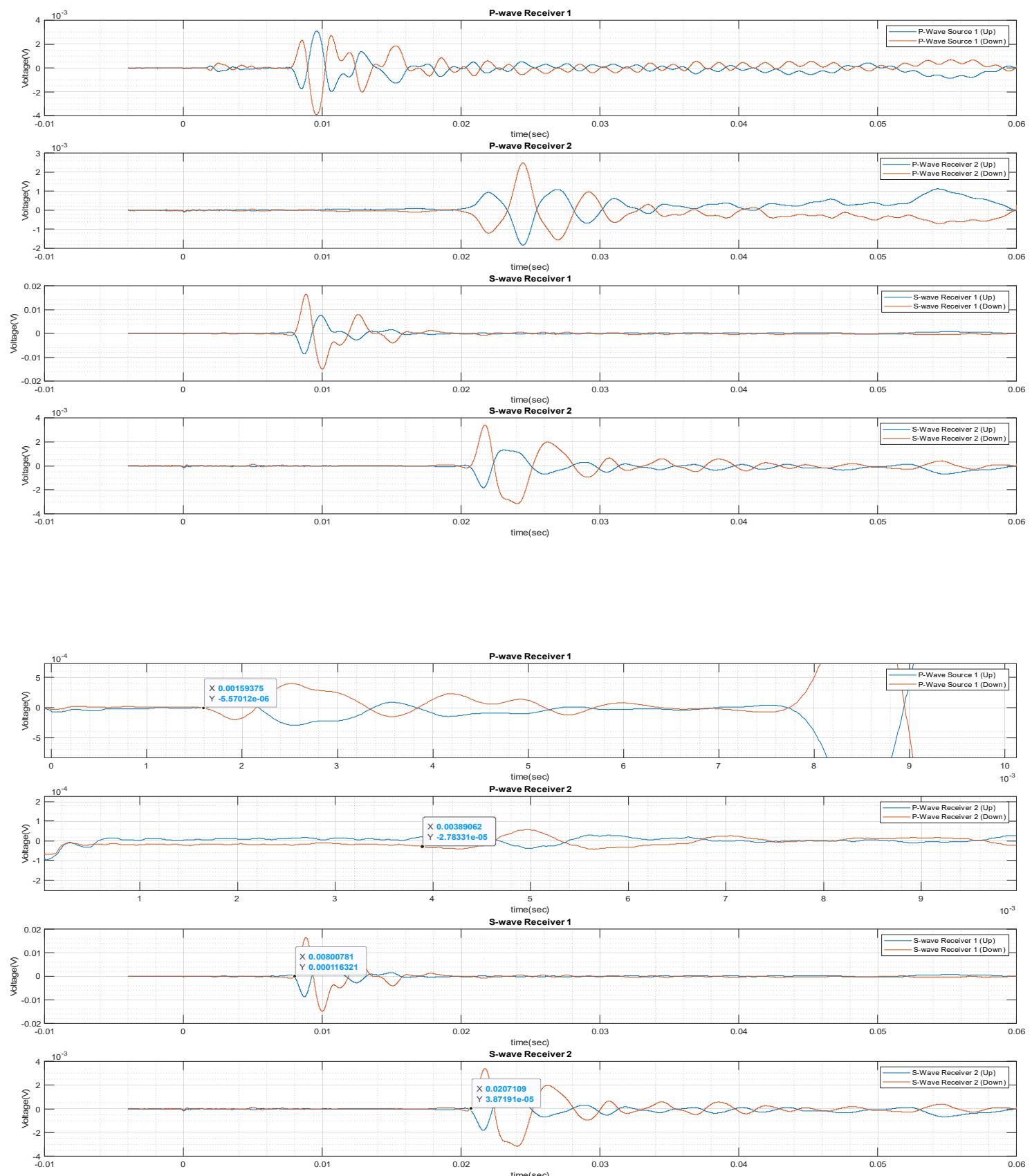

Data Set 35 (1.17.2020)

Run00271f

R4_1 

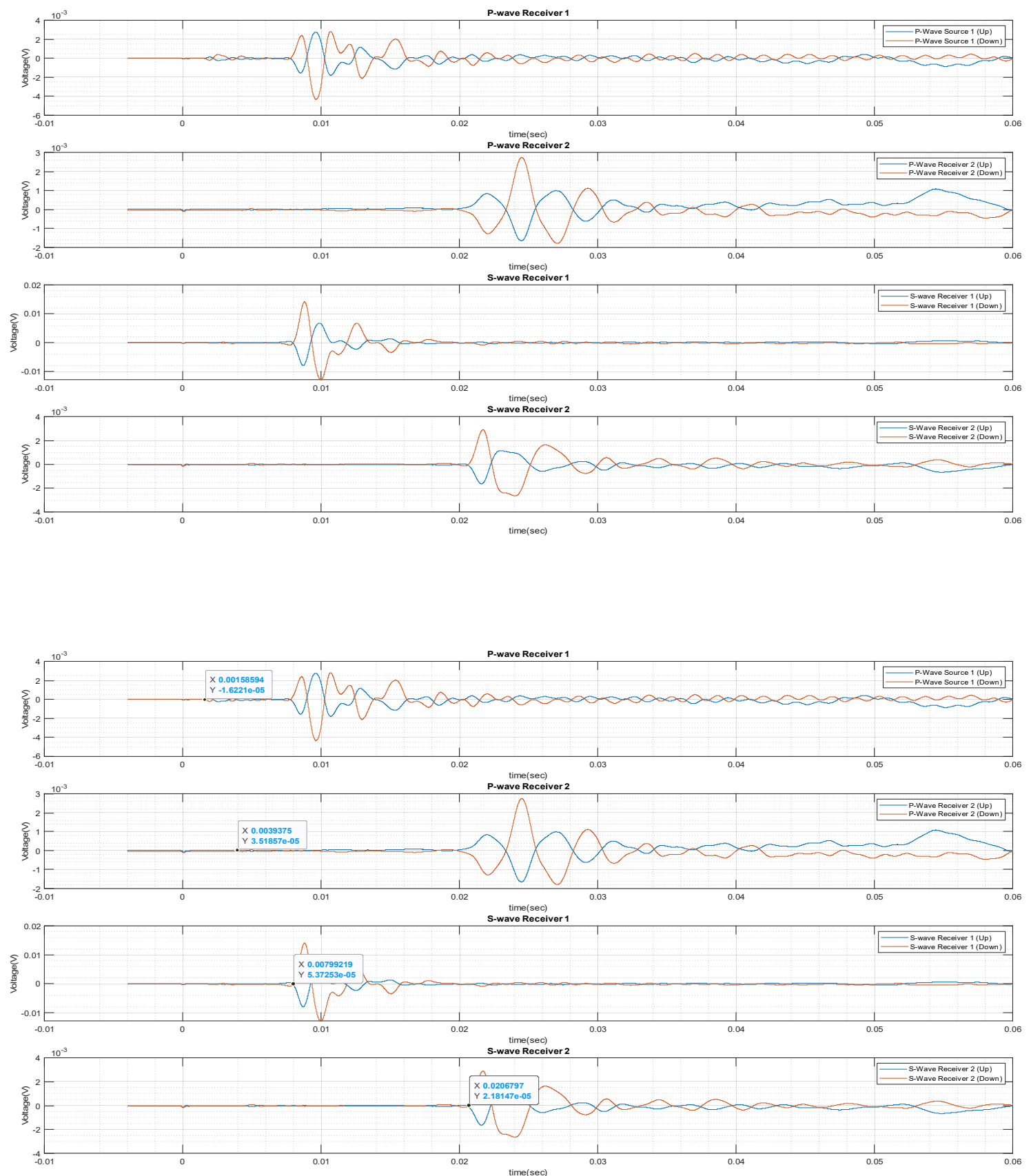

Data Set 35 (1.17.2020)

\section{Run00272f}

R4_2 

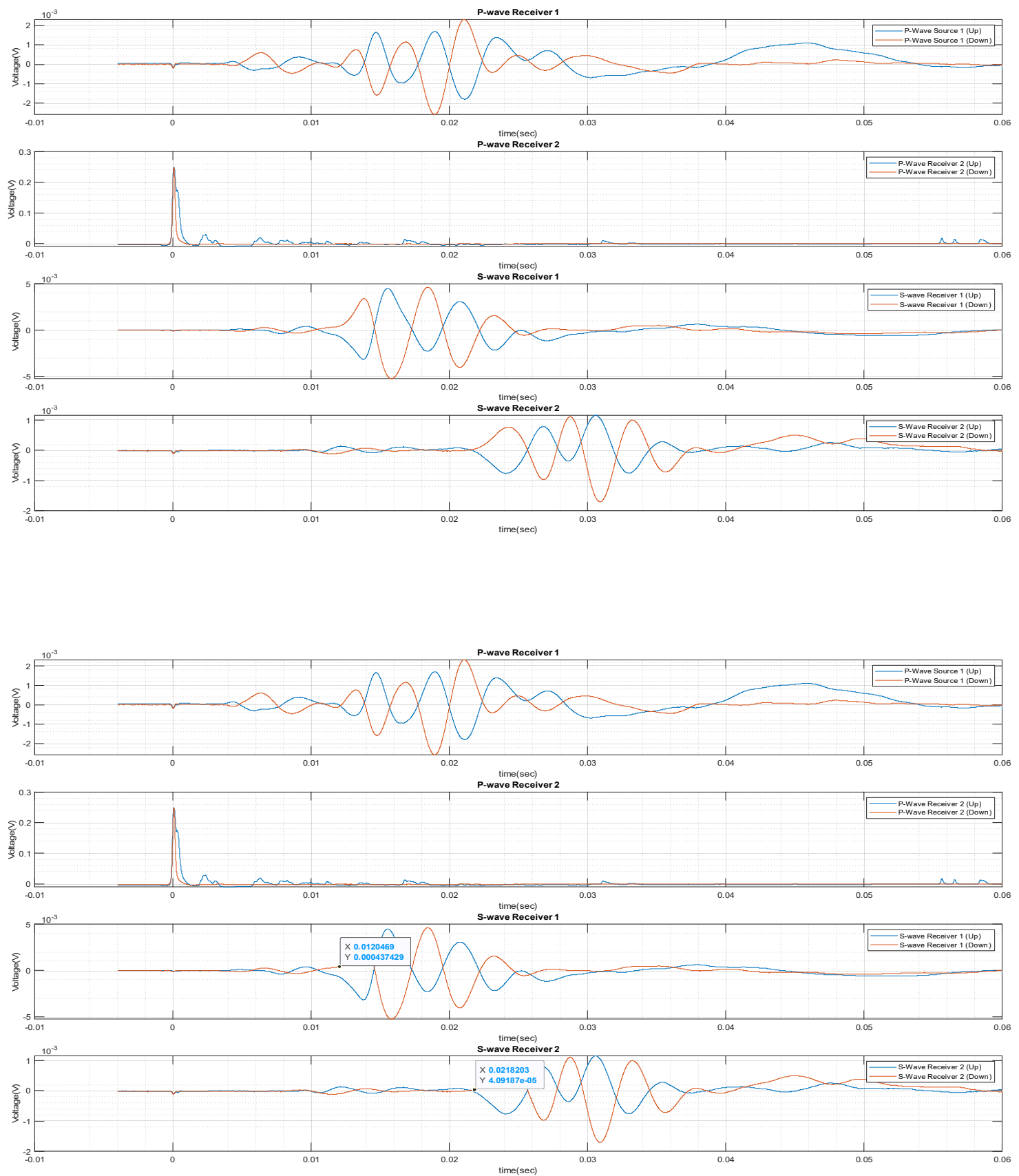

Data Set $36(2.21 .2020)$

Run00273f

R1_1 

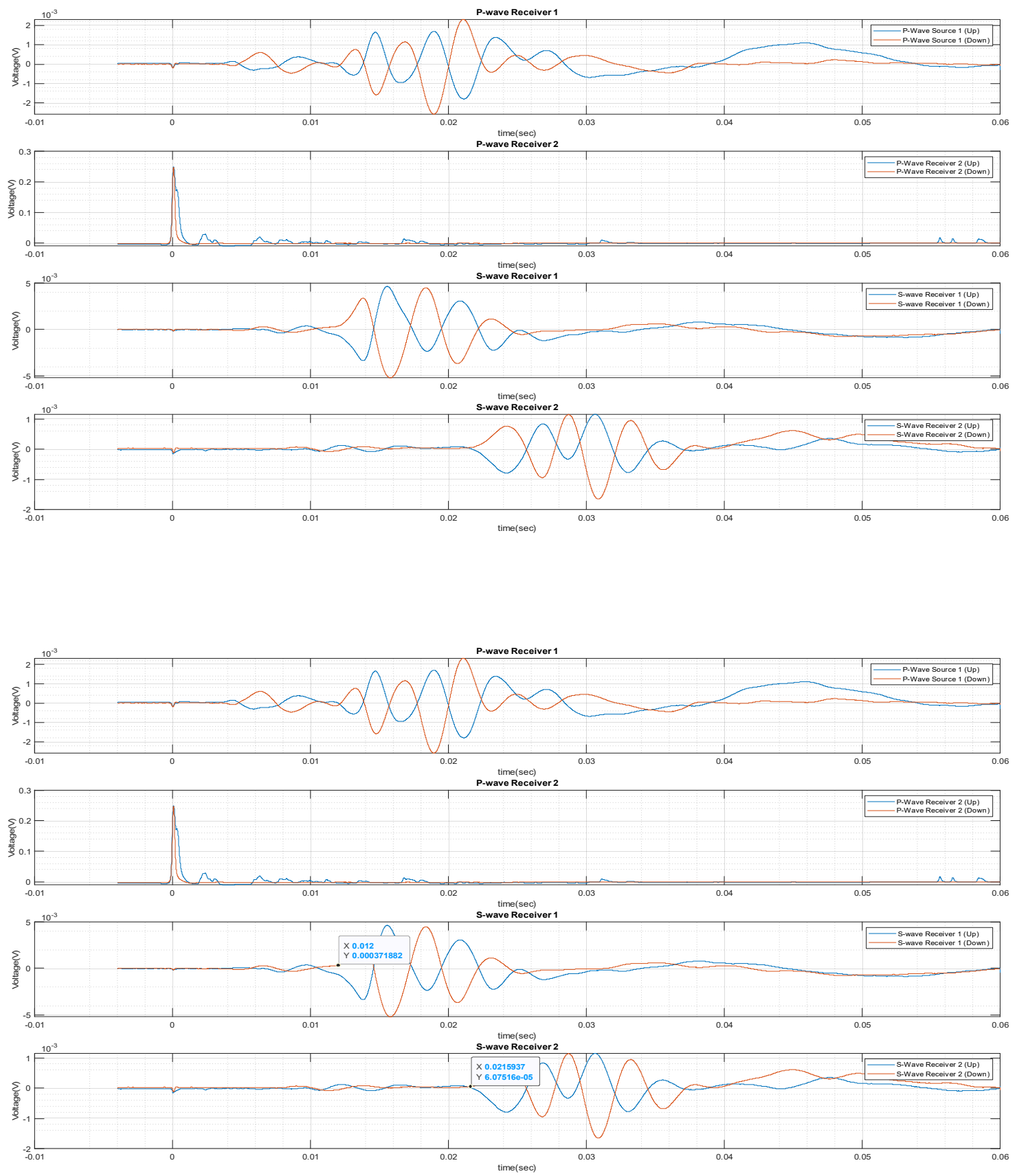

Data Set $36(2.21 .2020)$

\section{Run00274f}

R1_2 

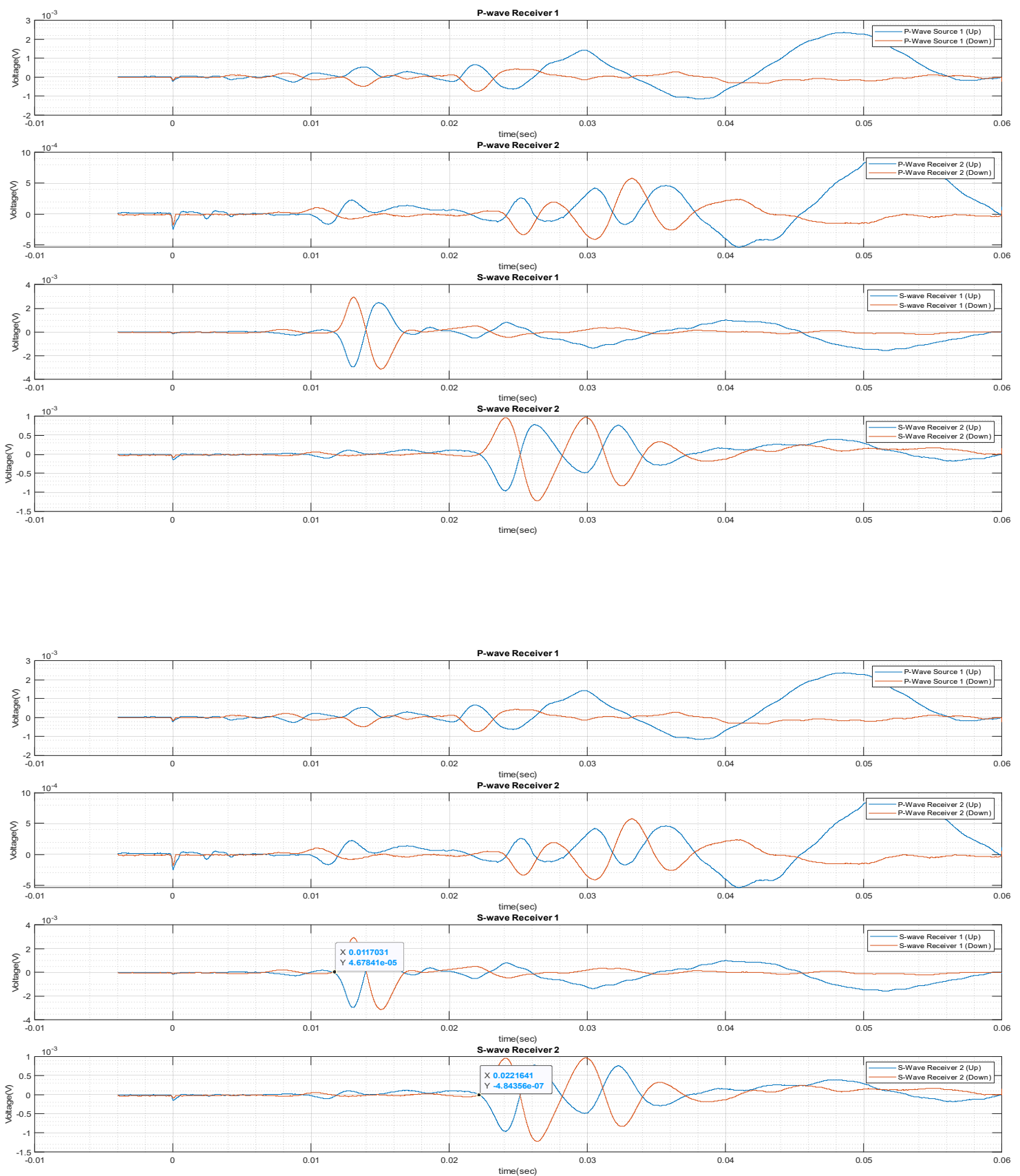

Data Set $36(2.21 .2020)$

Run00275f

R2_1 

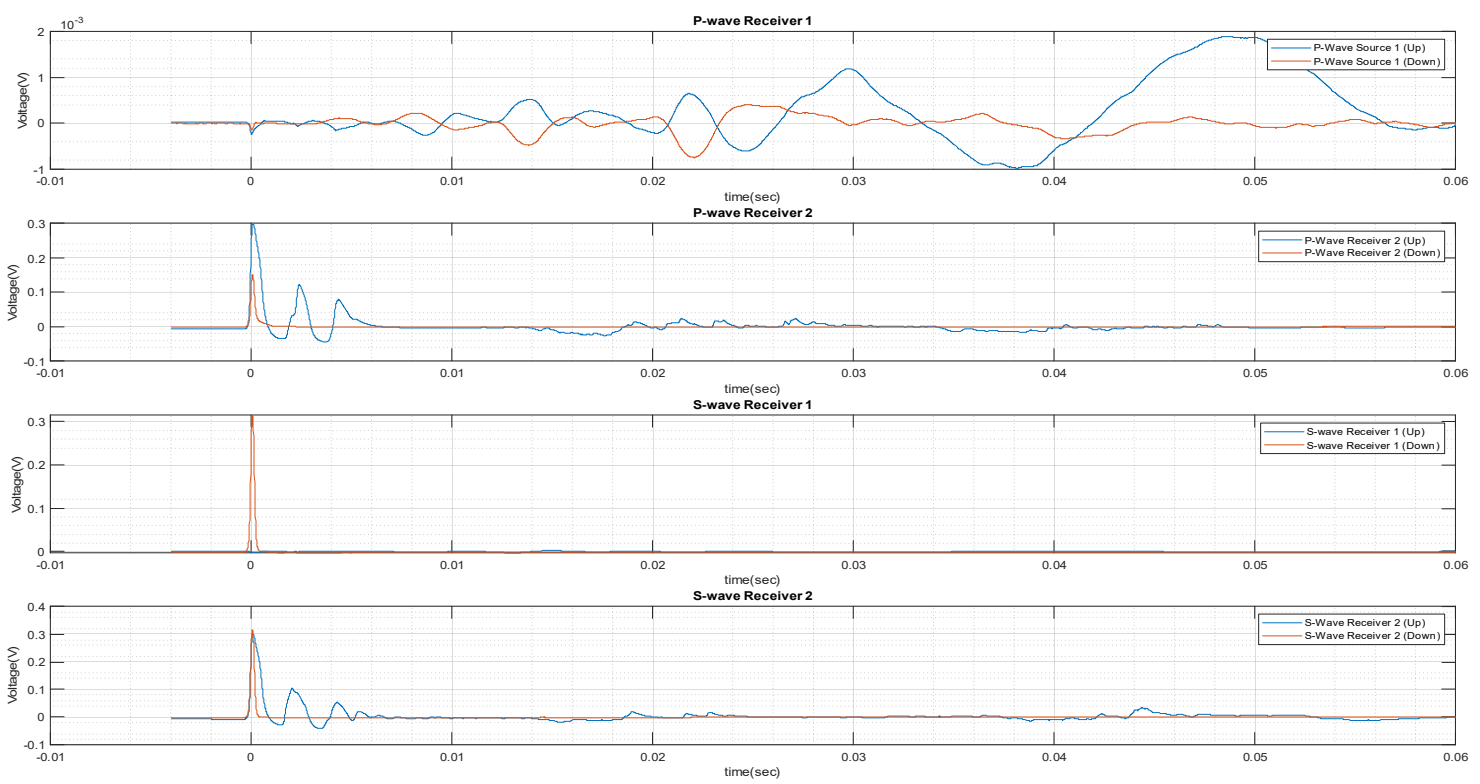

Cannot determine clear arrival times

Data Set 36

(2.21.2020)

Run00276f

R2_2 

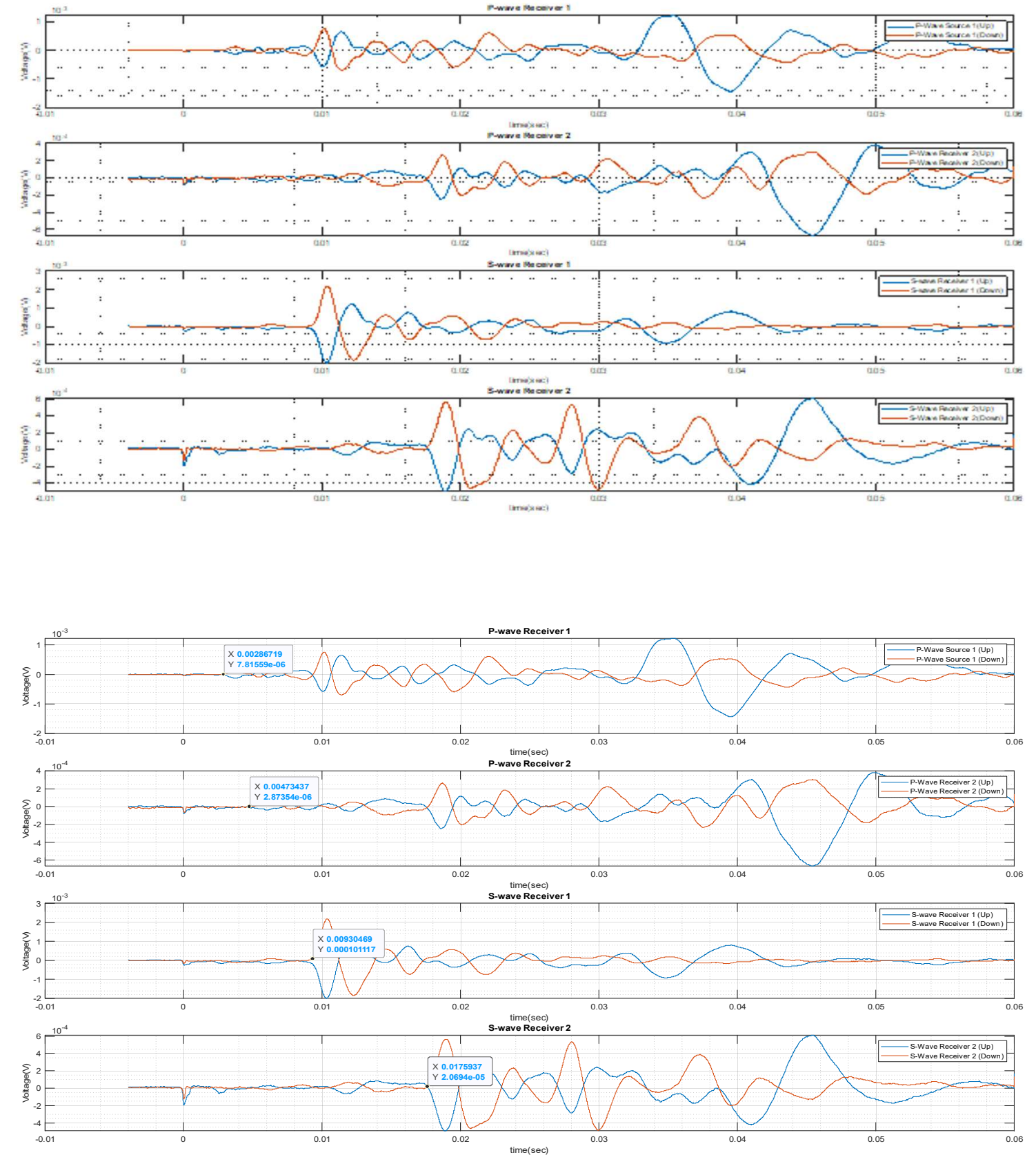

Data Set 36 (2.21.2020)

Run00277f

R3_1 

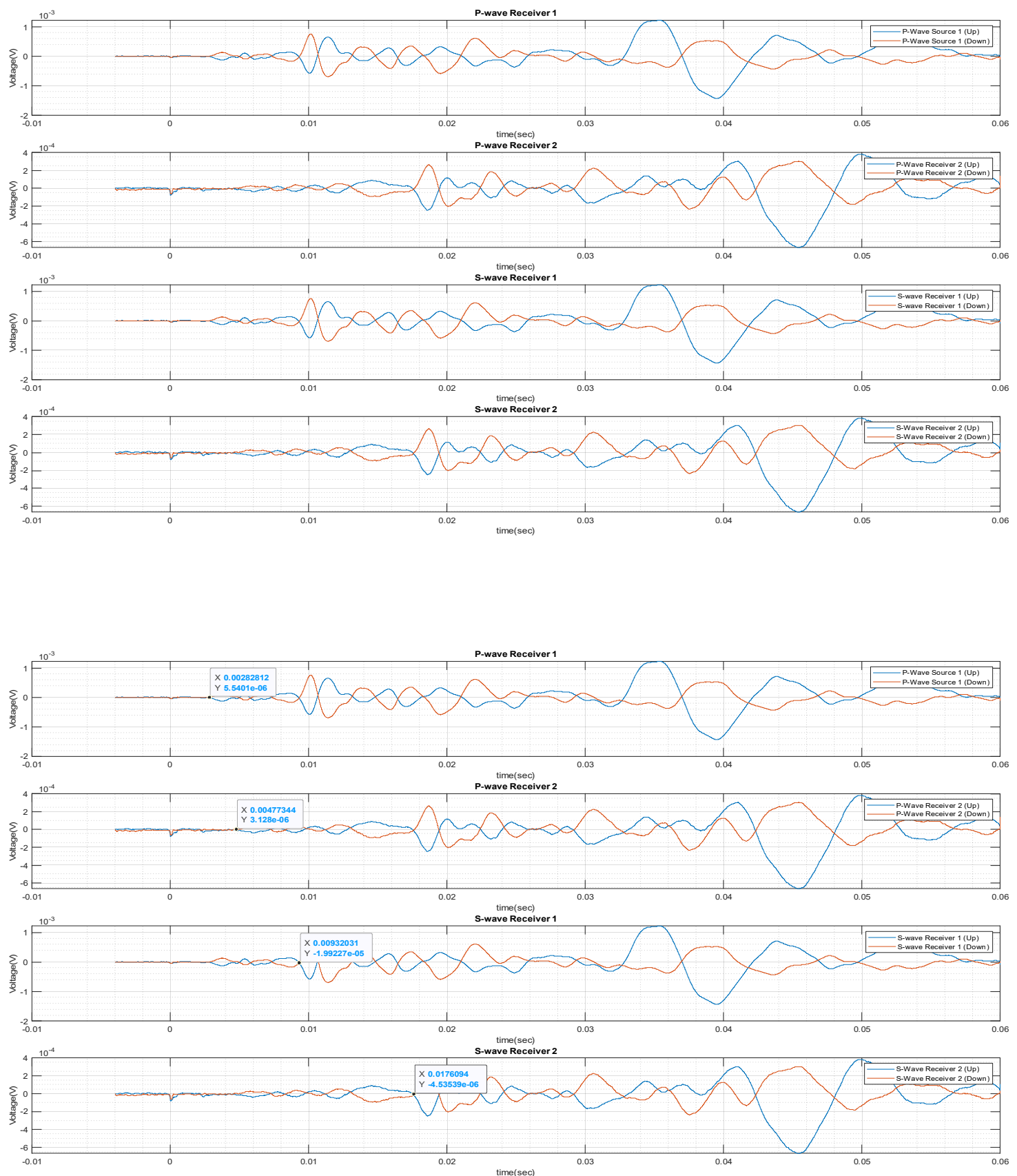

Data Set $36(2.21 .2020)$

\section{Run00278f}

R3_2 

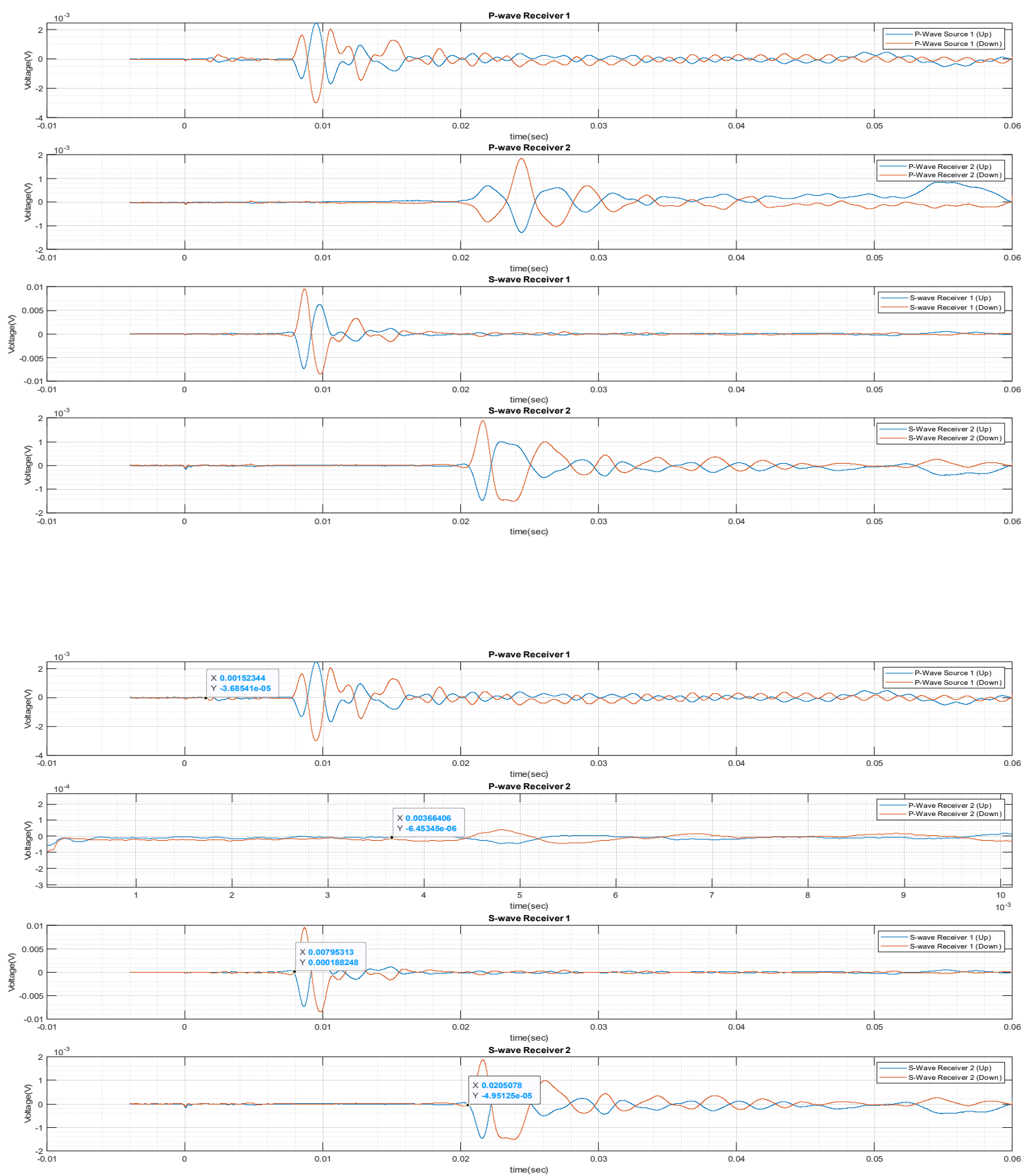

Data Set $36(2.21 .2020)$

Run00279f

R4_1 

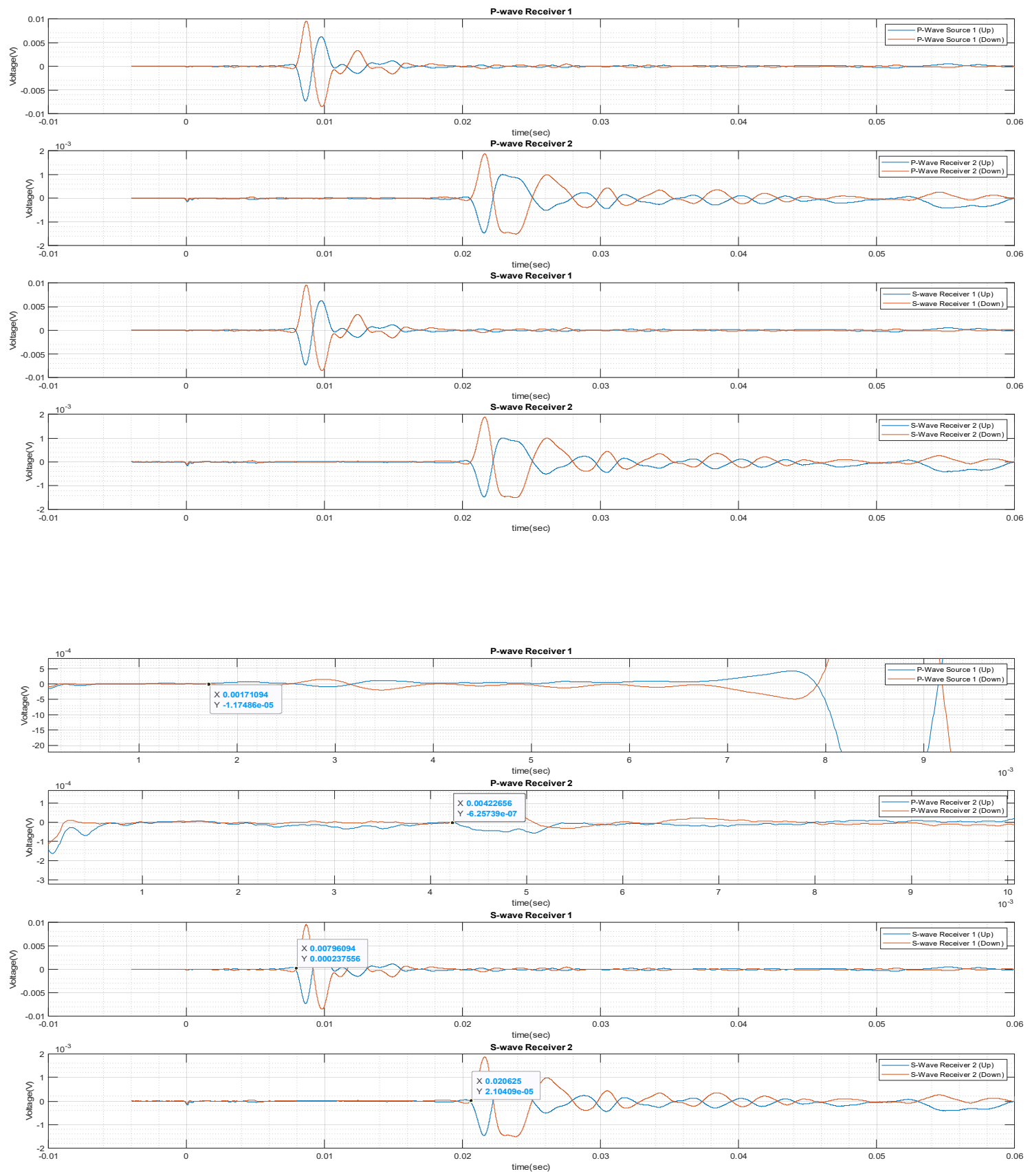

Data Set $36(2.21 .2020)$

Run00280f

R4_2 

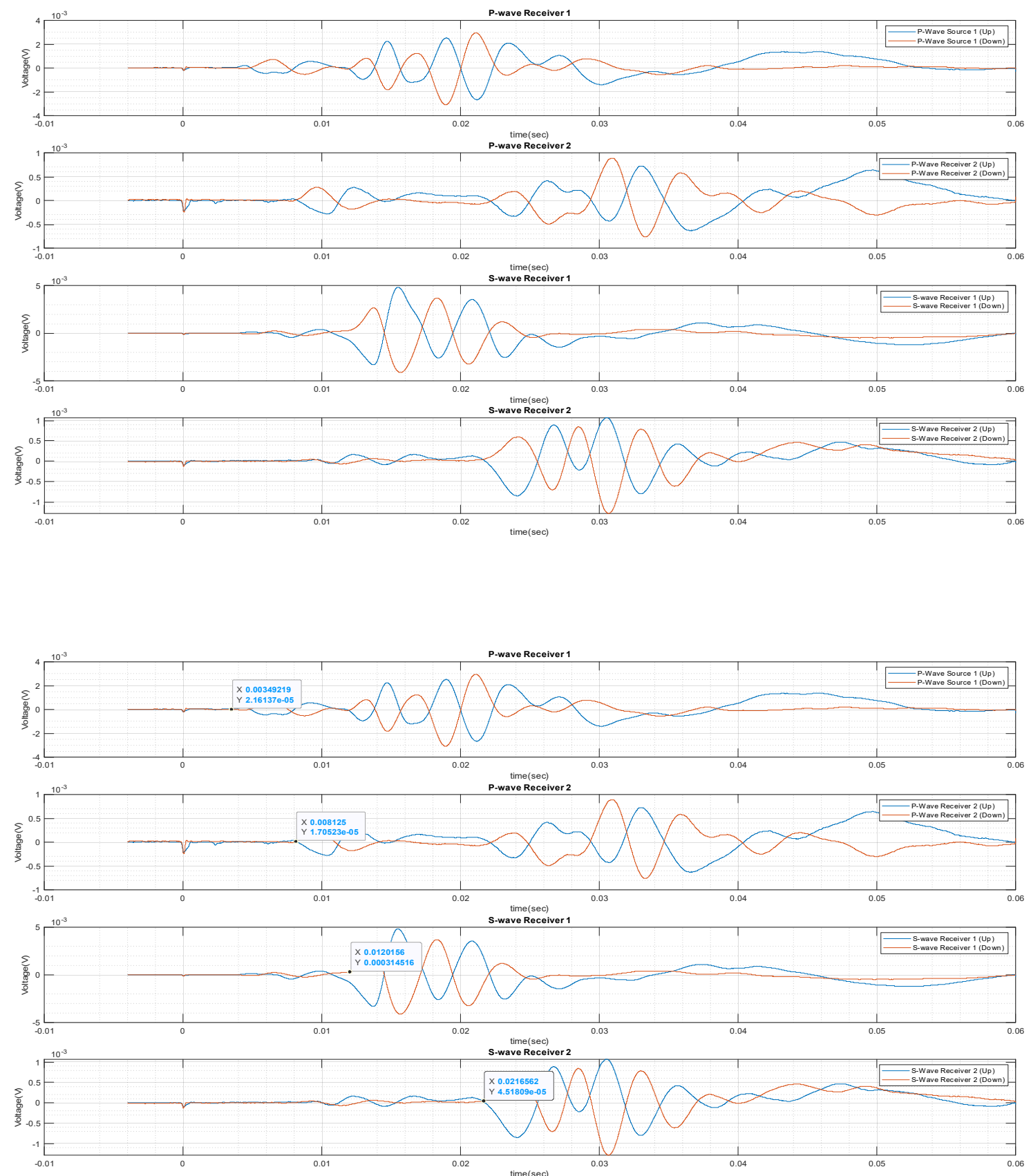

Data Set $37(2.28 .2020)$

Run00281f

R1_1 

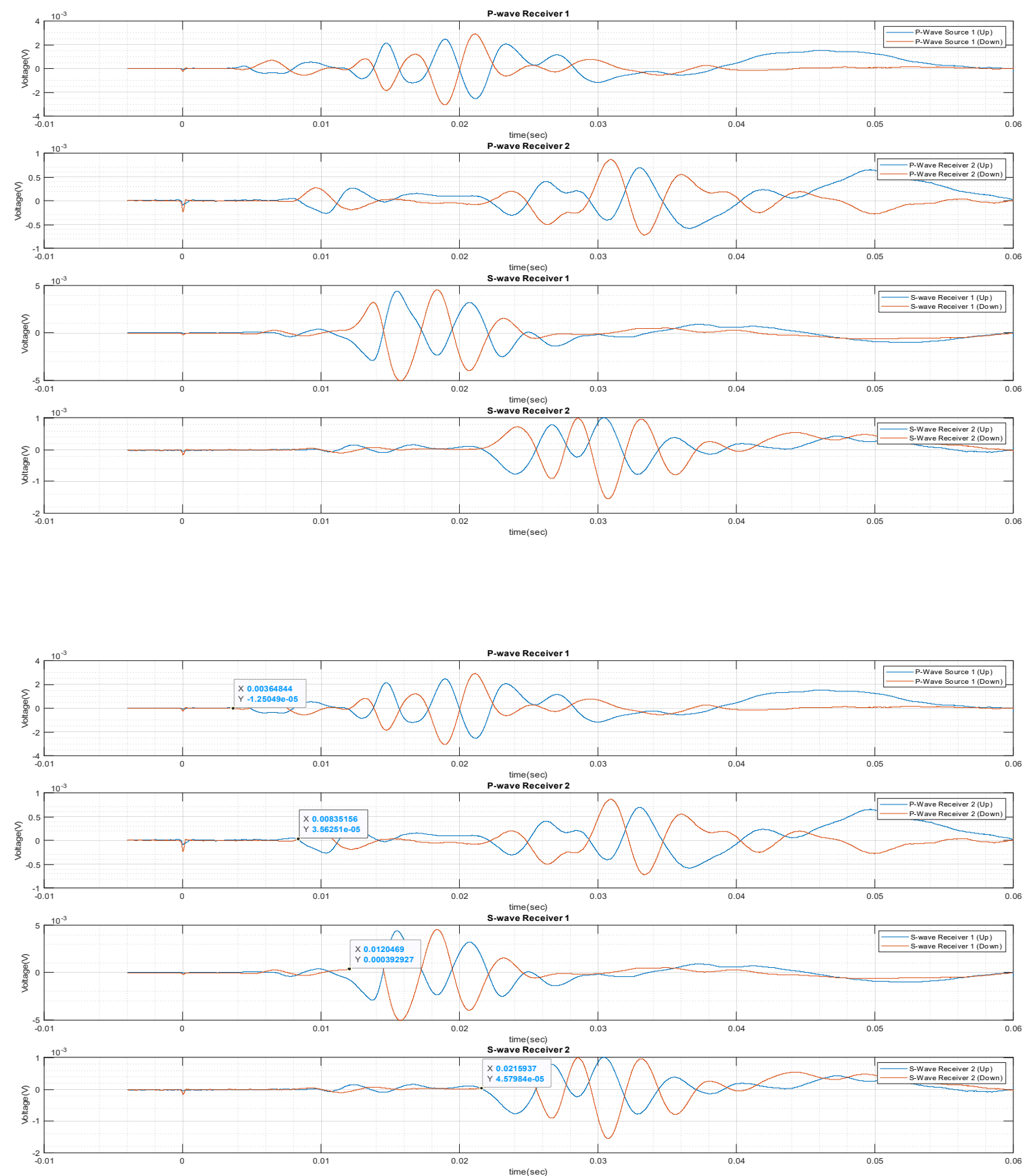

Data Set $37(2.28 .2020)$

Run00282f

R1_2 

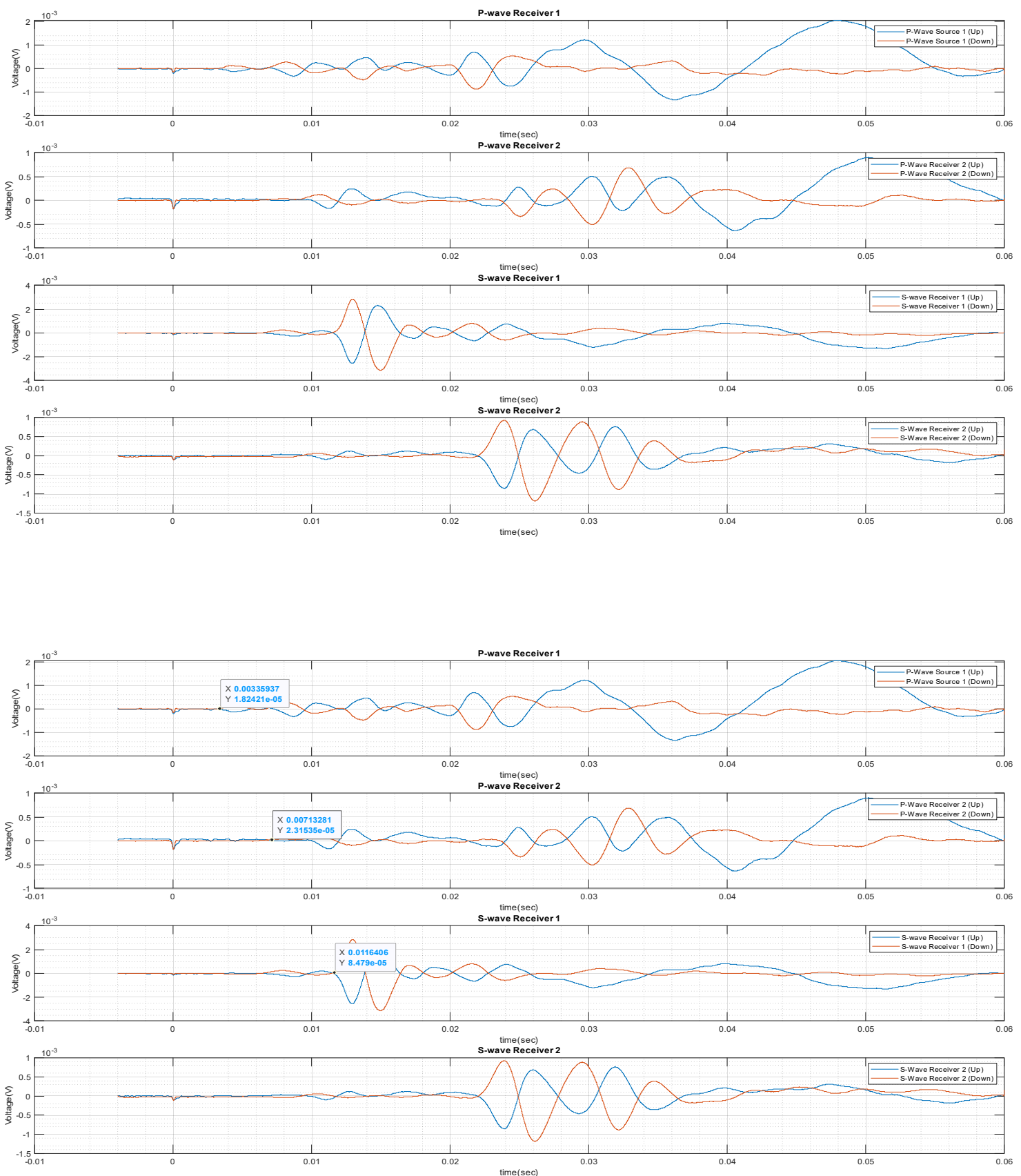

Data Set $37(2.28 .2020)$

Run00283f

R2_1 

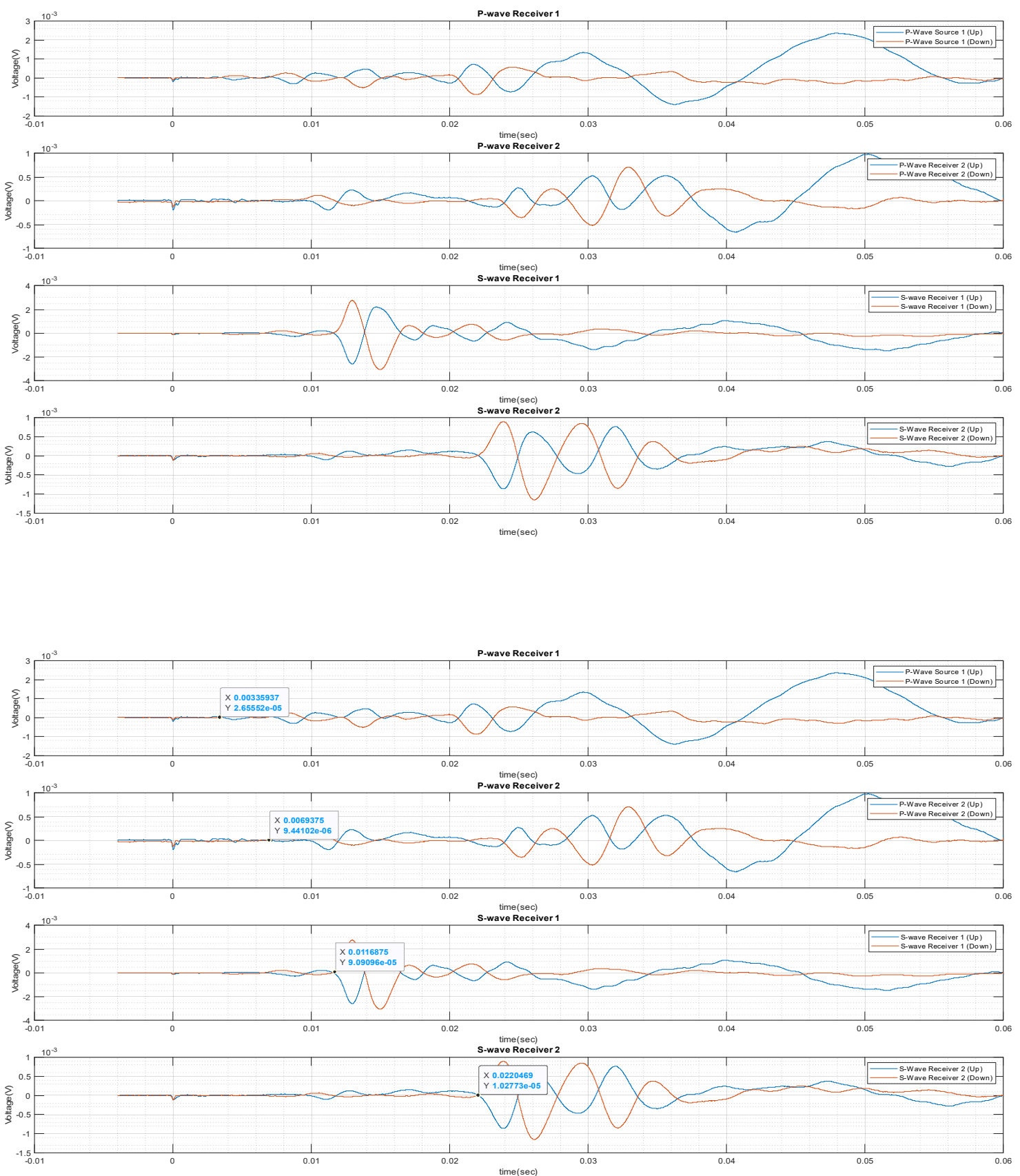

Data Set $37(2.28 .2020)$

Run00284f

R2_2 

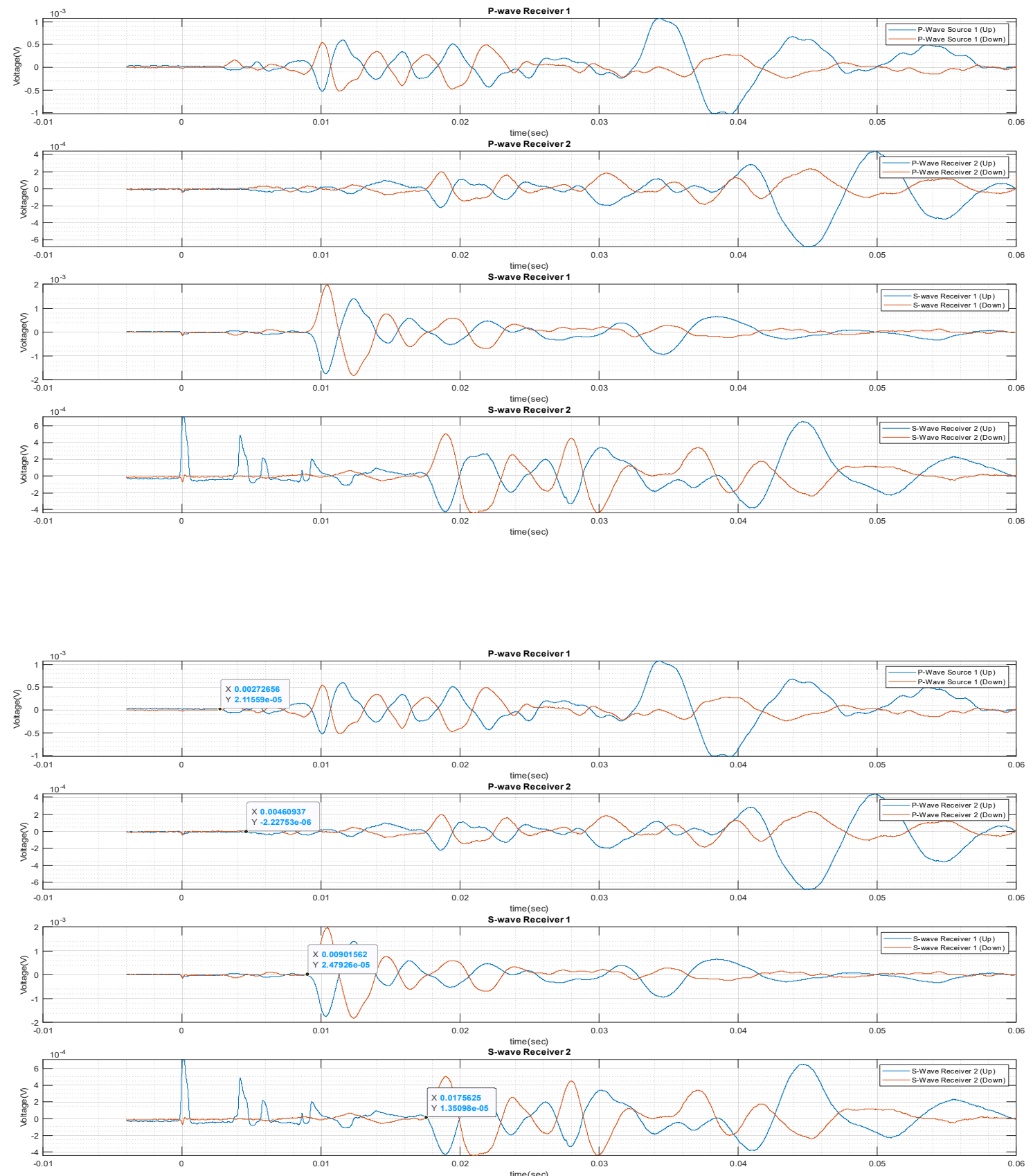

Data Set 37 (2.28.2020)

Run00285f

R3_1 

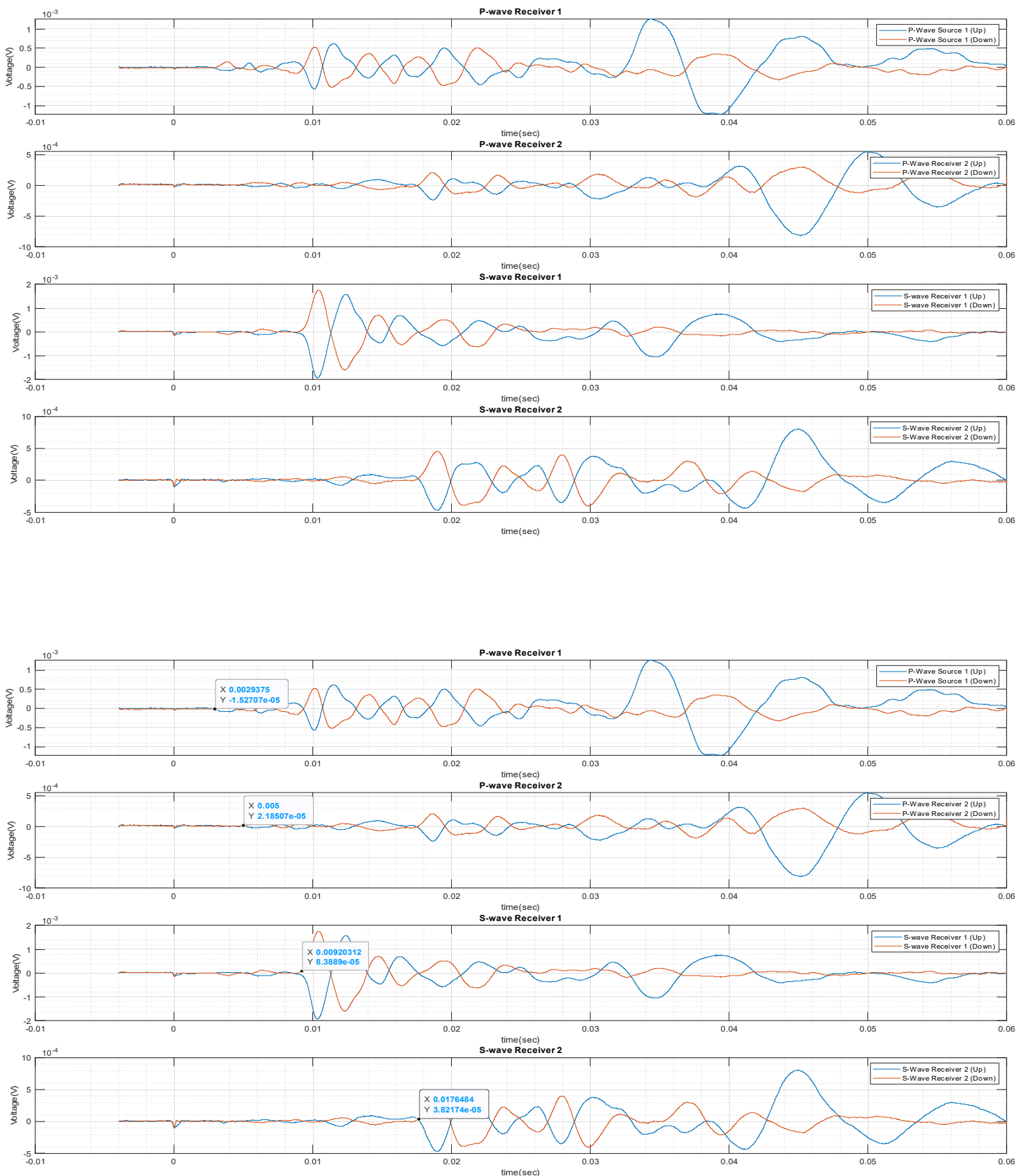

Data Set $37(2.28 .2020)$

Run00286f

R3_2 

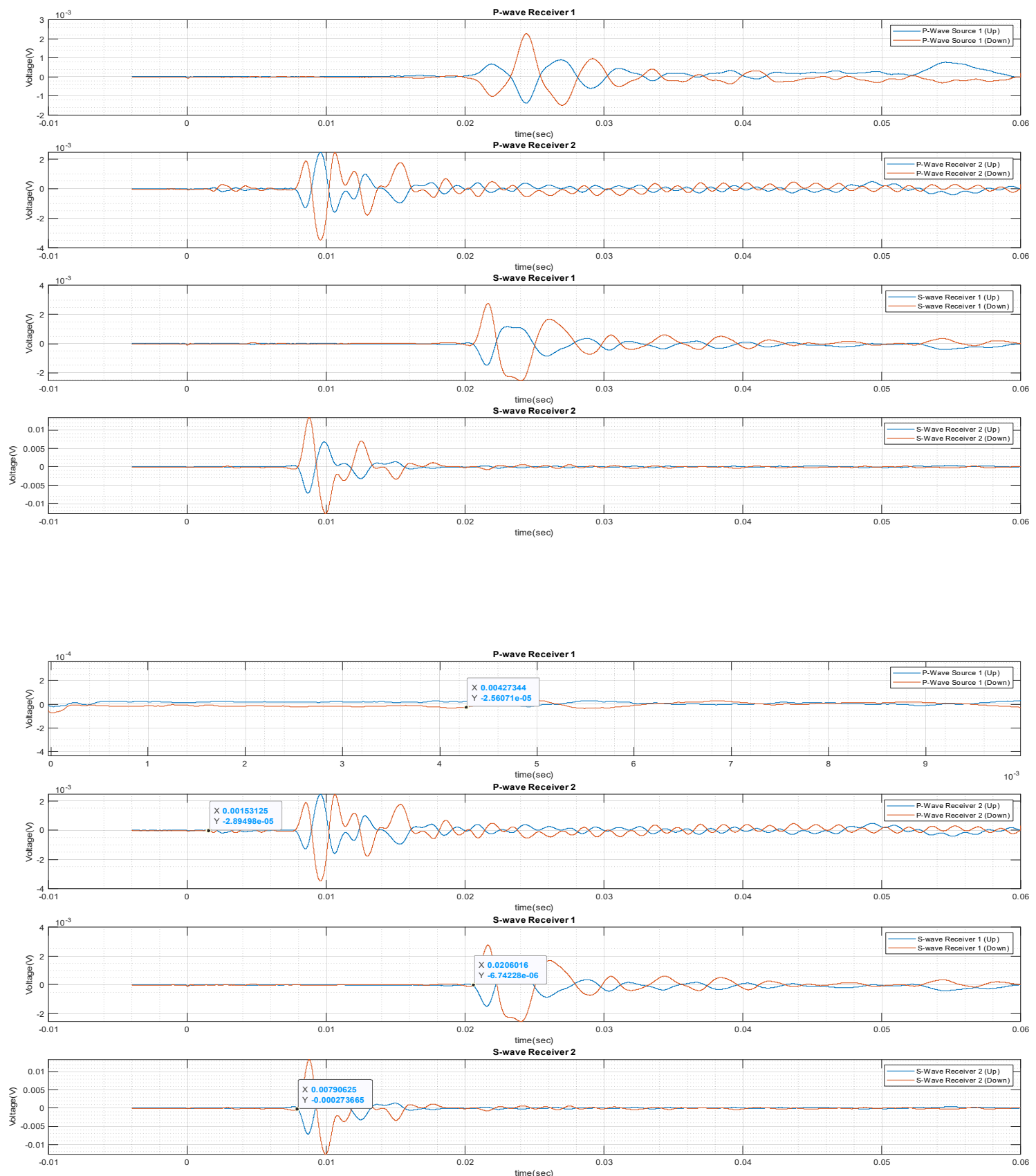

Data Set $37(2.28 .2020)$

Run00287f

R4_1 

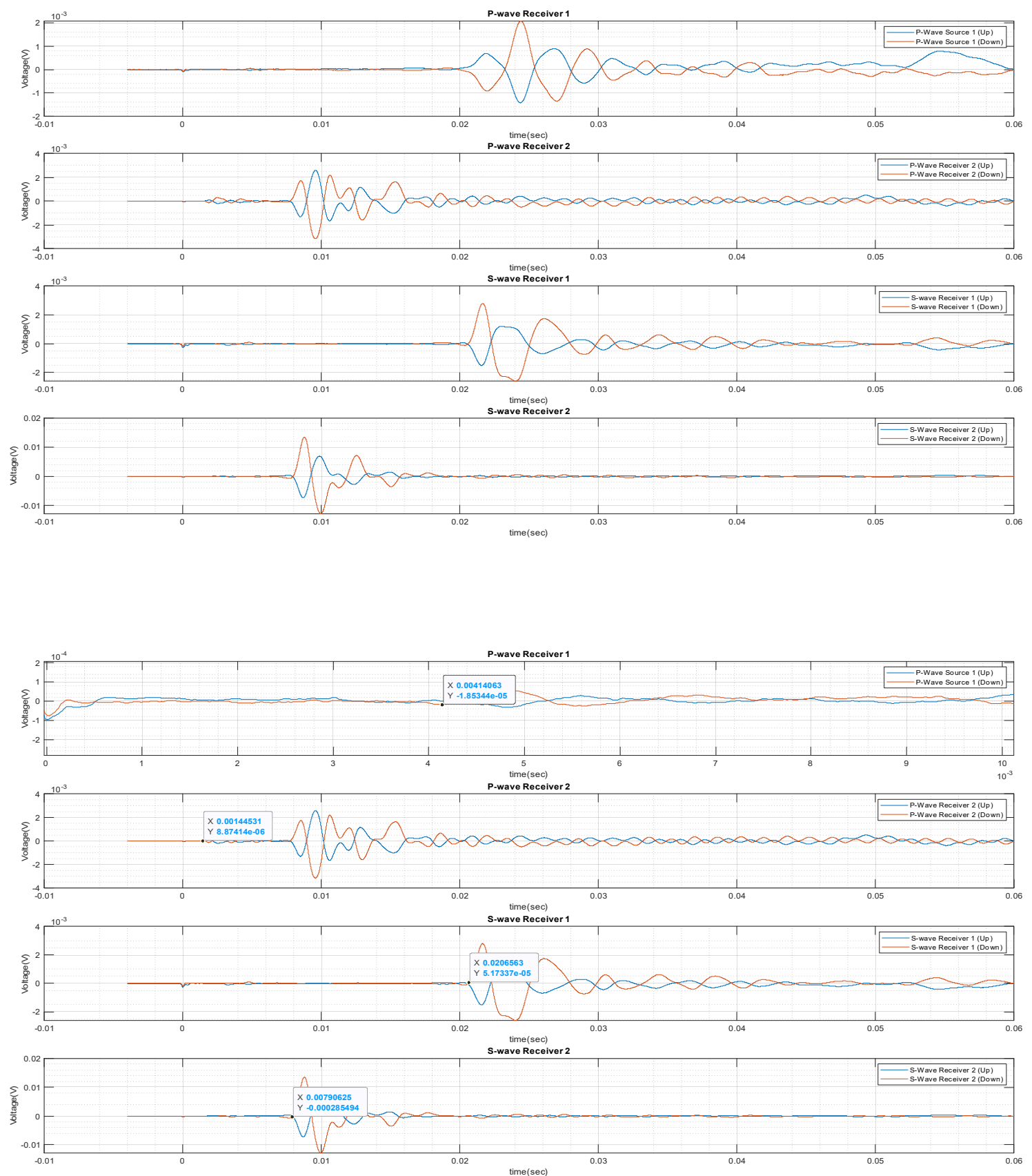

Data Set $37(2.28 .2020)$

Run00288f

R4_2 

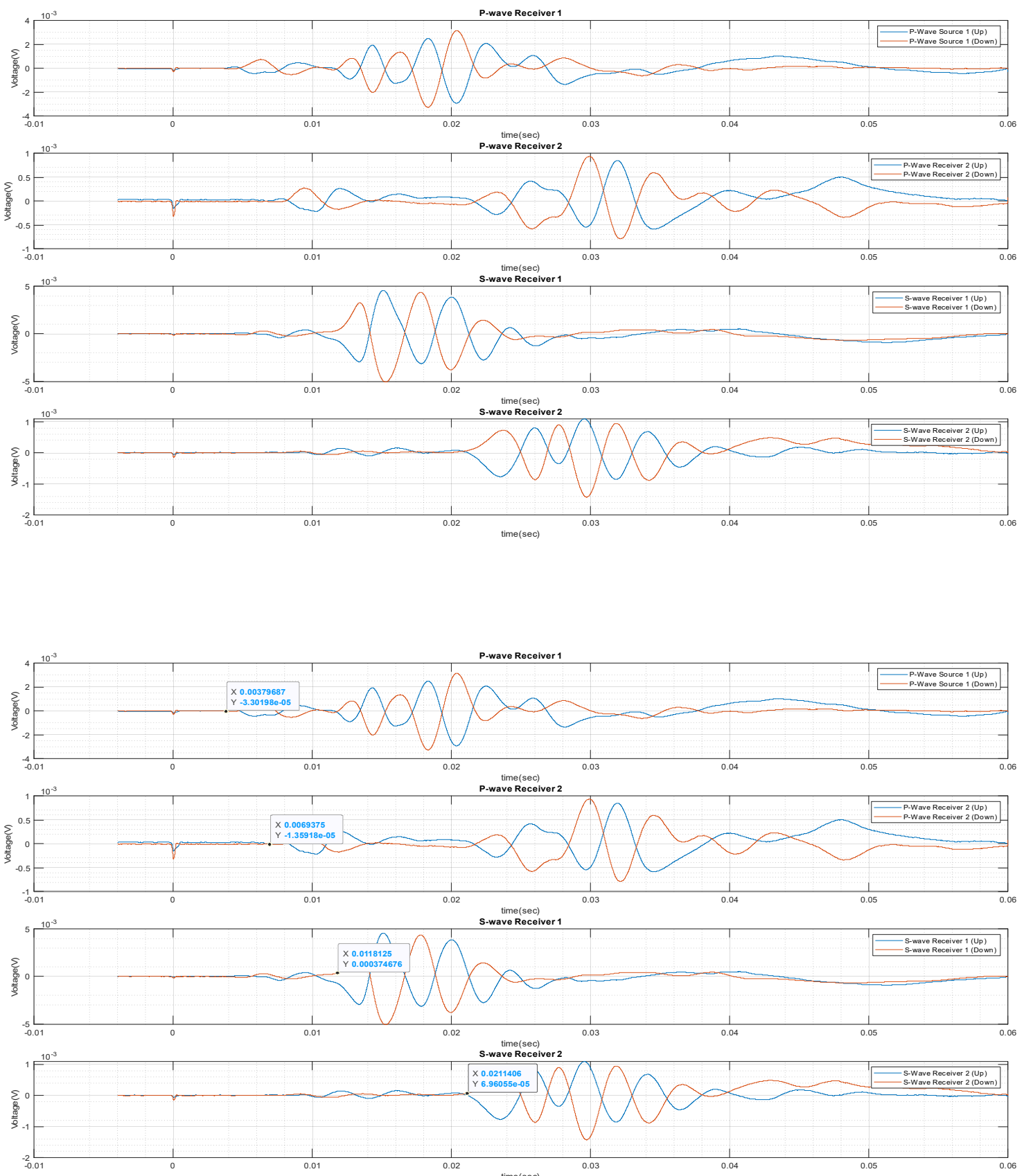

Data Set 38 (4.3.2020)

Run00289f

R1_1 

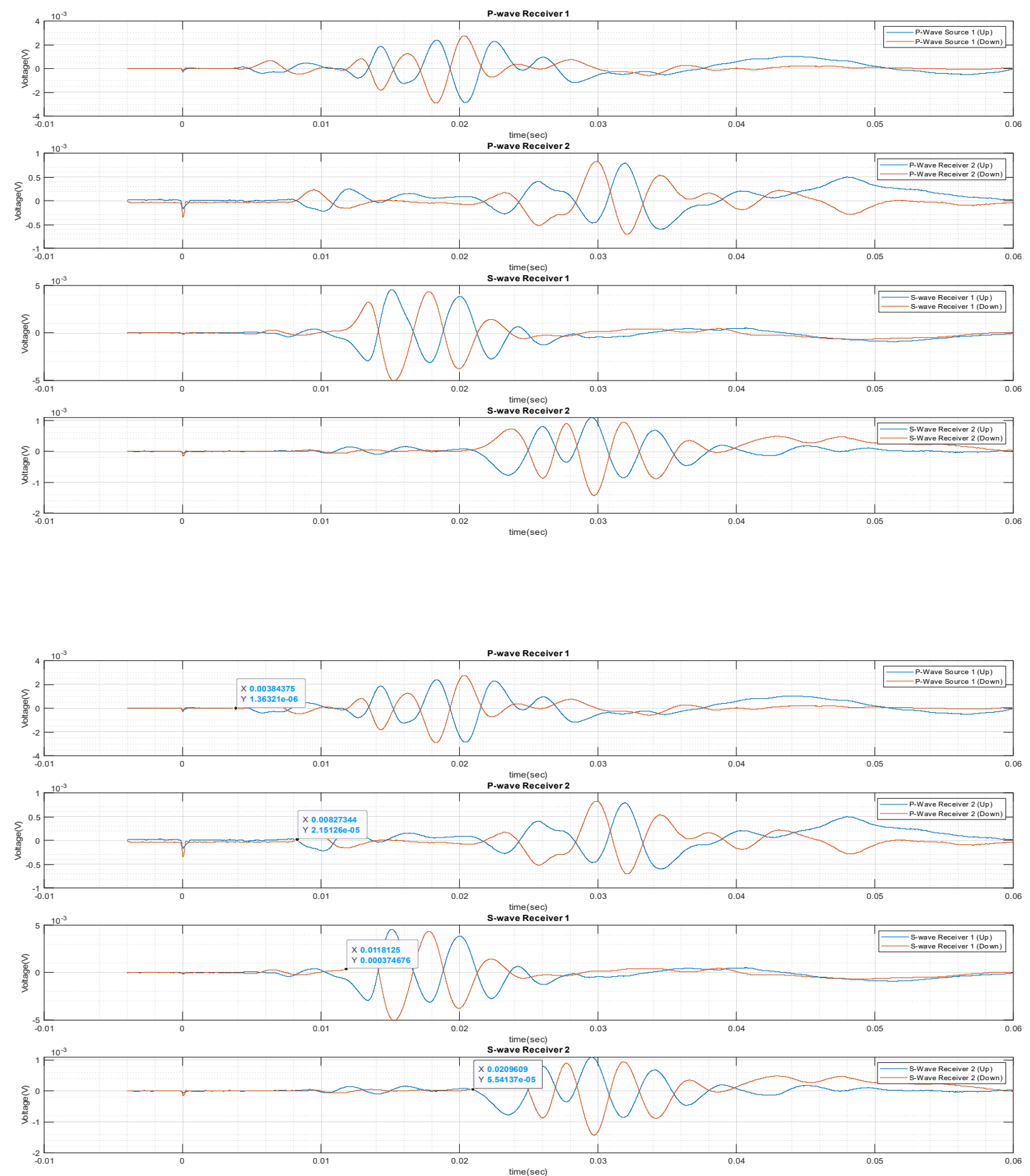

Data Set 38 (4.3.2020)

Run00290f

R1_2 

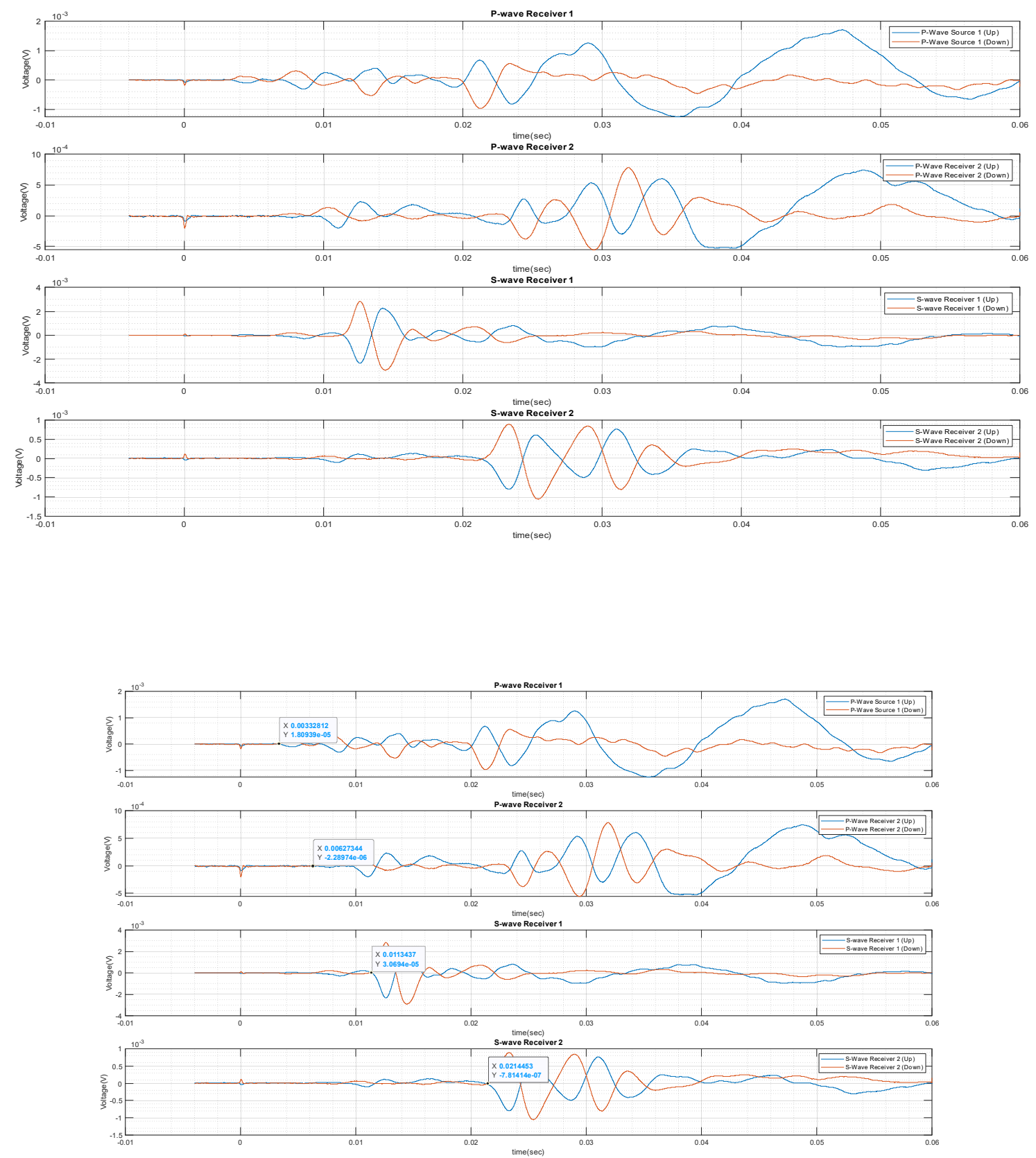

Data Set 38 (4.3.2020)

Run00291f

R2 1 

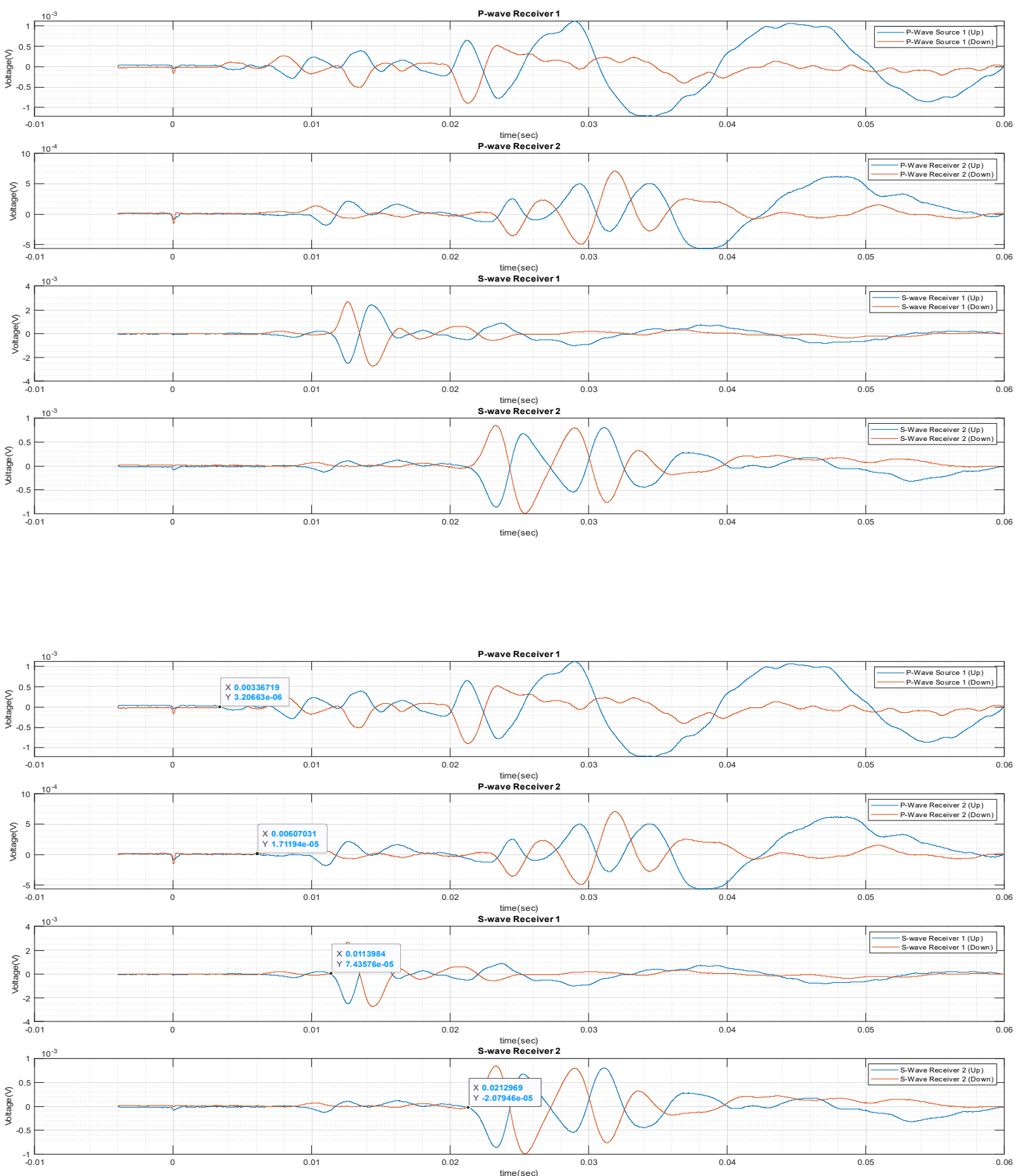

Data Set 38 (4.3.2020)

Run00292f

R2_2 

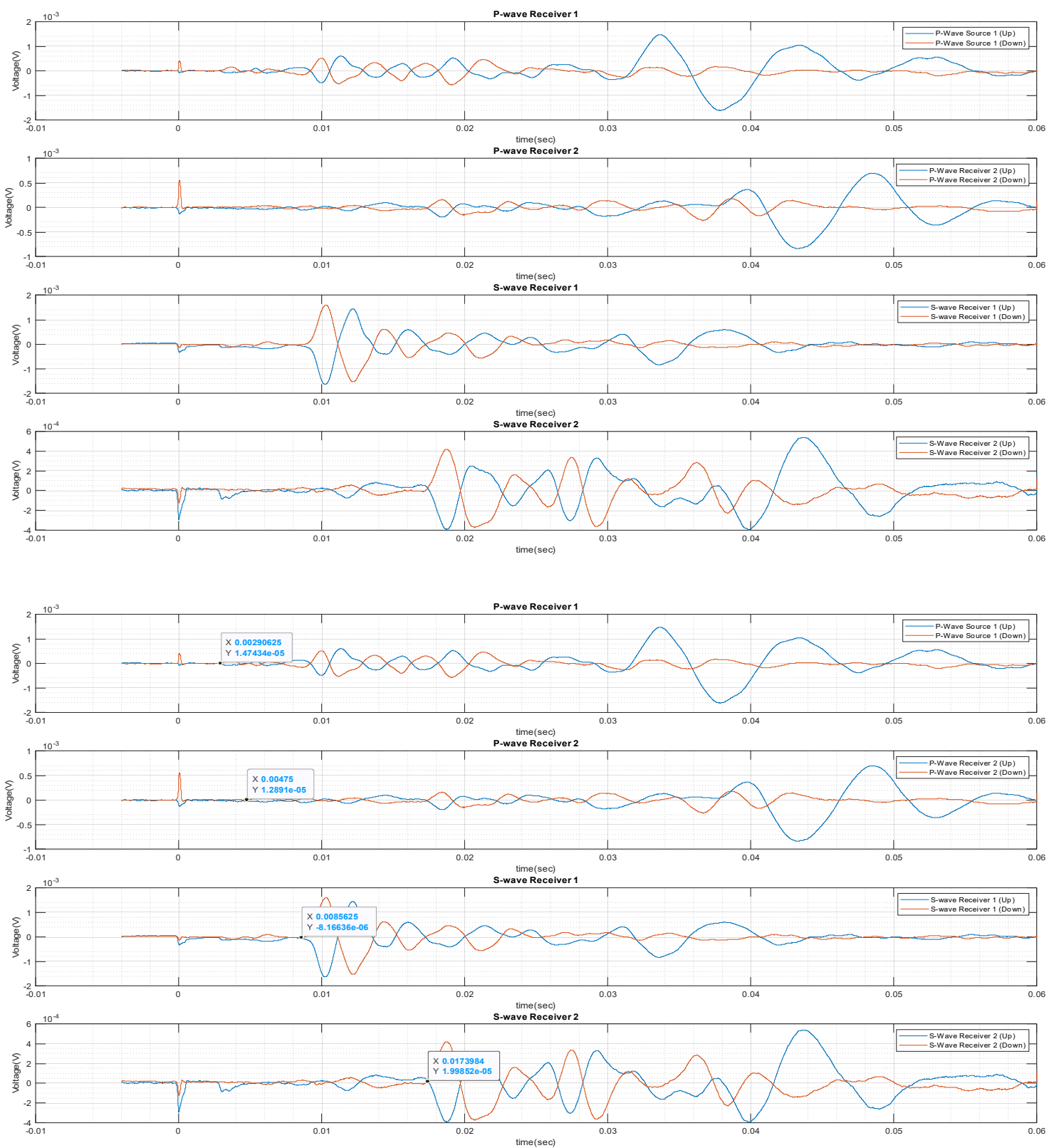

Data Set 38 (4.3.2020)

Run00293f

R3_1 

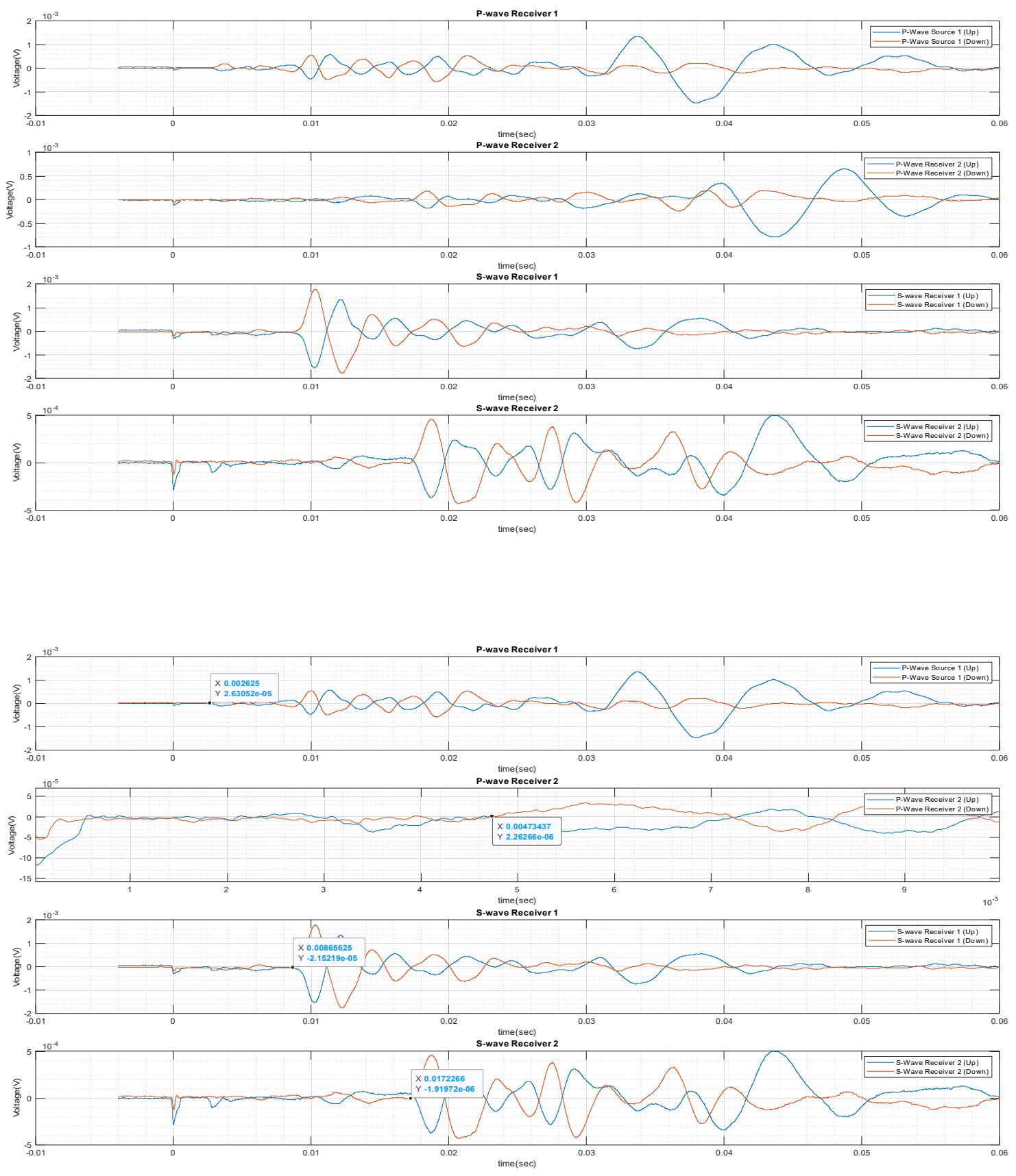

Data Set 38 (4.3.2020)

Run00294f

R3_2 

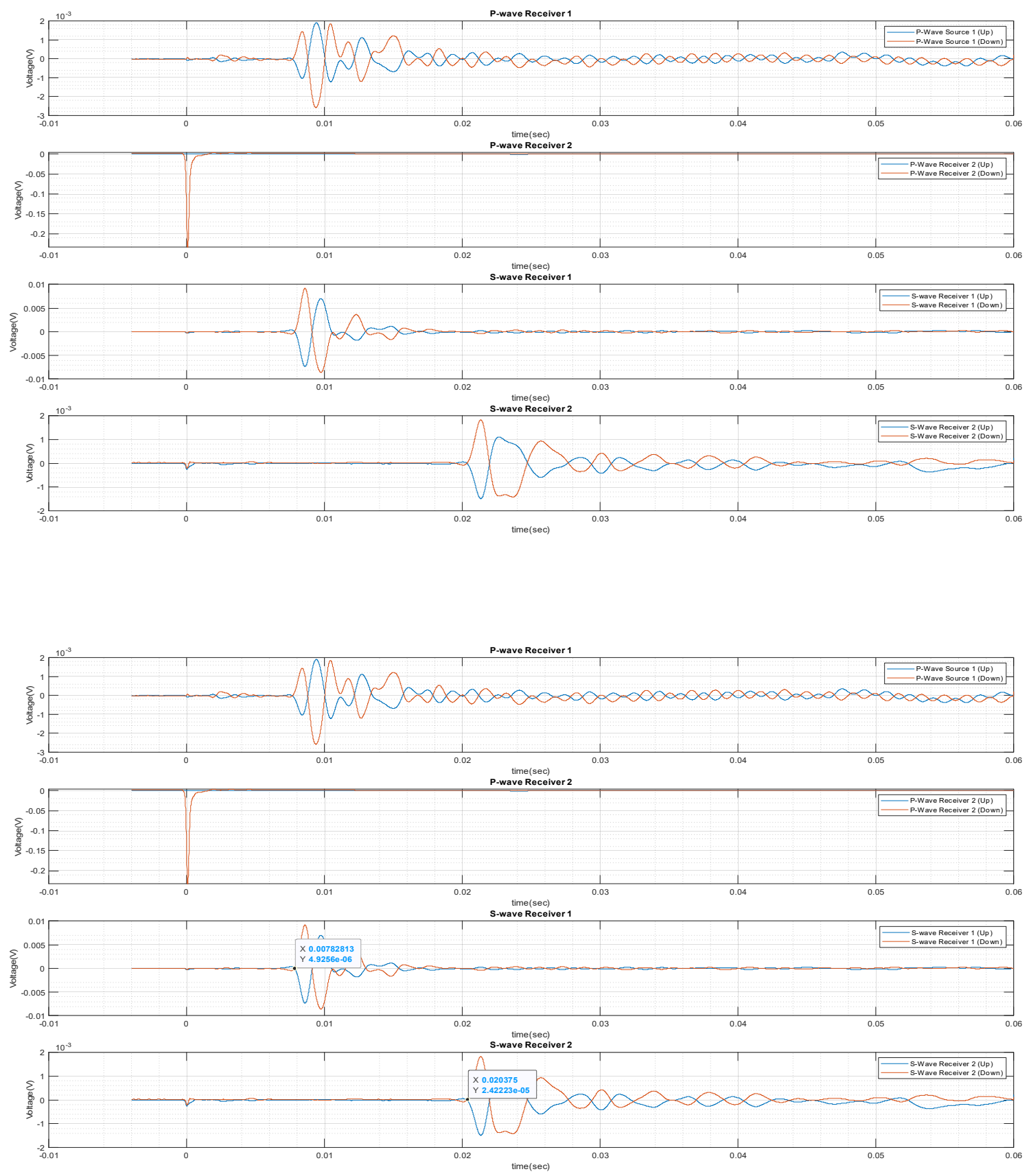

Data Set 38 (4.3.2020)

Run00295f

R4_1 

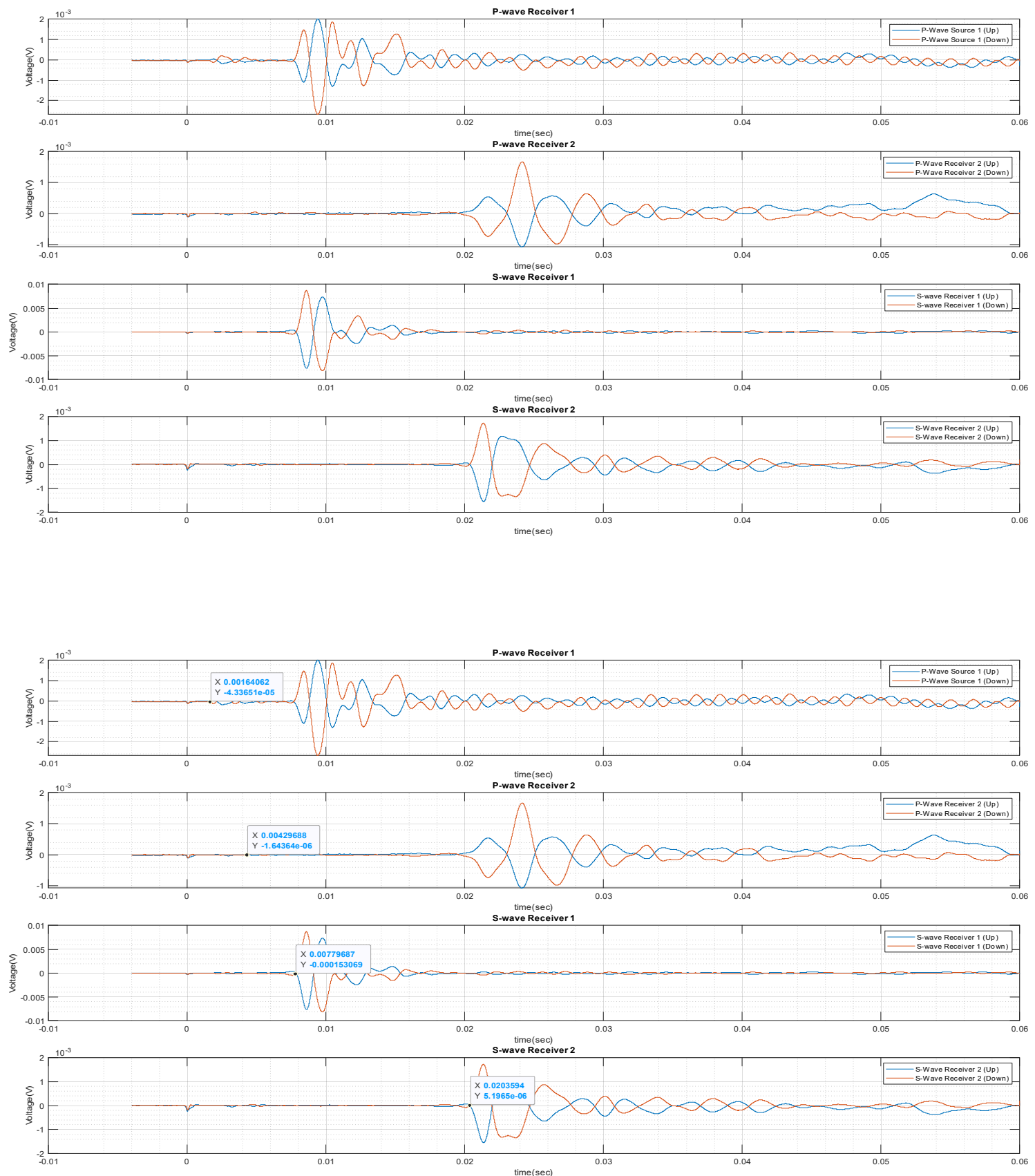

Data Set 38 (4.3.2020)

Run00296f

R4_2 

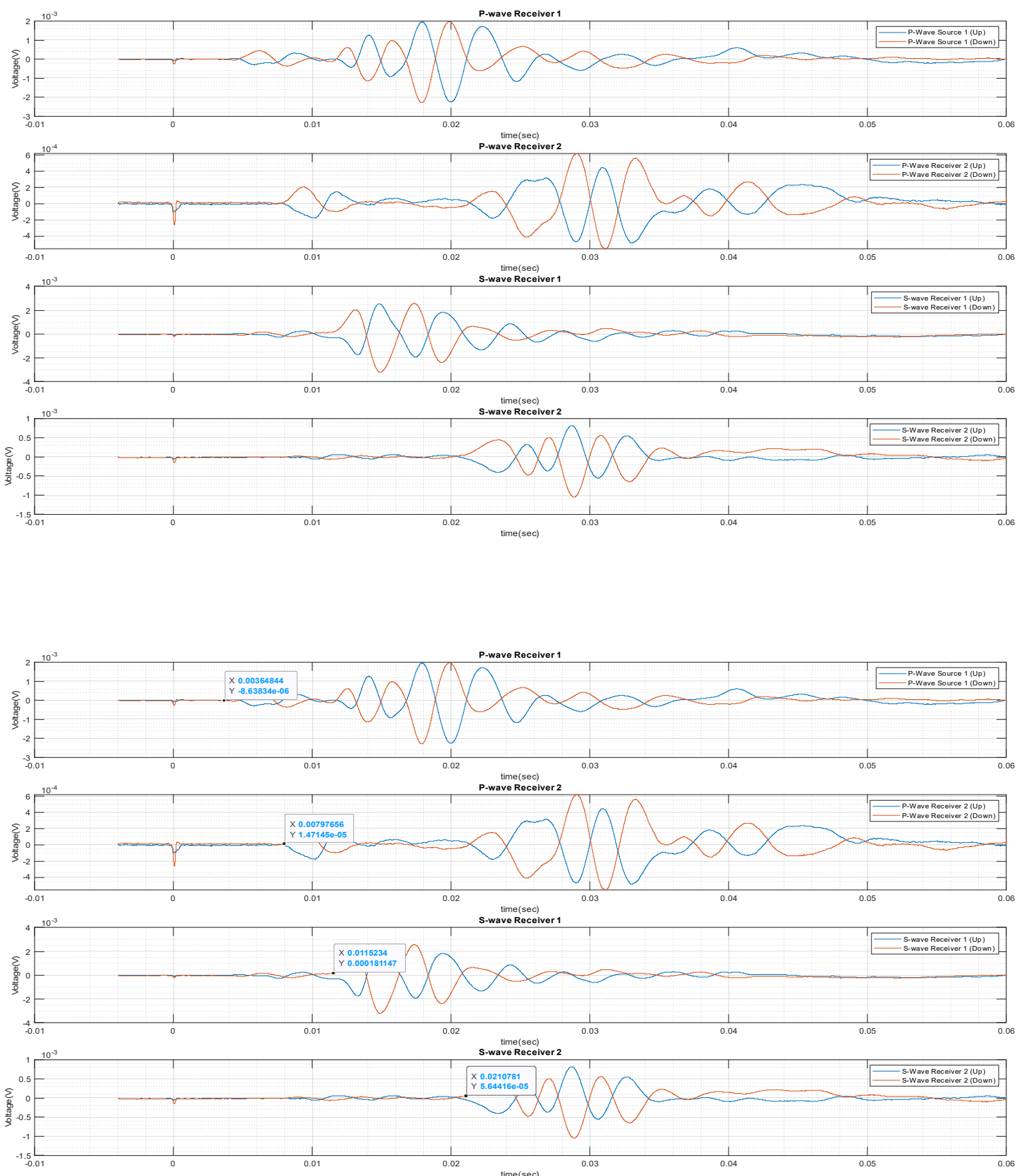

Data Set 39 (4.28.2020)

Run00297f

R1_1 

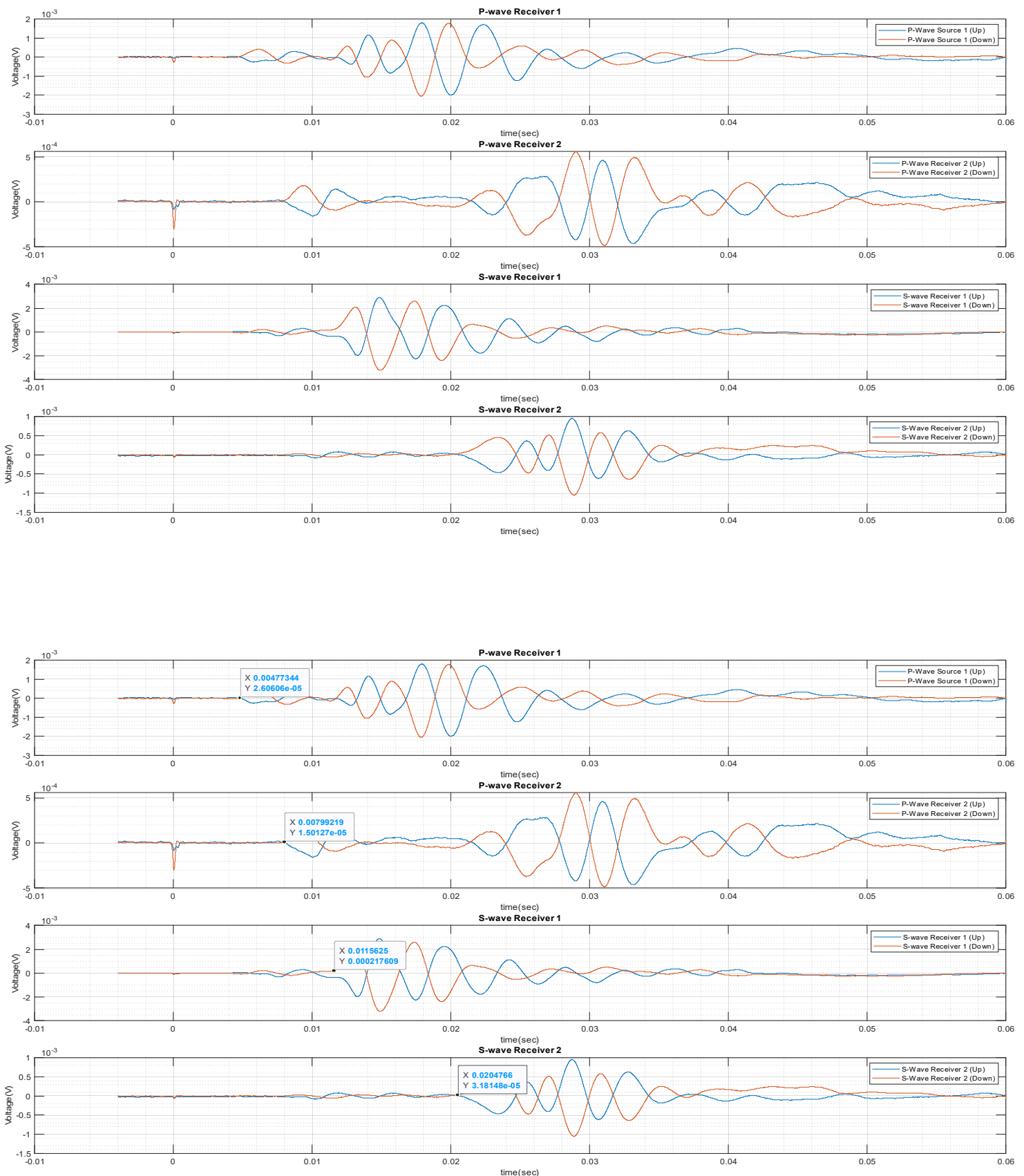

Data Set 39 (4.28.2020)

Run00298f

R1_2 

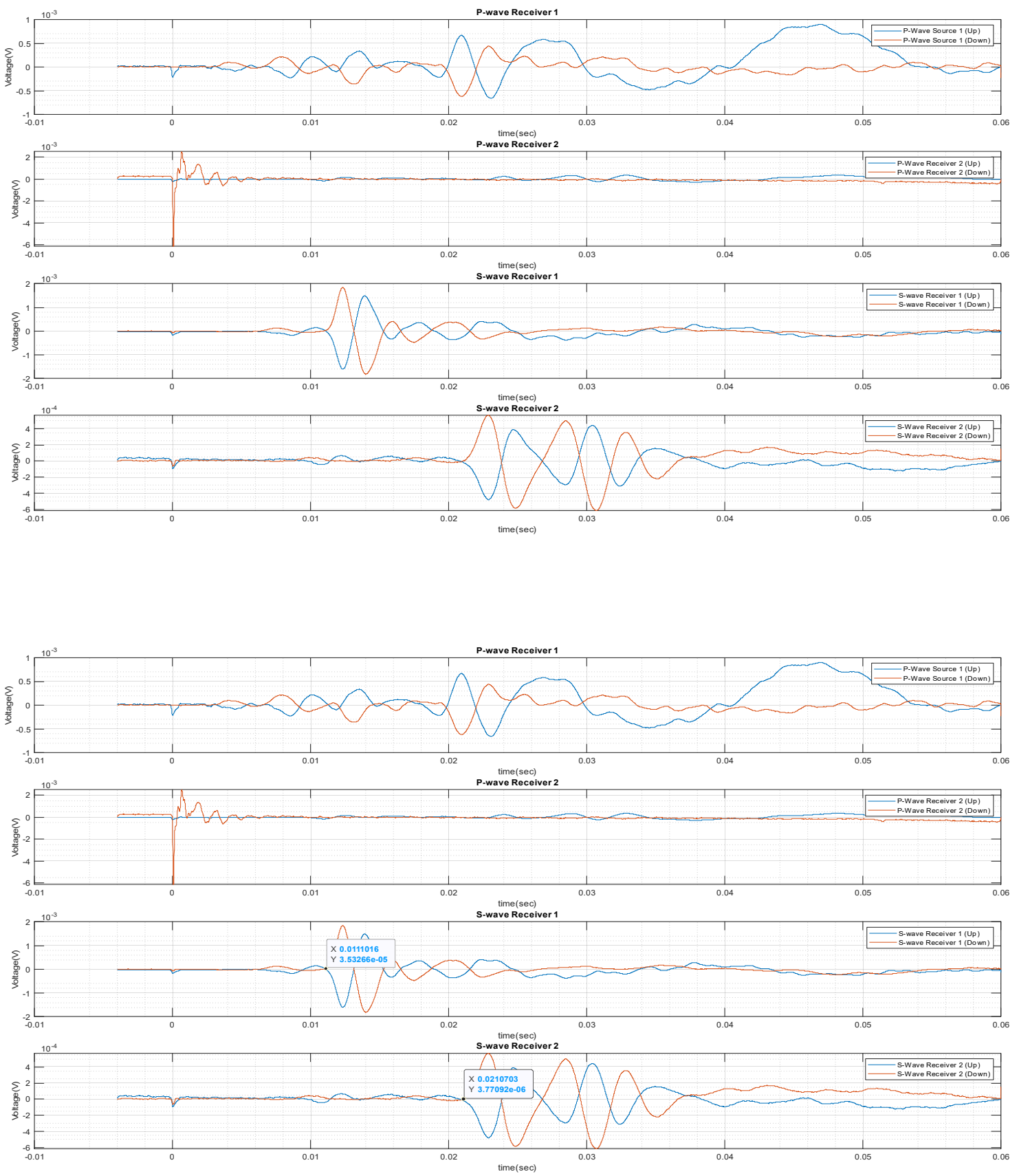

Data Set 39 (4.28.2020)

Run00299f

R2_1 

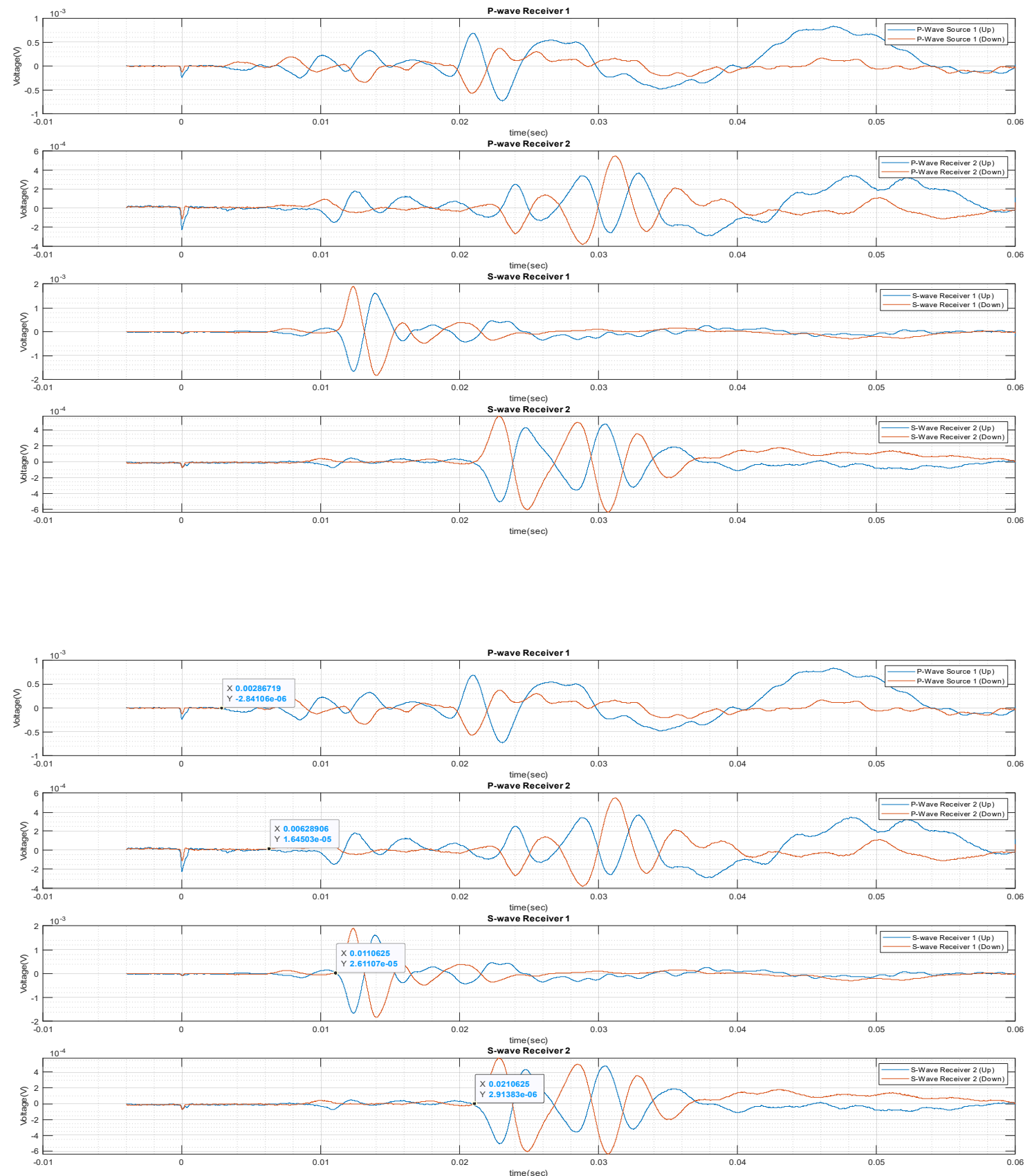

Data Set 39 (4.28.2020)

Run00300f

R2_2 

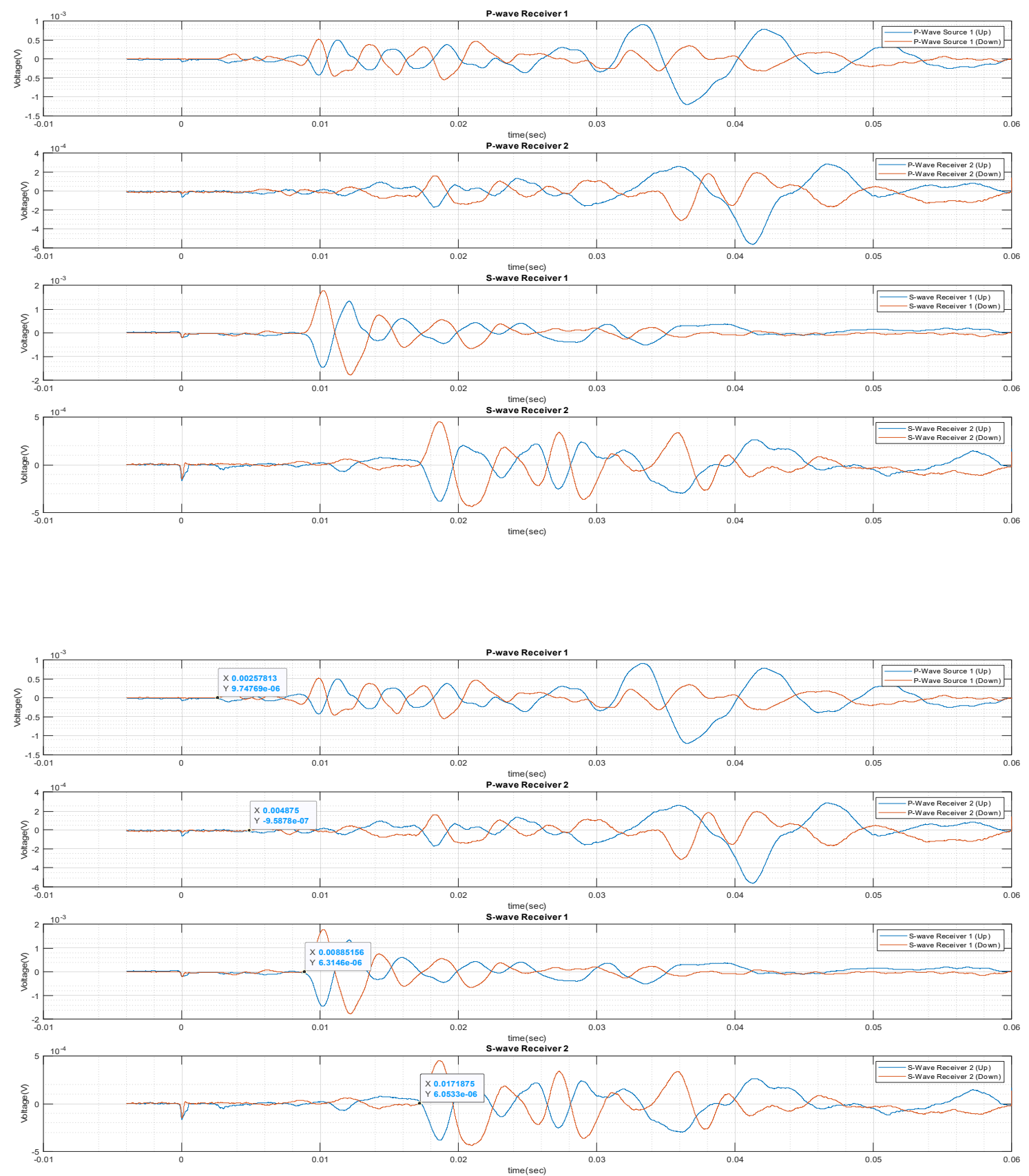

Data Set 39 (4.28.2020)

Run00301f

R3_1 

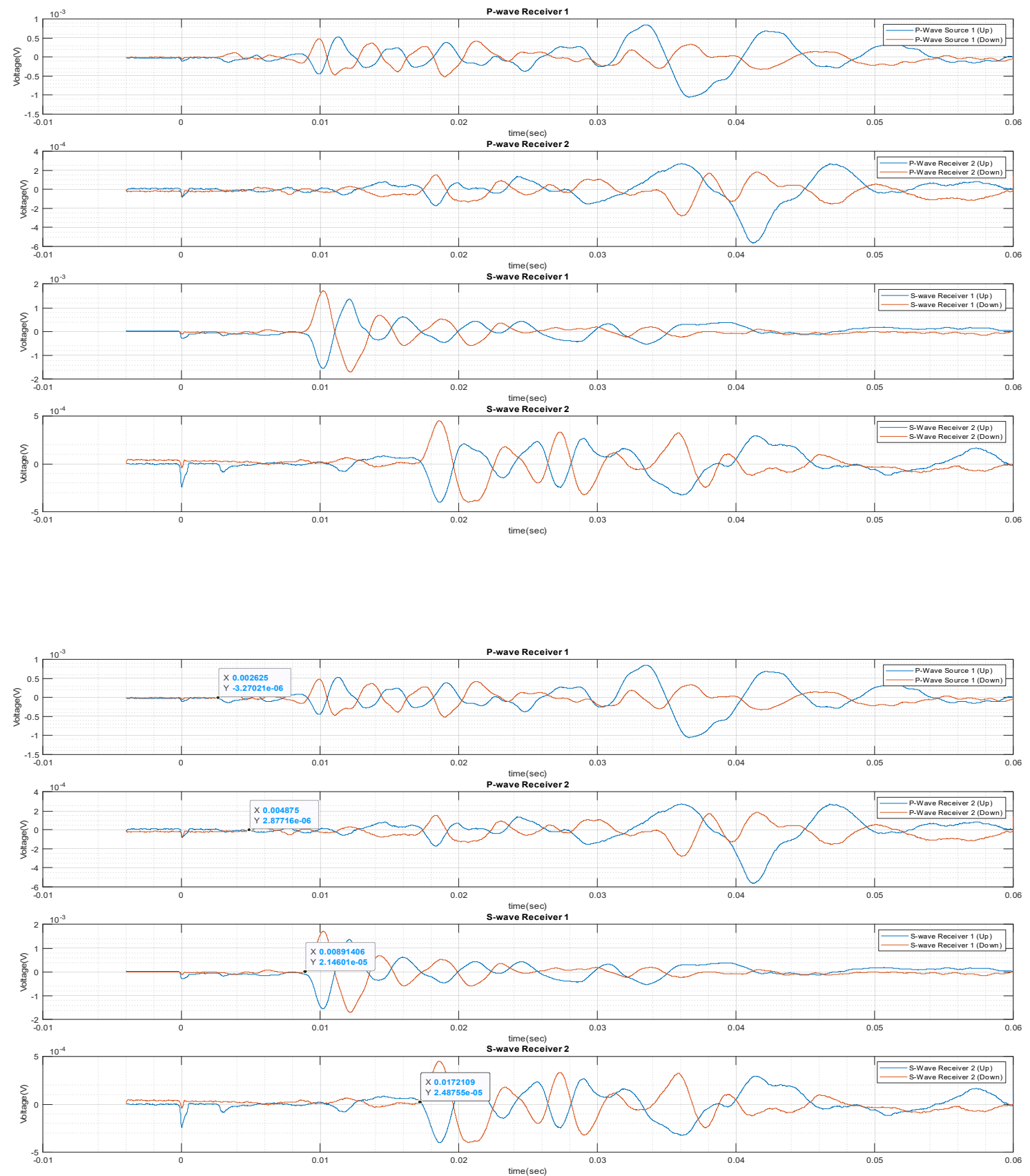

Data Set 39 (4.28.2020)

Run00302f

R3_2 

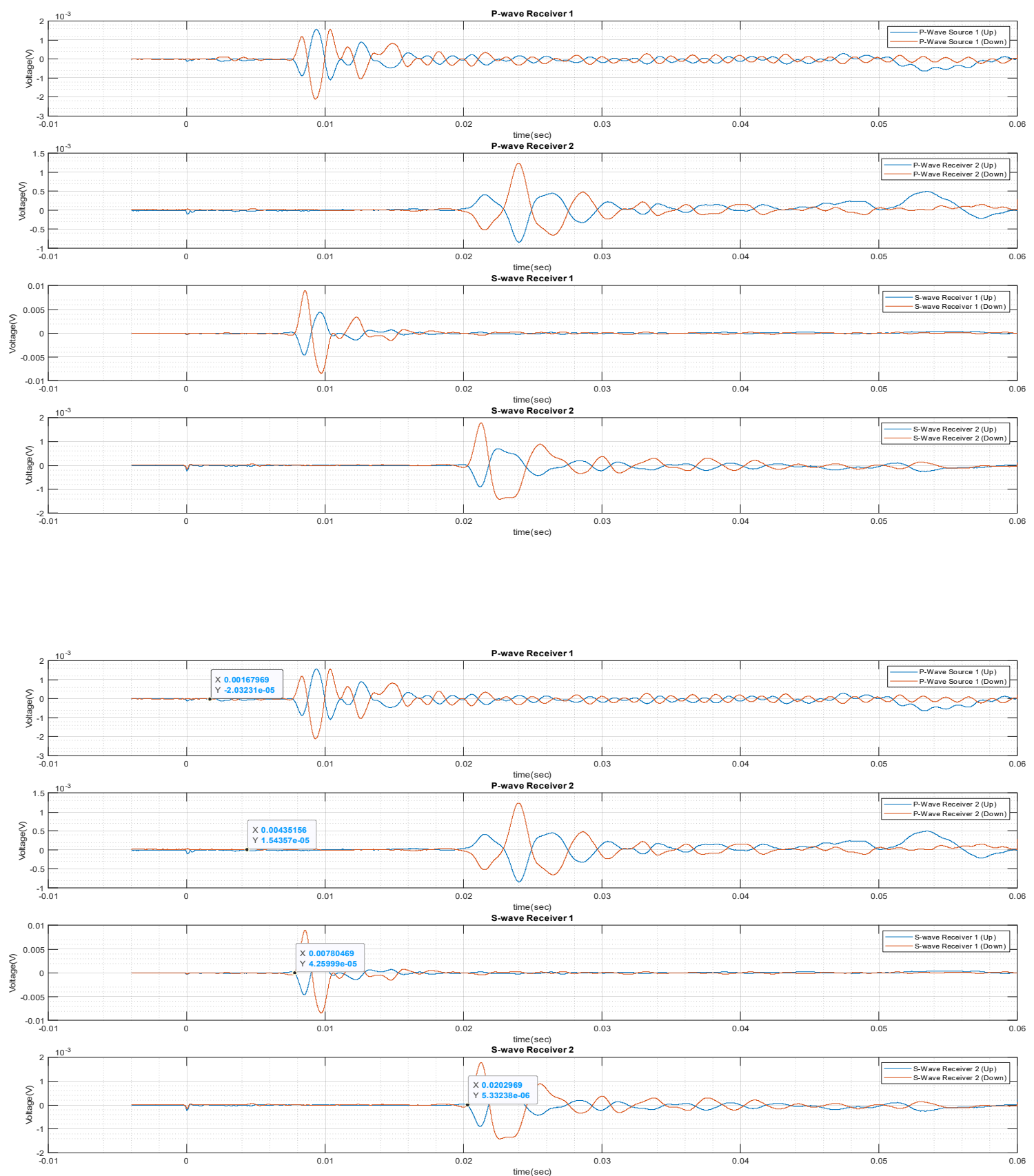

Data Set 39 (4.28.2020)

Run00303f

R4_1 

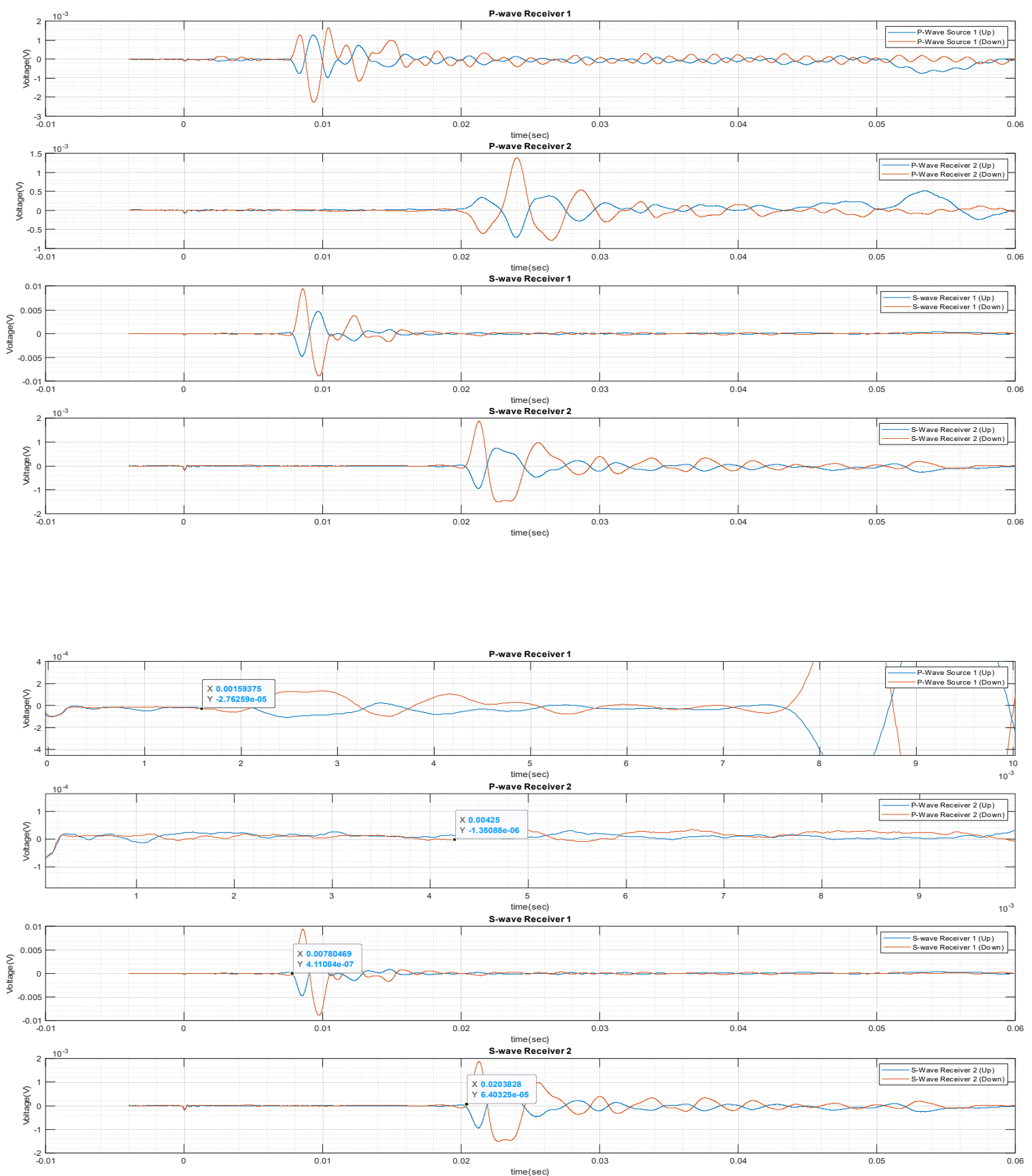

Data Set 39 (4.28.2020)

Run00304f

R4_2 

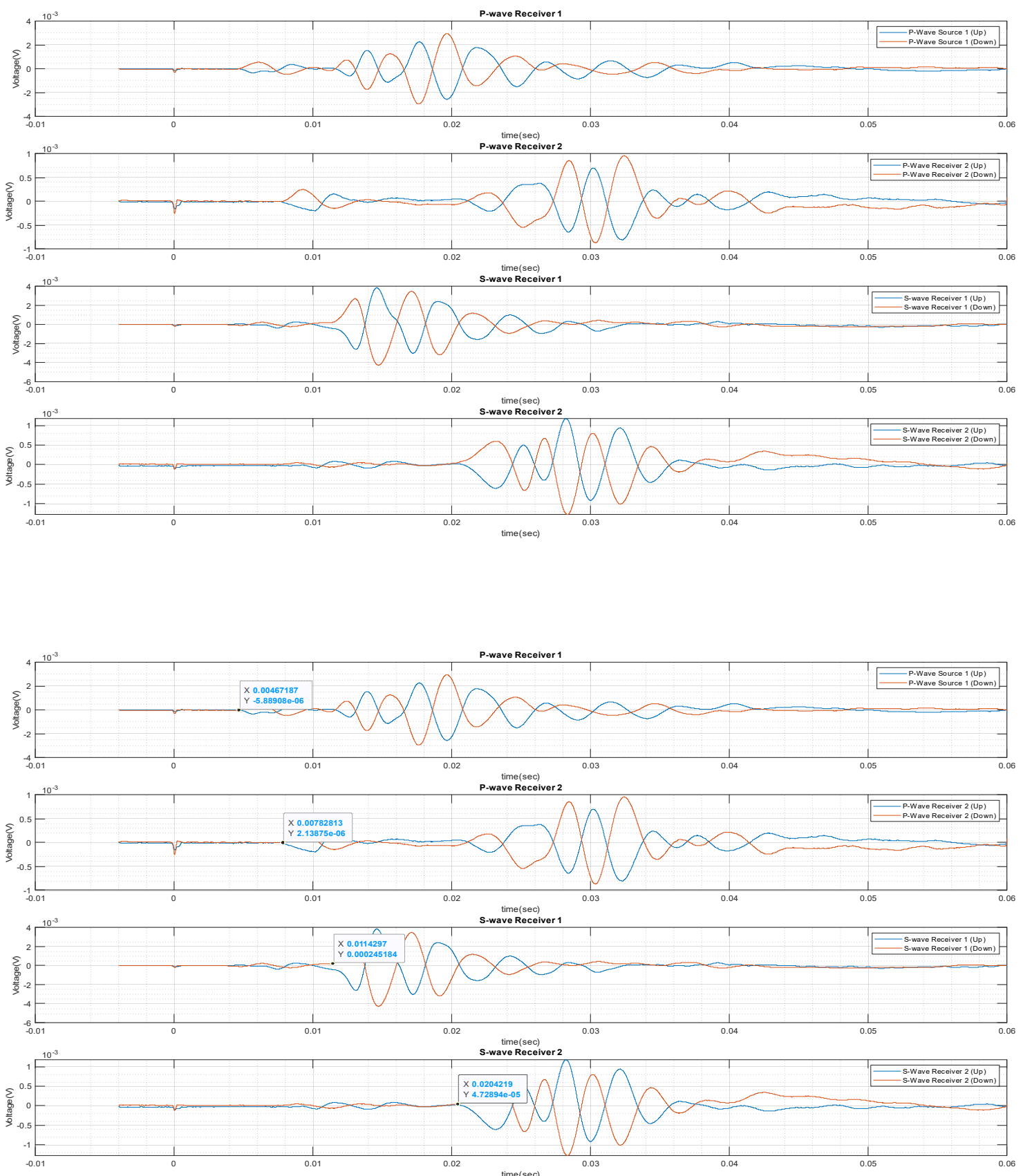

Data Set $40(5.29 .2020)$

Run00305f

R1_1 

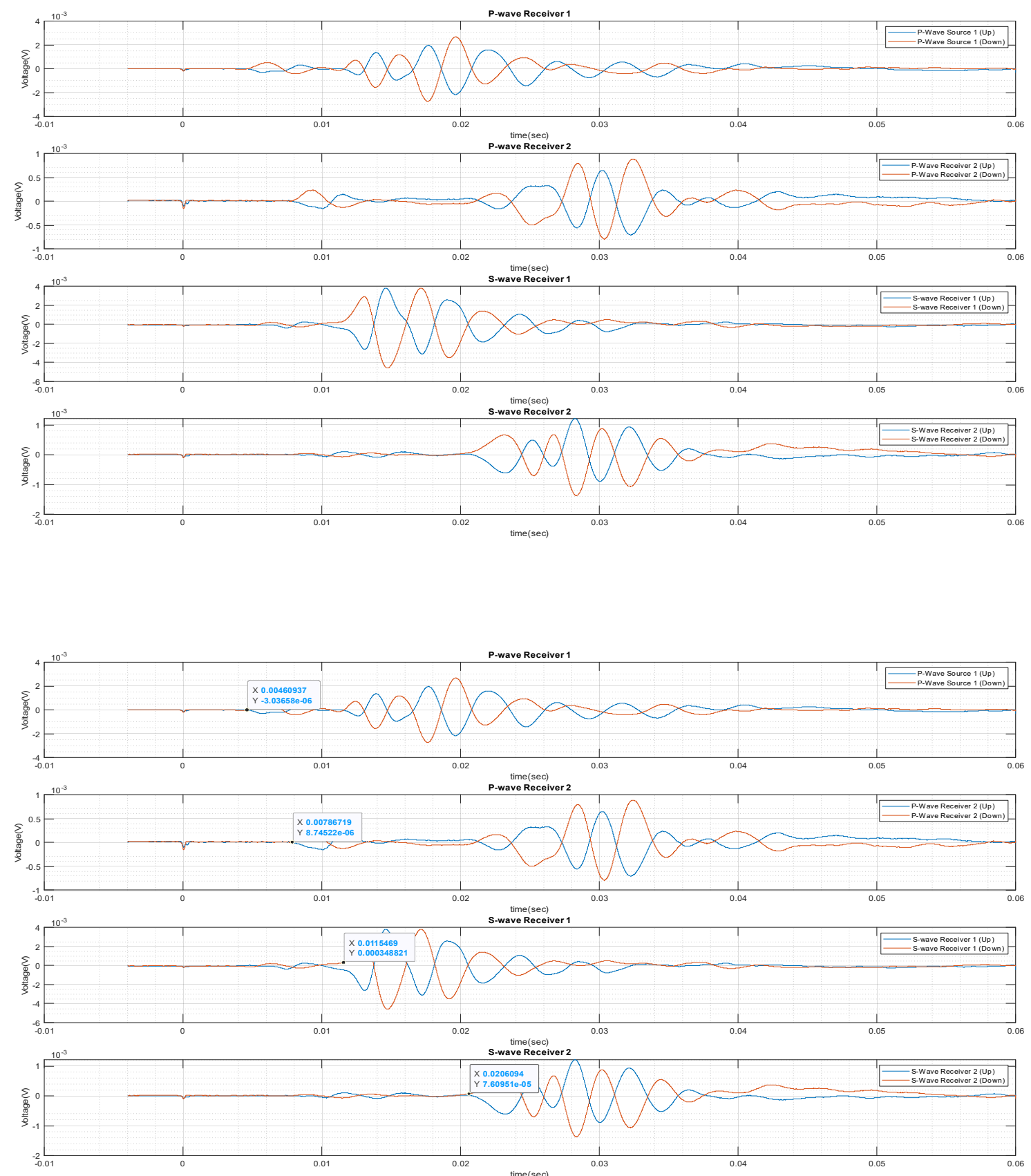

Data Set 40 (5.29.2020)

Run00306f

R1_2 

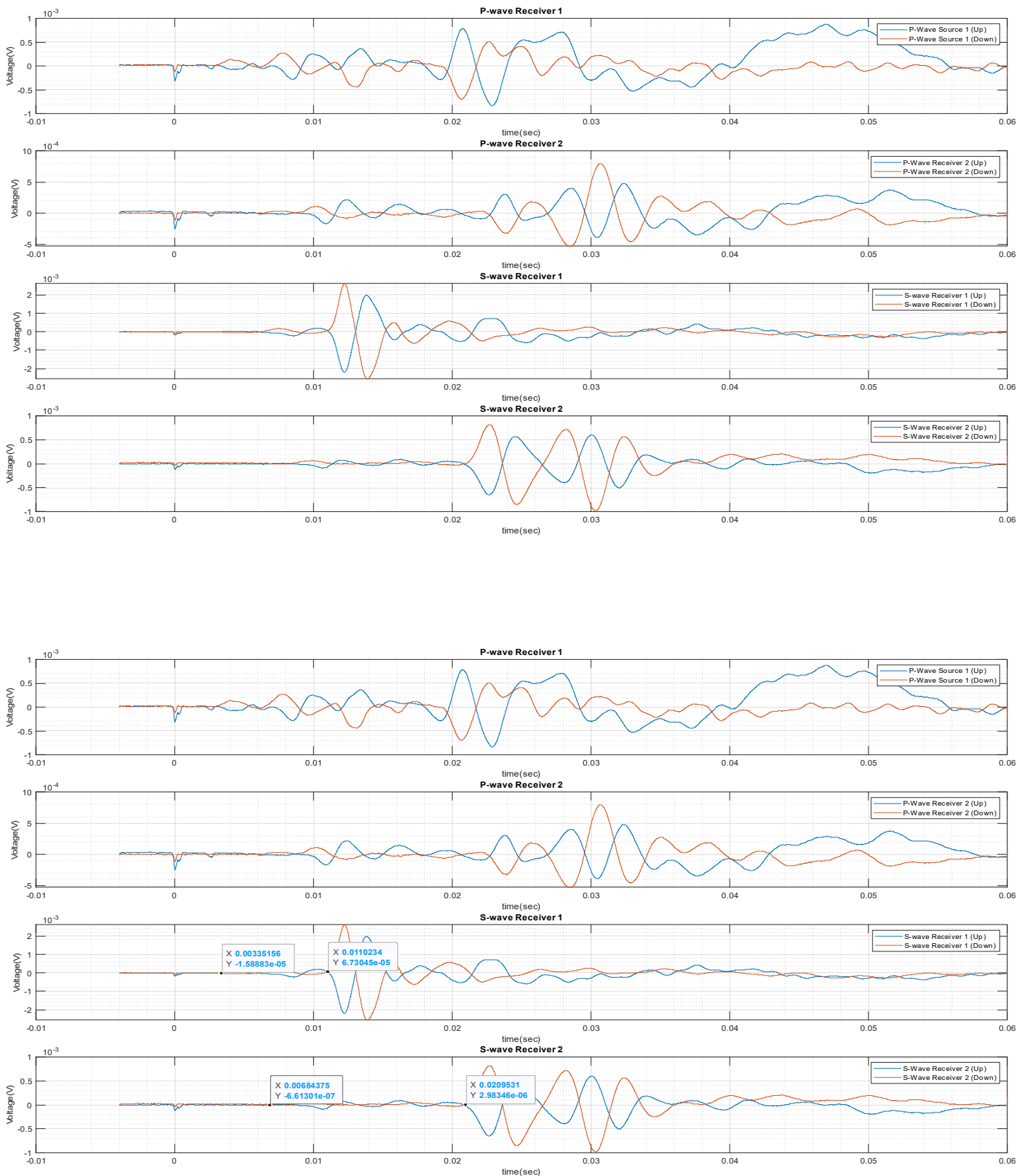

Data Set 40 (5.29.2020)

Run00307f

R2_1 

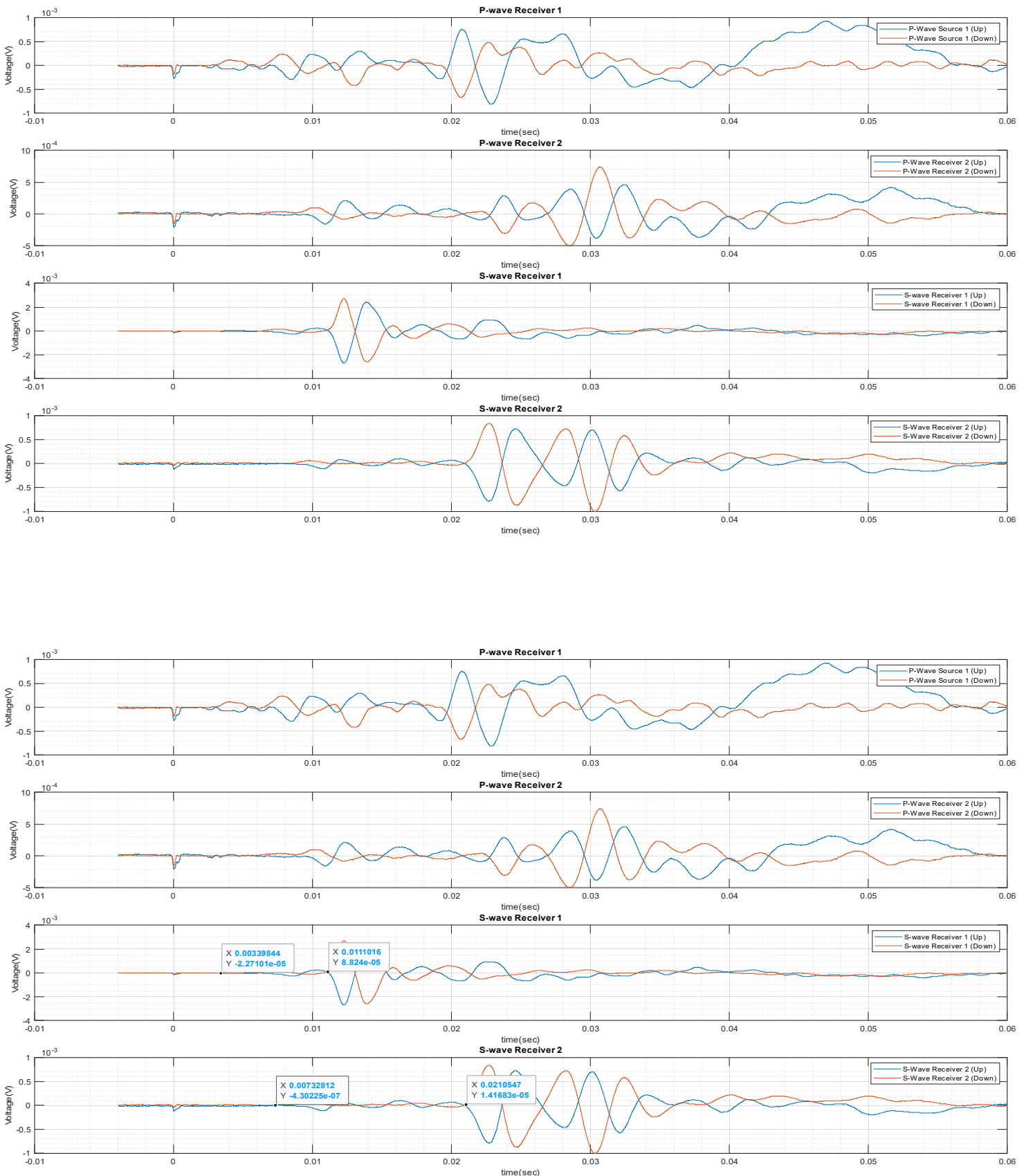

Data Set 40 (5.29.2020)

Run00308f

R2_2 

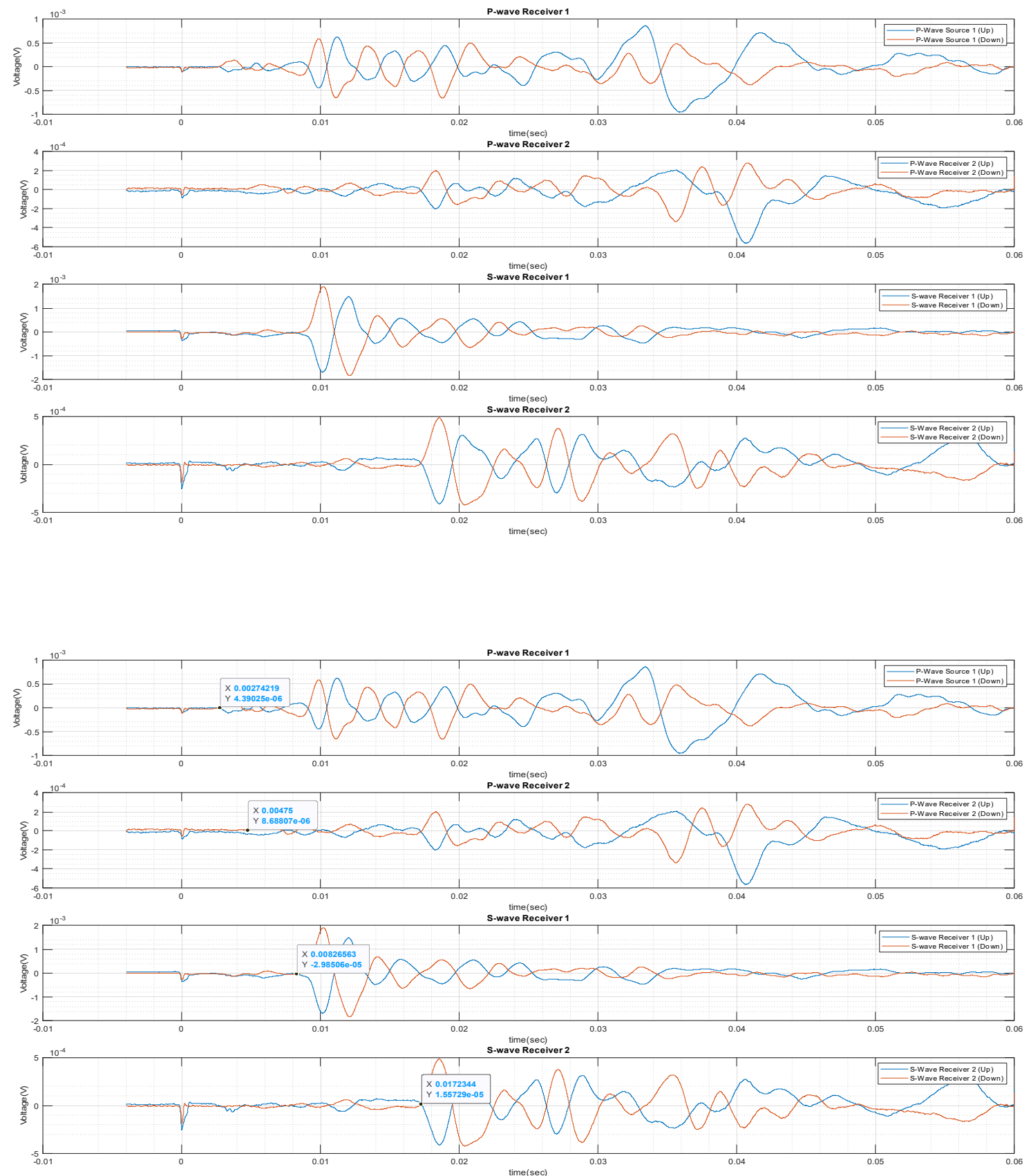

Data Set 40 (5.29.2020)

Run00309f

R3_1 

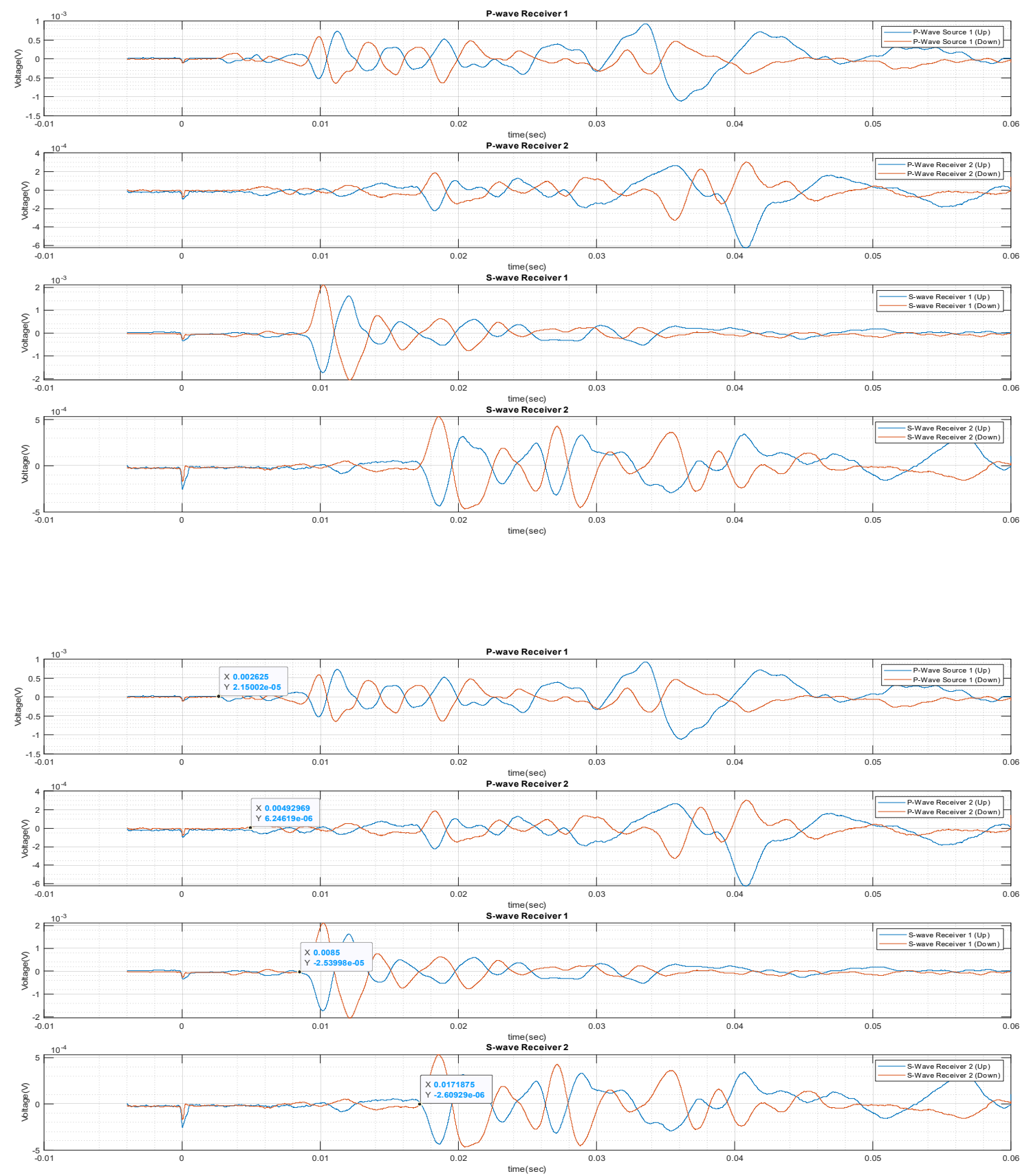

Data Set 40 (5.29.2020)

Run00310f

R3_2 

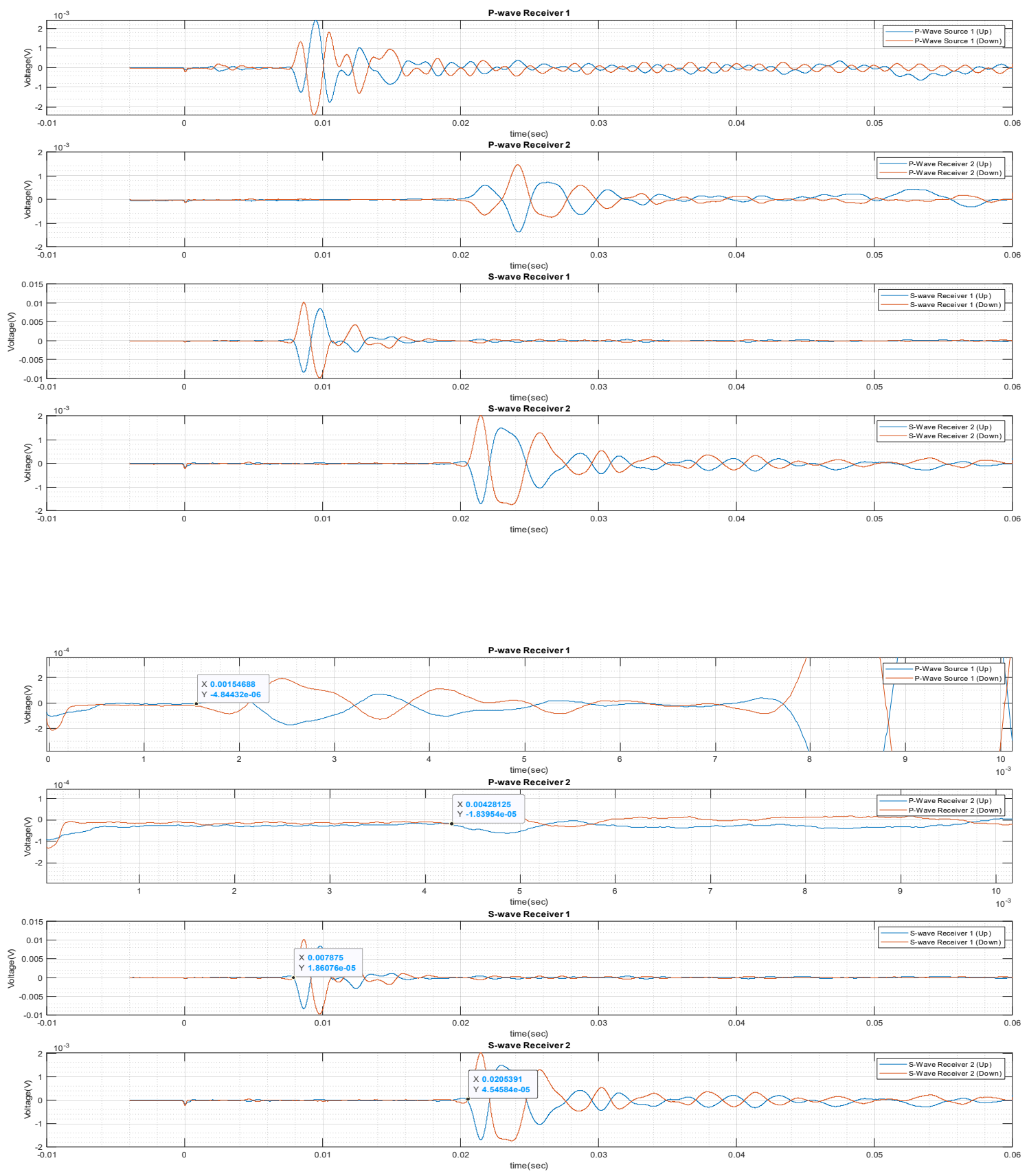

Data Set $40(5.29 .2020)$

Run00311f

R4_1 

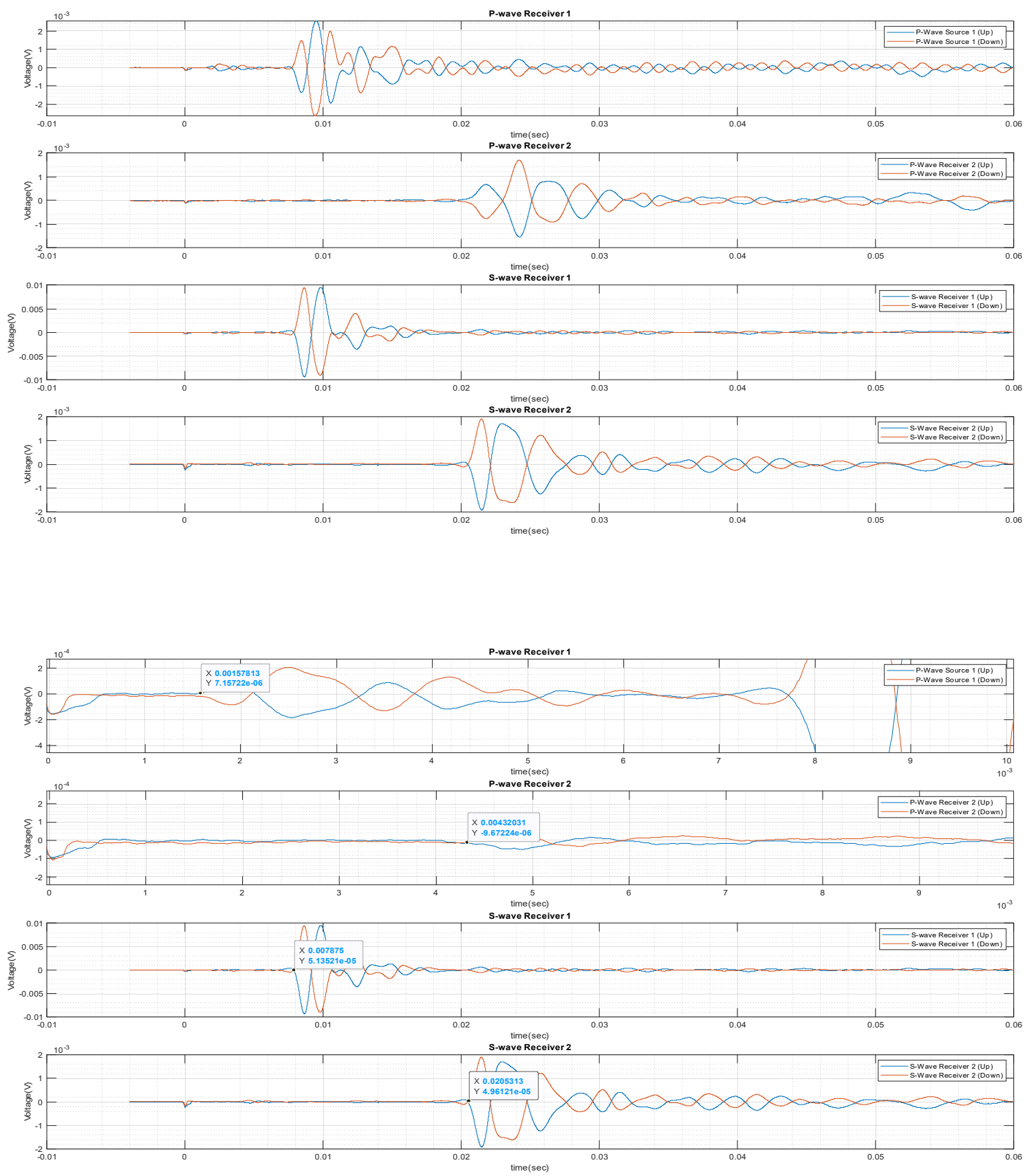

Data Set 40 (5.29.2020)

Run00312f

R4_2 

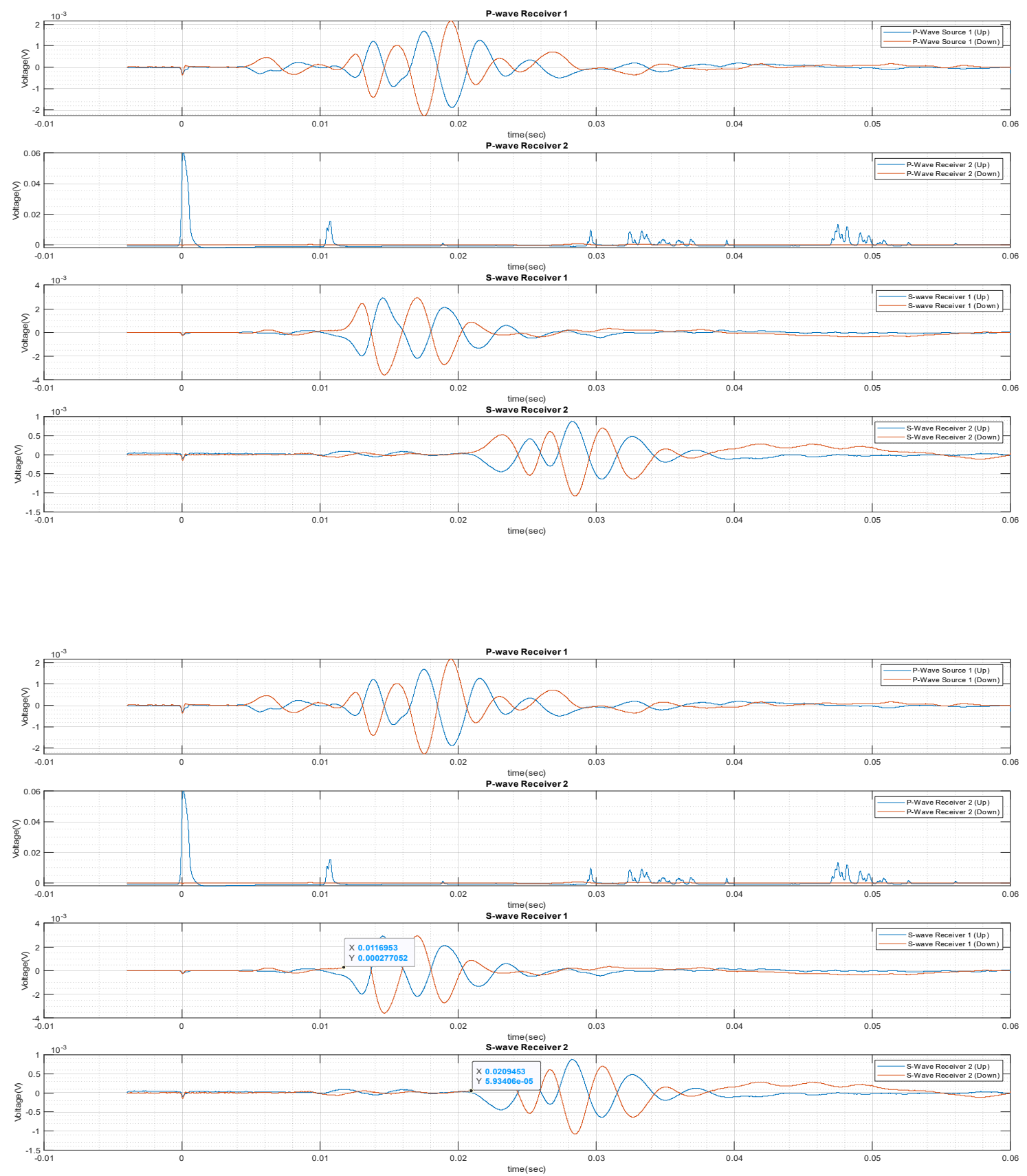

Data Set $41(6.21 .2020)$

Run00313f

R1_1 

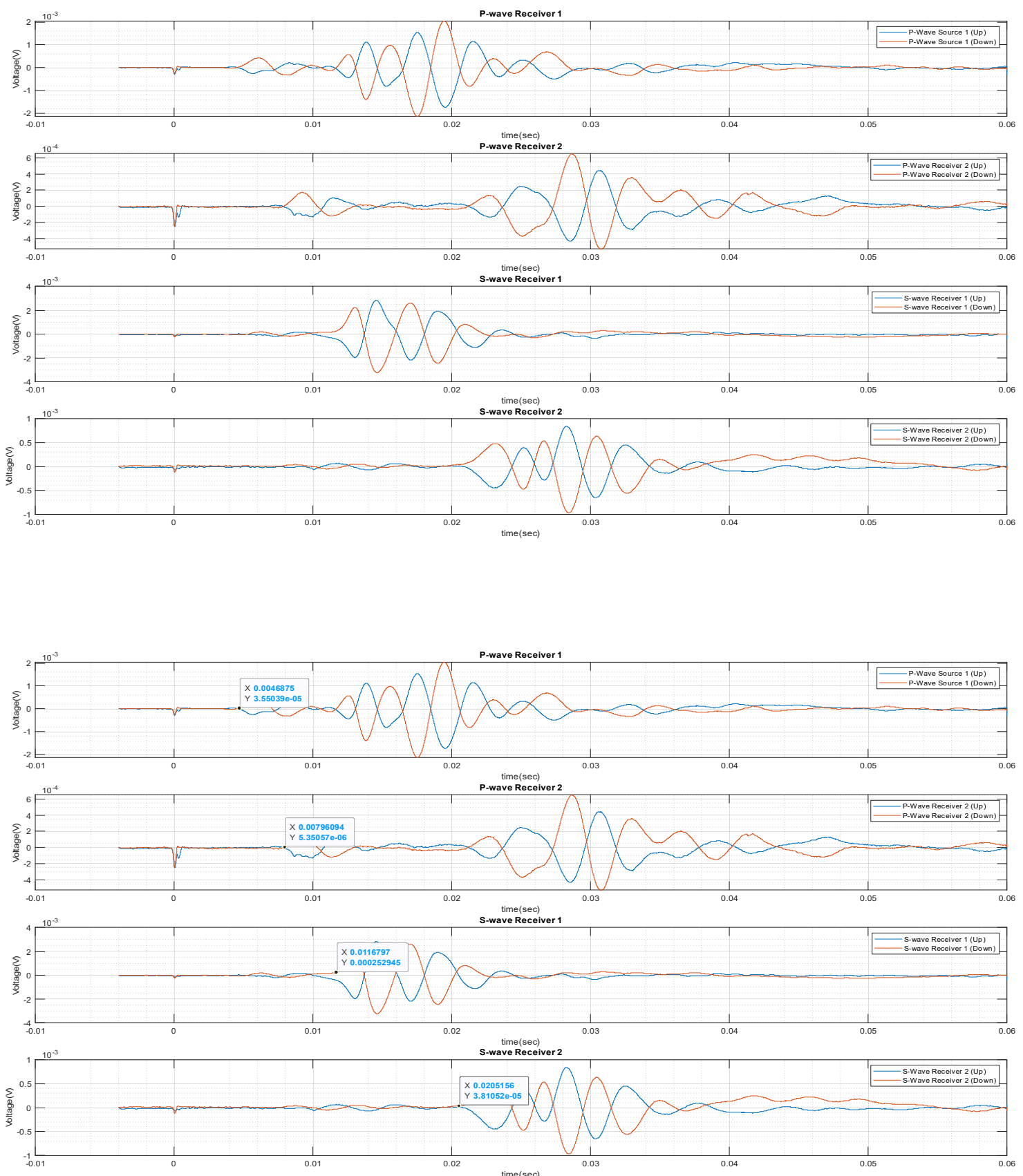

Data Set $41(6.21 .2020)$

Run00314f

R1_2 

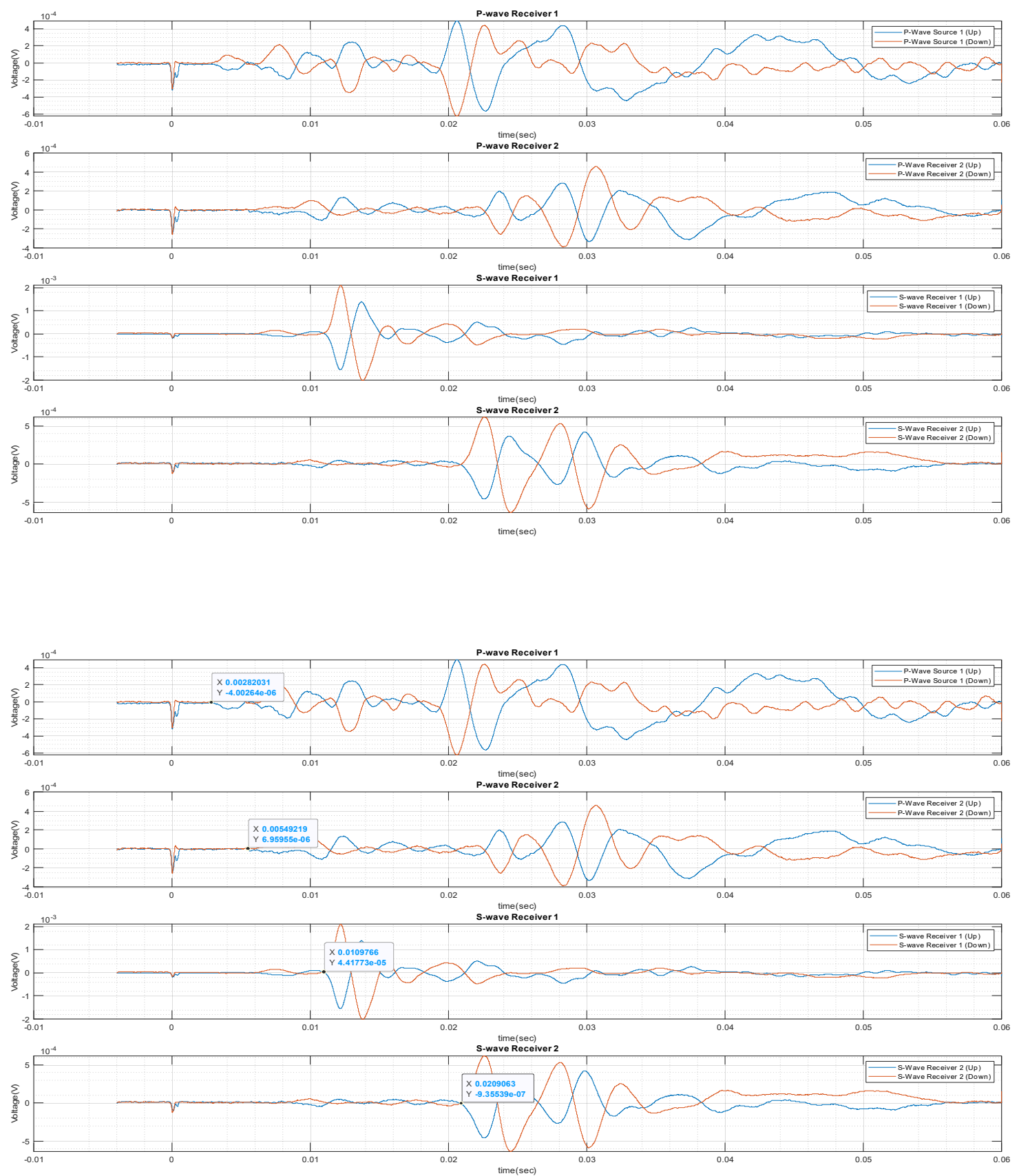

Data Set $41(6.21 .2020)$

Run00315f

R2_1 

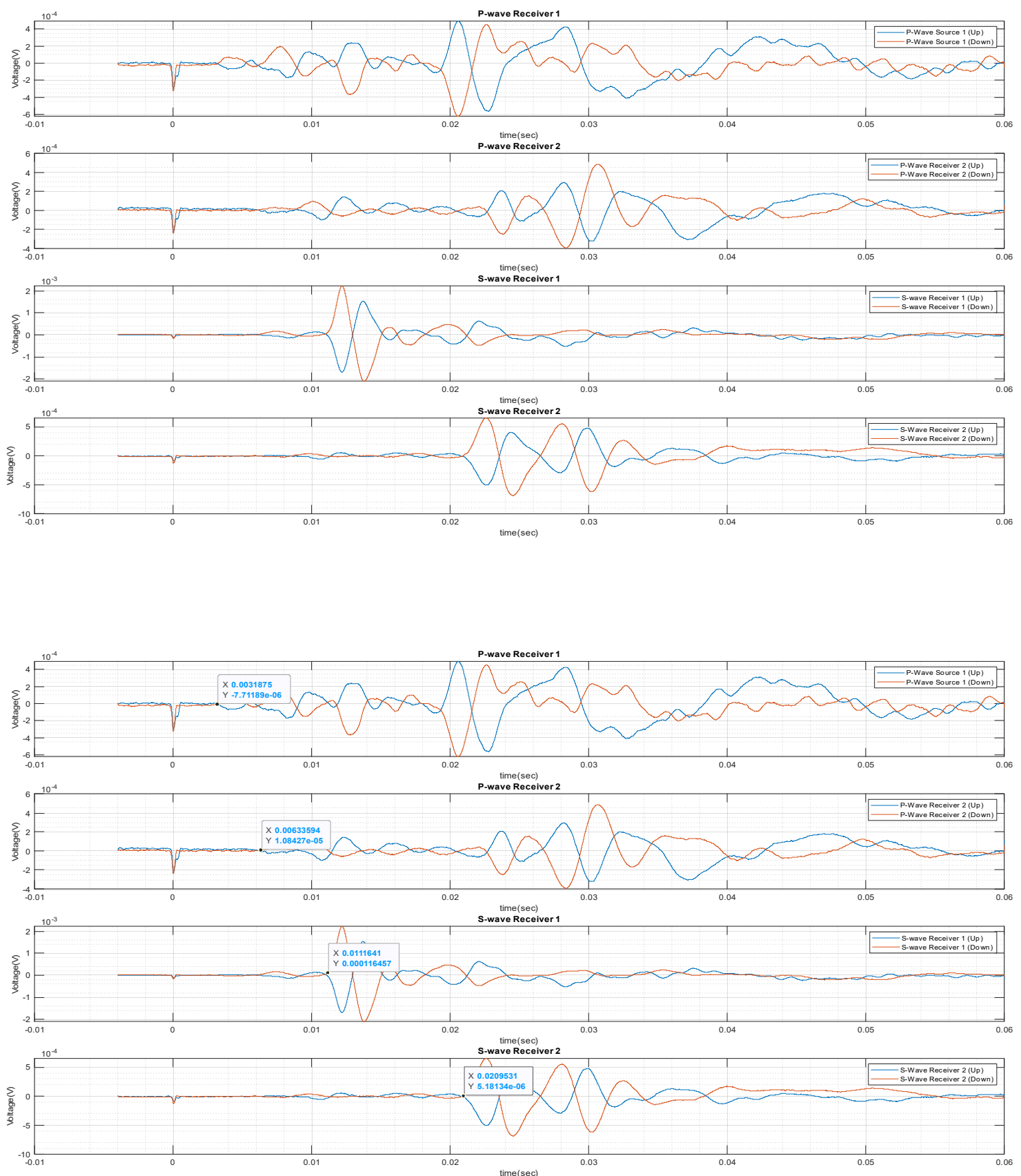

Data Set $41(6.21 .2020)$

Run00316f

R2_2 

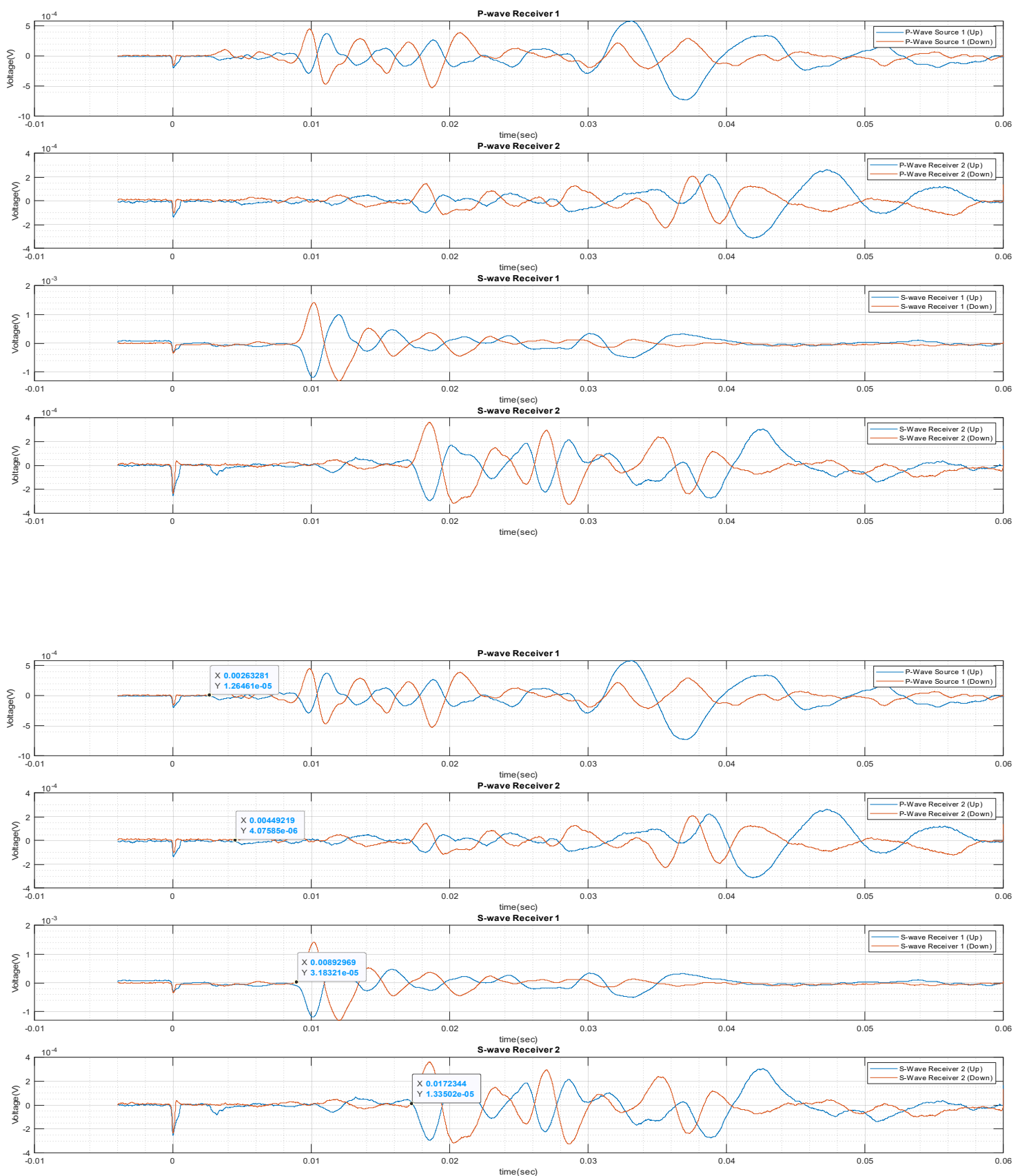

Data Set $41(6.21 .2020)$

Run00317f

R3_1 

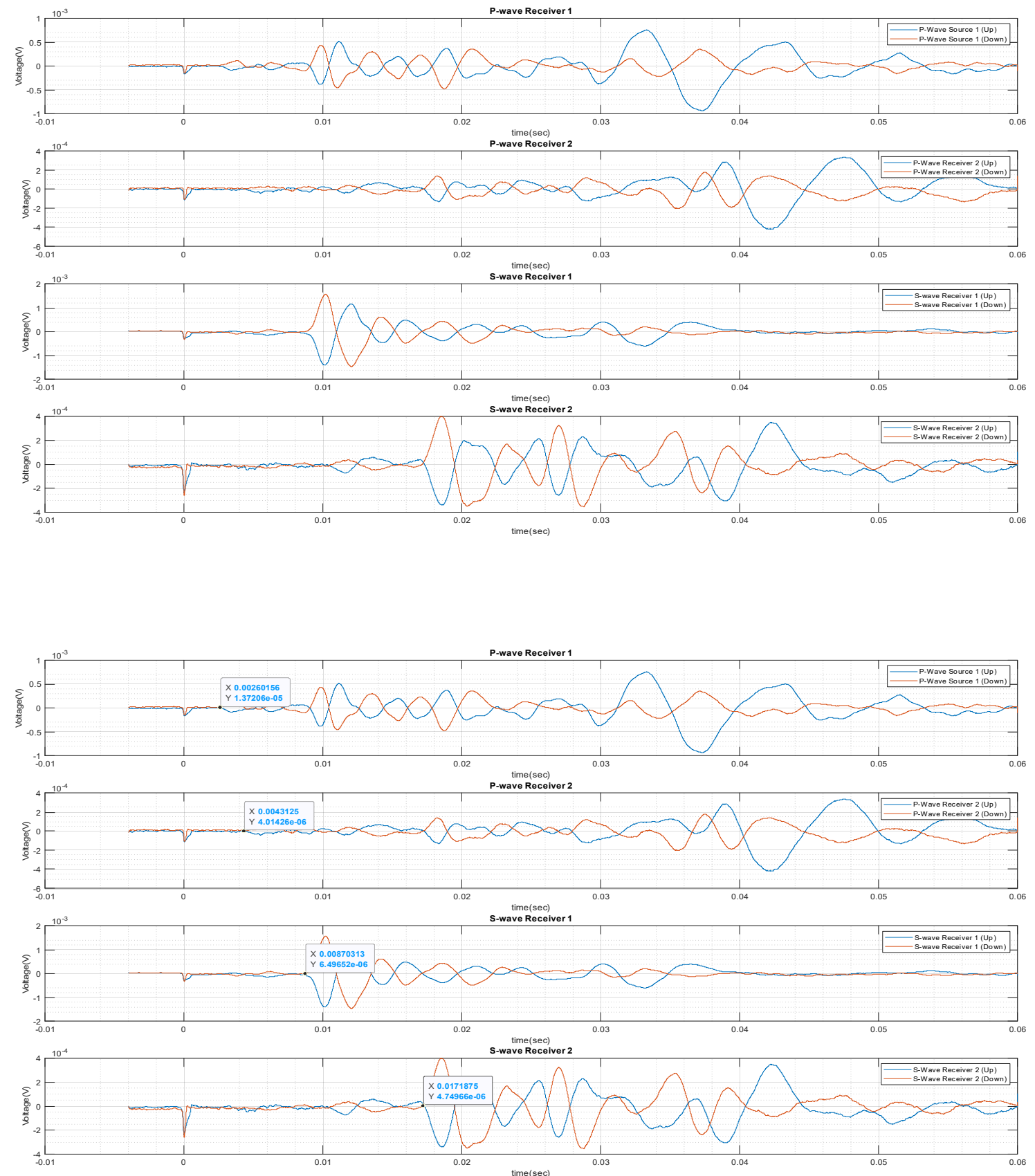

Data Set $41(6.21 .2020)$

Run00318f

R3_2 

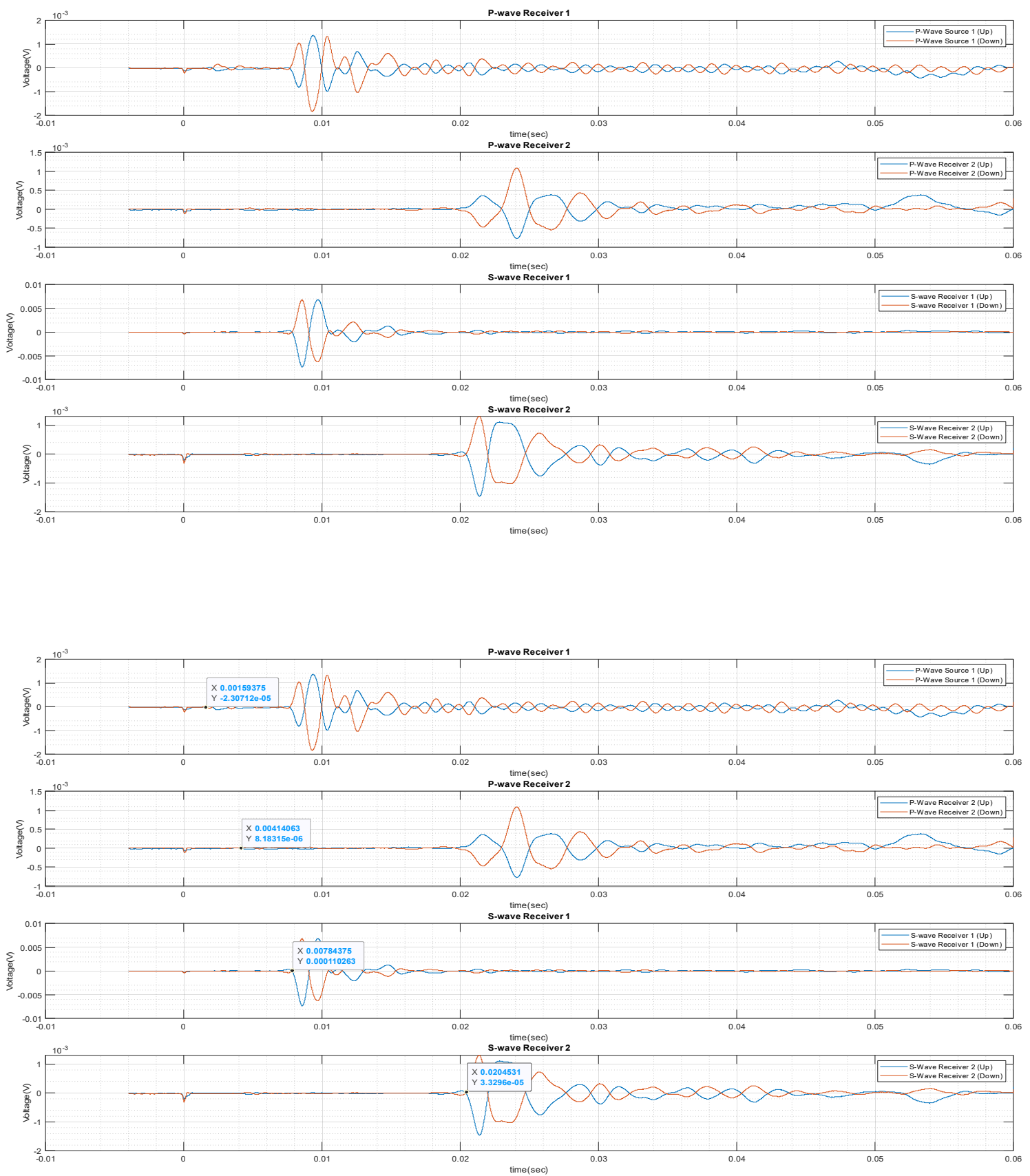

Data Set $41(6.21 .2020)$

Run00319f

R4_1 

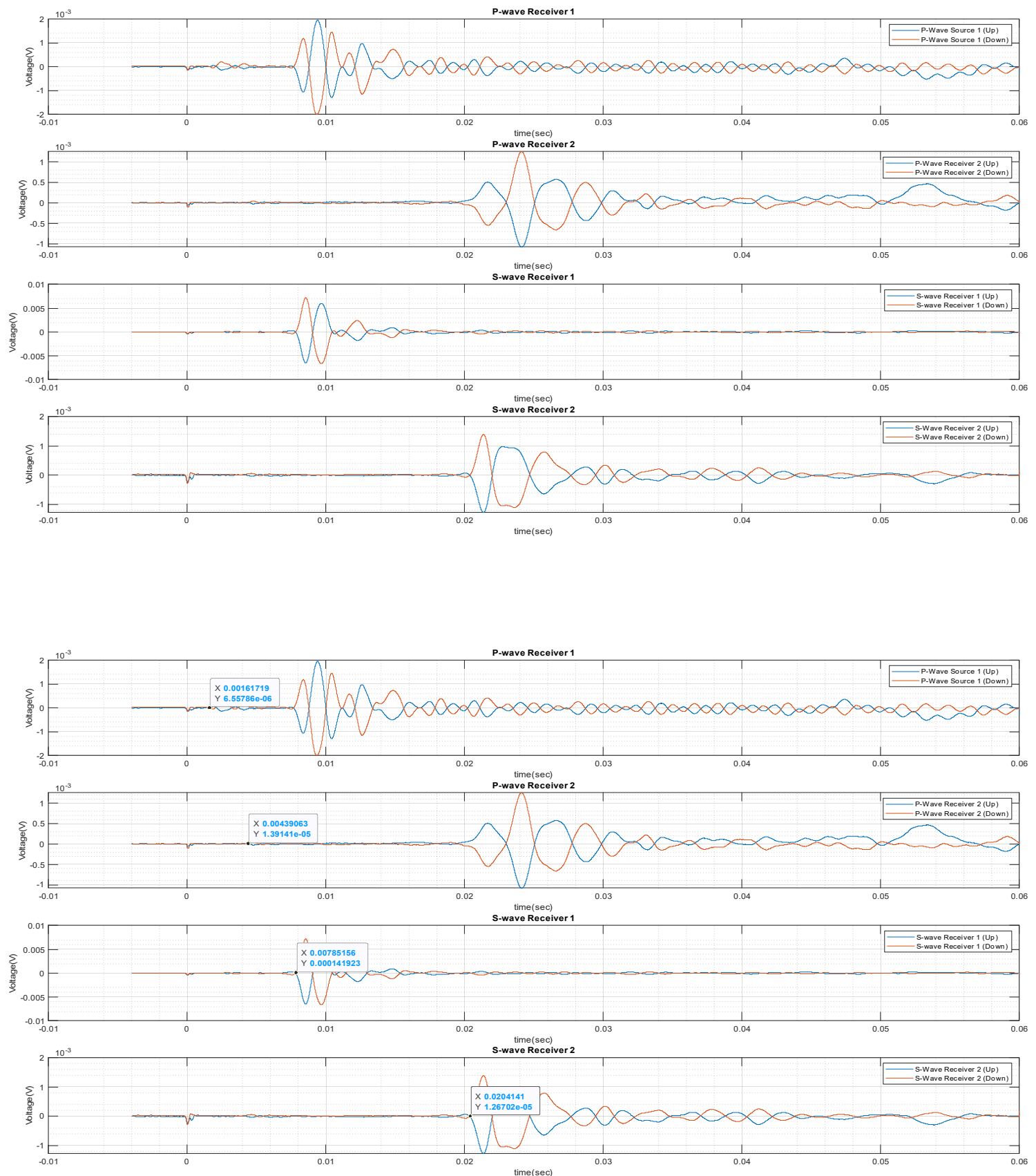

Data Set $41(6.21 .2020)$

Run00320f

R4_2 

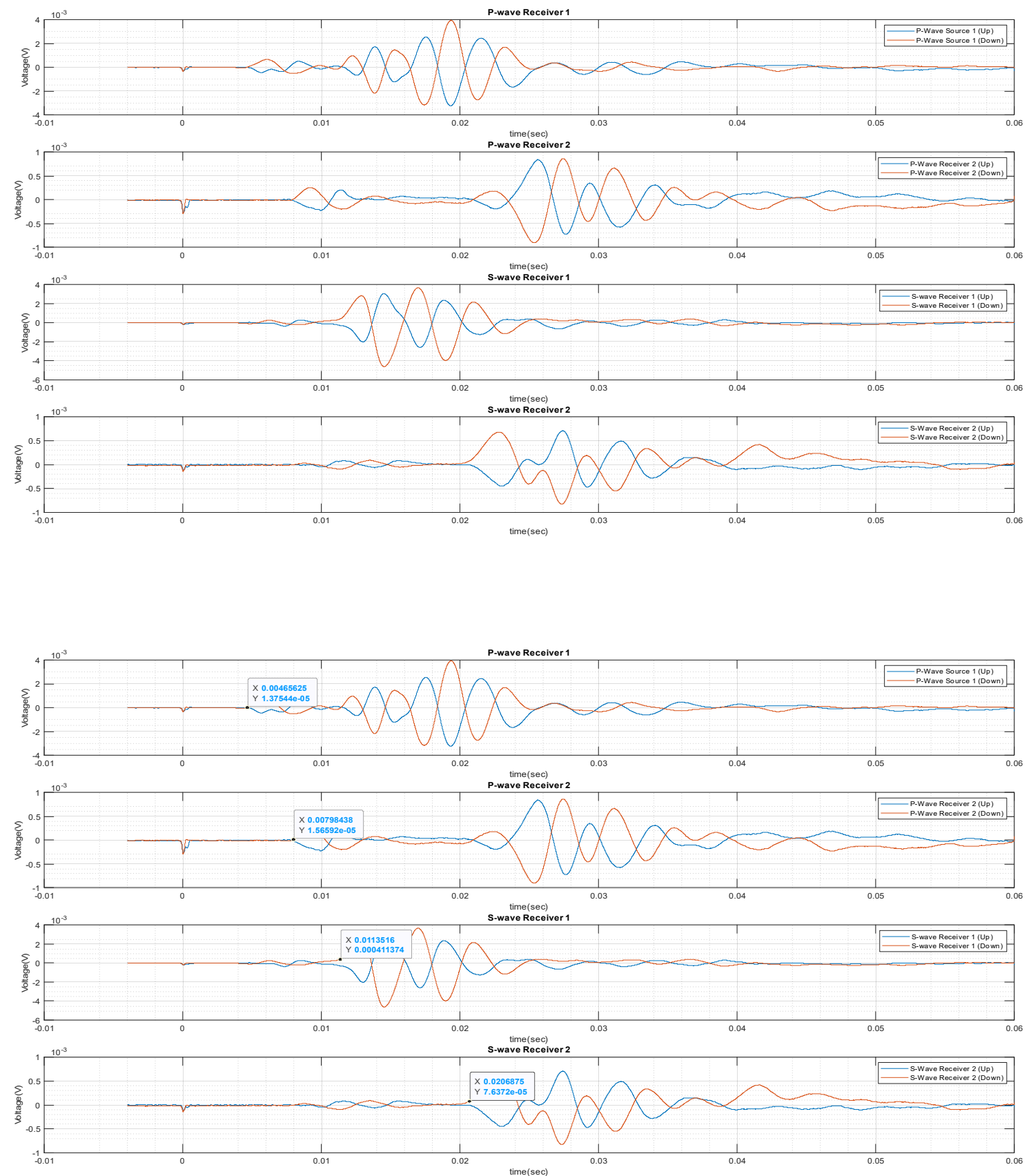

Data Set 42 (7.9.2020)

Run00321f

R1_1 

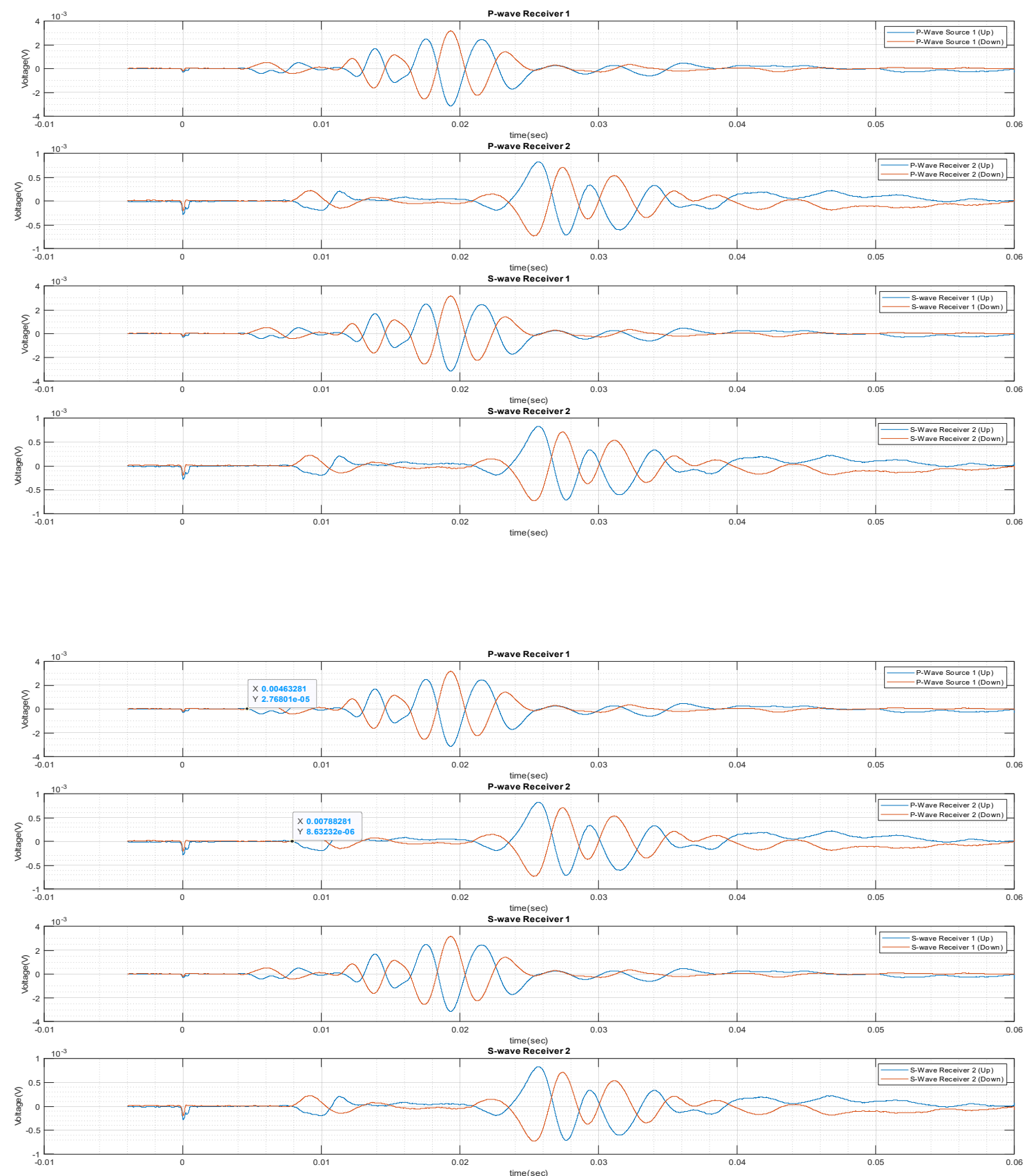

Data Set 42 (7.9.2020)

Run00322f

R1_2 

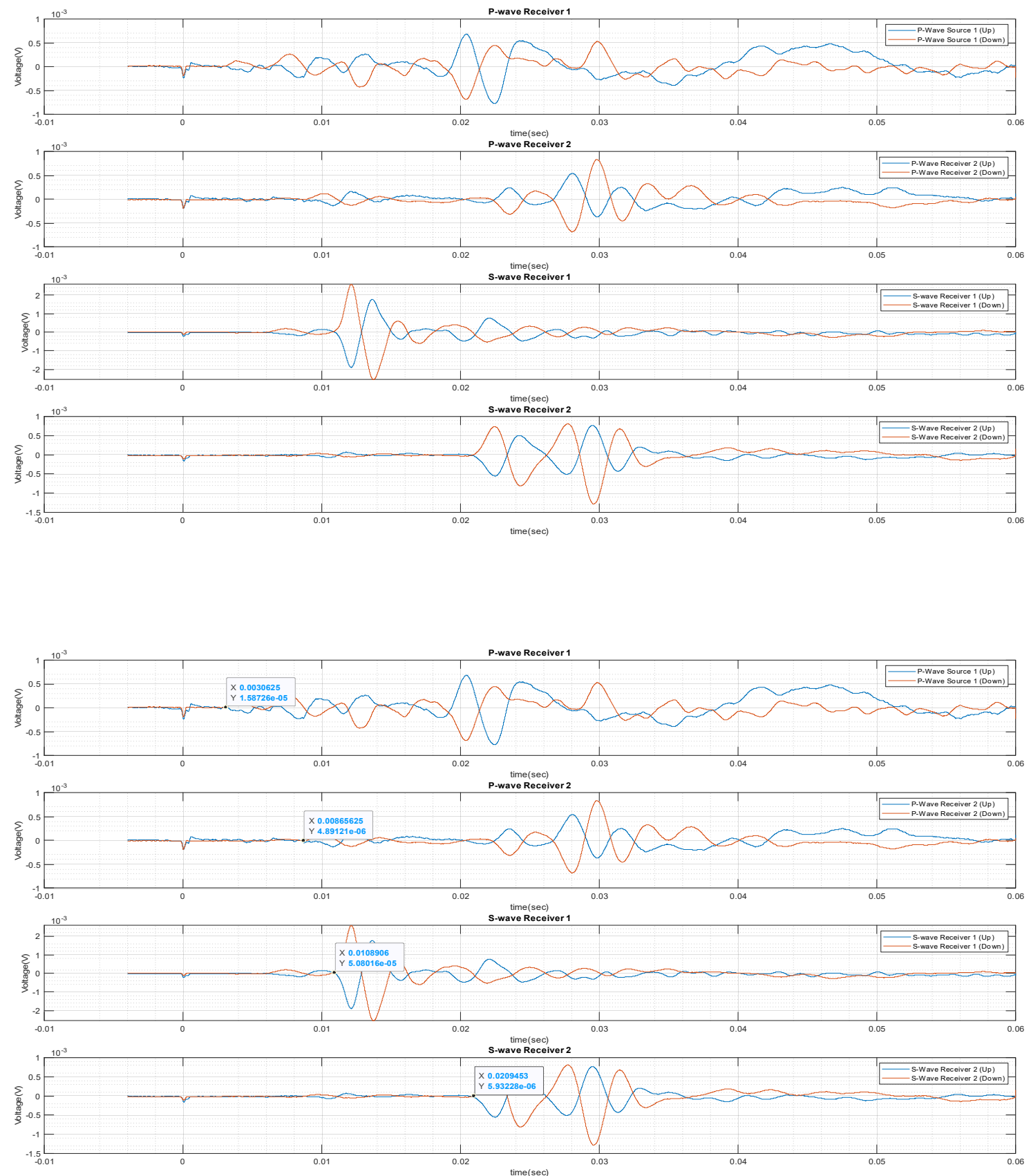

Data Set 42 (7.9.2020)

Run00323f

R2_1 

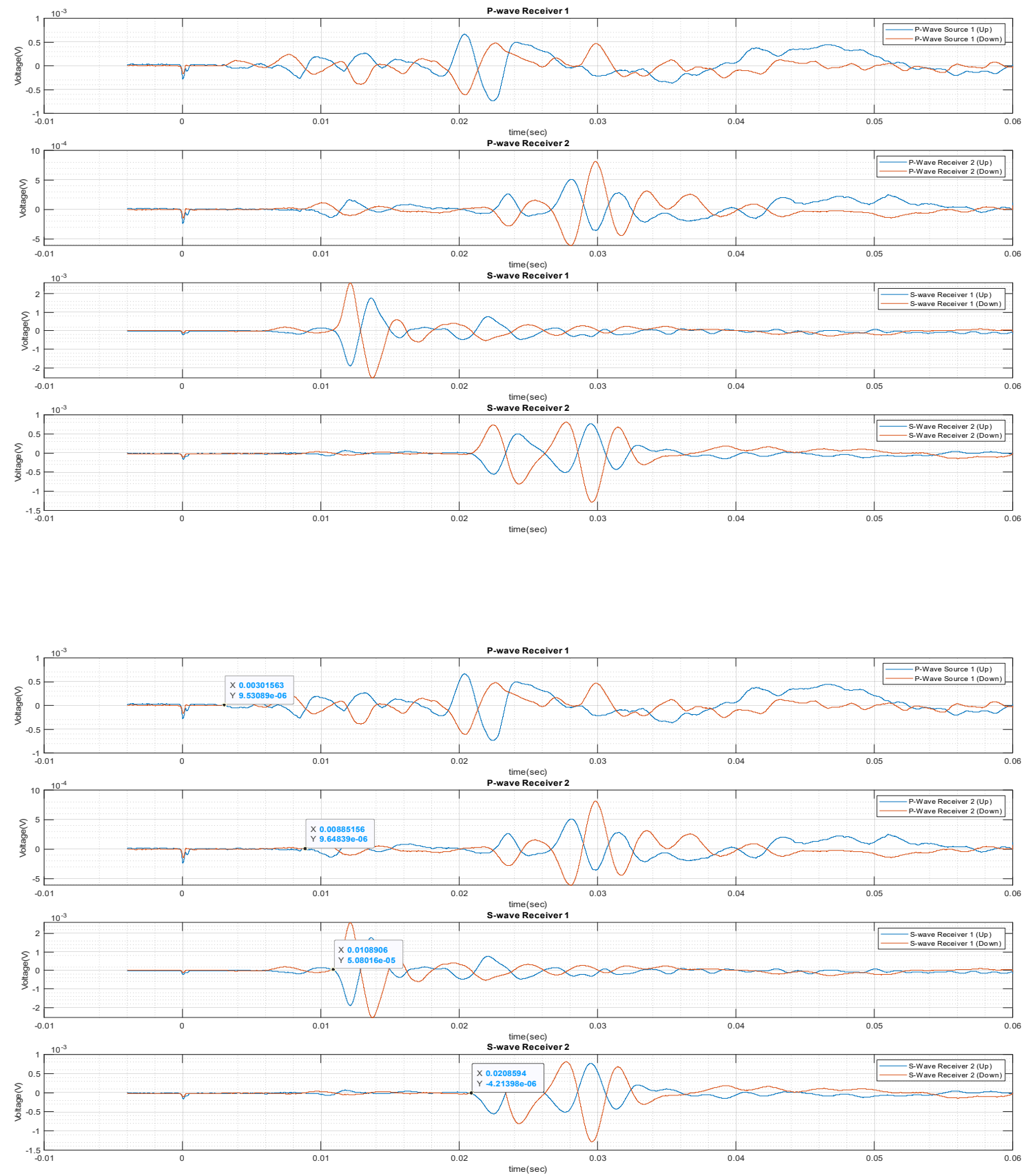

Data Set 42 (7.9.2020)

Run00324f

R2_2 

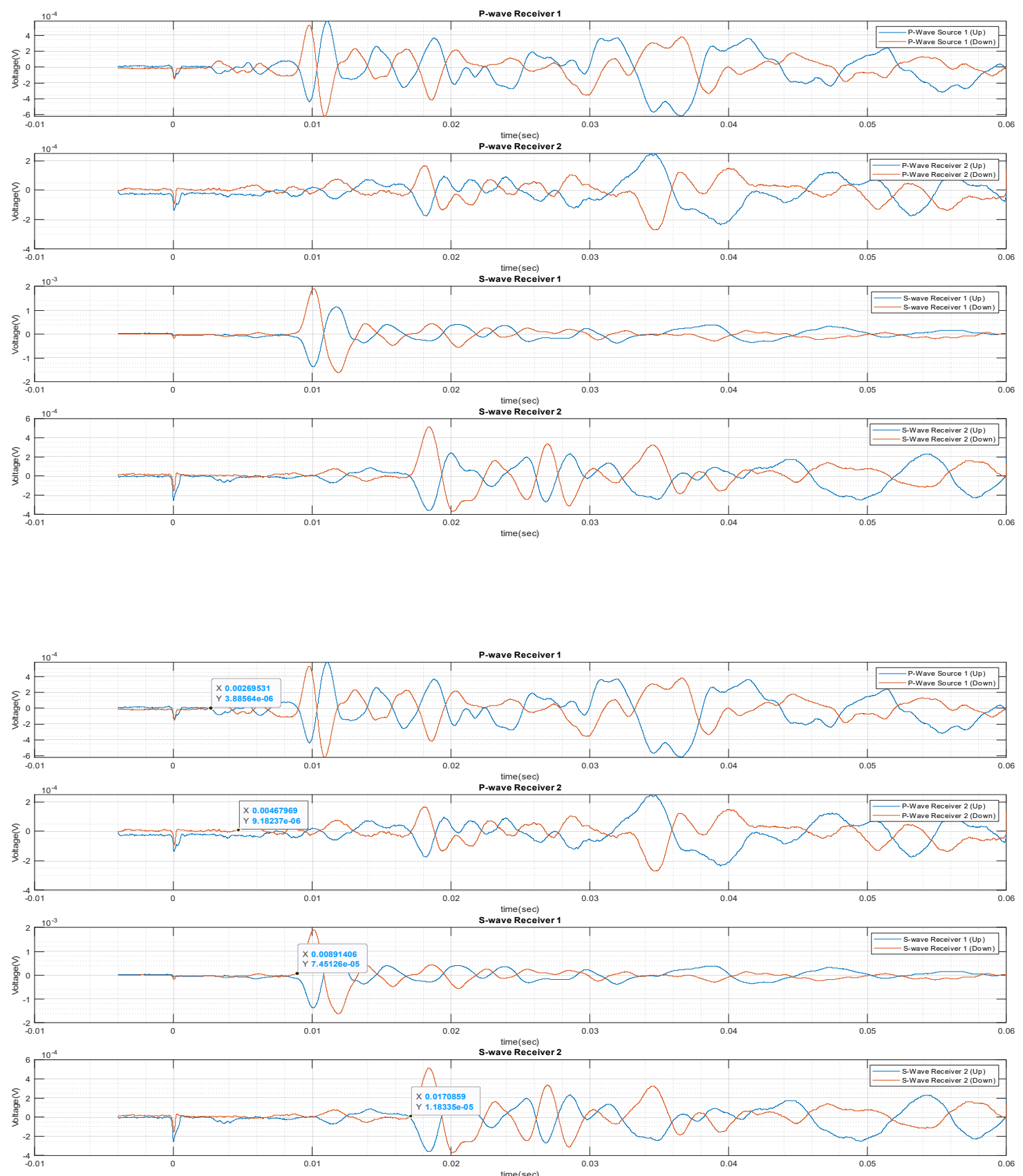

Data Set 42 (7.9.2020)

Run00325f

R3_1 

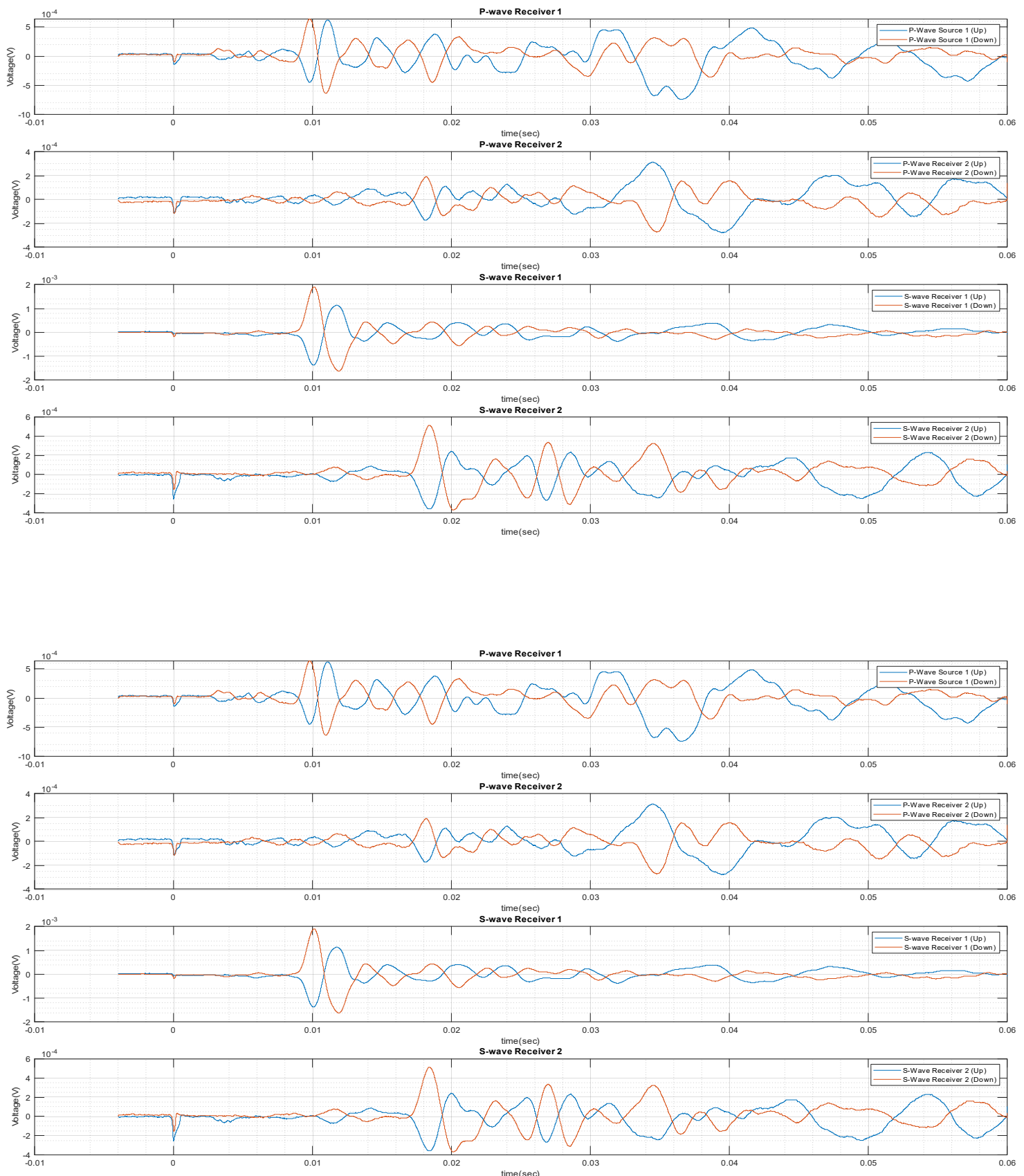

Data Set 42 (7.9.2020)

Run00326f

R3_2 

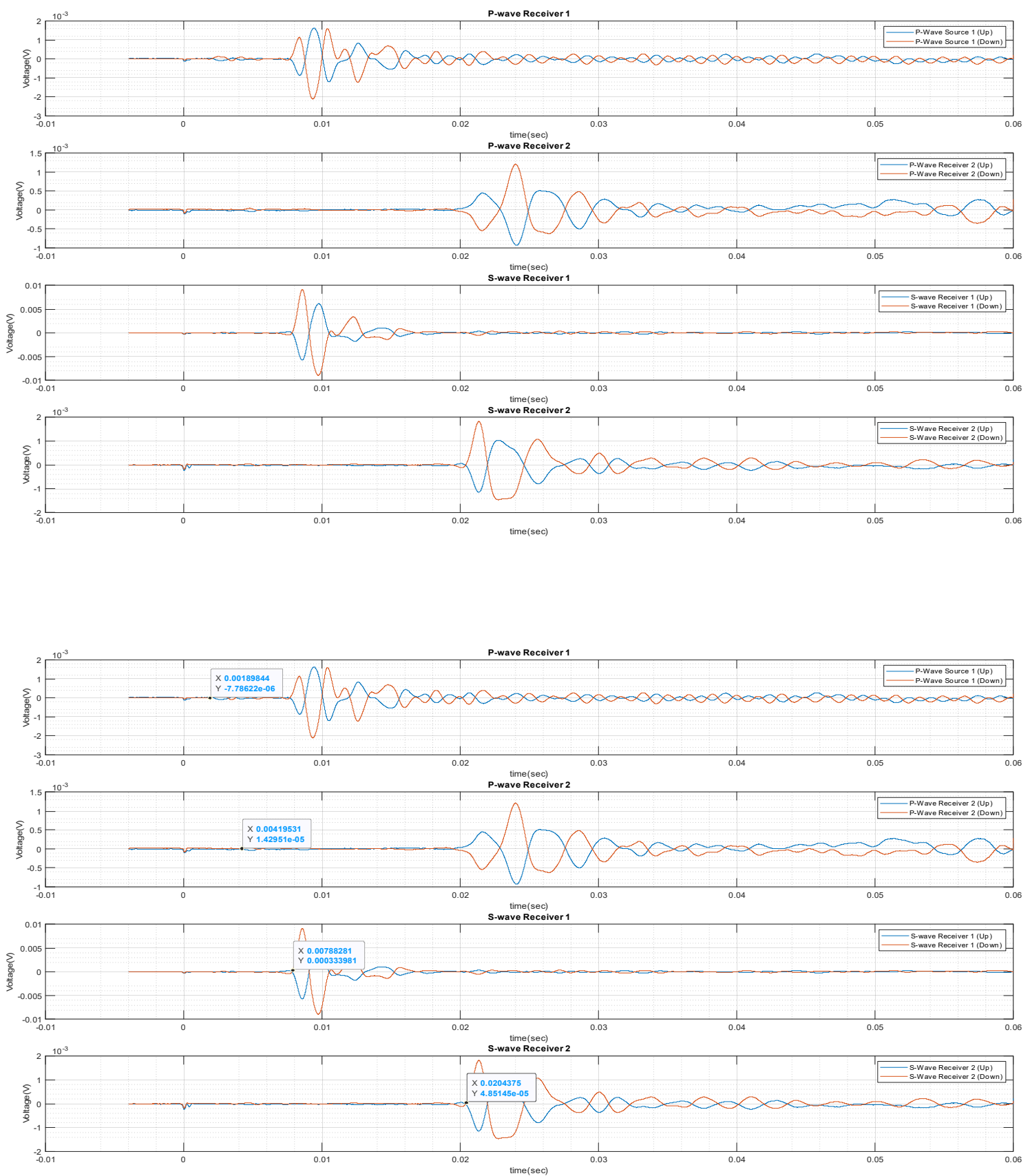

Data Set 42 (7.9.2020)

Run00327f

R4_1 

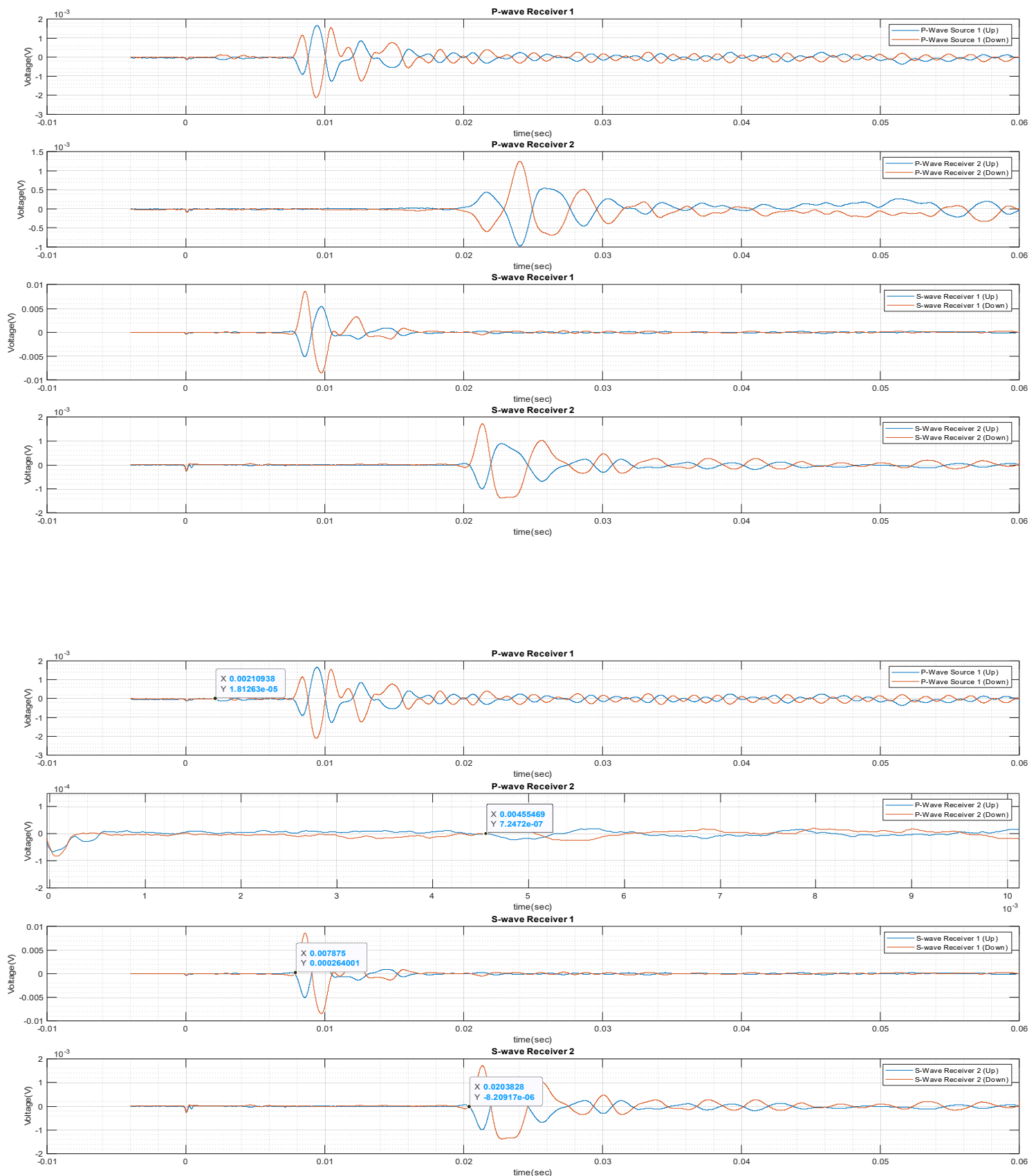

Data Set 42 (7.9.2020)

Run00328f

R4_2 

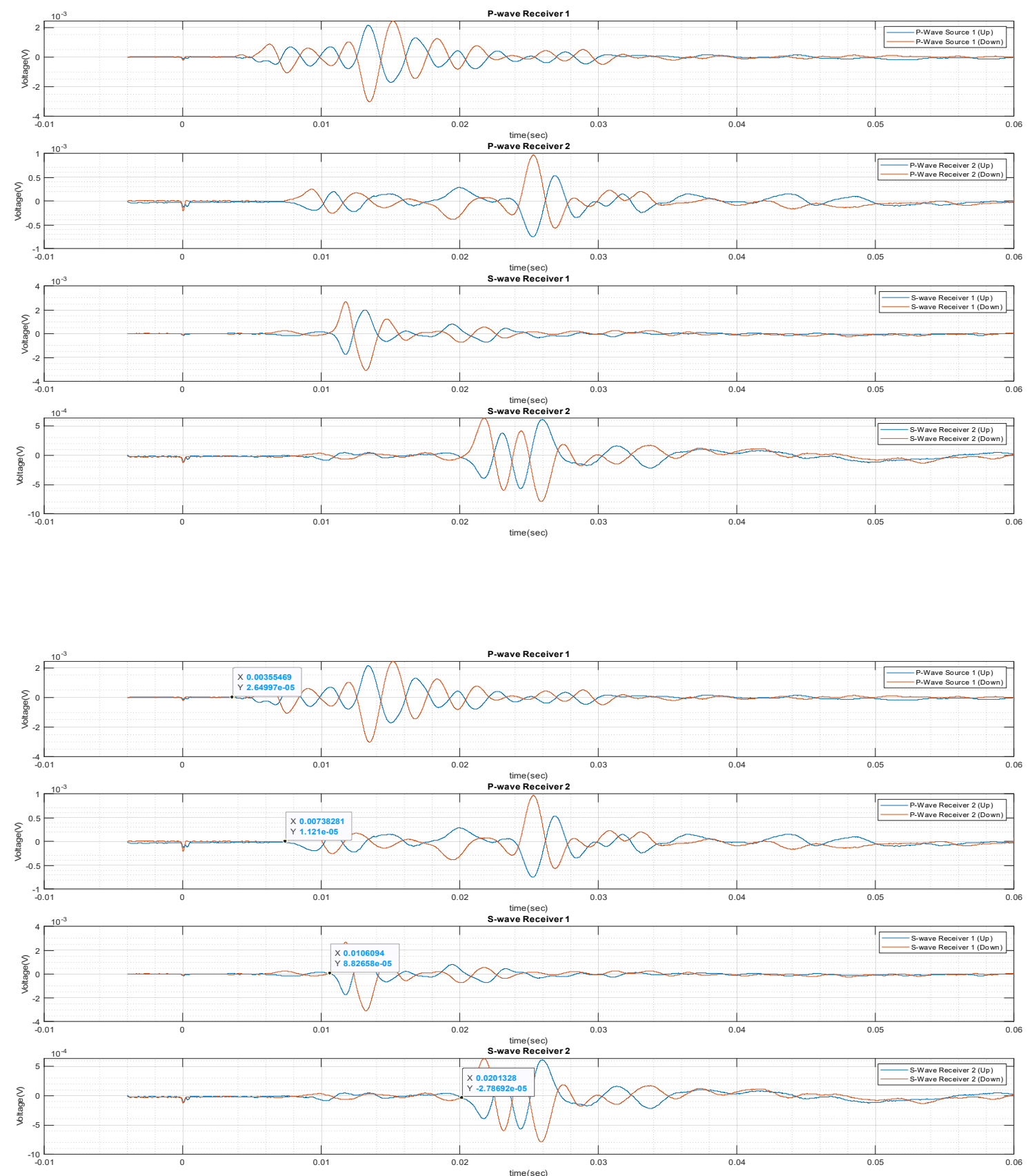

Data Set $43(8.11 .2020)$

Run00329f

R1_1 

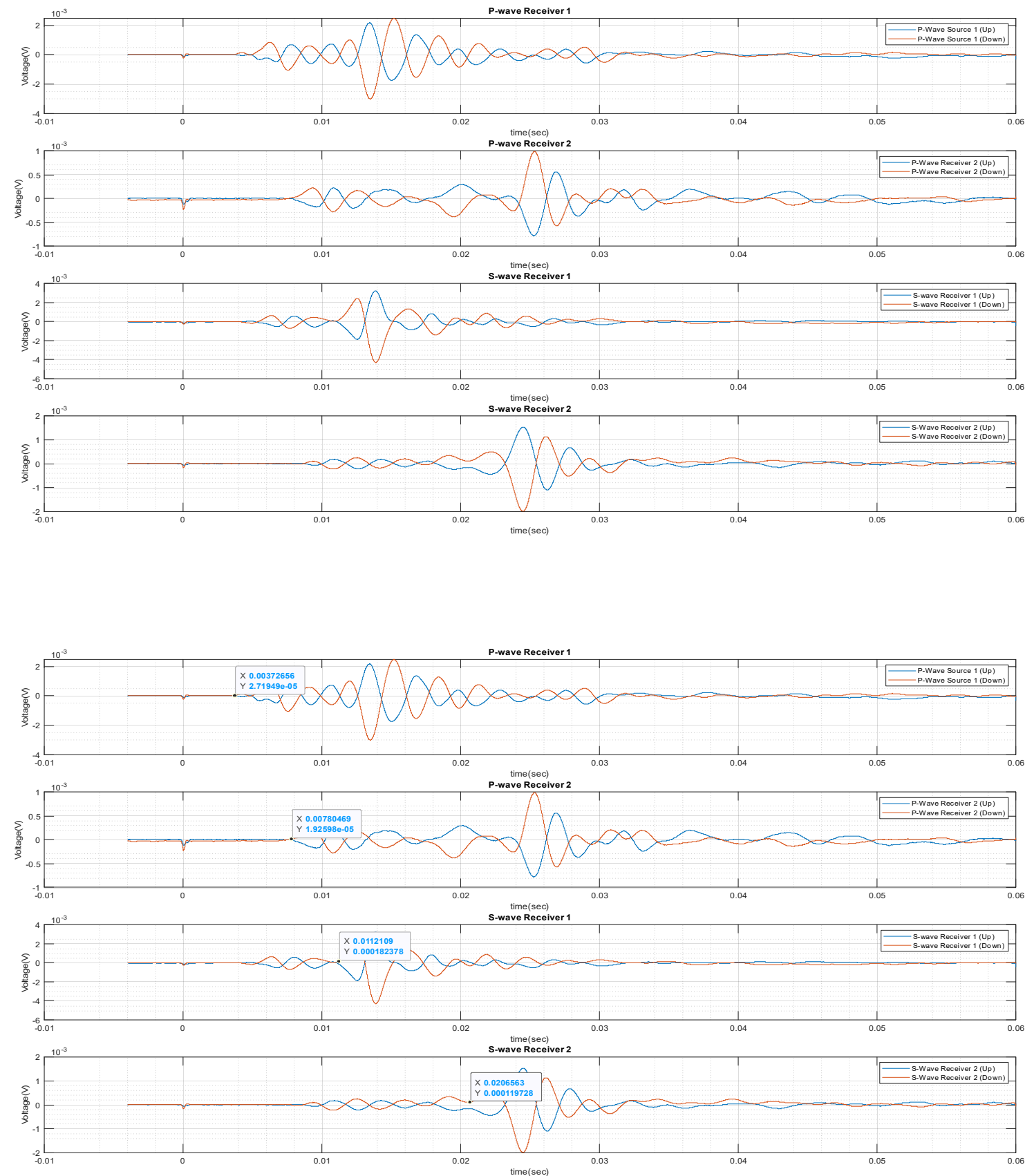

Data Set $43(8.11 .2020)$

Run00330f

R1_2 

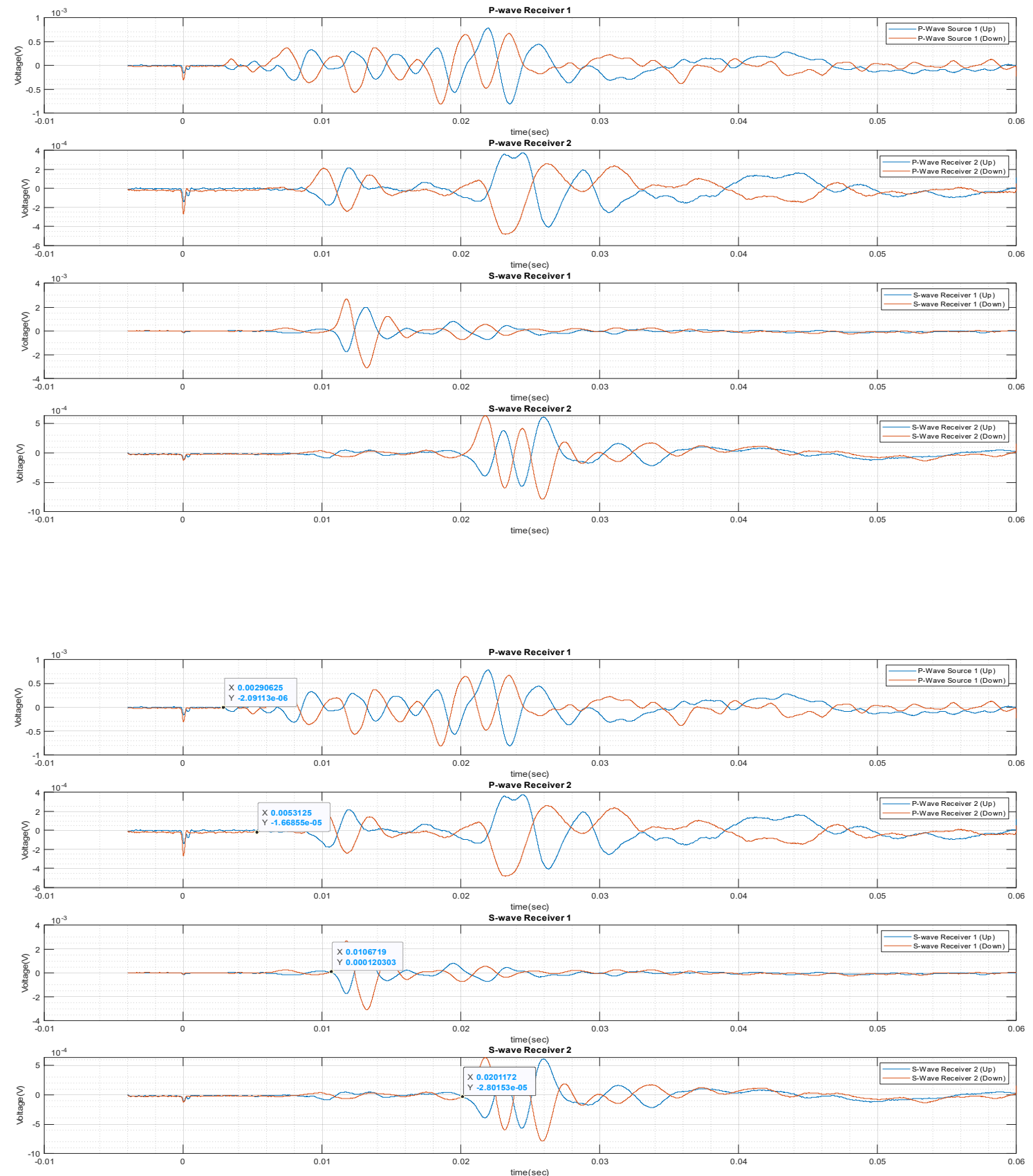

Data Set $43(8.11 .2020)$

Run00331f

R2_1 

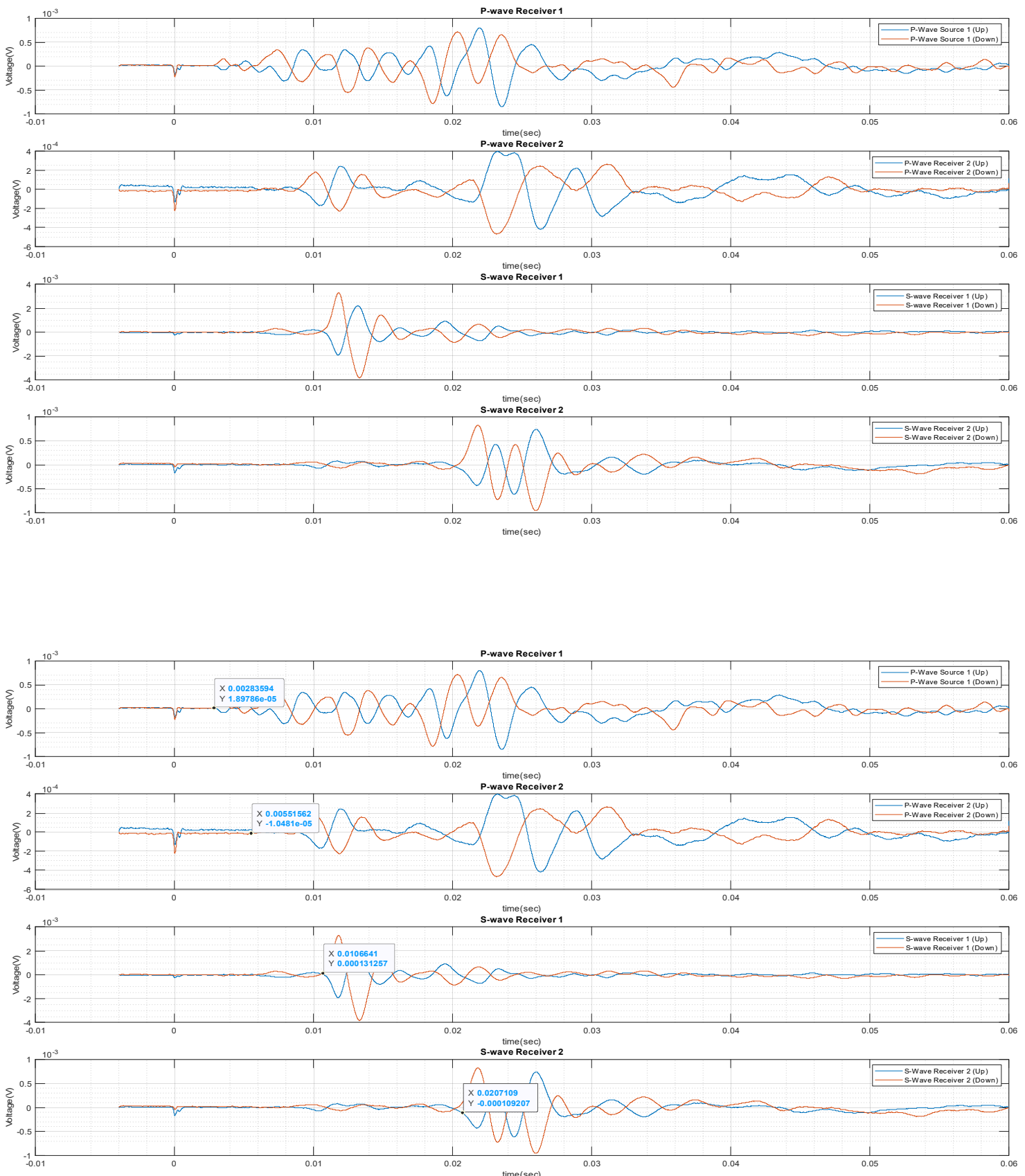

Data Set $43(8.11 .2020)$

Run00332f

R2_2 

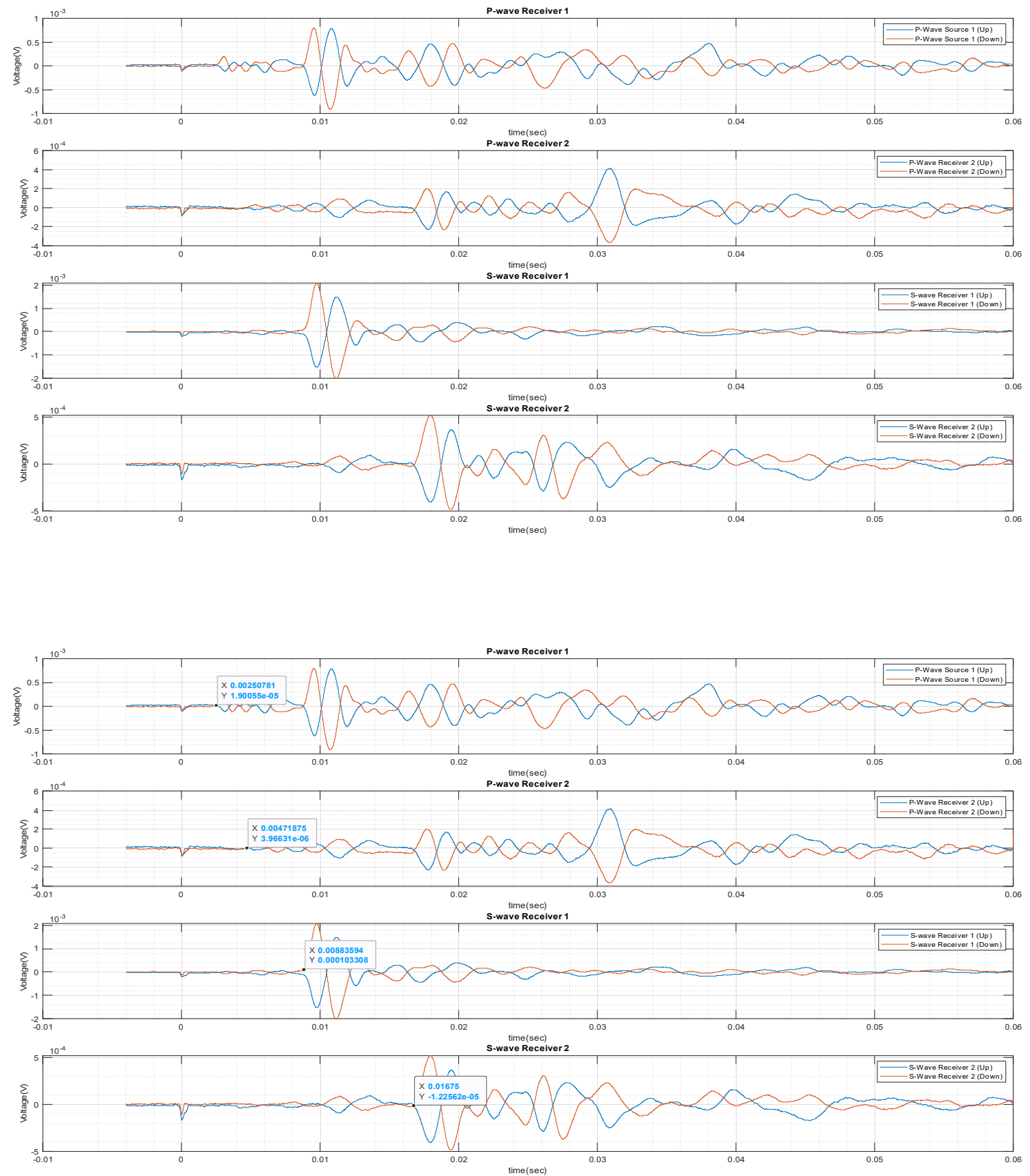

Data Set $43(8.11 .2020)$

Run00333f

R3_1 

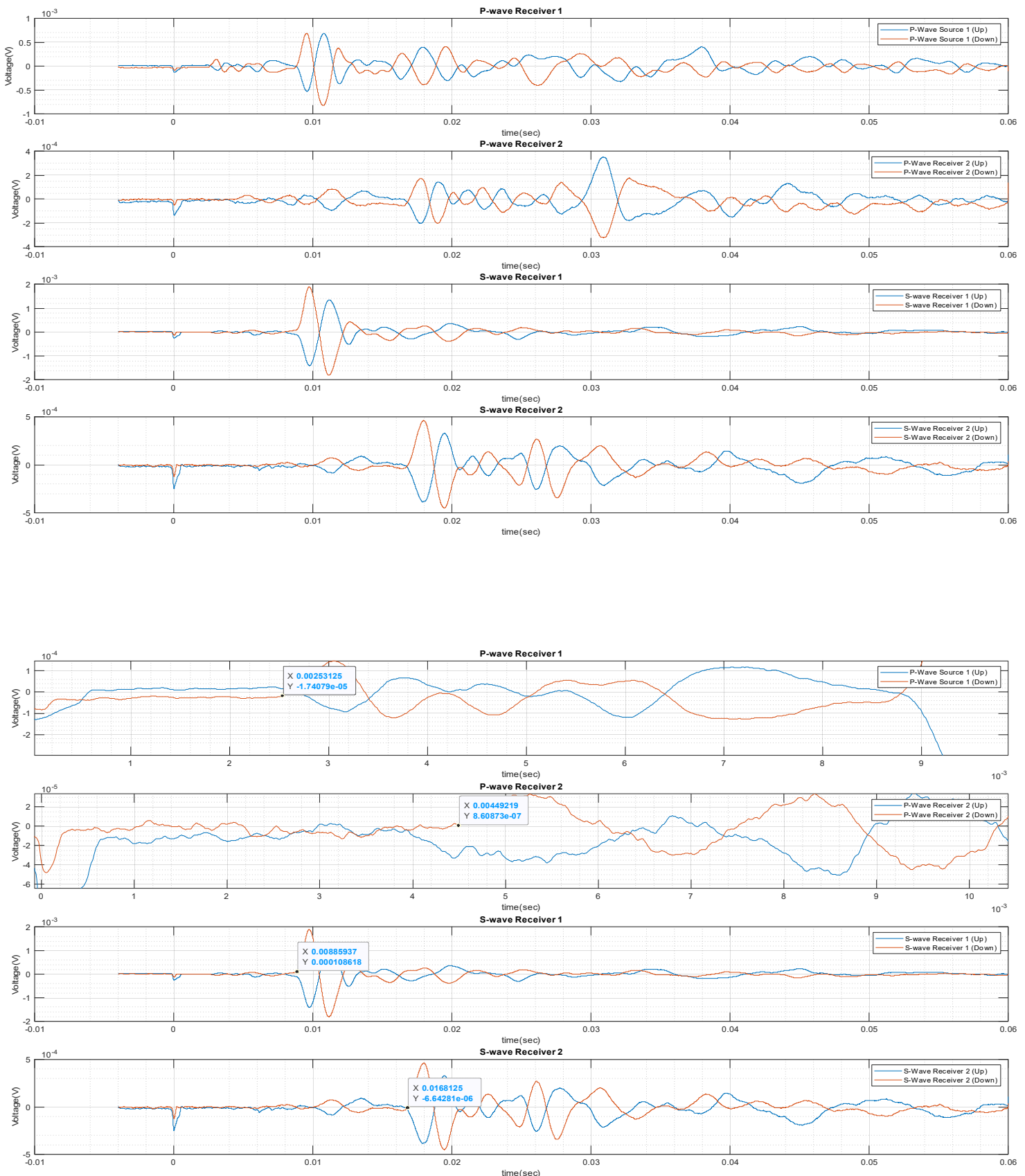

Data Set $43(8.11 .2020)$

Run00334f

R3_2 

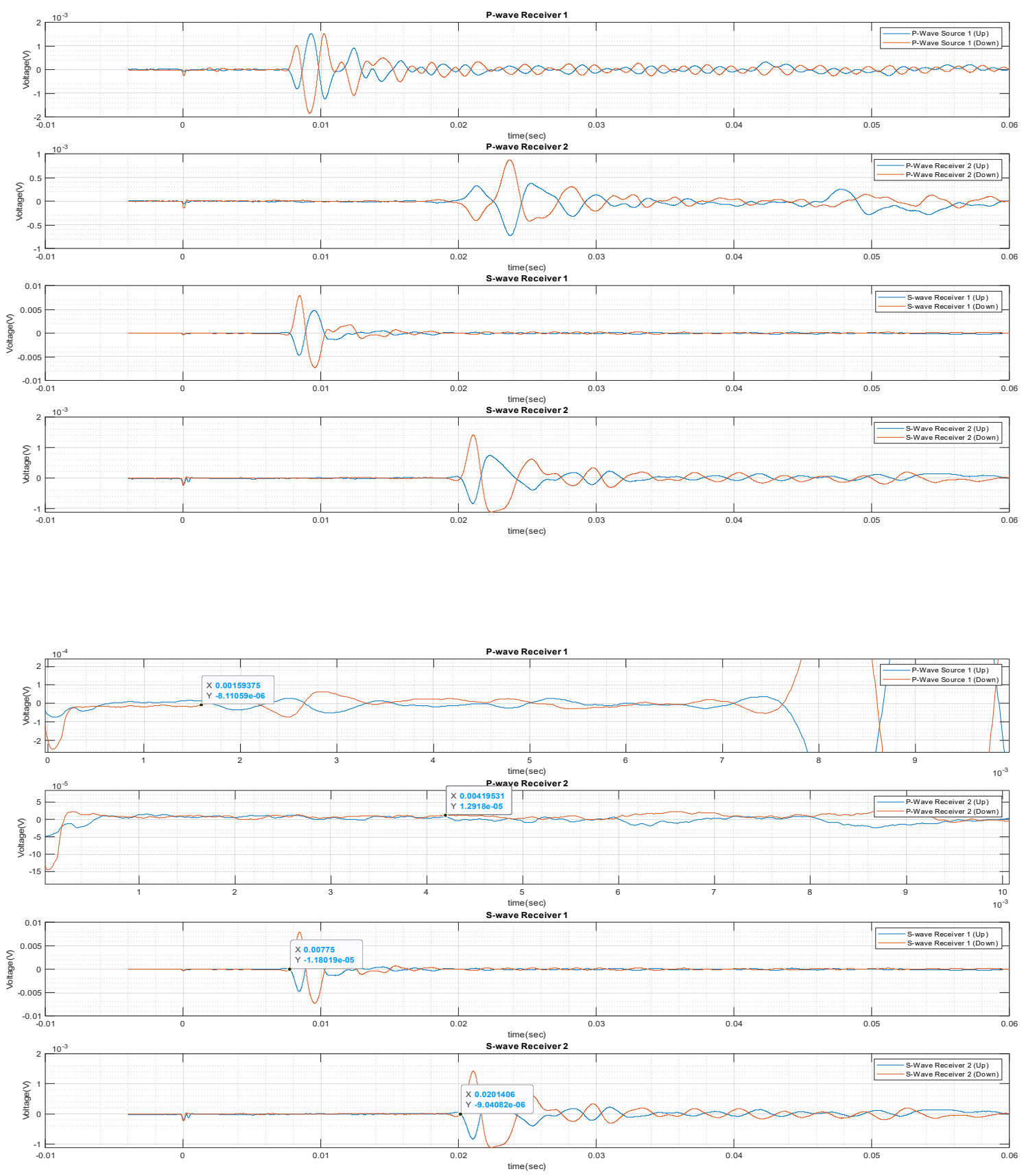

Data Set $43(8.11 .2020)$

Run00335f

R4_1 

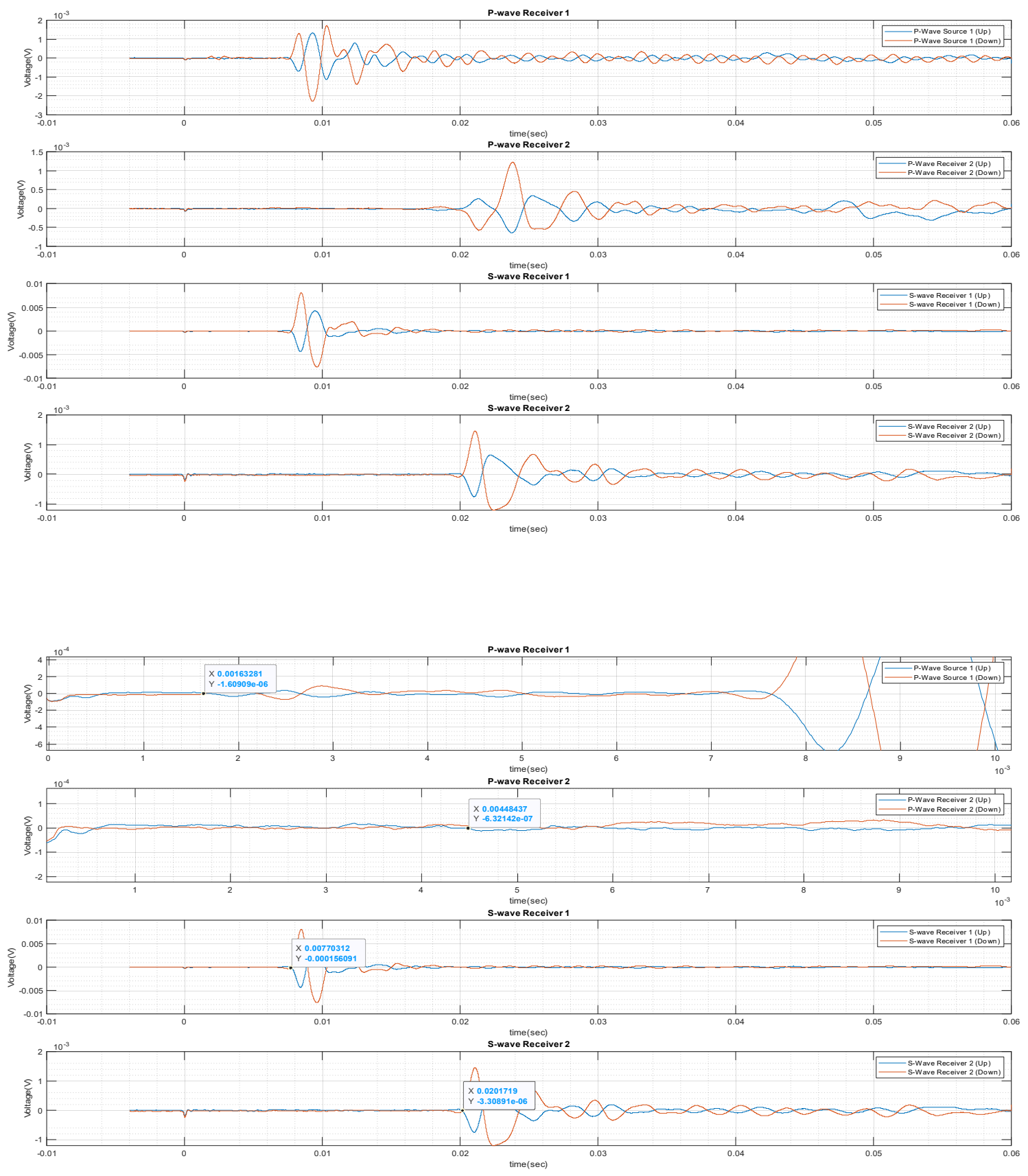

Data Set $43(8.11 .2020)$

Run00336f

R4_2 

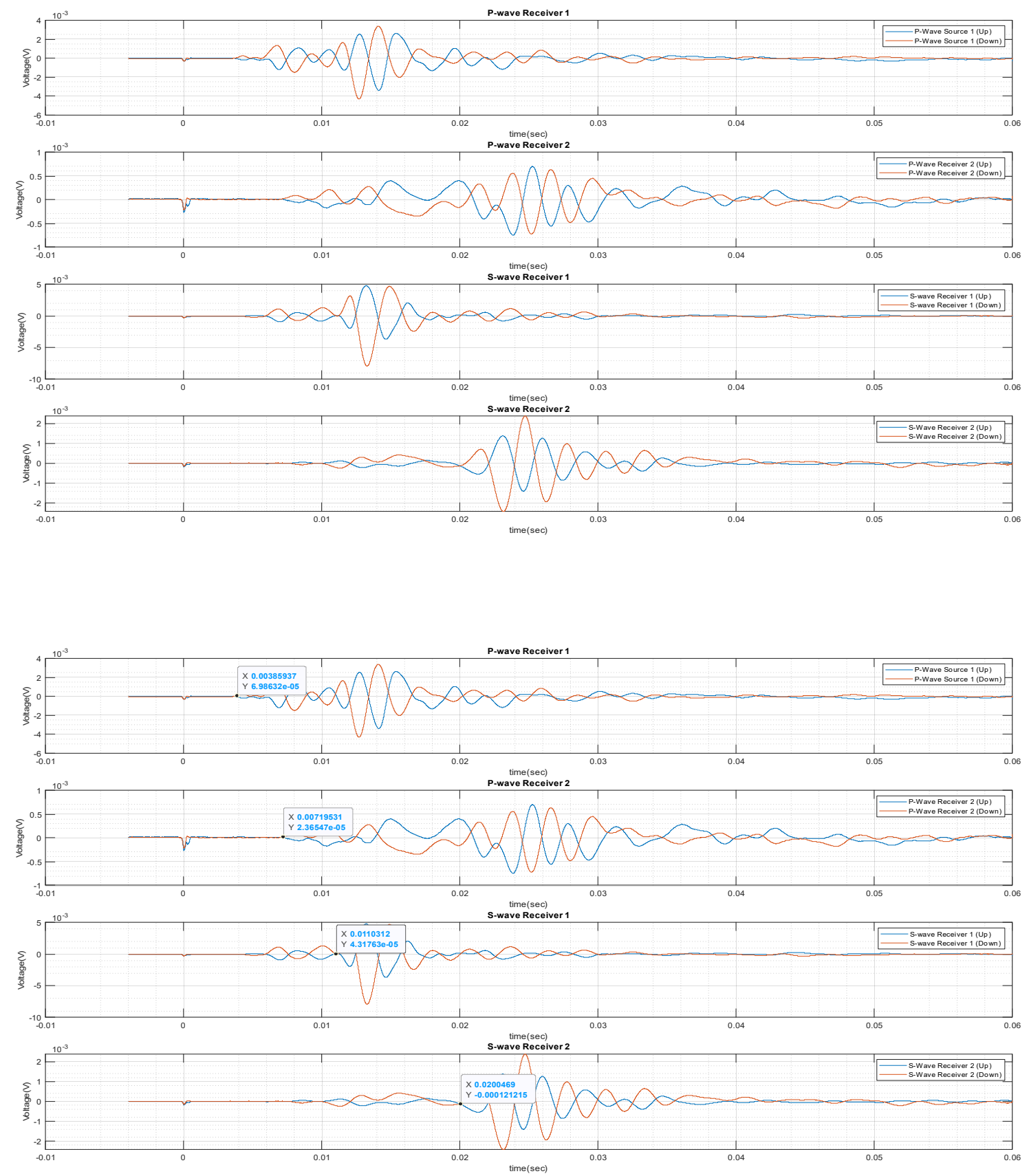

Data Set 44 (9.3.2020)

Run00337f

R1_1 

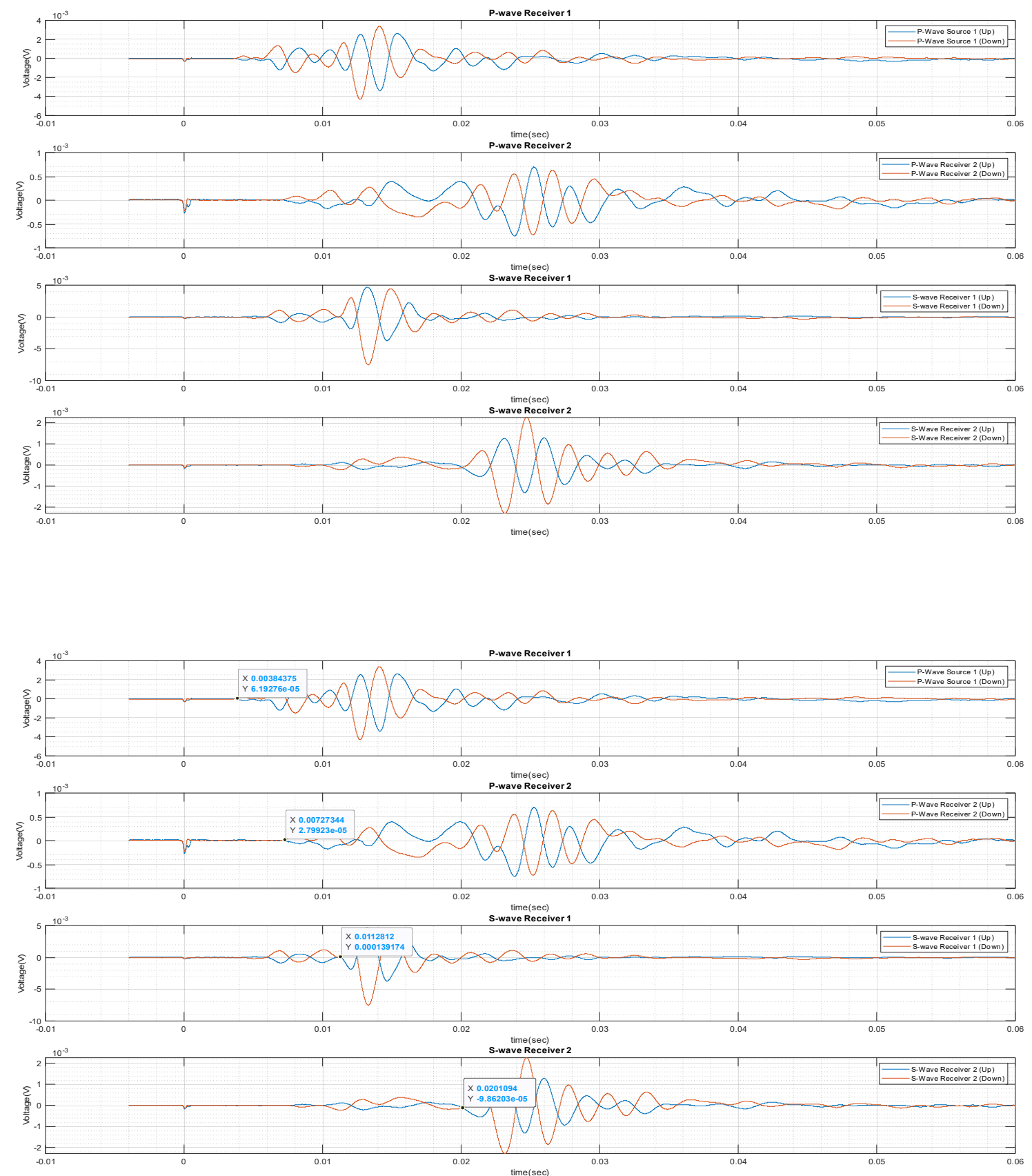

Data Set 44 (9.3.2020)

Run00338f

R1_2 

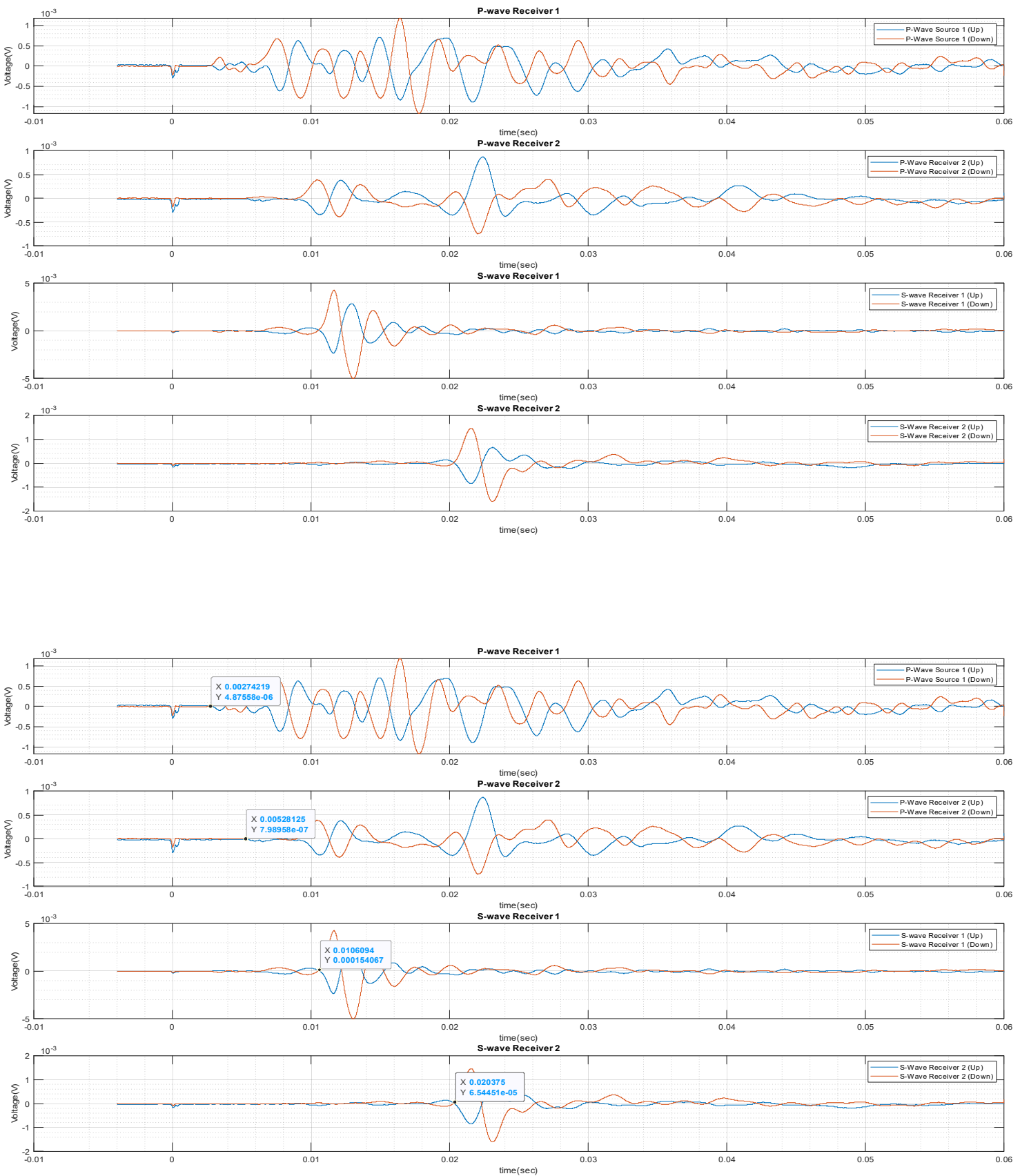

Data Set $44(9.3 .2020)$

Run00339f

R2 1 

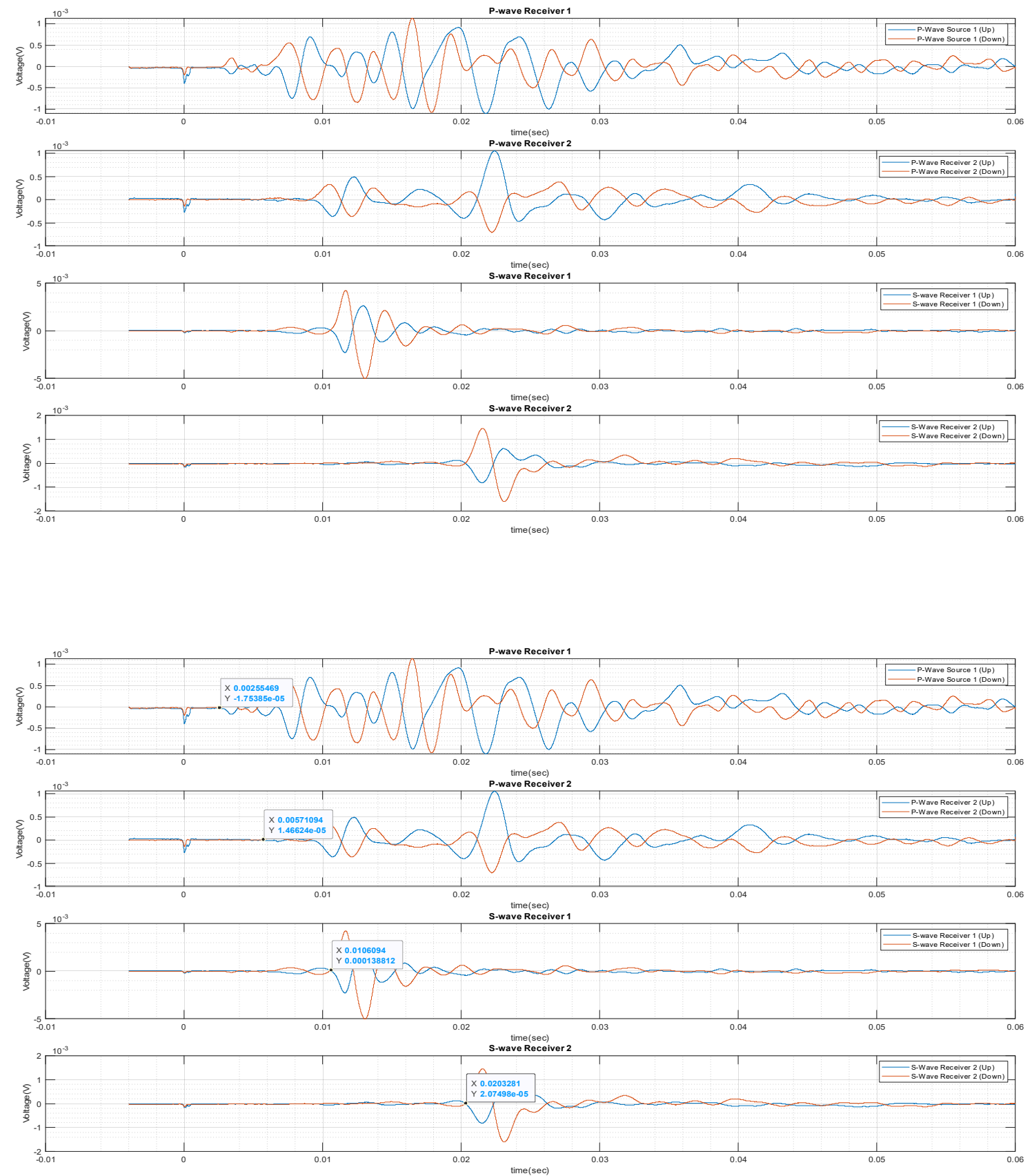

Data Set 44 (9.3.2020)

Run00340f

R2_2 

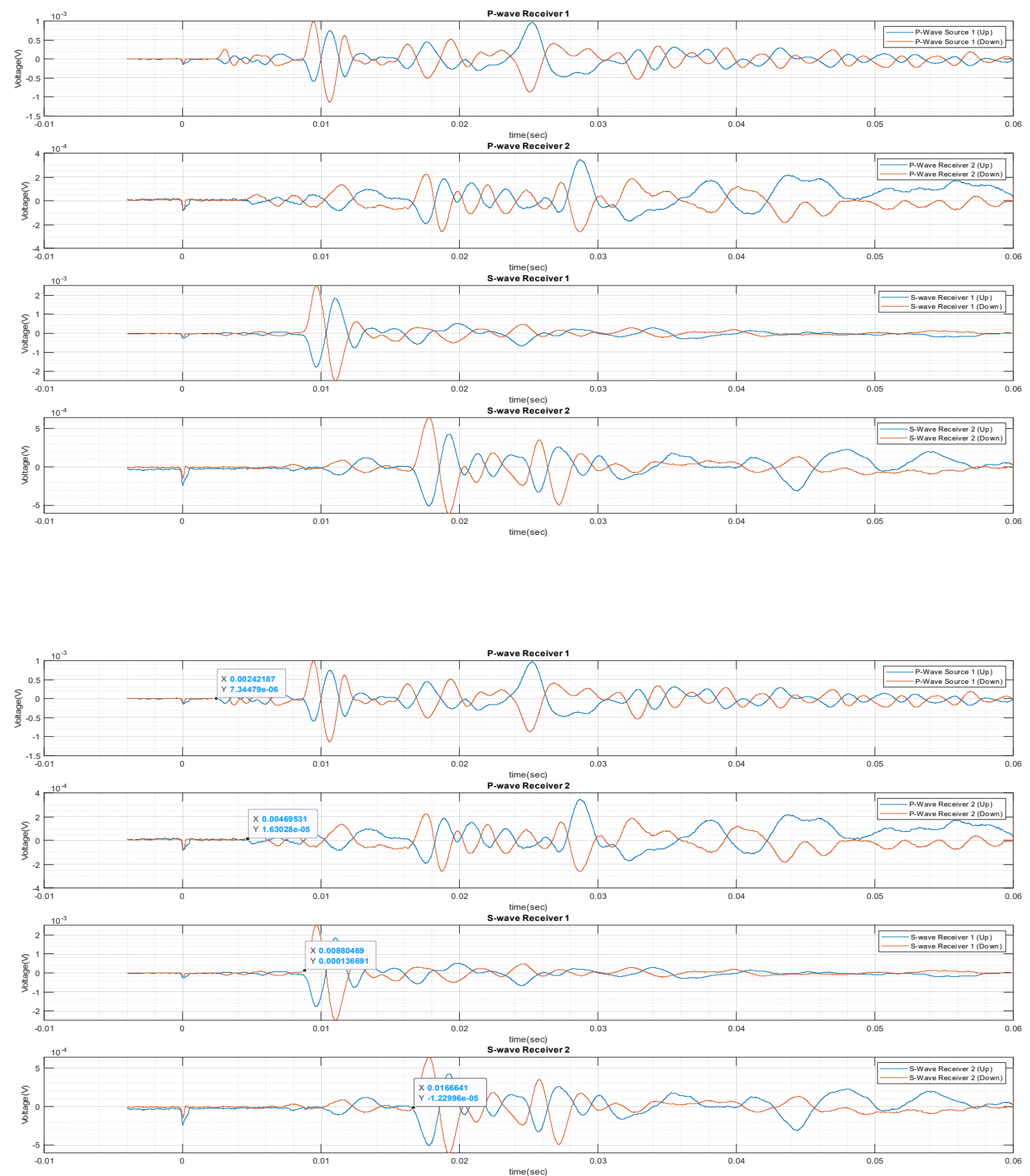

Data Set 44 (9.3.2020)

Run00341f

R3_1 

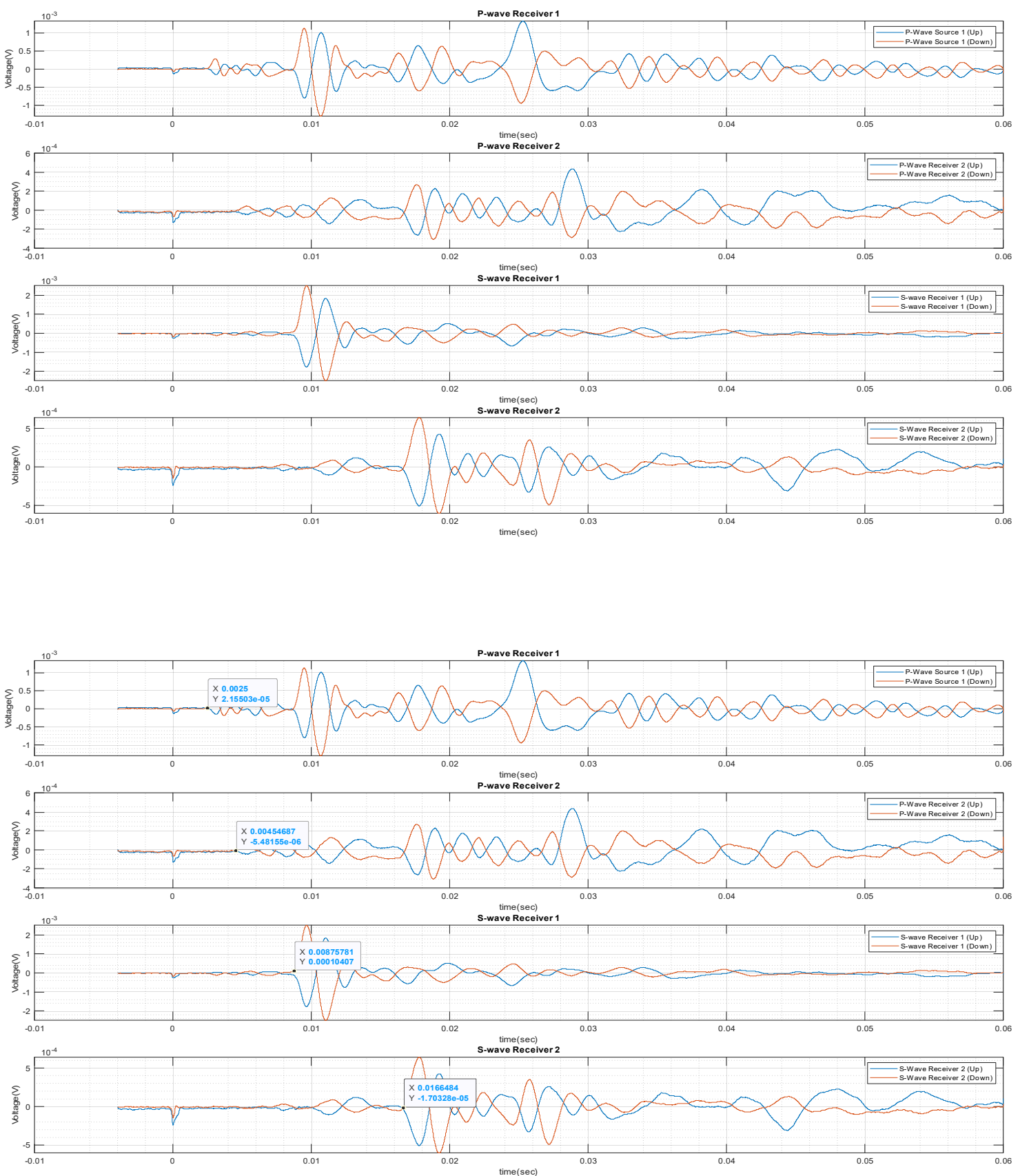

Data Set 44 (9.3.2020)

Run00342f

R3_2 

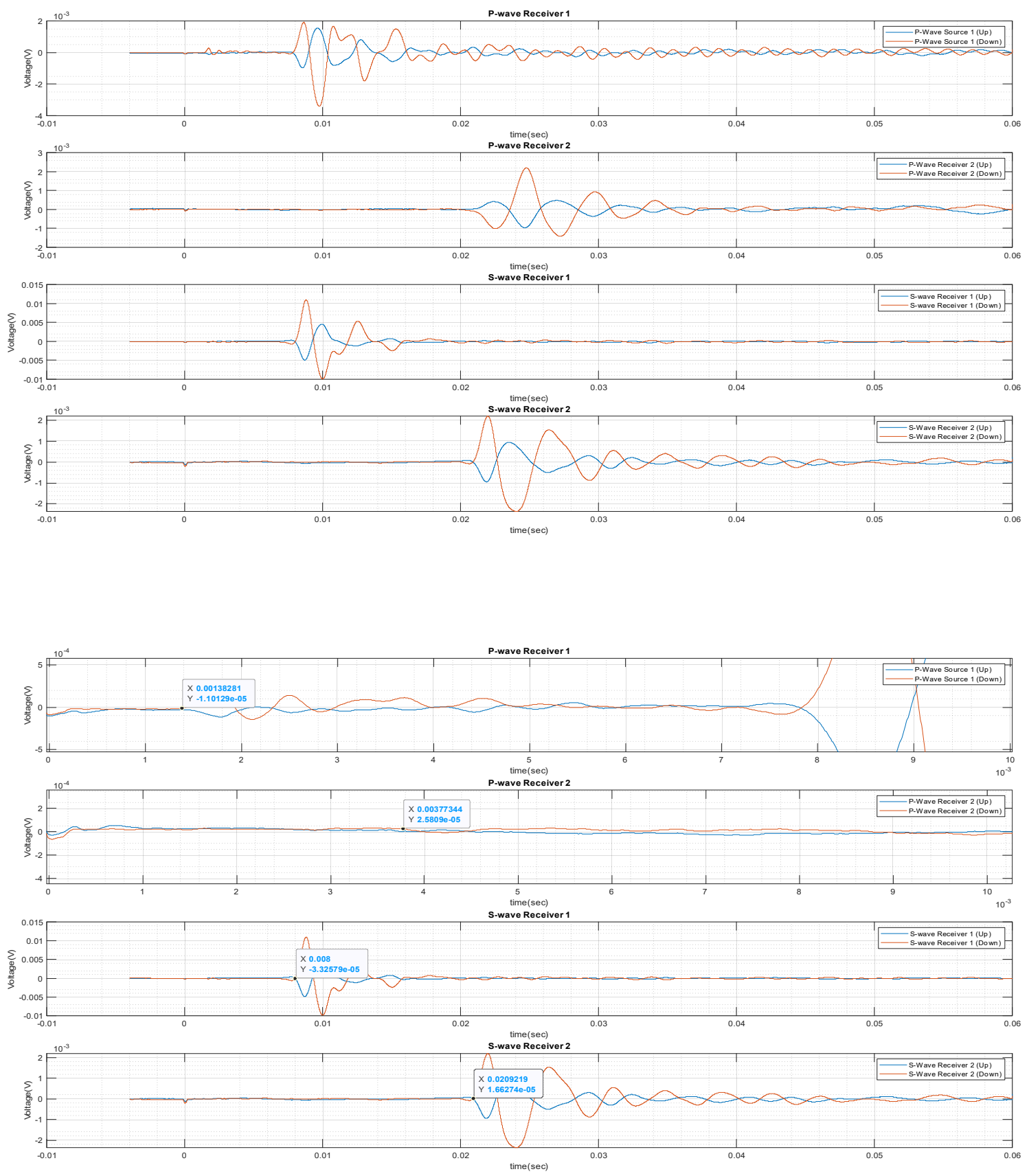

Data Set 44 (9.3.2020)

Run00343f

R4_1 

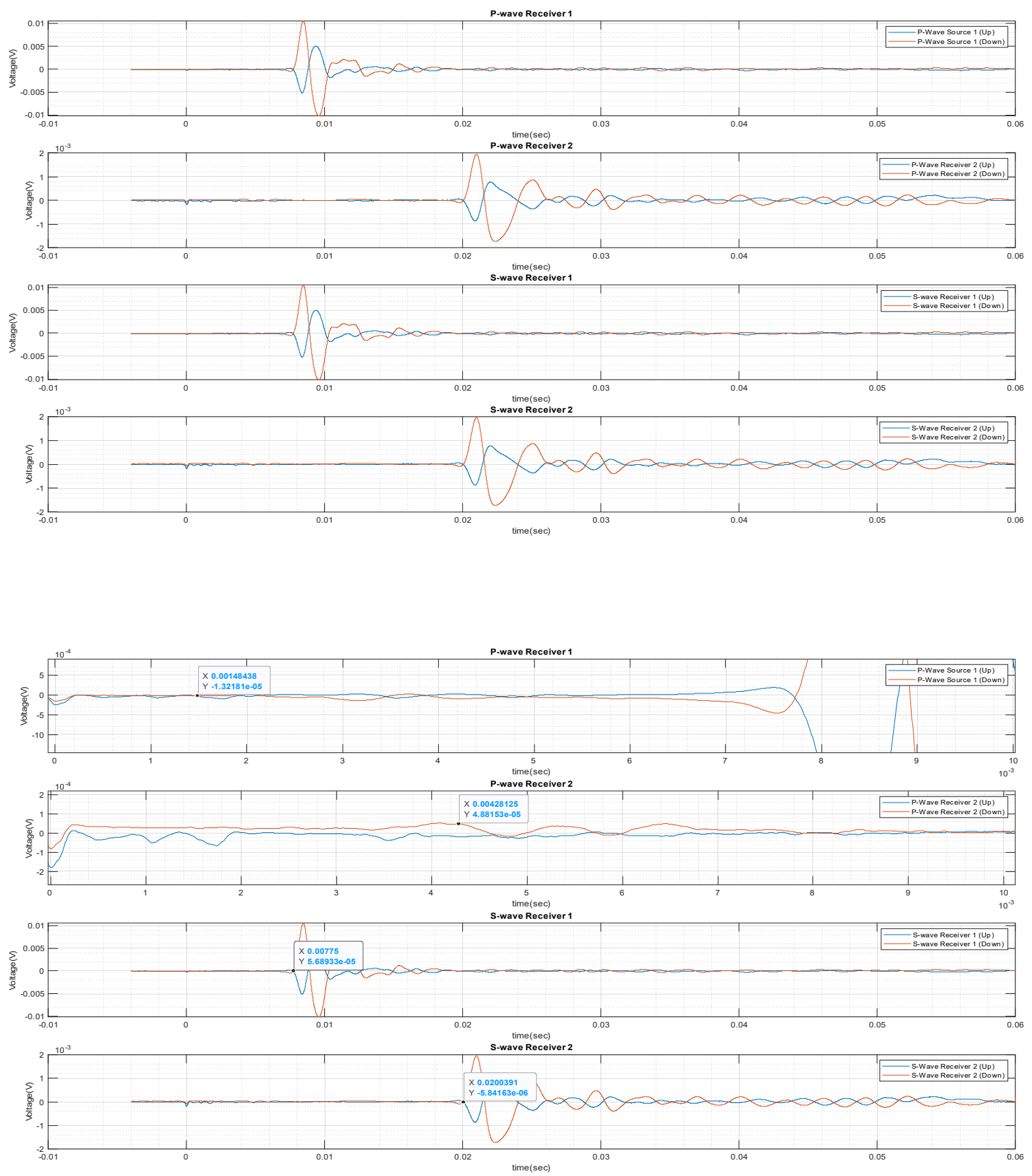

Data Set 44 (9.3.2020)

Run00344f

R4_2 

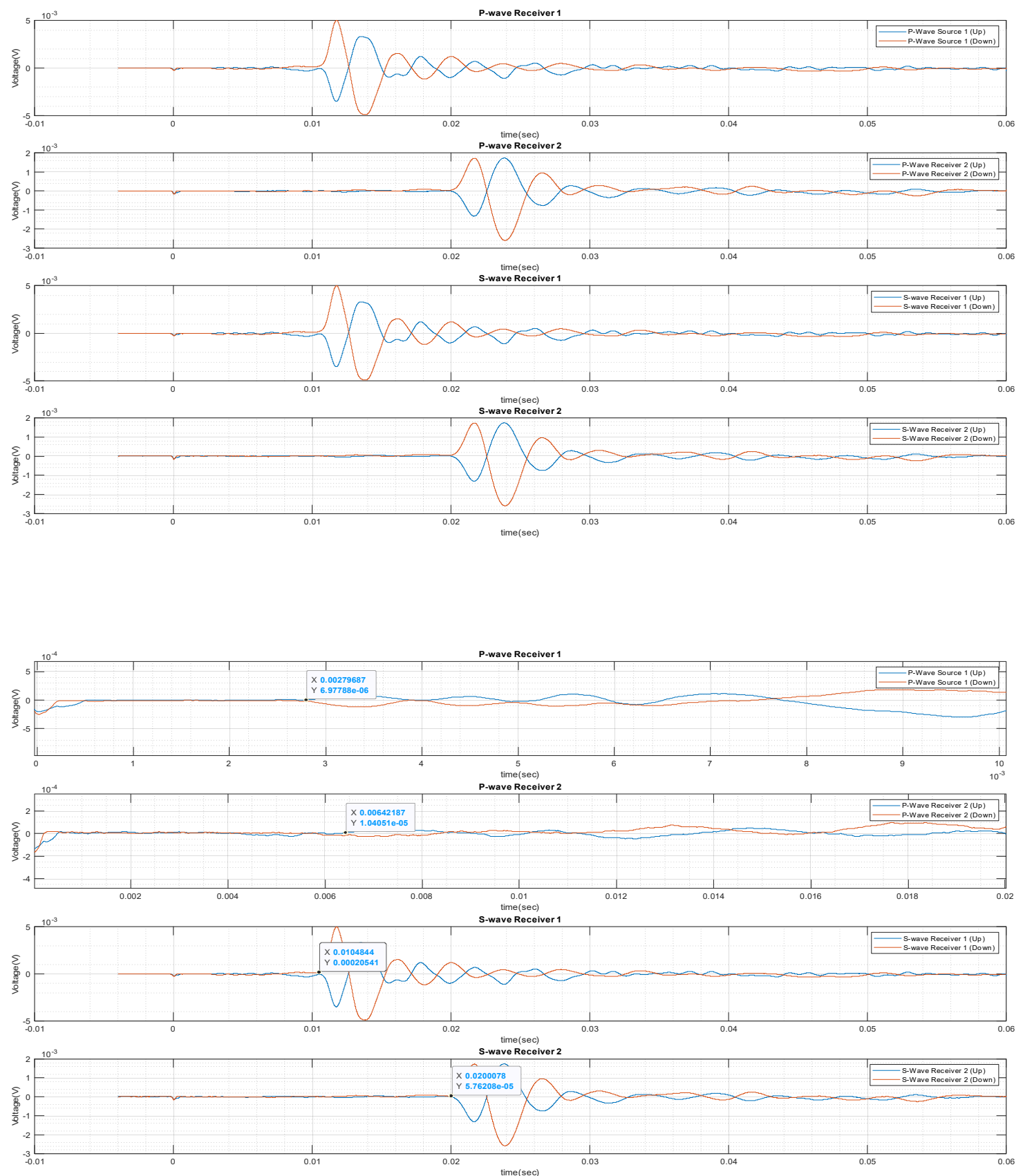

Data Set 45 (10.2.2020)

Run00345f

R1_1 

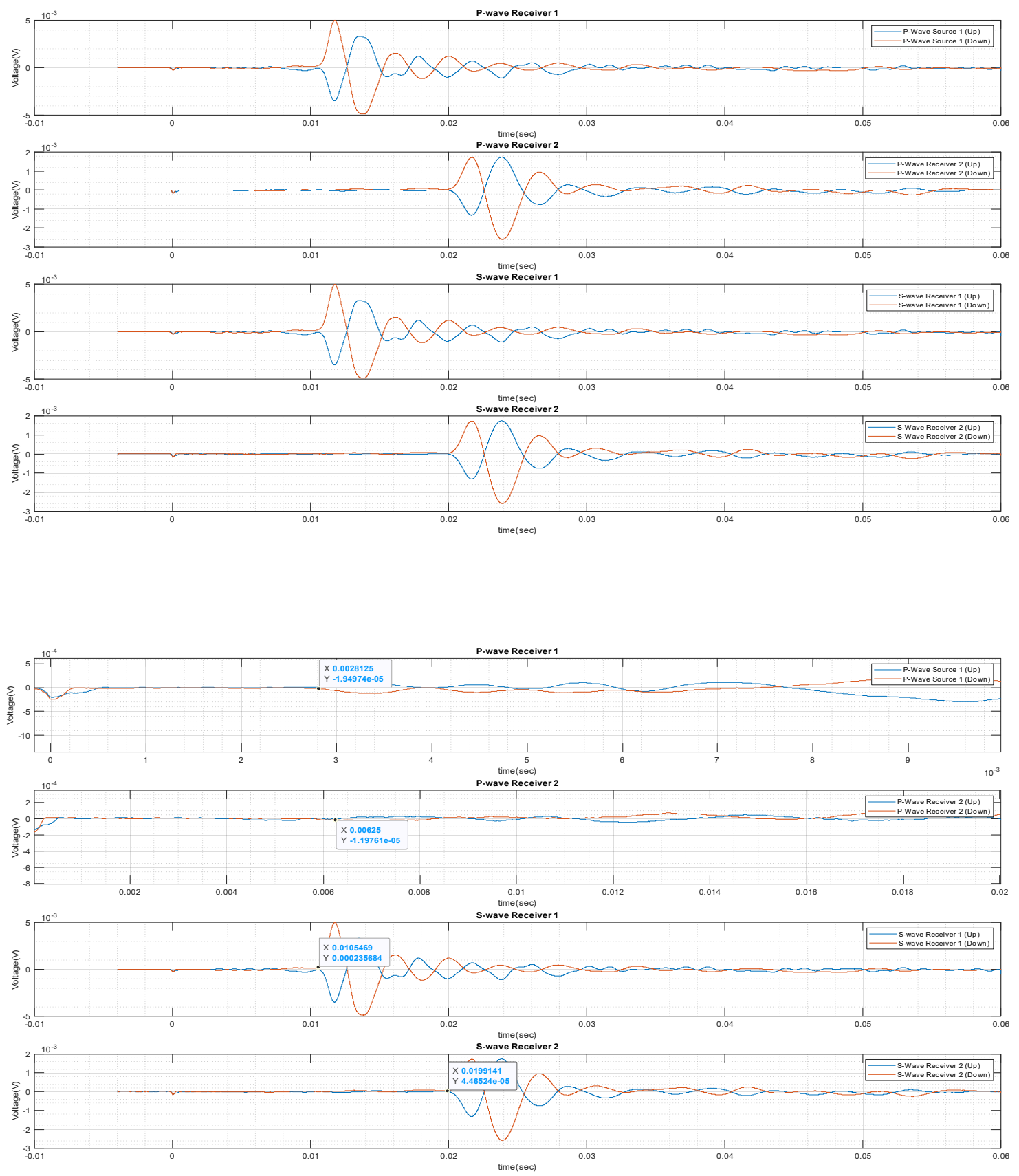

Data Set 45 (10.2.2020)

Run00346f

R1_2 

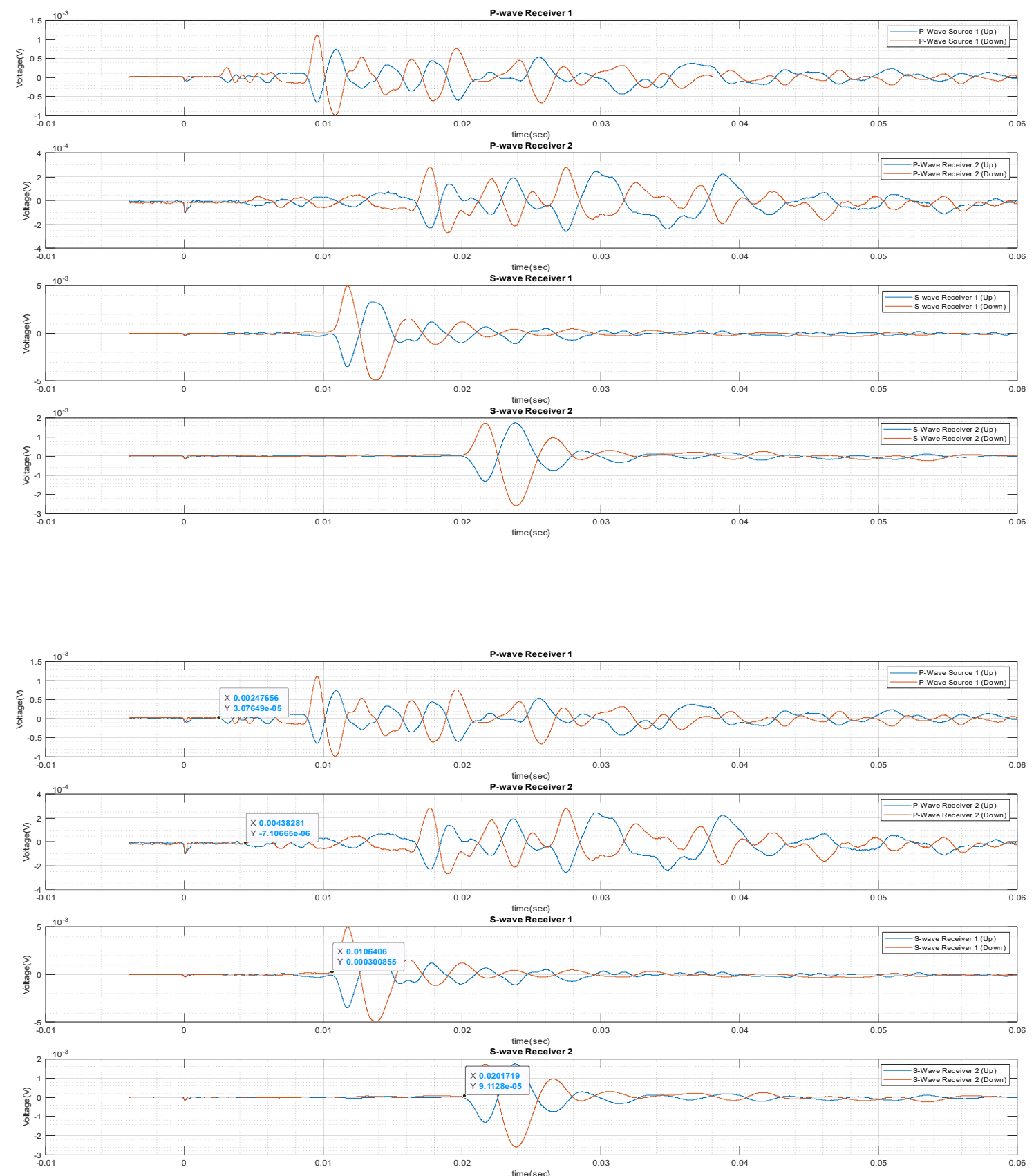

Data Set 45 (10.2.2020)

Run00347f

R2_1 

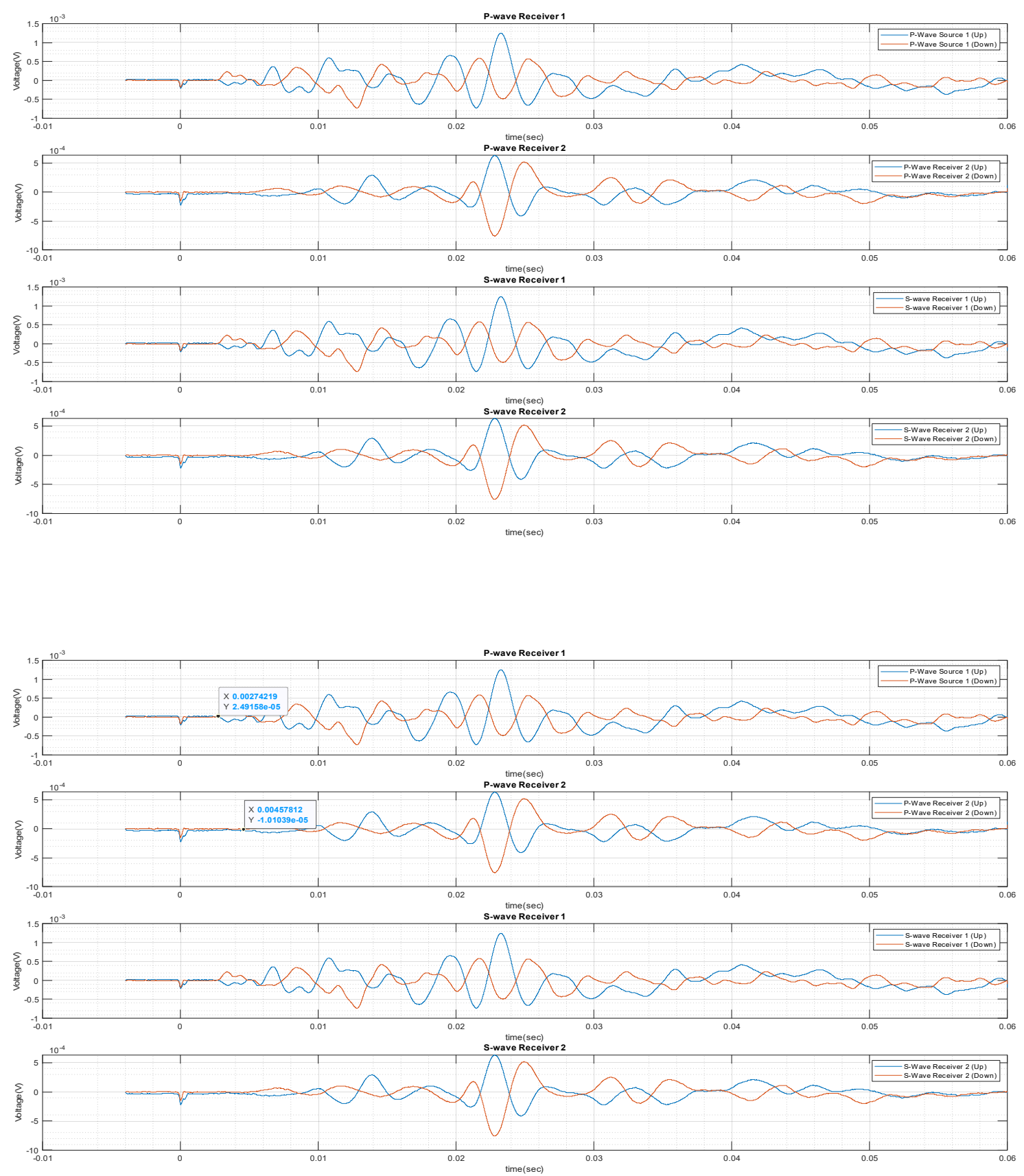

Data Set 45 (10.2.2020)

Run00348f

R2_2 

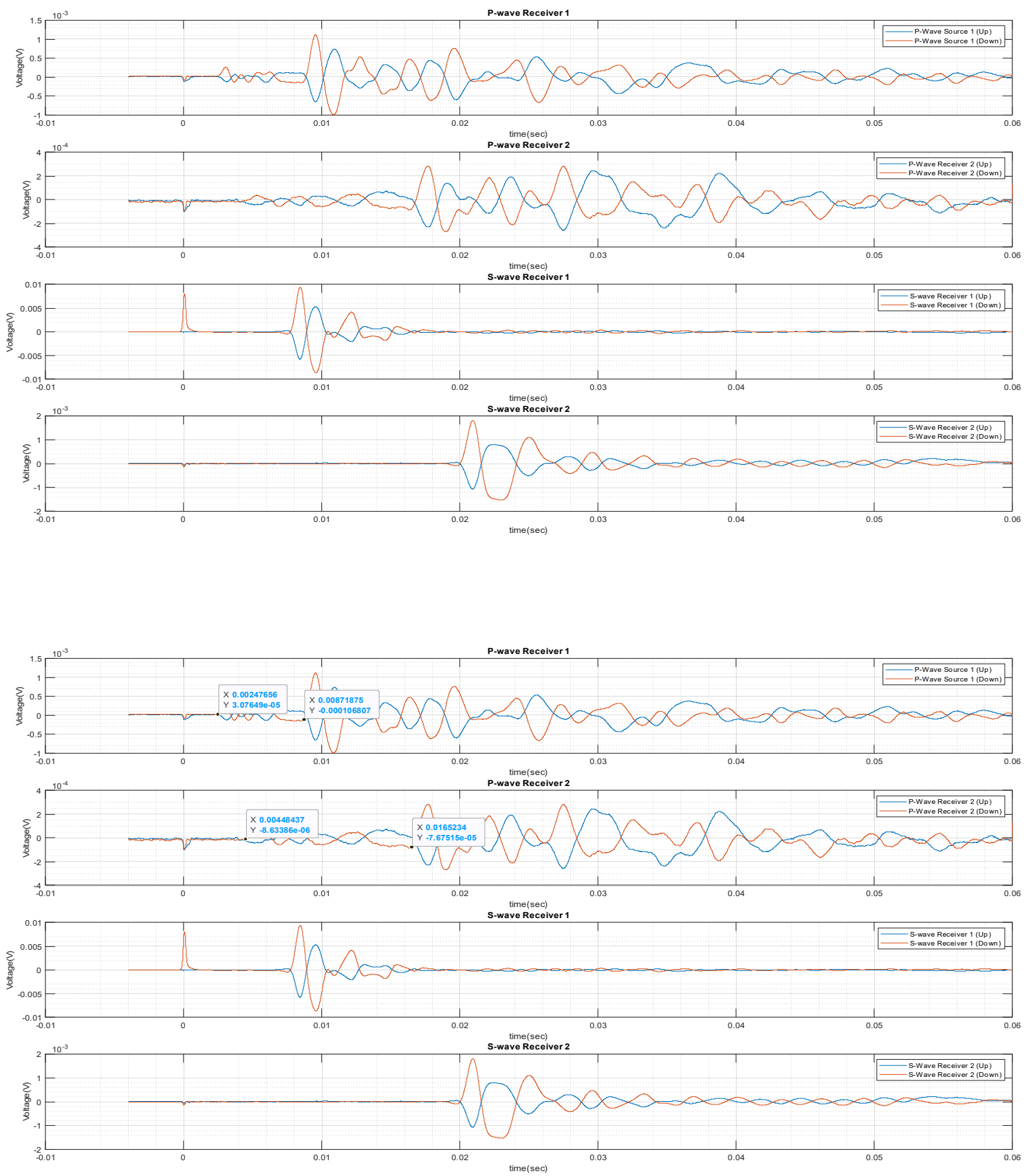

Data Set 45 (10.2.2020)

Run00349f

R3_1 

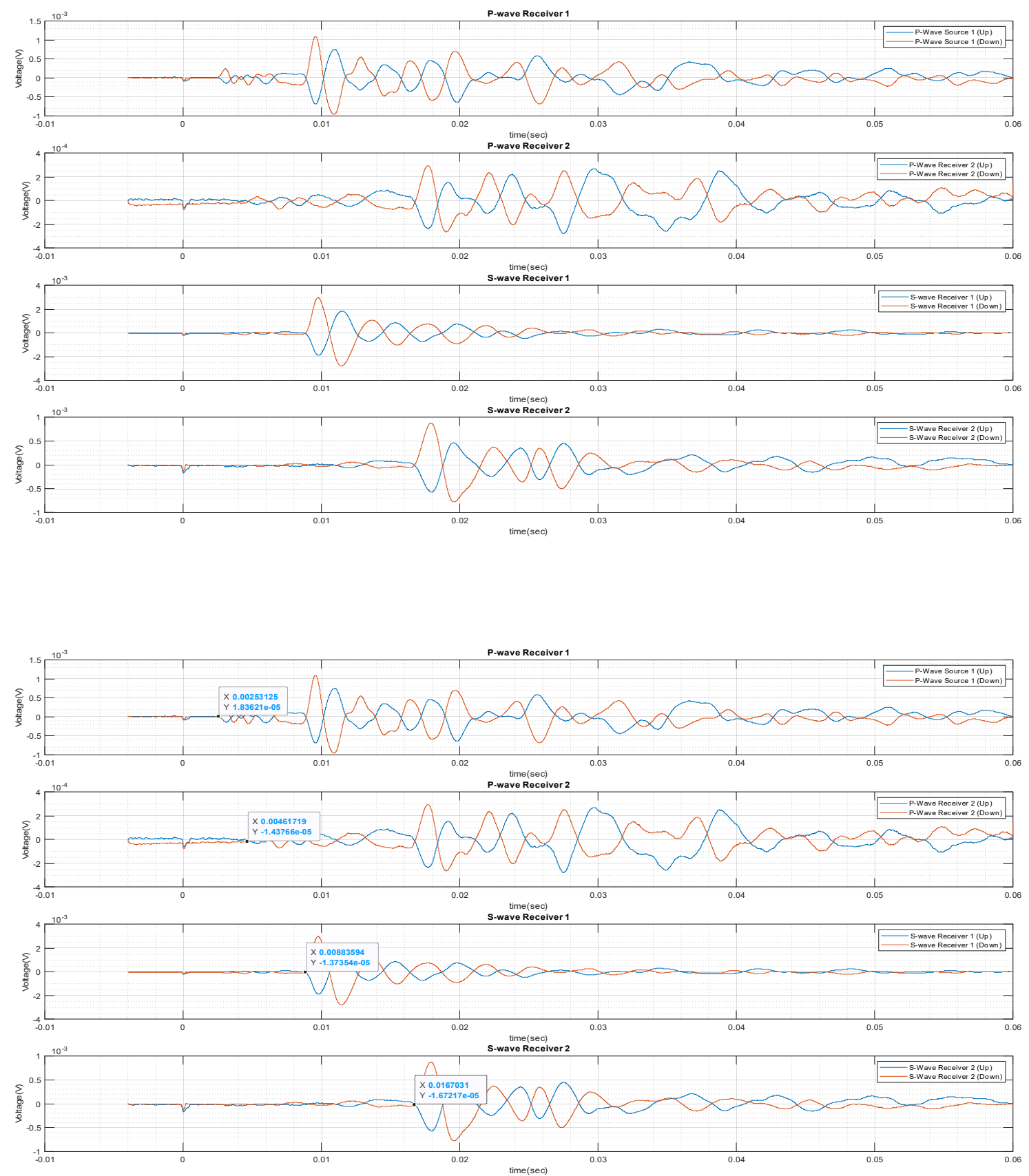

Data Set 45 (10.2.2020)

Run00350f

R3_2 

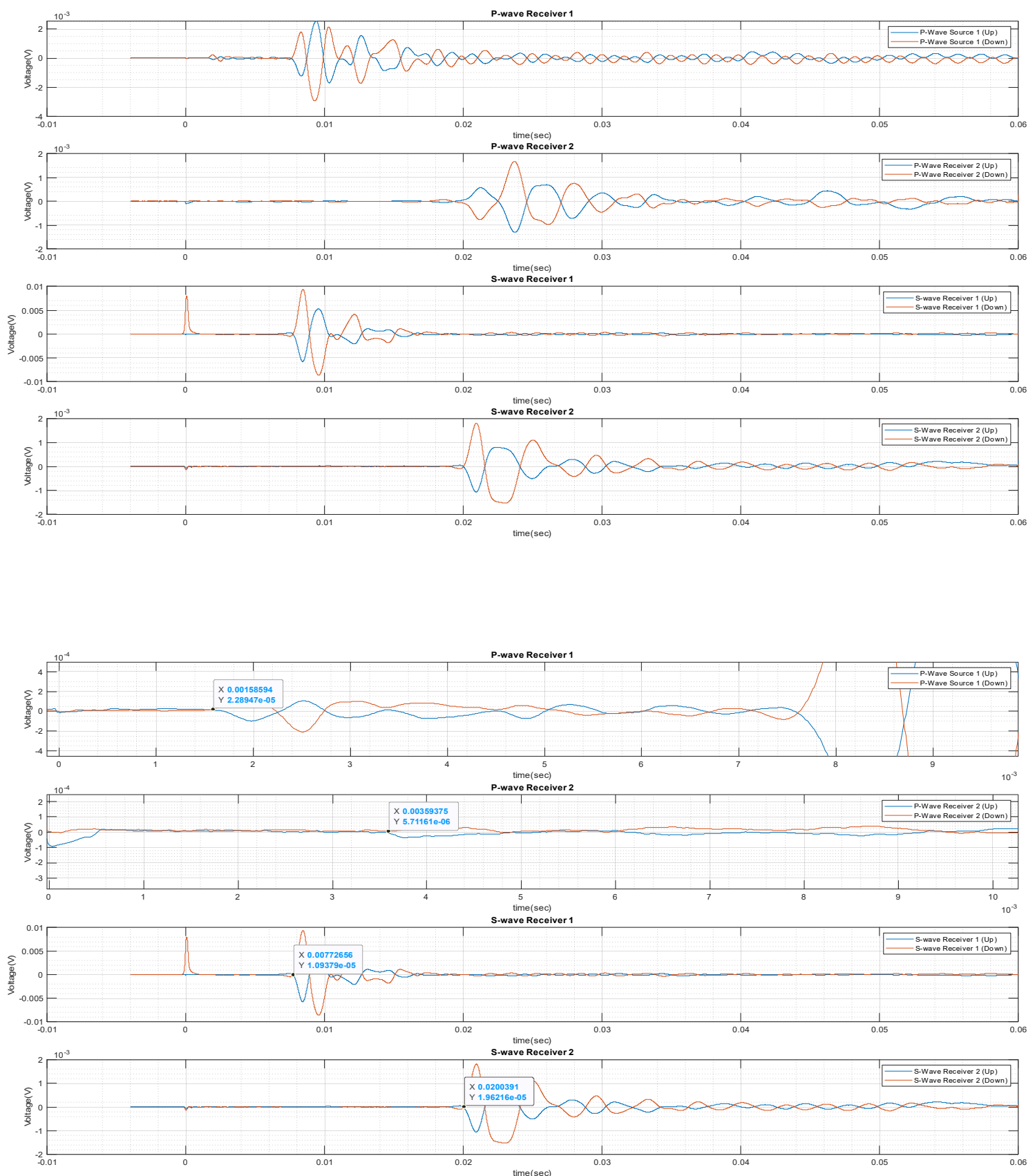

Data Set 45 (10.2.2020)

Run00351f

R4_1 

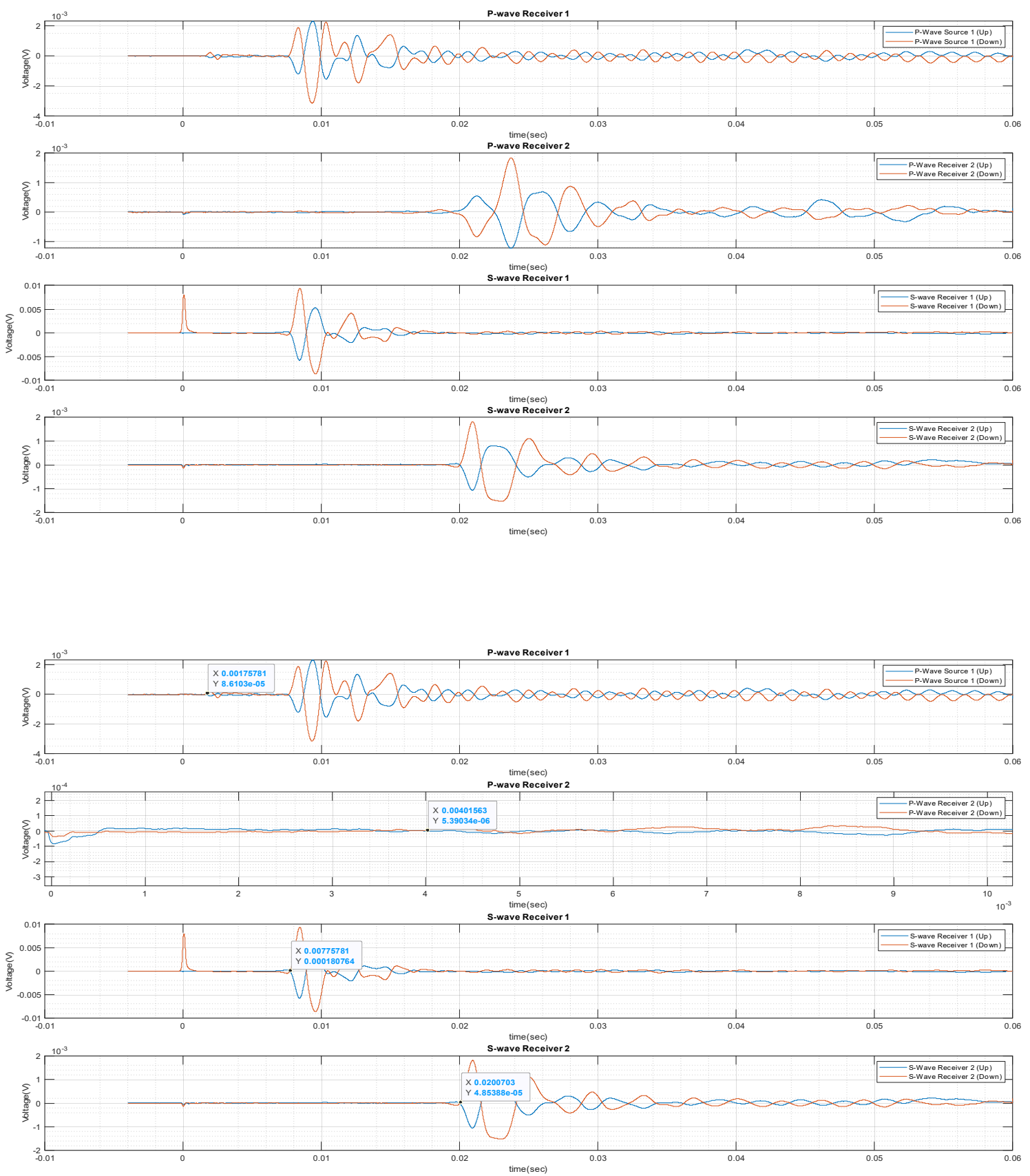

Data Set 45 (10.2.2020)

Run00352f

R4_2 

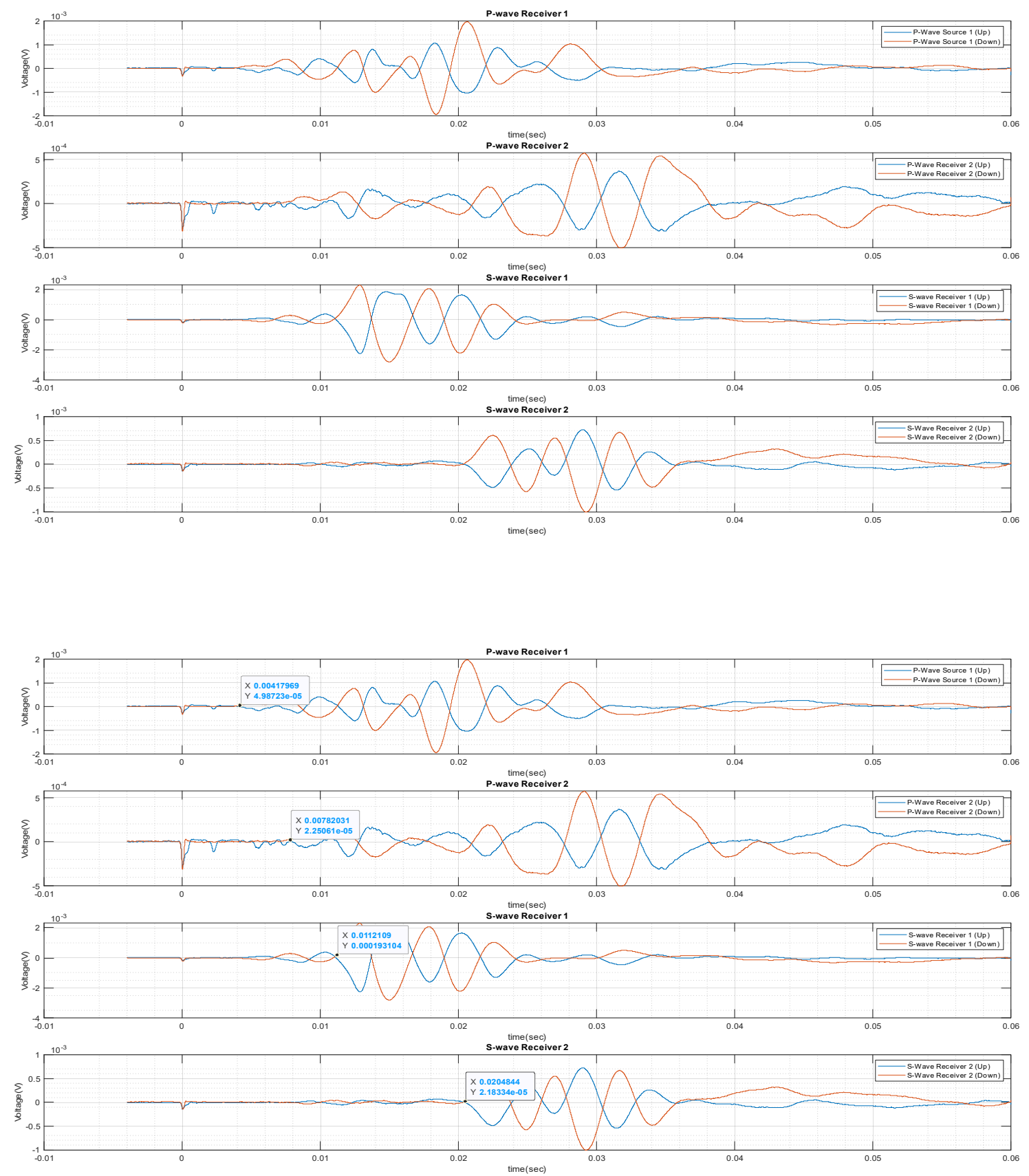

Data Set $46(11.21 .2020)$

Run00353f

R1_1 

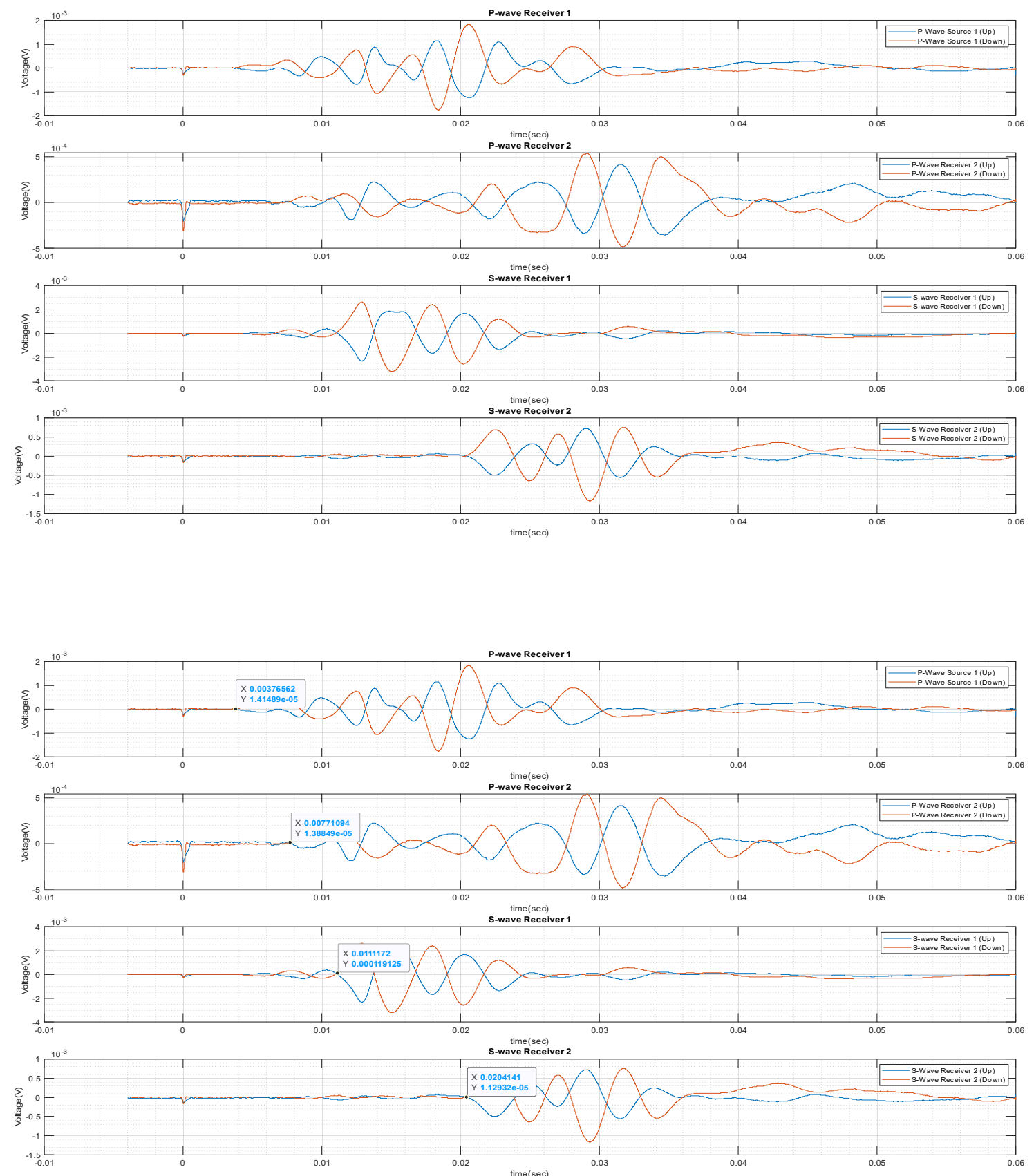

Data Set $46(11.21 .2020)$

Run00354f

R1_2 

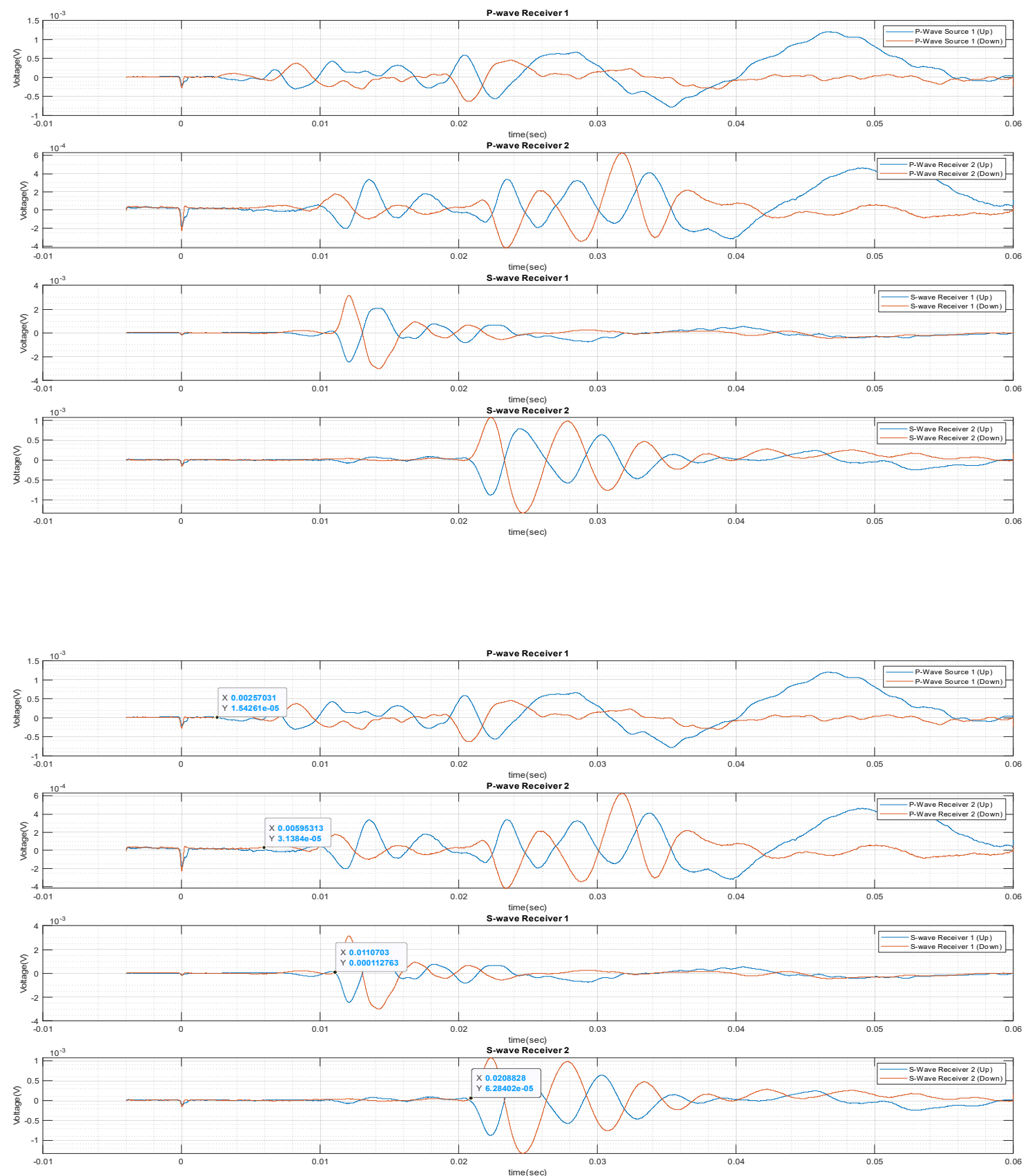

Data Set $46(11.21 .2020)$

Run00355f

R2_1 

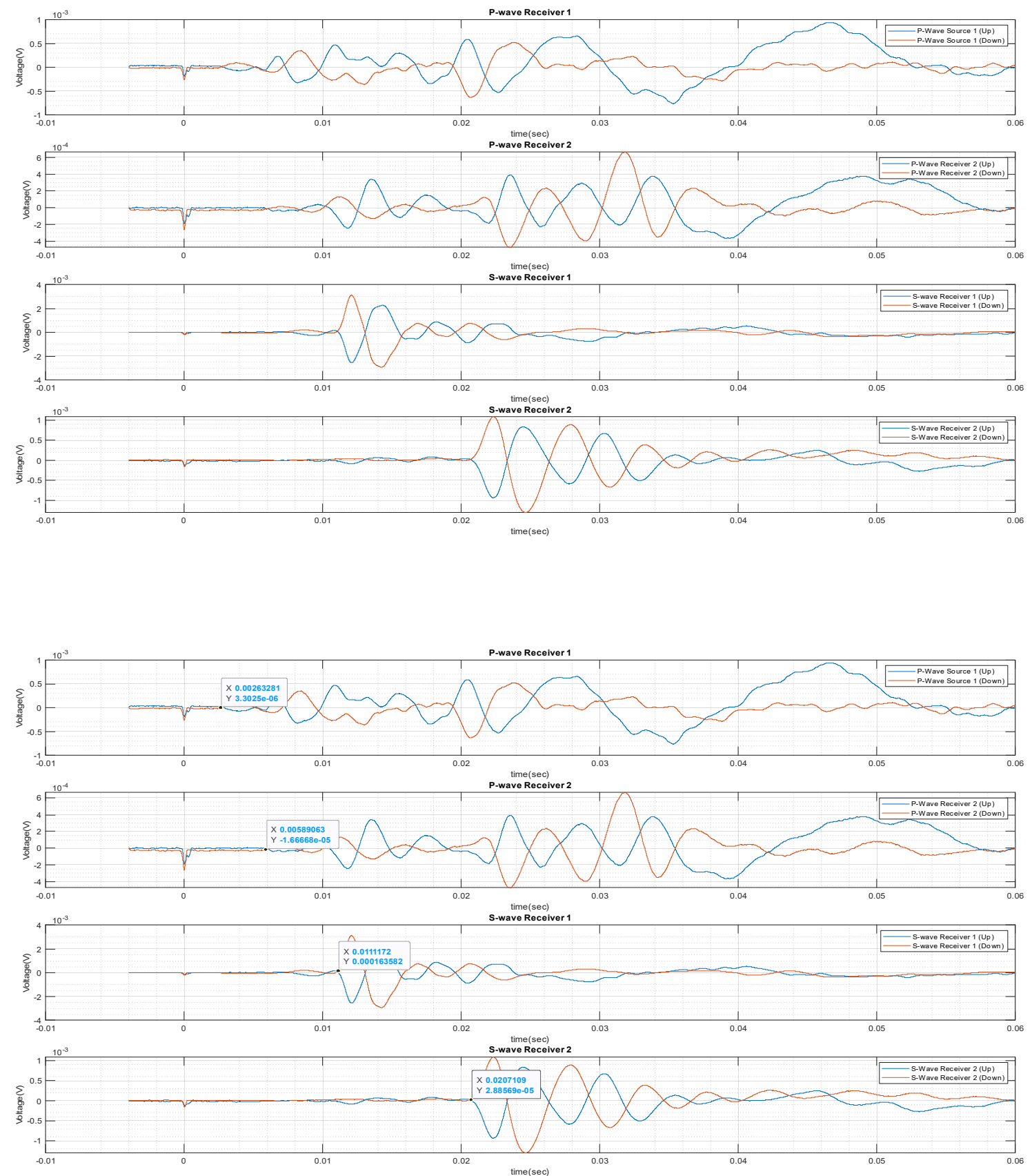

Data Set 46 (11.21.2020)

Run00356f

R2_2 

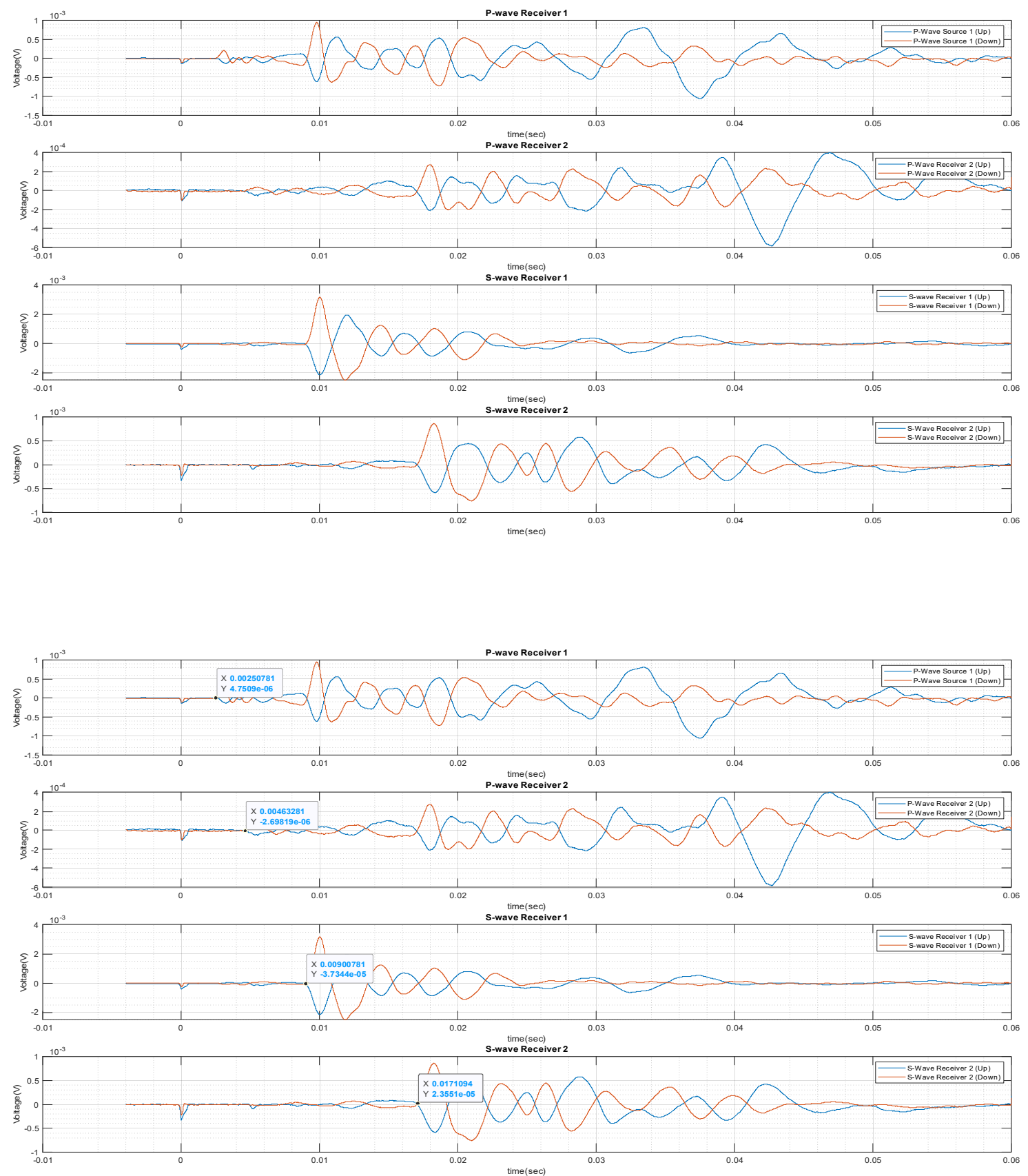

Data Set 46 (11.21.2020)

Run00357f

R3_1 

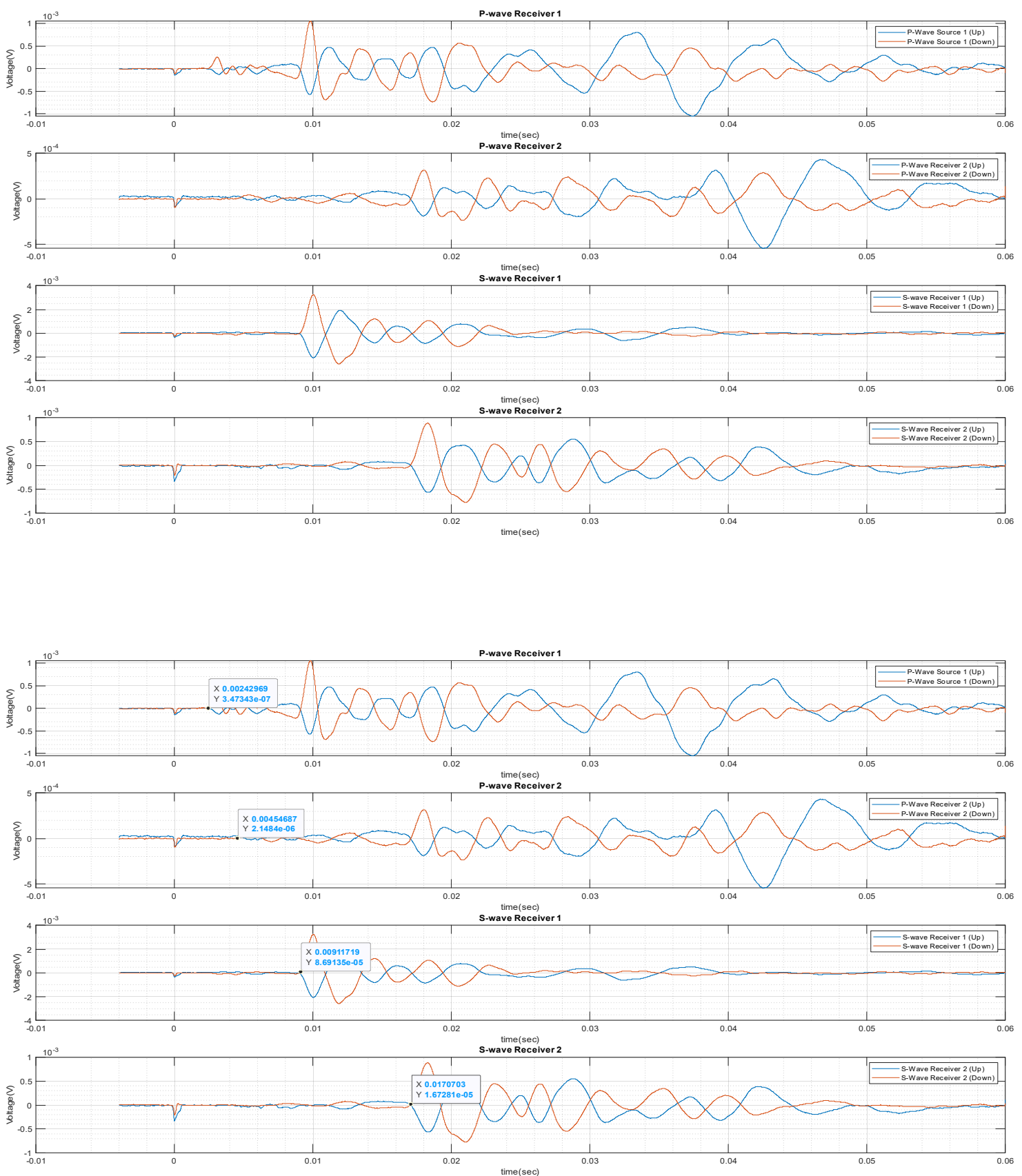

Data Set 46 (11.21.2020)

Run00358f

R3_2 

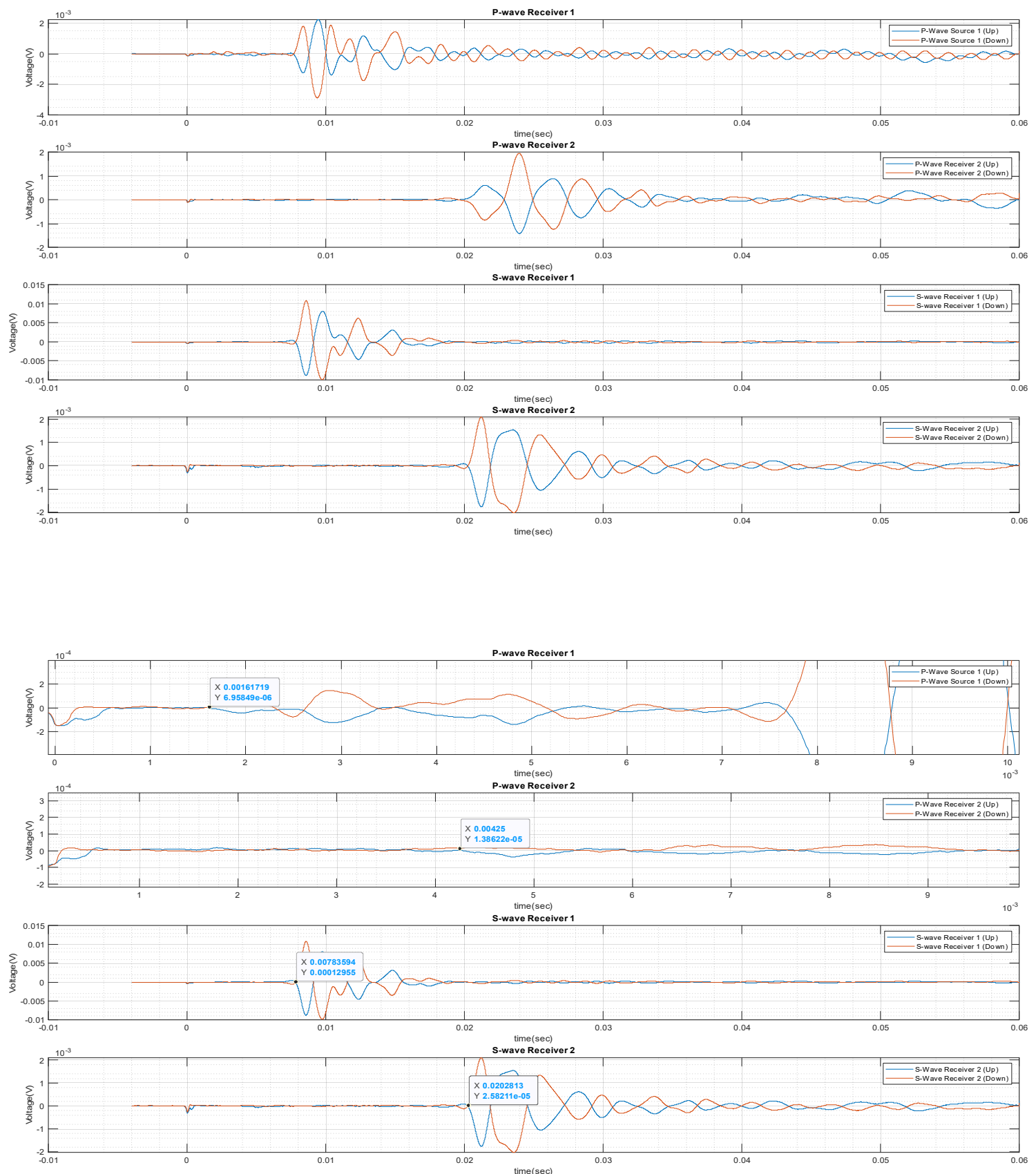

Data Set $46(11.21 .2020)$

Run00359f

R4_1 

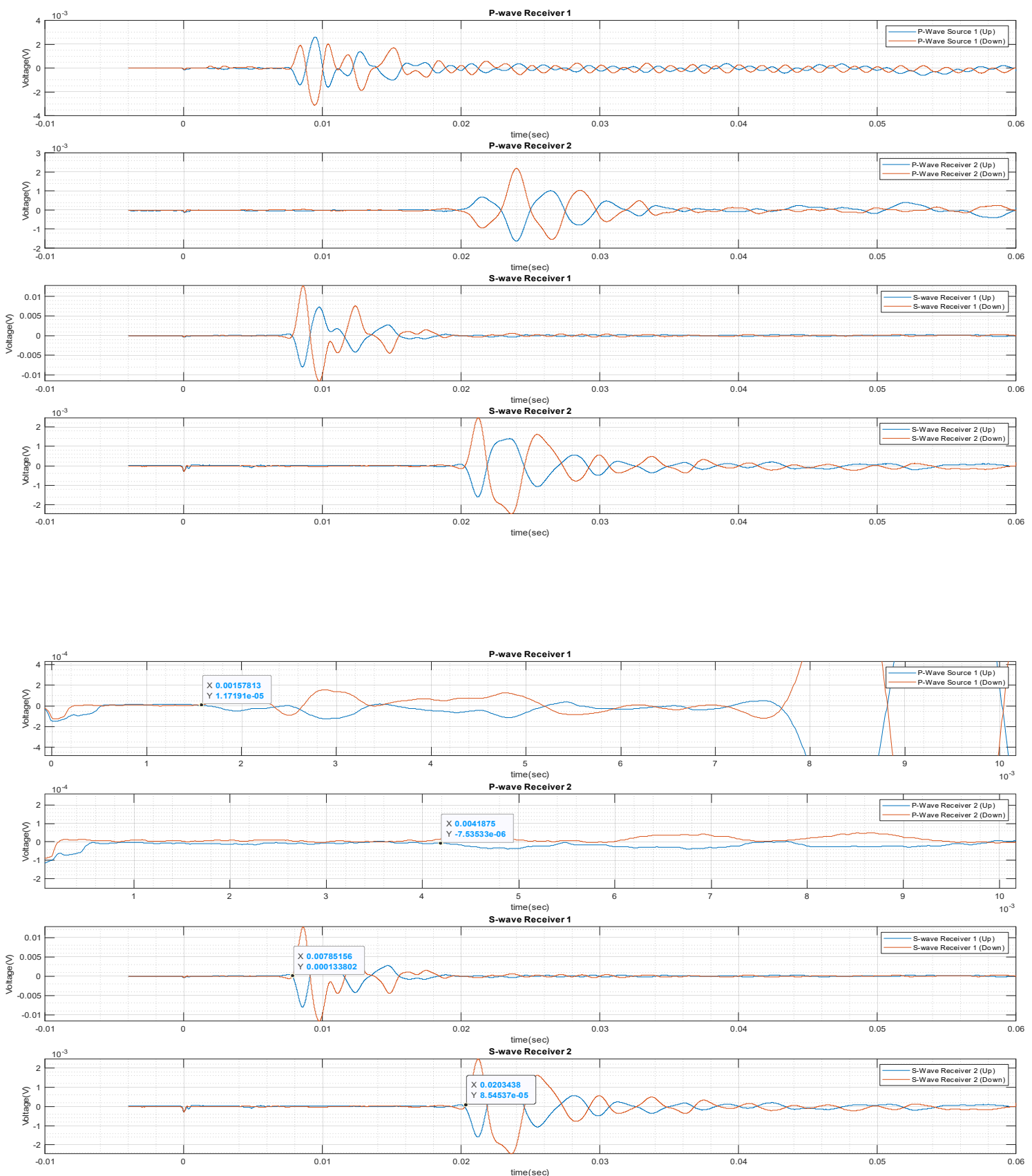

Data Set 46 (11.21.2020)

Run00360f

R4_2 

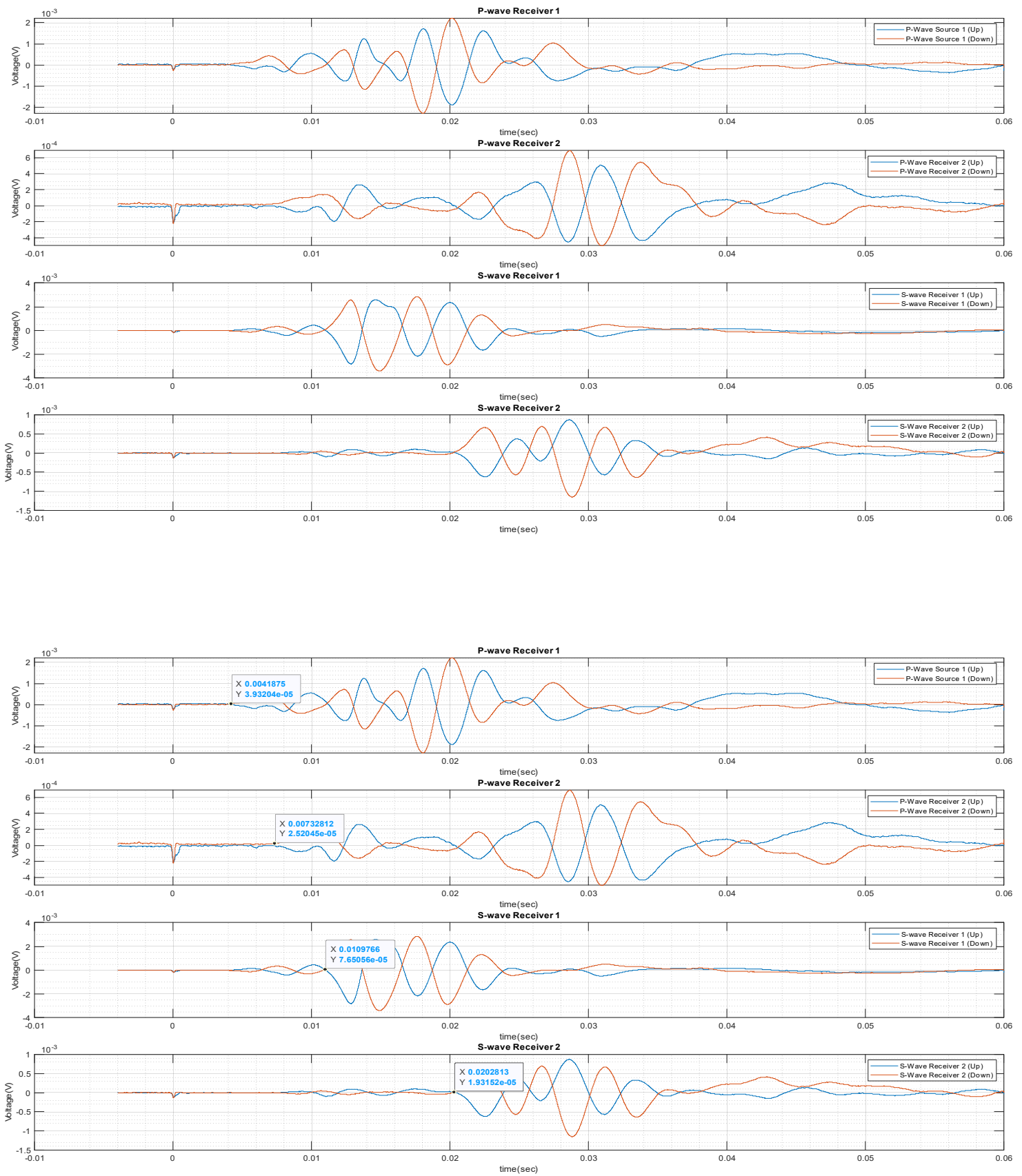

Data Set 47 (12.12.2020)

Run00361f

R1_1 

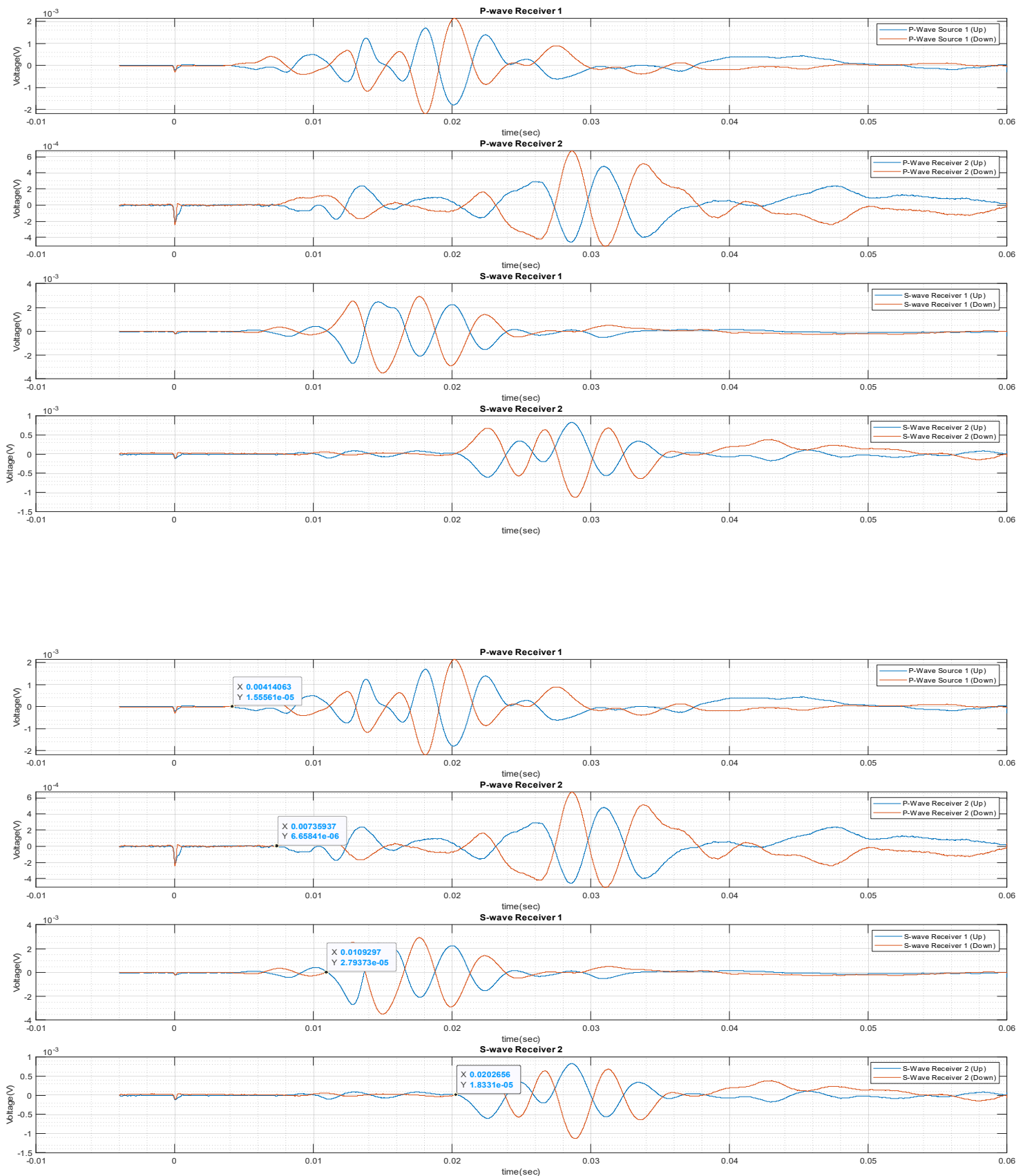

Data Set 47 (12.12.2020)

Run00362f

R1_2 

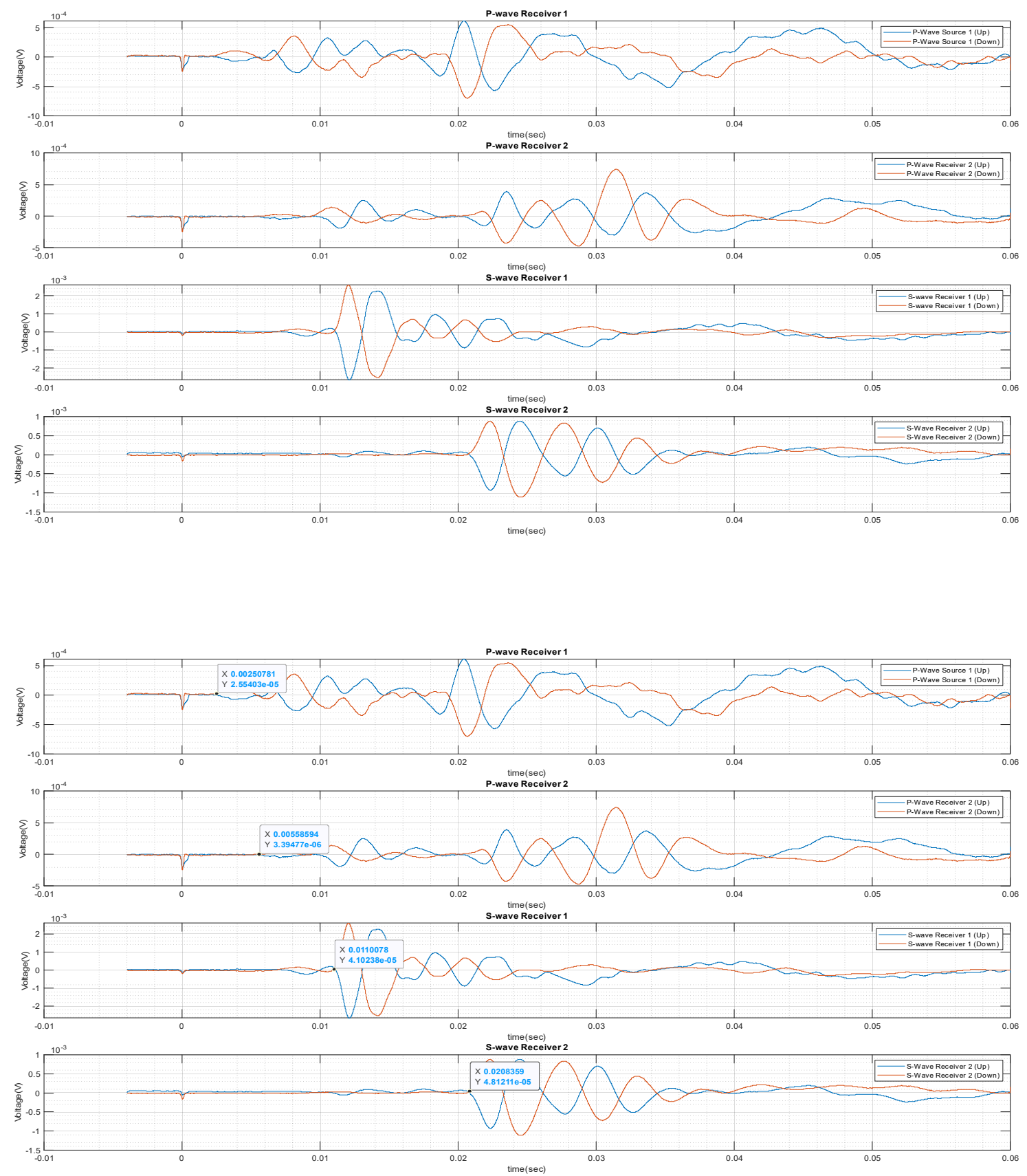

Data Set 47 (12.12.2020)

Run00363f

R2_1 

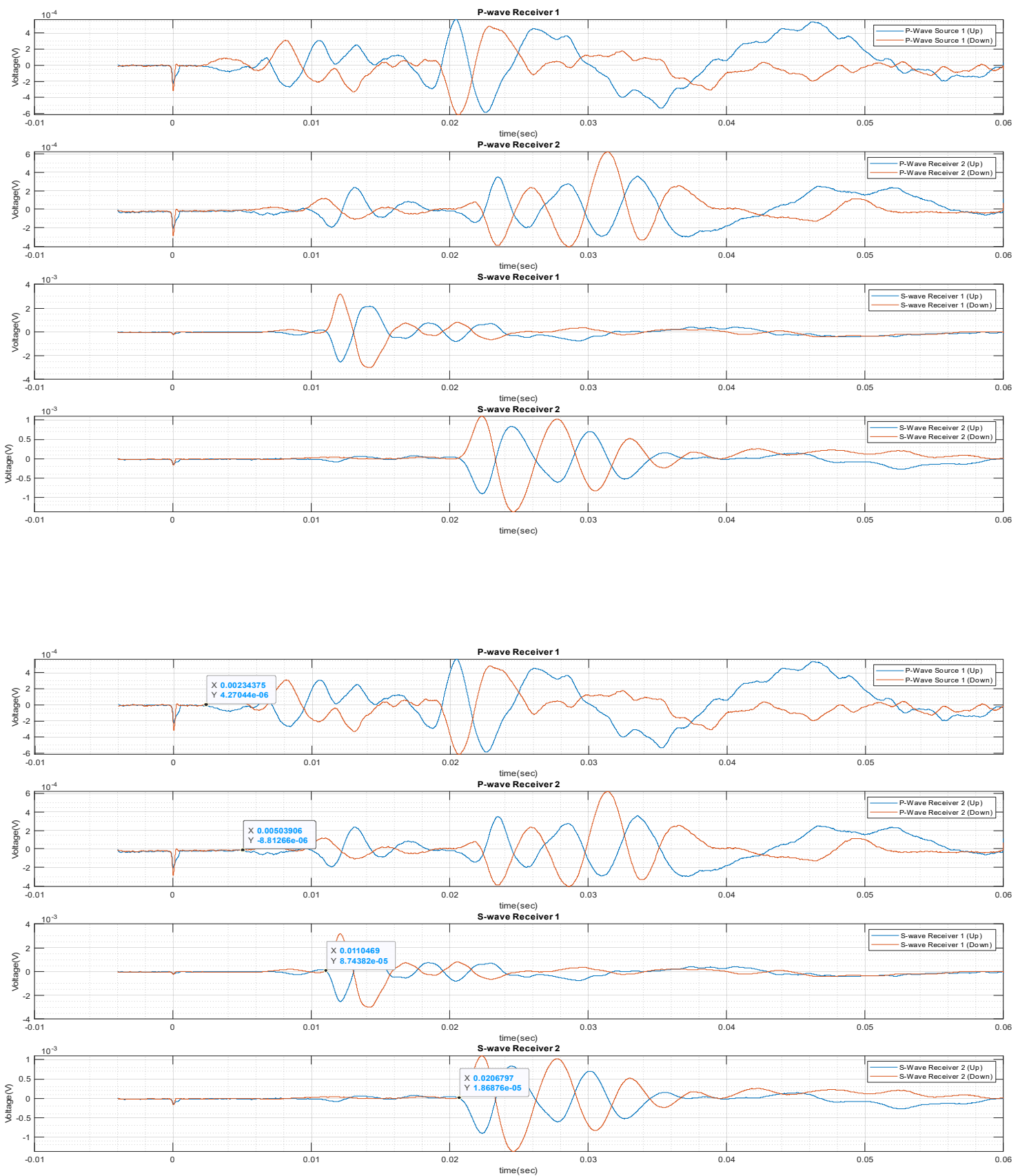

Data Set 47 (12.12.2020)

Run00364f

R2_2 

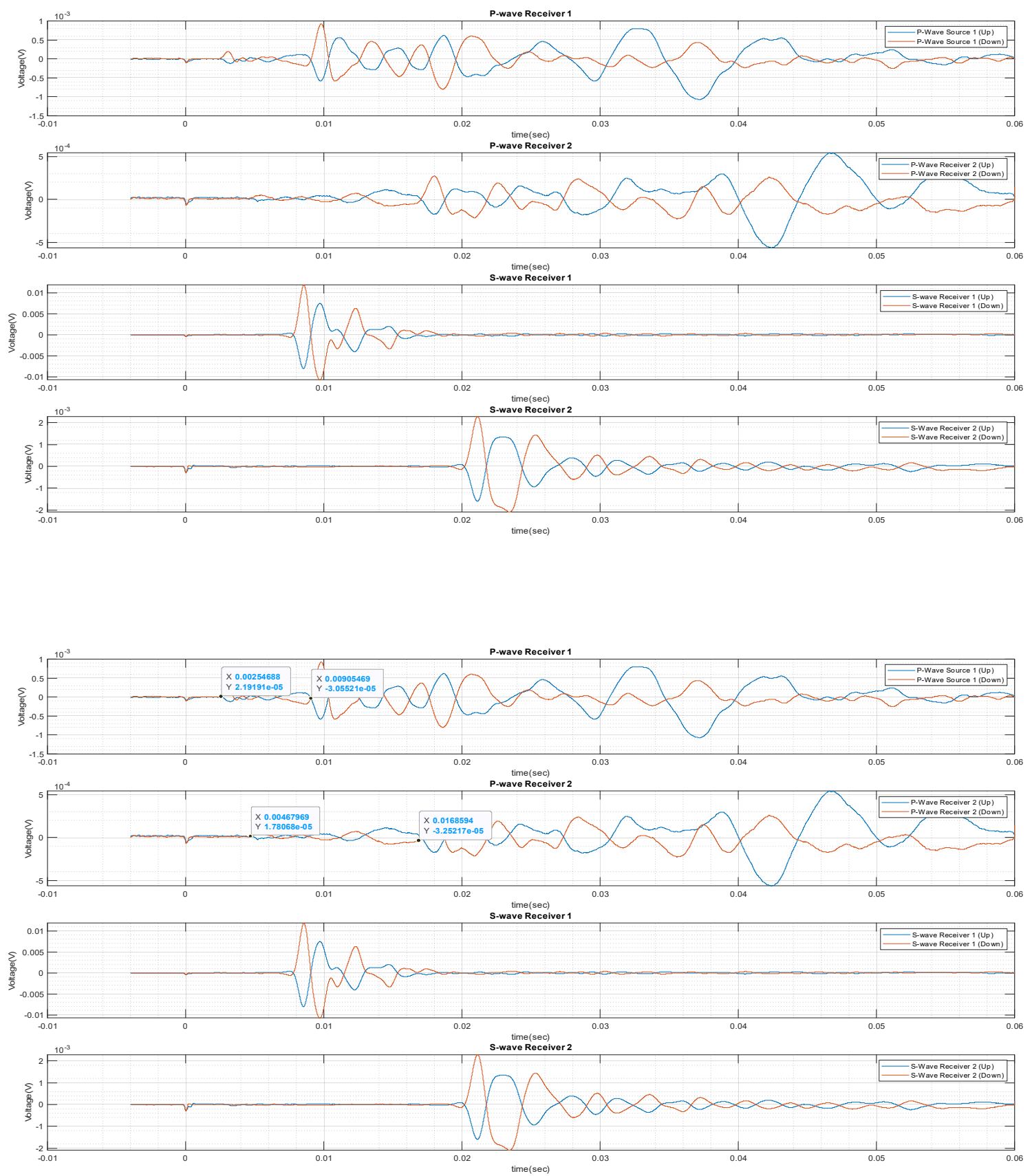

Data Set 47 (12.12.2020)

Run00365f

R3_1 

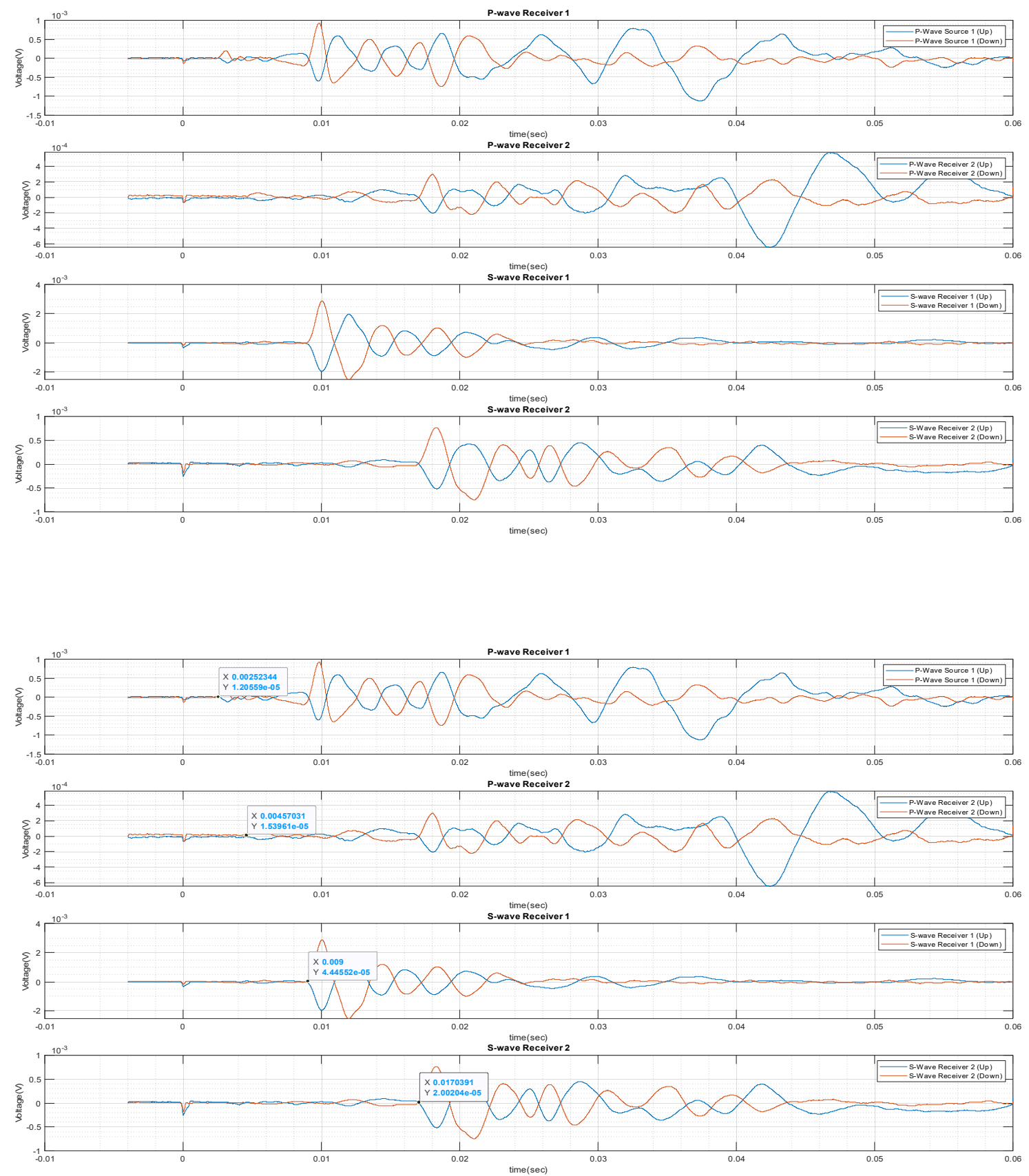

Data Set 47 (12.12.2020)

Run00366f

R3_2 

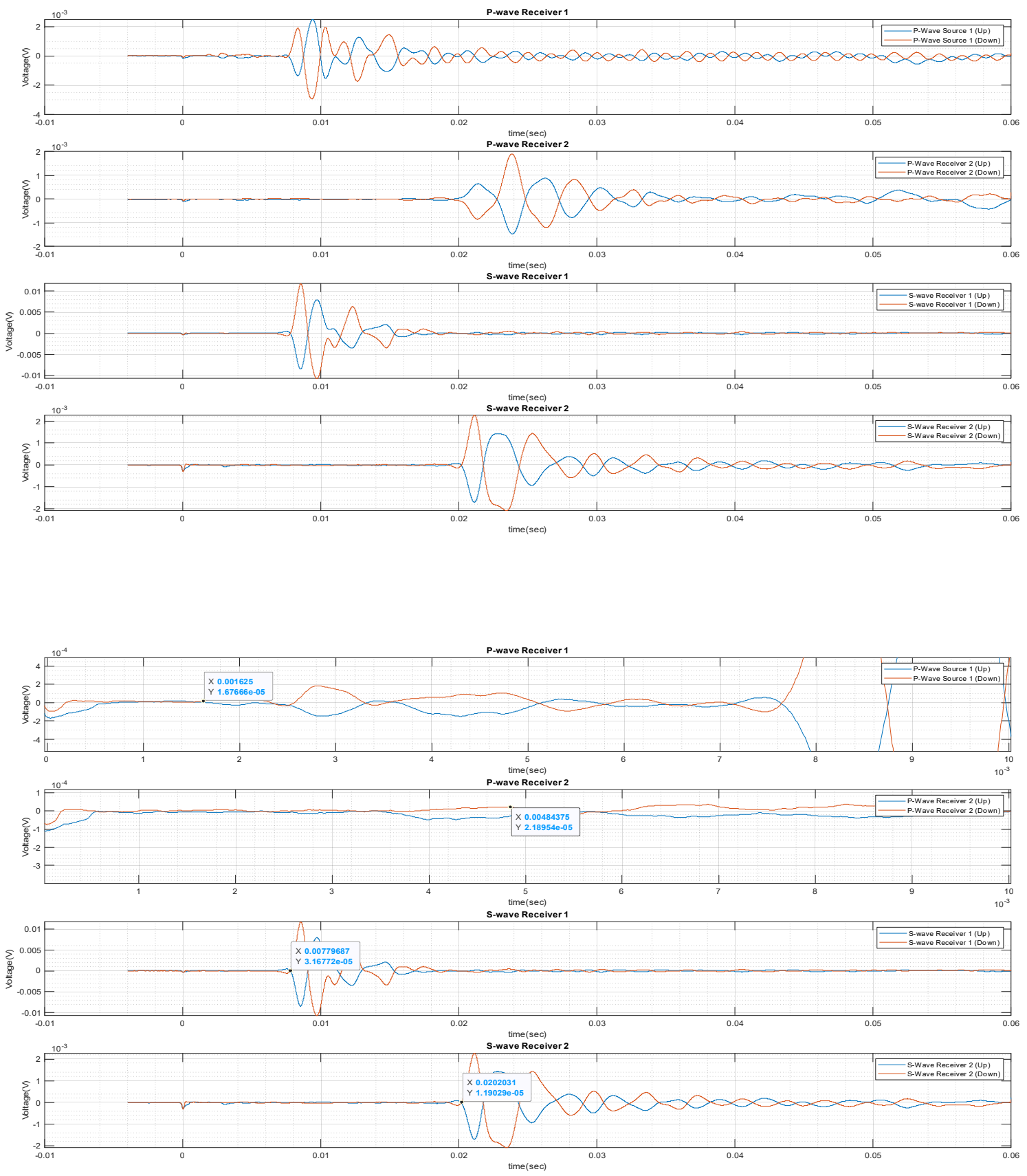

Data Set 47 (12.12.2020)

Run00367f

R4_1 

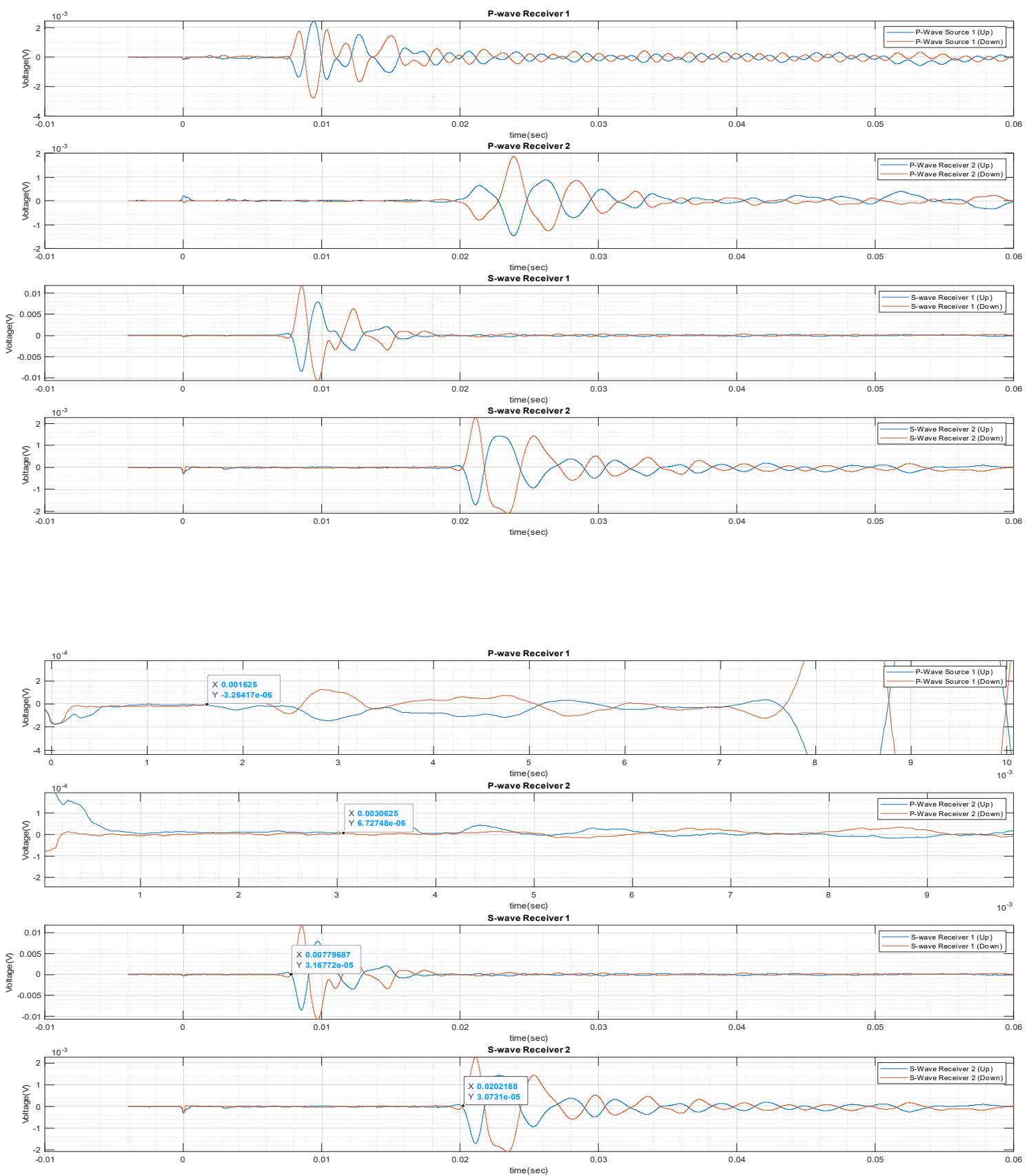

Data Set 47 (12.12.2020)

Run00368f

R4_2 

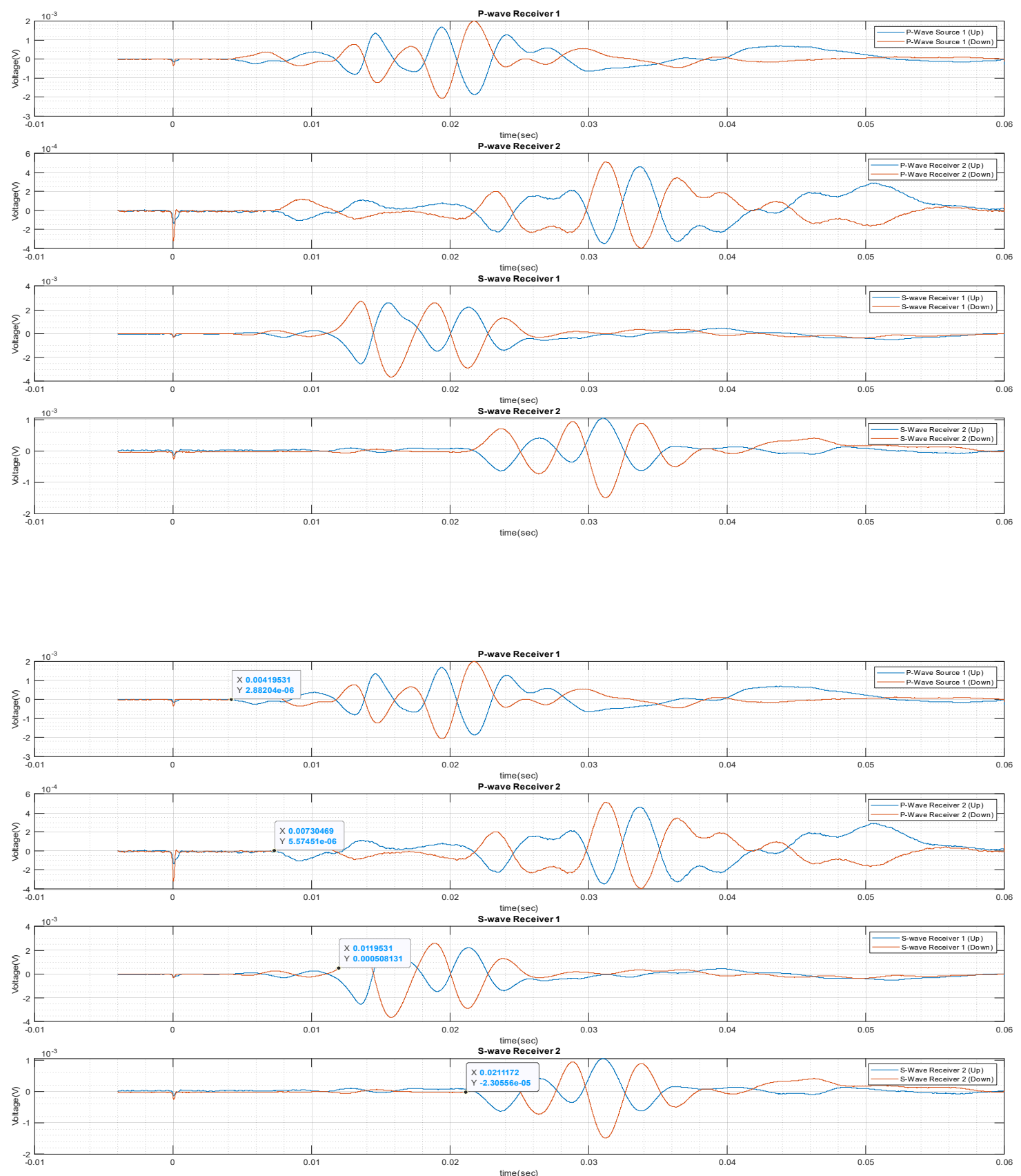

Data Set 48 (3.16.2021)

Run00369f

R1_1 

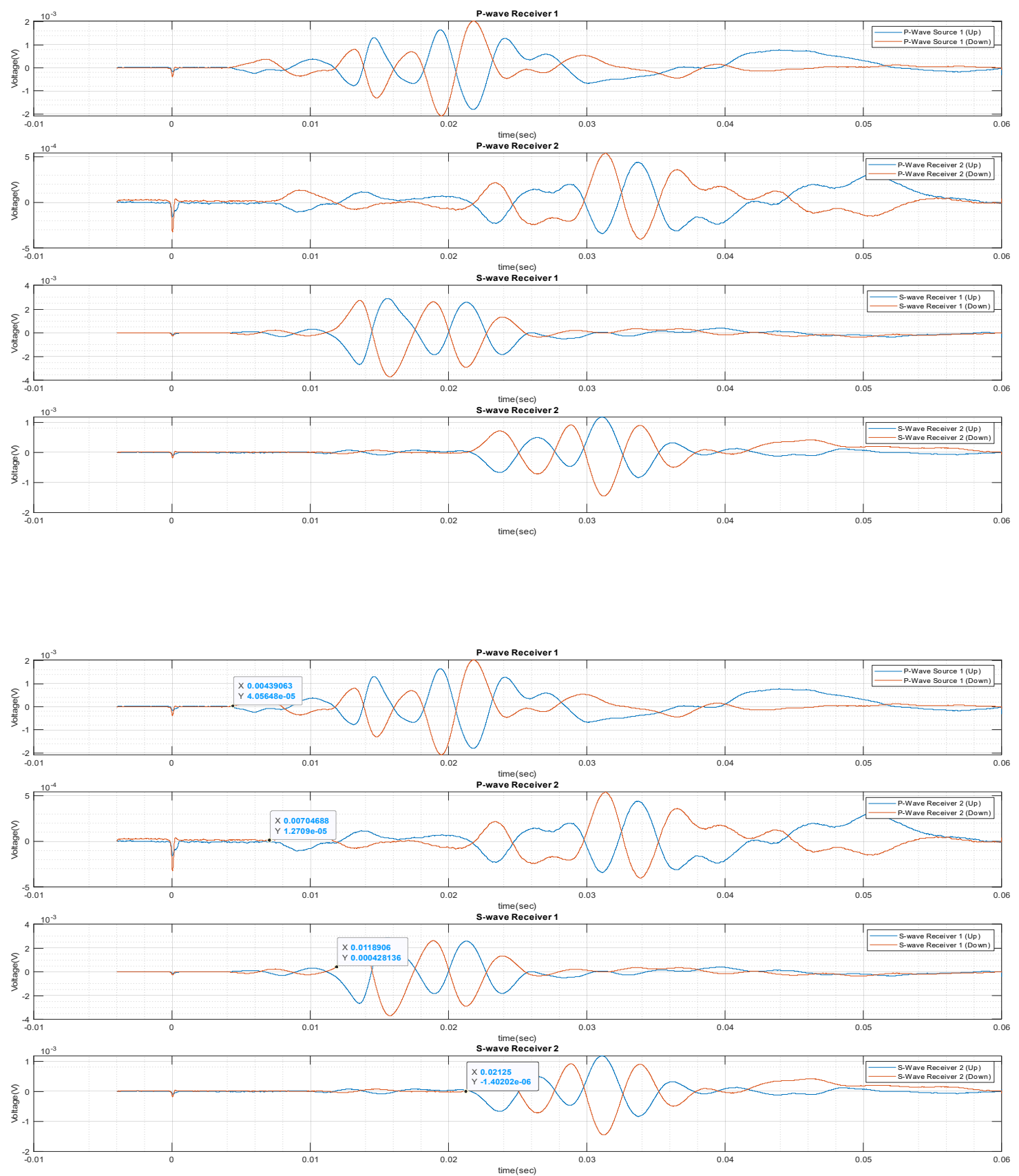

Data Set 48 (3.16.2021)

Run00370f

R1_2 

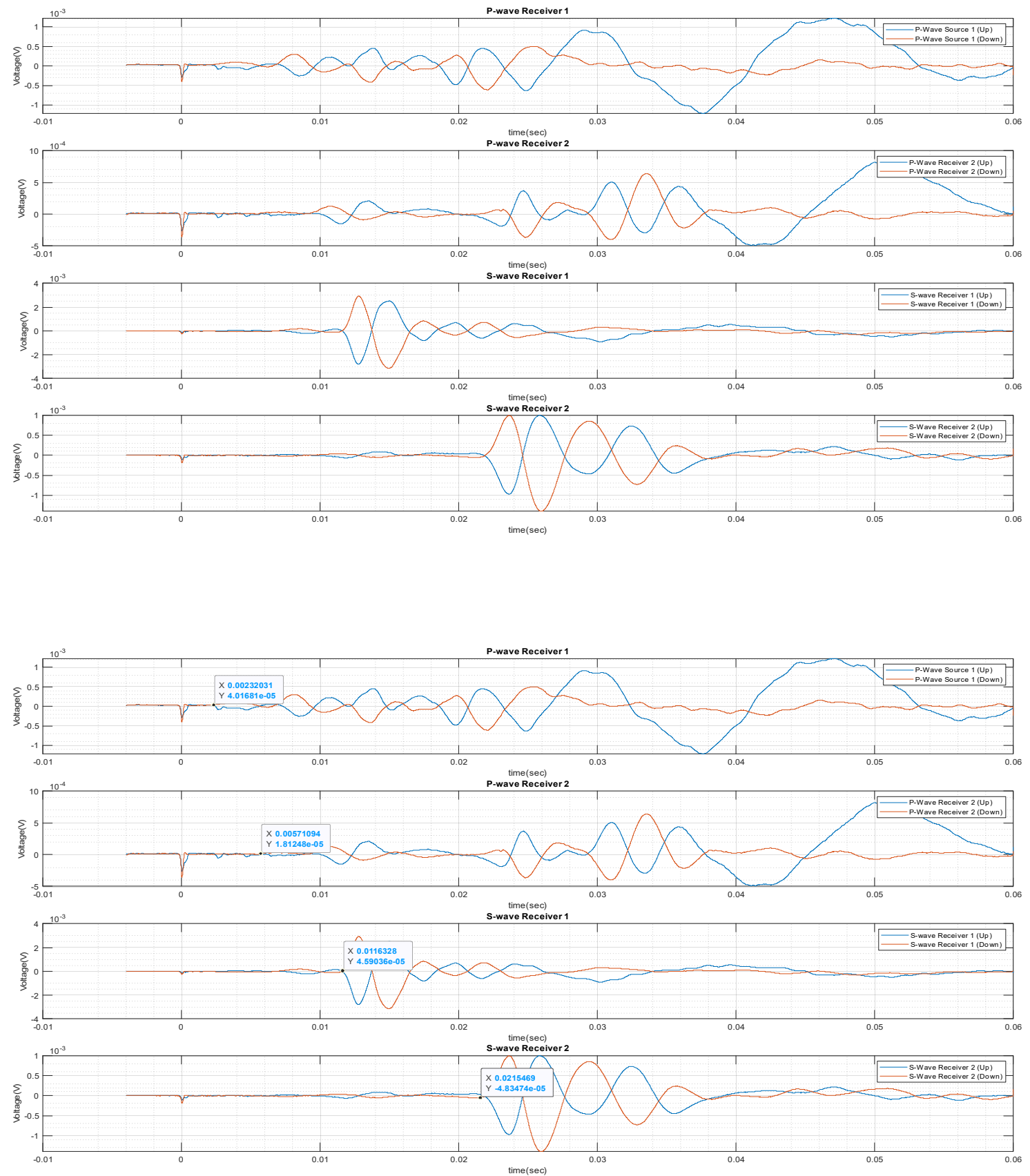

Data Set 48 (3.16.2021)

Run00371f

R2_1 

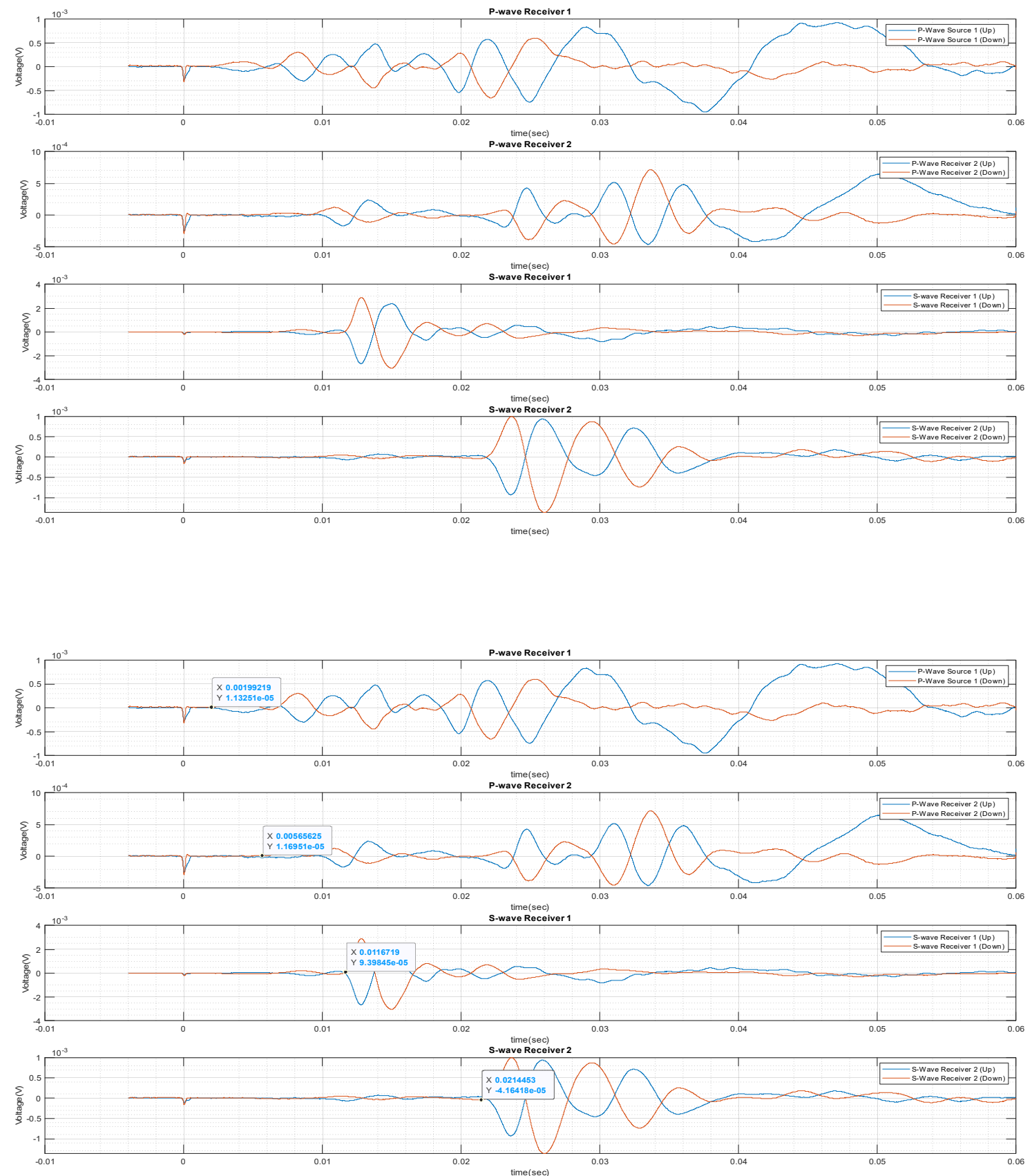

Data Set 48 (3.16.2021)

Run00372f

R2_2 

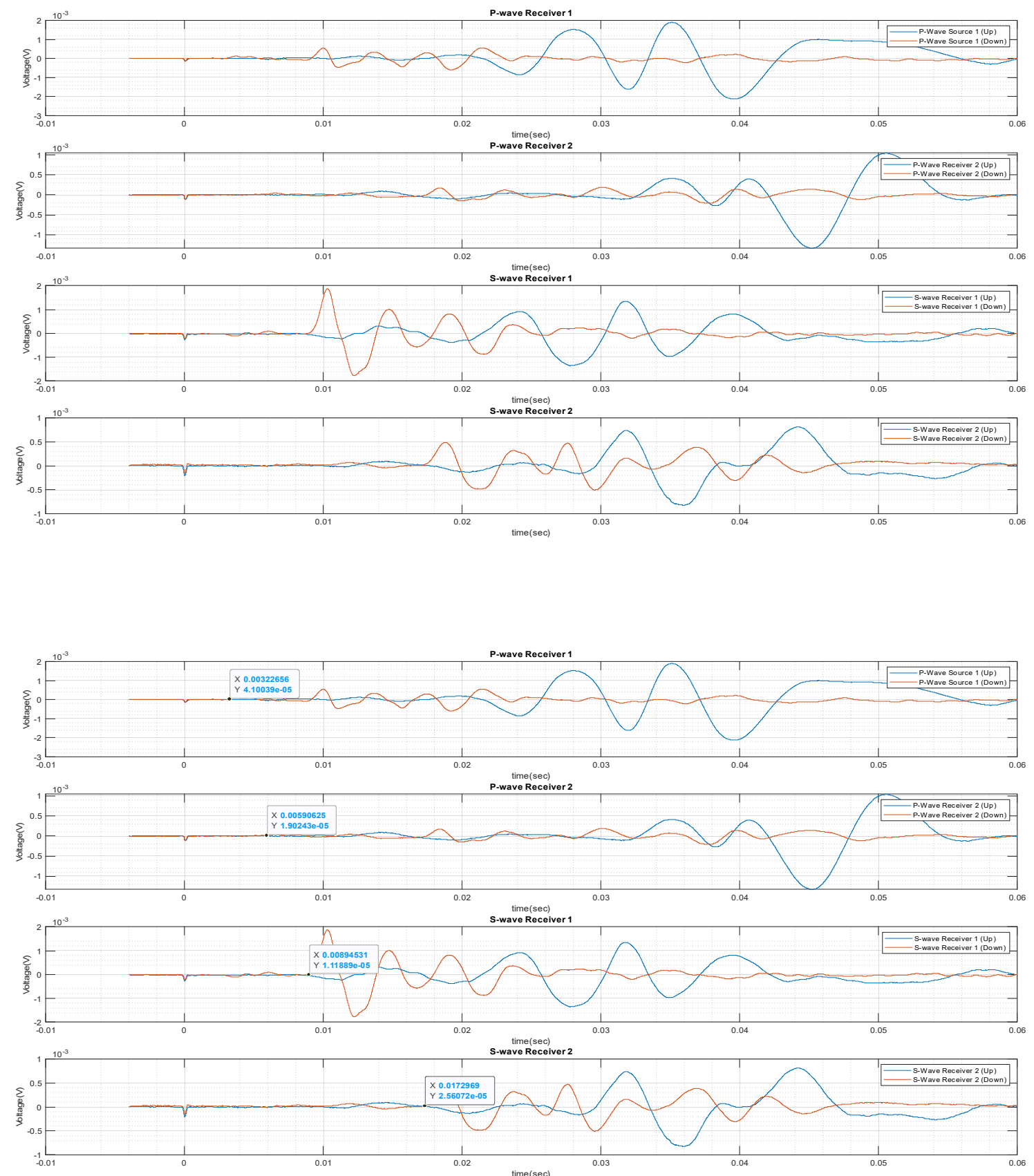

Data Set 48 (3.16.2021)

Run00373f

R3_1 

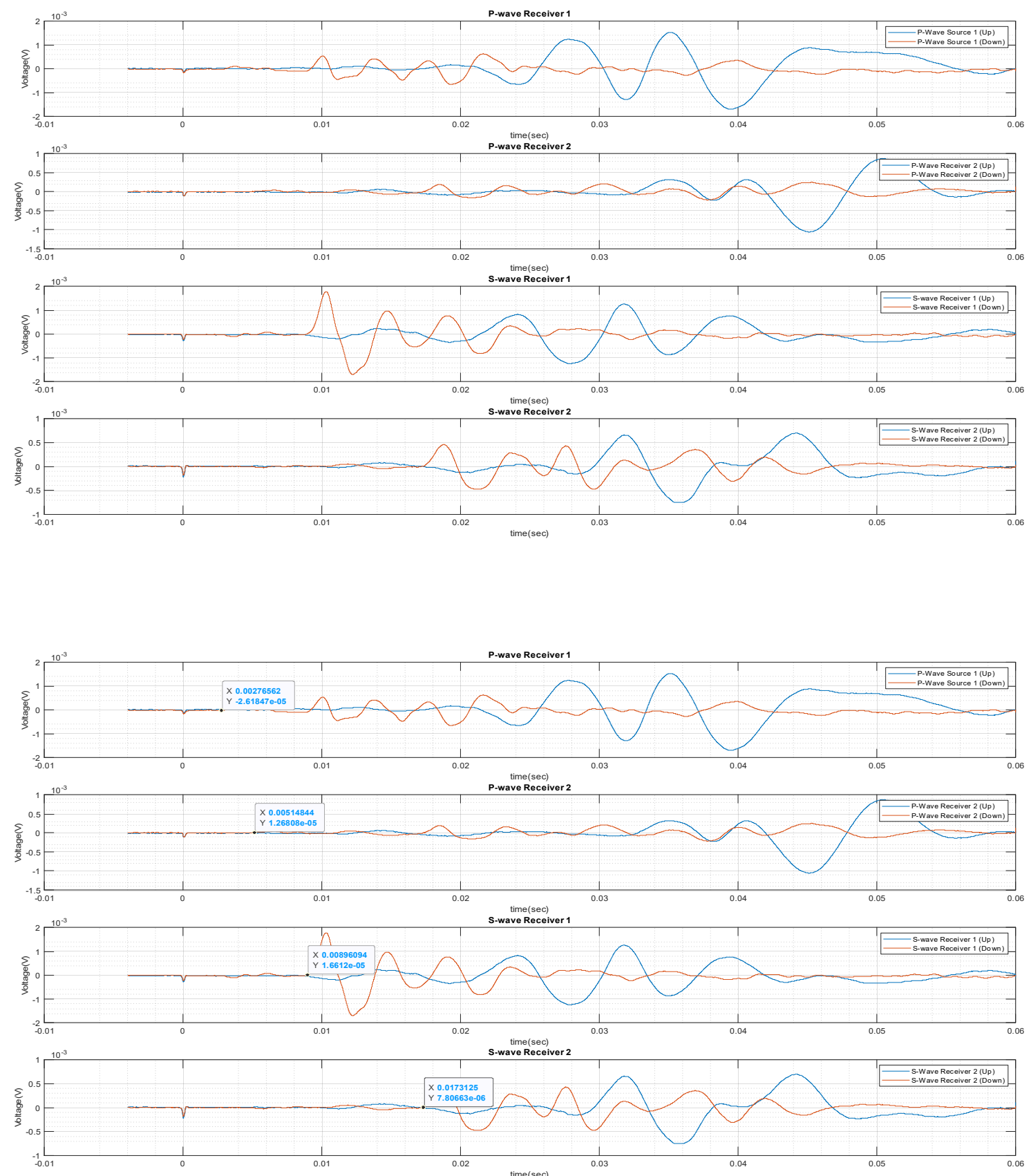

Data Set 48 (3.16.2021)

Run00374f

R3_2 

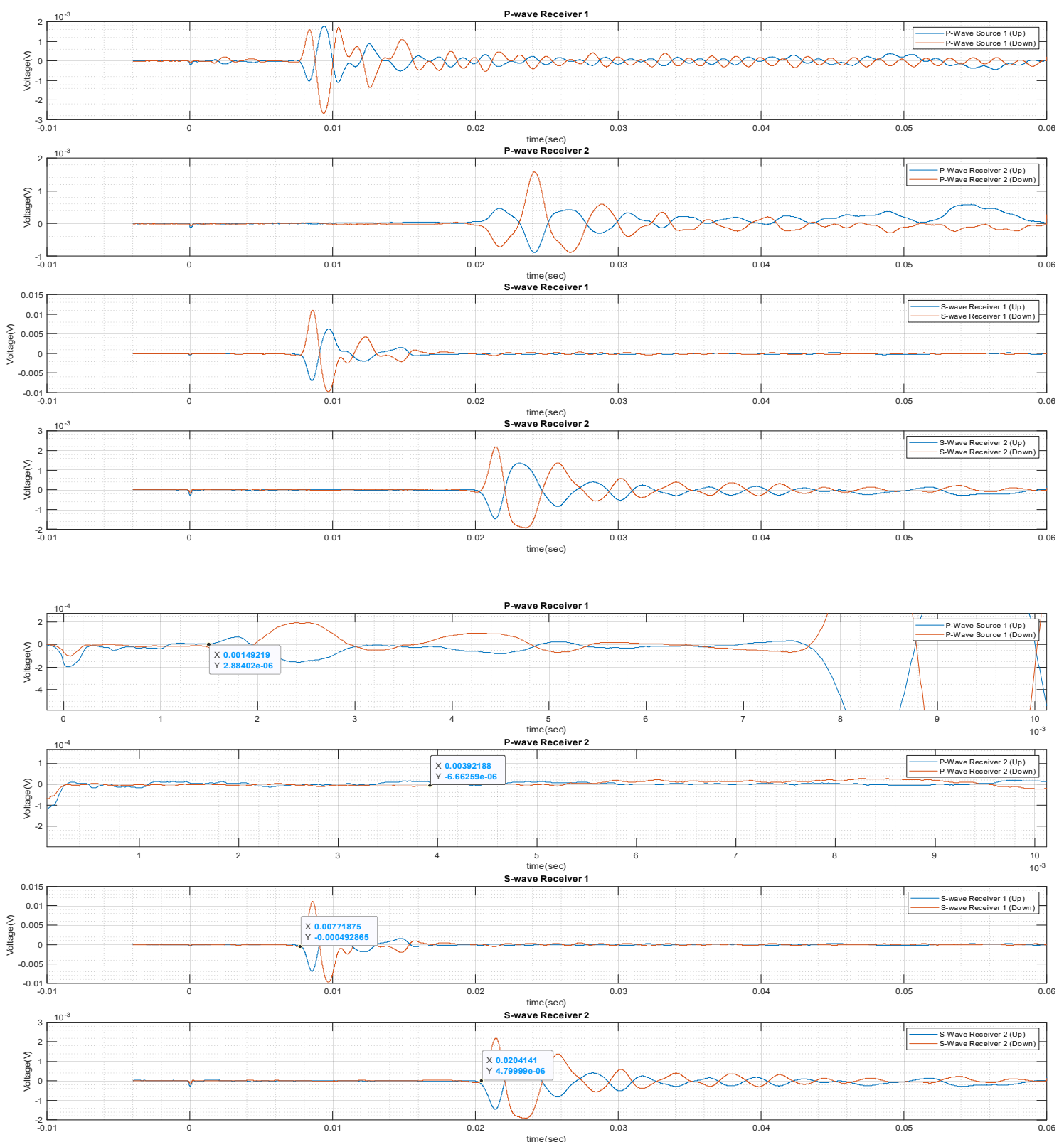

Data Set 48 (3.16.2021)

Run00375f

R4_1 

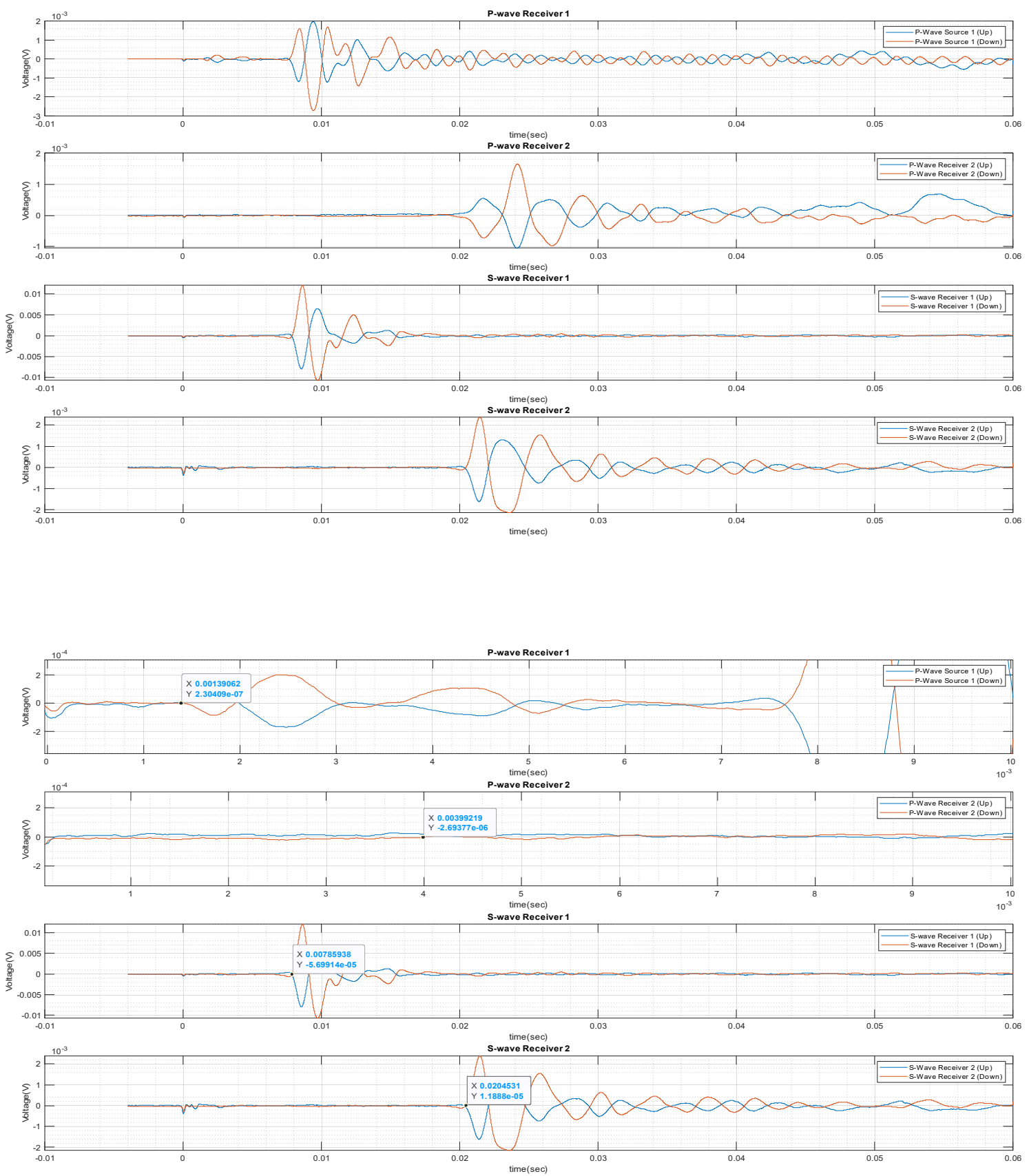

Data Set 48 (3.16.2021)

Run00376f

R4 2 